\title{
ARTIFICIAL NEURAL NETWORKS: A NEW METHOD FOR MINERAL-PROSPECTIVITY MAPPING
}

\author{
Warick Brown
}

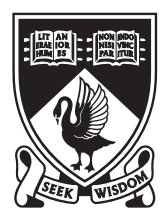

A thesis presented for the degree of

Doctor of Philosophy at the

University of Western Australia

Centre for Global Metallogeny

Department of Geology and Geophysics

Supervisors

Prof. David I. Groves

Prof. Tamás D. Gedeon

Dr Carl Knox-Robinson

Submitted: December 2002 
(c) Copyright 2002

by

Warick Brown 


\begin{abstract}
The results of a pilot and detailed case study show that neural networks are a powerful technique for integrating regional exploration data from GIS databases into mineral prospectivity maps. Neural networks are trained to indicate the presence or absence of deposits, using feature vectors formed by combining the values from co-registered grid cells in raster-based GIS thematic layers containing geological, geophysical, and geochemical data and binary target values.
\end{abstract}

In a pilot study, multilayer perceptron (MLP) neural networks, trained with a gradient descent, back-propagation algorithm, are used to estimate the favourability for disseminated intrusion-related, granite-hosted and related alluvial gold deposits in the Tenterfield 1:100,000 sheet area, New South Wales, Australia. The neural network method produces a geologically-plausible mineral prospectivity map similar, but superior, to fuzzy logic and weights-of-evidence maps of the same district. The results indicate that the use of neural networks for the integration of large multisource data sets used in regional mineral exploration, and for prediction of mineral prospectivity, offers several advantages over existing methods. These include the ability of neural networks to; 1) respond to critical combinations of parameters rather than increase the estimated prospectivity in response to each favourable parameter, 2) combine data sets without the loss of information inherent in existing methods, and 3) produce results that are relatively unaffected by redundant data, spurious data and data containing multiple populations. Statistical measures of map quality indicate that the neural network method performs as well as, or better than, existing methods, while using approximately a third less data than the weights of evidence method.

Factors affecting the performance of a neural network approach are investigated in detail in a case study, based on a large exploration database provided by Goldfields Exploration Pty Ltd. Neural networks are used to predict the potential for orogenic lode-gold deposits in the Archaean Kalgoorlie Terrane, Western Australia.

Three new methods are used to compare the quality of prospectivity maps produced using neural network, fuzzy systems and weights-of-evidence methods; 1) Spearman's and Kendall's rank correlation coefficients which, show the degree to which the conditional probability of occurrence of known deposits, increases with increasing prospectivity map class (for a nine-class prospectivity map), 2) $P\left(D / C_{k}\right)$, the posterior conditional probability of a deposit $D$, given that a cell occurs in an area corresponding to a particular map class $C_{k}$, and 3) $P\left(D / C_{k}\right) / P(D)$, the ratio of posterior conditional probability to the prior probability of a deposit. The area under receiver-operating characteristic (ROC) curves 
is also used as a measure of map quality.

A new statistical method, the cumulative bivariate J-function, and two methods developed in this study (i.e., interval-based bivariate J-function, and a plot of $(O-E) / E$, where $O$ is the observed and $E$ is the expected number of deposits in a buffer) are effective in identifying positive spatial associations between known deposit points and spatial features represented by line segments (e.g., faults and lithological contacts). These methods are also suitable for analysing spatial associations between deposit points and regions (e.g., magnetic anomalies) and point data (e.g., intersection of faults). However, the cumulative bivariate J-function is not well suited to measuring spatial associations for parameters that do not involve distances (e.g., density of faults or strike orientation of regional faults) or where positive or negative associations occur within discrete ranges of parameter values. A procedure for calculating the bivariate J-curve, in which the J-function is estimated for successive distance intervals rather than for cumulative distances, overcomes this problem. These methods, together with contrast of weights, chi-squared, and the Kolmogorov-Smirnov cumulative distribution function, are also used to estimate parameter values corresponding to the most favourable (prospective) regions for gold deposits.

Since the Kalgoorlie database comprises approximately 60 primary and derived GIS layers, a systematic method for selecting the most useful GIS layers as inputs to the neural networks is required. Ranking and selection criteria are compared on the basis of the generalization ability of the trained networks and statistical measures of the quality of the resultant prospectivity maps. The best criterion is $D \times(D / A)$, where $D$ is the percentage of known deposits in the favourable region of a temporary binary layer and $A$ is the percentage of the total map area occurring within the favourable region. Other methods are to select layers with the highest value of; 1) $D / A$ from each evidence category, 2) $D / A$ which also satisfies the criterion $D \geq \sim 20 \%$, and 3$) D \times(D / A)$ provided the criteria $D \geq \sim 20 \%$ and $D / A \geq \sim 3$ are satisfied.

The addition of random noise to augment the number of deposit patterns available for training (from approximately 50 to 1000) significantly improves the classification performance of trained networks and the quality of the resultant prospectivity maps. For example, using $\pm 40 \%$ uniform random noise, the classification performance for the independent test-set increases from $67.9 \%$ and $68.0 \%$ to $72.8 \%$ and $77.1 \%$ for overall and deposit patterns, respectively. Indices for the quality of the resultant prospectivity map, $D / A$ and $D \times(D / A)$ (where $D$ is the percentage of deposits and $A$ is the percentage of the total area for the highest prospectivity map-class), and area under an ROC curve, also 
increase from $8.2,105$, and 0.79 to $17.9,226$, and 0.87 , respectively. Increasing the size of the training-stop data set results in a further small increase in classification performance and map quality statistics. The method described here is not restricted to MLP networks, but could be applied to increase the number of training patterns for other types of neural networks or in other applications in which training data are rare, difficult or expensive to obtain.

Using fuzzy membership values as neural-network inputs is an effective method of combining subjective geological knowledge with empirical data in a neural network approach to mineral prospectivity mapping. Both subjective fuzzy-membership inputs and objective fuzzy-membership inputs, which are based on the statistical relationships between known gold deposits and the parameter in the GIS thematic layer, are used. Fuzzy-membership inputs represent a useful alternative to the 1-of-n coding commonly used for categorical inputs, particularly if there are a large number of classes.

Hybrid fuzzy-neural networks produce mineral prospectivity maps that clearly reflect the geology and structure in the input GIS maps. However, at the same time, they appear to smooth some of the noise and artifacts, which are apparent in neural-network maps, without the loss of information that is apparent in maps produced using the fuzzy systems. Thus, the hybrid fuzzy-neural systems have several advantages compared with either the neural-network or fuzzy systems, including; 1) ability to incorporate prior knowledge, 2) good generalization, reflected in best test-set classification performance, 3) very high values for map quality statistics, and 4) mineral prospectivity maps with low noise, no artifacts and a high level of geological detail. Fuzzy-membership inputs are applicable to a large range of subjective data (e.g., favourability of tectonic environment, host stratigraphy, or reactivation along major faults) currently used in regional exploration programs, but which would normally not be included as inputs in an empirical neural-network approach. 


\section{Contents}

1 Introduction $\quad 1$

1.1 Problem Statement . . . . . . . . . . . . . . . . . . . . . . 1

1.2 Generation and Statement of Hypothesis . . . . . . . . . . . . . . 3

1.3 Aim of the Research . . . . . . . . . . . . . . . . . . 4

1.3 .1 Primary Aims . . . . . . . . . . . . . . . . . 4

1.3 .2 Secondary Aims . . . . . . . . . . . . . . . 5

1.3 .3 Limitations . . . . . . . . . . . . . . . . . . 6

1.4 Research Approach . . . . . . . . . . . . . . . . . . . . 7

1.5 Organization of Thesis . . . . . . . . . . . . . . 8

1.6 Publications Based on this Study . . . . . . . . . . . . . . . . . . . 10

2 Review of the Literature $\quad 12$

2.1 Introduction . . . . . . . . . . . . . . . . . . . . . 12

2.2 Mineral-Prospectivity Mapping Methods . . . . . . . . . . . . . . . . . . . . 13

2.2 .1 Overview . . . . . . . . . . . . . . . . . . 14

2.2.2 Weights of Evidence . . . . . . . . . . . . . 15

2.2 .3 Fuzzy Systems . . . . . . . . . . . . . . . . . . . . . . 21

2.2.4 Mineral Systems Approach _. . . . . . . . . . . . . . . . 24

2.3 Geographic Information Systems _ . . . . . . . . . . . . . . . 28

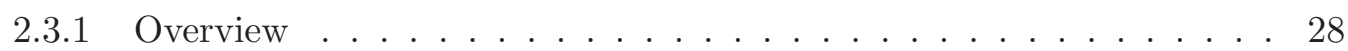

2.4 Neural Networks . . . . . . . . . . . . . . . . . . . . . . . . . . . 32

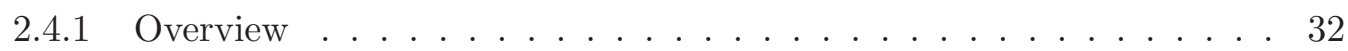

2.4.2 Applications of Neural Networks in the Geosciences . . . . . . . . 35

2.4.3 Application of Neural Networks to Spatial Analysis . . . . . . . . . . 38

2.4.4 Selection of Neural Network Architecture . . . . . . . . . . . . 45

2.4.5 Multilayer Perceptron (MLP) Neural Networks . . . . . . . . . . . . 46

2.4.6 Summary of Previous Work . . . . . . . . . . . . . . . . 54

2.5 Summary . . . . . . . . . . . . . . . . . . . 57 
3 Methods and GIS Databases $\quad 60$

3.1 Introduction . . . . . . . . . . . . . . . . . . . . . 60

3.2 Preparation of Mineral Prospectivity Maps . . . . . . . . . . . . . . . 61

3.2.1 Preparation of Derived Thematic Layers . . . . . . . . . . . . . . . 61

3.2.2 Neural Network Method . . . . . . . . . . . . . . . . . . . . . . . 64

3.2 .3 Assumptions . . . . . . . . . . . . . . . 70

3.3 Evaluating Neural Network Performance . . . . . . . . . . . . . . . . 72

3.3.1 Chi-Squared Test . . . . . . . . . . . . . . . . . 72

3.3 .2 Mean Square Error . . . . . . . . . . . . . . . . . . 73

3.3 .3 Confusion Matrix . . . . . . . . . . . . . . . . . 74

3.3.4 Receiver Operating Characteristic Curves . . . . . . . . . . . . 76

3.3.5 Harris and Pan's Measures of Performance . . . . . . . . . . . 78

3.4 Weights-of-Evidence Method . . . . . . . . . . . . . . . 80

3.4.1 Fuzzy Systems Method . . . . . . . . . . . . . . . . . 84

3.4.2 Comparing Mineral Prospectivity Map Quality . . . . . . . . . . . 85

3.5 Choice of GIS Databases for Pilot and Case Studies . . . . . . . . . . . . 89

3.5.1 Intrusion-Related and Placer Gold Deposits, Tenterfield, N.S.W. . . 89

3.5.2 Archaean Orogenic Gold Deposits, Kalgoorlie, W.A . . . . . . . . 90

3.6 Software . . . . . . . . . . . . . . . . . . . . . . . . . . . . . 91

3.6.1 Pre-Processing (C++ Programs) . . . . . . . . . . . . . . 91

3.6.2 Neural Network Simulation Packages . . . . . . . . . . . . . . . . . 92

3.7 Hardware . . . . . . . . . . . . . . . . . . . . . . . . 92

3.8 Summary . . . . . . . . . . . . . . . . . . . . . . . . . 92

4 Pilot Study based on Intrusion-Related, Granite-Hosted Gold Deposits, Tenterfield, New South Wales: Testing the Viability of an MLP Neural $\begin{array}{lr}\text { Network Method } & 96\end{array}$

4.1 Introduction . . . . . . . . . . . . . . . . . . 96

4.2 GIS Database . . . . . . . . . . . . . . . . . . . . . . 98

4.3 Geology . . . . . . . . . . . . . . . . . . . . 100

4.3 .1 Regional Geological Setting . . . . . . . . . . . . . . 100

4.3 .2 Mineralization . . . . . . . . . . . . . . . . 101

4.3 .3 Leucogranitoids . . . . . . . . . . . . . . . . . . . . . . . . . 101

4.3.4 Intrusion-Hosted Gold Mineralization . . . . . . . . . . . . . 103

4.3.5 Exploration Criteria . . . . . . . . . . . . . . . 105

4.4 Experimental Procedure . . . . . . . . . . . . . . . . . . . . 107

4.4.1 Preparation of Neural-Network Maps . . . . . . . . . . . . 107 
4.4.2 Preparation of Weights-of-Evidence Maps . . . . . . . . . . . . . . 113

4.4.3 Preparation of Fuzzy-Systems Maps . . . . . . . . . . . . . . . 118

4.4.4 Comparing Mineral Prospectivity Map Quality . . . . . . . . . . . . 124

4.5 Results . . . . . . . . . . . . . . . . . . . . . . . . . . . 124

4.6 Discussion . . . . . . . . . . . . . . . . . . . . . . . . . . . 128

4.6.1 Validation of the Neural Network Results . . . . . . . . . . . 128

4.6 .2 Interpretation of Output Values . . . . . . . . . . . . . . 128

4.6.3 Response to Critical Combinations of Favourable Parameters . . . . 129

4.6.4 Response to Redundant Input Parameters . . . . . . . . . . . . . . 131

4.6.5 Loss of Information through Re-Classification of Input Data . . . . . 131

4.6.6 Response to Irrelevant or Misleading Input Parameters . . . . . . . . 132

4.6.7 Comparison of Map Quality . . . . . . . . . . . . . . . . 133

4.7 Conclusion . . . . . . . . . . . . . . . . . . . . . 135

5 Compilation of Case Study GIS Database: Orogenic Gold Deposits, Kal$\begin{array}{lr}\text { goorlie Area, Western Australia } & 137\end{array}$

5.1 Introduction . . . . . . . . . . . . . . . . . . . . . . . . . . 137

5.2 Geology of Archaean Orogenic Gold Deposits . . . . . . . . . . . . . . . . 140

5.2 .1 Deposit Characteristics . . . . . . . . . . . . . . . 140

5.2 .2 Tectonic Setting . . . . . . . . . . . . . . . . . . 141

5.2.3 Timing of Gold Deposits within Orogens . . . . . . . . . . . . . . 142

5.2 .4 Structural Controls . . . . . . . . . . . . . . . . . . . . 142

5.2 .5 Host Rocks . . . . . . . . . . . . . . . . . . . . . . 146

5.2.6 Mechanism of Gold Precipitation . . . . . . . . . . . . . . 147

5.2.7 Simplified Model of an Orogenic Gold System . . . . . . . . . . . . 148

5.3 Exploration Criteria . . . . . . . . . . . . . . . . . . . . . . . 148

5.4 Primary GIS Layers . . . . . . . . . . . . . . . . . . . . . . . . 149

5.4 Geology Layer . . . . . . . . . . . . . . . . . . . 149

5.4 .2 Structural Layer . . . . . . . . . . . . . . . . . . . . 154

5.4 .3 Fold Axis Layer . . . . . . . . . . . . . . . . . . . . . . . . . . 154

5.4 .4 Aeromagnetic and Gamma-Ray Layer . . . . . . . . . . . . . . 155

5.4 .5 Gravity Layer . . . . . . . . . . . . . . . . . . . . 155

5.4 .6 Deposit Layer . . . . . . . . . . . . . . . . . . . . . . 155

5.4 .7 OZCHEM Database . . . . . . . . . . . . . . . . 156

5.4 .8 Rock Strength Data Sets . . . . . . . . . . . . . . 161

5.4 .9 Metamorphic Grade . . . . . . . . . . . . . . . . . . . 163

5.4 .10 Goldfields Pty Ltd Data Sets Not Used in Study . . . . . . . . . . 171 
5.5 Derived GIS Layers . . . . . . . . . . . . . . . . . . . . . . . 171

5.5.1 Simplified Geology Layers . . . . . . . . . . . . . . . . . . . . 172

5.5 .2 Binary Deposit Layer . . . . . . . . . . . . . . . . . . 173

5.5.3 Distance to Dolerite, Syenite, \& Feldspar Porphyry Layers . . . . . . 173

5.5.4 Distance to Lithological Contacts . . . . . . . . . . . . . . . . . . . . 174

5.5.5 Distance to Faults and Shear Zones . . . . . . . . . . . . . 175

5.5 .6 Distance to Fold Axes . . . . . . . . . . . . . . . . 176

5.5.7 Distance to Intersection of Regional Faults . . . . . . . . . . . 176

5.5.8 Distance to Magnetic Anomalies . . . . . . . . . . . . . . . . 176

5.5.9 Aeromagnetic First and Second Vertical Derivatives . . . . . . . 178

5.5 .10 Strike of Faults and Contacts . . . . . . . . . . . . . . . 178

5.5.11 Rheological Contrast . . . . . . . . . . . . . . . . . . . 180

5.5.12 Chemical Contrast . . . . . . . . . . . . . . . . 182

5.5.13 Favourable Contact Type . . . . . . . . . . . . . . . . . . 183

5.5.14 Favourable Lithology . . . . . . . . . . . . . . . . . . . . 187

5.5 .15 Density of Faults . . . . . . . . . . . . . . . . . . 191

5.5.16 Density of Lithological Contacts . . . . . . . . . . . . . . 191

5.5.17 Addition of No Data Cells to Derived Layers . . . . . . . . . . . . 191

5.5.18 Fuzzy Membership Layers . . . . . . . . . . . . . . . . . . 192

5.6 Summary . . . . . . . . . . . . . . . . . . . . . . . . . . . . 192

6 Feature Selection: Evaluating the predictive ability of potential input

GIS Layers $\quad \mathbf{2 2 5}$

6.1 Introduction . . . . . . . . . . . . . . . . . . . . . . 225

6.2 Statistical Methods used to Evaluate Layers . . . . . . . . . . . . . . . . 228

6.2 .1 Contrast of Weights . . . . . . . . . . . . . . . . . 228

6.2 .2 Chi-squared Statistic . . . . . . . . . . . . . . . . . . 231

6.2.3 Kolmogorov-Smirnov Cumulative Distribution Function . . . . . . . 231

6.2.4 Bivariate J-Curve (Cumulative) . . . . . . . . . . . . . . . . . 233

6.2.5 Bivariate J-Curve (Incremental) . . . . . . . . . . . . . . . 239

6.2.6 Difference Between Observed and Expected Number of Deposits . 245

6.2 .7 Average Distance between Spatial Objects . . . . . . . . . . . . . . 245

6.3 Results . . . . . . . . . . . . . . . . . . . . . . . . 247

6.3 .1 Discussion . . . . . . . . . . . . . . . . . 254

6.3 .2 Conclusions . . . . . . . . . . . . . . . . . 257 
7 Case Study: Application of MLP Neural Networks to the Prediction of Orogenic Gold Deposits

7.1 Introduction . . . . . . . . . . . . . . . . . . . . . . . 259

7.2 Method . . . . . . . . . . . . . . . . . . . . 261

7.3 Basic Approach Using an MLP Network . . . . . . . . . . . . . . . . . . 261

7.3.1 Measures of Performance and Prospectivity Map Quality . . . . . . 264

7.3.2 Training Pattern Selection . . . . . . . . . . . . . . . . 265

7.3 .3 Network Topology . . . . . . . . . . . . . . . . . . . 266

7.3.4 Training Algorithms . . . . . . . . . . . . . . . . . . 270

7.4 Input Data . . . . . . . . . . . . . . . . . . . . . 272

7.4 .1 Choice of Input GIS Layers . . . . . . . . . . . . . . . 272

7.4 .2 Number of Input GIS Layers . . . . . . . . . . . . . . . . . . 277

7.5 Input Data Transformations . . . . . . . . . . . . . . . . . . . 279

7.5.1 Coding Distance Inputs . . . . . . . . . . . . . . . . . . 279

7.5.2 Distance Thresholds for Fault Strike Inputs . . . . . . . . . . . . . 281

7.5.3 Distance Limits for Contrast Inputs . . . . . . . . . . . . . . . . . 284

7.6 Effect of Numbers of Deposit Patterns in Training Set . . . . . . . . . . . . 285

7.7 Increasing Training Set Size Using Random Noise . . . . . . . . . . . . . 288

7.7 .1 Previous Work . . . . . . . . . . . . . . . . . 290

7.7 .2 Training Data Sets . . . . . . . . . . . . . . . . . . 291

7.7.3 Augmentation of Training Data set with Noise . . . . . . . . . . . 291

7.7.4 Network Topology for Noise-Augmented Training Sets . . . . . . . . 292

7.7.5 Optimum Amount of Noise . . . . . . . . . . . . . . . . . . 293

7.7.6 Uniform and Normally-Distributed Random Noise . . . . . . . . . . 301

7.7.7 Use of Noise-Augmented Training and Training-Stop Sets . . . . . . 301

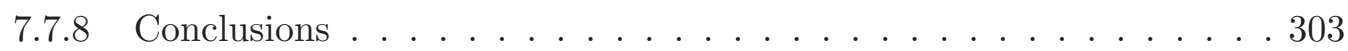

7.8 Input Data as Fuzzy-Membership Values . . . . . . . . . . . . . . . . . . 304

7.8.1 Objective Fuzzy-Membership Layers . . . . . . . . . . . . . . . 306

7.8.2 Subjective Fuzzy-Membership Layers . . . . . . . . . . . . . . . 307

7.8.3 Comparison of Methods for Coding Lithology Input . . . . . . . . . 308

7.8.4 Fuzzification Of All Neural Network Inputs . . . . . . . . . . . . . 311

7.8.5 Discussion: Use of Fuzzy Inputs in Neural Networks . . . . . . . . 316

7.8.6 Conclusions . . . . . . . . . . . . . . . . . . . . . . . 319

7.9 Prospectivity Maps . . . . . . . . . . . . . . . . . . . . . . . . . . 324

7.10 Summary . . . . . . . . . . . . . . . . . . . 326 
8 Summary and Conclusions

8.1 Contribution Demonstrated in this Thesis . . . . . . . . . . . . . . . 378

8.1.1 Developed Neural Network Mineral Prospectivity Method . . . . . . 378

8.1.2 Developed Methods for Comparing Prospectivity Maps . . . . . . . 379

8.1.3 Demonstrated Effectiveness of Neural-Network Approach . . . . . . 380

8.1.4 Compared Performance with Weights-of-Evidence \& Fuzzy Systems 381

8.1 .5 Developed Novel GIS Layers . . . . . . . . . . . . . . . . 382

8.1.6 Developed New Methods for Measuring Spatial Associations . . . . . 382

8.1.7 Quantitatively Assessed Exploration Criteria . . . . . . . . . . . . 384

8.1.8 Applied Neural Network Method to a Data Set Representative of those Used in Exploration . . . . . . . . . . . . . . . . . . . . 384

8.1.9 Investigated Factors Affecting Neural Network Method . . . . . . . . 385

8.1.10 Augmented Limited Training Data Using Random Noise . . . . . . . 387

8.1.11 Used Fuzzy Membership Values as Neural Network Inputs . . . . . . 388

8.1.12 Used Fuzzy Inputs to Create a Fuzzy-Neural Network . . . . . . . . 389

8.1.13 Compared Neural, Fuzzy-Neural Network, \& Fuzzy Systems . . . . . 389

8.2 Achievable Improvements to Existing Study . . . . . . . . . . . . . . . . . . 391

8.2.1 Combine Factors that Improved Performance . . . . . . . . . . . . 391

8.2 .2 Use a Leave-One-Out Training Strategy . . . . . . . . . . . . . . 391

8.2.3 Use of Fuzzy Inputs to Implement Narrow Distance Buffers . . . . . 392

8.2.4 Input Standardization, Target Values and Sigmoid Function . . . . . 392

8.2.5 Use of a Continuous Rather than Binary Target Variable . . . . . . 392

8.2.6 Elimination of Redundant Inputs . . . . . . . . . . . . . . . . . 393

8.2.7 Use of Self Organizing Maps for Improved Split of Training Data . . 393

8.3 Extensions to This Work . . . . . . . . . . . . . . . . . . . 394

8.3.1 Development of Rule-based Hybrid Fuzzy Systems . . . . . . . . . . 394

8.3.2 Estimates for Accuracy of Prediction . . . . . . . . . . . . . . . 394

8.3.3 Factors Affecting the Approximation of Outputs to Probabilities . . 394

8.3.4 Rule Extraction from Trained Neural Networks . . . . . . . . . . . . 395

8.3.5 Uncertainty of Mineral Prospectivity Estimates . . . . . . . . . . . . 396

8.4 Concluding Statement . . . . . . . . . . . . . . . . . 396

\section{References}

A Additional Mineral Prospectivity Mapping Methods: Review of Theory and Literature

A.1 Additional mineral prospectivity methods . . . . . . . . . . . . . . . . . . . 442

A.1.1 Boolean Algebra . . . . . . . . . . . . . . . . . . . 442 
A.1.2 Index-Overlay . . . . . . . . . . . . . . . . . . . . . 443

A.1.3 Multiple Linear Regression . . . . . . . . . . . . . . . . . . . 445

A.1.4 Multiple Discriminant Analysis . . . . . . . . . . . . . . . . 447

A.1.5 Cluster Analysis . . . . . . . . . . . . . . . . . . 449

A.1.6 Factor Analysis . . . . . . . . . . . . . . . . . . . . . . . . 449

A.1.7 Expert Systems . . . . . . . . . . . . . . . . 451

A.1.8 Logistic Regression . . . . . . . . . . . . . . . . . . 459

A.1.9 Dempster-Shafer Evidential Belief Theory . . . . . . . . . . . . . . 461

A.1.10 Decision Trees . . . . . . . . . . . . . . . . . . . 469

A.1.11 Canonical Correlation . . . . . . . . . . . . . . . . . . 472

A.2 Additional Neural Network Architectures . . . . . . . . . . . . . . . . . . . 474

A.2.1 Probabilistic Neural Networks . . . . . . . . . . . . . . . . . . . 474

A.2.2 General Regression Neural Networks . . . . . . . . . . . . . 483

A.2.3 Radial Basis Neural Networks . . . . . . . . . . . . . . . . . . . 487

A.2.4 Kohonen Neural Networks . . . . . . . . . . . . . . . . . . 490

A.2.5 Hybrid Fuzzy-Neural Network Systems . . . . . . . . . . . . . . 496

B Documentation of GIS Databases $\quad 511$

B.1 Directory Structure of GIS Databases on CD-ROM . . . . . . . . . . . . 511

B.2 Metadata for the GIS Data Sets . . . . . . . . . . . . . . . . . . . . 511

B.2.1 What are Metadata? . . . . . . . . . . . . . . . 511

B.2.2 Metadata Standards . . . . . . . . . . . . . . . . . 513

B.2.3 Metadata Format used in This Study . . . . . . . . . . . . . . . 514

B.3 Data Dictionaries . . . . . . . . . . . . . . . . . . . . . 514

B.3.1 Data Dictionaries for Spatial Attributes . . . . . . . . . . . 514

$\begin{array}{lc}\text { C Metadata for Tenterfield GIS Database } & 517\end{array}$

C.1 Location of the Tenterfield Data Set . . . . . . . . . . . . . . 517

C.2 Overview of Primary Thematic Layers . . . . . . . . . . . . . . . . 519

C.3 Metadata for Tenterfield GIS Database . . . . . . . . . . . . . . . . . . 521

C.4 Geological Data. . . . . . . . . . . . . . . . . . . . . . . . . 521

C.4.1 Core Metadata Elements for Geological Data . . . . . . . . . . . . 521

C.4.2 Data Dictionary for Regional Geology Layer . . . . . . . . . . . . . 523

C.4.3 Data Dictionary for Tenterfield Geology Layer . . . . . . . . . . . . 524

C.4.4 Data Dictionary for Geological Province Table . . . . . . . . . . . 525

C.4.5 Data Dictionary for Tenterfield Granite Suite Table . . . . . . . . 526

C.4.6 Data Dictionary for Tenterfield Dominant Lithology Table . . . . . . 528

C.4.7 Data Dictionary for Tenterfield Geological Age Table . . . . . . . . . 528 
C.4.8 Data Dictionary for Tenterfield Geological System Table . . . . . . . 530

C.5 Geophysical Data . . . . . . . . . . . . . . . . . . . . . . . 531

C.5.1 Core Metadata Elements for Geophysical Data . . . . . . . . . . . 531

C.6 Geochemical Data . . . . . . . . . . . . . . . . . . . . . 533

C.6.1 Core Metadata Elements for Geochemical Data . . . . . . . . . . . 533

C.6.2 Data Dictionary for Regional Geochemistry Layer . . . . . . . . . 536

C.7 Deposit Data . . . . . . . . . . . . . . . . . . 537

C.7.1 Core Metadata Elements for Tenterfield Deposit Location Data . . . 537

C.7.2 Data Dictionary for Tenterfield Mineral Deposits Layer . . . . . . . 539

C.7.3 Data Dictionary for Deposit Size Database Table . . . . . . . . . . . 541

C.7.4 Data Dictionary for Tenterfield Deposit Code Table . . . . . . . . . 541

C.8 Derived Thematic Layers . . . . . . . . . . . . . . . . . . . . 542

C.9 Derived Thematic Layers . . . . . . . . . . . . . . . . . 543

D Metadata for Kalgoorlie GIS Database $\quad 544$

D.1 Location of the Kalgoorlie Data Set . . . . . . . . . . . . . . . . 544

D.2 Overview of Primary Thematic Layers . . . . . . . . . . . . . . . . 545

D.3 Core metadata elements for Kalgoorlie GIS data set . . . . . . . . . . . 546

D.4 Geological Data. . . . . . . . . . . . . . . . . . . . . . . . . 548

D.4.1 Data Dictionary for Solid Geology Layer . . . . . . . . . . . . . 548

D.5 Rock Strength Data and Rheological Contrast Calculations . . . . . . . . . 550

D.5.1 Data for Rheological Contrast Calculations . . . . . . . . . . . . 550

D.5.2 Data Dictionary for Structural Geology Layer . . . . . . . . . . . 557

D.5.3 Data Dictionary for Fold Axis Layer . . . . . . . . . . . . . . 557

D.6 Geochemical Data . . . . . . . . . . . . . . . . . . 557

D.6.1 Data for Chemical Contrast Calculations _ . . . . . . . . . . 557

D.7 Geophysical Data . . . . . . . . . . . . . . . . . . . . . 618

D.7.1 Data dictionary for Gravity Layer . . . . . . . . . . . . 618

D.7.2 Metadata for Bouguer Gravity Anomaly Layer . . . . . . . . . . 618

D.7.3 Metadata for Aeromagnetic Survey Layer . . . . . . . . . . . . . 619

D.8 Deposit Data . . . . . . . . . . . . . . . . . . . . . 620

D.8.1 Data Dictionary for Deposit Layer . . . . . . . . . . . . . . 620

D.9 Metamorphic Grade . . . . . . . . . . . . . . . . . . . . . . . . 621

D.10 Favourable Contact-Type Data ～. . . . . . . . . . . . . . . . . . . . 622

D.11 Favourable Lithology Data . . . . . . . . . . . . . . . . . . . . . 634 
E Summary of Data Processing used in the Tenterfield Pilot Study $\quad 638$

E.1 Data Processing Procedure used in Pilot Study, Chp. 4 . . . . . . . . . . 638

E.1.1 Pre-processing . . . . . . . . . . . . . . . . . . 638

E.1.2 Neural Network Training . . . . . . . . . . . . . . . . . . . . 640

E.1.3 Post-processing . . . . . . . . . . . . . . . . . . . . . . 643

E.1.4 Miscellaneous . . . . . . . . . . . . . . . . . . . 645

$\begin{array}{lll}\text { F Summary of Matlab Scripts used in Kalgoorlie Case Study } & 646\end{array}$

G Evaluation of GIS Input Layers: Kalgoorlie Case Study $\quad 661$

G.1 Graphs used to evaluate spatial relationships in GIS layers . . . . . . . . . . 661

G.2 Ranking of potential GIS input layers . . . . . . . . . . . . . . . . . 710

H Results of Neural Network Experiments: Kalgoorlie Case Study $\quad 723$

I Results of Fuzzy Systems Experiments: Kalgoorlie Case Study $\quad 786$

$\begin{array}{lll}\text { J Explanation of ArcView GIS Layer Names } & \mathbf{7 9 1}\end{array}$

J.1 Explanation of ArcView GIS Layer Names . . . . . . . . . . . . . . . . 791 


\section{List of Tables}

2.1 An example of a fuzzy membership function . . . . . . . . . . . . . 22

2.2 Analysis of spatial data by neural networks . . . . . . . . . . . . . . . 44

2.3 Summary of mineral-prospectivity mapping methods . . . . . . . . . . . 56

3.1 Derived layers and the ARC/Info commands used to create them . . . . . 63

3.2 Confusion matrix for a classification decision involving two classes . . . . . 75

3.3 Receiver-operating-characteristic table . . . . . . . . . . . . . . 75

3.4 Summary of GIS databases used in this study . . . . . . . . . . . . . . . . . 89

3.5 Programs and software packages used in this study . . . . . . . . . . . 93

4.1 Number of patterns in the training data sets . . . . . . . . . . . . . . . . 110

4.2 Combining conditionally dependent maps in weights-of-evidence method . . 115

4.3 Class values and membership values for maps in the fuzzy-logic method . . 119

4.4 Classification accuracy for training, training-stop and test data sets . . . . . 126

4.5 Statistical measures of map quality . . . . . . . . . . . . . . . . . 128

4.6 Probability measures of map quality . . . . . . . . . . . . . . . . . 129

5.1 Exploration criteria for orogenic gold deposits in the Kalgoorlie Terrane . . 150

5.2 Conversion of geology map legend from 108 to 25 rock types . . . . . . . . . 152

5.3 Chemical reactivity layers . . . . . . . . . . . . . . . . . . 157

5.4 Chemical reactivity ratios for the Kalgoorlie study area . . . . . . . . . 157

5.5 Rock strength properties of rocks in the Kalgoorlie study area . . . . . . . . 165

5.6 Fault and shear zone types used to form derived GIS layers . . . . . . . . . 175

5.7 Rheological contrast layers evaluated as inputs for neural network . . . . . 182

5.8 Chemical contrast layers . . . . . . . . . . . . . . . . . . . . . 183

5.9 Favourable contact-type layers and parameters . . . . . . . . . . . 188

6.1 Methods used to evaluate potential neural network input GIS layers . . . . 228

6.2 Area cross-tabulation showing variables used to calculate contrast, $C_{W}$. . . 229

6.3 Favourable zones for Au deposits estimated with various statistical methods 244 
6.4 Favourable variable ranges in GIS layers . . . . . . . . . . . . . . . . 251

6.5 Layers selected as inputs in neural network experiments . . . . . . . . . . 255

7.1 Number of patterns in the training data sets . . . . . . . . . . . . . . 263

7.2 Effect of random split of training data into training sets . . . . . . . . 267

7.3 Effect of network topology: average performance . . . . . . . . . . . 268

7.4 Effect of network topology: best performance . . . . . . . . . . . . 268

7.5 Effect of 2 hidden layers on network topology: average performance . . . . 270

7.6 Effect of 2 hidden layers on network topology: best performance . . . . . . . 270

7.7 Effect of training algorithm: average performance . . . . . . . . . . . . 271

7.8 Effect of training algorithm: best performance . . . . . . . . . . . . . 272

7.9 Summary of sets of neural network input layers . . . . . . . . . . . . . 274

7.10 Effect of input selection criteria (10 layers): average performance . . . . . . 275

7.11 Effect of input selection criteria (10 layers): best performance . . . . . . . . 275

7.12 Effect of input selection criteria (17 layers): average performance . . . . . . 276

7.13 Effect of input selection criteria (17 layers): best performance . . . . . . . 276

7.14 Effect of number of input GIS layers: average performance . . . . . . . . . 278

7.15 Effect of number of input GIS layers: best performance . . . . . . . . . . 278

7.16 Effect of inverse and $\log$ distance inputs: average performance . . . . . . . 280

7.17 Effect of inverse and $\log$ distance inputs: best performance . . . . . . . . . . 280

7.18 Buffer limits applied to input GIS layers depicting strike and distance . . . 283

7.19 Effect of buffer limits on strike \& distance inputs: average performance . . 284

7.20 Effect of buffer limits on strike \& distance inputs: best performance . . . . 284

7.21 Effect of buffer limits on contrast layers: average performance . . . . . . . . 285

7.22 Effect of buffer limits on contrast layers: best performance . . . . . . . . . . 285

7.23 Training set composition: effect of number of deposit patterns . . . . . . . 287

7.24 Training set composition: augmentation with noise . . . . . . . . . . . . 291

7.25 Effect of network topology (noise-augmented data): ave. performance . . . 293

7.26 Effect of network topology (noise-augmented data): best performance . . . . 293

7.27 Effect of the amount of uniform random noise: average performance . . . . 295

7.28 Effect of the amount of uniform random noise: best performance . . . . . . 295

7.29 Effect of the amount of Gaussian random noise: average performance . . . . 296

7.30 Effect of the amount of Gaussian random noise: best performance . . . . . . 296

7.31 Effect of the type of noise: uniform versus Gaussian random noise: average performance . . . . . . . . . . . . . . . . . . . . . . . . . . .

7.32 Effect of the type of noise: uniform versus Gaussian random noise: best performance . . . . . . . . . . . . . . . . . . . . . . . . . . . . . 
7.33 Effect of expanding the stop data set: average results . . . . . . . . . . 303

7.34 Effect of expanding the stop data set: best results . . . . . . . . . . . . . 303

7.35 Comparison of the ability of layers to predict known gold deposits . . . . 308

7.36 Effect of different input GIS layers representing lithology: average performance . . . . . . . . . . . . . . . . . . . . . . . . . . . . . . . .

7.37 Comparison of neural networks with 3 and 10 fuzzy-membership inputs: average performance . . . . . . . . . . . . . . . . 312

7.38 Comparison of neural networks with 3 and 10 fuzzy-membership inputs: best performance . . . . . . . . . . . . . . . . . . 313

7.39 Comparison of neural networks with 3 and 17 fuzzy-membership inputs: average performance . . . . . . . . . . . . . . . . . . . 313

7.40 Comparison of neural networks with 3 and 17 fuzzy-membership inputs: best performance . . . . . . . . . . . . . . . . 314

7.41 Performance of fuzzy systems . . . . . . . . . . . . . . . . 315

A.1 Example values used to illustrate evidential belief theory concepts . . . . 464

B.2 Spatial attributes for ARC/Info point and polygon coverages . . . . . . . 515

B.3 Spatial attributes for ARC/Info line coverages . . . . . . . . . . . . 516

B.4 Spatial attributes for ArcView point, line and polygon coverages . . . . . . 516

C.1 Location of the Tenterfield GIS . . . . . . . . . . . . . . . . . . . 519

C.2 Primary thematic layers in the Tenterfield GIS database . . . . . . . . . . 520

C.3 ANZLIC core metadata elements for Tenterfield geological data . . . . . . . 521

C.4 Data dictionary for regional geology coverage . . . . . . . . . . . . . 524

C.5 Data dictionary for geology coverage . . . . . . . . . . . . . . . . . 524

C.6 Data dictionary for geological province database table . . . . . . . . . 525

C.7 Data dictionary for granite suite database table . . . . . . . . . . . . 526

C.8 Explanation of dominant lithology codes in attribute table . . . . . . . . . 526

C.9 Data dictionary for dominant lithology database table . . . . . . . . . . . 528

C.10 Data dictionary for geological age database table . . . . . . . . . . . 528

C.11 Explanation of age codes in attribute table . . . . . . . . . . . . . 529

C.12 Data dictionary for geological system database table . . . . . . . . . . . 530

C.13 ANZLIC core metadata elements for Tenterfield geophysical data . . . . . . 531

C.14 Survey specifications for the Tenterfield geophysical data set . . . . . . . . 533

C.15 ANZLIC core metadata elements for Tenterfield geochemical data . . . . . . 534

C.16 Data dictionary for regional geochemistry layer . . . . . . . . . . . . . 536

C.17 ANZLIC core metadata elements for Tenterfield deposit location data . . . 537 
C.18 Data dictionary for mineral deposits layer . . . . . . . . . . . . . . . . 540

C.19 Data dictionary for deposit size database table . . . . . . . . . . . . . . . 541

C.20 Explanation of deposit size attributes used in database table . . . . . . . . 541

C.21 Data dictionary for deposit code database table . . . . . . . . . . . . . . 542

C.22 Primary thematic layers in the Tenterfield GIS database . . . . . . . . . . . 542

C.23 Derived thematic layers in the Tenterfield GIS database . . . . . . . . . . . 543

D.1 Location of the Kalgoorlie study area . . . . . . . . . . . . . . . . . . 545

D.2 Primary thematic layers in the Kalgoorlie GIS database . . . . . . . . . 546

D.3 ANZLIC core metadata elements for the Kalgoorlie GIS database . . . . . . 546

D.4 Data dictionary for the geology coverage . . . . . . . . . . . . . . 550

D.5 Calculation of average rock strength properties: Kalgoorlie area . . . . . . . 551

D.6 Data dictionary for the Kalgoorlie structural layer . . . . . . . . . . . . 557

D.7 Data dictionary for the anticlinal-fold axis layer . . . . . . . . . . . . . 557

D.8 Calculation of chemical reactivity ratios: Kalgoorlie area . . . . . . . . . . 559

D.9 Data dictionary for gravity station layer . . . . . . . . . . . . . . 618

D.10 Metadata for Bouguer gravity anomaly layer . . . . . . . . . . . . . . . 619

D.11 Metadata for airborne magnetic survey layer _ . . . . . . . . . . . 620

D.12 Data dictionary for Kalgoorlie gold deposits layer . . . . . . . . . . . . . 621

D.13 Data dictionary for Kalgoorlie metamorphic grade layer . . . . . . . . . . 622

D.14 Data for favourable contact-type GIS layers based on P(D) . . . . . . . . . 623

D.15 Data for favourable contact-type GIS layers based on P(D)*TCG/cell . . . 629

D.16 Data to calculate favourable lithology GIS layer based on $\mathrm{P}(\mathrm{D}) \quad \ldots . . .635$

D.17 Data to calculate favourable lithology GIS layer based on P(D)× TCG/cell . 637

E.1 Inputs and outputs for program, merge_sls . . . . . . . . . . . . . . 639

E.2 Inputs and outputs for program, rand_select . . . . . . . . . . . . . . . . 640

E.3 Inputs and outputs for program, $b p$ Win . . . . . . . . . . . . . . . 641

E.4 Inputs and outputs for program $b p$ Win in test mode . . . . . . . . . . . . . 642

E.5 Inputs and outputs for program, bp Win in production mode . . . . . . . . . 643

E.6 Inputs and outputs for program, map_class . . . . . . . . . . . . 643

E.7 Inputs and outputs for program, map_qstats . . . . . . . . . . . . . . . 644

E.8 Inputs and outputs for program deposit_locns . . . . . . . . . . . . . 645

F.1 Summary of matlab functions and scripts . . . . . . . . . . . 646

G.1 Ranking of potential GIS input layers: categories . . . . . . . . . . . . . 711

G.2 Ranking of potential GIS input layers: D/A . . . . . . . . . . . . . 714

G.3 Ranking of potential GIS input layers: $\mathrm{D} \times(\mathrm{D} / \mathrm{A}) \ldots \ldots . \ldots 717$ 
G.4 Ranking of potential GIS input layers: $\mathrm{D} \times(\mathrm{D} / \mathrm{A}), \mathrm{D} / \mathrm{A} \geq \sim 3, \mathrm{D} \geq \sim 20 \% \ldots 720$

H.1 MLP 10-5-1, Inputs: cat., Traingdx . . . . . . . . . . . . . . . . . . . 724

H.2 MLP 10-5-1, Inputs: cat., Traingd, Random training pattern selection 1 . . 724

H.3 MLP 10-5-1, Inputs: cat., Traingd, Random training pattern selection 2 . 725

H.4 MLP 10-5-1, Inputs: cat., Traingd, Random training pattern selection 3 . . 725

H.5 MLP 10-5-1, Inputs: cat., Traingd, Random training pattern selection 4 . . 726

H.6 MLP 10-5-1, Inputs: cat., Traingd, Random training pattern selection 5 . . 726

H.7 MLP 10-5-1, Inputs: cat., Traingd, Random training pattern selection 6 . . 727

H.8 MLP 10-5-1, Inputs: cat., Traingd, Random training pattern selection 7 . . 727

H.9 MLP 10-5-1, Inputs: cat., Traingd, Random training pattern selection 8 . . 728

H.10 MLP 10-5-1, Inputs: cat., Traingd, Random training pattern selection 9 . . 728

H.11 MLP 10-5-1, Inputs: cat., Traingd, Random training pattern selection $10 \quad .729$

H.12 MLP 10-5-1, Inputs: cat., Swap VV and TV . . . . . . . . . . . . . . . . 729

H.13 MLP 10-20-1, Inputs: cat., Ndeps $=5$, Set 1, trainlm . . . . . . . . . 730

H.14 MLP 10-20-1, Inputs: cat., Ndeps $=5$, Set 2, trainlm . . . . . . . . . . 730

H.15 MLP 10-20-1, Inputs: cat., Ndeps $=5$, Set 3, trainlm . . . . . . . . . . 731

H.16 MLP 10-20-1, Inputs: cat., Ndeps $=5$, Set 4, trainlm . . . . . . . . . . 731

H.17 MLP 10-20-1, Inputs: cat., Ndeps $=5$, Set 5, trainlm . . . . . . . . . 732

H.18 MLP 10-20-1, Inputs: cat., Ndeps $=5$, Set 6, trainlm . . . . . . . . . . 732

H.19 MLP 10-20-1, Inputs: cat., Ndeps $=5$, Set 7, trainlm . . . . . . . . . . 733

H.20 MLP 10-20-1, Inputs: cat., Ndeps $=5$, Set 8, trainlm . . . . . . . . . 733

H.21 MLP 10-20-1, Inputs: cat., Ndeps $=5$, Set 9, trainlm . . . . . . . . . . 734

H.22 MLP 10-20-1, Inputs: cat., Ndeps $=5$, Set 10, trainlm . . . . . . . . . 734

H.23 MLP 10-20-1, Inputs: cat., Ndeps $=10$, Set 1, trainlm . . . . . . . . . . 735

H.24 MLP 10-20-1, Inputs: cat., Ndeps $=10$, Set 2, trainlm . . . . . . . . . 735

H.25 MLP 10-20-1, Inputs: cat., Ndeps $=10$, Set 3, trainlm . . . . . . . . . . 736

H.26 MLP 10-20-1, Inputs: cat., Ndeps $=10$, Set 4, trainlm . . . . . . . . . . 736

H.27 MLP 10-20-1, Inputs: cat., Ndeps $=10$, Set 5, trainlm . . . . . . . . . 737

H.28 MLP 10-20-1, Inputs: cat., Ndeps $=10$, Set 6, trainlm . . . . . . . . . 737

H.29 MLP 10-20-1, Inputs: cat., Ndeps $=10$, Set 7, trainlm . . . . . . . . . 738

H.30 MLP 10-20-1, Inputs: cat., Ndeps $=10$, Set 8, trainlm . . . . . . . . . 738

H.31 MLP 10-20-1, Inputs: cat., Ndeps $=10$, Set 9, trainlm . . . . . . . . . 739

H.32 MLP 10-20-1, Inputs: cat., Ndeps $=10$, Set 10, trainlm . . . . . . . . . 739

H.33 MLP 10-20-1, Inputs: cat., Ndeps $=20$, Set 1, trainlm . . . . . . . . . 740

H.34 MLP 10-20-1, Inputs: cat., Ndeps $=20$, Set 2, trainlm . . . . . . . . . . 740

H.35 MLP 10-20-1, Inputs: cat., Ndeps $=20$, Set 3, trainlm . . . . . . . . . . 741 
H.36 MLP 10-20-1, Inputs: cat., Ndeps $=20$, Set 4, trainlm . . . . . . . . . 741

H.37 MLP 10-20-1, Inputs: cat., Ndeps $=20$, Set 5, trainlm . . . . . . . . . . 742

H.38 MLP 10-20-1, Inputs: cat., Ndeps $=20$, Set 6, trainlm . . . . . . . . . 742

H.39 MLP 10-20-1, Inputs: cat., Ndeps $=20$, Set 7, trainlm . . . . . . . . . 743

H.40 MLP 10-20-1, Inputs: cat., Ndeps $=20$, Set 8, trainlm . . . . . . . . . 743

H.41 MLP 10-20-1, Inputs: cat., Ndeps $=20$, Set 9, trainlm . . . . . . . . . 744

H.42 MLP 10-20-1, Inputs: cat., Ndeps $=20$, Set 10, trainlm . . . . . . . . . 744

H.43 MLP 10-20-1, Inputs: cat., Ndeps $=40$, Set 1, trainlm . . . . . . . . . 745

H.44 MLP 10-20-1, Inputs: cat., Ndeps $=40$, Set 2, trainlm . . . . . . . . . . 745

H.45 MLP 10-20-1, Inputs: cat., Ndeps $=40$, Set 3, trainlm . . . . . . . . . 746

H.46 MLP 10-20-1, Inputs: cat., Ndeps $=40$, Set 4, trainlm . . . . . . . . . 746

H.47 MLP 10-20-1, Inputs: cat., Ndeps $=40$, Set 5, trainlm . . . . . . . . . . 747

H.48 MLP 10-20-1, Inputs: cat., Ndeps $=40$, Set 6, trainlm . . . . . . . . . 747

H.49 MLP 10-20-1, Inputs: cat., Ndeps $=40$, Set 7, trainlm . . . . . . . . 748

H.50 MLP 10-20-1, Inputs: cat., Ndeps $=40$, Set 8, trainlm . . . . . . . . . 748

H.51 MLP 10-20-1, Inputs: cat., Ndeps $=40$, Set 9, trainlm . . . . . . . . . 749

H.52 MLP 10-20-1, Inputs: cat., Ndeps $=40$, Set 10, trainlm . . . . . . . . . 749

H.53 MLP 10-20-1, Inputs: cat., Ndeps $=80$, Set 1, trainlm . . . . . . . . . 750

H.54 MLP 10-20-1, Inputs: cat., Ndeps $=80$, Set 2, trainlm . . . . . . . . . . 750

H.55 MLP 10-20-1, Inputs: cat., Ndeps $=80$, Set 3, trainlm . . . . . . . . . 751

H.56 MLP 10-20-1, Inputs: cat., Ndeps $=80$, Set 4, trainlm . . . . . . . . . . 751

H.57 MLP 10-20-1, Inputs: cat., Ndeps $=80$, Set 5, trainlm . . . . . . . . . . 752

H.58 MLP 10-20-1, Inputs: cat., Ndeps $=80$, Set 6, trainlm . . . . . . . . . 752

H.59 MLP 10-20-1, Inputs: cat., Ndeps $=80$, Set 7, trainlm . . . . . . . . . 753

H.60 MLP 10-20-1, Inputs: cat., Ndeps $=80$, Set 8, trainlm . . . . . . . . . 753

H.61 MLP 10-20-1, Inputs: cat., Ndeps $=80$, Set 9, trainlm . . . . . . . . . . 754

H.62 MLP 10-20-1, Inputs: cat., Ndeps $=80$, Set 10, trainlm . . . . . . . . . 754

H.63 MLP 10-20-1, Inputs: cat., Ndeps $=100$, Set 1, trainlm . . . . . . . . . 755

H.64 MLP 10-20-1, Inputs: cat., Ndeps $=100$, Set 2, trainlm . . . . . . . . . . 755

H.65 MLP 10-20-1, Inputs: cat., Ndeps $=100$, Set 3, trainlm . . . . . . . . 756

H.66 MLP 10-20-1, Inputs: cat., Ndeps $=100$, Set 4, trainlm . . . . . . . . 756

H.67 MLP 10-20-1, Inputs: cat., Ndeps $=100$, Set 5, trainlm . . . . . . . . . 757

H.68 MLP 10-20-1, Inputs: cat., Ndeps $=100$, Set 6, trainlm . . . . . . . . . 757

H.69 MLP 10-20-1, Inputs: cat., Ndeps $=100$, Set 7, trainlm . . . . . . . . 758

H.70 MLP 10-20-1, Inputs: cat., Ndeps $=100$, Set 8, trainlm . . . . . . . . . 758

H.71 MLP 10-20-1, Inputs: cat., Ndeps $=100$, Set 9, trainlm . . . . . . . . . 759

H.72 MLP 10-20-1, Inputs: cat., Ndeps $=100$, Set 10, trainlm . . . . . . . . 759 
H.73 MLP 10-20-1, Inputs: cat., Number of deposits in training set, trainlm . . . 760

H.74 MLP 10-0-1, Inputs: cat., trainlm . . . . . . . . . . . . . . . . . . 761

H.75 MLP 10-5-1, Inputs: cat., trainlm . . . . . . . . . . . . . . . . . 761

H.76 MLP 10-10-1, Inputs: cat., trainlm . . . . . . . . . . . . . . . 762

H.77 MLP 10-15-1, Inputs: cat., trainlm . . . . . . . . . . . . . . . . . 762

H.78 MLP 10-20-1, Inputs: cat., trainlm . . . . . . . . . . . . . . . . 763

H.79 MLP 10-25-1, Inputs: cat., trainlm . . . . . . . . . . . . . . . . 763

H.80 MLP 10-3-2-1, Inputs: cat., trainlm . . . . . . . . . . . . . . . . 764

H.81 MLP 10-7-3-1, Inputs: cat., trainlm . . . . . . . . . . . . . . . . 764

H.82 MLP 10-10-5-1, Inputs: cat., trainlm . . . . . . . . . . . . . . . 765

H.83 MLP 10-20-5-1, Inputs: cat., trainlm . . . . . . . . . . . . . . . . 765

H.84 MLP 10-20-1, Inputs: cat., traingd . . . . . . . . . . . . . . . . . 766

H.85 MLP 10-20-1, Inputs: cat., traingdx . . . . . . . . . . . . . . 766

H.86 MLP 10-0-1, Inputs: cat., trainlm, Noise, 10\%, uni. . . . . . . . . . . . . 767

H.87 MLP 10-10-1, Inputs: cat., trainlm, Noise, 10\%, uni. . . . . . . . . . . . . 767

H.88 MLP 10-15-1, Inputs: cat., trainlm, Noise, 10\%, uni. . . . . . . . . . . . 768

H.89 MLP 10-20-1, Inputs: cat., trainlm, Noise, 10\%, uni. . . . . . . . . . . . . 768

H.90 MLP 10-25-1, Inputs: cat., trainlm, Noise, 10\%, uni. . . . . . . . . . . . . 769

H.91 MLP 10-50-1, Inputs: cat., trainlm, Noise, 10\%, uni. . . . . . . . . . . . . 769

H.92 MLP 10-20-1, Inputs: cat., trainlm, Noise, 5\%, uni. . . . . . . . . . . . . 770

H.93 MLP 10-20-1, Inputs: cat., trainlm, Noise, 10\%, uni. . . . . . . . . . . . . 770

H.94 MLP 10-20-1, Inputs: cat., trainlm, Noise, 20\%, uni. . . . . . . . . . . . . 771

H.95 MLP 10-20-1, Inputs: cat., trainlm, Noise, 40\%, uni. . . . . . . . . . . . . 771

H.96 MLP 10-20-1, Inputs: cat., trainlm, Noise, 80\%, uni. . . . . . . . . . . . . 772

H.97 MLP 10-20-1, Inputs: cat., trainlm, Noise, 5\%, norm. . . . . . . . . . . . 772

H.98 MLP 10-20-1, Inputs: cat., trainlm, Noise, 10\%, normal . . . . . . . . . . 773

H.99 MLP 10-20-1, Inputs: cat., trainlm, Noise, 20\%, normal . . . . . . . . . . 773

H.100 MLP 10-20-1, Inputs: cat., trainlm, Noise, 40\%, normal . . . . . . . . . . . 774

H.101 MLP 10-20-1, Inputs: cat., trainlm, Noise, 80\%, normal . . . . . . . . . . . 774

H.102 MLP 10-10-1, Inputs: cat., trainlm, Noise 10\% uni., large train-test set . . 775

H.103 MLP 10-20-1, Inputs: cat., trainlm, Noise, 10\%, normal, large train-test set 775

H.104 MLP 10-20-1, Inputs: cat., trainlm, Noise, 40\%, uni., large train-test set . 776

H.105 MLP 17-20-1, Inputs: cat., trainlm . . . . . . . . . . . . . . . 776

H.106 MLP 10-20-1, Inputs: D/A, trainlm . . . . . . . . . . . . . . . 777

H.107 MLP 17-20-1, Inputs: D/A, trainlm . . . . . . . . . . . . . . . . 777

H.108 MLP 10-20-1, Inputs: D*D/A, trainlm . . . . . . . . . . . . . 778

H.109 MLP 17-20-1, Inputs: $\mathrm{D} * \mathrm{D} / \mathrm{A}$, trainlm . . . . . . . . . . . . 778 
H.110 MLP 10-20-1, Inputs: $\mathrm{D} * \mathrm{D} / \mathrm{A}$, vers.2, trainlm . . . . . . . . . . . . 779

H.111 MLP 17-20-1, Inputs: D*D/A, vers. 2, trainlm . . . . . . . . . . . . . 779

H.112 MLP 25-20-1, Inputs: all layers, trainlm . . . . . . . . . . . . . . . . 780

H.113 MLP 10-20-1, Inputs: cat., inverse distance, trainlm . . . . . . . . . . . . 780

H.114 MLP 10-20-1, Inputs: cat., log distance, trainlm . . . . . . . . . . . . . . 781

H.115 MLP 10-20-1, Inputs: cat., dist. \& strike narrow buffers, trainlm . . . . . . 781

H.116 MLP 10-20-1, Inputs: cat., dist. \& strike wide buffers, trainlm . . . . . . . 782

H.117 MLP 10-20-1, Inputs: cat., contrast narrow buffer, trainlm . . . . . . . . 782

H.118 MLP 14-20-1, Inputs: substitutes for geol., trainlm . . . . . . . . . . . . . 783

H.119 MLP 33-20-1, Inputs: 1-of-N coded geology., trainlm . . . . . . . . . . . 783

H.120 MLP 10-20-1, Inputs: cat., fuzzy inputs, trainlm . . . . . . . . . . . . . 784

H.121 MLP 10-20-1, Inputs: D* D/A, fuzzy inputs, trainlm . . . . . . . . . . . 784

H.122 MLP 17-20-1, Inputs: cat fuzzy inputs, trainlm . . . . . . . . . . . . . 785

H.123 MLP 17-20-1, Inputs: D* D/A, fuzzy inputs, trainlm . . . . . . . . . 785

I.1 Fuzzy, Inputs: 10, categories . . . . . . . . . . . . . . . . . . 787

I.2 Fuzzy, Inputs: 10, D* D/A . . . . . . . . . . . . . . . 788

I.3 Fuzzy, Inputs: 17, categories . . . . . . . . . . . . . . . . . . . . 789

I.4 Fuzzy, Inputs: 17, D* D/A . . . . . . . . . . . . . 790

J.1 Explanation of ArcView GIS Layer Names . . . . . . . . . . . . . . . . . 791 


\section{List of Figures}

1.1 Area of interest within task of regional mineral exploration . . . . . . . 2

2.1 Triangular and sigmoidal fuzzy membership functions . . . . . . . . . . . 24

2.2 Components and criteria in the mineral systems approach . . . . . . . . 27

2.3 Architecture of a 3-layer multilayer perceptron (MLP) neural network . . . 33

2.4 Suitability of AI, soft-computing, and statistical techniques for problem solving . . . . . . . . . . . . . . . . . . . . 34

2.5 Hierarchical neural network used to recognize hand-written digits . . . . . . 42

2.6 Example of the use of patterns of pixels used to train a network . . . . . . . 43

2.7 Computations performed in a single processing unit of a neural network . . 48

2.8 Block diagram representing the training process in a multilayer perceptron neural network . . . . . . . . . . . . . . . . . . . . . 4 49

2.9 Cross-section of a network error function . . . . . . . . . . . . . 50

2.10 Nomenclature for units and variables in the generalized delta rule . . . . . . 51

3.1 First pre-processing step is the conversion of layers to raster format . . . . . 64

3.2 Relationship between GIS layers and feature vectors used as inputs to the neural network. . . . . . . . . . . . . . . . . . 6 67

3.3 Data processing steps in the neural network method . . . . . . . . . . 71

3.4 Receiver-operating-characteristic (ROC) curves . . . . . . . . . . . . . 77

3.5 Measure of classification performance used by Harris and Pan (1999) . . . . 79

3.6 Measure of exploration performance used by Harris \& Pan (1999) . . . . . . 80

3.7 Processing steps to create weights-of-evidence mineral prospectivity map . . 82

3.8 Processing steps to create fuzzy-logic mineral prospectivity map . . . . . . 86

3.9 Fuzzy inference net used produce the mineral prospectivity map in the pilot study . . . . . . . . . . . . . . . . . . . 87

4.1 GIS thematic layers used as inputs in the pilot study . . . . . . . . . . . . 99

4.2 Location and simplified geology of the Tenterfield area . . . . . . . . . . . . 102

4.3 Simplified solid geology of the Tenterfield 1:100000 map sheet area . . . . 108 
4.4 Architecture of the MLP neural network used in the pilot study . . . . . . . 109

4.5 Weights-of-evidence mineral prospectivity maps, Tenterfield area, NSW. . . 116

4.6 Weights-of-evidence mineral prospectivity maps, Tenterfield area, NSW. . . 117

4.7 Fuzzy mineral prospectivity maps, Tenterfield 1:100000 map sheet area . . . 120

4.8 Fuzzy mineral prospectivity maps, Tenterfield 1:100000 map sheet area . . . 121

4.9 Fuzzy mineral prospectivity maps, Tenterfield 1:100000 map sheet area . . . 122

4.10 Fuzzy mineral prospectivity maps, Tenterfield 1:100000 map sheet area . . . 123

4.11 Prospectivity maps, Tenterfield 1:100000 area . . . . . . . . . . . . 127

4.12 Response of the fuzzy-logic and neural network methods to redundant input data . . . . . . . . . . . . . . . . . . . . . . . . . . . . . . . . .

4.13 Response of the weights-of-evidence and neural network methods to irrelevant input data . . . . . . . . . . . . . . . . . . . . . . . . . . . . . . . . . .

5.1 Location of the Kalgoorlie case study area. . . . . . . . . . . . . . . . . 139

5.2 Conversion of Goldfields data sets from Mapinfo to Matlab format . . . 151

5.3 Criteria used for subjective estimates of rock strength . . . . . . . . . . 163

5.4 Procedure to create layer of distance to intersection of NNW- \& NE-trending

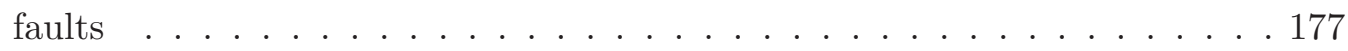

5.5 Procedure to create layer of distance to magnetic anomalies . . . . . . . . 179

5.6 Conversion of rock types in a solid geology layer to fuzzy member values . . 189

5.7 Kalgoorlie GIS database layer: solid geology . . . . . . . . . . . . . . . 196

5.8 Kalgoorlie GIS database layer: crustal \& regional-scale faults . . . . . . . 197

5.9 Kalgoorlie GIS database layer: major anticlinal \& synclinal fold-axes . . . . 198

5.10 Kalgoorlie GIS database layer: airborne magnetic survey . . . . . . . . . . . 199

5.11 Kalgoorlie GIS database layer: Bouguer gravity . . . . . . . . . . . . . 200

5.12 Kalgoorlie GIS database layer: Gold deposits (all deposits) . . . . . . . . . 201

5.13 Kalgoorlie GIS database layer: Gold deposits ( $\geq 1000 \mathrm{~kg}$ TCG) . . . . . . . 202

5.14 Kalgoorlie GIS database layer: fracture toughness . . . . . . . . . . . . 203

5.15 Kalgoorlie GIS database layer: metamorphic grade . . . . . . . . . . . . . . 204

5.16 Kalgoorlie GIS database layer: distance to porphyritic intrusives . . . . . . 205

5.17 Kalgoorlie GIS database layer: distance to lithological contacts . . . . . . . 206

5.18 Kalgoorlie GIS database layer: distance to crustal-scale faults . . . . . . . . 207

5.19 Kalgoorlie GIS database layer: distance to NNW regional-scale faults . . . . 208

5.20 Kalgoorlie GIS database layer: distance to NE regional-scale faults . . . . . 209

5.21 Kalgoorlie GIS database layer: distance to anticlinal axes . . . . . . . . . . 210

5.22 Kalgoorlie GIS database layer: distance to synclinal axes . . . . . . . . . . . 211

5.23 Kalgoorlie GIS database layer: distance to intersection of regional faults . . 212 
5.24 Kalgoorlie GIS database layer: distance to magnetic anomalies . . . . . . . 213

5.25 Kalgoorlie GIS database layer: strike of nearest crustal-scale fault . . . . . . 214

5.26 Kalgoorlie GIS database layer: strike of nearest NNW regional-scale fault . 215

5.27 Kalgoorlie GIS database layer: strike of nearest NE regional-scale fault . . . 216

5.28 Kalgoorlie GIS database layer: strike of nearest lithological contact . . . . . 217

5.29 Kalgoorlie GIS database layer: subjective rheological contrast (fuzzy) . . 218

5.30 Kalgoorlie GIS database layer: chemical contrast, $\mathrm{Fe}_{\mathrm{wt} \%} * \mathrm{Fe} /(\mathrm{Fe}+\mathrm{Mg}+\mathrm{Ca}) 219$

5.31 Kalgoorlie GIS database layer: chemical contrast, $\mathrm{Fe}_{2} \mathrm{O}_{3} /\left(\mathrm{Fe}_{2} \mathrm{O}_{3}+\mathrm{FeO}\right)$. . . 220

5.32 Kalgoorlie GIS database layer: chemical contrast, $\mathrm{Fe}_{2} \mathrm{O}_{3} / \mathrm{FeO}$. . . . . . . . 221

5.33 Kalgoorlie GIS database layer: favourable contact type based on $P(D)$. . . 222

5.34 Kalgoorlie GIS database layer: density of crustal- and regional-scale faults . 223

5.35 Kalgoorlie GIS database layer: density of lithological contacts . . . . . . . . 224

6.1 Graph of contrast $\left(C_{W}\right)$ versus cumulative distance to anticlinal axes . . . . 230

6.2 Graph of contrast $\left(C_{W}\right)$ versus cumulative distance to synclinal axes . . . 230

6.3 Graph of chi-squared statistic versus cumulative distance to anticlinal axes 232

6.4 Graph of chi-squared statistic versus cumulative distance to synclinal axes . 232

6.5 Graph of Kolmogorov-Smirnov CDF versus distance to anticlinal axes . . . 234

6.6 Graph of Kolmogorov-Smirnov CDF versus distance to synclinal axes . . . 234

6.7 Graph of bivariate J-function for distance to anticlinal axes . . . . . . . . . 237

6.8 Graph of bivariate J-function for distance to synclinal axes . . . . . . . . . 237

6.9 Plots for layer evaluation methods: distance to positive magnetic anomaly

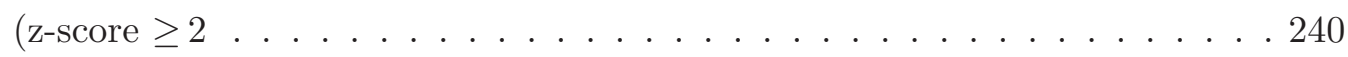

6.10 Graph of interval-based bivariate J-function for distance to anticlinal axes . 242

6.11 Graph of interval-based bivariate J-function for distance to synclinal axes . 242

6.12 Plots for layer evaluation methods: strike of crustal-scale shear zones . . . . 243

6.13 Graph of $(O-E) / E$ for versus distance to anticlinal axes . . . . . . . . . 246

6.14 Graph of $(O-E) / E$ for versus distance to synclinal axes . . . . . . . 246

6.15 Use of derived GIS layers to include conceptual knowledge in a neural network analysis . . . . . . . . . . . . . . . . . . . 257

7.1 Processing steps for neural network prospectivity mapping method . . . . . 262

7.2 Receiver-operating-characteristic (ROC) curve for a neural network prospec-

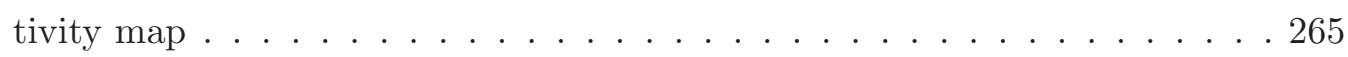

7.3 Histograms comparing raw and transformed distances to NE-trending faults 281

7.4 Relationship between numbers of map grid, deposit and training patterns . 286

7.5 Graphs of test-set performance and map quality vs no. of deposit patterns . 289

7.6 Schematic diagram of the use of noise in training a neural network . . . . . 292 
7.7 Detail of a neural network mineral prospectivity map for part of the Kalgoorlie Terrane . . . . . . . . . . . . . . . . . . . . . . 297

7.8 Detail of a neural network mineral prospectivity map for part of the Kalgoorlie Terrane . . . . . . . . . . . . . . . . . . 298

7.9 Detail of a neural network mineral prospectivity map for part of the Kalgoorlie Terrane . . . . . . . . . . . . . . . . . . . . . . . 299

7.10 Detail of a neural network mineral prospectivity map for part of the Kalgoorlie Terrane . . . . . . . . . . . . . . . . . . . . 300

7.11 Relationship of hybrid-fuzzy approaches to main mineral-prospectivity mapping methods . . . . . . . . . . . . . . . . . . . . . 305

7.12 Plot of $(O-E) / E$ versus rheological contrast (fuzzy membership) . . . . . 309

7.13 Schematic diagram of a hybrid, fuzzy-neural network system . . . . . . . . . 312

7.14 Histograms comparing outputs of neural and hybrid fuzzy-neural networks and fuzzy systems . . . . . . . . . . . . . . . . . . . . 318

7.15 Detail of a neural network mineral prospectivity map for part of the Kalgoorlie Terrane . . . . . . . . . . . . . . . . . . . . . . 321

7.16 Detail of a hybrid fuzzy, neural-network mineral prospectivity map for part of the Kalgoorlie Terrane . . . . . . . . . . . . . . . . . . 322

7.17 Detail of a fuzzy-system mineral prospectivity map for part of the Kalgoorlie Terrane . . . . . . . . . . . . . . . . . . . . . . . . . . . . . . . . . . .

7.18 ROC plots and activation histogram for 10-0-1 neural network (trainlm) . . 332

7.19 Neural network prospectivity map (topology: 10-0-1; input layers: 10, layer selection: categories,training algorithm: trainlm) . . . . . . . . . . . 333

7.20 ROC plots and activation histogram for 10-20-1 neural network (trainlm) . 334

7.21 Neural network prospectivity map (topology: 10-20-1; input layers: 10, layer selection: categories, training algorithm: trainlm) . . . . . . . . . 335

7.22 ROC plots and activation histogram for 10-20-1 neural network (traingdx) . 336

7.23 Neural network prospectivity map (topology: 10-20-1; input layers: 10, layer selection: categories, training algorithm: traingdx $\ldots . \ldots 337$

7.24 ROC plots and activation histogram for 10-20-1 neural network (trainlm),

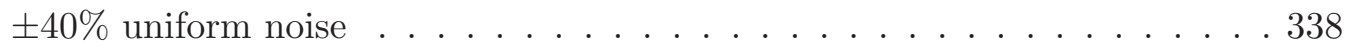

7.25 Neural network prospectivity map (topology: 10-20-1; input layers: 10, layer selection: categories, train. alg.: trainlm, noise: $\pm 40 \%$ uniform) . . . . 339

7.26 ROC plots and activation histogram for 10-20-1 neural network (trainlm),

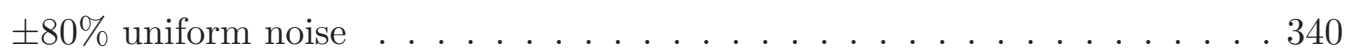

7.27 Neural network prospectivity map (topology: 10-20-1; input layers: 10, layer selection: categories, train. alg.: trainlm, noise: $\pm 80 \%$ uniform) . . . . 341 
7.28 ROC plots and activation histogram for 10-20-1 neural network (trainlm), $\pm 5 \%$ Gaussian noise . . . . . . . . . . . . . . . . . . 342

7.29 Neural network prospectivity map (topology: 10-20-1; input layers: 10, layer selection: categories, train. alg.: trainlm, noise: $\pm 5 \%$ Gaussian) . . . . 343

7.30 ROC plots and activation histogram for 10-20-1 neural network (trainlm), $\pm 10 \%$ Gaussian noise . . . . . . . . . . . . . . . . . . . . 344

7.31 Neural network prospectivity map (topology: 10-20-1; input layers: 10, layer selection: categories, training algorithm: trainlm, noise: $\pm 10 \%$ Gaus-

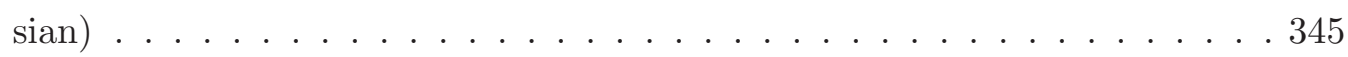

7.32 ROC plots and activation histogram for 10-20-1 neural network (trainlm), Input layer selection: D/A . . . . . . . . . . . . . . . . . 346

7.33 Neural network prospectivity map (topology: 10-20-1; input layers: 10, layer selection: D/A, training algorithm: trainlm) . . . . . . . . . . 347

7.34 ROC plots and activation histogram for 10-20-1 neural network (trainlm), Input layer selection: $\mathrm{D} *(\mathrm{D} / \mathrm{A}) \ldots \ldots 348$

7.35 Neural network prospectivity map (topology: 10-20-1; input layers: 10, layer selection: $\mathrm{D} *(\mathrm{D} / \mathrm{A})$, training algorithm: trainlm $) \ldots . . . . . .349$

7.36 ROC plots and activation histogram for 10-20-1 neural network (trainlm), Input layer selection: $\mathrm{D} *(\mathrm{D} / \mathrm{A}) A N D \mathrm{D} \geq \sim 20 \% A N D \mathrm{D} / \mathrm{A} \geq \sim 3 \% \ldots 350$

7.37 Neural network prospectivity map (topology: 10-20-1; input layers: 10, layer selection: $\mathrm{D} *(\mathrm{D} / \mathrm{A}) A N D \mathrm{D} \geq \sim 20 \% A N D \mathrm{D} / \mathrm{A} \geq \sim 3 \%$, training algorithm: trainlm) . . . . . . . . . . . . . . . . . . 351

7.38 ROC plots and activation histogram for 17-20-1 neural network (trainlm), Input layer selection: categories . . . . . . . . . . . . . . . . 352

7.39 Neural network prospectivity map (topology: 17-20-1; input layers: 17, layer selection: categories, training algorithm: trainlm) . . . . . . . . 353

7.40 ROC plots and activation histogram for 17-20-1 neural network (trainlm), Input layer selection: D/A . . . . . . . . . . . . . . . . 354

7.41 Neural network prospectivity map (topology: 17-20-1; input layers: 17, layer selection: D/A, training algorithm: trainlm) . . . . . . . . . 355

7.42 ROC plots and activation histogram for 17-20-1 neural network (trainlm), Input layer selection: $\mathrm{D} *(\mathrm{D} / \mathrm{A}) \ldots \ldots 356$

7.43 Neural network prospectivity map (topology: 17-20-1; input layers: 17, layer selection: $\mathrm{D} *(\mathrm{D} / \mathrm{A})$, training algorithm: trainlm $) \ldots . . \ldots 357$

7.44 ROC plots and activation histogram for 17-20-1 neural network (trainlm), Input layer selection: $\mathrm{D} *(\mathrm{D} / \mathrm{A}) A N D \mathrm{D} \geq \sim 20 \% A N D \mathrm{D} / \mathrm{A} \geq \sim 3 \% \ldots 358$ 
7.45 Neural network prospectivity map (topology: 17-20-1; input layers: 17, layer selection: $\mathrm{D} *(\mathrm{D} / \mathrm{A}) A N D \mathrm{D} \geq \sim 20 \% A N D \mathrm{D} / \mathrm{A} \geq \sim 3 \%$, training algorithm: trainlm $\ldots \ldots \ldots 359$

7.46 ROC plots and activation histogram for 25-20-1 neural network (trainlm), Input layer selection: all layers . . . . . . . . . . . . . . . . 360

7.47 Neural network prospectivity map (topology: 25-20-1; input layers: 25, layer selection: all, training algorithm: trainlm) . . . . . . . . . . 361

7.48 ROC plots and activation histogram for 10-20-1 neural network (trainlm), fuzzy inputs, layer selection: categories . . . . . . . . . . . . . . . 362

7.49 Neural network prospectivity map (topology: 10-20-1; input layers: 10 fuzzy membership, layer selection: categories, training algorithm: trainlm) . . . 363

7.50 ROC plots and activation histogram for 10-20-1 neural network (trainlm), fuzzy inputs, layer selection: $\mathrm{D} *(\mathrm{D} / \mathrm{A}) \ldots . . \ldots 364$

7.51 Neural network prospectivity map (topology: 10-20-1; input layers: 10 fuzzy membership, layer selection: $\mathrm{D} *(\mathrm{D} / \mathrm{A})$, training algorithm: trainlm) . . . . 365

7.52 ROC plots and activation histogram for 17-20-1 neural network (trainlm), fuzzy inputs, layer selection: categories . . . . . . . . . . . . . . 366

7.53 Neural network prospectivity map (topology: 17-20-1; input layers: 17 fuzzy membership, layer selection: categories, training algorithm: trainlm) . . . 367

7.54 ROC plots and activation histogram for 17-20-1 neural network (trainlm), fuzzy inputs, layer selection: $\mathrm{D} *(\mathrm{D} / \mathrm{A}) \ldots \ldots 368$

7.55 Neural network prospectivity map (topology: 17-20-1; input layers: 17 fuzzy membership, layer selection: $\mathrm{D} *(\mathrm{D} / \mathrm{A})$, training algorithm: trainlm) . . . . . 369

7.56 ROC plots and activation histogram for 10 input fuzzy system using gamma function $(\gamma=0.96)$, layer selection: categories . . . . . . . . . 370

7.57 Fuzzy systems prospectivity map (input layers: $10, \gamma=0.96$, layer selection:

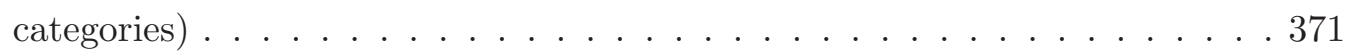

7.58 ROC plots and activation histogram for 10 input fuzzy system using gamma function $(\gamma=0.94)$, layer selection: $\mathrm{D} *(\mathrm{D} / \mathrm{A}) \ldots \ldots 772 \ldots \ldots \ldots$

7.59 Fuzzy systems prospectivity map (input layers: 10 fuzzy membership, $\gamma=$

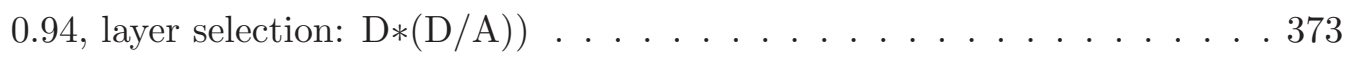

7.60 ROC plots and activation histogram for 17 input fuzzy system using gamma function $(\gamma=0.97)$, layer selection: categories . . . . . . . . . . . 374

7.61 Fuzzy systems prospectivity map (input layers: $17, \gamma=0.97$, layer selection: categories) . . . . . . . . . . . . . . . . . . . . 375

7.62 ROC plots and activation histogram for 17 input fuzzy system using gamma function $(\gamma=0.97)$, layer selection: $\mathrm{D} \times(\mathrm{D} / \mathrm{A}) \ldots \ldots . \ldots 376$ 
7.63 Fuzzy systems prospectivity map (input layers: 17, gamma $=0.97$, layer selection: $\mathrm{D} *(\mathrm{D} / \mathrm{A})) \ldots \ldots . \ldots . \ldots . \ldots . \ldots \ldots 77$

A.1 Form of simple IF-THEN rules in an expert system . . . . . . . . . . . . 451

A.2 Linking of rules in an expert system through forward chaining . . . . . . . . 452

A.3 Interpolation of a piecewise function in the PROSPECTOR expert system . 457

A.4 Form of the logistic function . . . . . . . . . . . . . . . . . 461

A.5 An example illustrating partial belief in evidence and support . . . . . . . . 462

A.6 Relationship between belief, uncertainty, doubt and plausibility . . . . . . . 465

A.7 Values of support used to illustrate Dempster's rule . . . . . . . . . . . . 466

A.8 Illustration of a decision tree . . . . . . . . . . . . . . . . . . 471

A.9 Example of the use of Bayes' decision rule for classification . . . . . . . . 475

A.10 Classification using a PNN . . . . . . . . . . . . . . . . . . . 477

A.11 Architecture of a probabilistic neural network . . . . . . . . . . . . . 479

A.12 Effect of smoothing parameter, $\sigma$, on classification in a PNN . . . . . . 480

A.13 Schematic illustration of the concept behind the GRNN network . . . . . . 483

A.14 Architecture of a general regression neural network . . . . . . . . . . . . 486

A.15 Architecture of a radial basis function neural network . . . . . . . . . 488

A.16 Division of input feature space by different neural network architectures . . 491

A.17 Architecture of a Kohonen neural network . . . . . . . . . . . . . . . . . . . 492

A.18 Fuzzy inference procedure for three rules and two premises . . . . . . . . 500

A.19 Methods for defuzzification of aggregated rule output membership function 504

A.20 ANIFS architecture for a two-input Sugeno fuzzy model . . . . . . . . . 506

C.1 Location of the Tenterfield GIS data set . . . . . . . . . . . . . . . 518

G.1 Plots for layer evaluation methods: favourable lithology (fuzzy) . . . . . . . 662

G.2 Plots for layer evaluation methods: subjective (fuzzy) rock competency . . 663

G.3 Plots for layer evaluation methods: subjective (classes) rock competency . . 664

G.4 Plots for layer evaluation methods: rock strength, Young's Modulus (CRC) 665

G.5 Plots for layer evaluation methods: rock strength, Young's Modulus (AMC) 666

G.6 Plots for layer evaluation methods: rock strength, UCS . . . . . . . . . 667

G.7 Plots for layer evaluation methods: rock strength, UCS . . . . . . . . . 668

G.8 Plots for layer evaluation methods: rock strength, UCS . . . . . . . . . . 669

G.9 Plots for layer evaluation methods: sulphidation index, $\mathrm{Fe} /(\mathrm{Fe}+\mathrm{Mg}+\mathrm{Ca}) \quad$. . 670

G.10 Plots for layer evaluation methods: sulphidation index, $\mathrm{Fe} \% * \mathrm{Fe} /(\mathrm{Fe}+\mathrm{Mg}+\mathrm{Ca}) 671$

G.11 Plots for layer evaluation methods: oxidation index, $\mathrm{Fe}_{2} \mathrm{O}_{3} /\left(\mathrm{Fe}_{2} \mathrm{O}_{3}+\mathrm{FeO}\right)$. . 672

G.12 Plots for layer evaluation methods: oxidation index, $\mathrm{Fe}_{2} \mathrm{O}_{3} /(\mathrm{FeO}) \ldots . .673$ 
G.13 Plots for layer evaluation methods: oxidation index, $\mathrm{Fe}^{2+} / \mathrm{Fe}^{3+} \ldots . . .674$

G.14 Plots for layer evaluation methods: distance to felsic porphyritic intrusions 675

G.15 Plots for layer evaluation methods: airborne total magnetic intensity . . . . 676

G.16 Plots for layer evaluation methods: Bouguer gravity anomalies . . . . . . 677

G.17 Plots for layer evaluation methods: distance to magnetic anomalies (+ve,

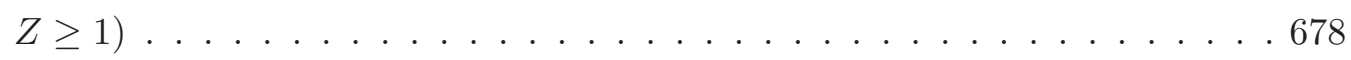

G.18 Plots for layer evaluation methods: distance to magnetic anomalies (+ve,

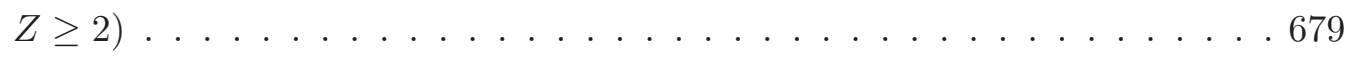

G.19 Plots for layer evaluation methods: distance to magnetic anomalies (-ve,

$Z \leq 1) \ldots \ldots \ldots \ldots$. . . . . . . . . . . . . . . . . . . . . . . . .

G.20 Plots for layer evaluation methods: distance to crustal-scale shear zones . . 681

G.21 Plots for layer evaluation methods: distance to NNW-trending regionalscale shear zones . . . . . . . . . . . . . . . . . . . . 6882

G.22 Plots for layer evaluation methods: distance to NE-trending regional faults 683

G.23 Plots for layer evaluation methods: distance to E-trending regional faults . 684

G.24 Plots for layer evaluation methods: strike of crustal-scale shear zones . . . . 685

G.25 Plots for layer evaluation methods: strike of NNW-trending regional-scale shear zones . . . . . . . . . . . . . . . . . . 6866

G.26 Plots for layer evaluation methods: strike of NE-trending regional-scale shear zones . . . . . . . . . . . . . . . . . . 687

G.27 Plots for layer evaluation methods: strike of E-trending regional-scale shear zones . . . . . . . . . . . . . . . . . . . . . . . 688

G.28 Plots for layer evaluation methods: distance to intersection of regional-scale

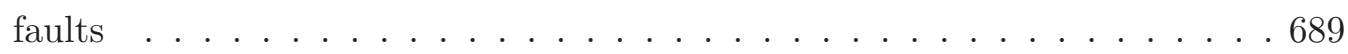

G.29 Plots for layer evaluation methods: subjective (fuzzy) rheological contrast . 690

G.30 Plots for layer evaluation methods: subjective (classes) rheological contrast 691

G.31 Plots for layer evaluation methods: rheological contrast, Young's Modulus

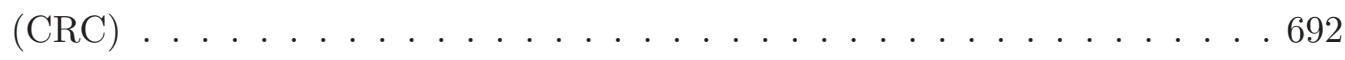

G.32 Plots for layer evaluation methods: rheological contrast, Young's Modulus

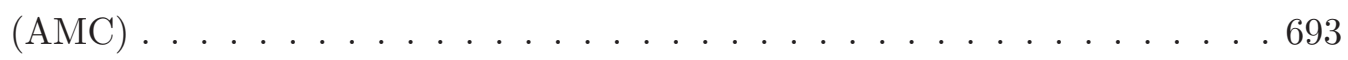

G.33 Plots for layer evaluation methods: rheological contrast, UCS . . . . . . . 694

G.34 Plots for layer evaluation methods: rheological contrast, UCS . . . . . . . 695

G.35 Plots for layer evaluation methods: rheological contrast, UCS . . . . . . . 696

G.36 Plots for layer evaluation methods: chemical contrast, $\mathrm{Fe} /(\mathrm{Fe}+\mathrm{Mg}+\mathrm{Ca})$. . . 697

G.37 Plots for layer evaluation methods: chemical contrast, $\mathrm{Fe} \% * \mathrm{Fe} /(\mathrm{Fe}+\mathrm{Mg}+\mathrm{Ca}) 698$

G.38 Plots for layer evaluation methods: chemical contrast, $\mathrm{Fe}_{2} \mathrm{O}_{3} /\left(\mathrm{Fe}_{2} \mathrm{O}_{3}+\mathrm{FeO}\right)$. 699

G.39 Plots for layer evaluation methods: $\mathrm{Fe}_{2} \mathrm{O}_{3} /(\mathrm{FeO}) \ldots \ldots . . . .700$ 
G.40 Plots for layer evaluation methods: chemical contrast, $\mathrm{Fe}^{2+} / \mathrm{Fe}^{3+} \ldots \ldots . .701$

G.41 Plots for layer evaluation methods: strike of lithological contacts . . . . . . 702

G.42 Plots for layer evaluation methods: distance to nearest lithological contact . 703

G.43 Plots for layer evaluation methods: density of regional faults . . . . . . . . 704

G.44 Plots for layer evaluation methods: lithological complexity . . . . . . . . . . 705

G.45 Plots for layer evaluation methods: favourable lithological contacts (fuzzy) . 706

G.46 Plots for layer evaluation methods: metamorphic grade zones . . . . . . . 707

G.47 Plots for layer evaluation methods: distance to anticlinal axes . . . . . . . 708

G.48 Plots for layer evaluation methods: distance to synclinal axes . . . . . . . . 709 


\section{Acknowledgements}

First, I must thank my supervisors, Prof. David Groves, Prof. Tom Gedeon, and Dr Carl Knox-Robinson, for all their help and advice throughout this study.

I am especially grateful to Stephen Gardoll for his assistance in the use of ARC/INFO and ARCVIEW, for ideas and suggestions on GIS and prospectivity mapping and his general enthusiasm.

Mr Robert Barnes of the Geological Survey of New South Wales is thanked for providing GIS data for the Tenterfield area. The GEODIPS GIS prospectivity analysis package written by Jusmady and Assoc. Prof. Geoff Taylor of the Department of Applied Geology, University of New South Wales, was used to create prospectivity maps presented in the pilot study. Its availability for this project is gratefully acknowledged.

After completing the pilot study based on the Tenterfield data, one of the difficulties was to find a suitable data set for a case study. My thanks go to Dr Scott Halley at Placer Dome (formerly Goldfields Exploration Pty Ltd) who solved this problem by providing the GIS data for the Kalgoorlie area and for sharing valuable insights on exploration for Archaean orogenic gold deposits.

Data were generously provided, in chronological order, by; Dr Chris Pilgram (Chief, Minerals Division) and Dr Kevin Cassidy of Geoscience Australia for kindly providing part of the Ozchem whole-rock geochemical database, Mr Max Lee (Australian Mining Consultants) for supplying rock strength data, Mr David Townsend (Geological Survey of Western Australia) for permission to use the Mines and Mineral Deposits Database (MINEDEX 2000), Mr Paul Hodkiewicz for a formatted and corrected version of MINDEX, Mr Steve Bandy (Manager, Geoscience Data Management, Geological Survey of Western) for a sample metadata file. I would also like to thank Dr Tony Zaknich and Dr Charles Willock for their advice and useful discussions.

Special thanks go to my friends Josef Holzschu, June Hill, and Elizabeth Colgan for their help at critical times and to Rob Stuart, Amanda Buckingham, and Steve Garwin for all those chats and motivational sessions (DIG - read red wine and pasta). Life during the thesis just wouldn't have been the same without you.

Support for this study in the form of a Australian Postgraduate Award, a grant from the Bicentennial Gold 88 Endowment Fund (administered by the Australasian Institute of Mining and Metallurgy) and Geoconferences W.A Inc. (1998) and J. H. Lord (1998) 
travel grants are gratefully acknowledged.

Dr Joe Leach is thanked for contributing the initial idea to include neural networks in this study.

Finally, to Hertha - thank you for all your patience and support, and particularly for putting up with these last two years. 


\section{Preface}

This study represents the first comprehensive study of the application of neural networks to mineral prospectivity mapping. At the time this study was commenced in 1997, there were only two published reports on the use of neural networks for mineral prospectivity mapping. No comparative studies of neural networks and statistical- or fuzzy systems-methods existed until 1999 and, even then, the methods are described only in general terms by the researchers. Little information existed to indicate which factors might be important in determining the performance of a neural network approach to mineral prospectivity mapping.

Consequently, much of the methodology for pre- and post-processing, selection of inputs, and the quantitative assessment of map quality needed to be developed and documented within this study. In addition, many alternative parameters for assessing the quality of the GIS layers selected as neural network inputs, and methods for assessing the quality of the output prospectivity maps, had to be tested in this study. This contrasts with the situation in more established fields, such as the classification of remotely-sensed images or visual information retrieval, where methodologies and metrics for evaluating performance are better established.

All of these factors have contributed to the length of this thesis. To assist the reader to assimilate this material, important aspects and results are presented as a summary section at the end of each chapter. 


\section{Chapter 1}

\section{Introduction}

\subsection{Problem Statement}

The development of faster, more efficient and objective Geographic Information Systems (GIS)-based methods for the integration and analysis of regional exploration data sets has a potentially important role in supporting the decision making process for geologists in terms of ground acquisition and the selection of exploration targets.

There is a need for such methods as the volume and complexity of regional data available to the exploration geologist is increasing rapidly from a variety of sources. For example, remote sensing, airborne geophysics (Hone et al., 1997) and large commercially-available geochemical databases compiled from open-file exploration reports (Beams, 1995) all contribute to the increasing volume of digital map data in exploration programs. New developments such as the use of airborne electromagnetic methods as a regolith and bedrock geological-mapping tool (Green \& Munday, 1998), airborne gravity gradiometers (Hannaford \& Opat, 1998) and differential vector magnetometers (Schmidt, 1998) are likely to continue this trend. As the amount of data increases, so too will the need for an effective means of integration and analysis (Cucuzza \& Goode, 1998).

GIS represent an ideal environment for the integration and analysis of map data (Wyborn et al., 1995b; Burrough \& McDonnell, 1998). Although GIS are emerging as an important technology in mineral exploration, the maximum benefit to be derived from the considerable time and cost of entering map data into the GIS are not realized if the system is only used to store and print paper maps. Despite the role of digital map data in exploration and the increasing difficulty of finding new world-class ore deposits, there has been relatively little research on methods to combine large regional data sets within a GIS in a way that can assist geologists. The process of combining digital map data from multiple sources 


\section{Problem: How to combine exploration data sets?}

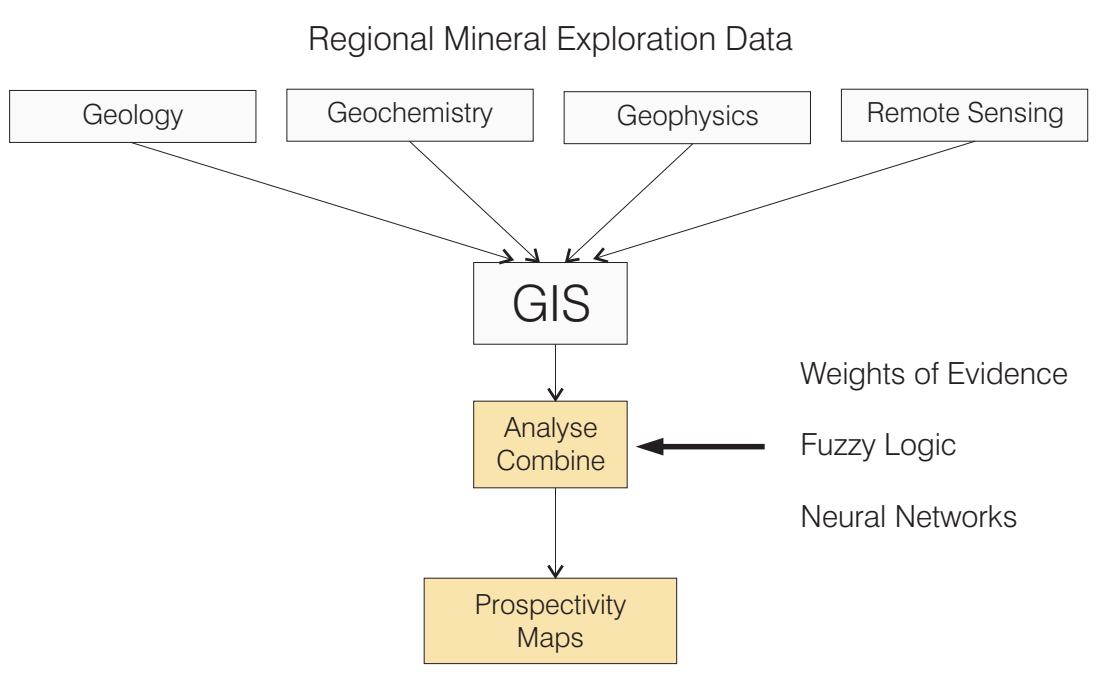

Figure 1.1. Illustration of how this study fits into the task of regional mineral exploration.

in order to produce a single mineral prospectivity map, depicting areas ranked according to their potential to host mineral deposits of a particular type, is the focus of this study (Fig 1.1).

Existing methods for producing prospectivity maps have some important limitations. Empirical statistically-based methods, for example, weights of evidence (WofE) based on Bayesian probability (Bonham-Carter et al., 1988), require a statistically significant number of mineral occurrences to estimate the relative importance of geological evidence associated with the deposits, and are, therefore, not applicable in poorly-explored areas containing few known deposits.

Conceptual methods, such as index overlay or fuzzy logic (An et al., 1991; Eddy et al., 1995), and the specific and interactive modules of the mineral systems approach developed by Wyborn et al. (1994c, 1995b), overcome this problem, but rely on a deposit expert to make subjective assessments. All these conceptual methods depend on the application of a deposit model to decisions about which geological parameters might be significant. The problem here is that new deposit types are still being discovered; for example, Olympic Dam (Woodall, 1994), and models for well-investigated deposit styles are constantly being revised in the light of new discoveries, such as, Century (Legge, 1995).

A further disadvantage of existing methods is that they are either exclusively conceptual (deposit-model-driven) or empirical (data-driven). On the other hand, Woodall (1994) has emphasized that discoveries are made when the right blend of conceptual and empirical 
evidence is applied. Part of the potential of a neural network system lies in the possibility of creating hybrid systems that enable the geologist's knowledge of deposit models and processes to be incorporated into the search for patterns and important relationships in very large data sets.

\subsection{Generation and Statement of Hypothesis}

Artificial neural networks ${ }^{1}$ offer a new approach to the problem of mineral-prospectivity mapping. Neural networks represent a type of adaptive computing system that can learn from the data. Properties of neural networks that make them particularly suitable for pattern recognition and classification include their ability to: 1) extract underlying patterns from a data set which may be imperceptible to humans and to standard statistical techniques, 2) require no statistical model for the data, 3) operate without the need for pre-existing knowledge (e.g. a deposit model), 4) operate at acceptable accuracy when the data are noisy or contains outliers, 5) perform well when the input parameters are inter-dependent and exhibit significant nonlinearity, 6) be flexible and retrained when new data become available, and 7) be used on large mixed data sets.

These features suggest that neural networks could be well suited to integration of mineral exploration data. By analogy to the way in which bands in remotely-sensed imagery are combined into composite images to create signatures for alteration minerals, digital geological, geophysical and geochemical maps can be superimposed in a GIS to produce an image from which the signatures of ore deposits can be extracted. The thrust of this study is that neural networks can be trained to recognize these signatures, and hence predict the location of new deposits of similar type. To test this concept, a neural network is used to integrate regional exploration data from a small GIS database into a single prospectivity map, and the performance of the neural-network approach is compared with Bayesian statistical and fuzzy-logic methods.

\footnotetext{
${ }^{1}$ The term artificial neural network is used to distinguish artificial neural networks implemented as computer software or hardware from the biological networks upon which they were originally based. Since only the former type is discussed in this paper, they are referred to as neural networks.
} 


\subsection{Aim of the Research}

\subsubsection{Primary Aims}

The main aims of this study are to;

1. investigate the application of a combined neural network GIS approach to the problem of integrating and analyzing geoscientific data sets to produce mineral prospectivity maps,

2. to evaluate the neural-network approach and quantitatively compare it with existing empirical and conceptual methods for mineral prospectivity analysis, and

3. test the general applicability and limitations of the neural-network approach using a GIS database typical of those used routinely by the mining industry in exploration.

One of the difficulties in this study is that little previous work has been done on the topic. At the time this study commenced, there was only one paper in the literature that described the use of neural networks to produce a mineral prospectivity map (Singer \& Kouda, 1996). Consequently, there is a huge range of aspects of the problem that could be investigated. What type of neural network architecture is best suited to the analysis of mineral prospectivity? What type of pre-processing should be performed? What training strategies can be used to cope with the enormous imbalance in the ratio of positive to negative training examples (i.e. deposits to barren cells)? How should the relative importance of input features be assessed? The question as to whether a neuralnetwork approach can work with a range of deposit types and GIS data sets in which the quality, extent of coverage, types of thematic layers and scale of data capture and compilation vary, is also important and can only be answered with case studies. How does the performance of a neural-network approach compare to existing methods is a question that is also addressed in this work.

The problem with all of this is a combinatorial one. Each network architecture could be trained with a variety of subsets of input features, network parameters (e.g. learning algorithms, topologies) and training strategies, (e.g. encoding of categorical input features using 1-of- $n$ or fuzzy membership values) and tested on different GIS databases. For each of the two databases analysed in this study, the results of a neural-network approach could be compared with examples of existing empirical and conceptual methods (the weights-ofevidence method and fuzzy logic are selected). This potentially amounts to a large number of mineral prospectivity analyses. 
To limit this scope, the weights-of-evidence methods is only applied to the database used in the pilot study. Recent work by Harris \& Pan (1999), Singer \& Kouda (1999a), and Kemp et al. (2001) suggests that probabilistic, general regression and radial basis function neural networks are suited to mineral exploration applications. However, the MLP network is the most commonly used type of neural network. It has powerful generalization abilities and is applicable to a wide range of applications. Consequently, an MLP network architecture is chosen for this study. MLP networks are used for experiments on the effect of input feature selection, training strategy, and the effect of incorporating expert knowledge on the performance of a neural-network approach to mineral-prospectivity mapping.

\subsubsection{Secondary Aims}

An important aspect of this work is to determine the most effective way of applying neural networks to the problem of mineral-prospectivity mapping.

One of the common criticisms of neural networks is that it is not possible to predict from theory what the most suitable network architecture, topology and parameters for a given problem should be. Also, before a neural network can be used to classify patterns, the raw data must be converted to a form suitable for processing by a neural network and attributes, known as features, which are relevant to solving a problem must be selected from the data. Pre-processing must also be adapted for each individual problem. Consequently, a secondary aim is to develop and evaluate a variety of different pre-processing strategies in order to improve neural network performance.

Since a vast number of different neural network architectures and learning algorithms exist today, another secondary aim is to test the suitability of selected types of neural networks for the task of estimating mineral prospectivity.

A general goal in this work is to make use of all the available sources of information in order to improve the mineral prospectivity analysis. In remote sensing applications, expert knowledge and ancillary information are important for generating reliable classifications (Milne, 1999). Novel techniques like stress mapping (Groves et al., 1997), shape analysis (Gardoll et al., 2000), and layers that depict essential components in a mineral system (Wyborn et al., 1994c) could be used to generate additional map layers to supplement the traditional geology, geochemistry and geophysics used in mineral exploration. As an example of this latter type of layer, features like hydrothermal alteration zones, metamorphic grade, favourable host-rock geochemistry, or zones with a high fracture-related permeability could be used to produce input layers suitable for processing by a neural net- 
work. Subjective geological knowledge of the type used in the fuzzy-logic method could be used as additional input to a neural network.

\subsubsection{Limitations}

Several ways in which the scope of this study has been limited are listed below.

1. Prospectivity mapping is only performed in two dimensions in the plane of geological maps. Although GIS can be extended to deal with three dimensions (Wyborn et al., 1996a), such systems are currently in the early research stages and are not commercially available. A three-dimensional approach on a regional scale for prospectivity analysis would also be difficult due to the very interpretive nature of cross sections at this scale and the limited availability of suitable data.

2. Although MLP neural networks form the basis for this study, a wide variety of neural network architectures have been developed since the mid-eighties. A review of six commonly-used architectures, which appear to have characteristics suitable for prospectivity analysis problems is presented in Appendix A. These networks cover a range of paradigms. Four networks (MLP, probabilistic, radial basis function, and general regression neural networks) require supervised training, in which training data comprise examples of both the inputs and the required outputs. This contrasts with the Kohonen neural networks which require unsupervised training. Here it is not necessary to know which class the patterns used in training belong to: that is, whether the patterns correspond to deposit locations or not. Only MLP networks and a hybrid fuzzy-neural network system similar to that developed by Kasabov \& Kozma (1999) are tested in Chapter 7.

3. This study does not attempt to apply neural networks to the analysis of geological patterns within a single two-dimensional plan or image. One of the great strengths of neural networks is their ability to recognize and classify patterns in two dimensional images. An example is the use of neural networks to recognize hand-written digits in post-codes (Le Cun et al., 1989). The recognition of roughly circular-shaped features in topographic or magnetic anomaly maps corresponding to kimberlite pipes is a geological example in which pattern recognition could be applied in a single map layer. Pattern analysis in geological maps to define analogues of the patterns of folded strata and rock-competency contrasts associated with deposits has been applied to Archaean lode-gold deposits in the Kalgoorlie area, Western Australia (Gardoll et al., 2000). Although neural networks could be applied to these two 
geological tasks, pattern recognition and image understanding represent very difficult problems in artificial intelligence and are the subject of intensive research in the fields of robotics and machine vision (Cawsey, 1998). Each one of these two examples would constitute a major investigation.

\subsection{Research Approach}

The research in this thesis consists of two main parts; a pilot study and a series of experiments based on a case study. The pilot study, based on intrusion-related gold deposits in the Tenterfield area, New South Wales (N.S.W.), was conducted to test the viability of using a neural network to produce a prospectivity map (Brown et al., 1997, 1999, 2000a).

The second part of this work consists of a case study based on Archaean lode-gold deposits in the Kalgoorlie area, W.A. Australia. The work is designed to test the general applicability and limitations of the neural network method on a large geoscience data set used in current exploration (Chapter 5).

The Kalgoorlie study area is the basis for a series of experiments aimed at improving the performance of the neural network method used in the pilot study. This investigation is based on the five main steps involved in solving a problem with a neural network as described by Zaknich (1999);

1. data collection,

2. pre-preprocessing,

3. selection and combination of attributes from data (feature extraction),

4. selection of an neural network type and topology, and

5. neural network training and testing.

As the databases in this study have been compiled at the University of New South Wales (Barnes, 1993; Jusmady \& Taylor, 1997) and Goldfields Exploration (now Placer Dome), an examination of steps 1 and 2 do not form part of this study.

It is generally recognized that the success of any neural network application depends, not only on the information supplied as input, but also, on how effectively the problem is presented to the network. Although neural networks are robust with respect to noise and can deal with irrelevant and confusing data, the training time is reduced and classification accuracy is improved if the task of the neural network is kept as simple as possible (Masters, 1993b; Zaknich, 1999). This can be achieved by performing as much work as possible 
in the initial pre-processing and feature selection stages. The effect of feature selection on network performance and on the quality of the resultant mineral prospectivity maps is investigated in Chapter 6. The possibility of improving the predictive quality of mineral prospectivity maps by incorporating subjective expert knowledge into the otherwise empirical neural-network approach is investigated in Chapter 5. The expert knowledge can be built into GIS layers that are derived from the primary data. The effect of using different types of training data and training strategies on network performance and map quality is also investigated in Chapter 7 .

Maps produced in this study, using the neural network method, are checked in two ways.

1. The neural-network maps are compared to those produced with the empirical weights of evidence and fuzzy-logic methods. A comparison with the weights-of-evidence method establishes whether the neural network result accounts for the major spatial relationships between map features and known deposits. Comparison with the fuzzylogic map shows whether the neural network accounts for current understanding of the factors important for controlling the location of mineralization.

2. A range of statistical and probability measures of prospectivity map quality are used to assess the effectiveness of different implementations of the neural network method.

\subsection{Organization of Thesis}

Chapter 2 discusses previous work in mineral-prospectivity mapping, the application of neural networks in mineral exploration, and other artificial intelligence and statistical techniques that could potentially be used for prospectivity analysis.

Chapter 3 outlines mineral prospectivity methods, GIS databases, software and hardware used in the thesis.

Chapter 4 describes a pilot study, based on intrusion related and placer gold deposits in the Tenterfield 1:100000 map sheet area, which tests the feasibility of using an MLP neural network to produce a mineral-prospectivity map.

Chapter 5 describes the Kalgoorlie study area, and the processing required to produce primary and derived GIS layers. A number of techniques for including geological knowledge and subjective judgement into GIS layers are demonstrated. These techniques include the use of novel derived GIS layers, which depict parameters that represent important controls on the location of mineralization. 
Chapter 6 applies a variety of statistical techniques to test the candidate GIS layers in order to select best predictors of known deposits as the inputs for neural networks.

Chapter 7 consists of a series of experiments based on the Kalgoorlie case study area. The experiments systematically test factors affecting the performance of neural networks and the quality of mineral prospectivity maps. Methods for improving the results and for overcoming limitations to the neural networks are also investigated.

Chapter 8 summarizes and discusses the key results of the work and includes suggestions for future work.

Appendix A explains the organization of the CD-ROM's, which contain all GIS databases used in the study, and describes metadata standards used to document the data.

Appendix B documents the Tenterfield GIS database used for the pilot study.

Appendix $\mathbf{C}$ documents the Kalgoorlie GIS database used for the case study.

Appendix D describes the neural network processing based on $\mathrm{C}++$ programs used in the pilot study.

Appendix E lists and briefly describes matlab functions and scripts used for the Kalgoorlie case study.

Appendix F contains graphs and tables used in the evaluation of candidate GIS input layers for the Kalgoorlie study area.

Appendix G contains tables documenting the detailed results of MLP neural network and fuzzy systems experiments based on the Kalgoorlie case study.

Appendix $\mathbf{H}$ contains tables documenting the detailed results of neural network and fuzzy systems experiments based on the Kalgoorlie case study.

Appendix I gives an explanation of the ArcVIEw layer names in the Kalgoorlie GIS database.

CD-ROM 1 contains the ARCVIEw database, $\mathrm{C}++$ programs used in the pilot study, and MATLAB programs used in the case study.

CD-ROM 2 contains input GIS layers and mineral prospectivity maps in GEOSOFT format and an interface for viewing the maps. 


\subsection{Publications Based on this Study}

This study is supported by the following publications, of which the author was the major contributor.

\section{Articles}

Brown, W. M., Gedeon, T. D. \& Groves, D. I. 2003. Use of noise to augment training data: a neural network method of mineral potential mapping in regions of limited known deposit examples. Natural Resources Research, 12, p. 141-151.

Brown, W. And Groves, D. I. \& Gedeon, T. D. 2003. Use of fuzzy membership input layers to combine subjective geological knowledge and empirical data in a neural network method for mineral potential mapping. Natural Resources Research, 12, p. 183-200.

Brown, W. M., Gedeon, T. D., Baddeley, A. J. \& Groves, D. I. 2002. Bivariate J-function and other graphical statistical methods help select the best predictor variables as inputs for a neural network method of mineral prospectivity mapping. In: Bayer, U. and Burger, H. and Skala, W. eds IAMG 2002 8th Annual Conference of the International Association for Mathematical Geology, vol. 1, pp. 263-268. International Association of Mathematical Geology.

Brown, W. M., Gedeon, T. D., Groves, D. I. \& Barnes, R. G. 2000. Artificial neural networks: a new method for mineral prospectivity mapping. Australian Journal of Earth Sciences, 47 757-770.

Brown, W. M., Gedeon, T. D. \& Barnes, R. G. 1999. The use of a multilayer feedforward neural network for mineral prospectivity mapping In: Gedeon, T., Wong, P., Halgamuge, S., Kasabov, N., Nauck, D., and Fukushima, K. eds ICONIP'99, Proceedings of the 6th International Conference of Neural Information Processing, vol. 1, pp. 160-165. IEEE, Piscataway.

\section{Submitted}

Brown, W. M., Gedeon, T. D. \& Groves, D. I. 2002. Use of noise to augment training data: a neural network method of mineral potential mapping in regions of limited known deposit examples. Natural Resources Research, in press.

Brown, W. M., Groves, D. I. \& Gedeon, T. D. 2002. Use of fuzzy membership input layers to combine subjective geological knowledge and empirical data in a neural network method for mineral potential mapping. Natural Resources Research, in press.

\footnotetext{
Abstracts

Brown, W. M., Gedeon, T. D. \& Groves, D. I. 2002. Use of noise to augment training data to compensate for lack of deposit examples in training a neural network for mineral potential mapping In: Kouda, R., Koike, K., Singer, D. \& Couglan, J. eds Abstracts and Programs for Seventh International Symposium on Mineral Exploration: Application of Neural Networks to the Earth Sciences, 20-21 August, NASA Moffett Field, Mountain View, California, pp. 9.

Brown, W. M., Groves, D. I. \& Gedeon, T. D. 2002. Use of fuzzy membership input layers to combine subjective geological knowledge and empirical data in a neural network method for mineral potential mapping In: Kouda, R., Koike, K., Singer, D. \& Couglan, J. eds Abstracts and
} 
Programs for Seventh International Symposium on Mineral Exploration: Application of Neural Networks to the Earth Sciences, 20-21 August, NASA Moffett Field, Mountain View, California, pp. $10-11$.

Brown, W. M., Gedeon, T. D., Groves, D. I. \& Barnes, R. G. 2000. Mineral prospectivity mapping: a comparison of MLP neural network, fuzzy logic and weights of evidence methods In: 15th Australian Geological Convention, Sydney, Abstracts, vol. 59, pp.63. Geological Society of Australia.

Brown, W. M., Taylor, G. R., Jusmady, Groves, D. I. \& Knox-Robinson, C. M. 1997. Application of artificial neural networks to prospectivity analysis in a GIS environment: a comparison with statistical and fuzzy-logic methods for Au and Sn deposits of the Tenterfield area, NSW. In: 14th Australian Geological Convention, Townsville, Abstracts, vol. 49, pp.57. Geological Society of Australia. 


\section{Chapter 2}

\section{Review of the Literature}

\section{$2.1 \quad$ Introduction}

In Chapter 1, the need for efficient methods of integration and analysis of multisource data sets is presented in the context of the large amounts of digital data currently generated in regional exploration programmes and the limitations of two of the most popular current methods; i.e. weights of evidence and fuzzy logic. It is the purpose of this chapter to examine previously-used methods and to summarize some of their strengths and weaknesses in order provide a background against which any potential neural network method can be compared.

It is also suggested in Chapter 1 that neural networks have many characteristics that appear to be particularly suited to geoscience applications and that neural networks in combination with a GIS represent a potentially useful new approach to mineral-prospectivity mapping. Since the GIS is an integral part of any mineral prospectivity method, key aspects of GIS that relate to this study are also briefly reviewed. This is followed by an examination of some neural network concepts relevant to the multilayer feedforward neural network, which is the main class of neural networks used in this study. Application of neural networks to geoscientific applications, particularly in spatial analysis and mineral exploration is also discussed.

A great variety of different types of neural networks exist. Although this chapter contains a review of the theory and applications relating to MLP neural networks, other architectures that are potentially useful for mineral-prospectivity mapping are described in Appendix A. 


\subsection{Mineral-Prospectivity Mapping Methods}

For the purpose of this review, mineral prospectivity or favourability mapping is defined as the process of making regional scale maps (1:50000 - 1:250,000) showing the relative potential to host a mineral deposit of a particular type. Singer \& Mosier (1988) suggested that the range of methods for regional assessment of mineral resources should be considered in terms of who is going to use the results and the required form of the output. This study is concerned with the way geologists involved in the early stages of regional exploration can combine and analyze geoscience data sets to make decisions about ground acquisition and target selection. Consequently, the focus of this review is on methods that

1. can be used to combine many different types of spatial geoscientific data sets,

2. produce output in map form,

3. estimate favourability according to geological rather than economic criteria,

4. apply to a single type or group of metallic mineral deposits, and

5. apply to regional-scale mineral exploration rather than land assessment.

Such criteria exclude mineral resource assessment undertaken for economic forecasting purposes. Some of these methods are described by Singer \& Mosier (1988), which they refer to as crustal abundance methods, cumulative-tonnage-frequency methods. These methods make an overall assessment of a large region and do not take account of local variations in geology and do not attempt to predict the probable location of individual deposits. For example, Allais (1957) used statistics on the deposit occurrences and values in France and Western United States to predict the expected number and value of deposits for a one million square kilometre area in the Algerian Sahara. The study was based on the following assumptions; 1) geologic conditions are uniform (in particular, that control and study areas are similar), 2) frequency of deposits follows a Poisson distribution, 3) value of deposits follows a log-normal distribution, and 4) nearly all deposits in the control areas have been discovered. The calculation of total endowment by Allais (1957) in terms of the value of contained metal required estimates of the number of deposits per square kilometre, and the grade and tonnage of deposits. By contrast, the goal in this study is much more limited; that is, to estimate the relative favourability of areas represented by grid cells to host mineralization of a specific type.

\footnotetext{
${ }^{0}$ Methods used in this study are reviewed in Chapter 2. The other main mineral prospectivity mapping methods are reviewed in Appendix A
} 
A list of the methods which fulfill the criteria listed above, and which are considered in this review, are shown in Table 2.3. Bonham-Carter (1994) distinguished two broad categories of mineral favourability mapping methods; data-driven (or empirical) and knowledgedriven (or conceptual) methods. These categories are indicated in Table 2.3 on page 56.

The historical development of mineral-prospectivity mapping methods is shown in Table 2.3, which lists methods according to the chronological order in which they were first applied to mineral-prospectivity mapping.

\subsubsection{Overview}

The initial work on integrating spatial geoscience data sets generally took an empirical or data-driven approach and was based on statistical methods (Table 2.3). A range of techniques are described in the literature, from simple statistical techniques, such as correlation coefficients and Boolean logic (Bates, 1959), to multivariate analysis techniques such as; multiple linear regression (Sinclair \& Woodsworth, 1970; Agterberg et al., 1972), cluster analysis (Briggs \& Press, 1977), multiple discriminant analysis (Prelat, 1977), a form of principal component analysis (Botbol et al., 1978), logistic regression (Chung \& Agterberg, 1980), Bayesian statistics combined with frequency distributions and an area of influence procedure (Singer \& Kouda, 1988), weights-of-evidence (WofE) (Bonham-Carter et al., 1988) and canonical correlation (Pan \& Harris, 1992).

Some earlier work dealt with conceptual approaches. For example, although the work of Bates (1959) was statistical, the technique used to integrate layers was Boolean logic . Harris (1973) combined subjective estimates of the number of deposits, grade and tonnages with statistics to estimate the total metal endowment. Koch et al. (1981) and Reddy \& Koch (1988) used a method involving a favourability score which is equal to a linear combination of geological variables in which the weighting factors were estimated by a subjective method. Both methods are equivalent to subjective overlay methods described by Bonham-Carter (1994). A different type of conceptual approach has been developed by Wyborn et al. (1994c, 1995b). Rather than focusing on the integration of existing data sets, the mineral systems approach involves identifying parameters considered essential for ore formation (e.g. source rock, reactive minerals, structures for fluid infiltration) and translating these parameters into mapping criteria or attributes that may be extracted form a regional-scale GIS database. It should be noted though that this approach to the creation of input layers could be incorporated into any of the methods shown in Table 2.3. 
Knowledge-driven or conceptual methods feature in later work and employ a range of artificial intelligence approaches ${ }^{1}$ such as expert systems ${ }^{2}$ (Duda et al., 1978; Katz, 1991; Reddy et al., 1992), Dempster-Shafer evidential belief theory (Moon, 1990), fuzzy systems (An et al., 1991), decision-tree induction systems (Reddy et al., 1992; Bui et al., 1998), and a hybrid statistical-fuzzy-logic approach (Knox-Robinson \& Groves, 1997a). Neural networks represent a data-driven approach within the field of artificial intelligence.

The following sections review the theory and literature describing mineral prospectivity methods which are directly used in this thesis. Other methods that have previously been used to predict the potential for mineral deposits are described in Appendix A. An excellent review of a variety of methods for analysing and combining geoscience data sets is given by Pan \& Harris (2000).

\subsubsection{Weights of Evidence}

Weights of evidence is a statistical method that uses a log-odds formulation of Bayesian probability to update prior probability estimates on the basis of evidence. The method was developed for medical diagnosis (Lusted, 1968; Aspinall, 1983; Spiegelhalter \& KnillJones, 1984) and later adapted to spatial data and mineral exploration by Bonham-Carter et al. (1988), Agterberg (1989c) and Agterberg et al. (1990). The following summary of the weights-of-evidence method is based on descriptions by Bonham-Carter et al. (1988, 1989) and Bonham-Carter (1994).

The weights-of-evidence method is based on the concept of prior and posterior probabilities. If each deposit is assumed to occupy a single cell (where data are in raster format), then the prior probability of any cell chosen at random containing a deposit is equal to the ratio of the number of deposit cell to the total number of cells:

$$
P(D)=\frac{n(D)}{n(T)}
$$

where $D$ represents the event that a cell contains a deposit and $T$ is the total number of cells in the map.

This prior probability can be updated using additional information, in the form of binary

\footnotetext{
${ }^{1}$ The term artificial intelligence has tended to be replaced with the terms intelligent computing or soft-computing.

${ }^{2}$ Expert systems are now more commonly referred to as knowledge-based systems.
} 
maps depicting the presence or absence of some parameter favourable for mineralization ${ }^{1}$ An example of a favourable map pattern in the case of exploration for skarn deposits could be "presence of limestone" or "magnetic anomaly". If $P(D \mid E)$ represents the conditional probability of a deposit occurring in a grid cell given the presence of an evidence pattern $E$ that is favourable for mineralization in a binary evidence map, then Bayes' rule states that:

$$
P(D \mid E)=\frac{P(E \mid D)}{P(E)} \cdot P(D)
$$

In equation 2.2 , the prior probability $P(D)$ is updated with the factor $P(E \mid D) / P(E)$ to give the posterior conditional probability, $P(D \mid E)$.

In order to extend this approach to multiple maps, an assumption of conditional independence between parameters with respect to deposits must be made; that is, the effects of the interactions between parameters used as evidence to modify the prior probability must be ignored. The approach is same as the one used in Bayesian updating of probabilities in expert systems described in equations A.22-A.26 on page 455. The discussion in the next section is based on the description by Stackhouse \& Boyd (1985). Bayes' rule for $N$ items of evidence (i.e. binary maps containing patterns favourable for mineralization) is:

$$
P\left(D \mid E_{1} \cap E_{2} \cap \ldots \cap E_{n}\right)=\frac{p\left(E_{1} \cap E_{2} \cap \ldots E_{n} \mid D\right)}{P\left(E_{1} \cap E_{2} \cap \ldots \cap E_{n}\right)} \times P(D)
$$

If events $E_{1}, E_{2}, \ldots, E_{n}$ are assumed to be conditionally independent then:

$$
P\left(E_{1} \cap E_{2} \cap \ldots \cap E_{n} \mid D\right)=P\left(E_{1} \mid D\right) \times P\left(E_{2} \mid D\right) \times \ldots \times P\left(E_{n} \mid D\right)
$$

Substituting equation 2.4 into the denominator on the right-hand side of equation 2.3

\footnotetext{
${ }^{1}$ Although weights of evidence has usually been applied as a binary method, it is not restricted to binary data. A 3-class geological map was used as an input in the first application of the weights-ofevidence method by Bonham-Carter et al. (1988) and a geological map with over 20 classes was used in a study of the mineral potential of the Slave Province (Bonham-Carter et al., 1998a,b). Multiclass maps can be used as inputs without reclassification in the weights-of-evidence module of the software package for ArCView called ArCSDMKemp et al. (2001). Bonham-Carter (pers. comm.) has pointed out that the main reason for reclassification is to enable robust weight estimates in data sets with a small number of known deposits.
} 
gives:

$$
P\left(H \mid E_{1} \cap E_{2} \cap \ldots \cap E_{n}\right)=\frac{P\left(E_{1} \mid D\right) \times P\left(E_{2} \mid D\right) \times \ldots \times P\left(E_{n} \mid D\right)}{P\left(E_{1} \cap E_{2} \cap \ldots \cap E_{n}\right)} \cdot P(D)
$$

Converting to odds involves taking the ratio of the probability that the event occurs to the probability that the event does not occur (i.e. odds $O=P / \bar{P}$ ). Converting equation 2.5 to odds gives;

$$
\begin{aligned}
\frac{P\left(D \mid E_{1} \cap E_{2} \cap \ldots \cap E_{n}\right)}{P\left(\bar{D} \mid E_{1} \cap E_{2} \cap \ldots \cap E_{n}\right)} & =\frac{P\left(E_{1} \mid D\right) \times P\left(E_{2} \mid D\right) \times \ldots \times P\left(E_{n} \mid D\right)}{P\left(E_{1} \mid \bar{D}\right) \times P\left(E_{2} \mid \bar{D}\right) \times \ldots \times P\left(E_{n} \mid \bar{D}\right)} \cdot \frac{P(D)}{P(\bar{D})} \\
O\left(D \mid E_{1}, E_{2}, \ldots, E_{n}\right) & =O(D) \cdot \frac{P\left(E_{1} \mid D\right)}{P\left(E_{1} \mid \bar{D}\right)} \times \frac{P\left(E_{2} \mid D\right)}{P\left(E_{2} \mid \bar{D}\right)} \times \ldots \times \frac{P\left(E_{n} \mid D\right)}{P\left(E_{n} \mid \bar{D}\right)}
\end{aligned}
$$

Taking the natural logarithm of both sides of equation 2.6 allows several factors $P\left(E_{n} \mid D\right) / P(E)$, corresponding to multiple evidence maps to be expressed as the sum of weights as follows;

$$
\begin{array}{r}
\ln \left[O\left(D \mid E_{1} \cap E_{2} \cap \ldots \cap E_{n}\right)\right]=\ln [O(D)]+\sum_{i-1}^{N} W_{i}^{ \pm} \\
\text {where } \quad W_{i}^{ \pm}= \begin{cases}W_{i}^{+}=\ln \left[\frac{P\left(E_{i} \mid D\right)}{P\left(E_{i} \mid \bar{D}\right)}\right] & \text { if pattern } E \text { present } \\
W_{i}^{-}=\ln \left[\frac{P\left(\bar{E}_{i} \mid D\right)}{P\left(\bar{E}_{i} \mid \bar{D}\right)}\right] & \text { if pattern } E \text { absent } \\
0 & \text { if data are missing }\end{cases}
\end{array}
$$

and $E_{i}$ represents evidence patterns that are useful as predictors of mineralization. Equation 2.8 is used to calculate prospectivity expressed as a posterior probability in the weights-of-evidence method. The intuitive appeal of the weights-of-evidence method lies in the fact that the contribution of each factor considered favourable for mineralization can be calculated in terms of a pair of weights for each input binary evidence map separately and then combined using equation 2.8 above. The output prospectivity map is presented in terms of probability values calculated from the posterior log-odds.

A binary predictor map is produced by reclassifying multiclass maps. Selected categories of categorical data (e.g. rock types) or, in the case of ratio data (e.g, magnetic intensity), a selected range of values or a group of class intervals, are re-labelled as being favourable for 
mineralization. All other categories or values are considered unfavourable. These areas are assigned values of one and zero, respectively. The choice of categories or cut-off value used to re-classify the multiclass map is made so that the spatial association between deposit points and pattern is maximized. The proximity relationships between deposits and linear map features such as faults and lithological contacts can be quantified by using the spatial overlay functionality of a GIS to create a series of buffer zones around the relevant lines.

Bonham-Carter (1994) described a method for selecting the class threshold which maximizes the spatial association between the predictor patterns and deposit points using a quantity called contrast. Contrast is defined as:

$$
C_{W}=W^{+}-W^{-}
$$

Weights are calculated for cumulative distances and plotted against cumulative distance. A peak in the contrast-cumulative distance graph can be used to choose an optimal cut-off distance for which the spatial association between the deposit points and the buffer pattern is strongest. Optimal cut-off values for classed ratio data like magnetic intensity and radiometric response are also selected using this procedure. Bonham-Carter (1994) used the standard deviation of contrast, $S(C)$, and the studentized contrast, $C / S(C)$, to check the reliability of the contrast for any particular threshold value of class. Bonham-Carter (1994) states that a significant contrast should have a studentized contrast value greater than about 1.5 to 2.0. Further methods for the quantification of spatial relationships between deposits and geological features are discussed by Knox-Robinson (1994).

A pair of weights is calculated for each binary predictor map using the conditional probability ratios estimated from area proportions (where data are stored in vector format) or numbers of cells (raster format). The positive weight is used in equation 2.8 when the favourable pattern is present and expresses how the prior probability is upgraded by the presence of the pattern. The negative weight is used when the pattern is absent and downgrades the prior probability. The procedure for calculating posterior probabilities differs slightly according to whether input maps in the GIS database are stored in a vector or raster format. For input maps stored in vector format, a unique conditions map is prepared showing the set of polygons representing all the overlap combinations of the binary input maps. The appropriate positive or negative weights are then added to the prior log-odds depending on whether the favourable pattern in each map is present or absent in the particular overlap combination. In raster mode, the overlap conditions are iteratively determined and appropriate weights added for each cell in the grid representing the study area. 
The weights-of-evidence method has been applied to mineral prospectivity studies of turbidite-hosted vein gold deposits in the Meguma Terrane, Nova Scotia (Bonham-Carter et al., 1988, 1989). A comparison of the weights-of-evidence and logistic regression methods was made by Agterberg et al. (1993), who applied the methods to the prediction of hydrothermal vents on the East Pacific Rise. They concluded that mutually inter-related evidence maps usually lead to an overestimate of the posterior probabilities because the conditional independence assumption is violated. They demonstrated that the logistic regression method is less sensitive to this problem. Watson \& Rencz (1989) used the weights-of-evidence method to combine geological, geophysical, and geochemical data and predict the prospectivity for gold deposits in the Bathurst area, New Brunswick, Canada. More recent studies include the estimation of mineral prospectivity for epithermal gold deposits in Western United States (Raines, 1999), porphyry copper deposits in the Philippines (Carranza \& Hale, 2002) and Queensland, Australia (Scott \& Dimitrakopoulos, 2001), base-metal deposits in Rajasthan, India (Venkataraman et al., 2000), orogenic gold deposits in Ontario, Canada (Harris et al., 2001), Carlin gold deposits in Nevada (Mihalasky, 1999), and continental-scale resource assessment (Raines \& Mihalasky, 2002). An example of an application of weights of evidence outside the area of economic geology is that of Daneshfar \& Benn (2002) who investigated the spatial relationship between seismicity an faults.

Advantages of the weights-of-evidence method include (Bonham-Carter et al., 1989);

1. weights are easier to interpret than coefficients in multiple regression equations,

2. missing data are easily handled, the weight for the missing data is set to zero so that it neither upgrades nor downgrades the prior probability,

3. all of the cells in a map are included in the analysis, not just those cells in the control areas as in other statistical methods,

4. it is simple to update the analysis if a new evidence map becomes available, and

5. the method allows an estimate of uncertainty to be made.

A difficulty with the weights-of-evidence method stems from the assumption of conditional independence with respect to deposits for evidence patterns. The requirement of conditional independence referred to above is rarely satisfied completely in geological data sets, particularly for those involving geochemical data (Bonham-Carter, 1994). To check the extent of the problem, chi-squared tests for pair-wise conditional independence are performed for each possible pair of input maps for cells where a deposit is present. Where a 
significant correlation between binary map parameters exists, either one of the dependent maps must be omitted or dependent maps can be combined using Boolean operators (AND or OR), multiple regression or principal component analysis (Bonham-Carter et al., 1988; Agterberg, 1992b; Bonham-Carter, 1994).

Bonham-Carter et al. (1989) and Bonham-Carter (1994) have also described an overall goodness-of-fit test that can be applied to check whether the assumption of conditional independence of the evidence patterns with respect to deposit points is satisfied. They suggest that the total number of deposits predicted by the posterior probability map should be no greater than 10-15\% larger than the observed number of deposits. For raster maps, the predicted number of deposits is given by equation 2.10 :

$$
N(D)_{\text {calc }}=\sum_{i=1}^{N} P_{i} N(A)_{i}
$$

where $N(D)=$ the predicted number of deposits, $P_{i}$ is the posterior probability of a deposit for the $i^{t h}$ unit cell (or polygon for vector maps)on the map, and $N(A)$ is the area of the polygon in terms of unit cells. A distinction should be made between the unit cell (which is the area for which the posterior probability has been calculated) and the raster cell size as they are not necessarily the same (Bonham-Carter, pers. comm.).

However, in a recent study with a data set for VMS deposits in the Chisel Lake area, Manitoba, Canada, Singer \& Kouda (1999a) demonstrated that although a chi-squared independence test indicated no significant correlation among variables, a test for the expected number of deposits demonstrated that the data clearly violate the independence assumption. Singer \& Kouda (1999a) performed simulations which showed that using variables with correlations of 1.0 or -1.0 can double the expected number of deposits and that small to moderate correlations with only 6 to 8 variables resulted in adverse effects. It should be noted that Agterberg \& Cheng (2002) have published a statistical test for conditional independence that is better than the omnibus test (where no information on statistical significance is possible) and the pairwise chi-squared test (Bonham-Carter, 1994) which breaks down if the number of deposits are small).

Another problem with the weights-of-evidence is that a considerable amount of the information available in the raw data, that might otherwise have contributed to the prospectivity estimate, is discarded prior to the integration step. This occurs when input maps 
that contain continuous variables or multiple classes are converted to binary maps ${ }^{2}$ Values below the threshold used to re-classify multiclass maps to binary maps play no role in determining prospectivity, and values above the threshold (included in the binary evidence map) are treated as though they are equal because they are assigned the same weight.

\subsubsection{Fuzzy Systems}

Fuzzy systems, like neural networks, are a computing paradigm that represents an attempt to build computer systems with a human-like ability to reason and make decisions based on data that are uncertain, imprecise or subjective (Kasabov, 1996). Whereas neural networks can learn from data, fuzzy systems require explicit statements of uncertain knowledge as inputs. Therefore, fuzzy systems represent a conceptual or knowledge-driven approach to mineral-prospectivity mapping (Bonham-Carter, 1994). Fuzzy set theory and fuzzy logic originated from the work of Zadeh (1965) and is widely used today in many disciplines of engineering, particularly in control applications.

In classical set theory, elements either belong to a set or they do not. In two-valued Boolean logic, propositions like "soil gold concentration is anomalous" is either true or false with no other choice in between. Fuzzy systems model uncertainty by allowing an element to belong to a set partially and fuzzy propositions include all the greyness between the two extremes. Crisp sets can be viewed as a special case of fuzzy set theory where only two membership values, 0 and 1 , are defined. The degree to which a variable belongs to the fuzzy set is defined by a fuzzy membership function:

$$
\mu_{A}(u): U \quad \longrightarrow \quad[0,1]
$$

where $\mu_{A}$ is a fuzzy membership function which maps values, $u$, to real numbers in the interval $[0,1]$ and $U$ is called the universe of discourse and represents the set of all possible values for the fuzzy variable. For example, for the fuzzy proposition "soil gold concentration is anomalous" , a fuzzy membership value of zero means that the raw parameter value is definitely not a member of the fuzzy set "anomalous", while a membership value of one means that the raw parameter value definitely is a member of the fuzzy set "anomalous". A fuzzy set $A$ is represented as a sequence of fuzzy membership degree-value pairs; $A=\left\{\mu_{A}(u) / \mu\right\}$, where the symbol "/" separates the membership degrees from the value

\footnotetext{
${ }^{2}$ Although a binary reclassification is usually applied, the WofE method is not restricted to binary data. See footnote on page 1 .
} 
of the fuzzy variables.

In practice, fuzzy membership values are usually assigned by an expert according to subjective judgement. An example of how a geologist might assign membership values to classes of gold soil concentration is given in Table 2.1 below.

Table 2.1. Example of the assignment of fuzzy membership values to soil gold concentration values. The membership values express the degree of membership of each of the assay values to the fuzzy set anomalous gold concentration. The set of paired, variable, values-membership function values represents a fuzzy membership function.

\begin{tabular}{|c|c|}
\hline $\begin{array}{c}\text { Gold concentration } \\
u(\mathrm{ppb})\end{array}$ & $\begin{array}{c}\text { Membership degree } \mu_{A} \\
\text { in fuzzy set A, " anomalous" }\end{array}$ \\
\hline \hline$<2$ & 0.0 \\
$2-5$ & 0.5 \\
$>5$ & 1.0 \\
\hline
\end{tabular}

Membership assignments take both the favourability for mineralization and the reliability of the data into account.

Fuzzy operators can be used to combine the fuzzy membership values from different fuzzy sets to produce a single new fuzzy-membership value. In mineral prospectivity studies, the GIS layers containing raw data are converted to layers containing fuzzy membership values. At each grid cell, the fuzzy membership value from each layer is combined. The resultant output fuzzy membership values represent the degree of membership of the fuzzy set "favourable for mineralization". The five main operators used in mineral prospectivity studies are listed below (Bonham-Carter, 1994);

$$
\begin{array}{ll}
\text { fuzzy AND } & \mu_{\text {comb }}=\min \left(\mu_{1}, \mu_{2}, \mu_{3}, \ldots, \mu_{n}\right) \\
\text { fuzzy OR } & \mu_{\text {comb }}=\max \left(\mu_{1}, \mu_{2}, \mu_{3}, \ldots, \mu_{n}\right) \\
\text { fuzzy algebraic product } & \mu_{\text {comb }}=\mu_{1} \times \mu_{2} \times \mu_{3} \times \ldots \times \mu_{n}=\prod_{i=1}^{N} \mu_{i} \\
\text { fuzzy algebraic sum } & \mu_{\text {comb }}=1-\left(\mu_{1} \times \mu_{2} \times \mu_{3} \times \ldots \times \mu_{n}\right) \\
& \mu_{\text {comb }}=1-\prod_{i=1}^{N}\left(1-\mu_{i}\right) \\
\text { fuzzy gamma operator } & \mu_{\text {comb }}=\left[1-\prod_{i=1}^{N}\left(1-\mu_{i}\right)\right]^{\gamma} \times\left(\prod_{i=1}^{N} \mu_{i}\right)^{1-\gamma}
\end{array}
$$

where $\gamma \in[0,1]$. Detailed descriptions of these fuzzy operators are given by Zimmermann (1984), Klir \& Folger (1988) and Bonham-Carter (1994). 
Fuzzy systems have been applied to mineral prospectivity mapping in a number of studies. Fuzzy systems have been applied to prospectivity studies on base-metal and iron deposits by An et al. (1991), silver-zinc deposits by Tabesh (1992), vein gold deposits by Donaldson (1996), MVT deposits by Eddy et al. (1995) and D'Ercole et al. (2000), orogenic lode-gold deposits by Knox-Robinson \& Wyborn (1997) and Knox-Robinson (2000), and epithermal gold deposits by Carranza \& Hale (1999). GIS layers containing raw data are converted to layers containing fuzzy membership values. At each grid cell, the fuzzy membership value from each layer is combined. The main fuzzy operators used to combine GIS input layers in mineral prospectivity studies are described by Bonham-Carter (1994). Output fuzzy membership values represent the degree of membership of the fuzzy set favourable for mineralization.

To determine which combination of fuzzy operators to use in order to combine the data layers, Eddy et al. (1995) reduced deposit model descriptions for MVT, sedimentary copper and sediment-hosted sulphide deposits to a series of simplified statements in which the essential parameters were linked with Boolean AND and OR operators. These logical statements were then applied to the data sets using equivalent fuzzy operators (from equations 2.12 to 2.16$)$.

In all of the studies referred to above (except for Knox-Robinson, 2000, who used a vectorial technique and Tabesh, 1992, who used fuzzy $A N D$ and $O R$ ), the gamma function (equation 2.16) was used in the final step to combine fuzzy membership values in the derived layers to produce a prospectivity map. Gamma values in the range [0.95-0.98] were used in these studies. The fuzzy-logic method overcomes the problem of applying a fixed cut-off values, which are used in the weights-of-evidence method to produce binary evidence maps, by assigning fuzzy membership values on a continuous scale from zero to one. However, a loss of information still occurs in the fuzzy-logic method during the conversion of raw data values to fuzzy membership values when single fuzzy-membership values are applied to a whole class or range of values, as in the studies by An et al. (1991) and Eddy et al. (1995). This is the same procedure as was applied in Table 2.1 above. Rather than assigning a single membership degree to a complete class of values, it is more common in engineering applications to use a continuous function to convert raw values to membership values over the complete range [0,1] (Jang \& Sun, 1995). This approach was used by Cheng \& Agterberg (1998), who combined weights of evidence with fuzzy sets to create a hybrid method, which allows the use of both subjective and objective definitions of a fuzzy membership function. Figure 2.1 shows two commonly-used membership functions.

There are two further problems with the fuzzy-logic method. First, the fuzzy membership 


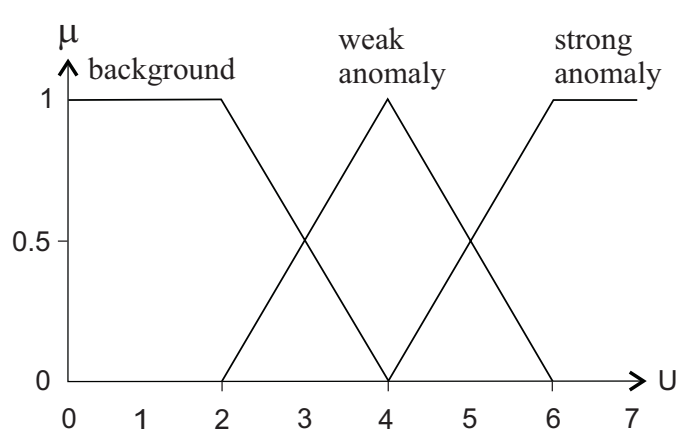

a)

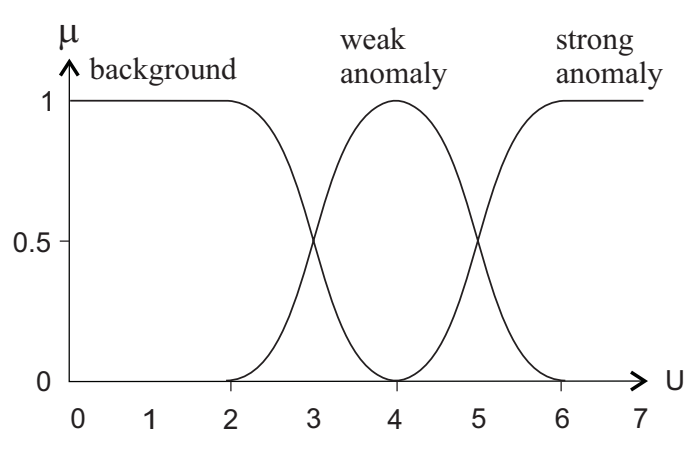

b)

Figure 2.1. Each figure shows three different fuzzy sets, background, weakly anomalous and strongly anomalous for the variable soil gold concentration. The fuzzy sets are defined using two commonly-used membership functions: A. triangular and B. sigmoidal.

values are often applied according to the degree to which parameter values conform to a deposit model, which itself may be flawed. Second, the assignment of fuzzy membership values is often entirely subjective (e.g. An et al., 1991; Eddy et al., 1995; Donaldson, 1996; D'Ercole et al., 2000). Although spatial analysis can provide a basis for estimating the fuzzy membership values as, for example, the use of statistically-determined fuzzy membership values by Knox-Robinson \& Wyborn (1997), subjective judgement is still used to determine the structure of the fuzzy inference net and to select the fuzzy operators used to combine the GIS layers. However, more recently, studies have been published by Luo \& Dimitrakopoulos (2003) and Porwal et al. (2003a) in which fuzzy membership functions are data driven. In the study by Porwal et al. (2003a), fuzzy membership functions are defined using piece-wise linear functions based on quantified spatial associations between map features and known deposits. The same approach is used in this study in Chapter 5 (5.5.14).

\subsubsection{Mineral Systems Approach}

The mineral systems approach proposed by Wyborn et al. (1994c, 1995b,a) is a conceptual method. Although it does not constitute a distinct mineral prospectivity method, it should be emphasized that this approach can also be embedded in the practice of prospectivity mapping, whatever implementation is being followed. The method is important because it provides a framework to view the relationship between the components of the deposit forming process and the kind of geological evidence that can be captured in a regional GIS. In addition to providing a generic approach to the selection of input GIS layers, Wyborn 
et al. (1995a) developed an extension to the ARC/INFO package that implements both conceptual and empirical mineral prospectivity mapping methods.

The mineral systems approach is based on the concept that an ore deposit results form an exceptional coincidence in space and time of ordinary mechanical and chemical processes and that these processes are mappable on a much larger scale (i.e. tens to hundreds of kilometres) than the ore deposit itself (Wyborn et al., 1994c). The utility of this concept is that the mineral system represents a far larger target for exploration that the actual ore deposit and the scale of the evidence of this mineral system is comparable to a regional scale GIS. Wyborn et al. (1994c) defined mineral systems as all geological factors that control the generation and preservation of mineral deposits and list the following six major components;

1. energy source to mobilize fluids and drive chemical reactions,

2. sources of transporting ligands,

3. sources of metals,

4. transport pathway,

5. trap zone (and ore deposit location) with a chemical and/or physical cause for mineral precipitation at the trap site, and

6. outflow zone.

An approach of this sort had been advocated as early as 1983 by Taylor \& Steven (1983). They proposed a structured subjective approach which involves the integration of all parameters that indicate the favourability for ore-forming processes, including known mineralization, geochemical and geophysical anomalies, rather than making statistical estimates. However, they did not describe how such an approach should be implemented.

In a later paper, Wyborn et al. (1995b) describe a mineral systems approach to evaluating mineral prospectivity that consists of three steps;

1. Define the essential ingredients that make up the components in Figure 2.2. For example, identify the specific form of the energy source (component 1 in Fig. 2.2) as a magmatic rather that a regional metamorphic event.

2. Construct a geoscientific GIS that incorporates these mappable geological criteria as searchable attributes in the GIS database. The GIS has to be constructed so that a metallogenic analysis is possible. In particular, such a GIS must contain consistent and validated attribute data and topologically correct layers. For example, granite attributes should include granite type (S- or I-type), degree of fractionation and oxidation state. Reactive minerals and rock types are also important; for example, 
Al-silicates (kaolinite, K-feldspar, and sericite), sulphides, carbonate, carbonaceous rocks, Fe-rich rocks (e.g. hematite BIF).

3. Develop conceptual methods to analyse the GIS for mineral potential that can be applied to 'greenfields 'areas.

The relationship between mineral systems components, essential ingredients (i.e. the specific form these components take in a particular system) and mappable criteria is shown in Figure 2.2. Wyborn et al. (1995b) contrast the mineral system approach, which considers the regional-scale features that contribute to the location of ore deposits, with deposit models, which are mainly concerned with characteristics of the deposits and focus on local-scale criteria.

Wyborn et al. (1995b) developed a menu-driven ARC/INFO extension that implements three different types of prospectivity analysis using a mineral systems approach, referred to as specific, interactive and generic modules.

The specific module allows the user to select a deposit type and specific criteria to find areas that match the criteria for that deposit type; for example, for Proterozoic graniterelated gold deposits, the system extracts all areas with graphite present, an age greater than $1700 \mathrm{Ma}$, and which are within a distance of $5 \mathrm{~km}$ of fractionated I-type granites.

The grid-based interactive module allows the user to choose their own model. The user can select layers and input criteria for each layer. Criteria can be combined using Boolean AND or OR and XOR operators. Each GIS layer is converted to a binary map with areas satisfying the criteria set to "one" and other areas set to "zero". Binary layers are combined using Boolean $A N D$, OR, or XOR so that the binary pixel value of the final map indicates whether there is a match between the data and the user specified criteria.

The generic module is also grid-based but, in contrast to the conceptual specific and interactive modules, it is an empirical method. This module allows the user to find all areas which have similar characteristics to a deposit or anomaly selected by the user. The system creates a buffer area around the selected point location. The user selects which attributes to use in the matching process. Layers are combined using the Boolean AND operator and the output is a binary map.

In contrast to the empirical multivariate techniques, discussed above, the conceptual mineral systems approach can be applied to areas where there are few known deposits or where no deposits are known. Multivariate methods require a minimum number of known 


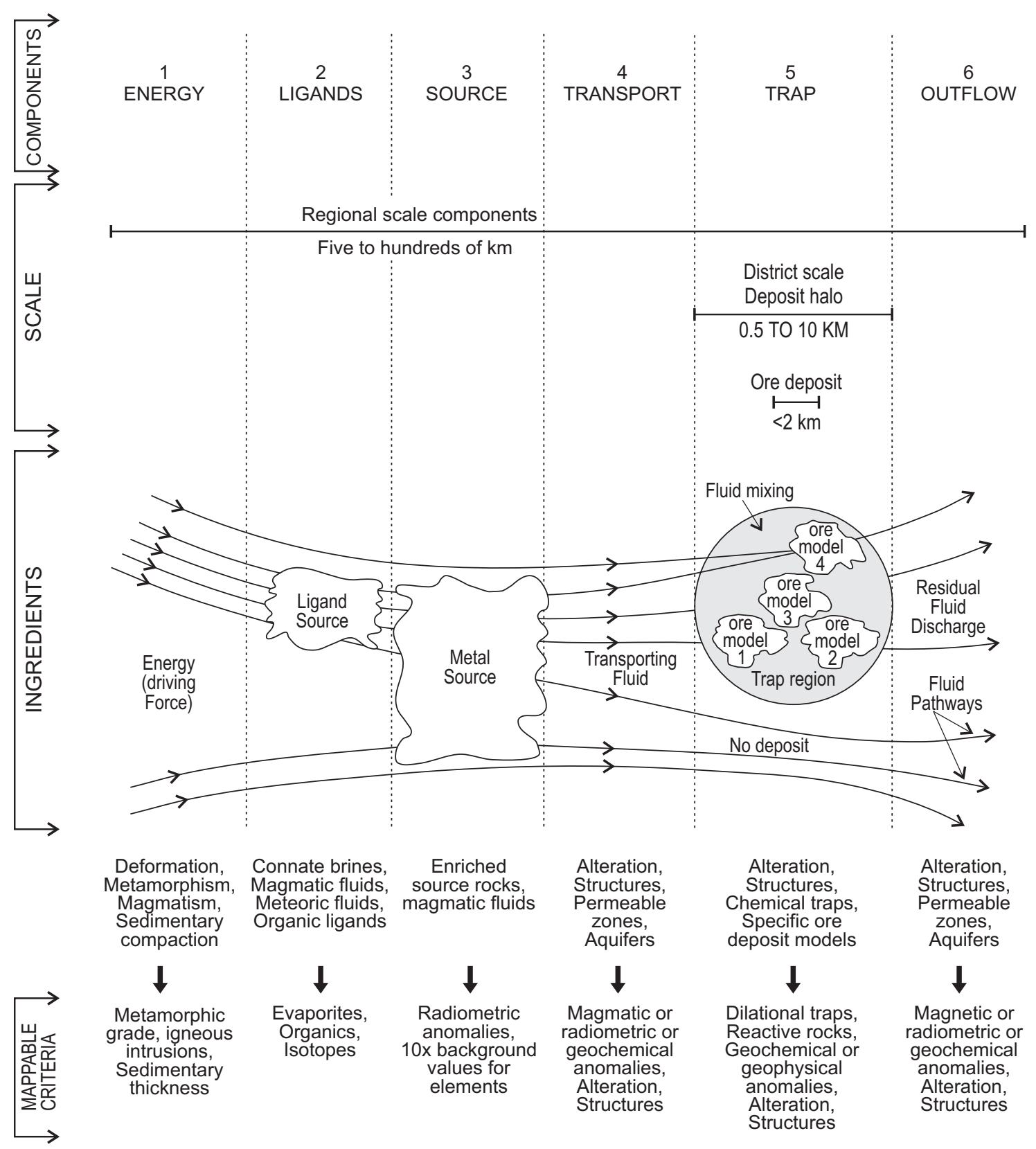

Figure 2.2. Components and criteria in the mineral systems approach developed by Wyborn et al. (1995b) 
deposits in order to perform a statistically valid analysis.

The difficulty with the mineral systems approach is that the criteria represent features that are not normally included in a regional GIS database. Consequently, new attribute fields, along with the corresponding data, must be collected and incorporated into the GIS database before undertaking this type of analysis. Another limitation of the method is the simple additive technique used to combine the layers and the fact that all layers have the same weight.

\subsection{Geographic Information Systems}

\subsubsection{Overview}

Geographic Information Systems (GIS) are systems that can capture, store, retrieve, transform, analyse and display both spatial (i.e. geographically referenced) and non-spatial data (Burrough \& McDonnell, 1998). GIS are important in this study because they are used to create the input and display the output of the mineral prospectivity methods. Although the majority of the mineral prospectivity studies referred to above were based on specially built image-processing systems (e.g. PROSPECTOR, CHARAN, SIMSAG, and FINDER), the availability of commercial GIS since the late 1980's means that at least some of the work of preparing input for current mineral prospectivity studies is performed in a GIS.

Development of GIS began with the cell-based SYMAP, GRID and IMGRID computer mapping and analysis systems developed at the Harvard School of Landscape Design, which were used for predicting urban growth (Burrough, 1986; Burrough \& McDonnell, 1998). This system produced grey-scale output by overprinting characters on a line printer. Early graduate students of the school went on to produce some of the commercial GIS in use today (e.g. Dangermond, ESRI, Sperry; ERDAS, and Tomlin; MAPS).

One of the main problems in the early development of GIS was the limitations imposed by computer graphics: for example, the PROSPECTOR system (described above) could only display a maximum of $128 \times 128$ pixels at a time when one LANDSAT image consisted of approximately $3000 \times 4000$ pixels (Fabbri \& Kasvand, 1988).

Factors that contributed to the development of GIS were the rapid improvement in the performance and a reduction in the cost of computers, particularly following the intro- 
duction of the first personal computers (e.g. Apple in 1977 and IBM in 1981 Faust et al., 1991) and the development of user-friendly graphical user interfaces (GUI) popularized by the Apple Macintosh computer. Success of the Earth Resources Technology Satellite (in 1972) and LANDSAT 4 and 5 (in 1982-84), and the availability of digital imagery also encouraged the development of GIS (Faust et al., 1991).

The storage of non-spatial attribute data is one of the features that distinguish GIS from image processing and CAD systems. The development of relational database systems, based on the model proposed by Codd (1970) and first developed by IBM in the early 1980's (Blasgen, 1981), was important. They were incorporated into GIS because they support a large range of types of database queries which do not all have to be anticipated in advance as part of the design of the database. Relational databases are structured like tables consisting of rows (which hold data records) and columns of fields (which hold attributes).

Underlying the relational database model is the process of normalization, which is concerned with defining the simplest structure for a given set of data. It deals with the dependency between attributes and prevents the loss of information when records are inserted or deleted.

Advantages of the relational data model are that it is the most flexible model and it can support a wide range of query types, and allows for the efficient update of data. Queries involving more than one table require tables to be liked via common attribute. This process is known as a relational join. Each record in one table is connected to a record in another table that shares the same value for a common item. However, data retrieval, in the relational model can be slow, since only common attributes between tables can be indexed.

A further disadvantage of the relational database model is that it is not an efficient method for storing spatial data because spatial data do not naturally conform to one of Codd's normal forms (Date, 1999). One of Codd's requirements for a relational database is that the rows can be changed without changing the meaning of the data, but obviously the order of the data does matter if rows contain $x y$-coordinate pairs for individual vertices along line segments. Also, in a GIS, it would be more efficient if data could be accessed as groups, each one comprising all the pairs of coordinates for a given line or all the line identifiers for the lines forming the boundary of a polygon. However, such a scheme would violate Codd's first normal form that all fields are atomic and cannot be decomposed (Zhou et al., 1997). 
A dual database model overcomes these difficulties by maintaining non-spatial attribute data as tables in a Relational Database Management System (RDBMS), but storing spatial data using conventional file techniques to ensure speed of access. This is the approach adopted in the ARC/Info GIS (Aronson \& Moorehouse, 1983). Commercially available RDBMS (e.g. INFO, ORACLE, INGRES, INFORMIX) are used for the storage of nonspatial attributes, whereas specialized file systems are used to represent the geometry and topology of spatial objects (Burrough \& McDonnell, 1998).

The most commonly used type of dual database is the arc-node-RDBMS model, which uses a full vector arc-node topology. ARC/INFO GIS used in this study is an example of this type of system, which can also operate in a raster mode (Burrough \& McDonnell, 1998).

\section{Data Structures}

At the highest level, data in a GIS are structured according to the cartographic model used in the Map Analysis Package developed by Tomlin $(1983,1990)$. The database is viewed as a collection of map layers which are all registered according to a common geographic coordinate system with each depicting a particular type of data or theme. Within map layers, two main data structures are used to represent spatial objects, i.e. raster and vector structures. In the raster model, thematic layers are divided into a grid of square cells. Each cell is represented by either; a coordinate pair and a set of attributes, or a single attribute whose cell position is recorded implicitly by its position within the twodimensional matrix of cells. In the vector model, the fundamental primitive is a point represented as a pair of coordinates. The points along a line (or arc), called vertices, define the shape of the arc. The end-points of an arc are called nodes. Polygons are represented as a series of $x y$-coordinates that enclose an area. Topological information, such as a list of the polygons to the left and right sides of each arc, is also stored by the system (Rhind \& Connolly, 1995).

Most GIS support the use of both vector and raster structures and enable data to be converted between the two formats. The merits of both systems have been discussed by Zhou (1994) and Burrough \& McDonnell (1998). The choice of which model to use is largely dictated by the type of data to be used. Discrete entities are best represented using the vector model whereas continuous fields (e.g. magnetic intensity survey data) are best represented by the raster model. Zhou (1994) notes that the raster model is required for the efficient manipulation and enhancement of digital images. Remotely-sensed imagery 
can only be efficiently processed in the raster format (Zhou, 1994). Burrough \& McDonnell (1998) state that, if each cell potentially represents a different value, then the simple raster structure is the most suitable one.

Another advantage of the raster model, in which each attribute is represented as a separate theme, is that mathematical operations performed on attributes of cells can be applied to whole maps of gridded data using the same algebraic notation. The process of producing a new attribute is represented as follows:

$$
A_{\text {new }}=f\left(A_{1}, A_{2}, A_{3}, \ldots, A_{n}\right)
$$

where $A_{i}$ represents the value of an attribute in the $i^{\text {th }}$ layer. This method can be extended to an entire map layer using a raster format as follows:

$$
M_{\text {new }}=f\left(M_{1}, M_{2}, M_{3}, \ldots, M_{n}\right)
$$

where $M_{i}$ represents the $i^{\text {th }}$ map layer. The method represented by equation 2.18 is referred to as map algebra (Tomlin, 1983, 1990).

\section{Data Modelling and Analysis Tools}

Data modelling and analysis tools provide a GIS with a functionality that goes beyond the digital and storage and production of maps. Detailed discussions of GIS analysis and modelling functions are given by Burrough (1986), Tomlin (1990), and Burrough \& McDonnell (1998). The application of GIS functions to mineral prospectivity analysis has been described in detail by Bonham-Carter (1994).

Examples of the type of GIS functions used in mineral prospectivity analysis include;

1. interpolation of point locations of geochemical analyses to polygons representing drainage basins,

2. creation of a series of buffers around fault lines,

3. overlaying a layer containing deposit point locations over fault buffers to determine the numbers of deposits at different distance intervals from faults, and

4. re-classification of a range of buffer intervals to form a binary pattern representing a favourable distance from faults. 
Wyborn et al. (1995a) and Hazell \& Wyborn (1995) have highlighted the issues and problems associated with creating GIS for metallogenic analysis or mineral-prospectivity mapping. According to these authors, such a GIS must;

1. contain attributes relevant to metallogenic analysis,

2. contain topologically correct coverages; for example, ones which do not contain any over- or undershoots, open polygons and gaps, and sliver polygons,

3. be compiled at a suitable scale. Wyborn et al. (1995a) suggested that the geological base map should be compiled at the scale of the original field photographs; for example, 1:25000, and

4. contain consistent and validated non-spatial data. Wyborn et al. (1995a) suggested the use of authority tables for stratigraphic names and rock codes. Roach (1994) has advocated the use of specially designed attribute structures for geological applications.

\subsection{Neural Networks}

\subsubsection{Overview}

Neural networks are a type of adaptive computing system that can learn from data and generalize from stored knowledge to produce appropriate outputs in response to new data. ${ }^{3}$ Although neural networks were inspired by studies of biological neurons they are more closely related to mathematical models like non-parametric pattern classifiers, non-linear filters and cluster algorithms, than models of neural or cognitive phenomena. Neural networks consist of simple processing elements, called neurons, interconnected by links associated with numeric coefficients or weights indicating the strength of each connection (Fig. 2.3).

Most computers today are based on the von Neumann model, and consist of one, or a few, complex CPUs and a central location for data. They perform actions that are determined by a program that remains fixed during run-time. In contrast, the large number of simple processors in a neural network can potentially be active at the same time, providing a model for massively parallel computation. Neural networks are adaptive in the sense that

\footnotetext{
${ }^{3}$ Computation with neural networks may be referred to elsewhere as parallel-distributed processing, neural computation, and neurocomputing.
} 


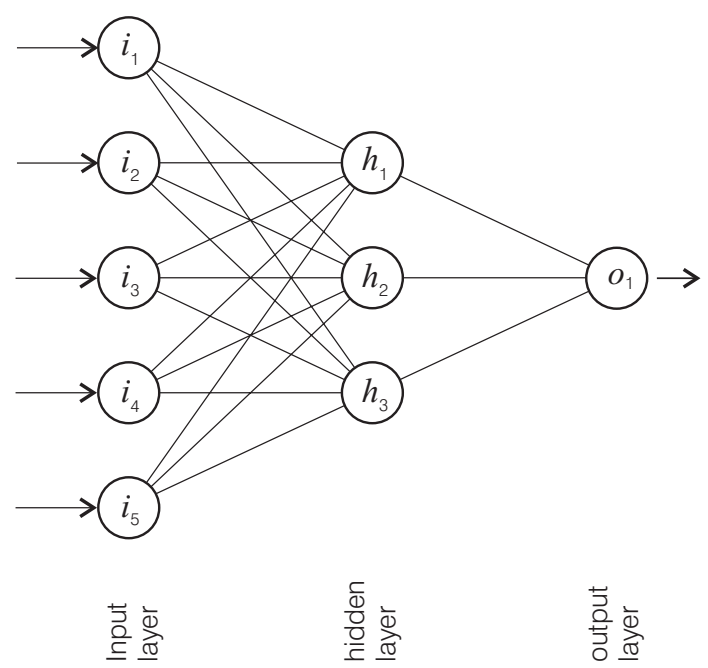

Figure 2.3. Architecture of a 3-layer multilayer perceptron (MLP) neural network. The network transforms a set of inputs $\left(x_{1} \ldots x_{3}\right)$ to a set of outputs $\left(y_{1} \ldots y_{3}\right)$. Three inputs and a single output are shown as an example only. Networks can have multiple outputs or two hidden-layers. Processing is performed by units called neurons (shown as circles), which are connected by links. Associated with each link is a numerical coefficient or weight. Learning is achieved by iteratively adjusting the weights so that the network produces the correct output for a given set of inputs for each example in the training data set.

knowledge is acquired through a process of learning from examples. Once trained, the stored knowledge is distributed throughout the network in the form of the weight values that have been modified as a result of learning. The globally complex behaviour of the network is determined by the pattern of connections linking the units and the values of the weights associated with those links. This type of knowledge representation is referred to as sub-symbolic and distinguishes neural networks from expert systems in which knowledge is stored as symbolic structures such as rules or decision trees.

\section{When to Use Neural Networks}

The question of which are appropriate types of problem to solve with neural networks has been addressed by a number of researchers. Kasabov (1996), writing from a knowledge engineering point of view, states that: 1) statistical methods are appropriate when data are available and the type of underlying relationship is known, 2) symbolic artificial intelligence (used in expert or rule-based systems) is suitable where well-defined rigid rules exist, 3) fuzzy systems are applicable when approximate, possibly contradictory, heuristic rules exist, and 4) neural networks are applicable where there is problem knowledge, including 


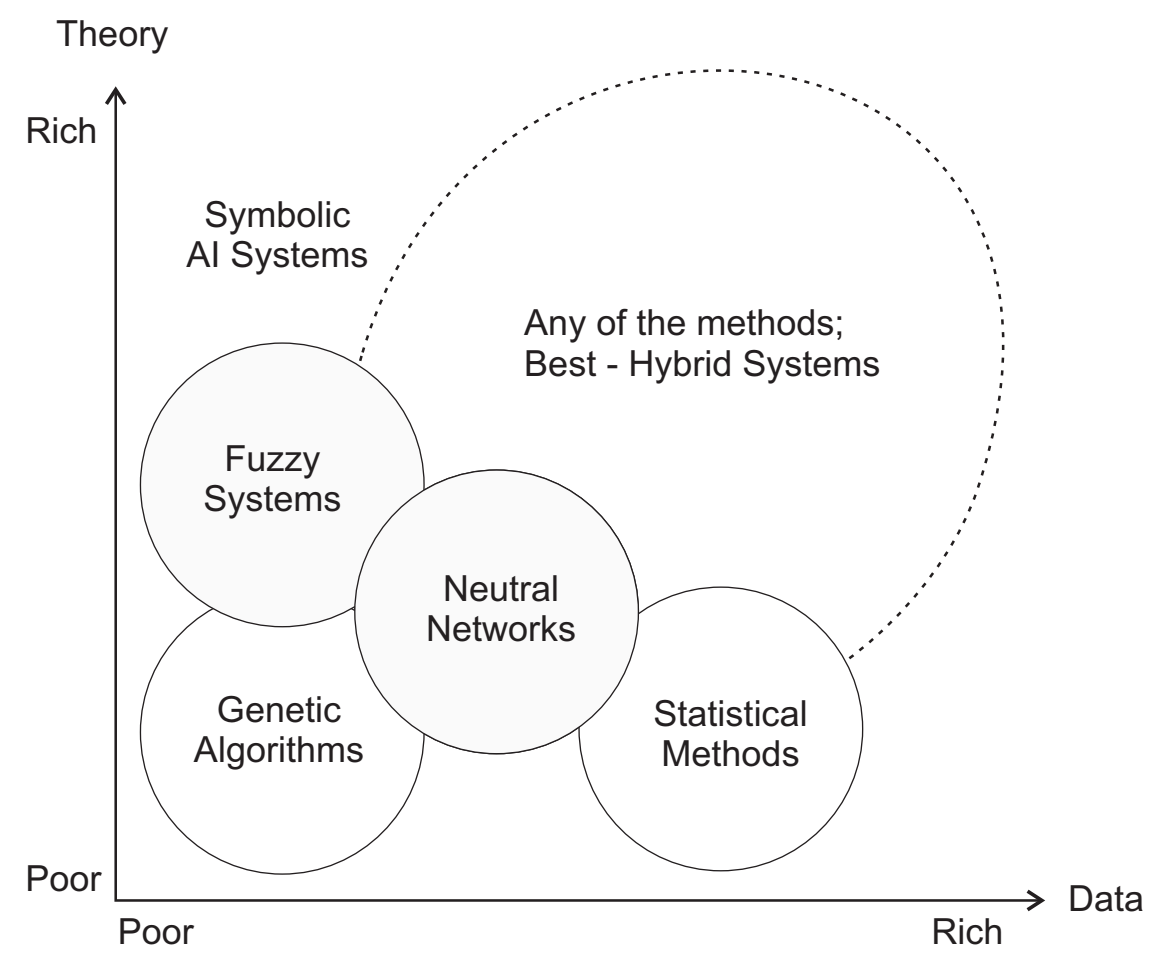

Figure 2.4. Diagram showing that the suitability of artificial intelligence (AI), soft computing (neural networks, fuzzy systems and genetic algorithms) and statistical techniques for solving a problem depends on the amount of data and theoretical knowledge available (after Kasabov, 1996; (c)MIT Press).

mainly data rather than rules, and where the nature of the underlying function relating the inputs to the outputs is unknown. Huang \& Williamson (1994) and Zaknich (1999) suggest that neural networks are suitable for problems with the following characteristics;

1. non-linearities,

2. high dimensionality; for example, large number of input parameters,

3. noisy data due to imperfect measurements,

4. inadequate conventional approaches due to problem complexity,

5. problem dependence on multiple interacting parameters, and

6. no known mathematical algorithm to determine the solution.

Figure 2.4 summarizes the relationship between the suitability of different methods where knowledge of the problem involves different degrees of theoretical knowledge, or rules, versus data. Neural networks, fuzzy systems and genetic algorithms (a paradigm based on an evolutionary analogy) are collectively known as soft computing techniques. 
Although the first, simple, neural network was developed in 1958 (Rosenblatt, 1958), there has been an upsurge of interest and development of neural networks since 1986, due to a combination of theoretical advances and improvements in the performance and the cost of computer hardware. Neural networks are now used in a very wide range of commercial and industrial applications. Descriptions of such systems are given by Dillon et al. (1997). Generic functions that neural networks can perform include (Patterson, 1996; Zaknich, 1999);

1. classification (e.g. identification of crop types from remotely sensed images),

2. function approximation (e.g. process control in manufacturing industry, typically where a result is dependent on multiple parameters that depend on one another and which are non-linear in their behaviour),

3. prediction (e.g.stock prices and customer credit worthiness for banks),

4. optimization (e.g. optimal location of pumping wells for the clean-up of contaminated aquifers), and

5. noise reduction (e.g. non-linear signal filtering to recover clean tone bursts from noisy Morse code and telemetry signals).

\subsubsection{Applications of Neural Networks in the Geosciences}

The reason for applying neural networks in geoscience problems is that they are known to be particularly well-suited to the task of pattern recognition (Bishop, 1995; Schalkoff, 1997). Geologists are also expert at recognizing pattern, especially where patterns are on one or two paper maps. However, due to the increasing volume of digital data now available in regional exploration, the problem can be viewed as one of extracting numeric patterns from multiple layers of digital map data and there may be 20 or more layers. In addition to their pattern recognition capabilities, neural networks have other attributes which are suited to processing geoscientific data. These include the ability to deal with;

1. uncertain and noisy data,

2. outliers in the data,

3. very complex distributions, including large deviations from normality and multimodal distributions,

4. multiple interdependent input parameters,

5. highly non-linear relationships between input and output parameters,

6. large heterogeneous data sets, and 
7. no prior knowledge or assumptions about the statistical distributions of the input data.

The potential of combining neural network and GIS technologies was recognized by Jeffrey \& Rossner (1986) and Ritter et al. (1988) soon after the revival of interest in neural networks that followed the development of learning algorithms for multilayer feedforward networks by Rumelhart et al. (1986b). Much of the subsequent geoscience research has focused on automated pattern recognition and the classification of remotely-sensed imagery (Decateur, 1989; Ritter \& Hepner, 1990). Applications include the classification of radar terrain images (Decateur, 1989), discrimination of snow-covered surfaces and cloud types in arctic areas (Key et al., 1989), and classification of sea-ice (Orlando et al., 1990) and land-cover types (Ritter \& Hepner, 1990).

Many of these studies demonstrated that neural networks could be used to combine large numbers of data sets to perform classification. For example, Benediktsson et al. (1993) used a Multilayer Perceptron (MLP) neural network to classify High Resolution Imaging Spectrometer (HIRIS) data to classify LANDSAT MSS and topographic data containing sixty variables. Seventeen input channels, consisting of Landsat Thematic Mapper (TM) and ancillary textural data, were used by Bruzzone et al. (1997) to classify a region with complex land-use. Recent studies of geographic and remote sensing applications of neural networks are presented in Lees (1996).

Non-spatial applications in which neural networks have been used as classifiers include seismic event discrimination (Dowla et al., 1990), first-arrival picking in seismic data (Wagner et al., 1990), and classification of lithofacies from wireline log data (Baldwin et al., 1990; Rogers et al., 1992; Cardon \& van Hoogstraten, 1995).

Neural networks have also been used for function approximation (i.e. the estimation of a continuous-valued output variables) in applications such as seismic inversion (Roethe \& Tarantola, 1991, 1995), prediction of porosity and permeability of petroleum reservoirs from wireline log data (Osborne, 1992; Wong et al., 1995b), and ore-grade estimation (Clarici et al., 1993; Denby \& Burnett, 1993). Guo \& Hansen (1992) used an MLP network to predict the lithology and location of lineaments from aeromagnetic data over the Pueblo Quadrangle, Colorado, USA. A similar approach has been adopted by Larsen (2000), who used spectral content, simple statistics and grey-level run-length distributions over a small moving window of cells in regional aeromagnetic data to classify lithology into similar areas in southern Greenland. Poulton et al. (1992) used a variety of neural network types to predict horizontal offset, depth, and conductivity of a sub-surface targets from 
high resolution ground EM data. Detailed examples of the use of neural networks in the environmental sciences and geosciences are given by Dowla \& Rogers (1995).

In contrast, there are very few studies that describe the application of neural networks to mineral exploration. All of those documented in the literature are briefly reviewed in the next paragraphs ${ }^{4}$. Studies involving the classification of non-spatial data include; 1) the use of MLP and Kohonen neural networks trained using fifty geological, mineral and structural parameters to classify gold deposits into grade-tonnage classes (Brodeur et al., 1992), 2) the classification of gossans into $\mathrm{Fe}$ and $\mathrm{Cu}$ deposit categories using the assay values of eight elements as inputs to an MLP neural network (Yudong, 1994), and 3) the use of a probabilistic neural network to classify mineral deposits into types defined by ore-deposit models. The network was trained using ore and alteration mineralogy and rock types (Singer \& Kouda, 1997b,a).

Several studies describe the use of neural networks for geological mapping. Schau et al. (1994) used a neural network to construct a reconnaissance bedrock-geology map of a 225 $\mathrm{km}^{2}$ area on the Melville Peninsula, Canada. The network was trained using satellite and airborne geophysical data and rock types determined from ground traverses. About 1\% of the total pixels were used to train the network.

In a similar study, An et al. (1994) used an MLP network, trained with input data from 17 bands of Landsat TM and airborne magnetic and radiometric survey data, to classify an area containing felsic and mafic rocks into areas with hydrothermal alteration and unaltered areas.

In a study comparing Dempster-Shafer evidential belief theory with neural network classifiers, Gong (1996) used Landsat TM, gravity, and airborne magnetic and gamma-ray survey data to produce a map showing the distribution of four rock types on the Melville Peninsula, Canada. Similar accuracies were achieved for the neural network and evidential reasoning methods (92.1\% and 89.3\% methods, respectively). Gong (1996) concluded that the neural-network map was more similar to manually produced geological maps and was less sensitive to noise than the evidential reasoning method.

Clare \& Cohen (2001) used a Kohonen neural network to identify patterns and anomalies in geochemical analyses for 33 elements in stream sediment samples from north-eastern NSW, Australia. The clusters are related to sub-catchment geology and topography, while

\footnotetext{
${ }^{4}$ Gahegan (1997) suggested using neural networks for pattern recognition and classification in mineral exploration, particularly for the interpretation of complex data sets such as airborne EM: however, no work was undertaken.
} 
the outliers generally represent sub-catchment basins containing major mineral occurrences and deposits. They produced maps which were coloured according to the degree of similarity between the individual catchment (grid cell values) and the trained neural network cluster vectors.

Although Bonham-Carter (1994) referred to neural networks as a possible empirical method for mineral-prospectivity mapping in his book in 1994, at the time this study commenced, the use of neural networks for mineral-prospectivity mapping had been described in only one study by Singer \& Kouda (1996). Singer \& Kouda (1996) used an MLP network to produce a map depicting the distance to the nearest Kuroko-type deposit. Training data consisted of the relative abundance of four alteration minerals in drill holes. The prediction accuracy over the entire data set (including the training data set) was $79 \%$. The use of a proprietary neural-network system, called PROSPECT EXPLORER, for the classification of anomalies in multisource mineral exploration data has been described also by Clare et al. (1997). The pilot study for this thesis, in which an MLP neural network method for producing mineral prospectivity maps is compared with fuzzy logic and weights of evidence, was reported by Brown et al. (1997). Two more recent studies in which probabilistic neural networks are used are described in the relevant section below.

Skabar et al. (2000) has applied a data-mining approach using an inductive symbolic learning technique combined with genetic algorithms and fuzzy membership functions to mineral-prospectivity mapping using exploration data for orogenic lode gold deposits in central Victoria and compared the results with those of an MLP network. Data mining involves the use of a variety of techniques that are applied in combination in order to search for relationships and complex patterns in large databases (Hair et al., 1998).

\subsubsection{Application of Neural Networks to Spatial Analysis}

\section{Spatial Data Without Use of Location}

Neural networks are used in a large variety of applications involving spatial data. Some of the ways in which neural networks have been used to process spatial data are shown in Table 2.2. The upper part of the table summarizes applications where data are spatial but the location or spatial relationships are implicit and do not form part of the networks inputs or outputs.

In many spatial data processing applications, the neural network inputs and outputs refer to a single location (i.e. a pixel in an image or cell in a GIS layer), but the output is not 
a function of the location of the data. The feature vectors merely belong to a location stored implicitly in a raster grid and thus can be processed independently of one another (refer to Table 2.2). Examples of this use of neural networks include the classification of land-cover types in remotely-sensed images (Key et al., 1989) and (Benediktsson et al., 1990a,b) and production of geological maps (Schau et al., 1994; An et al., 1994; Gong, 1996).

In the study by Singer \& Kouda (1996), proportions of four alteration minerals from drill core were used to predict the distance to VMS deposits in unexplored grid cells. Inputs to the network are processed without the location of the data being explicitly presented to the network; however, the network output is a function of location. This is because the development of alteration minerals is a function of proximity to the volcanic vent responsible for the formation of the VMS deposit. This represents an example of inverse modelling (1b in Table 2.2).

Dowla \& Rogers (1995) used a combination of neural networks and genetic algorithms to optimize possible well locations designed to clean up contaminated ground water. The problem was to select combinations of 23 potential extraction wells and five injection wells that could contain a contaminant plume. The 28 well locations were chosen by experts. The aim was to select a small subset of these locations which would be sufficient to achieve the remediation.

The solution consisted of three steps. First, ground water flow and solute transport models were used to predict; 1) whether the pumping pattern could contain the plume within its current boundaries (i.e. a binary decision), 2) the total mass of contaminant extracted, and 3 ) the cost. The mathematical model was only used to calculate these parameters for 325 well-site combinations because each calculation took 2-3 hours.

In the second step, these data were used as target outputs to train separate neural networks to predict the three parameters. Inputs consisted of binary values to indicate whether the corresponding well location is used for pumping or not. Once trained, the networks were able to speed up the prediction of the three parameters by an order of roughly one million.

In the third step, a genetic algorithm was used to produce 20000 generations of suitable well combinations. The measure of fitness for each member of the population was the sum of the three criteria (plume containment-yes or no, mass of contaminant extracted and cost). The neural networks were used to estimate the three criteria for each member of the population after each new generation was created. Four million subsets of the 268 million possible subsets of well patterns were evaluated in this way. 
This is an example of forward modelling, in which the inputs to the network refer to multiple locations. For example:

Mass of contaminant extracted, containment, cost $=f\left[\left(x_{1}, y_{1}\right), \ldots,\left(x_{n}, y_{n}\right)\right.$, well $_{1}, \ldots$, well $\left._{n}\right]$

where $\left(x_{i}, y_{i}\right)$ is the location of the $i^{\text {th }}$ well, $n$ is the number of wells and $w_{i}$ is a binary variable indicating if the $i^{\text {th }}$ well is operating or not. Although the output is a function of spatial location, the location information is not supplied to the network.

\section{Spatial Data and Location Data Explicitly Processed}

Spatial location is supplied directly to the network in cases where the inputs consist of;

1. $x, y$ coordinates,

2. neighbourhood or window containing contextual information, or

3. a pattern of pixels in 2-D.

Examples illustrating each of these three cases are discussed below.

\section{Use of $X Y$-Coordinates as Network Inputs}

The classic example where $x y$-coordinate data are used as inputs to a network is the two-spiral problem, which was devised by Lang \& Witbrock (1988) as a benchmark to evaluate the generalization capacity of neural networks with different architectures and training algorithms. To solve this problem, the network acts as a pattern classifier. Inputs to the network are the $x y$-coordinates of a point in a rectangle and the output is either 0.9 or 0.1 representing spiral A or spiral B, respectively.

Wong et al. (1998) used a modification of a radial basis function (RBF) network called an interpolation network to interpolate porosity measured in wells in a fluvial sandstone petroleum reservoir. Inputs used to train the network were the $x y$-coordinates of the well, a rough estimate of porosity obtained from a hand-drawn isoporosity map and a categorical variable to represent the type of depositional facies. The result of the analysis was a more reliable porosity map that reflected all the available geological knowledge (Table 2.2).

Another example of the use of spatial coordinates as inputs to a neural network is the work of Wong et al. (1998). Wong et al. (1998) used a combination of neural networks 
and geostatistics to map the top structure of an oil reservoir. A neural network was used to interpolate a predictable (trend) component of the spatial variation and kriging was used to model the noise (residual component). Inputs to the neural network consisted of the depth to the top of the petroleum reservoir determined from seismic data and the location of the data in terms of $x y$-coordinates. Target outputs used during training were the depths determined from wells.

\section{Window of Neighbouring Cells}

In the classification of remote sensing images, values from a neighbourhood or window of pixels surrounding the current pixel is moved across the image to provide textural or context information. For example, Ritter \& Hepner (1990) used an MLP network to classify land cover types. Input parameters were obtained from a $3 \times 3$ pixel moving window over images representing four bands of LANDSAT TM.

Cells in the neighbourhood of the current processing cell were also used in a study by Müller et al. (1998) in which a neural network was used to classify a back-scatter image mosaic from side scan sonar of the sea-floor. The resultant map divided the sea-floor into areas of foramniferal ooze, mixed sediments, and metamorphic basement and volcanic rocks. Textural features, consisting of 18 grey-level (pixel brightness) statistics derived from a $32 \times 32$ pixel window, were used as inputs to an MLP neural network. The correct classification of the training examples was known from sampling of the sea-floor. Müller et al. (1998) suggested that the method of feature extraction could be applied to patterns of geological and geophysical features related to targets in mineral exploration.

\section{Two-dimensional Patterns}

Two-dimensional patterns of pixels can also be used as inputs to neural networks. For example, Le Cun et al. (1989, 1990a,b) developed a series of hierarchical networks for the recognition of hand-written digits in postcodes. Grey-scale levels in a $28 \times 28$ image were used as inputs. Two types of hidden layers were used; 1) layers for feature detection, and 2) layers for averaging and sub-sampling. Digit shapes were recognized by detecting and combining local features in the input image.

Feature detection is performed in layers consisting of sets of neurons arranged in squares called feature maps (Fig. 2.5). Each unit in the feature map receives its input from a $5 \times 5$ window or kernel that is scanned over the image, moving one pixel at a time. Because 


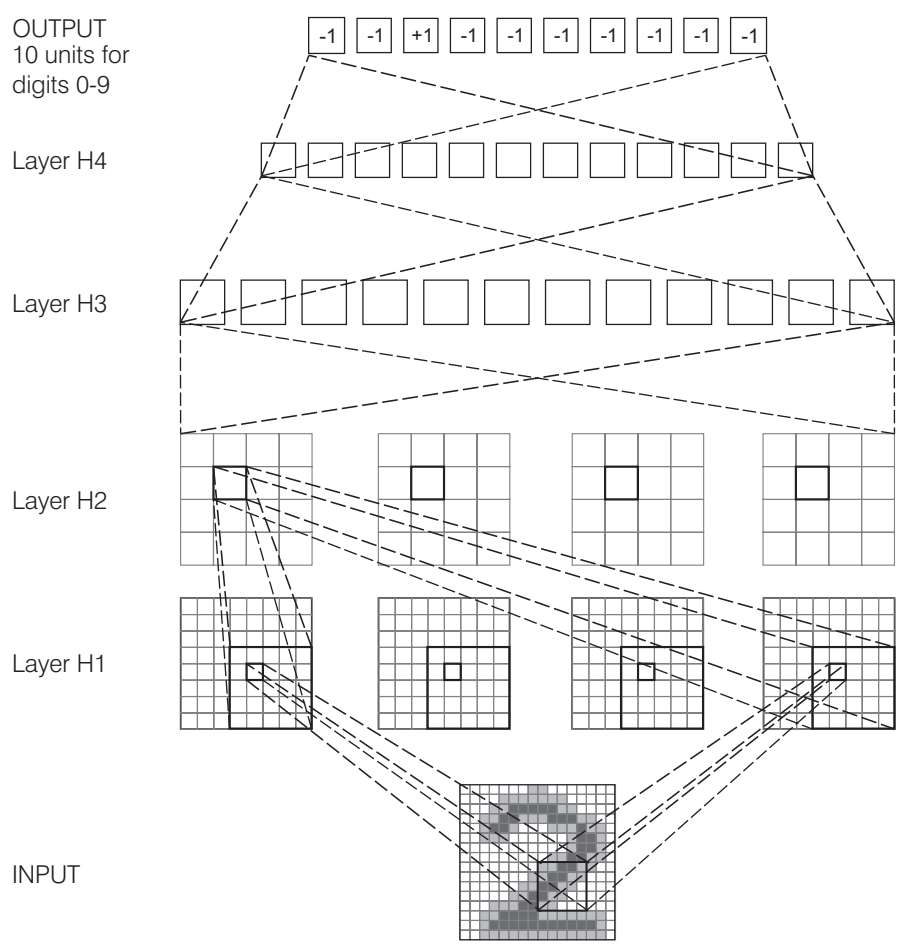

Figure 2.5. Hierarchical neural network used to recognize hand-written digits. Diagram adapted from Le Cun et al. (1990a).

the weights connecting the feature map units to each window location on the image are the same, the units in a feature map are constrained to perform the same operation on different parts of the image. This is useful because features of a digit could be distorted or displaced or some features could occur in different places in different characters. Multiple feature maps are used to extract different feature types from the image (layers $H_{1}$ and $H_{3}$ ). Sub-sampling and averaging is performed by layers $H_{2}$ and $H_{4}$ in Figure 2.5. A $4 \times 4$ window is moved across the corresponding feature maps in layer $H_{1}$ (so that the positions do not overlap), and the output of the four units is directed to just one unit in layer $\mathrm{H}_{2}$. The loss of resolution has the effect of increasing the level of abstraction and reduces the effect of distortion or translation of the input image on the representation of features in subsequent layers. Similar operations are performed in layers $H_{3}$ and $H_{4}$.

The output layer contains 10 units which represent digits 0 to 9 . If the input pattern corresponds to a 5 , then the $5^{\text {th }}$ output unit is set to +1 while the others are set to -1 . Implemented in hardware, the system was able to process 10-12 digits per second with a $92 \%$ success rate. ( $7 \%$ were rejected as indecipherable and $1 \%$ were incorrectly classified.)

Two-dimensional patterns of pixels were processed in a similar way by Zaknich (1997) in a study of the physical properties of aluminium hydroxide particles produced during 


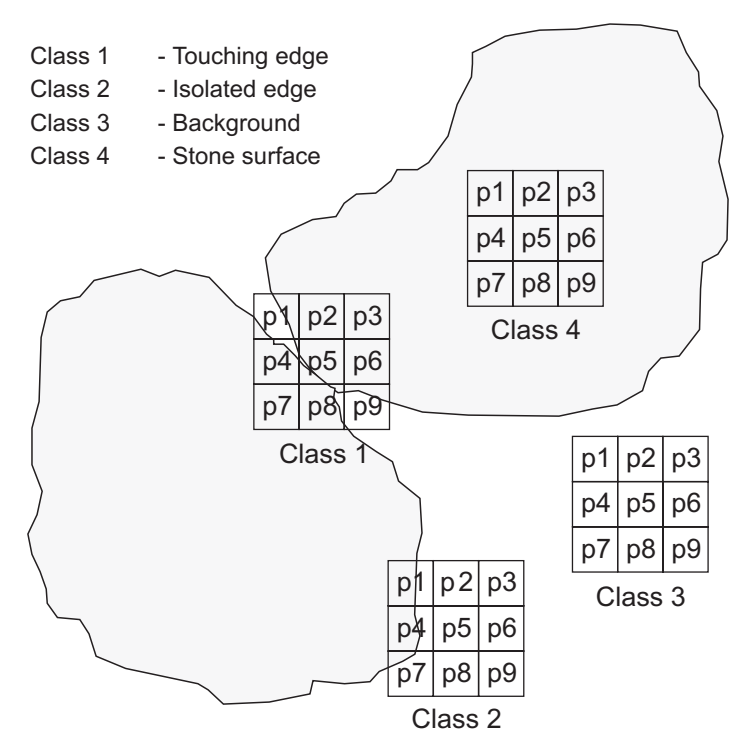

Figure 2.6. Example of the use of patterns of pixels used to train a network to recognize particle boundaries. Diagram adapted from Zaknich (1999)

the Bayer aluminium process. The task involved discriminating the boundaries between particles. Pixels in grey-scale SEM images were classified as belonging to either particle surfaces, particle edges or boundaries between particles (Fig. 2.6). A $5 \times 5$ moving window centred on the pixel to be classified provided 25 inputs to the neural network in the form of grey-scale pixel values. The output was presented as three different grey-scale values according to the class of the input pixel, thereby creating a new classified image.

A similar approach was used by Penn et al. (1993). He used a set of four edge patterns to train a neural network to detect geological boundaries in a single band LANDSAT TM image. Input to the neural network was obtained from a $9 \times 9$ moving window over a normalized grey-scale image and a value representing the contrast between the foreground and the background. The network was trained to output a one for patterns representing edges and a zero for all other patterns.

Zaknich (1995) used a different approach to recognize, count and measure the size of sodium oxalate crystals which form during the Bayer process used to refine aluminium. Shapes were classified using features such as perimeter, area, radius, geometric and central moments and best-fitting ellipses. 
Table 2.2. Analysis of spatial data by neural networks. All applications listed in the table involve spatially registered data. In the examples listed in the top part of the table, the location or pattern of the data in two-dimensional plane of the image or GIS thematic layer is not processed. In the bottom part of the table, spatial properties of the data, e.g. $x y$-coordinates or information about the neighbourhood around a cell, are used as inputs to the network.

\begin{tabular}{|c|c|c|c|}
\hline $\begin{array}{l}\text { No. of locations } \\
\text { referred to } \\
\text { in input }\end{array}$ & $\begin{array}{l}\text { Output } \\
=\mathrm{fn} \text { of } \\
\text { location? }\end{array}$ & Example & References \\
\hline \multicolumn{4}{|c|}{ Location not processed } \\
\hline \multirow[t]{2}{*}{ single location } & no & classification of remotely-sensed images & $\begin{array}{l}\text { Key et al. }(1989,1990) \text { Benedik- } \\
\text { tsson et al. (1990a,b) Gong } \\
(1996)\end{array}$ \\
\hline & yes & $\begin{array}{l}\text { prediction of distance to nearest VMS } \\
\text { deposit }\end{array}$ & Singer \& Kouda (1996) \\
\hline multiple locations & yes & $\begin{array}{l}\text { optimization of pumping well locations } \\
\text { for groundwater remediation }\end{array}$ & Dowla \& Rogers (1995) \\
\hline \multicolumn{4}{|c|}{ Location or $2-\mathrm{D}$ pattern processed } \\
\hline \multirow[t]{3}{*}{$x y$-coords } & yes & $\begin{array}{l}\text { interpolation of porosity measured in } \\
\text { wells in an oil reservoir }\end{array}$ & Wong et al. (1997b) \\
\hline & yes & $\begin{array}{l}\text { neural network-residual kriging to in- } \\
\text { terpolate the depth to top of oil reser- } \\
\text { voir }\end{array}$ & Wong et al. (1998) \\
\hline & yes & 2-spiral pattern recognition problem & Lang \& Witbrock (1988) \\
\hline \multirow{3}{*}{$\begin{array}{l}\text { textural features } \\
\text { determined from } \\
\text { moving window } \\
\text { in image }\end{array}$} & yes & $\begin{array}{l}\text { land-cover classification } \\
\text { of remotely-sensed images }\end{array}$ & Bruzzone et al. (1997) \\
\hline & yes & $\begin{array}{l}\text { classification of sonar } \\
\text { images of sea floor }\end{array}$ & Müller et al. (1998) \\
\hline & yes & $\begin{array}{l}\text { classification of cracks and scratches in } \\
\text { industrially treated surfaces }\end{array}$ & Schramm \& Spinnler (1993) \\
\hline \multirow{4}{*}{$\begin{array}{l}\text { input of all pixels } \\
\text { from moving } \\
\text { window(s) in } \\
\text { image }\end{array}$} & yes & $\begin{array}{l}\text { land-cover classification } \\
\text { of remotely-sensed images }\end{array}$ & $\begin{array}{l}\text { Ritter \& Hepner (1990) } \\
\text { Bischof et al. (1992) }\end{array}$ \\
\hline & yes & $\begin{array}{l}\text { recognition of hand-written } \\
\text { digits in postcodes }\end{array}$ & $\begin{array}{l}\text { Le Cun et al. }(1989,1990 \mathrm{a}, \\
\text { 1990b }\end{array}$ \\
\hline & yes & $\begin{array}{l}\text { detection of edges of } \mathrm{Al}(\mathrm{OH})_{3} \text { particles } \\
\text { in Bayer } \mathrm{Al} \text { refining process }\end{array}$ & Zaknich (1999) \\
\hline & yes & $\begin{array}{l}\text { detection of faults and rock unit bound- } \\
\text { aries in remotely-sensed images }\end{array}$ & Penn et al. (1993) \\
\hline
\end{tabular}




\subsubsection{Selection of Neural Network Architecture}

Although a large number of different types of neural networks exist, reflecting the different type of the units, connections, and learning and recall algorithms possible, a Multilayer Perceptron (MLP) neural network is used to test a neural-network approach to mineral prospectivity mapping in the pilot study presented in Chapter 4.

There are several reasons for this choice. An MLP neural network;

1. performs very well for a large variety of problem types,

2. has powerful learning and generalization capabilities (i.e. applicable to regression and classification problems) (Hornik, 1991; Masters, 1993b),

3. (with one hidden layer) is capable of approximating any continuous multivariate function to any desired accuracy, provided a sigmoidal (squashed S-shaped) activation function, appropriate network parameters, and a sufficient number of hidden units are used (Cybenko, 1989; Funahashi, 1989; Hornik et al., 1989), and

4. is likely to give better generalization performance in cases where only a few training samples are available. Clearly, this last characteristic is particularly important in mineral prospectivity applications where the training set size is severely limited due to the paucity of deposit patterns Masters (1993b).

A number of other neural network architectures that are potentially useful for the estimation of mineral prospectivity are examined in Appendix A. These are the probabilistic (PNN), general regression (GRNN), radial basis function (RBF) and self-organizing feature map (SOFM) or Kohonen neural networks and a hybrid neuro-fuzzy system.

Before describing the multilayer neural network (MLP) in detail, some neural network terminology is introduced and the differences between the main types of networks are briefly explained. Inputs to a neural network are selected according to their usefulness in predicting the output. In the case of a pattern recognition task, the output is the class to which the input pattern belongs. Inputs are presented to the network in the form of row vectors (i.e. $\mathbf{x}^{n}=\left(x_{1}, x_{2}, x_{3}, \ldots, x_{n}\right)$, where $n$ is the dimensionality). These are generally called input feature vectors, although the term feature generally refers to input parameters which have been pre-processed or combined in some way. If $n=3$, the input feature space is three-dimensional and inputs can be represented as points in space. Higher dimensional feature vectors are said to be located in hyperspace (Bishop, 1995).

MLP neural networks classify patterns by dividing multidimensional feature space representing input vectors into class regions using decision boundaries called hyperplanes. For 
two input variables, feature space is represented by a two-dimensional plane and hyperplanes are represented by lines. These lines are called decision boundaries (Fig. A.16). In contrast, PNN, GRNN, RBFNN's are all kernel, or receptive field classifiers because they partition feature space using radial basis functions that can be represented in 2-D as local circular areas of influence.

MLP, GRNN, and RBFNN neural networks learn with a strategy known as supervised training. This involves presenting the network with both the inputs and the corresponding correct output during training. In contrast, in unsupervised training used with the selforganising map (SOM or Kohonen network), only the input vectors are presented to the network, and the network creates its own classification of the patterns. The SOM network performs classification based on the labelled training examples (for which the classes are known) that are nearest to the unknown input vector.

The fuzzy-neural system represents a hybrid architecture that combines the ability of fuzzy systems to deal with imprecise or error-prone inputs with the pattern recognition capability of neural networks. This enables conceptual and empirical approaches to be combined by incorporating subjective geological knowledge into an otherwise data-driven neural network method.

\subsubsection{Multilayer Perceptron (MLP) Neural Networks}

\section{Architecture of MLP Networks}

An MLP neural network is a type of multilayer feedforward network containing perceptronlike processing units. An MLP network consists of input and output layers and one or two hidden layers (refer to Fig. 2.3 above). Unlike the hidden and output layer units, the input units do not perform any processing and only serve to connect the network to a source of input signals and pass these signals on to other units. It is the presence of the hidden layer, containing units with a non-linear activation function, that enables multilayer networks to solve non-linearly separable problems. Whereas the number of input and output units is dependent on the problem being solved and the training data that are available, the number of hidden-layer units must be determined by trial and error for each particular problem. The term feedforward refers to the fact that there are no feedback connections back to units of previous layers or connections between units within the same layer.

Each unit in the hidden and output layers of an MLP neural network performs the same series of simple operations involving multiplication and addition, as well as applying a 
non-linear transformation to the input signals it receives (Fig. 2.7).

In detail these three steps are performed as described below.

1. The net input to a neuron or processing unit is determined by multiplying each input signal by the coefficient or weight, representing the strength of the connection (which may be positive or negative).

2. The weighted inputs are summed:

$$
\text { net }_{j}=\sum_{i} w_{i j} o_{i}
$$

where $n e t_{j}$ is the net input to unit $j, w_{i j}$ is the weight of the connection from unit $i$ to unit $j$, and $o_{i}$ is the activation or output unit $i$ (refer to Fig. 2.7).

3. The output or activation of a neuron is the result of transforming the net input by a non-linear activation function:

$$
o=f(n e t)
$$

The activation function used in the MLP network in Chapter 4 is the sigmoidal(squashed S-shaped) logistic function:

$$
f(n e t)=\frac{1}{1+e^{-n e t}}
$$

where net is the weighted, summed input to the processing unit, calculated from equation 2.20 .

The output value $o$, for an individual processing unit is referred to as the activation and becomes the signal on all the connections fanning out from the unit. If the unit is in a hidden layer, then this output becomes the input for units at the other end of the links in the next layer.

\section{Learning in MLP Neural Networks}

Learning in MLP neural networks is achieved by modifying the connection weights while a set of training examples is repeatedly processed. This training method is referred to as supervised because each example specifies all the input parameters together with the desired outputs. The set of inputs to the network constitutes a real-valued vector, $\left(x_{1}, x_{2}, x_{3}, \ldots, x_{n}\right)$. Each vector component represents a feature that is considered to be useful in predicting the output ${ }^{5}$. As the MLP neural network used in Chapter 4 contains

\footnotetext{
${ }^{5}$ If multiclass nominal variables are being used then they should be recast as continuous numerical variables.
} 


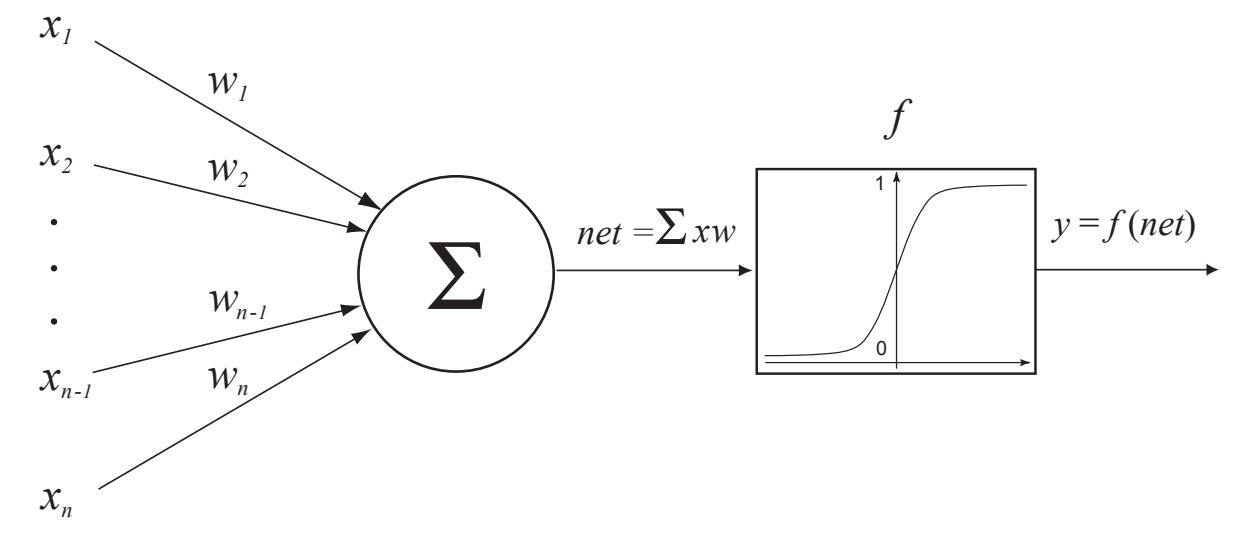

Figure 2.7. Computations performed in a single processing unit of a neural network. The input on each link is determined by multiplying the input signal by the weight associated with the link. Individual inputs are summed and then transformed by a non-linear sigmoidal activation function.

only a single output unit, the training data consist of a list of paired input vectors and a single scalar value representing the target or desired output.

During training, both the input feature vector and the target output value pairs are presented to the network (2.8). Initially, the weights are set to small random numbers (e.g. in the range $[-0.2,+0.2]$ ) and so, to begin with, the actual output is quite different to the target output.

The difference between the desired output and the output produced by the network represents the error. During training, this error signal is fed back into the network and forms the input to a learning algorithm. Learning proceeds by applying a learning algorithm to iteratively adjust the weight values in order to minimize this error the next time the pattern is presented to the network. The set of input and output pairs is called a training data set. Processing each pattern represents one cycle or epoch of training. Depending on the value of the random initial weights and other network parameters, such as the learning rate, hundreds or possibly several hundred thousand epochs of training may be required in order to reduce the network error to an acceptably small value. After training is completed, the weight values remain fixed. The network should then be able to generalize; that is, produce appropriate responses for inputs that have not been used during training. 


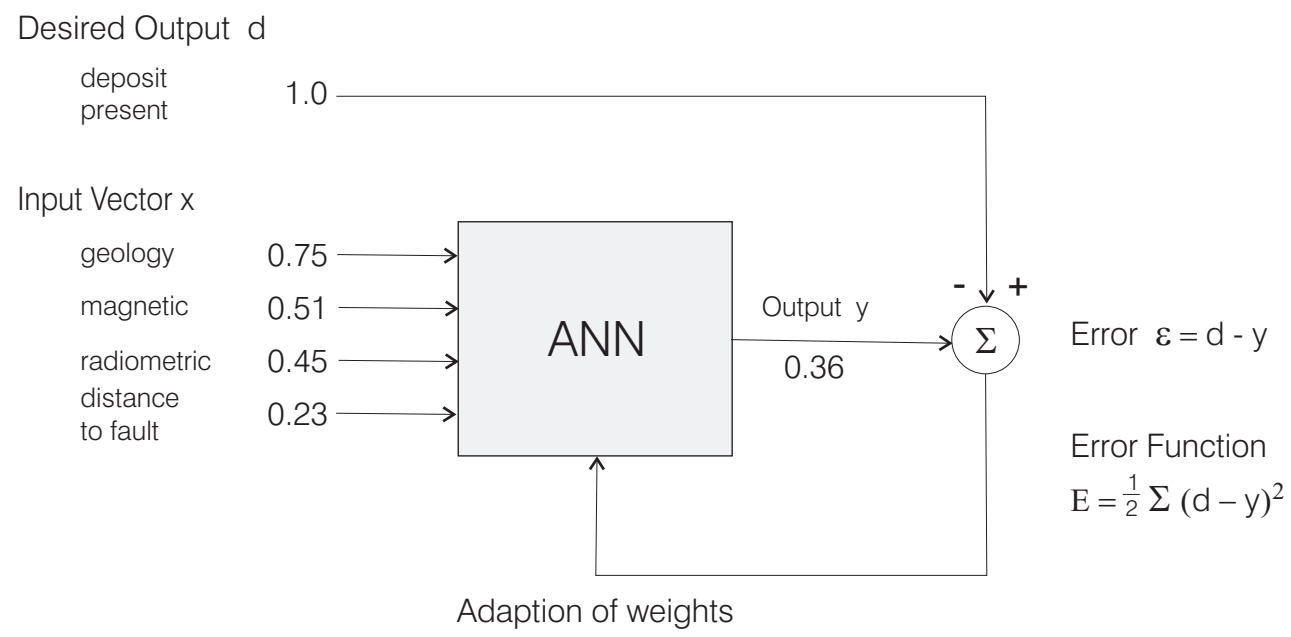

Figure 2.8. Block diagram representing the training process in a multilayer perceptron neural network (after Zaknich, 1999). The difference between the actual output produced by the network (y) and the desired output (d), supplied in the training data, represents the network error. The error-backpropagation algorithm iteratively modifies the connection weights in order to minimize the network error function.

\section{Error-Backpropagation Algorithm}

The learning algorithm used to minimize the network error during training in the MLP network in Chapter 4 is known as the error-backpropagation algorithm. The following discussion is based on descriptions of the algorithm by Rumelhart et al. (1986b), Rumelhart et al. (1986a), Beale \& Jackson (1990), Winston (1992), Hassoun (1995) and Zaknich (1999).

The algorithm was independently developed by Werbos (1974), and later by Parker (1985a) and Rumelhart et al. (1986b). The algorithm adjusts weights using a technique called gradient descent optimization. The concept behind the gradient descent algorithm is to make small changes to the weights proportional to the negative derivative of the error with respect to each weight for the current pattern of network inputs, as follows:

$$
\Delta w_{i j} \propto-\frac{\partial E_{p}}{\partial w_{i j}}
$$

where $w_{i j}$ is the weight on the link connecting unit $i$ to unit $j$ and $E_{p}$ is the network error for a single input pattern, $p^{6}$ The discussion that follows refers to the case where weights

\footnotetext{
${ }^{6}$ In this nomenclature, indices, $i, j, k$ refer to units in successive layers from the input towards the output (refer to Fig. 2.3).
} 


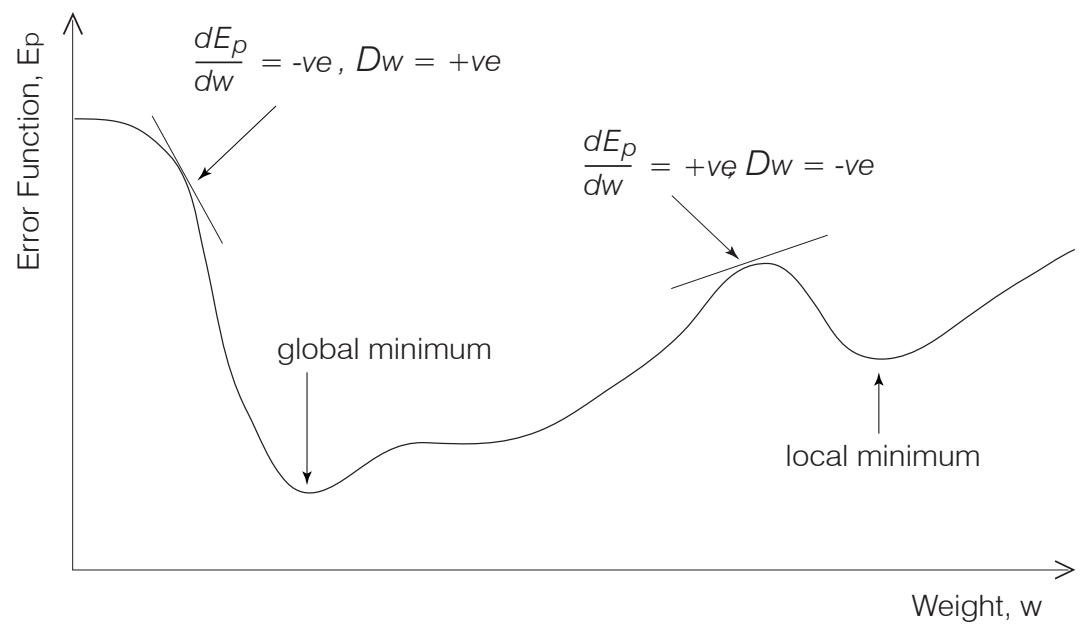

Figure 2.9. Effect of a single weight on the network error function (after Zaknich, 1999). At A where the slope i negative, the network error is reduced by a positive weight change. At B where the slope is positive, a negative weight change is required. A disadvantage of gradient-descent is that the network can converge to a local (D), rather than a global minimum (E).

are updated incrementally after processing each pattern.

The application of the weight update rule in equation 2.23 can be explained in terms of a hypothetical two-dimensional representation of an error function ${ }^{7}$, which describes the effect of a single weight on the network error at a single output unit (Fig. 2.9). In Figure 2.9, if the weight is too large, the gradient of the error function is positive and equation 2.23 indicates that the error can be reduced by subtracting a small amount from the weight. Conversely, if the gradient is negative, the error is reduced by increasing the weight by a small amount. Thus, weight changes are applied as follows:

$$
w_{i j}^{\text {new }}=w_{i j}^{\text {current }}+\Delta w_{i j}
$$

where $W_{i j}$ is the updated weight value for the weight connecting unit $i$ to unit $j$ and $w_{i j}^{\text {current }}$ is the current weight value.

One of the problems inherent in a gradient descent algorithm like error back-propagation is

\footnotetext{
${ }^{7}$ Since a real network contains many weights, the error function actually forms a complex surface in multidimensional weight space. If there were just two weights, the error function would form a surface, like a landscape with plateaus and deep valleys in multidimensional weight space.
} 


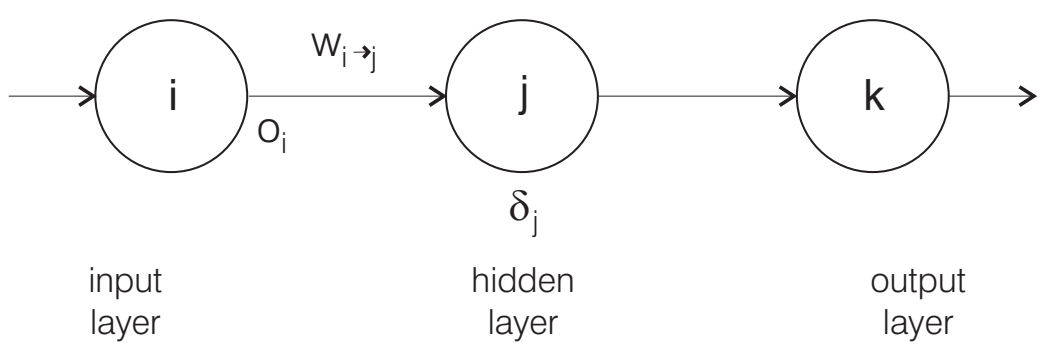

Figure 2.10. Nomenclature for units and variables in the generalized delta rule. The variables $o_{i}$ and $\delta_{j}$ refer to units at opposite ends of the link associate with weight $w_{i \rightarrow j}$ that is to be updated. $o_{i}$ is the output from unit $i$ that becomes one of the inputs to unit $j . \delta_{j}$ is the delta term for unit $j$.

that the network may converge to a local, rather than a global, minimum (Fig. 2.9). This depends partly on where training starts in weight space as a result of initializing weights to small random values. To avoid local minima, the training procedure is repeated using a different random set of initial weights each time.

A commonly-used error function is the sum of squares error. For a single input pattern, $p$, and a network with $N$ output units, the error function is given by:

$$
E_{p}=\frac{1}{2} \sum_{k=1}^{N}\left(d_{k}-o_{k}\right)^{2}
$$

where $E_{p}=$ error for pattern $p, d=$ desired output, $o=$ actual output and the subscript index $k$ denotes the $k^{t h}$ output unit. Rumelhart et al. (1986b) used this error function to evaluate the partial derivative in equation 2.23 and derive the propagation learning rule ${ }^{8}$ :

$$
\Delta w_{i j}=-k \frac{\partial E_{p}}{\partial w_{i j}}=\eta \delta_{j} o_{i}
$$

where $k$ is a proportionality constant, $E_{p}$ is the error for pattern $p, \Delta w_{i j}$ is the change in weight on the link from unit $i$ to unit $j, \eta$ is a proportionality constant called the learning rate, $\delta_{j}$ is the delta term for unit $j$ on the output end of the link and $o_{i}$ is the output from unit $i$ on the input end of the link (Fig. 2.3). The delta term, $\delta_{j}$ depends on unit $j$ and is proportional to; 1 ) the slope of the sigmoidal transformation function (at the point defined by the net input to unit $j$; Fig. 2.10), and 2) the network error due to unit $j$.

\footnotetext{
${ }^{8}$ The weight update rule used in the back-propagation algorithm is also known as the generalized delta rule
} 
The weight update rule that results from substituting equation 2.26 into equation 2.24 is:

$$
w_{i j}^{\text {new }}=w_{i j}^{\text {current }}+\eta \delta_{j} o_{i}
$$

For an output unit, the delta term can readily be determined since the delta term is proportional to the error-weight partial derivative (Equation 2.23) and the error for an output unit is given by equation eqn-netwk-error-fn. The delta term for an output unit is given by:

$$
\delta_{k}=f^{\prime}\left(\text { net }_{k}\right)\left(d_{k}-o_{k}\right)
$$

where the subscript $k$ refers to unit $k$ in the output layer, $d_{k}$ is the desired output, $o_{k}$ is the actual output, net $k$ is the total input to unit $k$, and $f^{\prime}$ is the derivative of the activation function shown in equation 2.22 .

The contribution a hidden unit makes to the network error is not clear. Although a learning rule for a single perceptron unit was known since about 1960 in the form of the least mean square rule (Widrow \& Hoff, 1960), no method for updating the weights of a hidden layer unit in multilayer perceptron neural networks was known until Rumelhart et al. (1986b) described the error backpropagation algorithm in 1986. They reasoned that, since the error of a hidden unit (e.g. unit $j$ in Fig. 2.3) is expressed through the output units (units $k$ in Fig. 2.3), the $\delta$ term for a hidden unit is proportional to the weighted sum of the $\delta$ terms for all the output units to which it is connected. The formula proposed by Rumelhart et al. (1986b) for the calculation of the delta term for units in a hidden layer is given by equation 2.29 :

$$
\delta_{k}=f^{\prime}\left(\text { net }_{k}\right) \sum_{k} \delta_{k} w_{j k}
$$

Equation 2.29 shows that the delta term for a hidden unit $j$ is defined recursively in terms of the delta values for the units in the output layer (denoted by the subscript, $k$ ). Consequently, the calculation of the delta terms for the output units must be propagated backwards in order to calculate delta terms for the hidden layer units. Hence the name error back-propagation algorithm. In order to evaluate delta terms for hidden and output layer units, the value of the derivative of the activation function, $f^{\prime}$, must be determined. The derivative for the logistic activation function (equation 2.30) used in the pilot study has the following simple form: 


$$
f^{\prime}=f(1-f)
$$

Substituting equation 2.30 in place of $f^{\prime}$, and then substituting equation 2.21 for $f$, allows equations 2.28 and 2.29 to be rewritten;

$$
\begin{aligned}
& \delta_{k}=o_{k}\left(1-o_{k}\right)\left(d_{k}-o_{k}\right) \\
& \delta_{j}=o_{j}\left(1-o_{j}\right) \sum_{k} \delta_{k} w_{j k}
\end{aligned}
$$

Equations 2.31, 2.32 and 2.27 are used to modify the weights after each presentation of a training pattern.

The error back-propagation consists of the following steps;

1. Calculate outputs $o_{i}, o_{j}, o_{k}$. In the forward pass, the input vector is presented to the network and the activations of the units are calculated using equations 2.20, 2.21 and 2.22 .

2. Calculate $\delta_{k}$ for output unit(s). At the output layer, the activations of the output units are then compared to the target outputs to give the error for each output unit. Delta terms are calculated for the output units according to equation 2.31.

3. Calculate $\delta_{j}$ for hidden units. In the backward pass through the network, delta terms for each hidden unit are determined recursively in terms of the output units directly connected to it using equation 2.32 .

4. Calculate weight changes. The weight updates are calculated by multiplying the delta terms for the unit on the output side of the link by the activation of the unit on the input side of the link, according to the delta rule given by equation 2.26.

5. Update weights using $w^{\text {new }}=w^{\text {old }}+\Delta w$.

In this way, the errors of the output units are propagated backwards. 


\subsubsection{Summary of Previous Work}

A summary of the main prospectivity mapping methods described in this Chapter and in Appendix A, together with the assumptions and data requirements, is given in Table 2.3 below.

The effectiveness of the multivariate statistical methods, such as multiple linear regression, multiple discriminant analysis, logistic regression and canonical correlation, are limited to varying degrees if the following underlying assumptions and requirements are not met;

1. relationship between the independent and dependent variables is linear (or in the case of logistic regression, a log-linear relationship is assumed; the Boolean and index-overlay methods also involve linear combinations of explanatory variables),

2. there is multivariate normality of independent variables,

3. homoscedasticity (constant variance over the range of the range of the independent variable is achieved, and

4. there is no significant multivariate collinearity between variables, or in the case of the weights-of-evidence method, conditional independence of binary input patterns with respect to deposit points (expert systems that use Bayesian updating of probabilities also require conditional independence of explanatory variables).

The assumption of linearity is the most serious limitation. If the underlying physical relationships are not linear, they cannot be adequately modelled using linear methods. Many physical relationships related to ore-forming processes are well documented and known to be highly non-linear. For example, three of the parameters used to describe the tendency of a substance to react with another, i.e. equilibrium constants, free energy and oxidation potential, are all non-linearly dependent on temperature (Krauskopf, 1979).

If the exact nature of a non-linear relationship is known, then it is sometimes possible to transform the variables to compensate for the non-linearity. However, Masters (1993b) notes that the type of non-linear relationship is generally not known and there are many types of non-linear relationships which can not be linearized by transformations.

Other limitations of the empirical methods previously discussed above include;

1. sensitivity to the inclusion of irrelevant variables in the model (e.g. cluster analysis),

2. the need for specially constructed GIS databases (e.g. mineral systems approach), 
3. the need to re-classify input data (i.e. conversion of continuous variable to multiclass variables) resulting in a loss of information (e.g. weights-of-evidence) ${ }^{9}$, and

4. large training sets (multiple linear regression, multiple discriminant analysis, logistic regression, canonical correlation and decision-tree induction systems).

Subjective methods include index-overlay, expert systems, fuzzy-logic, and the mineralsystems approach (see lower part of Table 2.3). These methods may be limited by their use of deposit models. Specifically, their performance may be adversely affected if;

1. undiscovered deposits are of the same type as that described by the deposit model in use, but have features that are significantly different to previously discovered deposits, (e.g. the Century Zn-Pb deposits; Legge, 1995),

2. undiscovered deposits may be of a different type to that described by the model, and

3. deposits of previously unknown types may exist (e.g, Olympic Dam Cu-Au-U-REE deposit at the time of discovery; Woodall, 1994).

These comments also apply to empirical methods. However, because conceptual methods involve an explicit definition of the ranges of variable values that are favourable, conceptual methods are likely to be more strongly affected by these problems than empirical methods. In addition, the result of any subjective method may be difficult to reproduce since experts are likely to make different assessments about the relative importance of the evidence.

\footnotetext{
${ }^{9}$ Although weights of evidence has usually been applied as a binary method, it is not restricted to binary data. For example, a geological map with over 20 classes was used in a study of the mineral potential of the Slave Province (Bonham-Carter et al., 1998a,b).
} 


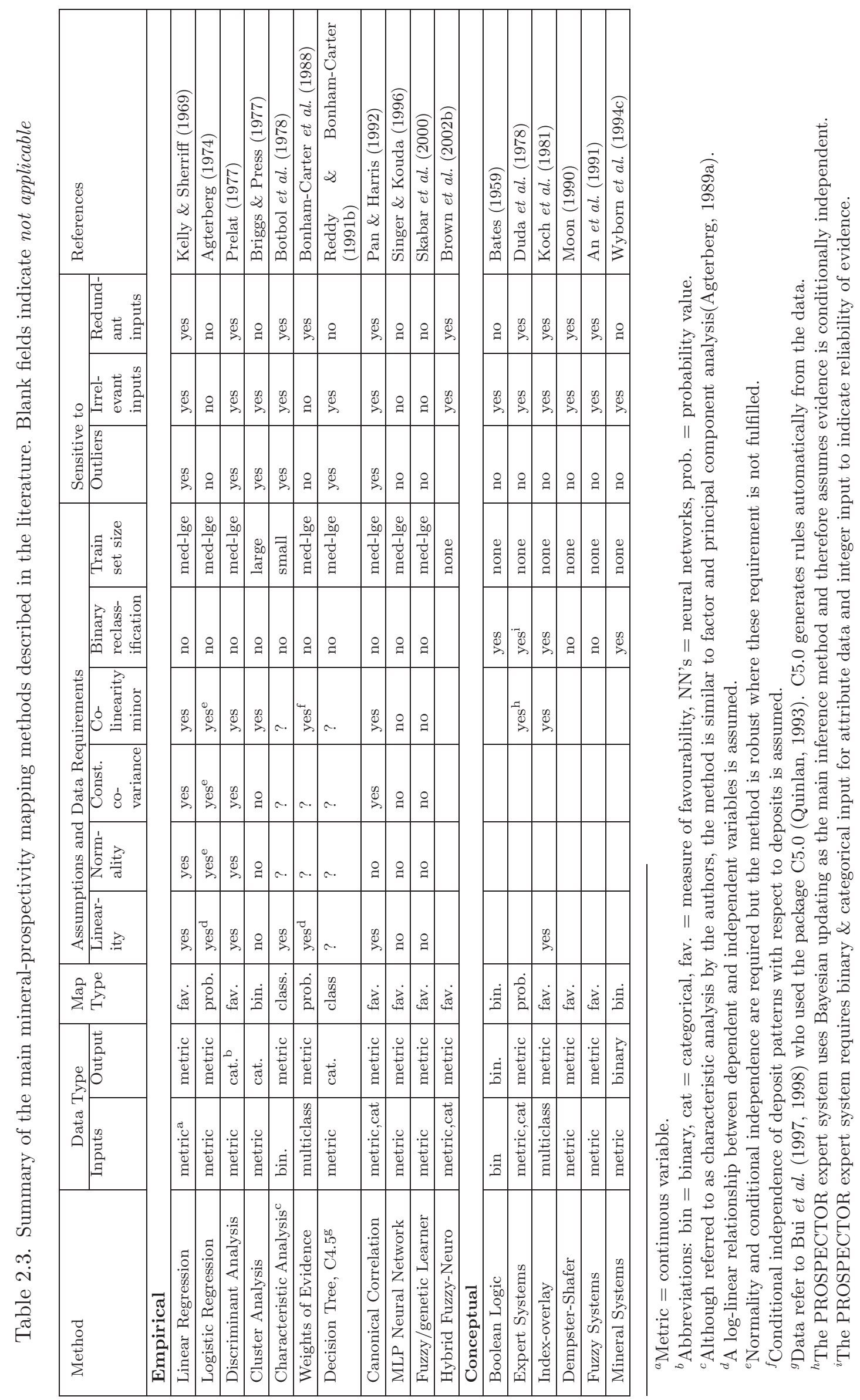




\subsection{Summary}

The main points discussed in Chapter 2 are listed below.

1. The purpose of mineral prospectivity maps is to estimate the relative favourability of areas represented by grid cells to host mineralization of a specific type.

2. Bonham-Carter (1994) distinguished two broad categories of mineral favourability mapping methods; that is, data-driven or empirical (e.g. weights of evidence) and knowledge-driven or conceptual methods (e.g. fuzzy systems). Neural networks represent a data-driven approach.

3. Weights of evidence (WofE) (Bonham-Carter et al., 1988) is the most widely-used statistical method for mineral prospectivity mapping. Evidence maps containing continuous variables are re-classified into binary or multiclass maps. In each evidence map, these classes representing ranges of variable values are converted to a pair of weights which express the degree to which the presence or absence of that class of values upgrades or downgrades the prior probability of a deposit, respectively. A prospectivity map displaying posterior probability values is created by combining all the weights corresponding to each cell location.

4. A difficulty with the WofE method is the assumption of conditional independence between parameters with respect to deposits. This requirement is rarely satisfied completely in geological data sets.

5. Where the assumption of conditional independence is seriously violated, either; 1 ) one of the dependent maps must be omitted or 2) dependent maps must be combined or 3) the logistic regression method can be applied (recommended when using ARCSDM). In the latter case, nominal multiclass maps are automatically treated as $1, \ldots, n$ variables.

6. Fuzzy systems are the most widely used, conceptual mineral-prospectivity mapping method. A fuzzy membership function is used to transform raw parameter values $u$, to real numbers in the interval $[0,1]$. In most mineral-prospectivity mapping applications, fuzzy membership values represent the degree to which the parameter belongs to the fuzzy set favourable for deposits.

7. In most studies documented in the literature, the gamma function (with gamma values in the range [0.95-0.98]) is used in the final integration step to combine fuzzy membership values to produce a prospectivity map. 
8. In most applications of the fuzzy-logic method to mineral prospectivity mapping, raw data values are converted to fuzzy membership values by assigning a single fuzzy-membership value to a whole class or range of raw values. This leads to a loss of information. In engineering applications, continuous fuzzy-membership functions are used to map raw values to membership values.

9. There are two further potential problems with the fuzzy-logic method; 1) fuzzy membership values are often applied according to the degree to which parameter values conform to a deposit model, which itself may be flawed, and 2) the assignment of fuzzy membership values, although often performed subjectively, could be performed objectively using the empirically-determined spatial associations between input features and known deposit points using methods described by Luo \& Dimitrakopoulos (2003) and Porwal et al. (2003b).

10. The mineral systems approach, proposed by Wyborn et al. (1994c), relates the essential ingredients of the deposit forming process to the type of geological evidence that is likely to have been captured in a regional-scale GIS.

11. A mineral system represents a far larger target for exploration than the actual ore deposit, and the scale of the parameters that define a mineral system is comparable to a regional-scale GIS.

12. Neural networks are suited to problems involving geoscience data because of their ability to deal with; 1) uncertain and noisy data, 2) outliers in the data, 3) very complex distributions, including large deviations from normality and multimodal distributions, 4) multiple interdependent input parameters, 5) highly non-linear relationships between input and output parameters, and 6) large heterogeneous data sets. In addition, they require no prior knowledge nor assumptions about the statistical distributions of the input data.

13. The multilayer perceptron (MLP) is the most generally applicable and by far the most widely used, type of neural network (Jang \& Sun, 1995)

14. MLP neural networks are chosen for this study because they; 1) perform very well for a large variety of problem types, 2) have powerful learning and generalization capabilities, and 3) are likely to give better generalization performance in cases where only a few training samples are available.

15. A problem with the gradient-descent algorithm used to train MLP neural networks is that the network may converge to a local, rather than a global, minimum. This depends partly on where training starts in weight space as a result of initializing 
weights to small random values. To avoid local minima, the training procedure is repeated using a different random set of initial weights each time. 


\section{Chapter 3}

\section{Methods and GIS Databases}

\subsection{Introduction}

This chapter describes the processing methods, databases and software environment used in this study. As stated in Chapter 1, the aims of the study are;

1. to develop a neural-network approach to integration of mineral exploration data and predict the favourability for mineralization,

2. quantitatively compare the results of a neural-network approach with those of other methods, and

3. test the applicability of a neural network method on variety of exploration data sets.

Aims one and two involve the preparation of mineral prospectivity maps and so the first part of this chapter outlines the procedures used in this study. Then, since many of the GIS map layers used as inputs represent modified rather than raw data, the types of derived layers and the methods used to produce them are summarized. This is followed by a description of the processing steps required to implement a neural-network approach to mineral prospectivity mapping.

The weights-of-evidence and fuzzy-logic methods are chosen to check the validity of the neural network results and to provide a basis for comparing the quality of mineral favourability maps produced in Chapter 4. These two methods are representatives of empirical and conceptual approaches, respectively, and are currently quite commonly used in industry. A brief description of the processing required to implement the weights-of-evidence and fuzzy-logic methods, together with a discussion of why they were chosen, forms a 
third section of this chapter. Although a fuzzy-logic inference net in which evidence maps are combined using fuzzy AND, OR and gamma functions is far from the most advanced fuzzy method available ${ }^{12}$, this simple fuzzy-logic method is used in this study because it is the main conceptual method for mineral prospectivity mapping in current use and therefore provides a good basis for comparison with neural networks. A variety of techniques are employed to assess the generalization and classification performance of trained neural networks and the most suitable methods for this study are reviewed.

There are very few reports in the literature that quantitatively compare the quality of mineral prospectivity maps produced by different methods ${ }^{3}$. Consequently, several approaches to this problem using both statistical and probability measures are developed and discussed.

In order to test the general applicability of a neural network method and to discover what factors may influence its performance, a case study is presented. The case study is based on a GIS database, which is in current use in an exploration program for an Archaean orogenic gold deposits. for a particular type of mineral deposit. The last part of the chapter gives a summary of the software and hardware used in this study.

\subsection{Preparation of Mineral Prospectivity Maps}

\subsubsection{Preparation of Derived Thematic Layers}

Map layers obtained by processing primary GIS thematic layers are called derived layers (e.g. a layer depicting distance to the nearest major fault can be derived from a primary structural layer). Derived layers are important because they represent a means of incorporating expert knowledge and encoding spatial relationships into the neural network input. It is clear that these categories can overlap if deposit knowledge indicates that certain spatial relationships are important requirements for ore formation.

Derived layers were prepared using the in-built functions of either the ARC/INFO or the ArCVIEW GIS systems. The choice of which system to use depended on the system in which the data were originally compiled and on the complexity of the derived layer required. ARC/INFo contains a larger range of in-built functions than ARCVIEW and is

\footnotetext{
${ }^{1}$ Kóczy, (pers. comm.)

${ }^{2}$ Rule-based and hybrid fuzzy systems are described in Section A.2.5 in Appendix A

${ }^{3}$ An exception is the study by Harris \& Pan (1999).
} 
more convenient to use because extensions can be written in the VISUAL BASIC programming language rather than the proprietary object-oriented language, Avenue, used in ARCView.

All operators, functions, statements and commands used to produce derived grid layers represent a type of map algebra operation (Tomlin, 1983, 1990) and result in the creation of a new map layer. The operation is applied to all locations in one or more input grids and has the following form:

$$
\text { newmap }=f\left(\operatorname{map}_{1}, \operatorname{map}_{2}, \ldots, \text { map }_{n}\right)
$$

The types of derived layers and the generic and specific GIS functions used to create them are summarized in Table 3.1.

Only a few simple derived layers were used in the pilot study in Chapter 4. Experiments dealing with ways in which feature selection ${ }^{4}$ and the incorporation of prior knowledge into the neural network can improve performance are described in Chapter 5.

\section{Conversion of Input Layers to Raster Format}

One pre-processing step performed within the GIS that is common to all neural network, weights-of-evidence and fuzzy-logic methods is the conversion of GIS thematic layers, such as image and ArCVIEw polygon coverages, to raster format (Fig. 3.1). Depending on the choice of cell size, some layers initially in raster format may need to be re-gridded in order to change the cell size to a common value.

All other processing in this study is based on raster GIS layers. A raster rather than a vector format was chosen because raster layers can more easily be converted to numerical patterns of the type used to train neural networks. In addition, only raster layers can be conveniently processed outside of the GIS using external programs and software packages. The use of raster layers allowed neural-network processing to be performed using $\mathrm{C}++$ programs and the C-like scripting language in MATLAB rather than the proprietary Avenue scripting language used in $\mathrm{ARCVIEW}^{5}$

An additional reason for using the raster format is that, although the vector format is

\footnotetext{
${ }^{4}$ feature selection is the process of transforming raw data so that it represents the essential information required for the neural network to perform the classification task (Bishop, 1995).

${ }^{5}$ Prior to the introduction of Microsoft Visual Basic in 1999, the only script language available in ARC/INFO was a proprietary interpreted language with limited capabilities called AML.
} 
Table 3.1. Examples of derived layers used to encode spatial relationships and prior knowledge into layers used as inputs. The main ARC/INFO functions used to create each layer are also shown, where applicable.

\begin{tabular}{|c|c|}
\hline Cell value in derived layer & ARC/INFO command ${ }^{a}$ \\
\hline \multicolumn{2}{|l|}{ Spatial Relationships } \\
\hline distance to nearest fault & eucdistance \\
\hline distance to nearest fold axis & eucdistance \\
\hline distance to nearest lithological boundary & eucdistance \\
\hline distance to fault/major lineament intersection & eucdistance \\
\hline distance to nearest deposit & eucdistance \\
\hline distance to nearest geochemical anomaly & eucdistance \\
\hline dip and strike of nearest fault & eucdistance \\
\hline distance to fault interval with strike $45^{\circ}$ & Visual Basic ${ }^{\mathrm{b}}$, eucdistance \\
\hline slope of top of lithological unit ${ }^{c}$ & slope \\
\hline derivatives of potential fields; e.g. gravity, magnetic ${ }^{\mathrm{c}}$ & slope, slope 2 \\
\hline density of faults in cell neighbourhood ${ }^{\mathrm{c}}$ & Visual Basic \\
\hline tonnes of contained metal in cell neighbourhood ${ }^{\mathrm{c}}$ & Visual Basic \\
\hline 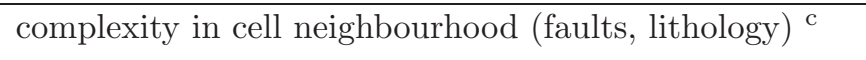 & focalvariety (grid, neighbourhood) \\
\hline \multicolumn{2}{|l|}{ Expert Knowledge } \\
\hline functions of parameters e.g. $U+T h,(U+T h) / m a g$ &,,$+- \times, /$ operators \\
\hline hydrothermal alteration; Landsat TM bands $5 / 7$ & / operator \\
\hline presence of reactive host rocks; e.g. $\mathrm{Fe} /(\mathrm{Fe}+\mathrm{Mg}) \mathrm{wt} \%$ & reclassification $^{\mathrm{d}}$ \\
\hline presence of alteration minerals & reclassification \\
\hline degree of fractionation \& oxidation of granitoids & reclassification \\
\hline age of rock units & reclassification \\
\hline thickness of lithological unit & - operator \\
\hline layer excluding faults formed after mineralization & ARCEDIT functions \\
\hline layer depicting low mean principal stress & external programs \\
\hline max. rock rheological contrast in cell neighbourhood ${ }^{\mathrm{c}}$ & focalmax (grid, neighbourhood) \\
\hline
\end{tabular}

${ }^{a}$ ARC/INFO commands, operators, functions and scripts

${ }^{b}$ Avenue code required to convert a structural layer showing the presence or absence of faults to one in which each cell represents the dip or strike of the fault along a specified interval, was developed by $\mathrm{S}$. Gardoll.

${ }^{c}$ These are focal functions that determine the value of the current cell from the values of cells around it within a small neighbourhood. The other functions and operators shown are local and only involve a single location.

${ }^{d}$ Reclassification is performed by applying the following conditional statement in an ARC/INFO GRID DO CELL processing loop to each cell in the grid: if(condition<statement list> else if $($ condition $)<$ statement list $>$ endif 


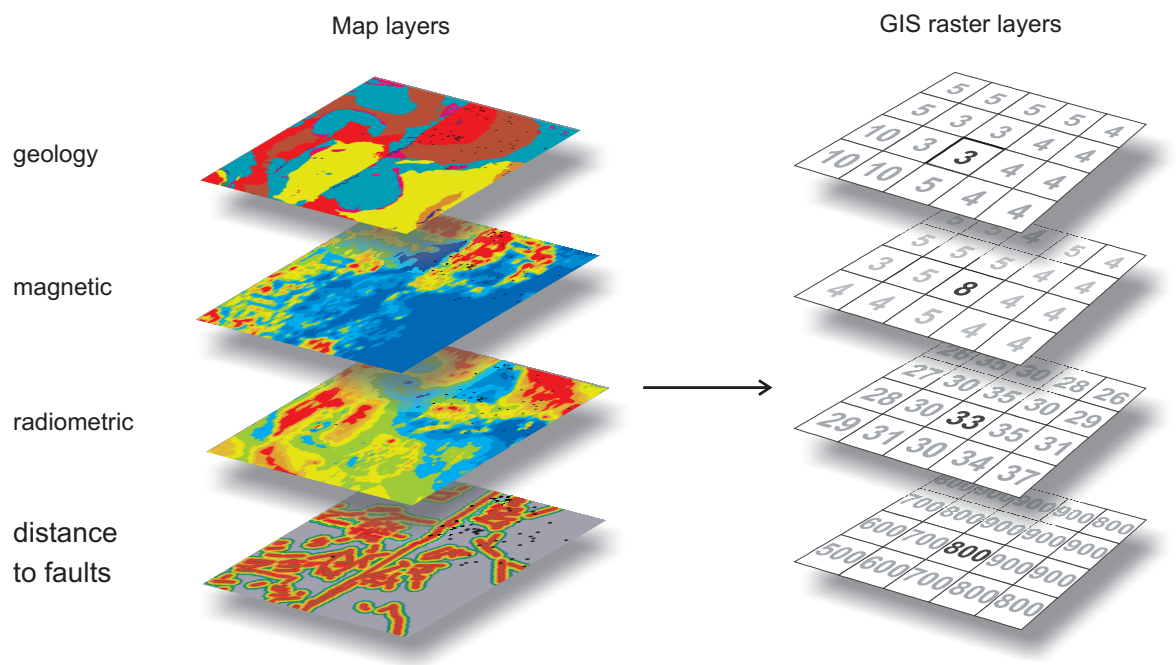

Figure 3.1. The first pre-processing step is the conversion of all GIS thematic layers (images and polygon coverages in vector format) to raster format. Layers initially in raster format may need to be converted to a consistent cell size.

preferable for layers containing discrete entities like polygons and lines, representing lithological units and faults respectively, the raster format is better suited for representing geochemical and geophysical data sets and satellite imagery. By far the majority of the input layers used in this study correspond to this latter group. Finally, a much greater variety of spatial analysis and image processing functions are available in most GIS for raster data (Burrough \& McDonnell, 1998).

\subsubsection{Neural Network Method}

The approach taken in this study was first to determine if and how a neural network could be used to predict prospectivity maps and then (based on the success of this first part) to investigate the factors which determine the quality of maps produced using neural networks.

In Chapter 2, it is shown that neural networks are a powerful type of model-free classifier and universal function approximator and that neural networks have many characteristics that are well suited to geoscience applications.

The investigation in Chapters 5-7 into how neural networks can best be applied to the problem of mineral prospectivity is based on the steps which are common to solving any problem with neural networks. These steps, described by Zaknich (1999) are: 
1. data collection,

2. raw data processing,

3. selection of a neural network type and topology,

4. feature extraction from pre-processed data, and

5. training and testing.

Although the use of GIS databases which have been compiled elsewhere eliminates the first two steps from consideration in this study, each of the other points forms the basis of a separate investigation in later chapters.

The problem of how best to apply neural networks to the problem of predicting mineral prospectivity is investigated by testing the factors that determine the performance of a neural-network approach. The factors and the chapters in which they are examined are;

1. type of neural network architecture (Chapter 7),

2. type and coding of features used as inputs (Chapter 5),

3. training strategy (training data set design, learning algorithm, model selection and classification threshold (Chapter 7), and

4. use of prior knowledge (Chapters $5 \& 7$ ).

Although many different types of neural networks exist, some of the main neural network architectures used in practical applications are examined in detail in Chapter 2 and in Appendix A. Of these, the Multilayer Perceptron (MLP) is the most generally applicable and by far the most widely-used type of neural network (Jang \& Sun, 1995). Consequently, it was selected for the pilot study described in Chapter 4. The neural network method described here is the general processing scheme that is applicable to neural network experiments to both the pilot and the case study (Chapters 4 \& 5-7). Details of the processing steps, which apply only to a specific experiment, are given in the relevant sections.

\section{Processing Steps}

Data collection, raw data processing, and some of the work of the feature extraction ${ }^{1}$ had already been performed in the production of the GIS thematic maps used as the starting point of this study.

The following processing steps are performed in the neural network method;

\footnotetext{
${ }^{1}$ Feature extraction is the one of the main subjects of Chapter 6.
} 
1. convert images, polygon coverages (vector format) and grids to ASCII raster format with the same cell size,

2. produce derived GIS thematic layers (feature extraction),

3. combine GIS thematic layers to produce input feature vectors,

4. perform pre-processing; scale input data to $[0,1]$,

5. create training data sets,

6. train neural network (and collect test-set performance statistics),

7. process entire study area using trained network,

8. scale and classify outputs,

9. produce prospectivity map, and

10. analyze prospectivity map quality.

The first two steps involving the preparation of derived layers and conversion of input layers to raster format are explained in Sections 3.2.1 and 3.2.1, respectively. The other steps are briefly explained in the section that follows.

1. Combine GIS thematic layers to produce input feature vectors. The key to applying a neural network to the solution of any problem is to formulate the problem as a series of numerical patterns of inputs and corresponding outputs that can be used to train the network ${ }^{1}$.

The way in which data stored in GIS thematic layers in raster format are converted to a series of patterns of inputs and outputs, that can be used to train an MLP neural network, is shown in Figure 3.2. Input GIS layers that depict features that are useful for predicting the location of mineralization are shown superimposed. Each layer represents a different data type (Fig. 3.2). The bottom layer represents an important additional layer. This depicts the locations of known deposits and occurrences of a particular type and is coded in binary form. The presence of mineral deposits or occurrences is indicated by a one, and barren locations by a zero. Viewing the stack of GIS layers from above, if a particular location corresponds to a deposit, then the string of numbers forms a feature vector, which can be viewed as a type of ore signature, just as the reflectance bands from a LANDSAT TM image can be used to give a signature for alteration minerals. The concept central to this thesis is that neural networks can be trained to recognize the signatures of known deposits and, once trained, predict the prospectivity of other areas. Thus, in Figure 3.2, the first four layers encoded as the input feature vector represent the input to the neural network (in both training and production modes) and the deposit layer values

\footnotetext{
${ }^{2}$ This is the situation if supervised training is used. For the unsupervised training scheme used for the Kohonen's Self-Organizing Feature Map, only the input patterns are presented to the network.
} 
represent the target output used to train the neural network. A paired input feature vector and scalar, target output value constitutes a single training pattern.

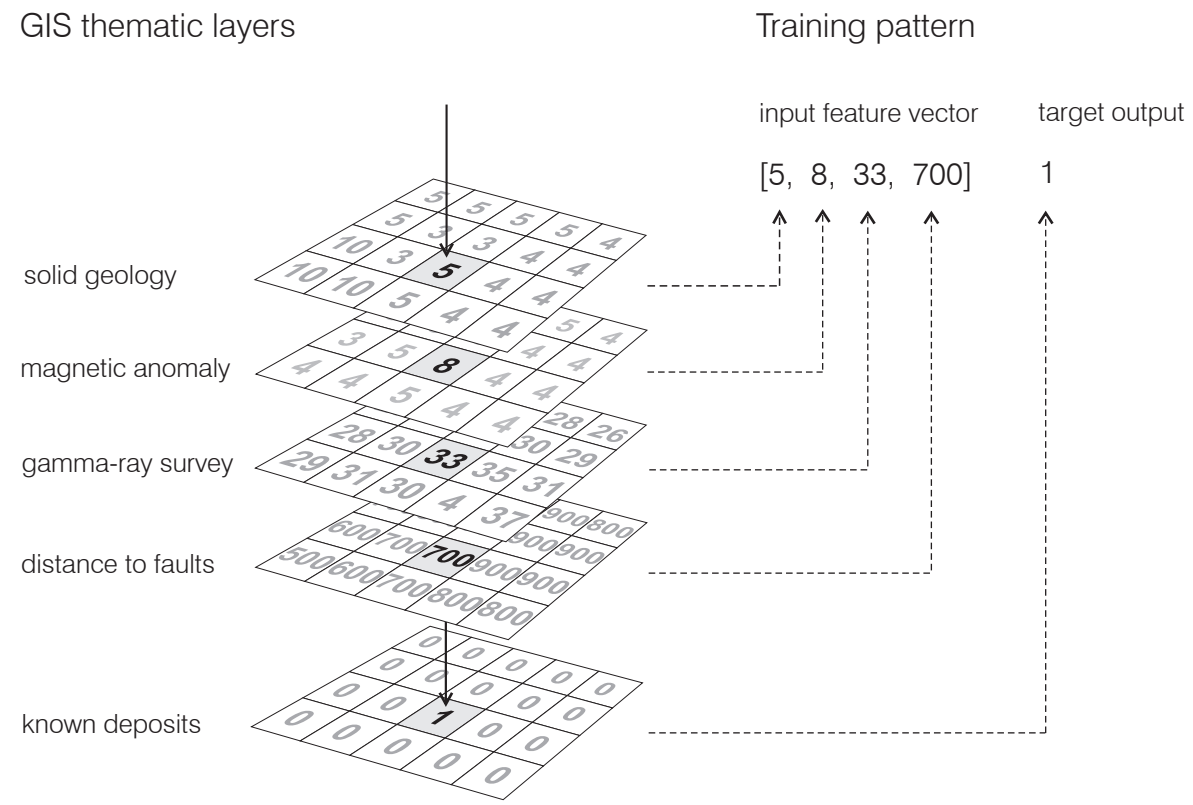

Figure 3.2. Relationship between GIS layers and feature vectors used as inputs to the neural network. For each cell location on the map grid, the components of the input feature vector for that location are set to the values stored in the thematic layers. Target vectors, which are used for training, contain a single component set to 1 if a deposit is present, otherwise the value is set to 0 .

2. Pre-processing. Although pre-processing and feature selection can have a significant effect on the generalization performance of a trained neural network, only basic pre-processing is described here. This topic is investigated in Chapter 6. Linear re-scaling of input variables to the range $[0,1]$ was always performed as a first pre-processing step for all neural network experiments according to equation 3.2:

$$
x=r\left(m-m_{\min }\right)+x_{\min }
$$

where

$$
r=\frac{x_{\max }-x_{\min }}{m_{\max }-m_{\min }}
$$

and

$m_{\max }$ is the maximum limit of the measured variable

$m_{\min }$ is the minimum limit of the measured variable

$x_{\max }$ is the maximum limit of the scaled variable, i.e. 1

$x_{\min }$ is the minimum limit of the scaled variable, i.e. 0 
If the logistic activation function is used in the output units of an MLP neural network (as in Chapter 4), then the practical limits of the outputs are 0.1 and 0.9. Training is facilitated if both the target output values used in training and the input values are scaled to the practical limits of the network output (Masters, 1993b). Bishop (1995) notes that it is particularly important to normalize the input variables of the radial basis function network, so that they span similar ranges, otherwise variables with significantly smaller ranges than the others will be ignored by the network. The scaling operation was performed once for the entire set of training data.

Scaling of inputs is also necessary because input variables can have significantly different ranges of values; for example, total magnetic intensity and the orientation of the nearest fault have values that differ by two orders of magnitude. If raw values were used as inputs, variables that typically have large values would have an unduly large effect on the network output compared to variables with small values. Scaling allows inputs to be recognized by the network to be of equal importance.

The creation of feature-vector-target-output pairs (step 1) and linear scaling (step 2) were performed using a single $\mathrm{C}++$ program, merge_sls. Details of the program are given in Appendix E and the code is included on the attached CD-ROM (vol. 1).

3. Create training data sets For network architectures in which a supervised training method is used (i.e. MLP, PNN, GRNN, and RBFNN), training data consist of input feature vectors paired with the corresponding correct output (referred to as target or desired outputs). Since only a single output was used in all feed-forward networks in this study, the target output is always a single value.

Training data were divided into roughly equal training, training-stop ${ }^{2}$ and test data sets. A number of authors have stressed the importance of using a completely independent data set for MLP networks in order to gain a true indication of the generalization (classification) performance of the trained network and warned of optimistic bias obtained from the test data set results (Masters, 1993b; Zaknich, 1999; Singer \& Kouda, 1999a). For the MLP network, the training data set is used to modify the connection weights, the training-stop data set is used to determine when to stop training and the test data set is used to assess the generalization performance of the network after it has been trained. In the case of probabilistic, general regression and radial basis function neural networks, each pattern in the training data set is stored

\footnotetext{
${ }^{2}$ The name training-stop data set is sometimes referred to as the stop set in tables in later chapters
} 
in the network in the form of weights connecting one of the hidden layer units. The training data set is used to modify network parameters and the test data set is used to check the performance of the trained network.

Training data patterns were selected randomly from cell locations over the entire study area. For experiments with the MLP neural network, approximately equal numbers of patterns representing deposit and non-deposit cells were randomly selected. The training data were then divided randomly between the training, trainingstop and test data sets.

Training data were selected (step 5) using a single $\mathrm{C}++$ program, rand_select. Details of the program are given in Appendix E and the code is listed on the attached CDROM (vol. 1).

4. Train and test neural network. The training scheme and adjustable parameters differ for each network type and are therefore described in the sections which document the individual experiments below.

The neural network used in the pilot study described in Chapter 4 was the $b p$ (backpropagation) program developed by McClelland \& Rumellhart (1988). All other experimentation was performed using the MATLAB software package in conjunction with the neural network and fuzzy tool boxes (i.e. add-on packages, Chapman, 1999).

5. Processing with the trained network. Once training is completed, the network parameters and weights are fixed. The network is then used to process feature vectors corresponding to the entire data set. Output values produced are stored as a grid. These values represent the activation of the single network output unit and are floating point values, which lie approximately in the range [0.05, 0.95].

6. Scale and classify outputs. The output values from the previous step were rescaled (using equation 3.2) to the range $[0,8]$ and then classified into integer intervals to produce a nine class prospectivity map. The scaling and classification are performed using a $\mathrm{C}++$ program, map_class, which is documented in Appendix E. A listing of the program code is given on the attached CD-ROM (vol. 1).

Map quality statistics were generated using the program, map_qstats, which takes the scaled, classified integer grid as input. This program calculates the chi-squared statistic, Spearman's and Kendall's rank correlation coefficients. A second program, deposit_locns is used to count the number of grid cells and deposits for each geological 
unit. These quantities are required to calculate the probability-based measures of map quality, described below in Section 3.4.2.

7. Prepare prospectivity map. Final map preparation, involving the assignment of colours to map classes, overlaying deposit points on the prospectivity maps and creating legends, scale bars, and grid coordinates, was performed with the GEOSOFT data processing package, OASIS MONTAJ.

In order to import the integer grid file from the previous step into GEOSOFT, the file was converted to GXF format (GEOSOFT, 1999) by inserting the appropriate header information at the start of the file before the data. GXF is a standard ASCII file format for exchanging gridded data among different software systems (ASEG, 1997). GXF Revision 3 implements the exchange of map projection information, which is specified using the Petrotechnical Open Software Corporation (POSC)/European Petroleum Survey Group (EPSG) projection data model and a standard set of geodesy parameters and values (EPSG, 1999). GXF files can be directly imported into the GEOSOFT package and displayed as a map.

The processing steps described above are shown schematically in Figure 3.3.

\subsubsection{Assumptions}

Several assumptions are common to all data-driven methods for estimating mineral prospectivity. These assumptions are listed below.

1. Undiscovered deposits have similar characteristics to known deposits. In particular, it is assumed that the regional-scale expressions of mineral systems associated with undiscovered deposits are similar to those of known deposits. Small ore deposits are far more abundant than large deposits and, therefore, training data sets are necessarily composed mainly of smaller deposits ${ }^{3}$. However, giant ore deposits are the targets of most exploration programs, particularly those of larger mining companies. Although there is much discussion about whether giant ore deposits have significantly different characteristics from smaller deposits (Cooke \& Pongratz, 2002), Phillips et al. (1996), among others, conclude that giant ore deposits are the result of a conjunction of a larger number of favourable factors than smaller deposits.

\footnotetext{
${ }^{3} \mathrm{~A}$ threshold of $1000 \mathrm{~kg}$ total contained gold is used in the Kalgoorlie case study in Chapter 7 .
} 


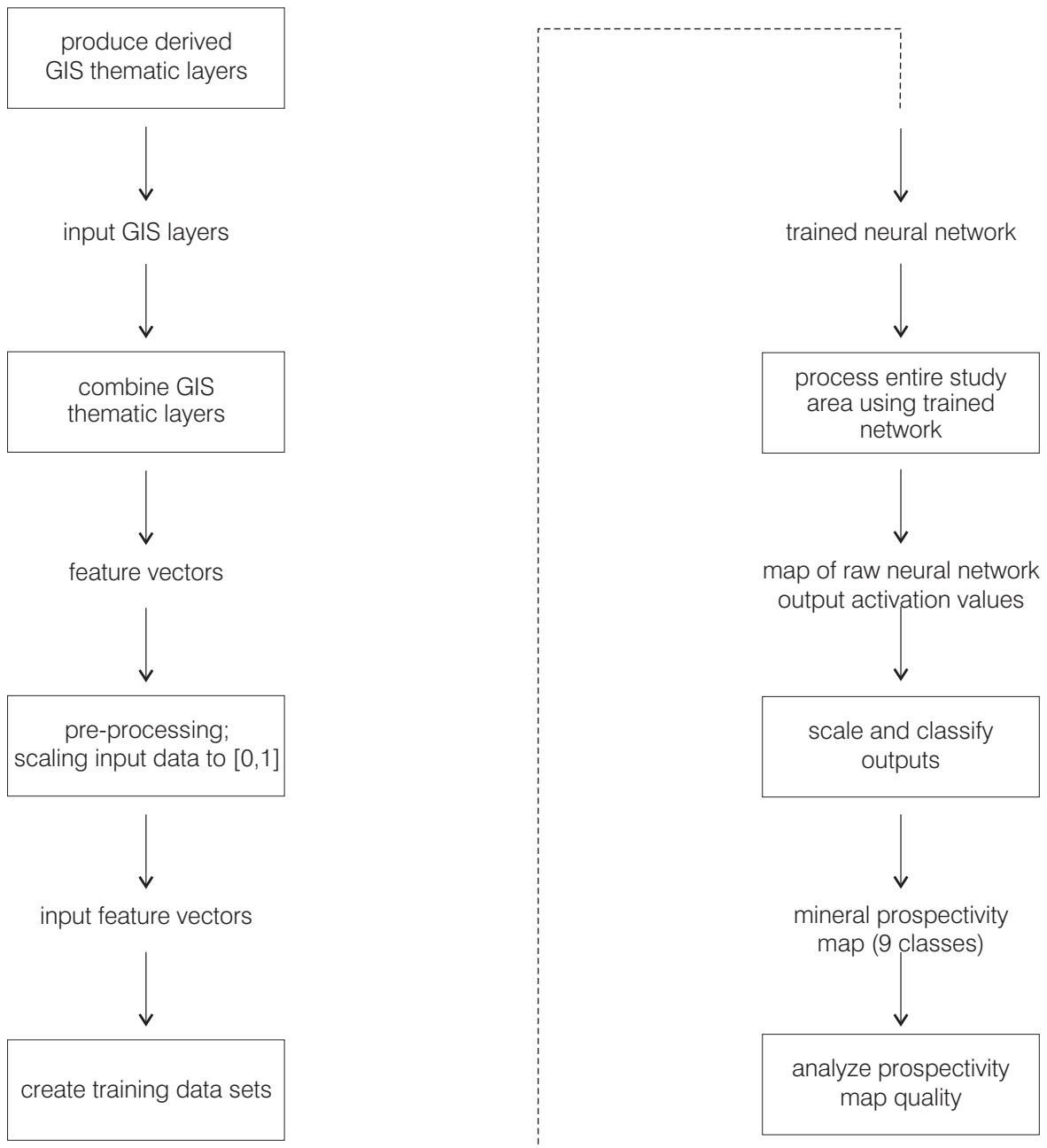

Figure 3.3. Schematic diagram of the data processing steps in the neural network method. 
2. GIS layers contain evidence for undiscovered deposits; that is, both the type of parameters and scale at which the parameters are captured in the GIS database are appropriate and sufficient to predict the location of the given deposit type.

3. The quality and degree of detail in the GIS layers is similar throughout the map area.

4. The data used for training are representative of the population of both discovered and undiscovered deposits, and cover the range of variation of all the parameters.

5. Grid cells selected to represent barren patterns, in the training set, do not contain any deposits of the type being sought. This is impossible to demonstrate without drilling. However, the rarity of deposits ensures that this assumption is generally true. The problem here is that the training patterns are commonly contradictory, in the sense that barren cells may possess nearly identical attribute values to mineralized cells, and yet the target value is significantly different.

Clearly, it is very difficult to satisfy all these assumptions in practice. However, for the purposes of this study, to test and compare a neural network method of prospectivity mapping with other methods, all data-driven systems and networks tested are subject to the same limitations because they use the same database. Despite these limitations, the use of a test data set that is independent of the training data can provide a useful comparative guide to the expected performance of the systems on completely new data.

\subsection{Evaluating Neural Network Performance}

Four of the main methods for evaluating the performance of neural networks are described in this section. These are the chi-squared (goodness-of-fit) test, mean square error, confusion matrix, and Receiver Operator Characteristic (ROC) curve. The last three of these methods measure the classification performance of the trained network, while the chisquared statistic is used to assess the quality of the prospectivity map produced using the trained network.

\subsubsection{Chi-Squared Test}

Bonham-Carter (1994))has recommended that the chi-squared statistic should be used in an exploratory way without reference to confidence levels in the case of spatial data. 
The chi-squared statistic is used in this study to assess the quality of prospectivity maps produced using different methods (Chapter 4).

The chi-squared statistic is calculated with the equation:

$$
\chi^{2}=\sum_{i=1}^{N} \frac{\left(O_{i}-E_{i}\right)^{2}}{E_{i}}
$$

where

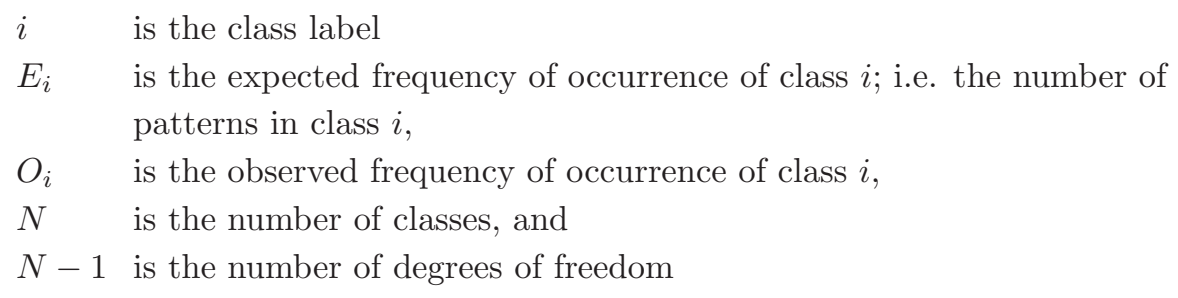

In studies where confidence levels are used, if the chi-squared value exceeds the tabulated value for a given level of confidence, then the null hypothesis (that the observed and expected frequency distributions are the same) is rejected.

In this study, the method is used to compare how well different classes in a nine-class prospectivity map predict the known deposits. Since the overall number of deposits occurring in the map area is known, the expected frequency of occurrence (i.e. prior probability) of deposits per grid cell can be calculated. Once this is known, the expected number of deposits can be calculated for each map class based on its area and compared with the actual number of known deposits occurring in that region.

\subsubsection{Mean Square Error}

The mean square error measures the error at the network output units in terms of the difference between the target output and the actual output calculated by the network. The mean square error is calculated according to equation 3.5;

$$
\begin{aligned}
E^{n} & =\frac{1}{K} \sum_{k=1}^{K}\left(y_{k}^{n}-t_{k}^{n}\right)^{2} \\
E & =\frac{1}{N} \sum_{n=1}^{N} E^{n}
\end{aligned}
$$

where 


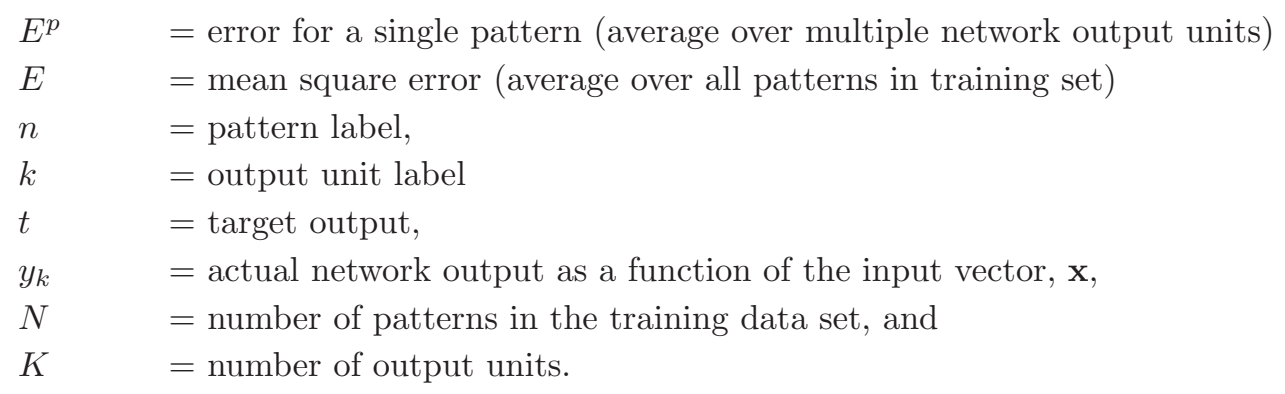

The mean square error (MSE) can be used to measure the performance of networks used for both classification and function approximation. The MSE is often used to assess performance during training. McClelland \& Rumellhart (1988) used a similar measure of error, namely the sum-of-squares error, to specify the training and test data set error for the bp program used in the pilot study in Chapter 4. The total sum-of-squares error (TSS) has the form:

$$
E=\frac{1}{2} \sum_{n=1}^{N} \sum_{k=1}^{K}\left(y_{k}^{n}-t_{k}^{n}\right)^{2}
$$

where the variables are the same as those for equation 3.5 shown above. Problems with the MSE and TSS error measures pointed out by Masters (1993b) include;

1. larger errors are emphasized, despite the fact that any error that leads to a misclassification could be important in a classification problem,

2. the measures do not provide any information about the type of errors, and

3. the measures do not indicate the expected frequency of misclassification.

For these reasons, the MSE and TSS error measures are only used during training and are not used to quote the classification performance of trained neural networks.

\subsubsection{Confusion Matrix}

A confusion matrix is a cross-tabulation of the true classification (rows) and the classification assigned by a system (columns) showing the number or percentages of correct and incorrect classifications for each class (Bonham-Carter, 1994). An example with just two classes is shown in Table 3.2 below.

The nomenclature, which is used commonly used in two-class classification problems is 
Table 3.2. A confusion matrix showing the four possible outcomes for a classification decision involving two classes.

\begin{tabular}{|cc||c|c|}
\hline \multicolumn{1}{|c||}{} & \multicolumn{2}{c|}{ Classified as } \\
& Class 1 & Class 2 \\
\hline \hline \multirow{2}{*}{ Class } & Class 1 & $C_{11}$ & $C_{12}$ \\
\cline { 2 - 3 } & Class 2 & $C_{21}$ & $C_{22}$ \\
\hline
\end{tabular}

Table 3.3. Receiver-operating-characteristic table showing nomenclature for a 2-class classification (after Zaknich, 1999).

\begin{tabular}{|cc||c|c|}
\hline \multicolumn{1}{|c||}{} & \multicolumn{2}{c|}{ Classified as } \\
& Positive & Negative \\
\hline \hline \multirow{2}{*}{ Class } & Positive & TP & FN \\
\cline { 2 - 3 } & Negative & FP & TN \\
\hline
\end{tabular}

shown in Table 3.3. The meanings of the abbreviations are shown below. If class 1 represents deposits and class 2 represents barren cells, then the elements have the following meanings;

$$
\begin{aligned}
& C_{11}=\text { number of deposit cells that were classified as deposits (True positive, TP) } \\
& C_{21}=\text { number of deposit cells that were classified as barren cells (False positive, FP) } \\
& C_{12}=\text { number of barren cells that were classified as deposit cells (False negative, FN) } \\
& C_{22}=\text { number of barren cells that were classified as barren cells (True negative, TN) }
\end{aligned}
$$

Entries in the diagonal positions are correct classifications, whereas all off-diagonal entries represent misclassifications. In a perfect classification, all non-diagonal entries would be zero. A confusion matrix is useful because it shows the type of misclassifications. For example, it is probably better to misclassify possibly barren cells as containing mineralization that it is to classify cells containing known deposits as barren. A confusion matrix would show if this occurs.

A number of single-value measures of classification performance, based on the quantities shown in Table 3.3, are commonly used. Most remote-sensing image classification studies; (e.g. Lees, 1996), use the overall correct classification rate defined by equation 3.8:

$$
\text { Overall correct }(\%)=\frac{\sum_{i=1}^{N}(\text { number correct for class } i)}{\text { total number of patterns }} \times \frac{100 \%}{1}
$$

The overall percentage correct measure is used in the pilot study in Chapter 4. In a study comparing PNNs and the weights-of-evidence methods for mineral favourability analysis, Singer \& Kouda (1999a) quoted the classification performance in terms of the classification error rate, which is defined as:

$$
\text { Classification error rate }(\%)=\frac{\mathrm{FN}+\mathrm{FP}}{\text { total cells }}
$$




\subsubsection{Receiver Operating Characteristic Curves}

The receiver-operating-characteristic (ROC) curve shows how classification performance varies with the threshold value used to divide feature vectors into two classes on the basis of activation at the output unit of the neural network (Zaknich, 1999). When a network is used to distinguish between cells belonging to deposit and non-deposit classes, a decision threshold must be applied to the activation values produced at the output unit. For the MLP neural network studied in Chapter 4, the output activation was approximately in the range $[0.05,0.95]$. Patterns associated with an output activation greater or equal to the arbitrarily chosen threshold of 0.5 were classified as deposits.

The quantities referred to in the following discussion of ROC curves are those shown in Table 3.3. The probability of making the four types of classification in Table 3.3 depends on the decision threshold and is illustrated in Figure 3.4a below (Masters, 1993b; Zaknich, 1999). The left-hand and right-hand curves show the probability distributions for the output unit activation for barren and deposit cells, respectively. Areas corresponding to the true positive (TP), false positive (FP), true negative (TN), and false negative (FN) probabilities are also shown in Figure 3.4a.

These quantities are related in the following way. If the threshold (indicated by the vertical line at $T_{h}$ in Fig. 3.4a) for the classification of a pattern as a deposit is very high, then very few barren cells will be mistaken for deposits (FP probability is low) but many cells that are actually deposits may be incorrectly classified as barren (FN probability is high). Conversely, if the threshold is very low ( $T_{l}$ in Fig. 3.4a), a large number of barren patterns will be classified as deposits (FP probability is high) and most deposits will be correctly identified as deposits (TP probability is high.

The following quantities are required in order to represent ROC curves. The true positive rate is defined as;

$$
\text { True positive ratio }=\frac{T P}{T P+F N}
$$

$T P+F N$ equals the total number of deposit patterns.

The false positive ratio is defined as:

$$
\text { False positive ratio }=\frac{F P}{F P+T N}
$$




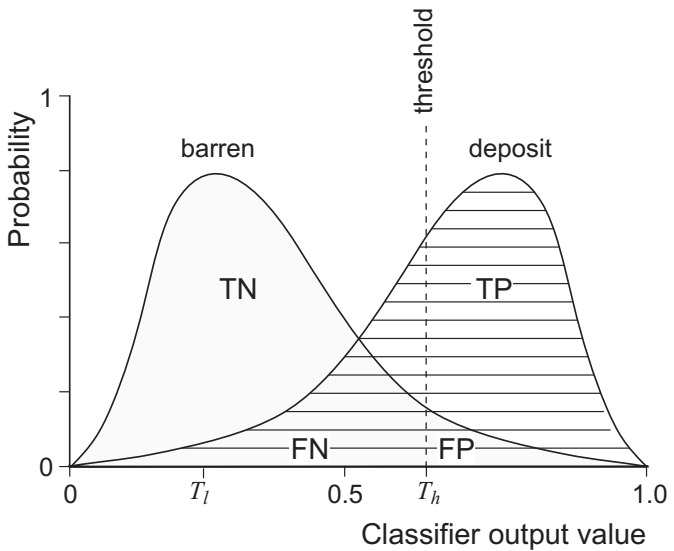

a)

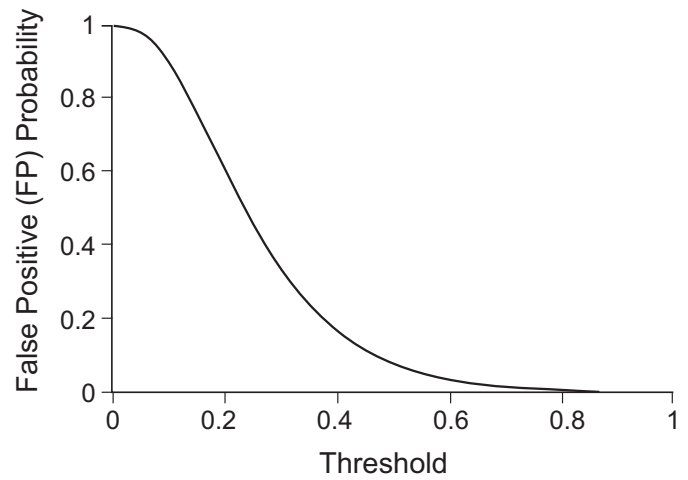

c)

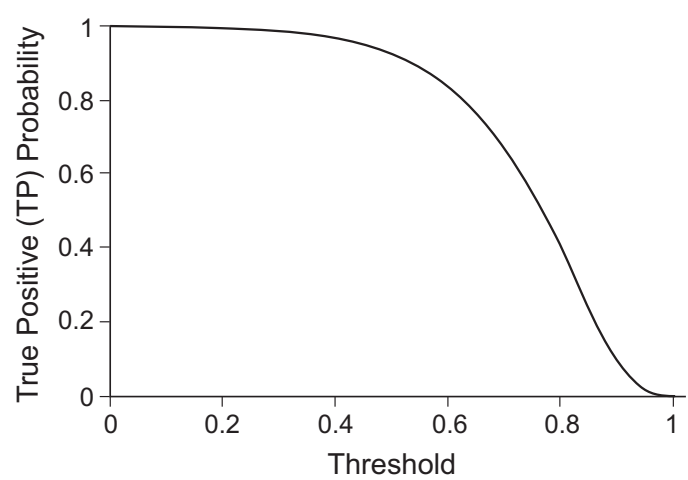

b)

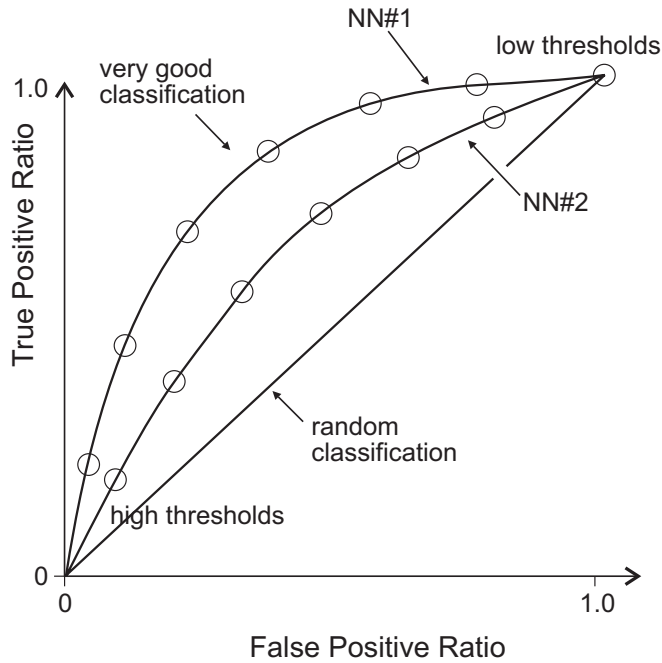

d)

Figure 3.4. Receiver-operating-characteristic (ROC) curves (modified from Masters, 1993b; Zaknich, 1999. Abbreviations: $\mathrm{FN}=$ false negative, $\mathrm{FP}=$ false positive, $\mathrm{NN}=$ neural network, $\mathrm{TN}=$ true negative, $\mathrm{TP}=$ true positive). 
where $F P+T N$ equal the total number of barren patterns. These two parameters are plotted in Figures 3.4b and c, respectively. Thus, there are contradictory requirements; a low threshold is needed for a high true positive ratio (i.e. to make sure the deposits are not missed) and a high threshold is needed for a low false positive ratio (i.e. to make sure the barren cells are not mistaken for deposits).

The ROC curve is generated by plotting the true positive and false positive ratios together for a range of threshold values (Fig. $3.4 \mathrm{~d}$ ). Curves very close to the top left corner indicate a very good discrimination of classes. On the other hand, a straight line with a slope equal to one corresponds to a situation where no discrimination occurs. To get a straight line, true positive and false positive ratios would have to be equal for all threshold values. The two curves in Figure 3.4a would have to completely overlap corresponding to a network that produces random output activation values in response to deposit and barren patterns (Masters, 1993b).

The area under the ROC curve is used as a measure of classification performance and varies from a minimum of 0.5 to a maximum of 1.0. Masters (1993b) has noted that the shape of the curves are also important because networks with very different performance can produce curves with equal areas.

\subsubsection{Harris and Pan's Measures of Performance}

Harris \& Pan (1999) use a variation of these ideas to represent the performance of different methods for mineral favourability mapping. They plot the percentage correct classification for mineralized and barren cells versus the probability value used as a threshold to make the classification. (Fig. 3.5). A good prospectivity model is one for which the two curves are as close to $100 \%$ correct classification for a large range of threshold values.

Harris \& Pan (1999) used a second measure of classification performance, which they refer to as exploration performance. The exploration task is viewed as one of selecting the optimum threshold for allocating cells to the mineralized class and it is assumed that all cells classified by the system as mineralized are retained as ground for further exploration. Harris \& Pan (1999) measure performance in terms of the percentage of total cells that must be retained to ensure that different percentages of mineralized cells are retained when various cutoff probabilities for mineralization are applied to neural network output probabilities. Their approach results in a plot that is nearly identical to the ROC curve due to the very small proportion of the total number of cells represented by deposits. Since the terms $T P$ and $F N$ in the ROC equation 3.11 refer to deposits, they are exceedingly 

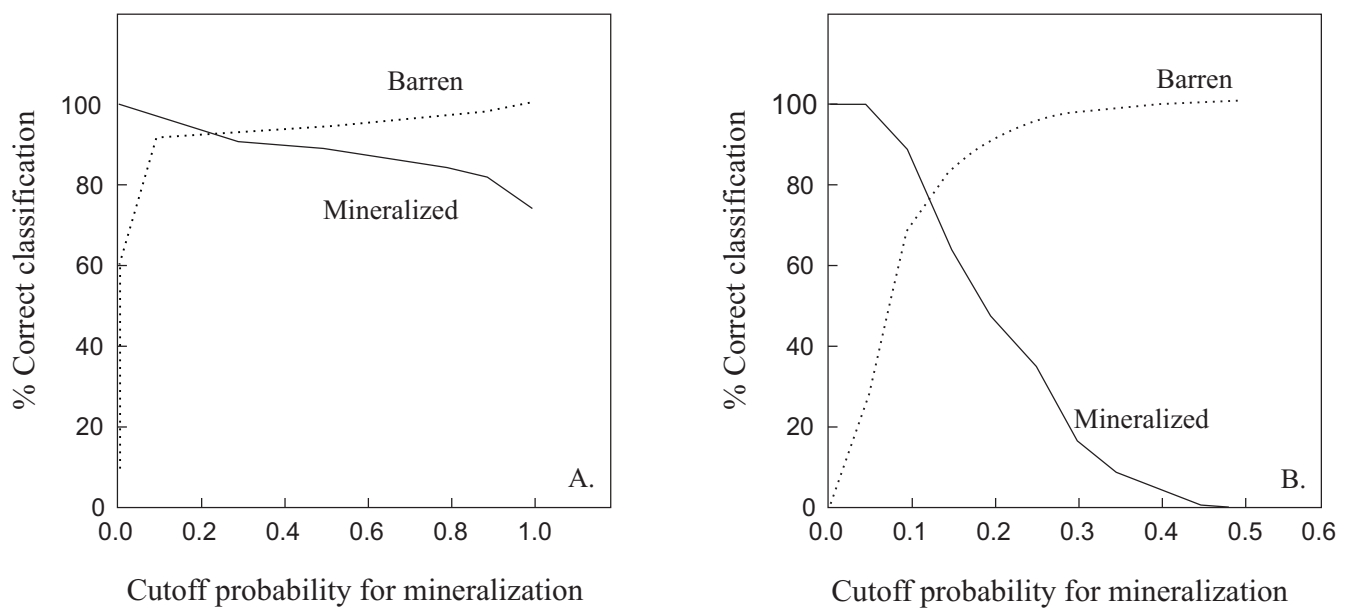

Figure 3.5. Classification of input feature vectors as mineralized and barren using a) probabilistic neural network (PNN) and b) logistic regression methods (Harris \& Pan, 1999). Classification performance is measured in terms of the percentages of mineralized cells that are correctly classified because their probabilities of mineralization exceed a threshold ( $x$-axis) and barren cells that are correctly rejected (solid and dashed lines, respectively). Note that at high threshold values, a large fraction of both mineralized and barren cells are correctly classified by the PNN, whereas the fraction of mineralized cells correctly classified by logistic regression is relatively low.

small relative to the terms $T N$ and $F P$, which refer to barren cells. If the deposit terms are omitted, the ROC equation 3.11 is identical to equation 3.13 .

The percentage of mineralized cells that are 'retained' is defined as;

$$
y=\frac{\text { no. of mineralized cells classified as mineralized }}{\text { total no. of mineralized cells }} \cdot \frac{100 \%}{1}=\frac{T P}{T P+F N} \cdot \frac{100 \%}{1}
$$

and the total number of cells retained is defined as:

$$
x=\frac{\text { total no. of cells classified as mineralized }}{\text { total no. of cells }} \cdot \frac{100 \%}{1}=\frac{T P+F P}{T P+F N+F P+T N} \cdot \frac{100 \%}{1}
$$

The interpretation of the curves in Figure 3.6 is similar to that in Figure 3.4d. To illustrate the use of this plot, suppose that two methods represented by curves 1 and 2 are to be compared and the goal is to retain at $90 \%$ of the mineralized cells in a region. Because curve 1 lies to the top left of curve 2, this means that method 1 allows the $90 \%$ of the 

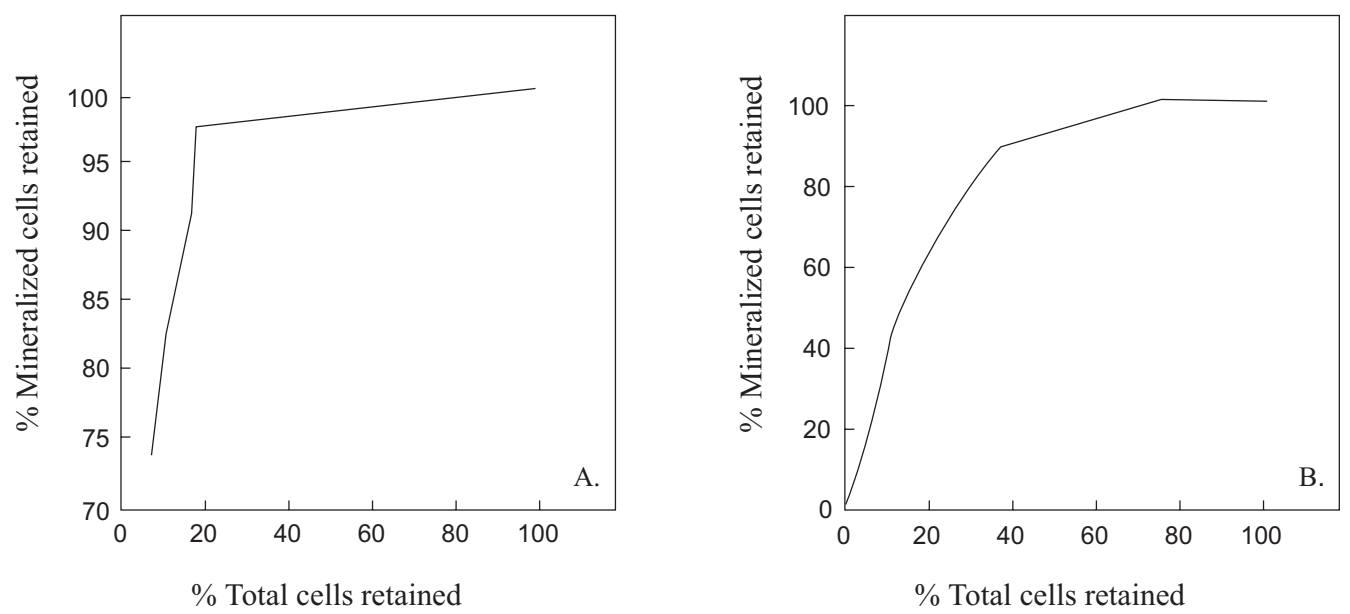

Figure 3.6. Measure of exploration performance used by Harris \& Pan (1999) for a) probabilistic neural network (PNN) and b) logistic regression methods. In Harris and Pan's model for exploration decision-making, cells for which the probability of mineralization exceeds a threshold are retained for exploration and those that fall below the threshold are rejected. A good classifier for exploration decision-making is one that accurately classifies both mineralized and barren cells so that only a small fraction of the total cells must be retained in order to retain a high proportion of the mineralized cells.

mineralized cells to be retained using a smaller total number of cells (and therefore at lower cost) than method 2.

\subsection{Weights-of-Evidence Method}

A literature survey of previously-used mineral prospectivity methods in Chapter 2 reveals that logistic regression and the weights-of-evidence method offer some advantages compared to other multivariate techniques that are based a linear model of the data. In particular, the logistic and weights-of evidence methods are better able to model non-linear relationships and are less sensitive to the degree to which the assumptions of normality and heteroscedasticity are satisfied than other methods. Although the weights-of-evidence method is subject to problems related to the conditional dependence of input data sets, it is simple to implement, easy to interpret, and, because of this, it is more commonly used than logistic regression.

Maps were prepared according to the weights-of-evidence method using the GEODIPS GIS prospectivity analysis package developed by Jusmady \& Taylor (1997). 


\section{Processing}

The weights-of-evidence method used in this study has been described in detail by BonhamCarter (1994) and is only summarized here. The following steps were performed;

1. Test association of deposit points with patterns in evidence maps,

2. Create binary maps,

3. Create a unique conditions map and table,

4. Test for conditional independence,

5. Combine and eliminate dependent maps, and

6. Create prospectivity map.

These steps are shown in Figures 3.7 and are briefly explained below.

1. Test association of deposit points with patterns in evidence maps. The degree to which deposit points are associated with features in the input GIS thematic layers is tested in order to select a threshold value or class to convert multiclass maps to binary maps. Geodips produces a table containing; classes, number of deposit points in each class, weights $\left(\mathrm{W}^{+}\right.$and $\left.\mathrm{W}^{-}\right)$, weight contrast $\left(C_{w}=W^{+}-W^{-}\right)$, standard deviation of the weights and the weight contrast values and the studentized value of the contrast.

The studentized value of the contrast is the ratio of the contrast divided by the standard deviation of the contrast. The studentized value of contrast indicates the reliability of the contrast value. A Student $\left(C_{w}\right)$ value of 1.00 indicates a very high degree of uncertainty while higher values indicate more reliable values of contrast.

For continuous variables, weights and contrast are determined cumulatively (from the minimum to maximum value) and in reverse cumulative order at successive thresholds. The table is used to select the appropriate threshold value to use in order to convert a multiclass map into a binary one. In most cases, the choice of an appropriate threshold from the class-weight tables is subjective. Generally, the class with the maximum $C_{w}$ or the last class in a sequence of high $C_{w}$ values before an abrupt decrease in the value of $C_{w}$ was selected as the threshold. Where these criteria could not be applied, or where the choice was not clear, a threshold with a combination of a high $C_{w}$ and a $\operatorname{Student}\left(C_{w}\right)$ above 1.5 was chosen. Steps 1 to 2 are repeated until an optimal reclassification scheme is determined that maximizes the predictive effect of the binary evidence map.

2. Create binary maps. After suitable classes are selected in input maps containing 


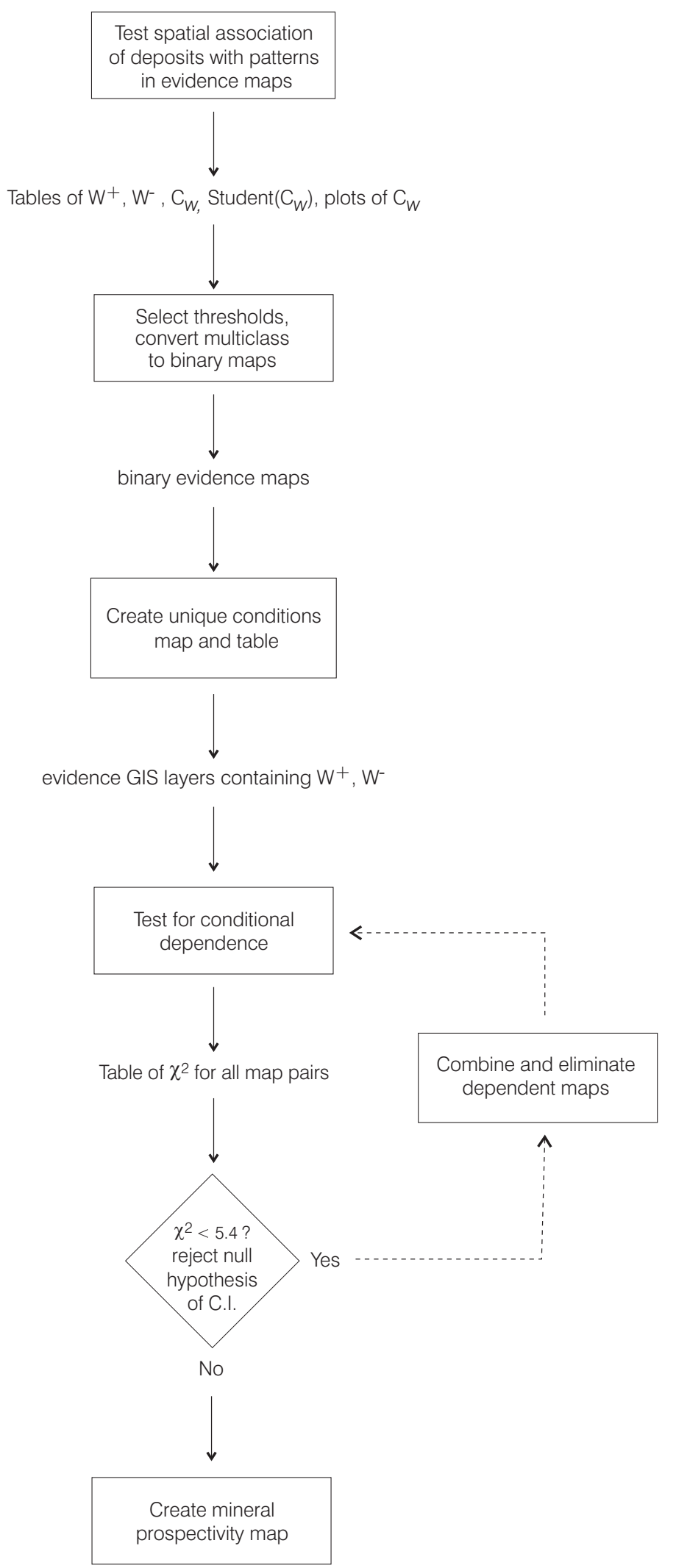

Figure 3.7. Processing steps used to create weights-of-evidence mineral prospectivity maps in pilot study 
discrete entities, or a suitable threshold is selected for maps depicting a continuous field, binary evidence maps are created by reclassifying the multiclass map.

3. Create a unique conditions map and table. In the GEODIPS system, used to implement the weights-of-evidence method in the pilot study in Chapter 4, a unique conditions map and table are produced. All binary evidence maps are processed to create a map in which the cell values are codes representing a particular combination of binary evidence patterns that resulted from overlaying the binary maps. For example, for the case where there are just two binary input maps, each containing a binary pattern, which can either be present or absent, there are just four possible combinations of patterns (referred to as unique conditions). For each unique condition, the presence or absence of the favourable pattern in the binary map is stored as a code, the positive and negative weights are summed according to whether the pattern is present or absent, and the resulting value is stored in a table, together with the total number of deposit points associated with that combination of patterns. The table is used in the next step.

The MATLAB code, written to perform the calculations for the weights-of-evidence method in the rest of the study, does not employ a unique conditions map or table. Instead, each binary evidence map is replaced by one containing a positive weight, where the binary pattern is present, and a negative weight where the pattern is absent. Then, at each grid location, weights in the different map layers are added to the log of the prior probability of a deposit to determine the posterior probability of a deposit.

4. Test for conditional independence. A table is produced that shows values of the chi-squared statistic $\left(\chi^{2}\right)$ for all combinations of map pairs . Map pairs resulting in a $\chi^{2}$ value above the limit of 5.4, at the $95 \%$ confidence level for one degree of freedom, are conditionally dependent (Bonham-Carter, 1994). ${ }^{4}$

5. Combine and eliminate dependent maps. For example, in the Tenterfield pilot study, maps corresponding to the $\mathrm{K}$ and total count gamma-ray channels were discarded. Maps for the $\mathrm{U}$ and Th channels were combined by multiplying them together. This method produced the strongest association between the resultant map pattern and the known deposit points by emphasizing leucogranites of the Stanthorpe granite group, which produce a high radiometric response and are the

\footnotetext{
${ }^{4}$ A new 'omnibus' test developed by Agterberg \& Cheng (2002) and tested by Thiart et al. (2003b,a) can also be used for pairwise testing and is superior to chi-squared and Kolmogorov-Smirnov tests in cases with a small number of deposit points.
} 
main hosts of disseminated and placer deposits (Barnes et al., 1995). Steps 1-4 must be repeated for new maps that have been created as a result of combining conditionally dependent ones.

6. Create prospectivity map. The posterior probability of a deposit is calculated for each grid cell using Equation 2.8 (Chapter 2), and the data stored in the unique conditions table. Equation 2.8 gives the $\log$ of the posterior odds, which is then converted to a probability value. The steps to create the final map are the same as those described for the neural network method (step 7 on page 70 ).

\subsubsection{Fuzzy Systems Method}

Conceptual methods referred to in Chapter 2 include statistical-subjective methods (Harris \& Carrigan, 1981) and Dempster-Shafer evidential belief theory, expert systems, decisiontrees inference systems, fuzzy-logic and hybrid statistical-fuzzy-logic methods, and adaptive neuro-fuzzy inference systems. The fuzzy-logic method was chosen as a example of a conceptual system because it has been widely used (Eddy et al., 1995; Donaldson, 1996; Burgess, 1997). and is relatively easy to implement compared to the other methods. The pre- and post-processing steps are the same as those for the weights-of-evidence method explained in Chapter 3 above. The procedure used has been described in detail by Bonham-Carter (1994), and therefore only a short summary is given here.

\section{Processing}

The fuzzy-logic procedure consists of five steps (Fig. 3.8);

1. Classify raw values and assign fuzzy membership values. Fuzzy membership values, in the range $[0,1]$, are assigned to classes of cell values for each input map layer. The membership values are assigned subjectively, according to how favourable the parameter values are for mineralization. Classes of variable values and the corresponding membership values are shown in Table 4.3 (Chapter 4).

2. Create map layers containing fuzzy membership values. A new fuzzy membership map layer was created for each varaible using values in Table 4.3.

3. Design fuzzy-logic net. Maps containing membership values are combined using map algebra functions (e.g. addition, subtraction, multiplication, and division) or fuzzy membership operators (fuzzy AND, fuzzy OR, algebraic sum, algebraic prod- 
uct and the gamma operator; Zimmermann, 1984; An et al., 1991; Bonham-Carter, 1994). The fuzzy-logic inference net refers to the combination of primary and derived map layers and the choice of map algebra and fuzzy operators used to combine maps. The fuzzy inference net used in the pilot study is shown in Figure 3.9.

4. Combine layers to create favourability map. At each grid cell location, fuzzy membership values from different map layers were combined using fuzzy operators to produce a single output membership value representing the overall favourability for mineralization. The procedure is the same as that described in point 7 on page 7 .

5. Model selection. A variety of different fuzzy membership value assignments, inference nets, choice of fuzzy operators for the final map combination step, and, in the case of the gamma operator, different values of the gamma operator (in the range $0-1$ ), were tested. The prospectivity map that best accounts for the known deposit locations, while minimizing the area of the highest prospectivity classes, was chosen as the final fuzzy-logic model.

The relationship between the deposit points and the magnetic intensity map is ambiguous, and therefore the magnetic map was not used as an input map in the fuzzy-logic and weights-of-evidence methods. The map algebra addition-operator was used to combine the gamma-ray U- and Th-channel maps. The fuzzy gamma operator with $\gamma=0.95$ was used to combine membership values in the final step (refer to Fig. 3.9).

\subsubsection{Comparing Mineral Prospectivity Map Quality}

This section describes work performed as part of this pilot study to determine how to assess the results of the different prospectivity mapping methods. In order to quantitatively compare the quality of maps produced using different methods, verification of the results using some form of ground truth would normally be required. This is not feasible here, since prospectivity maps do not represent a classification of an extensive and continuously measurable category, such as agricultural crop type, that can be readily checked. Instead, prospectivity maps were quantitatively compared by measuring the extent to which high prospectivity areas of the maps include the locations of known mineral deposits and occurrences. A variety of statistical and probability indices of map quality was used.

The chi-squared statistic measures the extent to which observed and expected numbers of deposits differ. As noted previously, although the chi-squared statistic cannot be used to make statistical tests of significance of spatial associations, it can provide explorative and 


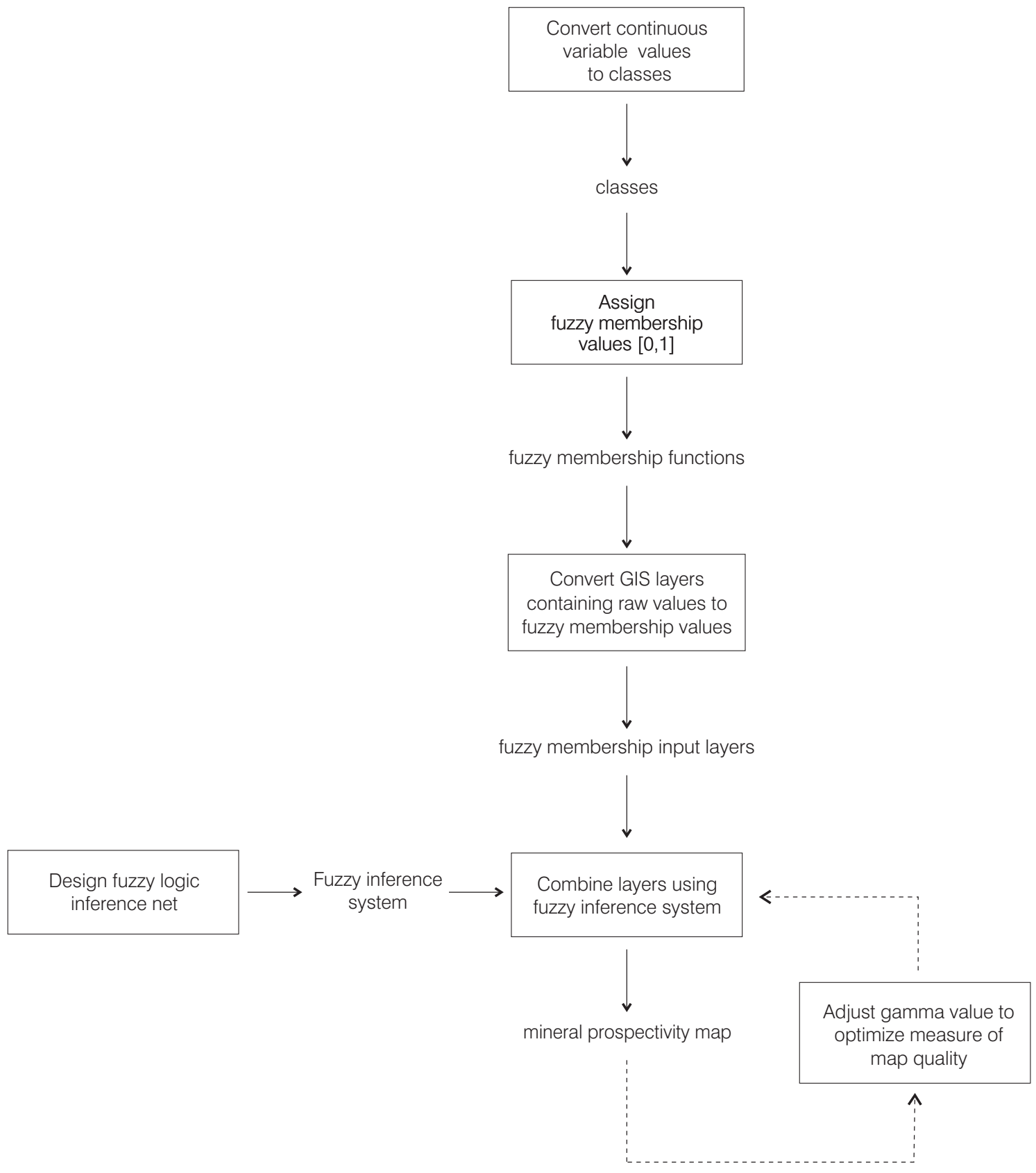

Figure 3.8. Processing steps used to create fuzzy-logic mineral prospectivity maps in pilot study 


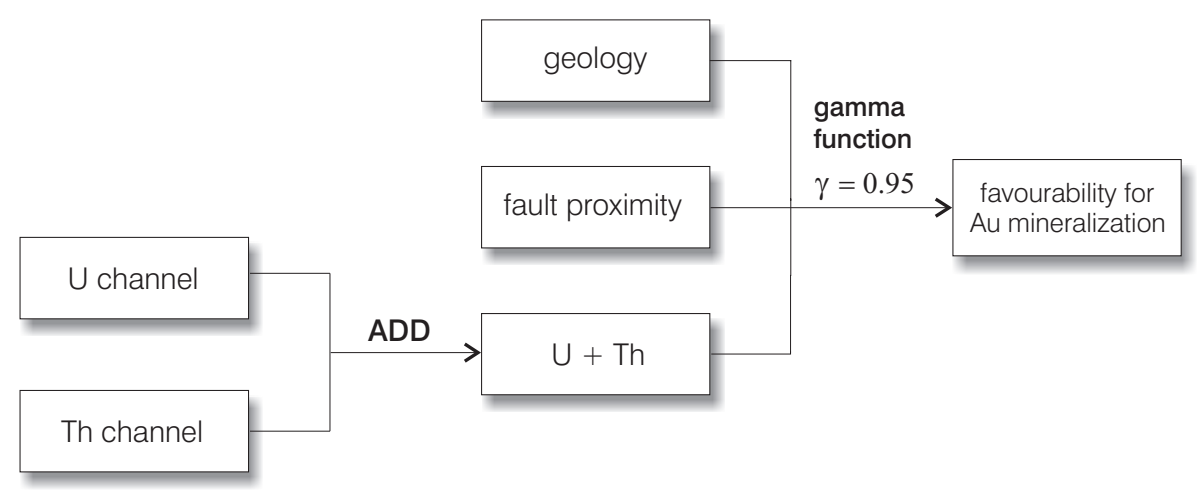

Figure 3.9. Fuzzy inference net used in the mineral prospectivity map shown in Fig. 4.11 b.

descriptive measures of spatial correlation (Bonham-Carter, 1994) and is used here as a measure of the quality of a prospectivity map.

The observed number of deposits in the map classes with the highest favourability should be much higher than the number that would be expected if the map class areas were distributed randomly with respect to deposits. The chi-squared statistic has a lower limit of zero when the patterns of the map class areas and deposits are completely independent of one another, and it increases in value as the differences between observed and expected numbers of deposits become larger. A problem with the chi-squared statistic is that it is only sensitive to the total difference between observed and expected numbers of deposits for all the map classes and not to whether it is the higher prospectivity map classes that contain higher than expected numbers of the known deposits.

The non-parametric rank-order tests of Spearman's $\rho$ and Kendall's $\tau$ were used to measure the degree of correlation between the probability of occurrence of known mineral deposits with prospectivity map class. Both tests measure the degree of independence between random variables. The hypotheses for a one-tailed test of positive correlation between variables are (Macfarlane, 1998);

$H_{0}: X_{i}$ and $Y_{i}$ are mutually independent, i.e. there is no correlation $H_{1}$ : there is a tendency for larger values of $\mathrm{X}$ and $\mathrm{Y}$ to be paired together

Spearman's and Kendall's rank correlation coefficients were chosen because they are suitable where a monotonic, rather than a strictly linear, trend is of interest, and they are robust with respect to outliers and inexact measurement (Rock, 1988). For both these statistics, a value of zero indicates that no correlation exists and a value of one indicates 
a perfect correlation.

Conditional probabilities $P\left(D / C_{k}\right)$ of the occurrence of a known mineral deposit, given the grid cell is located within a particular prospectivity class $C_{k}$ were calculated for each prospectivity map class, using the numbers of grid cells. Each deposit is assumed to occupy only a single cell. A disadvantage of conditional probabilities is that they are very small numbers and depend on the total number of known deposits in a region.

The ratio of the conditional probability of a deposit $P\left(D / C_{k}\right)$, given that the area of interest is restricted to a particular prospectivity-map class $C_{k}$, to the prior probability of a deposit, $P(D)$ results in numbers that can be more simply compared (Brown et al., 2000a). This ratio, $P\left(D / C_{k}\right) / P(D)$, is equivalent to an intuitive notion of a successful prospectivity map; that is, a high prospectivity map class should define an area in which a high proportion of the known deposits $\left(n\left(D_{C_{k}}\right) / n\left(D_{\text {total }}\right)\right)$ are predicted in a small proportion of the total map area $\left(n\left(C_{k}\right) / n(T)\right)$. This can be expressed as the following ratio:

$$
\frac{n\left(D_{C_{k}}\right) / n\left(D_{\text {total }}\right)}{n\left(C_{k}\right) / n(T)}=\frac{P\left(D / C_{k}\right)}{P(D)}
$$

where $n\left(D_{C_{k}}\right)$ represents the number of deposits in map class $C_{k}, n\left(D_{\text {total }}\right)$ is the total number of deposits, $n(C)$ is the area of map class $C_{k}$, and $n(T)$ is the total area. It is interesting to note that Mihalasky \& Bonham-Carter (2001) use the same ratio as the one shown in Equation 3.14 but refer to it as the normalized density, $\rho_{i}$ for class $i$. When the unit cell is infinitely small, $\rho$ is related to weights by:

$$
W=\ln (\rho)
$$

They use $\rho$ to measure the spatial association between lithodiversity-map classes and deposit points. Mihalasky \& Bonham-Carter (2001) have pointed out that $\rho$ as a measure of association is skewed because the range of $\rho$ for negative associations is compressed into $[0,1]$, whereas the range for positive associations is $[1,+\infty]$. They solve this by using the natural $\operatorname{logarithm}$ of $\rho$ which is symmetrical about the null value of $\ln \rho=0$.

If a prospectivity map is to be useful, then the probability of discovery of a deposit in the highest group of prospectivity map classes should be significantly upgraded compared to selecting a grid cell at random. Consequently, the ratio in Equation 3.14 should be greater than one for high prospectivity-map classes. Conversely, map classes representing 
the lowest prospectivity classes should have ratios significantly less than one, indicating that the probability of discovery of a deposit is much lower than average. In the middle group of map classes, the ratio should be approximately equal to one, indicating that cells in these areas are no more likely to contain a deposit than cells chosen at random.

Another simple measure that can be used to assess the assess the final map is the contrast, $C_{W}=W^{+}-W^{-}$, and the studentized contrast, $C_{W} / S\left(C_{W}\right)$, for each prospectivity-map class (Bonham-Carter, pers. comm.; where $S(C)$ is the standard deviation of contrast).

\subsection{Choice of GIS Databases for Pilot and Case Studies}

This section provides a summary of the source, location and content of the GIS databases used in this study, together with the reasons for using the database and a description of the role the database plays in the study. GIS databases used in this study are summarized in Table 3.4 below. Detailed descriptions and metadata for the two databases are given in Appendices C and D.

Table 3.4. Summary of GIS databases used in this study. The Tenterfield database is used for the pilot study in Chapter 4 and the Kalgoorlie database is used for experiments described in Chapters 5 to 7 .

\begin{tabular}{|l|l|l|l|l|l|}
\hline Database & Deposit Type & Location & Rows $\times$ Cols & Cell Size $^{\mathrm{a}}$ & Deposits $^{\mathrm{b}}$ \\
\hline \hline Tenterfield & Intrusion-related Au & Tenterfield, N.E. NSW & $280 \times 240$ & $200 \mathrm{~m}$ & 63 \\
Kalgoorlie & Archaean lode-Au & Eastern Goldfields, WA & $1140 \times 1100$ & $100 \mathrm{~m}$ & 100 \\
\hline
\end{tabular}

${ }^{a}$ Rows, columns and grid size after converting all GIS layers to a standard size

${ }^{b}$ Total includes deposits and mineral occurrences

\subsubsection{Intrusion-Related and Placer Gold Deposits, Tenterfield, N.S.W.}

The Tenterfield database was compiled by Robert Barnes at the NSW Geological Survey in Armidale (Barnes, 1993; Barnes et al., 1995; Barnes, 1995; Barnes \& Suppel, 1995; Barnes, 1997a,b). The data set corresponds to the Tenterfield 1:100000 map sheet area (9339), an area, located in north-eastern NSW.

Although a wide variety of mineralization types exist in the area (e.g. Mo-W-Bi, $\mathrm{Sn} \pm \mathrm{W}$, and Sn placer), intrusion-related primary and associated placer gold deposits are the 
largest group (63 deposits) and were chosen for the pilot study in Chapter 4 . The area corresponds to part of the southern portion of the New England Orogen.

GIS thematic layers supplied as ARCVIEW polygon coverages and BIL images include solid geology (compiled at 1:100000 and 1:250000 scale), regional faults and shear zones, a regional stream-sediment geochemistry survey, and airborne magnetic and gamma-ray survey (1986 data on $1500 \mathrm{~m}$ flight-line spacing) and mineral deposits and occurrences. Data were converted to a raster grid with 280 rows $\times 240$ columns and a $200 \mathrm{~m}$ cell size.

The database was chosen for the pilot study in Chapter 4 because;

1. there is complete coverage over the area for all data sets,

2. there are abundant gold deposits and mineral occurrences (63 primary and placer),

3. the data are in the public domain,

4. there was no major post-mineralization structural deformation or metamorphism.

The Tenterfield database is not suitable for the detailed investigations in other chapters because;

1. the data were not collected specifically to explore for intrusion-related gold deposits,

2. the resolution of the geophysics data is low collected in 1986 using a flight-line spacing of $1500 \mathrm{~m}$,

3. geophysics data are only available as grey-scale images, and

4. the regional faults and shear zone data are incomplete, as indicated by the number of faults apparent as topographic features on a high resolution DEM produced after the compilation of the geological map (unpublished data of the NSW Geological Survey).

A detailed description of the GIS database is given in Appendix C.

\subsubsection{Archaean Orogenic Gold Deposits, Kalgoorlie, W.A}

The Kalgoorlie data set was provided by Dr Scott Halley at Placer Dome (formerly Goldfields Exploration Pty Ltd). The data set is currently being used for exploration for Archaean lode-gold deposits in the Kalgoorlie district.

The area covers part of the Bardoc Tectonic Zone within the Norseman-Wiluna belt of the 
Yilgarn Block. The data set covers an approximately square area with sides of $110 \mathrm{~km}$ and corresponds to the eastern half of the Kalgoorlie 1:250000 geological map sheet (SH51-9). Data compilation was mainly performed at a scale of 1:100000. In raster format, the map gird consists of 1140 rows $\times 1100$ columns with a cell size of $100 \mathrm{~m}$.

Data consist of solid geology (a compilation of mapping by the Geological Survey of Western Australia and Goldfields Exploration geologists using field mapping and aeromagnetic interpretation), structure, Bouguer gravity anomaly, total magnetic intensity (RTP), drill-hole geochemistry, and regolith geochemistry. All vector data layers were originally supplied in MAPINFO format.

This database is used for experiments in the methodology part of the thesis because;

1. it is a current and high-quality compilation and contains one of the best syntheses of geological mapping presently available,

2. it was compiled specifically for exploration for Archaean lode-gold deposits,

3. there is a large amount of expertise within the Centre for Global Metallogeny on this deposit type,

4. the database contains a large number of gold deposits and occurrences, and

5. the deposit database is well attributed and includes tonnage and grade information.

A detailed description of the GIS database is given in Appendix D.

\subsection{Software}

\subsubsection{Pre-Processing $(\mathrm{C}++$ Programs $)$}

Pre-processing steps shown in Figure 3.3 were performed with a series of $\mathrm{C}++$ programs. The functions performed by these programs and other commercial software used in the project are shown in Table 3.5. The code for the $\mathrm{C}++$ programs is listed in the attached CD-ROM (vol.1), and the processing procedure for the MLP network is documented in Appendix E. All input and output data supplied to the $\mathrm{C}++$ programs are in ASCII format. 


\subsubsection{Neural Network Simulation Packages}

A teaching package called the GIS prospectivity analysis package (GEODIPS), developed by Jusmady \& Taylor (1997), was used to implement the weights-of-evidence and fuzzylogic methods in the pilot study in Chapter 4 only. A tutorial description of the GEODIPS package is given by Taylor (1997) All other neural network processing was performed using the MATLAB software package. Experiments in Chapters 6-7 were conducted using MATLAB, which is a software package suited to manipulating large arrays, an extension package referred to as the Neural network Toolbox, and functions written in a C-like script language.

\subsection{Hardware}

Most of the work in this study was performed using a Dell 410 Precision workstation with a $650 \mathrm{MHz}$ Intel Pentium III processor, $512 \mathrm{MB}$ of ECC RAM, $32 \mathrm{MB}$ of video memory and a 27.2 GB hard drive. Peripherals included external 40 GB and 80 GB IEEE 1394/Firewire hard drives. The main requirement for this study is a large main memory. This allowed all the large matrices representing GIS map layers (up to 18 a time) ) to be stored in RAM simultaneously, rather have to be transferred from disk using paged memory. The ARC/INFO GIS was run on a Sun Sparc 20 workstation.

\subsection{Summary}

The main points discussed in Chapter 3 are listed below.

1. Derived layers (layers derived from primary geoscientific data) are important because they represent a means of incorporating expert knowledge into the neural network input.

2. In this study, derived layers were prepared using the in-built functions of either the ARC/INFO and ARCVIEw GIS systems.

3. All GIS thematic layers in vector format (i.e., polygon coverages in ARC/INFO) were converted to raster format prior to further processing because; 1) a raster format is compatible with neural network packages, and 2) most derived GIS layers and geophysical data are stored in raster format. 
4. The main processing steps in a neural network approach to mineral prospectivity mapping are; 1) convert GIS data to ASCII raster format, 2) produce derived GIS thematic layers (feature extraction), 3) combine GIS layers to create input feature vectors, 4) scale data to range $[0,1], 5)$ create training data sets, 6) train network and collect test-set performance statistics, 7) process entire study area using trained network, 8) scale and classify outputs, 9) produce prospectivity map, and 10) analyze the quality of that prospectivity map.

5. In the pilot study, performance of the trained network is assessed using; 1) the mean square error, and 2) the percentage correct classification of an independent test set.

6. In the case study, the area under a receiver operating characteristic (ROC) curve is used as an additional measure of the classification performance of the trained

Table 3.5. Programs and software packages used in this study

\begin{tabular}{|c|c|}
\hline Processing step & Program Name \\
\hline \multicolumn{2}{|l|}{ GIS Pre-Processing } \\
\hline Clipping and re-gridding (geophysics) & ERMAPPER \\
\hline Conversion to raster format & Mapinfo, Arc/Info, ArcView \\
\hline Create derived layers & ARCVIEW \\
\hline \multicolumn{2}{|l|}{ Neural Network Pre-Processing ${ }^{\mathrm{a}}$} \\
\hline Create feature vectors, scaling & merge_sls \\
\hline Create training data sets & rand_select \\
\hline Scale and classify output & map_class \\
\hline Calculate map quality statistics & map_qstats \\
\hline Calculate deposit statistics & deposit_locns \\
\hline \multicolumn{2}{|l|}{ Data integration } \\
\hline Neural network processing & MATLAB, $\mathrm{bp}^{\mathrm{b}}$ \\
\hline Weights-of-Evidence & MATLAB, GEODIPS ${ }^{c}$ \\
\hline Fuzzy Logic & Matlab, GeOdips \\
\hline \multicolumn{2}{|l|}{ Post-Processing } \\
\hline Preparation and display of maps & GEODIPS Oasis Montaj \\
\hline
\end{tabular}

\footnotetext{
${ }^{a}$ These are $\mathrm{C}++$ programs. Listings are given on the attached CD-ROM (vol. 1).

${ }^{b}$ The $b p$ program is a MLP neural network trained with the back-propagation algorithm and was written by McClelland \& Rumellhart (1988).

${ }^{c}$ The GEODIPS software package was developed by Jusmady \& Taylor (1997) and Taylor (1997).
} 
network on an independent test set. The area is calculated using all of the data (rather than the test set only) and varies from a minimum of 0.5 to a maximum of 1.0 .

7. A plot designed by Harris \& Pan (1999) is also used as a measure of classification performance in the case study. The plot shows the percentage of total cells that must be retained to ensure that different percentages of mineralized cells are retained when various cutoff probabilities for mineralization are applied to neural-network output probabilities.

8. Weights-of-evidence maps and fuzzy-systems maps in the pilot study were prepared using the GEODIPS GIS prospectivity analysis package developed by Jusmady \& Taylor (1997).

9. Prospectivity maps produced using different methods are quantitatively compared by measuring the extent to which high prospectivity areas of the maps account for the locations of known deposits.

10. The following measures of map quality are used to compare mineral prospectivity maps produced using different methods; 1) the chi-squared statistic, which measures the extent to which the observed number of deposits in the highest prospectivity map class differs from the number of deposits expected due to chance, 2) Spearman's $\rho$ and Kendall's $\tau$ rank correlation coefficients which are used as measures of the correlation between increasing prospectivity map class and the probability of occurrence of known deposits within the region corresponding to that class, and 3) $P\left(D / C_{k}\right) / P(D)$, which represents the ratio of the conditional probability of finding a deposit given that the cell is located in the highest prospectivity class (i.e., in a prospectivity map converted to nine-classes) compared to the prior probability of finding a deposit for the map area as a whole.

11. The ratio $P\left(D / C_{k}\right) / P(D)$ is a measure of the performance of the prospectivity map. The ratio represents the posterior conditional probability that a known deposit is located in the area corresponding to class $k$ of the prospectivity map to the prior probability of a deposit (i.e., the probability that any cell selected at random contains a deposit), It is equivalent to the ratio, $D / A$, where $D=$ the percentage of the total number of deposits located in the highest prospectivity class and $A=$ the percentage of the total area of the map represented by the highest prospectivity class.

12. The pilot study is based on intrusion-related primary gold and associated placer gold deposits in the Tenterfield area of north-eastern NSW. The map grid has 280 rows 
$\times 240$ columns and a $200 \mathrm{~m}$ cell size. This database was chosen because; 1) there is complete coverage over the area for all data sets, 2 ) there are abundant deposits and mineral occurrences (63 primary and placer), 3) the data are in the public domain, and 4) there was no major post-mineralization structural deformation, such that the map represents the geological scenario at the time of primary gold mineralisation.

13. The case study is based on a database provided by Goldfields Exploration Pty Ltd., used in a current exploration program for orogenic lode-gold deposits in the Archaean Kalgoorlie Terrane, Western Australia. The map gird consists of 1100 rows $\times 1100$ columns with a cell size of $100 \mathrm{~m}$. The database was chosen because; 1) it is a current, comprehensive and high-quality compilation, and 2) the timing of gold is late within the deformational history of the host terrane, and, therefore, the geometry of the structures associated with deposits is essentially similar to that at the time of mineralization. Consequently, spatial relationships between the structures and known gold deposits displayed in the geological map can be analyzed using a GIS to predict the location of new gold deposits.

14. Experiments in Chapters 6-7 were performed using MATLAB, which is a software package suited to manipulating large arrays. 


\section{Chapter 4}

\section{Pilot Study based on}

\section{Intrusion-Related, Granite-Hosted}

\section{Gold Deposits, Tenterfield, New}

\section{South Wales: Testing the Viability of an MLP Neural Network}

\section{Method}

\subsection{Introduction}

The pilot study described in this chapter was performed to test whether it is possible to produce a multiclass mineral-prospectivity map using a neural network, and if so, then how it compares to established methods. Although Bonham-Carter (1994) refers to neural networks as a possible method for mineral-prospectivity mapping, there are very few papers on the use of neural networks in mineral exploration, and only two papers described the use of neural networks for mineral prospectivity at the time this study commenced (Singer \& Kouda, 1996; Clare et al., 1997). In the case of Clare et al. (1997), a proprietary commercial system is briefly reported and no information is given on the type of network used, methods or exact nature of the output from the network.

It is difficult to assess from the study by Singer \& Kouda (1996) whether neural networks 
could be used to produce mineral prospectivity maps similar to those produced with the weights-of-evidence and fuzzy-logic methods. The reason for this is that both the deposit type and the study area appear to represent special cases, which allowed Singer \& Kouda (1996) to express mineral prospectivity in terms of the distance to the nearest VMS deposit rather than as probability or favourability that a cell contains mineralization.

Singer \& Kouda (1996) trained an MLP neural network using semi-quantitative X-ray analyses of four minerals in drill holes to predict the distance to Kuroko-type massive sulphide deposits in the Hokuroku district, Japan. In that study, the neural network is used as a function approximator rather than as a classifier, since the target output is a continuous variable representing the distance to the nearest ore deposit. The results were presented in the form of a map showing contours indicating the distance to ore deposits. Contours were not shown for areas greater that three kilometres from any drill hole. It is possible to present the results in this way because the abundance of the alteration minerals (used as input variables) display a distance relationship on a regional scale to the sites of hydrothermal vent on the sea-floor that originally produced the deposits (Agterberg et al., 1993).

In contrast, the weights-of-evidence method and fuzzy-logic methods produce results in the form of the probability of occurrence of deposits and a fuzzy membership value in the range $[0,1]$, indicating favourability for mineralization. Data used to determine weights in the weights-of-evidence method and to validate models in the fuzzy-logic method are the presence or absence of deposits.

The area analysed by Singer \& Kouda (1996) is unusually well explored. The Hokuroko district is the most thoroughly studied Kuroko-bearing district in the world. This has important implications for the application of neural networks. First, the density of drill holes is such that it is possible to be very sure that no undiscovered deposits exist in areas used to obtain training data, and therefore, that the distances used in the training data are correct. Generally, areas for which mineral prospectivity maps are prepared are not so well explored, and therefore, it is not possible to have the same degree of confidence that some of the apparently barren areas selected for use as training data do not contain deposits. Second, in most areas under exploration, the amount of training data available for deposits are very small relative to non-deposit data. This pilot study was intended to determine whether a neural-network approach could be applied to a more general situation in which;

1. GIS data sets typical of those used in mineral exploration are used,

2. the area contains known deposits but has not been the subject of intense scientific 
exploration or study, and

3. output in the form of a map showing probability or favourability for mineralization is required.

A particular problem related to this last point is whether a neural network method can be used to produce a multiclass mineral prospectivity map. The problem is that if the presence and absence of deposits are used as target values to train a neural network, the network is being required to perform as a binary classifier. However, in this mode, the output of the network should approach one and zero for inputs corresponding to deposits and non-deposit areas, respectively. This presents a problem if the task is to produce a multiclass (and therefore multicoloured) mineral prospectivity map, since the output must be in the form of a continuous variable that spans a wide range of values. This pilot study was intended to resolve some of these problems and compare the performance of a neural-network approach to the weights-of-evidence and fuzzy-logic methods in producing mineral prospectivity maps.

\subsection{GIS Database}

The GIS database for the trial study corresponds to the area of the Tenterfield 1:100000 sheet (map 9339), and was compiled by the Geological Survey of New South Wales (Barnes, 1993; Barnes et al., 1995). Metadata, including the sources, preparation of the data layers and an explanation of the attributes and codes used in each layer, are given in Appendix C. An explanation of the metadata standards used to document the GIS data in this thesis is given in Appendix B.

The database comprises the following thematic layers; solid geology, regional-scale faults, airborne total magnetic intensity (classed-image data), airborne gamma-ray spectrometry (U, Th, K and total count, classed-image data) and deposit locations (Fig. 4.2). The mineral deposit layer includes mineral occurrences, and small and medium sized mines. The Tenterfield 1:100,00 map grid contains 69,440 cells in 280 rows and 248 columns. Each cell in the grid data represents a 200 metre square on the ground. The Tenterfield GIS database is well suited for a pilot study to test the application of neural networks to prospectivity mapping for the reasons listed below.

1. The data are well documented and in the public domain.

2. The area has a uniform and complete coverage for all thematic layers. 


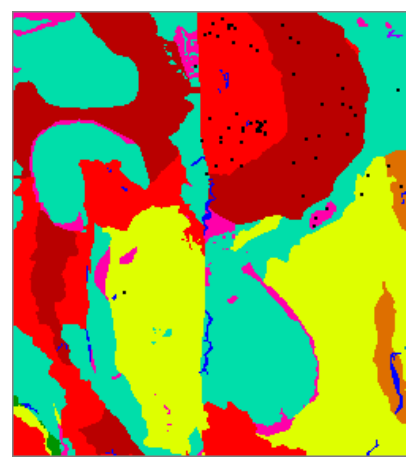

(a)

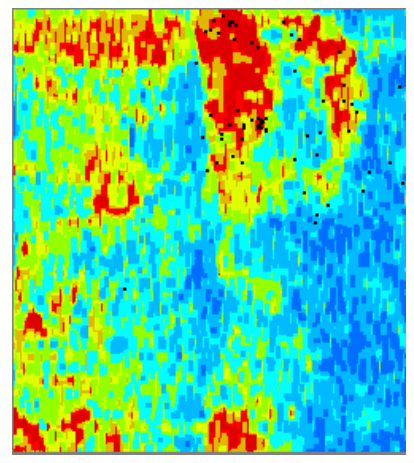

(d)

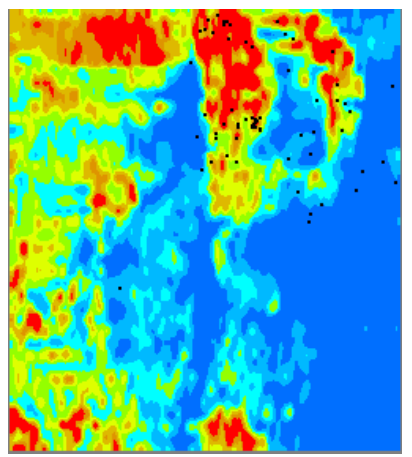

(g)

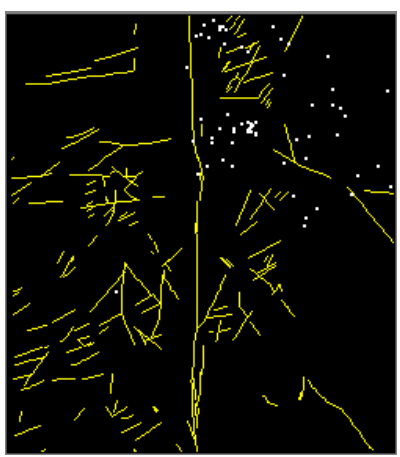

(b)

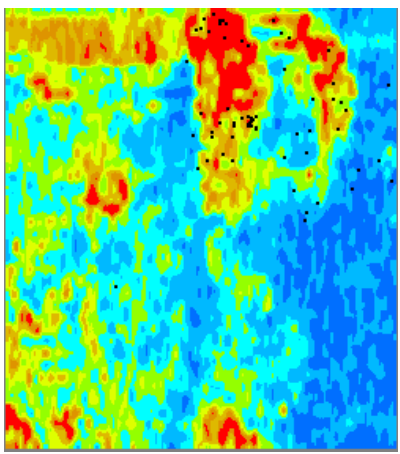

(e)

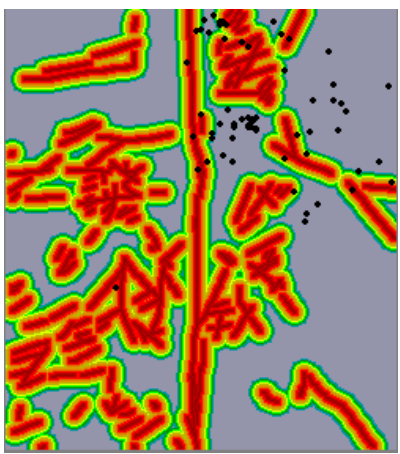

(h)

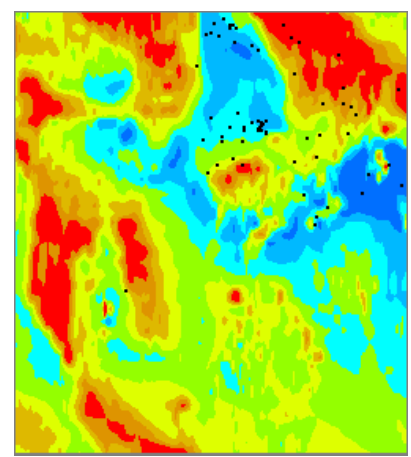

(c)

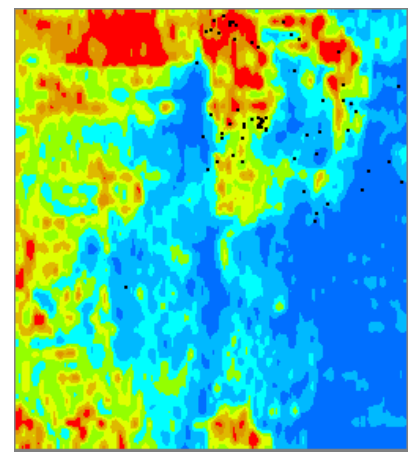

(f)

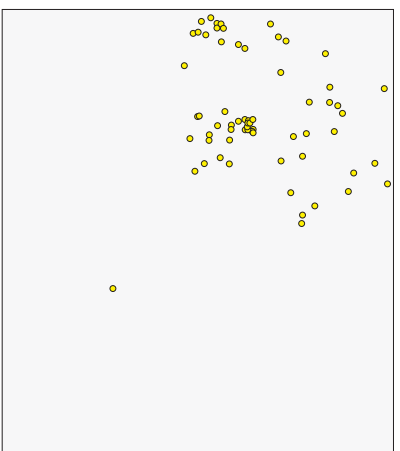

(i)

Figure 4.1. GIS thematic layers used as inputs in the pilot study: a) simplified solid geology, b) major faults and shear zones, c) total magnetic intensity (RTP), d) U channel gamma-ray survey data (classed), e) Th-channel, f) K-channel, g) total count, h) $200 \mathrm{~m}$ fault buffers (only first 5 shown), i) deposit points; vertical crosses = primary, intrusion-related, disseminated, Au deposits, diagonal crosses $=$ placer deposits, and open circles $=$ disseminated deposits with unknown form). 
3. There are a sufficiently large number of mineral deposits and occurrences to provide training data for the neural network method and to apply the statistical weights-ofevidence method used to provide a comparison.

4. Although some of the older sedimentary basement sequences are highly deformed, Permo-Triassic granites (which are the main hosts to mineralization) and volcanic units are relatively undeformed. Thus, present-day structures are a good guide to those that existed when the deposits formed. This is an important requirement for applying the fuzzy-logic method, because it is based on a deposit model and relies on a correspondence between the spatial relationships of geological structures and the features of the deposit model. This correspondence would be obscured in an area that had undergone strong post-mineralization structural deformation.

5. The thematic layers in the Tenterfield GIS are representative of those that would be available in a typical regional exploration program.

A disadvantage of the data set is that the geophysical data (airborne magnetic and four gamma-ray survey channels) are only available as grey-scale images. Consequently, the raw data have been converted to the range [0, 255]. Although this represents a potential disadvantage in the case of neural network analysis, because information available in the raw data are reduced, it does not represent a problem for the weights-of-evidence method, and the implementation of the fuzzy-logic method used in this pilot study, since both methods involve steps where the input data are reduced to classes.

\subsection{Geology}

\subsubsection{Regional Geological Setting}

The study area is located in the southern portion of the New England Orogen, N.S.W. (Fig. 4.2). The area of the New England Orogen was part of a major Devonian-Permian fore-arc basin-accretionary prism complex that formed during westward subduction of the paleo-Pacific plate beneath a convergent Australian continental margin (Cross et al., 1987; Blake \& Murchey, 1988; Cawood \& Leitch, 1985). Extensive synorogenic CarboniferousPermian S-type and postorogenic Permian-Triassic I-type granitoids intrude the complexly deformed siliclastic sedimentary sequences that form the accretionary complexes (Gilligan \& Barnes, 1990). A major period of igneous activity occurred in the Permian-Early Triassic producing Middle- to Late Permian intermediate to felsic calc-alkaline volcanic rocks, 
represented by the Wandsworth Volcanic Group in the study area, and comagmatic felsic to intermediate granitoids (Barnes \& Willis, 1989). The sedimentary and volcanic rocks were intruded by Late-Permian granodiorites and Triassic leucogranites during a Middle Permian to Early Triassic episode of plutonism. They were emplaced late in the orogenic sequence following major thrusting and folding in the New England Orogen (Barnes et al., 1995). Several hundred plutons extend throughout the central New England area, forming the New England Batholith (Shaw \& Flood, 1981).

The study area consists of three main lithostratigraphic units: 1) a Silurian to Carboniferous metasedimentary and volcanic basement, 2) the Mid to Late Permian Wandsworth Volcanic Group, comprising intermediate to felsic volcanic rocks and comagmatic felsic intrusions, and 3) Late Permian to Early Triassic leucogranitoids. The main granitoids in the subject area are those of the Clarence River and Moonbi Suites and an undifferentiated group of leucogranitoids.

\subsubsection{Mineralization}

The New England Orogen contains a wide variety of magmatic, hydrothermal and granitederived mineralization. The leucogranites of the Moonbi Supersuite are the most important hosts to mineralization in this area. They contain the important Timbarra gold deposits as well as deposits of tin, tungsten, molybdenum and bismuth. Additional commodities include silver, arsenic, copper, lead, fluorite, beryl and topaz (Gilligan \& Barnes, 1990; Blevin \& Chappell, 1992).

\subsubsection{Leucogranitoids}

The Permian-Triassic (245-238 Ma) high-K leucogranites of the Tenterfield-Stanthorpe region form a distinct group within the Moonbi Supersuite, and are informally referred to as the Stanthorpe Granite group by Mustard (1999). The granites previously have been referred to as leucoadamellites, and were grouped separately from the Moonbi Plutonic Suite by previous workers (Barnes \& Willis, 1989; Barnes, 1993; Barnes et al., 1995). They are highly fractionated I-type granitoids and formed as high level intrusions. The intrusions of the Stanthorpe Granite group contain chilled zones and porphyritic phases and are intruded by pegmatite and numerous gently-dipping and flat-lying aplite dykes. These features suggest that the present weathering surface intersects the upper levels of the granite bodies. Mustard (1999) recognized three main granite types within the 

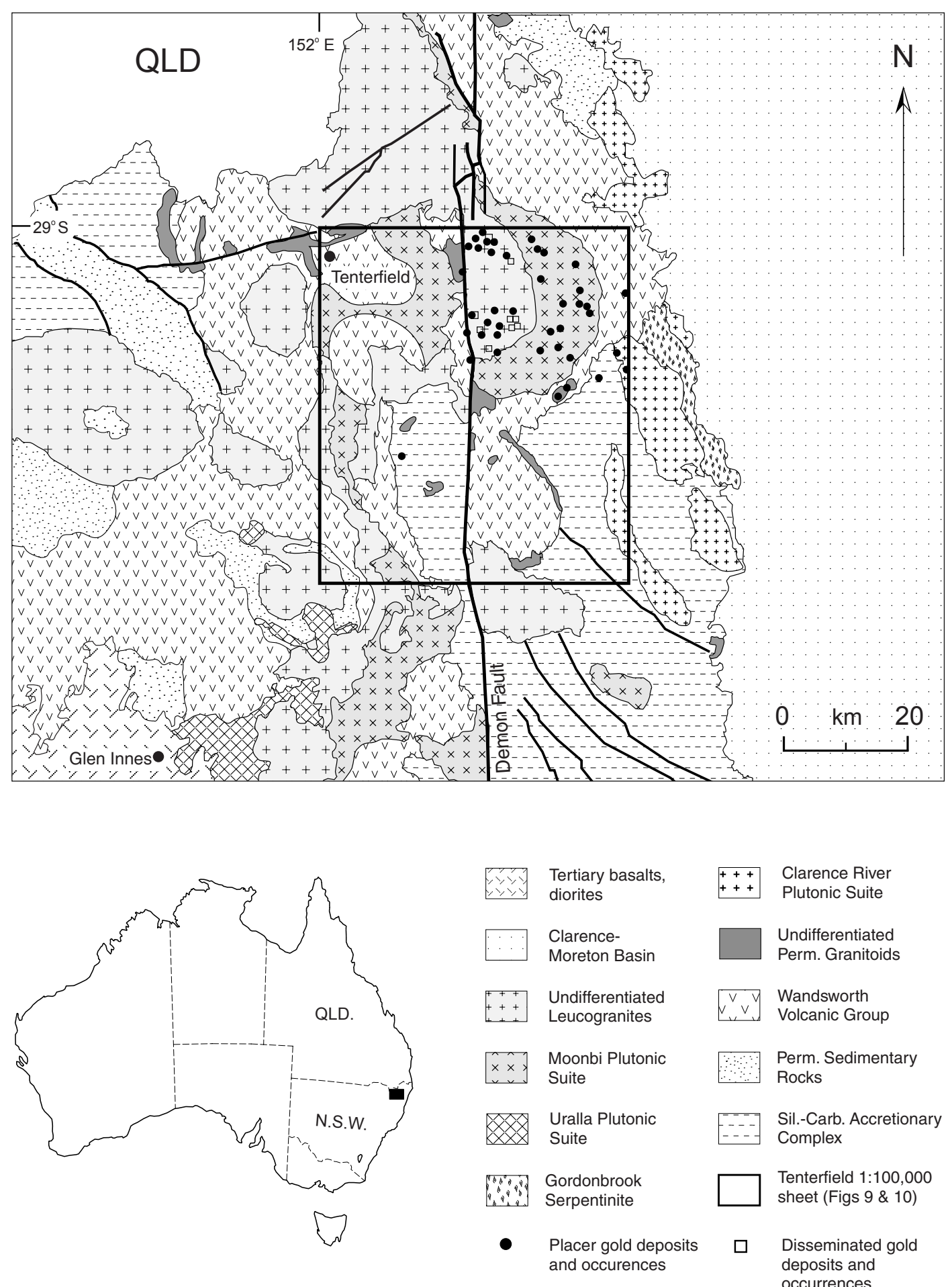

Figure 4.2. Location and simplified geology of the Tenterfield 1:100000 sheet area, adapted from an unpublished regional digital map supplied by the Geological Survey of New South Wales. 
Stanthorpe Granite group, termed the Bungulla (referred to by Barnes et al., 1995 as the Bungulla Porphyritic Adamellite), Stanthorpe and Ruby Creek types. These form a compositional and textural continuum, and are interpreted by Mustard (1999) to represent a differentiated magma series.

The mid-Triassic Stanthorpe Granite has intruded the Bungulla Granite and lies directly over the top of the Bungulla Granite in the Timbarra area. The Stanthorpe Granite, in plan view, forms a semi-circular body east of the Demon Fault in the north of the study area (Fig. 4.2).

\subsubsection{Intrusion-Hosted Gold Mineralization}

The Stanthorpe Granite hosts gold mineralization as low-grade disseminations within poorly-defined irregular lenses near the upper surface of the granite body (e.g. TimbarraPoverty Point deposits) and as minor sheeted veins. The Timbarra deposits contain a resource of $14 \mathrm{Mt}$ at an average grade of $0.82 \%$ (de Graaf, 1999). Gold is located within, or immediately below, the aplitic carapice in the Surface Hill granite, which is a late-stage fractionated phase of the Stanthorpe granite (Mustard, 1999). The granite is described by Simmons et al. (1996) as a magnetite-series, metaluminous to peraluminous granite, which comprises two porphyritic to equigranular phases cut by pegmatite, fine-grained granite, and highly fractionated microgranite dykes. Barnes et al. (1995) report that, throughout the area in which the Stanthorpe granite type is exposed, gold is also locally structurally controlled along fractures, joints and faults. Previously, gold has been mined from pegmatite sheets. Many alluvial and eluvial gold deposits occur in the Stanthorpe Granite.

A characteristic feature of the disseminated deposits in the Timbarra goldfield is that the gold is very fine-grained ( $<1$ to $100 \mu \mathrm{m}$; Bampton, 1988, Mustard, 1999). Gold is located in miarolytic cavities, disseminations between granitic minerals, and in dissolution cavities within K-feldspar (Simmons et al., 1996).

Numerous alluvial gold occurrences are scattered across the Bungulla Granite, which forms an arcuate body on the eastern margin of the Stanthorpe Adamellite (Barnes et al., 1995). Forty-seven secondary, thirteen primary and three spatially-associated granitoid-hosted gold deposits of unknown form were selected as the basis for the prospectivity analysis in this study.

The use of placer deposits in the training data is reasonable because of the very close 
genetic and spatial association of those placer and primary deposits. Gold in the placer deposits in the Stanthorpe Adamellite was derived from the disseminated deposits. Bampton (1988) concluded that the alluvial gold from the Stanthorpe Adamellite had not been transported very far, because the faceted morphology of some grains reflects the shape of crystals around which they had deposited. The fineness of the alluvial and primary gold reported by Barnes et al. (1995), the flat and poorly-drained top of the plateau formed by the Stanthorpe Adamellite, and the fact that much of the historical production from the Timbarra-Poverty Point goldfield came from eluvial deposits also supports this conclusion. Thus, locations of these disseminated gold deposits are likely to be favourable indicators for undiscovered primary as well as secondary deposits.

Similarly, the use of both primary mineral occurrences and deposits, which contain predominantly disseminated gold within a Late Permian to Early Triassic leucogranitoid host, in the training data set is reasonable because both groups appear to represent the same population. A number of studies show that the alluvial deposits were derived from the primary deposits. In addition, gold present in alluvial deposits formed very locally, and appears to have formed at a similar time (Barnes et al., 1988; Barnes \& Willis, 1989; Barnes et al., 1995). The largest deposit in the area, Timbarra-Poverty Point, is quite small and low grade (de Graaf, 1999), and Barnes et al. (1995) report that it appears very similar to a large number of smaller deposits and occurrences throughout the Tenterfield area. Although these studies have shown that variation exists in terms of the form of mineralization (e.g. disseminations, sheeted veins), and accessory mineral assemblages, the disseminated gold deposits have been distinguished from the epigenetic $\mathrm{Au} \pm \mathrm{Sb} \pm \mathrm{Hg}$ mineralization, exemplified by the Lionsville-Solferino deposits to the east of Tenterfield sheet area, and from the Drake-style $\mathrm{Cu} \pm$ base metal $\pm \mathrm{Ag} \pm \mathrm{Au}$ epithermal mineralization located to the north of the area.

The Timbarra and other similar disseminated gold deposits in the Stanthorpe granite group belong to a recently proposed, globally widespread class of intrusion-related gold deposits, which are associated with $\mathrm{W}$-Sn-bearing magmatic provinces. The Au-Bi-Te-Mo signature, spatial association with a Sn-W province and felsic intrusions with intermediate oxidation states (spanning the boundary between the ilmenite-magnetite series), the sheeted-vein and disseminated mineralization style, close association of gold with bismuth and tectonic setting at a cratonic margin are all characteristic features of this type of deposit (Sillitoe, 1991; Hollister, 1992; Lang et al., 1997; Thompson et al., 1999; Lang et al., 2000).

A group of primary and closely-associated alluvial deposits near the north-west contact of 
the Dumbudgery Creek Granodiorite (Clarence River Plutonic Suite) were excluded from the prospectivity analysis because they represent a genetically different group to those described above. The deposits belong to the historic Lionsville-Solferino goldfields. The primary deposits represent a type of $\mathrm{Au} \pm \mathrm{Ag} \pm \mathrm{W} \pm \mathrm{Sb} \pm \mathrm{Hg}$ epigenetic mineralization, which grades from gold to gold-antimony to antimony in a south-easterly direction. Quartz reefs, veins and breccia fills occur in faults and shear zones, which cut all rock types. Barnes et al. (1988) refer to these deposits as metahydrothermal, because their relationship to the granites is unclear.

\subsubsection{Exploration Criteria}

Exploration criteria for intrusion-related gold deposits, proposed by Lang et al. (2000), mainly on the basis of their studies of deposits in the Yukon and eastern Alaska, include the following observations.

1. Prospective intrusions correspond to a short time interval within a protracted and commonly episodic period of magmatism.

2. Prospective intrusions are mostly metaluminous to weakly peraluminous, calc-alkalic and subalkalic, with the inferred oxidation state on the boundary between strongly oxidized magnetite-series and strongly reduced ilmenite-series intrusions, although intrusions at Timbarra are considered by Lang et al. (2000) to be a more highly oxidized exception due to emplacement into older oxidized calc-alkaline intrusions,

3. A high degree of differentiation is favourable. For intrusions in the Fairbanks area of Alaska, McCoy et al. (1997) suggest that most gold is associated with intrusions with a differentiation index above $80 . \mathrm{A} \mathrm{Fe}_{2} \mathrm{O}_{3} / \mathrm{FeO}$ value below 0.5 is favourable (Mutschler et al., 1985; Leveille et al., 1988).

4. In the Yukon, pre-existing faults and fractured zones adjacent to intrusive contacts are favourable sites for mineralization.

5. Deposits formed at greater than $1.5 \mathrm{kbar}$ do not have a large lateral extent. Lang et al. (2000) estimated that Timbarra formed at a depth of about $5 \mathrm{~km}$; that is, at approximately 1.5 kbar.

6. Age of the host rock is unimportant.

7. The geochemical signature recorded by Lang et al. (2000) in deeper systems from the Yukon and eastern Alaska consists of $\mathrm{Au}-\mathrm{Bi}-\mathrm{W} \pm \mathrm{As} \pm \mathrm{Te} \pm \mathrm{Mo}$, whereas Au occurs 
together with Mo, Bi, and Sn in the leucogranites of the Tenterfield area (Bampton, 1988).

8. Deeper systems, like Timbarra, display a very good correlation between Au and both $\mathrm{Bi}$ and Te.

9. Bismuth geochemical anomalies are located proximal to deposits, whereas As is more widely dispersed and yields large geochemical haloes.

10. The most prospective systems were first recognized from associated placer deposits.

Unfortunately, much of the data required to apply the criteria of Lang et al. (2000), such as the geochemistry and geochronology of the granitoids, are lacking for the study area. In detailing the exploration potential of the Tenterfield 1:100000 sheet area, Barnes et al. (1995) noted the following aspects of gold mineralization that have implications for exploration.

1. Gold occurs as large low-grade disseminations in poorly-defined irregular lenses in the upper part of the granite bodies, and is widespread throughout the Stanthorpe granite group.

2. Other leucogranites contain very low-grade gold in association with tin, molybdenum, bismuth and tungsten, and therefore have moderate potential for low-grade high-tonnage gold deposits.

3. Gold mineralization occurs in close proximity to cogenetic aplite dykes within the Stanthorpe granite group.

4. The Stanthorpe granite has very low magnetic intensities.

5. The Bungulla granite is associated with high magnetic intensities.

6. Leucogranitoids have high radiometric responses in all channels.

7. Intrusions of the Bungulla granite by the Stanthorpe granite or locations where the Stanthorpe granite underlies the Bungulla granite at shallow depth, identified by the magnetic intensity images, may indicate prospective areas for disseminated gold mineralization. 


\subsection{Experimental Procedure}

\subsubsection{Preparation of Neural-Network Maps}

\section{Pre-Processing of Input Data}

All thematic layers in the GIS database were converted to raster format prior to further processing. The original solid geology layer contained 41 rock units, many of which contain zero or very few deposits. The reliability of probability estimates used in the weights-ofevidence method depends on the existence of a statistically significant number of known deposits. Therefore, units belonging to the same rock suite or stratigraphic group were combined, resulting in a simplified geology layer consisting of 12 rock units (Fig 4.3). The GIS database layer containing regional faults was converted to a grid in which the cell values indicate the distance to the nearest fault.

The thematic layers in the Tenterfield GIS database were combined into a set of input feature vectors. At each cell location in the Tenterfield 1:100000 sheet grid, values from each thematic layer were combined to form a vector as shown in Chapter 3 (Fig. 3.2). These vectors, known as input feature vectors, formed the input to the neural network.

Linear scaling, based on the maximum and minimum values contained in each thematic layer, was applied to all input feature vectors. The logistic activation function, which is used in the hidden and output layer units of a neural network, has a squashed Sshape shown in Figure 2.7. The output values of this function approach limits of 1 and 0 asymptotically. Although the actual output produced by the network can theoretically reach these limits, in practice, the values that can be achieved by the network are limited to the range $[0.1,0.9]$. Consequently, training is facilitated if the target values are scaled to this range (Masters, 1993b). The input values were also scaled to this range. Masters (1993b) demonstrated that this improves the stability of the network and reduces the time required for training, since the network does not need to adjust weights to extreme values to compensate for the diverse range of the input data.

Apart from the preparation of the map layers, all processing was performed outside the GIS using a series of utility programs written in $\mathrm{C}++$ and the neural network simulation program, bp (McClelland \& Rumellhart, 1988). Data processing in the neural network method consisted of three main stages: 1) pre-processing of data to a format suitable as inputs to the neural network, 2) training and processing the entire data set after training, and 3) post-processing to convert the output values to a map. The data processing pro- 
cedures are shown schematically in Figures 3.3, 3.7, and 3.8. $\mathrm{C}++$ programs used in the neural network method in this chapter are listed and briefly explained in Appendix E and the code is included on the CD-ROM (vol.1) that accompanies the thesis.
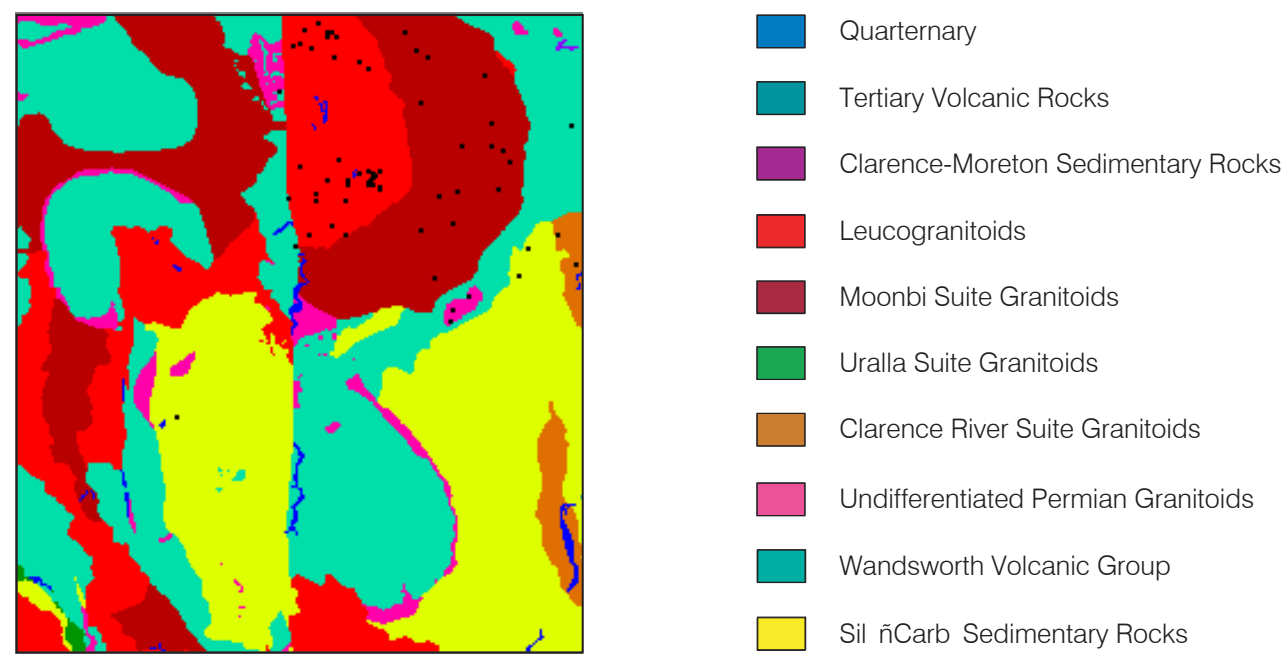

Figure 4.3. Simplified solid geology of the Tenterfield 1:100000 map sheet area. The three main rock units in the area are a Silurian-Carboniferous accretionary complex (yellow), the EarlyPermian Wandsworth Volcanic group (light green), and a series of Late-Permian to Early-Triassic granitoids (red colours). The two leucogranitoids in the north-east of the area (inner semi-circle: Stanthorpe Adamellite, and Bungulla Porphyritic Adamellite: outer semi-circle) are the main hosts to mineralization.

\section{Input Layers}

The 18 input units shown in Figure 4.4 correspond to; Quaternary alluvium, Tertiary volcanic rocks, Clarence-Moreton Basin sedimentary rocks, undifferentiated leucogranitoids (Stanthorpe Granite), granitoids of the Moonbi, Uralla, and Clarence River Plutonic Suites, undifferentiated Permian granitoids, undifferentiated Permian porphyritic granitoids, rocks of the Wandsworth Volcanic Group, Permian sedimentary rocks, SilurianCarboniferous sedimentary rocks, uranium, thorium, potassium and total count channels of airborne gamma-ray survey data, airborne magnetic data (total magnetic intensity, reduced to pole) and distance to the nearest fault.

\section{Input Coding}

The solid geology GIS layer represents the only categorical data type used as an input to the neural network in this study. A 1-of-n coding scheme was applied to the solid geology 


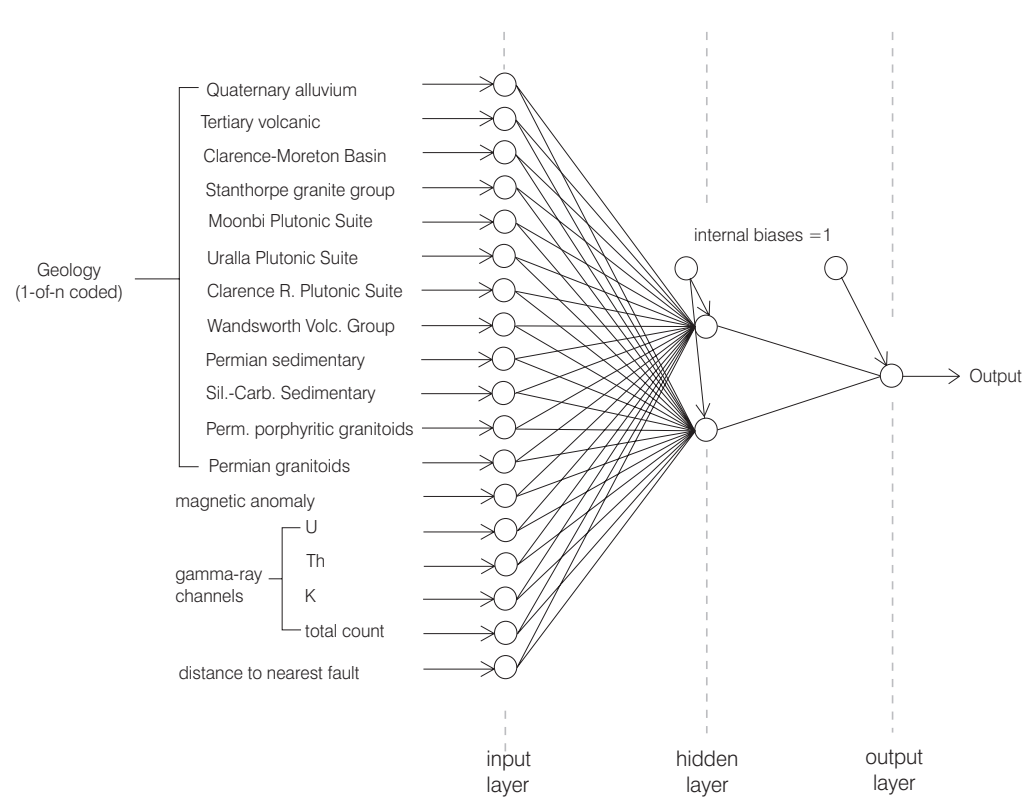

Figure 4.4. Architecture of the MLP neural network used in the pilot study. The network has an 18-2-1 topology (where the numbers refer to input, hidden, and output units, respectively). Each map layer in the GIS database was assigned an input unit except the geology layer, for which a 1-of-n coding scheme was used.

layer, so that each of the 12 rock types in the simplified geology layer was assigned a separate input layer unit in the neural network. For example, if the current rock type is basalt, then the input unit for basalt is set to one and all other input units allocated to rock types are set to zero. A single input unit was assigned to each of the magnetic, four radiometric and fault-proximity layers. Binary coding was used for the single output unit, with one and zero representing the presence and absence of deposits, respectively.

\section{Training Data}

Training data were randomly divided into training, training-stop ${ }^{1}$ and test sets $^{2}$. As noted in Chapter 3, the training data set is used to adapt the weights in the network and the training-stop data set is used to determine when to stop training (see description of neural network training in Section 4.4.1). The test data set plays no role in training and is

\footnotetext{
${ }^{1}$ The name training-stop data set is sometimes referred to as the stop set in tables in subsequent chapters

${ }^{2}$ There is some confusion in the literature concerning the nomenclature of these data sets. Some authors refer to the data set used to determine when to stop training as the test set whereas others refer to this as the validation set. If only two data sets are used, the data sets are sometimes referred to as the training and validation sets. However, the second set in this case is not independent because training is stopped at the point at which the error for the data set is at a minimum value. Consequently, the nomenclature used here is call the data set used to determine when to stop training as the training-stop data set and the data set used to assess the generalization performance of the network after training as the test set.
} 
used assess the generalization performance of the trained network when new data are processed. Only the independent test data can provide a guide to the performance of the trained network on new data, since the network is biased to perform well on the training and training-stop data sets (Masters, 1993b).

Although the ratio of deposit to non-deposit cells in the Tenterfield grid is approximately 1:1000, the ratio of deposit to non-deposit vectors used in the training set is close to 1:1. This is necessary because the learning algorithm minimizes the TSS error over all the patterns in the training set. If deposit patterns were represented in the training set in the same proportion as they appear in the total data population, the learning algorithm would optimize the performance for non-deposit patterns (Masters, 1993b). The network would not learn to recognize the rare deposit patterns at all or would perform very poorly for this class of patterns. Learning in MLP networks is improved by selecting equal numbers of training vectors from each class irrespective of the a priori probability of occurrence of the classes (Zaknich, 1999). Consequently, the training, training-stop and test data sets were randomly assigned one third of the available deposit vectors (21 each), and two non-deposit vectors were randomly selected for each of the 12 main rock units, giving a total of 24 non-deposit vectors in each training set. Table 4.1 shows the composition of the training sets.

Table 4.1. Number of patterns in the training data sets

\begin{tabular}{|l|c|c|c|}
\hline Class & Training set & Stop set & Test set \\
\hline \hline Deposit & 21 & 21 & 21 \\
\hline Barren & 24 & 24 & 24 \\
\hline Total & 45 & 45 & 45 \\
\hline
\end{tabular}

Since there are only 63 gold deposits of the required type in the Tenterfield area, compared with 69,377 non-deposit cells. Since the size of the training data sets are limited by the number of known deposits, the available training data is too small for a third of the data to be reserved as a test data set in the statistically-based weights-of-evidence method. The weights-of-evidence method includes a test for correlations between evidence maps. Using only deposit cells, the chi-squared statistic is calculated for all possible pairs of input layers. However, at least five deposits are required in each of the four possible overlap combinations involving the pair of binary maps (e.g. pattern 1 present, pattern 2 absent). Otherwise the chi-squared test is very unreliable. To avoid this problem, all of the available data was used to estimate the value of the the weights in the weights-of-evidence method. 
The training patterns were presented to the network in a randomly permutated order.

\section{Network Topology and Parameters}

The number of units in the hidden layer of the network was determined experimentally. A heuristic for estimating the number of hidden units that works for many practical problems has been suggested by Masters (1993b). The number of units should decrease from the input to the output layer with the number of hidden layer units roughly given by:

$$
h=\sqrt{m n}
$$

where $h=$ number of hidden units, $m=$ number of input units, and $n=$ number of output units.

Applying this rule of thumb to the network here, which has 18 input units and a single output, suggests that four hidden units should be used. If too many hidden layer units used in combination with a small training set, this can result in a network that is too complex, leading to over-fitting of the data (Bishop, 1995). In order to minimize the effective complexity of the network, as few hidden units as possible were used. Consequently, the procedure used here was to experiment with one to five hidden units using identical training sets and network parameters. The smallest number of hidden units giving the best generalization performance on the test data set was used. This procedure resulted in a 18-2-1 network topology.

A constant learning rate of 0.5 was used for all networks. Weights were randomly initialized in the range $[-0.5,+0.5]$. The values of the momentum parameter (used to add a fraction of the previous weight change to the current weight change) and the weight-update frequency (i.e. whether the weights are updated after the presentation of each training pattern or after all the patterns in the training set are processed) are indicated in Table 4.4.

\section{Neural Network Training}

A series of networks were trained using ten different random sets of initial weights. The purpose of using different random initial weights is to prevent the network converging at a local rather than a global minimum TSS error in multidimensional weight space (Fig. 2.9). Whether this has occurred can only be determined if training with some other set of random initial weights results in a significantly lower TSS error for both the 
training and test sets. Thus, the weights in the network after training is completed depend on the starting point in multidimensional weight space, which is determined by the set of initial weights. Each of these random initializations effectively results in a different trained network. In each case the initial weights were saved. In some cases, training was repeated for a given set of initial weights using different network parameters (Table 4.4). Training runs with identical random initial weights are labelled with the same run number in Table 4.4.

Although the training error decreases during training, the test error initially decreases to a minimum and then begins to increase as training continues. This occurs because the network starts to learn the idiosyncrasies of the training set. To avoid this, the procedure known as early stopping was used (Bishop, 1995). The total sum of squares (TSS) error was monitored for both the training and test data sets and training is stopped at the number of epochs corresponding to the minimum TSS error for the test data set. To achieve this, the test data set is processed and the TSS error is recorded at intervals of 10 or 100 training cycles (depending on the rate at which the error is decreasing). Weights are not modified during the test data set cycle. This continued until a clear increase in the TSS error for the test data set occurs. Once the number of cycles corresponding to the minimum test set error is known, the values of the weights are reset to their random initial values and the network is re-trained for the optimal number of cycles using the training data set only. The neural network giving the best classification performance for the independent test data set was used to process the input vectors for the entire Tenterfield grid (network 10a in Table 4.4 .

\section{Post-Processing}

Output values, produced by the trained network used to process the complete Tenterfield data set, were re-scaled to the range $[0,8]$ and then classified into integer values to produce a nine-class prospectivity map. The percentages of correctly classified deposits and nondeposit cells were determined by applying a threshold of 0.5 to the activation of the single output unit. Cells corresponding to values greater or equal to 0.5 were classified as deposits, otherwise the cells were classified as barren.

A $\mathrm{C}++$ program, map_class was used to convert the output file produced by the neural network simulation package, bp, consisting of a list of floating point values in the range $[0.1,1.0]$, to an integer grid with values representing nine prospectivity classes (code included on CD-ROM (vol. 1)). Inputs were scaled to a range $[0,1]$. 
The integer grid file was imported into the geoscience data processing package called OASIS MONTAJ (also known by the company name, GEOSOFT) in order to assign colours to map classes and to overlay deposit points on the prospectivity maps (Fig. 3.3).

\subsubsection{Preparation of Weights-of-Evidence Maps}

As a means of validating and comparing the performances of the neural network method, mineral prospectivity maps were prepared according to the empirical weights-of-evidence and conceptual fuzzy-logic methods described in sections 3.4 and 3.4.1 using the GEODIPS GIS prospectivity-analysis package developed by Jusmady \& Taylor (1997).

The input maps depicting solid geology, buffered regional faults and shear zones (25 buffer zones at $200 \mathrm{~m}$ intervals), airborne total magnetic intensity, U, Th, K and total count gamma-ray survey data channels were reclassified using threshold classes which maximize the spatial association between the evidence pattern and deposit point pattern (known deposits). Thresholds were selected from the tables of weights and contrasts produced by the GEODIPS system.

Pairwise tests of all possible binary evidence maps from the weights-of-evidence method showed that the assumption of conditional independence is violated for nearly all map pairs. Only the proximity-to-faults map did not exceed the $\chi^{2}$ limit of 5.4 at the $95 \%$ confidence level for 1 degree of freedom. This means the other binary map pairs are not conditionally independent, which would lead to the predicted number of deposits being significantly greater than the observed number of deposits (Bonham-Carter, 1994; Singer \& Kouda, 1999a). The following steps were taken in order to reduce the conditional dependence;

1. The solid geology map was re-classified to produce a new binary evidence map. The map algebra operations used to produce the initial and modified binary solid geology maps are as follows;

Geol_1 $=$ recode_classIf Class ${ }_{\text {old }}=$ Leucogranitoid, Moonbi Suite Granitoids

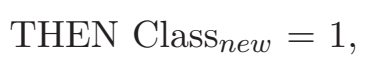
ELSE Class $_{n e w}=0$ )

Geol_2 $=$ recode_classIff Class old $_{2}=$ Leucogranitoid, Moonbi Suite Granitoids, Permian Granitoids, Clarence River Suite

THEN Class $_{\text {new }}=1$,

ELSE Class $_{n e w}=0$ ) 
2. All airborne gamma-ray survey maps except the one for the uranium channel were discarded. The U-channel map was retained because it was associated with the highest value of $W^{+}$. This did not solve the problem as the U-channel binary radiometric map is conditionally dependent on the geology and magnetic maps. Since leucogranitoids associated with gold deposits are highly fractionated (Blevin \& Chappell, 1996a,b) and have high radiometric responses (Barnes, 1993), a number of combinations of the uranium- and thorium-channel radiometric and magnetic maps were tested. The $U \times T h$ map showed a combination of a strongest association with known deposit and occurrence points and a lower degree of variability between adjacent cells in the raster grid.

3. The airborne total magnetic intensity map was discarded because the statistical relationship between magnetic intensity and deposit points is unclear. The magnetic intensity data were not used in the final weights-of-evidence map that is compared with the fuzzy-logic and neural network methods in the conclusions section below.

The steps above resulted in the seven original input maps being reduced to the following three maps; bin_flt, bin_geol2, bin_uxth and magnetic. Since these new derived geology and radiometric map pairs were also conditionally dependent, different ways of combining the maps using the Boolean $A N D$ and $O R$ operators were tested. The operations that were used to combine the maps are shown in terms of map algebra in Table 4.2 below.

In each case, the weight calculated for the binary output map in Table 4.2 was combined with the weight for the binary proximity-to-fault map, bin_flt to create a prospectivity map. The resulting maps are shown in Figures 4.5 and 4.6. The prospectivity map that best accounted for the known deposit locations, while minimizing the area of the highest prospectivity classes, was chosen as the final map to be compared with the fuzzy-logic and neural network methods in the results section below (Fig. 4.5b; add2). 
Table 4.2. Map algebra operations used to combine conditionally dependent input maps in weights-of-evidence method. All maps in Figures 4.5, 4.6 and 4.11 were produced using the layer depicting the distance to the nearest major fault or shear zone as an additional input.

\begin{tabular}{|l||l|}
\hline Map & Map algebra operation \\
\hline \hline add1 & geology $+(\mathrm{U} \times \mathrm{Th})+$ magnetic \\
\hline add2 & geology $+(\mathrm{U} \times \mathrm{Th})+$ magnetic $^{\mathrm{a}}$ \\
\hline $\max 1$ & $\min [$ geology, $(\mathrm{U} \times \mathrm{Th})]$ \\
\hline $\max 2$ & $\min \left[\right.$ geology, $\left.(\mathrm{U} \times \mathrm{Th})^{\mathrm{b}}\right]$ \\
\hline $\max 3$ & $\min [$ geology, $(\mathrm{U} \times \mathrm{Th})$, magnetic $]$ \\
\hline $\min 1$ & $\min [$ geology, $(\mathrm{U} \times \mathrm{Th})]$ \\
\hline $\min 2$ & $\min \left[\right.$ geology, $\left.(\mathrm{U} \times \mathrm{Th})^{\mathrm{b}}\right]$ \\
\hline $\min 3$ & $\min [$ geology, $(\mathrm{U} \times \mathrm{Th}) /$ magnetic $]$ \\
\hline $\min 4$ & $\min \left[\right.$ geology, $(\mathrm{U} \times \mathrm{Th})^{\mathrm{b}} /$ magnetic $]$ \\
\hline $\min 5$ & $\min [$ geology, $(\mathrm{U} \times \mathrm{Th})$, magnetic] \\
\hline $\min 6$ & $\min [$ geology, $(\mathrm{U} \times \mathrm{Th} \times \mathrm{K}) /$ magnetic] \\
\hline $\min 7$ & $\min ($ geology, $(\mathrm{U}+\mathrm{Th})]$ \\
\hline
\end{tabular}

\footnotetext{
${ }^{a}$ In add1 the geology binary input map consists of leucogranites only whereas in add2 it consists of additional lithological units; i.e. Permian Undifferentiated Granitoids, Moonbi Granite and Clarence River Granite.

${ }^{b}$ The $U \times T h$ evidence map was recoded using a different set of class thresholds to produce a new binary map.
} 


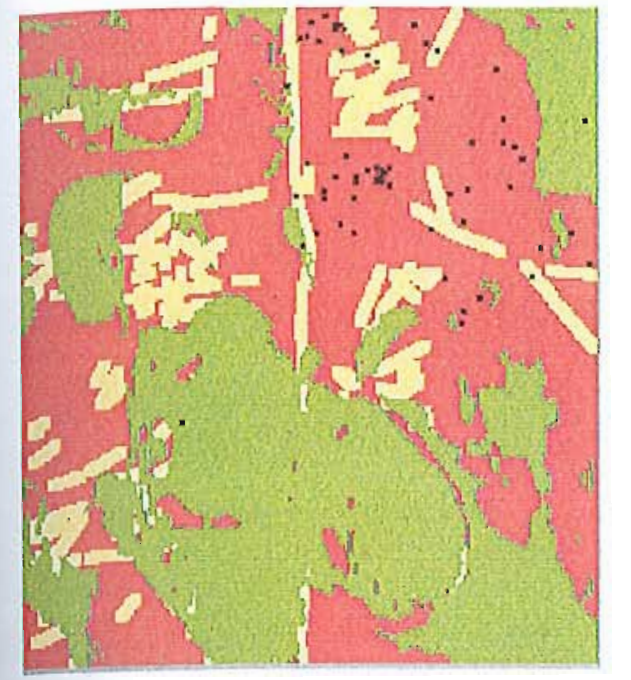

(a) add1

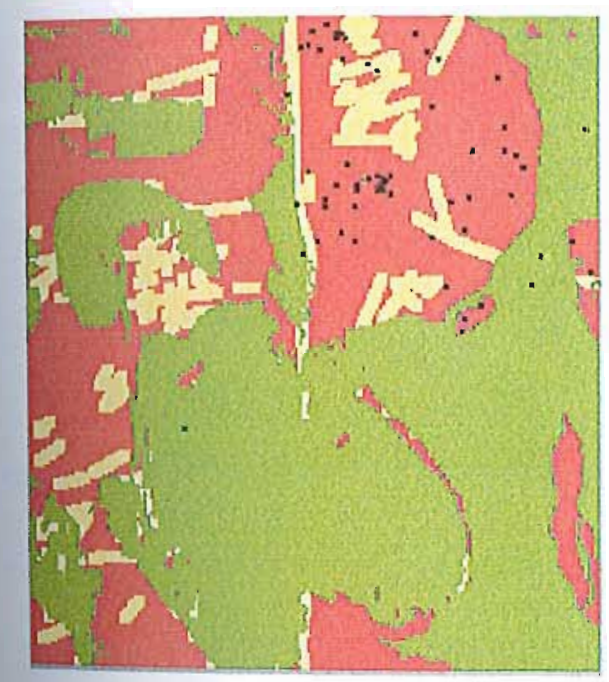

(c) $\max 1$

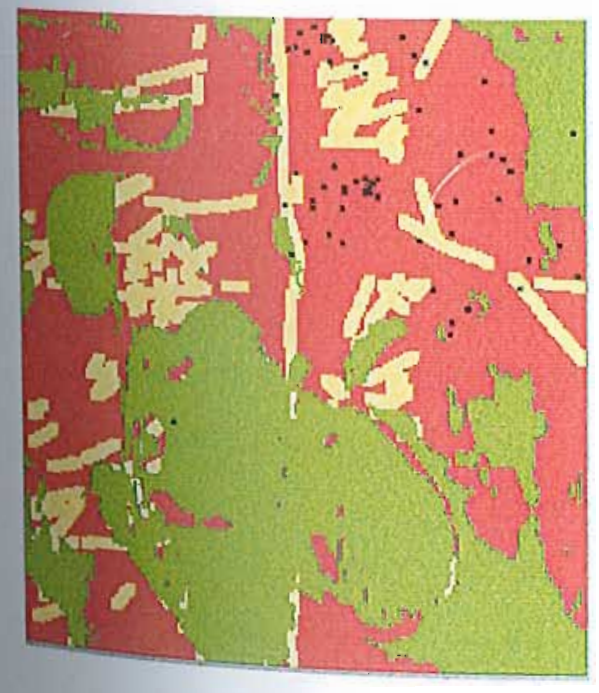

(e) $\max 3$
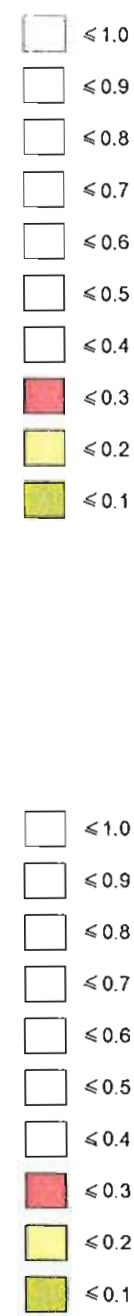

$\square \leqslant 1.0$
$\square \leqslant 0.9$
$\square \leqslant 0.8$
$\square \leqslant 0.7$
$\square \leqslant 0.6$
$\square \leqslant 0.5$
$\square \leqslant 0.4$
$\square \leqslant 0.3$
$\square \leqslant 0.2$
$\square \leqslant 0.1$

\footnotetext{
Figure 4.5. Weights-of-evidence mineral prospectivity maps for intrusion-related dissemito Tated gold mineralization, Tenterfield 1:100000 map sheet area, north-eastern NSW. Refer to Table 4.2 on page 115 for an explanation of the map names.
} 


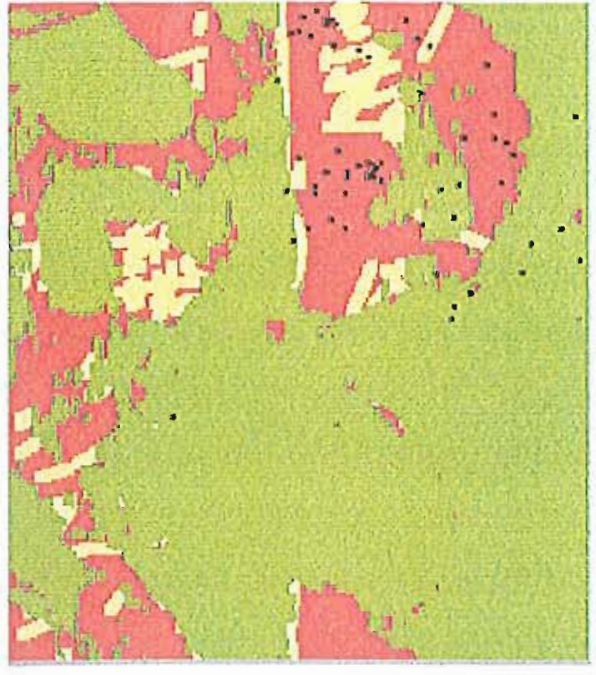

(a) $\min 2$

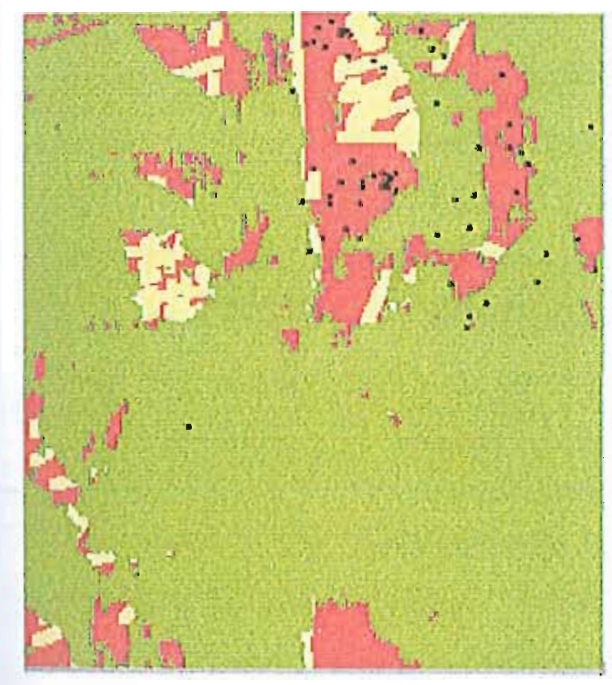

(c) $\min 4$

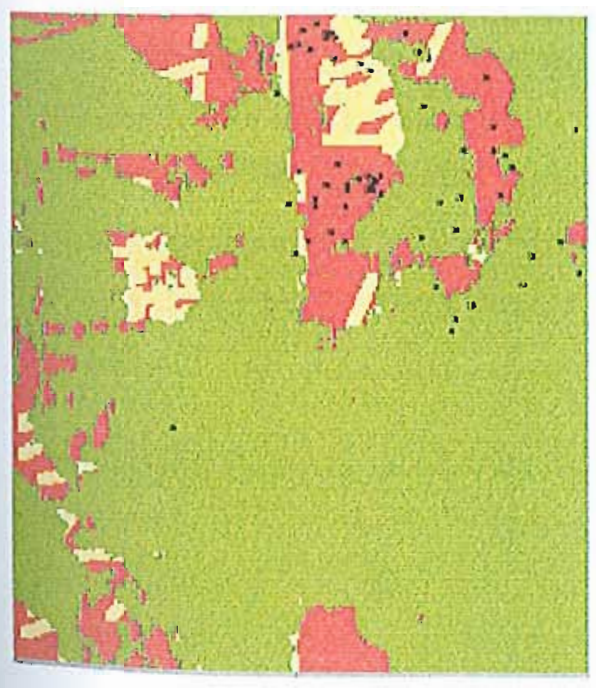

(e) $\min 6$

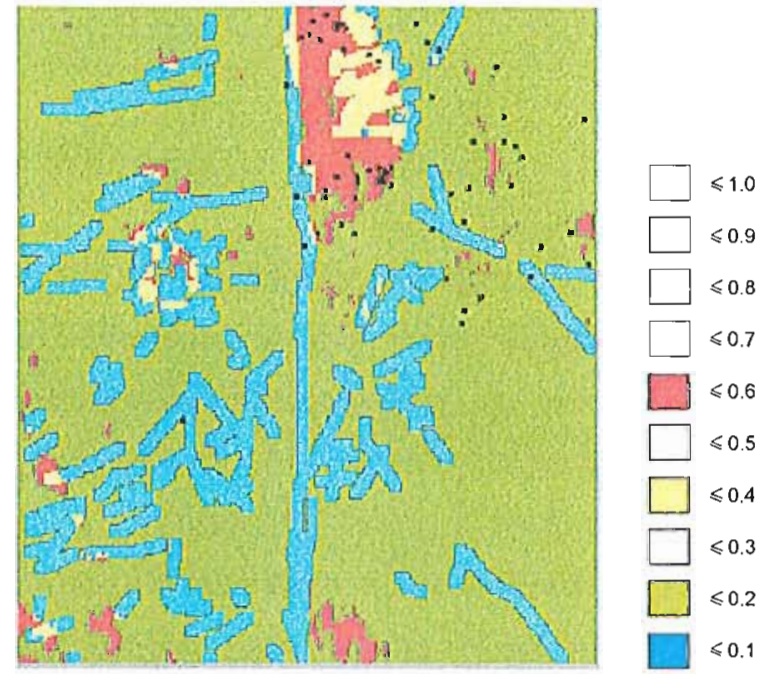

(b) $\min 3$

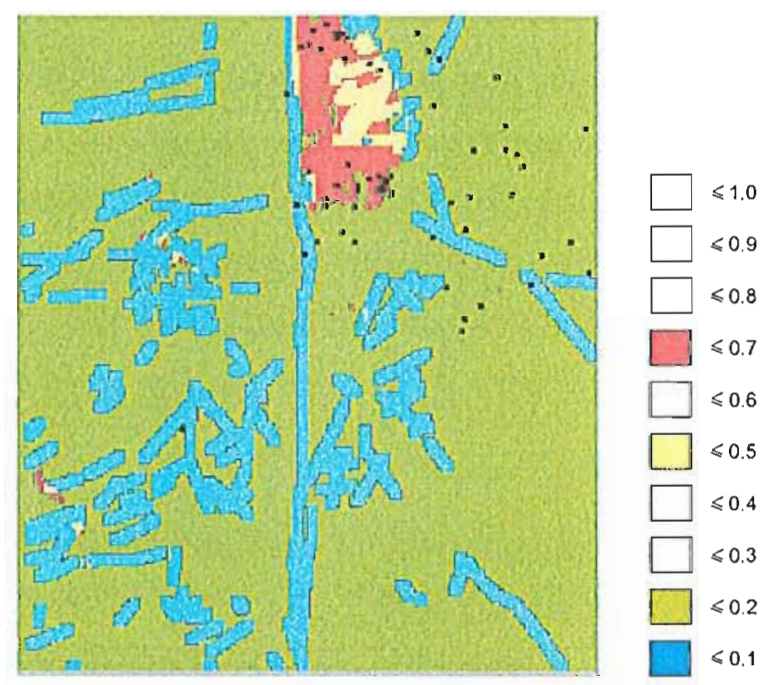

(d) $\min 5$

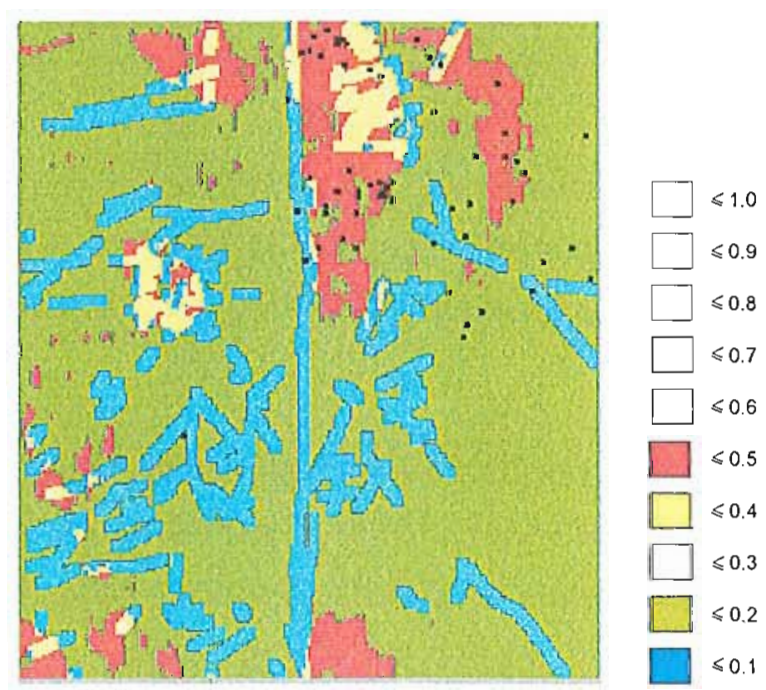

(f) $\min 7$

Figure 4.6. Weights-of-evidence mineral prospectivity maps for intrusion-related disseminated gold mineralization, Tenterfield 1:100000 map sheet area, north-eastern NSW. Refer to Table 4.2 on page 115 for an explanation of the map names. 


\subsubsection{Preparation of Fuzzy-Systems Maps}

The GEODIPS GIS prospectivity analysis package, developed by Jusmady \& Taylor (1997), was also used to prepare maps based on the fuzzy-logic method. Fuzzy membership values, in the range $[0,1]$, were assigned on the basis of subjective judgement according to how favourable the parameter values are for mineralization. Parameter value classes and the corresponding membership values are shown in Table 4.3 below. These are the final values chosen after experimentation. Some of the membership values were varied so that the two highest prospectivity map classes included as many of the known mineral deposits in as small an area as possible. A new layer containing fuzzy membership values was created for each input map using the values in Table 4.3.

A variety of different fuzzy membership value assignments, inference nets, and choices of fuzzy operators for the final map combination step were tested. In the case of the gamma operator, a range of values of gamma (in the range $[0,1]$ ) was tested. The results of the tests showing the mineral prospectivity maps together with the inference net, are shown in Figures 4.7-4.10. The relationship between the deposit points and the magnetic intensity map was ambiguous, and therefore the magnetic map was not used as an input map in the fuzzy-logic and weights-of-evidence methods.

The areas of the fuzzy systems maps, which represent the highest favourability, are very sensitive to small changes in gamma in the range $0.90 \leq \gamma \leq 1.0$. Large gamma values result in larger areas being assigned a high prospectivity due to the effect of the fuzzy algebraic sum component in the gamma function (see equation 2.15 and the left-hand factor in equation 2.16). For example, in Figure 4.10b $(\gamma=0.95)$, the highest prospectivity class (favourability index $\geq 0.90$ ) connects the two main groups of deposit points in the northern part of the map in a north-south direction, whereas in Figure 4.9b $(\gamma=0.90)$, the two groups of deposits are separated by a lower prospectivity area. The second map represents a more conservative estimate of prospectivity, in which the gap between deposit points (shown in yellow-green) is assigned the next lower prospectivity class (favourability $\geq 0.80)$.

Modifying the fuzzy membership values and the inference net also has a significant effect on the resulting prospectivity map. The effect of slight differences in the fuzzy membership functions and inference nets (for a given gamma value) can be illustrated by comparing Figures $4.7 \mathrm{a}, 4.7 \mathrm{~b}$ and $4.10 \mathrm{~b}$ ( $\gamma=0.95$ for the three maps). The effect of using a different 
fuzzy inference net is illustrated by comparing Figure 4.7a with Fig. 4.7b. The difference between the fuzzy-logic schema for Figures $4.7 \mathrm{a}$ and $4.7 \mathrm{~b}$ is that separate $\mathrm{U}$ and Th inputs are used in Figure 4.7a, whereas summed $U+T h$ inputs are used in Figure 4.7b.

The effect of using different values in the fuzzy membership functions is evident in Figures $4.7 \mathrm{~b}$ and $4.10 \mathrm{~b}$. For Figure 4.7b (and Fig. 4.7a), fuzzy membership values for the fault buffer classes where distance to the nearest fault $\leq 2000 \mathrm{~m}$ are set to 0.1 , whereas a value of 0.5 is used in Figure 4.10b. The same fuzzy inference net is used in both cases.

The pre- and post-processing steps are the same for as those described for the weights-ofevidence method in Chapter 3 above.

The prospectivity map that best accounted for the known deposit locations, while minimizing the area of the highest prospectivity classes, was chosen as the final fuzzy-logic model. (Fig. 4.7c). This fuzzy-logic map is compared with the weights-of-evidence and neural-network maps in the results section in Figure 4.11b. It was produced by using the gamma fuzzy-membership operator $(\gamma=0.95)$ in the final integration step. The fuzzy inference net corresponding to this map is shown in Figure 4.11b. The map algebra addition-operator was used to combine the gamma-ray U- and Th-channel maps.

Table 4.3. Class values and fuzzy membership values for input maps in the fuzzy-logic method. Abbreviations: Quat. = Quaternary, Clarence Moreton $=$ Clarence Moreton Basin sedimentary rocks, Stanthorpe Granite $=$ Granite $=$ Granite group, Gtds $=$ granitoids, PS $=$ Plutonic Suite, Perm. $=$ Permian, Sed. $=$ sedimentary rocks, $\mathrm{Gp}=$ Group.

\begin{tabular}{|l||l||l|c||c|c||c|c|c|c|}
\hline \multicolumn{2}{|l||}{ Geological units } & \multicolumn{2}{l||}{ Distance to faults } & \multicolumn{2}{l||}{ U image } & \multicolumn{2}{l|}{ Th image } & \multicolumn{2}{l|}{ U + Th } \\
\hline Class & $\mathrm{m} / \mathrm{ship}$ & Class & $\mathrm{m} / \mathrm{ship}$ & Class & $\mathrm{m} / \mathrm{ship}$ & Class & $\mathrm{m} / \mathrm{ship}$ & Class & $\mathrm{m} / \mathrm{ship}$ \\
\hline \hline Quat. Alluvium & 0.10 & $<200$ & 0.10 & 1 & 0.10 & 1 & 0.20 & 1 & 0.1 \\
\hline Tertiary Volcanic & 0.00 & $200-<400$ & 0.10 & 2 & 0.20 & 2 & 0.20 & 2 & 0.1 \\
\hline Clarence-Moreton & 0.00 & $400-<600$ & 0.10 & 3 & 0.20 & 3 & 0.25 & 3 & 0.1 \\
\hline Stanthorpe Granite & 0.95 & $600-<800$ & 0.10 & 4 & 0.40 & 4 & 0.40 & 4 & 0.3 \\
\hline Bungulla Granite & 0.95 & $800-<1000$ & 0.10 & 5 & 0.70 & 5 & 0.60 & 5 & 0.3 \\
\hline Uralla Suite Gtds & 0.00 & $1000-<1200$ & 0.10 & 6 & 0.80 & 6 & 0.80 & 6 & 0.3 \\
\hline Clarence River PS & 0.10 & $1200-<1400$ & 0.10 & 7 & 0.90 & 7 & 0.80 & 7 & 0.3 \\
\hline Undiff. Perm. Gtds & 0.40 & $1400-<1600$ & 0.10 & 8 & 0.90 & 8 & 0.90 & 8 & 0.6 \\
\hline Wandsworth Volc.Gp & 0.10 & $1800-<2000$ & 0.10 & & & & & 10 & 0.6 \\
\hline Permian Sed. & 0.10 & $>2000$ & 0.75 & & & & & $11-14$ & 0.9 \\
\hline Sil.-Carb. Sed. & 0.10 & & & & & & & $15-16$ & 0.8 \\
\hline
\end{tabular}



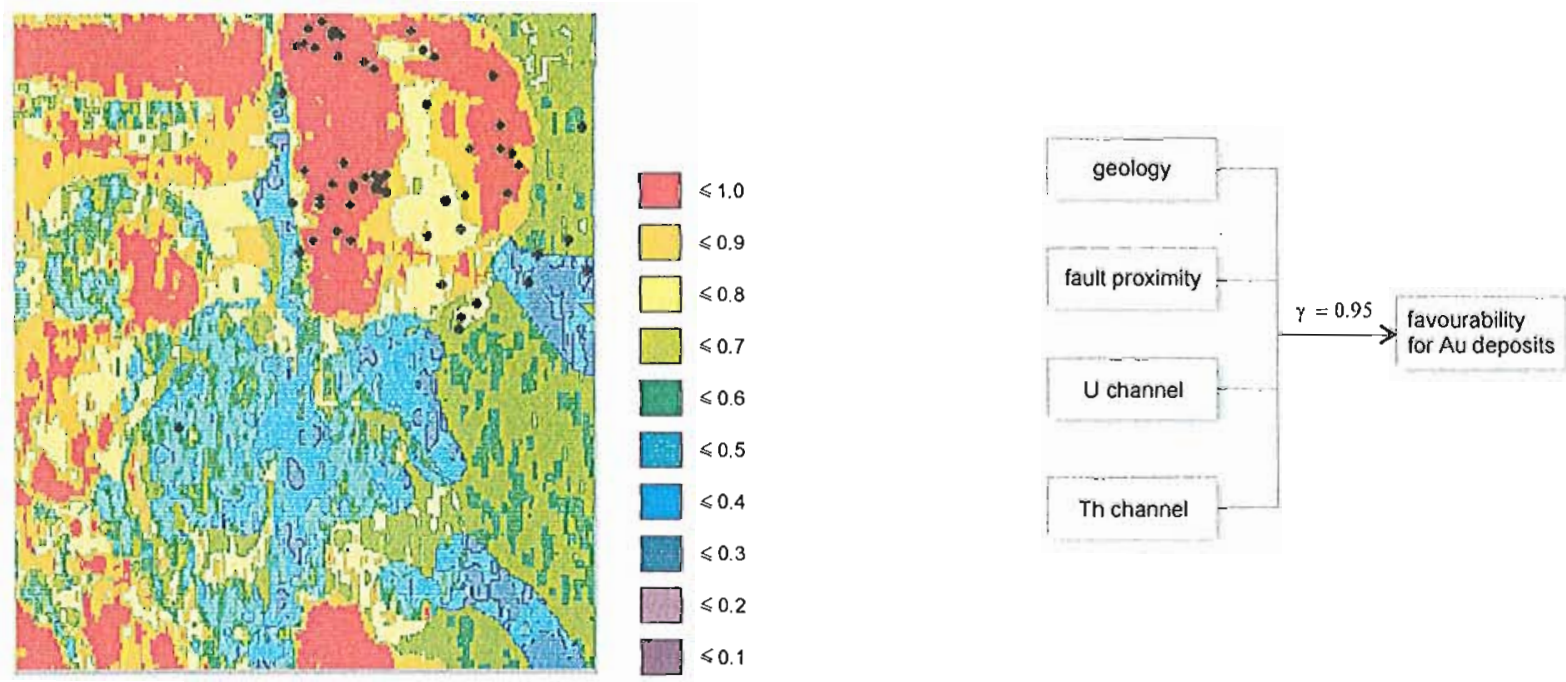

(a) $\gamma=0.95,4$ inputs. Fuzzy membership values in Table 4.3 cxccpt distance-to-faults layer (class $>2000 \mathrm{~m}=0.70$, rest $=0.05$ instead of 0.75 and 0.01 , respectively).
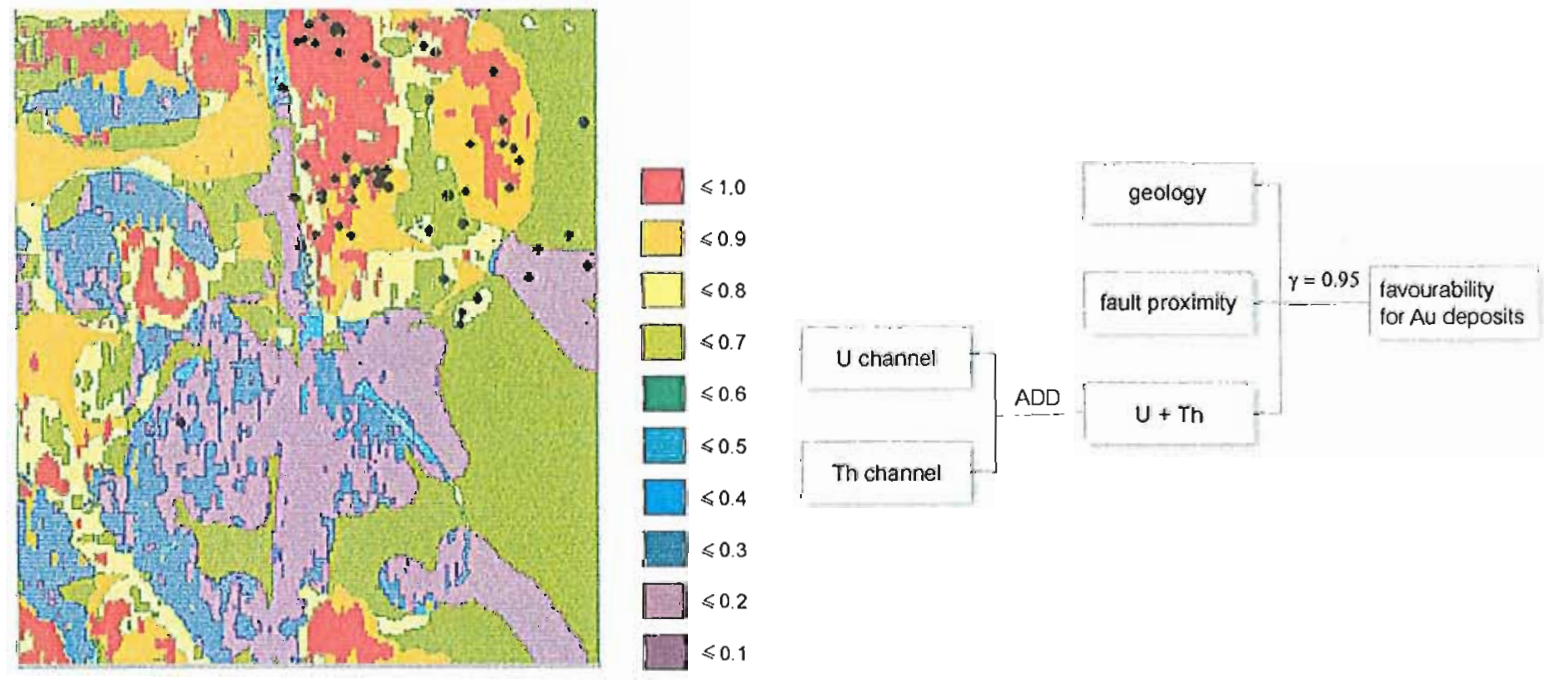

(b) $\gamma=0.95,4$ inputs. Fuzzy membership values in Table 4.3 except UplusTh layer (classes 1-6 $=0.1$ instead of $0.1,0.1,0.3,0.3, \& 0.3$ and classes $7-11=0.2$ instead of $0.3,0.6,0.6,0.6, \& 0.9$ ).
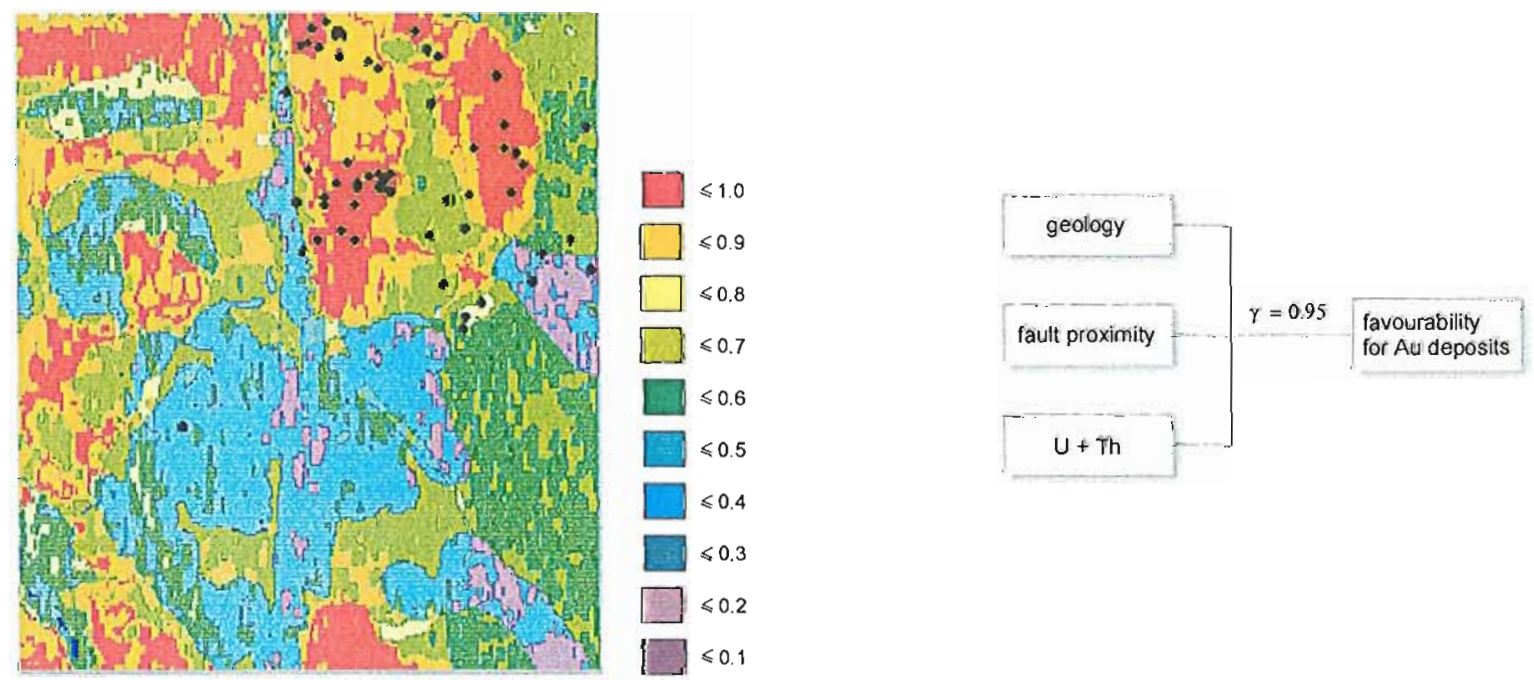

(c) $\gamma=0.95,3$ inputs. Fuzzy membership values in Table 4.3 except dist.-to-faults layer (class $>2000 \mathrm{~m}$ $=0.90$, instead of 0.75 ) and geology layer (Leuco- \& Moonbi Granites $=0.90$ instead of 0.75).

Figure 4.7. Fuzzy mineral prospectivity maps for the Tenterfield 1:100000 map sheet area. 


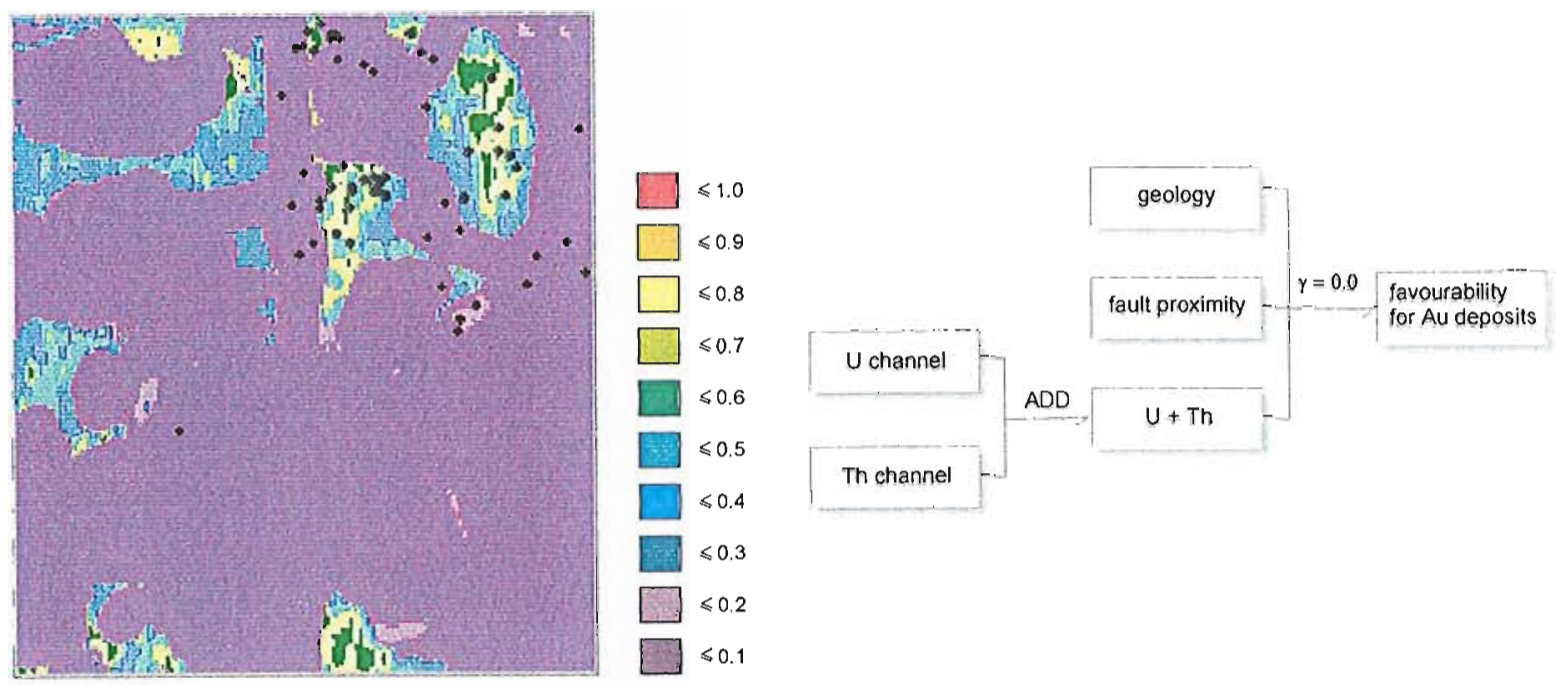

(a) $\gamma=0.0,4$ inputs. Fuzzy membership values are those given in Table 4.3 except for the distanceto-faults layer (class $<2000 \mathrm{~m}=0.50$ instead of 0.1 ).
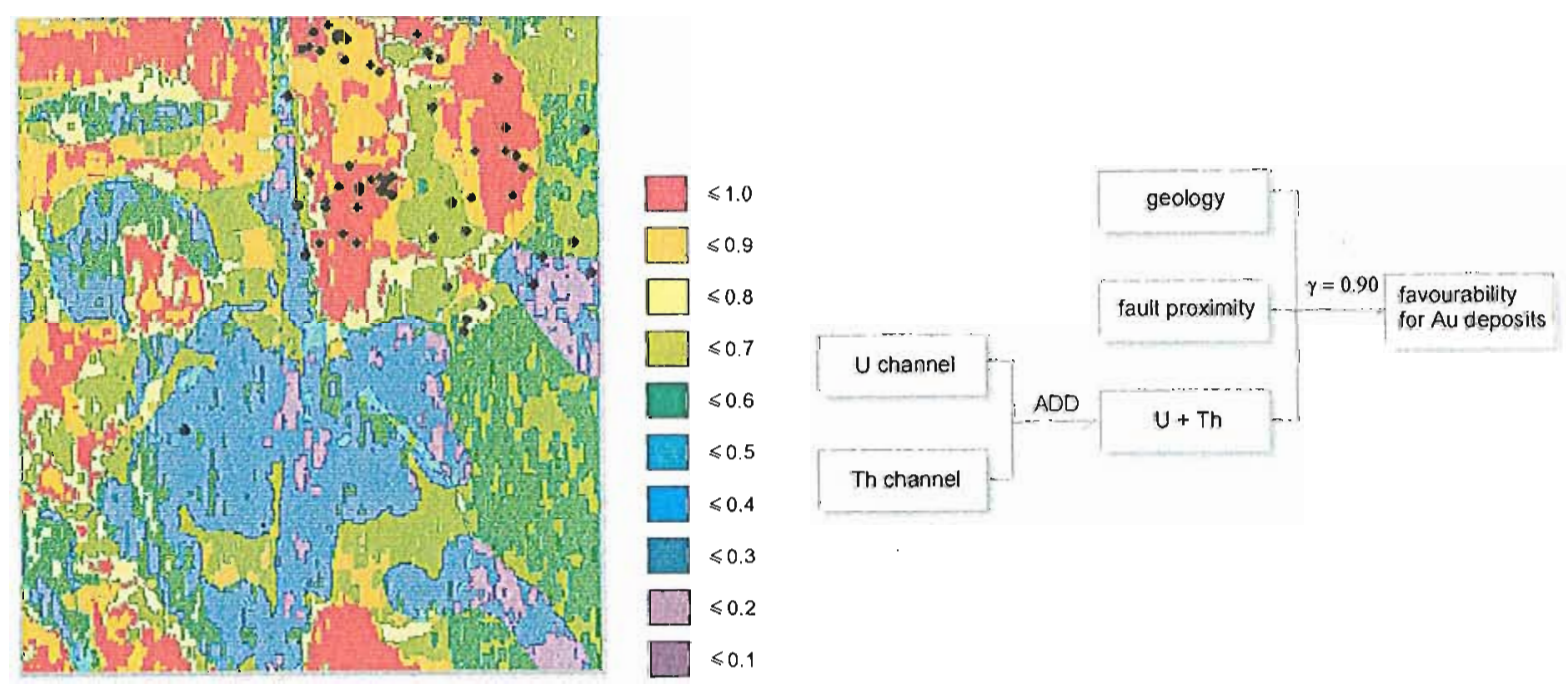

(b) $\gamma=0.90,4$ inputs. Fuzzy membership values are those given in Table 4.3 except for the distanceto-faults layer (class $<2000 \mathrm{~m}=0.50$ instead of 0.1 ).
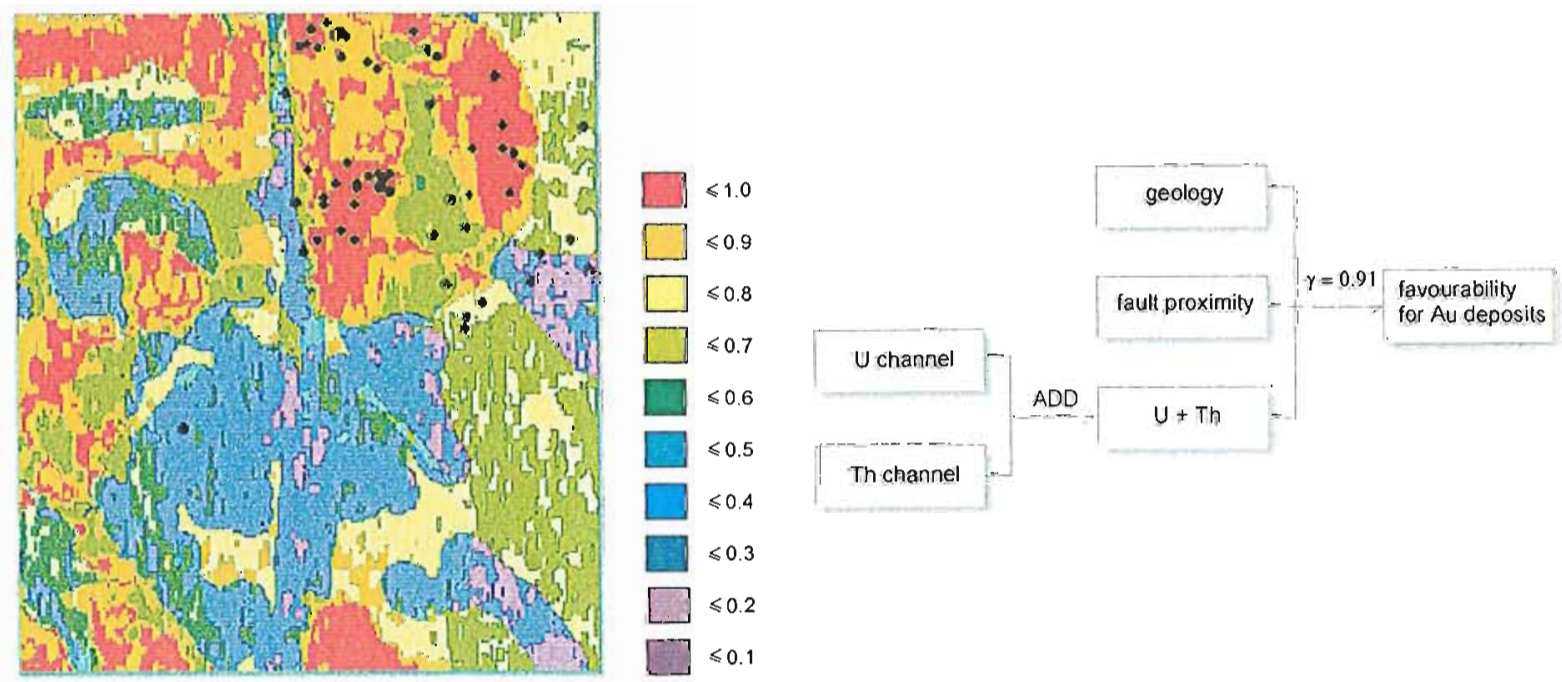

(c) $\gamma=0.91,4$ inputs. Fuzzy membership values are those given in Table 4.3 except for the distanceto-faults layer (class $<2000 \mathrm{~m}=0.50$ instead of 0.1 ).

Figure 4.9. Fuzzy mineral prospectivity maps for the Tenterfield 1:100000 map sheet area. 

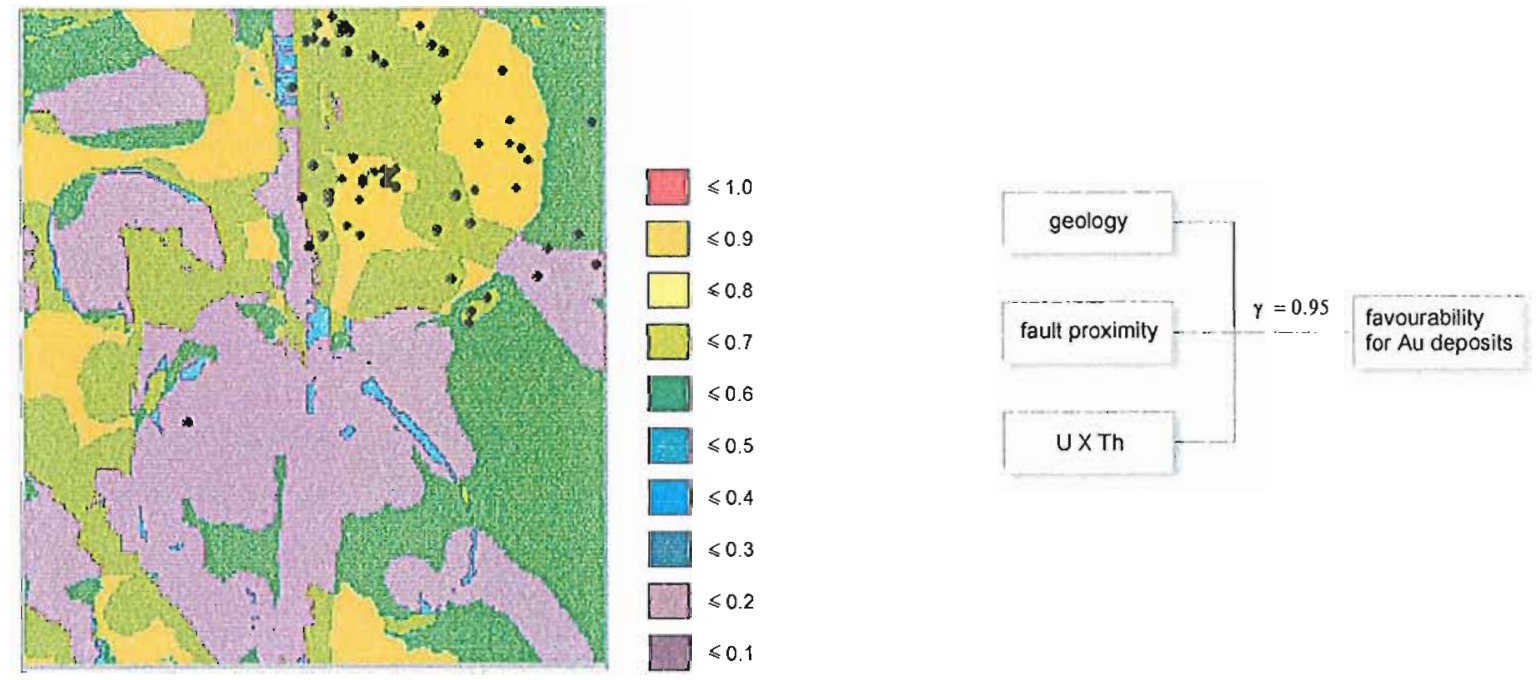

(a) $\gamma=0.95,3$ inputs. Fuzzy membership values are those given in Table 4.3 except for the distanceto-faults layer (class $>2000 \mathrm{~m}=0.70$, rest $=0.05$ instead of 0.75 , rest $=0.1$, respectively).
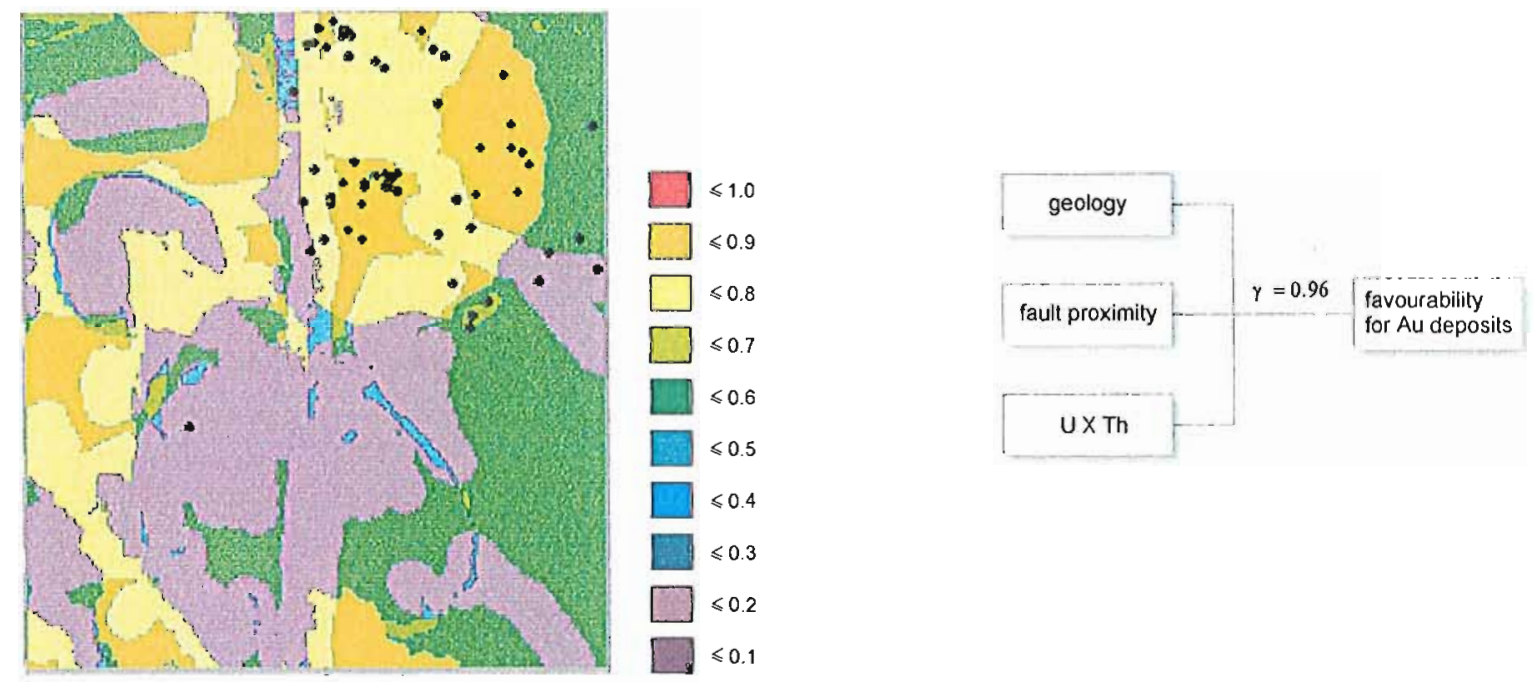

(b) $\gamma=0.96,3$ inputs. Fuzzy membership values are those given in Table 4.3 except for the distanceto-faults layer (class $>2000 \mathrm{~m}=0.70$, rest $=0.05$ instead of 0.75 and 0.01 , respectively).
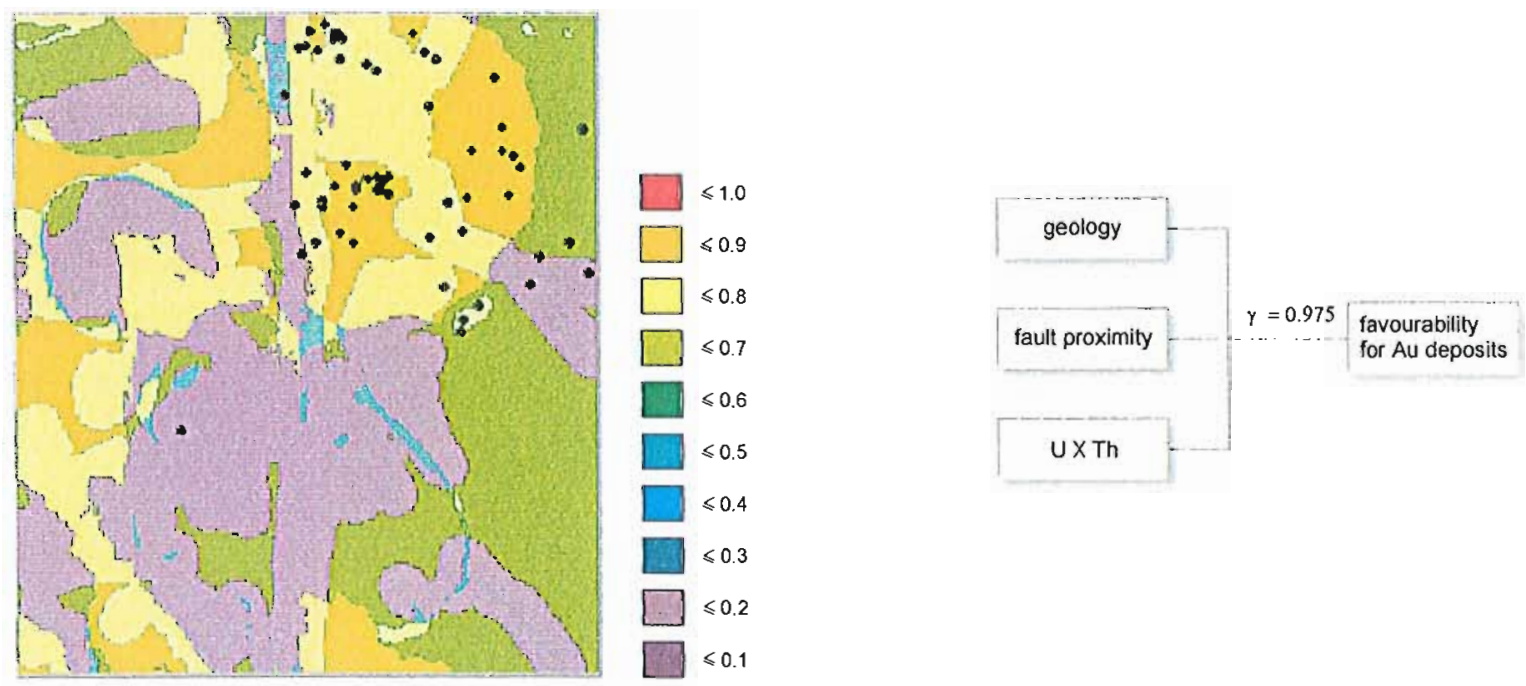

(c) $\gamma=0.975,3$ inputs. Fuzzy membership values are those given in Table 4.3 except for the distanceto-faults layer (class $>2000 \mathrm{~m}=0.70$, rest $=0.05$ instead of 0.75 and 0.01 , respectively).

Figure 4.8. Fuzzy mineral prospectivity maps for the Tenterfield 1:100000 map sheet area. 


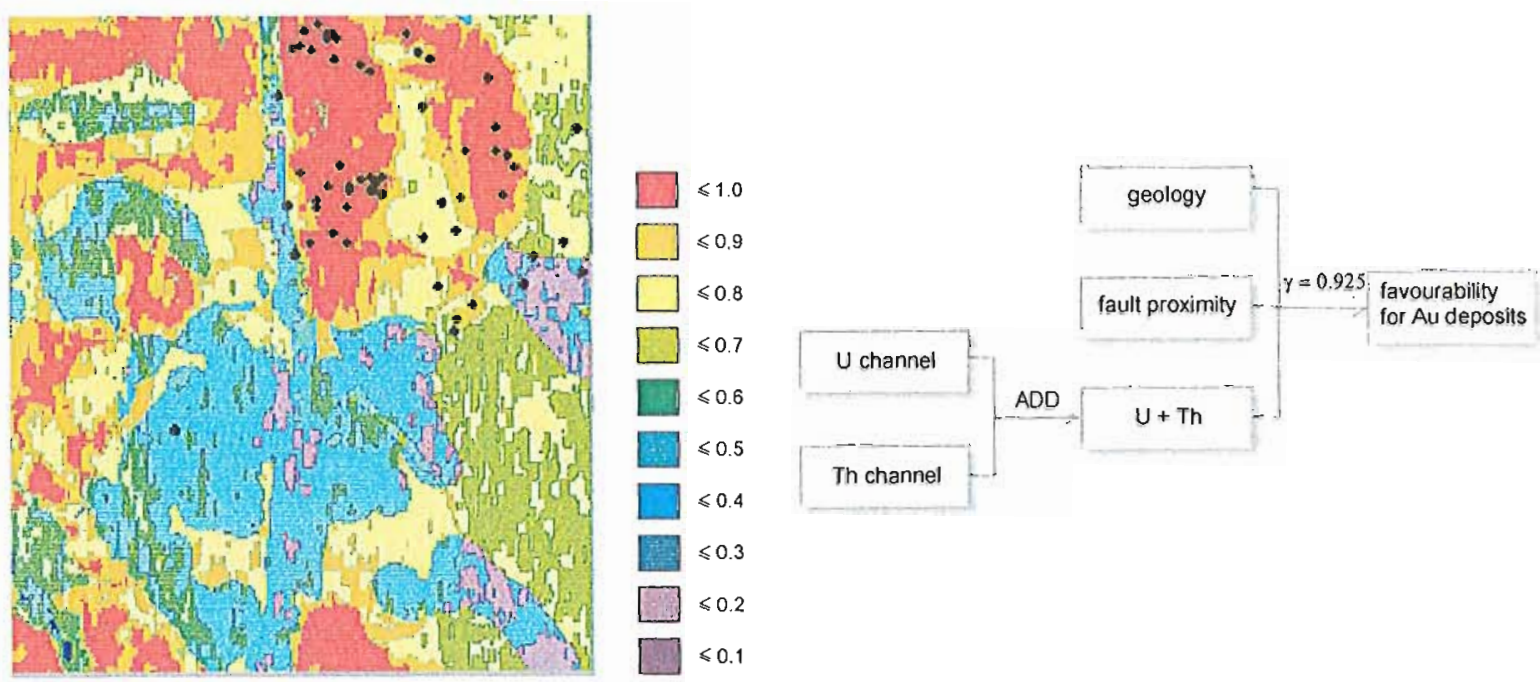

(a) $\gamma=0.925,4$ inputs. Fuzzy membership values are those given in Table 4.3 except for the distanceto-faults layer (class $<2000 \mathrm{~m}=0.50$ instead of 0.1 ).
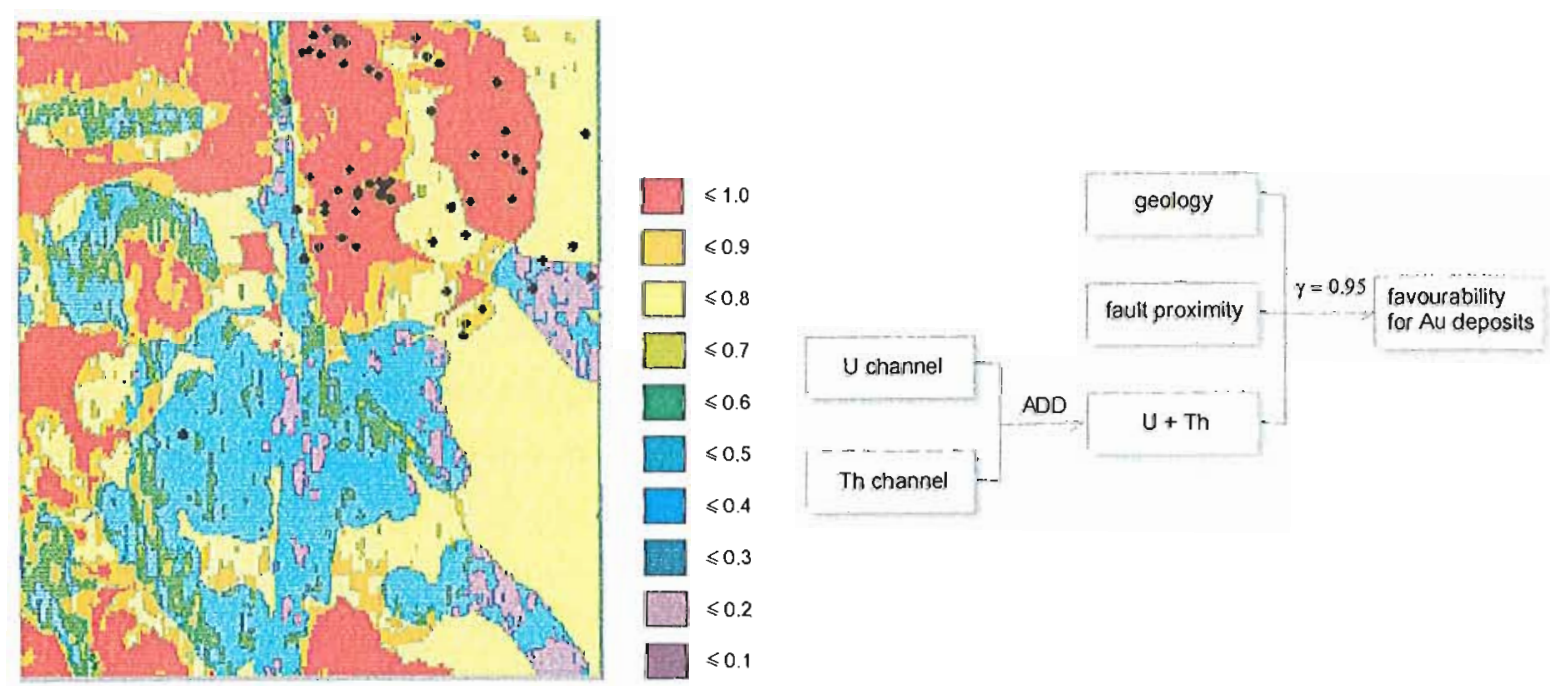

(b) $\gamma=0.95,4$ inputs. Fuzzy membership values are those given in Table 4.3 except for the distanceto-faults layer (class $<2000 \mathrm{~m}=0.50$ instead of 0.1 ).
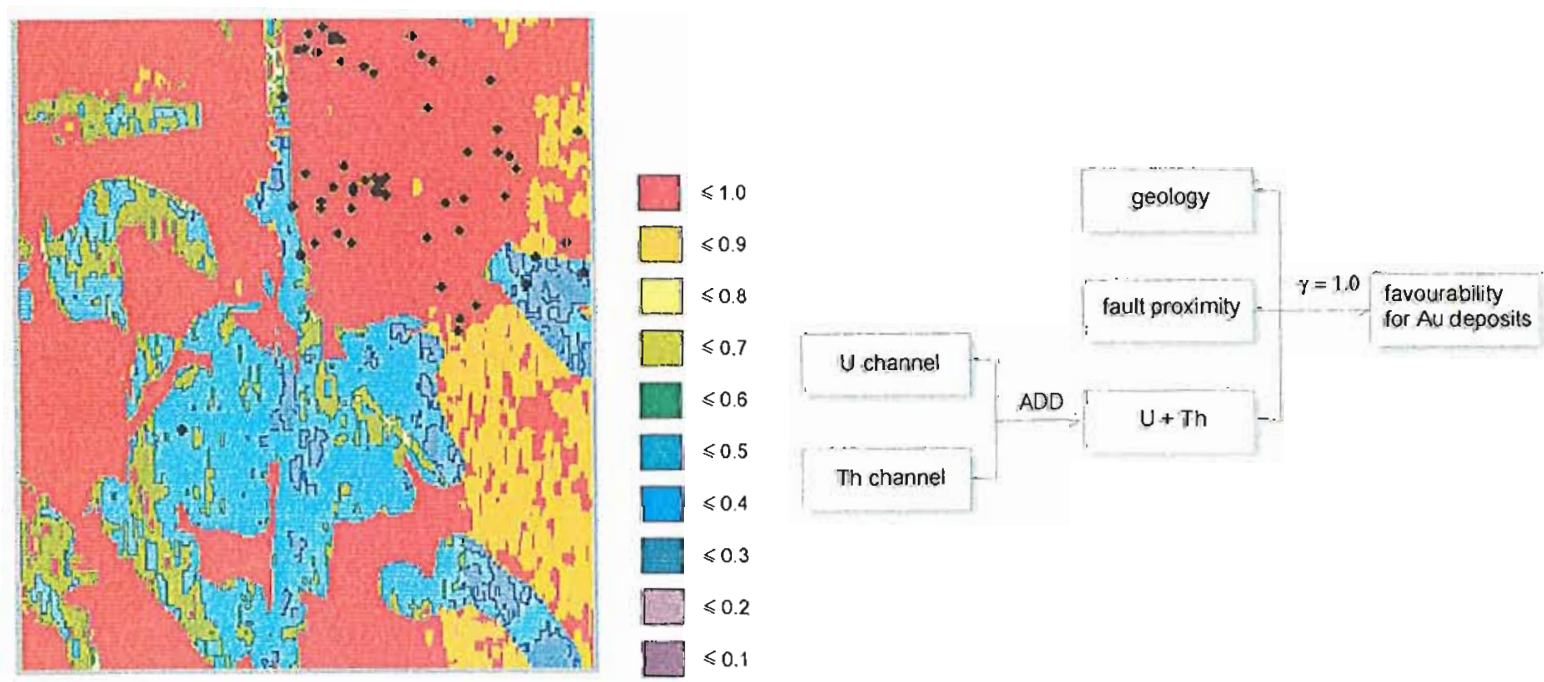

(c) $\gamma=1.0,4$ inputs. Fuzzy membership values are those given in Table 4.3 except for the distanceto-faults layer (class $<2000 \mathrm{~m}=0.50$ instead of 0.1 ).

Figure 4.10. Fuzzy mineral prospectivity maps for the Tenterfield 1:100000 map sheet area. 


\subsubsection{Comparing Mineral Prospectivity Map Quality}

Several statistical and probability measures were used to compare the quality of maps produced using the different methods. These are explained in Chapter 3.

The chi-squared statistic measures the extent to which observed and expected numbers of deposits differ. It is used here as a measure of the quality of a prospectivity map. Due to autocorrelation in spatial data and the fact that clustering of deposit cells means that deposits do not represent independent samples Bonham-Carter (1994) has recommended that the chi-squared statistic be used without reference to confidence limits.

For each class within a prospectivity map, the chi-squared statistic measures the degree to which the numbers of deposits differs from the number that would be expected if the distribution of the deposits through the different prospectivity map class areas were random. Due to the limitations of the chi-squared statistic explained in Section 3.3.1. Spearman's and Kendall's rank correlation coefficients are also used as measures of map quality. These statistics are very similar and are used to determine whether the probability of the occurrence of known deposits increases monotonically with increasing prospectivity map class. For both these statistics, a value of zero indicates that no correlation exists and a value of one indicates a perfect correlation (Stoodley et al., 1980; Harnett, 1982; Rock, 1988).

Conditional probabilities of the occurrence of a known mineral deposit, given the grid cell is located within a particular prospectivity class and the ratio of the conditional probability, $P\left(D / C_{k}\right) / P(D)$, of a deposit (given a prospectivity class $k$ ), to the probability of a deposit for the map area as a whole, were calculated. The probability ratio increases as the proportion of known deposits increases in comparison to the proportion of the total area occupied by the prospectivity class decreases. Consequently, the ratio should increase from a value smaller than one to a number greater than one with increasing map prospectivity class.

The fuzzy-logic and neural-network maps were reclassified from nine to four classes of favourability prior to calculating the probability-based statistics to allow a more direct comparison with the weights-of-evidence map, which, due to problems of conditional independence, contains only four prospectivity classes.

\subsection{Results}

Mineral prospectivity maps produced using the weights-of-evidence, fuzzy-logic and neural network methods are shown together with known gold occurrence and deposit points 
in Figure 4.11. Both the neural network and fuzzy-logic favourability results are classified into nine prospectivity classes. The weights-of-evidence map (Fig. 4.11a) displays only three colours corresponding to three discrete probability levels because input maps were combined to avoid problems of conditional dependence. Converting the values into classes gave a four-class prospectivity map. However, one of the classes (class 3 in Fig. 4.11 a and Table 4.6) does not correspond to any cells on the map.

Table 4.4 shows the percentages of correct classifications for training, training-stop and test sets for training runs using different random initial weights. Values over the threshold of 0.5 are classified as deposit vectors and those below 0.5 as non-deposit vectors. An estimate of how well the network performs at generalization is provided by the error rate for the classification of deposit and non-deposit vectors from the test data set. The trend of successively lower success rates for training, training-stop and test data sets shown in Table 4.4 is typical for back-propagation-trained MLP networks, and reflects the degree to which each of the data sets was used in training the network.

Values of the statistics used to quantify map quality are shown in Tables 4.5 and 4.6. The chi-squared statistic and Spearman's and Kendall's rank correlation coefficients are shown for each of the three prospectivity-mapping methods. Table 4.6 shows probabilitybased measures of map quality in which favourability values for the fuzzy-logic and neural network methods are reclassified from nine to four classes. Although no cells in the weights-of-evidence map correspond to class 3 , in order to calculate the statistics shown in Tables 4.5 and 4.6, it was assumed that just one cell value corresponds to this class. The upper part of Table 4.6 shows the conditional probability that a cell contains a known deposit, given that the area of interest is restricted to a particular prospectivity class. The lower part of Table 4.6 shows the ratio of conditional probability for a given map class area to the prior probability for the study area as a whole. 
Table 4.4. Classification accuracy for deposit and non-deposit vectors in training, training-stop and test data sets. ${ }^{\mathrm{a}}$ A series of networks were trained using ten different sets of random initial weights. Training was repeated, in some cases, using the same set of initial weights, but different network parameters. The weight sets are labelled numerically and repeated training runs are labelled alphabetically.

\begin{tabular}{|c|c|c|c|c|c|c|}
\hline \multirow{2}{*}{$\begin{array}{l}\text { Training } \\
\text { run }\end{array}$} & \multirow{2}{*}{$\begin{array}{l}\text { Weight } \\
\text { update }\end{array}$} & \multirow[t]{2}{*}{ Momentum } & \multirow{2}{*}{$\begin{array}{l}\text { Training } \\
\text { cycles }\end{array}$} & \multicolumn{3}{|c|}{ Classification results (\% correct) } \\
\hline & & & & Training & $\begin{array}{l}\text { Training- } \\
\text { stop }\end{array}$ & Test \\
\hline $1 \mathrm{a}$ & batch & 0.0 & 206 & 83 & 76 & 71 \\
\hline $1 b$ & sequential & 0.0 & 1536 & 93 & 83 & 74 \\
\hline $1 c$ & sequential & $0.5 \rightarrow 0.0^{\mathrm{c}}$ & 700 & 95 & 81 & 74 \\
\hline 2 & sequential & 0.0 & 700 & 93 & 81 & 74 \\
\hline 3 & sequential & $0.7 \rightarrow 0.0^{\mathrm{c}}$ & 4278 & 95 & 79 & 76 \\
\hline 4 & sequential & 0.0 & 65 & 79 & 74 & 74 \\
\hline $5 \mathrm{a}$ & sequential & 0.0 & 300 & 90 & 81 & 76 \\
\hline $5 \mathrm{~b}$ & sequential & 0.5 & 195 & 90 & 81 & 74 \\
\hline $5 c$ & batch & 0.3 & 104 & 83 & 79 & 74 \\
\hline 6 & batch & 0.3 & 250 & 90 & 79 & 76 \\
\hline $7 \mathrm{a}$ & batch & 0.3 & 28 & 79 & 74 & 71 \\
\hline $7 \mathrm{~b}$ & sequential & 0.3 & 39 & 79 & 74 & 71 \\
\hline $8 \mathrm{a}$ & batch & 0.3 & 1186 & 95 & 83 & 74 \\
\hline $8 \mathrm{~b}$ & batch & $0.3 \rightarrow 0.0^{\mathrm{c}}$ & 1704 & 95 & 79 & 74 \\
\hline $8 c$ & sequential & 0.3 & 2050 & 95 & 79 & 74 \\
\hline $9 a^{d}$ & sequential & 0.3 & 817 & 93 & 79 & 71 \\
\hline $9 \mathrm{~b}$ & sequential & 0.0 & 400 & 90 & 79 & 74 \\
\hline $10 a^{e}$ & sequential & 0.0 & 33 & 84 & 79 & 84 \\
\hline $10 b^{f}$ & sequential & 0.0 & 200 & 79 & 77 & 79 \\
\hline
\end{tabular}

${ }^{a} \mathrm{~A}$ threshold of 0.5 was applied to the network output values in order to make a classification. Inputs corresponding to an output below 0.5 were classified as non-deposits; otherwise the vector was classified as a deposit.

${ }^{b}$ batch $=$ weights update after each training set cycle, sequential $=$ after each pattern

${ }^{c}$ Momentum was reduced during training within the range shown.

${ }^{d}$ Stopped training before minimum TSS error for test data set was reached.

${ }^{e}$ This network was used to process vectors from the entire Tenterfield map grid.

${ }^{f}$ Eight deposit and eight non-deposit training patterns taken from the test data set and added to the training data set. 


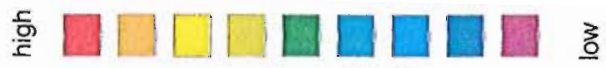
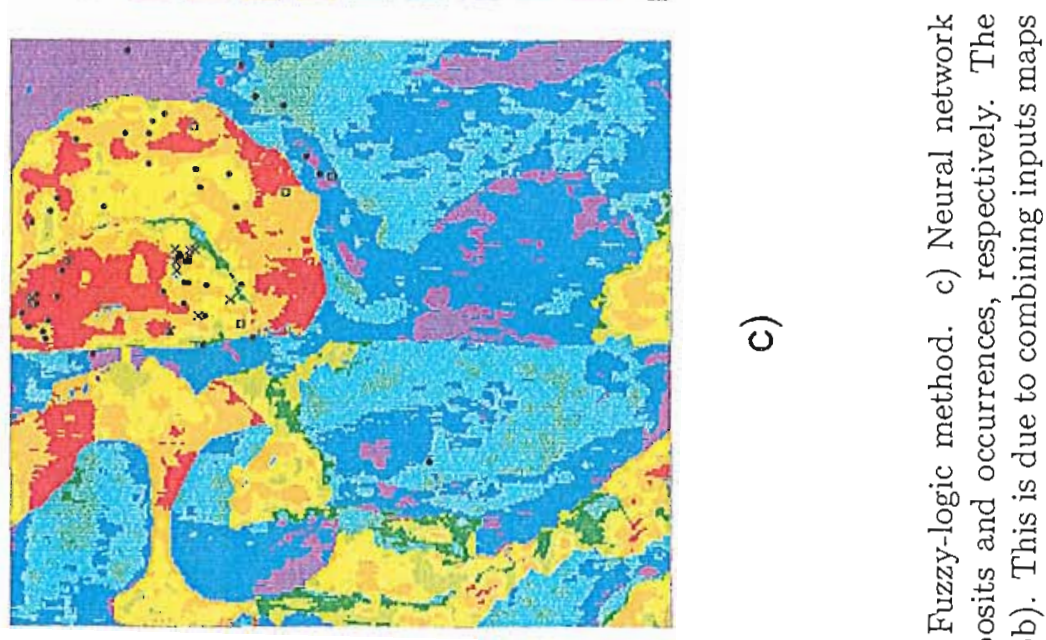

.

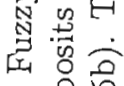

วิ

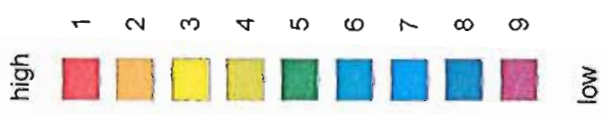

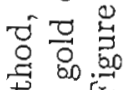

实

兽逸
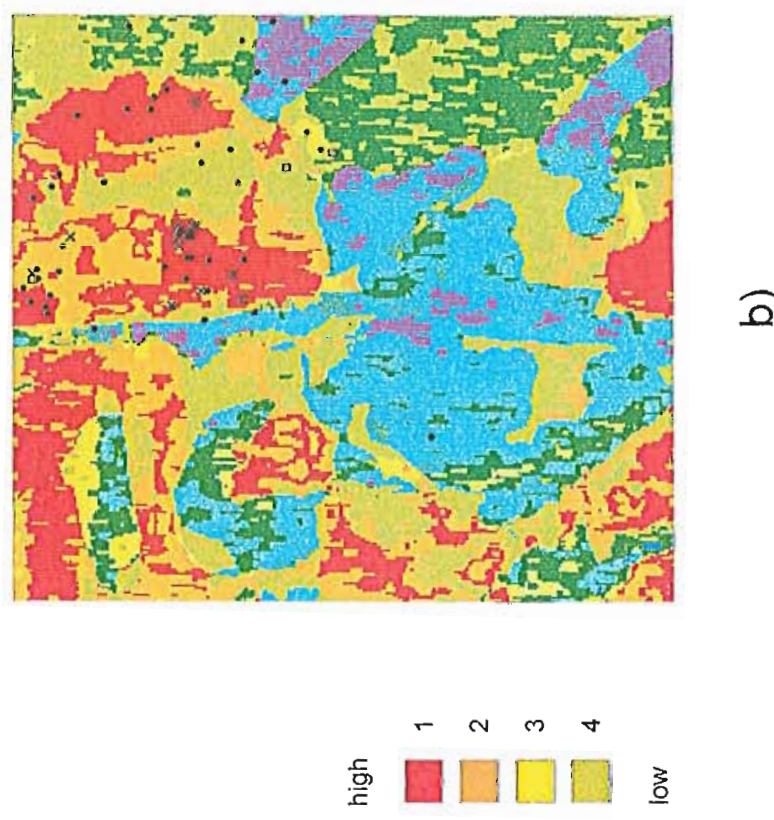

อ

若芯

峲灾

岁鸹密

喜哮

敢总总

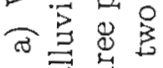

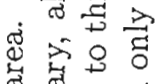

$\infty$ b

8 影

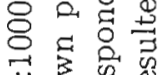

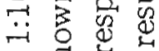

믐ㄷㅇ용

密若品

봉웡ㅇㅇㅇ

\& 8

类

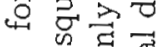

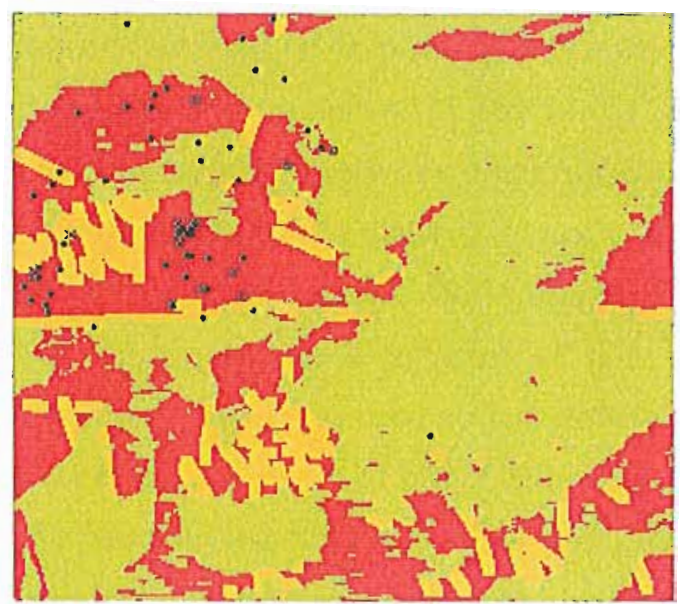

ํํㅇำ

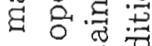

密马 范

矛 की

ชั

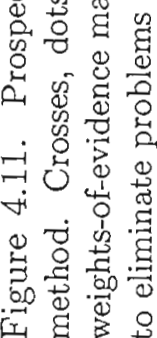


Table 4.5. Statistical measures of map quality. Chi-squared statistic, and Spearman's and Kendall's rank correlation coefficients, for prospectivity maps produced using different methods

\begin{tabular}{|c||c|c|c|}
\hline Statistic & Wts of Evidence & Fuzzy Logic & Neural Network \\
\hline \hline Chi-squared & 66 & 63 & 83 \\
\hline Spearman's $\rho$ & 0.80 & 0.50 & 0.93 \\
\hline Kendall's $\tau$ & 0.67 & 0.53 & 0.83 \\
\hline
\end{tabular}

\subsection{Discussion}

\subsubsection{Validation of the Neural Network Results}

The close similarity of the neural-network map to the fuzzy-logic map, and the broad similarity to the weights-of-evidence map, indicates that the neural network method produces geologically meaningful results. The similarity of the neural network and conceptuallybased fuzzy-logic maps indicates that the neural network result conforms to current understanding of geological factors important for gold mineralization in the Tenterfield area. The broad similarity between the neural network and empirically-derived weights-of-evidence maps indicates that the neural network result accounts for observed spatial relationships between known gold deposits and the parameters of the GIS database.

\subsubsection{Interpretation of Output Values}

The output of the neural network is suitable for producing multiclass prospectivity maps. Although the network was trained as a classifier using binary target values $(0=$ deposit absent and 1 = deposit present), it is possible to produce a multiclass map because the trained network produces output values in the range $[0.1,0.9]$. Rescaled to the range $[0,1]$, the network output approximates the posterior conditional probability $p\left(C_{\text {deposit }} \mid \mathbf{x}\right)$ that the input vector $\mathrm{x}$ belongs to the class of deposits $C_{\text {deposit }}$ given that the input vector is $\mathbf{x}$ (White, 1989; Richard \& Lippmann, 1991; Bishop, 1995). The output of the trained network can also be interpreted as a measure of how similar the input vector is to a composite of all deposit vectors presented to the network during training. The degree of similarity can also serve as a measure of how favourable the area is for mineral deposits of the type included in the training data set. This interpretation of the network output fits with the intuitive notion that a prospective area has characteristics that closely resemble 
Table 4.6. Probability measures of map quality for prospectivity maps produced using different methods

\begin{tabular}{|c||c|c|c|}
\hline Map Class & Wts of Evidence & Fuzzy Logic $^{\mathrm{a}}$ & Neural Network $^{\mathrm{a}}$ \\
\hline \hline$P\left(D / C_{k}\right) \times 10^{4}$ & & & \\
4 & 2.53 & 2.85 & 2.60 \\
\hline 3 & 0.00 & 1.65 & 2.15 \\
\hline 2 & 8.67 & 6.89 & 11.66 \\
\hline 1 & 23.45 & 18.91 & 26.74 \\
\hline \hline$P\left(D / C_{k}\right) / P(D)$ & & & 0.29 \\
\hline 4 & 0.28 & 0.31 & 0.24 \\
\hline 3 & 0.00 & 0.18 & 1.29 \\
\hline 2 & 0.96 & 0.76 & 2.95 \\
\hline 1 & 2.58 & 2.08 & \\
\hline
\end{tabular}

\footnotetext{
${ }^{a}$ The fuzzy-logic and neural-network maps were reclassified from nine to four classes to enable a direct comparison with the weights-of-evidence map.
}

areas known to contain mineral deposits. Since the training data comprise both deposits and mineral occurrences, what is being estimated here is favourability for mineralization rather than for deposits. In order to analyse these two groups separately, a GIS database with larger numbers of both deposits and occurrences would be required.

\subsubsection{Response to Critical Combinations of Favourable Parameters}

The main difference between the prospectivity maps is that the neural network contains much-smaller high-prospectivity areas (classes 1 and 2 in Fig. 4.11c) compared to the other two maps (Fig. 4.11a \& b). This is interpreted to be due to the ability of the neural network to respond in a highly non-linear way to combinations of input parameters, so that a high favourability value is only assigned to areas where a combination of the critical favourable parameters is present. This contrasts with the weights-of-evidence and fuzzy-logic methods, where, for a given grid location, each favourable input-parameter value automatically results in an increase in the prospectivity estimate. For example, if three parameters are favourable for mineralization and one parameter is essential for mineralization, then, for an area where only the three favourable parameters are present, the overall favourability estimated by the weights-of-evidence and fuzzy-logic methods is 

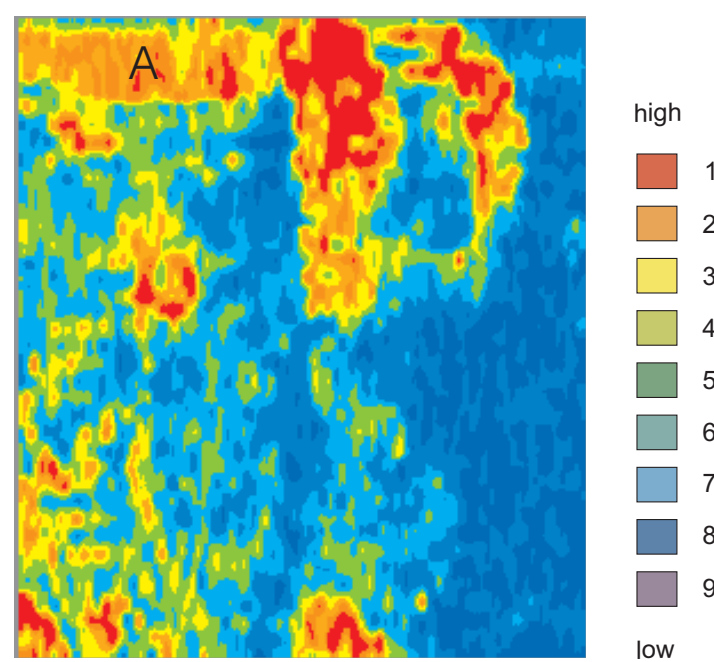

(a) Airborne gamma-ray survey, Th-channel.

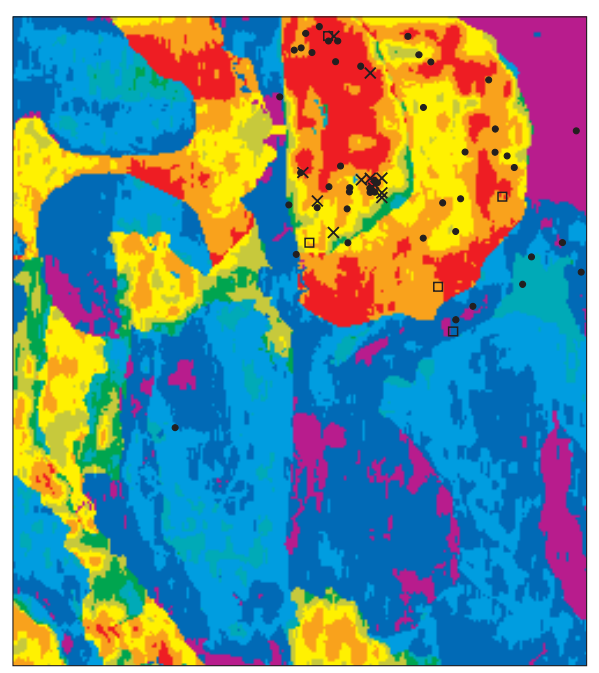

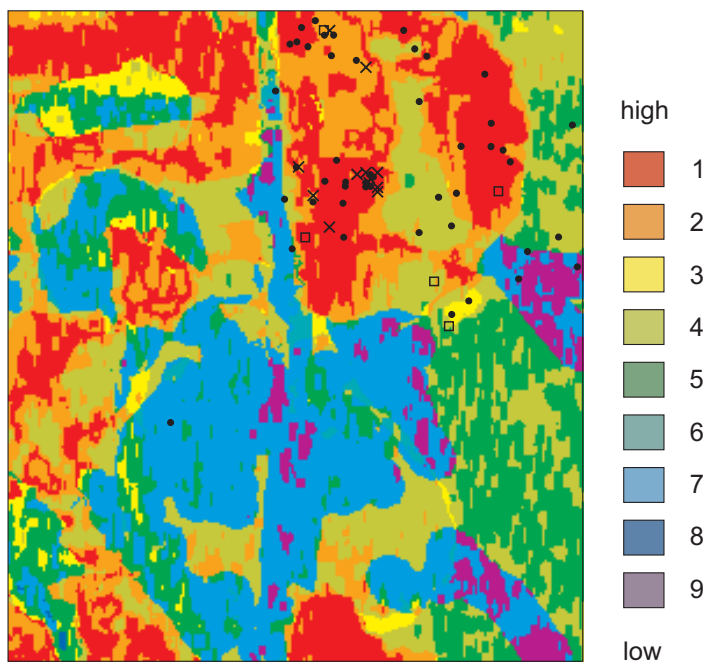

(b) Fuzzy-logic mineral prospectivity map.

Figure 4.12. Contrasting response of the fuzzy-logic and neural network methods to input maps containing highly correlated or redundant data. Gold deposit types: crosses $=$ primary, dots $=$ alluvial and squares $=$ undifferentiated. The high prospectivity band in the north-eastern part of the fuzzy-logic map (b) is due to the U- and Th-channel airborne gamma-ray input maps, which contain high counts in the area labelled A. The fuzzylogic method is sensitive to inputs containing similar or redundant information. The high prospectivity band is absent in the neuralnetwork map because the neural network method is quite robust with respect to redundant inputs.

(c) Neural network mineral prospectivity map for the Tenterfield 1:100000 map sheet area.

high. The estimated favourability would be high despite the absence of the one essential parameter. The fuzzy-logic method is quite sensitive to data layers that are strongly correlated; that is, contain similar information and produce spuriously-high favourability estimates. Such data are, however, common in data sets used for geological exploration, particularly those involving geochemistry. 


\subsubsection{Response to Redundant Input Parameters}

The broad high prospectivity band in the north-eastern part of the fuzzy-logic map appears to be an example of the additive character of the fuzzy-logic method for combining evidence maps (red and orange area in Fig. 4.12b). The high favourability estimate is the result of high radiometric responses in all channels of the gamma-ray survey data (Fig. 4.12a).

This area is not highlighted in the neural-network map (Fig. 4.12c), where both a favourable leucogranite host and a high radiometric response are required to produce a high favourability estimate. The high prospectivity band doesn't appear in the weights-of-evidence map (Fig. 4.11a) because gamma-ray survey map layers, which contain very similar information, violate the assumption of conditional independence and were therefore converted to a single input layer. The uranium and thorium channels were combined and the potassium and total count channels were discarded.

A neural-network approach overcomes problems related to the assumption of conditional independence in the weights-of-evidence method. To deal with conditional dependence, dependent data sets must either be rejected or combined in some way. However, Boolean and multiple regression methods of combining data sets reduce multiple parameters to a single parameter and therefore result in a loss of information. However, Masters (1993b) warns that, although the first few principal components may explain the majority of the variation in the data, the information that is important for the classification may reside in the last principal component.

\subsubsection{Loss of Information through Re-Classification of Input Data}

The neural network method allows data sets to be combined without the loss of information inherent in the weights-of-evidence and some implementations of the fuzzy-logic method. Although the weights-of-evidence method can be used with multiclass maps (Bonham-Carter et al., 1988, 1998a,b; Bonham-Carter \& Agterberg, 1999), in many studies multiclass evidence maps are converted to a binary format prior to integration (e.g. Watson:1990;Raines:1999;Venkataraman:2000;Harris:2001;Scott:2001). Values below the threshold used to reclassify multiclass maps to binary maps play no role in determining prospectivity, and values above the threshold (included in the binary evidence map) are treated as though they are equal because they are assigned the same weight. Thus, information available in the raw data, which might otherwise have contributed to the prospectivity estimate, may be lost prior to integration. 
The fuzzy-logic method overcomes the problem of applying a fixed cut-off value by assigning fuzzy membership values to raw data on a continuous scale from zero to one. However, loss of information also occurs with the fuzzy-logic method if single fuzzy-membership values are applied to a whole class or range of values, as in the case of studies by An et al. (1991), Eddy et al. (1995), Donaldson (1996), D'Ercole (1998) and in this pilot study. In contrast to the weights-of-evidence, and most implementations of the fuzzy-logic method, the neural network method does not require ratio data to be classed prior to combining data sets.

\subsubsection{Response to Irrelevant or Misleading Input Parameters}

Inconsistent or misleading data are unlikely to represent as serious a problem for neural networks as for statistical methods, such as the weights-of-evidence methods and combinations of statistical and fuzzy-logic methods. The GIS thematic layer containing regional faults and lineaments is an example of a possibly spurious data set (Fig 4.13a). An examination of the digital elevation model (DEM) for the Tenterfield area reveals many major lineaments not present in the structural layer. Thus, the structural map represents incomplete information. The weak inverse relationship between deposit points and faults that emerged from the statistical analysis performed in the weights-of-evidence method is likely to be misleading. Subjective judgement was used in estimating the cut-off value used to convert the multiclass map showing distances to the nearest fault, to a binary evidence map.

The effect of this input on the estimated prospectivity is clearly illustrated in the weightsof-evidence prospectivity map in the form of elongate zones of moderate prospectivity throughout the map (Fig 4.13b). In contrast, the neural network prospectivity map does not show any evidence that major faults have played an important role in determining prospectivity (Fig 4.13c). A property of neural networks is the ability to respond to spurious input data by adjusting the weights connected to the relevant input to very low values, and thereby reducing or eliminating the contribution made by those data to the output. 


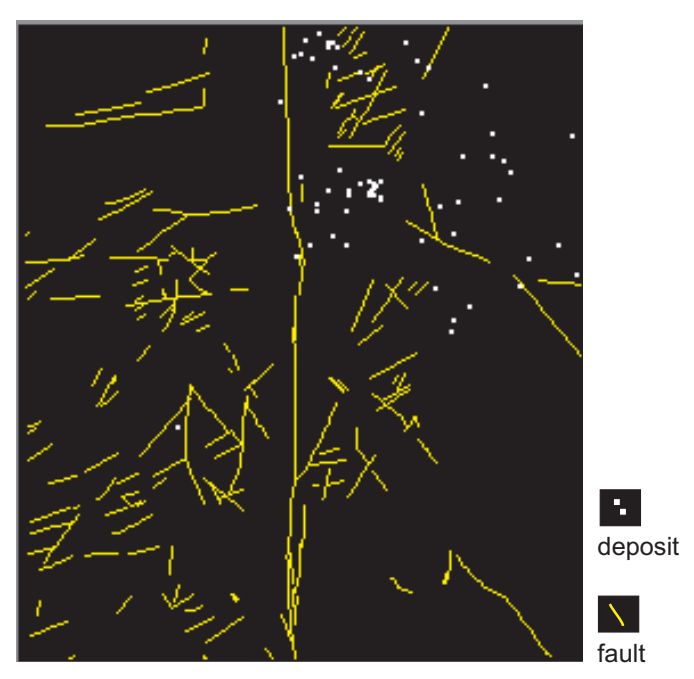

(a) Regional-scale faults and shear zones.
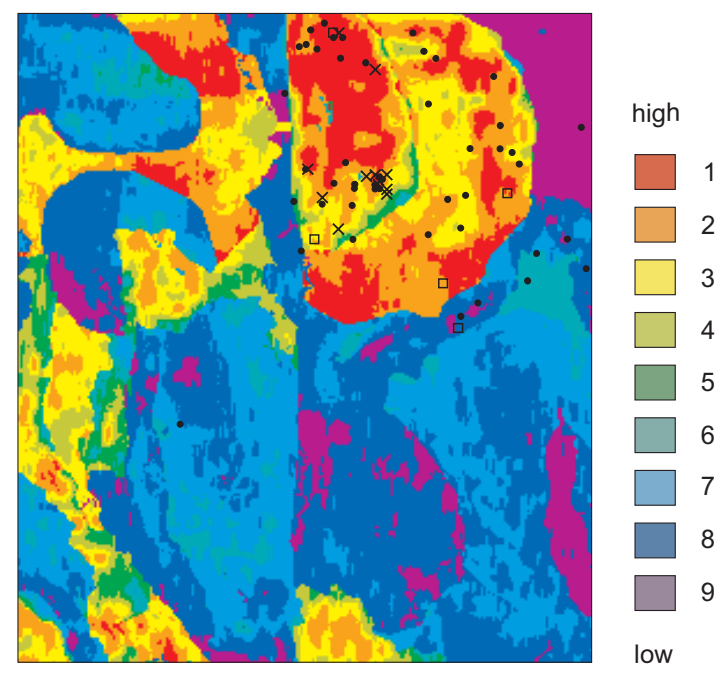

(c) Neural network mineral prospectivity map for the Tenterfield 1:100000 map sheet area.

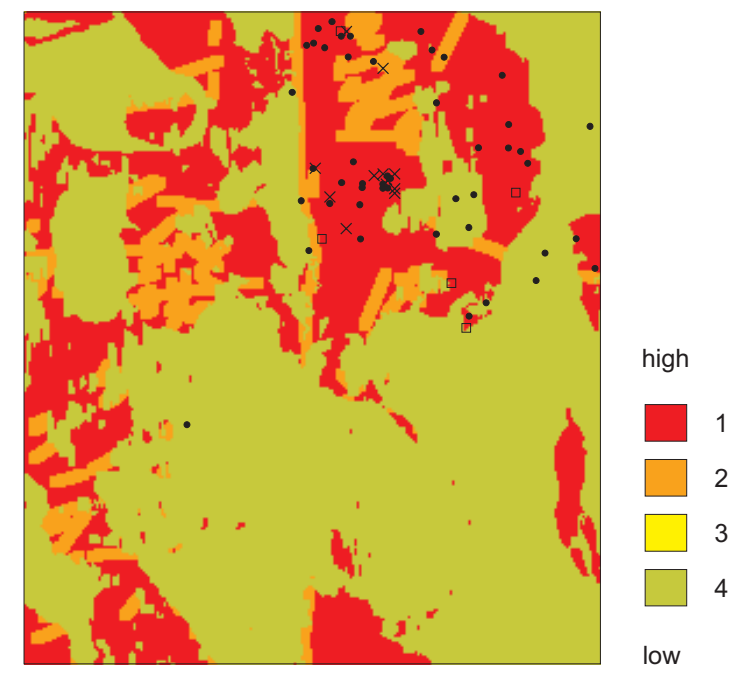

(b) Weights-of-evidence mineral prospectivity map.

Figure 4.13. Contrasting response of the WofE and neural network methods to input maps containing spurious or irrelevant data. Gold deposit types: crosses $=$ primary, dots $=$ alluvial and squares $=$ undifferentiated. The weak inverse relationship between faults and deposit points apparent in a) is misleading because the structural map does not include many of the faults that are apparent on a recent high resolution DEM produced by the NSW Geological Survey. Note that the WofE map reflects the effect of the structural input map in the form of orange bands where the prospectivity has been significantly downgraded due to proximity to faults. In contrast, the neural-network map does not show any evidence that proximity to faults has played an significant role in determining mineral prospectivity.

\subsubsection{Comparison of Map Quality}

The statistical measures of prospectivity map quality (Table 4.5) are all highest for the neural-network map. The chi-squared statistic indicates greater differences between number of deposits observed in the class areas of the neural-network map than those that would be expected if the class areas were located at random, compared with maps produced by the fuzzy-logic and weights-of-evidence methods. The larger values of both Spearman's and Kendall's rank correlation coefficients show that there is a stronger correlation be- 
tween map class and probability of a cell containing a known deposit than for the other two methods.

The probability and probability ratio measures of map quality (Table 4.6) indicate that the highest prospectivity map classes in the neural-network map are stronger predictors of deposits than the highest prospectivity map classes in the fuzzy-logic map, and slightly stronger than those in the weights-of-evidence map. As noted above, in an ideal prospectivity map, the ratio $P\left(D / C_{k}\right) / P(D)$ should be greater than one in the high prospectivity class, approximately equal to one for the middle prospectivity class, and considerably less than one for the low prospectivity class (where $C_{k}$ represents map class $k$; Brown et al., 2000b,a). Mihalasky \& Bonham-Carter (2001) interpret a normalized density measure, $\rho$ for lithodiversity classes in a similar way. However, since the ranges for negative and positive spatial associations have unequal ranges $([0,1]$ and $[1,+\infty]$, respectively) they use the natural logarithm of $\rho$ so that negative and positive spatial associations are symmetrical about the null value of $\ln \rho=0$.

The prospectivity map produced using the neural network method is based on approximately two thirds of the data that are used in the weights-of-evidence method. Approximately one third of the available data were reserved as a test data set in the neural network method, and used to check the generalization performance of the network after it had been trained. Only one third of the available data, the training data set, were used directly in training the network and a further third, the test data set, were used indirectly. The test data set played no role in training. In contrast, all of the available deposit points were used in the weights-of-evidence method, as there were an insufficient number of deposit and occurrence points available to allow a third of the data to be reserved as a test data set.

One important issue that has not been addressed here is the uncertainty associated with the prospectivity map. Bonham-Carter has noted that one of the advantages of the weightsof-evidence method over other methods such as logistic regression is that uncertainty can be estimated using the; 1) variance due to the weights (usually controlled by the number of deposits), 2) variance due to missing data (Agterberg et al., 1990) and 3) variance such as kriging variance and other sources of error in the explanatory variables (pers. comm.;Bonham-Carter \& Agterberg, 1999). To calculate the variances due to the weights, Bonham-Carter (1994); Bonham-Carter \& Agterberg (1999) use the expressions from Bishop et al. (1975): 


$$
\begin{gathered}
\rho^{2}\left(W^{+}\right)=\frac{1}{B_{j} \cap D}+\frac{1}{B_{j} \cap \text { barD }} \text { and } \\
\rho^{2}\left(W^{-}\right)=\frac{1}{b a r B_{j} \cap D}+\frac{1}{b a r B_{j} \cap \operatorname{bar} D} \text { and }
\end{gathered}
$$

which assume that the number of deposit cells is large. The uncertainty at each cell is given by Bonham-Carter et al. (1989); Bonham-Carter \& Agterberg (1990) as:

$$
\rho^{2}\left(P_{\text {post }}\right)=\left[\frac{1}{b a r D}+\sum_{j=1}^{m} \rho^{2}\left(W_{j}^{k}\right)\right] * P_{\text {post }}^{2}
$$

where $1 / D$ is the variance of the prior odds $O(D)$ (based on the assumption that the prior probability of a deposit is given by $D / T)$, D is the number of deposit cells, $\mathrm{T}$ is the total number of cells, and $B_{j}$ is the number of cells with the favourable pattern present in the $j$-th binary input map. Bars indicate that the favourable or deposit patterns are absent. Bonham-Carter \& Agterberg (1999) has also pointed out that, because standard deviation of the posterior logit is correlated with the logit magnitude, the studentized ratio of the posterior logit, $\ln \left(O_{p} o s t\right) / s\left(\ln \left(O_{p} o s t\right)\right)$, is a better measure of uncertainty than the standard deviation.

Although these methods can not be applied to prospectivity estimates made with neural networks, a Monte Carlo simulation could be applied to obtain uncertainty estimates (Section 8.3.5, page 396).

\subsection{Conclusion}

A neural-network approach, using a simple implementation of the most commonly-used neural network architecture (i.e. the multilayer perceptron), is successful in integrating geological and geophysical data sets, representative of those used in a regional exploration program, to produce a mineral prospectivity map. The neural-network map is consistent with both the spatial relationships in the data and with geological knowledge about factors important for gold mineralization in the Tenterfield area. The results of this study indicate that the neural network method has several advantages over existing methods. These include the ability to: 
1. respond to critical combinations of parameters, rather than automatically increasing the prospectivity due to all favourable parameters,

2. combine data sets without the loss of information inherent in existing methods, and

3. produce results that are relatively unaffected by redundant data, spurious data and data containing multiple populations.

Statistical measures used to compare map quality indicate that the neural network method performs as well, or better, than existing methods while using approximately a third less data than the weights-of-evidence method.

As noted in Section 3.5.1, the Tenterfield database is not ideal for a detailed investigation. A larger more detailed and recently compiled database, which is being used for exploration for Archaean orogenic lode-gold deposits around Kalgoorlie is used for all further investigations in the methodology part of the thesis. The advantage of this database is that it is representative of geoscience GIS databases used in modern, large-scale regional exploration programs. 


\section{Chapter 5}

\section{Compilation of Case Study GIS Database: Orogenic Gold Deposits, Kalgoorlie Area, Western Australia}

\subsection{Introduction}

This chapter describes the layers in a GIS database for Archaean orogenic lode-gold deposits in the Kalgoorlie area of Western Australia. It is used in subsequent chapters as the basis for experiments on a neural-network approach to mineral prospectivity mapping. All the layers referred to are included on the attached CD-ROM (vol.2) in both the ARCVIEW and MATLAB directories ${ }^{1}$. Layers that are used as inputs to neural networks in experiments in Chapter 7 are shown in Figures 5.7-5.35 at the end of the chapter.

Although the results of Chapter 4 illustrate the effectiveness of Multi-Layer Perceptron (MLP) neural networks for combining exploration data into prospectivity maps and highlight several advantages of neural networks over the weights-of-evidence and a simple fuzzy-systems method, the Kalgoorlie data set can provide a more realistic test of a neural network method because the controls on the location of Archaean lode-gold deposits

\footnotetext{
${ }^{1}$ ArCVIEW layer names differ from those in MATLAB in some cases due to the restriction on file-name length in ARCVIEW. In all cases, however, the number (1-46) in the first part of the name is the same in both ArCView and Matlab directories
} 
are better understood and possibly more complex than those for intrusion-related disseminated gold deposits in the Tenterfield area. For example, the location of lode-gold deposits in the Kalgoorlie area depends on a complex combination of structural and host-rock rheology and geochemistry, whereas gold deposits in the Tenterfield are largely restricted to two main lithological units, which represent the most highly-differentiated intrusions and can be discriminated on the basis of the high airborne radiometric responses in the uranium, thorium and total-count channels. In contrast, the Kalgoorlie area contains gold deposits that formed at a wide range of crustal depths $(\sim 5->15 \mathrm{~km})$, with correspondingly varied metamorphic grades, structural styles, host rocks and mineralogy (Groves, 1993; Groves et al., 1998). It is therefore likely that there are wide variations in the data that characterize deposits and that multiple deposit populations exist within the multi-dimensional data.

The Kalgoorlie data set was chosen because;

1. the data set was recently compiled (i.e. 2000-2001),

2. it is in current use in exploration by Placer Dome,

3. it is based on a single recent high-resolution airborne geophysical survey (flight-line spacing: $200 \mathrm{~m}$ ) that covers the entire study area,

4. the data set consists of a variety of data types (geology, structure, airborne magnetic, radiometric drill-hole geochemistry, and Bouguer gravity anomaly maps),

5. the Kalgoorlie area has been well explored and has been well mapped,

6. the solid geology and structural geology maps, the primary sources for many of the derived GIS layers, were produced by interpretation of airborne magnetic survey maps and existing geological maps by an experienced geologist (Dr Scott Halley, Placer Dome) so that the interpretation is uniform and the geological legend is consistent across the study area, and

7. the area contains a large number of known gold deposits available for training neural networks.

The Kalgoorlie study area covers an approximately $100 \times 100 \mathrm{~km}$ area located in the Norseman-Wiluna greenstone belt of the Yilgarn Block, Western Australia (Fig. 5.1). Metadata for the GIS data are given in Appendix D. The Kalgoorlie data set is typical of the sort of geoscience data sets used by companies in modern exploration programs and provides a good test of the general applicability of the neural-network approach to mineral prospectivity mapping. 


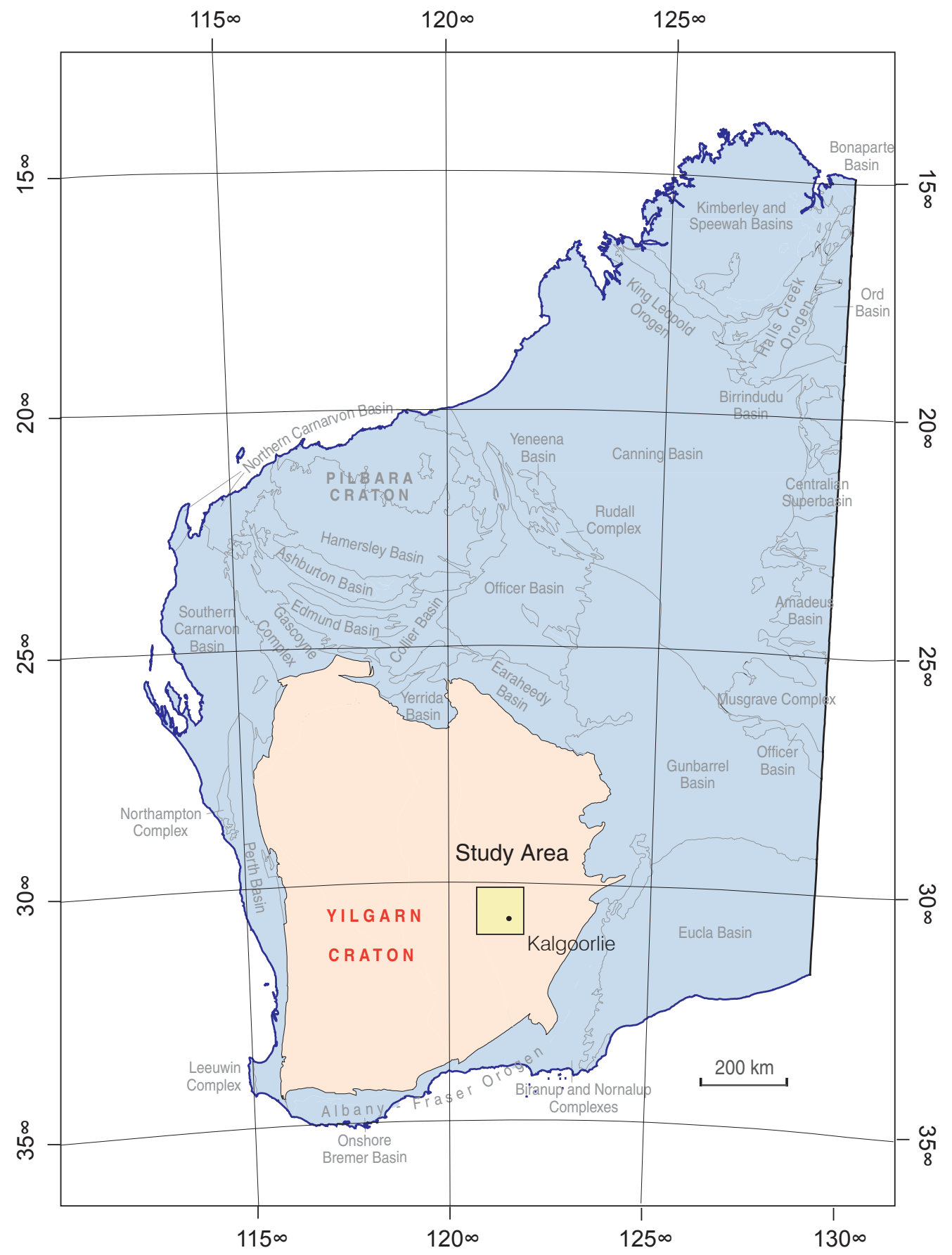

Figure 5.1. Location of the Kalgoorlie case study area. The data set covers an approximately $110 \times 110 \mathrm{~km}$ area within Norseman-Wiluna greenstone belt of the Yilgarn Block near Kalgoorlie, Western Australia. The northern margin of the area is about $25 \mathrm{~km}$ north of Bardoc and the southern margin is located approximately $12 \mathrm{~km}$ south of Coolgardie. The area corresponds to the eastern half of the Kalgoorlie 1:250000 geological map sheet (SH51-9), with a small proportion of the data set extending into the Kurnalpi, Boorabbin and Widgiemooltha 1:250000 sheet areas (SH51-10, SH51-13, \& SH51-14). 


\subsection{Geology of Archaean Orogenic Gold Deposits}

The purpose of this section is to describe the main features of orogenic lode-gold deposits, particulary those in the Kalgoorlie area of the Norseman-Wiluna belt of the Yilgarn Block, Western Australia, and to summarize the essential features of the deposit model relevant to exploration on a regional scale. In the following section, these features are translated into exploration criteria that are likely to be captured in the sort of data that are contained in a regional-scale GIS database or which can be obtained from the primary GIS data layers and incorporated explicitly as a derived layer.

\subsubsection{Deposit Characteristics}

Orogenic lode-gold deposits are a widespread epigenetic type of gold deposit, which form structurally-hosted quartz-dominant vein systems and are located in Archaean greenstonegranitoid and Phanerozoic sedimentary-rock dominated (e.g. slate) belts within deformed metamorphic terranes (Groves et al., 2000). Although orogenic lode-gold deposits can be hosted in a large variety of rocks and their structural style, alteration, ore mineralogy, and metamorphic grade is also variable, they are now recognized as a coherent group with a common origin (Groves et al., 1995, 2000), in which the deposit characteristics vary due to the large range of crustal depths and temperatures over which the deposits can form (i.e. $180^{\circ} \mathrm{C}$ at $2-5 \mathrm{~km}$ depth to $>700^{\circ} \mathrm{C}$ at $15-20 \mathrm{~km}$ depth; the crustal continuum model; Groves et al., 1992; Groves, 1993).

Gold mineralization occurs in quartz vein systems, which in greenschist-facies host rocks contain $\leq 3-5 \%$ sulphides and $\leq 5-15 \%$ carbonates. Arsenopyrite is the most common sulphide in metasedimentary country rocks and pyrite and pyrrhotite predominate in igneous host rocks. In addition to the quartz veins, gold can be located in sulphidized wall rocks. Gold:silver ratios are normally about 10 but range to less than 1 .

Lateral alteration zoning is on the scale of centimetres to tens of metres. At greenschist facies, alteration zones are enriched in $\mathrm{CO}_{2}$, S, K, $\mathrm{H}_{2} \mathrm{O}, \mathrm{SiO}_{2}, \pm \mathrm{Na}$ and LILE, leading to the development of carbonates (ankerite, dolomite, or calcite) alkali metasomatism (sericitization, fuchsite, biotite, K-feldspar), and albitization. Mafic minerals are highly chloritized.

Vein-forming fluids in orogenic gold deposits are low salinity $\mathrm{H}_{2} \mathrm{O}-\mathrm{CO}_{2} \pm \mathrm{CH}_{4} \pm \mathrm{N}_{2}$ fluids with a high $\mathrm{CO}_{2}$ content and minor $\mathrm{H}_{2} \mathrm{~S}$ content. The high $\mathrm{CO}_{2}$ content is responsible for widespread carbonate alteration zones and the low chloride $(<\sim 6 \mathrm{wt} \%$ equiv. $\mathrm{NaCl})$ 
content is responsible for high $\mathrm{Au}: \mathrm{Ag}$ ratios and a lack of base metal enrichment and low Sn content in orogenic deposits. Although O- and H-isotope work indicates a deep crustal source for the fluids, it is not known if the fluids are metamorphic, magmatic or from a mantle-fluid source (Groves et al., 2000).

\subsubsection{Tectonic Setting}

Orogenic lode-gold deposits are associated with either plate subduction below continental crust (accretionary terranes) or the collision of terrains on to older blocks of continental crust (collisional terranes). An important aspect of the tectonic setting is association of orogenic deposits with large thermal anomalies due to thrusting of the crust due to thickening of relatively radiogenic crustal material in the orogen core or to upwelling of the asthenosphere (Goldfarb et al., 2001). Tectonic setting and depth of formation distinguish them from other gold deposit types. For example, epithermal-vein, gold-rich porphyry and skarn deposits form at shallow depths (commonly $<3 \mathrm{~km}$ ) in both island and continental arcs in compressional to extensional regimes. In continental arcs in which these deposit types coexist, porphyry and epithermal gold deposit types are sited above, and landward of, orogenic lode-gold deposits (Groves et al., 1998). Intrusion-related gold deposits have many similar characteristics to orogenic lode gold deposits (Sillitoe, 1991; Thompson et al., 1999) and intrusion-related gold mineralization does occur in Archaean granitoids (Qui \& McNaughton, 1999). However the tectonic setting appears to be different to that of orogenic deposits. Intrusion-related gold deposits are located in old continental crust well to the landward side of convergent plate boundaries (e.g. continental-margin Andean batholiths). Additionally, the deposits show a clear association with magmatic intrusions, occurring as sheeted veins and disseminated mineralization within plutons or their contact metamorphic aureoles. This type of deposit is also associated with regional extension and form at relatively shallow depths $(<6 \mathrm{~km})$.

There is a heterogeneous distribution of ages of orogenic lode-gold deposits. Peaks in the number of deposits occur around 2800-2555 Ma (a period which includes the giant Kalgoorlie and Timmins gold fields) and at 2100-1800 Ma (including the Ashanti and Homestake gold fields, Goldfarb et al., 2001) attributed to during episodes of, possibly plume-driven, crustal growth while the Earth was hotter (before ca. $1800 \mathrm{Ma}$ ), and subsequently preserved in the interiors of cratons. Abundant major gold provinces also exist with ages from ca. 450 to ca. $50 \mathrm{Ma}$. and include the gold fields of the circum-Pacific and the giant gold placers. 


\subsubsection{Timing of Gold Deposits within Orogens}

Both structural and geochronological studies show that the timing of gold deposition is typically late within the tectonic evolution of its host terranes. A variety of structural studies indicate, that the relative timing of gold is slightly later than regional metamorphism, plutonism, and early phases of orogenic deformation in mid-crustal level greenschist facies host rocks. At deeper levels in the crust, closer to the source of the gold-bearing fluids, mineralization is earlier and may be coeval with metamorphism and magmatism (Groves, 1995). In both the Yilgarn and elsewhere, gold was generally deposited during the $D_{2}$ or $D_{3}$ events in a $D_{1}-D_{4}$ deformational sequence.

Absolute ages for gold mineralization in the Yilgarn have been established from premineralization granitoid and felsic porphyry dykes and sills that are cut by gold-bearing veins, post-mineralization cross-cutting dykes and sills, which are not mineralized, and by direct dating of gold deposits using alteration minerals. These data show that most of the gold was deposited from 2645-2630 Ma (Yeats \& McNaughton, 1997), a period which corresponds to a widespread gold-mineralizing event in the Yilgarn. Most of the deposits were formed 60-70 m.y. after the end of widespread basic-ultrabasic volcanism and towards the end of the craton-wide emplacement of felsic magmas.

Late timing of gold within the deformational history of the host terranes has important implications for the use of GIS-based methods in mineral exploration. It means that the geometry of the structures associated with deposits (i.e. faults and shear zones) and rock sequences hosting the deposits on geological maps are essentially similar to those that existed during mineralization. It is significant that the location of ore deposits are related to repeated and predictable structural geometries. In addition, Hronsky et al. (1990) record that the vast majority of the gold-hosting structures in the Yilgarn are near vertical. Consequently, it means the spatial relationships between the structures and known deposits displayed in a geological map can be analysed using a GIS to predict the location of new deposits (Groves et al., 2000).

\subsubsection{Structural Controls}

Regional structures are the main structural control on the location of orogenic deposits. Although a broad spatial association between crustal- and regional-scale first-order structures exists, these structures rarely host gold deposits. Deposits are generally located in nearby second and third-order structures (Eisenlohr et al., 1989; Witt, 1993; Groves et al., 2000). Possible reasons for the lack of mineralization associated with first-order structures, 
which are generally crustal to regional shear zones, are that the translational movements along these shear zones did not produce permeability or that the shear zones are barriers to fluid flow (S.Halley, pers.comm., 2001)

Examples of favourable structural sites include;

1. Large, linear greenstone belts with major bounding shear zones which are favourable because the high structural permeability associated with thin bands of relatively reactive rock (compared to the surrounding granitoids) can result in high fluid-rock ratios. Reactivation of the major bounding structures may create second and thirdorder structures, and associated dilational zones, which are potential sites of gold mineralization.

2. Suitably-oriented structures in heterogenous stress fields around rigid granitoid plutons and complex batholith margins (e.g. Knight et al., 1993; Ojala et al., 1993; Phillips et al., 1996; Groves et al., 2000).

3. Pressure shadows at the terminations of elongate batholiths (Groves et al., 1995).

4. Irregularities in strike (and large rheological contrasts) along crustal and regionalscale faults or shear zones (Sibson, 1987; Phillips et al., 1996; Groves et al., 2000). According to Hronsky et al. (1991), only minor variations in strike along shear zones are required to produce a heterogenous stress distribution and thereby affect fluid flow. This is due to potential for compressional and dilational zones within the same domain (Groves et al., 2000).

5. Dilational jogs along strike-slip faults and overstepping faults (Hagemann et al., 1992).

6. Dilational zones where shear zones and faults are more gently dipping than elsewhere, for example, Granny Smith (Ojala et al., 1993) and Sons of Gwalia (Smith \& Gardoll, 1997) deposits.

7. Cross faults between first-order faults (Goldfarb et al., 1997).

8. Reactivated, locked-up fold-thrust structures and tightly-folded, overturned anticlines. The giant Golden Mile deposit, Kalgoorlie, is located in a zone where earlyformed thrust faults, involving competent units surrounded by less competent units, were later folded and faulted (Groves et al., 1995; Phillips et al., 1996).

9. Structurally isolated blocks of more competent volcanic rocks surrounded by less competent volcanic or sedimentary rocks. These blocks are formed where late NE- 
trending faults cross-cut NW-trending competent units enclosed in less competent units (Fig. 4, Groves et al., 2000). Isolated blocks of this type at all scales represent favourable sites for gold mineralization since the competent units within these blocks preferentially fail and become sites of enhanced structural permeability. Groves et al. (2001) have suggested that the cross-cutting faults tend to act as barriers to fluid flow, thereby greatly increasing fluid-flux ratios required to form larger deposits.

10. Isolated curvilinear blocks, which are closed off at one or both ends may also form where early thrust faults within competent units, which are enclosed by less competent units are later folded and faulted. Fluids reach supralithostatic pressure in these blocks causing fracturing in competent units and the formation forming large vein arrays; the anomalous fluid fluxes produced in such blocks can produce giant ore deposits; for example, Mt Charlotte, Golden Mile deposits (Ridley \& Mengler, 2000), Kalgoorlie and Timmins, Canada (Fig. 5, Groves et al., 2000).

11. Anticlinal hinges and domes formed by doubly plunging anticlines. In the Kalgoorlie Terrane, the location of anticlines are often inferred to be between synclines. Anticlinal axes are favourable sites for mineralization because they are often the sites of thrust faults and therefore may represent areas where fluids have been focussed. Anticlinal folds may also have the effect of trapping upward moving fluids if the overlying lithologies (e.g. shale) form an impermeable cap.

12. Dykes and small bodies of syenite and porphyritic intermediate to mafic intrusions are, in places, associated with gold mineralization (Halley, Groves, Cassidy, pers. comm., 2000). Halley (2000; pers. comm.), argues that porphyritic felsic intrusions, which contain up to $200 \mathrm{ppb}$ gold, have moved gold from the lower crust to higher levels and that later events pump fluid through these to produce deposits. Alternatively, most of the porphyritic intrusions are quartz-bearing and therefore very brittle. Consequently, they may correspond to sites of selective failure and high structural permeability.

13. Large to giant deposits are more common in greenschist facies terranes than in higher-grade metamorphic settings (Phillips et al., 1996). This may be due the greater effectiveness of mean stress variations as a fluid focussing mechanism at depths corresponding to greenschist facies metamorphism (Ridley, 1993) or, as Sibson (1987) has proposed, that fluid fluctuations in faults may be greatest at these crustal depths.

14. Magnetite-bearing rocks may be particularly favourable hosts for gold deposition. Phillips et al. (1996) has suggested that while Fe-silicate-rich (chlorite, actinolite) 
host rocks greatly facilitate the destabilization of gold complexes in solution (Neall \& Phillips, 1987), the presence of magnetite may promote gold deposition. Carbonation of magnetite to form siderite leads to oxidation of the fluid, in contrast to carbonation of Fe-silicates which do not involve a change in redox state. Reaction of the oxidized fluid would lead to gold deposition by lowering the activity of reduced sulphur species which complex with gold. Evidence from ore and alteration mineral assemblages suggests that the fluids which deposited gold in the Golden Mile Dolerite were relatively oxidized and that magnetite was relatively abundant compared with other deposits in the same terrane. The reactions envisaged by Phillips et al. (1996) are shown below;

$$
\begin{aligned}
& 2\left(\mathrm{Fe}^{2+} 2 \mathrm{Fe}^{3+}\right) \mathrm{O}_{4}+6 \mathrm{HAu}(\mathrm{HS})_{2}+0.5 \mathrm{CO}_{2} \rightleftharpoons 6 \mathrm{Au}+6 \mathrm{Fe}^{2+} \mathrm{S}_{2}+9 \mathrm{H}_{2} \mathrm{O} \\
& \mathrm{H}_{2} \mathrm{~S}+2 \mathrm{O}_{2} \rightleftharpoons \mathrm{SO}_{4}^{2-}+2 \mathrm{H}^{+}
\end{aligned}
$$

Alteration zones containing leucoxene and pyrite formed by the replacement of magnetite (see the first of the two reactions shown above) extend well beyond the carbonate alteration. Groves et al. (2001) have noted that this destruction of magnetite leads to a magnetically depleted core of the deposit and that some deposits have a magnetic rim due to the precipitation of magnetite by spent fluids. Strong positive and negative aeromagnetic anomalies may indicate sites of magnetite production and destruction, respectively. Although ore-forming fluids produce strong carbonate alteration, it is so widespread throughout the Norseman-Wiluna greenstone belt that the presence of alteration cannot be used as a simple vector to ore.

Further structural geometries that are favourable for gold deposition have been identified using a technique called stress mapping. This is a computer-based structural analysis that models paleo-stress patterns using the geometry of the lithological units, regional-scale structures and estimates of rock strength and fault friction properties and the far-field stress orientation (Holyland \& Ojala, 1997; Groves et al., 2000). The low stress anomalies (low mean stress and low minimum principal stress) identified using this technique correlate well with the location of all the known gold fields in the Kalgoorlie Terrane (Groves et al., 2000).

A requirement of this method is that fluid pressures locally exceed lithostatic pressures resulting in focussing of fluids, generally upwards, to zones of low mean stress. Such zones are commonly sites of selective failure, high fluid flux and resultant gold deposition (Groves et al., 1997). Evidence for high or supralithostatic pressures occurs throughout 
the Yilgarn block at all crustal depths in the form of flat extensional veins containing crack-seal textures

Gold deposits associated with low stress anomalies were identified in the following locations (Groves et al., 2000);

1. changes in strike of first-order faults from dominant the NNW to a NW trend,

2. intersections of two or more first-order faults, and

3. intersections of first- and second-order faults.

Groves et al. (1995, 2000) noted that reactivation of suitably-oriented, pre-existing structures in response to a change in the far-field stress direction may also provide favourable sites for gold deposition and suggest that multiple episodes in response to repeated reactivation may be an important ingredient in the formation of giant ore deposits.

At a mine scale, work by Hronsky et al. (1990) and Witt (1993) demonstrates that all kinematic types of structure such as strike-slip, oblique-slip, reverse, and normal faults may host gold mineralization. These local structures containing the gold mineralization vary with crustal depth (from brittle-ductile faults to ductile shear zones), lithology of host rocks, and rheological contrasts between the host and enclosing rocks units. In ductile sequences, where fluid flow was mainly within a fault or shear zone, structures vary from tensional quartz veins and breccias, quartz-vein sets, laminated quartz reefs and broad ductile shear zones. In more competent rocks, where fluid flow was in sub-vertical zones of fractured rock, gold is generally hosted in tensional quartz-vein sets.

\subsubsection{Host Rocks}

Highly competent and chemically reactive host rocks are the most common hosts to gold mineralization. On a regional scale, most of the gold deposits are sited in competent units or at contacts between layers of contrasting competency, which are oriented at high angles to the maximum principal far-field stress. Ridley (1993) has noted that the reason for this is that the more competent units tend to fail selectively in this orientation and hence become sites of increased structural permeability and fluid focussing. In the Yilgarn, this means that competent layers, which are oriented between NNW and NNE, are selectively mineralized, since the far-field maximum principal stress at the time of gold mineralization is estimated to have been approximately E-W (Phillips \& Groves, 1983; Witt, 1993). According to this criterion, most of the greenstone belt is prospective as most of the units have this orientation. 
Although a number of reports suggest that rheological contrasts across lithological boundaries are an important control on the location of larger deposits (Groves et al., 1995, 1997, 2000; Holyland \& Ojala, 1997), there have been few studies that test this hypothesis on a regional scale. One study by Yun (2000) confirms that this concept does apply well to the Kalgoorlie Terrane. Yun (2000) used subjective estimates of the relative competency, based on the degree of fabric development and modal mineralogy and ranked 15 rock types using integers in the range, 1-5. For example, BIF, porphyry, quartz dolerite and basalt were ranked as very competent (1) and ultramafic schists as very incompetent (5).

Rocks with high Fe and/or high $\mathrm{Fe} /(\mathrm{Fe}+\mathrm{Mg})$ such as dolerite, tholeiitic basalt, Banded Iron Formation (BIF), and some mafic porphyries are also the most chemically reactive host rocks and therefore the most likely to be mineralized. However, in the NorsemanWiluna Belt, banded iron formations are negligible and mafic rocks such as dolerite sills are associated with most large deposits (Phillips \& Groves, 1983; Phillips et al., 1996). Much of the Golden Mile Dolerite especially the granophyre has high $\mathrm{Fe} /(\mathrm{Fe}+\mathrm{Mg}+\mathrm{Ca}$ ) (Phillips, 1986).

Iron-rich rocks react with the ore-bearing fluids to produce pyrite, thereby destabilizing the reduced sulphur complexes (see equations 5.2.4 \& 5.2.4 above; Phillips \& Groves, 1983; Mikucki, 1998) and depositing gold. Due to the high $\mathrm{CO}_{2}$ content of the fluids, reaction with calcium and magnesium-rich wall rocks will form Fe-bearing carbonates (i.e. ankerite and dolomite) which prevents the formation of Fe-sulphides leading to gold deposition. For this reason, BIF and Fe-rich mafic rocks represent ideal host rocks for gold deposition, as evidenced by the sometimes extreme sulphide alteration in places (Groves et al., 2000), because iron content is very high $(\sim 40-55 \%)$ and calcium contents are very low.

\subsubsection{Mechanism of Gold Precipitation}

The main mechanism for the precipitation of gold is fluid-wall-rock reaction Reduced Srich fluids containing the $\mathrm{Au}(H S)_{2}$ complex react with Fe-bearing host rocks (Phillips \& Groves, 1983; Mikucki, 1998). Gold deposition is thought to occur through coupled reactions such as (Mikucki, 1998):

$\mathrm{FeO}_{\text {rock }}+2 \mathrm{H}_{2} \mathrm{~S}^{\circ} \rightleftharpoons \mathrm{FeS}_{2 \text { pyrite }}+\mathrm{H}_{2} \mathrm{O}+\mathrm{H}_{2}(\mathrm{~g})$

and

$\mathrm{Au}(\mathrm{HS})_{2}+\mathrm{H}^{+}+1 / 2 \mathrm{H}_{2}(\mathrm{~g}) \rightleftharpoons \mathrm{Au}+2 \mathrm{H}_{2} \mathrm{~S}^{\circ}$ 
Phase separation due to brittle failure and a concomitant sudden reduction of pressure and fluid mixing of ore fluid with near surface fluids are other possible means by which gold can be precipitated. However, they appear to have played a minor role in the Yilgarn (Groves et al., 1997; Mikucki, 1998).

\subsubsection{Simplified Model of an Orogenic Gold System}

A simplified model of an orogenic gold system is one in which an anomalous heat source in the middle crust (due to mantle plume activity, subduction of a oceanic arc, subduction roll-back or mantle delamination) causes upward migration of low-salinity fluids carrying reduced sulphur species through first-order crustal-scale and regional-scale channel ways, which are associated with heterogenous stress fields. These primary structures may focus fluids into second and third -order structures. Large fluid fluxes and high fluid-rock ratios in thick units of competent, chemically-reactive host rocks are required for the formation of large gold deposits. High structural permeability results from complex geometries in volcanic rocks and strong contrasts in rock strength (so that a competent unit fails and is surrounded by other less competent units that do not). In the Yilgarn, reactive host rocks are generally mafic with high-Fe or high $\mathrm{Fe} /(\mathrm{Fe}+\mathrm{Mg}+\mathrm{Ca})$ contents. The main mechanism of gold deposition is through fluid-wall rock reactions in which the iron-rich minerals in the host rocks react with sulphide complexes which carry gold in solution to form Fe-sulphides (Phillips \& Groves, 1983; Mikucki, 1998).

\subsection{Exploration Criteria}

This purpose of this section is to derive exploration criteria based on the model for orogenic deposits described above. Only criteria that are applicable to regional-scale exploration and which can be applied to the data available for the Kalgoorlie study area are considered here.

The approach used in this study was developed by Wyborn et al. (1994c, 1995b) and involves converting the essential ingredients of a mineral systems model to features that are likely to have been mapped and compiled in a regional scale GIS database. Wyborn emphasized the need to consider the entire mineralizing system, including source region, metal and ligand sources, pathways, and trap zone, and outflow zone rather than just the characteristics of the deposit itself, as the mineral system provides a larger target and is more applicable to the type of data that are captured in a regional-scale GIS. 
Regional-scale exploration criteria are listed together with a description of how the criteria are modelled as a GIS map layer in Table 5.1 below.

\subsection{Primary GIS Layers}

This section gives a brief explanation of the primary data used in the Kalgoorlie case study. The data set comprises geology, gravity and airborne geophysics, a composite LANDSAT Thematic Mapper image and whole-rock geochemical analyses from soil samples and drill holes. These data sets were supplied by Dr Scott Halley, Placer Dome. The data sets were compiled in the period 1999-2000 and were part of an active exploration program. Additional data sets, such as the deposit and regional whole-rock geochemical databases and the rock strength data, came from other sources, which are explained briefly below. Detailed information, such as metadata and data dictionaries for the digital GIS data sets, are given in Appendix D. Digital copies of all ArCVIEw GIS layers and Matrab arrays are included on the CD-ROM (vol.2) attached to the thesis.

\subsubsection{Geology Layer}

The solid geology GIS layer was compiled in November 2000 and incorporates a combination of mapping by Goldfields Exploration geologists and an interpretation of a highresolution airborne magnetic survey (200 m flight-line spacing). The GIS layer supplied by Goldfields Exploration Pty Ltd maps comprises a number of non-contiguous areas along the NNE-trending Bardoc Tectonic Zone (BTZ; refer to Fig. 5.1 in Appendix D).

Solid geology and structural geology maps supplied by Goldfields Exploration Pty Ltd in MapInfo format were imported first into ARCVIEW and then clipped to conform to the boundaries of the Kalgoorlie study area. The solid geology GIS layer was then exported from ARCVIEW as a plain ASCII file, which was then imported into MATLAB (a software package containing the neural network programs). A similar procedure was applied to other data sets (except for geophysics data sets). The steps are illustrated in Figure 5.2.

Once the data were imported into ARCVIEw, the legend used by Goldfields Exploration Pty Ltd was simplified from 108 to 25 rock types (Table 5.2 and Section 5.5.1 below). 
Table 5.1. Regional-scale exploration criteria for orogenic gold deposits in the Kalgoorlie Terrane

\begin{tabular}{|c|c|}
\hline Exploration Criteria & Corresponding GIS Database Layer \\
\hline Proximity to crustal \& regional-scale faults & $\begin{array}{l}\text { Distance to nearest crustal fault } \\
\text { Distance to nearest regional NNW fault } \\
\text { Distance to nearest regional NNE fault }\end{array}$ \\
\hline Heterogenous stress fields around granitoid margins & Not modelled \\
\hline Pressure shadows at ends of elongate batholiths & Not modelled \\
\hline Irregularites in strike of faults or shear zones & Strike of nearest fault, types $1-4$ \\
\hline Dilational jogs along strike-slip faults & Strike of nearest fault, types $1-4$ \\
\hline Dilational zones where dip of fault locally flattens & Not modelled \\
\hline Cross faults between first-order faults & $\begin{array}{l}\text { Distance to intersection of crustal and regional } \\
\text { faults }\end{array}$ \\
\hline Intersections of two or more first-order faults & As above \\
\hline Intersections of first- and second-order faults & As above \\
\hline $\begin{array}{l}\text { Reactivated locked-up fold-thrust structures \& tightly- } \\
\text { folded, overturned anticlines }\end{array}$ & Not modelled \\
\hline $\begin{array}{l}\text { Structurally isolated blocks (late NE faults cross-cut NW } \\
\text { trending competent units) }\end{array}$ & Not modelled \\
\hline $\begin{array}{l}\text { Anticlinal hinges \& domes formed by doubly plunging } \\
\text { anticlines }\end{array}$ & Distance to nearest regional-scale anticlinal axis \\
\hline $\begin{array}{l}\text { Syenite intrusions (weak association with gold mineral- } \\
\text { ization) }\end{array}$ & $\begin{array}{l}\text { Binary syenite layer (i.e.; presence-absence of } \\
\text { syenite } \\
\text { Distance to nearest syenite intrusion }\end{array}$ \\
\hline $\begin{array}{l}\text { Porphyritic felsic intrusions (weak association with gold } \\
\text { mineral- } \\
\text { ization) }\end{array}$ & $\begin{array}{l}\text { Binary porphyritic felsic intrusion layer } \\
\text { Distance to nearest porphyritic felsic intrusion }\end{array}$ \\
\hline $\begin{array}{l}\text { Quartz dolerite \& leucocratic dolerite (highly prospective } \\
\text { host rocks) }\end{array}$ & $\begin{array}{l}\text { Binary quartz dolerite/leucocratic dolerite layer } \\
\text { Distance to nearest quartz dolerite/leucocratic } \\
\text { dolerite }\end{array}$ \\
\hline $\begin{array}{l}\text { Isolated curvilinear blocks formed by folding \& faulting } \\
\text { of early thrust faults }\end{array}$ & Not modelled \\
\hline Most prospective metamorphic grade is greenschist facies & Metamorphic-grade map \\
\hline $\begin{array}{l}\text { Chemically reactive host rocks with high } \mathrm{Fe} \text { and/or high } \\
\mathrm{Fe} /(\mathrm{Fe}+\mathrm{Mg}+\mathrm{Ca})\end{array}$ & $\begin{array}{l}\text { Maps of } \mathrm{Fe}^{3+} / \mathrm{Fe}^{2+}, \quad \mathrm{Fe}_{t} \text { ot } /\left(\mathrm{Fe}_{t o t}+\mathrm{Mg}+\mathrm{Ca}\right), \\
\mathrm{Fe}_{t} \text { ot } * \mathrm{Fe}_{t} \text { ot } /\left(\mathrm{Fe}_{\text {tot }}+\mathrm{Mg}+\mathrm{Ca}\right), \& \mathrm{Fe}_{2} \mathrm{O}_{3} /\left(\mathrm{Fe}_{2} \mathrm{O}_{3}+\right. \\
\mathrm{FeO}) \text { ratios }\end{array}$ \\
\hline Highly competent host rocks & $\begin{array}{l}\text { Subjective rock strength, Young's modulus, UTS, } \\
\text { UCS \& fracture toughness }\end{array}$ \\
\hline $\begin{array}{l}\text { Host rock sequences containing layers with strong com- } \\
\text { petency contrasts }\end{array}$ & $\begin{array}{l}\text { Maps with rheological contrast at nearest litho- } \\
\text { logical boundary Cell values }=\text { absolute value of } \\
\text { difference in rock strength at nearest rock bound- } \\
\text { ary }\end{array}$ \\
\hline Alteration related to ore-forming fluids & $\begin{array}{l}\text { Positive \& negative magnetic anomaly maps show } \\
\text { domains of magnetite destruction \& production }\end{array}$ \\
\hline $\begin{array}{l}\text { Changes in strike of first-order faults from dominant the } \\
\text { NNW to a NW trend }\end{array}$ & Strike of nearest fault, types $1-4$ \\
\hline $\begin{array}{l}\text { Reactivated crustal \& regional-scale faults and shear } \\
\text { zones }\end{array}$ & Not modelled \\
\hline
\end{tabular}




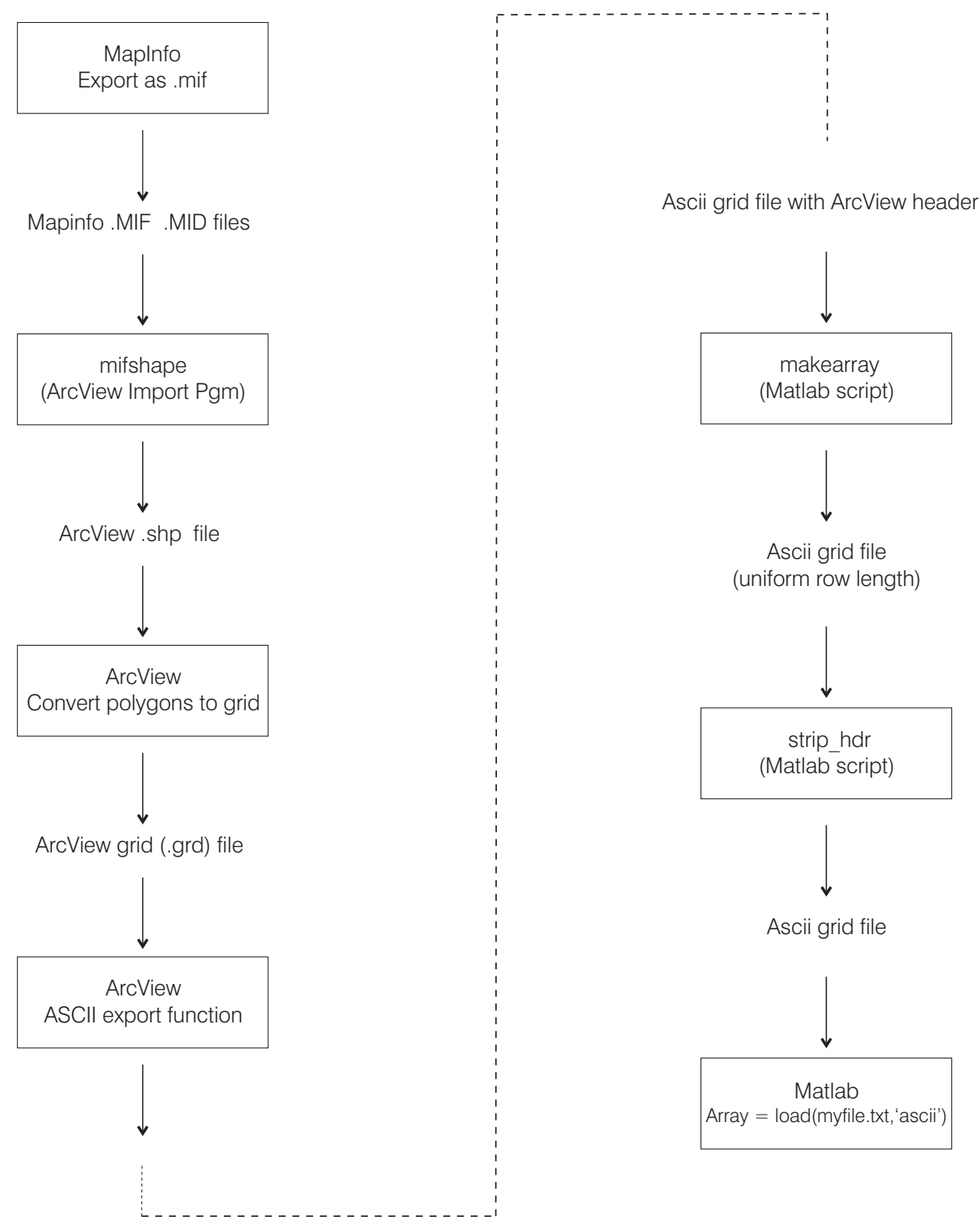

Figure 5.2. Steps required to convert data sets obtained from Goldfields Pty Ltd in MAPINFO vector GIS format to MATLAB arrays. 
Table 5.2: Simplified rock type legend for the solid geology GIS layer. The geological legend used by Goldfields Exploration Pty Ltd was reduced to 25 rock types (listed in order of simplified legend rock names).

\begin{tabular}{|c|c|c|c|c|}
\hline $\begin{array}{l}\text { New } \\
\text { Code }\end{array}$ & $\begin{array}{l}\text { Simplified Legend } \\
\text { ( } 25 \text { rock types) }\end{array}$ & $\begin{array}{l}\text { Old } \\
\text { Code }\end{array}$ & $\begin{array}{l}\text { Goldfields Exploration Pty Ltd legend } \\
\text { (108 rock types) }\end{array}$ & Count \\
\hline $\begin{array}{l}\mathrm{Hm} \\
\mathrm{Hm} \\
\mathrm{Hm} \\
\mathrm{Hm} \\
\mathrm{Hm} \\
\mathrm{Hm} \\
\mathrm{Hm} \\
\mathrm{Hm}\end{array}$ & $\begin{array}{l}\text { Amphibolite (Undivided) } \\
\text { Amphibolite (Undivided) } \\
\text { Amphibolite (Undivided) } \\
\text { Amphibolite (Undivided) } \\
\text { Amphibolite (Undivided) } \\
\text { Amphibolite (Undivided) } \\
\text { Amphibolite (Undivided) } \\
\text { Amphibolite (Undivided) } \\
\text { Amphibolite (Undivided) }\end{array}$ & $\begin{array}{l}\text { Hof } \\
\text { Hpc } \\
\text { Homc } \\
\text { Hom } \\
\text { Homh } \\
\text { Hm } \\
\text { Hou } \\
\text { Houh } \\
\text { Hpt }\end{array}$ & $\begin{array}{l}\text { Alkali-rich Ortho Amphibolite (f.g. felsic) } \\
\text { Calcic Para Amphibolite (diopside almandine) } \\
\text { Fe-rich Ortho Amphibolite (c.g. mafic) } \\
\text { Fe-rich Ortho Amphibolite (f.g mafic) } \\
\text { Fe-rich Ortho Hornfels (mafic derived) } \\
\text { Mafic Amphibolite (undivided) } \\
\text { Mg-rich Ortho Amphibolite (f.g. ultramafic) } \\
\text { Mg-rich Ortho Hornfels (ultramafic derived) } \\
\text { Para Amphibolite (mafic) }\end{array}$ & 11346 \\
\hline $\begin{array}{l}\mathrm{Mb} \\
\mathrm{Mb} \\
\mathrm{Mb} \\
\mathrm{Mb}\end{array}$ & $\begin{array}{l}\text { Basalt } \\
\text { Basalt } \\
\text { Basalt } \\
\text { Basalt }\end{array}$ & $\begin{array}{l}\text { Mb } \\
\text { Mbbx } \\
\text { Mb- } \\
\text { mg } \\
\text { Mbp } \\
\text { Mbx } \\
\text { Mby } \\
\text { M }\end{array}$ & $\begin{array}{l}\text { Basalt } \\
\text { Mafic Pyroclastic Breccia } \\
\text { Mount Goode Basalt } \\
\text { Porphyritic Basalt (plagioclase phyric) } \\
\text { Undivided Basaltic Autoclastic Rocks } \\
\text { Undivided Mafic Pyroclastic Rock } \\
\text { Undivided Mafic Rocks (often altered) } \\
\end{array}$ & 117529 \\
\hline Shcc & Carbonaceous Shale & Shcc & Carbonaceous Shale & 22755 \\
\hline Sct & Chert & Sct & Chert & 0 \\
\hline $\begin{array}{l}\mathrm{Sc} \\
\mathrm{Sc} \\
\mathrm{Sc} \\
\mathrm{Sc} \\
\mathrm{Sc}\end{array}$ & $\begin{array}{l}\text { Conglomerate } \\
\text { Conglomerate } \\
\text { Conglomerate } \\
\text { Conglomerate } \\
\text { Conglomerate }\end{array}$ & $\begin{array}{l}\text { Sc } \\
\text { Scpf } \\
\text { Scxm } \\
\text { Scxp } \\
\text { Scvm }\end{array}$ & $\begin{array}{l}\text { Conglomerate } \\
\text { Jones Creek Conglomerate } \\
\text { Monomict Conglomerate } \\
\text { Polymict Conglomerate (Kurrawang) } \\
\text { Volc.-derived Conglomerate (mafic-u/mafic) }\end{array}$ & 8530 \\
\hline $\begin{array}{l}\mathrm{Md} \\
\mathrm{Md} \\
\mathrm{Md} \\
\mathrm{Md}\end{array}$ & $\begin{array}{l}\text { Dolerite } \\
\text { Dolerite } \\
\text { Dolerite } \\
\text { Dolerite }\end{array}$ & $\begin{array}{l}\text { Md } \\
\text { Mdl } \\
\text { Mb-m } \\
\text { Mdp }\end{array}$ & $\begin{array}{l}\text { Dolerite } \\
\text { Leucocratic Dolerite (pyroxene, phyric) } \\
\text { Magnetic Basalt } \\
\text { Porphyritic Dolerite (plagioclase, phyric) }\end{array}$ & 15161 \\
\hline $\begin{array}{l}\text { Pf } \\
\text { Pf } \\
\text { Pf } \\
\text { Pf } \\
\text { Pf } \\
\text { Pf } \\
\text { Hgf }\end{array}$ & $\begin{array}{l}\text { Feldspar Porphyry } \\
\text { Feldspar Porphyry } \\
\text { Feldspar Porphyry } \\
\text { Feldspar Porphyry } \\
\text { Feldspar Porphyry } \\
\text { Feldspar Porphyry } \\
\text { Felsic Gneiss } \\
\end{array}$ & $\begin{array}{l}\text { Pfbh } \\
\text { Pf } \\
\text { Pfqb } \\
\text { Pfqh } \\
\text { Pfq } \\
\text { Pfh } \\
\text { Hgf }\end{array}$ & $\begin{array}{l}\text { Feldspar Hornblende Biotite Porphyry } \\
\text { Feldspar Porphyry } \\
\text { Feldspar Quartz Biotite Porphyry } \\
\text { Feldspar Quartz Hornblende Porphyry } \\
\text { Feldspar Quartz Porphyry } \\
\text { Plagioclase } \pm \text { Hornblende Porphyry } \\
\text { Felsic Gneiss }\end{array}$ & 14998 \\
\hline $\begin{array}{l}\mathrm{Mg} \\
\mathrm{Mg} \\
\mathrm{Mg} \\
\mathrm{Mg} \\
\mathrm{Mg} \\
\mathrm{Mg} \\
\mathrm{Mg} \\
\mathrm{Mg} \\
\mathrm{Mg} \\
\mathrm{Mg}\end{array}$ & $\begin{array}{l}\text { Gabbro } \\
\text { Gabbro } \\
\text { Gabbro } \\
\text { Gabbro } \\
\text { Gabbro } \\
\text { Gabbro } \\
\text { Gabbro } \\
\text { Gabbro } \\
\text { Gabbro } \\
\text { Gabbro }\end{array}$ & $\begin{array}{l}\mathrm{Mg} \\
\mathrm{Mg} 1 \\
\mathrm{Mg} 2 \\
\mathrm{Mg} 3 \\
\mathrm{Mg} 5 \\
\mathrm{Mg} 6 \\
\mathrm{Mg} 6 \mathrm{~A} \\
\mathrm{Mgl} \\
\mathrm{Mgm} \\
\mathrm{Mgp}\end{array}$ & $\begin{array}{l}\text { Gabbro } \\
\text { Gabbro - Kundana unit } 1 \\
\text { Gabbro - Kundana unit } 2 \\
\text { Gabbro - Kundana unit } 3 \\
\text { Gabbro - Kundana unit } 5 \\
\text { Gabbro - Kundana unit } 6 \\
\text { Gabbro - Kundana unit } 6 \mathrm{~A} \\
\text { Leucocratic Gabbro (pyroxene, phyric) } \\
\text { Melanocratic Gabbro - amphibole rich } \\
\text { Porphyritic Gabbro (plagioclase phyric) }\end{array}$ & 56944 \\
\hline $\begin{array}{l}\text { Gt } \\
\text { Gt }\end{array}$ & $\begin{array}{l}\text { Granitoid } \\
\text { Granitoid }\end{array}$ & $\begin{array}{l}\text { Gr } \\
\text { Gt }\end{array}$ & $\begin{array}{l}\text { Granite } \\
\text { Granitoid }\end{array}$ & 331673 \\
\hline
\end{tabular}


Table 5.2: continued

\begin{tabular}{|c|c|c|c|c|}
\hline $\begin{array}{l}\text { New } \\
\text { Code }\end{array}$ & $\begin{array}{l}\text { Simplified Legend } \\
\text { (25 rock types) }\end{array}$ & $\begin{array}{l}\text { Old } \\
\text { Code }\end{array}$ & $\begin{array}{l}\text { Goldfields Exploration Pty Ltd legend } \\
\text { (108 rock types) }\end{array}$ & Count \\
\hline $\begin{array}{l}\mathrm{Gt} \\
\mathrm{Gt} \\
\mathrm{Gt} \\
\mathrm{Gt} \\
\mathrm{Gt} \\
\mathrm{Gt} \\
\mathrm{Gt} \\
\mathrm{Gt}\end{array}$ & $\begin{array}{l}\text { Granitoid } \\
\text { Granitoid } \\
\text { Granitoid } \\
\text { Granitoid } \\
\text { Granitoid } \\
\text { Granitoid } \\
\text { Granitoid } \\
\text { Granitoid }\end{array}$ & $\begin{array}{l}\text { Gdi } \\
\text { Gmo } \\
\text { Gp } \\
\text { Gmoq } \\
\text { Gsy } \\
\text { Gsdi } \\
\text { Gto } \\
\text { Gdi- } \\
\text { wee }\end{array}$ & $\begin{array}{l}\text { Granodiorite } \\
\text { Monzonite } \\
\text { Pegmatite } \\
\text { Quartz Monzonite (cf Adamellite) } \\
\text { Syenite } \\
\text { Syenodiorite } \\
\text { Tonalite } \\
\text { Weebo Granodiorite }\end{array}$ & \\
\hline $\begin{array}{l}\text { Sif } \\
\text { Sif }\end{array}$ & $\begin{array}{l}\text { Iron Formation } \\
\text { Iron Formation }\end{array}$ & $\begin{array}{l}\text { Bif } \\
\text { Sif }\end{array}$ & $\begin{array}{l}\text { Banded Iron Formation } \\
\text { Iron Formation }\end{array}$ & 0 \\
\hline $\begin{array}{l}\mathrm{Mmb} \\
\mathrm{Mmb}\end{array}$ & $\begin{array}{l}\text { Magnesian Basalt } \\
\text { Magnesian Basalt }\end{array}$ & $\begin{array}{l}\mathrm{Mmb} \\
\mathrm{Mbu}\end{array}$ & $\begin{array}{l}\text { Magnesian Basalt (variolitic } \pm \text { pyx.) } \\
\text { Mafic Ultramafic (Greenstone) }\end{array}$ & 49249 \\
\hline $\begin{array}{l}\text { Perm } \\
\text { Pmd } \\
\text { Pmd }\end{array}$ & $\begin{array}{l}\text { Permian Sedimentary Rock } \\
\text { Proterozoic Dolerite } \\
\text { Proterozoic Dolerite }\end{array}$ & $\begin{array}{l}\text { Perm } \\
\text { Pmd } \\
\text { Pd }\end{array}$ & $\begin{array}{l}\text { Permian Sedimentary Rock } \\
\text { Proteroic Dolerite } \\
\text { Proterozoic Mafic \& Ultramafic Dyke }\end{array}$ & $\begin{array}{l}- \\
- \\
-\end{array}$ \\
\hline $\begin{array}{l}\text { Mdq } \\
\text { Qv }\end{array}$ & $\begin{array}{l}\text { Quartz Dolerite } \\
\text { Quartz Vein }\end{array}$ & $\begin{array}{l}\text { Mdq } \\
\text { Qv }\end{array}$ & $\begin{array}{l}\text { Quartz Dolerite } \\
\text { Stacked Plutonic-Style Lodes }\end{array}$ & 117 \\
\hline $\begin{array}{l}\text { Hpq } \\
\text { Us } \\
\text { Us } \\
\text { Us } \\
\text { Hpqh }\end{array}$ & $\begin{array}{l}\text { Quartzite } \\
\text { Serpentinite } \\
\text { Serpentinite } \\
\text { Serpentinite } \\
\text { Si-Rich Hornfels }\end{array}$ & $\begin{array}{l}\text { Hpq } \\
\text { Usk } \\
\text { Uspd } \\
\text { Us } \\
\text { Hpqh }\end{array}$ & $\begin{array}{l}\text { Quartzite } \\
\text { Serpentinised Komatiite } \\
\text { Serpentinised Peridotite } \\
\text { Serpentinite } \\
\text { Si-rich Hornfels (qtz, minor Al-silicate) }\end{array}$ & 15489 \\
\hline $\begin{array}{l}\text { Utc } \\
\text { Utc }\end{array}$ & $\begin{array}{l}\text { Talc-Chlorite Ultramafic } \\
\text { Talc-Chlorite Ultramafic }\end{array}$ & $\begin{array}{l}\mathrm{Uac} \\
\mathrm{Utc}\end{array}$ & $\begin{array}{l}\text { Amphibole-Chlorite Ultramafic Rock } \\
\text { Talc-Chlorite Ultramafic Rock }\end{array}$ & 589 \\
\hline $\mathrm{Zu}$ & Ultramafic Schist & $\mathrm{Zu}$ & Ultramafic schist & 2 \\
\hline $\begin{array}{l}\text { Zls } \\
\text { Zls } \\
\text { Zls } \\
\text { Zls } \\
\text { Zls }\end{array}$ & $\begin{array}{l}\text { Undiff'd Felsic Schist } \\
\text { Undiff'd Felsic Schist } \\
\text { Undiff'd Felsic Schist } \\
\text { Undiff'd Felsic Schist } \\
\text { Undiff'd Felsic Schist }\end{array}$ & $\begin{array}{l}\text { Zac } \\
\mathrm{Zf} \\
\mathrm{Zm} \\
\mathrm{Zs} \\
\mathrm{Zls}\end{array}$ & $\begin{array}{l}\text { Actinolite Schist } \\
\text { Fuchsite schist } \\
\text { Muscovite schist } \\
\text { Sericite schist } \\
\text { Undifferentiated Felsic Schists }\end{array}$ & 15 \\
\hline $\begin{array}{l}\text { Fv } \\
\text { Fv } \\
\text { Fv } \\
\text { Fv } \\
\text { Fv } \\
\text { Fv } \\
\text { Fv } \\
\text { Fv } \\
\text { Fv } \\
\text { Fv }\end{array}$ & $\begin{array}{l}\text { Undivided Felsic Volcanic } \\
\text { Undivided Felsic Volcanic } \\
\text { Undivided Felsic Volcanic } \\
\text { Undivided Felsic Volcanic } \\
\text { Undivided Felsic Volcanic } \\
\text { Undivided Felsic Volcanic } \\
\text { Undivided Felsic Volcanic } \\
\text { Undivided Felsic Volcanic } \\
\text { Undivided Felsic Volcanic } \\
\text { Undivided Felsic Volcanic }\end{array}$ & $\begin{array}{l}\text { Svcf } \\
\text { Fd } \\
\text { Fpbx } \\
\text { Ftc } \\
\text { Svff } \\
\text { Svmf } \\
\text { Ft } \\
\text { Fvx } \\
\text { Fvy } \\
\text { Fv }\end{array}$ & $\begin{array}{l}\text { Coarse-grained Fragmental } \\
\text { Dacite (even grained) } \\
\text { Felsic Pyroclastic Brecia (agglomerate) } \\
\text { Felsic Tuff (crystal lithic) } \\
\text { Fine-grained Fragmental } \\
\text { Medium-grained Fragmental } \\
\text { Metamorphosed Felsic Tuff (lava dominant) } \\
\text { Undivided Felsic Autoclastic Rocks } \\
\text { Undivided Felsic Pyroclastic Rocks } \\
\text { Undivided Felsic Volcanic Rocks }\end{array}$ & 18131 \\
\hline $\begin{array}{l}\text { Ia } \\
\text { Iv } \\
\text { Ia } \\
\text { Iv } \\
\text { Iv }\end{array}$ & $\begin{array}{l}\text { Undivided Int. Volcanic } \\
\text { Undivided Int. Volcanic } \\
\text { Undivided Int. Volcanic } \\
\text { Undivided Int. Volcanic } \\
\text { Undivided Int. Volcanic }\end{array}$ & $\begin{array}{l}\text { Ia } \\
\text { Ipbx } \\
\text { Ifp } \\
\text { Ivx } \\
\text { Iv }\end{array}$ & $\begin{array}{l}\text { Andesite (even grained) } \\
\text { Intermediate Pyroclastic Breccia } \\
\text { Porphyritic Andesite (plag. phyric biotite) } \\
\text { Undivided Intermediate Autoclastic Rocks } \\
\text { Undivided Intermediate Volcanic Rock }\end{array}$ & 384 \\
\hline $\begin{array}{l}\mathrm{S} \\
\mathrm{S} \\
\mathrm{S}\end{array}$ & $\begin{array}{l}\text { Undivided Sed. Rock } \\
\text { Undivided Sed. Rock } \\
\text { Undivided Sed. Rock }\end{array}$ & $\begin{array}{l}\text { Sslh } \\
\text { Shl } \\
\text { Sha }\end{array}$ & $\begin{array}{l}\text { Interbedded Siltstone - Shale } \\
\text { Shale } \\
\text { Shale (altered) }\end{array}$ & 56462 \\
\hline
\end{tabular}


Table 5.2: continued

\begin{tabular}{|c|c|c|c|c|}
\hline $\begin{array}{l}\text { New } \\
\text { Code }\end{array}$ & $\begin{array}{l}\text { Simplified Legend } \\
\text { ( } 25 \text { rock types) }\end{array}$ & $\begin{array}{l}\text { Old } \\
\text { Code }\end{array}$ & $\begin{array}{l}\text { Goldfields Exploration Pty Ltd legend } \\
\text { (108 rock types) }\end{array}$ & Count \\
\hline $\begin{array}{l}\mathrm{S} \\
\mathrm{S} \\
\mathrm{S}\end{array}$ & $\begin{array}{l}\text { Undivided Sed. Rock } \\
\text { Undivided Sed. Rock } \\
\text { Undivided Sed. Rock }\end{array}$ & $\begin{array}{l}\text { Ssl } \\
\text { San } \\
\text { S }\end{array}$ & $\begin{array}{l}\text { Siltstone } \\
\text { Undivided Arenite (sandstone, matrix }<15 \% \text { ) } \\
\text { Undivided Sedimentary Rocks }\end{array}$ & \\
\hline $\begin{array}{l}\mathrm{U} \\
\mathrm{U} \\
\mathrm{U} \\
\mathrm{U} \\
\mathrm{U}\end{array}$ & $\begin{array}{l}\text { Undivided Ultramafic Rock } \\
\text { Undivided Ultramafic Rock } \\
\text { Undivided Ultramafic Rock } \\
\text { Undivided Ultramafic Rock } \\
\text { Undivided Ultramafic Rock }\end{array}$ & $\begin{array}{l}\text { Uk } \\
\text { Upd } \\
\text { Upx } \\
\text { Uxa } \\
\text { U }\end{array}$ & $\begin{array}{l}\text { Komatiite } \\
\text { Peridotite (olivine cumulate) } \\
\text { Pyroxenite } \\
\text { Ultramafic Autobreccia } \\
\text { Undivided Ultramafic Rocks }\end{array}$ & 95067 \\
\hline $\begin{array}{l}? \\
?\end{array}$ & $\begin{array}{l}\text { Unknown/Unclassified } \\
\text { Unknown/Unclassified }\end{array}$ & $\begin{array}{l}\mathrm{X} \\
?\end{array}$ & $\begin{array}{l}\text { Mylonite } \\
\text { Unknown/Unclassified Unit }\end{array}$ & 217 \\
\hline $\begin{array}{l}\text { Svg } \\
\text { Svg } \\
\text { Svg } \\
\text { Svg } \\
\text { Svg } \\
\text { Svg }\end{array}$ & $\begin{array}{l}\text { Volcanogenic Rock } \\
\text { Volcanogenic Rock } \\
\text { Volcanogenic Rock } \\
\text { Volcanogenic Rock } \\
\text { Volcanogenic Rock } \\
\text { Volcanogenic Rock }\end{array}$ & $\begin{array}{l}\text { Sfw } \\
\text { Ivs } \\
\text { Sw } \\
\text { Svfw } \\
\text { Svw } \\
\text { Svg }\end{array}$ & $\begin{array}{l}\text { Feldspathic Wacke ( }>75 \% \text { feldsp. }<25 \% \\
\text { lithic) } \\
\text { Intermediate Volcanogenic Sedimentary Rock } \\
\text { Undivided Wacke }(15-75 \% \text { matrix }) \\
\text { Volcanic Feldspathic Wacke ( } \% \text { qtz }<\text { feldsp.) } \\
\text { Volcanic Wacke } \\
\text { Volcanogenic Sediments }\end{array}$ & 167123 \\
\hline
\end{tabular}

\subsubsection{Structural Layer}

The primary structural GIS layer is a synthesis of published mapping by the Geological Survey of Western Australia (Swager, 1989; Swager \& Griffin, 1990; Swager et al., 1990), mapping by company geologists at Goldfields Exploration Pty Ltd, and an interpretation of airborne magnetic data by Dr Scott Halley. The final structural GIS coverage is the result of combining the Kalgoorlie area and the BTZ structural maps using the same procedure described for the geology layer above. The structural GIS layer, faults.shp containing all faults, shear zones and magnetic trend lines (ARCVIEW vector format) is shown in Figure 5.8 below.

\subsubsection{Fold Axis Layer}

GIS layers, which show the ground traces of major anticlinal and synclinal axes are based on an interpretation by Prof. David Groves of geology and structural maps of the Kalgoorlie area (Swager \& Griffin, 1990; Swager et al., 1990; Yun, 2000). Whilst many of the synclinal locations are mapped, the site of anticlinal hinge zones are generally the locations of thrust faults and are inferred. The fold-axis layer, (folds.shp; ARCVIEW vector format) is shown in Figure 5.9 below. 


\subsubsection{Aeromagnetic and Gamma-Ray Layer}

The airborne geophysics survey covering the Kalgoorlie study area is a high-resolution (200 m flight-line spacing) multi-client survey flown by World Geoscience in 1989. The data comprise total magnetic intensity (TMI) with reduction-to-pole (RTP) corrections, first vertical derivative of magnetic intensity, and radiometric survey data (i.e. U, Th, K and total-count channels). Due to poor outcrop and the widespread and thick regolith layer in the Kalgoorlie area, the radiometric data were not used. The total magnetic intensity layer, $15 \_m \_r t p$ is a raster layer which was converted from a ERMAPPER file (.ers; BIL format) by importing it into Geosoft. ${ }^{1}$ The data set were re-gridded to convert the original $25 \mathrm{~m}$ grid spacing to $100 \mathrm{~m}$ and then converted from a GEOSOFT .grd file to an ArCVIEW binary raster grid file, which was then imported into ARCVIEW (Fig. 5.10).

\subsubsection{Gravity Layer}

The gravity GIS layer shows Bouguer gravity anomalies calculated from data collected by AGSO in 1971. The original data has an $11 \mathrm{~km}$ station spacing, which was re-gridded to a 100 metre grid by Dr Mike House, geophysicist, Goldfields Pty Ltd. The Bouguer anomaly layer, l12_grav, was converted from an ERMAPPER .ers file to an ARCVIEW grid using the procedure described for the TMI magnetic layer above (Fig. 5.11).

\subsubsection{Deposit Layer}

The locations and sizes of gold deposits in the Kalgoorlie study area were obtained from the Mines and Mineral Deposits database (MINEDEX) compiled by the Geological Survey of Western Australia (Townsend et al., 2000). The data relevant to gold deposits in the Kalgoorlie study area were extracted from the original ACCESS 97 database and converted to an ArcView point coverage by Paul Hodkiewicz and Stephen Gardoll at the Centre for Global Metallogeny, University of Western Australia.

For each deposit, an estimate of pre-mining resource (kg total contained gold, TCG) was calculated by adding the largest resource category corresponding to the Australian Institute of Mining and Metallurgy (AusIMM) JORC code (inferred, indicated, and measured) available to any historic and recent production. Hodkiewicz (2002) notes that, although

\footnotetext{
${ }^{1}$ The software package referred to as GEOSOFT is the OASIS montaj geoscience data processing and analysis system (Release 4.3) produced by GEOsoft.
} 
pre-1980 production was typically from underground operations that could only support average grades greater than $\sim 30 \mathrm{~g} / \mathrm{t}$, current large-scale open pit operations now mine average grades of less than $5 \mathrm{~g} / \mathrm{t}$. Consequently, Hodkiewicz concluded that a combination of historic production and current resources is the best way to provide order-of-magnitude estimates of the district-scale pre-mining gold endowment for the comparison purposes of a mineral prospectivity study (see ARCVIEW vector format layers deps_all.shp and deps_1000tcg, which show all gold deposits and occurrences, and deposits with resource of $\geq 1000 \mathrm{~kg}$ TCG, respectively in Figs. 5.12 and 5.13).

\subsubsection{OZCHEM Database}

Whole-rock geochemical data were used to calculate five different indices representing the chemical reactivity of potential host rocks in the Kalgoorlie region.

The data used to calculate the chemical ratios are a subset of a spatial representation of the Australian Government Survey Organization's (AGSO) OZCHEM whole-rock geochemistry database (Kilgour \& Hazell, 1998). The Yilgarn Block data set comprises 4473 analyses, which were obtained from the database of the CSIRO Division of Exploration Geoscience, PhD theses, rocks collected as part of joint AGSO-Geological Survey of Western Australia (GSWA) mapping projects, and from the bottom of AGSO seismic shot holes.

The database was supplied as an Microsoft ExCEL spreadsheet and an ArCVIEW shape file showing sample locations. Table D.8 in Appendix D shows the OZCHEM analyses used to calculate the reactivity ratios for each rock type in the solid geology legend. The ratio values were calculated in an EXCEL spreadsheet and then exported as an ASCII file together with AMG coordinates of the sample locations. The ASCII file was imported into ArcView. Using the ARCVIEw spatial join function, the attributes of the point data set containing the chemical ratios were added to the geology attribute table. The chemical ratio attributes were then used to create raster GIS layers corresponding to each of the five indices. In a further step, chemical contrasts at lithological boundaries were calculated to produce a derived GIS layer. The procedure is described in Section 5.5.12 below.

The chemical ratios tested together with the corresponding layer names (refer to the GIS database on the attached CD-ROM (vol.2)) are shown in Table 5.3. In the first two ratios, $\mathrm{Fe} /(\mathrm{Fe}+\mathrm{Mg}+\mathrm{Ca})$ is a ratio of weight percentages of metals and Fe represents the total iron; i.e. $\mathrm{Fe}^{2+}+\mathrm{Fe}^{3+}$. Work by Yun (2000) using the first two ratios has shown 
that chemical reactivity of the host rocks is one of the parameters useful in estimating the prospectivity for orogenic gold deposits. The two ratios describe how much iron is available in the host rock for sulphidation reactions (see Equations 5.2.6 \& 5.2.6) which are proposed as the primary means of gold precipitation (Mikucki, 1998). Magnesium and calcium in the host rocks inhibit the precipitation of gold by reducing the amount of iron due to reaction with the $\mathrm{CO}_{2}$ - rich fluids to produce carbonates (e.g. calcite, dolomite, magnesite or in low $\mathrm{Fe} /(\mathrm{Fe}+\mathrm{Mg}+\mathrm{Ca})$ conditions, ankerite). In addition to the ratio of iron to iron, magnesium and calcium, the total amount of iron is important. Consequently, total iron in weight percent was multiplied by the $\mathrm{Fe} /(\mathrm{Fe}+\mathrm{Mg}+\mathrm{Ca})$ ratio in row 1 of Table 5.3. The last two ratios in Table 5.3 show the $\mathrm{Fe}^{3+} / \mathrm{Fe}^{2+}$ ratios as oxide and molar ratios, respectively, and are included to check if the oxidation state of the rocks could be used to estimate the prospectivity for gold mineralization. The layers, $133_{-} l_{-}$ch2 -5 are in the ARCVIEw directory on the attached CD-ROM (vol.1).

Table 5.3. Chemical reactivity layers, which were evaluated as potential input GIS layers for neural network analyses

\begin{tabular}{|l|l|}
\hline Layer name & Rock chemical reactivity ratio \\
\hline \hline 13_l_ch1 & $\mathrm{Fe}_{\text {wt\% }} \times \mathrm{Fe}_{\text {total }} /\left(\mathrm{Fe}_{\text {total }}+\mathrm{Mg}+\mathrm{Ca}\right)$ \\
\hline 13_l_ch2 & $\mathrm{Fe}_{\text {total }} /\left(\mathrm{Fe}_{\text {total }}+\mathrm{Mg}+\mathrm{Ca}\right)$ \\
\hline 13_l_ch3 & $\mathrm{Fe}_{2} \mathrm{O}_{3} /\left(\mathrm{Fe}_{2} \mathrm{O}_{3}+\mathrm{FeO}\right)$ \\
\hline 13_l_ch4 & $\mathrm{Fe}_{2} \mathrm{O}_{3} /(\mathrm{FeO})$ \\
\hline 13_l_ch5 & $\mathrm{Fe}^{3+} / \mathrm{Fe}^{2+}$ \\
\hline
\end{tabular}

The values of these five different methods of calculating a chemical reactivity index based on iron ratios are shown in Table 5.4 below.

Table 5.4: Chemical reactivity ratios for the Kalgoorlie study area, arranged in order of decreasing $\mathrm{Fe} \times \mathrm{Fe} /(\mathrm{Fe}+\mathrm{Mg}+\mathrm{Ca})$.

\begin{tabular}{|l|l|l|l|l|l|l|}
\hline $\begin{array}{l}\text { Rock } \\
\text { Code }\end{array}$ & Rock Type/Description & $\begin{array}{l}\mathrm{Fe}_{w t \%} \times \\
\frac{\mathrm{Fe}}{\mathrm{Fe}+\mathrm{Mg}+\mathrm{Ca}}\end{array}$ & $\frac{\mathrm{Fe}}{\mathrm{Fe}+\mathrm{Mg}+\mathrm{Ca}}$ & $\frac{\mathrm{Fe}_{2} \mathrm{O}_{3}}{\mathrm{Fe}_{2} \mathrm{O}_{3}+\mathrm{FeO}}$ & $\frac{\mathrm{Fe}_{2} \mathrm{O}_{3}}{\mathrm{FeO}^{3+}}$ & $\frac{\mathrm{Fe}^{2+}}{\mathrm{Fe}^{2+}}$ \\
\hline \hline Bif & Banded Iron Formation & 22.65 & 0.78 & 0.54 & 1.15 & 1.04 \\
\hline Sif & Iron Formation & 22.65 & 0.78 & 0.54 & 1.15 & 1.04 \\
\hline Md & Dolerite & 4.75 & 0.44 & 0.25 & 0.62 & 0.56 \\
\hline Mdl & Leucocratic Dolerite & 4.75 & 0.44 & 0.25 & 0.62 & 0.56 \\
\hline Mb-m & Magnetic Basalt & 4.75 & 0.44 & 0.25 & 0.62 & 0.56 \\
\hline
\end{tabular}


Table 5.4: continued

\begin{tabular}{|c|c|c|c|c|c|c|}
\hline $\begin{array}{l}\text { Rock } \\
\text { Code }\end{array}$ & Rock Type/Description & $\begin{array}{l}\mathrm{Fe}_{w t \%} \times \\
\frac{\mathrm{Fe}}{\mathrm{Fe}+\mathrm{Mg}+\mathrm{Ca}}\end{array}$ & $\frac{\mathrm{Fe}}{\mathrm{Fe}+\mathrm{Mg}+\mathrm{Ca}}$ & $\frac{\mathrm{Fe}_{2} \mathrm{O}_{3}}{\mathrm{Fe}_{2} \mathrm{O}_{3}+\mathrm{FeO}}$ & $\frac{\mathrm{Fe}_{2} \mathrm{O}_{3}}{\mathrm{FeO}}$ & $\frac{\mathrm{Fe}^{3+}}{\mathrm{Fe}^{2+}}$ \\
\hline Mdp & Porphyritic Dolerite & 4.75 & 0.44 & 0.25 & 0.62 & 0.56 \\
\hline Sslh & Interbedded Sst/Shale $^{1}$ & 4.11 & 0.68 & 0.37 & 0.78 & 0.70 \\
\hline Ssl & Siltstone & 4.11 & 0.68 & 0.37 & 0.78 & 0.70 \\
\hline Zf & Fuchsite Schist & 3.54 & 0.31 & 0.17 & 0.20 & 0.18 \\
\hline $\mathrm{Zm}$ & Muscovite Schist & 3.54 & 0.31 & 0.17 & 0.20 & 0.18 \\
\hline Zs & Sericite Schist & 3.54 & 0.31 & 0.17 & 0.20 & 0.18 \\
\hline $\mathrm{Zls}$ & Undiff. Felsic Schists & 3.54 & 0.31 & 0.17 & 0.20 & 0.18 \\
\hline $\mathrm{Sc}$ & Conglomerate & 3.30 & 0.38 & 0.14 & 0.38 & 0.34 \\
\hline Scxm & Monomict Conglomerate & 3.30 & 0.38 & 0.14 & 0.38 & 0.34 \\
\hline S & Undivided Sed. Rocks & 3.30 & 0.38 & 0.14 & 0.38 & 0.34 \\
\hline Shcc & Carbonaceous Shale & 3.02 & 0.45 & 0.71 & 2.44 & 2.19 \\
\hline $\mathrm{Mg}$ & Gabbro & 2.58 & 0.29 & 0.13 & 0.15 & 0.13 \\
\hline $\operatorname{Mg} 1$ & Gabbro - Kundana unit 1 & 2.58 & 0.29 & 0.13 & 0.15 & 0.13 \\
\hline Mg2 & Gabbro - Kundana unit 2 & 2.58 & 0.29 & 0.13 & 0.15 & 0.13 \\
\hline Mg3 & Gabbro - Kundana unit 3 & 2.58 & 0.29 & 0.13 & 0.15 & 0.13 \\
\hline $\operatorname{Mg} 5$ & Gabbro - Kundana unit 5 & 2.58 & 0.29 & 0.13 & 0.15 & 0.13 \\
\hline Mg6 & Gabbro - Kundana unit 6 & 2.58 & 0.29 & 0.13 & 0.15 & 0.13 \\
\hline $\operatorname{Mg} 6 \mathrm{~A}$ & Gabbro - Kundana unit 6A & 2.58 & 0.29 & 0.13 & 0.15 & 0.13 \\
\hline $\mathrm{Mgl}$ & Leucocratic Gabbro & 2.58 & 0.29 & 0.13 & 0.15 & 0.13 \\
\hline Mgm & Melanocratic Gabbro & 2.58 & 0.29 & 0.13 & 0.15 & 0.13 \\
\hline Mgp & Porphyritic Gabbro & 2.58 & 0.29 & 0.13 & 0.15 & 0.13 \\
\hline Hof & K-rich Ortho Amphibolite & 2.52 & 0.29 & 0.17 & 0.21 & 0.19 \\
\hline $\mathrm{Hpc}$ & Ca-rich Para Amphibolite & 2.52 & 0.29 & 0.17 & 0.21 & 0.19 \\
\hline $\mathrm{Hm}$ & Mafic Amphibolite & 2.52 & 0.29 & 0.17 & 0.21 & 0.19 \\
\hline $\mathrm{Mb}$ & Basalt & 2.38 & 0.29 & 0.18 & 0.24 & 0.22 \\
\hline Mbbx & Mafic P/clastic Breccia & 2.38 & 0.29 & 0.18 & 0.24 & 0.22 \\
\hline Mbmg & Mount Goode Basalt & 2.38 & 0.29 & 0.18 & 0.24 & 0.22 \\
\hline Mbp & Porphyritic Basalt & 2.38 & 0.29 & 0.18 & 0.24 & 0.22 \\
\hline Mbx & Basaltic A/clastic Rock & 2.38 & 0.29 & 0.18 & 0.24 & 0.22 \\
\hline Mby & Mafic P/clastic Rock & 2.38 & 0.29 & 0.18 & 0.24 & 0.22 \\
\hline M & Undivided Mafic Rocks & 2.38 & 0.29 & 0.18 & 0.24 & 0.22 \\
\hline
\end{tabular}


Table 5.4: continued

\begin{tabular}{|c|c|c|c|c|c|c|}
\hline $\begin{array}{l}\text { Rock } \\
\text { Code }\end{array}$ & Rock Type/Description & $\begin{array}{l}\mathrm{Fe}_{w t \%} \times \\
\frac{\mathrm{Fe}}{\mathrm{Fe}+\mathrm{Mg}+\mathrm{Ca}}\end{array}$ & $\frac{\mathrm{Fe}}{\mathrm{Fe}+\mathrm{Mg}+\mathrm{Ca}}$ & $\frac{\mathrm{Fe}_{2} \mathrm{O}_{3}}{\mathrm{Fe}_{2} \mathrm{O}_{3}+\mathrm{FeO}}$ & $\frac{\mathrm{Fe}_{2} \mathrm{O}_{3}}{\mathrm{FeO}}$ & $\frac{\mathrm{Fe}^{3+}}{\mathrm{Fe}^{2+}}$ \\
\hline Sct & Chert $^{2}$ & 2.14 & 0.30 & 0.31 & 0.69 & 0.62 \\
\hline $\mathrm{X}$ & Mylonite $^{2}$ & 2.14 & 0.30 & 0.31 & 0.69 & 0.62 \\
\hline$?$ & Unknown/Unclassified unit ${ }^{2}$ & 2.14 & 0.30 & 0.31 & 0.69 & 0.62 \\
\hline Upx & Pyroxenite & 2.12 & 0.24 & 0.14 & 0.18 & 0.16 \\
\hline $\mathrm{Mbu}$ & Mafic U/mafic (Greenstone) & 1.74 & 0.21 & 0.18 & 0.24 & 0.21 \\
\hline $\mathrm{Mmb}$ & Magnesian Basalt & 1.74 & 0.21 & 0.18 & 0.24 & 0.21 \\
\hline San & Undivided Arenite & 1.61 & 0.33 & 0.28 & 0.42 & 0.38 \\
\hline Hou & Mg-rich Ortho Amphibolite & 1.56 & 0.21 & 0.14 & 0.16 & 0.15 \\
\hline Houh & Mg-rich Ortho Hornfels & 1.56 & 0.21 & 0.14 & 0.16 & 0.15 \\
\hline $\mathrm{Sfw}$ & Feldspathic Wacke & 1.53 & 0.39 & 0.40 & 0.96 & 0.86 \\
\hline Ivs & Int. Volcanogenic Sed. Rock & 1.53 & 0.39 & 0.40 & 0.96 & 0.86 \\
\hline $\mathrm{Sw}$ & Undivided Wacke & 1.53 & 0.39 & 0.40 & 0.96 & 0.86 \\
\hline Svfw & Volcanic Feldspathic Wacke & 1.53 & 0.39 & 0.40 & 0.96 & 0.86 \\
\hline Svw & Volcanic Wacke & 1.53 & 0.39 & 0.40 & 0.96 & 0.86 \\
\hline Svg & Volcanogenic Sed. Rock & 1.53 & 0.39 & 0.40 & 0.96 & 0.86 \\
\hline Zac & Actinolite Schist & 1.49 & 0.20 & 0.17 & 0.20 & 0.18 \\
\hline Gsdi & Syenodiorite & 1.39 & 0.31 & 0.30 & 0.51 & 0.46 \\
\hline Mdq & Quartz Dolerite & 1.27 & 0.30 & 0.41 & 0.72 & 0.65 \\
\hline Utc & Talc-Chl. U/mafic Rock & 1.26 & 0.17 & 0.21 & 0.26 & 0.24 \\
\hline $\mathrm{Uac}$ & Amph.-Chl U/mafic Rock & 1.23 & 0.17 & 0.16 & 0.20 & 0.18 \\
\hline Shl & Shale & 1.16 & 0.44 & 0.37 & 0.78 & 0.70 \\
\hline Sha & Shale (altered) & 1.16 & 0.44 & 0.37 & 0.78 & 0.70 \\
\hline Homc & Fe-Ortho Amphibolite (c.g.) & 1.15 & 0.20 & 0.40 & 0.67 & 0.61 \\
\hline Hom & Fe-Ortho Amphibolite (f.g.) & 1.15 & 0.20 & 0.40 & 0.67 & 0.61 \\
\hline Homh & Fe-rich Ortho Hornfels & 1.15 & 0.20 & 0.40 & 0.67 & 0.61 \\
\hline Hpt & Para Amphibolite (mafic) & 1.15 & 0.20 & 0.40 & 0.67 & 0.61 \\
\hline $\mathrm{U}$ & Undivided Ultramafic Rock & 0.93 & 0.13 & 0.29 & 0.49 & 0.44 \\
\hline $\mathrm{Hpq}$ & Quartzite & 0.91 & 0.29 & 0.38 & 0.62 & 0.56 \\
\hline Usk & Serpentinised Komatiite & 0.90 & 0.12 & 0.11 & 0.15 & 0.13 \\
\hline Uspd & Serpentinised Peridotite & 0.90 & 0.12 & 0.11 & 0.15 & 0.13 \\
\hline $\mathrm{Us}_{\mathrm{s}}$ & Serpentinite & 0.90 & 0.12 & 0.11 & 0.15 & 0.13 \\
\hline
\end{tabular}


Table 5.4: continued

\begin{tabular}{|c|c|c|c|c|c|c|}
\hline $\begin{array}{l}\text { Rock } \\
\text { Code }\end{array}$ & Rock Type/Description & $\begin{array}{l}\mathrm{Fe}_{w t \%} \times \\
\frac{\mathrm{Fe}}{\mathrm{Fe}+\mathrm{Mg}+\mathrm{Ca}}\end{array}$ & $\frac{\mathrm{Fe}}{\mathrm{Fe}+\mathrm{Mg}+\mathrm{Ca}}$ & $\frac{\mathrm{Fe}_{2} \mathrm{O}_{3}}{\mathrm{Fe}_{2} \mathrm{O}_{3}+\mathrm{FeO}}$ & $\frac{\mathrm{Fe}_{2} \mathrm{O}_{3}}{\mathrm{FeO}}$ & $\frac{\mathrm{Fe}^{3+}}{\mathrm{Fe}^{2+}}$ \\
\hline $\mathrm{Zu}$ & Ultramafic Schist & 0.89 & 0.14 & 0.22 & 0.18 & 0.16 \\
\hline Uk & Komatiite & 0.88 & 0.13 & 0.39 & 0.73 & 0.66 \\
\hline Uxa & Ultramafic Autobreccia & 0.88 & 0.13 & 0.39 & 0.73 & 0.66 \\
\hline Gto & Tonalite & 0.84 & 0.34 & 0.42 & 0.75 & 0.67 \\
\hline Upd & Peridotite & 0.83 & 0.12 & 0.30 & 0.52 & 0.47 \\
\hline $\mathrm{Fd}$ & Dacite (even grained) & 0.82 & 0.29 & 0.38 & 0.76 & 0.68 \\
\hline Ia & Andesite (even grained) & 0.80 & 0.25 & 0.43 & 2.25 & 2.02 \\
\hline Ifp & Porphyritic Andesite & 0.80 & 0.25 & 0.43 & 2.25 & 2.02 \\
\hline Scvm & Volc.-derived Conglomerate & 0.70 & 0.23 & 0.25 & 0.52 & 0.47 \\
\hline $\operatorname{Hgf}$ & Felsic Gneiss & 0.69 & 0.36 & 0.46 & 1.02 & 0.92 \\
\hline Gdi & Granodiorite & 0.69 & 0.32 & 0.44 & 0.89 & 0.80 \\
\hline Gdiwee & Weebo Granodiorite & 0.69 & 0.32 & 0.44 & 0.89 & 0.80 \\
\hline Pfbh & Felds. Hbl. Bio. Porphyry & 0.67 & 0.40 & 0.40 & 0.78 & 0.70 \\
\hline $\mathrm{Pf}$ & Feldspar Porphyry & 0.67 & 0.40 & 0.40 & 0.78 & 0.70 \\
\hline Pfqb & Feldspar Qtz Bio. Porphyry & 0.67 & 0.40 & 0.40 & 0.78 & 0.70 \\
\hline Pfqh & Feldspar Qtz Hbl. Porphyry & 0.67 & 0.40 & 0.40 & 0.78 & 0.70 \\
\hline Pfq & Feldspar Qtz Porphyry & 0.67 & 0.40 & 0.40 & 0.78 & 0.70 \\
\hline Gmo & Monzonite & 0.67 & 0.30 & 0.59 & 1.45 & 1.31 \\
\hline Pfh & Plag. \pm Hbl. Porphyry & 0.67 & 0.40 & 0.40 & 0.78 & 0.70 \\
\hline Hpqh & Si-rich Hornfels & 0.67 & 0.61 & 0.86 & 6.33 & 5.70 \\
\hline Gsy & Syenite & 0.65 & 0.38 & 0.54 & 1.95 & 1.76 \\
\hline Svcf & Fragmental (c.g) & 0.63 & 0.37 & 0.40 & 1.62 & 1.46 \\
\hline Fpbx & Felsic Pyroclastic Breccia & 0.63 & 0.37 & 0.40 & 1.62 & 1.46 \\
\hline Ftc & Felsic Tuff (crystal lithic) & 0.63 & 0.37 & 0.40 & 1.62 & 1.46 \\
\hline Svff & Fragmental (f.g.) & 0.63 & 0.37 & 0.40 & 1.62 & 1.46 \\
\hline Svmf & Fragmental (m.g.) & 0.63 & 0.37 & 0.40 & 1.62 & 1.46 \\
\hline $\mathrm{Ft}$ & Meta-. Felsic Tuff \& Lava & 0.63 & 0.37 & 0.40 & 1.62 & 1.46 \\
\hline Fvx & Undiv. Fels. A/clastic Rock & 0.63 & 0.37 & 0.40 & 1.62 & 1.46 \\
\hline Fvy & Undiv. Fels. P/clastic Rock & 0.63 & 0.37 & 0.40 & 1.62 & 1.46 \\
\hline $\mathrm{Fv}$ & Undivided Felsic Volc. Rock & 0.63 & 0.37 & 0.40 & 1.62 & 1.46 \\
\hline Ipbx & Int. $\mathrm{P} /$ clastic Breccia & 0.56 & 0.42 & 0.38 & 0.63 & 0.57 \\
\hline
\end{tabular}


Table 5.4: continued

\begin{tabular}{|l|l|l|l|l|l|l|}
\hline Rock & Rock Type/Description & $\begin{array}{l}\mathrm{Fe}_{w t \%} \times \\
\frac{\mathrm{Fe}}{\mathrm{Fe}+\mathrm{Mg}+\mathrm{Ca}}\end{array}$ & $\frac{\mathrm{Fe}}{\mathrm{Fe}+\mathrm{Mg}+\mathrm{Ca}}$ & $\frac{\mathrm{Fe}_{2} \mathrm{O}_{3}}{\mathrm{Fe}_{2} \mathrm{O}_{3}+\mathrm{FeO}}$ & $\frac{\mathrm{Fe}_{2} \mathrm{O}_{3}}{\mathrm{FeO}^{3+}}$ & $\frac{\mathrm{Fe}^{2+}}{\mathrm{Fe}^{2+}}$ \\
\hline Ivx & Undiv. Int. A/clastic Rock & 0.56 & 0.42 & 0.38 & 0.63 & 0.57 \\
\hline Iv & Undivided Int. Volc. Rock & 0.56 & 0.42 & 0.38 & 0.63 & 0.57 \\
\hline Gt & Granitoid & 0.50 & 0.37 & 0.46 & 1.03 & 0.93 \\
\hline Gr & Granite & 0.48 & 0.40 & 0.43 & 0.82 & 0.74 \\
\hline Scpf & Jones Creek Conglomerate & 0.36 & 0.34 & 0.44 & 0.78 & 0.70 \\
\hline Scxp & Polymict Conglomerate & 0.36 & 0.34 & 0.44 & 0.78 & 0.70 \\
\hline Gmoq & Quartz Monzonite & 0.35 & 0.36 & 0.51 & 1.40 & 1.26 \\
\hline Gp & Pegmatite & 0.07 & 0.31 & 0.67 & 2.00 & 1.80 \\
\hline Perm & Permian Sedimentary Rock & -999 & -999 & -999 & -999 & -999 \\
\hline Pmd & Proterozoic Dolerite & -999 & -999 & -999 & -999 & -999 \\
\hline Pd & Proterozoic Dykes & & -999 \\
\hline
\end{tabular}

\subsubsection{Rock Strength Data Sets}

Although it is generally recognized that competency contrasts across lithological boundaries are an important control on the location of deposits, there are little data on how to model these rheological contrasts. Yun's study (Yun, 2000) employed subjective estimates and Holyland's work (Holyland et al., 1993; Groves et al., 1997) is based on proprietary software and few details on how the calculations are performed have been published. To help determine the best way to model rheological contrast, a number of different rock strength parameters were tested (i.e. two different compilations of Young's Modulus, uniaxial compressive and tensile strength, and fracture toughness). Subjective estimates of rock competency, expressed as classes and fuzzy membership values, were also used to prepared layers depicting rheological contrasts at lithological boundaries layers. These rheological-contrast layers are described in Section 5.5.11 below.

\footnotetext{
${ }^{1}$ Abbreviations: a/clastic $=$ autoclastic, amphib. = amphibolite, bio = biotite, c.g. = coarse-grained, fels. $=$ felsic, f.g. $=$ fine-grained, hbl = hornblende, int. = intermediate, m.g. = medium-grained, meta$=$ metamorphosed, $\mathrm{p} /$ clastic $=$ pyroclastic, plag. $=$ plagioclase, $\mathrm{qtz}=$ quartz, $\mathrm{sst}=$ sandstone, sed. $=$ sedimentary, undiff. = undifferentiated, undiv. = undivided, \& u $/$ mafic $=$ ultramafic.

${ }^{2}$ Chemical ratios for these rock units represent area weighted averages for all rock units.

${ }^{3}$ Rock units formed after gold mineralization $(\sim 2.6 \mathrm{Ga})$ are represented in raster format GIS layers with the value -999 , which is the default no data value for ARCView.
} 


\section{Measured Rock Strength and Deformational Properties}

Rock strength and deformational data for Young's modulus, uniaxial compressive and tensile strengths and fracture toughness were provided by M. Lee (unpublished data from Australian Mining Consultants, 2000; abbreviated as AMC). Some of the data supplied by AMC originated from Szwedzicki \& Jiang (1994). Although these data refer to rocks from mines in the Yilgarn Terrane, many of the 108 rock types in the simplified lithology legend derived from the solid geology map obtained from Goldfields Exploration Pty Ltd were not available. In such cases, an estimate of the rock strength properties was made selecting a rock type with similar deformational properties. The parameter values used to calculate the rheological contrast at lithological boundaries represent the average value in the AMC data set for a specific rock type from different mine sites. In addition, Young's Modulus data from a compilation of rock properties by Hatheway \& Kiersch (1989) have also been tabulated (Table 5.5).

\section{Subjective Estimates of Rock Competency}

There is an overall lack of rock strength properties for many specific rock types in the Kalgoorlie Terrane. The properties that are available commonly apply only to a particular unit with a specific alteration assemblage and fracture density at a particular mine. Thus, subjective estimates of relative rock strength were also made in this study.

Rocks in the detailed geology legend (108 rock types) are ranked according to their tendency for brittle or ductile failure, based on the field observations by Prof. David Groves. The primary criterion used to estimate rock competency on a regional-scale is silica content and mineralogy. The following minerals are associated with decreasing strength (from left to right);

$$
\text { quartz } \rightarrow \text { plagioclase } \rightarrow \text { hornblende } \rightarrow \text { biotite } \rightarrow \text { sericite } \rightarrow \text { talc }
$$

Quartz-rich rocks are more competent than rocks which are rich in mafic minerals. In addition to silica content, subjective estimates of the relative competency of host rocks are based on (in decreasing order of importance); modal mineralogy, grain size, fabric development, texture, and metamorphic grade. For example, banded iron formation and basalt are ranked as very competent, whereas ultramafic schists are ranked as very incompetent under greenschist facies conditions. There may be reversals in rheology at amphibolite facies. Figure 5.3 illustrates how the criteria were applied. 


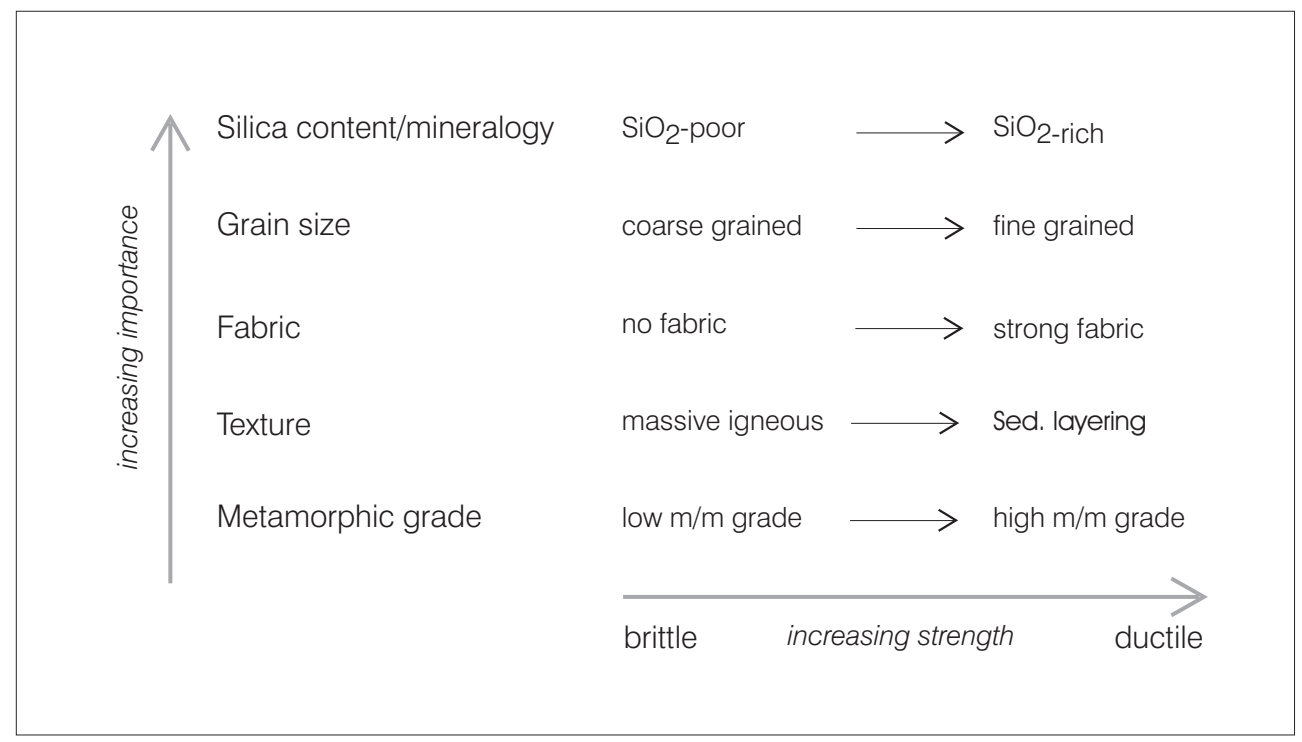

Figure 5.3. Criteria used for subjective estimates of rock strength. The importance of the criterion decreases from top to bottom and the competency of rocks decreases from left to right. Provided that the rocks are compared under conditions of equal lithostatic and hydrostatic pressures and temperature and assuming the rock does not contain pre-existing fractures, silica content and modal mineralogy are the most significant factors in determining the rock strength

Rock units later in age than gold deposits were assigned the value -9999 and excluded from rheology contrast calculations. The steps used to produce the rheology contrast layers are described in Section 5.5.11 below.

A percentile transformation was then applied and the values scaled to the range $[0,1]$. The resultant parameter is a fuzzy membership value. Two GIS rheology layers were prepared; one containing a continuous, fuzzy-membership variable (layer $12 \_l_{-} 1$ ), and the other containing values in the range $[1,10]$ representing ten classes of rock competency (layer $12 l_{2}$ 2 2). Values in the subjectively estimated layers for each rock type are shown in Table 5.5 below. The rock strength layers, 12_l_rh1-5 correspond to the subjective fuzzy membership layer, subjective ten-class layer, Young's modulus (AMC data), Young's modulus (Hatheway \& Kiersch, 1989 data), uniaxial compressive strength, tensile strength, and fracture toughness and are included in the ARCVIEW directory on the attached CDROM (vol. 1). The layer, l2_l_rh5, depicting fracture toughness is shown in Figure 5.14

\subsubsection{Metamorphic Grade}

A metamorphic-grade map was prepared from a combination of maps by Binns et al. (1976) and Witt (1993). Scanned images of the maps, which covered different parts of 
the Kalgoorlie study area, were digitized in ARCVIEw. Boundaries of the metamorphic domains were adjusted slightly so that they matched the crustal-scale faults and shear zones of the solid geology map of the study area. There are four classes of rock in the metamorphic-grade map, which were assigned the numeric codes shown in brackets; granite (0), amphibolite (1), upper greenschist (2), and lower greenschist facies (3). The numeric codes were assigned so that the code increases with increasing favourability for gold deposits. A raster version of the metamorphic grade map is shown in Figure 5.15. 


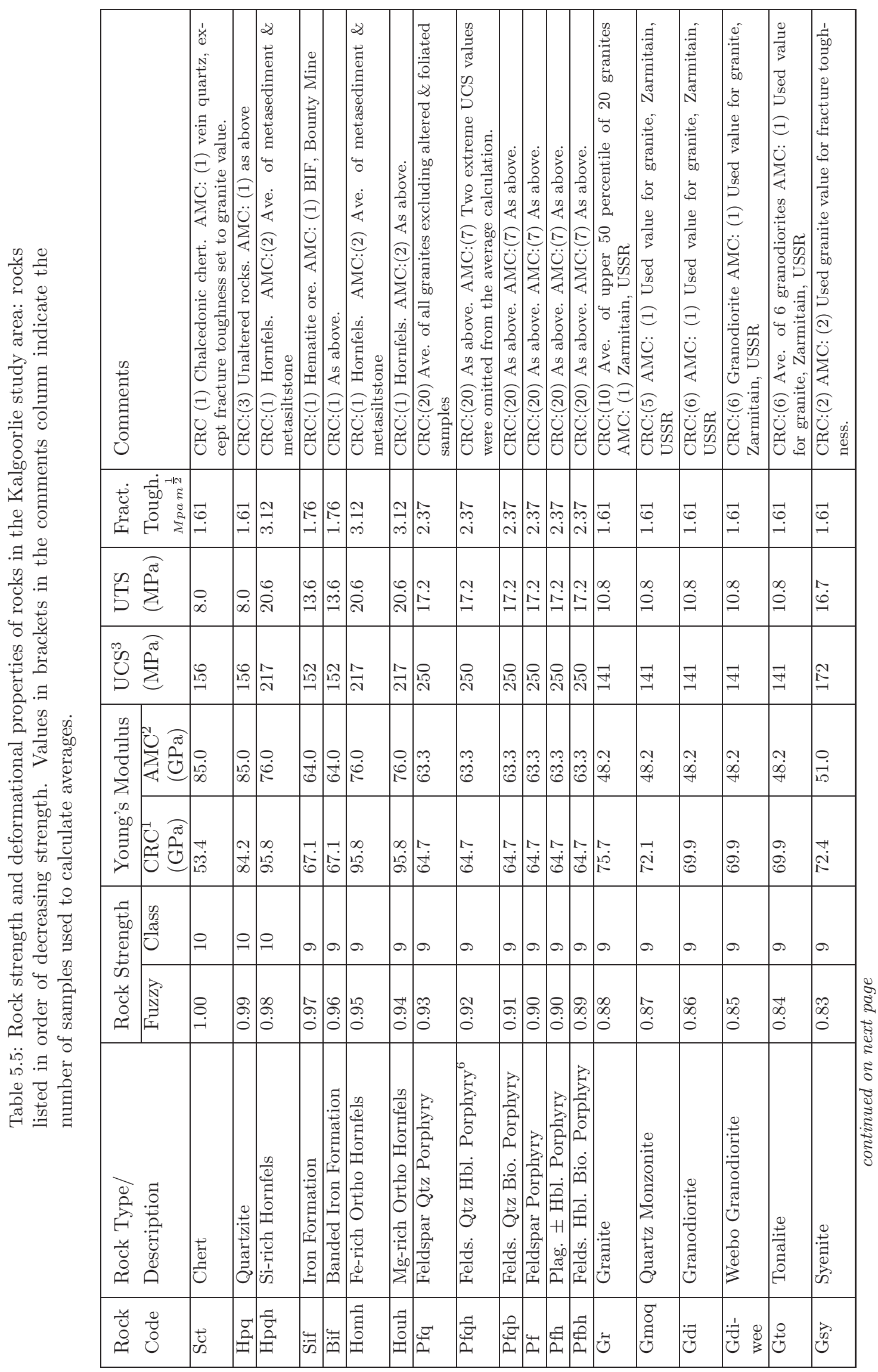




\begin{tabular}{|c|c|c|c|c|c|c|c|c|c|c|c|c|c|c|c|c|c|}
\hline 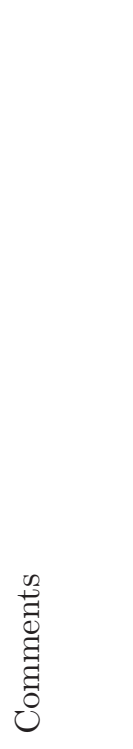 & 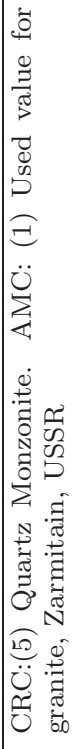 & 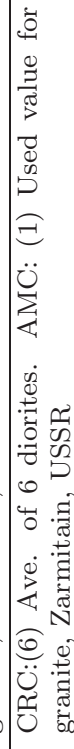 & 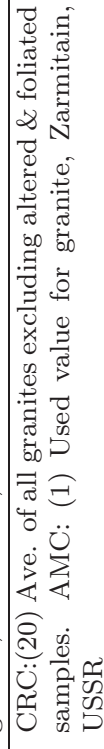 & 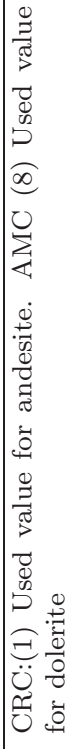 & 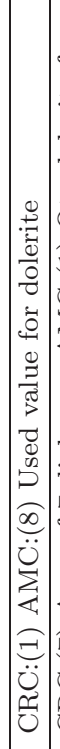 & 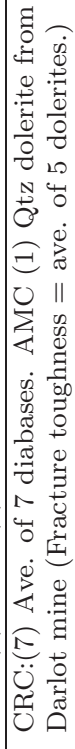 & 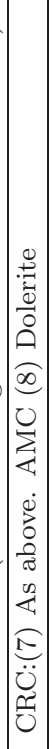 & 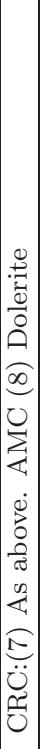 & 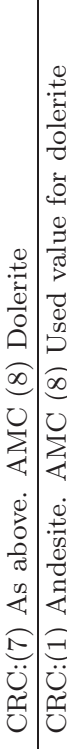 & 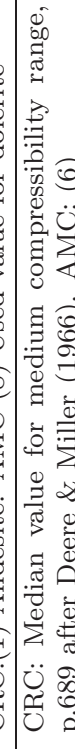 & 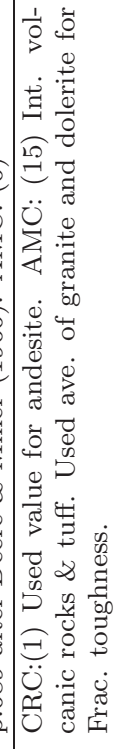 & 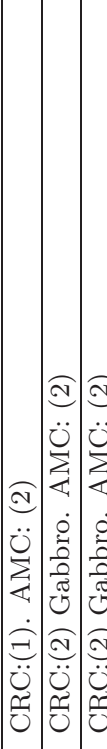 & 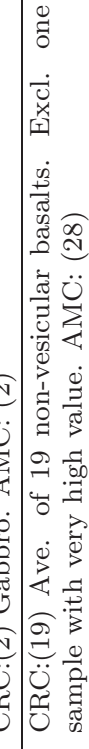 & 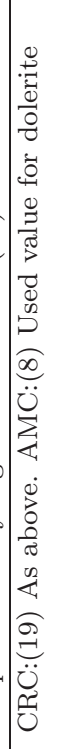 & 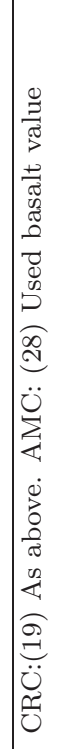 & 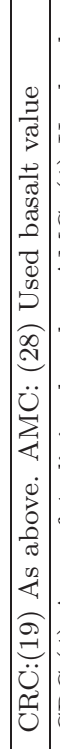 & 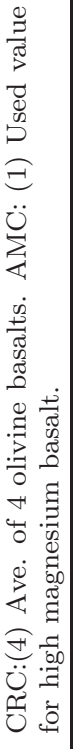 \\
\hline 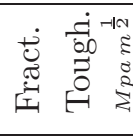 & $\vec{~}$ & - & E. & $\begin{array}{l}8 \\
\dot{2} \\
i \\
i\end{array}$ & & 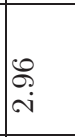 & 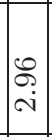 & $\begin{array}{l}8 \\
\stackrel{2}{2} \\
i\end{array}$ & 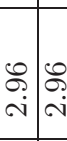 & : & & 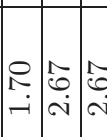 & 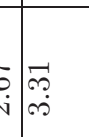 & 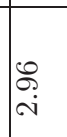 & $\vec{m}$ & $\vec{s}$ & $\begin{array}{l}\qquad 8 \\
\infty \\
\infty \\
\infty\end{array}$ \\
\hline 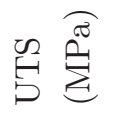 & 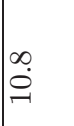 & 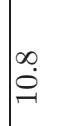 & 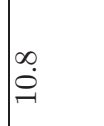 & $\begin{array}{r}\wedge \\
\ddot{\theta}\end{array}$ & $\begin{array}{r}1 \\
\dot{\theta} \\
\hdashline\end{array}$ & ?יב & $\begin{array}{r}-1 \\
\dot{\theta} \\
-1\end{array}$ & $\begin{array}{l}r \\
\stackrel{\sim}{0} \\
-\end{array}$ & 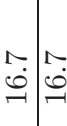 & 家 & $\stackrel{0}{\dot{\Delta}}$ & 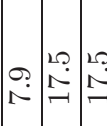 & ¿̊. & $\stackrel{\wedge}{\leftrightarrow}$ & :̈. & $\vec{\curvearrowright}$ & $\stackrel{9}{\bullet}$ \\
\hline$\overbrace{0}^{\infty} \overbrace{}^{\pi}$ & $\exists$ & $\exists$ & $\exists$ & $\stackrel{\omega}{N}$ & & ลิ & $\frac{20}{\stackrel{2}{*}}$ & $\begin{array}{l}\stackrel{2}{\mathrm{~N}} \\
\end{array}$ & $\stackrel{10}{\sim} \frac{10}{\sim}$ & 施 & $\exists$ & 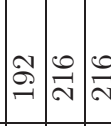 & $\vec{a}$ & $\frac{20}{N}$ & $\vec{\sigma}$ & $\vec{\sigma}$ & సै \\
\hline 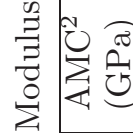 & 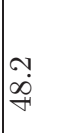 & 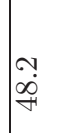 & $\mid \begin{array}{l}\mathcal{N} \\
\dot{\alpha} \\
\dot{q}\end{array}$ & ?ִ & $\left(\begin{array}{c}\infty \\
\dot{\infty}\end{array} \mid\right.$ & $\mid \begin{array}{l}20 \\
09 \\
0\end{array}$ & $\begin{array}{r}m \\
\dot{\infty} \\
\dot{\infty}\end{array}$ & $\begin{array}{l}\infty \\
\infty \\
\infty \\
\infty\end{array}$ & \begin{tabular}{l|l}
$\dddot{m}$ \\
$\dot{\infty}$ \\
$\dot{\infty}$
\end{tabular} & : & $\mid \begin{array}{l}\infty \\
\substack{10 \\
10}\end{array}$ & 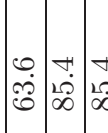 & $\dot{i} \vec{i} \dot{i}$ & $\ddot{\infty}$ & $\underset{i}{\stackrel{i}{i}}$ & $\left|\begin{array}{l}0 \\
\stackrel{i}{i}\end{array}\right|$ & $\begin{array}{l}\dddot{\infty} \\
\infty \\
\infty\end{array}$ \\
\hline 吾 & $\vec{i}$ & $\begin{array}{l}\infty \\
\dot{0} \\
\dot{\theta}\end{array}$ & $\begin{array}{l}1 \\
+ \\
\dot{b}\end{array}$ & $\mid \begin{array}{l}0 \\
\dot{1} \\
0\end{array}$ & $\left|\begin{array}{l}0 \\
\dot{0} \\
\dot{0}\end{array}\right|$ & $\begin{array}{l}\dot{\theta} \\
\dot{8}\end{array}$ & $\mid \begin{array}{l}\dot{\theta} \\
\dot{8}\end{array}$ & $\begin{array}{l}\dot{\theta} \\
\dot{8} \\
\dot{8}\end{array}$ & 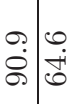 & $\dot{0}$ & $\begin{array}{l}0 \\
\dot{H} \\
\dot{H}\end{array}$ & 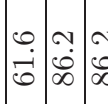 & 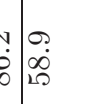 & $\mid \begin{array}{l}9 \\
0 \\
0 \\
10\end{array}$ & 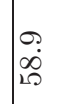 & $\mid \begin{array}{l}2 \\
0 \\
20 \\
10\end{array}$ & $\begin{array}{l}\dot{0} \\
\dot{8}\end{array}$ \\
\hline 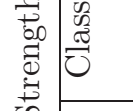 & $\sigma$ & $\sigma$ & $\sigma$ & $\infty$ & $\infty$ & $\infty$ & $\infty$ & $\infty$ & $\infty \mid \infty$ & $\infty$ & $\infty$ & 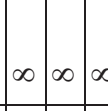 & O 1 & 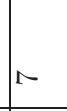 & N & 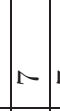 & 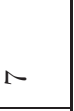 \\
\hline 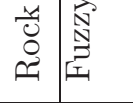 & $\begin{array}{l}\hat{1} \\
\infty \\
0 \\
0\end{array}$ & $\begin{array}{l}\vec{\infty} \\
0 \\
0\end{array}$ & 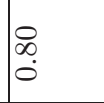 & R & $\left|\begin{array}{l}\infty \\
0 \\
0 \\
0\end{array}\right|$ & $\begin{array}{l}\hat{N} \\
0 \\
0\end{array}$ & & $\begin{array}{l}10 \\
1 \\
0\end{array}$ & $\begin{array}{lll}\mathbb{1} \\
\vdots \\
0\end{array}$ & $: \begin{array}{l}\hat{N} \\
\hat{N}\end{array}$ & $\stackrel{E}{E}$ & \begin{tabular}{l|l|l}
$r$ & 0 \\
0 & 0 & 0 \\
0 & 0 & 0 \\
0
\end{tabular} & : & 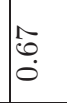 & : & $\mid$ & 苞 \\
\hline 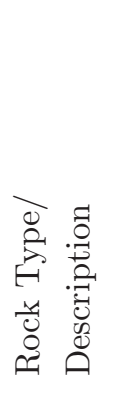 & 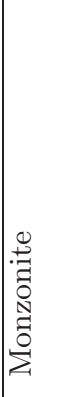 & 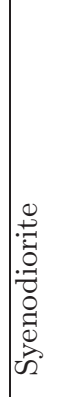 & 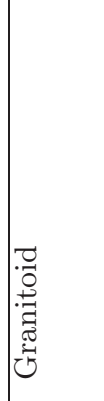 & 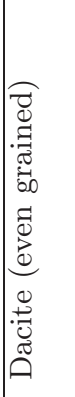 & 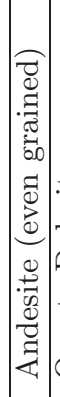 & 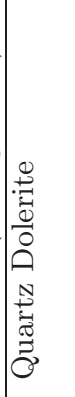 & 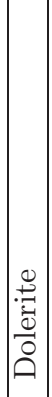 & 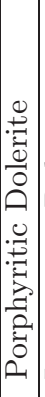 & 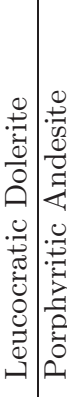 & 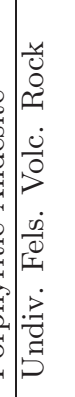 & 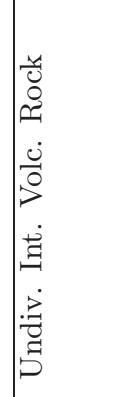 & 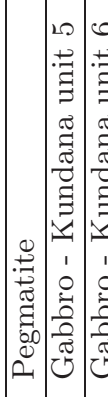 & 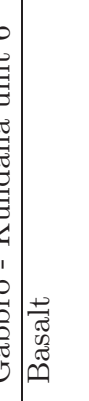 & 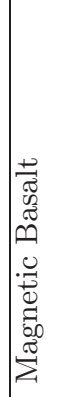 & 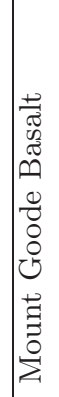 & 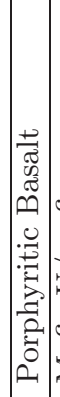 & 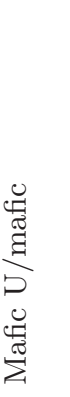 \\
\hline 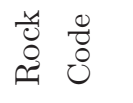 & 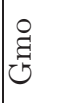 & 苟 & $\vec{U}$ & & & 吾 & & & & 在 & \pm & $\overrightarrow{0}$ & 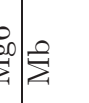 & $\frac{1}{\sum}$ & & $\vec{\Sigma}$ & \\
\hline
\end{tabular}




\begin{tabular}{|c|c|c|c|c|c|c|c|c|c|c|c|c|c|c|c|c|c|c|c|}
\hline 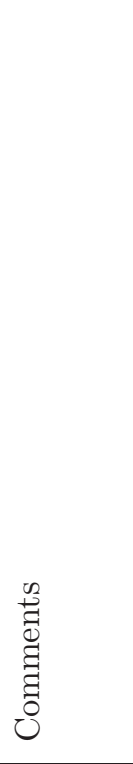 & 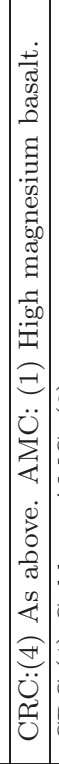 & & 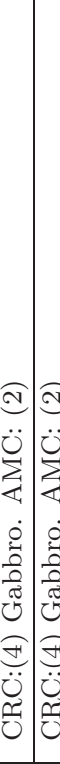 & 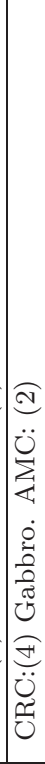 & 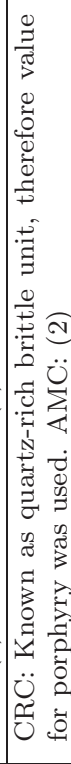 & 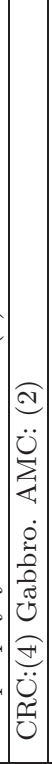 & 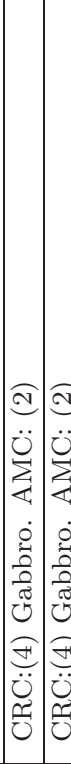 & 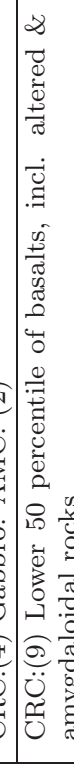 & 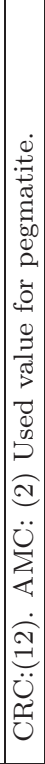 & 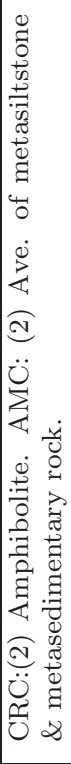 & 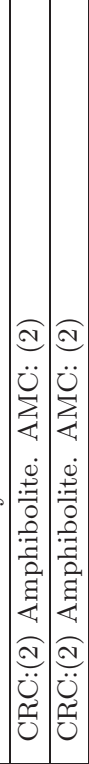 & 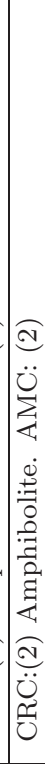 & 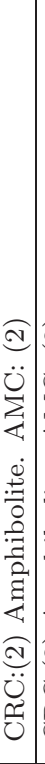 & & 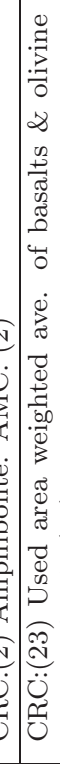 & 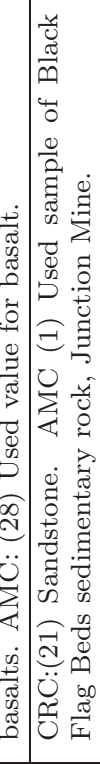 & 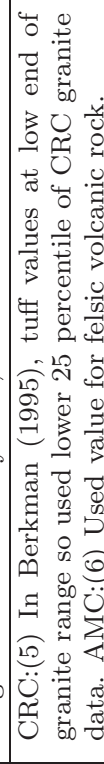 & 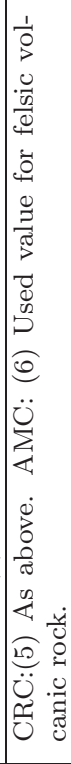 & 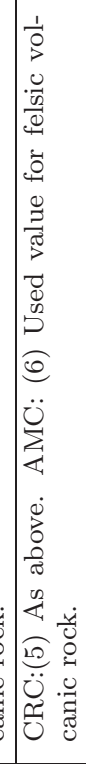 \\
\hline 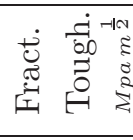 & $\mid \begin{array}{l}1 \\
0 \\
\infty \\
\infty \\
0\end{array}$ & & 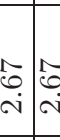 & 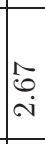 & & & 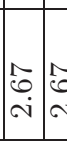 & $\hat{j}$ & & 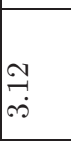 & 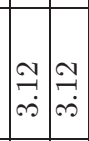 & & & $y=$ & $\overrightarrow{\dot{m}}$ & 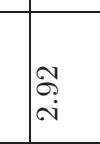 & $\stackrel{f}{\sim}$ & $\stackrel{f}{\stackrel{f}{-}}$ & I্ \\
\hline 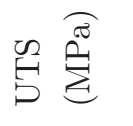 & $\begin{array}{l}0 \\
\dot{0} \\
-\end{array}$ & & $\begin{array}{l}\qquad \\
\dot{u} \\
\dot{u}\end{array}$ & $\stackrel{\bullet}{\stackrel{0}{-}}$ & $\begin{array}{l}\stackrel{0}{ } \\
\underset{+}{+}\end{array}$ & & $\begin{array}{ll}0 & 0 \\
\dot{I} & =\end{array}$ & $\dot{t}$ & مج & $\begin{array}{l}0 \\
\dot{N} \\
\dot{N}\end{array}$ & 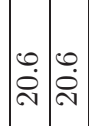 & & 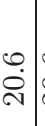 & & 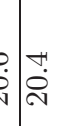 & $\overrightarrow{\mathrm{N}}$ & $\stackrel{\ominus}{\stackrel{9}{g}}$ & $\stackrel{\circ}{\dot{\rho}}$ & $\stackrel{\ominus}{\stackrel{9}{\rightarrow}}$ \\
\hline 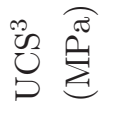 & 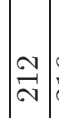 & & \begin{tabular}{l|l}
0 \\
$\stackrel{N}{N}$ & 0 \\
\end{tabular} & $\frac{\bullet}{\sim}$ & $\stackrel{\leftrightarrow}{\sim}$ & & $\underset{N}{0} \frac{0}{\sigma}$ & $\vec{\sigma}$ & :̊ & $\vec{\sim}$ & $\stackrel{\vec{N}}{\vec{\sim}}$ & & $\stackrel{-}{-}$ & & $\vec{g}$ & $\frac{\partial}{\vec{N}}$ & î & 总 & 㫌 \\
\hline 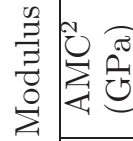 & $\begin{array}{c}\infty \\
\infty \\
\infty \\
\infty \\
\infty\end{array}$ & & $\begin{array}{lll}0 & 0 \\
i & 0 \\
i & 0\end{array}$ & & 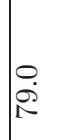 & & \begin{tabular}{l|l}
0 & 0 \\
$\dot{i}$ & 0 \\
$i$
\end{tabular} & ${ }_{i}^{i}$ & $\begin{array}{l}0 \\
\dot{\theta}\end{array}$ & $\dot{0}$ & \begin{tabular}{|c|c|}
0 & 0 \\
$\dot{i}$ & $\dot{i}$ \\
$i$ & $\dot{i}$
\end{tabular} & & $\begin{array}{l}0 \\
\dot{0}\end{array}$ & & 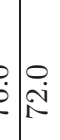 & 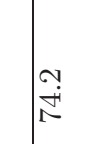 & $\underset{0}{\stackrel{0}{0}}$ & ְि & 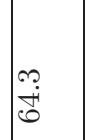 \\
\hline 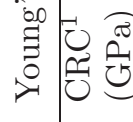 & & نे & 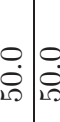 & & 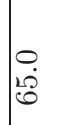 & & $\begin{array}{l}0 \\
\dot{i} \\
\left.i\right|^{2}\end{array}$ & 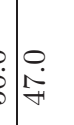 & 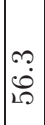 & $\begin{array}{l}\infty \\
0 \\
0 \\
0\end{array}$ & \begin{tabular}{l|l}
$\infty$ & $\infty$ \\
0 \\
$\dot{0}$ & 0 \\
0
\end{tabular} & & $\begin{array}{l}\infty \\
\dot{0} \\
\dot{0}\end{array}$ & $\begin{array}{l}0 \\
\dot{b} \\
\dot{b}\end{array}$ & \begin{tabular}{l|l}
$\dot{0}$ \\
$\dot{b}$
\end{tabular} & 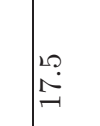 & ְ. & ஜே & 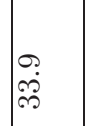 \\
\hline 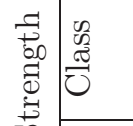 & 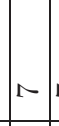 & & $\wedge$ & r & $N$ & $\wedge$ & 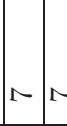 & -1 & 0 & 0 & 00 & 0 & 0 & 0.0 & 0 & 10 & 10 & 20 & 10 \\
\hline 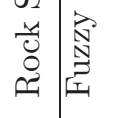 & $\vec{b}$ & & $\begin{array}{lll}\substack{0 \\
0} & 0 \\
0 & 0 & 0\end{array}$ & : & & & 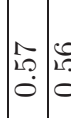 & $\begin{array}{l}: \\
:\end{array}$ & 索 & 苟 & 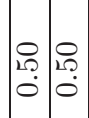 & & 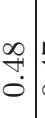 & & b. & I & 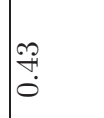 & F & F \\
\hline 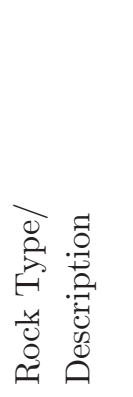 & 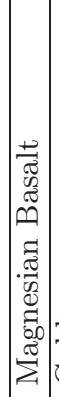 & & 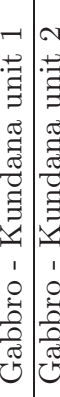 & 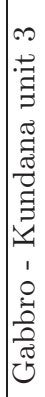 & 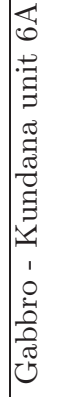 & 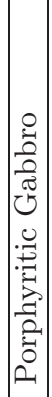 & 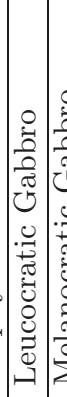 & 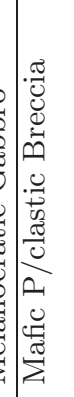 & 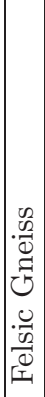 & 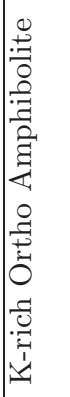 & 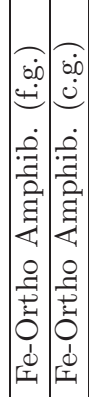 & 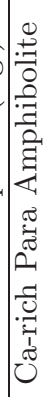 & 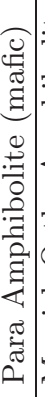 & & 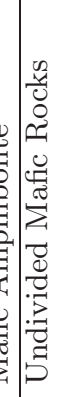 & 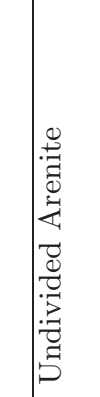 & 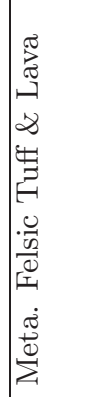 & 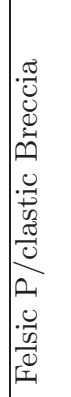 & 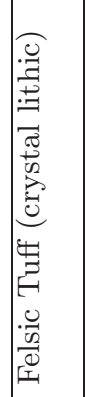 \\
\hline 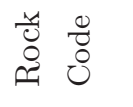 & छे & & & & 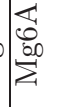 & & $\bar{x}_{10}^{00}$ & : & & 岁 & : & & & & & 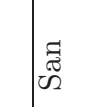 & 鿒 & $\begin{array}{l}\frac{x}{2} \\
\frac{2}{2} \\
\frac{1}{2}\end{array}$ & 节 \\
\hline
\end{tabular}




\begin{tabular}{|c|c|c|c|c|c|c|c|c|c|c|c|c|c|c|c|c|c|}
\hline 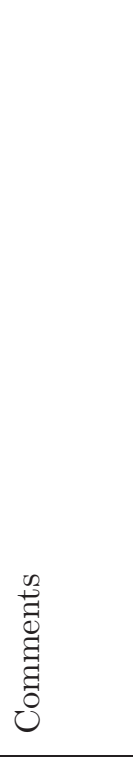 & 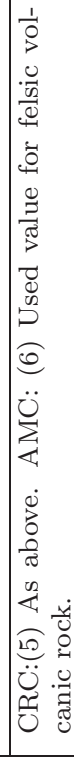 & 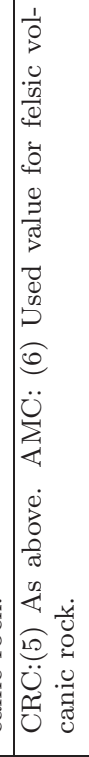 & 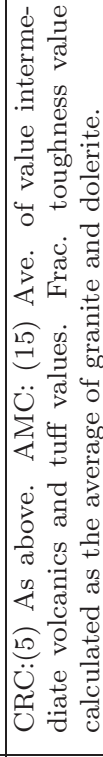 & 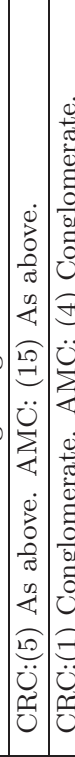 & 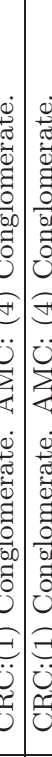 & 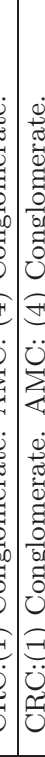 & 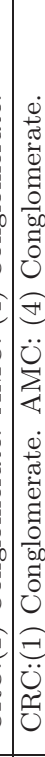 & 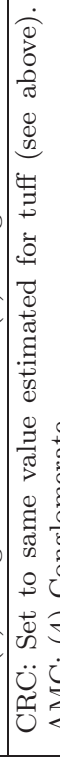 & 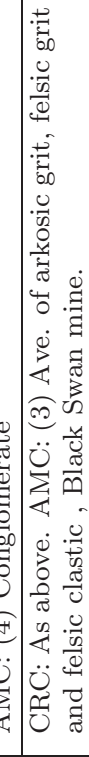 & 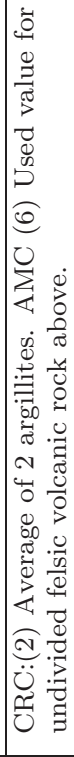 & 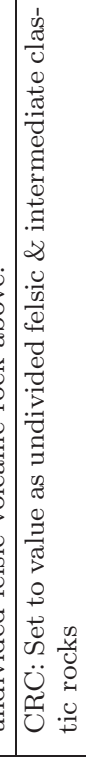 & 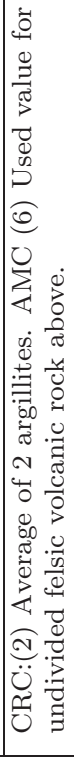 & 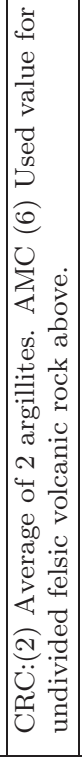 & 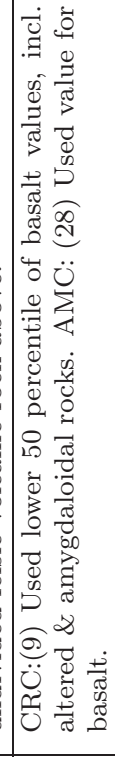 & 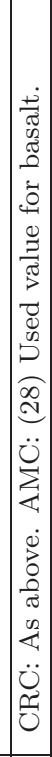 & 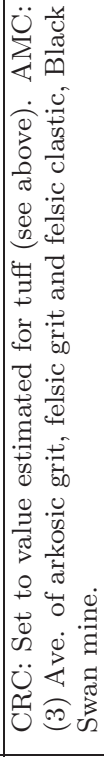 & 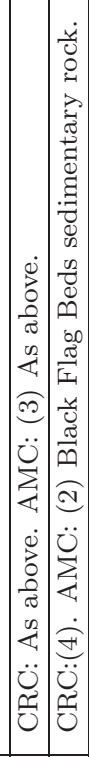 \\
\hline 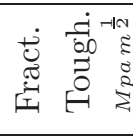 & $\underset{-}{\stackrel{7}{-}}$ & $\stackrel{\mathcal{F}}{\underset{\sim}{\rightarrow}}$ & 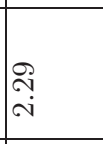 & 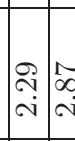 & 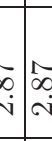 & $\begin{array}{c}\infty \\
\infty \\
\infty \\
i \\
\sim\end{array}$ & 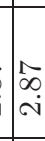 & & $\stackrel{0}{\stackrel{-}{-}}$ & Fै & 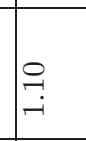 & $\stackrel{\stackrel{F}{\sharp}}{\rightarrow}$ & $\underset{\sim}{\stackrel{f}{二}}$ & $\vec{m}$ & के & $\stackrel{0}{=}$ & 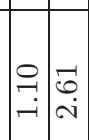 \\
\hline 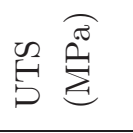 & $\stackrel{0}{9}$ & $\stackrel{0}{\dot{\theta}}$ & $\stackrel{0}{\stackrel{\leftrightarrow}{\dot{N}}}$ & 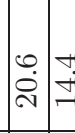 & & $\stackrel{+}{+}$ & $\stackrel{I}{-}$ & $\stackrel{H}{\longrightarrow}$ & $\begin{array}{l}\mathcal{y} \\
\stackrel{\leftrightarrow}{9}\end{array}$ & $\stackrel{\circ}{\stackrel{0}{\rightarrow}}$ & $\begin{array}{l}\tilde{y} \\
\ddot{\varphi}\end{array}$ & $\stackrel{\circ}{\dot{\rho}}$ & 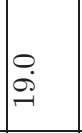 & ஜே. & $\begin{array}{l}\overrightarrow{1} \\
\stackrel{\leftrightarrow}{\mathbf{N}}\end{array}$ & \begin{tabular}{l}
\multirow{2}{*}{} \\
$\vdots$ \\
\hdashline
\end{tabular} & 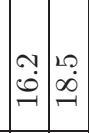 \\
\hline 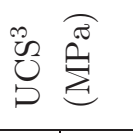 & î & i⿱乛龰 & $\exists$ & $\exists$ & & $\exists$ & $\exists$ & & $\stackrel{\cong}{\cong}$ & 飺 & ָิ & 总 & 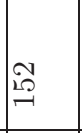 & $\vec{\sigma}$ & $\vec{\sigma}$ & ָิ & 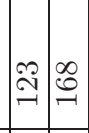 \\
\hline 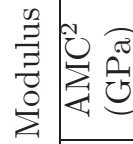 & Pִ & & $\begin{array}{l}\infty \\
\substack{10 \\
10} \\
10.0\end{array}$ & \begin{tabular}{l|l}
$\infty$ & - \\
1 & 10 \\
10 & 10 \\
10
\end{tabular} & & 20 & $\frac{1}{10}$ & & $\stackrel{0}{\overrightarrow{0}}$ & 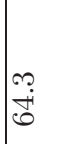 & $\begin{array}{l}0 \\
\dot{\theta}\end{array}$ & جִ & 承 & $\underset{i}{\stackrel{i}{i}}$ & $\begin{array}{l}\vec{i} \\
\vec{i} \\
\mathrm{i}\end{array}$ & $\mid \begin{array}{l}0 \\
\dot{\theta}\end{array}$ & \begin{tabular}{l|l}
0 \\
$\dot{\vec{H}}$
\end{tabular} \\
\hline 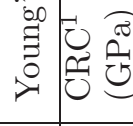 & $\underset{m}{\infty}$ & $\begin{array}{l}\dot{p} \\
\dot{m} \\
\ddot{m}\end{array}$ & $\stackrel{\dddot{m}}{\dddot{m}}$ & 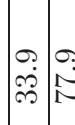 & & 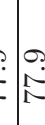 & $\frac{2}{R}$ & ڤ̊. & 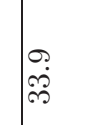 & ஓ̆丶 & 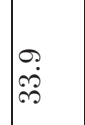 & 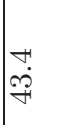 & $\stackrel{\ddot{\leftrightarrow}}{\stackrel{\leftrightarrow}{\leftrightarrow}}$ & 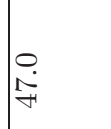 & 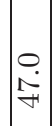 & $\ddot{œ}$ & 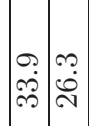 \\
\hline 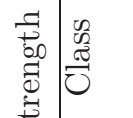 & 10 & 10 & 10 & 1020. & . 10. & 20 & 10 & 20 & H & $\forall$ & r & $r$ & $\nabla$ & $\nabla$ & + & P & 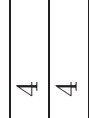 \\
\hline 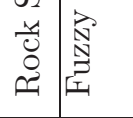 & f & 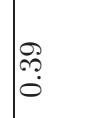 & 文 & 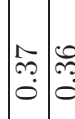 & & : & 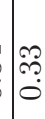 & ?ֶ. & $\overrightarrow{0}$ & ๑̊: & 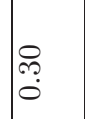 & श्: & $\mid$\begin{tabular}{l}
$\infty$ \\
\multirow{2}{*}{} \\
\hdashline
\end{tabular} & ָ̃ & $\mid \begin{array}{c}0 \\
\stackrel{2}{*} \\
0\end{array}$ & 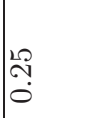 & స̂. \\
\hline 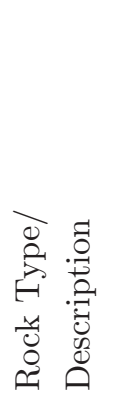 & 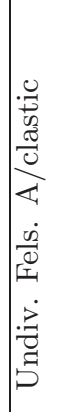 & 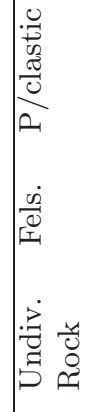 & 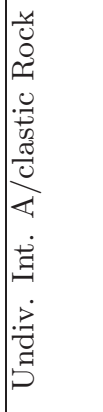 & 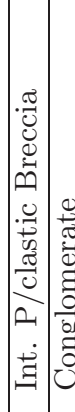 & 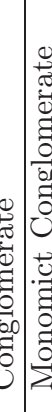 & 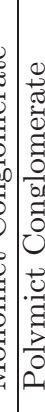 & 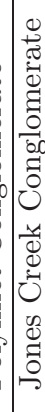 & 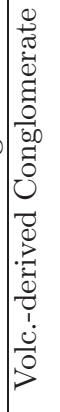 & 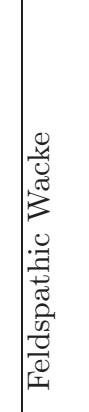 & 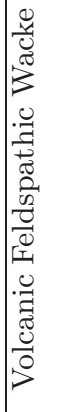 & 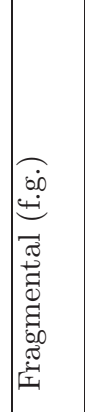 & 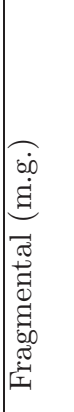 & 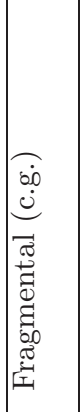 & 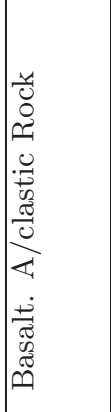 & 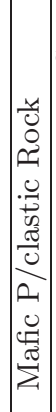 & 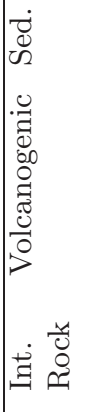 & 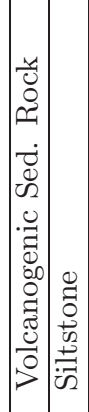 \\
\hline 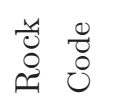 & 荍 & 5 & $\stackrel{x}{\Delta}$ & ن. & 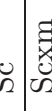 & 2 & & 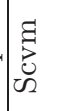 & $\frac{3}{\omega 2}$ & $\sum_{5}^{3}$ & 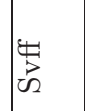 & 苛 & 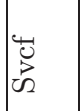 & 炎 & & 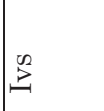 & 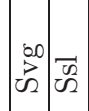 \\
\hline
\end{tabular}




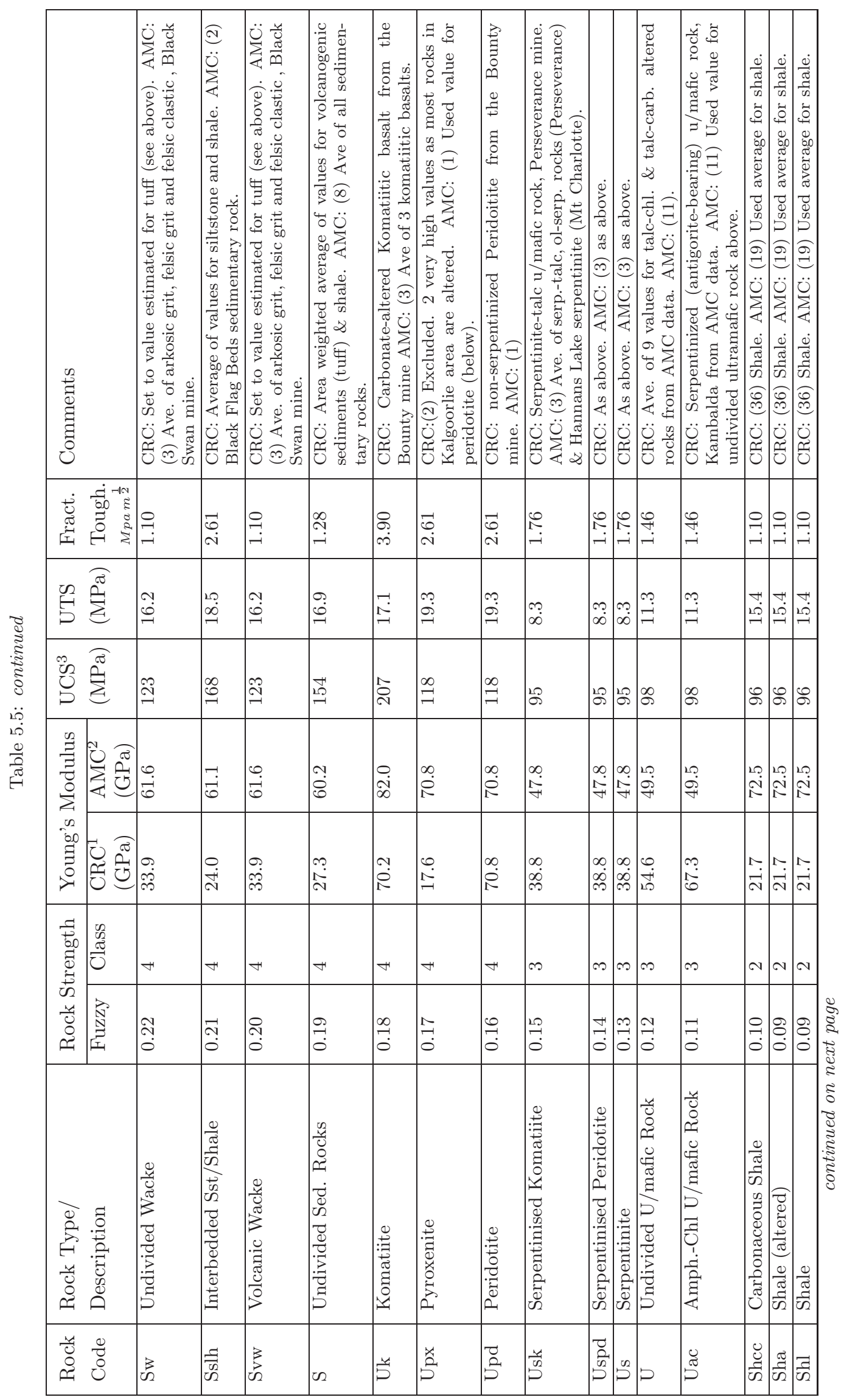




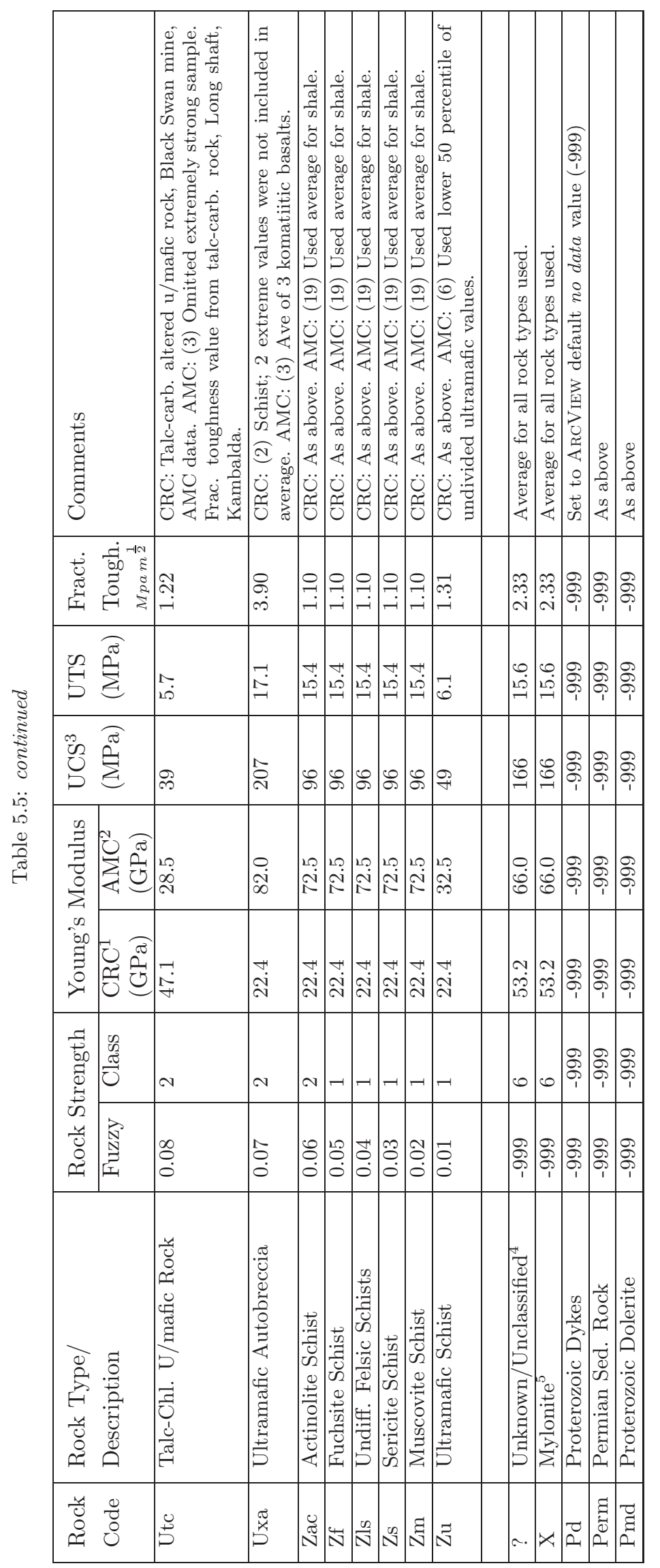

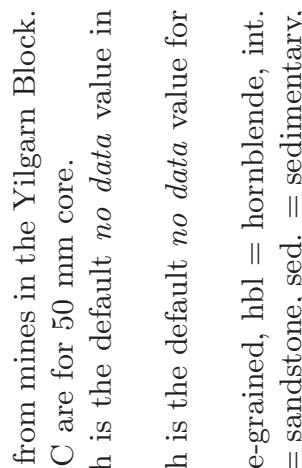

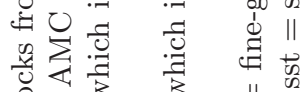

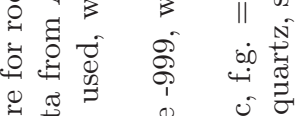

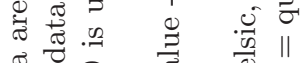

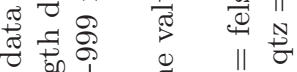

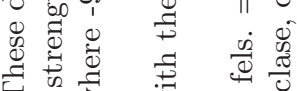

$\therefore 3$ i 80

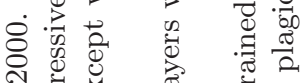

궁

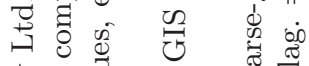

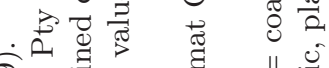

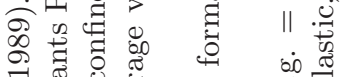

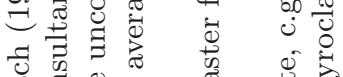

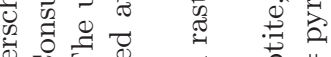

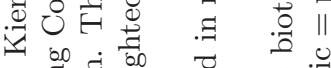

$\$ . 尹$

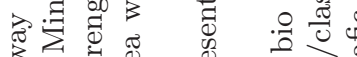

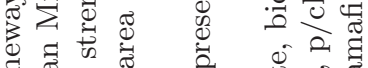

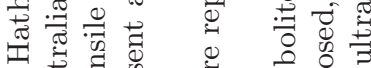

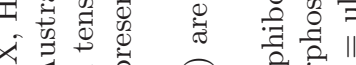

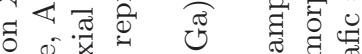

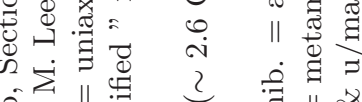

Lी

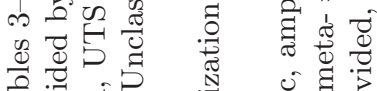

बह

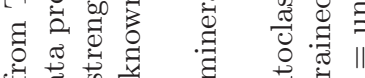

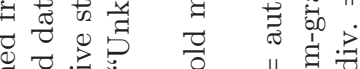

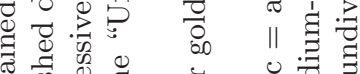

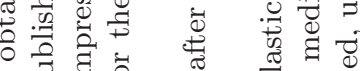

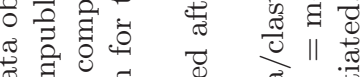

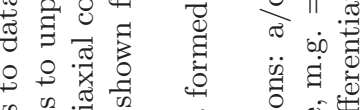

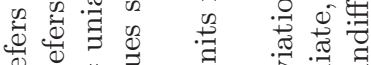

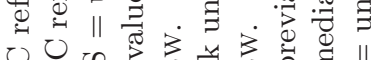

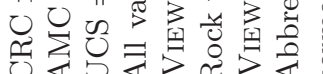

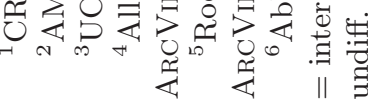




\subsubsection{Goldfields Pty Ltd Data Sets Not Used in Study}

Primary GIS layers described above represent all the available data sets that provide uniform coverage over the complete complete study area and which are considered to be useful in delineating prospective areas for orogenic gold deposits at a regional scale.

A regolith geochemistry data set, containing point locations for 13,503 auger holes together with the maximum gold concentration in ppb, covers a $23 \times 7 \mathrm{~km}$ north-south strip from Broad Arrow to Bardoc, and a percussion drill hole geochemistry data set containing the maximum gold and arsenic concentrations for 112,366 drill hole locations along the Bardoc Tectonic Zone were not included in this study, because they did not cover the entire study area.

In order to include data sets with incomplete coverage, a series of neural networks would have to be constructed so that parts of the study area where ten layers were available could be processed using a network with ten inputs and areas where nine data sets were available would be processed using a network with nine inputs and so on. An alternative would be to devise a method of filling in the missing areas with values that would be neither favourable nor unfavourable. However, a simple average is not suitable because background geochemical values, for example, vary with different lithologies. The problem of how to deal with missing data in a neural network analysis is an important one since most exploration data sets consist of areas of detailed information that do not all overlap. However, this issue requires further research and will most likely involve considerably more software development and is therefore the scope of the present study.

Two other data sets were available but not used in this study. These are a gamma-ray survey and a composite LANDSAT image. Both these data sets provide information about the surface mineralogy and, in the Kalgoorlie area where very little outcrop exists, the data sets are mainly useful for identification of regolith layers.

\subsection{Derived GIS Layers}

This section describes how the main set of layers that were used as inputs to the neural network analyses were prepared. All the GIS layers that form the inputs to neural networks except the metamorphic grade and deposits layers, are derived by processing the primary GIS layers described above in order to make an exploration criterion or ingredient of the mineral systems model for orogenic gold deposits more explicit. For example, the 
structural layer contains a variety of faults and shear zones, although it is known that most deposits in the Kalgoorlie area are associated with crustal-scale NW-trending shear zones. Consequently, rather than present the network with a binary map indicating the presence of absence of faults, faults were separated into separate GIS layers according to scale and orientation and converted so that each cell in a new raster format map represents the proximity to the nearest fault of the specific type displayed in that GIS layer. Raw input parameters that have been modified in this way are referred to as features in the pattern recognition literature. The more effectively the input features presented to a neural network capture the underlying process responsible for the output variable (in this case, the favourability of a cell location for a gold deposit), the more effectively the network can learn the patterns in the data.

\subsubsection{Simplified Geology Layers}

The legend used by Goldfields Exploration Pty Ltd for the Bardoc Tectonic Zone and Kalgoorlie maps was simplified from 108 to 25 rock types (Table 5.2). Only 22 rock types appear on the legend, however, because lithological units corresponding to three of the rock types do not occur within the Kalgoorlie study area (i.e. as units mapped at 1:100000 scale; chert,iron formation, felsic gneiss and quartzite) and two units are not shown because they post-date gold mineralization (i.e. Proterozoic Dolerite and Permian Sedimentary Rock are represented as no data $=-999$, Table 5.2).

The simplified geology layer was converted to raster format and exported as an ASCII file. The ASCII file was imported into MATLAB (geol_25.mat) and used to create other derived layers, i.e. the 1-of-n-coded geology and favourable geology layers, described below. The simplified geology layer comprising 22 rock types is shown in Figure 5.7.

A raster-format, solid-geology map with 55 rock types was also derived from the original 108 rock-type, solid-geology layer (l1_l_ass_t55_nohdr.asc). This map was used to produce the distance-to-magnetic-anomaly and density-of-lithological-contacts layers described below. It was not used in the neural network experiments described in Chapter 7 directly, because the statistical relationship between deposits and each of the 55 lithologies is less reliable due to the fact that many rock types contain fewer than five known deposits. 


\subsubsection{Binary Deposit Layer}

The primary deposit layer is a point data set in vector format. This was converted to a binary raster layer. As a preliminary step, deposits containing a resource of at least $1000 \mathrm{~kg}$ total contained gold were selected and a new attribute to store binary values was added to the database table attached to the vector deposit layer. All records for this field were initially set to one using the Field Calculator function. Converting the vector layer to a grid file (using a built-in ARCVIEw function) then resulted in a grid in which all deposits were represented by ones (124_d_bin1000; CD-ROM, vol.1). However, barren cells all contained a special No Data value (-9999) by default. Barren cells were converted to zeros using the ARCVIEw Map Calculator function and the following command;

([l24_d_bin1000].IsNull.Con(0.AsGrid,[l24_d_bin1000]))

\subsubsection{Distance to Dolerite, Syenite, \& Feldspar Porphyry Layers}

Binary GIS layers showing the proximity to the nearest contact for several particularly favourable rock types and a potentially favourable rock type, were created from the solid geology layer. Binary layers were created in ARCVIEw by adding a new attribute field to the solid-geology shape file table, using the built-in field calculator function to select polygons with a simplified rock code = "pf" (Table 5.2) and then creating a grid theme based on the new attribute field. Binary grids contain 1's to indicate the presence of the favourable rock type and all the remaining cells contain zeros.

Quartz dolerite and leucocratic dolerite were combined into a binary layer (GIS layer l18_l_mdql on the attached CD-ROM, vol.1). These are particulary favourable host rocks as quartz dolerite forms a strongly mineralized unit within the thick Golden Mile Dolerite, which hosts the giant Golden Mile deposit. In addition, quartz dolerite and leucocratic dolerite are relatively competent, brittle units (Table 5.5) and both, particularly the leucocratic dolerite, have high $\mathrm{Fe}_{\text {tot }} \times \mathrm{Fe} /(\mathrm{Fe}+\mathrm{Mg}+\mathrm{Ca})$ (Phillips, 1986, and Table 5.4).

Feldspar porphyry units (feldspar porphyry, feldspar-quartz-biotite porphyry, feldsparquartz-hornblende porphyry, feldspar-quartz porphyry, plagioclase \pm hornblende porphyry) were also combined to form a binary layer (GIS layer $l_{4}-l-p f$ on the attached CD-ROM, vol. 1). As noted above (in Section 5.2.4) porphyritic intermediate to mafic intrusions are in places associated with gold mineralization and may indicate a connection between ore fluids with the lower crust (S.Halley, pers.comm., 2000). Also, porphyritic intrusions are 
quartz-bearing and therefore very brittle (Table 5.5)

A binary layer was also created by combining syenite and syenodiorite units (GIS layer l19_l_gsy on the attached CD-ROM, vol.1). Robert (1997) has noted that, in the Abitibi greenstone belt in Canada, gold ore bodies occur within and along the margins of syenitic stocks, and along faults and lithological contacts away from any intrusions. Orebodies in such positions are interpreted to represent proximal to distal components of large magmatic-hydrothermal systems centred on, and possibly genetically related to, composite syenitic stocks. A similar association has also been suggested by D. Groves (pers. comm., 2000). Syenite and synenodiorite are also relatively competent and brittle rock types (Table 5.5).

The binary rock-type layers were used to create a new set of layers, in which each cell value contains the distance to the nearest lithological contact of one of the selected rock types. The following procedure was used; first the analysis mask was set to an existing raster layer, in which all the no data areas are flagged with value -9999. Second, the zero values in the binary grids were converted to nulls (i.e. a special value used by ARCVIEW to signify no data, using the following map calculator command in the ARCVIEW analysis menu (where the porphyry layer $l_{4} l_{-}$p $f$ is used as an example);

$$
\left(\left[l_{4} l_{-} p f_{-} d i s t\right]=0 . \text { AsGrid }\right) . \operatorname{SetNull}\left(\left[l_{4} \_l \_p f_{-} d i s t\right]\right) .
$$

The third step was to convert the binary layer to a layer containing distances using the ArcVIEW analysis function find distances. The last step in the procedure was to convert the resultant temporary file to a permanent grid file (i.e. layers $l_{4} l_{-} p f_{-}$dist shown in Figure 5.16, l18_mdql_dist, and 19_gsy_dist).

By overlaying the known deposit points over the quartz dolerite and syenite layers, it was possible to determine that, although an association of deposit points and the favourable lithologies appears to exist at a local scale, these rock types have not been mapped in units large enough to appear in more than one or two locations at the regional scale of the solid geology. Consequently, these two binary maps were discarded as possible input layers for neural network analysis.

\subsubsection{Distance to Lithological Contacts}

Previous prospectivity studies on orogenic gold deposits (Knox-Robinson, 1994; Yun, 2000) have both shown that proximity to lithological boundaries is an important factor control- 
ling the location of deposits. Depending on the adjacent rock types, lithological boundaries may be the site of rheological and a chemical contrasts. The GIS layer representing the distance to the nearest lithological contact (139_c_dist) was created from the solid geology layer (i.e. geology.shp; a polygon layer in vector format) using the procedure described in Section 5.5.3 (Fig. 5.17).

\subsubsection{Distance to Faults and Shear Zones}

Separate layers were created from the primary structural layer (faults.shp) for each of the fault and shear zone types, shown in Table 5.6.

Table 5.6. Characteristics of fault and shear zone types in primary GIS layer faults.shp used to form separate derived GIS layers.

\begin{tabular}{|l|l|l|l|}
\hline $\begin{array}{l}\text { Fault } \\
\text { Type }\end{array}$ & $\begin{array}{l}\text { Strike } \\
\text { Direction }\end{array}$ & $\begin{array}{l}\text { Definition } \\
\text { (Deformation event) }\end{array}$ & $\begin{array}{l}\text { Attribute value } \\
\text { in faults.shp }\end{array}$ \\
\hline \hline crustal & NNW & $\geq 100 \mathrm{~km}, \mathrm{D}_{2}{ }^{1}$ & shear \\
\hline regional & NNW & $\geq 10 \mathrm{~km}, \mathrm{D}_{2}$ & shear \\
\hline local & NE & $\leq 10 \mathrm{~km}$, dextral, $\mathrm{D}_{3}{ }^{2}$ & fault \\
\hline local & $\mathrm{E}$ & $\leq 10 \mathrm{~km}$, sinistral & fault \\
\hline
\end{tabular}

Crustal- and regional- scale structures were separated from the other faults on the basis of length, whilst the two classes of faults were separated on the basis of orientation. Crustal-scale faults and shear zones are defined as those greater than or equal to $100 \mathrm{~km}$ in length and regional faults as those at least $10 \mathrm{~km}$ in length. An additional attribute called faultclass was added to a copy of the faults layer (struct_types.shp). All the line segments corresponding to a particular type of fault/shear zone were then selected individually using the ARCVIEw select-feature tool and then a numeric value was assigned to the faultclass attribute for the selected features using the field-edit function. Later, all the records for each of the fault/shear zone types were selected and converted to raster-format layers (i.e. grid files 120_s_crust, 121_s_shear, l22_s_flt_ne, 123_s_flt_e). The procedure to convert these

\footnotetext{
${ }^{1} \mathrm{D}_{2}$ refers to upright folding an high-angle reverse faulting in a structural evolution of the Yilgarn Block, which spans events $\mathrm{D}_{E}, \mathrm{D}_{1}-\mathrm{D}_{4}$.

${ }^{2} \mathrm{D}_{3}$ to $\mathrm{D}_{4}$ refer to sinistral and/or dextral oblique-slip faulting and reactivation of earlier formed faults.

${ }^{3}$ The east-west oriented faults appear correspond to the emplacement of Proterozoic dolerite dykes and therefore post-date the main period of gold mineralization. These faults could, however, be associated with the remobilization of earlier deposited gold.
} 
single theme raster grids to distance grids (120_f1_dist, 121_f2_dist, 122_d3_dist, 123_d4_dist) was the same as that for the lithological contacts described above (5.5.3). The layers are shown in Figures 5.18-5.20 below.

\subsubsection{Distance to Fold Axes}

The position of major anticlinal and synclinal axes were obtained by combining existing maps produced by Swager \& Griffin (1990); Swager et al. (1990), Yun (2000) and Goldfields Exploration Pty Ltd. The line segments were traced over the existing maps to create a new vector format layer; folds.shp. In a second step, the attribute tables were queried to select all the anticlines and synclines in separate steps to create temporary raster grids containing either just anticlines or synclines and then in a third step, distance grids were created using the same procedure described for the lithological contacts above (5.5.3). The name of the grid files containing the distance to anticlinal and synclinal axes are l46_dist_anti and l46_dist_sync, respectively (Fig. 5.21 and 5.22).

\subsubsection{Distance to Intersection of Regional Faults}

The procedure to create a layer representing the distance to the nearest intersection of a NNW-trending and NE-trending regional fault 5.4 consists of two parts. The first step was to create a layer showing the intersection of the two fault types using layers $121 \_s_{-} f 2$ and 122_s_d3 in ARCVIEW. The result of this step is a temporary file (Map Query, also save as a permanent grid; 132_s_f2f3) which was then used as an input to the ARCVIEW analysis function, find distance to produce the distance grid, l33_s_f23gdst (Fig. 5.23).

\subsubsection{Distance to Magnetic Anomalies}

An inspection of the deposit layer superimposed over the total magnetic intensity map (15_m_rtp) in ARCVIEW revealed that there is an association of deposits with magnetic anomalies, but that the deposits are located close to the anomalies and not everywhere within the anomalies. Consequently, proximity to magnetic anomalies appeared to be a more useful predictor of deposit locations.

Since the total magnetic intensity values depend on the average or background values, and these in turn depend on the magnetic susceptibility of the rock, the approach used here was to calculate the z-score for each rock type separately; i.e. the difference between each 
1. Create $\mathrm{f} 2-\mathrm{d} 3$ fault intersection grid

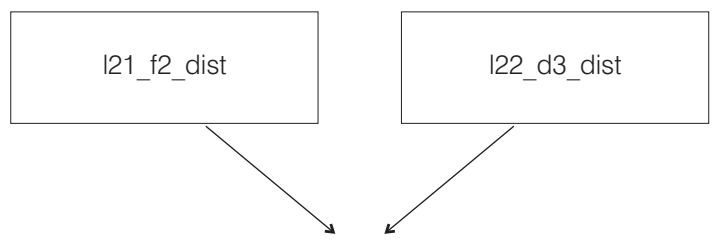

Analysis -> Map Query:

$\left.\left(\left[121 \_f 2 \_d i s t\right]\right)<100\right)$ AND $\left.\left(\left[122 \_d 3 \_d i s t\right]\right)<100\right)$

Map Query 1

(temporary grid)

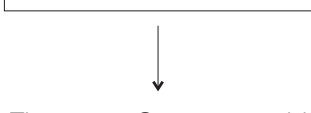

Theme -> Convert to grid

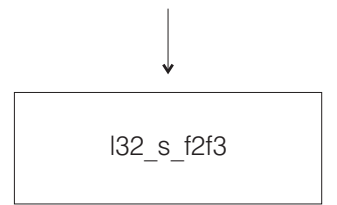

2. Create distances to nearest f2-d3 fault intersection grid

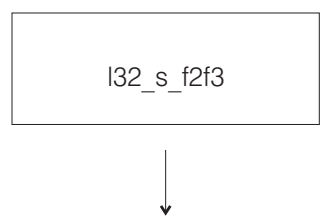

Analysis -> Map Calculator:

([Map Query 1] = 0.AsGrid).SetNull([Map Query 1])

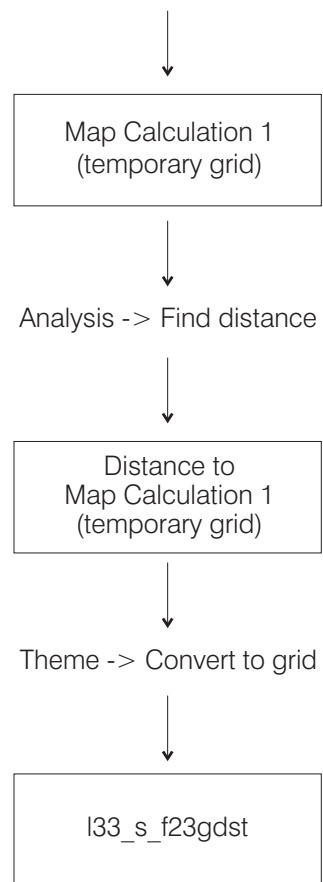

Figure 5.4. Procedure used to create a GIS raster layer showing distance to the nearest intersection of regional NNW- \& NE-trending faults. The steps refer to ARCVIEW 3.1 commands. 
cell value and the average value expressed as numbers of of standard deviations. A solid geology layer with 55 rock types was used for this purpose. The z-scores were calculated for each rock type according to the following formula:

$$
Z=\frac{x_{\text {rock }}-\mu_{\text {rock }}}{s_{\text {rock }}}
$$

The procedure to produce the distance-to-magnetic-anomaly maps comprised three steps;

1. calculate z-scores,

2. create a set of binary anomaly maps, and

3. calculate distance grids.

Different threshholds were used in each map to define the anomalies. Figure 5.5 shows the processing steps in detail. Distance-to-magnetic-anomaly maps, 114_mz-1_dist, l14_mz1_dist, and 114_mz2_dist, were created for positive and negative anomalies using z-score threshold values of $-1,+1$, and +2 , respectively.

\subsubsection{Aeromagnetic First and Second Vertical Derivatives}

Layers showing the first and second vertical derivative of the airborne total-magnetic intensity survey-data were created in the GEosoft Oasis Montaj software package builtin functions (see layers $16 \_\mathrm{m} \_1 \mathrm{vd}$ and $17 \_\mathrm{m} \_2 \mathrm{vd}$ in the ARCVIEW directory on the attached CD-ROM, vol. 1).

\subsubsection{Strike of Faults and Contacts}

The fault-strike layers, as well as the contact-strike, rheological and chemical contrast layers, were produced by Stephen Gardoll using ARC/InFo. Fault-strike layers were processed using an AML script written by Dr Carl Knox-Robinson (see Knox-Robinson et al., 1996, p. 5-13). The following steps were required;

1. import faults.shp (ARCVIEW shape file) into ARC/Info,

2. convert shape file to ARC/INFO polygon coverage (.pat file) using the shapearc command,

3. execute ARC/INFO build command to establish polygon topology, 
1. Calculate Z-scores
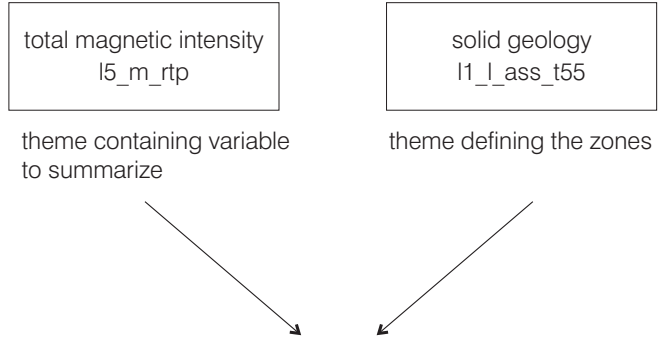

theme defining the zones

Analysis -> Summarize zones

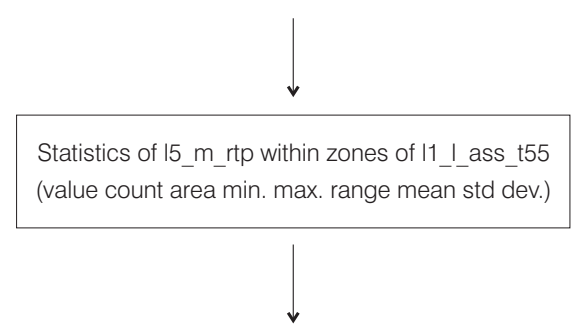

join stats table to geology table (via common value field)

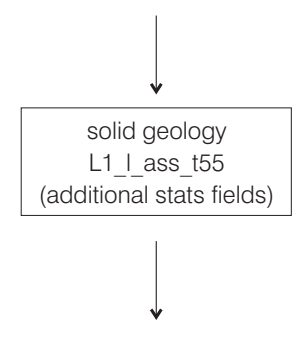

analysis - map calculator

([15_m_rtp] - [l1_I_ass_t55.Mean]) / [11_I_ass_t55.Std])

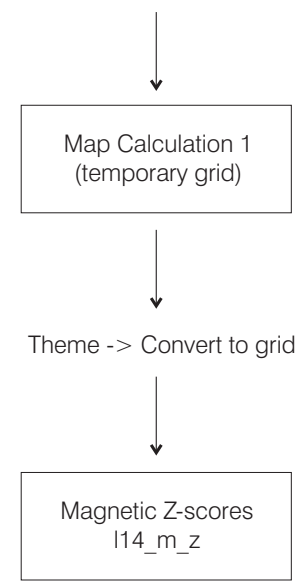

\section{Create binary anomaly maps} using Z-score threshold

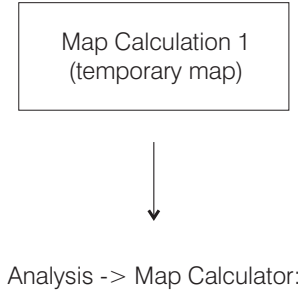

([Map Calculation 1] > = 1.AsGrid)

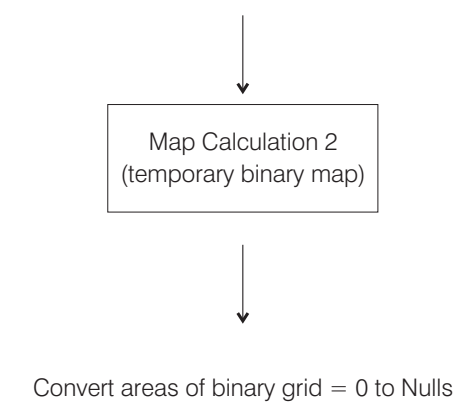

Analysis -> Map Calculator:

([Map Calculation 1] $=$ 0.AsGrid).SetNull([Map Calculation 1] $)$

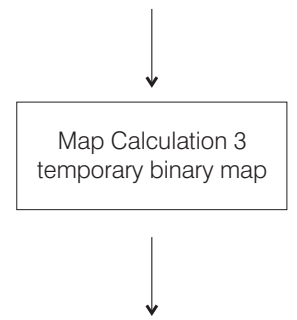

3. Create distance grid

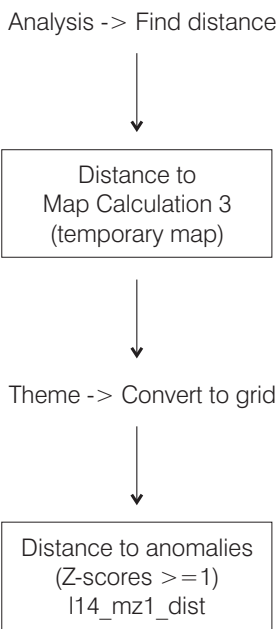

Figure 5.5. Procedure used to create a GIS raster layer showing distance to the nearest magnetic anomaly. Magnetic anomalies are defined in terms of z-scores. Note; the value field used to create a relational join between the summary statistics and geology tables is a unique identifier for rock type. 
4. execute AML to apply the ARCEDIT spline command, which inserts vertices into the line segments representing the faults. The grain parameter was set to produce vertices at intervals of 100 metres. As a result of the spline command, all the attributes of the original line segment are retained. A new attribute was added to the table to store the strike angles (additem command), which are calculated in the next step. The AML reads the arc attribute table (.aat) file associated with the line coverage; for each original fault-line segment, the script reads the coordinates of each pair of vertices defining the 100 metre segments and calculates the orientation of the each segment using the trigonometric identity; $\arctan \theta=$ opposite/adjacent and stores the strike value,

5. create distance grid by applying ARC/INFO command, eucdistance, "assign by value". The output of this command is a raster grid. Instead of setting the value of each cell to the distance to the nearest fault as in the standard command, cell values are set to the strike of the nearest 100 metre fault segment, and

6. re-calculate all strikes in the range $\left[180^{\circ}-360^{\circ}\right]$ to the equivalent angle in the range $\left[0^{\circ}-170^{\circ}\right]$. Angles were converted to avoid two identically oriented line segments (i.e. contacts or faults) being described by two different angles (e.g. $0^{\circ}$ is equivalent to $\left.180^{\circ}\right)$.

Layers showing the strike of crustal-scale, NNW-trending, NE-trending and E-trending regional-scale faults and lithological contacts (128_s_f1str, 129_s_f2str, 130_s_f3str, 131_s_f4str, and 139_c_str, respectively) are shown in Figures 5.25-5.27.

\subsubsection{Rheological Contrast}

Rheological-contrast layers were prepared from the seven rock-strength parameters described in Section 5.4.8 above. These are; subjective estimates (expressed as 10 classes and fuzzy membership values), Young's modulus, uniaxial compressive strength, uniaxial tensile strength and fracture toughness. Two different data compilations were used for Young's modulus; those of Hatheway \& Kiersch (1989) and M. Lee (unpublished data from Australian Mining Consultants Pty Ltd, 2000). Rheological contrast layers, 136_l_rh1-6, are listed in Table 5.7 below. All these layers are derived from the vector-format, solidgeology layer, geology.shp, which contains the rock strength properties in an attribute table. Units post-dating the main gold mineralization (2645-2630 Ma; Yeats \& McNaughton, 1997), such as Proterozoic Dolerite dykes and Permian sedimentary rocks, are assigned a value used in ARCVIEW and ARC/INFO to signify no data, and excluded from rheology 
contrast calculations. Rheological-contrast layers were prepared in ARC/INFO using a similar procedure to that described for the contact-type layer above. Raw data used to calculate the rheological contrasts are given in Appendix D.5.

Each cell in the rheological-contrast layers represents the absolute value of the difference between the rock strengths at the nearest lithological boundary. The fuzzy membership values represent the favourability of the rock as a host for gold deposits due to the likelihood the rock will fail in a brittle manner, where surrounding rocks do not, thus resulting in structural permeability and influx of ore-bearing fluids.

The following procedure was used to create the rheological contrast layers;

1. import solid geology shape file (geology.shp) from ArCVIEw into ARC/INFo and convert to polygon coverage geology using the shapepoly command,

2. build new coverage for lines and polygons,

3. add lrheol, rrheol, \& rh1-7 (rheological contrast) fields to arc attribute table (.aat file),

4. fill the lrheol field with the rock-strength parameter values of the rock types on the left-hand side of rock boundaries as follows;

a) link the rock strength information in the geology polygon attribute table (.pat file) to the arc attribute table, which describes the lines, using the geology\# field (default polygon identifier in the polygon attribute table) to create a relation join to the lpoly\# field (containing identifier of left polygon) in the arc attribute table (.aat file). Both the .pat and .aat files refer to the solid geology coverage, b) set the lrheol (left-hand rock type) equal to the rheology parameter in the polygon attribute table (e.g. Young's Modulus or fracture toughness etc.),

5. remove relational link,

6. repeat the step above to populate the rrheol field with values of the rheology parameter on the right-hand side of the contacts,

7. calculate rheological contrasts (stored in attribute fields $r h 1-7$ ) by finding the difference between rock strength parameter values at contacts, for example as follows;

$$
\text { rh1 }=\mid \text { rrheol }- \text { lrheol } \mid
$$

8. convert polygon coverage to grid using the $r h 1-7$ attribute fields to supply the values, and 
9. create distance grid using ARC/INFO command, eucdistance, "assign by value". Instead of setting the value of each cell to the distance to the nearest fault as in the standard command, cell values are set to the rock strength contrast at the nearest lithological contact.

Table 5.7. Rheological contrast layers, which were evaluated as potential input GIS layers for neural network analyses

\begin{tabular}{|l|l|}
\hline Layer name & Rock strength or deformational property \\
\hline \hline 136_l_rh1 & Subjective estimate, fuzzy membership \\
\hline 136_lrh2 & Subjective estimate, 10 classes \\
\hline 136_lrh3 & Young's modulus (Hatheway \& Kiersch, 1989) \\
\hline 136_lrh4 & Young's modulus (Lee, 2000) \\
\hline 136_lrh5 & Uniaxial compressive strength (Lee,2000) \\
\hline 136_l_rh6 & Uniaxial tensile strength (Lee, 2000) \\
\hline 136_l_rh7 & Fracture toughness (Lee, 2000) \\
\hline
\end{tabular}

${ }^{a}$ M. Lee, unpublished data from Australian Mining Consultants Pty Ltd, 2000

\subsubsection{Chemical Contrast}

A set of raster layers representing the difference in the chemical reactivity at the nearest lithological contact was prepared using the chemical ratios summarized in Table 5.4 (Section 5.4.7) above. Data for the calculations were obtained from AGSO's OZCHEM whole-rock geochemical database (Table D.8, Appendix D).

The procedure used to create the chemical contrast layers is the same as that for the rheological contrast layers described above, except that lrheol, rrheol, and rh1-7 fields are replaced by lchem, rchem, and ch1-5, respectively. Like the rheological contrast layers, the chemical-contrast calculations were performed using data stored as attributes (ch1-5) in the solid geology layer, geology.shp. The ratios and the GIS database layer names are shown in Table 5.8 below. The first two ratios are molar ratios. The layers are shown in Figures 5.30, 5.31 and 5.32. 
Table 5.8. Chemical contrast layers, which were evaluated as potential input GIS layers for neural network analyses

\begin{tabular}{|l|l|}
\hline Layer name & Chemical ratio \\
\hline \hline $137 \_$_ch1 & $\mathrm{Fe}_{\text {wt } \%} * \mathrm{Fe}_{\text {total }} /\left(\mathrm{Fe}_{\text {total }}+\mathrm{Mg}+\mathrm{Ca}\right)$ \\
\hline $137 \_$l_ch2 & $\mathrm{Fe}_{\text {total }} /\left(\mathrm{Fe}_{\text {total }}+\mathrm{Mg}+\mathrm{Ca}\right)$ \\
\hline $137 \_$_ch3 & $\mathrm{Fe}_{2} \mathrm{O}_{3} /\left(\mathrm{Fe}_{2} \mathrm{O}_{3}+\mathrm{FeO}\right)$ \\
\hline $137 \_$_ch 4 & $\mathrm{Fe}_{2} \mathrm{O}_{3} / \mathrm{FeO}$ \\
\hline $137 \_$_ch5 & $\mathrm{Fe}^{3+} / \mathrm{Fe}^{2+}$ \\
\hline
\end{tabular}

\subsubsection{Favourable Contact Type}

The main mechanism of gold deposition for orogenic lode-gold deposits in the Yilgarn is through fluid-wall rock reactions in which the iron-rich minerals in the host rocks react with sulphide complexes, which carry gold in solution, to form Fe-sulphides (Phillips \& Groves, 1983; Mikucki, 1998). Consequently, upward migrating hydrothermal fluids may precipitate gold where there is a change in host rock. Further, fluid migration will occur into dilational zones of high fracture-related permeability, which tend to occur where rheological contrasts are high. Thus, the combination of rock types at lithological boundaries may play two roles in the localization of gold deposits.

The favourable contact-type GIS layer is a raster layer in which cell values represent the combination of rock types which occur at the nearest lithological boundary. Since the combinations represent a nominal data type, these rock-type combinations must be converted to a ratio data type. Using a version of the solid geology layer with 25 rock types as a starting point for processing gives a total of 134 rock-type combinations. Assigning a numerical code to each type is not appropriate for a parameter to be used as an input to a neural network, since the network would treat a pair of lithologies with a code of 10 as twice as large as a pair with the code 5. Clearly, if the two contact types are similarly prospective, such a coding scheme is misleading. Due to the large number of combinations, it was not feasible to use 1-of-n coding. This coding scheme was used for the small number of lithologies (12) used in the pilot study in Chapter 4. Instead, the association of known deposits with the different contact rock-type combinations was used as a statistical basis for converting the contact type categories to fuzzy membership values. The fuzzy membership values express the favourability of areas, for which the nearest contact is of a certain type, to host orogenic gold deposits. 
The favourable contact-type layer was created in the following three main steps;

1. create the layer showing the nearest contact-type,

2. calculate statistical relationships between known deposits and contact types, and

3. convert to a fuzzy membership layer using statistics.

In the last step, the contact-type names were replaced by fuzzy membership values indicating the degree of favourability of the contact-type.

The nearest-contact-type layer was created using the same procedure as the one used to create the rheological layers described above. The contact-type layer is created by linking the rock name attribute associated with the polygons with the information about left and right neighbours of each polygon that is stored for all arcs in ARC/INFO. A unique name was generated for each contact type using an AML script. Each contact-type name consists of abbreviations for the pair of rock types arranged in alphabetical order. Cell values were then set equal to the concatenated rock codes for the lithologies at the nearest contact. Using a simplified legend with 25 rock types for the solid geology GIS layer results in 134 rock-type combinations. The procedure for ranking layers and for assigning fuzzy membership values for the contact-type layer is the same as that described for the favourable geology layer above.

Since there are some important differences the procedure is listed below. The steps are;

1. import solid geology shape file (geology.shp) from ArCView into ARC/Info and convert to polygon coverage geology using the shapepoly command,

2. build new coverage for lines and polygons,

3. add lrocktype, rrocktype, \& contact_type fields to arc attribute table (.aat file),

4. fill the lrocktype field with the names of the rock types on the left-hand side of rock boundaries as follows;

a) link the rock name information in the geology polygon attribute table (.pat) to the arc attribute table to create a relation join to the lpoly\# (field containing identifier of left polygon) in the arc attribute table (.aat file). Both the .pat and .aat files refer to the solid geology coverage,

b) set the lrocktype (left-hand rock type) equal to the value of the rock name field $(r t-25-d e s c)$ in the polygon attribute table,

5. remove relational link, 
6. repeat the step above to populate the rrocktype field with the name of the rock type on the right-hand side of the contact,

7. populate contact_type field by concatenating the lrocktype and rrocktype fields. This step results in two contact_type values for each contact type (e.g. basalt-granite and granite-basalt values are both generated for the same contact type).

The AML script to generate a unique name for each contact type is functionally equivalent to the following code fragment;

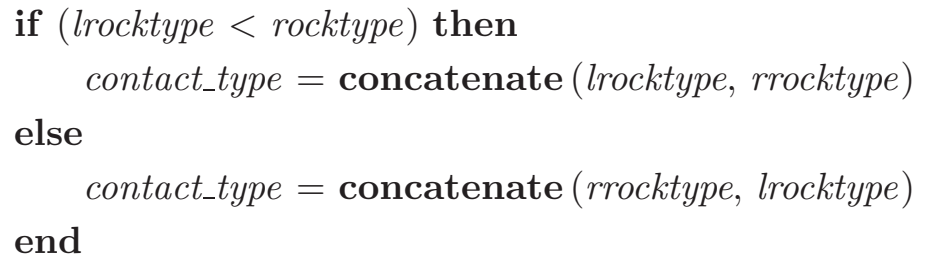

where rrocktype and lrocktype are processed as strings. This step resulted in a set of unique values where the contact types are expressed in alphabetic order of the rock types,

8. convert polygon coverage to grid using the contact_type attribute field to supply the values, and

9. create a distance grid using ARC/INFO command, eucdistance(assign by value). Cell values are set to the concatenated rock codes of the lithologies at the nearest contact type (e.g. MbGt, where $\mathrm{Mb}=$ magnesian basalt and $\mathrm{Gt}=$ granite).

Two contact-type maps were prepared; one based on a solid geology rock-type legend comprising 25 rock types (135_l_rt25) and second based on a 55 rock-type legend (135_l_rt55). The latter map was not used for further calculations because there were too few known deposits associated with the resultant 235 contact types,

The maps of nearest contact-type were converted to a form suitable for input to a neural network by converting the nominal variable (e.g MbGt for a basalt-granitoid contact) to numerical fuzzy membership values. Although subjective judgement was important, the assignment of fuzzy membership values was based on the statistical relationship of known deposits with areas for which the nearest contact lithologies were of a certain type. The following steps were required to convert the GIS contact-type layer to a MATLAB array;

1. Export the ARCVIEW database table for nearest-contact-type layer, 135_l_rt25 as a 
DBF file and import into EXCEL. This results in a table (rt25_value_count_codes.xls) with numerical codes and concatenated strings representing contact-types (e.g. 6, GtMb for a granitoid-basalt contact).

2. Create a table that contains rock codes together with the rock descriptions in ARCVIEw. Open attribute table for geology.shp coverage, and use the field-summarize function. Export the table as a DBF file and import into EXCEL.

3. Add rock names to EXCEL table with numerical and textural contact-type codes.

4. Run the Matlab function, deps_cont_type. For each region comprising cells with the same nearest contact type, the following statistics were calculated; the number of cells, deposits, total contained gold (TCG, kg), probability that a cell contains a deposit $(\mathrm{P}(\mathrm{D}))$, ratio of the percentage of the total deposits to the percentage of the total area (D/A), the ratio of the TCG to area, average TCG per cell, and the product of the probability of a $\mathrm{P}(\mathrm{D}) \times \mathrm{TCG} /$ cell. The function only calculates statistics for cells that are located within a user-specified maximum distance from the contacts. This was set to $200 \mathrm{~m}$ or $1300 \mathrm{~m}$ depending on the layer (see explanation below). Inputs to the deps_cont_type function were the geology (11_l_ass_t25), contact-type (135_l_rt25), binary deposits (124_d_all_bin), tonnes-contained-gold (125_d_tcg2), and distance-to-nearest-lithological-contact (139_c_dist) layers. Only gold deposits containing a resource of $1000 \mathrm{~kg}$ total contained metal were used for these calculations. The function outputs an ASCII file, deps_cont_type_log containing a table of the parameters listed above for each contact type. This table was imported and added to the EXCEL spreadsheet containing the contact codes and names.

5. Rank the contact-types in order of decreasing probability of a deposit $P(D)$. A layer was also created based on ranking the layers using the product of probability of a deposit and the total contained gold $(P(D) \times T C G /$ cell $)$.

6. Select contact types that are definitely favourable for deposits and assign these a fuzzy membership value of 1.0.

The dolerite-porphyry contact type is arbitrarily chosen as the lowest ranking contact type to be assigned a fuzzy membership value of 1.0 for both the $P(D)$ and $P(D) \times$ $T C G /$ cell ranking criteria. Eight of the top ten ranked contact-types based on $P(D)$ and all of the top ten contact-types based on $P(D) \times T C G /$ cell include at least one of the following rock types; feldspar porphyry, quartz dolerite, dolerite, andesite or carbonaceous shale. These are the lithological units with the highest probability of containing a known deposit ( $\geq 1000 \mathrm{~kg}$, TCG; Tables D.16 and D.17, 
Appendix D). Contact-types that do not contain any known deposits were assigned a fuzzy membership value of 0.1 .

The occurrence of orogenic gold deposits in a wide variety of host rocks throughout the Yilgarn Block, (which includes the Kalgoorlie Terrane containing the study area), suggests that none of the host rocks that were present prior to the main phase of gold mineralization should be excluded as potential hosts for gold deposits. Therefore, a minimum fuzzy membership value of 0.1 rather than 0.0 was used (Carranza \& Hale, 1999).

A linear interpolation was made between these limits in order to assign fuzzy membership values for all other contact types. The calculations were performed in EXCEL spreadsheets (refer to Tables D.14 and D.15 in Appendix D).

7. The fuzzy membership values determined in the previous step were then used as constants in the MATLAB functions (favcontact25_pr0110.m, and favcontact25_pxtcg0110.m) which created favourable contact-type layers based on $P(D)$ and $P(D) \times($ ave.TCG/cell), respectively, by reclassifying the contact-type layer, 135_l_rt25.

As the distance increases, the effect of the rock types at the nearest lithological boundary on prospectivity must diminish and eventually become irrelevant. In order to model this, fuzzy membership values were set to 0.1 for cells beyond a certain distance to the nearest contact. Distance limits are based on maximum and minimum estimates determined using a variety of statistical tests described in Section 6.3 in Chapter 6. Layers using limits of 200 and 1300 metres were prepared using the MATLAB functions referred to above. These distances correspond to the minimum and maximum estimates obtained for the distance within which proximity to lithological boundaries upgrades the prospectivity for orogenic gold deposits $(\geq 1000 \mathrm{~kg}$ TCG; Table 6.4 below.

The favourable contact-type layers together with the parameters used to create them, are shown in Table 5.9 below.

\subsubsection{Favourable Lithology}

Rock types in the solid geology GIS layer, like the contact-types discussed above, represent a nominal data type. Two methods were used to convert the solid geology GIS layer into a numerical form suitable for input to a neural network; 1) conversion to fuzzy membership 
Table 5.9. Candidates for favourable contact-type GIS layers and the parameters used to rank them. Cells located beyond maximum distance from the nearest lithological boundary were assigned the minimum fuzzy membership (i.e. 0.1 for $200 \mathrm{~m}$ buffer limit; 0.0 for $1300 \mathrm{~m}$ buffer limit).

\begin{tabular}{|l|l|l|l|l|}
\hline Layer name & $\begin{array}{l}\text { Parameter used } \\
\text { to rank contacts }\end{array}$ & $\begin{array}{l}\text { Distance } \\
\text { limit }(\mathrm{m})\end{array}$ & $\begin{array}{l}\text { M/ship if } \\
\text { dist._limit }\end{array}$ & $\begin{array}{l}\text { Matlab function used } \\
\text { to create layer }\end{array}$ \\
\hline \hline 144_favcont25_pr0110_200 & P(deposit) & 200 & 0.1 & favcontact25_pr0110_200 \\
\hline 144_favcont25_pxtcg0110_200 & P(deposit)* TCG & 200 & 0.1 & favcontact25_pxtcg0110_200 \\
\hline 144_favcont25_pr_1300 & P(deposit) & 1300 & 0.0 & favcontact25_pr0110_1300 \\
\hline 144_favcont25_pxtcg_1300 & P(deposit)* TCG & 1300 & 0.0 & favcontact25_pxtcg0110_1300 \\
\hline
\end{tabular}

values, and 2) 1-of-n coding. These methods are discussed in the sections below.

\section{Conversion of Solid Geology to Fuzzy Membership Layer}

Rock-type codes in the solid geology layer are converted to fuzzy-membership values using the following steps:

1. calculate statistical relationships between known deposits and lithological units

2. rank layers and set definitely favourable rock types to a fuzzy membership to 1.0 and unfavourable rock types to 0.1 , and

3. convert to a fuzzy membership layer using statistics;

a) calculate fuzzy membership values between 0.1 and 1.0 by linear interpolation, and

b) use the fuzzy membership values calculated above to re-classify the solid geology layer.

The first step, in the list above, was performed using the MATLAB function (deps_litho.m), which calculates number of cells, deposits, total contained gold (TCG), TCG/cell, probability of deposits $(P(D))$, and $P(D) \times T C G /$ cell for each rock type. The linear interpolations referred to in step 3a were performed in ExCEL spreadsheets (refer to Tables D.16 and D.17 in Appendix D, page 635). The step 3b was performed with the MatLaB function favgeol25_pr, which created the favourable lithology layers $11_{-}$favgeol25_pr0110 and l1_favgeol_pxtcg. 


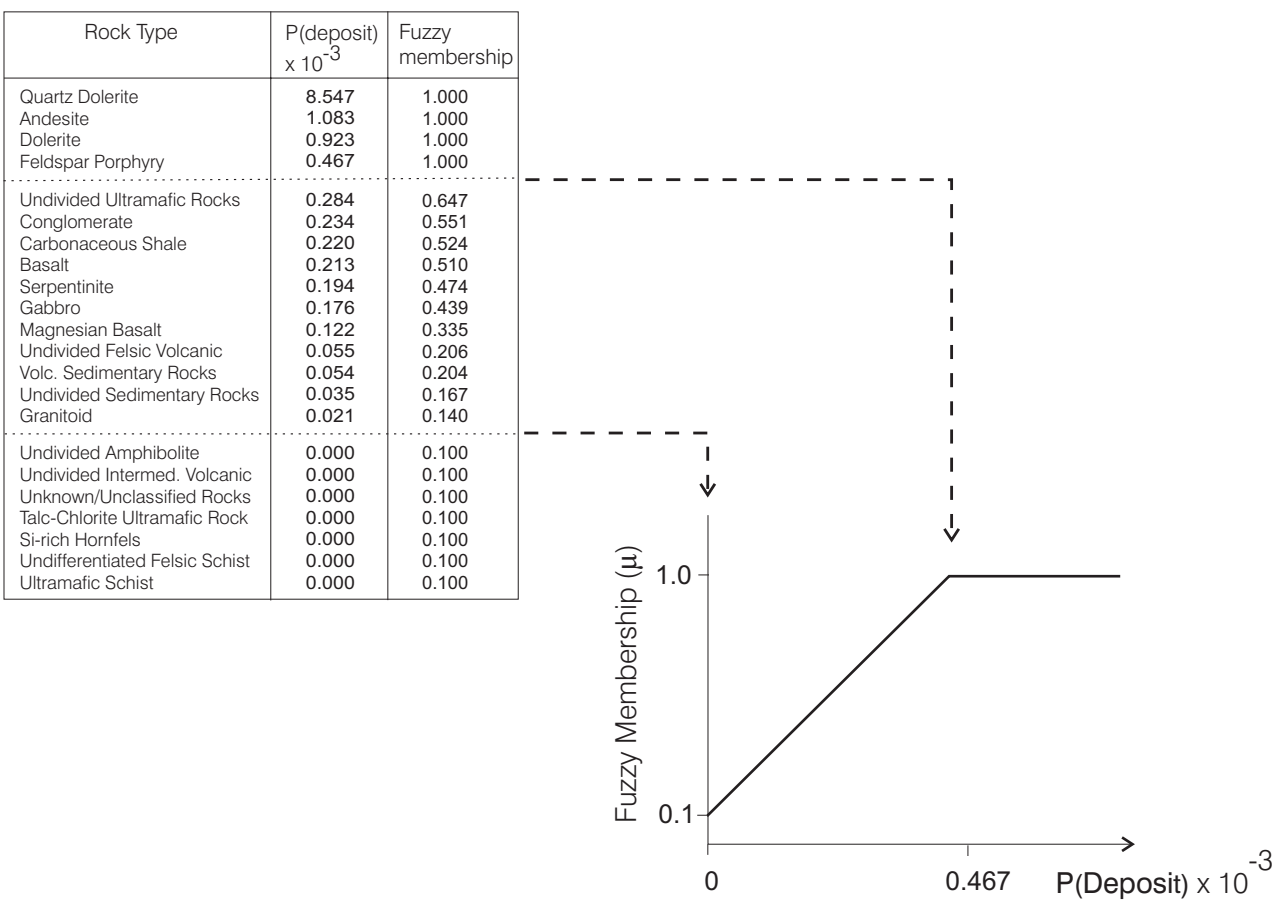

Figure 5.6. Conversion of host-rock classes in an input GIS layer to fuzzy member values. Assignment of fuzzy membership values is based on a ranking of rock types in order of the probability of containing a known deposit, $(P(D)$. Definitely favourable and definitely unfavourable rock types are assigned fuzzy membership values of 1.0 and 0.1 , respectively. $P(D)$ and fuzzy membership values associated with these groups are then used to construct a triangular fuzzy membership function, which is then used to perform a linear interpolation of the values for the rock types ranked between the end-member groups.

Two fuzzy membership layers are created. The first layer, l1_favgeol25_pr0110, is based on ranking rock types according to the probability that the rock type contains a known gold deposit. Definitely favourable and unfavourable host rocks were assigned fuzzy membership values of 1.0 and 0.1 , respectively. The second layer is based on a ranking rock types according to $\mathrm{P}(\mathrm{D}) \times \mathrm{TCG} /$ cell. A minimum value of 0.1 is used for this layer because no rock type is completely unfavourable as a host to gold mineralization (Section 6). Carranza \& Hale (1999) have recently used a similar approach in a fuzzy systems estimate of the potential for epithermal gold mineralization in the Philippines. These steps and the triangular fuzzy membership function used in this study are illustrated schematically in Figure 5.6. The procedure is the same as that used for the fuzzy contact-type layer above, except that it was not necessary to process topological information from the vector coverage in ARC/INFO in order to first create a derived layer. 


\section{Conversion of Solid Geology to 1-of-n Coding Scheme}

In 1-of-n coding, a separate neural network input is assigned to each class in the data; in this case rock types. In order to indicate that the current input corresponds to basalt, the input unit corresponding to basalt is set to one and all the other units are set to zero (Masters, 1993b). Since the simplified version of the Goldfields Pty Ltd lithology legend contained 25 rock types of which 22 occurred in the study area, the 1-of-n coding scheme results in 22 inputs assigned to rock-type. Thus, the first 22 components of the resultant feature vector representing an input pattern belong to the 1-of-n code (Fig. 3.2).

There are two disadvantages to this scheme. First, the number of network inputs is significantly increased, particularly if there are a large number of classes in the data, leading to a consequent increase in the number of interconnections between input neurons and hidden layer neurons. For small training-set sizes, the large number of weights associated with the connections can result in over-learning (i.e. learning the idiosyncrasies rather than the underlying patterns in the data). This reduces the ability of the trained network to generalize to new patterns not presented during training (Masters, 1993b; Singer \& Kouda, 1999a). Second, to code the entire solid-geology layer containing 25 rock types effectively results in the creation of 25 new input layers, which requires a very large amount of main computer memory. For example, 512 MB RAM used in this study is not sufficient for layers consisting of $1140 \times 1100$ cells.

Simplification of the original mapping legend used by Goldfields Exploration Pty Ltd for the Yilgarn Block from 108 to 25 rock types was a necessary preliminary step in order to apply a 1-of-n coding scheme to the solid geology layer. Although simplification of the legend is not required if fuzzy-membership values are used, a geology layer containing 108 rock types would probably result in less reliable results because many rock types contain less than five known deposits. Due to the large number of classes in the contact-type layer (134 rock-type combinations using a geology legend with 25 rock types), it is not feasible to use a 1-of-n coding scheme for this layer.

Although conversion of the rock-type codes in the solid geology layer to 1-of-n coding was performed at the same time the other layers were combined into feature vectors, merging of cell values from the different input GIS layers to form feature vectors had to be performed in two steps in which each half of the map area was processed separately. These steps were performed in MATLAB as a batch job (make_train_batch_1ofn). Processing to code the rock types in 1-of-n format involved the following steps;

1. convert rock type codes to a contiguous sequence using MatlaB function, geol- 
codes_kal to give the array geol_22,

2. combine all layers other than geology to create input patterns (MATLAB script, merge_cat_10), and

3. insert 1-of-n codes into feature vectors created in previous step (MATLAB function, $a d d_{-}$geol1ofn).

\subsubsection{Density of Faults}

A layer depicting fault density was prepared by calculating the total length of faults in a $5.1 \times 5.1 \mathrm{~km}$ moving window and assigning that value to the central cell (442_f123den $5 \mathrm{k}$ in Figure 5.34). Three vector-format fault layers containing crustal-scale, and NNE-trending and NE-trending regional-scale faults (i.e. 120_s_crust, 121_s_shear and 121_s_flt_ne), were combined using the Map Calculator function in ARCVIEW, converted to a binary grid file (faults $=1$, other $=0$ ), and exported as an ASCII file. The ASCII file was imported into Matlab where ARCView header lines were removed using a Matlab script, strip_hdr.m and the function, fault_density. $m$ was used to perform a $2 \mathrm{D}$ convolution which counted the cells in the processing window, which correspond to faults and assigned the total to the central cell. A $51 \times 51$ cell window size allowed for a central cell and 25 cell in each orthogonal direction.

\subsubsection{Density of Lithological Contacts}

A layer representing a measure of lithological complexity in the area around each cell was created by an integer count of lithological contacts in the neighbourhood of each cell (l43_lithdiv in Figure 5.35). A solid geology layer containing a legend comprising 55 rock types (11_l_ass_t55) was imported into MATLAB using the same procedure as explained above in Section 5.5.15. Within MatlaB, the function, litho_diversity.m was used to count the total length of lithological contacts along rows and down columns within a $5.1 \times 5.1 \mathrm{~km}$ moving processing window. The algorithm, described by Bonham-Carter (1994), checks if rock types in adjacent cells are different.

\subsubsection{Addition of No Data Cells to Derived Layers}

Areas where data were missing in the geological map supplied by Goldfields Exploration were flagged using a special No Data value recognized in ARCVIEW. Since analysis func- 
tions used to create the derived layers produced output values for all cells within the boundaries of the study area, the No Data values had to be added to the derived layers. This was achieved using the primary solid geology map, in which No Data cells were correctly flagged and the ARCVIEW map calculator function. The following command was used;

([l1_ass_t25].IsNull).SetNull([l46_dist_anti])

where the derived layer showing the distance to anticlinal axes has been used as an example.

\subsubsection{Fuzzy Membership Layers}

A set of fuzzy membership layers, corresponding to the derived layers described above, were prepared using parameter values that define the most favourable regions for deposits. These favourable ranges of parameter values were determined using methods discussed in the next section and are shown in Table 6.4,

Triangular fuzzy membership functions were used to convert the raw data from the GIS layers to values representing the fuzzy membership degree. The processing was performed in the MATLAB environment using the scripts, layer2fav and layer2fav_batch.

\subsection{Summary}

The main points discussed in Chapter 5 are listed below.

1. Archaean orogenic gold deposits in the Yilgarn Craton formed over a wide range of crustal depths and, consequently, have correspondingly varied metamorphic grades, structural styles, host rocks and mineralogy.

2. Timing of gold deposition was late within the deformation history of the host terrane. Consequently, the geometry of the structures associated with deposits and rock sequences hosting the deposits that are represented in geological maps are essentially similar to those that existed during gold mineralization. As a result: 1) the locations of gold deposits are related to repeated and predictable structural geometries, and 2) spatial relationships between the structures and known deposits determined from 
geological maps can be analyzed in a GIS to predict the location of new gold deposits (Groves et al., 2000).

3. Favourable areas for gold deposits include: 1) large linear greenstone belts with major bounding shear zones, 2) irregularities in strike (and large rheological contrasts) along crustal and regional-scale faults or shear zones, 3) dilational jogs along strike-slip faults, 4) cross faults between first-order faults, 5) changes in strike of first-order faults from a dominant NNW to a NW trend, 6) intersections of two or more faults, 7) anticlinal hinges or domes formed by doubly plunging anticlines, 8) structurally isolated blocks of more competent volcanic rocks surrounded by less competent volcanic or sedimentary rocks, 9) greenschist facies terranes rather than higher-grade metamorphic settings, 10) dykes and small bodies of syenite and porphyritic intermediate to mafic intrusions, particularly those surrounded by magnetite alteration, 11) rheological contrasts across lithological boundaries, and 12) chemically reactive host rocks, that is, mafic rocks and rocks with high Fe and/or high $\mathrm{Fe} /(\mathrm{Fe}+\mathrm{Mg}+\mathrm{Ca})$, such as tholeiitic basalt and banded iron formation (BIF), and some mafic porphyries.

4. The main mechanism of gold deposition in orogenic lode-gold deposits in the Yilgarn Block is fluid-wall rock reactions in which the iron-rich minerals in the host rocks react with sulphide complexes, which carry gold in solution, to form pyrite.

5. Destruction of magnetite (due to reactions involving the replacement of magnetite by pyrite and leucoxene) leads to a magnetically depleted core of some deposits, while some deposits have a magnetic rim due to the precipitation of magnetite by spent ore fluids. Strong positive and negative aeromagnetic anomalies may indicate sites of magnetite production and destruction, respectively.

6. Primary GIS data layers supplied by Goldfields Exploration are; solid geology (108 rock types), faults and shear zones, aeromagnetic and gamma-ray survey data, and Bouguer gravity anomalies. Layers and data derived from other sources are; traces of regional-scale anticlines, deposits and mineral occurrence database, and the OZCHEM whole-rock geochemistry database.

7. Chemical reactivity of host rocks is modelled using two sulphidation ratios; $\mathrm{Fe} /(\mathrm{Fe}+\mathrm{Mg}+\mathrm{Ca})$ and $\mathrm{Fe}_{\text {total }}(\mathrm{wt} \%) \times \mathrm{Fe} /(\mathrm{Fe}+\mathrm{Mg}+\mathrm{Ca})$ and three oxidation ratios; $\mathrm{Fe}_{2} \mathrm{O}_{3} /\left(\mathrm{Fe}_{2} \mathrm{O}_{3}+\mathrm{FeO}\right), \mathrm{Fe}_{2} \mathrm{O}_{3} / \mathrm{FeO}$, and $\mathrm{Fe}^{3+} / \mathrm{Fe}^{2+}$. These ratios are calculated from the OZCHEM whole-rock geochemistry data. Quantities are weight percentages of metals where oxides are not shown. 
8. Competency contrasts across lithological boundaries are an important control on the location of deposits.

9. To determine the best way to model rheological contrast, the following rock strength parameters were tested; two different compilations of Young's Modulus, uniaxial compressive and tensile strength, fracture toughness, and subjective estimates expressed as classes and fuzzy membership values.

10. The effect of using derived rather than primary GIS layers is to emphasize exploration criteria and make the ingredients of the mineral systems model for orogenic gold deposits more explicit. This process of modifying the raw input parameters to emphasize the underlying relationship between the neural network inputs and the outputs is referred to as feature extraction.

11. The original geological legend was simplified from 108 to 25 rock types. This is because the statistical relationship between gold deposits and host rock types cannot be reliably determined from the original legend as many rock types do not host any known gold deposits.

12. The aeromagnetic intensity map was converted to a layer depicting the distance to positive magnetic anomalies. Anomalies were defined as cells with a z-score $\geq 2$, where $z=(x-\mu) / s$, and $x=$ raw value, $\mu=$ average total magnetic intensity and $s=$ standard deviation for a single rock type.

13. Rheological-contrast layers represent the absolute values of the difference between rock strengths at the nearest lithological boundary.

14. Rheological contrast values were calculated in ARC/INFO by linking the information about the polygons that are located to the left and right of any line that is stored in the arc (i.e., line) attribute table (i.e., spatial database) together with the rock strength data stored in the polygon attribute table (i.e. non-spatial database). Chemical contrast and nearest-contact-type layers were produced in the same way.

15. The favourable contact-type layer is a raster layer in which cell values represent the favourability of the two adjacent rock types at the nearest lithological boundary to host gold deposits. Favourability is expressed as fuzzy membership values.

16. To model the decreasing effect on prospectivity of the rock types at the nearest lithological boundary as distance increases, fuzzy membership values were set to 0.1 for cells beyond a certain distance to the nearest contact. Distance limits of 200 and 1300 metres are based on minimum and maximum estimates determined using 
a variety of statistical tests described in Section 6.3 in Chapter 6.

17. Favourable contact-type and favourable host-rock type layers are objective fuzzy membership layers which are based on the statistical relationships between known deposits and the respective parameters.

18. A subjective fuzzy-membership layer was created to represent rheological contrast. Rocks in the detailed geology legend (108 rock types) were subjectively ranked according to their tendency for brittle or ductile failure based on field observations.

19. The fuzzy membership values for the rheological contrast layer represent the favourability of the rock to host gold deposits due to the likelihood that the rock will fail brittley where surrounding rocks do not, thus resulting in structural permeability and influx of ore-bearing fluids.

20. A 1-of-n coding scheme is used to convert the categorical rock-type data in the geology GIS layer to a form suitable for input to a neural network. Disadvantages of this method are that; 1) the number of network inputs is significantly increased, leading to the possibility of overfitting of the data for small training data sets, and 2) the 1-of-n layers consume a lot of computer memory, especially if there are large numbers of rock types.

21. A layer representing density of lithological contacts is used as a measure of lithological complexity. A 2D convolution was used to determine the number of lithological contacts in a $5 \times 5 \mathrm{~km}$ window around each cell. This method was also used to calculate a layer representing the density of regional-scale faults. 


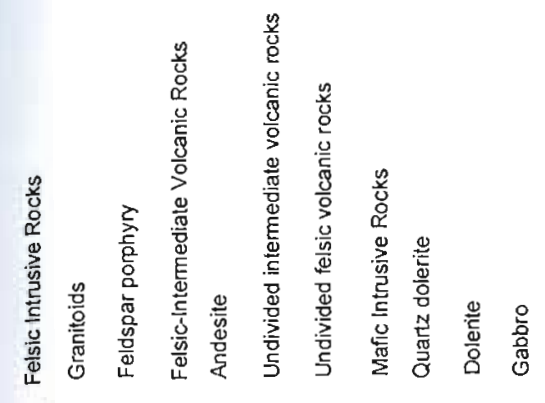

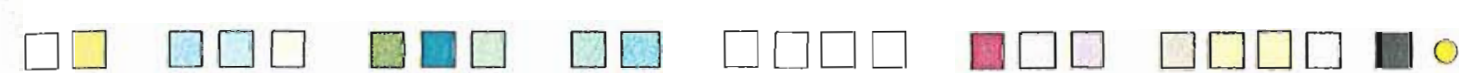

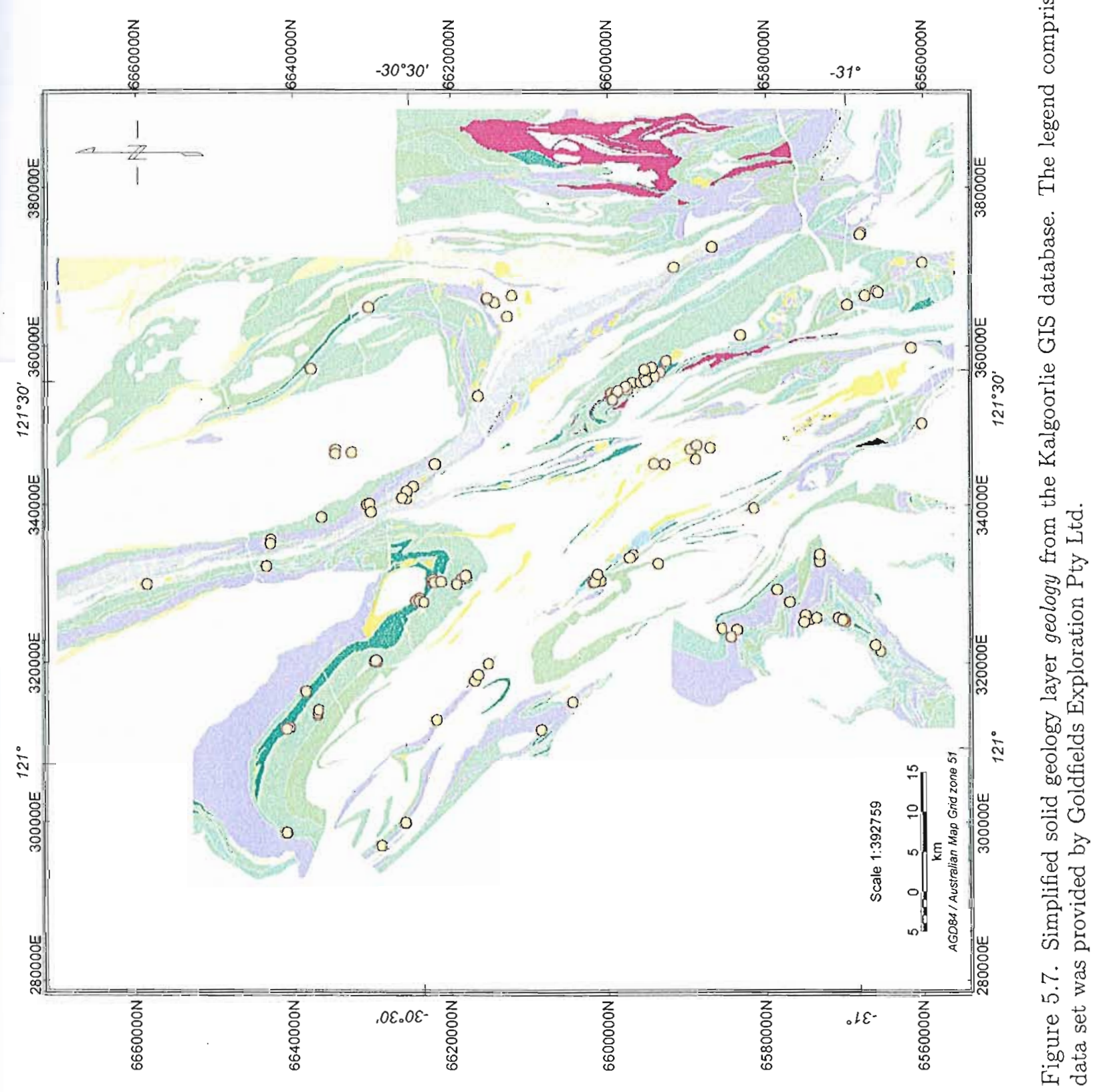




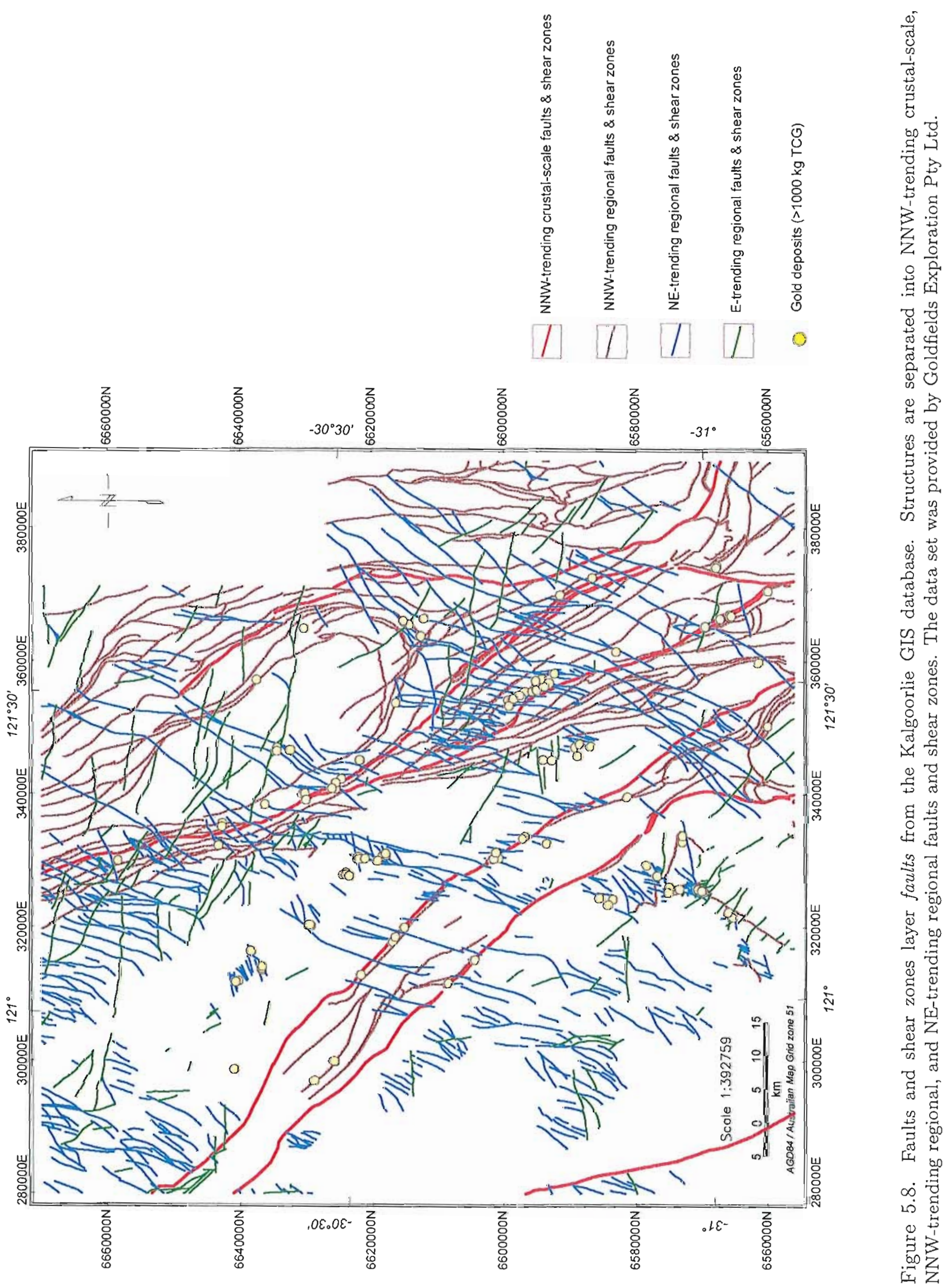



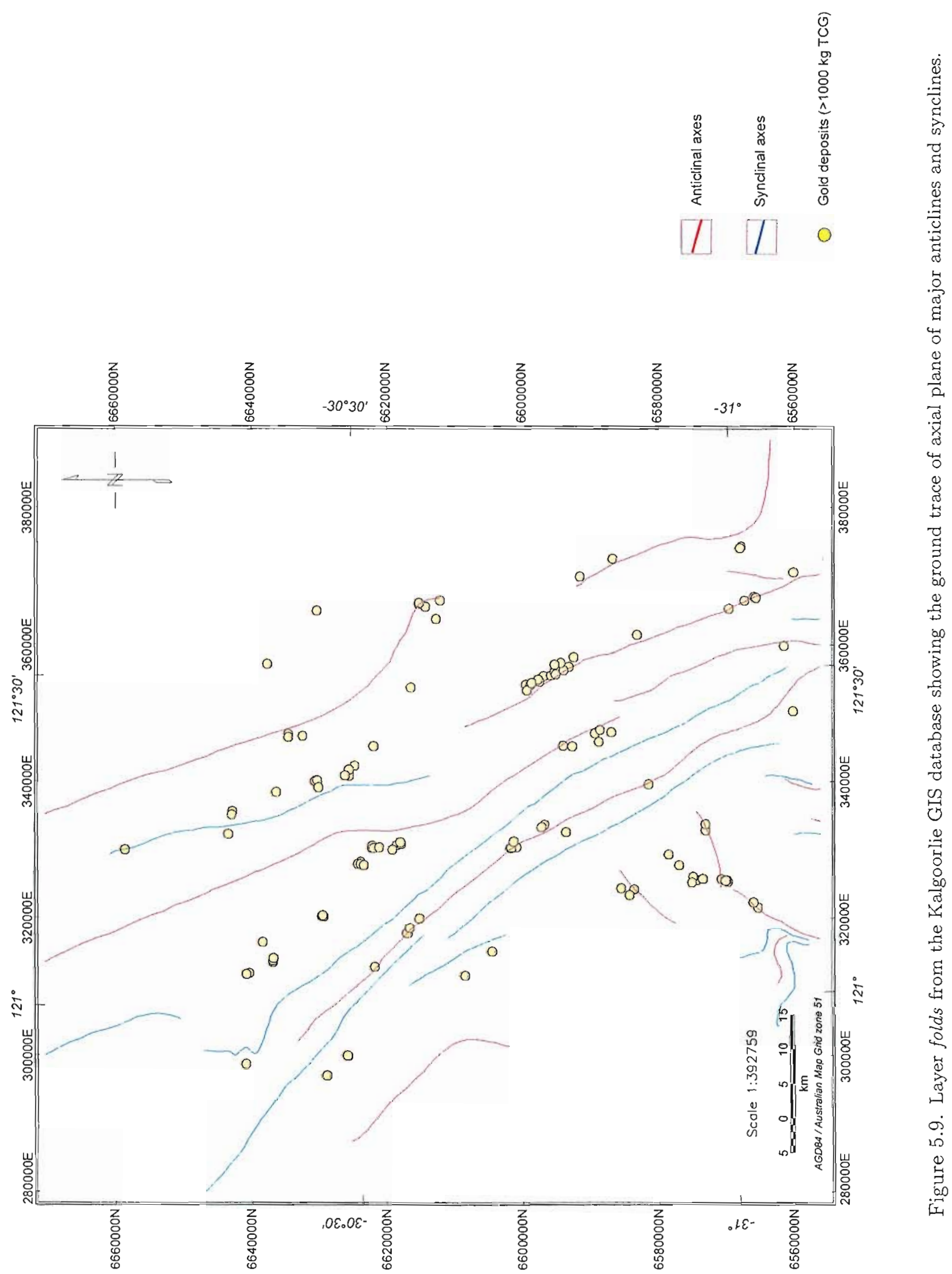


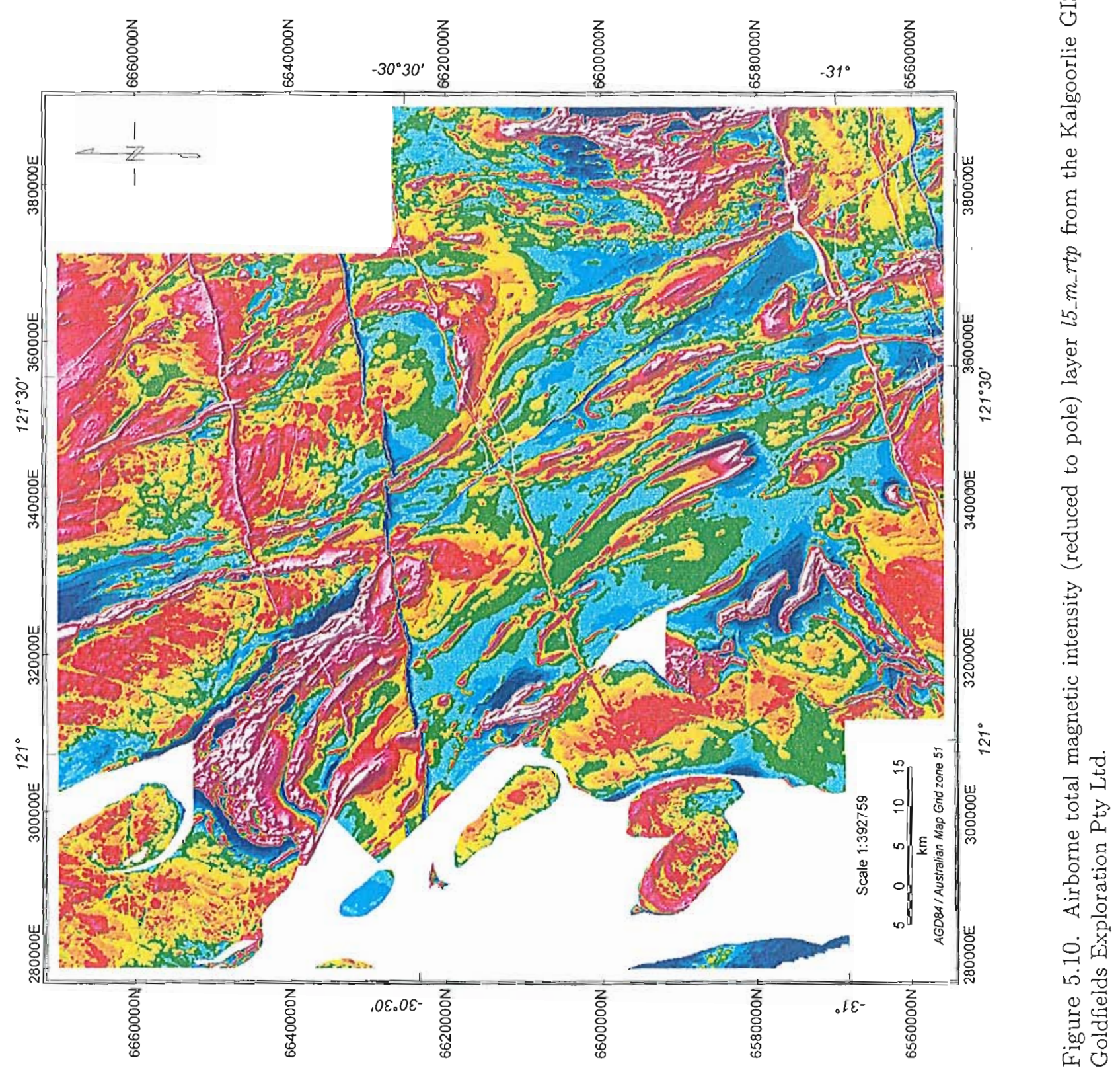




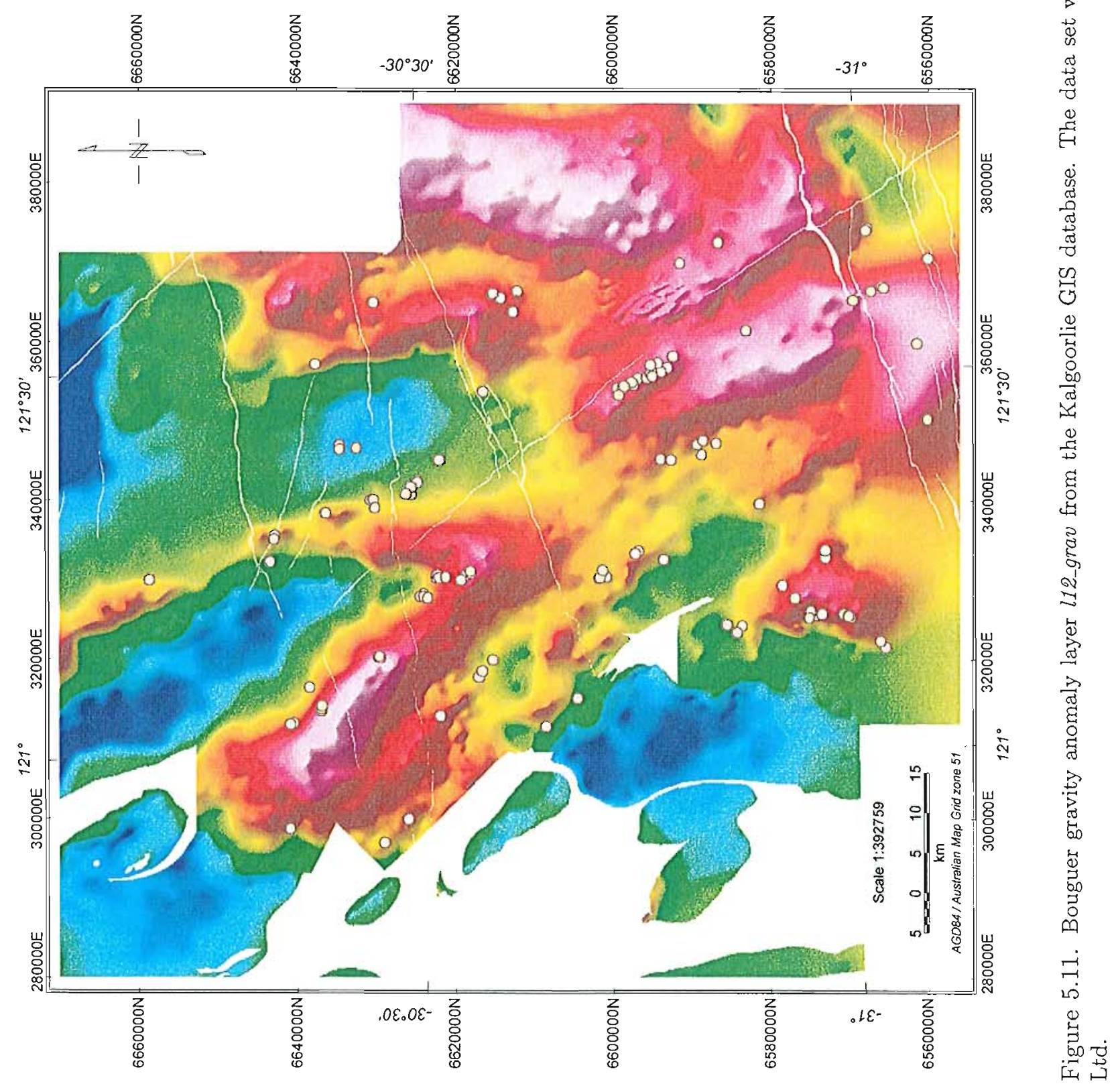




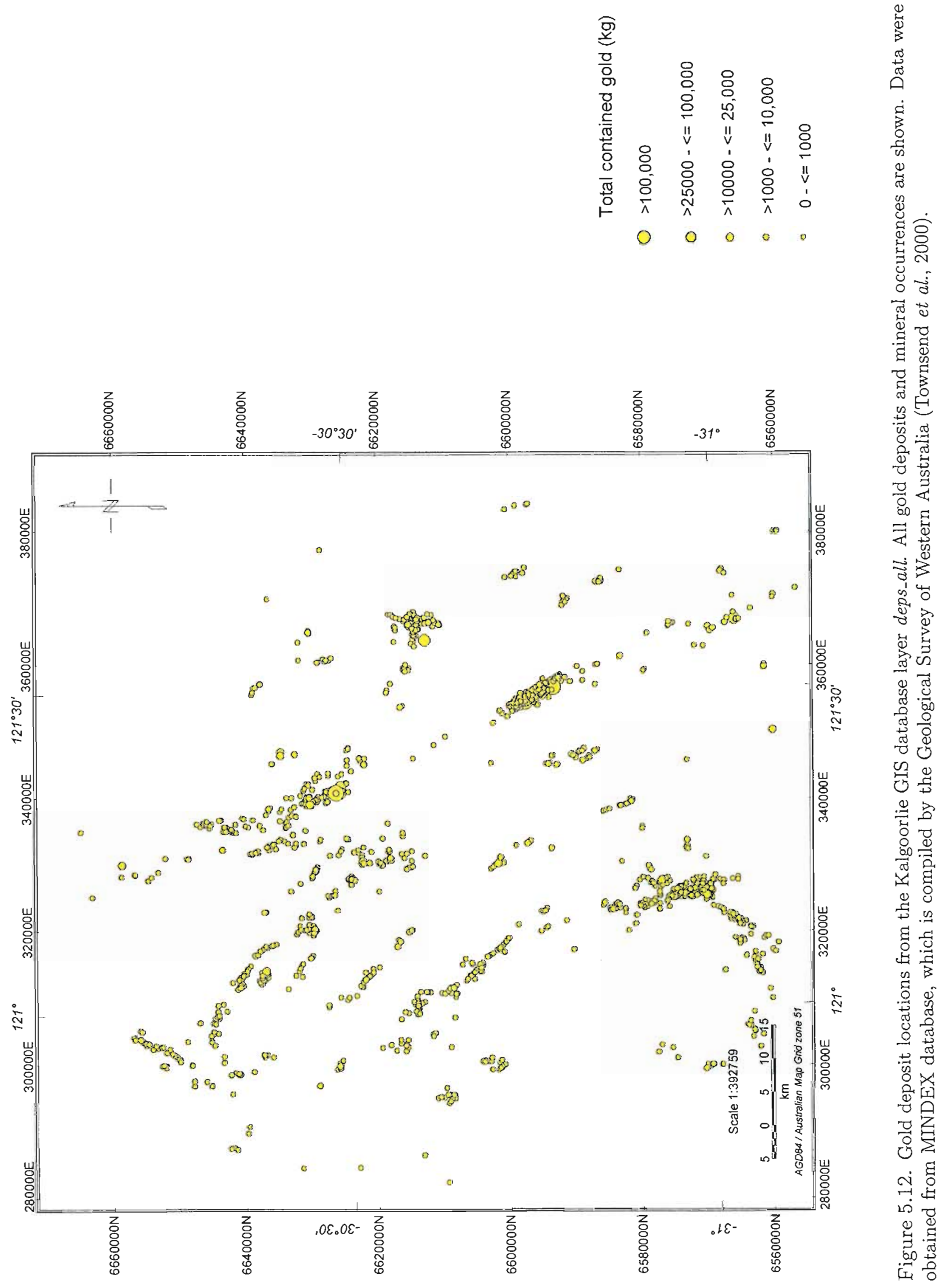




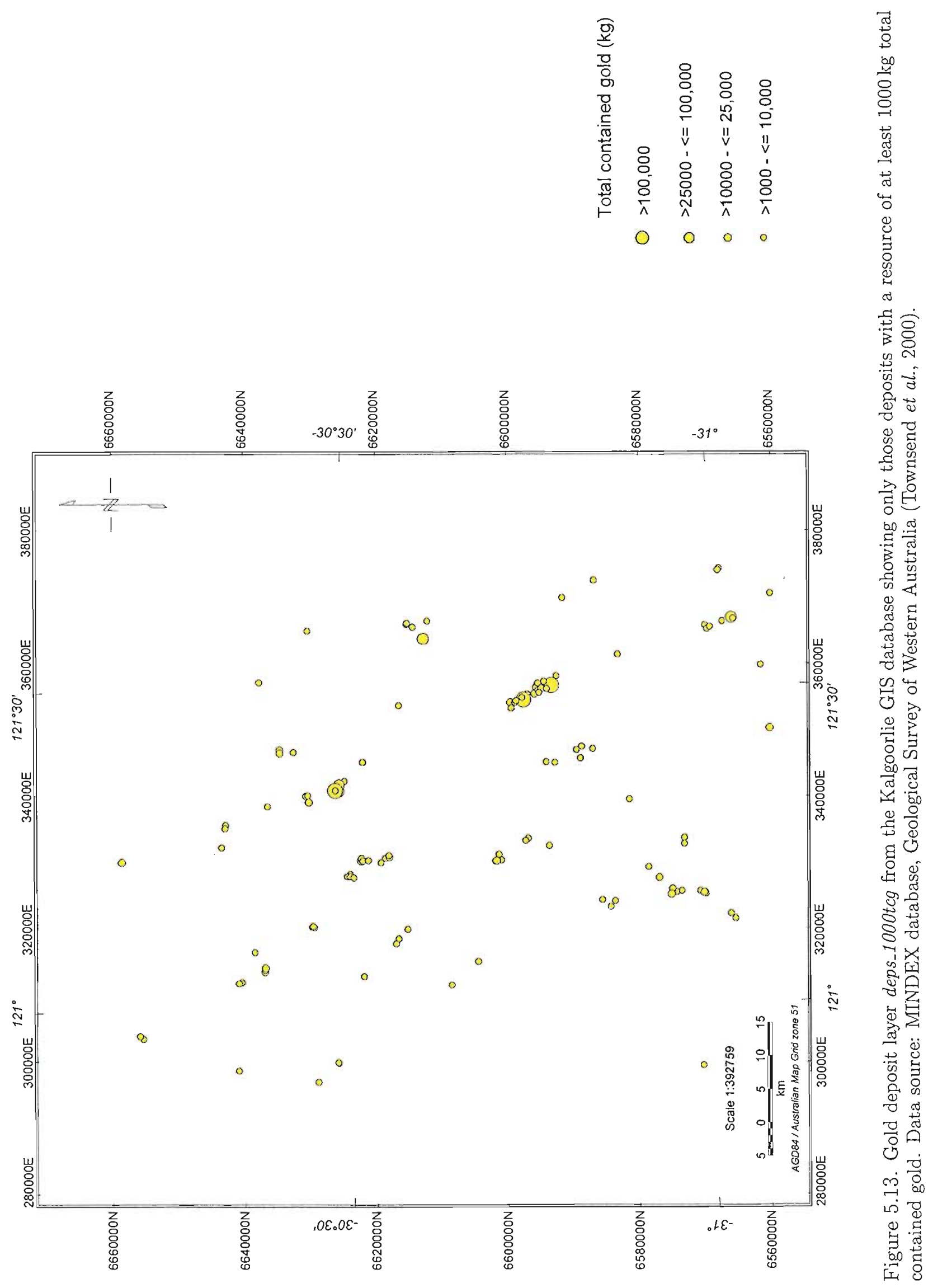




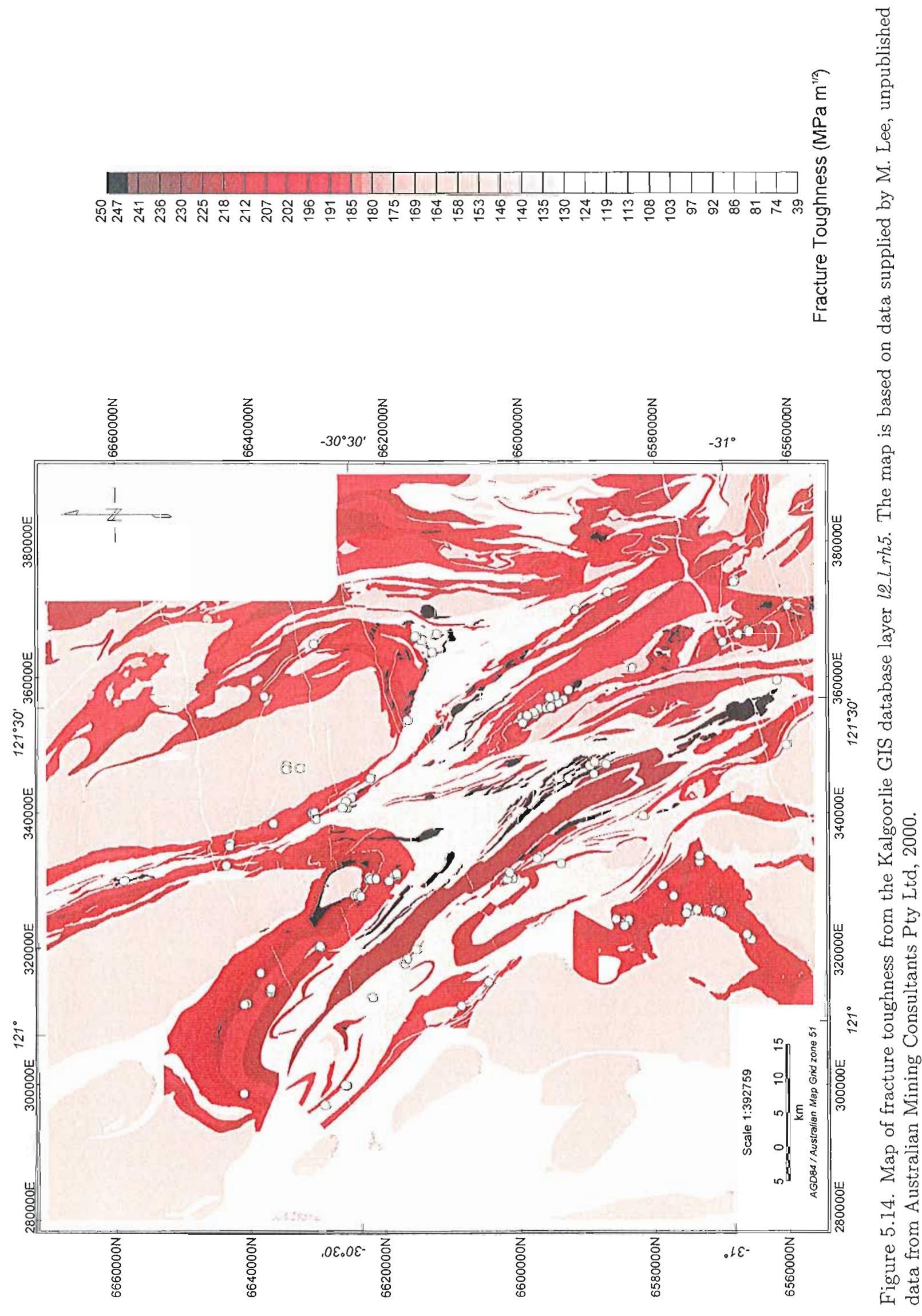




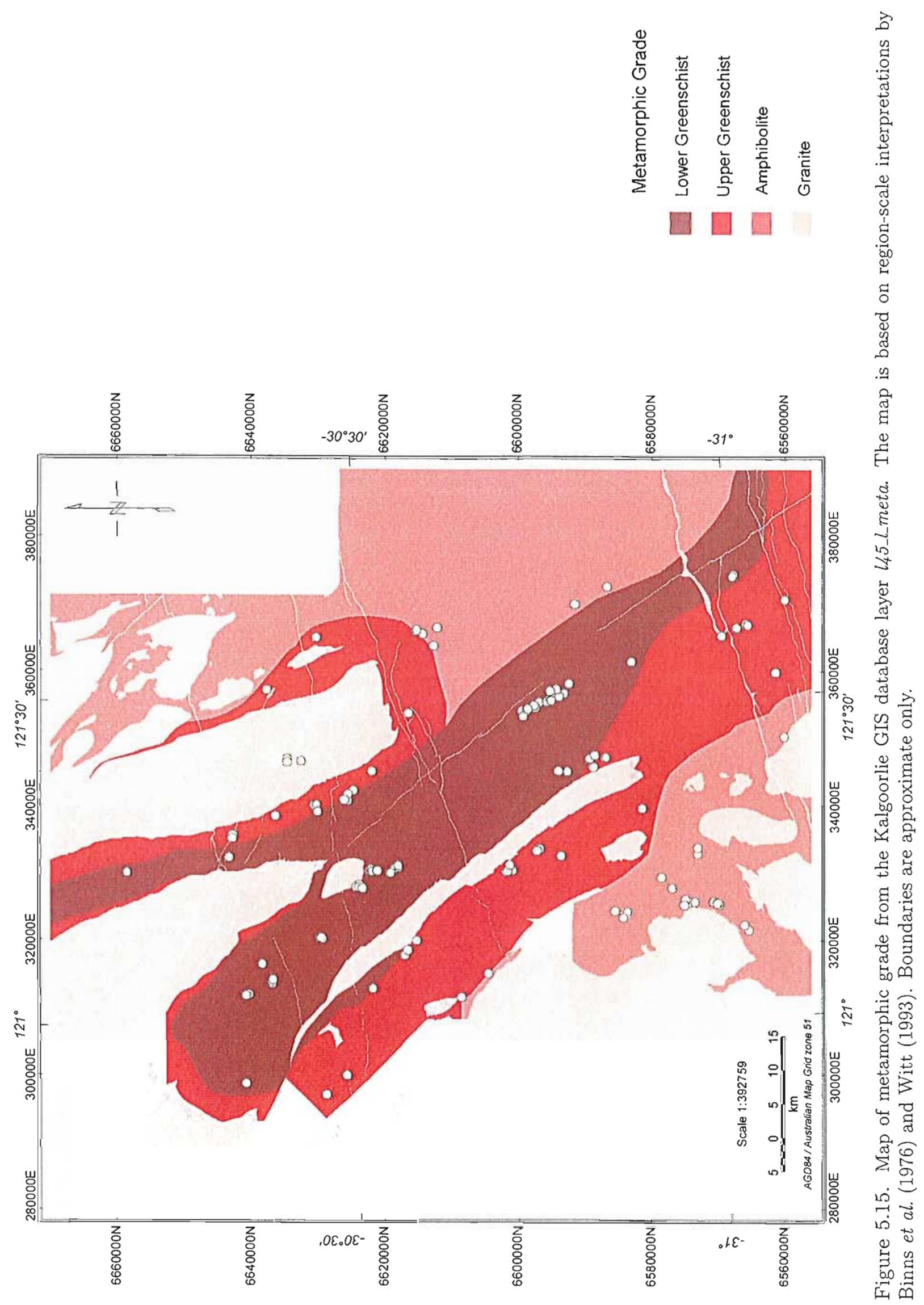




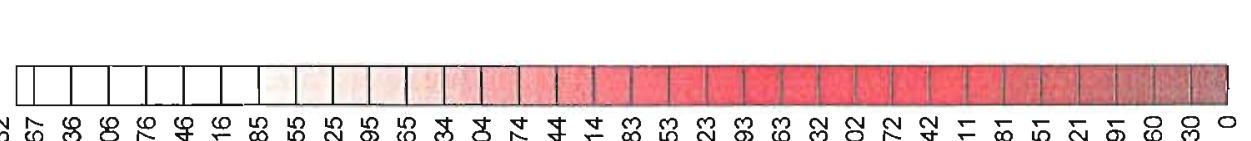

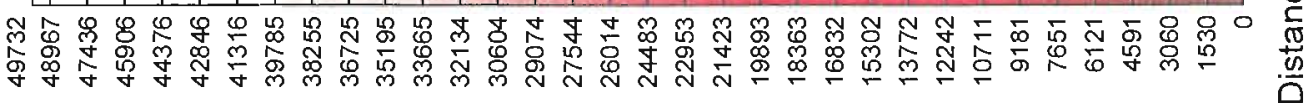

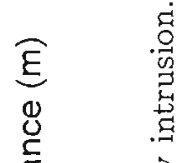

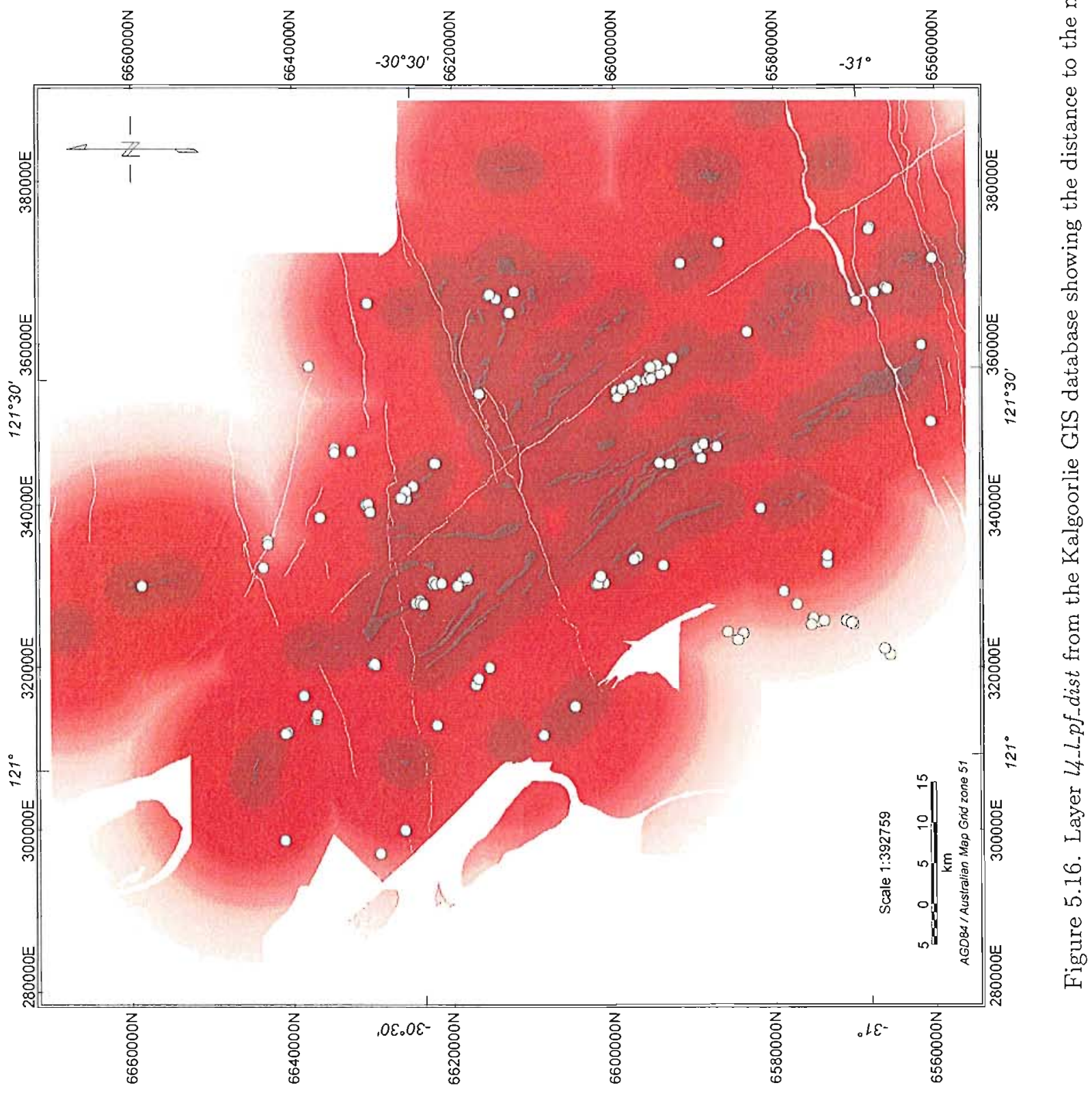




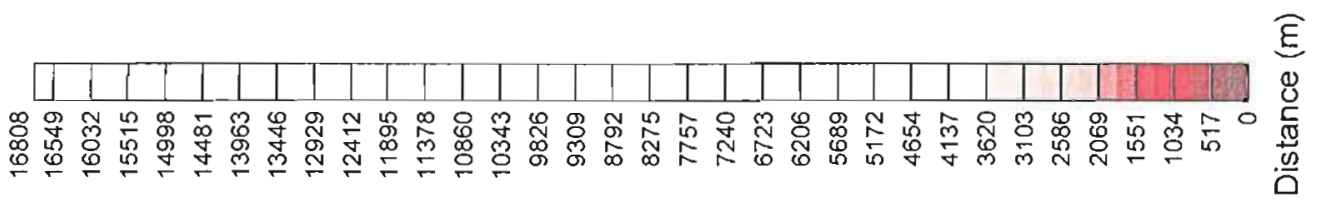

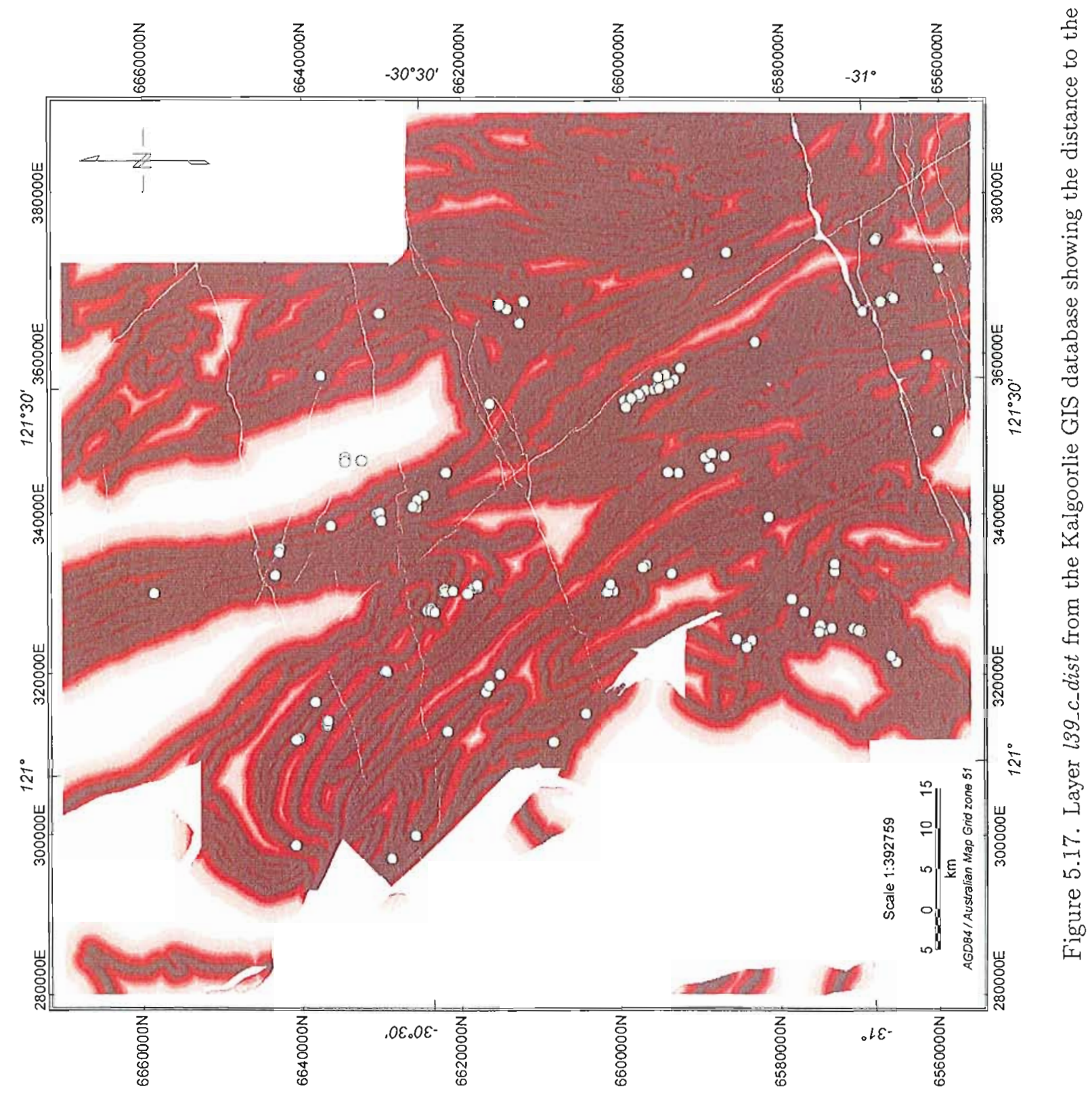




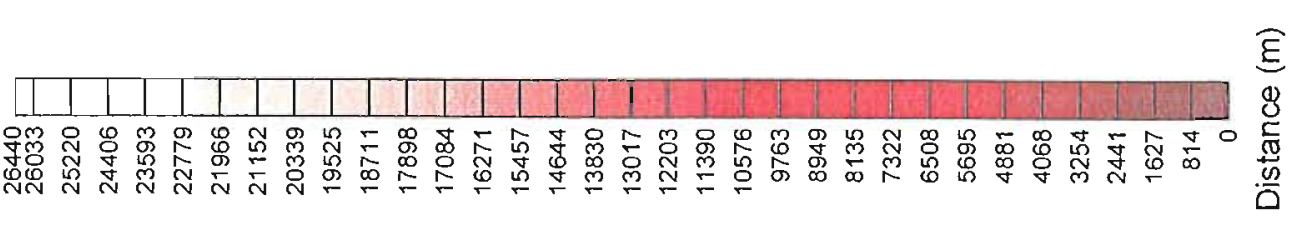

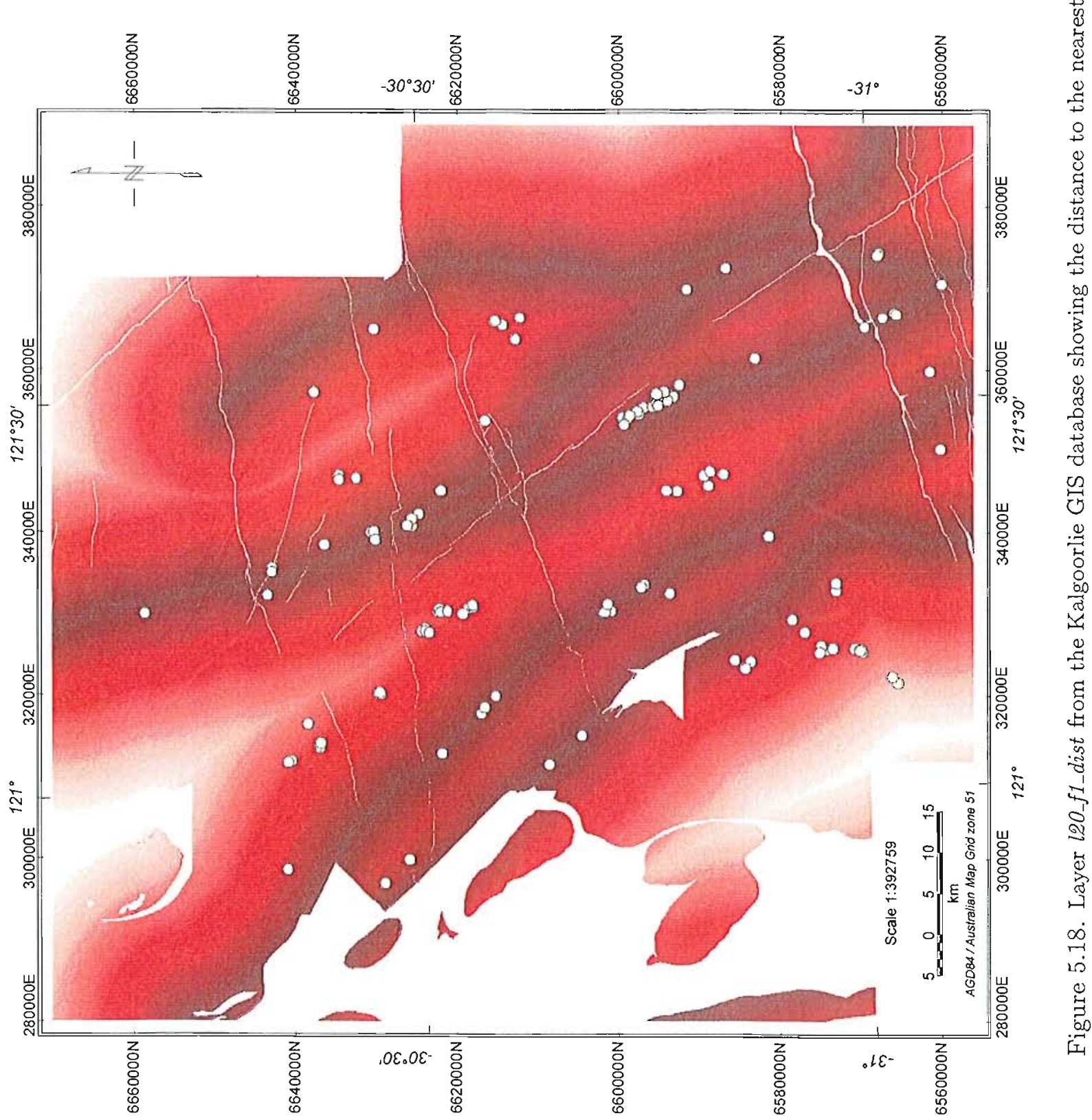




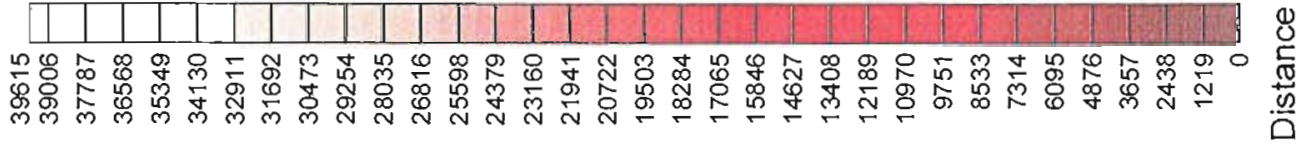

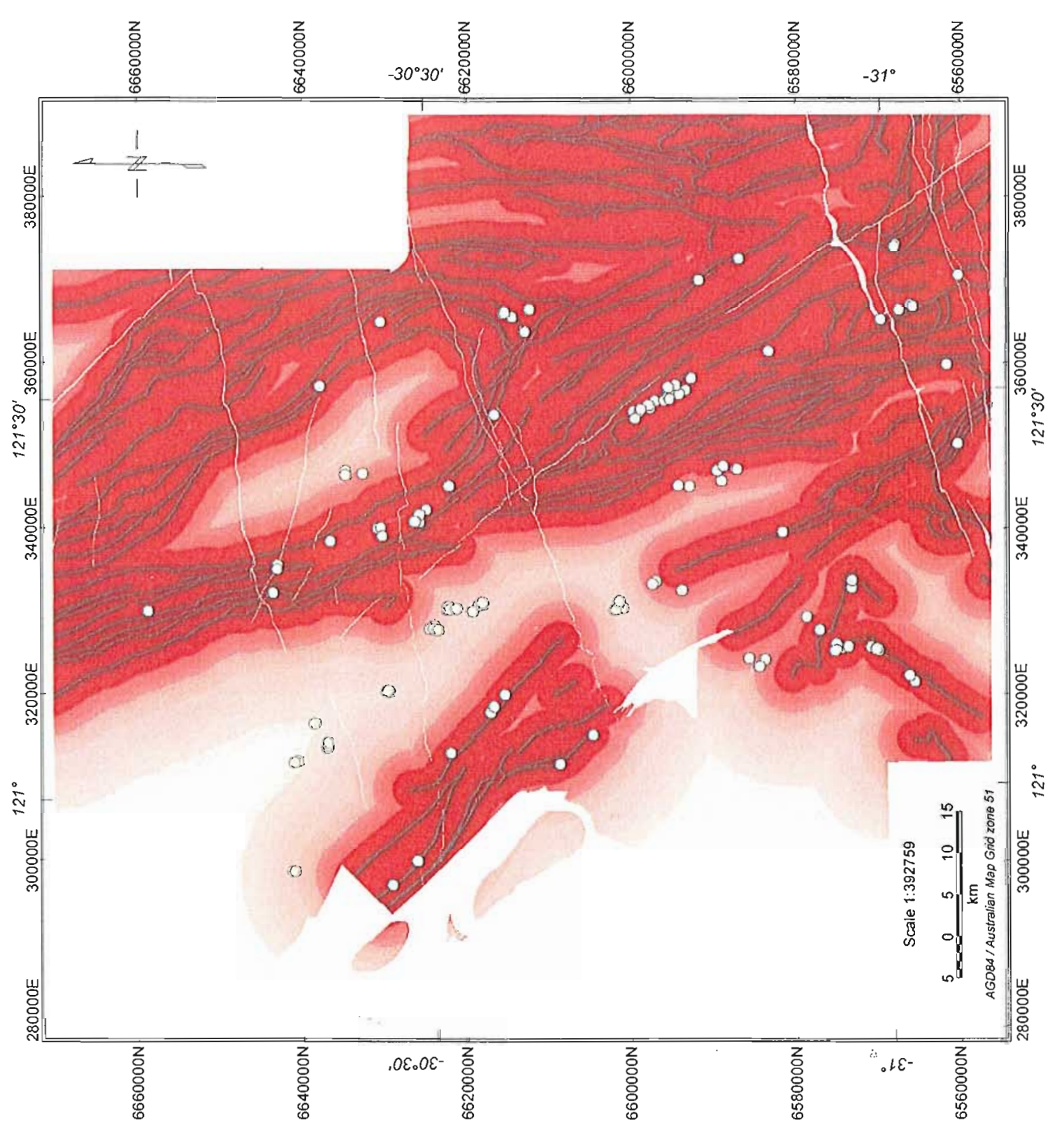




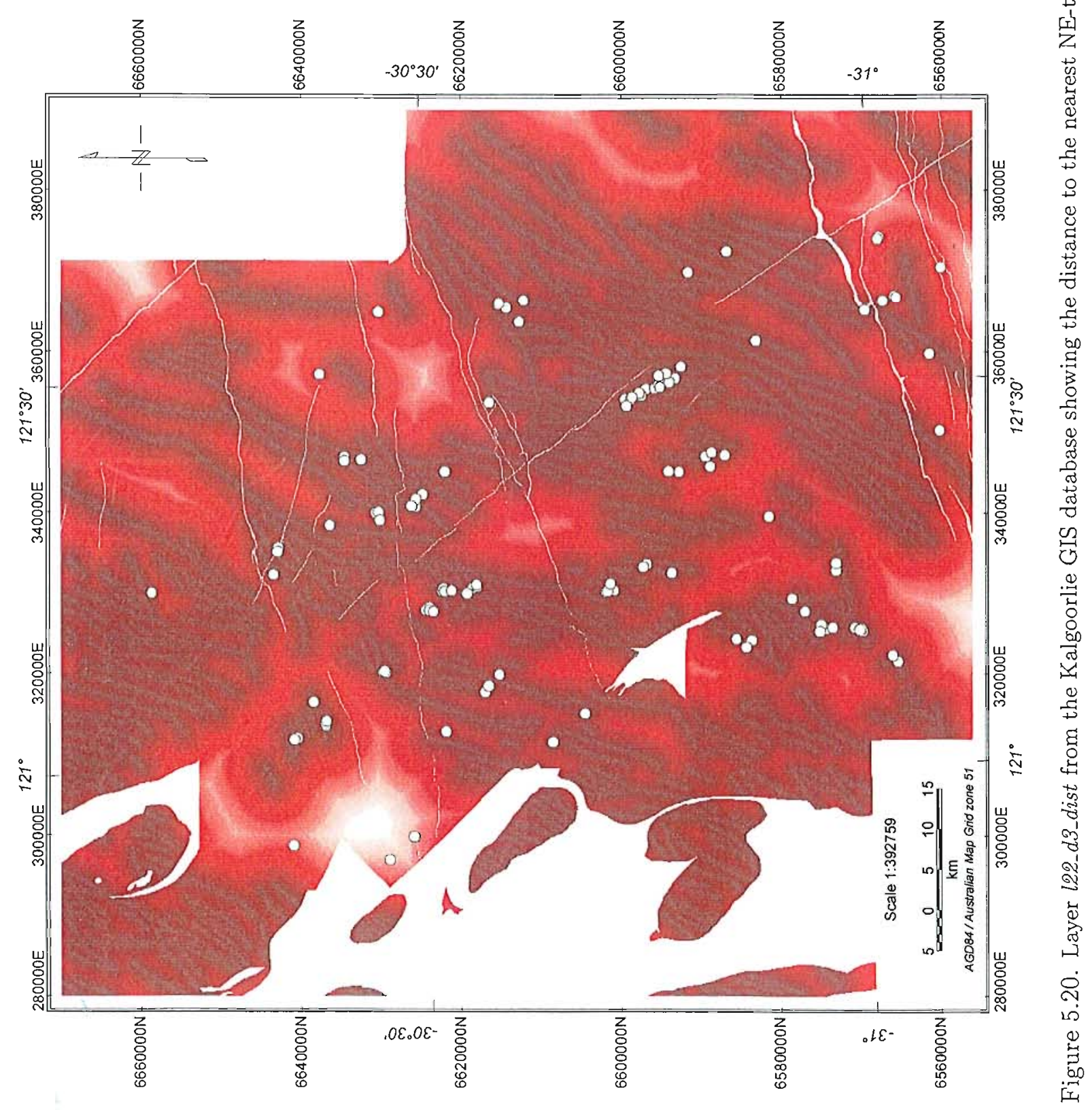




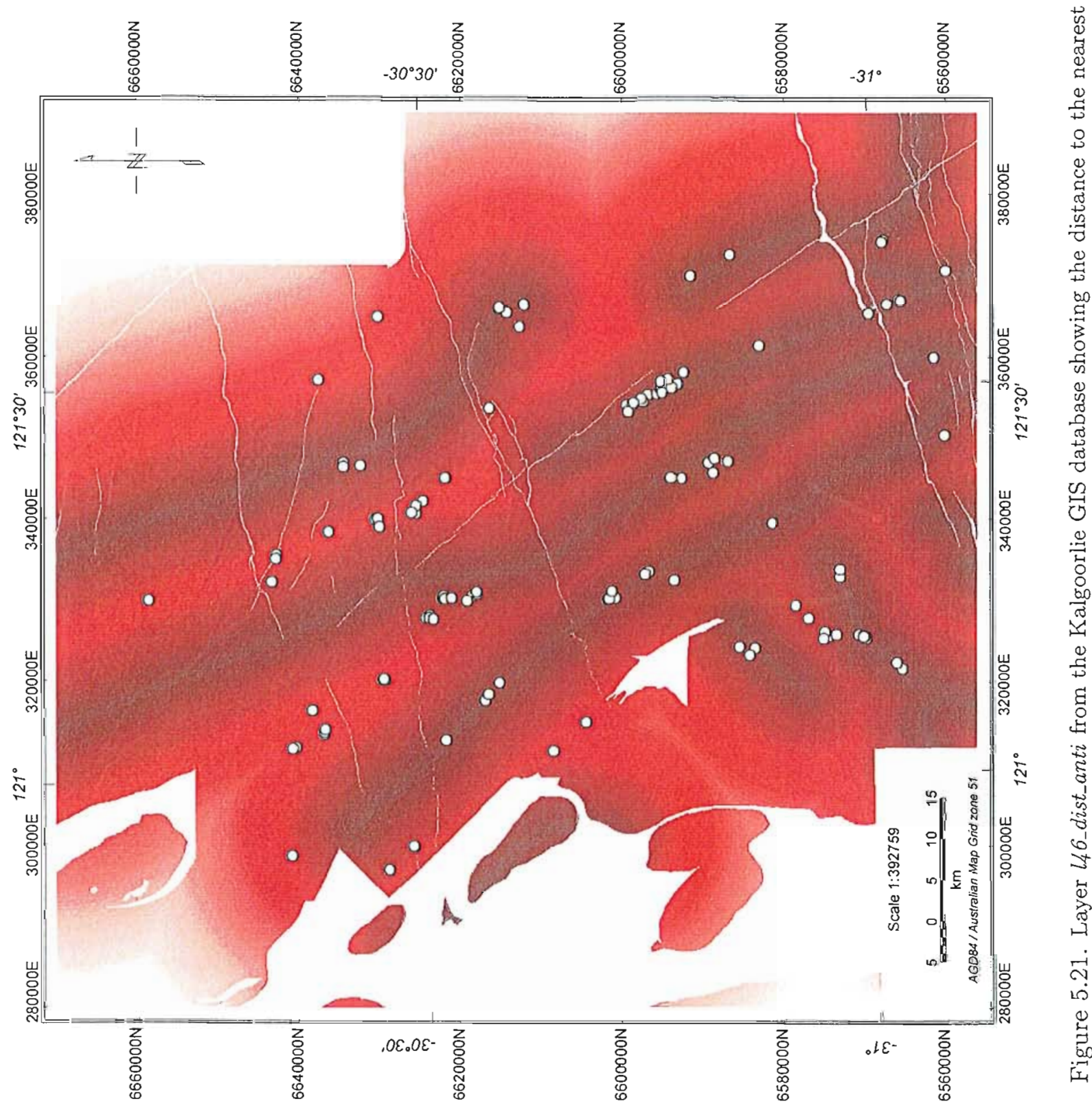




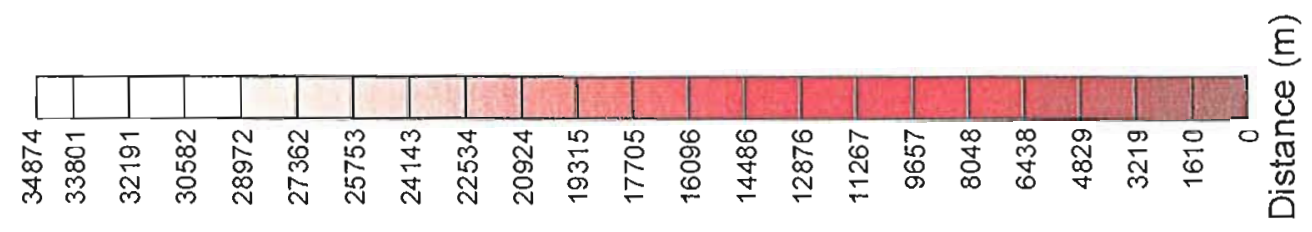

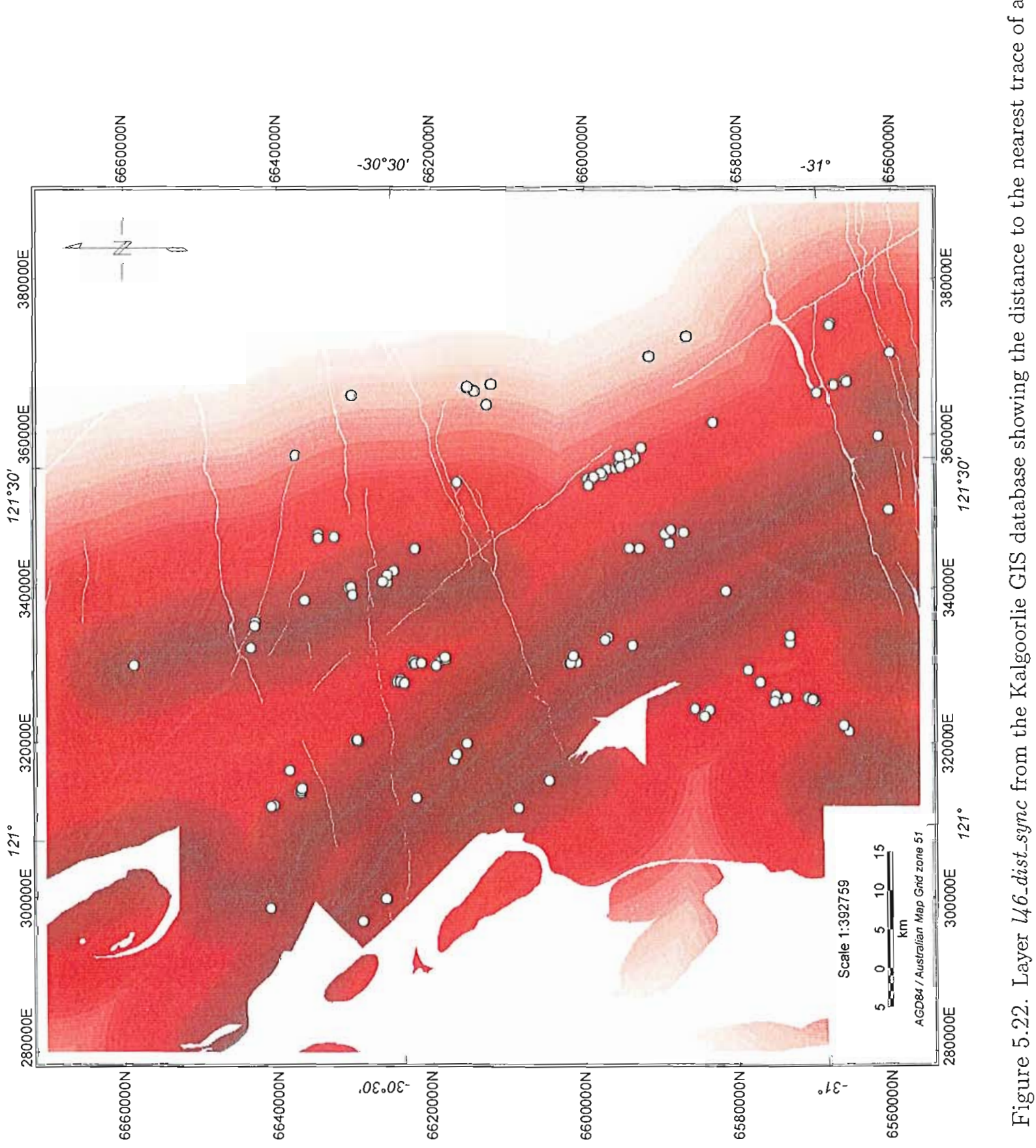

$\dot{\vec{x}}$

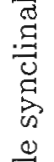



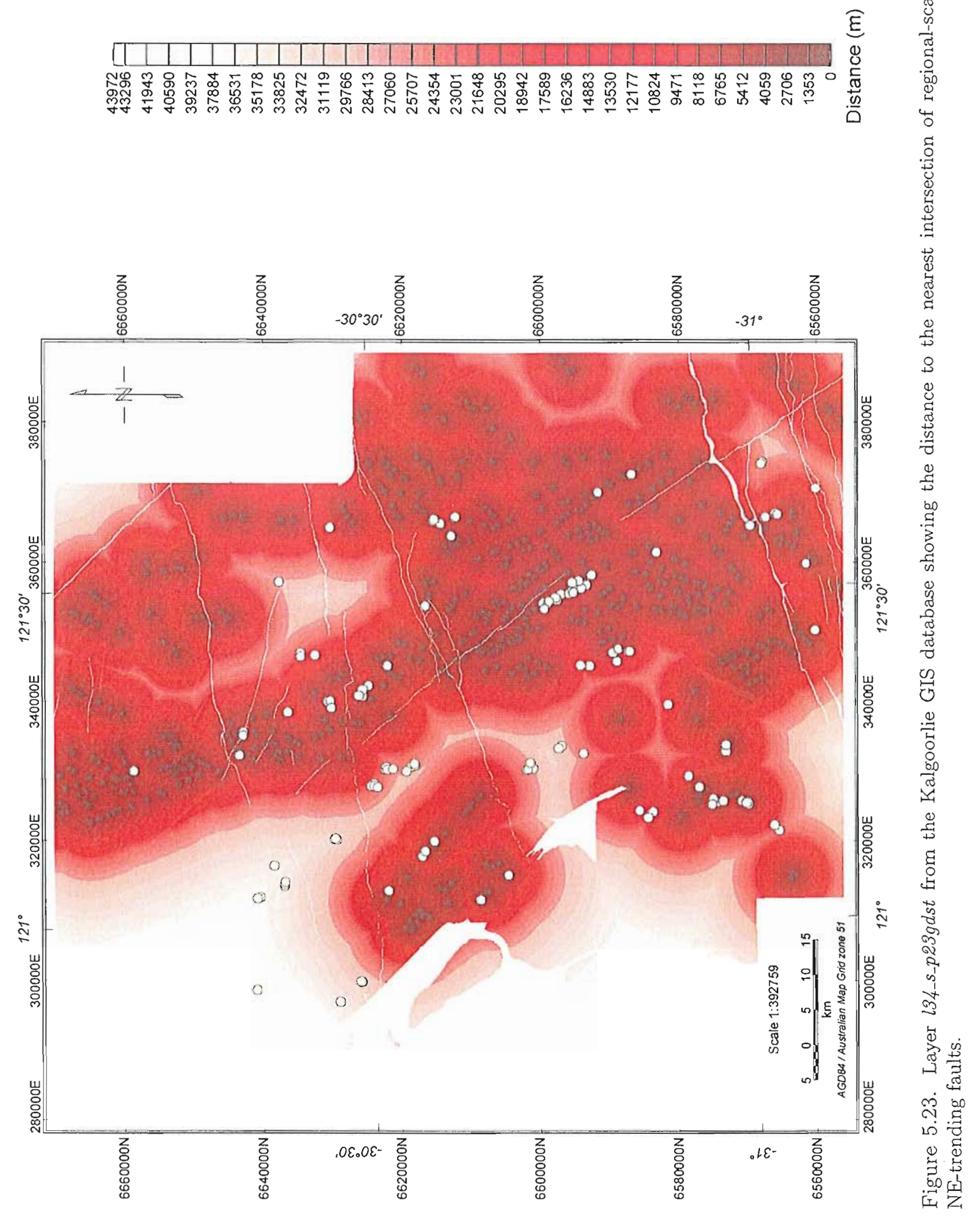


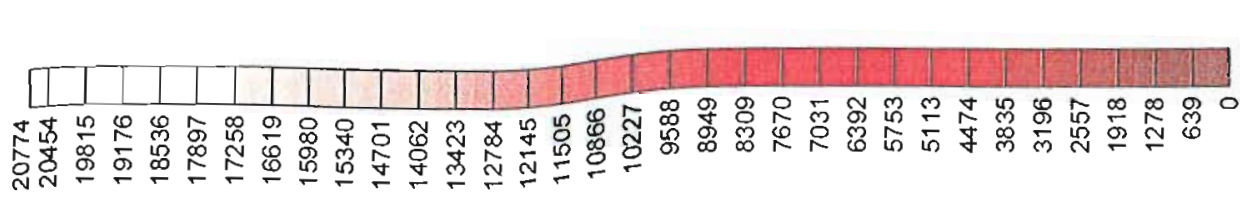

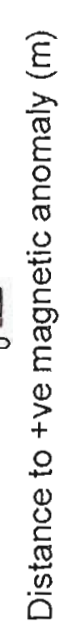

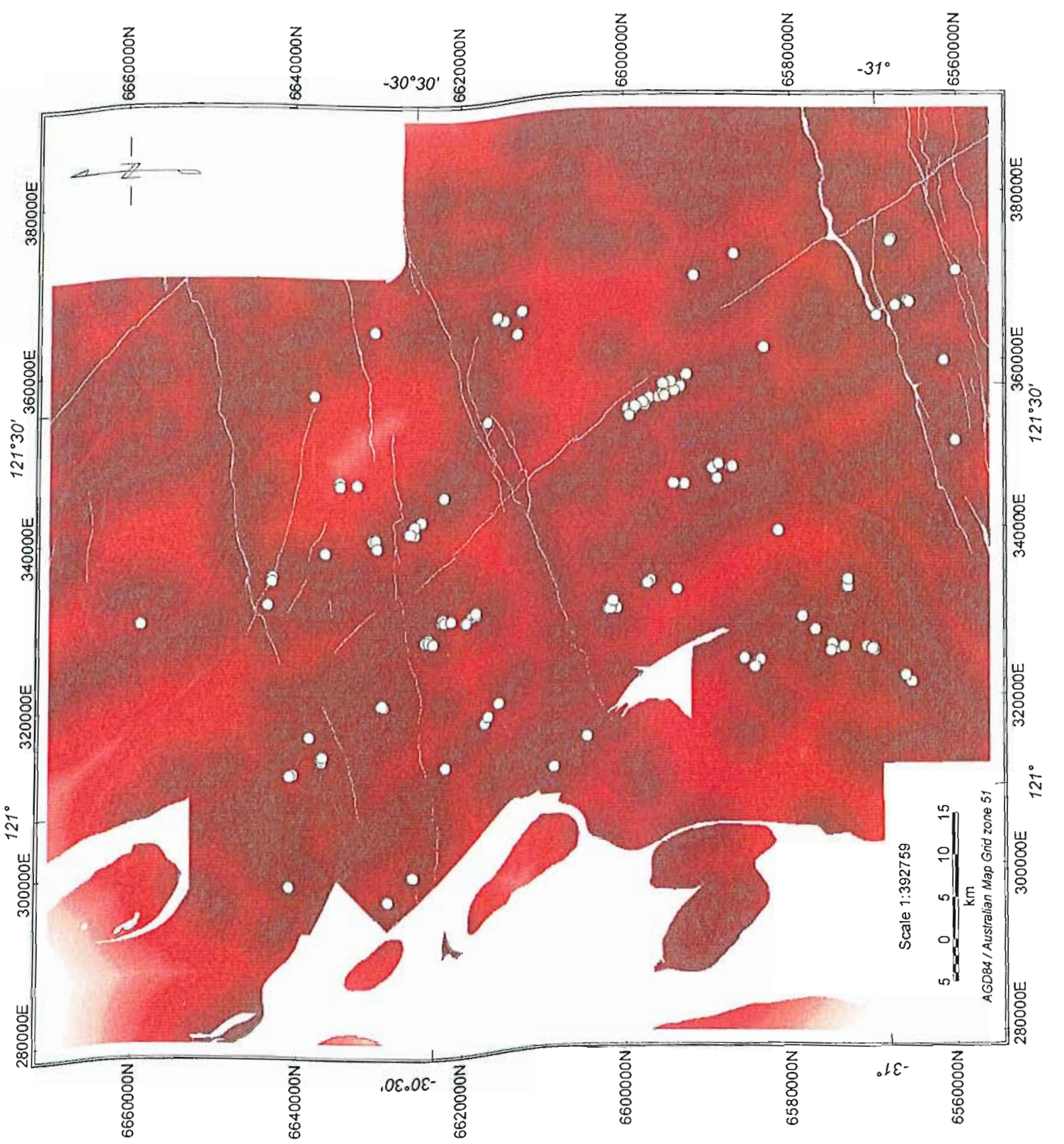

: का 可 กิ 范 की , O 墕 . 总 . 동 E. 40

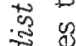
of है घ \& $\rightarrow$ 50 त्త 过 20 员 㟢 $\overbrace{0 \rightarrow}^{\circ}$ 


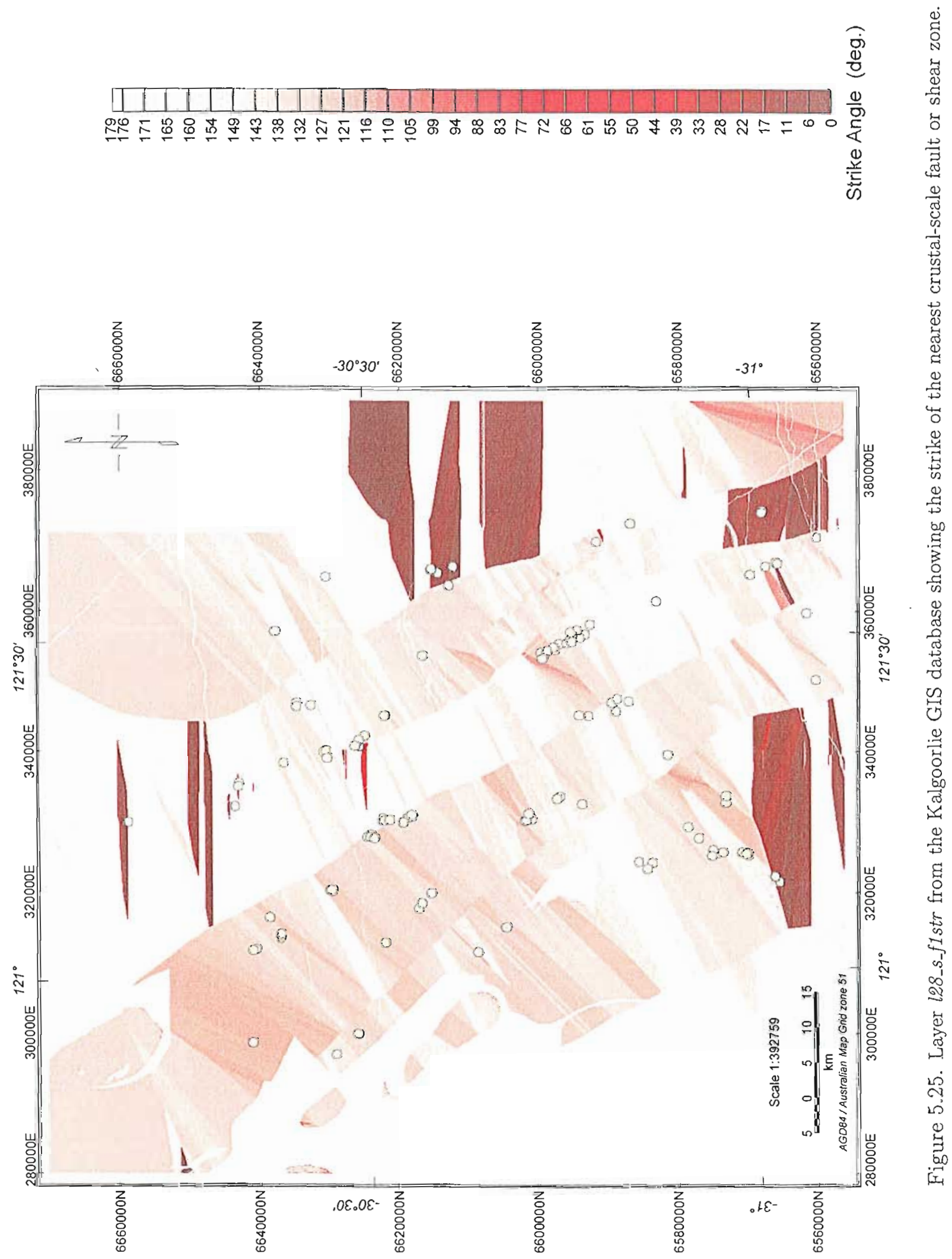




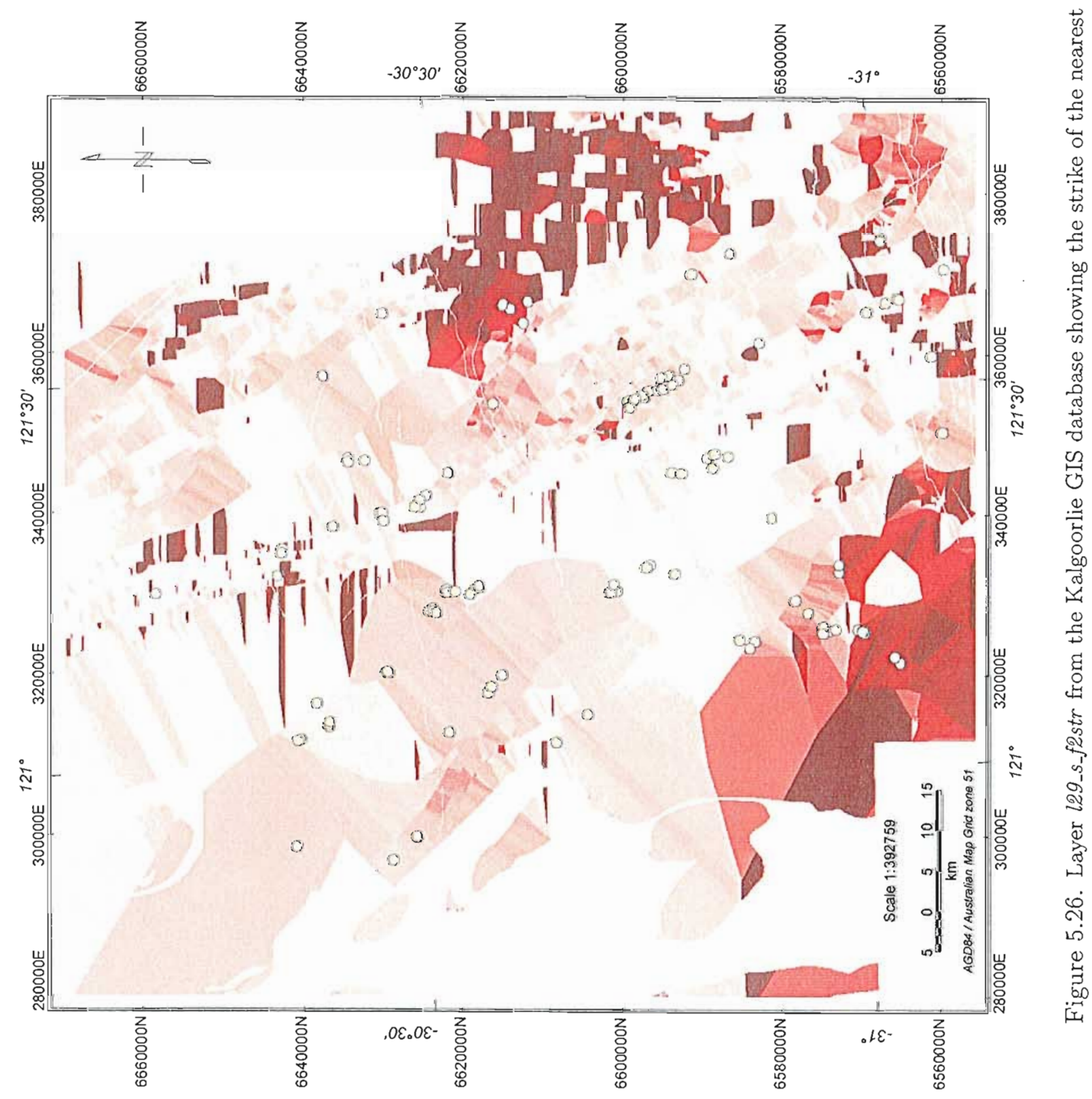




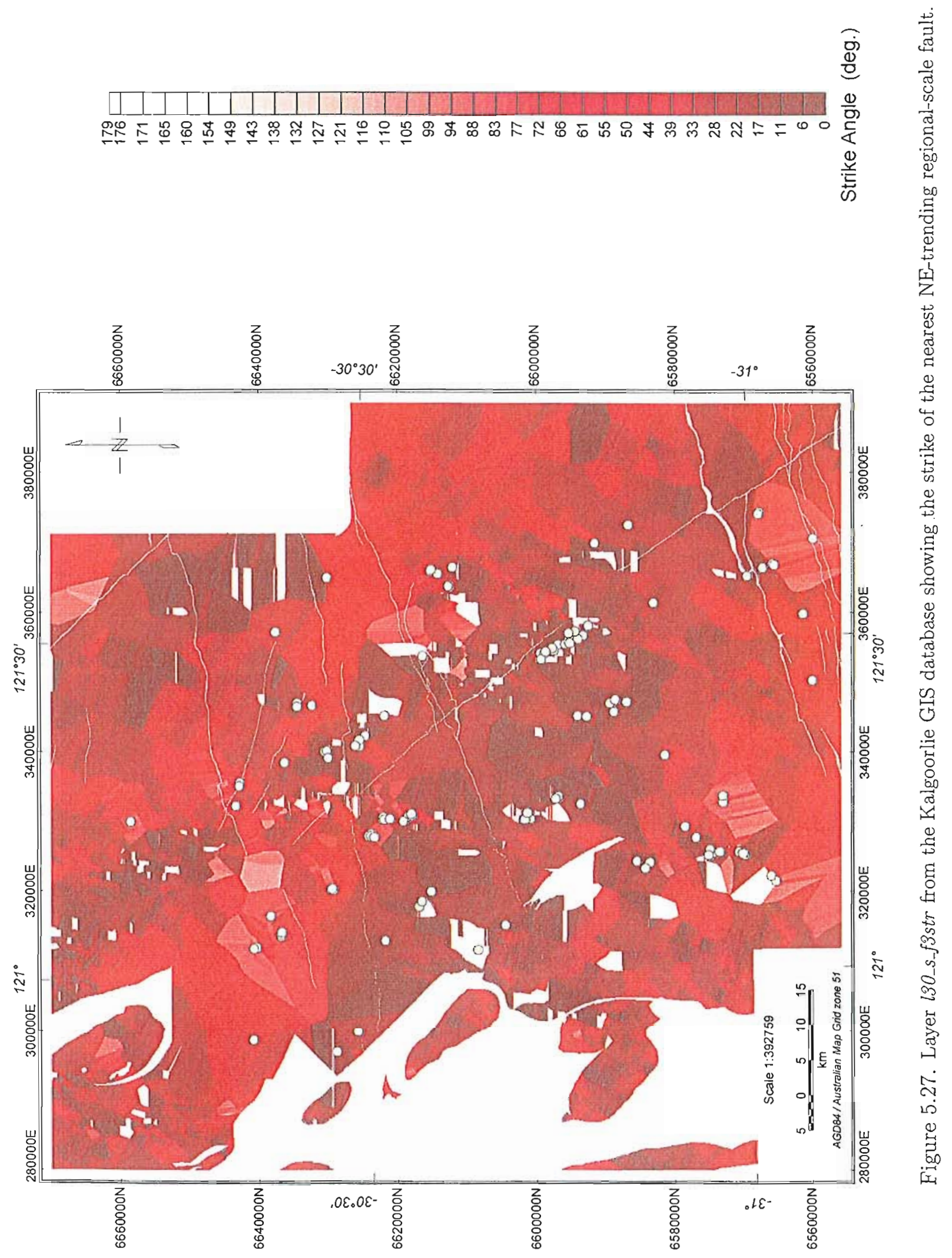




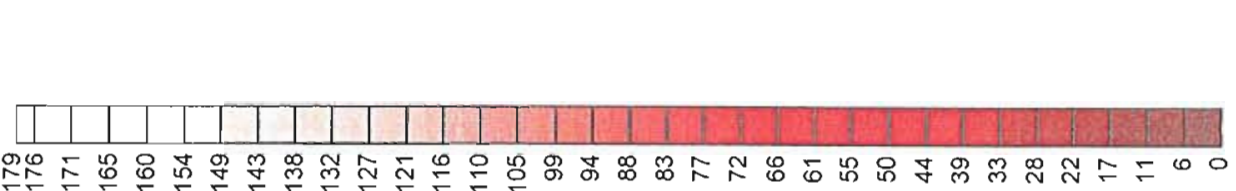

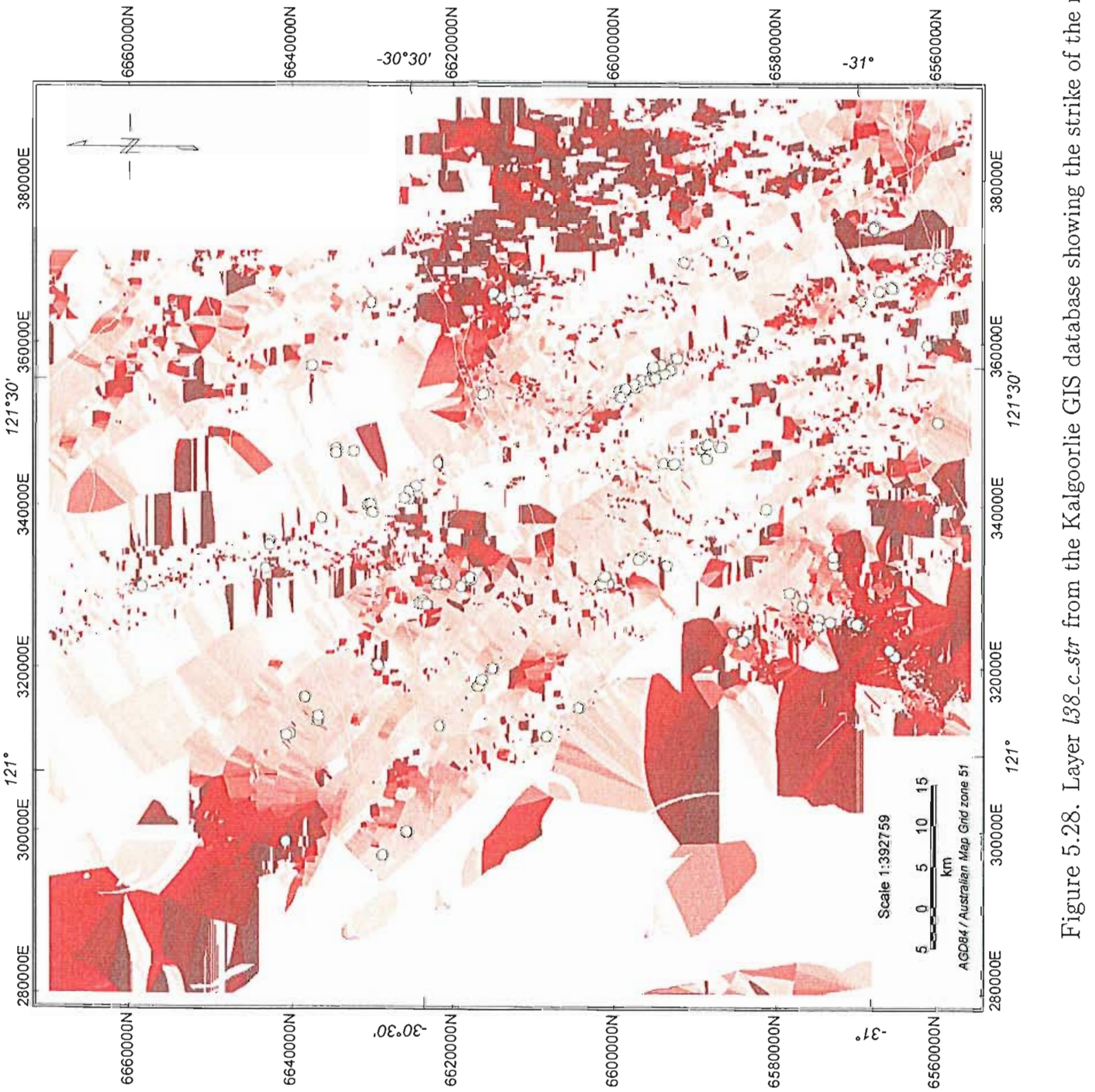




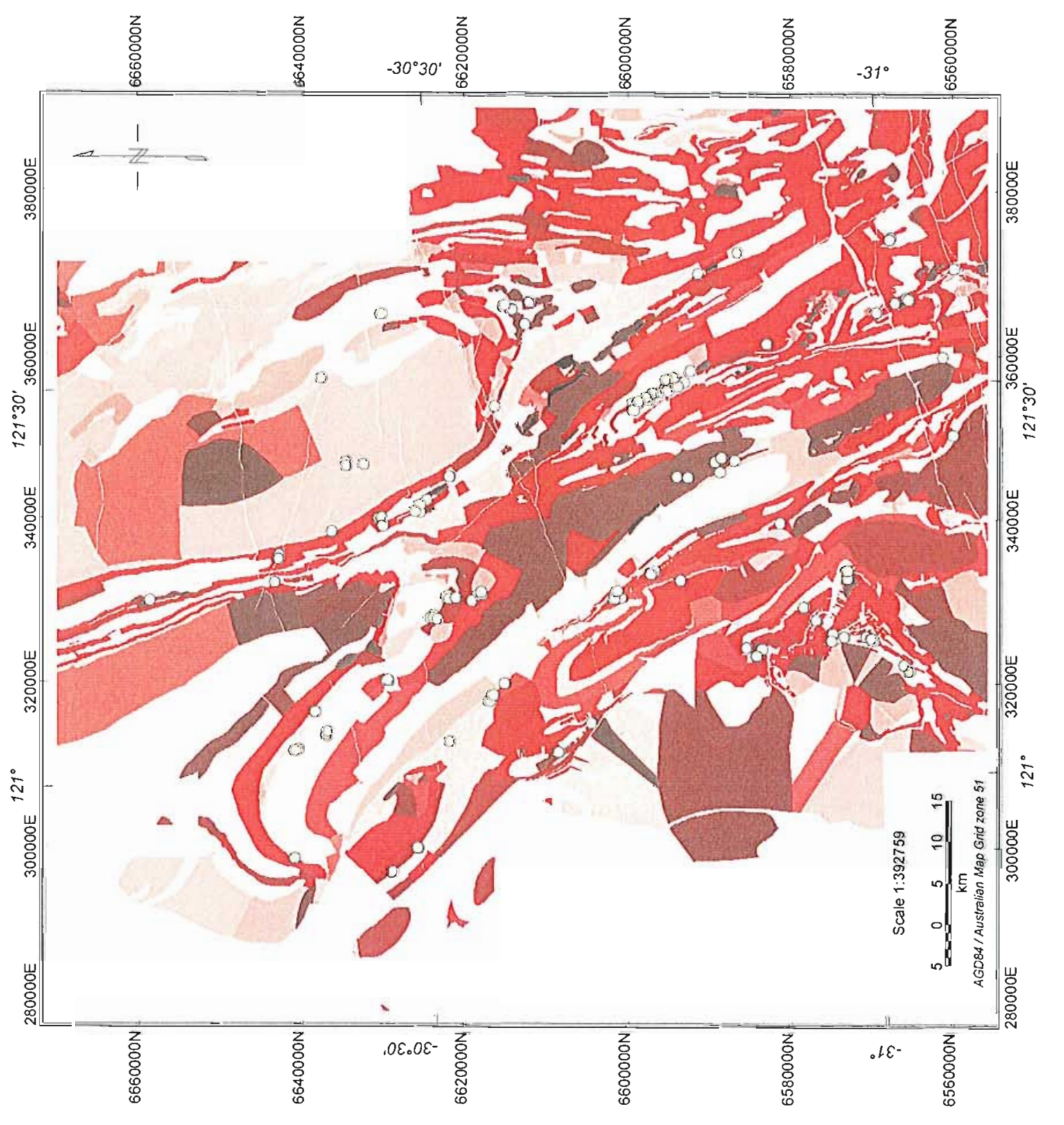

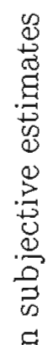

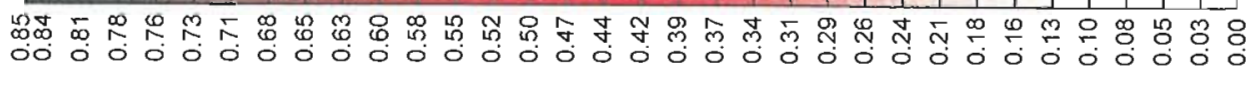

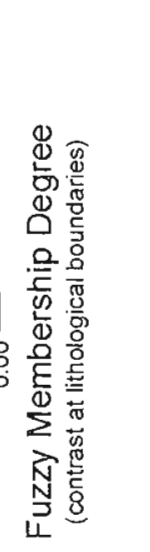



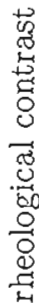

章离

搴 穵 象 空 范 bo 运 8 密 ह 8 넌 로 공 ก 产灾

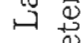
จे द्ध เा 8 号䓀 


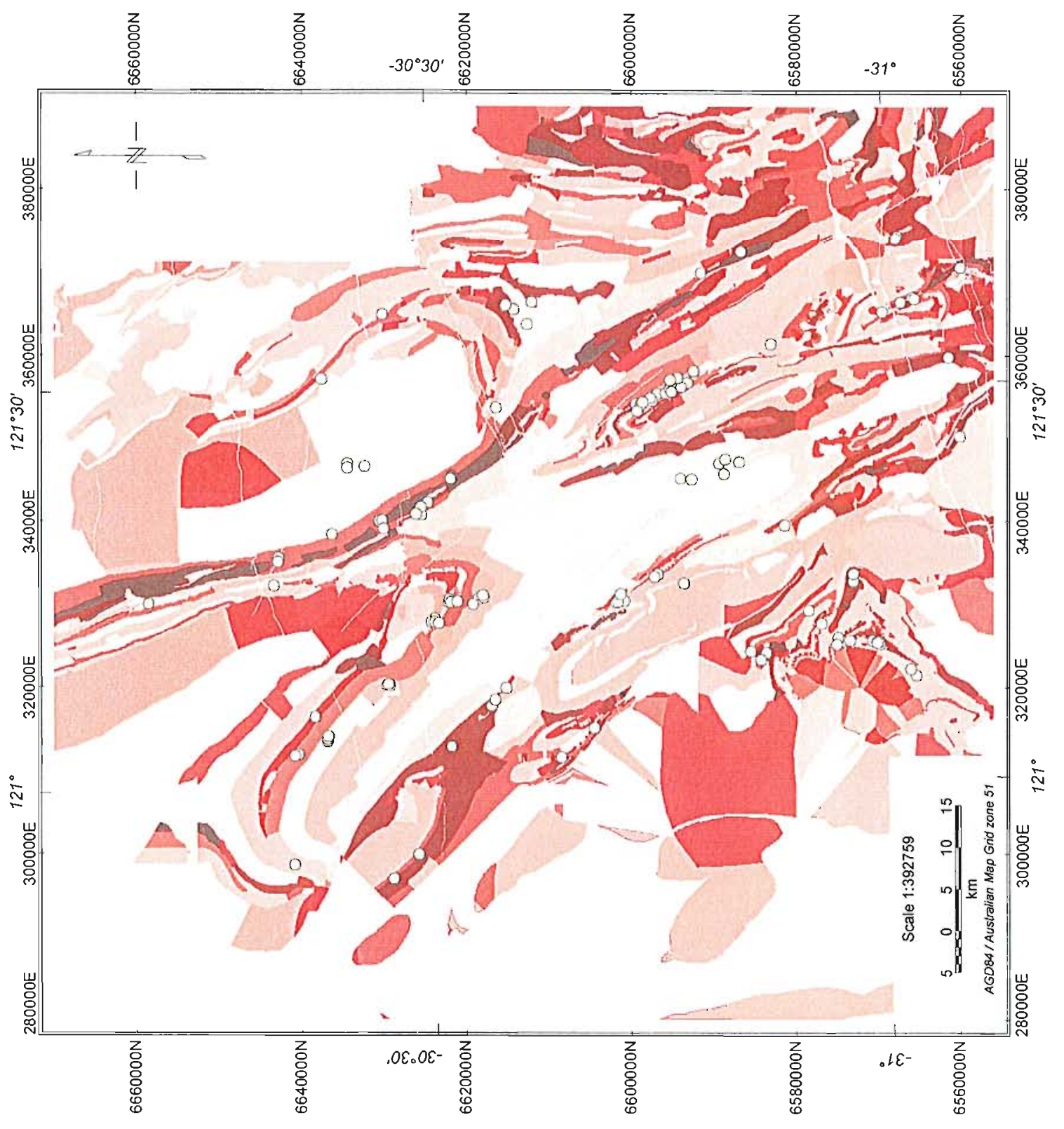




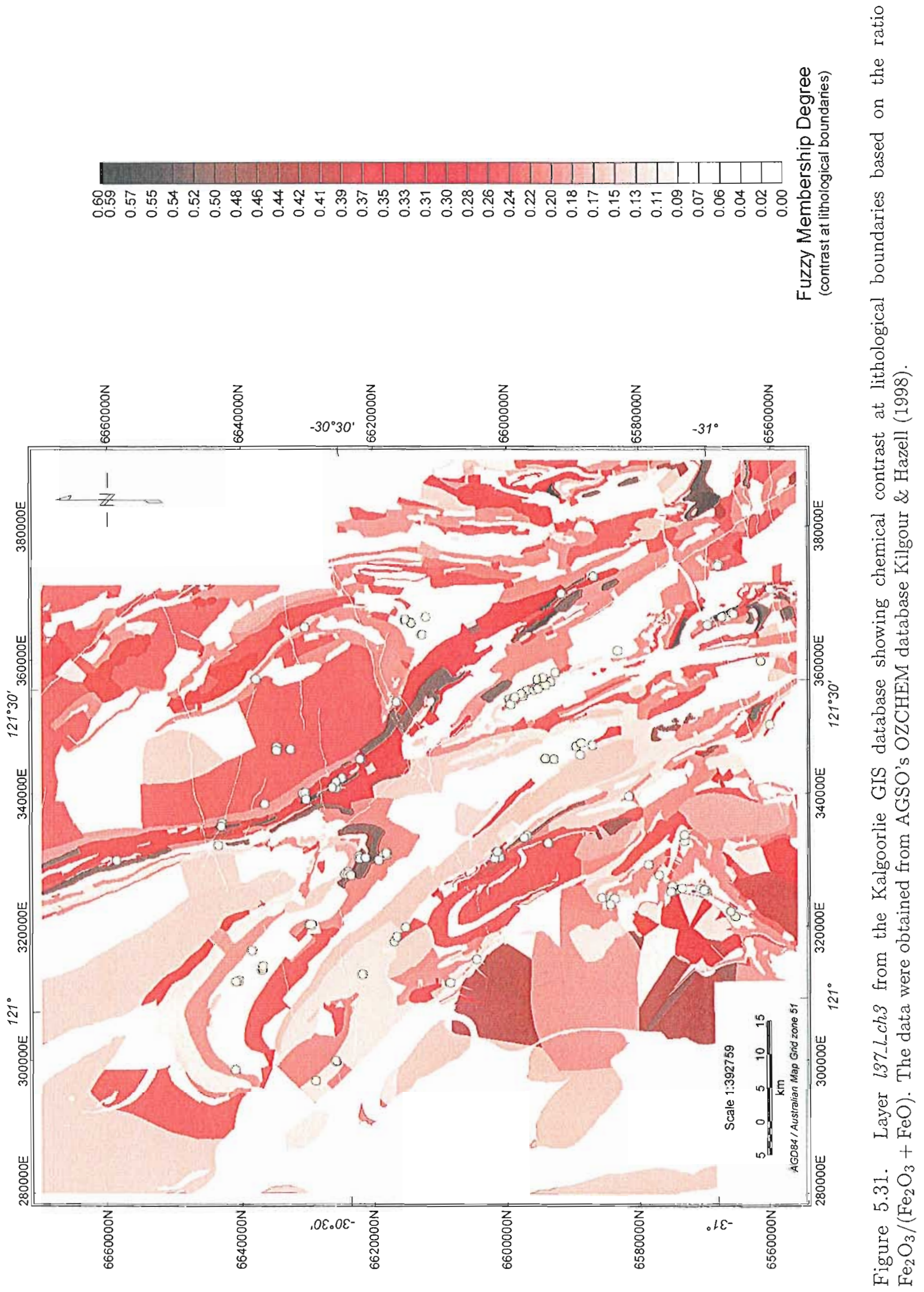




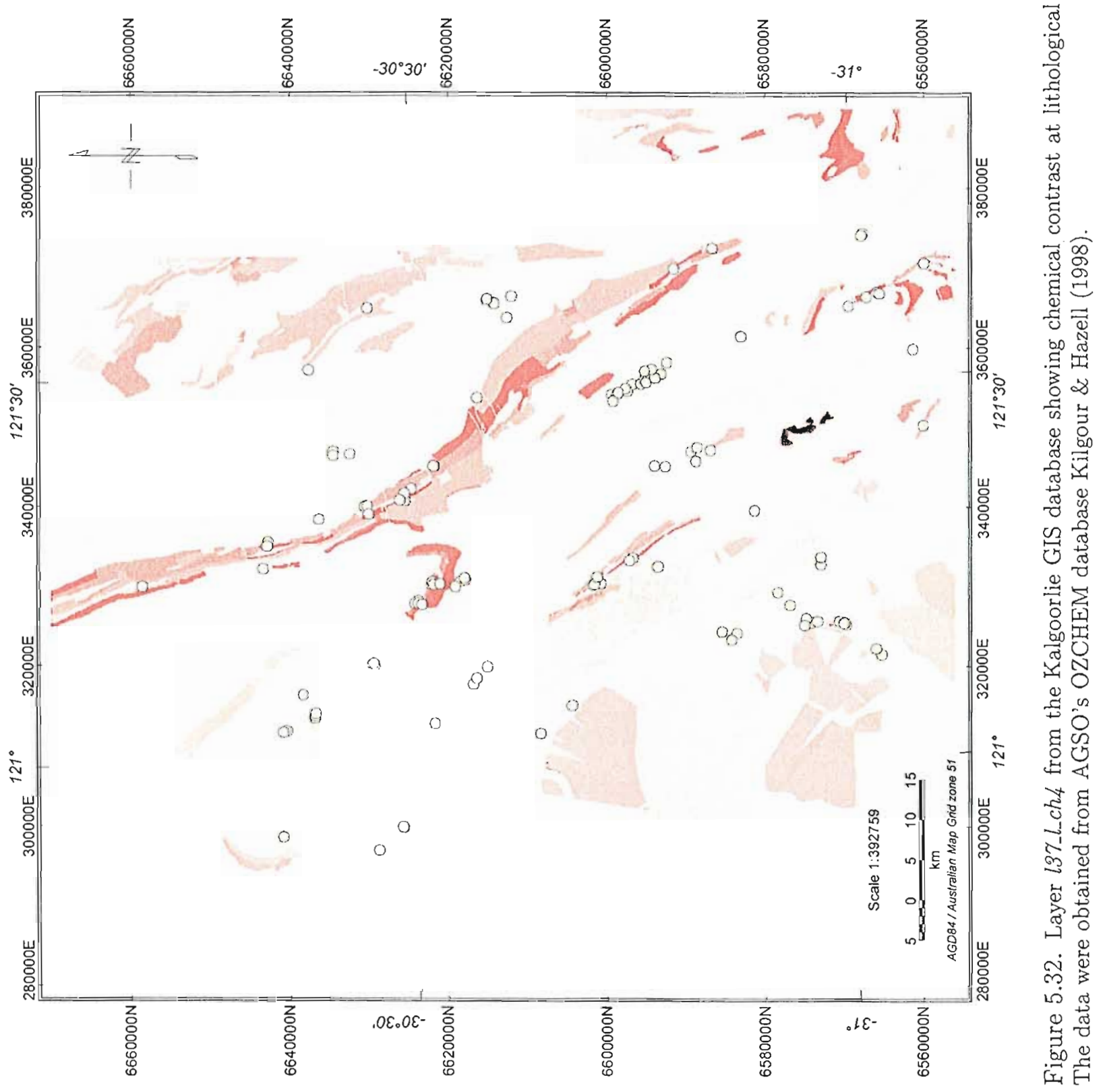




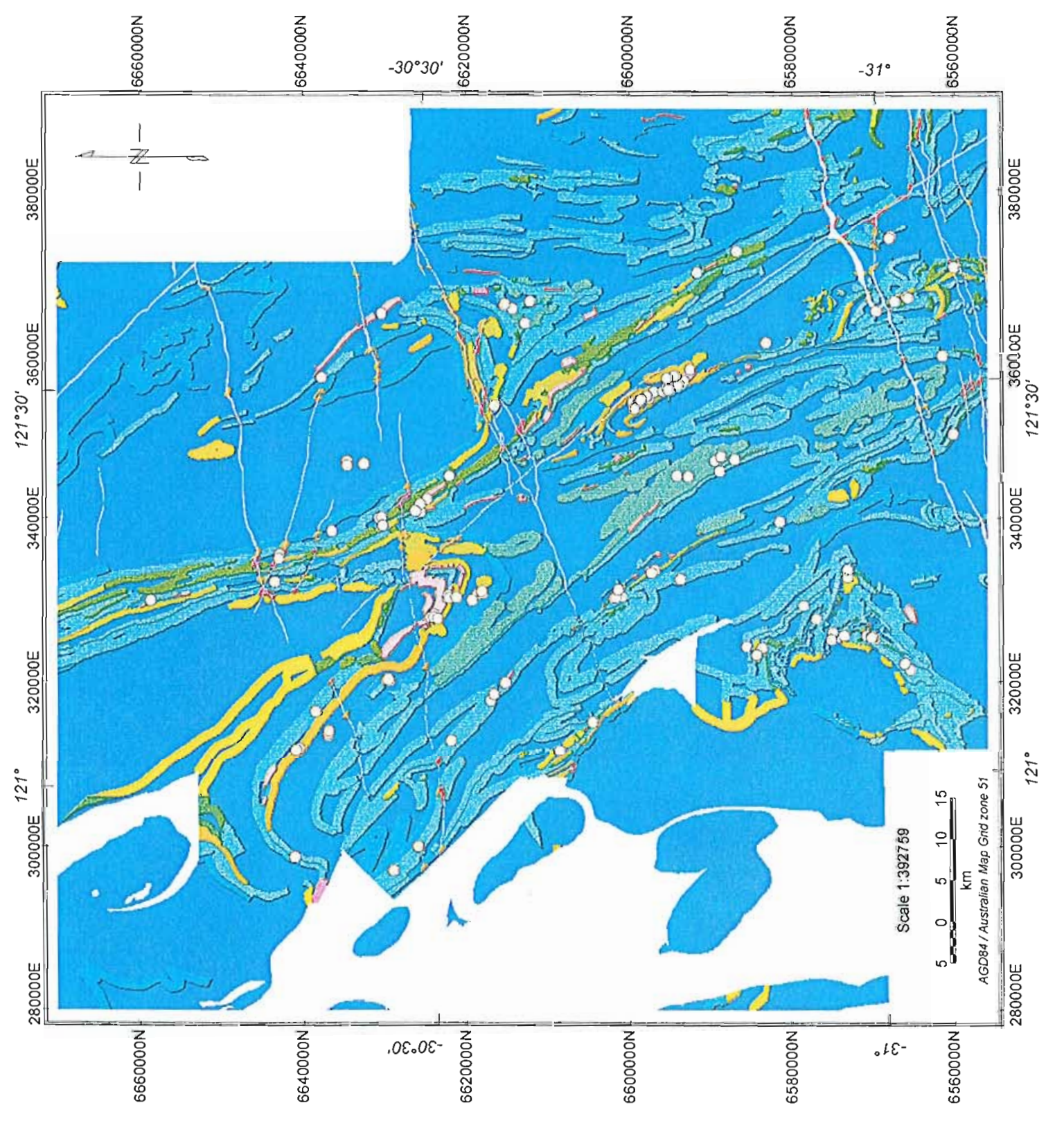

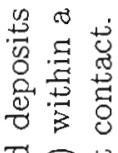

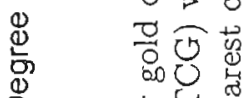

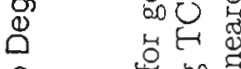

은 $\quad 0$ 里

ते

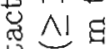

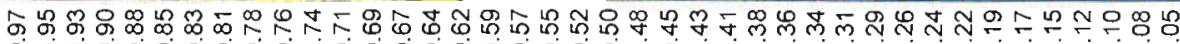

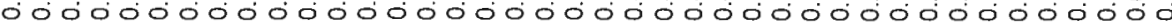

ह
文
건

$8+\frac{0}{8} 8$

पू

宅 ป

द द्व

密总要

एक

空

(⿻)丨

氙茍

तु

연

$\Phi$ 峁

क्षे

责

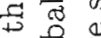

잉영

. 013

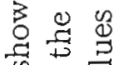

ข

\%

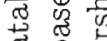

$\frac{0}{0}, 0$

ᄋ ड़ है

$0 \%$

总总

8 零

त्ठ.

论言芯

造苍泀

छ

용 \&

$\rightarrow \rightarrow 0$

ह $\mathrm{N} \Omega$

讨瓜

है क

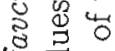

$\forall$ 零

요

䎡点

$\infty$ द्व

एक द्व

怘趂 㐘出 


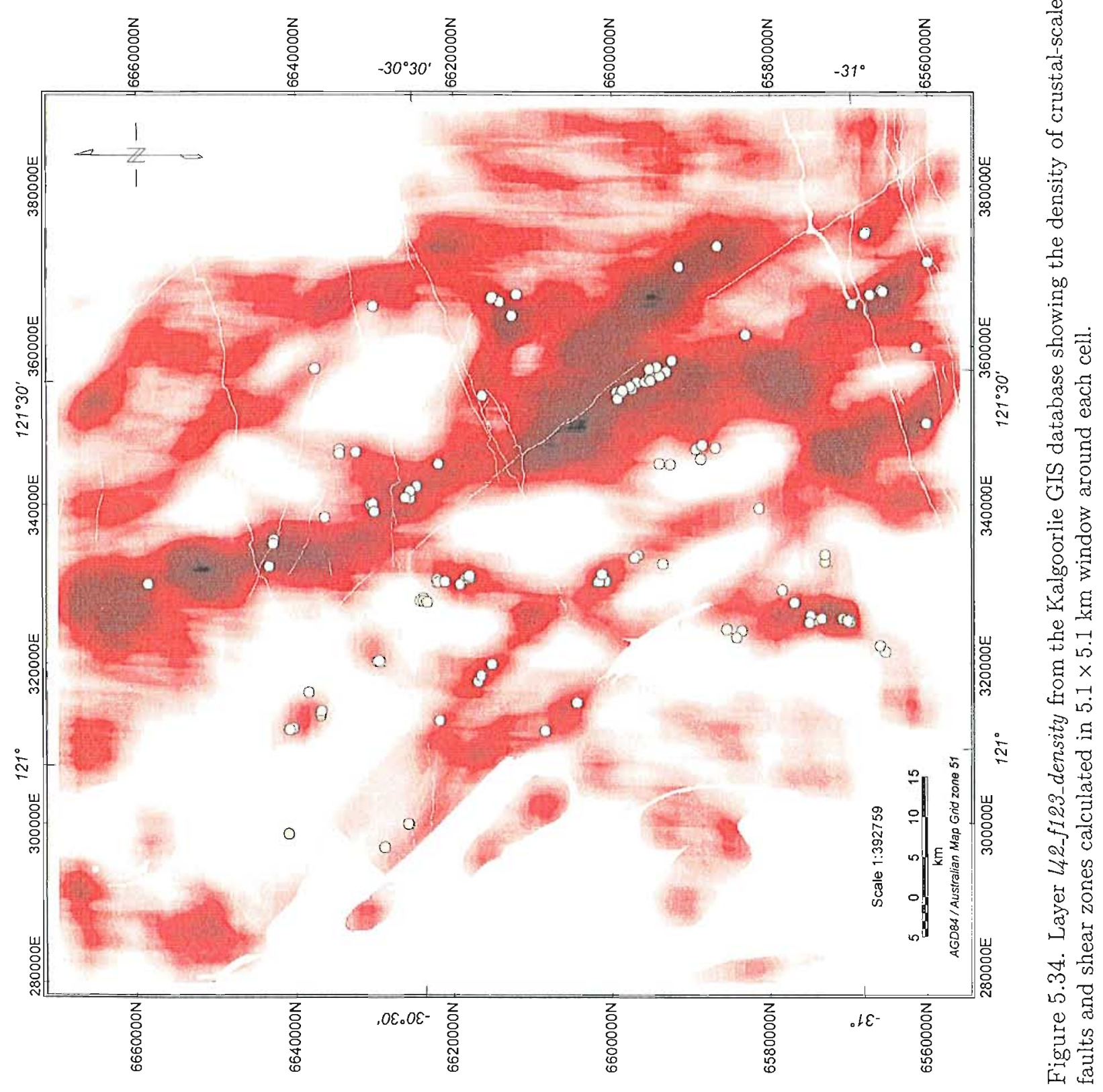




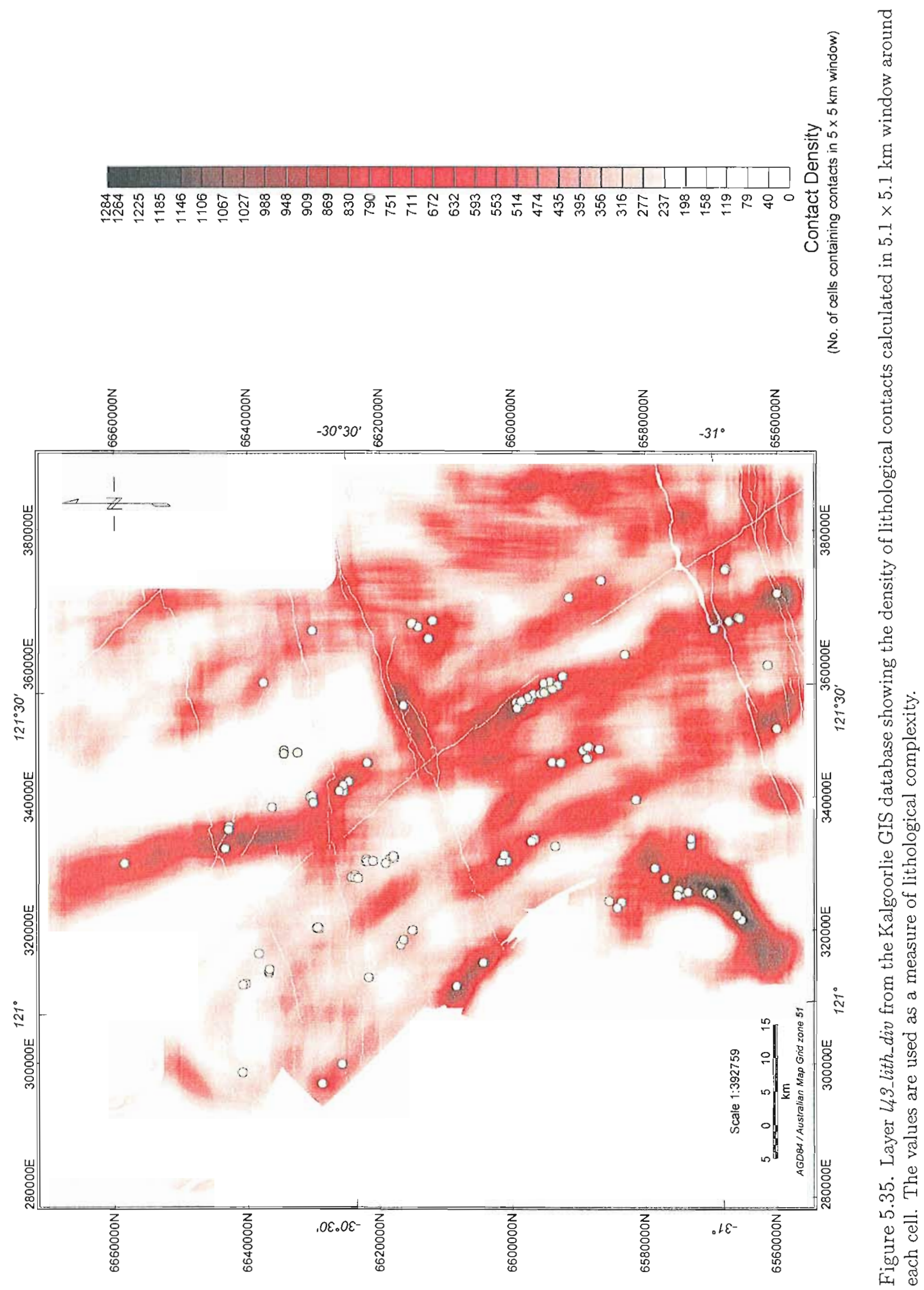




\section{Chapter 6}

\section{Feature Selection: Evaluating the predictive ability of potential input GIS Layers}

\subsection{Introduction}

The purpose of this chapter is to extract and empirically evaluate features in order to select the most useful ones to be used as neural network inputs in Chapter 7. Thus, the task consists of two parts;

1. pre-processing and feature extraction, and

2. feature selection.

Pre-processing and feature extraction can often greatly improve a pattern classification system, particularly if prior knowledge can be incorporated into the inputs (Bishop, 1995). Consequently, much of this chapter is devoted to the preparation of derived GIS layers in order to represent the essential ingredients of the mineral systems in terms of the regionalscale GIS data that are available. For example, rather than present the network with a binary layer indicating the presence or absence of faults, groups of faults which are related to different deformation events are divided into separate layers. Layers are then transformed so that cell values represent the distance to the nearest fault of a specific type. The basic principles are to avoid encrypting the underlying structure of the data and to avoid using irrelevant inputs (Gedeon, 1996). 
Feature selection is the problem of choosing a small subset of distinguishing features that are most useful as inputs. It is widely agreed that data encoding and feature selection are of paramount importance for any learning algorithm (Kohavi \& Frasca, 1994; Kohavi, 1995; Gedeon, 1996; Piramuthu, 1996). Kohavi \& Frasca (1994) have shown that, in practical situations, using a subset of the core features may lead to superior performance.

The approach taken in this study is to apply statistical methods for measuring the spatial association of known deposit points and spatial features in order to assess the predictive value of 60 possible input GIS layers. The methods used are contrast of weights $\left(C_{w}\right.$, Bonham-Carter, 1994), the chi-squared statistic $\left(\chi^{2}\right)$, and the Kolmogorov-Smirnov cumulative distribution function (KS). Three new methods are also introduced. These are the cumulative and interval-based calculations of the bivariate J-function and plots of $(O-E) / E$ (where $\mathrm{O}$ is the observed and $\mathrm{E}$ is expected number of deposits in a buffer; refer to Sections 6.2.6 and 6.2.4). The latter two methods were developed in this study. The best GIS layers selected using these methods were then used as inputs in the neural networks experiments in the following chapters.

One of the problems in modelling mineral systems at a regional scale in order to predict the prospectivity for ore deposits is the choice of potential input layers. There are many different ways to interpret the essential ingredients of a mineral systems model in terms of features that are likely to have been mapped and compiled in a regional-scale GIS database. In addition, each of these ingredients can be represented as GIS layers in different ways. For example, Archaean orogenic gold deposits form selectively where fluids have been focussed into dilatant zones or zones of high structural permeability, which, in turn, are produced by heterogenous stress fields in areas with complex geometries and strong contrasts in rock strength. Such areas could be modelled using layers representing proximity to faults, shear zones, lithological contacts or anticlinal hinges, segments of faults and shear zones with favourable strike orientations, density of lithological contacts, favourability of rock types at the nearest lithological boundary, and chemical or rock-strength contrasts at lithological boundaries. Rheological contrast could be modelled using subjective estimates of rock competency in the form of fuzzy membership values, Young's Modulus, uniaxial compressive strength, uniaxial tensile strength or fracture toughness. The aim is to select only the GIS layers containing the features, which are the best predictors of the known gold deposits in order to ensure that the neural networks correctly learn the underlying patterns in the data.

A sensitivity analysis was not a feasible method in this study as there are 60 potential input GIS layers. In a sensitivity analysis, all the layers are used to predict the known deposits 
and then, as each layer is subtracted (and later replaced), it is noted whether the predictive performance of the resultant prospectivity map increases or decreases (e.g. Knox-Robinson et al., 1996). Only those layers that improve the performance are subsequently used as inputs to the analysis.

It has been suggested that principal component analysis could be useful in reducing the number of inputs (P. Wong, pers. comm.). However, Masters (1993b) has warned that it may be that the information required by a network to perform a classification may be contained in the last principle component; i.e. the one that accounts for the smallest percentage of the variance of the data and therefore the most likely to be discarded.

The approach used in this study was to;

1. test each layer separately to determine the layers and ranges of variable values that show a positive relationship with known deposits, using plots of contrast of weights, chi-squared statistic, $(O-E) / E$ (refer to Section 6.2.6), and the bivariate J-function,

2. use the bivariate J-function and the Kolmogorov-Smirnov cumulative distribution function plots to determine if associations are statistically significant,

3. use the favourable ranges of variables (e.g. distance to nearest fault; 0-800 m) to create binary maps,

4. rank binary maps in terms of their ability to predict the 120 known deposits (with a resource of at least $1000 \mathrm{~kg}$ TCG), and

5. select a group of 10 or 20 of the best layers.

It is important to note that the binary maps referred to in step 3 above are only used for the purpose of ranking layers and are not used as inputs to neural networks. The methods used to test the layers are summarized Table 6.1 below and are described in separate sections below.

The strong spatial relationship between gold deposits ( $\geq 1000 \mathrm{~kg}$ TCG) and proximity to regional-scale anticlinal axes is used to illustrate the methods described in the following sections. To illustrate the response of each method to a strong negative spatial relationship, a graph showing proximity to synclinal axes is also shown. 
Table 6.1. Methods used to evaluate potential neural network input GIS layers.

\begin{tabular}{|l|l|}
\hline Statistical Method & Significance Test \\
\hline Contrast of Weights & no \\
\hline Kolmogorov-Smirnov Cumulative Distribution Function & yes \\
\hline Chi-Squared statistic & no \\
\hline$(\mathrm{O}-\mathrm{E}) / \mathrm{E}(\text { Bar graph })^{\mathrm{a}}$ & no \\
\hline Bivariate J-function (Cumulative) & yes \\
\hline Bivariate J-function (Bar graph) & yes \\
\hline
\end{tabular}

${ }^{a} \mathrm{O}=$ observed \& $\mathrm{E}=$ expected number of deposits.

\subsection{Statistical Methods used to Evaluate Layers}

\subsubsection{Contrast of Weights}

Bonham-Carter (1994) used plots of the contrast, $C_{W}$, versus cumulative distance or some other variable represented in a predictive input map to determine the optimal threshold values to use in creating binary maps required in the weights-of-evidence method. Contrast is normally expressed as the difference between the positive and negative weights:

$$
C_{W}=W^{+}-W^{-}
$$

The expression used to calculate the contrast is derived by substituting the right-hand terms in equations 6.2 and 6.3 into equation 6.1. The weights, $W^{+}$and $W^{-}$, are defined as;

$$
\begin{aligned}
& W^{+}=\ln O(A \mid D)-\ln O(A)=\ln \left(\frac{T_{11} T_{2}}{T_{21} T_{1}}\right) \\
& W^{-}=\ln O(A \mid \bar{D})-\ln O(A)=\ln \left(\frac{T_{12} T_{2 \cdot}}{T_{22} T_{1}}\right)
\end{aligned}
$$

where $A$ represents the event favourable pattern present, $\bar{A}$ is favourable pattern absent, $D$ is deposit pattern present, and $T_{i j}$ stands for areas measured in numbers of cells (Table 6.2). The odds, $\mathrm{O}$, are related to the corresponding probabilities according to the formula, $O=P /(1-P)$. 
Contrast curves were produced together with chi-squared statistic curves (explained in the next section) by exporting GIS layers from ARCVIEW as ASCII files, importing the grid into MATLAB and running the function, chi_wts3. The algorithm in function chi_wts3 generates contrast-cumulative distance curves by determining the areas associated with the cross-tabulation patterns (shown in Table 6.2) within 50 successively increasing buffers. The buffers are gradually increased in size until the entire distance or variable range is covered. Only deposits with a resource of at least $1000 \mathrm{~kg}$ TCG are used. It was assumed for the purpose of these calculations that each deposit occupies a single $100 \mathrm{~m}$ cell.

Contrast, $C_{W}=W^{+}-W^{-}$, shows the overall effect resulting from the competing influences of how much being within a zone or range of variable values upgrades the prospectivity for a deposit (expressed in $W^{+}$) and how much not being in a particular zone downgrades the prospectivity (expressed in $W^{-}$). In cases where proximity to some spatial feature is a strong control on mineralization, the contrast-distance curve contains a peak. The position of this peak is used to determine which areas or variable values of the map are the most prospective. For example, the distance to the nearest trace of an anticlinal axis is shown in Figure 6.1 below. There is a peak at approximately $1400 \mathrm{~m}$, which is interpreted to mean that the effect of being close to an anticlinal axis is greatest within the buffer zone from 0 to $1400 \mathrm{~m}$. For comparison with an example of where there is a negative spatial relationship, the graph of contrast versus cumulative distance to synclinal axes is shown in Figure 6.2.

Although distance is used as the independent variable in the preceding examples, contrast curves and plots for all the other methods used to evaluate layers were generated using the variables in all the potential input GIS layers.

Table 6.2. Area cross-tabulation showing variables used to calculate contrast, $C_{W}$ where patterns overlap. $\mathrm{T}_{.1}$ and $\mathrm{T}_{.2}=$ column totals, $\mathrm{T}_{1}$ and $\mathrm{T}_{2 .}=$ row totals, and $\mathrm{T}_{. .}=$grand total.

\begin{tabular}{|lrc|cc|}
\hline & \multicolumn{4}{c}{ Deposit } \\
& & $\mathrm{D}$ & $\bar{D}$ & \\
Favourable & $\mathrm{A}$ & $T_{11}$ & $T_{12}$ & $T_{1 .}$ \\
\cline { 3 - 5 } Evidence & $\bar{A}$ & $T_{21}$ & $T_{22}$ & $T_{2 .}$ \\
& & & & \\
& & $T_{.1}$ & $T_{.2}$ & $T_{. .}$ \\
\hline
\end{tabular}




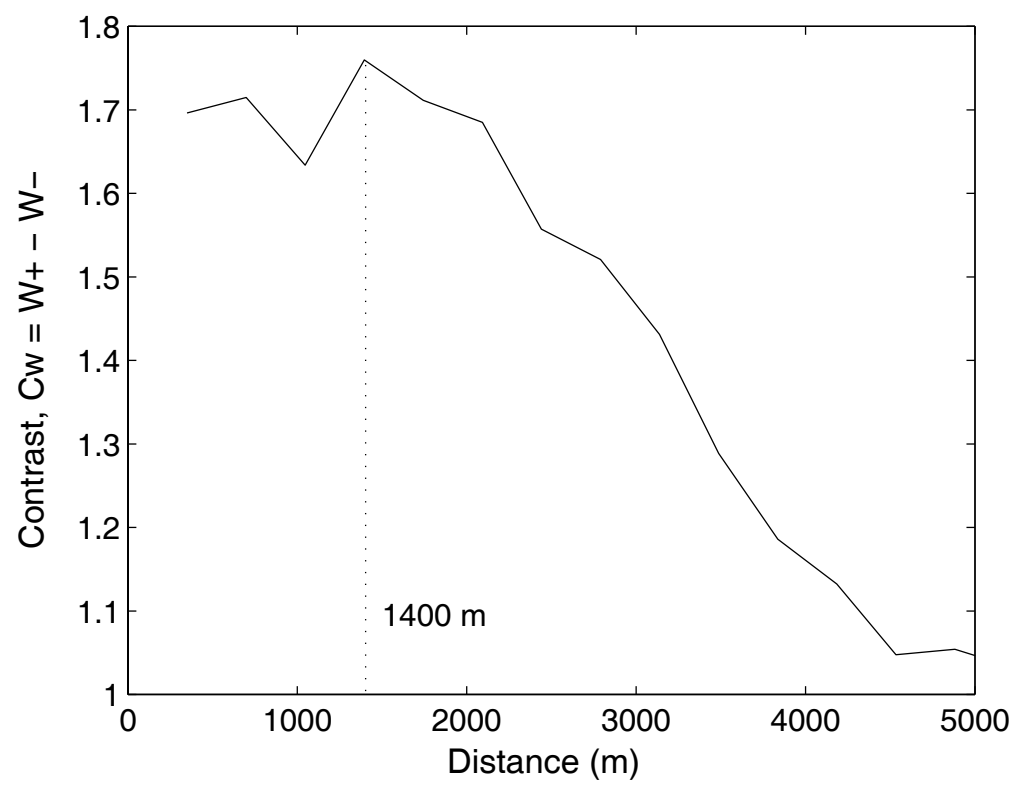

Figure 6.1. Graph of contrast versus cumulative distance to the ground traces of regional-scale anticlinal axes. The peak in the contrast curve for distance to the anticlinal axes at $1400 \mathrm{~m}$ is interpreted to indicate that the effect of proximity to an anticlinal axis in upgrading the favourability for gold deposits is at a maximum within a buffer interval of 0-1400 m.

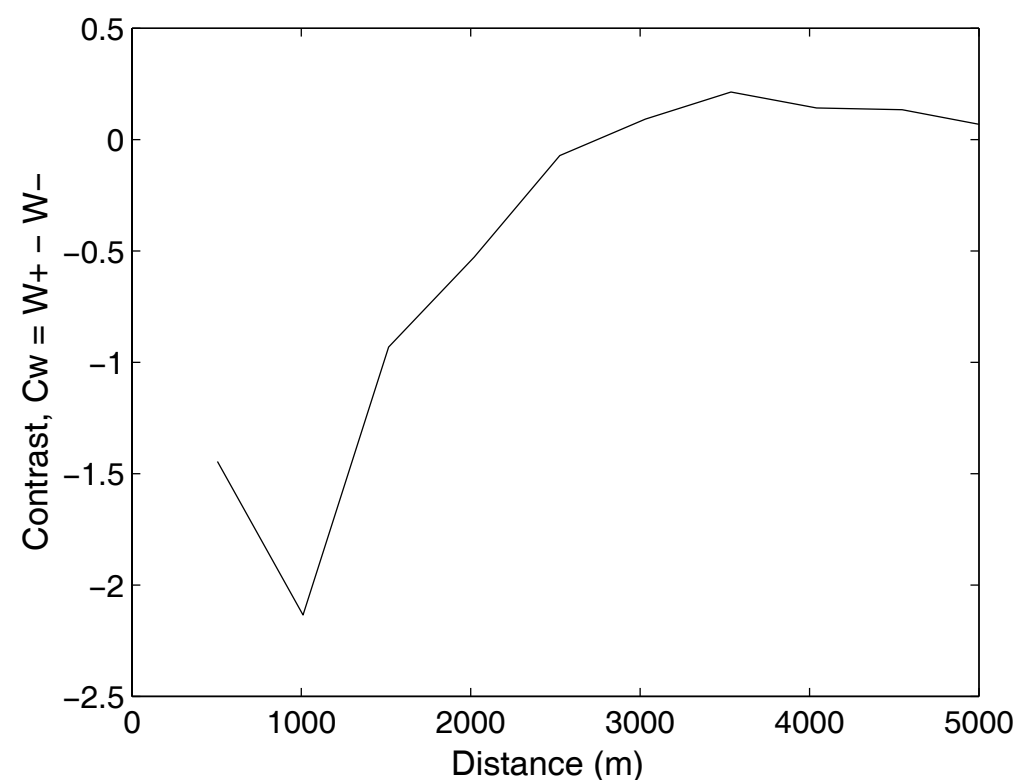

Figure 6.2. Graphs of contrast versus cumulative distance to the ground traces of regional-scale synclinal axes. 


\subsubsection{Chi-squared Statistic}

The chi-squared statistic $\left(\chi^{2}\right)$, described in Section 3.3.1 (see equation 3.4), was used to determine if a particular range of variable values (e.g. buffer distance) is favourable for gold deposits. Calculations were based on the numbers of cells corresponding to the overlap conditions shown in Table 6.2 and were performed using the same method as for contrast calculations described above (MATLAB function, chi_wts2). The graphs of the chi-squared statistic versus cumulative distance to the nearest anticlinal and synclinal axis are shown in Figures 6.3 and 6.4 below. The peak at approximately $1400 \mathrm{~m}$ indicates that locations within a buffer zone from 0 to $1400 \mathrm{~m}$ are the most favourable for deposits. A problem with the chi-squared statistic plot is that both positive and negative spatial relationships result in a positive peak (Fig. 6.3). A further difficulty, with both the contrast and the chi-squared statistic plots, is that, for many variables, there are no definite peaks.

\subsubsection{Kolmogorov-Smirnov Cumulative Distribution Function}

The Kolmogorov-Smirnov (KS) test measures goodness-of-fit between an observed and a theoretical cumulative frequency distribution (Harnett, 1982). In this study, the expected or theoretical distribution, $\hat{F}(x)$, is the cumulative frequency distribution of deposits that would be expected if deposits are randomly distributed and independent of the spatial feature under investigation. The observed distribution, $\hat{G}(x)$, is the distribution of deposits that is associated with ranges of a spatial variable in a GIS grid (e.g. distance from crustalscale faults). The KS-test is defined by:

$H_{0}$ : the data follow the specified distribution

$H_{a}$ : the data do not follow the specified distribution

The Kolmogorov-Smirnov test statistic, $D$, is based on the maximum absolute difference between the value of the theoretical and empirical cumulative distribution functions and is given by:

$$
D=\max _{j}\left\{\left|G\left(x_{j}\right)-F\left(x_{j}\right)\right|\right\}
$$

The theoretical function is generated by a Monte Carlo simulation in which locations for 1000 "deposit points" are generated according to a uniform random distribution. The test statistic is compared to a critical value from a statistical table (Harnett, 1982). If the 


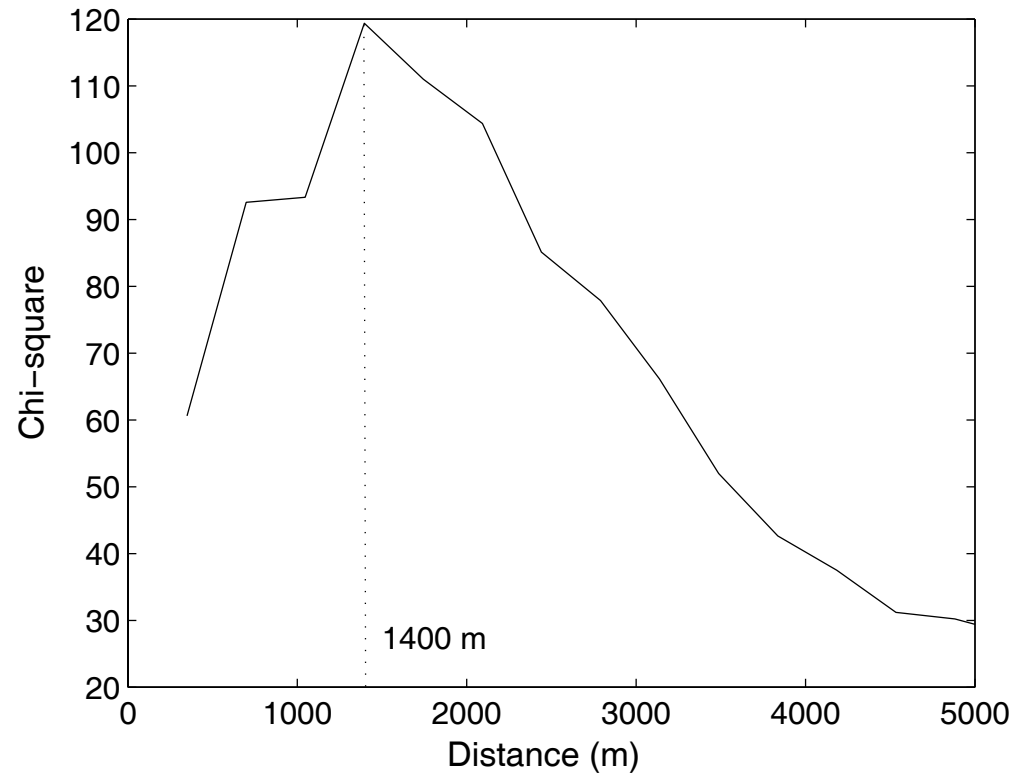

Figure 6.3. Graph of chi-squared statistic versus cumulative distance to the ground traces of regional-scale anticlinal axes. The peak in the chi-squared statistic curve for distance to the anticlinal axes at $1400 \mathrm{~m}$ is interpreted to indicate that the effect of proximity to an anticlinal axis in upgrading the favourability for gold deposits is at a maximum within a buffer interval of 0-1400 $\mathrm{m}$.

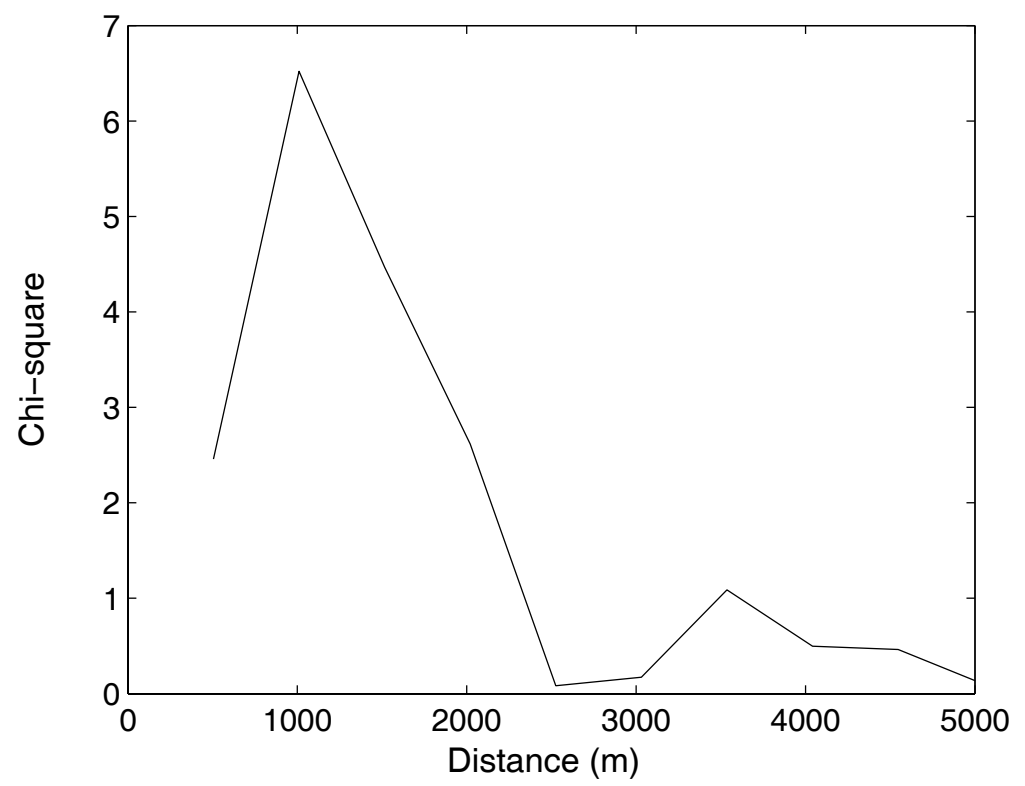

Figure 6.4. Graph of chi-squared statistic versus cumulative distance to the ground traces of regional-scale synclinal axes. 
test statistic is larger than the critical value, it follows that the deposits are not randomly distributed and are therefore associated with the spatial feature in some way. For numbers of data values, $N>35, D_{\text {critcal }}$ was calculated according to the formula:

$$
D=\frac{A}{\sqrt{N}}
$$

where $\mathrm{A}=1.63$ for a p-value of $0.01(99 \%)$ and $\mathrm{N}$ is the number of deposits. A one-tailed test was used since D is always positive. The calculations and plots were performed using the MatLaB function $k s$ according to an algorithm described by Thesen (2001).

Graphs showing the KS-test curves for the distance to anticlinal and synclinal axes are shown in Figures 6.5 and 6.6. In Figure 6.5, $D_{\text {test }}=0.39>D_{\text {critical }}=0.15$ which is strong evidence that there is a significant association between proximity to regional-scale anticlinal axes and gold deposits. It is important to note that this maximum $D_{\text {test }}$ value occurs at a distance of approximately $2 \mathrm{~km}$.

\subsubsection{Bivariate J-Curve (Cumulative)}

This section describes the use of a nonparametric summary statistic called the bivariate J-function for measuring dependence in bivariate processes $(X, Y)$, where $X$ is a point process and $Y$ is a spatial process of line segments or a more general random set (van Lieshout \& Baddeley, 1997; Foxall \& Baddeley, 2002).

The association between deposit points and lineaments has been the subject of a number of studies. Berman $(1977,1986)$ used the Kolmogorov-Smirnov test statistic to evaluate the spatial association between a set of deposit points and a set of lines representing lineaments. The method is a parametric one in which the distance of deposits and the nearest lineament is measured and the resulting observed distribution is compared with a distribution that would be expected if the points and lines occurred at random (i.e. the expected distribution). This method was applied to uranium deposits and Landsat lineaments in the Pine Creek Geosyncline by Huntington \& Green (1978) and Simpson et al. (1980). Berman's model assumes that lines are uniformly (Poisson) distributed and independent. However, in practice, this assumption is too restrictive because lines are often clustered. To overcome this problem associated with assuming a Poisson line distribution, Bonham-Carter (1984) obtained an expected distribution using a Monte Carlo simulation to generate a large set of uniformly distributed points as well as using the actual lines on the geological map. This method was applied to gold deposits and Landsat lineaments from the Timmins-Kirkland Lake area, Ontario (Bonham-Carter, 1984, 1985) and the Meguma Group, Nova Scotia (Bonham-Carter et al., 1985). 


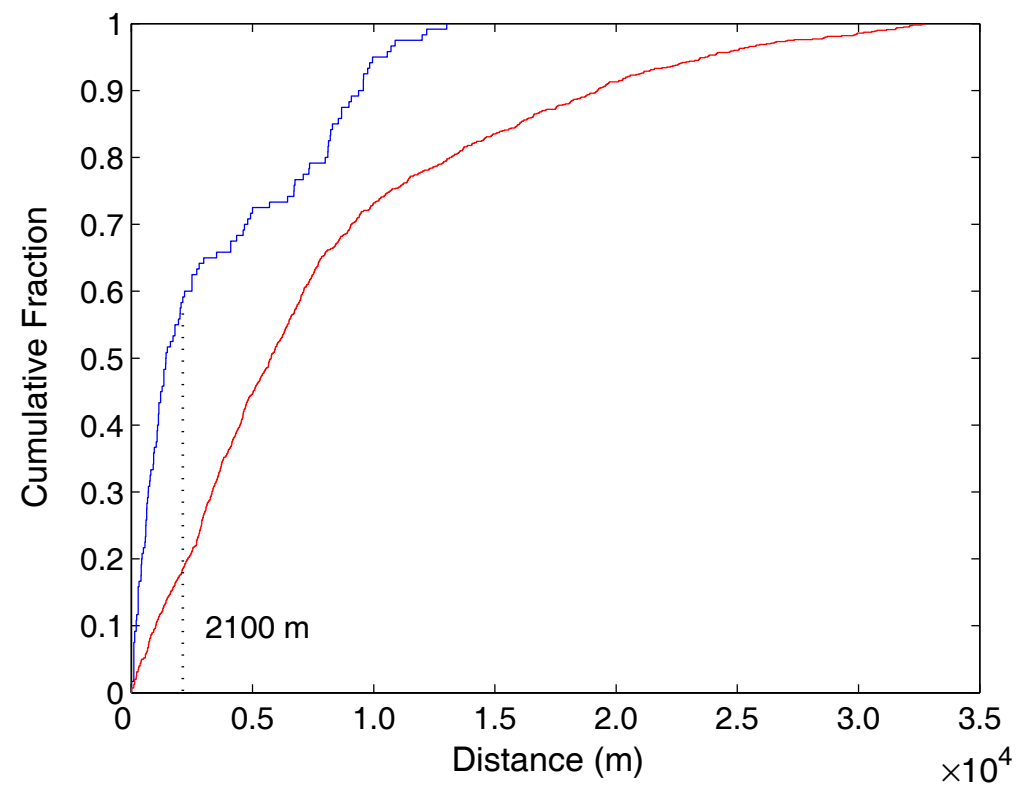

Figure 6.5. Graph of Kolmogorov-Smirnov (KS) cumulative distribution function versus distance to the ground traces of regional-scale anticlinal axes. The maximum difference between the curves representing the empirical (blue) and theoretical (red) distributions at $2100 \mathrm{~m}$ is interpreted to indicate that the effect of proximity to an anticlinal axis in upgrading the favourability for gold deposits is at a maximum within a buffer interval of 0-2100 $\mathrm{m}$.

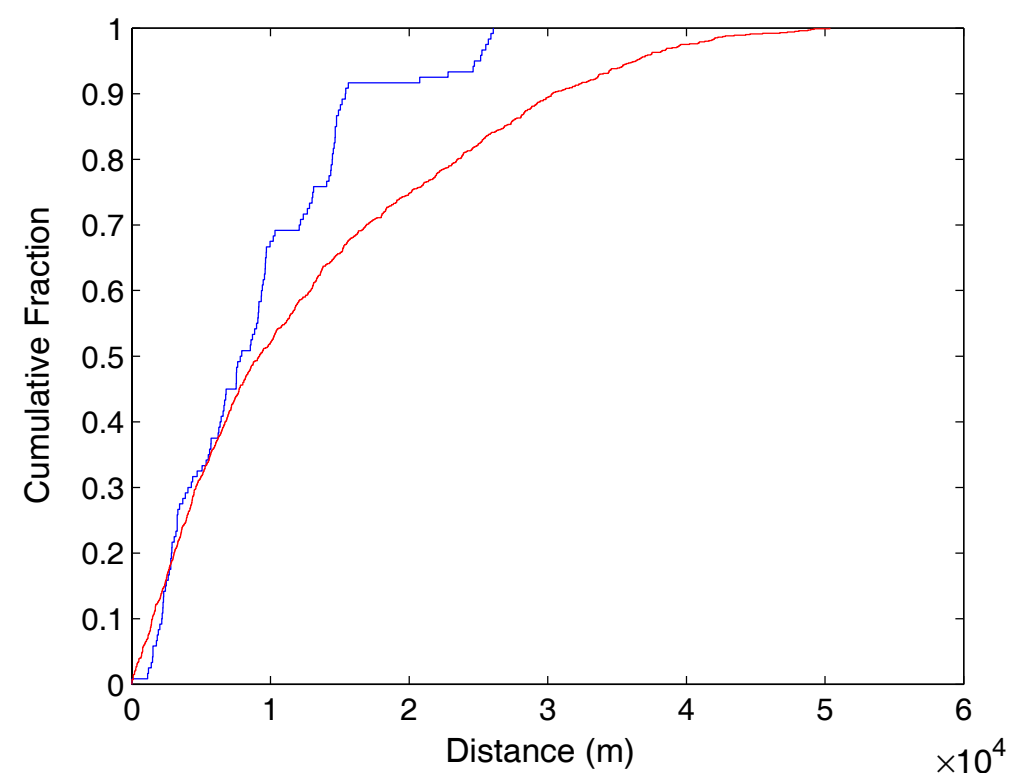

Figure 6.6. Graph of Kolmogorov-Smirnov cumulative distribution function versus distance to the ground traces of regional-scale synclinal axes. 
In this study, the bivariate J-function was used to identify positive spatial associations between known orogenic lode-gold deposits and the variables in a range of potential GIS layers in order to select layers to be combined into prospectivity maps. Simulated estimates of the J-function and a Monte Carlo test provide information about the significance of spatial relationships. The cumulative J-curve and a bar graph, based on an intervalbased calculation of the J-function, are compared with plots of $(O-E) / E$ (where $O$ is the observed and $E$ is expected number of deposits in a buffer), cumulative contrast of weights, $C_{W}$, and the Kolmogorov-Smirnov cumulative distribution function.

As well as dealing with spatial features represented by line segments such as faults and lithological contacts, the method was extended as part of this study to apply to regions within raster GIS layers such as the proximity to magnetic anomalies, or proximity to the intersection of faults (i.e. layers l14_mz2_dist \& 133_s_f23gdst, respectively) and to relationships between deposit points and variables that do not involve distances, such as the density of faults or rheological contrast at the nearest lithological boundary; i.e, layers, 136_l_rh1-6 (Brown et al., 2002c).

The bivariate J-function is defined by Foxall \& Baddeley (2002) as:

$$
J_{X, Y}=\frac{1-G_{X, Y(r)}}{1-F_{Y}}
$$

for all $r \geq 0$ such that $F_{Y}(r)<1$, where

$$
F_{Y}(r)=P\{d(z, Y)<r\}
$$

and

$$
G_{X, Y}(r)=P\{d(x, Y)<r\}
$$

where $F_{Y}(r)$ is the cumulative distribution function of the distance $d(z, Y)$ from any point $z$ to the nearest point of the spatial process $Y$ (e.g. fault pattern) and $G_{Y}(r)$ is the cumulative distribution function of the distance from a point of $\mathrm{X}$ (e.g. gold deposit) to the nearest point of $Y$ (e.g. fault lines). It is assumed that $Y$ is a random set. In a GIS containing deposits and faults, the value of the function $F$ can be regarded as the fraction of the area of the region lying within a distance of $r$ units from the nearest fault and the value of $\mathrm{G}$ as the fraction of the deposits located within $r$ units from the nearest fault.

The method involves calculating a cumulative distribution curve defined by the J-function. In the case of a raster GIS layer depicting the distance to the nearest regional fault, Equation 6.9 becomes:

$$
J_{X, Y}=\frac{1-P(\text { cell } \leq \text { distance } r \text { to fault } \mid \text { deposit })}{1-P(\text { cell } \leq \text { distance } r \text { to fault })}
$$


The numerator represents the probability that no fault occurs within a distance $r$, given the cell contains a large deposit and the denominator represents the probability that there is no fault within distance $r$. Values of $J_{X, Y}(r)$ close to 1 suggest independence of $X$ (e.g. deposit points) and the spatial feature $Y$ (e.g. faults), while values less than 1 suggest a positive association or clustering between $X$ and $Y$ and values greater than 1 suggest a negative association. Computations were performed in MatLaB.

The cumulative probability functions in the numerator and denominator in Equation 6.9 above were calculated in MATLAB using the function $j_{-}$function_dist (see attached CDROM (vol.1) by counting the deposit cells and all cells within 100 buffer zones. For example, zones of successively increasing distance from faults and shear zones. ${ }^{1}$

A Monte Carlo test is applied to determine the significance of a relationship. The procedure is to generate 99 simulations using the same number of randomly located points as there are gold deposits and plot the pointwise maximum and minimum of the simulated $\mathrm{J}$ estimates. There are 120 gold deposits in the study area. Only deposits with a resource of at least $1000 \mathrm{~kg}$ total contained metal were considered because evidence for smaller deposits is unlikely to have been incorporated in a regional-scale GIS database. At the regional scale of the study area (approximately $100 \times 100 \mathrm{~km}$ ), deposits can be treated as a point data set. X- and Y-coordinates for the simulations were selected from a uniform random distribution so that these "deposit" points correspond to the null hypothesis, i.e. that there is no spatial association between the deposit points and faults. At a 100 evenly spaced values of distance, $r$, the maximum and minimum of the simulated $\hat{J}_{X, Y}(r)$ estimates are plotted, giving a pointwise Monte Carlo test with a p-value of $2 \%$.

Examples of the use of the bivariate J-function to measure the spatial association between gold deposits and anticlinal and synclinal axes are shown in Figures 6.7 and 6.8. The $\mathrm{X}$-axes represent the distance to the trace of the nearest anticlinal and synclinal axis, respectively. In both figures, the blue curves (solid line) represents the estimate of the bivariate J-function for the empirical data $\hat{J}_{X, Y}(r)$, the horizontal dotted line shows the expected value of $J_{X, Y}(r) \equiv 1$ for the case where deposit points and anticlinal axes are independent, and the upper and lower red (dashed) curves represent the pointwise critical envelopes of $J_{X, Y}(r)$ estimated from 99 simulations of the data.

If the empirical curve (blue solid line) lies wholly within the envelopes (red dashed lines), then the null hypothesis (no spatial association) would be accepted. If the observed

\footnotetext{
${ }^{1}$ Separate cumulative and the stepwise versions of the bivariate J-function were written to deal with variables that do not involve distance such as strike angles.
} 


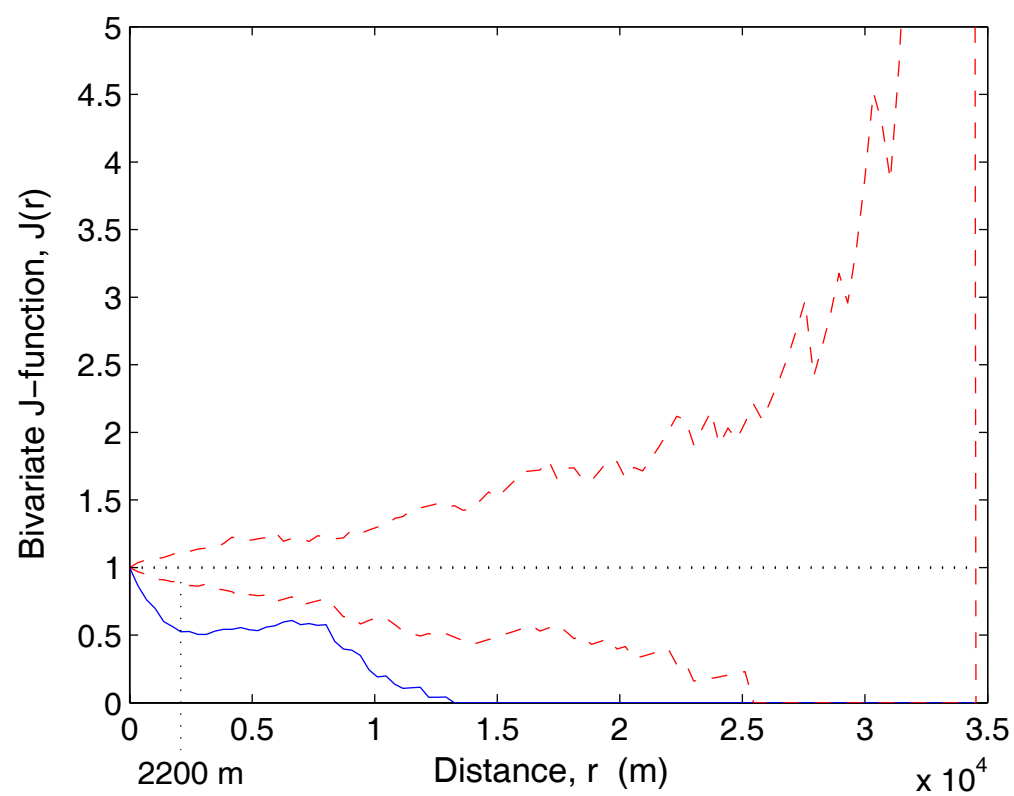

Figure 6.7. Graph of bivariate J-function for distance to the ground traces of regional-scale anticlinal axes. The maximum difference between the lower empirical curve (blue) and the lower critical envelope (dashed red curve) at $2200 \mathrm{~m}$ is interpreted to indicate that the effect of proximity to an anticlinal axis in upgrading the favourability for gold deposits is at a maximum within a buffer interval of 0-2200 m.

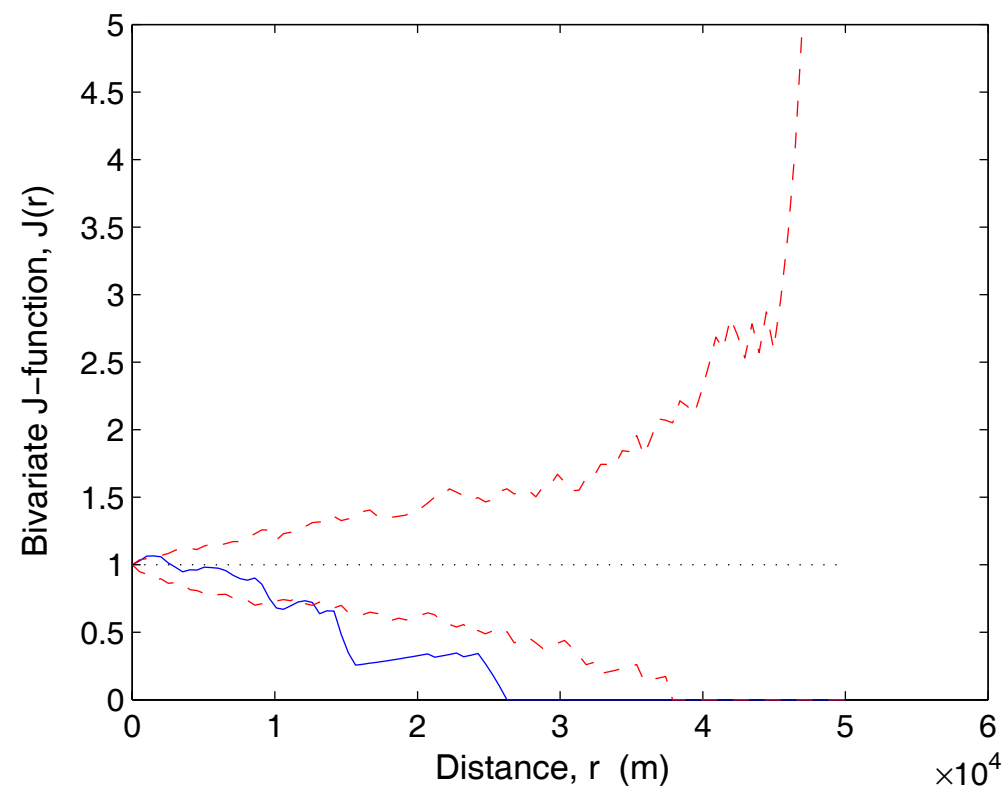

Figure 6.8. Graph of bivariate J-function for distance to the ground traces of regional-scale synclinal axes. 
J-curve (blue solid line) extends well below the envelope created by the lower dashed curve, then this is taken as strong evidence that gold deposits are associated with faults. The bivariate J-curve in Figure 6.7 is an example of a strong positive spatial association. Studies of regional-scale controls on the location of orogenic gold deposits have shown that proximity to major anticlinal axes significantly upgrades prospectivity for mineralization (Groves et al., 2000, 2001).

If the observed J-curve extends well above the upper dashed curve, then some negative or inhibitory relationship exists. Figure 6.8 shows the bivariate J-curve for the proximity to regional-scale synclinal axes. For distances below about $10 \mathrm{~km}$, the empirical (blue solid) curve lies within the envelope of the simulated curves, indicating that no spatial association exists. The positive association at distances greater than $10 \mathrm{~km}$ is due to the fact that anticlines are located between the synclines. The curves show an increasing deviation from unity (horizontal line in Fig. 6.7) at high values of $r$ since the variability increases with $r$.

Edge corrections were not performed. However, recent work by Baddeley et al. (2000) on the J-function for univariate point patterns suggests that the bivariate J-function is likely to be relatively insensitive to edge effects. Edge effects arise because the distance to the nearest line segment cannot be correctly estimated for points located close to the borders of the map if the nearest segment lies outside the map boundary.

In additional to spatial associations between deposit points and features represented by line segments (e.g. faults and lithological contacts), the bivariate J-function was also useful for analysing spatial associations between deposit points and regions (e.g. magnetic anomalies) and point data (e.g. intersection of faults). In both cases, layers representing proximity to the features were used as the inputs to the analysis. Figure 6.9 shows plots corresponding to all of the methods described in Section 6.2 for a GIS layer depicting distance to positive magnetic anomalies (layer: l14_mz2_dist, attached CD-ROM (vol.2).

In general, the methods discussed above are all capable of identifying spatial associations between deposits and layer variables provided the type of the spatial relationship is a simple one involving some inverse or direct proportionality. Examples of this type of relationship are; 1) proximity to linear and point map features (e.g. faults \& magnetic anomalies, respectively), where favourability is high at low values and decreases with distance, and 2) density of faults, or sulphidation index, where the maximum variable value is most favourable for deposits. Trends of this type are usually clearly revealed in the cumulative version of the bivariate J-function, chi-squared, contrast, and Kolmogorov-Smirnov tests, 
all of which which are based on cumulative curves. ${ }^{2}$.

The cumulative bivariate J-function is not well suited to measuring spatial associations for variables that do not involve distances, such as the density of faults, or rheological contrast at the nearest lithological boundary and strike orientation of regional faults: the latter relationship is shown in Figure 6.12. Cumulative plots (e.g. KolmogorovSmirnov cumulative distribution function) generally do not deal very well with spatial relationships where favourable variable values occur in several discrete intervals. Only the $(O-E) / E$ and interval-based bivariate J-function plots, which are calculated at successive discrete intervals, provided useful information for this type of relationship. The association of deposits around segments of regional faults with a particular range of strike angles (possibly due to the existence of dilational zones at the time of mineralization) is a good example of this type of relationship (Fig. 6.12).

\subsubsection{Bivariate J-Curve (Incremental)}

In order to overcome the problems involving the use of the bivariate J-function for spatial relationships that do not involve distances, or where positive associations exist within discrete ranges of variable values, a procedure for calculating the bivariate J-curve was developed in which the J-function is estimated for successive distance intervals rather than for cumulative distances (Brown et al., 2002c). For example, in the case of proximity to anticlinal axes, the bivariate J-function was calculated for each 349-metre distance interval. This distance interval was chosen so that there are approximately 100 intervals along the $\mathrm{X}$-axis, resulting in a graph that shows detailed trends but is not excessively noisy. Calculations and plots were performed in MATLAB using the function $j_{-}$function15_stepwise_dist (see attached CD-ROM (vol.1). Examples of the interval-based J-curve are shown in Figures 6.10 and 6.11 .

This interval calculation of the J-function was successfully applied to variables other than distance. Figure 6.12 shows the interval-based calculation of the J-function for the strike orientation of crustal-scale faults. As for the cumulative J-curve, the solid line represents the estimate of the bivariate J-function for the empirical data $\hat{J}_{X, Y}(r)$, the horizontal line shows the expected value of $J_{X, Y}(r)$ for the case where deposit points and the spatial variable are independent, and the grey bars show the maximum and minimum values for

\footnotetext{
${ }^{2}$ Contrast and chi-squared methods do not have to be plotted as cumulative curves, but experience has shown that non-cumulative plots tend to be very noisy due large fluctuations in the number of deposits in the discrete buffer zones (see examples of the step-wise bivariate J-function curve)
} 


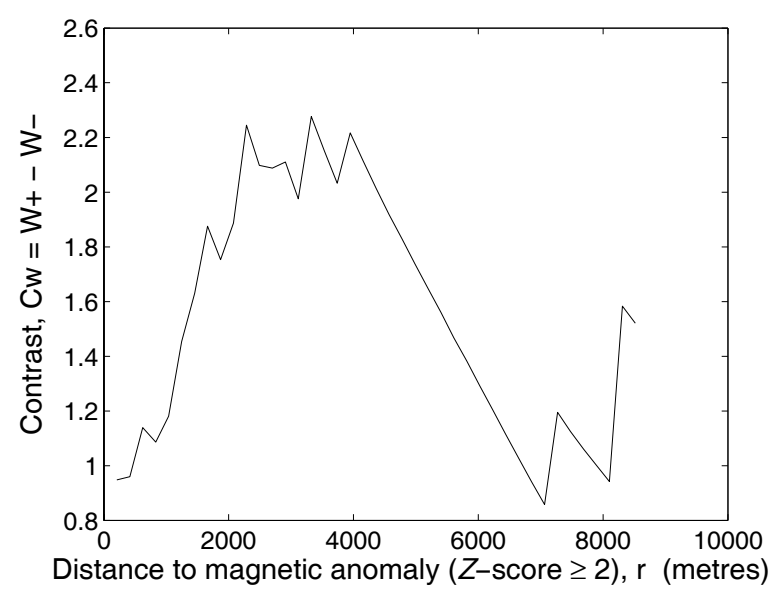

(a) Contrast, $C_{W}$

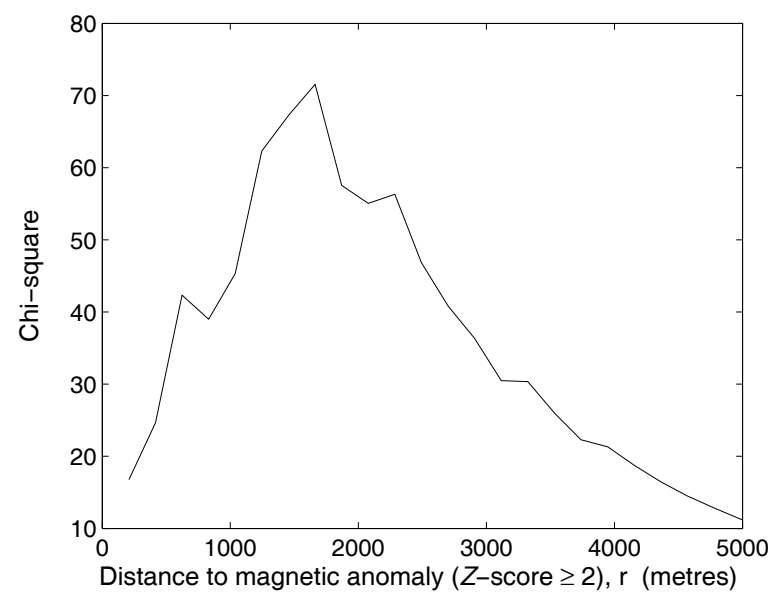

(c) Chi-squared statistic

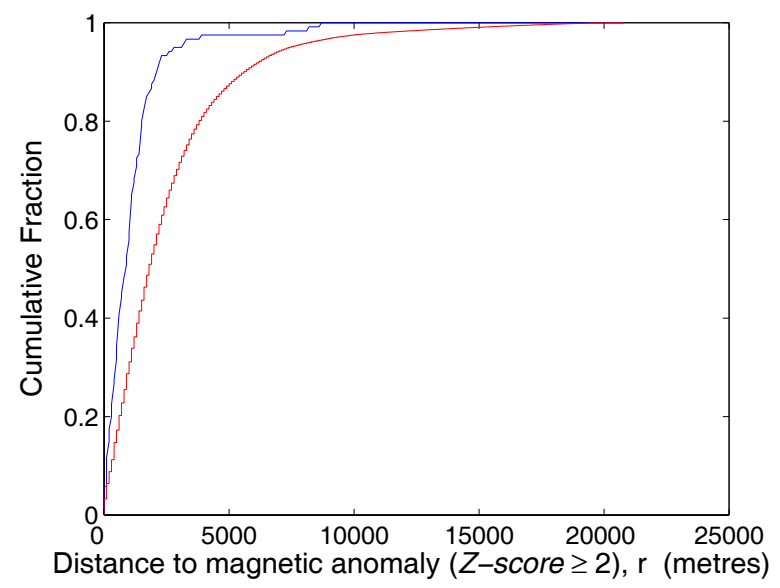

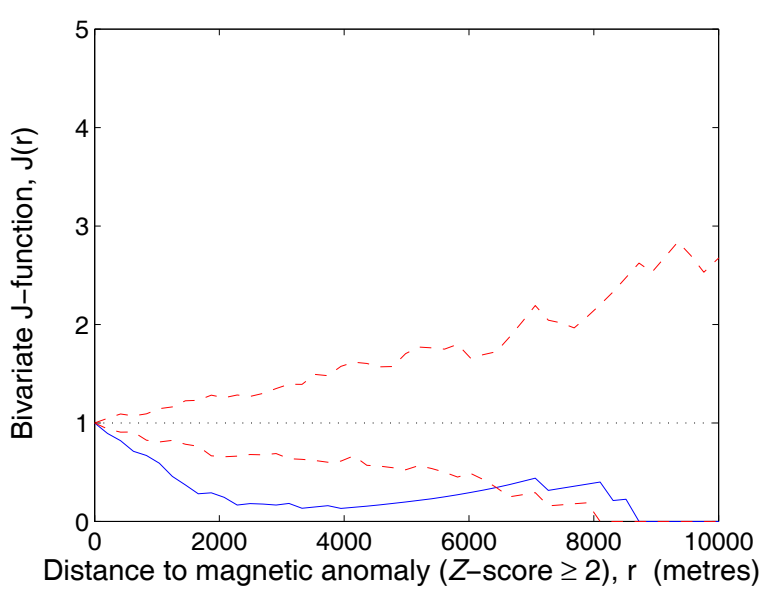

(b) Bivariate J-function (cumulative)

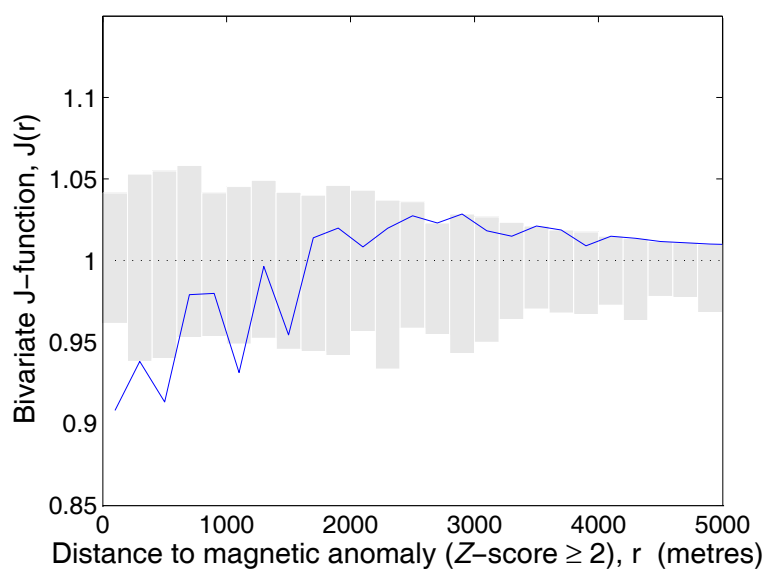

(d) Bivariate J-function (interval-based)

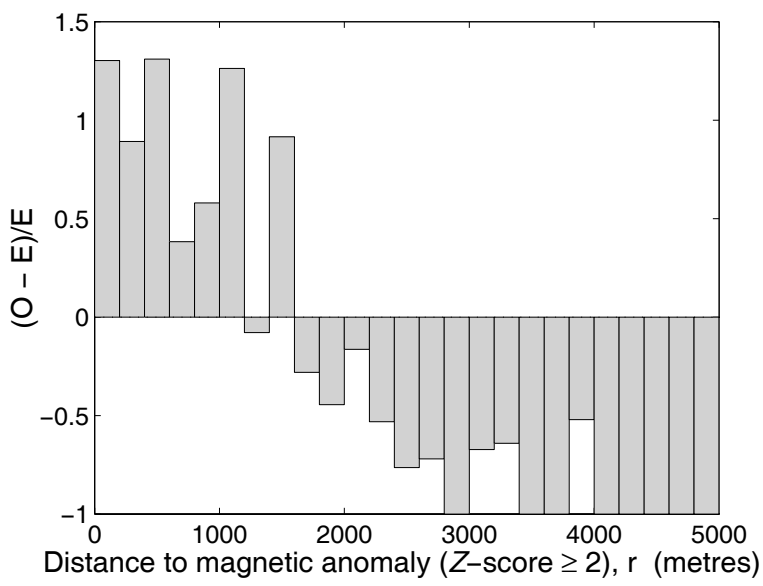

(f) $(O-E) / E$

(e) Kolmogorov-Smirnov test

Figure 6.9. Plots used to examine the relationship between deposits and the distance to positive magnetic anomalies (z-score $\geq 2$ ) based on the GIS layer l14_mz2_dist. In b), d) \& e), the empirical curve is shown in blue and the theoretical curve is shown in red. 
99 simulations of the data at each interval. The interpretation of the stepwise J-function bar graphs is the same as that explained above.

\section{Comparison with Other Graphical Methods}

The results obtained using the bivariate J-functions were compared with other methods for measuring spatial relationships. Plots of contrast of weights (Bonham-Carter, 1994), chisquared, a bar graph of the $(O-E) / E$ (where $O=$ observed number of deposits in a region corresponding to an interval on the $\mathrm{X}$-axis and $E=$ expected number of deposits for a uniform random distribution) and the Kolmogorov-Smirnov (KS) cumulative distribution function for the distance to anticlinal axes are shown in Figures 6.1, 6.3, 6.5, 6.7, 6.10 and 6.13. The KS-curve shows the cumulative fraction of deposits versus distance to anticlinal axes (Fig. 6.5). The upper curve (blue) represents the observed distribution and the lower curve (red) represents a simulated uniform random distribution.

Bonham-Carter (1994) used the position of the peak in cumulative plots of contrast $\left(C_{w}\right)$ to determine which areas of the map are the most prospective. For example, the peak in the contrast plot for anticlinal axes (Fig. 6.1) is interpreted to mean that proximity to the anticlinal axes has a maximum effect in upgrading the prospectivity for deposits within the buffer zone from 0 to $1400 \mathrm{~m}$. In the $(O-E) / E$ plot (Fig. 6.13), the excess in the number of known deposits over those that would be expected if the deposits had a uniform random distribution falls off rapidly between 1200 and 1800 metres. The most favourable zone in this case was estimated to be from 0 to 1800 metres from anticlinal axes. A degree of subjective judgement is required in the estimation of the cut-off distances for the most prospective zones. This approach was applied to the other methods for measuring spatial associations. A comparison of the results for each of the methods is given in Table 6.3 which shows estimates of the most prospective distance to anticlinal axes and strike orientations of crustal-scale faults. Distances have been rounded off to 100 metres, corresponding to the grid cell size, and angles are shown to the nearest $10^{\circ}$, which corresponds to the bar-graph intervals for the $(O-E) / E$ and interval-based bivariate J-function plots.

All of the methods provide evidence for the strong positive nature of the relationship between gold deposits and proximity to anticlinal axes. Although the bivariate J- and KSplots are not ideally suited to identifying variable ranges in which the spatial association is maximized, the points on the $\mathrm{X}$-axis corresponding to the maximum difference between the observed and simulated curves (Figs $6.5 \& 6.7$ ) are interpreted to indicate the distance at which the effect of upgrading the prospectivity for gold deposits is maximized. These 


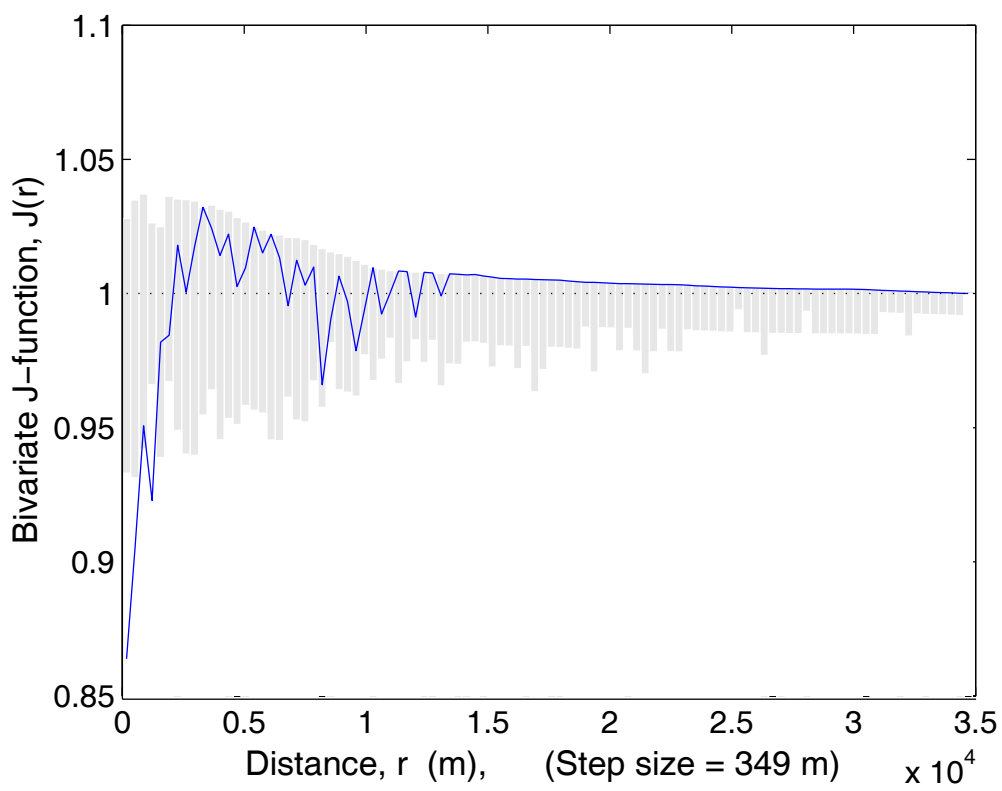

Figure 6.10. Graph of interval-based bivariate J-function for distance to the ground traces of regional-scale anticlinal axes. The distance at which the empirical curve (blue) is located below the lower critical envelope (grey bars below $y=1$ ) is $1400 \mathrm{~m}$. This is interpreted to indicate that the effect of proximity to an anticlinal axis in upgrading the favourability for gold deposits is at a maximum within a buffer interval of $0-1400 \mathrm{~m}$.

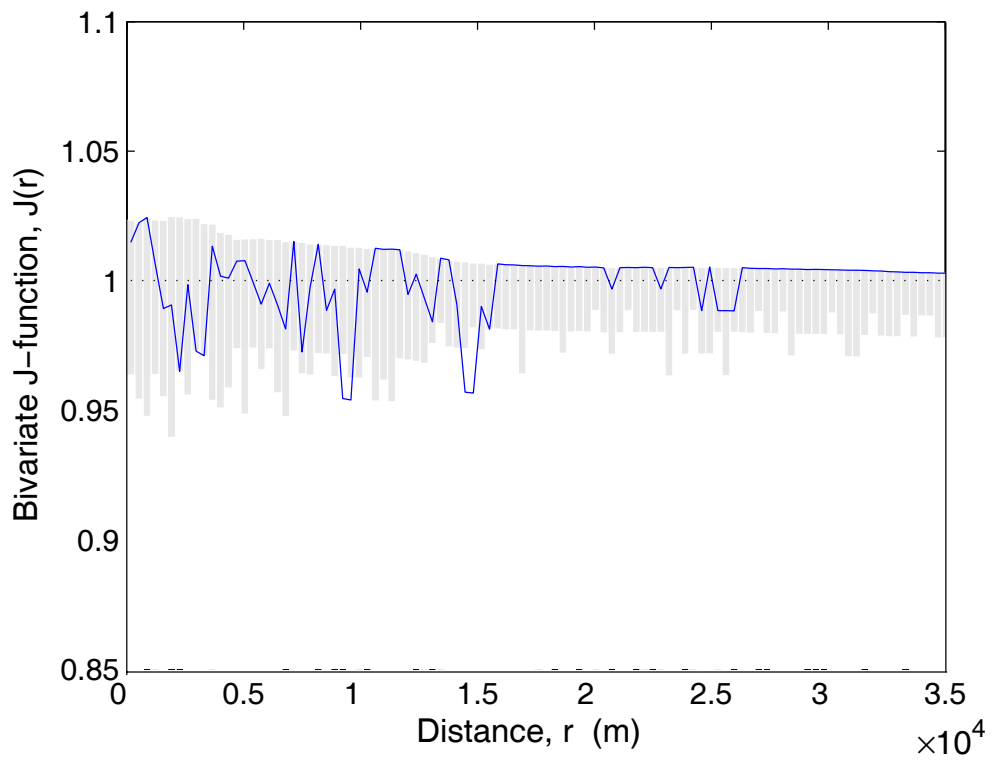

Figure 6.11. Graph of interval-based bivariate J-function for distance to the ground traces of regional-scale synclinal axes. 


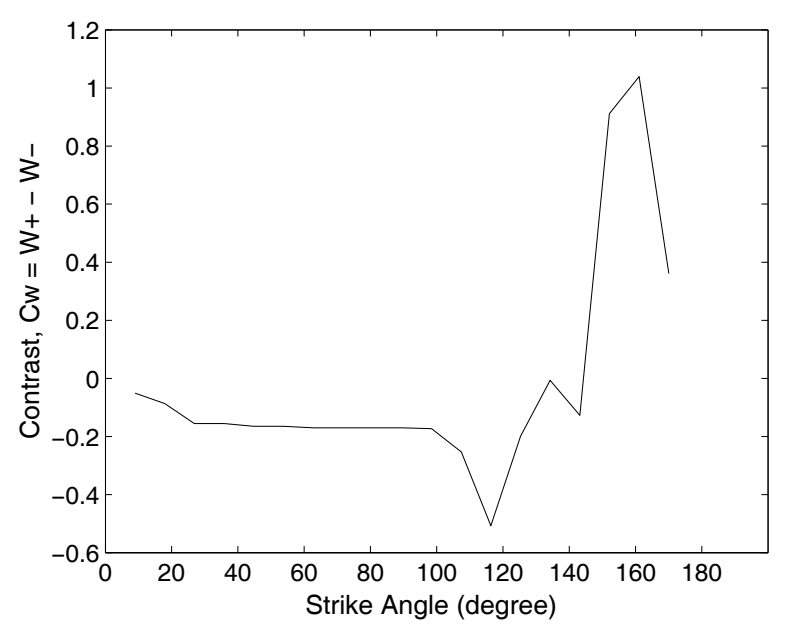

(a) Contrast, $C_{W}$

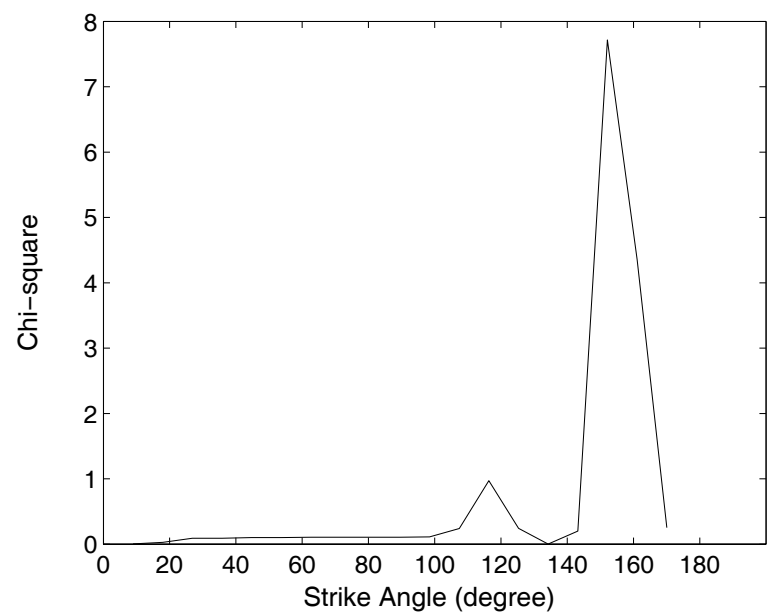

(c) Chi-squared

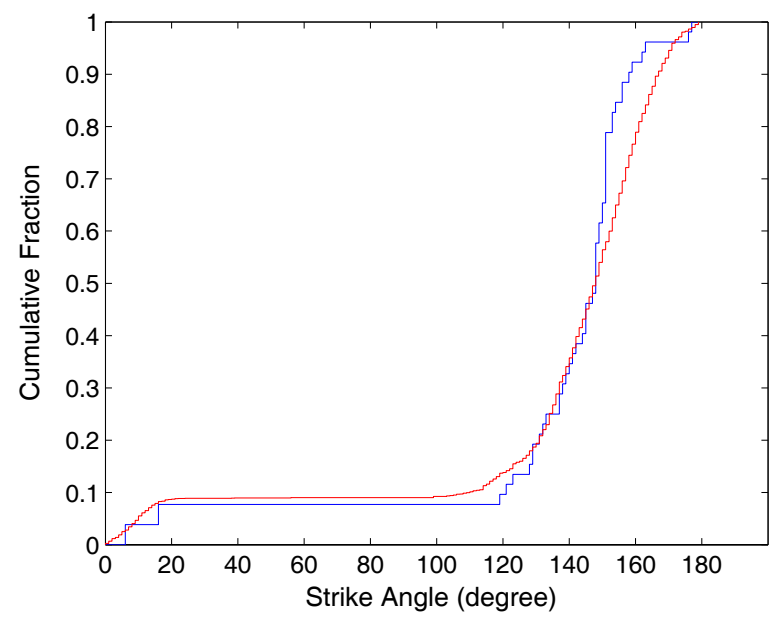

(e) Kolmogorov-Smirnov test

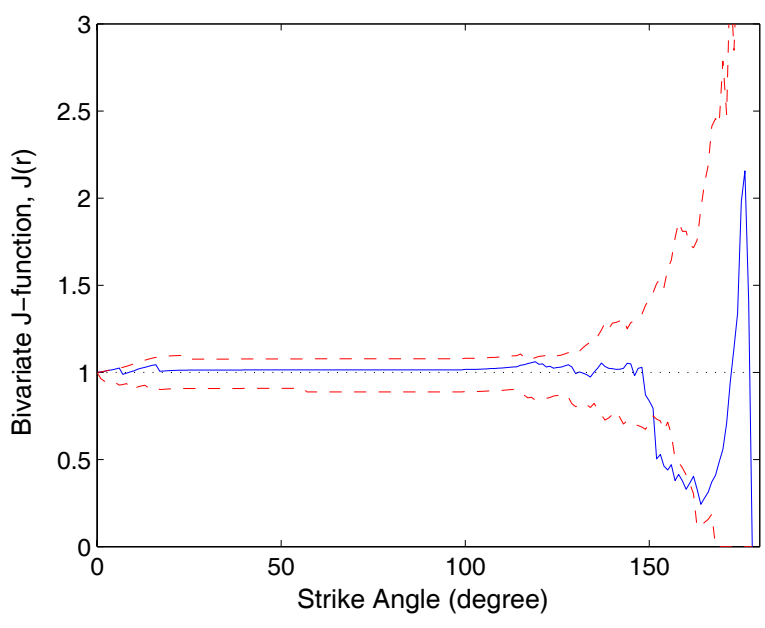

(b) Bivariate J-function (cumulative)

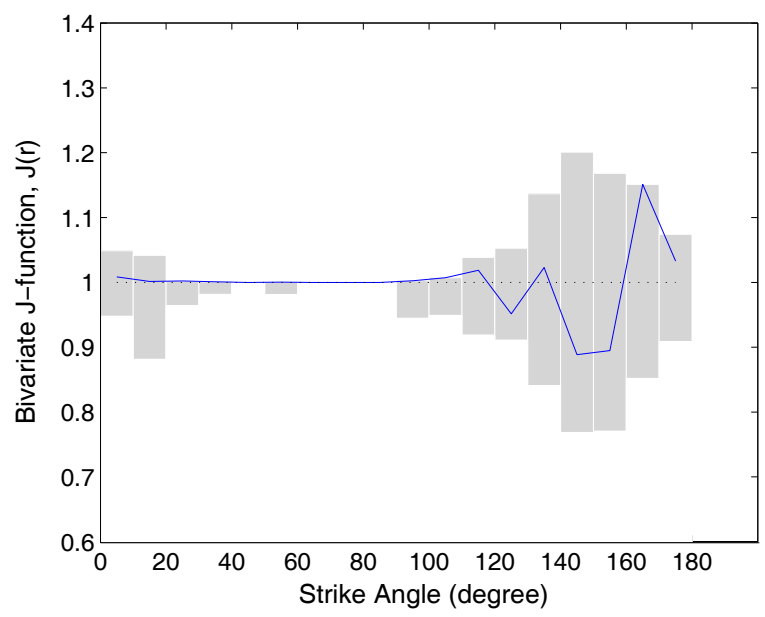

(d) Bivariate J-function (interval-based)

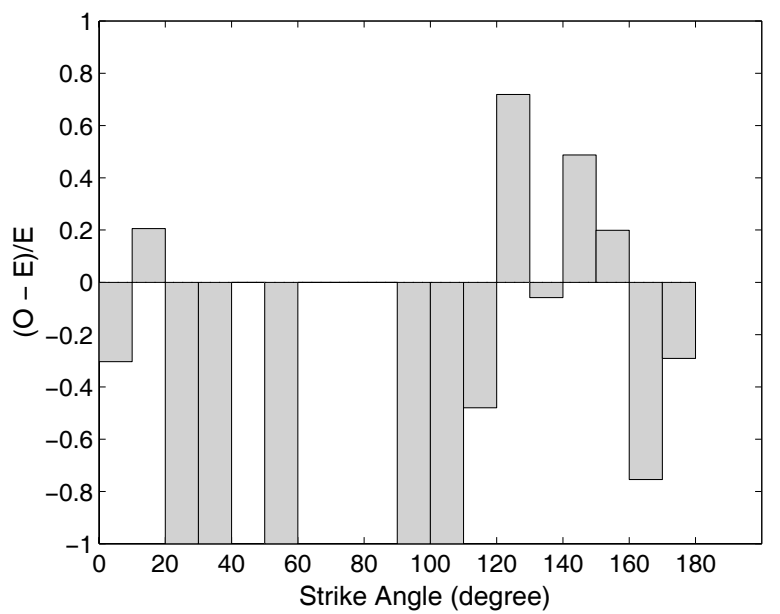

(f) $(O-E) / E$

Figure 6.12. Plots used to examine the relationship between gold deposits and the strike of crustal-scale shear zones. The layer analysed (128_s_f1str) shows the strike angle of the nearest fault reduced to a range $0-179^{\circ}$. In b), d) \& e), the empirical curve is shown in blue and the theoretical curve is shown in red. 
distances are also shown in Table 6.3. It was not possible to estimate the most favourable strike angles using the Kolmogorov-Smirnov graph. The results agree reasonably well, although estimates for distances to anticlinal axes based on the J- and KS-curves are higher than for the other methods. There is also reasonably good agreement between methods for the strike of regional NNW-trending faults. However, the positive association for strike angles in the range of $10-20^{\circ}$, identified by the contrast of weights, chi-squared, and $(O-E) / E$ methods, was not identified in plots of the cumulative and interval-based J-functions and the Kolmogorov-Smirnov plot. Results for all the GIS layers tested are shown in Table 6.4 below.

Table 6.3. Favourable zones for gold deposits estimated using different statistical methods. Columns show the different statistical methods. Abbreviations: $(O-E) / E=$ bar graph of observed $(O)$ versus expected $(E)$ numbers of known deposits versus distance or strike angle, $\chi^{2}=$ chi-squared, $C_{W}=$ contrast of weights, KS $=$ Kolmogorov-Smirnov cumulative distribution function, J-fn (cumul.) = bivariate J-function calculated as a cumulative function, and J-fn (int.) = bivariate J-function calculated for each interval. The first row shows the results for distance to the nearest anticlinal axis. The second row shows the results for a GIS layer in which cell values represent the strike orientation of the nearest crustal-scale fault or shear zone. Angles were converted to unique orientations in the range $[0,179]$.

\begin{tabular}{|l|l|l|l|l|l|l|}
\hline GIS layer & $C_{W}$ & $\chi^{2}$ & KS-test & $\begin{array}{l}J \text {-func. } \\
\text { cumulative }\end{array}$ & $\begin{array}{l}J \text {-func. } \\
\text { interval }\end{array}$ & $(O-E) / E$ \\
\hline \hline Anticlinal axes $(\mathrm{m})$ & $0-1400$ & $0-1400$ & $0-2100$ & $0-2200$ & $0-1400$ & $0-1800$ \\
\hline $\begin{array}{l}\text { Strike of nearest } \\
\text { crustal fault }\left(^{\circ}\right)\end{array}$ & 10,20 & 10 & $-^{a}$ & & & $10-20$ \\
\hline
\end{tabular}

${ }^{a}$ It was not possible to estimate particular intervals corresponding to positive associations using the KS-curve.

Estimates of favourable strike orientations show reasonably good agreement between the interval-based J-function, $(O-E) / E$ and cumulative J-function methods although, as noted above, the latter is not generally useful where favourable variable values occur in discrete ranges.

The bivariate J-function (calculated both as a cumulative curve and over intervals) is a useful method for identifying spatial relationships between deposit point patterns and other spatial features. An advantage of both forms of the J-function over other methods, such as contrast of weights, chi-squared, KS-curves and $(O-E) / E$, is that the Monte Carlo test provides a graphical indication of the statistical significance of the spatial relationships. The interval-based calculation of the J-curve (represented as a bar graph) is useful for 
determining the nature of spatial relationships involving variables other than distance and where positive or negative associations occur within discrete ranges of variable values.

\subsubsection{Difference Between Observed and Expected Number of Deposits}

A graphical method developed in this study to assess the relationship between deposit points and spatial features is to plot a bar graph of the function, $y=(O-E) / E$, where $O$ $=$ observed number of deposits in regions corresponding to an interval on the $\mathrm{X}$-axis and $E$ $=$ expected number of deposits for uniform random distribution. Calculations were made in Matlab using the function $x c h i$. The interval size is user-definable and was chosen to produce a graph that yields detailed trends but is not excessively noisy. An interval size of 200 metres was used for layers involving proximity relationships and approximately 50 intervals for other variables (Figs 6.13 and 6.14).

Variables such as distance to positive magnetic anomalies or density of faults differ from most distance relationships involving faults because deposits are associated with the maximum rather than the minimum value of the variable. For this type of GIS layer, the variable was transformed to give the difference between the raw value and maximum value (i.e. most favourable value). This transformation was applied as a pre-processing step in MATLAB using the function rescale_big_is_fav. The transformed variable was then used in the contrast, chi-squared, and J-function plots. Layers which were processed in this way are indicated in the right-most column in Table 6.4 (Section 6.3) below.

\subsubsection{Average Distance between Spatial Objects}

The trends in the plots showing distance on the X-axis cannot always be meaningfully interpreted beyond a certain maximum distance. The reason for this is that, as the distance from a particular linear feature, for example, the ground trace of a particular anticlinal axis increases, the distance to some other favourable feature such as a crustalscale shear zone or a different anticlinal axis increases. Significant trends are those that are apparent at distances that are less than about two or three kilometres. Therefore, the X-axis in some of plots shown in Appendix G have been truncated in order to show the trends at small distances more clearly. The average distance between linear and point features was calculated in MATLAB using the function median_dist (Table 6.4). 


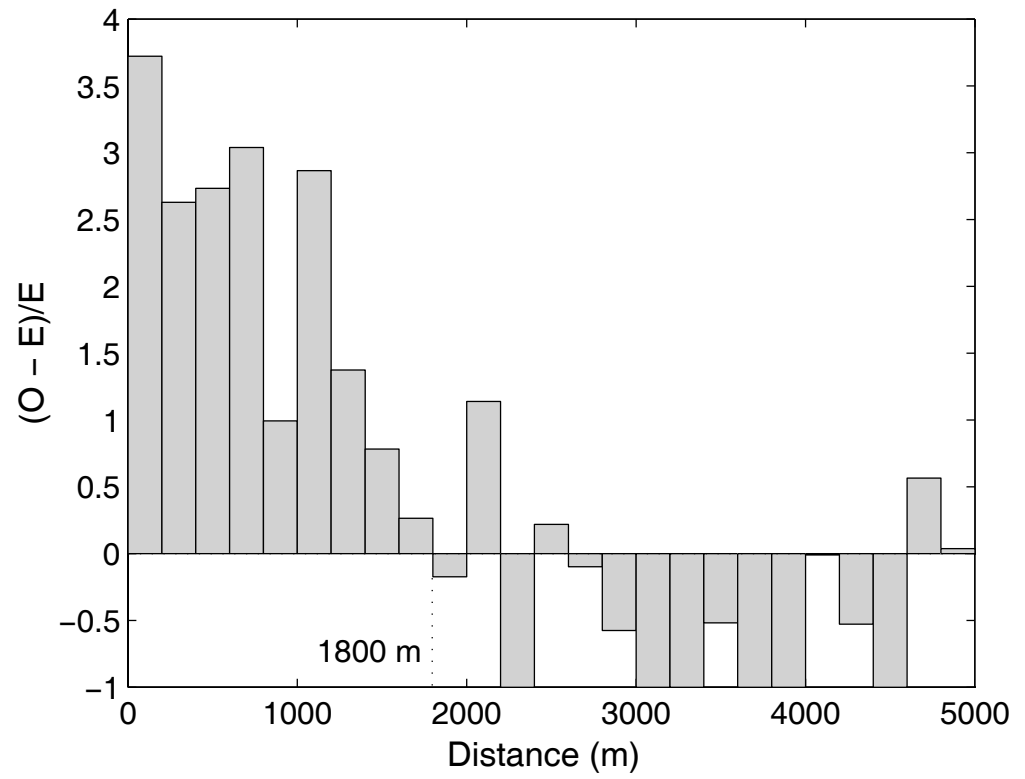

Figure 6.13. Graph of $(O-E) / E$ versus distance to the ground traces of regional-scale anticlinal axes. The decline in excess of observed deposits over the number of deposits expected for a uniform random distribution of deposits for the anticlinal axes at between 1200 and $1800 \mathrm{~m}$ is interpreted to indicate that the effect of proximity to an anticlinal axis in upgrading the favourability for gold deposits is at a maximum within a buffer interval of $\sim 0-1800 \mathrm{~m}$.

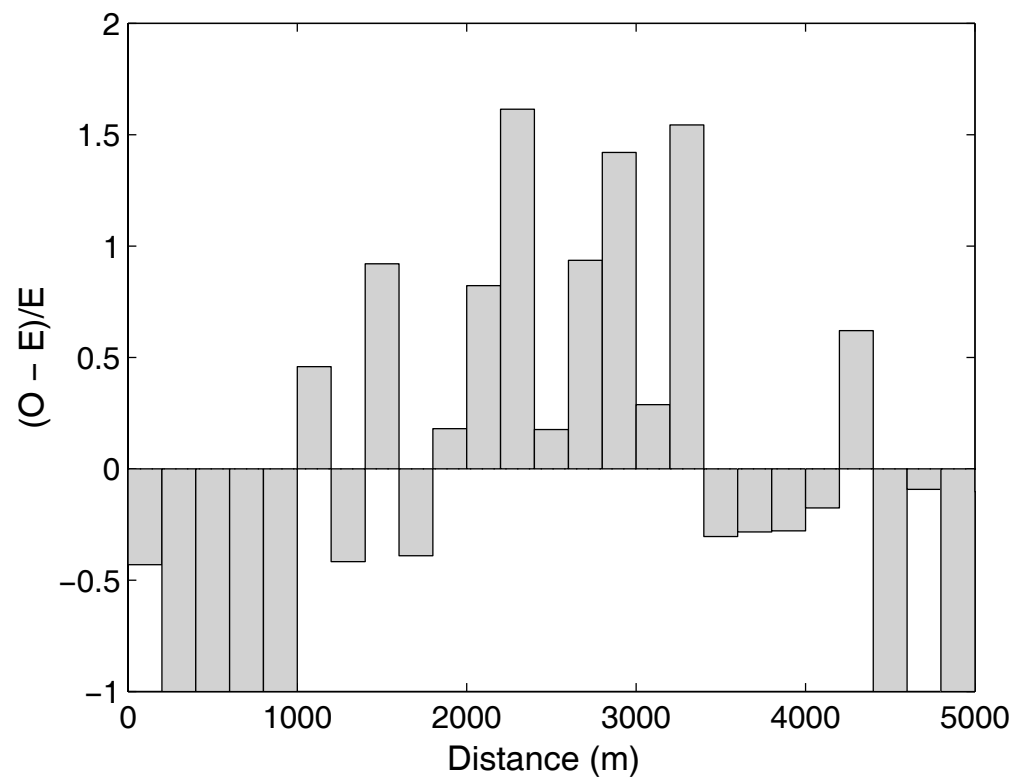

Figure 6.14. Graph of $(O-E) / E$ versus distance to the ground traces of regional-scale synclinal axes. 


\subsection{Results}

Fifty-nine potential input GIS layers were created as a result of the work in Chapter 5 (Tables G.1-G.4 in Appendix G). Layers that performed best at predicting the known deposits were selected as inputs for neural networks in the experiments in Chapter 7.

The selection process consisted of two parts. First, the layers were tested using the methods described above to determine: 1) the ranges of variable values that are most favourable for deposits, and 2) the range of variable values over which the positive spatial associations are statistically significant. Plots of contrast $\left(C_{W}\right)$, chi-squared $\left(\chi^{2}\right)$, and $(O-E) / E$ were used to identify the positive associations and the Kolmogorov-Smirnov (KS) cumulative distribution function and bivariate J-function were used to determine if the associations are statistically significant.

Second, the thresholds defining the most favourable variable values for gold deposits were used to produce temporary binary layers which were ranked according to the ability to predict known deposits. For the purpose of ranking potential input GIS layers, capture efficiency ratios (see explanation below) are calculated using a temporary binary layer in which the region corresponding to the most favourable classes or range of variable values are set to a value of one and the remaining area to zero. Calculations were performed in MATLAB using the function, mapstats_binary. The following four sets of criteria were used to rank the layers;

1. layer from each evidence category with highest $D / A$ value,

2. $D / A$ AND $D \geq \sim 20 \%$,

3. $D \times(D / A)$, and

4. $D \times(D / A) A N D D \geq \sim 20 \% A N D D / A \geq \sim 3$,

where $D$ is the percentage of known deposits in the favourable region of the binary layer and $A$ is the percentage of the total map area occurring within the favourable region.

The first method was to select one layer from each evidence category. The best layer within each category is considered to be the one that contains the largest proportion of the known deposits in the smallest proportion of the total area of the map; i.e. the largest value of the capture efficiency ratio $D / A$. A minimal set of 10 layers and a group of 17 layers including all available evidence types was chosen in this way.

In the second method, layers were selected with the highest value of the capture efficiency ratio $D / A$, where the binary favourable region also contains approximately $20 \%$ of all the 
known deposits. A problem with this ratio is that the value can be high if just two or three deposits occur in a very small area. In order to account for this, the ratio $D / A$, is multiplied by the percentage of known deposits that occur in the favourable region of the binary map, giving the statistic $D \times(D / A)$. This was used as the criterion in the third method. A fourth method of ranking the layers was tested, in which a combination of criteria was applied. Layers were ranked according to the $D \times(D / A)$ statistic, but only layers with $D \geq \sim 20 \%$ and $D / A \geq \sim 3$ were chosen. Sets of 10 and 17 layers, corresponding to each of the four selection methods, were selected as inputs to neural networks.

Table 6.4 shows the ranges of variable values in which there is a positive association with deposits. For the bivariate J-function, there is also a column showing the variable value corresponding to the maximum difference between the empirical curve and the lower critical envelope created using uniform random data. Although the cumulative bivariate J-function curve is not well suited to determining the zone in which the spatial association is maximized, the points on the $\mathrm{X}$-axis corresponding to the maximum difference between the observed and simulated curves (Fig. 6.7) are shown in Table 6.4. These results agree reasonably well with the other methods, although estimates for distances to anticlinal axes, based on the J- and KS-curves, are higher than for the other methods.

For the Kolmogorov-Smirnov (KS) test, only the $D$ statistic and the critical value of $D$ are shown, since the KS-test was only used to determine whether the variable values for known deposits are different from a uniform random distribution. Blank fields indicate that there is no interval in which there was a clearly a strong positive association. In all cases, except for the strike of the nearest E-trending regional fault (layer, 131_s_f 4 str), the test statistic exceeded the critical value of D; i.e. $D_{\text {test }}>D_{\text {critical }}$, indicating that the relationship between the particular variable in the GIS layer and the deposit points are significantly different from a uniform random distribution $(p$-value $=0.01)$.

The results shown in Table 6.4 provide quantitative evidence for the importance of structural controls on the location of orogenic gold deposits. In creating the temporary binary layers, the threshold value defining the most favourable region for gold deposits was set to the variable value on the $\mathrm{X}$-axis at which the observed number of deposits falls below the number that would be expected due to a uniform random distribution (i.e. $y=0$ on the $(O-E) / E$ bar graph). The bivariate J-function plot was used to confirm that the positive association with deposits is statistically significant within the interval from zero to the particular distance (Fig. 6.7).

The following intervals, in which the effect of proximity to a linear or point feature max- 
imized, were defined; crustal-scale faults $(0-2400 \mathrm{~m})$, NNW-trending regional faults (0$2400 \mathrm{~m})$, NE-trending regional faults $(0-800 \mathrm{~m})$, E-trending regional faults $(0-800 \mathrm{~m})$, regional-scale anticlinal axes $(0-1800 \mathrm{~m})$, lithological contacts $(0-200 \mathrm{~m})$, distance to positive magnetic anomalies ( $z \geq 2,0-1600 \mathrm{~m}$ ), the intersection of regional-scale NNW- and NE-trending faults $(0-1200 \mathrm{~m})$, and felsic porphyritic intrusions $(0-1400 \mathrm{~m})$.

Selective mineralization also appears to occur proximal to segments of regional faults and shear zones with particular orientations. The following favourable strike angles were determined from $(O-E) / E$ bar graphs and then modified according to which intervals were statistically significant according to the bivariate J-function plots; crustal-scale faults (10$\left.20^{\circ} \& 130-160^{\circ}\right)$, NNW-trending regional-scale faults $\left(30-40^{\circ}\right)$, NE-trending regional-scale faults $\left(70-80^{\circ} \& 140-170^{\circ}\right)$, and E-trending regional-scale faults $\left(80-90^{\circ} \& 140-150^{\circ}\right)$.

The plots also show the importance of high values of rock competency. The following favourable ranges were identified;

1. rock strength: Young's Modulus ( $~ 80 \geq x \leq 95$ GPa; Hatheway \& Kiersch, 1989 compilation), uniaxial compressive strength ( $\geq 190 \mathrm{MPa})$,

2. sulphidation index: $F e_{t o t}(w t \%) \times F e_{t o t} /\left(F e_{t o t}+M g+C a\right)(\geq \sim 4.3)$,

3. rheological contrast: fuzzy membership ( $\geq 0.7)$, subjective classes $(\geq 4)$, Young's Modulus (62.3-69.2 GPa, Hatheway \& Kiersch, 1989 compilation), uniaxial compressive strength (148-169, MPa), and fracture toughness $(\geq 2.3)$,

4. chemical contrast: $F e_{t o t} /\left(F e_{t o t}+M g+C a\right)(0.20-0.35), F e_{t o t}(w t \%) \times F e_{t o t} /\left(F e_{t o t}+\right.$ $\mathrm{Mg}+\mathrm{Ca})(\geq 4.0), \mathrm{Fe}_{2} \mathrm{O} /\left(\mathrm{Fe}_{2} \mathrm{O}_{3}+\mathrm{FeO}(0.42-0.60)\right.$,

5. regional fault density: NNW- and NE-trending $(300-600 \mathrm{~m})$,

6. lithological complexity: $(710-1220 \mathrm{~m})$, and

7. oxidation index: $\mathrm{Fe}_{2} \mathrm{O} /\left(\mathrm{Fe}_{2} \mathrm{O}_{3}+\mathrm{FeO}\right)(0.11-0.26)$

The association of deposits with high values of Young's Modulus and rheological contrast was not as clear using the detailed AMC data set (M. Lee, unpublished data from Australian Mining Consultants Pty Ltd, 2000) as the trend obtained from the compilation of Hatheway \& Kiersch (1989) This is despite the fact that the AMC data set was based on samples from mines within the study area. Part of the problem with the AMC data could be due to the high degree of variability in the samples tested due to alteration in the vicinity of the mines.

Table 6.5 shows the sets of 10 and 17 layers selected using each of the four sets of ranking criteria discussed above. The following layers are used in at least one of the sets of inputs: 1) distance to porphyritic felsic intrusions, 2) distance to crustal-scale faults and 
shear zones (defined as those exceeding $100 \mathrm{~km}$ in length), 3) distance to positive aeromagnetic anomalies, 4) distance to nearest lithological boundary, 5) distance to intersection of NNW- and NNE-trending faults and shear zones, 6) distance to nearest ground trace of a regional-scale anticlinal axis, 7) strike of nearest crustal scale fault or shear zone, 8) strike of nearest NNE-trending regional-scale shear zone, 9) strike of nearest NNWtrending regional-scale shear zone, 10) strike of nearest lithological contact, 11) chemical contrast between rock units at the nearest lithological boundary (sulphidation index based on the ratio, $\left.\mathrm{Fe}_{\text {total }} w t \% \times \mathrm{Fe} /(\mathrm{Fe}+\mathrm{Mg}+\mathrm{Ca})\right)$, 12) chemical contrast based on the ratio, $\left.\mathrm{Fe}_{2} \mathrm{O}_{3} /\left(\mathrm{Fe}_{2} \mathrm{O}_{3}+\mathrm{FeO}\right), 13\right)$ density of crustal and regional-scale faults and shear zones (calculated using a $5 \times 5 \mathrm{~km}$ moving window), and 14) density of lithological contacts (used as a measure of lithological heterogeneity), and 15) favourable geology (subjective fuzzy membership), 16) favourable lithological contact-types (objective fuzzy membership), and 17) favourable rheological contrast (subjective fuzzy membership). A binary deposit layer depicting orogenic lode-gold deposits with a resource of $\geq 1000 \mathrm{~kg}$ total contained metal is used to provide the target or desired output values required to train the networks.

These layers were used as inputs in neural networks described in Chapter 7. The details of the rankings of the layers using each of the four methods are shown in Tables G.1, G.2, G.3 and G.4 in Appendix G. 


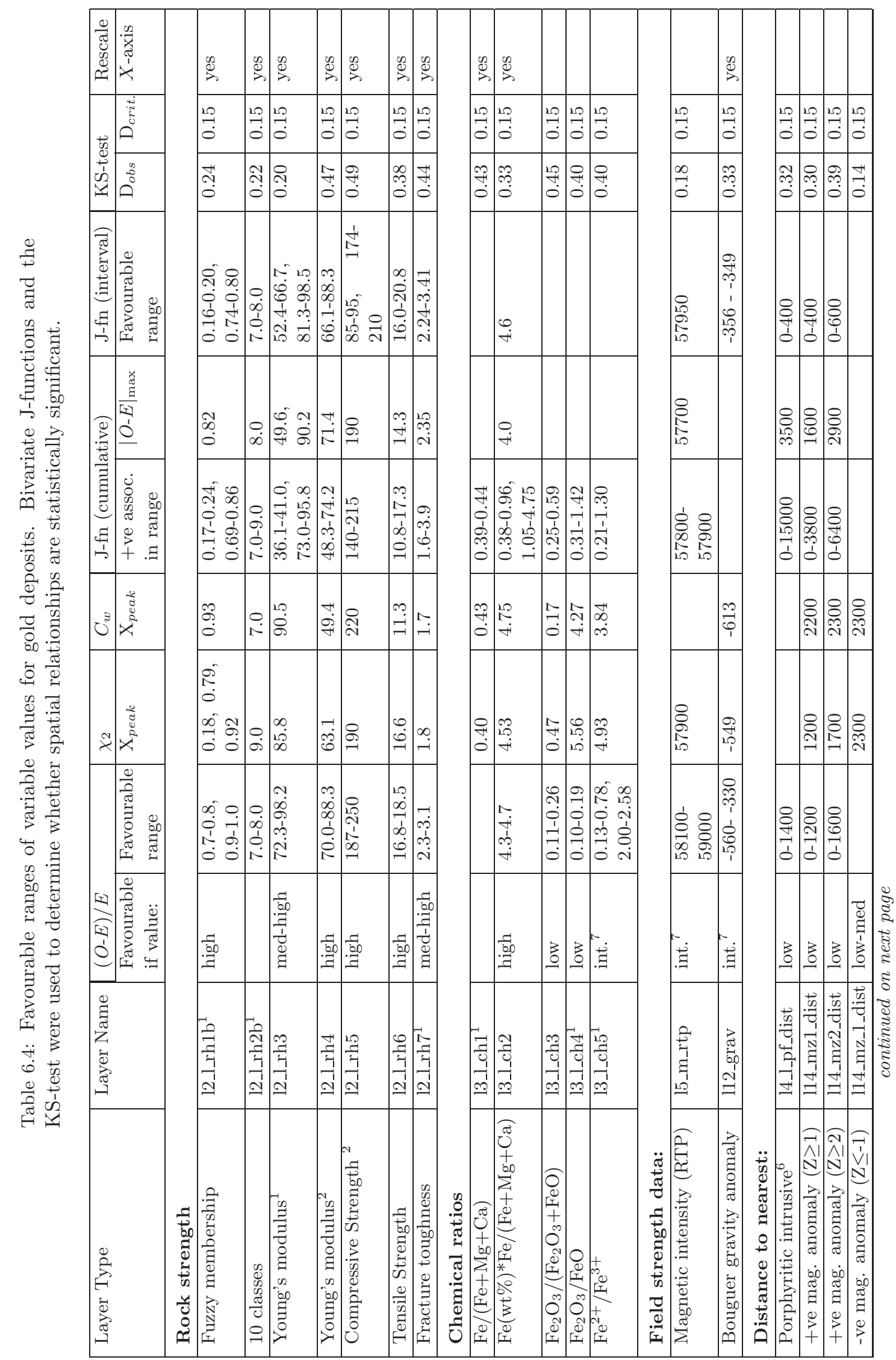




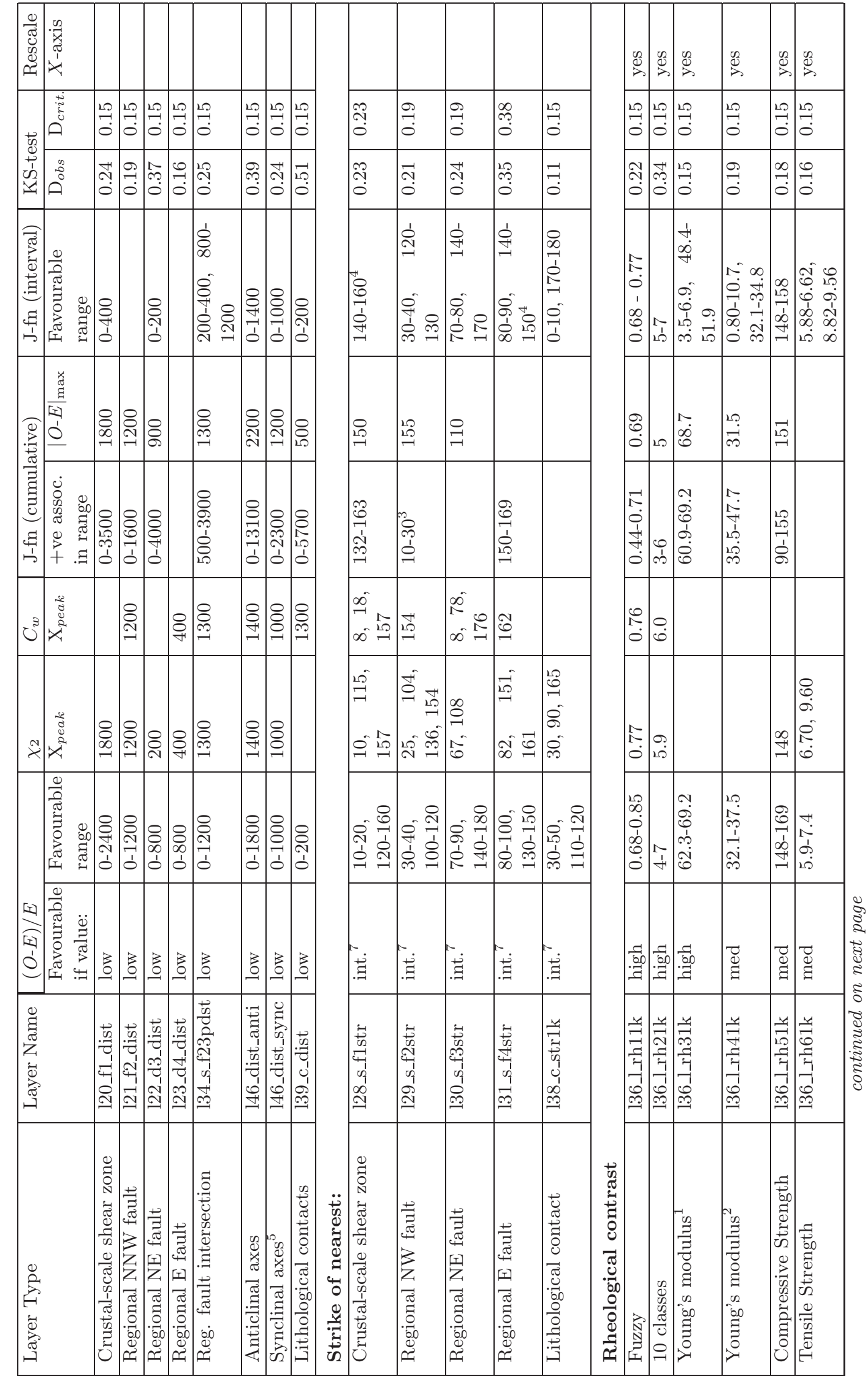




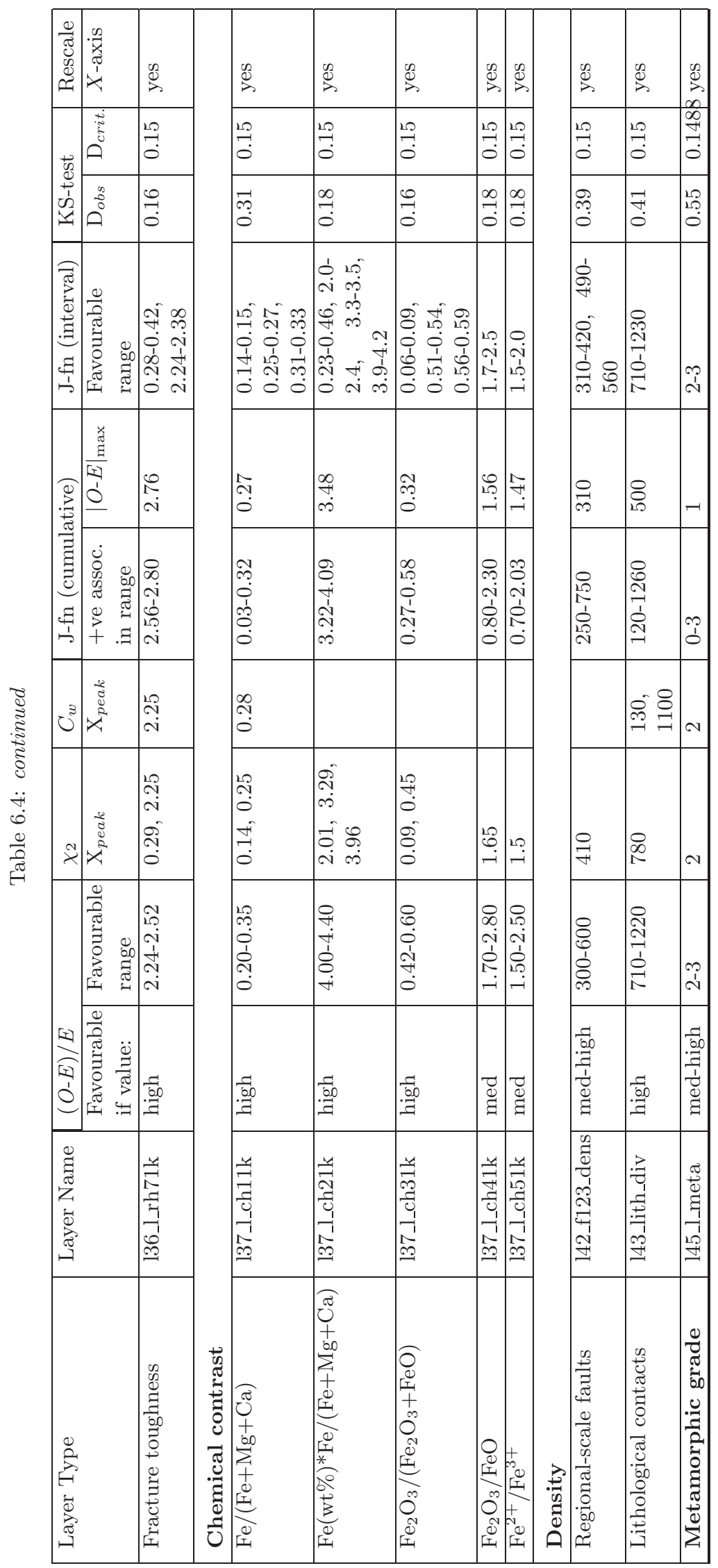

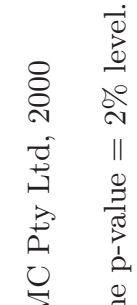

$\sum \quad \dot{0}$

का त

苟 苟

Ð

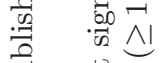

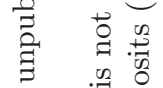

\begin{tabular}{ll}
7 & 0 \\
0 & 0 \\
\hline & 0
\end{tabular}

穴 䒼

3.

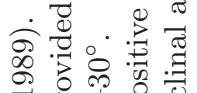

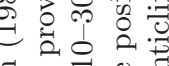

듕

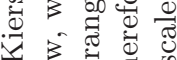

의

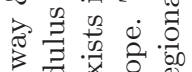

讨

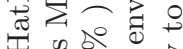

炛

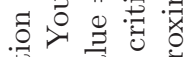

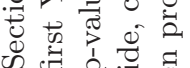

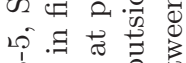

कि

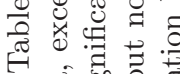

도.

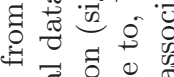

赵.

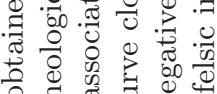

웠

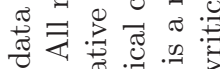

$\ddot{0} \ddot{0} 0$.

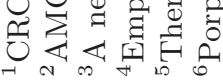




\subsubsection{Discussion}

\section{Use of Layer-Evaluation Methods to Test Exploration Criteria}

Methods used in this chapter for analysing the spatial relationships between known deposits and variables provide a means of testing whether exploration criteria are generally applicable on a regional scale and provide quantitative information that is useful for applying the criteria in exploration programs. For example, a number of exploration geologists in the Yilgarn have suggested that felsic porphyritic intrusions are favourable for orogenic gold mineralization at a local scale, because these bodies indicate a connection between the faults or shear zones and a deep crustal source and are therefore likely to have also acted as conduits for gold-bearing fluids. However, it has not been previously established if this is a useful exploration criterion on a regional scale. The analysis methods described above show that, at distances less than $400 \mathrm{~m}$ (and possibly up to $1400 \mathrm{~m}$ ), the favourability for gold deposits is significantly increased (see Table 6.4). Clearly, derived layers incorporating empirical exploration criteria are an important means of incorporating geological knowledge into a prospectivity analysis.

\section{Conditional Independence of Input Variables}

Kohavi \& Frasca (1994) have shown that useful feature subsets are not necessarily maximally independent sets. This is one of the reasons why input layers have not been tested to check if they are conditionally independent with respect to deposits. An additional reason is that the results of Chapter 4 suggest that neural networks are less affected by problems of conditional independence than either the weights-of-evidence or fuzzy systems. Recent work by Singer \& Kouda (1999a) who compared the weights-of-evidence method with probabilistic neural networks supports this. Singer \& Kouda (1999a) concluded that probabilistic neural networks have no problem in dealing with correlated variables.

\section{Use of Derived GIS Layers to Include Geological Knowledge in a Neural Net- work Analysis}

The use of GIS layers containing fuzzy membership values is another effective method of combining subjective geological knowledge with empirical data in a neural-network approach to mineral prospectivity mapping. 
Table 6.5. Layers selected as inputs for neural networks using four different criteria (see text for explanation). A set of 10 and 17 layers (shown in shaded boxes) was selected using each method.

\begin{tabular}{|c|c|c|c|c|c|}
\hline \multirow[t]{2}{*}{ Layer Type } & \multirow[t]{2}{*}{ Layer Name } & \multicolumn{4}{|l|}{ Selection Criteria } \\
\hline & & $\begin{array}{l}\text { Evidence category } \\
\text { highest } \mathrm{D} / \mathrm{A} A N D \\
\mathrm{D} \geq \sim 20 \%\end{array}$ & $\mathrm{D} / \mathrm{A}$ & $\mathrm{D} *(\mathrm{D} / \mathrm{A})$ & $\begin{array}{l}\mathrm{D} *(\mathrm{D} / \mathrm{A}) A N D \\
\mathrm{D} \geq \sim 20 \% A N D \\
\mathrm{D} / \mathrm{A} \geq \sim 3 \%\end{array}$ \\
\hline \multicolumn{6}{|l|}{ Rock strength } \\
\hline Compressive Strength $^{1}$ & 12_l_rh5 & & & 10 & \\
\hline \multicolumn{6}{|l|}{ Distance to nearest: } \\
\hline Porphyritic intrusive $^{2}$ & 14_l_pf_dist & 10 & 10 & 10 & 10 \\
\hline +ve mag. anomaly, $\mathrm{z} \geq 2$ & 114_mz2_dist & 17 & 17 & 10 & \\
\hline Crustal-scale shear zone & 120_f1_dist & 10 & 10 & & 17 \\
\hline Regional NE fault & 122_d3_dist & 17 & 17 & 10 & 10 \\
\hline Regional E fault & 123_d4_dist & & & & \\
\hline Reg. fault intersection & 134_s_f23pdst & 17 & 17 & 17 & 17 \\
\hline Anticlinal axes & 146_dist_anti & 10 & 17 & 10 & 10 \\
\hline Synclinal axes & 146_dist_sync & & & & \\
\hline Lithological contacts & 139_c_dist & 17 & 17 & 10 & \\
\hline \multicolumn{6}{|l|}{ Strike of nearest: } \\
\hline Crustal-scale shear zone & 128_s_f1str & & 17 & 17 & 10 \\
\hline Regional NW fault & 129_s_f2str & & & 17 & \\
\hline Regional NE fault & 130_s_f3str & 10 & 10 & 17 & 10 \\
\hline Lithological contact & 138_c_str1k & 17 & 10 & & 17 \\
\hline \multicolumn{6}{|c|}{ Rheological contrast } \\
\hline Fuzzy & 136_l_rh11k & 10 & 10 & 17 & 10 \\
\hline \multicolumn{6}{|l|}{ Chemical contrast } \\
\hline $\mathrm{Fe} /(\mathrm{Fe}+\mathrm{Mg}+\mathrm{Ca})$ & 137_l_ch11k & 10 & 10 & 17 & 17 \\
\hline $\mathrm{Fe}_{2} \mathrm{O}_{3} /\left(\mathrm{Fe}_{2} \mathrm{O}_{3}+\mathrm{FeO}\right)$ & 137_l_ch31k & 17 & & & \\
\hline $\mathrm{Fe}_{2} \mathrm{O}_{3} / \mathrm{FeO}$ & 137_l_ch41k & & 10 & & 17 \\
\hline \multicolumn{6}{|l|}{ Density } \\
\hline Regional-scale faults & 142_f123_density & 10 & 17 & 10 & 10 \\
\hline Lithological contacts & 143_lith_div & 10 & 10 & 10 & 10 \\
\hline Metamorphic grade & 145_l_meta & 17 & & 10 & \\
\hline \multicolumn{6}{|c|}{ Fuzzy membership Layers } \\
\hline Fav. geology & l1favgeol $25 \mathrm{pr}^{1}$ & 10 & 10 & 17 & 10 \\
\hline Fav. contact, $\mathrm{P}($ deposit $)$ & 144 favcnt $25 \mathrm{pr}^{1}$ & 10 & 10 & 10 & \\
\hline
\end{tabular}

\footnotetext{
${ }^{1}$ Data provided by M. Lee, AMC Pty Ltd, unpublished data, 2000

${ }^{2}$ Porphyritic felsic intrusion.

${ }^{3}$ Abbreviated name $=$ GIS layer name used in ArcView, text, \& CD-ROM: 11favgeol25pr = 11_favgeolt25_pr0110, 144favcnt25pr = 11_favcont25_pr0110_200.
} 
Two types of fuzzy membership layers have been used:

1. objective, and

2. subjective layers.

\section{Objective fuzzy membership layers}

In order to create the favourable geology and favourable lithological contact layers, categorical data were converted to fuzzy membership values. This conversion was based on the statistical relationships between known gold deposits and rock types in a solid geology GIS layer, and a layer representing the rock-type combination at the nearest lithological boundary, respectively.

The use of fuzzy membership rather than categorical inputs is useful for MLP networks, particularly if there are a large number of classes in the categorical layer. Categorical data are normally converted to numerical form using 1-of-n coding and this leads to a large number of additional input connections, which, in combination with small training data set sizes, could lead to over-fitting of the training data and poor generalization (see Section 5.5.14).

\section{Subjective Fuzzy Membership Layers}

Rheological contrast at lithological boundaries was modelled using a fuzzy membership layer, in which the assignment of fuzzy membership values, although based on geological field observations, was subjective. On a regional scale, most large gold deposits in the Kalgoorlie area are sited in structurally isolated, thick competent units or at contacts between layers of contrasting competency, which are oriented between NNW and NNE. The reason for this is that the more competent units tend to fail selectively in this orientation and hence become sites of increased structural permeability and fluid focusing. Subjective estimates of the relative competency of host rocks are based on the degree of silica content, modal mineralogy, grain size, fabric development, texture, and metamorphic grade. For example, banded iron formation and basalt are ranked as very competent whereas ultramafic schists are ranked as very incompetent. All rock types are assigned a ranking which was used to create a GIS layer containing cell values corresponding to the competency contrast at the nearest lithological boundary.

An additional application for the methods used to evaluate potential input layers described in this chapter is to create objective fuzzy membership layers which are based on statistically measured spatial relationships rather than subjective judgement. The results of evaluating input GIS layers shown in Table 6.4 were used in this way to create the fuzzy 
systems and hybrid neural-network fuzzy systems that are compared with neural networks in Chapter 7. The approach used here could potentially be applied to a large range of subjective data (e.g. favourability of tectonic environment, host stratigraphy, or reactivation along major faults) currently used in regional exploration programs, but which would normally not be employed in an empirical neural-network approach.

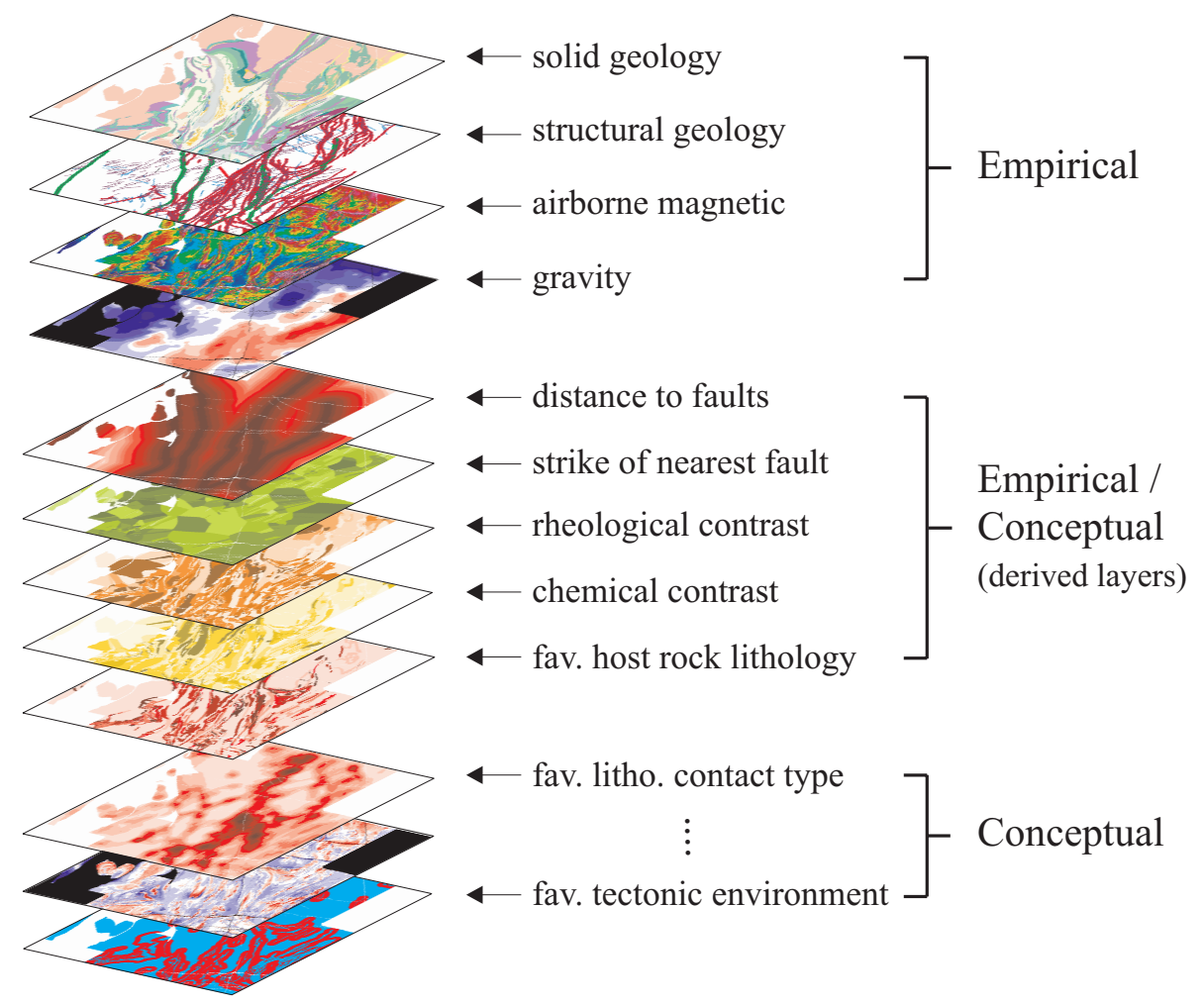

Figure 6.15. Use of derived GIS layers to include conceptual knowledge in a neural network analysis. The first type of derived layers is the conventional type, which represent exploration criteria that are based on some aspect of a mineral systems model. In the second and third groups of layers, subjective geological knowledge is presented to the neural network as fuzzy membership layers.

\subsubsection{Conclusions}

The main results are listed below.

1. Methods used to identify spatial associations between deposits and layer variables (contrast of weights, chi-squared, KS-curve, two types of bivariate J-function, and $(O-E) / E$ plots) agree generally very well provided the type of the spatial relationship is a simple one involving some inverse or direct proportionality. 
2. Different methods for selecting layers produce very similar results. Although only 3 layers are common to the set of 10 layers selected using the four methods, 13 layers are common to the four 17-layer sets.

3. The ranking of potential input layers shows that the most useful layers for predicting the location of deposits are not composed of raw data, but contain quite explicit representations of essential ingredients of the mineral systems model; e.g. the top ranking layer (according to three different criteria; $D / A, D \times(D / A)$, and $D \times(D / A)$ $A N D \mathrm{D} / \mathrm{A} \geq \sim 3, A N D \mathrm{D} \geq \sim 20 \%$ ) depicts favourable lithological contacts expressed as fuzzy membership values. Other examples are the layers representing density of faults and density of lithological contacts (i.e a measure of lithological diversity).

4. $(O-E) / E$ and interval-based bivariate J-function plots are more useful than methods involving cumulative curves in cases where the most favourable variable values for deposits occur over discrete intervals.

5. The bivariate J-function is a useful new non-parametric statistical method for examining spatial relationships that provides information about the existence, nature (positive or negative) and statistical significance of spatial relationships between a point pattern (e.g. deposits) and some other linear or point feature.

6. The cumulative bivariate J-function was modified so that the function is calculated for successive intervals (e.g. distance buffers).

7. Layer-evaluation methods described in this chapter are very useful for quantitatively testing the regional applicability of exploration criteria.

8. Fuzzy membership layers allow geological knowledge to be incorporated into an otherwise data-driven neural network method.

9. Two types of fuzzy membership layers were created; 1) favourable lithology and contact type layers based on the statistics of the spatial relationships between deposits and the variable values, and 2) rock-competency and the rheological contrast layers based on subjective geological judgement.

10. Methods for the evaluation of input layers, particularly the $(O-E / E)$ bar graph, can provide a statistical basis for the preparation of input layers used in fuzzy systems (Chapter 7). 


\section{Chapter 7}

\section{Case Study: Application of MLP Neural Networks to the Prediction of Orogenic Gold Deposits}

\section{$7.1 \quad$ Introduction}

The purpose of this chapter is to test a neural-network approach to mineral prospectivity mapping using the Kalgoorlie GIS data set and to investigate the parameters that determine performance of the trained networks and the quality of the resultant prospectivity maps. The Kalgoorlie data set is larger and more complex than the one used in the pilot study in Chapter 4, and is representative of the type of data set used routinely in mineral exploration ${ }^{1}$.

The work of this Chapter builds on the results of the previous four chapters; the neural network method for prospectivity mapping developed in Chapter 4 is used together with GIS layers described in Chapter 5 which were analysed and selected using methods developed in Chapter 6. Only those layers which are the best predictors of orogenic gold deposits are used as inputs to the neural networks which form the basis of experiments described in this chapter. Methods used to measure the generalization performance of the trained neural networks ${ }^{2}$ are explained in Section 3.3 of Chapter 3 and Section 4.4.4 of

\footnotetext{
${ }^{1}$ The Tenterfield data set contains 69440 cells in 280 rows $\times 248$ columns, whereas the Kalgoorlie GIS data set grid contains 1254000 cells in 1140 rows $\times 1100$ columns and is therefore over 20 times larger.

${ }^{2}$ Mean square error (MSE), chi-squared statistic, correlation coefficients ( $\left.\rho \& \tau\right), P(D \mid$ map class), and ROC curves are used as measures of network performance and map quality
} 
Chapter 4 .

The approach to the neural network experiments adopted in this chapter was to use a small number of input layers and a simple training algorithm and then progressively attempt to improve the performance of the basic network by altering a single parameter at a time. In a final section, the performance of the Multilayer Perceptron neural networks are compared with a simple fuzzy system and a hybrid fuzzy-neural network.

Parameters examined in this chapter are as follows;

1. effect of random initial weights,

2. effect of random training set selection;

a) random split of training patterns into training, training-stop, \& test sets,

3. training set size;

a) effect of number of deposit patterns used in training set,

4. network parameters;

a) network topology; e.g. 5 or 10 hidden layers,

b) training algorithms; e.g. use of momentum or adaptive learning rate,

5. augmentation of training sets using random noise;

a) network topology for larger noise-augmented training sets,

b) optimum maximum percentage of noise to add or subtract,

c) uniform random versus normally-distributed random noise,

6. input data;

a) choice of input GIS layers,

b) number of input GIS layers,

7. input data transformations;

a) use of log or inverse of distance instead of raw distance values,

b) 1-of-n coded lithology versus favourable lithology as fuzzy membership values,

c) limits on distance at which variables that depend on proximity to contacts or faults are considered to have an effect; e.g. chemical contrast of host rocks at a lithological boundary, and

8. input data as fuzzy-membership values. 


\subsection{Method}

The data processing required to combine a set of GIS layers (in ASCII format) into a prospectivity map consist of six main steps;

1. create a feature vector for each grid cell location,

2. create training data sets,

3. train a set of networks,

4. process feature vectors for entire map using a trained network,

5. produce prospectivity maps, and

6. analyse map quality.

These steps and the programs used to perform them in this study are shown in detail in Figure 7.1.

All of the data processing described in this chapter was performed using the MATLAB software package. Matlab provides a range of mathematical, matrix algebra and neural network functions that run in a GUI environment. Built-in neural network functions are provided to perform tasks such as creating a network with a topology, which can be specified by the user, network training and graphing the network error during training.

Other tasks, such as combining layers to produce a set of feature vectors (patterns) for each grid cell on the map and creating the training data sets, had to be written especially for this project. MATLAB includes a scripting language, which closely resembles the $\mathrm{C}$ programming language.

The Matlab functions and scripts, which are referred to in Figure 7.1 and the rest of this chapter are of this type. All functions and scripts written as part of this study are listed and briefly explained in Appendix F and included on the CD-ROM (vol. 1).

\subsection{Basic Approach Using an MLP Network}

Initially, only ten input layers, selected according to the category selection criteria, are used in experiments. Selection criteria are explained in Section 6.3. In later experiments layers sets of 10 and 17 layers, selected using $D / A, D \times(D / A)$ and a combination of criteria $(D \times(D / A), D \geq \sim 20 \%$ and $D / A \geq \sim 3)$, are used.

The training data sets are created by randomly dividing the 120 patterns, corresponding 


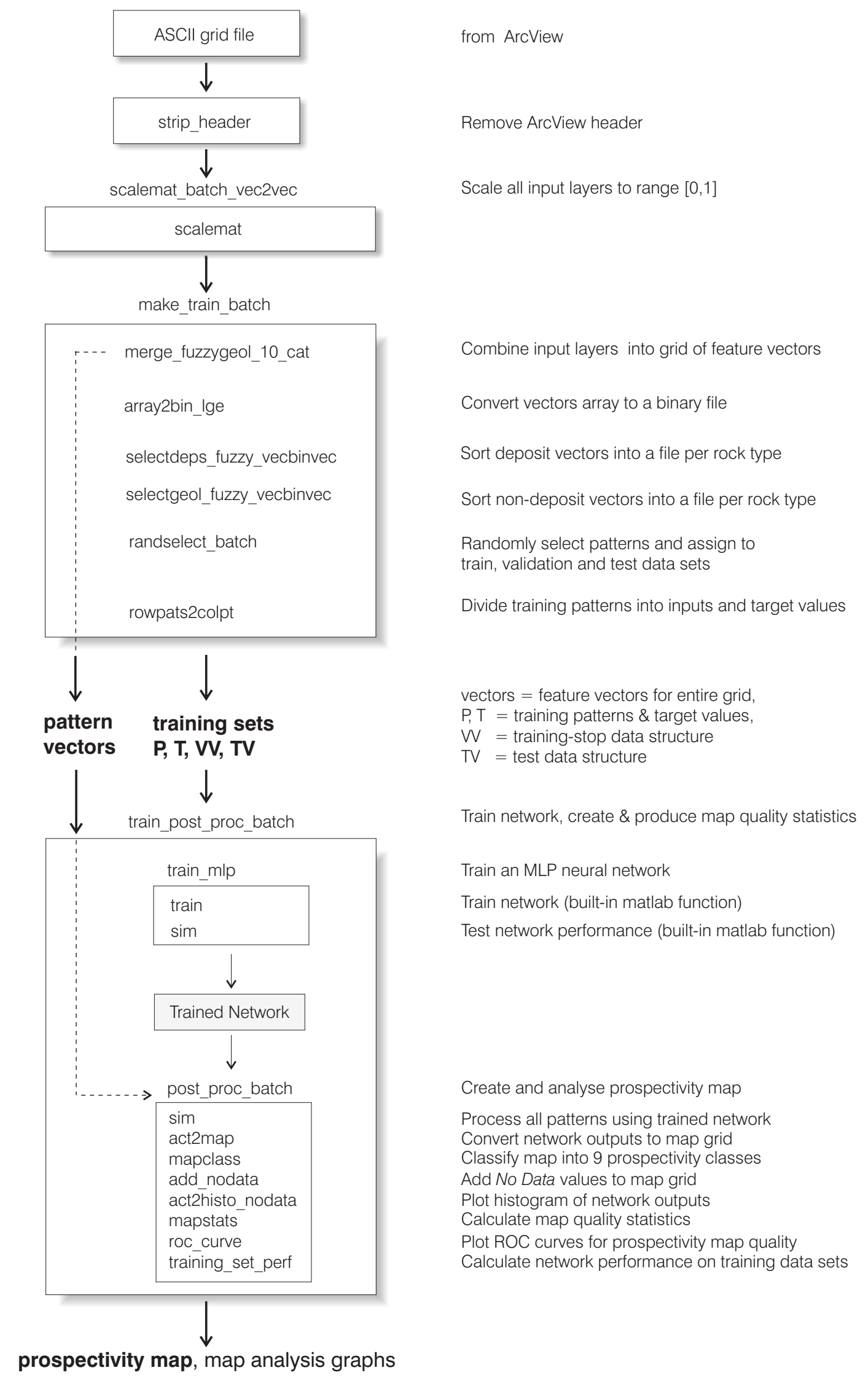

Figure 7.1. Data processing required to combine a set of GIS layers (in ASCII format) into a prospectivity map using a neural network. 
to deposits containing at least $1000 \mathrm{~kg}$ TCG, approximately equally between the training, training-stop and test sets. The training data set is used to adapt the network weights. The training-stop set is used in a cross-validation procedure with early stopping in order to avoid over-fitting the data (Morgan \& Bourland, 1990; Wang et al., 1994). The test data set plays no role in training, but is used as a guide to the ability of the trained network to generalize when new data are processed. The random selection of patterns is performed so that the gold deposits from each host rock-type in the solid geology GIS layer are divided approximately equally between the three training sets. Approximately matching numbers of barren patterns are also randomly selected for each rock type. Thus, the deposit and barren patterns are represented in the training sets approximately equally. In cases where there were too few deposit patterns to divide them equally, the patterns are always assigned to the training set, training-stop set, and test set, in that order. Training patterns are split randomly into training, training-stop and test sets. Table 7.1 shows the composition of the training sets.

Table 7.1. Number of patterns in the training data sets

\begin{tabular}{|l|c|c|c|}
\hline Class & Training set & Stop set & Test set \\
\hline \hline Deposit & 46 & 39 & 35 \\
\hline Barren & 53 & 49 & 46 \\
\hline Total & 99 & 88 & 81 \\
\hline
\end{tabular}

Most experiments in this chapter involved training a set of ten networks. Since the networks are initialized with random connection weights, which produces some variation in the performance of the trained networks, the average results of these 10 networks are shown as the bottom line in each of the tables. In each experiment, the ten networks were initialized with the same set of random weights in order to ensure that variations in performance are due to differences in experimental parameters rather than random initial weights.

The tables in this chapter summarize the experimental results which listed in full in Appendix H and I. Consequently, the rows in the tables in Appendix H generally represent the results for individual neural networks, whereas the rows in the tables in this chapter generally represent the average results for ten networks. It is clearly stated in the following text if a table differs in this respect.

Experimental parameters, such as the criteria used to select the input layers, training algorithm, training set size, and whether rock types are represented by equal numbers 
of patterns in the training set or whether the training patterns represent the rock types according to proportion of known deposits hosted in the rock type, are included in the header information for the tables shown in Appendix H. In this chapter, this information is given in the text. Unless otherwise stated, input layers were selected using the category selection criteria. The four sets of input layers obtained using each of the selection criteria are shown in Table 6.5.

\subsubsection{Measures of Performance and Prospectivity Map Quality}

Network performance is measured in two ways using; 1) the percentage of correct classifications for the independent test data set, and 2) measures of the quality of the mineral prospectivity set prepared using the trained network. An arbitrary threshold of 0.5 is used to classify patterns in the test-set. Patterns associated with network output activations greater or equal to 0.5 are classified as deposits and those less than 0.5 are classified as barren cells. The measures of map quality are based on the degree to which the highest prospectivity class (in a nine-class prospectivity map) accounts for the known gold deposits (where TCG $\geq 1000 \mathrm{~kg}$ ). The statistics used to measure map quality are: 1) Spearman's correlation coefficient, which indicates the degree of correlation between increasing prospectivity map class and the probability that the region contains a known deposit, 2) the area under a receiver-operating-characteristic (ROC) curve (Fig. 7.2), and 3 ) the capture efficiency ratios, $D / A$ and $D \times(D / A)$, where $D$ is the percentage of known deposits in highest prospectivity class in a 9-class prospectivity map, in which the output activation values from the neural network are divided into nine classes, and $A$ is the percentage of the total map area corresponding to the highest prospectivity class (see Section 6.3 above).

A ROC curve is generated by plotting the true positive (TP) versus false positive (FP) ratios, over the range of threshold values $([0,1])$ used to make the classification (where TP $=$ true positive, $T N=$ true negative, $F P=$ false positive, $F N=$ false negative, $\mathrm{TP}$ ratio $=T P /(T P+F N)$ and FP ratio $=F P /(F P+T N)$; Uncini et al., 1990; Zaknich, 1999). The area under the ROC curve is used as a measure of classification performance, and varies from a minimum of 0.5 to a maximum of 1.0. The receiver operating characteristic curve is discussed in Section 3.3.4. 


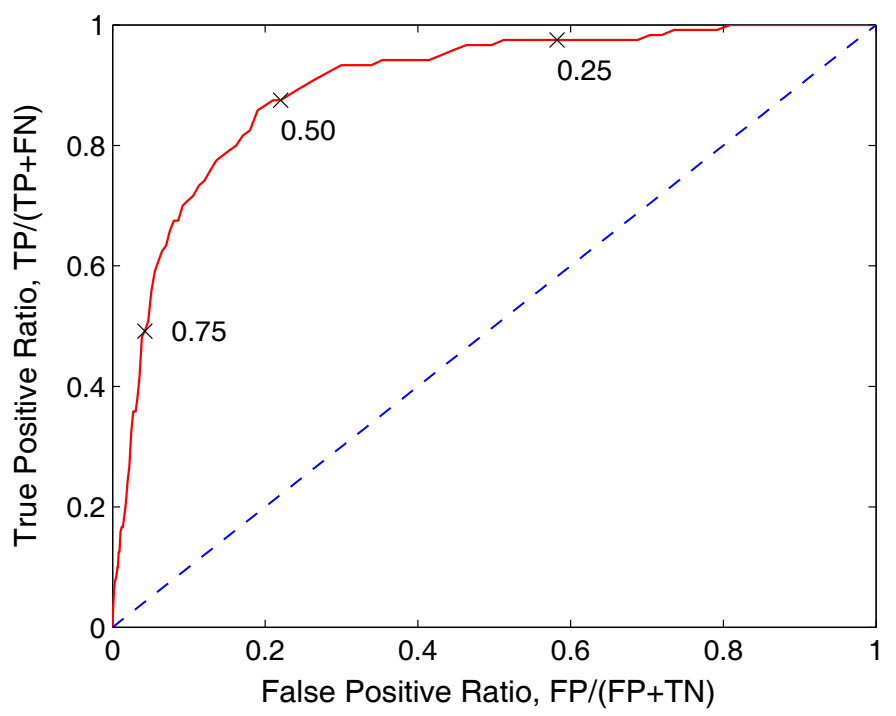

Figure 7.2. Receiver-operating-characteristic (ROC) curve for a neural network prospectivity map. The curve shows how the true- and false positive ratios vary with the threshold value applied to the network output in order to classify cell patterns as either deposits or barren. The area under the curve is an indicator of system performance and varies between 0.5 for a random classifier and 1.0 for perfect discrimination.

\subsubsection{Training Pattern Selection}

Several aspects of neural network training involve random selection and could potentially affect the performance of the trained network and the quality of the mineral prospectivity map. These are the:

1. random selection of barren patterns for training set from map grid,

2. random split of training data in to training, training-stop, and test sets, and

3. random initial weights assigned to network prior to training.

In order to minimize the influence of the random selection of barren patterns, patterns were randomly selected from locations corresponding to each rock type separately and then grouped together. Deposit patterns were identified and grouped according to the host-rock type and then an approximately equal number of barren patterns from the same rock type were randomly selected. The numbers of barren patterns were only approximately equal, since some host-rocks do not contain any known deposits.

Differences in the data populations represented by the training, stop, and test sets gives rise to differences in the performance of the trained network for the three sets. If the three training sets were statistically identical, then the classification performance of the trained 
network on each of the three sets would be the same (Masters, 1993b). The random split of data into the training, stop and test sets is only likely to be important when the amount of training data is small. This is a problem in the application of neural networks to mineral prospectivity mapping due to the limit that the relatively very small number of known deposits (together with the need to represent deposit and barren patterns in approximately equal numbers) places on the size of the training set.

In this study, this problem is dealt with by testing a number of random splits and choosing the split that results in the best network performance. This split was then used for all further experiments. An alternative approach is to use a self-organizing map to classify input feature vectors and then select data from each class according to the quantization errors (i.e. Euclidean distance of feature vectors from the centroid of the class; Wong et al., 1996a).

Table 7.2 shows the result of training ten networks on ten different random splits of the available training data. The same set of random initial weights was used for each split of the training data. Networks with five hidden layer units were used for this initial experiment since these gave the best performance in the Tenterfield pilot study (Chapter 4). The gradient-descent backpropagation training algorithm with an adaptive learning rates and momentum (function traingdx in MATLAB) and input layers selected on the basis of the categories criteria (i.e.; layers representing each each evidence category with highest $D / A$ value, 6.3$)$ were used.

Statistical similarity of the training data sets should result in similar performance of the trained network for each of the three data sets. Selections 5,8 , and 3 are quite similar in terms of overall pattern classification performance. However, the percentage of deposit patterns correctly identified is similar or worse than that expected due to chance (i.e. $50 \%$ ). Consequently, set 10 was chosen for use in further experiments because: 1) the classification results for the stop and test sets are reasonably similar (73.9\% and $67.9 \%)$, and 2) this set gave the best classification performance for deposit patterns.

\subsubsection{Network Topology}

\section{Single Hidden Layer}

Whereas the number of input units are determined by the dimensionality of the input vectors and the number of output units are determined by the problem and are therefore 
Table 7.2. Effect of random split of training data into training sets. All networks were trained starting with the same set of initial random weights.

\begin{tabular}{|c|l|l|l|l|l|r|r|r|l|}
\hline \multirow{2}{*}{$\begin{array}{c}\text { Training set } \\
\text { selection }\end{array}$} & \multicolumn{4}{|l|}{ Training Sets } & \multicolumn{4}{l|}{ Test Set } & \multicolumn{4}{l|}{ Map Quality Statistics } \\
\cline { 2 - 10 } & Train & Stop & All & Deps & Barren & $\rho$ & D/A & D*(D/A $)^{1}$ & ROC \\
\hline \hline 1 & 75.8 & 80.7 & 63.0 & 42.9 & 78.3 & 0.93 & 19.7 & 262.3 & 0.81 \\
\hline 2 & 78.8 & 65.9 & 60.5 & 48.6 & 69.6 & 0.95 & 11.6 & 155.1 & 0.72 \\
\hline 3 & 72.7 & 69.3 & 65.4 & 42.9 & 82.6 & 0.98 & 19.3 & 160.6 & 0.79 \\
\hline 4 & 70.7 & 59.1 & 64.2 & 51.4 & 73.9 & 0.44 & 0.0 & 0.0 & 0.70 \\
\hline 5 & 73.7 & 72.7 & 75.3 & 57.1 & 89.1 & 0.98 & 18.5 & 169.2 & 0.80 \\
\hline 6 & 63.6 & 50.0 & 51.9 & 71.4 & 37.0 & -0.07 & 0.0 & 0.0 & 0.53 \\
\hline 7 & 68.7 & 62.5 & 72.8 & 48.6 & 91.3 & 0.98 & 15.4 & 179.1 & 0.71 \\
\hline 8 & 70.7 & 67.1 & 70.4 & 51.4 & 84.8 & 0.98 & 12.4 & 31.0 & 0.75 \\
\hline 9 & 83.8 & 75.0 & 74.1 & 62.9 & 82.6 & 0.98 & 14.7 & 208.4 & 0.81 \\
\hline 10 & 84.9 & 73.9 & 67.9 & 65.7 & 69.6 & 0.93 & 8.9 & 148.8 & 0.79 \\
\hline
\end{tabular}

${ }^{a} \mathrm{D} *(\mathrm{D} / \mathrm{A})=\mathrm{D} *(\mathrm{D} / \mathrm{A}) A N D \mathrm{D} \geq \sim 20 \% A N D \mathrm{D} / \mathrm{A} \geq \sim 3 \%$

fixed, the number of hidden units and layers are variable and determine the number of weights (i.e. free parameters) in the network. The network requires a certain minimum number of weights in order to learn the patterns in the data and fit the data well. If the number of weights is too large and the number of patterns available for training is too small, over-fitting of the data may occur. This section shows the results of experiments to determine the optimum number of hidden units and layers.

Ten networks were trained for each topology. The same set of initial weights is used for each experiment, so that any differences in the results are due to changes in the experimental parameters rather than the random starting point in multidimensional weight space. The average results for networks with a single hidden layer of neurons are shown in Table 7.3. Networks, which gave the best overall test-set classification results, are shown in Table 7.4.

The results in Table 7.3 show that average performance varies only slightly with the number of hidden layer units. However, a 10-20-1 topology (20 hidden-layer neurons) gave the highest overall test-set performance (percentage correct classification. This applies to both the average of 10 networks (Table 7.3) and for a single network (Table 7.3). A 10-15-1 architecture gave the best percentage correct test-set performance for deposit patterns.

In Table 7.4, which shows the single network with the best overall test-set performance, 
Table 7.3. Effect of MLP network topology on average classification performance \& map quality. Each row represents the addition of five hidden neurons. The rows represent averages for ten networks.

\begin{tabular}{|l|l|l|l|l|l|c|c|c|c|}
\hline \multirow{2}{*}{$\begin{array}{c}\text { Network } \\
\text { Topology }\end{array}$} & \multicolumn{4}{|l|}{ Training Sets } & \multicolumn{4}{l|}{ Test Set } & \multicolumn{4}{l|}{ Map Quality Statistics } \\
\cline { 2 - 11 } & Train & Stop & All & Deps & Barren & $\rho$ & D/A & D*(D/A) & ROC \\
\hline \hline $10-0-1$ & 77.6 & 72.3 & 66.3 & 72.0 & 62.0 & 0.94 & 23.4 & 331.3 & 0.77 \\
\hline $10-5-1$ & 80.3 & 71.3 & 67.2 & 66.3 & 67.8 & 0.94 & 13.9 & 119.0 & 0.78 \\
\hline $10-10-1$ & 83.3 & 72.1 & 66.3 & 68.0 & 65.0 & 0.95 & 8.9 & 91.7 & 0.78 \\
\hline $10-15-1$ & 84.1 & 72.7 & 65.4 & 72.9 & 59.8 & 0.98 & 8.4 & 92.0 & 0.79 \\
\hline $10-20-1$ & 84.4 & 72.7 & 67.9 & 68.0 & 67.8 & 0.97 & 8.2 & 105.3 & 0.79 \\
\hline $10-25-1$ & 89.7 & 73.2 & 66.5 & 70.8 & 63.3 & 0.97 & 6.1 & 141.7 & 0.80 \\
\hline
\end{tabular}

Table 7.4. Effect of MLP network topology on the best classification performance \& map quality. Each row represents the addition of five hidden neurons.

\begin{tabular}{|l|l|l|l|l|l|l|l|l|l|}
\hline \multirow{2}{*}{$\begin{array}{c}\text { Training set } \\
\text { selection }\end{array}$} & \multicolumn{4}{|l|}{ Training Sets } & \multicolumn{4}{l|}{ Test Set } & \multicolumn{4}{|l|}{ Map Quality Statistics } \\
\cline { 2 - 10 } & Train & Stop & All & Deps & Barren & $\rho$ & D/A & D*(D/A) & ROC \\
\hline \hline $10-0-1$ & 74.8 & 73.9 & 69.1 & 71.4 & 67.4 & 0.95 & 28.1 & 328.0 & 0.75 \\
\hline $10-5-1$ & 84.9 & 77.3 & 72.8 & 82.9 & 65.2 & 1.00 & 7.8 & 109.8 & 0.83 \\
\hline $10-10-1$ & 81.8 & 69.3 & 71.6 & 62.9 & 78.3 & 0.88 & 5.2 & 8.7 & 0.84 \\
\hline $10-15-1$ & 88.9 & 76.1 & 72.8 & 65.7 & 78.3 & 0.95 & 6.6 & 141.9 & 0.82 \\
\hline $10-20-1$ & 88.9 & 72.7 & 76.5 & 77.1 & 76.1 & 0.90 & 9.6 & 256.7 & 0.82 \\
\hline $10-25-1$ & 95.0 & 73.9 & 70.4 & 82.9 & 60.9 & 0.97 & 5.1 & 208.7 & 0.82 \\
\hline
\end{tabular}


there is a trend of increasing performance with increasing numbers of hidden-layer neurons up to and including 20 hidden neurons. The performance of networks with 25 hidden neurons is slightly lower both for the average of 10 networks (Table 7.3) and best the single network (Table 7.4) suggesting that the optimal number of hidden-layer neurons is less than 25. Although too many hidden units can lead to over-fitting of the data resulting in poorer generalization, these results clearly show that the optimal number hidden-layer neurons is approximately 20. ${ }^{3}$ It is interesting to note that the highest values of the map quality statistics $D / A, D \times(D / A)$ were obtained with a network without any hidden-layer neurons. However, this topology resulted in the poorest correct classification rate for a single network (Table 7.4).

\section{Two Hidden Layers}

Whereas networks with single hidden layers (i.e. two layers of weights) can generate decision boundaries which partition, for example two-dimensional, input space into a single convex regions, networks with two hidden layers can generate arbitrary, non-convex and disjoint decision regions (Lippmann, 1989; Bishop, 1995). This can be an advantage in solving some problems. Optical character recognition problems are an example of an application area where networks with two or more hidden layers are often used (e.g. Fig. 2.5; Le Cun et al., 1989).

A series of networks with two hidden layers was tested using the same procedure as that described above. The networks have the same total number of hidden units as networks in the previous experiment (see Tables 7.3 and 7.4). The results for the networks with two hidden units are shown in Tables 7.5 and 7.6.

Networks with two hidden layers produced similar results to those with a single hidden layer, except that the average values for percentage correct classification of barren patterns was higher and the capture efficiency ratios, $D / A$ and $D \times(D / A)$, were slightly higher. This slight advantage is outweighed by the fact that many networks with two hidden layers had a tendency to generate relatively bimodal prospectivity estimates. In these cases, the bulk of known deposits are located in either low or high prospectivity map classes. This resulted in the lower values of Spearman's correlation coefficient, $\rho$ (i.e. the correlation between the prospectivity map class for a nine class map and the probability of finding a known deposit; see networks with topology 10-3-2-1, 10-10-5-1 and 10-20-5-1 in Table 7.5).

\footnotetext{
${ }^{3}$ It is actually the number of adaptable weights (degrees of freedom or effective complexity) in relation to the number of training examples that is significant (Bishop, 1995).)
} 
Table 7.5. Effect of MLP network topology on average classification accuracy \& map quality for networks with two hidden layers.

\begin{tabular}{|l|l|l|l|l|l|l|l|l|l|}
\hline \multirow{2}{*}{$\begin{array}{c}\text { Network } \\
\text { Topology }\end{array}$} & \multicolumn{4}{|l|}{ Training Sets } & \multicolumn{4}{l|}{ Test Set } & \multicolumn{3}{l|}{ Map Quality Statistics } \\
\cline { 2 - 11 } & Train & Stop & All & Deps & Barren & $\rho$ & D/A & D*(D/A) & ROC \\
\hline \hline $10-3-2-1$ & 78.6 & 72.4 & 65.6 & 62.0 & 68.3 & 0.79 & 15.1 & 229.1 & 0.75 \\
\hline $10-7-3-1$ & 80.5 & 71.3 & 68.0 & 59.4 & 74.6 & 0.93 & 10.5 & 206.1 & 0.79 \\
\hline $10-10-5-1$ & 81.6 & 72.1 & 68.3 & 64.3 & 71.3 & 0.81 & 11.6 & 107.4 & 0.77 \\
\hline $10-20-5-1$ & 81.1 & 70.2 & 64.6 & 66.3 & 63.3 & 0.87 & 18.4 & 159.1 & 0.76 \\
\hline
\end{tabular}

Table 7.6. Effect of MLP network topology on best classification accuracy \& map quality for networks with two hidden layers.

\begin{tabular}{|l|l|l|l|l|l|r|r|r|l|}
\hline \multirow{2}{*}{$\begin{array}{c}\text { Network } \\
\text { Topology }\end{array}$} & \multicolumn{4}{|l|}{ Training Sets } & \multicolumn{4}{l|}{ Test Set } & \multicolumn{4}{l|}{ Map Quality Statistics } \\
\cline { 2 - 11 } & Train & Stop & All & Deps & Barren & $\rho$ & D/A & D*(D/A) & ROC \\
\hline \hline $10-3-2-1$ & 87.9 & 79.6 & 71.6 & 60.0 & 80.4 & 0.83 & 3.0 & 181.4 & 0.79 \\
\hline $10-7-3-1$ & 80.8 & 73.9 & 72.8 & 57.1 & 84.8 & 0.98 & 6.0 & 29.9 & 0.84 \\
\hline $10-10-5-1$ & 74.8 & 71.6 & 75.3 & 62.9 & 84.8 & 0.97 & 16.0 & 200.1 & 0.79 \\
\hline $10-20-5-1$ & 90.9 & 72.7 & 71.6 & 65.7 & 76.1 & 0.97 & 10.6 & 354.1 & 0.85 \\
\hline
\end{tabular}

These results may be explained by the empirical observation that networks with more than one hidden layer are prone to converging to local minima (Masters, 1993b; Duda et al., 2001).

A 10-20-1 topology (i.e. 10 inputs, 20 hidden neurons and 1 output) was selected for use in further experiments as this gives the best generalization performance, as indicated by both the average and best percentage correct classifications for the independent test set.

\subsubsection{Training Algorithms}

A range of training algorithms are available within the MATLAB neural network tool-box, which was used for the experiments described in this chapter. Most of the algorithms are designed to improve the speed at which networks reache convergence to a global minimum. Rather than test all the algorithms, the approach adopted here was to use the standard gradient-descent backpropagation algorithm, a gradient-descent algorithm with an adaptive learning rate and momentum (Hagan \& Menhaj, 1994), and the Levenberg- 
Marquardt training algorithm, which is fastest algorithm available in MATLAB (Hagan \& Menhaj, 1994).

All training algorithms described in this study are applied in batch mode; i.e. weights are updated only after the errors for all the patterns in the training data set have been calculated. A 10-20-1 network topology was used for the following experiments.

Although the average test-set classification (generalization) results are very similar, the standard and adaptive gradient-descent algorithms produced significantly better values for the capture efficiency statistics, $D / A$ and $D \times(D / A)$ (Table 7.7) than the LevenbergMarquardt training algorithm. With the exception of the $D \times(D / A)$ value for the traingdx algorithm, this also applies to the networks that produced the best overall test-set results (Table 7.8).

An average (for ten networks) of 3051, 301, and 11 iterations were required for training using the standard gradient-descent, adaptive gradient-descent and Levenberg-Marquardt algorithms, respectively (refer to Tables H.84, H.85, and H.78 in appendix H). The advantage of the Levenberg-Marquardt algorithm is that it can converge ten to one hundred times faster than the adaptive and standard gradient-descent algorithms, respectively (Hagan \& Menhaj, 1994). In addition, a network trained with the Levenberg-Marquardt algorithm gave the best overall test-set performance. Consequently, the Levenberg-Marquardt training algorithm was chosen for experiments in the sections that follow.

Table 7.7. Effect of training algorithm on average classification accuracy \& map quality. Training algorithms: trainlm = Levenberg-Marquardt, traingd = gradient descent, traingdx = gradient descent with adaptive learning rate and momentum. Network topology: 10-20-1, layer selection criteria: categories.

\begin{tabular}{|l|l|l|l|l|l|c|c|l|l|}
\hline \multirow{2}{*}{$\begin{array}{c}\text { Training } \\
\text { Algorithm }\end{array}$} & \multicolumn{4}{|l|}{ Training Sets } & \multicolumn{4}{l|}{ Test Set } & \multicolumn{3}{l|}{ Map Quality Statistics } \\
\cline { 2 - 10 } & Train & Stop & All & Deps & Barren & $\rho$ & D/A & D*(D/A) & ROC \\
\hline \hline trainlm & 84.4 & 72.7 & 67.9 & 68.0 & 67.8 & 0.97 & 8.2 & 105.3 & 0.79 \\
\hline traingd & 82.7 & 70.6 & 68.3 & 70.3 & 66.7 & 0.98 & 20.1 & 162.5 & 0.78 \\
\hline traingdx & 80.1 & 69.4 & 68.0 & 69.1 & 67.2 & 0.96 & 21.4 & 152.5 & 0.78 \\
\hline
\end{tabular}


Table 7.8. Effect of training algorithm on best classification accuracy \& map quality.

\begin{tabular}{|l|l|l|l|l|l|c|c|l|l|}
\hline \multirow{2}{*}{$\begin{array}{c}\text { Networks } \\
\text { Topology }\end{array}$} & \multicolumn{4}{|l|}{ Training Sets } & \multicolumn{4}{l|}{ Test Set } & \multicolumn{4}{|c|}{ Map Quality Statistics } \\
\cline { 2 - 11 } & Train & Stop & All & Deps & Barren & $\rho$ & D/A & D*(D/A) & ROC \\
\hline \hline trainlm & 88.9 & 72.7 & 76.5 & 77.1 & 76.1 & 0.90 & 9.6 & 256.7 & 0.82 \\
\hline traingd & 81.8 & 75.0 & 72.8 & 71.4 & 73.9 & 0.98 & 42.1 & 315.7 & 0.81 \\
\hline traingdx & 82.8 & 71.6 & 72.8 & 77.1 & 69.6 & 1.00 & 19.9 & 148.9 & 0.78 \\
\hline
\end{tabular}

\subsection{Input Data}

\subsubsection{Choice of Input GIS Layers}

The purpose of this section is to test different methods for selecting neural network inputs and the effect of using different numbers of inputs on the generalization ability of the trained networks and quality of prospectivity maps. Criteria used to rank the layers are;

1. layer from each evidence category with highest $D / A$ value,

2. $D / A A N D D \geq \sim 20 \%$,

3. $D \times(D / A)$, and

4. $D \times(D / A) A N D D \geq \sim 20 \% A N D D / A \geq \sim 3$,

where $D$ is the percentage of known deposits in the favourable region of a temporary binary layer and $A$ is the percentage of the total map area occurring within the favourable region. The favourable region was determined by graphing a series of statistics described in Section 6.2. The selection of the input layers is described in 6.3. Sets of 10 and 17 layers, corresponding to each of the four selection methods, were selected as inputs to neural networks (Table 6.5). Details of the rankings of the layers using each of the four methods are shown in Tables G.1, G.2, G.3 and G.4 in Appendix G.

Similar results were obtained for the different sets of 10 inputs, except for the layers selected according to the $D / A$ criterion, which gave significantly poorer average and best values of the capture efficiency ratios, $D / A$ and $D \times(D / A)$ and a lower average percentage of correct classification of deposit patterns (Table 7.10). The lower performance of the $D / A$ layer set may be due to the absence of the distance-to-anticlinal-axes and densityof-faults layers and the inclusion of two chemical-ratio layers i.e. the sulphidation index, $\mathrm{Fe}_{\text {tot }}(\mathrm{wt} \%) \times \mathrm{Fe}_{\text {tot }} /\left(\mathrm{Fe}_{\text {tot }}+\mathrm{Mg}+\mathrm{Ca}\right)$, and oxidation index, $\mathrm{Fe}_{2} \mathrm{O}_{3} / \mathrm{FeO}$. 
The similarity of the other results is not surprising considering the high proportion of layers in each selection that are the same (i.e. compared to the categories selection in column 1 of Table 7.9, the input layer sets selected using the $D / A, D \times(D / A)$ and modified $D \times(D / A)$ criteria share 8,5 , and 8 out of 10 layers, respectively). The set of input layers selected using the $D \times(D / A)$ criterion gave slightly better average and best classification performance for the test set overall, higher values for the area under the ROC curves, and significantly better classification performance for deposit patterns than the other criteria (Tables $7.10 \& 7.11$ ). 


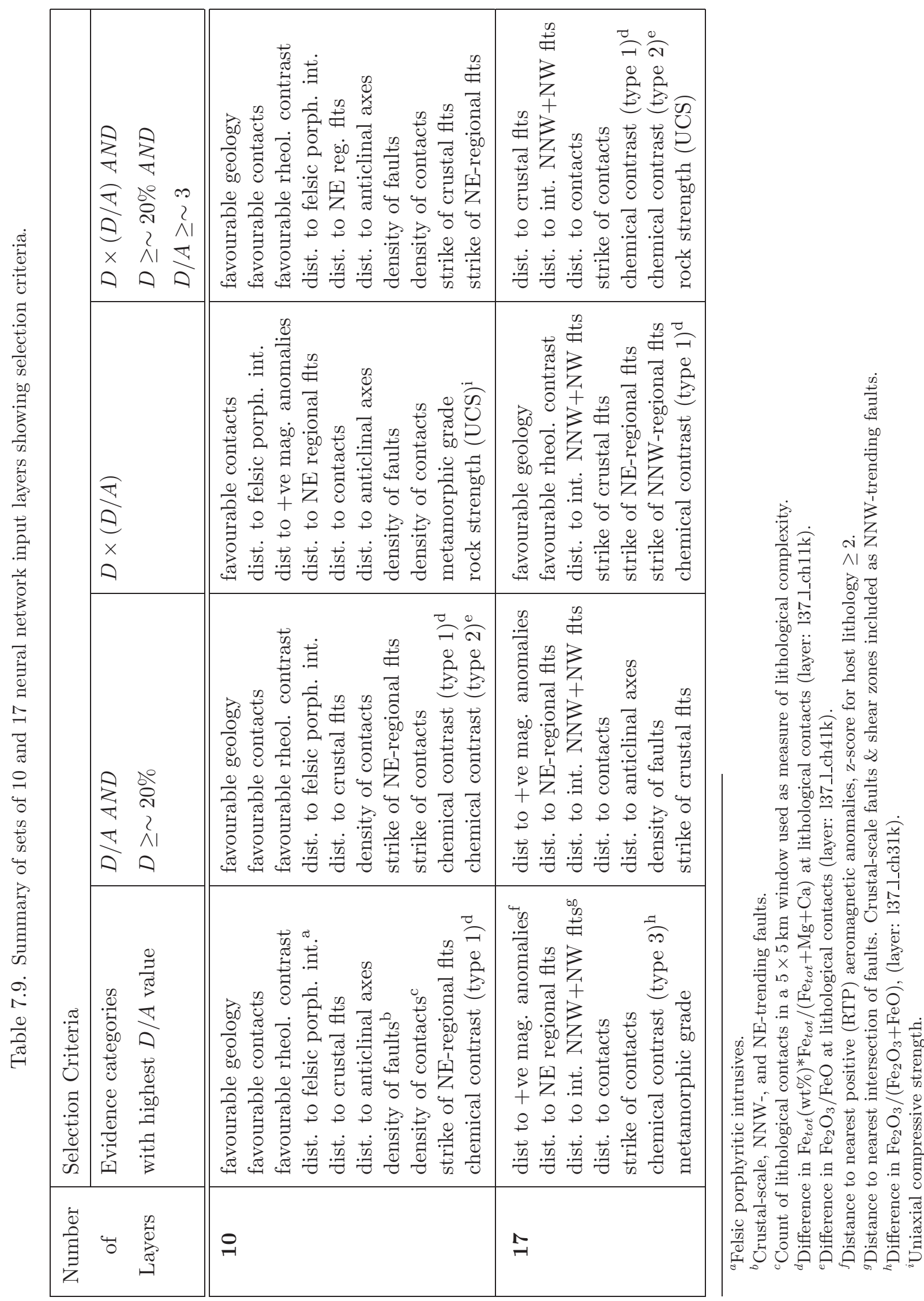


Different selection criteria for 17 layers also produced similar average results (Table 7.12). Compared to the set of layers selected according to the category criterion, the $D / A$, $D \times(D / A)$ and modified $D \times(D / A)$ layer sets (labelled in tables as DDA2) share 15, 14, and 14 out of 17 layers, respectively. The layer set selected with the $D \times(D / A)$ criterion produced slightly better average classification performance for the test set overall than the other criteria. The $D \times(D / A)$ criterion also produced significantly higher values for the capture efficiency ratios, $D / A$ and $D \times(D / A)$, than other criteria.

Results for the networks giving the best overall test-set performance for 17 inputs are similar for all the layer selection criteria but the other statistics did not show any consistent trends (Table 7.13). The categories criteria produced a significantly higher percentage of correct classifications for deposit patterns than the other criteria. Consequently, sets of layers selected using the categories and $D \times(D / A)$ criteria were chosen for use in experiments dealing with the augmentation of training data set with noise and fuzzy membership inputs below.

Table 7.10. Effect of criteria used to select 10 input GIS layers on average training set performance \& map quality. Network topology: 10-20-1, training algorithm: trainlm.

\begin{tabular}{|l|l|l|l|l|l|c|c|c|c|}
\hline \multirow{2}{*}{$\begin{array}{l}\text { Selection } \\
\text { criteria }\end{array}$} & \multicolumn{4}{|l|}{ Training Sets } & \multicolumn{4}{l|}{ Test Set } & \multicolumn{4}{l|}{ Map Quality Statistics } \\
\cline { 2 - 11 } & Train & Stop & All & Deps & Barren & $\rho$ & D/A & D*(D/A) & ROC \\
\hline \hline categories & 84.4 & 72.7 & 67.9 & 68.0 & 67.8 & 0.97 & 8.2 & 105.3 & 0.79 \\
\hline $\mathrm{D} / \mathrm{A}$ & 80.3 & 70.5 & 69.1 & 64.0 & 73.0 & 0.94 & 5.5 & 54.2 & 0.77 \\
\hline $\mathrm{D} *(\mathrm{D} / \mathrm{A})$ & 87.9 & 76.9 & 72.1 & 75.1 & 69.8 & 0.95 & 7.4 & 109.0 & 0.84 \\
\hline $\mathrm{DDA} 2^{a}$ & 84.9 & 72.6 & 69.9 & 67.1 & 72.0 & 0.93 & 12.1 & 111.2 & 0.80 \\
\hline
\end{tabular}

${ }^{a} \mathrm{DDA} 2=\mathrm{D} *(\mathrm{D} / \mathrm{A}) A N D \mathrm{D} \geq \sim 20 \% A N D \mathrm{D} / \mathrm{A} \geq \sim 3 \%$

Table 7.11. Effect of criteria used to select 10 input GIS layers on best training set performance \& map quality. Network topology: 10-20-1, training algorithm: trainlm.

\begin{tabular}{|l|l|l|l|l|l|c|c|c|l|}
\hline \multirow{2}{*}{$\begin{array}{l}\text { Selection } \\
\text { criteria }\end{array}$} & \multicolumn{4}{|l|}{ Training Sets } & \multicolumn{4}{l|}{ Test Set } & \multicolumn{4}{|l|}{ Map Quality Statistics } \\
\cline { 2 - 10 } & Train & Stop & All & Deps & Barren & $\rho$ & D/A & D*(D/A) & ROC \\
\hline \hline categories & 88.9 & 72.7 & 76.5 & 77.1 & 76.1 & 0.90 & 9.6 & 256.7 & 0.82 \\
\hline $\mathrm{D} / \mathrm{A}$ & 78.8 & 70.5 & 72.8 & 65.7 & 78.3 & 0.95 & 5.7 & 9.5 & 0.81 \\
\hline $\mathrm{D} *(\mathrm{D} / \mathrm{A})$ & 92.9 & 81.8 & 77.8 & 82.9 & 73.9 & 0.98 & 5.1 & 104.5 & 0.87 \\
\hline $\mathrm{DDA} 2^{a}$ & 81.8 & 72.7 & 76.5 & 77.1 & 76.1 & 1.00 & 11.4 & 133.5 & 0.83 \\
\hline
\end{tabular}

${ }^{a} \mathrm{DDA} 2=\mathrm{D} *(\mathrm{D} / \mathrm{A}) A N D \mathrm{D} \geq \sim 20 \%$ AND $\mathrm{D} / \mathrm{A} \geq \sim 3 \%$ 
Table 7.12. Effect of criteria used to select 17 input GIS layers on average training set performance \& map quality. Network topology: 17-20-1, training algorithm: trainlm.

\begin{tabular}{|l|l|l|l|l|l|c|c|c|c|}
\hline \multirow{2}{*}{$\begin{array}{l}\text { Selection } \\
\text { criteria }\end{array}$} & \multicolumn{4}{|l|}{ Training Sets } & \multicolumn{4}{l|}{ Test Set } & \multicolumn{4}{|c|}{ Map Quality Statistics } \\
\cline { 2 - 11 } & Train & Stop & All & Deps & Barren & $\rho$ & D/A & D*(D/A) & ROC \\
\hline \hline categories & 82.0 & 70.8 & 71.0 & 68.9 & 72.6 & 0.88 & 6.4 & 49.1 & 0.78 \\
\hline $\mathrm{D} / \mathrm{A}$ & 87.5 & 76.1 & 71.6 & 72.6 & 70.9 & 0.94 & 4.6 & 52.9 & 0.80 \\
\hline $\mathrm{D} *(\mathrm{D} / \mathrm{A})$ & 87.8 & 77.4 & 73.1 & 70.3 & 75.2 & 0.95 & 9.0 & 117.0 & 0.83 \\
\hline $\mathrm{DDA} 2^{a}$ & 84.2 & 75.6 & 71.6 & 72.0 & 71.3 & 0.95 & 7.4 & 93.7 & 0.81 \\
\hline
\end{tabular}

${ }^{a} \mathrm{DDA} 2=\mathrm{D} *(\mathrm{D} / \mathrm{A}) A N D \mathrm{D} \geq \sim 20 \% A N D \mathrm{D} / \mathrm{A} \geq \sim 3 \%$

Table 7.13. Effect of criteria used to select 17 input GIS layers on best training set performance \& map quality. Network topology: 17-20-1, training algorithm: trainlm.

\begin{tabular}{|l|l|l|l|l|l|r|r|r|l|}
\hline \multirow{2}{*}{$\begin{array}{c}\text { Selection } \\
\text { criteria }\end{array}$} & \multicolumn{4}{|l|}{ Training Sets } & \multicolumn{4}{l|}{ Test Set } & \multicolumn{4}{|c|}{ Map Quality Statistics } \\
\cline { 2 - 11 } & Train & Stop & All & Deps & Barren & $\rho$ & D/A & D*(D/A) & ROC \\
\hline \hline categories & 82.8 & 79.6 & 76.5 & 88.6 & 67.4 & 1.00 & 9.7 & 72.5 & 0.83 \\
\hline D/A & 88.9 & 72.7 & 76.5 & 80.0 & 73.9 & 0.97 & 7.5 & 111.7 & 0.79 \\
\hline $\mathrm{D} *(\mathrm{D} / \mathrm{A})$ & 90.9 & 83.0 & 75.3 & 82.9 & 69.6 & 0.98 & 13.2 & 231.4 & 0.86 \\
\hline $\mathrm{DDA}^{a}$ & 81.8 & 79.6 & 79.0 & 77.1 & 80.4 & 1.00 & 8.0 & 73.4 & 0.83 \\
\hline
\end{tabular}

${ }^{a} \mathrm{DDA} 2=\mathrm{D} *(\mathrm{D} / \mathrm{A}) A N D \mathrm{D} \geq \sim 20 \% A N D \mathrm{D} / \mathrm{A} \geq \sim 3 \%$ 


\subsubsection{Number of Input GIS Layers}

In general, networks trained with 17 layers gave slightly better average test-set classification results than networks trained with 10 layers (Table7.14). This is generally true for all layer selection criteria and applies to the classification performance for the test set overall, and deposit and barren patterns separately. Two reasons why such a large increase in the number of input variables leads to only a slight increase in the average classification performance (i.e. generalization ability) are: 1) that the sets of 10 inputs may already include all the variables that are truly important for distinguishing deposit and barren classes, and 2) the smaller number of weights, corresponding to a network with 10 inputs, are more likely to be well represented by a very small training data set, such as the one used here, than 17 inputs.

Both explanations relate to the the trade-off between adding input features to improve classification and the limiting the dimensionality of the feature space so that the degree to which the training set represents the multidimensional feature space is not too sparse. It is well known that a classification problem may be solved through the addition of appropriate new features (thus creating a hyperplane in a higher dimension). The classic example is the exclusive-OR (XOR) problem which is not solvable in two dimensions but is solvable in three dimensions (McClelland \& Rumellhart, 1988). However, Bishop (1995) has pointed out that increasing the number of input variables whilst keeping the training-set size fixed means that the higher-dimensional feature space is more sparsely represented - a problem known as the curse of dimensionality. This increases the risk that the network may not learn the patterns in the data reliably.

For comparison purposes, a set of 25 layers, which includes all the input variables selected using the four criteria, was also tested. The average performance for the 25-input set is between that of the 10- and 17-layer sets. The values of the map quality statistics $(\rho, D / A$, $D \times(D / A)$, and area under the ROC curve) for the 25-input set were generally slightly poorer than those obtained for 10- and 17-inputs. The map quality statistics produced by the best network with 25 inputs were poor compared to the other networks (Table 7.15). This is due to the fact that the highest prospectivity map class (in the 9-class produced by this network) did not contain any of the known deposits.

Comparison of the best test-set performance for networks trained with 10 and 17 inputs, selected using the modified $D \times(D / A)$ (labelled in tables as DDA2), and categories criteria shows that 17 input layers produce significantly better performance than that obtained by using 10 inputs or 25 (i.e. all available) input variables. 
Table 7.14. Effect of number of input GIS layers on average training set performance \& map quality. Network topology: 10-20-1 or 17-20-1, training algorithm: trainlm.

\begin{tabular}{|l|l|l|l|l|l|c|c|c|c|}
\hline \multirow{2}{*}{$\begin{array}{l}\text { No. of Layers, } \\
\text { Selection criteria }\end{array}$} & \multicolumn{3}{|l|}{ Training Sets } & \multicolumn{3}{l|}{ Test Set } & \multicolumn{4}{l|}{ Map Quality Statistics } \\
\cline { 2 - 10 } & Train & Stop & All & Deps & Barren & $\rho$ & D/A & D*(D/A) & ROC \\
\hline \hline 10, categories & 84.4 & 72.7 & 67.9 & 68.0 & 67.8 & 0.97 & 8.2 & 105.3 & 0.79 \\
17, categories & 82.0 & 70.8 & 71.0 & 68.9 & 72.6 & 0.88 & 6.4 & 49.1 & 0.78 \\
\hline 10, D/A & 80.3 & 70.5 & 69.1 & 64.0 & 73.0 & 0.94 & 5.5 & 54.2 & 0.77 \\
17, D/A & 87.5 & 76.1 & 71.6 & 72.6 & 70.9 & 0.94 & 4.6 & 52.9 & 0.80 \\
\hline 10, D*D/A & 87.9 & 76.9 & 72.1 & 75.1 & 69.8 & 0.95 & 7.4 & 109.0 & 0.84 \\
17, D*D/A & 87.8 & 77.4 & 73.1 & 70.3 & 75.2 & 0.95 & 9.1 & 117.0 & 0.83 \\
\hline 10, DDA2 & 84.9 & 72.6 & 69.9 & 67.1 & 72.0 & 0.93 & 12.1 & 111.2 & 0.80 \\
17, DDA2 & 84.2 & 75.6 & 71.6 & 72.0 & 71.3 & 0.95 & 7.4 & 93.7 & 0.81 \\
\hline 25, all & 89.2 & 74.2 & 70.1 & 71.1 & 69.4 & 0.91 & 6.0 & 52.5 & 0.81 \\
\hline
\end{tabular}

${ }^{a} \mathrm{DDA} 2=\mathrm{D} *(\mathrm{D} / \mathrm{A}) A N D \mathrm{D} \geq \sim 20 \% A N D \mathrm{D} / \mathrm{A} \geq \sim 3 \%$

Table 7.15. Effect of number of input GIS layers on best training set performance \& map quality. Network topology: 10-20-1 or 17-20-1, training algorithm: trainlm.

\begin{tabular}{|l|l|l|l|l|l|l|r|r|r|}
\hline \multirow{2}{*}{$\begin{array}{l}\text { No. of Layers, } \\
\text { Selection criteria }\end{array}$} & \multicolumn{2}{|l|}{ Training Sets } & \multicolumn{3}{l|}{ Test Set } & \multicolumn{4}{l|}{ Map Quality Statistics } \\
\cline { 2 - 10 } & Train & Stop & All & Deps & Barren & $\rho$ & D/A & D*(D/A) & ROC \\
\hline \hline 10, categories & 88.9 & 72.7 & 76.5 & 77.1 & 76.1 & 0.90 & 9.6 & 256.7 & 0.82 \\
17, categories & 82.8 & 79.6 & 76.5 & 88.6 & 67.4 & 1.00 & 9.7 & 72.5 & 0.83 \\
\hline 10, D/A & 78.8 & 70.5 & 72.8 & 65.7 & 78.3 & 0.95 & 5.7 & 9.5 & 0.81 \\
17, D/A & 87.5 & 76.1 & 71.6 & 72.6 & 70.9 & 0.94 & 4.6 & 52.9 & 0.80 \\
\hline 10, D*D/A & 92.9 & 81.8 & 77.8 & 82.9 & 73.9 & 0.98 & 5.0 & 104.5 & 0.87 \\
17, D*D/A & 90.9 & 83.0 & 75.3 & 82.9 & 69.6 & 0.98 & 13.2 & 231.4 & 0.86 \\
\hline 10, DDA2 & 81.8 & 72.7 & 76.5 & 77.1 & 76.1 & 1.00 & 11.4 & 133.5 & 0.83 \\
17, DDA2 & 81.8 & 79.6 & 79.1 & 77.1 & 80.4 & 1.00 & 8.0 & 73.4 & 0.83 \\
\hline 25, all & 87.9 & 77.3 & 74.1 & 71.4 & 76.1 & 0.38 & 0.0 & 0.0 & 0.83 \\
\hline
\end{tabular}

${ }^{a} \mathrm{DDA} 2=\mathrm{D} *(\mathrm{D} / \mathrm{A}) A N D \mathrm{D} \geq \sim 20 \% A N D \mathrm{D} / \mathrm{A} \geq \sim 3 \%$ 


\subsection{Input Data Transformations}

\subsubsection{Coding Distance Inputs: Raw Values versus Inverse and Log}

Learning in a neural network is facilitated if the relationships between the input and output variables are expressed as explicitly as possible (Masters, 1993b; Zaknich, 1999). For example, the closer a location is to a regional fault and shear zone, the more prospective it is. Thus, if all other parameters are equal, a smaller distance should result in a higher estimate of prospectivity. Consequently, learning may be improved if the inverse of the distance input is used instead of the raw input so that larger distances give larger prospectivity estimates.

The inverse of all input layers depicting proximity relationships was calculated using MATLAB functions, inv_layer and inv_scale_batch_vec2vec (see attached CD-ROM, vol.1). Layers that were transformed in this way depict the distance to the nearest:

1. felsic porphyritic intrusion,

2. positive aeromagnetic (RTP) anomaly (where z-score for host lithology $\geq 2$ ),

3. crustal-scale fault or shear zone,

4. NE-trending regional-scale fault or shear zone,

5. lithological contact, and

6. trace of anticlinal axis.

Since $\log _{10}(0)$ is undefined, distances of zero were treated as $100 \mathrm{~m}$. The cell size is $100 \mathrm{~m}$, so this corresponds to the uncertainty for distances values. As a final step, the inputs were scaled to the range $[0,1]$.

Tables 7.16 and 7.17 show the results for the transformed distance inputs together with the results for raw distances (first row). The average results for all three inputs are quite similar. However, networks with transformed distance inputs produced slightly poorer test set classification and map quality results compared to the network with the raw inputs. The $1 /(x)$ and is $\log _{10}(x)$ inputs produce significantly poorer values of the capture efficiency statistic, $D / A$. For networks that produced the best overall test-set classification results (Table 7.17), the transformed inputs also produced significantly poorer correct classification rates for the test set overall and barren cells, and both capture efficiency ratios. Since the results indicate that raw distances produce better results, no transformations were performed in the experiments shown below. 
The decrease in network performance for networks trained using transformed-distance inputs may be due to the fact that the transformations actually obscure the relationship between the network input and the output. In ARCVIEw, the calculation of Euclidean distances based a raster grid result in an irregular distribution of distances. This is due to the geometry of the grid, in which only certain discrete inter-cellular distances are possible. Whilst this quantization of distances is less significant at large distances, the results of Chapter 6 (Table 6.4) show that most of the gold deposits are located relatively close to faults, anticlinal axes and contacts. Both the inverse and log-transformations actually emphasize this irregular distribution of small distances. This effect is illustrated in Figures $7.3 \mathrm{~b} \& \mathrm{c}$ which show the inverse and log-transformed distances to the nearest NE-trending regional-scale faults and the distance interval 0-800 $\mathrm{m}$ which is the most most prospective zone for gold deposits. interval .

Table 7.16. Effect of using inverse and log transformations of distance inputs on average results. Network topology: 10-20-1, training algorithm: trainlm, layer selection criteria: categories.

\begin{tabular}{|l|l|l|l|l|l|l|l|l|l|}
\hline \multirow{2}{*}{$\begin{array}{l}\text { Distance } \\
\text { input format }\end{array}$} & \multicolumn{4}{|l|}{ Training Sets } & \multicolumn{4}{l|}{ Test Set } & \multicolumn{4}{l|}{ Map Quality Statistics } \\
\cline { 2 - 10 } & Train & Stop & All & Deps & Barren & $\rho$ & D/A & D*(D/A) & ROC \\
\hline \hline$x$ & 84.4 & 72.7 & 67.9 & 68.0 & 67.8 & 0.97 & 8.2 & 105.3 & 0.79 \\
\hline $1 /(x)$ & 87.8 & 79.0 & 67.0 & 66.5 & 67.4 & 0.87 & 5.4 & 77.7 & 0.75 \\
\hline $\log _{10}(x)$ & 88.7 & 77.1 & 67.3 & 65.7 & 68.5 & 0.94 & 5.3 & 100.3 & 0.76 \\
\hline
\end{tabular}

Table 7.17. Effect of using inverse and $\log$ transformations of distance inputs on best results. Network topology: 10-20-1, training algorithm: trainlm, layer selection criteria: categories.

\begin{tabular}{|l|l|l|l|l|l|l|l|l|l|}
\hline \multirow{2}{*}{$\begin{array}{l}\text { Distance } \\
\text { input format }\end{array}$} & \multicolumn{4}{|l|}{ Training Sets } & \multicolumn{4}{l|}{ Test Set } & \multicolumn{4}{l|}{ Map Quality Statistics } \\
\cline { 2 - 10 } & Train & Stop & All & Deps & Barren & $\rho$ & D/A & D*(D/A) & ROC \\
\hline \hline$x$ & 88.9 & 72.7 & 76.5 & 77.1 & 76.1 & 0.90 & 9.6 & 256.7 & 0.82 \\
\hline $1 /(x)$ & 82.8 & 72.7 & 71.6 & 77.1 & 67.4 & 0.90 & 3.1 & 26.0 & 0.76 \\
\hline $\log _{10}(x)$ & 90.9 & 77.3 & 70.4 & 74.3 & 67.4 & 0.98 & 4.9 & 77.2 & 0.82 \\
\hline
\end{tabular}




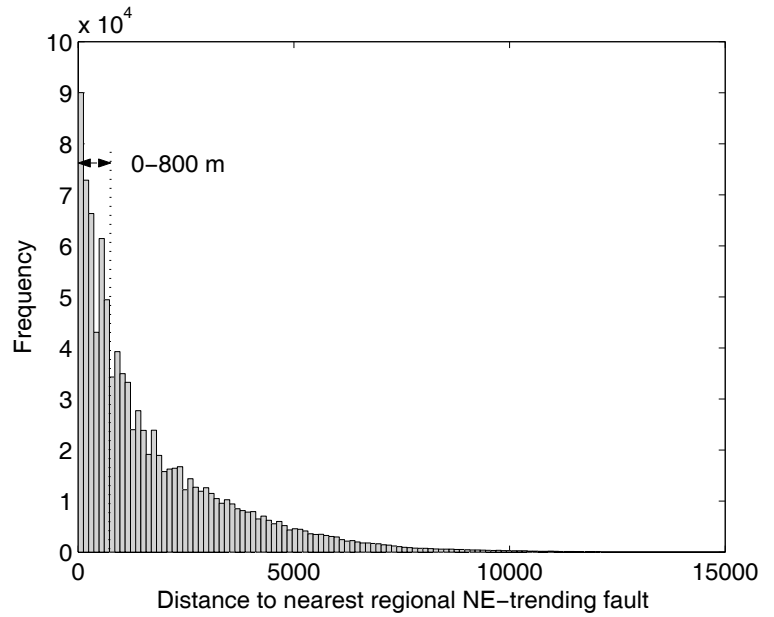

(a) Neural network: 3 fuzzy membership inputs.

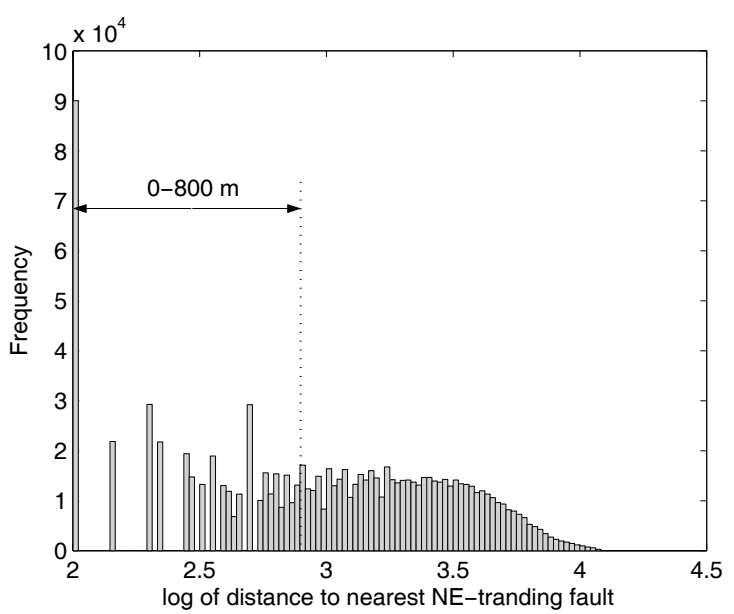

(c) Hybrid fuzzy-neural network: 17 fuzzy inputs.

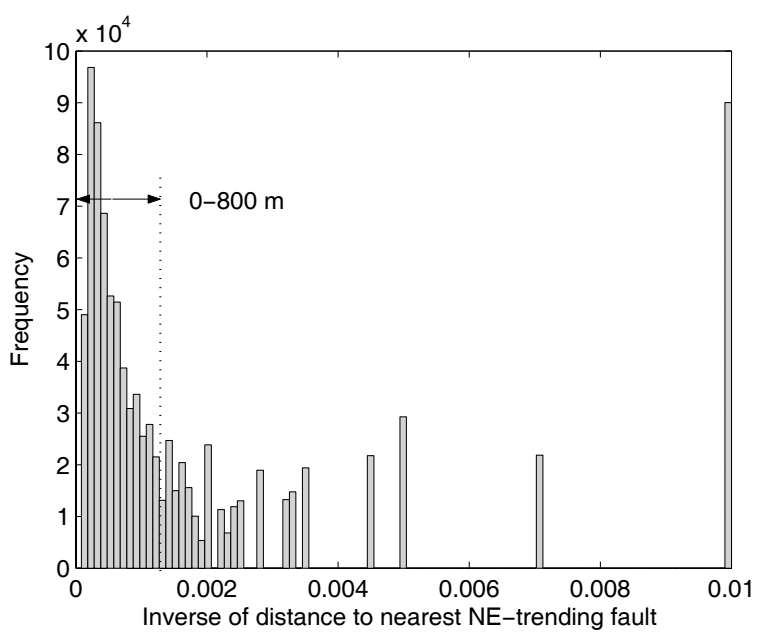

(b) Neural network: 3 fuzzy membership inputs.

Figure 7.3. Histograms comparing raw and transformed distances to NE-trending faults; a) raw data, b) inverse and c) logarithm of distance - For transformations, zero distances were converted to $100 \mathrm{~m}$ (grid cell size). All histograms compiled using 100 bins. The most favourable distance from regional-scale NE-trending faults for gold deposits is approximately $0-800 \mathrm{~m}$ (Table 6.4). The fact that the inverse and log transformations emphasize the irregular distribution of data resulting from the grid-based Euclidean distance calculation may explain why better performance was obtained from networks trained with raw data.

\subsubsection{Distance Thresholds for Fault Strike Inputs}

Experiments in this section address the question of whether network performance could be improved if buffer limits were placed on layers that involve distance to linear features such as faults and lithological contacts. The motivation for this comes from the concept that although, for example, proximity to a fault upgrades the mineral prospectivity for a cell that is close to the fault, there must be a distance beyond which the existence of the fault has no effect on the prospectivity. It should be noted here that the $2 \mathrm{D}$-prospectivity analysis can be applied successfully to the Yilgarn Craton successfully because: 1) regionalscale structural features are the main control on the location of large gold deposits, 2) gold is very late in the sequence of kinematic events, so that geometries revealed in a solid 
geology map are similar to those at the time of gold deposition, and 3) major structures associated with gold are generally steeply dipping (Groves et al., 1997, 2000).

In order to model this concept, cell values at a distance exceeding the limit were set to a background value. In the case of proximity layers (listed in Section 7.5.1 above) the cells beyond the buffer limit were set to the maximum distance. For layers representing the strike orientation of the nearest fault or shear zone, i.e. layers showing the strike of:

1. crustal-scale faults and shear zones,

2. NE-trending regional faults and shear zones, and

3. lithological contacts,

the strike was set to the mean for the entire data set.

Buffer limits were estimated by interpreting plots of $(O-E) / E$ (where $O=$ observed number of deposits in a region corresponding to an interval on the $\mathrm{X}$-axis and $E=$ expected number of deposits for a uniform random distribution). The distance threshold was set to the distance on the $(O-E) / E$ bar graph where the $(O-E) / E$ value consistently falls below zero. Bivariate J-function plots (Section 6.2.4) were used to confirm that the positive association with deposits is statistically significant within intervals that appear to be favourable.

Wide and narrow buffer limits were estimated for each of the three types of faults and lithological contacts. The wide buffer limits are based on the values shown in column 4 of Table 6.4. Distances for wide buffer limits are $100 \mathrm{~m}$ greater than the values shown in Table 6.4 because Y-intercepts on the $(O-E) / E$ bar graphs were taken as the middle of the bar at which the $\mathrm{Y}$-value falls below zero rather than the start of the interval as quoted in the table. Narrow buffers were based on a partly subjective estimate of the distance at which the value of $(O-E) / E$ starts to decrease significantly. The same limits were used as the inflection points and the $y=0$ intercepts in the triangular fuzzy membership functions described below.

Cells beyond the distance limits were set to zero in the case of layers depicting proximity and strike angles were set to the mean strike angle for the type of fault and shear zone represented in the layer. Processing was performed in MATLAB using functions reclass_dist_strike_narrow_buf and reclass_dist_strike_wide_buf. The buffer limits are shown in Table 7.18 below. 
Table 7.18. Input GIS layers for which distance transformations were applied. At distances greater than the buffer limit, cell values were set to the maximum distance and mean strike for distance and strike orientation layers, respectively.

\begin{tabular}{|l|l|l|l|}
\hline \multirow{2}{*}{$\begin{array}{l}\text { Layer } \\
\text { type }\end{array}$} & Layer name & \multicolumn{2}{l|}{ Buffer limit (m) } \\
\cline { 3 - 4 } & in ArcVIEW & Narrow & Wide \\
\hline \hline Distance Layers & 14_l_pf_dist & 400 & 1500 \\
\hline Felsic porphyry & 114_mz2_dist & 200 & 1700 \\
\hline +ve magnetic anomalies & 120_f1_dist & 400 & 2500 \\
\hline Crustal-scale faults & 122_d3_dist & 200 & 900 \\
\hline NE regional faults & 139_c_dist & 200 & 500 \\
\hline Lithological contacts & 146_dist_anti & 800 & 2300 \\
\hline Anticlinal axes & 134_s_f23pdst & 200 & 1300 \\
\hline Intersection of faults & & \\
\hline Strike Orientation Layers & 128_s_f1str & 600 & 1600 \\
\hline \hline Crustal-scale faults & 129_s_f2str & 600 & 1200 \\
\hline NNW regional faults & 130_s_f3str & 500 & 500 \\
\hline NE regional faults & 138_c_str1k & 200 & 500 \\
\hline Lithological contacts & 136_l_rh11k-71k & 200 & n.a. \\
\hline Contrast Layers & 137_l_ch11k-51k & 200 & n.a. \\
\hline \hline Rheological & \\
\hline Chemical & & \\
\hline
\end{tabular}

Narrow and wide buffers cause a significant decrease in the generalization ability of the trained networks. This is indicated by the classification performance for the test-set overall and deposit patterns and applies to both the average and best results (Tables 7.19 \& 7.20). However, the use of narrow buffers does produce a significant increase in the average values of the capture efficiency statistics, $D / A$ and $D \times(D / A)$, and the $D / A$ value for the networks that gave the best overall test-set classification (Table 7.20). It is possible that the abrupt change in the distance and strike values, in cells located at distances exceeding the buffer limits in the transformed layers, inhibit efficient learning and therefore generalization. The higher capture efficiency values for the narrow buffers may be due to a decrease in the prospectivity estimates cells located beyond the buffer limits. The bulk of these cells would be barren and therefore result in fewer barren cells being falsely identified as deposits (i.e. fewer false positives). This would result in lower areas $(A$ in $D / A$ and $D \times(D / A))$ for 
high prospectivity map classes and therefore higher values of $D / A$ and $D \times(D / A))$.

Table 7.19. Effect of applying distance limits in input GIS layers depicting strike and distance on average performance. Layers depicting distance were set to the maximum distance and layers depicting strike angle of the nearest fault or shear zone were set to the mean strike for distances $\geq$ limit. Network topology: 10-20-1, training algorithm: trainlm, layer selection criteria: categories.

\begin{tabular}{|l|l|l|l|l|l|l|r|l|l|}
\hline \multirow{2}{*}{$\begin{array}{l}\text { Buffer } \\
\text { type }\end{array}$} & \multicolumn{4}{|l|}{ Training Sets } & \multicolumn{4}{l|}{ Test Set } & \multicolumn{4}{l|}{ Map Quality Statistics } \\
\cline { 2 - 11 } & Train & Stop & All & Deps & Barren & $\rho$ & D/A & D*(D/A) & ROC \\
\hline \hline no buffers & 84.4 & 72.7 & 67.9 & 68.0 & 67.8 & 0.97 & 8.2 & 105.3 & 0.79 \\
\hline narrow buffers & 84.8 & 76.6 & 66.4 & 59.7 & 71.5 & 0.95 & 10.4 & 230.5 & 0.80 \\
\hline wide buffers & 88.7 & 74.1 & 62.2 & 59.7 & 64.1 & 0.91 & 5.8 & 147.4 & 0.79 \\
\hline
\end{tabular}

Table 7.20. Effect of applying distance limits in input GIS layers depicting strike and distance on best performance. Layers depicting distance were set to the maximum distance and layers depicitng strike angle of the nearest fault or shear zone were set to the mean stike for distances $\geq$ limit. Network topology: 10-20-1, training algorithm: trainlm, layer selection criteria: categories.

\begin{tabular}{|l|l|l|l|l|l|r|r|r|l|}
\hline \multirow{2}{*}{$\begin{array}{l}\text { Buffer } \\
\text { type }\end{array}$} & \multicolumn{4}{|l|}{ Training Sets } & \multicolumn{4}{l|}{ Test Set } & \multicolumn{4}{l|}{ Map Quality Statistics } \\
\cline { 2 - 11 } & Train & Stop & All & Deps & Barren & $\rho$ & D/A & D*(D/A) & ROC \\
\hline \hline no buffers & 88.9 & 72.7 & 76.5 & 77.1 & 76.1 & 0.90 & 9.6 & 256.7 & 0.82 \\
\hline narrow buffers & 84.9 & 76.1 & 71.6 & 62.9 & 78.3 & 0.93 & 13.1 & 229.9 & 0.82 \\
\hline wide buffers & 89.9 & 83.0 & 67.9 & 68.6 & 67.4 & 0.92 & 5.6 & 74.2 & 0.82 \\
\hline
\end{tabular}

\subsubsection{Distance Limits for Rheological and Chemical Contrast Inputs}

Narrow buffer limits were also applied to the rheological and chemical contrast layers used in networks with 10 inputs, where the variables were selected according to the categories criterion. The rheological contrast layer is based on subjectively estimated fuzzy membership values (layer 136_l_rh11k, described in sections 5.4 .8 \& 7.8.2) and the chemical contrast layer is based on the ratio, $\mathrm{Fe}_{t o t}(\mathrm{wt} \%)^{*} \mathrm{Fe}_{t o t} /\left(\mathrm{Fe}_{t o t}+\mathrm{Mg}+\mathrm{Ca}\right)$ (layer 137_l_ch11k, described in Section 5.4.7). Contrast was set to zero for cells located outside the buffer limits. Reclassification of the input layers was performed in MATLAB with the function reclass_dist_contrast_nrrow_buf. The results are shown in Tables 7.21 and 7.22.

In contrast to the distance and strike layers (described above), the average results for the contrast layers indicate that the use of narrow buffers has little effect on generalization. 
However, for networks with the best test-set classification performance, the use of narrow buffers resulted in lower percentage correct classification of the test-set (overall and deposit patterns) but a significant increase in the average $D \times(D / A)$ value. The average results show a significantly higher value for the capture efficiency ratio, $D \times(D / A)$.

Table 7.21. Effect of applying distance limits in input GIS layers depicting rheological and chemical contrast on average performance. Contrast was set to zero for cells where distance to the nearest lithological contact $\geq$ limit. Network topology: 10-20-1, training algorithm: trainlm, layer selection criteria: categories.

\begin{tabular}{|l|l|l|l|l|l|l|l|l|l|}
\hline \multirow{2}{*}{$\begin{array}{l}\text { Input } \\
\text { layers }\end{array}$} & \multicolumn{3}{|l|}{ Training Sets } & \multicolumn{3}{l|}{ Test Set } & \multicolumn{4}{l|}{ Map Quality Statistics } \\
\cline { 2 - 11 } & Train & Stop & All & Deps & Barren & $\rho$ & D/A & D*(D/A) & ROC \\
\hline \hline no buffers & 84.4 & 72.7 & 67.9 & 68.0 & 67.8 & 0.97 & 8.2 & 105.3 & 0.79 \\
\hline narrow buffers & 87.5 & 74.3 & 67.0 & 66.6 & 67.4 & 0.96 & 9.2 & 142.9 & 0.80 \\
\hline
\end{tabular}

Table 7.22. Effect of applying distance limits in input GIS layers depicting rheological and chemical contrast on best performance. Contrast was set to zero for cells where distance to the nearest lithological contact $\geq$ limit. Network topology: 10-20-1, training algorithm: trainlm, layer selection criteria: categories.

\begin{tabular}{|l|l|l|l|l|l|c|c|c|c|}
\hline \multirow{2}{*}{$\begin{array}{l}\text { Input } \\
\text { layers }\end{array}$} & \multicolumn{4}{|l|}{ Training Sets } & \multicolumn{4}{l|}{ Test Set } & \multicolumn{4}{l|}{ Map Quality Statistics } \\
\cline { 2 - 11 } & Train & Stop & All & Deps & Barren & $\rho$ & D/A & D*(D/A) & ROC \\
\hline \hline no buffers & 88.9 & 72.7 & 76.5 & 77.1 & 76.1 & 0.90 & 9.6 & 256.7 & 0.82 \\
\hline narrow buffers & 78.8 & 65.9 & 70.4 & 62.9 & 76.1 & 0.95 & 17.6 & 117.1 & 0.80 \\
\hline
\end{tabular}

\subsection{Effect of Numbers of Deposit Patterns in Training Set}

In Chapter 7, it was noted that one of the main limitations of the weights-of-evidence method for mineral prospectivity mapping is the requirement of a statistically significant number of known deposits. Within the geosciences, neural networks have most frequently been applied to remote-sensing and geophysics problems in which large amounts of data are available. In contrast, the paucity of deposit training patterns compared to the very large number of patterns that correspond to barren cells potentially limits the application of neural networks in mineral prospectivity mapping to well explored areas where many known deposits are available for training. The experiments in this section address the question of how many deposit training examples are required to correctly train a neural 
network for this type of application.

In order to ensure that the network correctly learns the patterns in the data, the training data should sample the entire range of variation in the data population and adequately represent feature vectors that are located close to decision boundaries in multidimensional feature space. Both these requirements are difficult to fulfil because there are so few deposit examples relative to barren patterns. An additional problem arising from the gradientdescent backpropagation algorithm (Rumelhart et al., 1986a) used to train multilayer perceptron (MLP) networks is that minimization of the network error function requires that there are approximately equal numbers of training examples from each class (i.e. deposit and barren cells) irrespective of the actual a priori probability of occurrence of the classes (Parikh et al., 1999; Zaknich, 1999; Fig. 7.4). In most cases, the solution to this problem is to collect more data. However, this is expensive and time consuming in the case of ore deposits and, even in areas very well endowed with mineral resources, map grid cells containing deposits are very rare compared to barren cells. Thus, the overall size of the training data sets is limited by the small number of known deposits.

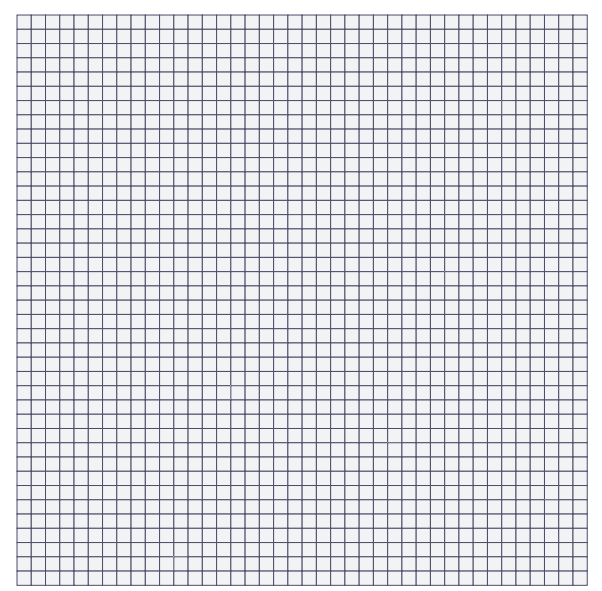

Study Area

$1,254,000$ cells

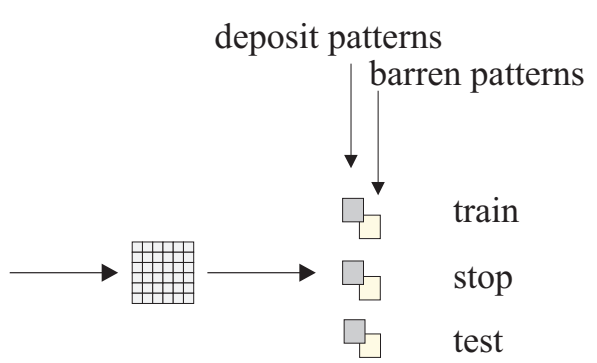

Training Data

120 deposits

Figure 7.4. Schematic illustration of the relationship between numbers of map grid, deposit and training set patterns. The paucity of patterns representing known deposits limits the size of training sets for a neural network method in which supervised training and a training algorithm based on the minimization of an error function over all the training patterns is used. 


\section{Method}

A series of training data sets were prepared using 5, 10204080 and 100 deposit patterns in order to test the way in which the limited number of deposit patterns available for training affects the performance of the trained network. For each of these numbers of deposit patterns, ten training data sets are created by randomly allocating deposits to each of the three training sets. Only the 120 known deposits with a total resource of at least $1000 \mathrm{~kg}$ gold are used to train the networks. Where possible, the training and training-stop data were allocated the same number of deposit patterns. If this was not possible, the training-stop and test data sets were allocated equal numbers of deposits. For each experiment, the total number of deposit and barren patterns used to train the networks were the same. The composition of the training sets is shown in Table 7.23.

Table 7.23. Composition of training data sets used to test effect of the number of deposit patterns in the training set on network generalization performance and prospectivity map quality. Patterns represent cells containing orogenic gold deposits and barren cells from raster GIS data for the Kalgoorlie Terrane.

\begin{tabular}{|c|c|c|c|c|c|c|}
\hline Number of & \multicolumn{2}{|l|}{ Training set } & \multicolumn{2}{l|}{ Stop set } & \multicolumn{2}{l|}{ Test set } \\
\hline deposits & Deposits & Barren & Deposits & Barren & Deposits & Barren \\
\hline \hline 5 & 5 & 5 & 5 & 5 & 110 & 110 \\
\hline 10 & 10 & 10 & 10 & 10 & 100 & 100 \\
\hline 20 & 20 & 20 & 20 & 20 & 80 & 80 \\
\hline 40 & 40 & 40 & 40 & 40 & 40 & 40 \\
\hline 80 & 80 & 80 & 20 & 20 & 20 & 20 \\
\hline 100 & 100 & 100 & 10 & 10 & 10 & 10 \\
\hline
\end{tabular}

\section{Results and Discussion}

The results are shown in Figures 7.5, which show the percentage correct classification for the test set overall, deposit patterns in the test set, and $D \times(D / A)$ and area under the ROC curve as measures of the quality of the prospectivity map, respectively. Each point on the plots represents the average of ten networks for each of the ten training data sets; i.e. 100 networks.

All of the plots show; 1) a trend of significantly increasing generalization ability of the networks and map quality with increasing numbers of deposit patterns and training data set size, and 2) that the rate at which performance increases levels off above 40 deposit patterns. In the case of the test-set patterns, the percentage correct classification rate 
levels off at 80 deposit patterns and for test-set deposit patterns, the percentage correct classification rate levels off at about 20 deposit patterns (see Figs 7.5a \& b). Parikh et al. (1999) obtained similar results for experiments using unequal class sizes for patterns corresponding to normal and fault conditions. In their data, the rate of network performance improvement decreases as a 1:1 ratio is approached between the numbers of patterns corresponding to the two classes. It should be noted that in these experiments, training sets contained equal numbers of deposit and barren patterns.

The slight drop in performance for 100 deposit patterns indicated in Figures 7.5a and c may be due to the fact that there are only 20 patterns in the training-stop and test data sets. Due to decreasing size of the test data sets, the reliability of the results decreases with increasing numbers of deposit patterns.

The observation that above approximately 40 deposit patterns, the effect of increasing deposit patterns decreases as deposit patterns are added is important because it suggests that 40 deposit patterns is approximately the minimum number of deposit patterns required for training. Clearly, this number is dependent on the characteristics of the data set and the degree to which the input variables are predictive of the deposits but it provides a rough guide to the number of deposit patterns required to reliably train a neural network for this sort of problem. The standard (not noise-augmented) training data set used in this chapter contains 46 deposit patterns. In contrast, the training data set in the Tenterfield pilot study contained only 21 deposit patterns.

\subsection{Increasing Training Set Size Using Random Noise}

It is clear from the foregoing section the prediction of mineral prospectivity represents a difficult problem to solve using neural networks due to the lack of abundant deposit training data. This problem potentially excludes or limits the application of neural networks to new exploration areas where there are few known deposits. A possible solution might be to include the the abundant smaller deposits in the training data set (1327 orogenic lode gold deposits and occurrences exist within areas for which solid geology data are available). However, there are good geological and GIS-related reasons for training networks on larger deposits (i.e. those with $\geq 1000 \mathrm{~kg}$ total contained gold). First, ore systems responsible for minor deposits and occurrences are smaller and therefore less likely to be associated with geological features that would be captured in a regional-scale GIS database than those associated with large deposits. Second, if the large deposits represent the type of targets that any useful neural network system would be expected to identify then training target 


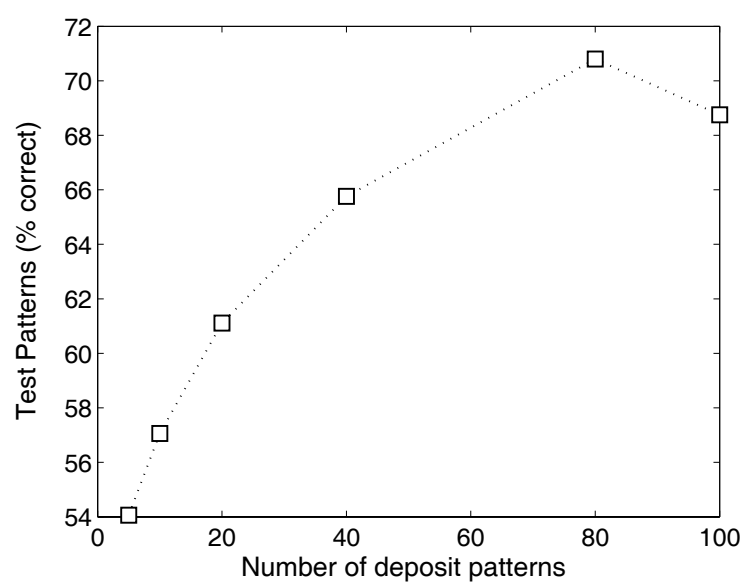

(a) Test-set classification: overall

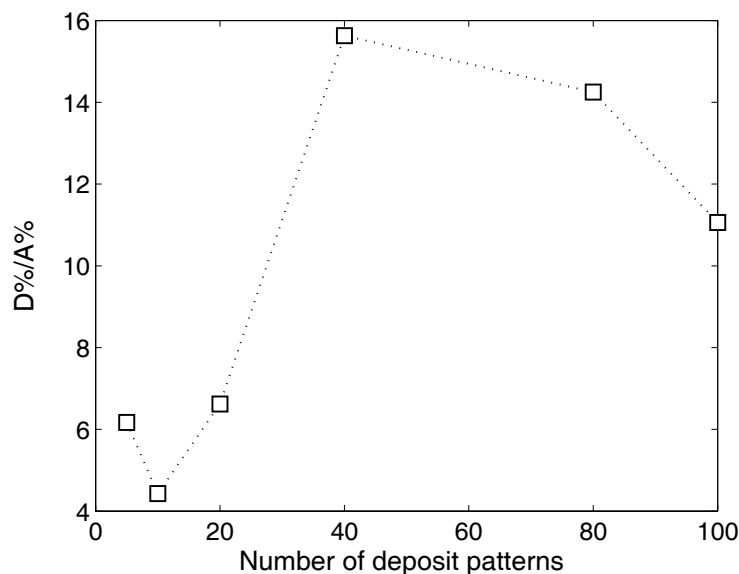

(c) Map quality statistic D/A.

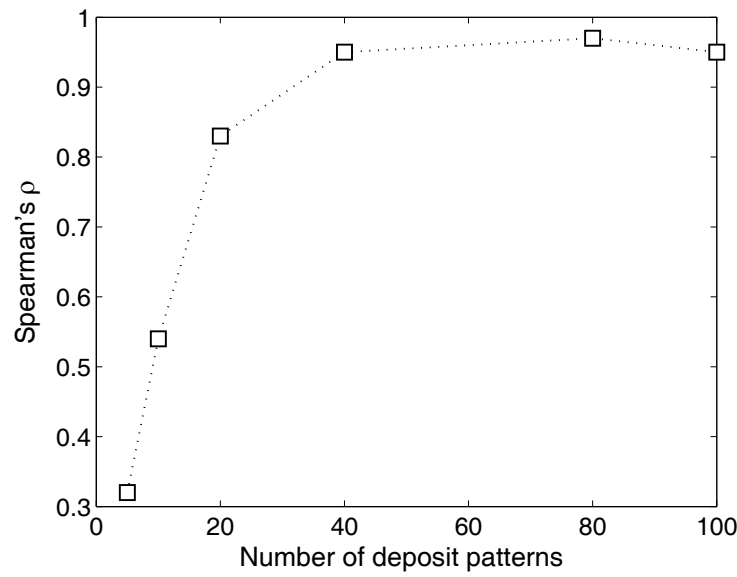

(e) Correlation of map class \& P(Deposit)

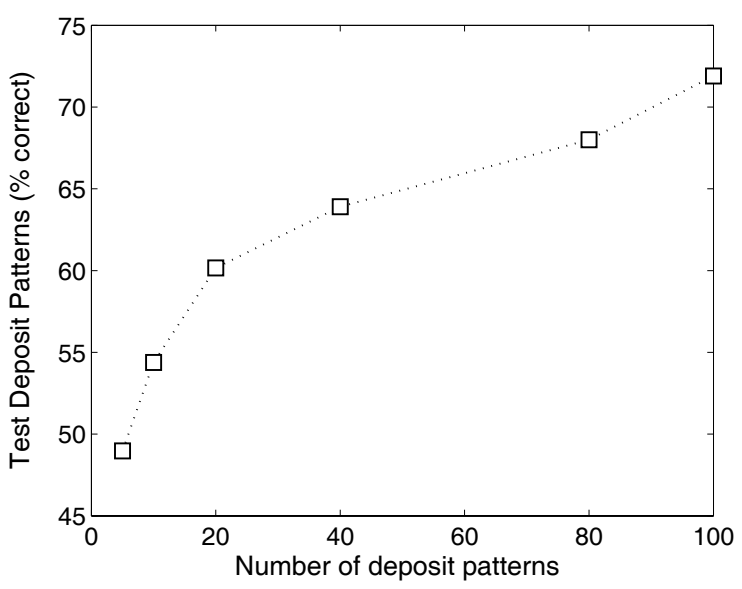

(b) Test-set classification: deposits

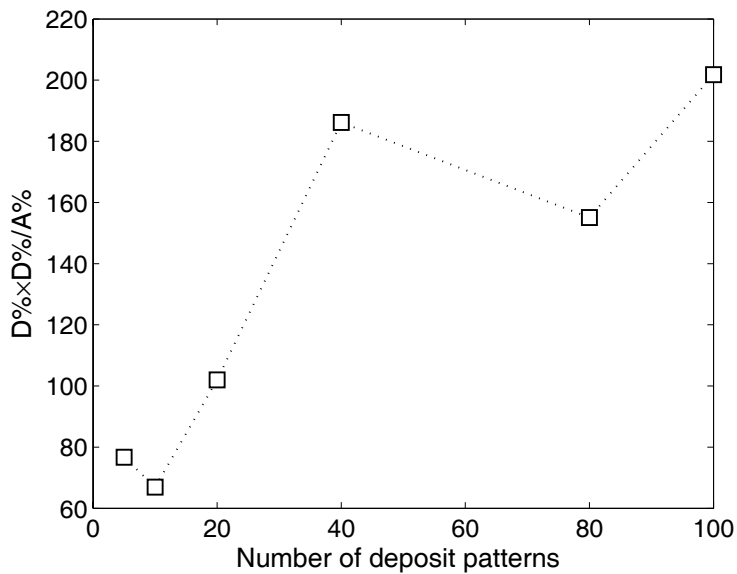

(d) Map quality statistic, $\mathrm{D} \times \mathrm{D} / \mathrm{A}$.

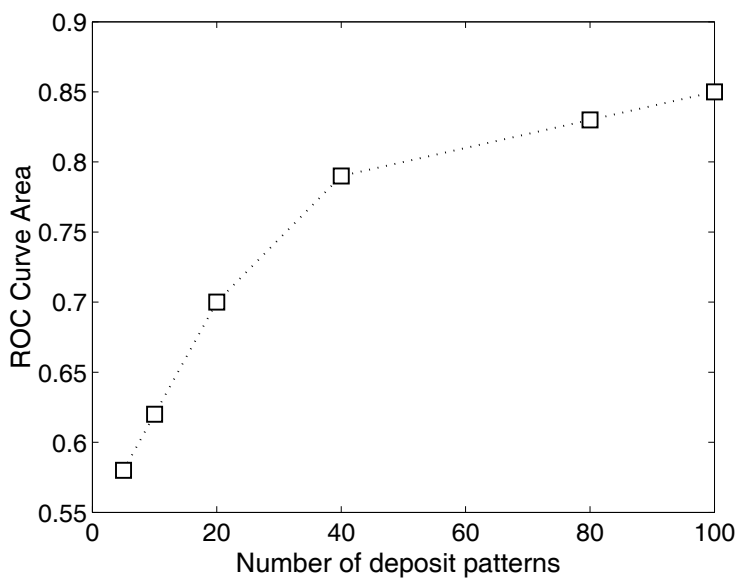

(f) Area under ROC curve.

Figure 7.5. Graphs of test set performance and map quality indices versus the number of deposit patterns in the training data set. Figures a) \& b) refer to classification of the independent test-set patterns by the trained network. Figures c)-f) are measures of the quality of the prospectivity map. Figures c) \& d) refer to the highest prospectivity class in the prospectivity maps, where D $=\%$ of total known deposits and $\mathrm{A}=\%$ of total map area. Figures e) \& f) are calculated using all areas in the map. All graphs show that the performance of the network increases with the number of deposit patterns. 
data should consist of these deposits.

This and the following section (Section 7.8) present two very different approaches to compensating for the paucity of deposit examples. The approach, described in this section involves a technique for increasing the number of deposit patterns available for training. The other approach is to add knowledge to an otherwise data-driven neural network method in the form of fuzzy-membership input layers.

\subsubsection{Previous Work}

A number of studies show that adding noise to the input data, outputs, weight connections, and weight changes can improve the generalization ability of trained networks (Sietsma \& Dow, 1991; Holmstrom \& Koistinen, 1992; Matsuoka, 1992; Clay \& Sequin, 1992; Krogh \& Hertz, 1992). An (1996) demonstrated that input noise is effective in improving the generalization performance for both regression (function approximation) and classification problems. Cho et al. (1998) described a method for generating "virtual training samples" and reported a $10 \%$ improvement in prediction performance for networks trained using a combination of virtual and real samples. Bishop (1995) shows that the addition of noise is equivalent to error regularization, and Reed et al. (1992, 1995) compare the effects of adding noise on the generalization performance to error regularization, sigmoid gain scaling (i.e. reducing the slope of the sigmoid function), and target smoothing. More recently, Wang \& Principe (1999) investigated the effect of injecting noise into the target output on learning speed and the ability to avoid local minima. Most papers concentrate on the effect of noise on the generalization ability.

Comparatively little work has been done on the composition of the training sets and how to deal with problems where the number of training examples representing different classes is significantly different. Parikh et al. (1999) studied the performance of a MLP classifier in a condition monitoring and fault diagnosis application, in which data representing normal operation are readily available compared to fault data. Such data are often difficult or very expensive to obtain. They showed that the highest overall network performance is obtained if roughly equal numbers of samples from the normal and fault classes are used for training. 


\subsubsection{Training Data Sets}

All input variables are scaled to the range $[0,1]$. As noted above, the requirement to have approximately equal numbers of training patterns from both deposit and barren classes means that training set size is limited by the number of known deposits. Since the GIS data are captured at a regional scale, only the 120 deposits with a total contained gold resource $>1000 \mathrm{~kg}$, together with an approximately equal number of barren patterns can be used to train and test the neural networks. The original training data (i.e. those without added noise) are created by randomly splitting the deposit patterns into three approximately equal groups; that is, training, training-stop and test sets. Deposits from each rock-type in the solid geology GIS layer are divided approximately equally between the three training sets. A number of barren patterns approximately equal to the deposit patterns is also randomly selected for each rock type. There are slightly more barren than deposit patterns because some rock types do not contain any known deposits. The composition of the training sets is shown in Table 7.24. Training was performed with cross validation and early stopping to avoid over-fitting the data.

Table 7.24. Number of patterns in the training sets in which the training set was expanded by adding random noise to the original training data patterns.

\begin{tabular}{|l|c|c|c||c|c|c||c|c|c|}
\hline \multirow{2}{*}{$\begin{array}{l}\text { Pattern } \\
\text { type }\end{array}$} & \multicolumn{3}{|l||}{ Original set } & \multicolumn{3}{|c||}{ Noise (train set) } & \multicolumn{3}{c|}{ Noise (train + stop sets) } \\
\cline { 2 - 10 } & Training & Stop & Test & Training & Stop & Test & Training & Stop & Test \\
\hline \hline Deposit & 46 & 39 & 35 & 1058 & 39 & 35 & 1058 & 897 & 35 \\
\hline Barren & 53 & 49 & 46 & 1053 & 49 & 46 & 1053 & 895 & 46 \\
\hline Total & 99 & 88 & 81 & 2111 & 88 & 81 & 2111 & 1792 & 81 \\
\hline
\end{tabular}

\subsubsection{Augmentation of Training Data set with Noise}

In order to augment the original training data sets, random noise is added to each component of the original deposit feature vectors in the training data set to create 22 new patterns from each original pattern. Random vectors, with a dimensionality equal to the number of inputs, are added repeatedly to each original feature vector in the training set (Fig. 7.6). This ratio of new to original patterns results in 1058 deposit patterns compared with 46 in the original training set. Matching numbers of new barren patterns were randomly selected from the map grid so that the numbers of deposit and barren patterns are approximately equal. Equal numbers of barren patterns are selected from each host-rock type (Table 7.24). 


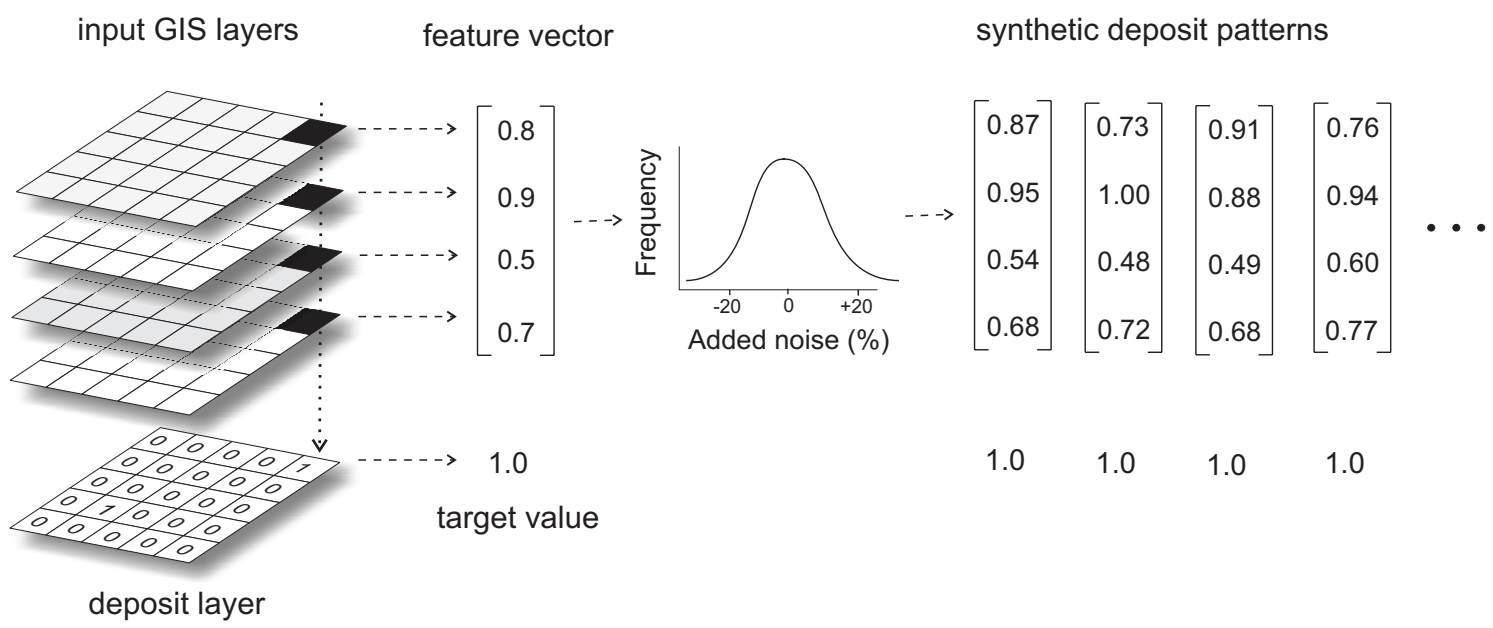

Figure 7.6. Schematic diagram of data processing steps used to augment the training data set through the addition of random noise to deposit patterns to create multiple new patterns. Twentytwo new deposit patterns were created from each original pattern to increase the number of patterns in the training set from 46 to 1058.

Training data sets were created with the MATLAB function add_noise_equal.m (see CDROM, Vol.1). For experiments to determine the optimum amount and type of noise, the training-stop and test data sets are not changed. In a final experiment, the training-stop data set is also expanded using additive noise.

\subsubsection{Network Topology for Noise-Augmented Training Sets}

This section shows the results of experiments to determine the optimum number of hidden layer units for networks trained with the noise-augmented training set. Since the noiseaugmented training data set is approximately twenty times greater than in the original training set, it is important to check if more hidden units are required to learn the patterns in the data set.

Tables 7.25 and 7.26 show that a 10-20-1 topology gave the highest correct-classification rates for the noise-augmented test set overall (2111 patterns) and for deposit patterns in the test set. This applies to both the average performance for 10 networks and to the single networks that gave the best overall test-set performance. Consequently, a 10-20-1 topology was used for all further noise experiments. The same number of hidden-layer units was found to give the best performance for the standard training data-set (99 patterns) in 
Section 7.3.3. The fact that the network topologies means that differences in performance are due to the different training-set sizes.

Table 7.25. Effect of MLP network topology on average classification accuracy \& map quality for large noise-augmented data sets. Noise: $\pm 10 \%$ uniform, training algorithm: trainlm, 10 input layers, layer selection criteria: categories.

\begin{tabular}{|l|l|l|l|l|l|c|c|c|c|}
\hline \multirow{2}{*}{$\begin{array}{c}\text { Network } \\
\text { Topology }\end{array}$} & \multicolumn{4}{|l|}{ Training Sets } & \multicolumn{4}{l|}{ Test Set } & \multicolumn{4}{l|}{ Map Quality Statistics } \\
\cline { 2 - 10 } & Train & Stop & All & Deps & Barren & $\rho$ & D/A & D*(D/A) & ROC \\
\hline \hline $10-0-1$ & 74.3 & 66.5 & 69.9 & 67.1 & 72.0 & 0.98 & 9.0 & 83.4 & 0.75 \\
\hline $10-10-1$ & 81.2 & 75.1 & 71.9 & 64.3 & 77.6 & 0.98 & 13.5 & 142.8 & 0.85 \\
\hline $10-15-1$ & 81.8 & 74.8 & 71.6 & 70.6 & 72.4 & 0.97 & 15.4 & 175.4 & 0.84 \\
\hline $10-20-1$ & 83.1 & 72.7 & 72.2 & 72.3 & 72.2 & 0.97 & 13.7 & 160.5 & 0.85 \\
\hline $10-25-1$ & 83.1 & 73.0 & 70.1 & 65.7 & 73.5 & 0.98 & 18.5 & 152.3 & 0.85 \\
\hline $10-50-1$ & 84.5 & 71.4 & 70.6 & 66.0 & 74.1 & 0.97 & 13.4 & 165.7 & 0.84 \\
\hline
\end{tabular}

Table 7.26. Effect of MLP network topology on best classification accuracy \& map quality for large noise-augmented data sets. Noise: $\pm 10 \%$ uniform, training algorithm: trainlm, 10 input layers, layer selection criteria: categories.

\begin{tabular}{|l|l|l|l|l|l|c|c|c|c|}
\hline \multirow{2}{*}{$\begin{array}{c}\text { Network } \\
\text { Topology }\end{array}$} & \multicolumn{4}{|l|}{ Training Sets } & \multicolumn{4}{l|}{ Test Set } & \multicolumn{4}{|c|}{ Map Quality Statistics } \\
\cline { 2 - 11 } & Train & Stop & All & Deps & Barren & $\rho$ & D/A & D*(D/A) & ROC \\
\hline \hline $10-0-1$ & 74.0 & 77.3 & 71.6 & 68.6 & 73.9 & 0.98 & 14.7 & 441.2 & 0.80 \\
\hline $10-10-1$ & 85.2 & 76.1 & 76.5 & 57.1 & 91.3 & 0.98 & 9.7 & 48.7 & 0.84 \\
\hline $10-15-1$ & 85.5 & 73.9 & 75.3 & 68.6 & 80.4 & 0.95 & 6.1 & 20.2 & 0.86 \\
\hline $10-20-1$ & 84.0 & 75.0 & 76.5 & 80.0 & 73.9 & 0.98 & 9.7 & 96.6 & 0.86 \\
\hline $10-25-1$ & 86.6 & 70.5 & 75.3 & 74.3 & 76.1 & 0.98 & 20.5 & 68.3 & 0.86 \\
\hline $10-50-1$ & 92.0 & 72.7 & 77.8 & 74.3 & 80.4 & 0.92 & 7.2 & 29.8 & 0.87 \\
\hline
\end{tabular}

\subsubsection{Optimum Amount of Noise}

Experiments were performed using $\pm 5, \pm 10 \pm 20 \pm 40$ and $\pm 80 \%$ noise in an attempt to determine the optimal amount of noise. Two different types of noise, uniform random and normally-distributed or Gaussian random noise, were also tested. In the case of uniform random noise, the percentages represent the maximum amount added or subtracted. For normally-distributed noise, the standard deviation was set to a fraction of the variable 
value represented by the percentage. The mean was set to the value of the component in the original feature vector.

Tables 7.27 and 7.28 show the results of experiments using various amounts of uniform random noise. The same network topology (10-20-1) and set of initial weights was used for each of the 10 networks trained for each noise level. Each row in Table 7.27 shows the average results for 10 networks, whereas Table 7.28 shows the network which gives the best overall test-set performance. In both tables, the results for the original training set without added noise is shown in the first row.

The average results (Table 7.27) show that the increased training data-set size, due to the addition of noise, results in a significant improvement in generalization and most measures of map quality. The percentage classification of the test set, both capture efficiency ratios, $D / A, D \times(D / A)$ and the area under the ROC, are significantly increased. The average values of $D / A$ and $D \times(D / A)$ are approximately doubled. The results for $40 \%$ noise represent a very significant improvement in the percentage correct classification for deposit patterns (i.e. from $68.0 \%$ to $77.1 \%$ ), although, for most other noise levels, there is only a slight improvement.

The best test-set results (Table 7.28) vary considerably with different amounts of added noise. In many cases, the classification performance for barren cells and the $D \times(D / A)$ statistic are poorer than for the network trained without added noise (top row in Table 7.28). However, the results for $\rho, D / A$ and the area under the ROC are significantly improved and the correct classification of test-set deposit patterns for the $40 \%$ noise level show a similarly large improvement as displayed in the average results (i.e. from $77.1 \%$ to 85.7\%). A notable feature of the average results is that there appears to be a weak inverse relationship between correct classification performance for deposit and barren patterns in the test set (see for example the results for $\pm 20 \%$ and $\pm 40 \%$ in Table 7.27 ).

Examples of mineral prospectivity maps for part of the Kalgoorlie Terrane, Western Australia, showing the potential for orogenic lode-gold deposits, are given in Figures 7.7-7.8. The maps were created using a 10-20-1 MLP neural network, which was trained with a training data set expanded from 46 to 1058 deposit patterns through the addition of $\pm 40 \%$ and $\pm 80 \%$ uniform random noise. The effect of training the network with patterns created with $\pm 80 \%$, rather than $\pm 40 \%$, noise is to reduce the size of areas estimated to have high prospectivity. A similar difference exists between maps which were produced using $\pm 5 \%$ and $\pm 10 \%$ normally-distributed noise (Figs $7.9 \& 7.10$, respectively). For comparison, the neural network with 10 inputs (layer selection criteria:categories), which 
was trained without noise and which gave the best overall test-set performance, is shown in Figure 7.15.

Table 7.27. Effect of the amount of uniform random noise used to augment the training data set on average performance. Network topology: 10-20-1, training algorithm: trainlm, layer selection criteria: categories.

\begin{tabular}{|c|l|l|l|l|l|l|l|l|l|}
\hline \multirow{2}{*}{$\begin{array}{c}\text { Noise } \\
\text { max.(\%) }\end{array}$} & \multicolumn{2}{|l|}{ Training Sets } & \multicolumn{4}{l|}{ Test Set } & \multicolumn{4}{|c|}{ Map Quality Statistics } \\
\cline { 2 - 10 } & Train & Stop & All & Deps & Barren & $\rho$ & D/A & D*(D/A) & ROC \\
\hline \hline \pm 0 & 84.4 & 72.7 & 67.9 & 68.0 & 67.8 & 0.97 & 8.2 & 105.3 & 0.79 \\
\hline \pm 5 & 83.3 & 74.1 & 74.1 & 70.3 & 77.0 & 0.98 & 17.3 & 202.2 & 0.86 \\
\hline \pm 10 & 83.4 & 74.1 & 72.4 & 71.4 & 73.0 & 0.97 & 15.3 & 178.8 & 0.85 \\
\hline \pm 20 & 85.2 & 74.6 & 71.6 & 66.6 & 75.4 & 0.97 & 18.9 & 237.5 & 0.85 \\
\hline \pm 40 & 82.5 & 73.2 & 72.8 & 77.1 & 69.6 & 0.99 & 17.9 & 226.4 & 0.87 \\
\hline \pm 80 & 86.5 & 73.9 & 71.5 & 68.9 & 73.5 & 0.98 & 14.6 & 188.2 & 0.84 \\
\hline
\end{tabular}

Table 7.28. Effect of the amount of uniform random noise used to augment the training data set on best performance. Network topology: 10-20-1, training algorithm: trainlm, layer selection criteria: categories.

\begin{tabular}{|c|l|l|l|l|l|r|r|r|r|}
\hline \multirow{2}{*}{$\begin{array}{c}\text { Noise } \\
\text { max.(\%) }\end{array}$} & \multicolumn{4}{|l|}{ Training Sets } & \multicolumn{4}{l|}{ Test Set } & \multicolumn{4}{|c|}{ Map Quality Statistics } \\
\cline { 2 - 10 } & Train & Stop & All & Deps & Barren & $\rho$ & D/A & D*(D/A) & ROC \\
\hline \hline \pm 0 & 88.9 & 72.7 & 76.5 & 77.1 & 76.1 & 0.90 & 9.6 & 256.7 & 0.82 \\
\hline \pm 5 & 81.3 & 80.7 & 79.0 & 74.3 & 82.6 & 0.98 & 13.2 & 164.8 & 0.89 \\
\hline \pm 10 & 84.0 & 75.0 & 76.5 & 80.0 & 73.9 & 0.98 & 9.7 & 96.6 & 0.86 \\
\hline \pm 20 & 82.7 & 75.0 & 76.5 & 80.0 & 73.9 & 0.95 & 16.7 & 152.7 & 0.82 \\
\hline \pm 40 & 82.9 & 75.0 & 76.5 & 85.7 & 69.6 & 1.00 & 14.4 & 216.0 & 0.90 \\
\hline \pm 80 & 92.9 & 79.6 & 80.3 & 74.3 & 84.8 & 0.97 & 11.0 & 247.3 & 0.86 \\
\hline
\end{tabular}


Table 7.29. Effect of the amount of normally-distributed random noise used to augment the training data set on average performance. Network topology: 10-20-1, training algorithm: trainlm, layer selection criteria: categories.

\begin{tabular}{|c|l|l|l|l|l|l|l|l|l|}
\hline \multirow{2}{*}{$\begin{array}{c}\text { Noise } \\
\text { max.(\%) }\end{array}$} & \multicolumn{4}{|l|}{ Training Sets } & \multicolumn{4}{l|}{ Test Set } & \multicolumn{4}{|c|}{ Map Quality Statistics } \\
\cline { 2 - 10 } & Train & Stop & All & Deps & Barren & $\rho$ & D/A & D*(D/A) & ROC \\
\hline \hline \pm 0 & 84.4 & 72.7 & 67.9 & 68.0 & 67.8 & 0.97 & 8.2 & 105.3 & 0.79 \\
\hline \pm 5 & 83.1 & 72.8 & 72.8 & 71.4 & 73.9 & 0.94 & 12.9 & 143.0 & 0.84 \\
\hline \pm 10 & 83.4 & 73.6 & 73.8 & 66.9 & 79.1 & 0.92 & 13.7 & 131.2 & 0.86 \\
\hline \pm 20 & 84.3 & 75.0 & 73.1 & 69.7 & 75.7 & 0.93 & 17.9 & 321.5 & 0.87 \\
\hline \pm 40 & 84.2 & 77.1 & 71.2 & 68.6 & 73.3 & 0.93 & 13.1 & 149.8 & 0.84 \\
\hline \pm 80 & 84.8 & 67.4 & 64.7 & 47.7 & 77.6 & 0.86 & 19.1 & 109.9 & 0.76 \\
\hline
\end{tabular}

Table 7.30. Effect of the amount of normally-distributed random noise used to augment the training data set on best performance. Network topology: 10-20-1, training algorithm: trainlm, layer selection criteria: categories.

\begin{tabular}{|c|l|l|l|l|l|r|r|r|l|}
\hline \multirow{2}{*}{$\begin{array}{c}\text { Noise } \\
\text { max.(\%) }\end{array}$} & \multicolumn{4}{|l|}{ Training Sets } & \multicolumn{4}{l|}{ Test Set } & \multicolumn{4}{|c|}{ Map Quality Statistics } \\
\cline { 2 - 10 } & Train & Stop & All & Deps & Barren & $\rho$ & D/A & D*(D/A) & ROC \\
\hline \hline \pm 0 & 88.9 & 72.7 & 76.5 & 77.1 & 76.1 & 0.90 & 9.6 & 256.7 & 0.82 \\
\hline \pm 5 & 83.9 & 77.3 & 79.0 & 85.7 & 73.9 & 0.97 & 19.0 & 269.0 & 0.87 \\
\hline \pm 10 & 87.5 & 71.6 & 80.3 & 80.0 & 80.4 & 0.98 & 12.0 & 130.4 & 0.85 \\
\hline \pm 20 & 88.4 & 78.4 & 77.8 & 82.9 & 73.9 & 1.00 & 13.2 & 439.7 & 0.90 \\
\hline \pm 40 & 85.7 & 78.4 & 77.8 & 82.9 & 73.9 & 0.98 & 8.9 & 110.8 & 0.87 \\
\hline \pm 80 & 86.8 & 68.2 & 69.1 & 62.9 & 73.9 & 0.97 & 16.3 & 81.6 & 0.76 \\
\hline
\end{tabular}




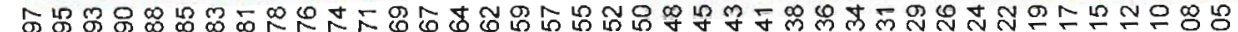

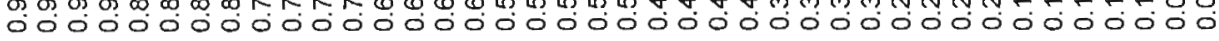

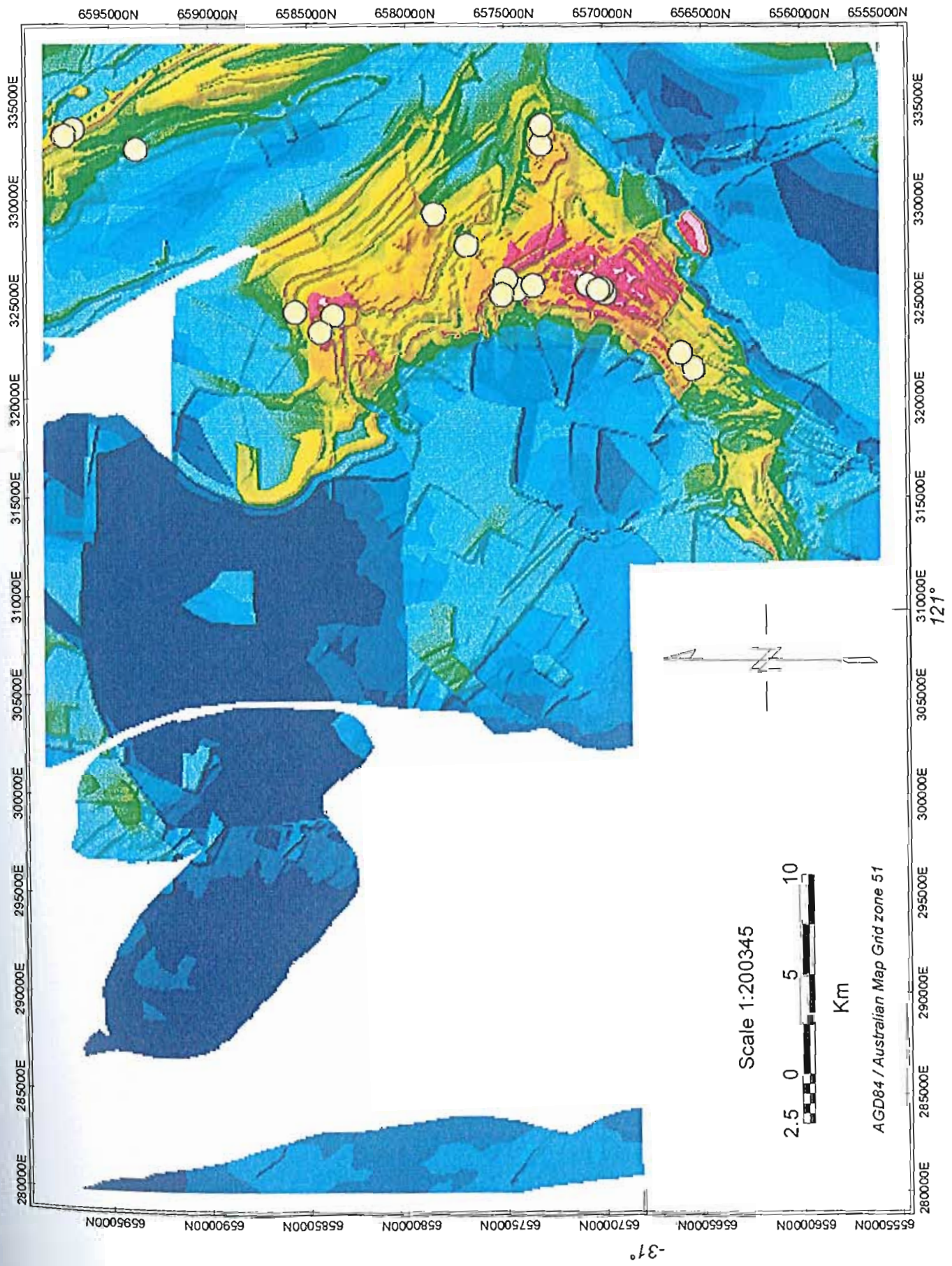

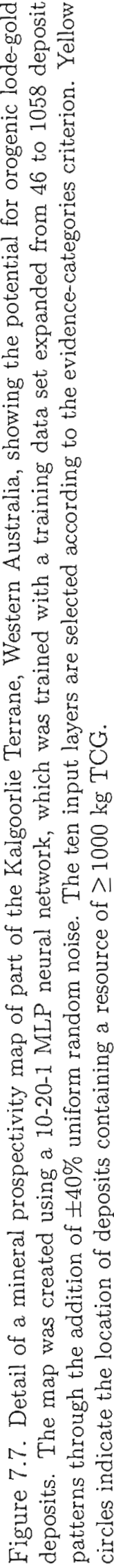




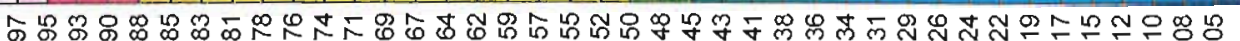

000000000000000000000000000000000000000
$6595000 \mathrm{~N} \quad 6590000 \mathrm{~N}$

$6585000 \mathrm{~N}$

$6580000 \mathrm{~N}$

$6575000 \mathrm{~N}$

$6570000 \mathrm{~N}$

$6565000 \mathrm{~N}$

$6560000 \mathrm{~N} \quad 6555000 \mathrm{~N}$

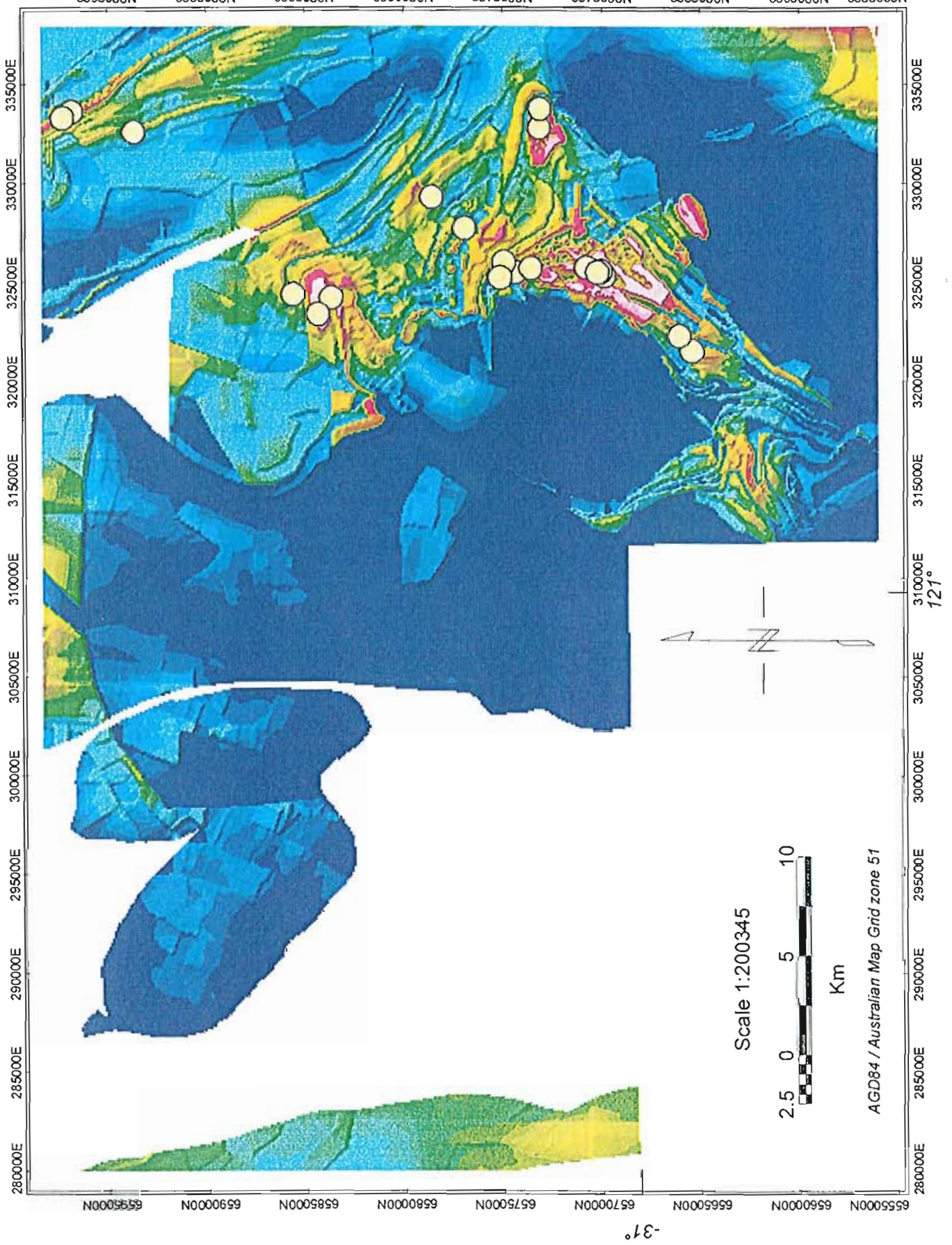

믕 용

of

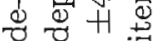

0 它

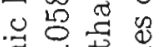

苟南古

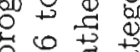

邹

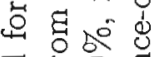

$\pi$

?

di

品落,

类 80

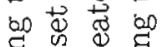

\%

की

- bo 8

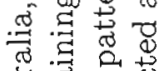

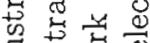

त 0

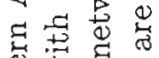

曹

3 菧吉㲾

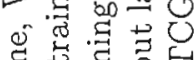

तु

氙蛋

स 4 क $\$$

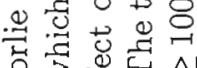

○出

क्ष

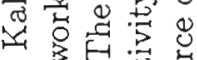

\&

虫

可

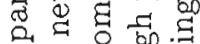

岁 म

它密要

茫密

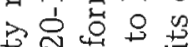

号宫

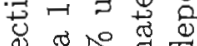

की no 5 प्र

参骂市苍

正然.

त्ञ

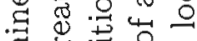

छ्व 0 :

๙ 30

岁满。

$=0 \frac{1}{4} \frac{\pi}{4}$

苍范

ค 흘

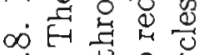

人 को

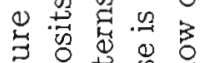

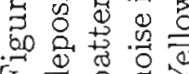




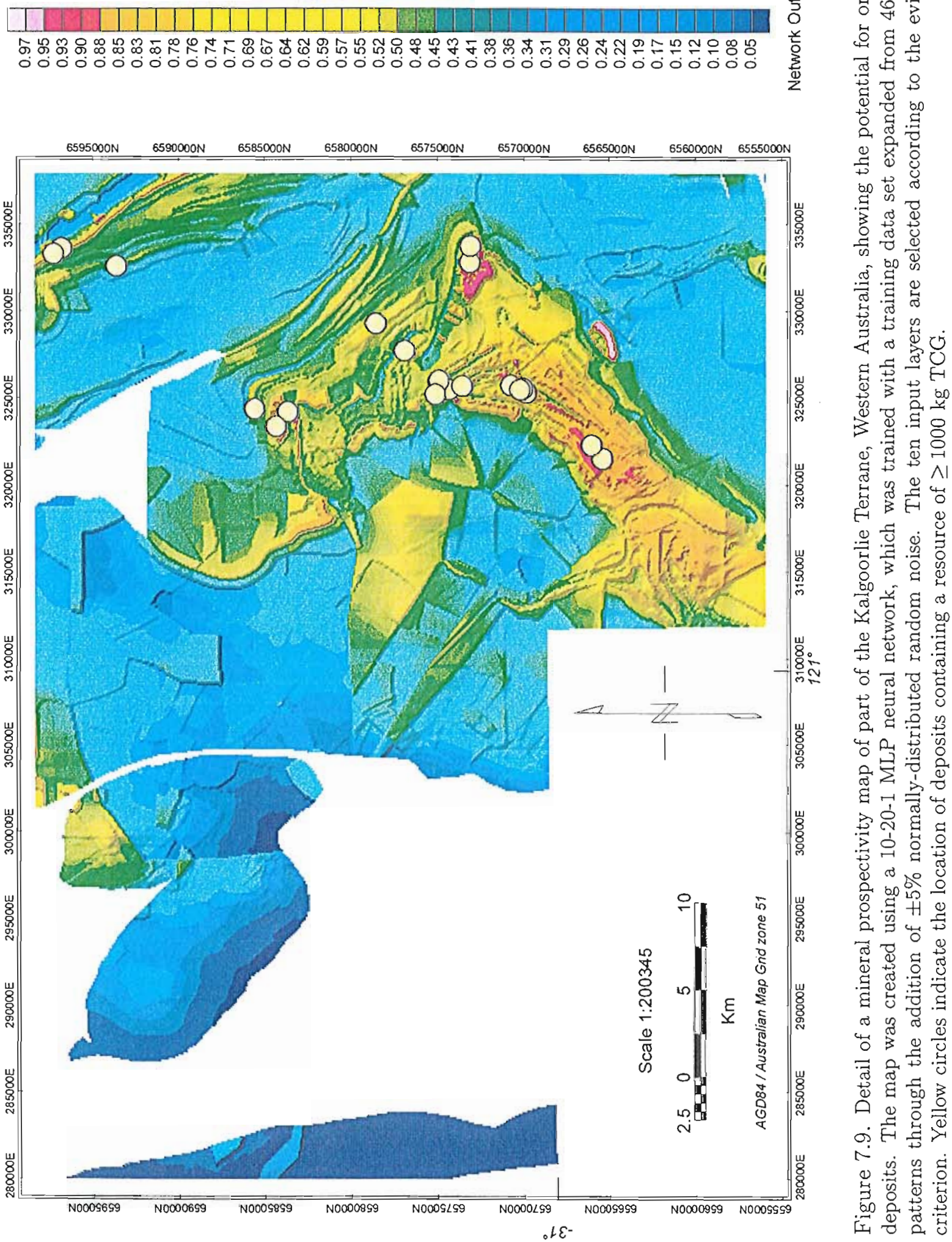




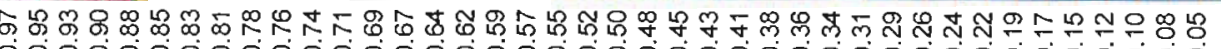

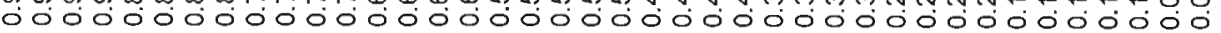

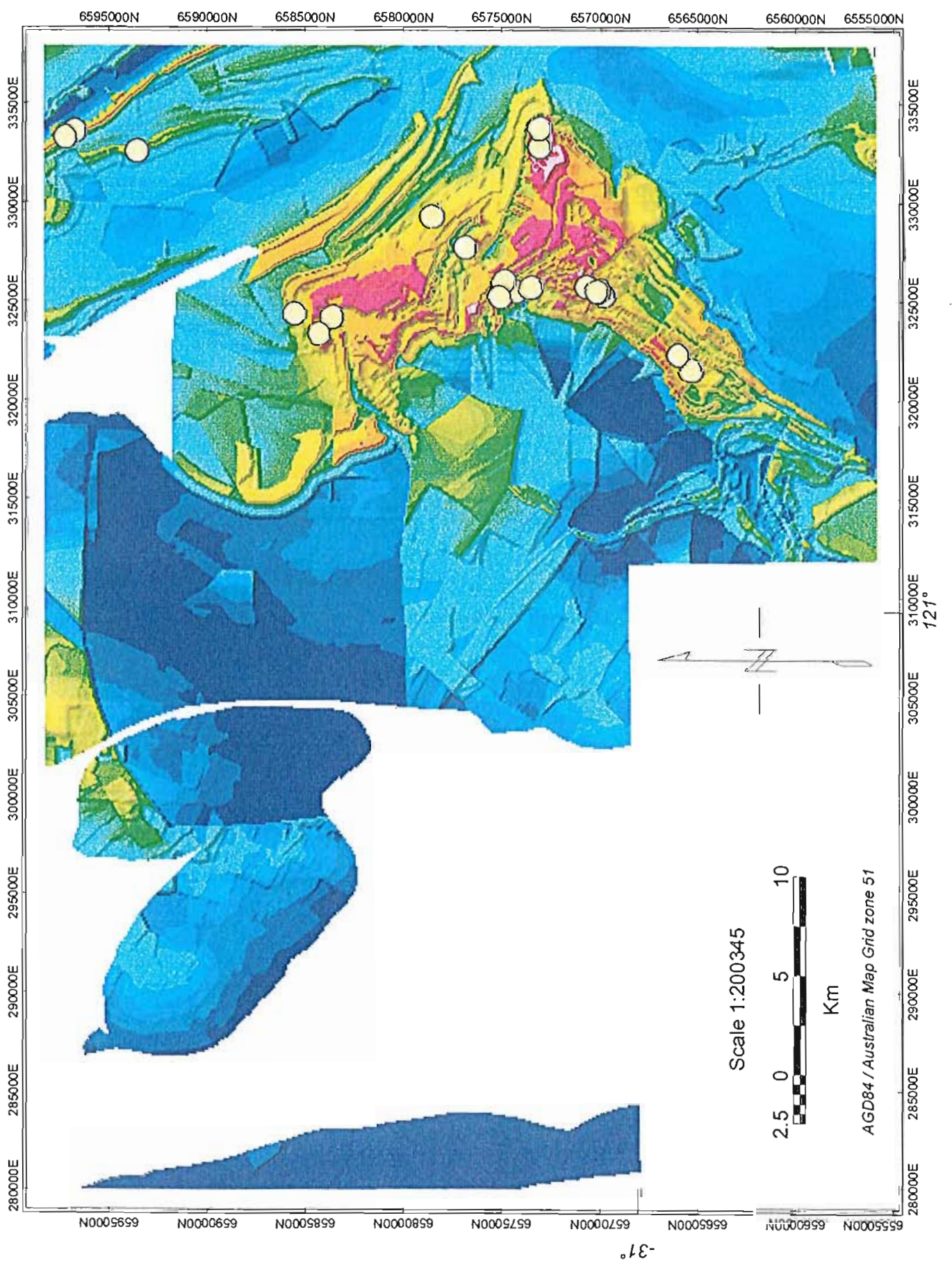

궁 60 完し

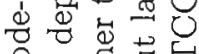
$\odot \infty$ 击

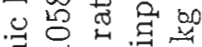




\subsubsection{Uniform and Normally-Distributed Random Noise}

The experiments described above are repeated to check the effect of using normallydistributed rather than uniform random noise (Tables $7.31 \& 7.32$ ). The addition of normally-distributed noise improves the average overall test-set performance as well as measures of map quality, $D / A, D \times(D / A)$, and the area under the ROC curve. However, the percentage correct classification for test-set deposit patterns, and the correlation between probability of a deposit and increasing prospectivity map class (Spearman's $\rho$ ), are generally poorer than the results obtained without using noise (Table 7.31). An exception is for $\pm 5 \%$ noise, where the correct classification rate for deposit patterns increases from $68.0 \%$ to $71.4 \%$.

To allow a comparison between uniform and normally-distributed noise, results from the previous Tables, 7.27, 7.28, and 7.29 and 7.30, have been combined into Tables 7.31 and 7.32 , respectively.

Despite the fact that the average results for normally-distributed noise are generally poorer than those for uniform random noise, the results for the networks with the best test-set classification performance using $\pm 5 \%, \pm 10 \%$ and $\pm 20 \%$ normally-distributed noise are slightly better than those for uniform noise. The network with $\pm 10 \%$ normally-distributed noise gave the best combination of overall test-set and test-set deposit pattern classification performance of all the networks shown in Tables 7.27-7.30.

\subsubsection{Use of Noise-Augmented Training and Training-Stop Sets}

Although the patterns in the training data set must provide the network with a representative sample of the variation in the data population that the trained network will be required to process, the training-stop data set also determines the generalization ability of the trained network. In this last experiment, the same procedure used to augment the number of deposit patterns is applied to the training-stop data set, so that the size of both the training and training-stop sets are increased. Training data sets were created with the MATLAB function add_noise_equal_bigVV.m (see CD-ROM, Vol.1). The composition of the training-data sets is shown in Table 7.24.

The noise levels giving the best test-set performance for using uniform and normallydistributed random noise are repeated with both noise-augmented training and trainingstop data sets. Ten networks are trained using $\pm 40 \%$ uniform random noise and $\pm 10 \%$ normally-distributed noise. The results are shown in Tables 7.33 and 7.34. For $\pm 40 \%$ 
Table 7.31. Comparison of uniform and normally-distributed random noise showing the average test-data-set performance. Network topology: 10-20-1, training algorithm: trainlm, layer selection criteria: categories.

\begin{tabular}{|l|l|l|l|l|l|l|l|l|l|}
\hline \multirow{2}{*}{$\begin{array}{l}\text { Noise } \\
\text { max.(\%) }\end{array}$} & \multicolumn{3}{|l|}{ Training Sets } & \multicolumn{3}{l|}{ Test Set } & \multicolumn{3}{l|}{ Map Quality Statistics } \\
\cline { 2 - 10 } & Train & Stop & All & Deps & Barren & $\rho$ & D/A & D*(D/A) & ROC \\
\hline \hline \pm 5 uni. & 83.3 & 74.1 & 74.1 & 70.3 & 77.0 & 0.98 & 17.3 & 202.2 & 0.86 \\
\pm 5 norm. & 83.1 & 72.8 & 72.8 & 71.4 & 73.9 & 0.94 & 12.9 & 143.0 & 0.84 \\
\hline \pm 10 uni. & 83.4 & 74.1 & 72.4 & 71.4 & 73.0 & 0.97 & 15.3 & 178.8 & 0.85 \\
\pm 10 norm. & 83.4 & 73.6 & 73.8 & 66.9 & 79.1 & 0.92 & 13.7 & 131.2 & 0.86 \\
\hline \pm 20 uni. & 85.2 & 74.6 & 71.6 & 66.6 & 75.4 & 0.97 & 18.9 & 237.5 & 0.85 \\
\pm 20 norm. & 84.3 & 75.0 & 73.1 & 69.7 & 75.7 & 0.93 & 17.9 & 321.5 & 0.87 \\
\hline \pm 40 uni. & 82.5 & 73.2 & 72.8 & 77.1 & 69.6 & 0.99 & 17.9 & 226.4 & 0.87 \\
\pm 40 norm. & 84.2 & 77.1 & 71.2 & 68.6 & 73.3 & 0.93 & 13.1 & 149.8 & 0.84 \\
\hline \pm 80 uni. & 86.5 & 73.9 & 71.5 & 68.9 & 73.5 & 0.98 & 14.6 & 188.2 & 0.84 \\
\pm 80 norm. & 84.8 & 67.4 & 64.7 & 47.7 & 77.6 & 0.86 & 19.1 & 109.9 & 0.76 \\
\hline
\end{tabular}

Table 7.32. Comparison of uniform and normally-distributed random noise showing the results for the networks with the best test-data-set performance. Network topology: 10-20-1, training algorithm: trainlm, layer selection criteria: categories.

\begin{tabular}{|l|l|l|l|l|l|r|r|r|l|}
\hline \multirow{2}{*}{$\begin{array}{l}\text { Noise } \\
\text { max.(\%) }\end{array}$} & \multicolumn{4}{|l|}{ Training Sets } & \multicolumn{4}{l|}{ Test Set } & \multicolumn{4}{l|}{ Map Quality Statistics } \\
\cline { 2 - 10 } & Train & Stop & All & Deps & Barren & $\rho$ & D/A & D*(D/A) & ROC \\
\hline \hline \pm 5 uni. & 81.3 & 80.7 & 79.0 & 74.3 & 82.6 & 0.98 & 13.2 & 164.8 & 0.89 \\
\pm 5 norm. & 83.9 & 77.3 & 79.0 & 85.7 & 73.9 & 0.97 & 19.0 & 269.0 & 0.87 \\
\hline \pm 10 uni. & 84.0 & 75.0 & 76.5 & 80.0 & 73.9 & 0.98 & 9.7 & 96.6 & 0.86 \\
\pm 10 norm. & 87.5 & 71.6 & 80.3 & 80.0 & 80.4 & 0.98 & 12.0 & 130.4 & 0.85 \\
\hline \pm 20 uni. & 82.7 & 75.0 & 76.5 & 80.0 & 73.9 & 0.95 & 16.7 & 152.7 & 0.82 \\
\pm 20 norm. & 88.4 & 78.4 & 77.8 & 82.9 & 73.9 & 1.00 & 13.2 & 439.7 & 0.90 \\
\hline \pm 40 uni. & 82.9 & 75.0 & 76.5 & 85.7 & 69.6 & 1.00 & 14.4 & 216.0 & 0.90 \\
\pm 40 norm. & 85.7 & 78.4 & 77.8 & 82.9 & 73.9 & 0.98 & 8.9 & 110.8 & 0.87 \\
\hline \pm 80 uni. & 92.9 & 79.6 & 80.3 & 74.3 & 84.8 & 0.97 & 11.0 & 247.3 & 0.86 \\
\pm 80 norm. & 86.8 & 68.2 & 69.1 & 62.9 & 73.9 & 0.97 & 16.3 & 81.6 & 0.76 \\
\hline
\end{tabular}


uniform noise, there is a small improvement in the percentage of correct classifications for the test-set (overall, deposit and barren patterns) and in the capture-efficiency statistic, $D \times(D / A)$. In the case of the normally-distributed noise, there is a small increase in the overall test-set performance and correlation coefficient $(\rho)$, and a large increase in the classification performance for deposit patterns and both capture-efficiency statistics $D / A$ and $D \times(D / A)$. There is no increase in the best test-set results for either uniform or normally-distributed noise

Table 7.33. Effect of expanding the stop data set in addition to the training set using random noise: average results. Network topology: 10-20-1, training algorithm: trainlm, layer selection criteria: categories.

\begin{tabular}{|l|l|l|l|l|l|l|l|l|l|}
\hline \multirow{2}{*}{$\begin{array}{l}\text { Network } \\
\text { Topology }\end{array}$} & \multicolumn{3}{|l|}{ Training Sets } & \multicolumn{3}{l|}{ Test Set } & \multicolumn{4}{l|}{ Map Quality Statistics } \\
\cline { 2 - 10 } & Train & Stop & All & Deps & Barren & $\rho$ & D/A & D*(D/A) & ROC \\
\hline \hline \pm 40, uniform & 82.5 & 73.2 & 72.8 & 77.1 & 69.6 & 0.99 & 17.9 & 226.4 & 0.87 \\
\pm 40, uni.,lge stop & 85.9 & 77.8 & 73.5 & 77.4 & 70.4 & 0.98 & 14.7 & 296.2 & 0.87 \\
\hline \pm 10, normal & 83.4 & 73.6 & 73.8 & 66.9 & 79.1 & 0.92 & 13.7 & 131.2 & 0.86 \\
\pm 10, nml.,lge stop & 86.2 & 76.4 & 74.1 & 76.6 & 72.2 & 0.98 & 15.0 & 245.6 & 0.87 \\
\hline
\end{tabular}

Table 7.34. Effect of expanding the stop data set in addition to the training set using random noise: best results. Network topology: 10-20-1, training algorithm: trainlm, layer selection criteria: categories.

\begin{tabular}{|l|l|l|l|l|l|l|l|l|l|}
\hline \multirow{2}{*}{$\begin{array}{l}\text { Network } \\
\text { Topology }\end{array}$} & \multicolumn{3}{|l|}{ Training Sets } & \multicolumn{4}{l|}{ Test Set } & \multicolumn{4}{l|}{ Map Quality Statistics } \\
\cline { 2 - 10 } & Train & Stop & All & Deps & Barren & $\rho$ & D/A & D $*(\mathrm{D} / \mathrm{A})$ & ROC \\
\hline \hline \pm 40, uniform & 82.9 & 75.0 & 76.5 & 85.7 & 69.6 & 1.00 & 14.4 & 216.0 & 0.90 \\
\pm 40, uni.,lge stop & 82.9 & 79.8 & 76.5 & 85.7 & 69.6 & 1.00 & 14.4 & 216.0 & 0.90 \\
\hline \pm 10, normal & 87.5 & 71.6 & 80.3 & 80.0 & 80.4 & 0.98 & 12.0 & 130.4 & 0.85 \\
\pm 10, nml.,lge stop & 87.5 & 76.7 & 80.3 & 80.0 & 80.4 & 0.98 & 12.0 & 130.4 & 0.85 \\
\hline
\end{tabular}

\subsubsection{Conclusions: Augmentation of Training Data with Random Noise}

The use of random noise to create additional synthetic deposit-patterns helps overcome the lack of deposit patterns that limits the training set sizes for neural networks trained using a backpropagation training algorithm. In particular, the following results are obtained: 
1. Training with the larger noise-augmented data sets results in significantly increased classification accuracy for the test set (overall) and test-set deposit patterns, area under the ROC curve and capture efficiency $(D / A$ and $D \times(D / A))$ statistics.

2. The best results are obtained using an MLP network with a 10-20-1 topology and $\pm 40 \%$ noise.

3. Uniform random noise generally gives better average results than normally-distributed noise.

4. Networks with best overall test-set and test-set deposit classification performance are $\pm 5, \pm 10 \& \pm 20$.

5. Increasing the amount of uniform random noise from $\pm 40 \%$ to $\pm 80 \%$ reduces the size of areas estimated to have high prospectivity.

6. Increasing the size of the training-stop data set yields a small increase in average overall test-set and test-set deposit-pattern classification performance.

The method described here could be applied to increase the number of patterns available for training in other applications in which training data are rare, difficult or expensive to obtain.

\subsection{Input Data as Fuzzy-Membership Values}

Different approaches to mineral prospectivity are needed depending on the type of exploration area and the availability and reliability of data compared to geological knowledge available. In well-explored areas where there are large amounts of data, statistical methods like weights of evidence and neural networks are appropriate (Fig. 7.11). In poorlyexplored areas, containing few known deposits and where little reliable data are available, a purely subjective method like fuzzy systems is appropriate (An et al., 1991; BonhamCarter, 1994; Eddy et al., 1995; Wyborn et al., 1995b). Ideally, lessons learned from mature exploration areas should be applied to new areas and, conversely, new conceptual models or exploration criteria need to be applied to well-explored areas. Woodall (1994) notes that exploration success depends on the correct blend of empiricism and conceptual thinking. Fuzzy weights of evidence and hybrid fuzzy-neural networks are examples of systems which combine elements from both conceptual and empirical approaches (Fig. 7.11). The fuzzy-neural system represents a hybrid architecture that combines the ability of fuzzy systems to deal with imprecise or error-prone inputs with the pattern recognition 
capability of neural networks. This enables conceptual and empirical approaches to be combined by incorporating subjective geological knowledge into an otherwise data-driven neural network method.

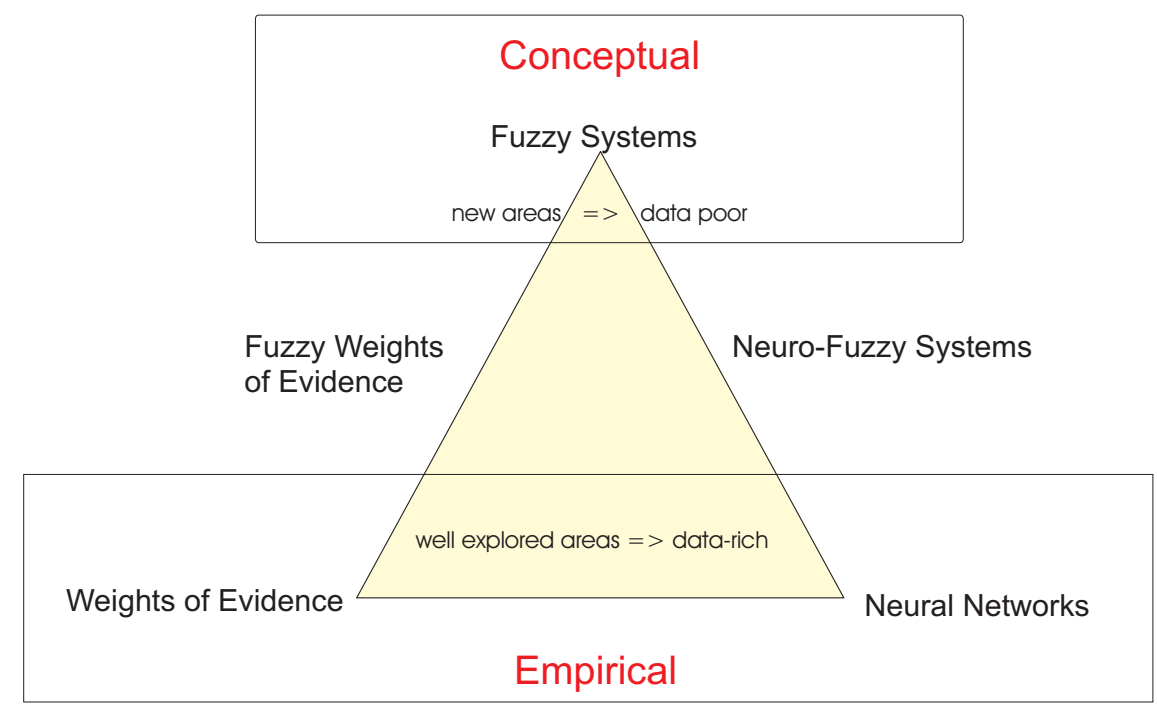

Figure 7.11. Relationship of hybrid fuzzy-neural-network systems and fuzzy weights-of-evidence approach to main empirical and conceptual methods for mineral prospectivity mapping. Both weights-of-evidence and neural network methods require large amounts of data, commonly available in well-explored areas, whereas fuzzy systems, which incorporate geological knowledge, are more suited to new areas where few data are available.

Since the early 1990's, there has been considerable interest in methods of combining neural network and fuzzy-logic approaches. Much of the research has been directed towards solving complex non-linear control problems in engineering (Jang \& Sun, 1995; Kasabov, 1996). The reason for combining the two methods is that they appear to complement one another. Whilst both methods can deal with uncertain and imprecise inputs, neural networks represent a data-driven approach whereas fuzzy systems represent a conceptual approach. Neural networks can detect very complex patterns in data, but fuzzy systems are able to incorporate expert knowledge, which neural networks cannot do directly. The benefits of combining the two approaches have been demonstrated in a number of studies. Wong et al. (1998) used a subjective geological layer as an input to a neural network to interpolate porosity data obtained from well-sites over the area of a fluvial sandstone oil reservoir. They showed that the addition of the subjective geological knowledge significantly improved the quality of porosity estimates. Huang et al. (1996) used a fuzzy neural network to estimate permeability in a petroleum reservoir and showed that the fuzzy neural network resulted in faster convergence, and a lower test-set error, than an MLP network which was trained using the backpropagation algorithm. Three main ways of com- 
bining neural networks and fuzzy systems are described in the literature (Gupta \& Rao, 1994; Jang \& Sun, 1995; Kasabov, 1996). These are 1) fuzzy neurons, 2) multi-modular hybrid neural network-fuzzy systems, and 3) adaptive network-based fuzzy inference systems (ANFIS). This study demonstrates a very simple form of hybrid fuzzy-neural-network system system of the second type.

Using GIS layers, in which the cell values represent fuzzy membership values, is one method of combining subjective geological knowledge with empirical data in a neuralnetwork approach to mineral prospectivity mapping. In this section, this approach to forming a simple hybrid fuzzy-neural networks system is investigated and compared with the results for an MLP neural network. The hybrid systems are used to combine up to 17 GIS layers to predict the potential for orogenic gold deposits.

Two types of fuzzy membership layers are used in this study. In the first type of layer, statistical relationships between known gold deposits and the variable in the GIS thematic layer are used to determine the fuzzy membership values. For example, GIS layers depicting solid geology and rock-type combination at the nearest type of lithological boundary for each cell (i.e. categorical data) are converted to fuzzy membership layers representing favourable lithologies and favourable lithological boundaries. In the second type of fuzzy membership layer, the assignment of fuzzy membership values, although based on geological field data, is subjective. The most effective layer representing rheological contrast at the nearest lithological boundary is modelled in this way. In each of the layers, the fuzzy membership values represent the degree to which the raw variable values belong to the fuzzy set favourable for orogenic lode gold deposits.

\subsubsection{Objective Fuzzy-Membership Layers}

The solid geology and contact-type layers are examples of objective fuzzy membership layers because they are converted to fuzzy membership values using the statistical association between the classes and known deposits (containing $\geq 1000 \mathrm{~kg}$ TCG).

Two fuzzy membership layers are created; one where fuzzy membership values are based on ranking rock types according to the probability of at least one deposit per cell and a second layer based on ranking rock types according to $P(D) \times T C G /$ cell.

The two favourable host-rock layers are compared using capture efficiency ratios, $D / A$ and $D \times(D / A)$, where $D$ refers to the percentage of known deposits in the area corresponding to the favourable region, and $A$ refers to the percentage of the total map area represented 
by the region. The second ratio is used because the ratio $D / A$ may be high if the most favourable region contains just a few deposits and the area of the region happens to be small. The ratios are measures of the degree to which the most favourable region in the map accounts for the known deposits.

In order to compare the two different methods used to create the favourable contact layers, the favourable region is arbitrarily defined as the upper quartile of membership values (i.e. 0.75-1.0). In the favourable geology layer based on ranking rock types according to the probability of deposits, the favourable region contains $19 \%$ of the deposits in $3 \%$ of the area. This compares to $29 \%$ of the deposits in approximately $10 \%$ of the area for the layer based on $(P(D) \times T C G) /$ cell (Table 7.35). Values of $D / A$ and $D \times(D / A)$ for the layer based on the probability of deposits, $P(D)$ and $P(D) \times T C G /$ cell are 6.0 and 116 versus 3.0 and 86, respectively (Table 7.35). The layer based on the probability of deposits was therefore selected as the input to the neural networks.

\subsubsection{Subjective Fuzzy-Membership Layers}

A variety of statistical methods, described in Chapter 6, were used to test the strength and statistical significance of associations between the seven GIS layers representing rheologicalcontrast and known gold deposits. Methods used comprise plots of cumulative and interval-based bivariate J-curves, contrast of weights (Bonham-Carter, 1994), chi-squared, $(O-E) / E$ (where $O=$ observed number of deposits in a region corresponding to an interval on the $\mathrm{X}$-axis and $E=$ expected number of deposits for a uniform random distribution) and the Kolmogorov-Smirnov (KS) cumulative distribution function.

The subjective fuzzy rheological-contrast layer shows the strongest association with known deposits and was therefore selected as the layer to use as a neural network input. A plot of $(O-E) / E$ (where $O=$ observed number of deposits in a region corresponding to an interval on the $\mathrm{X}$-axis and $E=$ expected number of deposits for a uniform random distribution) versus favourable rheological contrast, represented using the fuzzy-membership values, is shown in Figure 7.12.

The reason for the weak association between known deposits and estimates of rheological contrast based on the quantitative data may be due to the fact that rock strength properties for many rock types in the study area are not available and are therefore approximated by using data for similar rocks. In addition, tabulated data from Hatheway \& Kiersch (1989) mainly refers to unaltered, massive specimens, whereas rocks in the Kalgoorlie Terrane are altered and deformed to varying degrees. Although the compilation from M.Lee 
Table 7.35. Comparison of the ability of alternative rock-type, contact-type and rheological contrast layers to predict known orogenic gold deposits in the Kalgoorlie Terrane of Western Australia

\begin{tabular}{|l|r|r|r|l|}
\hline $\begin{array}{l}\text { Layer Type or } \\
\text { variable }\end{array}$ & $\begin{array}{c}\text { Deposits } \\
(\%)\end{array}$ & $\begin{array}{c}\text { Area } \\
(\%)\end{array}$ & $D / A$ & $\mathrm{D} *(\mathrm{D} / \mathrm{A})$ \\
\hline Favourable geology & 19.2 & 3.2 & 6.0 & 116 \\
\hline $\mathrm{P}(\mathrm{D})$ & 29.2 & 9.8 & 3.0 & 86 \\
\hline $\mathrm{P}(\mathrm{D}) \times$ TCG/cell & \multicolumn{5}{|l|}{} \\
\hline Favourable contact-type & 15.8 & 0.6 & 25.2 & 398 \\
\hline $\mathrm{P}(\mathrm{D}) 0-200 \mathrm{~m}^{\text {a }}$ & 18.3 & 1.0 & 18.0 & 330 \\
\hline $\mathrm{P}(\mathrm{D}) \times$ TCG/cell 0-200 m & 17.5 & 1.2 & 14.3 & 251 \\
\hline $\mathrm{P}(\mathrm{D}) 0-1300 \mathrm{~m}^{\mathrm{b}}$ & 21.7 & 1.9 & 11.3 & 246 \\
\hline $\mathrm{P}(\mathrm{D}) \times$ TCG/cell 0-1300 m & \multicolumn{5}{|l|}{} \\
\hline Rheological Contrast & 20.0 & 3.7 & 5.5 & 110 \\
\hline Subjective fuzzy membership & 37.5 & 14.9 & 2.5 & 94 \\
\hline Subjective 10 classes & 2.5 & 0.2 & 11.9 & 30 \\
\hline Young's modulus ${ }^{\mathrm{c}}$ & 12.5 & 1.9 & 6.8 & 85 \\
\hline Young's modulus ${ }^{\mathrm{d}}$ & 4.2 & 0.2 & 20.3 & 85 \\
\hline Uniaxial compressive strength & 12.5 & 4.6 & 2.7 & 34 \\
\hline Uniaxial tensile strength & 10.0 & 2.2 & 4.5 & 45 \\
\hline Fracture toughness &
\end{tabular}

\footnotetext{
${ }^{a}$ Fuzzy membership values set to 0.1 at distance $>200 \mathrm{~m}$ in order to model the decreasing effect of proximity to a lithological boundary with distance. Limit is based on the minimum estimate obtained using a variety of statistical tests used to measure the spatial association of known deposits with proximity to lithological contacts. Refer to Table 6.4.

${ }^{b}$ Fuzzy membership values set to 0.1 at distance $>1300 \mathrm{~m}$. See footnote above.

${ }^{c}$ CRC: data obtained from Hatheway \& Kiersch (1989). See Section D.5.1 in Appendix D.

${ }^{d}$ AMC: data provided by M.Lee (unpublished data from Australian Mining Consultants Pty Ltd, 2000). See Section D.5.1 in Appendix D.
}

(unpublished data from Australian Mining Consultants, 2000) is obtained from mine sites in the Yilgarn Craton, the data refer only to a small number of locations.

\subsubsection{Comparison of Fuzzy Membership with Other Methods for Rep- resenting Lithology Input}

Different methods of representing host-rock lithology as inputs to a neural network are compared in Table 7.36. Host-rock lithology is represented using: 1) 1-of-n coding, 2) 


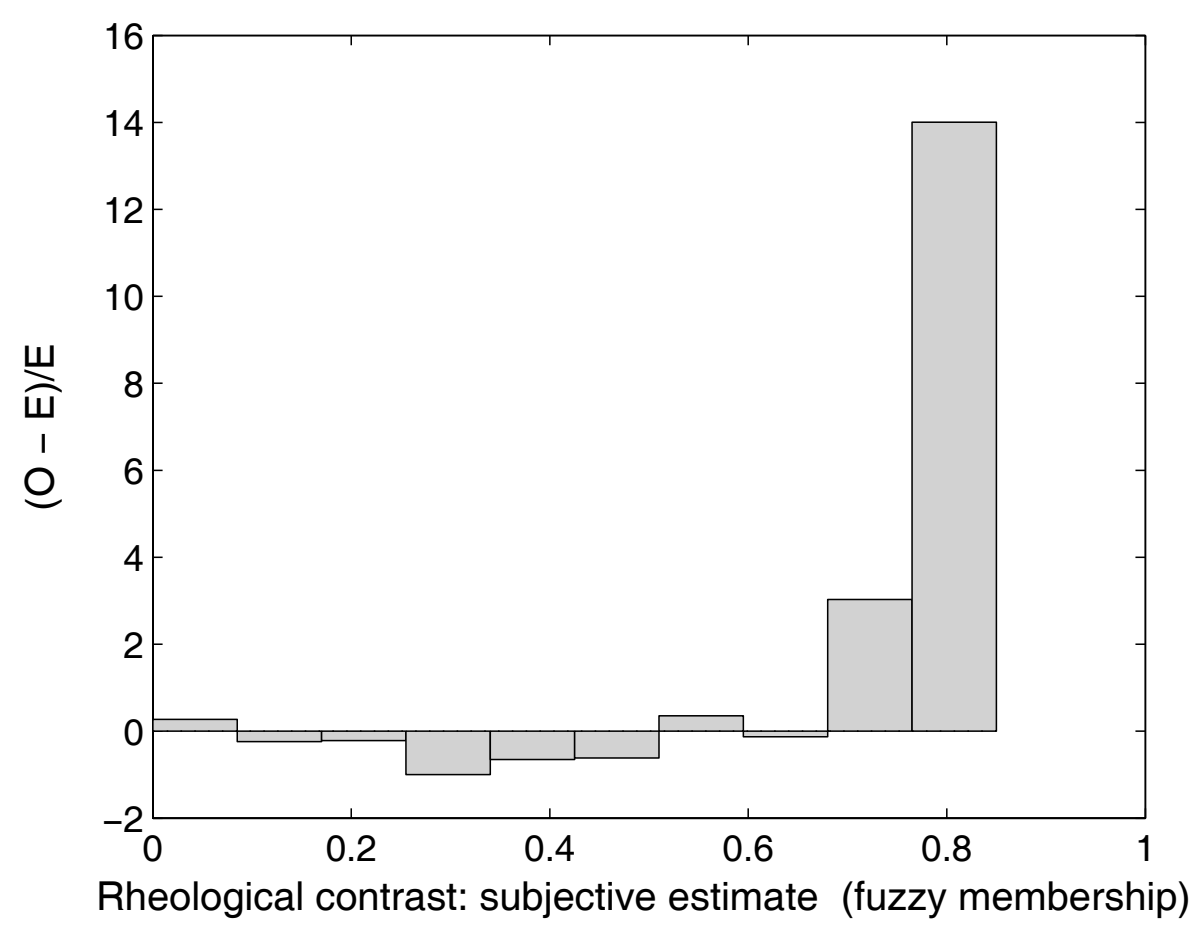

Figure 7.12. Plot of $(O-E) / E$ versus subjective fuzzy membership values for rheological contrast, where $O=$ observed number of deposits in a region corresponding to an interval on the $\mathrm{X}$-axis and $E=$ expected number of deposits for a uniform random distribution of deposits. The rheological contrast layer containing subjectively estimated fuzzy membership values shows the strongest association with known deposits of all the rheological contrast layers and is therefore selected as the layer to use as a neural network input. Other rock strength variables tested were Young's modulus (two different data compilations), uniaxial tensile and compressive strength, and fracture toughness.

three rock strength layers (Young's modulus ${ }^{4}$, uniaxial compressive strength \& fracture toughness, and layers for the sulphidation index, $\mathrm{Fe}_{(\text {total wt } \% \text { oxides })} \times \mathrm{Fe} /(\mathrm{Fe}+\mathrm{Mg}+\mathrm{Ca})$, and the oxidation ratio, $\mathrm{Fe}_{2} \mathrm{O}_{3} /\left(\mathrm{Fe}_{2} \mathrm{O}_{3}+\mathrm{FeO}\right)$, and 3) fuzzy membership values.

Networks with 1-of-n coded and fuzzy host-rock inputs have 10 inputs, whereas the networks, in which rock-strength and chemical-ratio inputs are used to represent the rock type have 14 inputs. In both cases, the other 9 inputs are the same and include the favourable contact-type and favourable rheological contrast input layers, described above. For this experiment, GIS layers which have the highest value of the capture efficiency ratio, D/A, for each evidence-category were selected as inputs to the neural network. These input-layer selection criteria are abbreviated in the table captions below as categories.

${ }^{4}$ data from M. Lee, Australian Mining Consultants Pty Ltd (AMC), 2000 
Although the network performance statistics are generally similar for both methods, 1-of-n coding produced slightly better average classification performance for test-set, and map quality statistics, and approximately $40 \%$ higher values for the capture efficiency statistic, $D \times(D / A)$. In contrast, the best test-set results (see second last line of Table 7.36) are obtained using fuzzy membership values. In addition, the $D \times(D / A)$ value is much higher for the network with the fuzzy membership inputs compared to the one with 1-of-n coded geology inputs. As noted above, 1-of-n coding is more difficult to use for problems involving a large number of classes (if patterns are stored as arrays in memory during processing) due to the large amount of main computer memory required. The similarity of results suggests that fuzzy membership values are a viable alternative for categorical data inputs to a neural network in this domain.

Table 7.36. Effect of using 1-of-n coding, 5 rock strength and chemical ratio layers, and fuzzy membership values to represent host-rock lithology for input to a neural network on the test-set performance. The following rock strength and chemical ratios were used to represent lithology; Young's Modulus, uniaxial compressive strength, fracture toughness, sulphidation index, $\mathrm{Fe}_{\text {totalwt\% }} \times \mathrm{Fe} /\left(\mathrm{Fe}_{\text {total }}+\mathrm{Mg}+\mathrm{Ca}\right)$, \& oxidation ratio, $\mathrm{Fe}_{2} \mathrm{O}_{3} /\left(\mathrm{Fe}_{2} \mathrm{O}_{3}+\mathrm{FeO}\right)$. Each row represents the average of 10 networks. Values for training and test set performance represent the percentages of correctly classified patterns. Network topology: 10-20-1, training algorithm: trainlm, layer selection criteria: GIS layers which have the highest value of the capture efficiency ratio, $D / A$, for each evidence-category. These criteria are abbreviated in subsequent tables as categories.

\begin{tabular}{|c|c|c|c|c|c|c|c|c|c|}
\hline \multirow{2}{*}{$\begin{array}{l}\text { Lithology } \\
\text { input type }\end{array}$} & \multicolumn{2}{|c|}{ Training Sets } & \multicolumn{3}{|c|}{ Test Set } & \multicolumn{4}{|c|}{ Map Quality Statistics } \\
\hline & Train & Stop & All & Deps & Barren & $\rho$ & $\mathrm{D} / \mathrm{A}$ & $\mathrm{D} *(\mathrm{D} / \mathrm{A})$ & $\mathrm{ROC}$ \\
\hline \multicolumn{10}{|c|}{ Average performance } \\
\hline Fuzzy m/ship ${ }^{a}$ & 84.4 & 72.7 & 67.9 & 68.0 & 67.8 & 0.97 & 8.2 & 105.3 & 0.79 \\
\hline Rheol.+ Chem. ${ }^{b}$ & 86.6 & 72.9 & 69.4 & 69.1 & 69.6 & 0.90 & 9.1 & 148.7 & 0.79 \\
\hline 1-of-n coding ${ }^{c}$ & 87.9 & 78.2 & 68.5 & 71.5 & 66.3 & 0.98 & 9.0 & 147.4 & 0.81 \\
\hline \multicolumn{10}{|c|}{ Best performance } \\
\hline Fuzzy m/ship & 88.9 & 72.7 & 76.5 & 77.1 & 76.1 & 0.90 & 9.6 & 256.7 & 0.82 \\
\hline Rheol.+ Chem. & 86.9 & 69.3 & 71.6 & 77.1 & 67.4 & 0.95 & 10.3 & 197.1 & 0.81 \\
\hline 1-of-n coding & 93.9 & 79.3 & 72.5 & 76.5 & 69.6 & 0.97 & 8.0 & 200.0 & 0.82 \\
\hline
\end{tabular}

${ }^{a}$ Fuzzy membership values. Network topology: 10-20-1

${ }^{b}$ Rheology and chemical ratio layers. Network topology: 14-20-1

${ }^{c}$ Network topology: 33-20-1 


\subsubsection{Fuzzification Of All Neural Network Inputs}

An extension of the concept of using fuzzy membership values in order to represent categorical data types as inputs is to use fuzzy membership values for all the network inputs. Such a system effectively represents a simple form of hybrid fuzzy-neural network system (Fig. 7.13). A series of networks were tested in which all 10 or 17 inputs are converted to fuzzy membership values. Triangular fuzzy membership functions were used to convert the scaled raw data to membership values (refer to Fig. 5.6). The thresholds above which all raw data values are mapped to a fuzzy membership value of 1.0, or below which the raw data values are mapped to 0.1 , are determined using plots of $(O-E) / E$, such as that shown in Figure 7.12. Reclassification of the original raster GIS layers is performed in MATLAB using the functions layer2fav and layer2fav_batch. Since all neural network inputs are scaled to the range $[0,1]$, the fuzzy membership inputs require no special preprocessing.

Results for neural networks with 10 inputs, in which 1, 3 and 10 inputs are represented as fuzzy membership values, are shown in Table 7.37. Systems for which all 10 inputs are presented as fuzzy membership values represent a form of hybrid fuzzy-neural network. Each row represents the average for ten networks. The top half of each table shows the results for inputs selected using the evidence-category criteria and the lower half of each table shows the results for inputs selected using the $D \times(D / A)$ criteria. Table 7.38 shows the results for the neural network, hybrid fuzzy-neural network and fuzzy system with the best percentage of correct classifications for the independent test data. Although networks and fuzzy systems, which are grouped according to the layer selection criteria, have different numbers of fuzzy and standard scaled inputs, each system uses the same total number of inputs and the input variables correspond to the same set of GIS layers. Tables 7.39 and 7.40 are similar, but show the average and best results for networks using 17 inputs.

Networks with 1, 3, and 10 fuzzy inputs produce similar results for the percentage correct classification of the test-set data. However, those with 1 and 3 fuzzy inputs generally performed slightly better than the equivalent networks with 10 fuzzy inputs (i.e. those with the same number of inputs and selection criteria). An exception is for 10 input layers selected according to the evidence categories criteria, where the average test-set classification percentage is $67.9 \%$ and $70.5 \%$ for three and ten fuzzy inputs, respectively (top of Table 7.37). 
input GIS layers

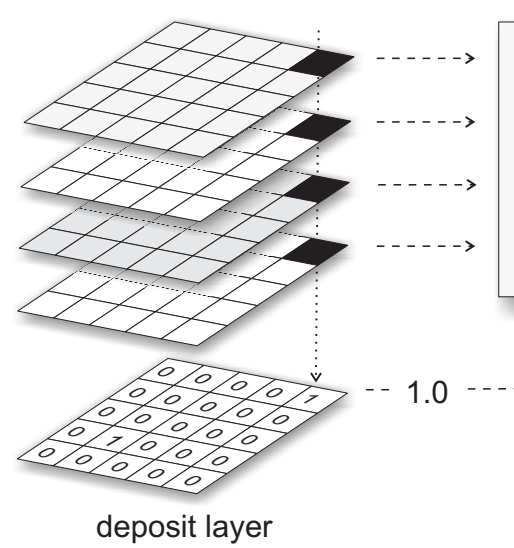

mineral prospectivity map

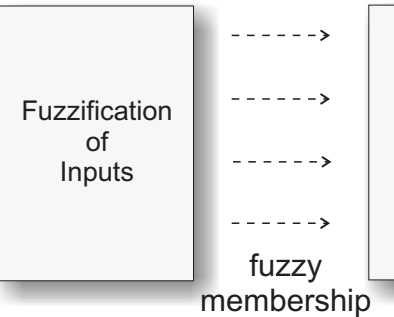

values

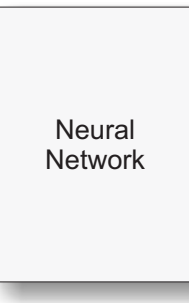

$\hat{\imath}$

output

activation

values

target value

Figure 7.13. Schematic diagram of a hybrid, fuzzy-neural network system. Some or all of the network inputs may be fuzzy membership values.

In addition, the highest overall test-set classification accuracies were obtained with networks using only fuzzy inputs (i.e. $79.0 \%$ for the best 10 input network with layers selected with the category criterion, Table 7.38), and $79.0 \%$ for the best 17 input network with layers selected using the $D \times(D / A)$ criterion, (bottom of Table 7.40).

The fuzzy-neural networks with both 10 and 17 inputs generally gave better results (i.e. average and best) for the two capture efficiency ratios and the area under the ROC curves. For example, the values of these statistics for the average of 10 networks with 10 inputs (selection criteria: $D \times(D / A))$ are 7.4, 109.0, and 0.84 for 3 fuzzy inputs compared with 14.4, 264.3 and 0.86 for ten fuzzy inputs (see lower half of Table 7.37).

Table 7.37. Comparison of neural networks with 3 and 10 fuzzy-membership inputs (i.e. latter is a hybrid fuzzy-neural network): average results. Network topology: 10-20-1, training algorithm: trainlm.

\begin{tabular}{|c|c|c|c|c|c|c|c|c|c|}
\hline \multirow{2}{*}{$\begin{array}{l}\text { Number of } \\
\text { fuzzy inputs }\end{array}$} & \multicolumn{2}{|c|}{ Training Sets } & \multicolumn{3}{|c|}{ Test Set } & \multicolumn{4}{|c|}{ Map Quality Statistics } \\
\hline & Train & Stop & All & Deps & Barren & $\rho$ & $\mathrm{D} / \mathrm{A}$ & $\mathrm{D} *(\mathrm{D} / \mathrm{A})$ & $\mathrm{ROC}$ \\
\hline \multicolumn{10}{|c|}{ Layer selection criteria: categories } \\
\hline 3 & 84.4 & 72.7 & 67.9 & 68.0 & 67.8 & 0.97 & 8.2 & 105.3 & 0.79 \\
\hline 10 & 84.3 & 75.2 & 70.5 & 66.9 & 73.3 & 0.94 & 9.7 & 133.4 & 0.83 \\
\hline \multicolumn{10}{|c|}{ Layer selection criteria: $\mathrm{D} \times(\mathrm{D} / \mathrm{A})$} \\
\hline 3 & 87.9 & 76.9 & 72.1 & 75.1 & 69.8 & 0.95 & 7.4 & 109.0 & 0.84 \\
\hline 10 & 87.2 & 78.4 & 69.1 & 71.4 & 67.4 & 0.97 & 14.4 & 264.3 & 0.86 \\
\hline
\end{tabular}


Table 7.38. Comparison of neural networks with 3 and 10 fuzzy-membership inputs (i.e. latter is a hybrid fuzzy-neural network), and a fuzzy system with 10 inputs: best results. Network topology: 10-20-1, training algorithm: trainlm.

\begin{tabular}{|c|c|c|c|c|c|c|c|c|c|}
\hline \multirow{2}{*}{$\begin{array}{l}\text { Number of } \\
\text { fuzzy inputs }\end{array}$} & \multicolumn{2}{|c|}{ Training Sets } & \multicolumn{3}{|c|}{ Test Set } & \multicolumn{4}{|c|}{ Map Quality Statistics } \\
\hline & Train & Stop & All & Deps & Barren & $\rho$ & $\mathrm{D} / \mathrm{A}$ & $\mathrm{D} *(\mathrm{D} / \mathrm{A})$ & $\mathrm{ROC}$ \\
\hline \multicolumn{10}{|c|}{ Layer selection criteria: categories } \\
\hline 3 & 88.9 & 72.7 & 76.5 & 77.1 & 76.1 & 0.90 & 9.6 & 256.7 & 0.82 \\
\hline 10 & 86.9 & 80.7 & 79.0 & 71.4 & 84.8 & 0.90 & 10.3 & 120.6 & 0.86 \\
\hline 10 fuzzy $(\gamma=0.96)$ & 69.7 & 73.9 & 67.9 & 65.7 & 69.6 & 1.00 & 160.6 & 267.7 & 0.87 \\
\hline \multicolumn{10}{|c|}{ Layer selection criteria: $\mathrm{D} \times(\mathrm{D} / \mathrm{A})$} \\
\hline 3 & 92.9 & 81.8 & 77.8 & 82.9 & 73.9 & 0.98 & 5.0 & 104.5 & 0.87 \\
\hline 10 & 82.8 & 84.1 & 74.1 & 85.7 & 65.2 & 0.98 & 19.5 & 519.0 & 0.89 \\
\hline 10 fuzzy $(\gamma=0.94)$ & 67.7 & 72.7 & 70.4 & 62.9 & 76.1 & 1.00 & 68.2 & 397.7 & 0.89 \\
\hline
\end{tabular}

Table 7.39. Comparison of neural networks with 3 and 17 fuzzy-membership inputs (i.e. latter is a hybrid fuzzy-neural network): average results. Network topology: 10-20-1, training algorithm: trainlm.

\begin{tabular}{|l|l|l|l|l|l|l|l|l|l|}
\hline \multirow{2}{*}{$\begin{array}{l}\text { Number of } \\
\text { fuzzy inputs }\end{array}$} & Training Sets & \multicolumn{3}{l|}{ Test Set } & \multicolumn{3}{l|}{ Map Quality Statistics } \\
\cline { 2 - 10 } & Train & Stop & All & Deps & Barren & $\rho$ & D $/ A$ & D $*(\mathrm{D} / \mathrm{A})$ & ROC \\
\hline \hline
\end{tabular}


Table 7.40. Comparison of neural networks with 3 and 17 fuzzy-membership inputs (i.e. latter is a hybrid fuzzy-neural network), and a fuzzy system with 17 inputs: best results. Network topology: 10-20-1, training algorithm: trainlm.

\begin{tabular}{|c|c|c|c|c|c|c|c|c|c|}
\hline \multirow{2}{*}{$\begin{array}{l}\text { Input } \\
\text { type }\end{array}$} & \multicolumn{2}{|c|}{ Training Sets } & \multicolumn{3}{|c|}{ Test Set } & \multicolumn{4}{|c|}{ Map Quality Statistics } \\
\hline & Train & Stop & All & Deps & Barren & $\rho$ & $\mathrm{D} / \mathrm{A}$ & $\mathrm{D} *(\mathrm{D} / \mathrm{A})$ & $\mathrm{ROC}$ \\
\hline \multicolumn{10}{|c|}{ Layer selection criteria: categories } \\
\hline 3 & 82.8 & 79.6 & 76.5 & 88.6 & 67.4 & 1.00 & 9.7 & 72.5 & 0.83 \\
\hline 17 & 91.9 & 76.1 & 70.4 & 68.6 & 71.7 & 1.00 & 21.6 & 541.0 & 0.88 \\
\hline 17 fuzzy $(\gamma=0.97)$ & 71.7 & 76.1 & 70.4 & 51.4 & 84.8 & 0.98 & 266.4 & 887.8 & 0.89 \\
\hline \multicolumn{10}{|c|}{ Layer selection criteria: $\mathrm{D} \times(\mathrm{D} / \mathrm{A})$} \\
\hline 3 & 90.9 & 83.0 & 75.3 & 82.9 & 69.6 & 0.98 & 13.2 & 231.4 & 0.86 \\
\hline 17 & 93.9 & 80.7 & 79.0 & 71.4 & 84.8 & 0.97 & 15.6 & 349.9 & 0.88 \\
\hline 17 fuzzy $(\gamma=0.97)$ & 70.7 & 73.9 & 71.6 & 54.3 & 84.8 & 0.98 & 199.8 & 665.9 & 0.89 \\
\hline
\end{tabular}

Increasing the number of inputs from 10 to 17 does not result in a significant improvement in performance nor map quality (Tables $7.37 \& 7.38$ versus $7.39 \& 7.40$ ). Better recognition of the deposit patterns in the test data set is achieved with networks using layers selected according to the $D \times(D / A)$ criterion than with the categories criterion. Networks using the $D \times(D / A)$ set of layers also generally give significantly higher values for the measures of prospectivity map quality (i.e. Spearman's correlation coefficient, $D / A, D \times(D / A)$, and area under the ROC curve).

For comparison purposes, maps are prepared using simple fuzzy systems, in which the same input layers were combined using the gamma function (Zimmermann, 1984). Maps are created and tested for values of the gamma parameter in the range $[0.0,1.0]$ in 0.01 increments. Results for gamma values in the range $[0.9,1.0]$ are shown in Table 7.41 below. The results corresponding to the best test-set classification performance are shown in Tables 7.38 and 7.40 .

The generalization performance of the fuzzy systems is significantly poorer than the neural networks and the hybrid fuzzy-neural networks. For example, the systems with 10 inputs give average results of $76.5 \%, 79.0 \%$, and $67.9 \%$ correct classification of the test set and $77.1 \%, 71.4 \%$, and $65.7 \%$ correct classification of the deposit patterns in the test set, respectively (Table 7.37). The fuzzy system does yield some extremely high values of the capture efficiency ratios, $D / A, D \times(D / A)$ (e.g. 266.4 and 887.8, respectively, for 17 inputs, category selection criterion; see Table 7.40). 
Table 7.41. Performance of fuzzy systems using the gamma function for a range of gamma values: 10 and 17 input layers,layer selection criteria: categories.

\begin{tabular}{|c|c|c|c|c|c|c|c|c|c|}
\hline \multirow{2}{*}{$\begin{array}{c}\text { Gamma } \\
\text { value }(\gamma)\end{array}$} & \multicolumn{2}{|c|}{ Training Sets } & \multicolumn{3}{|c|}{ Test Set } & \multicolumn{4}{|c|}{ Map Quality Statistics } \\
\hline & Train & Stop & All & Deps & Barren & $\rho$ & $\mathrm{D} / \mathrm{A}$ & $\mathrm{D} *(\mathrm{D} / \mathrm{A})$ & $\mathrm{ROC}$ \\
\hline \multicolumn{10}{|c|}{10 layers, cat. $^{a}$} \\
\hline 0.94 & 62.6 & 71.6 & 65.4 & 22.9 & 97.8 & 0.95 & 348.5 & 580.9 & 0.87 \\
\hline 0.95 & 65.7 & 76.1 & 61.7 & 28.6 & 87.0 & 0.98 & 292.5 & 487.5 & 0.87 \\
\hline 0.96 & 69.7 & 73.9 & 67.9 & 65.7 & 69.6 & 1.00 & 160.6 & 267.7 & 0.87 \\
\hline 0.97 & 58.6 & 60.2 & 56.8 & 94.3 & 28.3 & 0.98 & 95.8 & 159.7 & 0.87 \\
\hline 0.98 & 56.6 & 48.9 & 48.2 & 97.1 & 10.9 & 0.98 & 51.1 & 127.7 & 0.87 \\
\hline \multicolumn{10}{|c|}{10 layers, $\mathrm{D} *(\mathrm{D} / \mathrm{A})$} \\
\hline 0.94 & 67.7 & 72.7 & 70.4 & 62.9 & 76.1 & 1.00 & 68.2 & 397.7 & 0.89 \\
\hline 0.95 & 72.7 & 73.9 & 65.4 & 80.0 & 54.4 & 0.98 & 88.5 & 811.3 & 0.89 \\
\hline 0.96 & 58.6 & 56.8 & 63.0 & 100.0 & 34.8 & 0.96 & 87.4 & 1019.6 & 0.89 \\
\hline 0.97 & 49.5 & 48.9 & 48.2 & 100.0 & 8.7 & 0.92 & 65.6 & 928.7 & 0.89 \\
\hline 0.98 & 48.5 & 46.6 & 44.4 & 100.0 & 2.2 & 0.85 & 29.8 & 546.7 & 0.89 \\
\hline \multicolumn{10}{|c|}{17 layers, cat. } \\
\hline 0.94 & 54.6 & 56.8 & 59.3 & 5.7 & 100.0 & 1.00 & 744.6 & 620.5 & 0.89 \\
\hline 0.95 & 55.6 & 61.4 & 60.5 & 8.6 & 100.0 & 1.00 & 744.6 & 1241.0 & 0.89 \\
\hline 0.96 & 58.6 & 65.9 & 64.2 & 20.0 & 97.8 & 1.00 & 321.2 & 535.3 & 0.89 \\
\hline 0.97 & 71.7 & 76.1 & 70.4 & 51.4 & 84.8 & 0.98 & 266.4 & 887.8 & 0.89 \\
\hline 0.98 & 51.5 & 53.4 & 53.1 & 100.0 & 17.4 & 0.92 & 109.5 & 547.2 & 0.89 \\
\hline \multicolumn{10}{|c|}{17 layers, $\mathrm{D} *(\mathrm{D} / \mathrm{A})$} \\
\hline 0.94 & 55.6 & 61.4 & 60.5 & 8.6 & 100.0 & 0.47 & 0.0 & 0.0 & 0.89 \\
\hline 0.95 & 57.6 & 64.8 & 61.7 & 11.4 & 100.0 & 0.53 & 0.0 & 0.0 & 0.89 \\
\hline 0.96 & 61.6 & 67.1 & 63.0 & 22.9 & 93.5 & 0.53 & 0.0 & 0.0 & 0.89 \\
\hline 0.97 & 70.7 & 73.9 & 71.6 & 54.3 & 84.8 & 0.98 & 199.8 & 665.9 & 0.89 \\
\hline 0.98 & 50.5 & 52.3 & 50.6 & 100.0 & 13.0 & 0.96 & 117.2 & 781.4 & 0.89 \\
\hline
\end{tabular}

${ }^{a}$ cat. = categories. Input layers selected using category criteria.

This highlights the limitation of using summary statistics like capture efficiency ratios. The very high values of $D / A$, and $D \times(D / A)$ are due to the fact that the region corresponding to the highest prospectivity map class contains a small number of deposits in a very small proportion of the map (i.e. 4 deposits in 123 grid cells for the 17 categories input fuzzy system shown in the top part of Table 7.40). However, the bulk of the deposits occur in the region corresponding to classes 5-7 (i.e. 104 deposits in 482464 cells). By comparison, the hybrid fuzzy-neural network with the same inputs (Table 7.40), generates a map with 
the largest number of deposits (30) in the area corresponding to class 9 (11355 cells), the highest prospectivity area. In this prospectivity map, in which the prospectivity values are divided into 9 classes, the bulk of deposits are located in the area corresponding to classes 7-9 (i.e. 73 deposits in 82,258 cells). Details of the statistics for each neural network and fuzzy system are given in the file mapstats on the attached CD-ROM (vol.1).

\subsubsection{Discussion: Use of Fuzzy Inputs in Neural Networks}

The better map quality statistics, but poorer test-set classification performance, for fuzzy systems and hybrid fuzzy-neural networks with 10 and 17 fuzzy inputs compared to neural networks with 1 and 3 fuzzy inputs may be due to the way in which the two types of tests differ $^{5}$. Good performance in terms of the map quality statistics, $D / A, D \times(D / A)$ and area under the ROC curve, depends on capturing a high proportion of the known deposits in a small proportion of the total map area.

In maps produced with fuzzy systems and, to a lesser degree, hybrid fuzzy-neural network systems, high relative prospectivity values are assigned to a much smaller proportion of the area than in maps produced with neural networks. This is evident in Figure 7.14, which shows histograms of the output values produced by a neural network, a hybrid fuzzy-neural and fuzzy systems for the maps covering the entire study area. For fuzzy systems, this may be due to the high values of the gamma parameter (0.94-0.97) used in the gamma function which is used to combine input layers.

The gamma function is a combination of two functions; the fuzzy algebraic product and fuzzy algebraic sum. At very high values of the gamma parameter, the gamma function is dominated by the fuzzy algebraic sum component of the function and is therefore additive in character; that is, favourable input values result in an output that is larger than any of the inputs. Bonham-Carter (1994) notes that the gamma value, at which the gamma function behaves in an increasing or decreasing way, is data dependent.

The following evidence suggests that the fuzzy systems which produced the best overall test-set performance (Tables $7.38 \& 7.40$ ) combined the inputs in an increasing way; 1) very large increases in the percentage correct classification for deposit patterns occur for 10- and 17-input fuzzy systems at gamma values above 0.92 and 0.96 , respectively (see statistics for individual prospectivity maps on attached CD-ROM (vol.1). Gamma values

\footnotetext{
${ }^{5}$ Similar results were obtained using narrow buffers applied to layers depicting distance and contrast (Tables 7.19-7.22).
} 
in the systems quoted in Tables $7.38 \& 7.40$ range from 0.94-0.97, and 2) the 10-input fuzzy systems (layer selection: categories, $\gamma=0.96$ and layer selection: $D \times(D / A), \gamma=0.94$ ) and 17-input systems (layer selection: categories, $\gamma=0.97$ and layer selection: $D \times(D / A)$, $\gamma=0.97$ ) produced minimum output values of $0.26,0.17,0.26$, and 0.26 , respectively (see histograms in Figure 7.14). However, locations where there is a conjunction of high input values are very rare. Consequently, cells that are assigned very high prospectivity values are also very rare.

The percentage correct classification results for the independent test-set is a measure of the ability of the trained neural network system to generalize from the patterns learnt during training to new patterns. For the fuzzy systems, it is a guide as to how well the system can distinguish between deposit and barren cells on the basis of the deposit model implicit in the definitions of the fuzzy membership functions.

The poorer generalization performance of the fuzzy, and to a lesser extent, the fuzzy hybrid systems may be due to the way in which the fuzzy membership functions, which define the favourable variables, simplify and smooth out spatial relationships. Compared to a neural network, in which no information is provided about how the inputs must be interpreted in order to produce the target output, the membership functions in fuzzy systems narrow the definition of what constitutes a highly favourable location for gold deposits.

Since a certain proportion of the known deposits conform to the model represented by the fuzzy membership functions, this, combined with the small number of cell locations which are assigned high output values, leads to good performance in terms of the capture efficiency ratios. However, deposits which do not fit this simpler model, may not be recognized and therefore contribute to the poorer test-set classification (i.e. generalization) performance for systems in which the inputs consist of fuzzy membership layers compared to neural networks.

The strength of the neural network method is that it is predictive. Although the neuralnetwork approach is effective in highlighting areas of known mineralization, new prospective areas are also identified. For example, some very high prospectivity areas within a narrow folded lithological unit in the south of the Kalgoorlie study area (Fig. 7.25; $366700 \mathrm{E}, 6561360 \mathrm{~N}$ ) and in scattered patches north of Kalgoorlie in the middle of the study area (Fig. 7.25; 351040 E, 6607100 N). The predictive nature of the neural-network map is due to the ability of the network to generalize from patterns contained in the training data.

Mineral prospectivity maps, produced using a neural network with three fuzzy inputs, a 


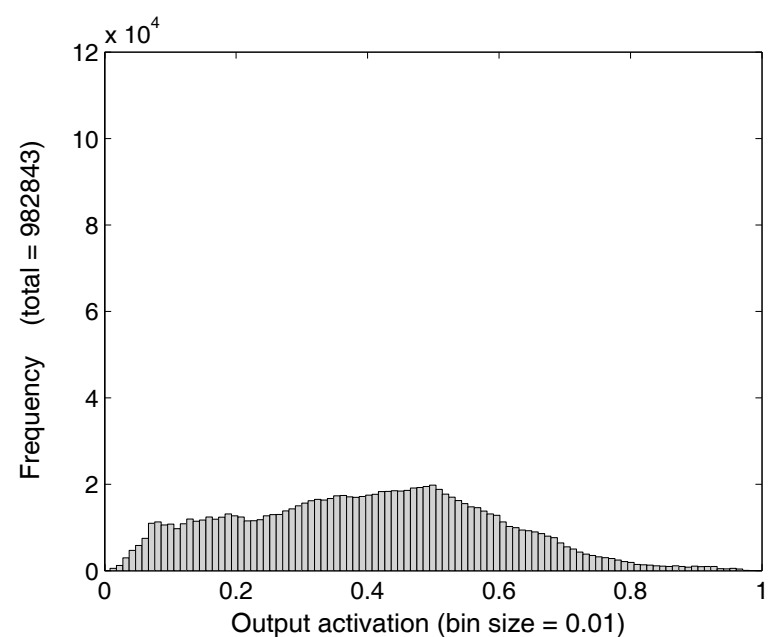

(a) Neural network: 3 fuzzy membership inputs.

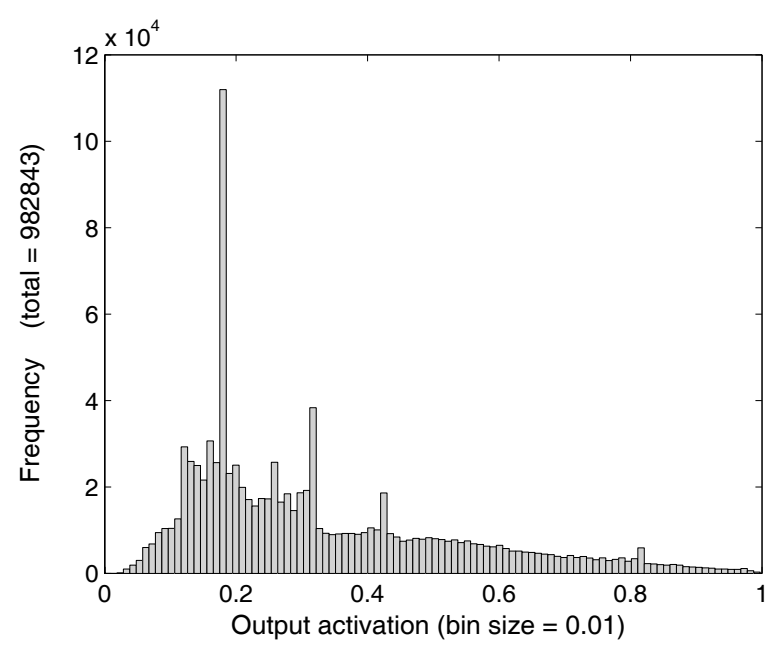

(c) Hybrid fuzzy-neural network: 17 fuzzy inputs.

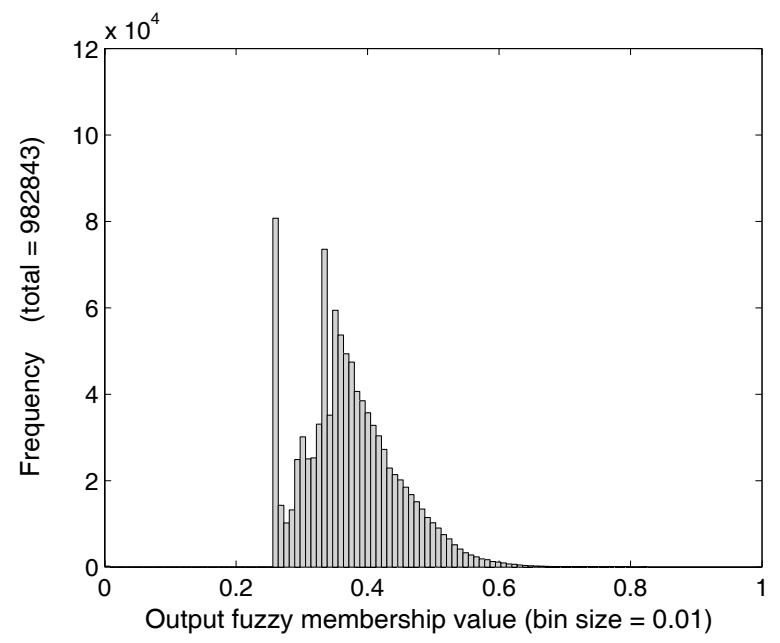

(e) Fuzzy system: 17 inputs, $\gamma=0.97$.

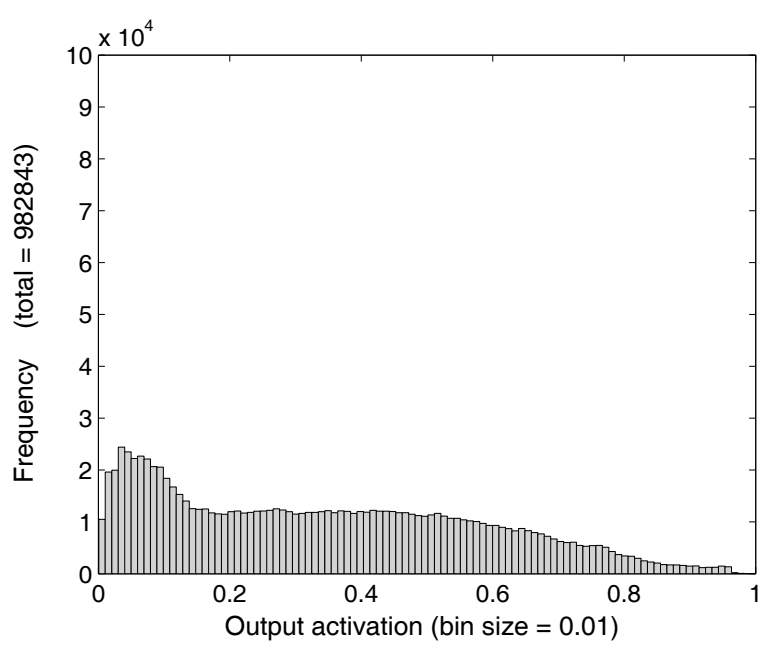

(b) Neural network: 3 fuzzy membership inputs.

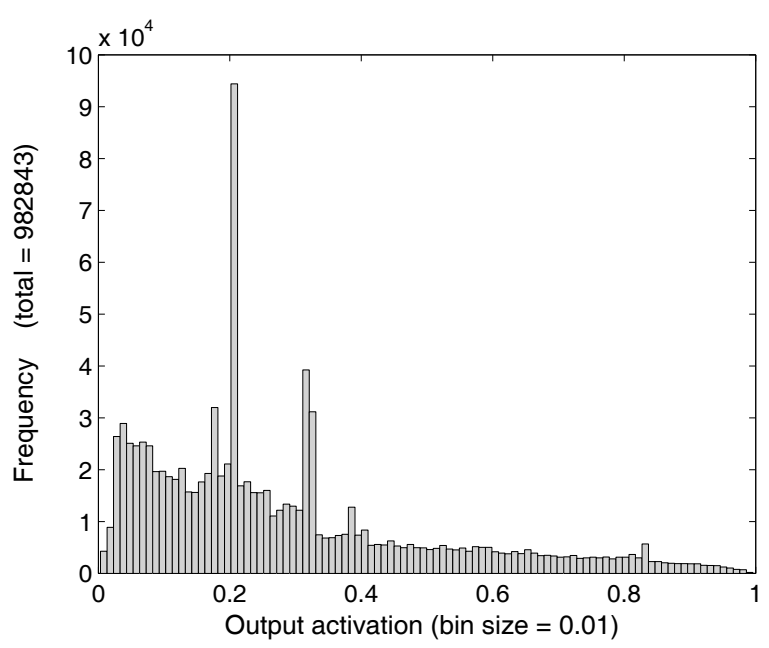

(d) Hybrid fuzzy-neural network: 17 fuzzy inputs.

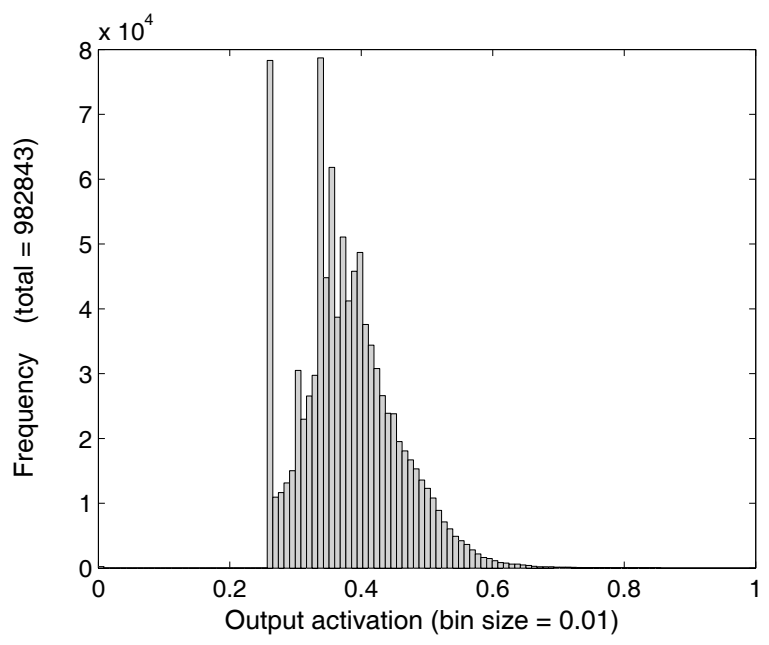

(d) Fuzzy system: 17 inputs, $\gamma=0.97$.

Figure 7.14. Histograms comparing outputs of the a) neural network, 2) hybrid fuzzy-neural network, and 3) fuzzy system $(\gamma=0.97)$ that produced the best overall test-set classification performance (see Table 7.40). The left and right columns show results for systems in which the input layers were selected using the categories and $D \times(D / A)$ criteria, respectively. 
hybrid fuzzy-neural network and a fuzzy system using the gamma function, are shown in Figures 7.15-7.17. Only a small portion of the study area is shown as the data for this study were provided by Goldfields Exploration Pty Ltd and exploration is still being conducted in other areas. All maps account reasonably well for the known gold deposits. Those containing at least $1000 \mathrm{~kg}$ TCG are shown as yellow circles.

The three maps show a trend of decreasing detail from the neural network to the hybrid fuzzy-neural network and fuzzy system. The fuzzy systems map (Fig. 7.17) shows only a small range of variation in the estimated prospectivity compared to the other two maps. There is little evidence of geological features, such as faults, lithological units or boundaries, reflected in the map. The most geological information appears in the neural-network map (Fig. 7.15), although some of the detail represents artifacts resulting from the way in which some of the input layers are prepared. For example, layers representing the strike orientation of the nearest fault or rheological contrast at the nearest lithological boundary contain triangular zones corresponding to a single value. These zones widen away from the linear feature from which the cell values are derived. The use of fuzzy membership values smooths these abrupt transitions, thus simplifying the map and making it appear more geological.

Inputs affecting the prospectivity estimate are most clearly represented in the hybrid fuzzy-neural-network map (Fig. 7.16). The elongate shapes in the north-west represent NNE-trending faults and shear zones, and lines corresponding to lithological boundaries are evident along the eastern margin of the map. This map appears to be the best tradeoff of the three maps in terms of sensitivity to the detail in the input GIS layers while displaying overall trends in the data without a lot of noise or loss of information.

\subsubsection{Conclusions: Use of Fuzzy Membership Layers as Inputs}

The use of both objective (i.e. statistically based) and subjectively derived fuzzy-membership layers is a useful means of incorporating geological knowledge in an otherwise data-driven neural network method of combining geoscience data sets to produce mineral prospectivity maps.

Objective fuzzy-membership layers, in which the fuzzy-membership values are based on the statistical relationships have been used to represent solid geology and rock-type at the nearest lithological boundary. This type of fuzzy-membership input represents a useful alternative to the 1-of-n coding, that is normally used to encode categorical data as neural network inputs, particularly if there are a large number of classes in the data (e.g. 134 
rock-type combinations in the contact-type layer).

It is shown that, in some cases (e.g. rheological contrast), subjectively estimated inputs may be superior to objective data if those data are inconsistent or not extensive enough to be representative at a regional scale. The use of fuzzy membership values for some or all of the inputs to a neural network results in similar performance to conventional data.

Presenting all inputs to the network as fuzzy membership values results in slightly decreased generalization performance, as indicated by the test-set classification performance, but significantly increased values of statistics used to measure the quality of the resultant mineral prospectivity map. 


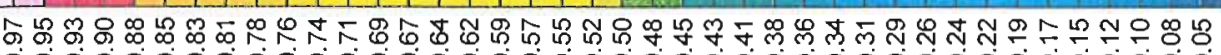

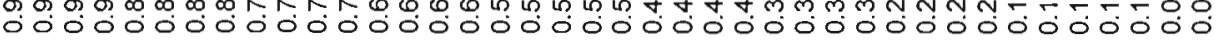

\section{$艹$
0
5
0
5
0
0
0
0}

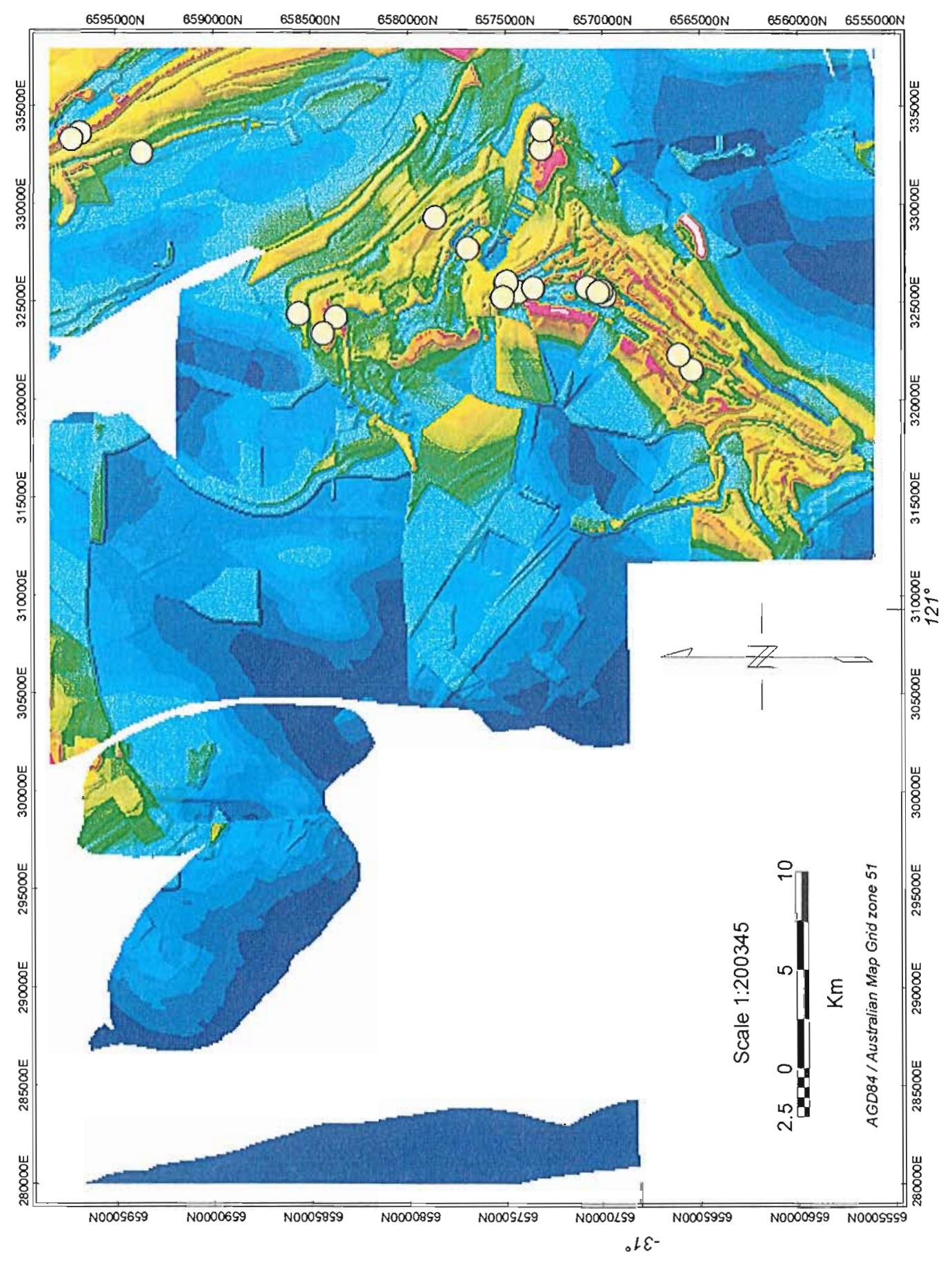

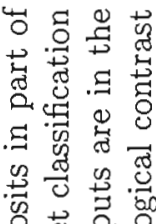

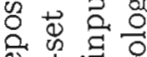

के

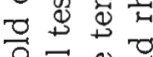

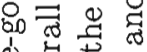

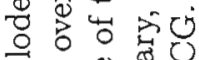

范然

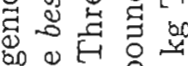

00 눙

용

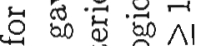

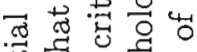

矛声寻

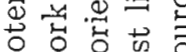

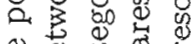

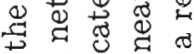

舟要过范

¿

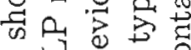

空是 8

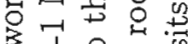

엉옹ㅇㅇㅇ

a

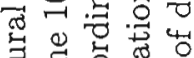

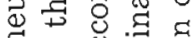

7 bo

ऽ.

可

马

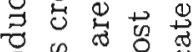

8 象

范第出至

응

范要焉

总合言.

踏

猔

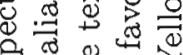

की

号品它.

ब 8 i

要要灵

类 70

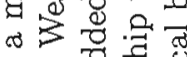

पे 80

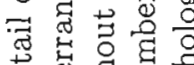

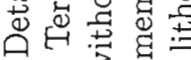

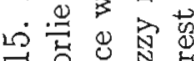

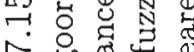
० बह है 氙式 so \& 讷专造 

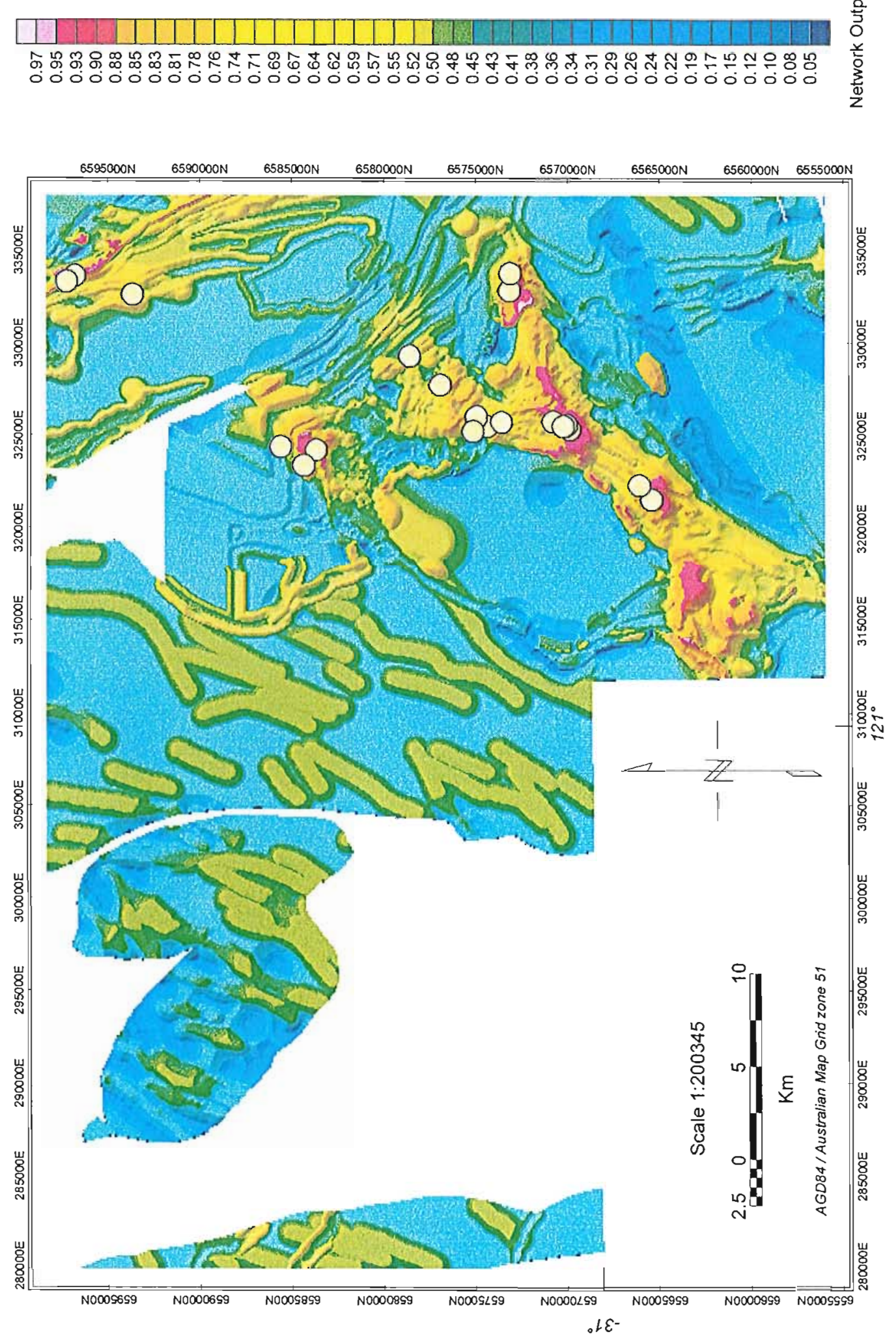

믕 잃

i 5

음ำ

品志志

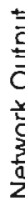

客

융 त्ञ

호ㅇㅝㅛ

त

$\vec{z} . \exists$

造范

ㄴ.

\&

bo

胥

过

की

运空。

金帘

aे

車为

害苔

$1 \cdot \overrightarrow{0}$

西要

己

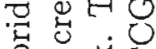

蛋出

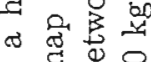

편 द्व

ED $\wedge$

ठ․

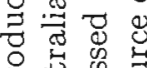

牙要考

㛡员总

वृ द्व

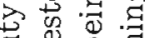

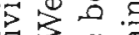

至

की

究

क 可

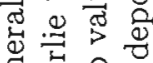

范

a

๙

$=$ 咅

苍㟧忞㱏

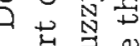
$\dot{0}$

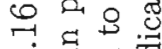

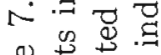
可产式 我骂 总 


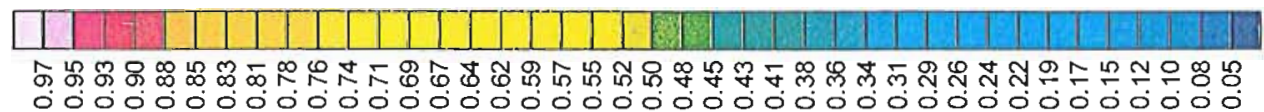

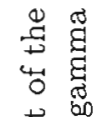

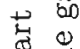

$\stackrel{\pi}{2}$

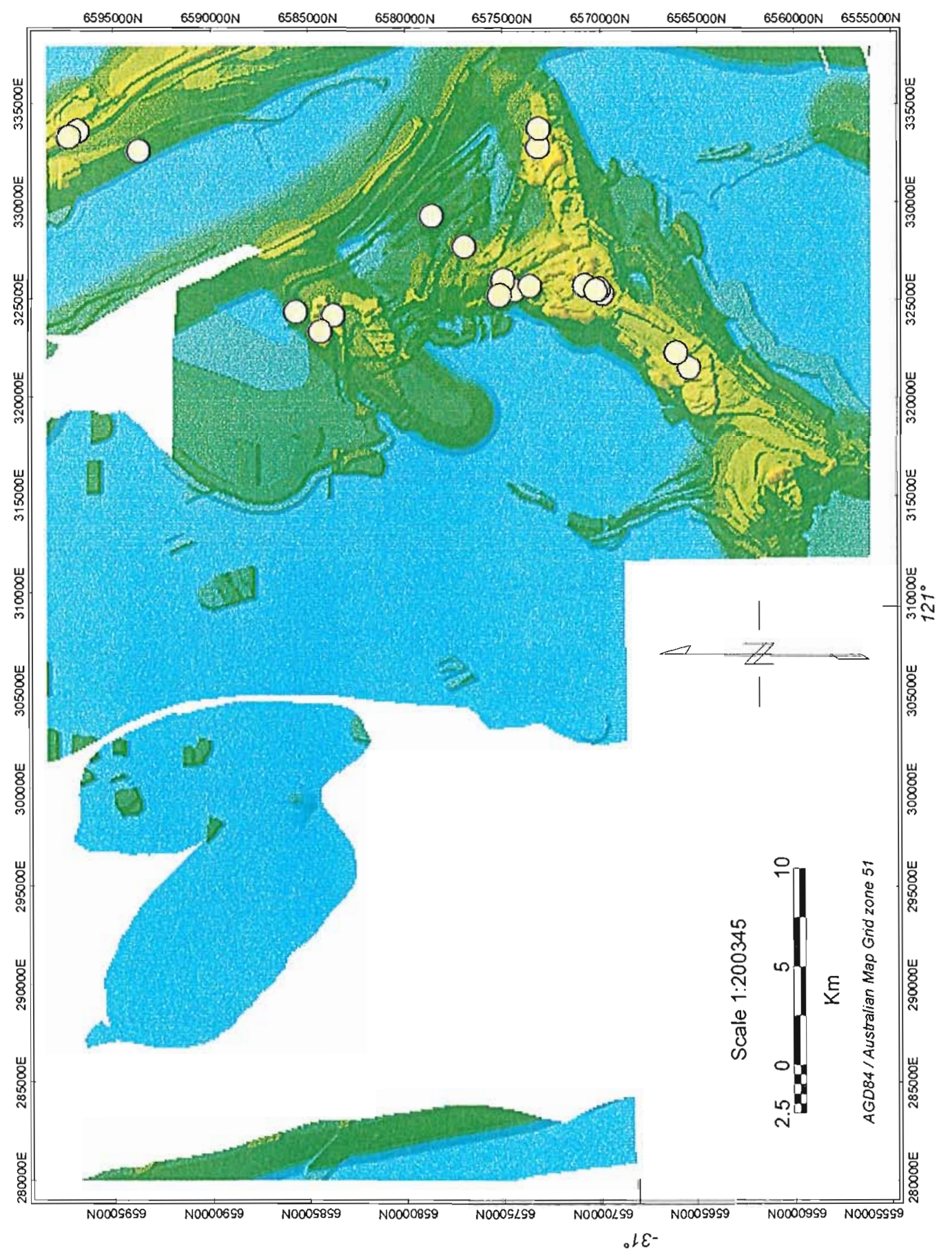

$\Xi$ bo

$\stackrel{n=1}{=}$

.

\&.

뭉

$\therefore 8$

車

응 8

겅

$\sin$

80.4

oิ

过

蛋

(1)

.

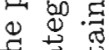

需要

\& \&

胥

总亮

द्व

出岁

它

灾通

䙳

๘

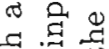

웅

芩 80

$\cong$.

명

岩要

完.

类

密

$5 \%$

氙

ठ

员

픙

पे

:

g

क क

范管

त्] है

范

대

$\rightarrow$ \&

동

용.

60. 
Prospectivity maps produced using hybrid fuzzy-neural networks clearly reflect the geology and structure in the input GIS maps, but, at the same time, appear to smooth some of the noise in the input maps without the loss of information that is apparent in maps produced using the fuzzy systems.

The methods used here could potentially be applied to a large range of subjective data (e.g. favourability of tectonic environment, host stratigraphy, or reactivation along major faults) currently used in regional exploration programs, but which would not normally be included as inputs in an empirical neural-network approach.

\subsection{Prospectivity Maps}

This section contains graphs used to assess map quality together with the corresponding prospectivity maps for neural networks, hybrid fuzzy-neural networks, and fuzzy systems referred to in the text above.

The following plots are shown:

1. Percentage correct classification for deposit and barren patterns versus classification threshold,

2. Percentage of deposit cells correctly classified versus percentage of total cells classified as mineralized,

3. ROC curve: true positive versus false positive ratio for classification thresholds in the range $[0,1]$,

4. True positive and false positive probability for deposit and barren cells, respectively versus threshold, and

5. Histogram of neural network and fuzzy system outputs for all map patterns.

The first two plots have been used by Harris \& Pan (1999) to measure model and exploration performance, respectively. Detailed descriptions are given in Section 3.3.5. The plots are useful for comparing the relative performance of different prospectivity models. In the first type of plot, the threshold that maximizes the correct classification of deposit and barren patterns corresponds to the intersection of the two curves (Fig. 7.18a). The greater the area under the curves, the better the performance of the model. Such a curve would resemble the inverted letter "J", and have an inflection point that is close to one of 
the top corners of the plot. An example of a model with very good generalization and map quality statistics is shown in Figure 7.24. The corresponding network results are shown in Table 7.28 above.

The second plot represents the measure of exploration performance used by Harris \& Pan (1999) and shows the percentage of deposit versus total cells captured for classification thresholds in the range $[0,1]$. An example is shown in Figure 7.18b. It represents a scenario in which all the map cells, where a network output (or the output fuzzy membership values in a fuzzy system) exceeds a threshold, are classified as deposits and are retained for further exploration. Depending on the threshold, different proportions of deposits and barren cells are incorrectly classified. A very effective model would allow a very high proportion of deposits to be retained in a very small area. Such a model would result in a J-shaped curve similar to the one in Figure 7.24. Again, the greater the area under the curve, the better the model. This plot represents the way the capture efficiency statistic, $D / A$ varies according to the threshold used for classification. Although it appears to be identical to the ROC curve, it is derived from a different formula. The equivalence is discussed in Section 3.3.5.

The third type of plot is the ROC curve (e.g. Fig. 7.18c). The area under the ROC curve is also a measure of the classification performance of the system. ROC curves are explained in detail in Section 3.3.4.

In the fourth type of plot represents TP and FP probability versus classification threshold. The red curve is the same as that in the first type of plot and represents the fraction of deposits which are correctly identified (e.g. Fig. 7.18d). For any given threshold, the larger the fraction of deposits correctly identified as deposits (and the smaller the percentage of barren cells falsely identified as deposits), the better the model. In general, the greater the area under the TP curve (red), the smaller the area under the FP curve, and the greater the space between the two curves, the better the model.

Histogram plots illustrate why some models can produce good generalization performance but average or poor results for the capture efficiency statistics, $D / A$, and $D \times(D / A)$ (e.g. Fig. 7.18e). Networks or systems with good generalization generally produce high capture efficiency ratios if the histogram is strongly skewed towards the low end of the output range $(X$-axis). In this case the frequency of high prospectivity cells is very low. This corresponds to an intuitive notion of prospectivity; that is, that cells representing the conjunction of a high number of favourable variable values must be very rare compared to cells where only one or two variables are favourable. The interpretation of the histograms is discussed in Section 7.8.5. 


\subsection{Summary}

The main points discussed in Chapter 7 are listed below.

1. The approach to the neural network experiments was to use a simple training algorithm and then progressively attempt to improve the performance of the basic network by altering a single parameter at a time.

2. Ten input layers, selected according to the category selection criterion, were used in initial experiments.

3. Training sets were created by randomly dividing 120 patterns, corresponding to gold deposits containing at least $1000 \mathrm{~kg}$ TCG, approximately equally between training, training-stop and test sets. Gold deposits from each host rock-type in the solid geology GIS layer are divided approximately equally between the three training sets. Approximately matching numbers of barren patterns were also randomly selected for each rock type. Deposit and barren patterns are represented in the training sets approximately equally.

4. The average results for ten networks are reported in order to account for the variation in the performance of the trained networks due to random initial connection-weights.

5. In each experiment, ten networks were initialized with the same set of random weights in order to ensure that the variation in performance is due to differences in the patterns allocated to the training, stop and test sets.

6. Performance of trained networks are measured using the percentage of correct classifications for the independent test data set (i.e. overall, deposit and barren patterns separately).

7. The quality of mineral prospectivity maps is measured using; 1) Spearman's correlation coefficient, which indicates the degree of correlation between increasing prospectivity map class and the probability that the region contains a known deposit, 2) the area under a receiver-operating-characteristic (ROC) curve, and 3) the capture efficiency ratios, $D / A$ and $D \times(D / A)$, where $D=\%$ of known deposits in highest prospectivity class in a 9 -class prospectivity map and $A=\%$ of total map area for the highest class.

8. Compared with networks with a single hidden layer, networks with two hidden layers produce slightly higher average percentage-correct classification values for test-set patterns (overall) and barren test-set patterns, and significantly higher values for capture efficiency ratios, $D / A$ and $D \times(D / A)$. Values for Spearman's correlation 
coefficient (indicating the correlation between increasing prospectivity map class and the probability of a deposit) are lower for networks with two hidden layers than for one hidden layer.

9. Networks with two hidden layers commonly produce relatively bimodal prospectivity estimates. In this case, the bulk of known deposits are located in either low or high prospectivity map-classes.

10. A 10-20-1 topology (i.e. 10 inputs, 20 hidden neurons and 1 output) was selected for use in further experiments because this gives the best generalization performance, as indicated by both the average and best percentage correct classifications for the independent test set.

11. The standard and adaptive gradient-descent algorithms produce significantly better values for the capture efficiency statistics, $D / A$ and $D \times(D / A)$, than the LevenbergMarquardt training algorithm. All algorithms produce similar average test-set classification (generalization) results.

12. The Levenberg-Marquardt algorithm converges approximately ten to one hundred times faster than the adaptive and standard gradient-descent algorithms, respectively.

13. The Levenberg-Marquardt algorithm also gives the best overall test-set performance. Consequently, this algorithm was chosen for subsequent experiments.

14. Sets of 10 and 17 input layers were tested as neural network inputs. The following criteria were used to rank and select the inputs; 1) layer from each evidence category with highest $D / A$ value, 2) $D / A$ and $D \geq \sim 20 \%, 3) D \times(D / A)$, and 4$) D \times(D / A)$ and $D \geq \sim 20 \%$ and $D / A \geq \sim 3$, where $D=\%$ of known deposits in the favourable region of the binary layer and $A=\%$ of the total map area occurring within the favourable region.

15. Similar generalization and map quality results were obtained for the sets of 10 inputs selected using the category, $D \times(D / A)$, and modified $D \times(D / A)$ criteria. Layers selected according to the $D / A$ criterion produce significantly poorer results.

16. The set of 10 input layers selected using the $D \times(D / A)$ criterion result in slightly better average and best classification performance for the test set overall, higher values for the area under the ROC curves, and significantly better classification performance for deposit patterns, than the evidence-category, $D / A$, and modified $D \times(D / A)$ criteria 
17. The set of 17 inputs selected with the $D \times(D / A)$ criterion produce the highest average test-set (overall) classification performance and significantly higher average values for the capture efficiency ratios, $D / A$ and $D \times(D / A)$, than other criteria.

18. In general, networks trained with 17 layers give slightly better average test-set classification results than networks trained with 10 layers.

19. Layers selected using the category and $D \times(D / A)$ criteria were used for experiments with random noise and fuzzy inputs.

20. Networks trained with $1 /(x)$ and $\log _{10}(x)$ transformed distance inputs produce poorer results compared to those without any transformation.

21. Buffer limits were placed on layers that involve distance to linear features, such as faults and lithological contacts, to model the concept that the existence of a fault or contact can have no effect on the prospectivity beyond a certain distance limit. Cell values at distances exceeding the limit were set to the maximum distance, and, in strike orientation layers, the strike angle was set to the mean for the entire map grid.

22. Buffer limits were estimated by interpreting plots of $(O-E) / E$ (where $O=$ observed number of deposits in a region and $E=$ expected number of deposits for a uniform random distribution).

23. The use of both narrow and wide buffers causes a significant decrease in the generalization ability of the trained networks.

24. The use of narrow buffers produces a significant increase in the average values of the capture efficiency statistics, $D / A$ and $D \times(D / A)$.

25. Narrow buffer limits were also applied to the rheological and chemical contrast layers but have little effect on generalization. Contrast was set to zero for cells located outside the buffer limits.

26. The overall size of the training data sets is limited by the small number of known gold deposits.

27. Plots of the percentage correct classification, for the test set overall, deposit patterns in the test set, and $D \times(D / A)$ and area under the ROC curve, show a trend of significantly increasing generalization-ability and map quality with increasing numbers of deposit patterns and training data set size.

28. The addition of random noise to augment the number of deposit patterns available for training (from approximately 50 to 1000) significantly improves the classification 
performance of trained networks and the quality of the resultant prospectivity maps. The average values of $D / A$ and $D \times(D / A)$ are approximately doubled.

29. The results for $\pm 40 \%$ noise represent a very significant improvement in the percentage correct classification for deposit patterns (i.e. from $68.0 \%$ to $77.1 \%$ ).

30. The effect of training the network with patterns created with $\pm 80 \%$, rather than $\pm 40 \%$, noise is to reduce the size of areas estimated to have high prospectivity. There is a similar difference between maps produced using $\pm 5 \%$ and $\pm 10 \%$ normallydistributed noise.

31. Average results for normally-distributed noise are generally poorer than those for uniform random noise. However, networks trained using $\pm 5 \%, \pm 10 \%$ and $\pm 20 \%$ normally-distributed noise result in slightly better, best test-set classification results than those trained with uniform noise.

32. The network with $\pm 10 \%$ normally-distributed noise, that gives the best overall test-set performance, also produces the best test-set deposit pattern classificationperformance of all the networks tested, that is, $80.3 \%, 80.0 \%$, and $80.4 \%$ for the overall, deposit, and barren test-set patterns, respectively.

33. Increasing the size of the training-stop data set results in a further small increase in classification performance and map quality statistics compared to increasing the size of the training set only.

34. The subjective fuzzy rheological-contrast layer shows the strongest association with known deposits and was therefore selected as the layer to use as a neural network input.

35. Fuzzy membership values and 1-of-n coding of host-rock geology produce similar network performance statistics. However, the best test-set results are obtained using fuzzy membership values. The $D \times(D / A)$ value (measure of map quality) is much higher for the network with the fuzzy membership than 1-of-n coded geology input.

36. The results above suggest that fuzzy membership values are a viable alternative to 1-of-n coding for categorical data inputs to a neural network in this domain.

37. An extension of the concept of using fuzzy membership values, in order to represent categorical data types as inputs, is to use fuzzy membership values for all the network inputs. Such a system effectively represents a simple form of hybrid fuzzy-neural network system.

38. Networks with 1, 3, and 10 fuzzy inputs produce similar results for the percentage 
correct classification of the test-set data.

39. The highest overall test-set classification accuracies are obtained with networks using only fuzzy inputs; that is, $79.0 \%$ for the best 10 input network with layers selected with the category criteria, and $79.0 \%$ for the best 17 input network with layers selected using the $D \times(D / A)$ criterion.

40. Better recognition of the deposit patterns in the test data set is achieved with networks using layers selected according to the $D \times(D / A)$ criterion than with the categories criterion. This applies to both average and best test-set classification performance, and 10 and 17 inputs.

41. Networks using the $D \times(D / A)$ set of layers also generally give significantly higher values for the measures of prospectivity map quality (i.e. Spearman's correlation coefficient, $D / A, D \times(D / A)$, and area under the ROC curve).

42. The generalization performance of the fuzzy systems is significantly poorer than for neural networks and hybrid fuzzy-neural networks.

43. The poorer generalization performance of the fuzzy and, to a lesser extent, the fuzzyneural network, systems may be due the way that fuzzy membership functions in fuzzy systems narrow the definition of what constitutes highly favourable locations for a gold deposit. This excludes many deposits which do not fit this simpler model.

44. Fuzzy systems and hybrid fuzzy-neural networks produce better map quality statistics than neural networks with the same inputs.

45. Very high values of map quality statistics, $D / A$, and $D \times(D / A)$, for maps produced using fuzzy systems are due to the fact that the region corresponding to the highest prospectivity map class contains a small number of deposits in a very small proportion of the map area. However, for a 9-class prospectivity map, the bulk of the deposits are located in prospectivity classes 5-7. In contrast, a hybrid fuzzy-neural network system, with the same 10 inputs, generates a map in which the bulk of the deposits are located in map classes 7-9.

46. Histograms of the system output values suggest that the higher capture efficiency ratios, $D / A$, and $D \times(D / A)$, produced by fuzzy and, to a lesser degree, hybrid fuzzy-neural network systems, are due to the fact that high prospectivity values are assigned to a much smaller proportion of the map than in maps produced with neural networks. 
47. A comparison of prospectivity maps produced with neural network, fuzzy and hybrid fuzzy-neural network systems shows that the hybrid system produces the best tradeoff in terms of sensitivity to the detail in the input GIS layers, while displaying overall trends in the data without significant noise nor loss of information. 


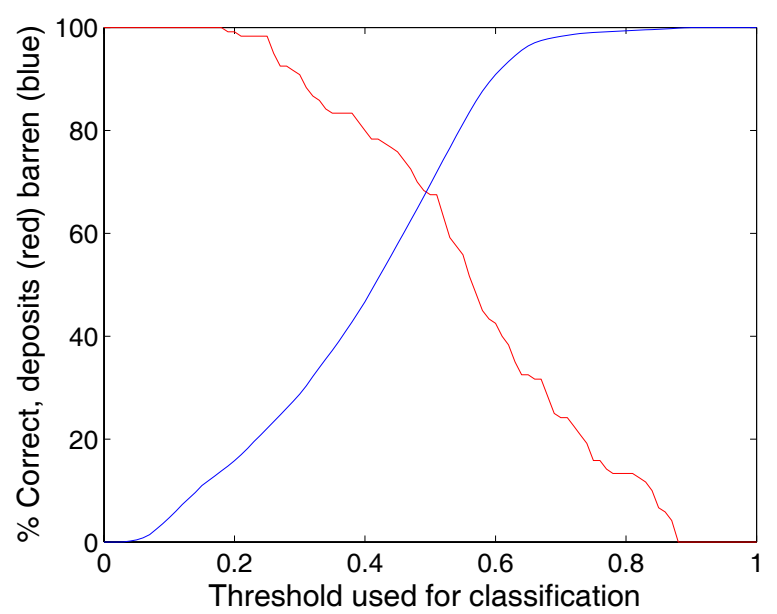

a) Classification accuracy versus threshold.

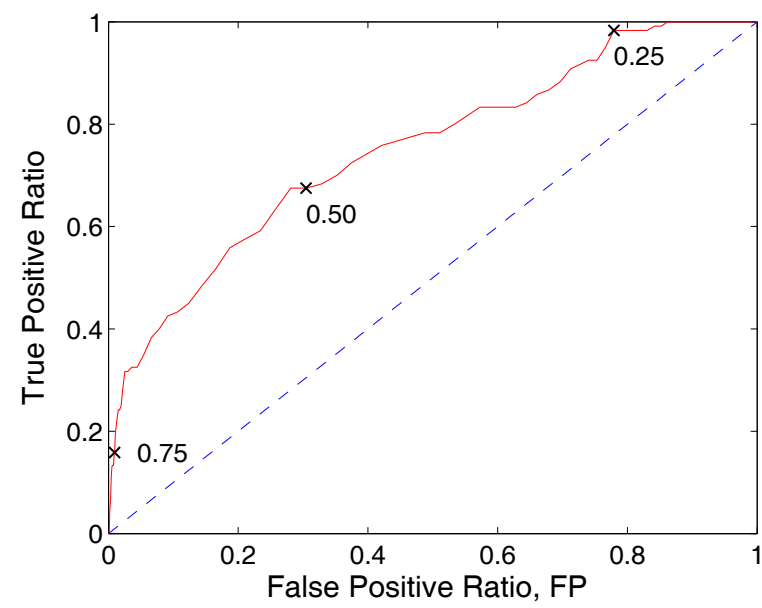

c) ROC curve showing threshold values.

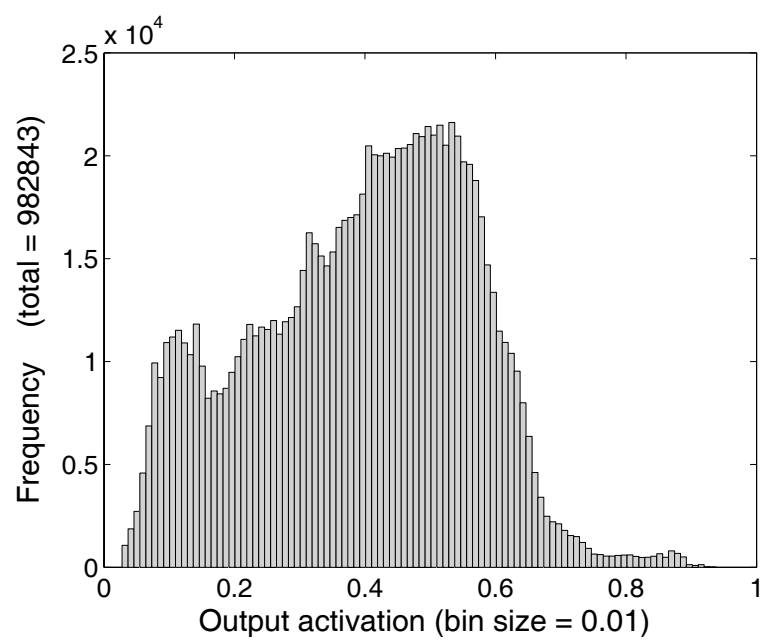

(e) Histogram of output activations of trained networks.

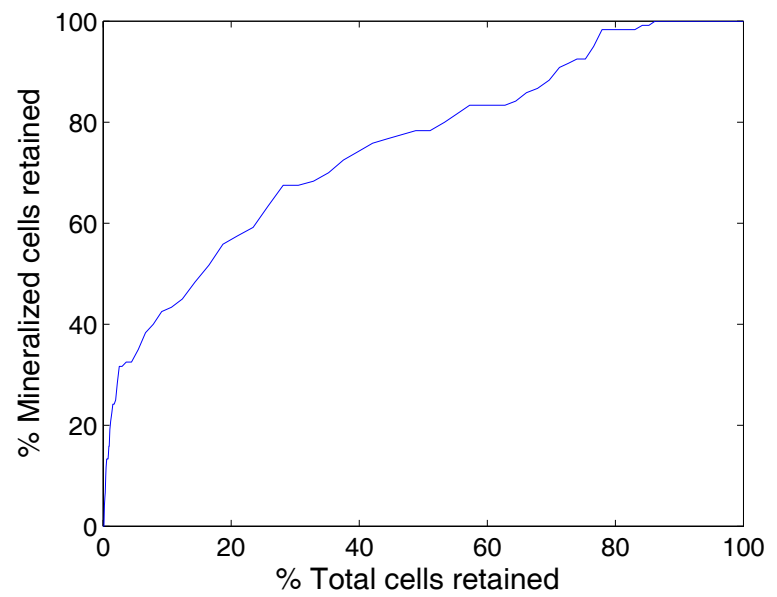

b) Percentage of deposit versus total cells captured for classification thresholds in the range $[0,1]$.

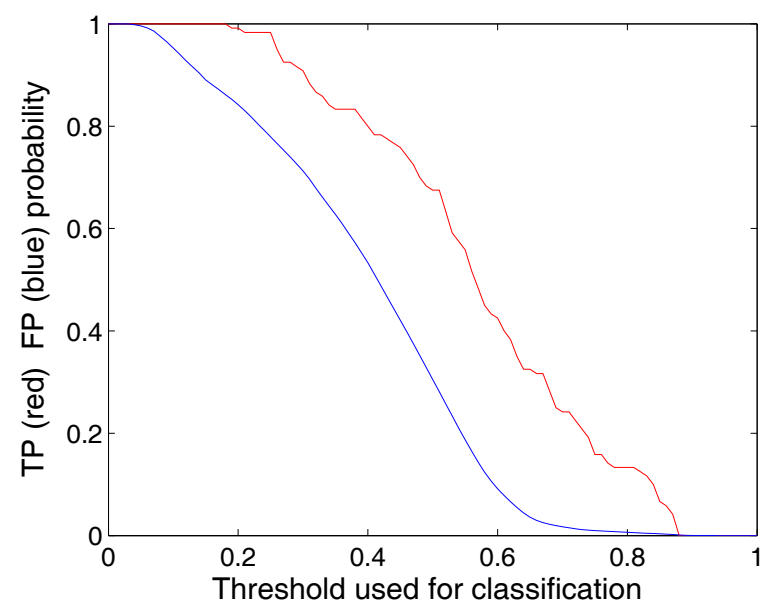

d) Proportion of correct and false deposit classification vs classification threshold.

Figure 7.18. Classification performance of the 10-0-1 neural network with the best test-set classification accuracy. Input layer selection: categories. Learning algorithm: trainlm. 


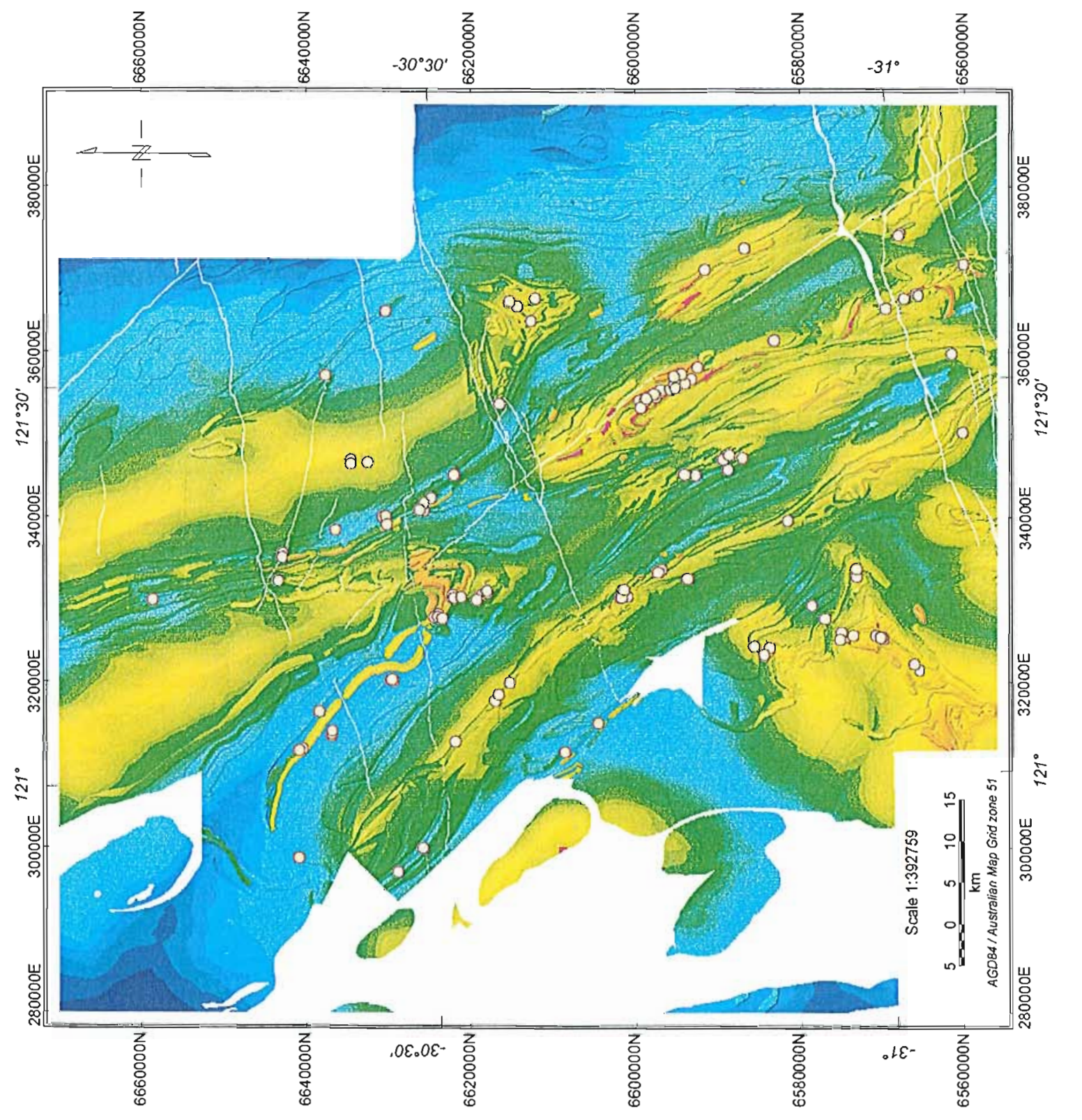

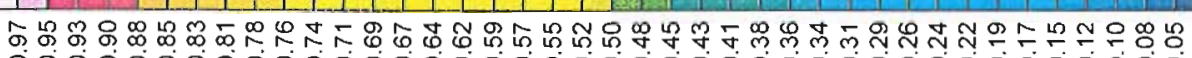

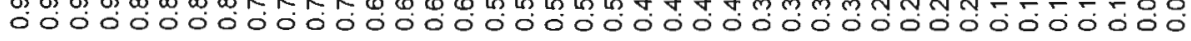

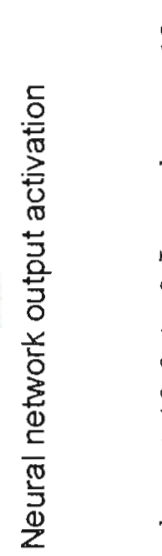

$\ddot{0}$

营

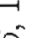

is

$0_{1}^{1}$

岁

穷

覂

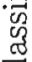

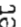

竎

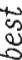

\&

营

농

号

ঢ’

ᄋ

로

要

范

马ํ.

동

.

零

을.

द्व

总

壱

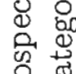

范

م.

จ.

원

常 


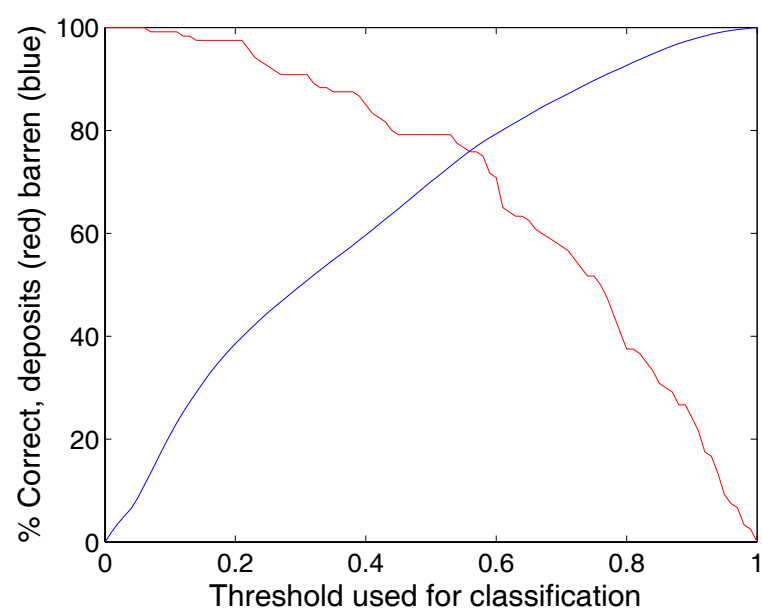

a) Classification accuracy versus threshold.

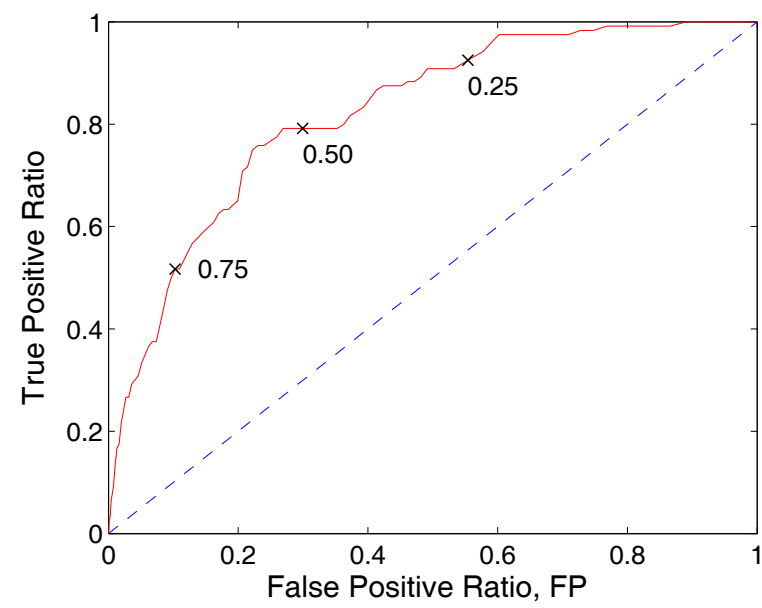

c) ROC curve showing threshold values.

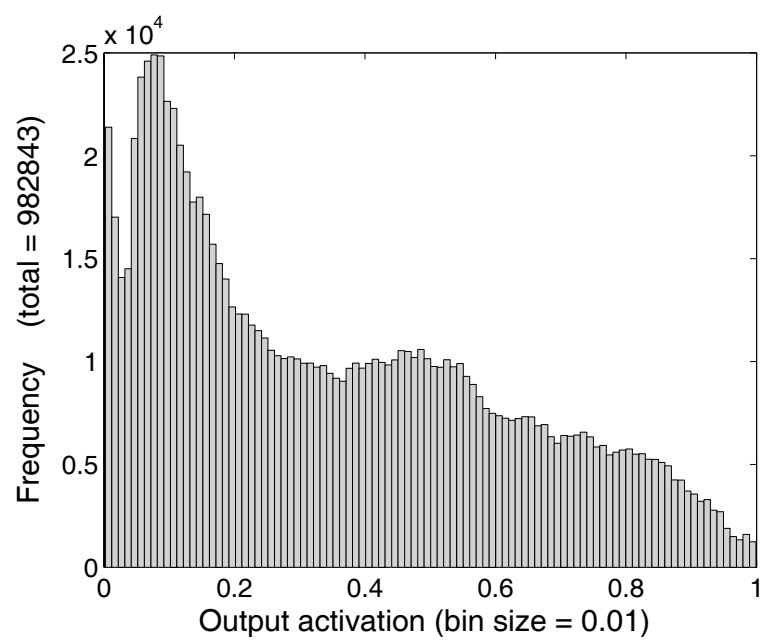

(e) Histogram of output activations of trained networks.

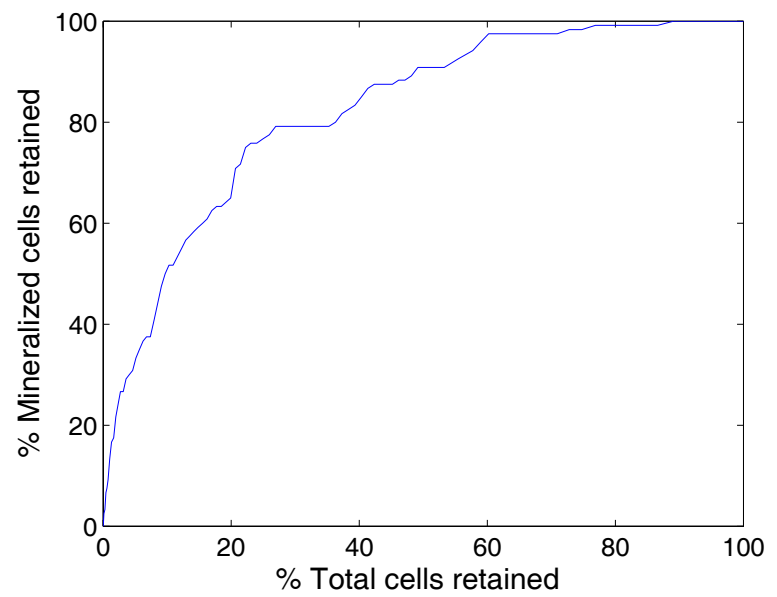

b) Percentage of deposit versus total cells captured for classification thresholds in the range $[0,1]$.

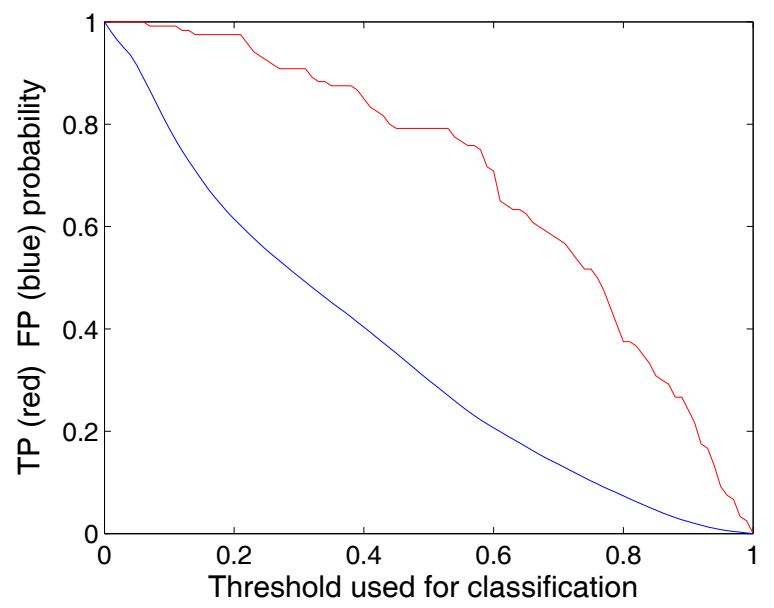

d) Proportion of correct and false deposit classification vs classification threshold.

Figure 7.20. Classification performance of the 10-20-1 neural network with the best test-set classification accuracy. Input layer selection: categories. Learning algorithm: trainlm. 


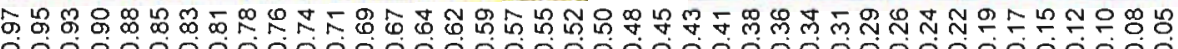

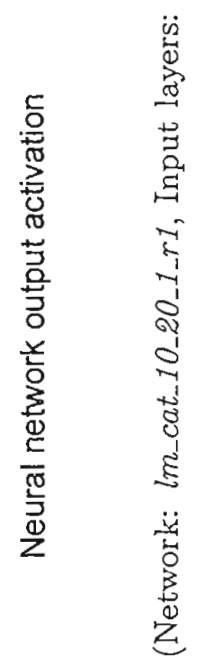

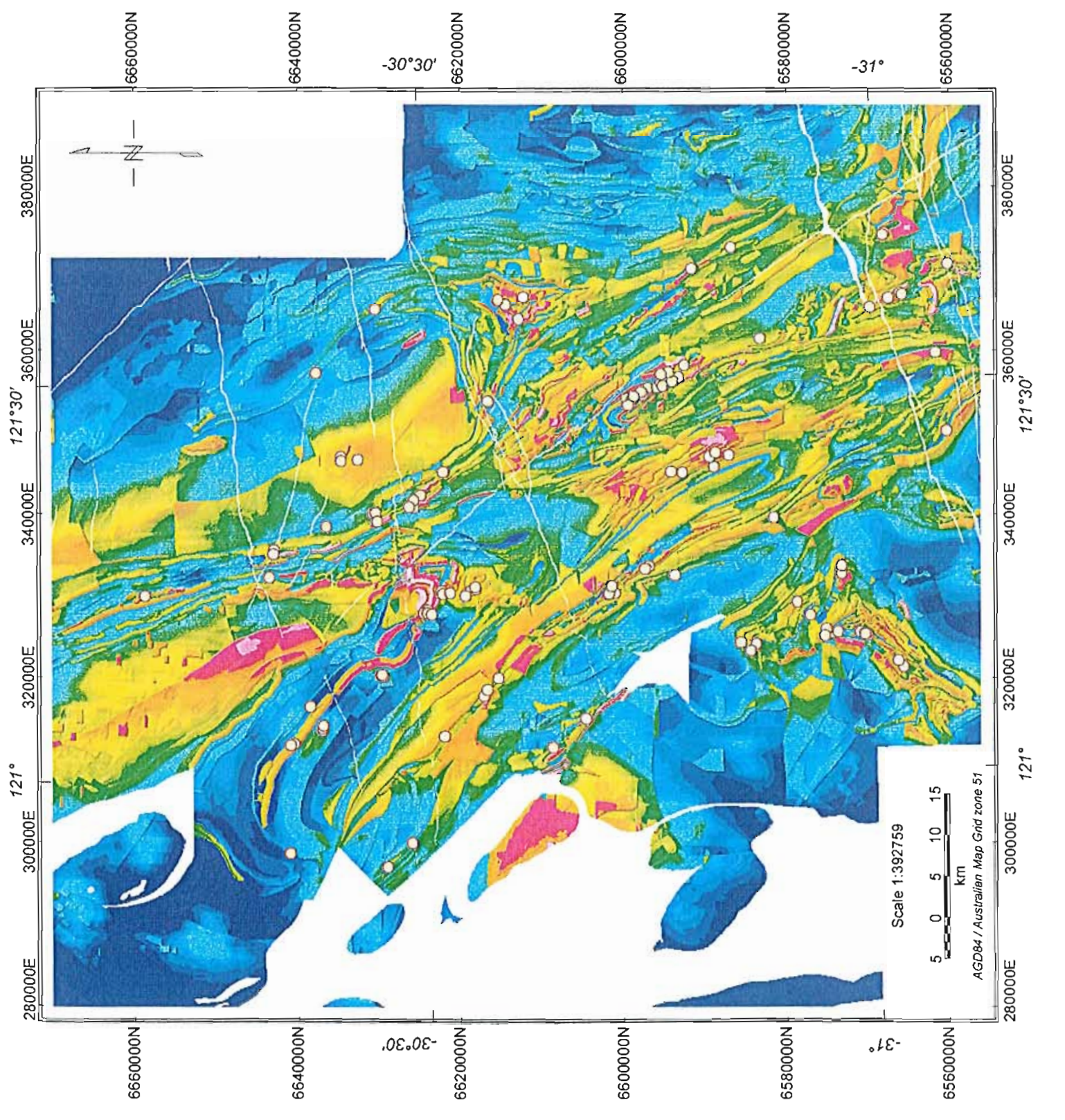

.

岕

剀

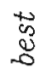

$\stackrel{9}{4}$

要

$\rightarrow$

离

뭉

용

윰

혼

bo

量高

$\exists$

\%

.

要

․․․․

용

घ)

总

용

迅

号

D.

का

N.

(1)

0 


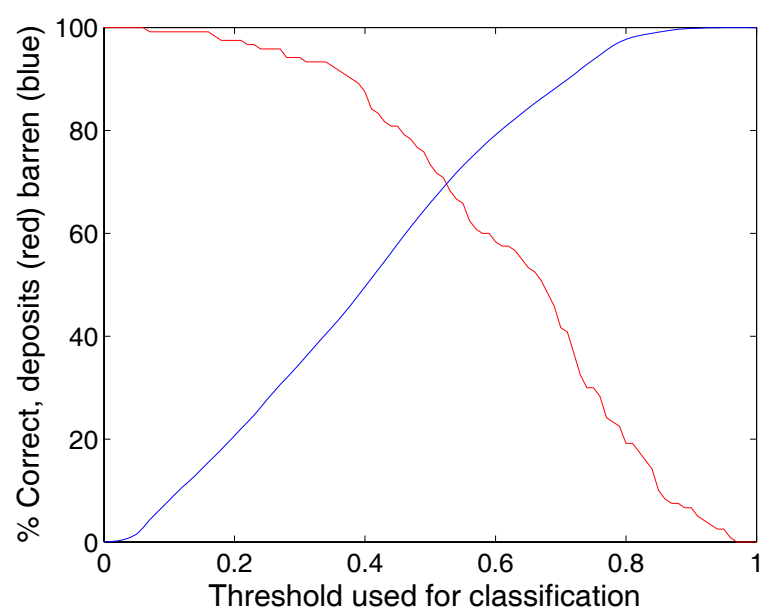

a) Classification accuracy versus threshold.

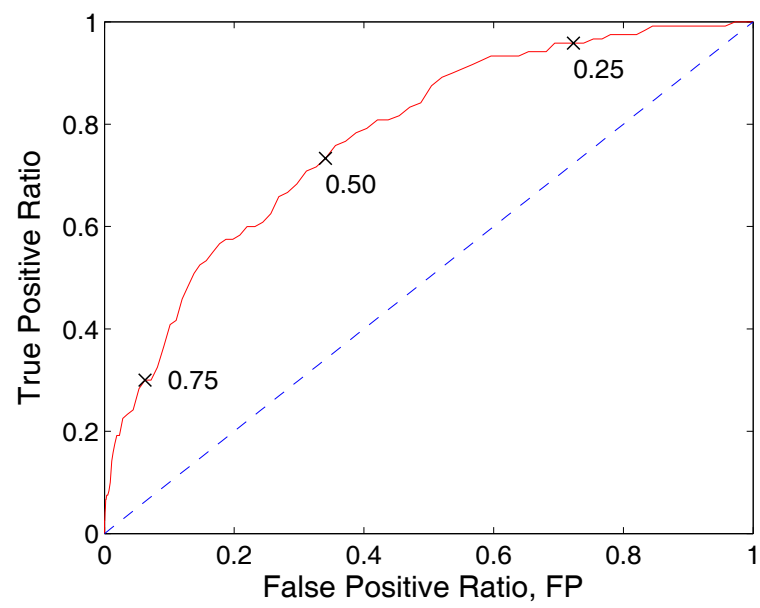

c) ROC curve showing threshold values.

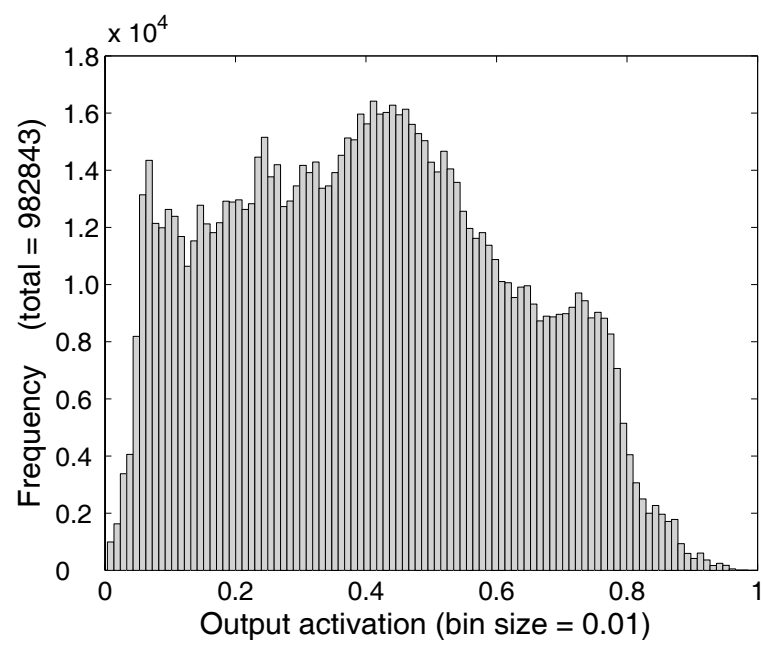

(e) Histogram of output activations of trained networks.

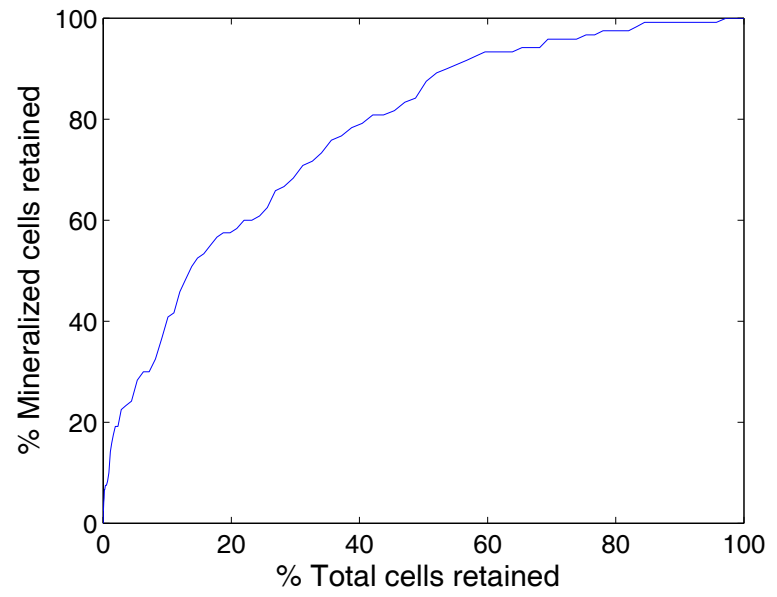

b) Percentage of deposit versus total cells captured for classification thresholds in the range $[0,1]$.

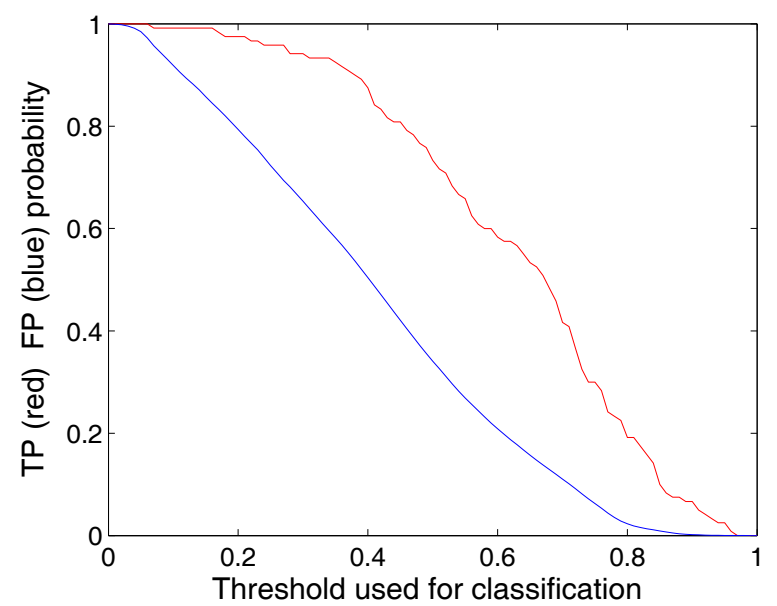

d) Proportion of correct and false deposit classification vs classification threshold.

Figure 7.22. Classification performance of the 10-20-1 neural network with the best test-set classification accuracy. Input layer selection: categories. Learning algorithm: gradient descent with adaptive learning rate and momentum (traingdx). 


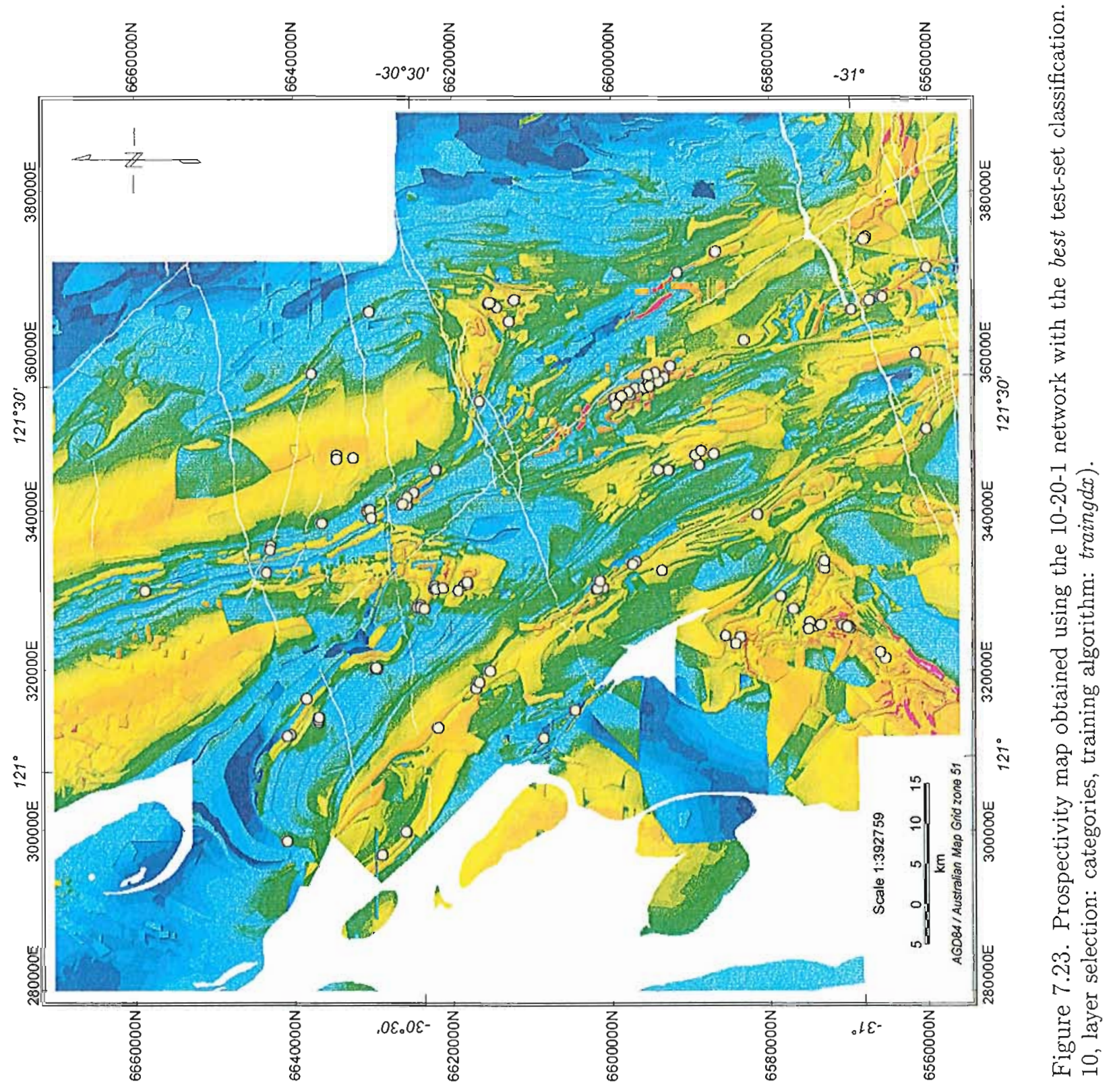




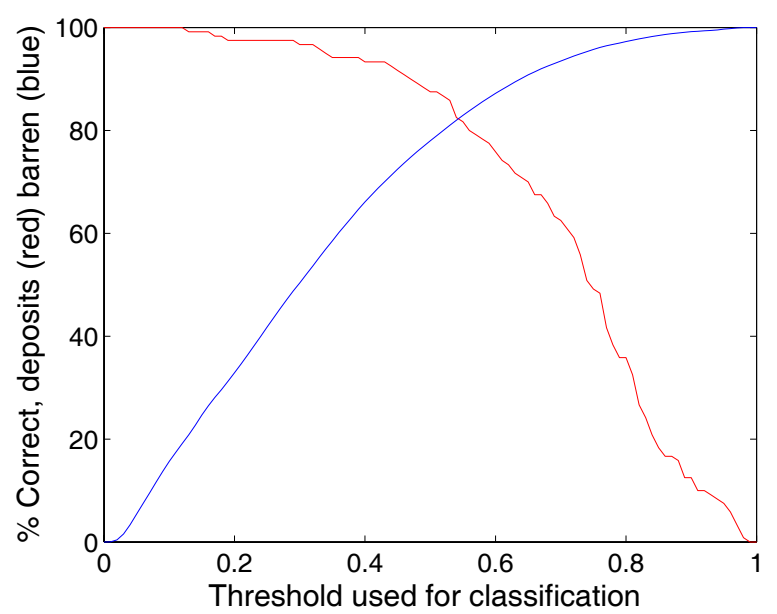

a) Classification accuracy versus threshold.

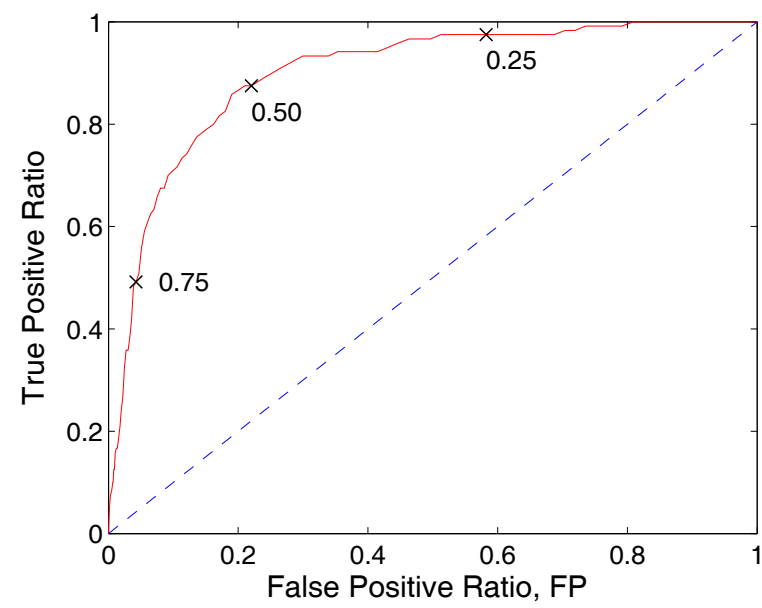

c) ROC curve showing threshold values.

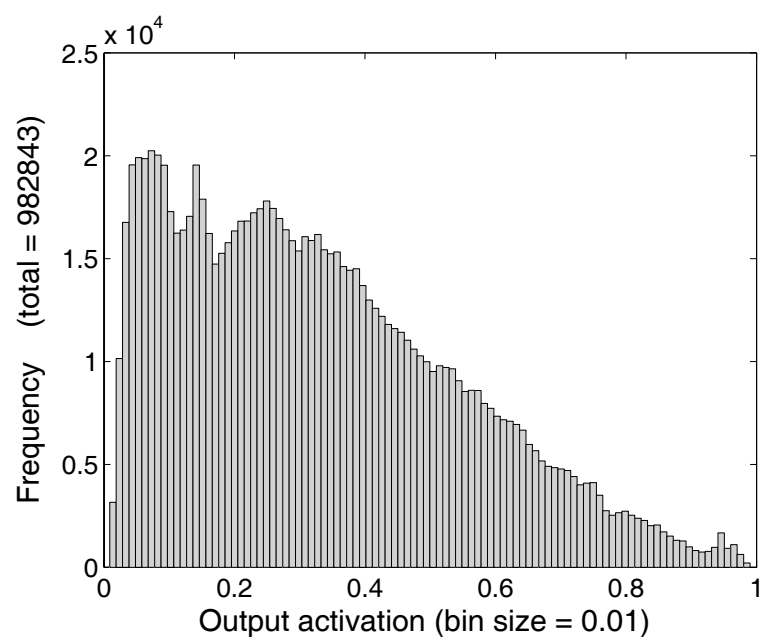

(e) Histogram of output activations of trained networks.

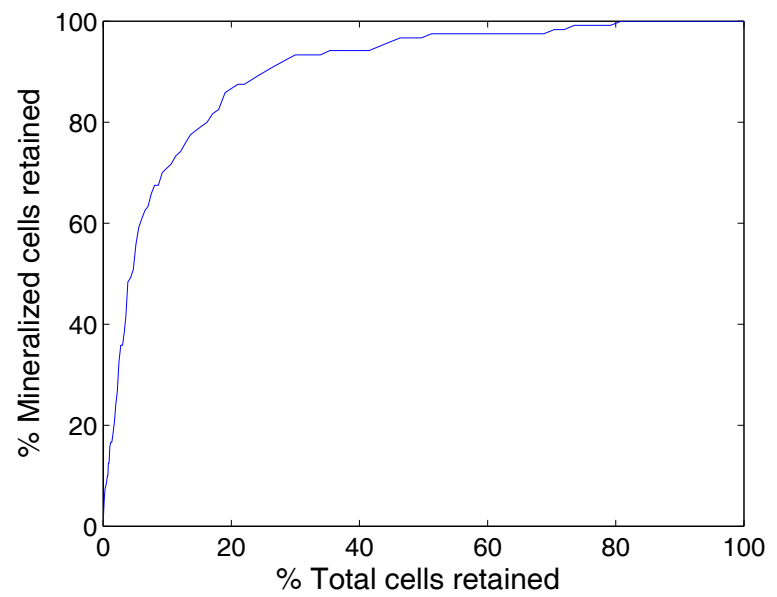

b) Percentage of deposit versus total cells captured for classification thresholds in the range $[0,1]$.

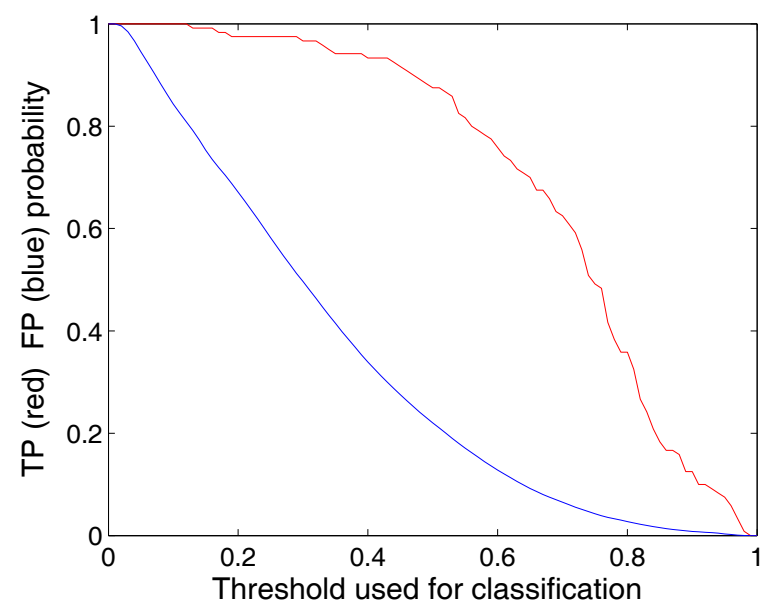

d) Proportion of correct and false deposit classification vs classification threshold.

Figure 7.24. Classification performance of the 10-20-1 neural network with the best test-set deposit classification accuracy. The training data set was expanded using $\pm 40 \%$ uniform random noise. Input layer selection: categories. Learning algorithm: trainlm. 


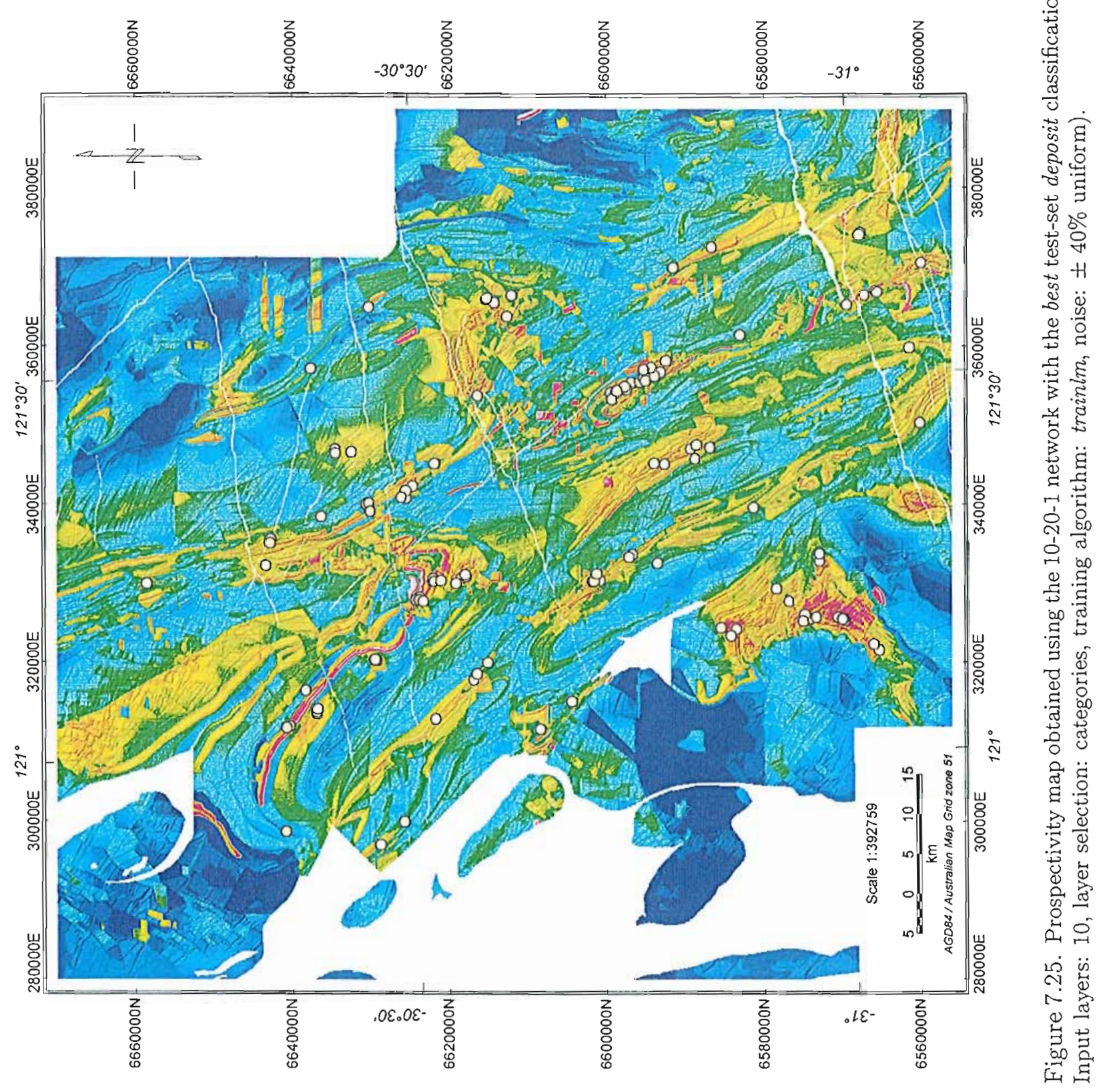




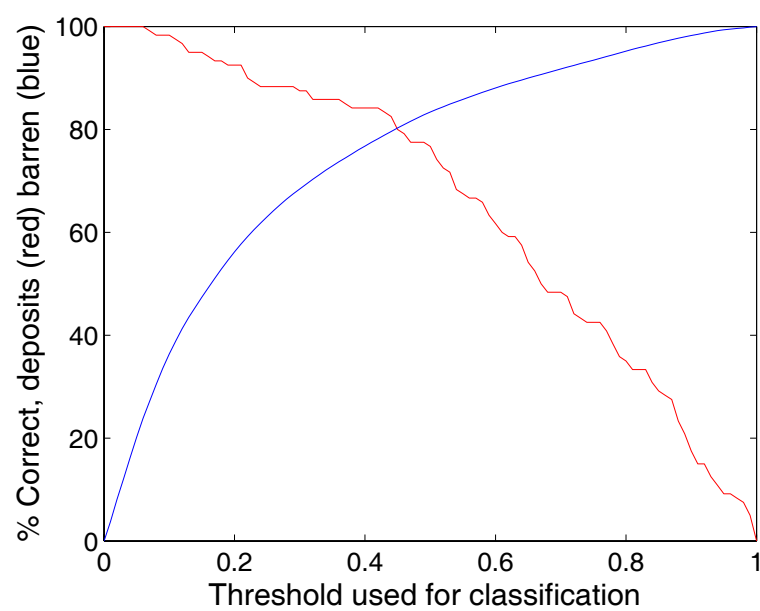

a) Classification accuracy versus threshold.

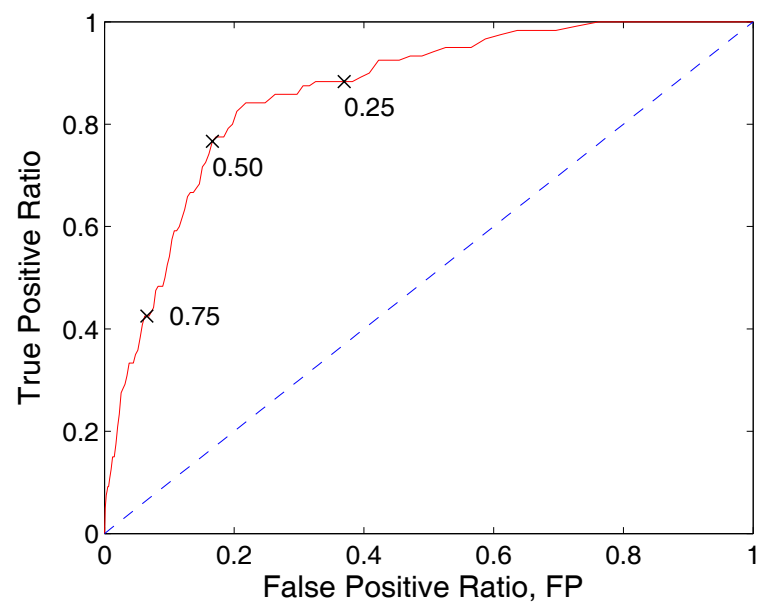

c) ROC curve showing threshold values.

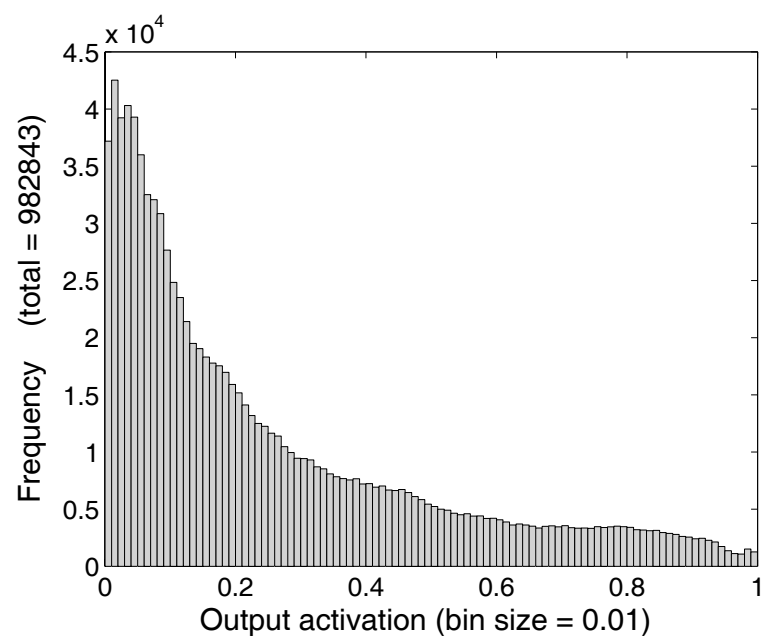

(e) Histogram of output activations of trained networks.

Figure 7.26. Classification performance of the 10-20-1 neural network with the best test-set classification accuracy. The training data set was expanded using $\pm 80 \%$ uniform random noise. Input layer selection: categories. Learning algorithm: trainlm.

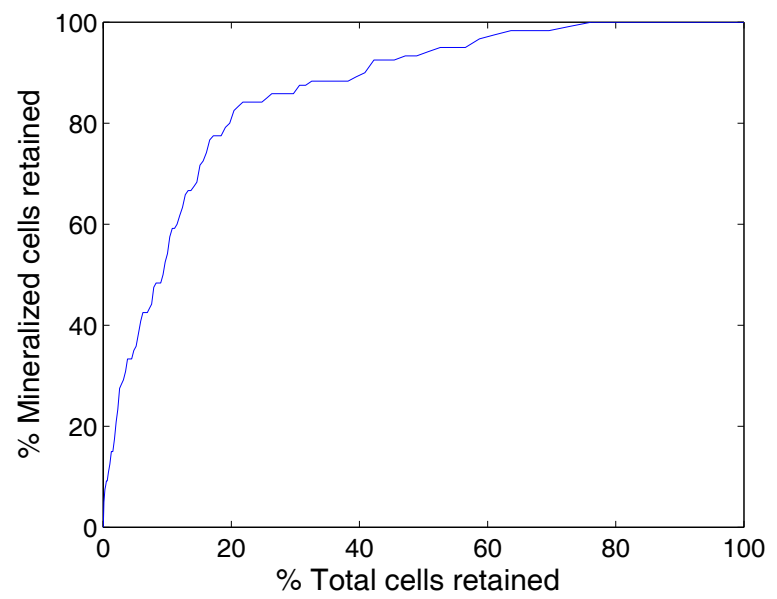

b) Percentage of deposit versus total cells captured for classification thresholds in the range $[0,1]$.

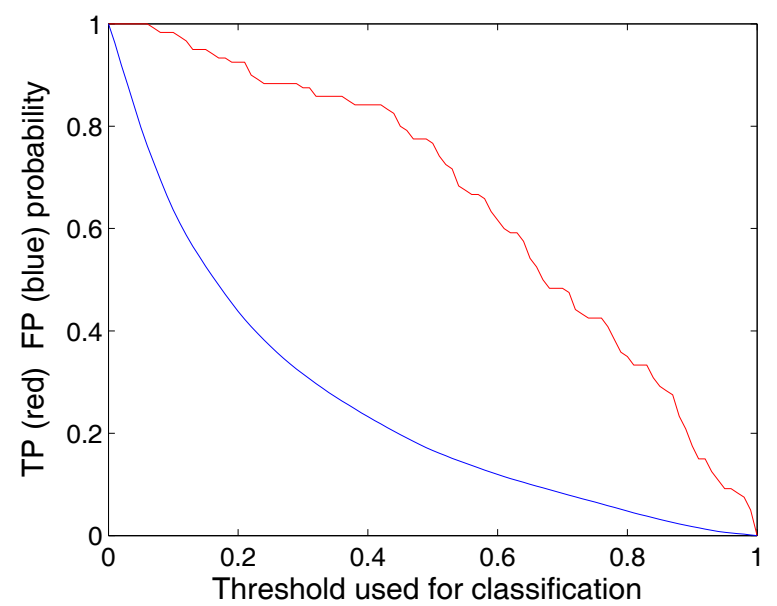

d) Proportion of correct and false deposit classification vs classification threshold. 


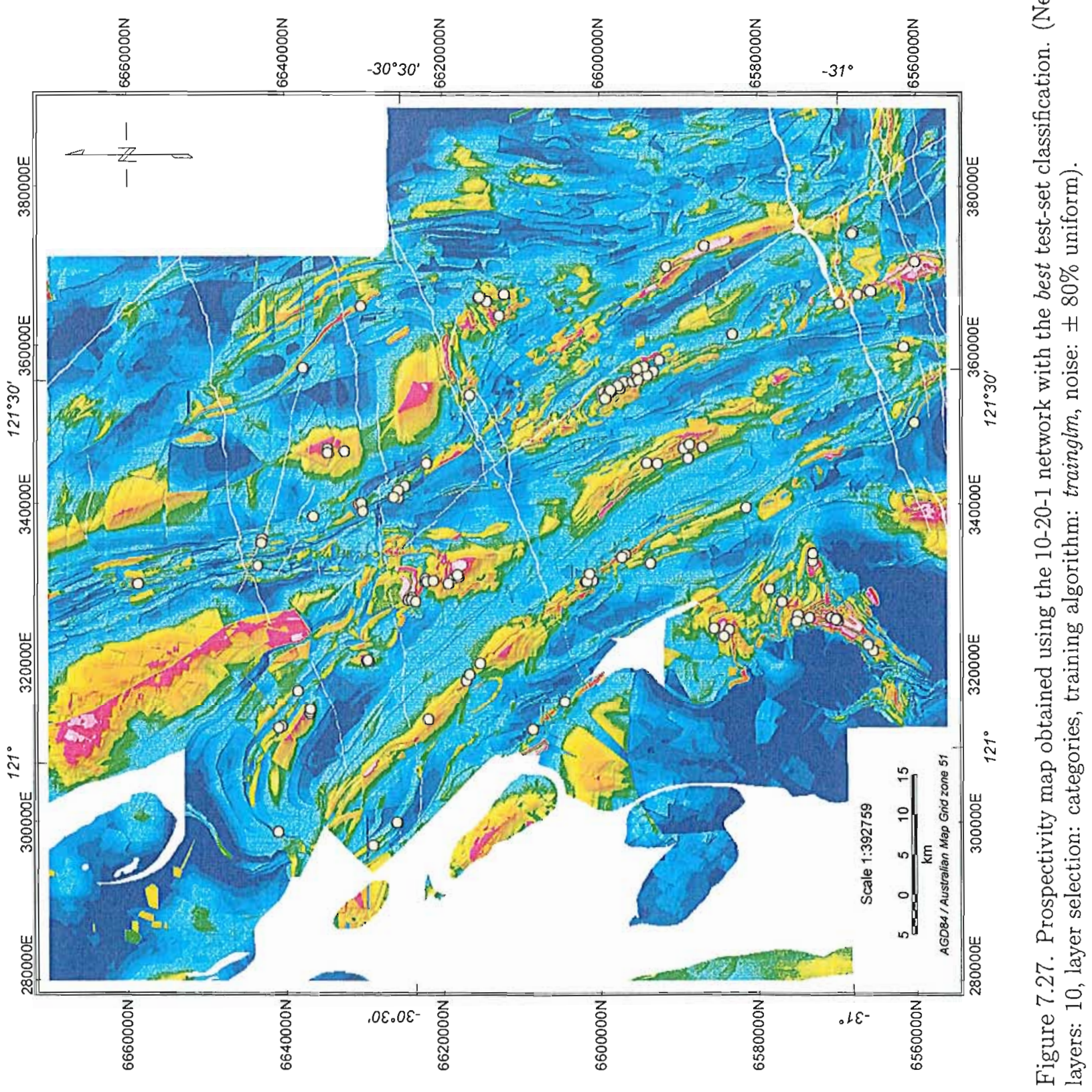

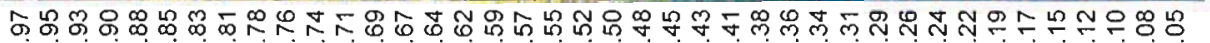

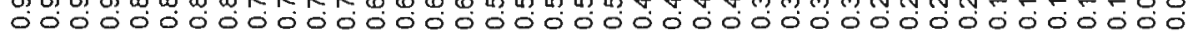

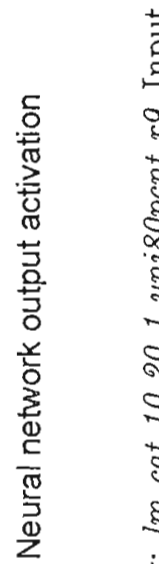

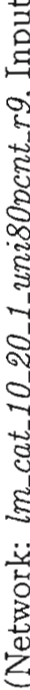

.9

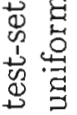

芯

$\stackrel{\Xi}{\leftrightarrows} H$

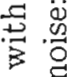

है

$\stackrel{0}{\square}$

$\rightarrow+$

尺े

곤

논

:

व

๑ ڤ

है

志

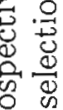

ค.

$\therefore$ 胥

$N \subseteq$

永㐘 


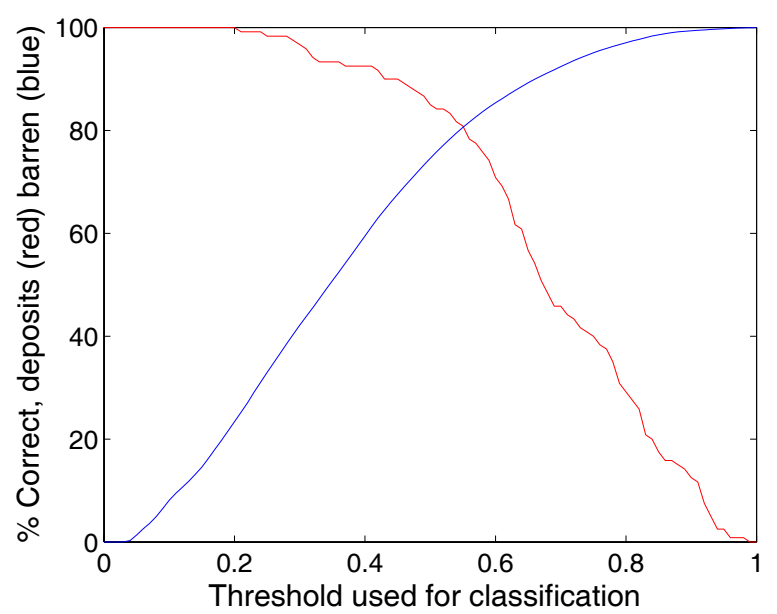

a) Classification accuracy versus threshold.

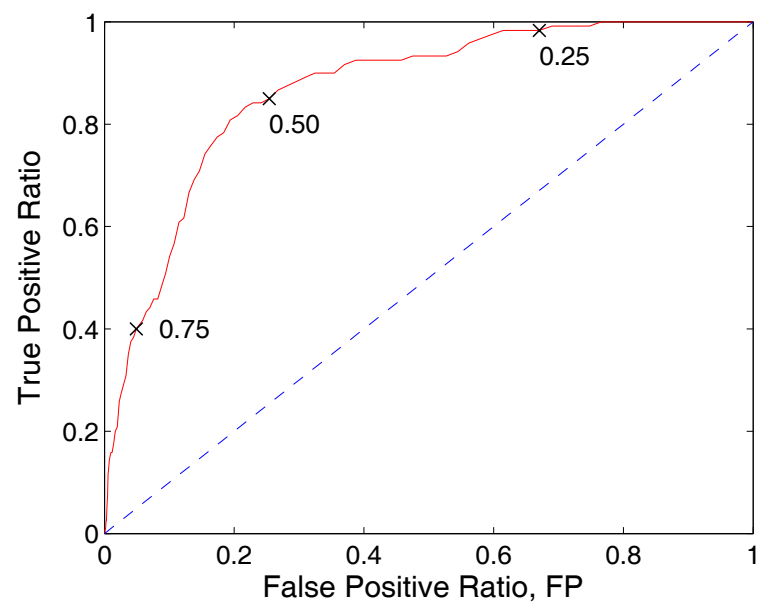

c) ROC curve showing threshold values.

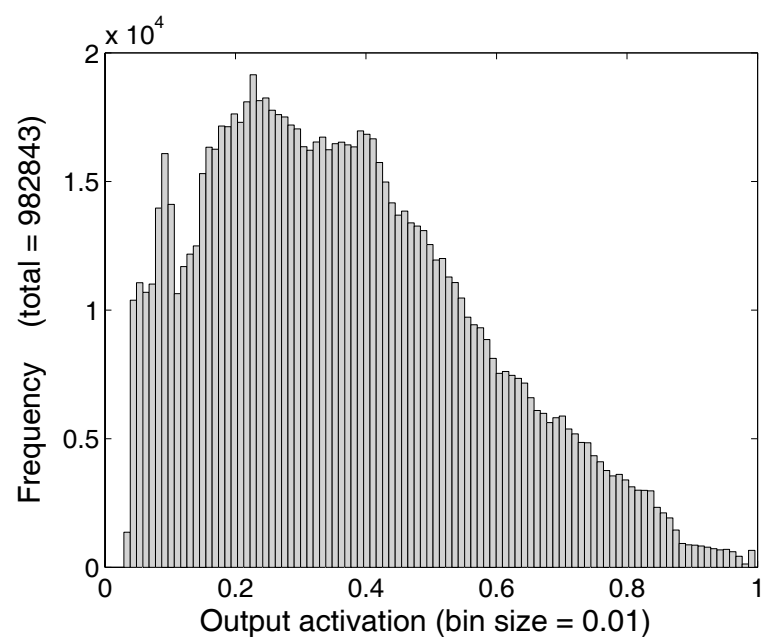

(e) Histogram of output activations of trained networks.

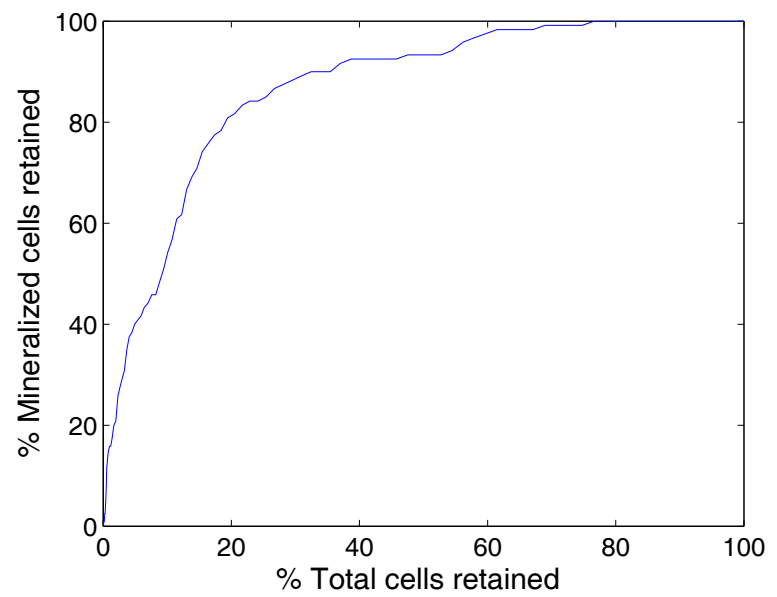

b) Percentage of deposit versus total cells captured for classification thresholds in the range $[0,1]$.

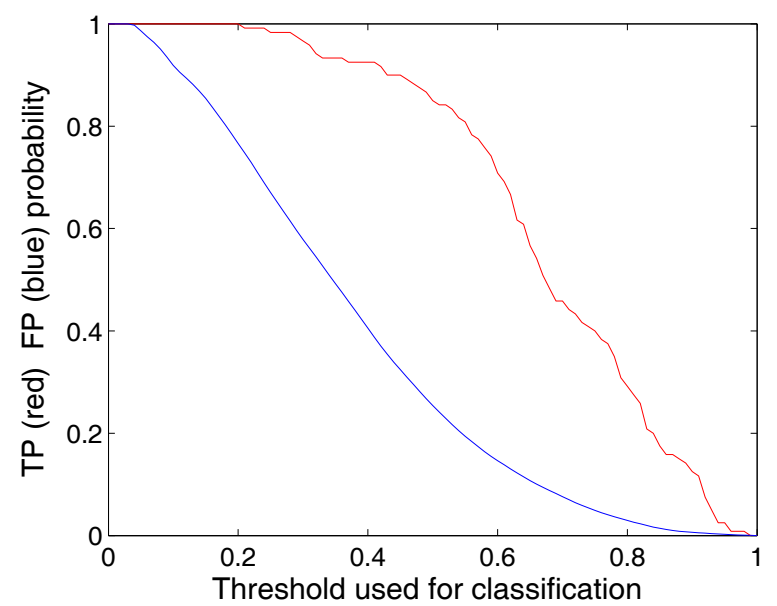

d) Proportion of correct and false deposit classification vs classification threshold.

Figure 7.28. Classification performance of the 10-20-1 neural network with the best test-set deposit classification accuracy. The training data set was expanded using $\pm 5 \%$ normally-distributed random noise. Input layer selection: categories. Learning algorithm: trainlm. 


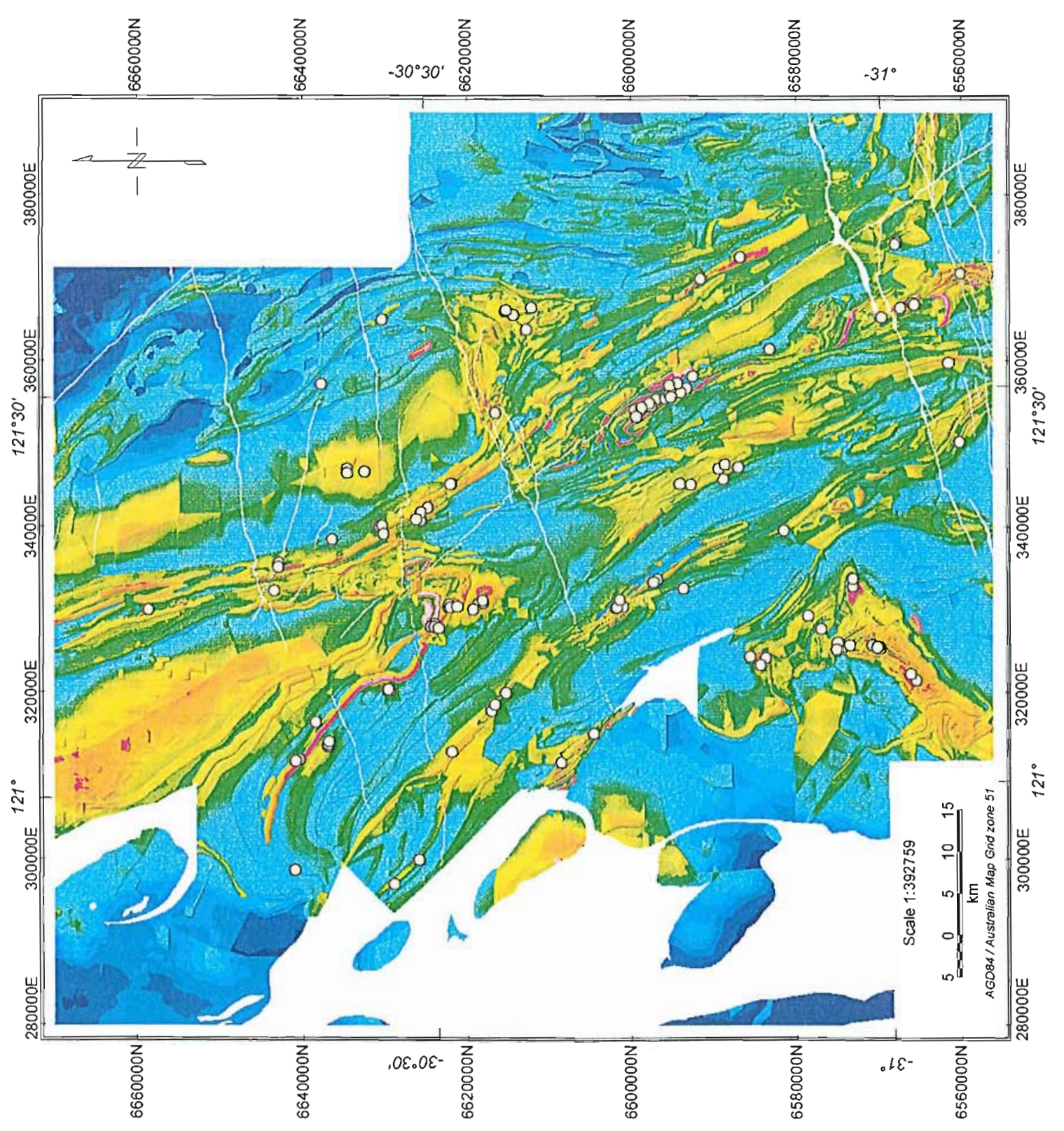

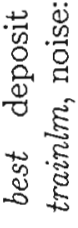

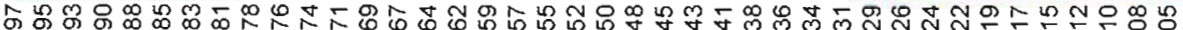

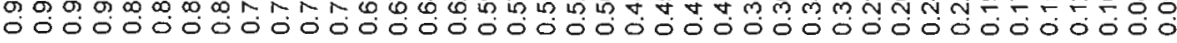

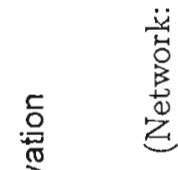

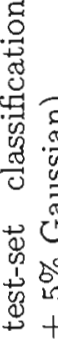

$+1$

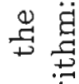

要要

产葛

范

$\rightarrow$

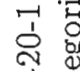

常离

总.

赵

氙

完

承

융

要

总

ह

है

की है

i

है

จิ จิ

总 


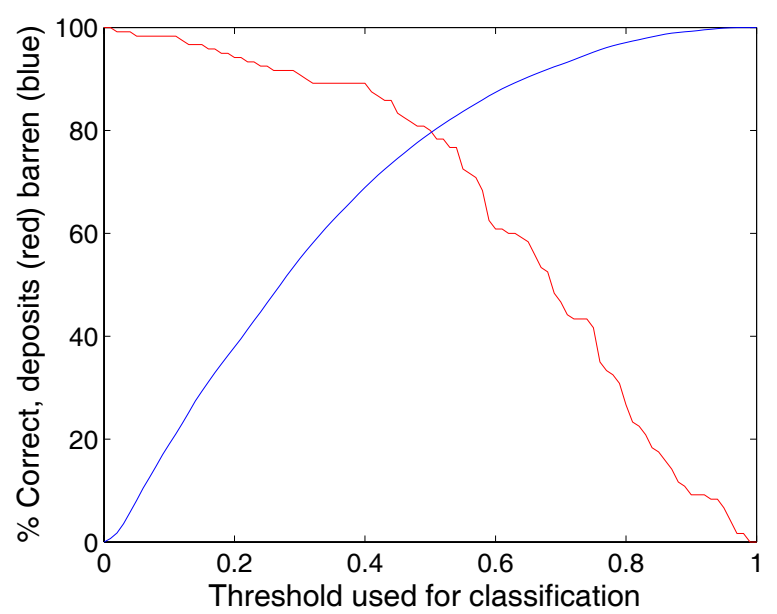

a) Classification accuracy versus threshold.

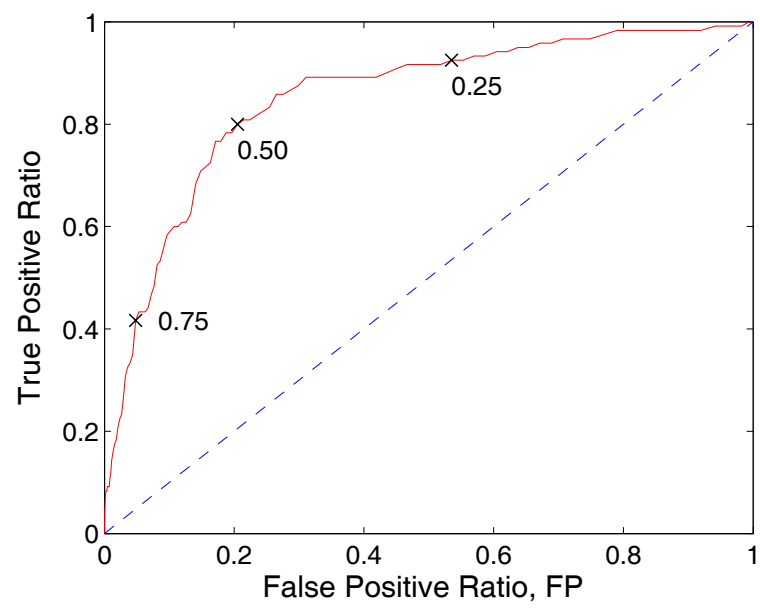

c) ROC curve showing threshold values.

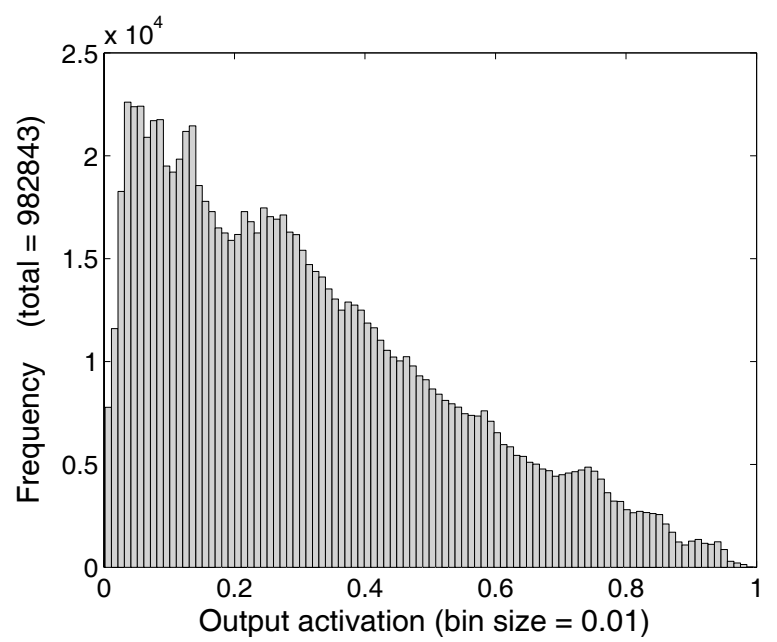

(e) Histogram of output activations of trained networks.

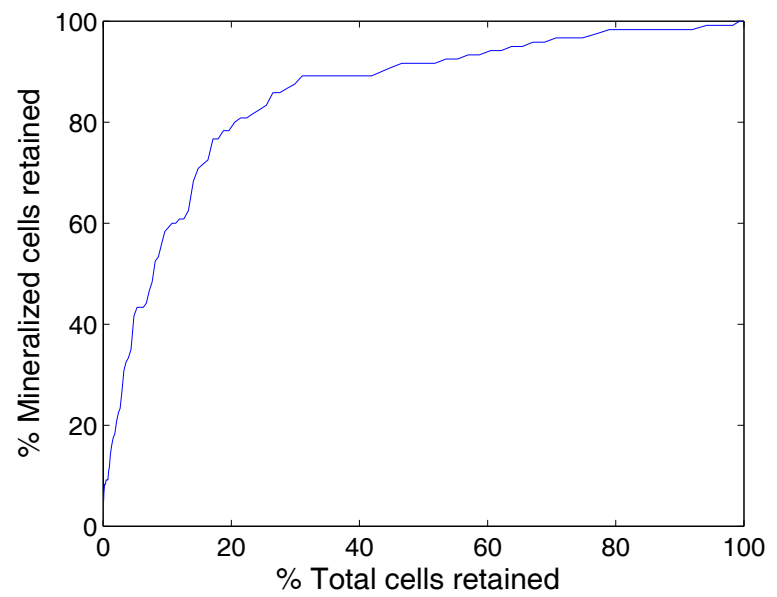

b) Percentage of deposit versus total cells captured for classification thresholds in the range $[0,1]$.

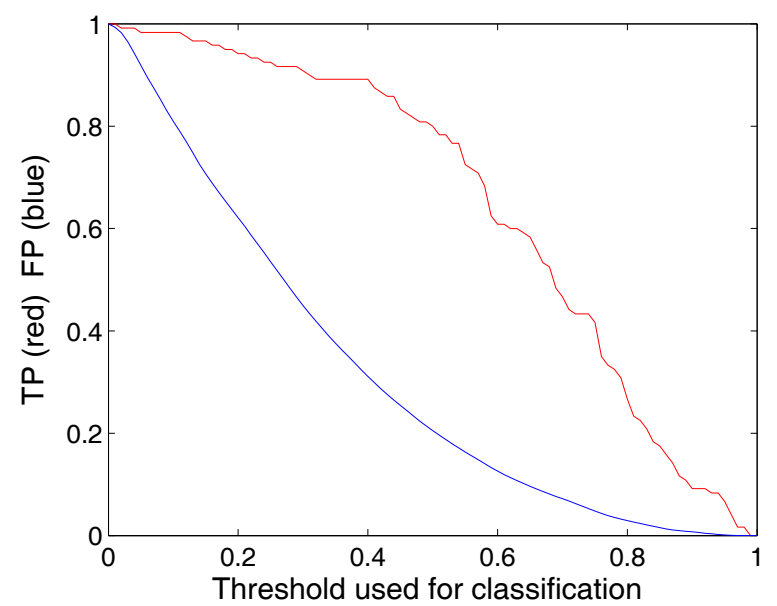

d) Proportion of correct and false deposit classification vs classification threshold.

Figure 7.30. Classification performance of the 10-20-1 neural network with the best test-set classification accuracy. The training data set was expanded using $\pm 10 \%$ normally-distributed random noise. Input layer selection: categories. Learning algorithm: trainlm. 


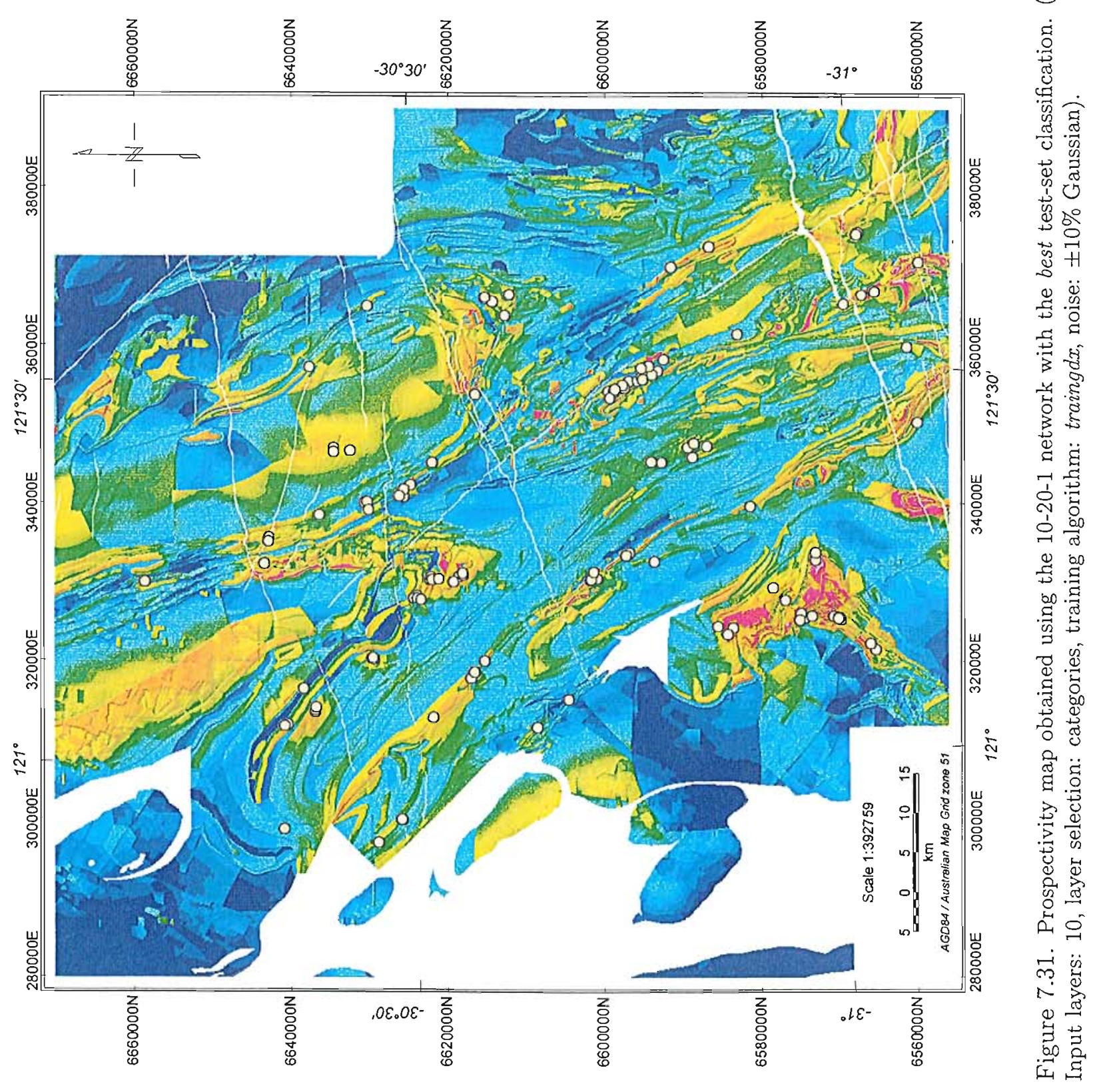




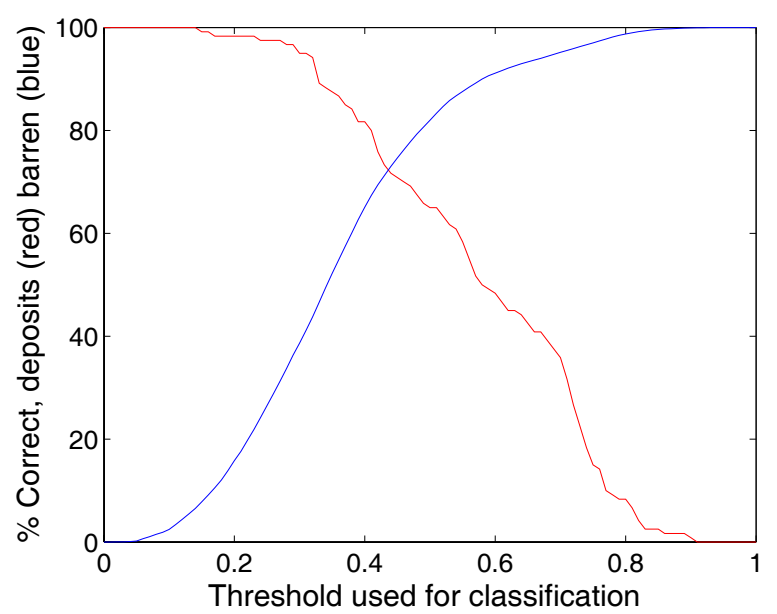

a) Classification accuracy versus threshold.

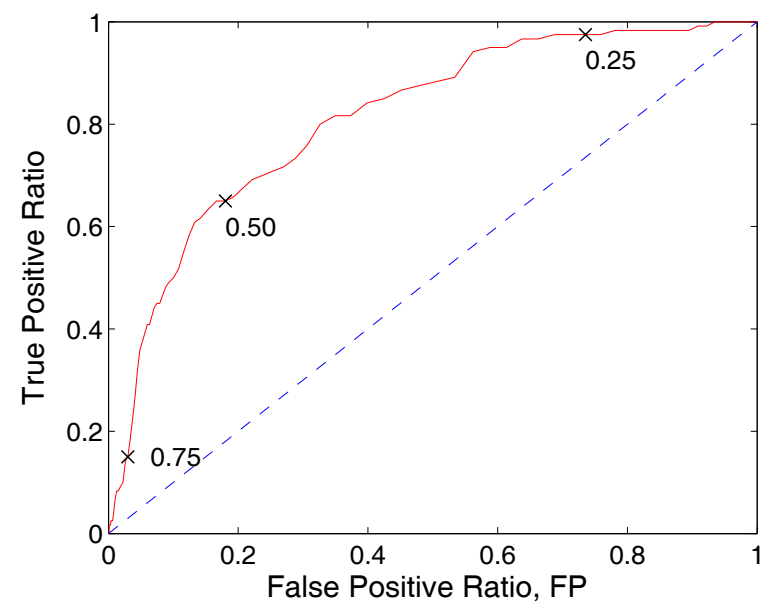

c) ROC curve showing threshold values.

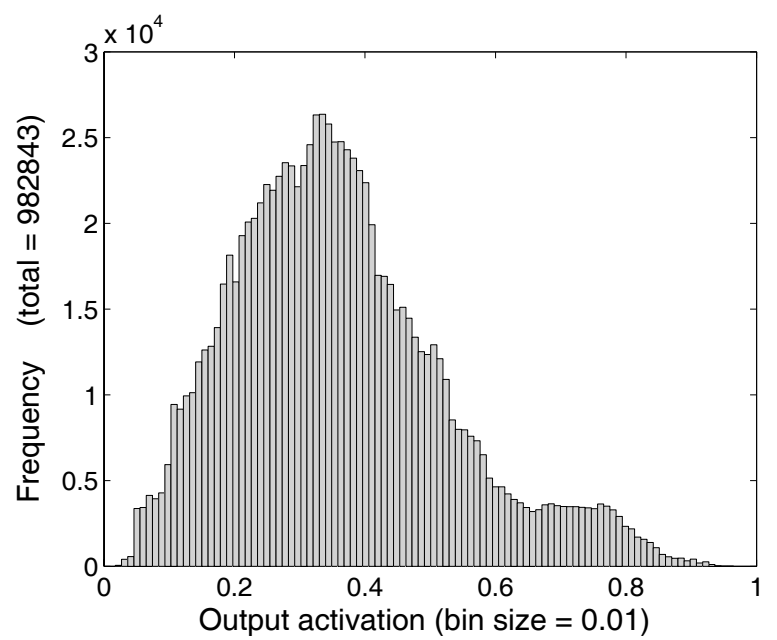

(e) Histogram of output activations of trained networks.

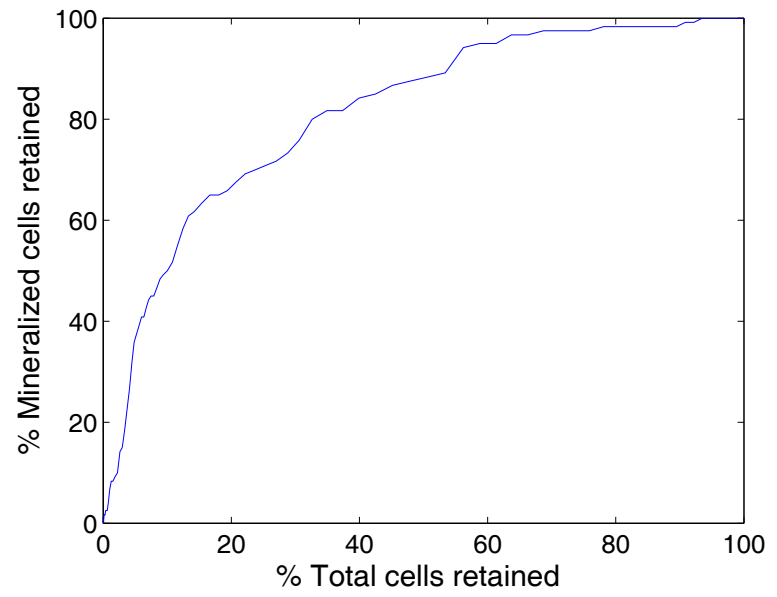

b) Percentage of deposit versus total cells captured for classification thresholds in the range $[0,1]$.

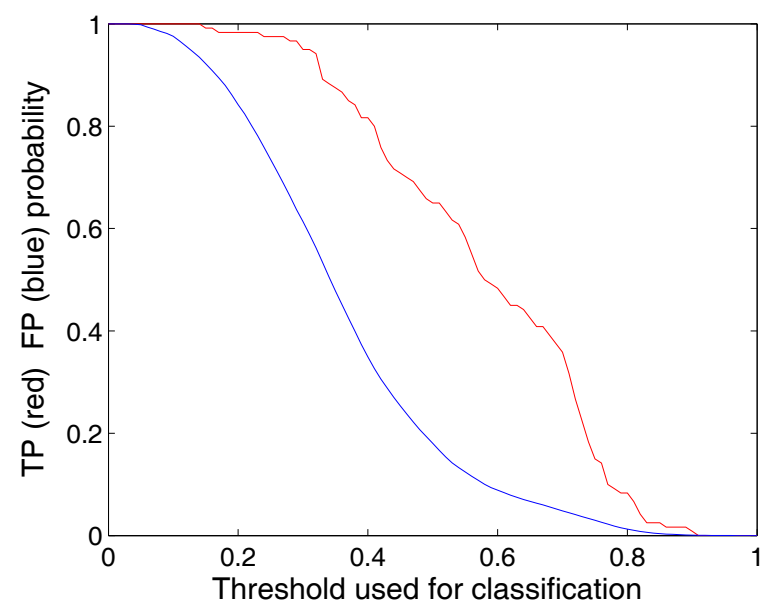

d) Proportion of correct and false deposit classification vs classification threshold.

Figure 7.32. Classification performance of the 10-20-1 neural network with the best test-set classification accuracy. Learning algorithm: trainlm. Input layer selection criteria: D/A. 


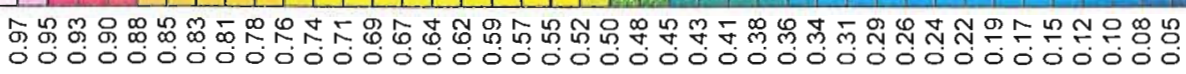

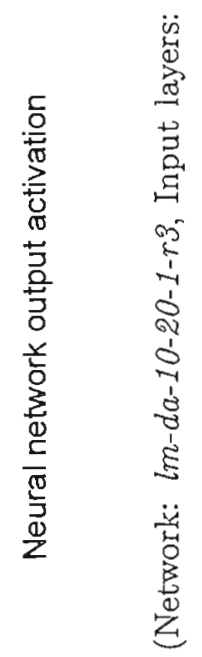

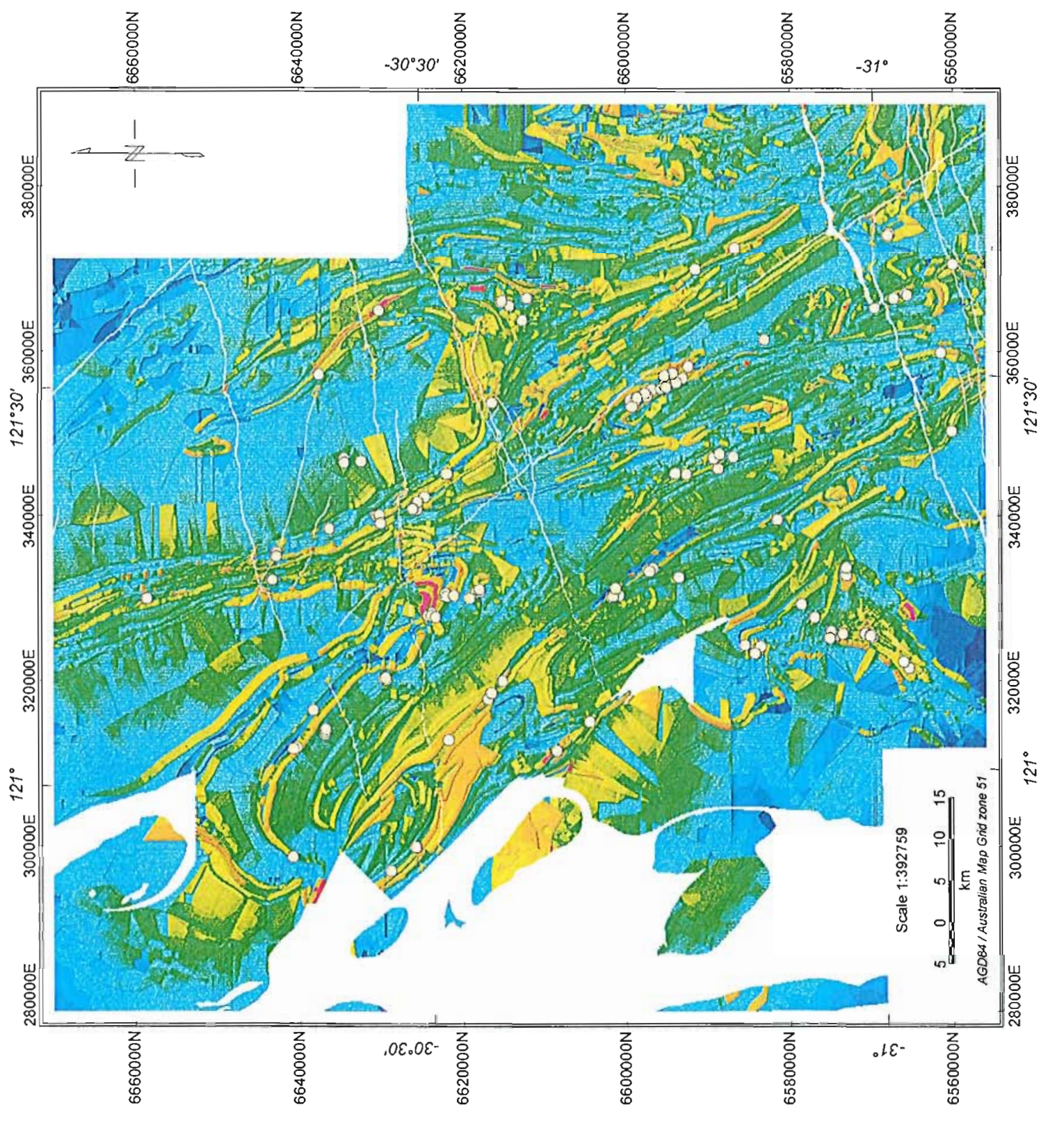

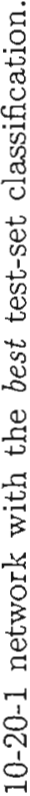

$\frac{1}{8}$

,

窎

¿

弯

露

일

द्व.

密

造

a

号.

中. 尊

ஸ்

i

(1) 先

党蛋 


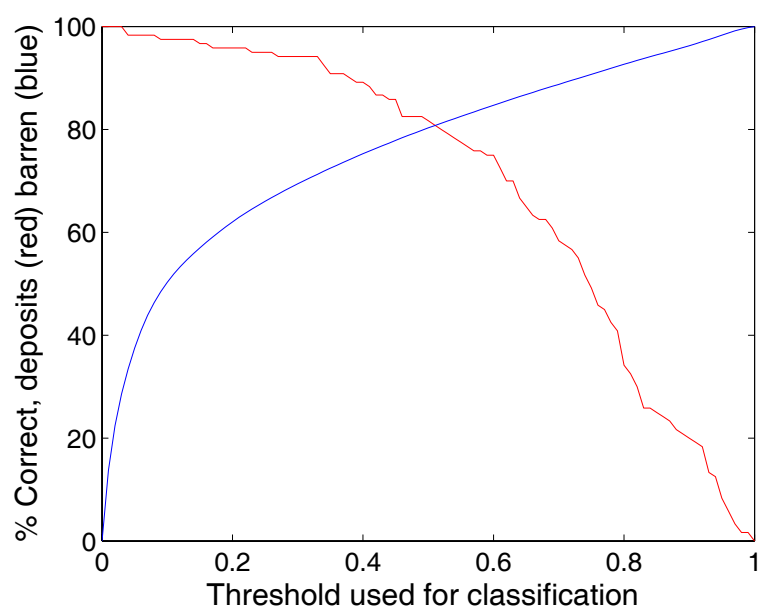

a) Classification accuracy versus threshold.

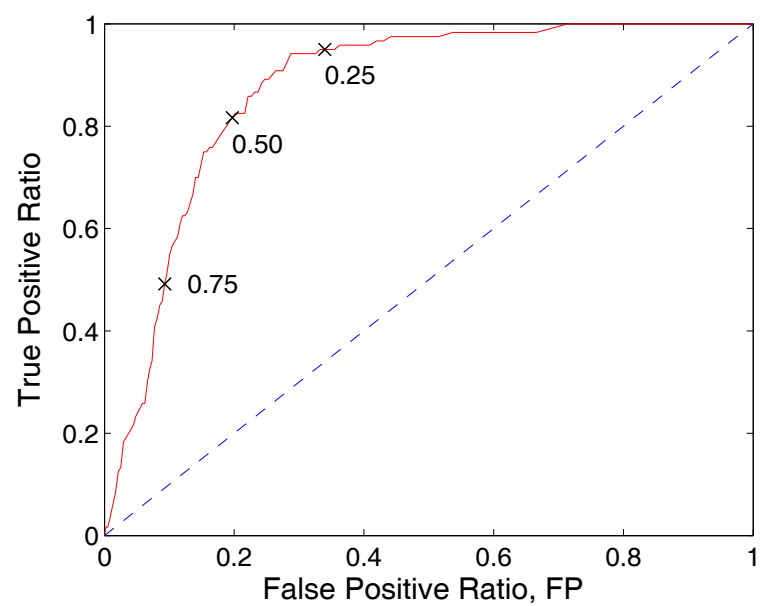

c) ROC curve showing threshold values.

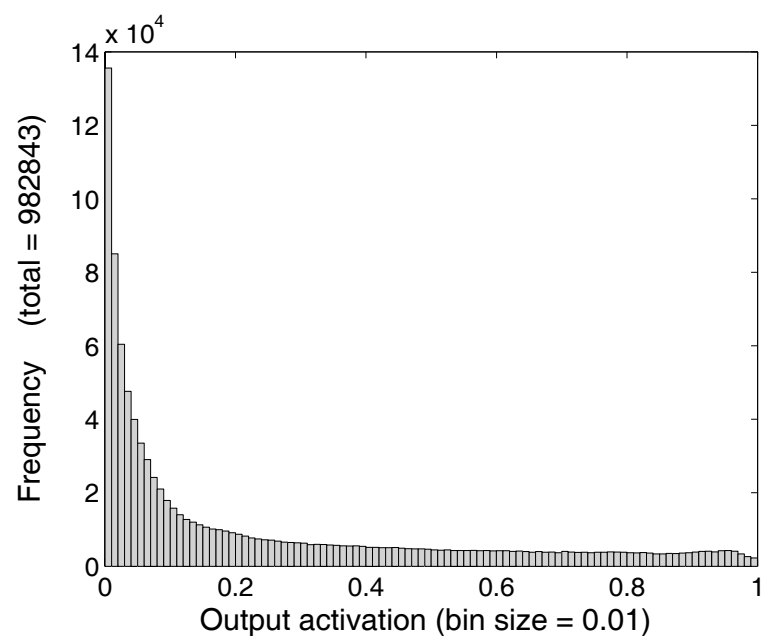

(e) Histogram of output activations of trained networks.

Figure 7.34. Classification performance of the 10-20-1 neural network with the best test-set classification accuracy. Learning algorithm: trainlm. Input layer selection criteria: $D *(D / A)$.

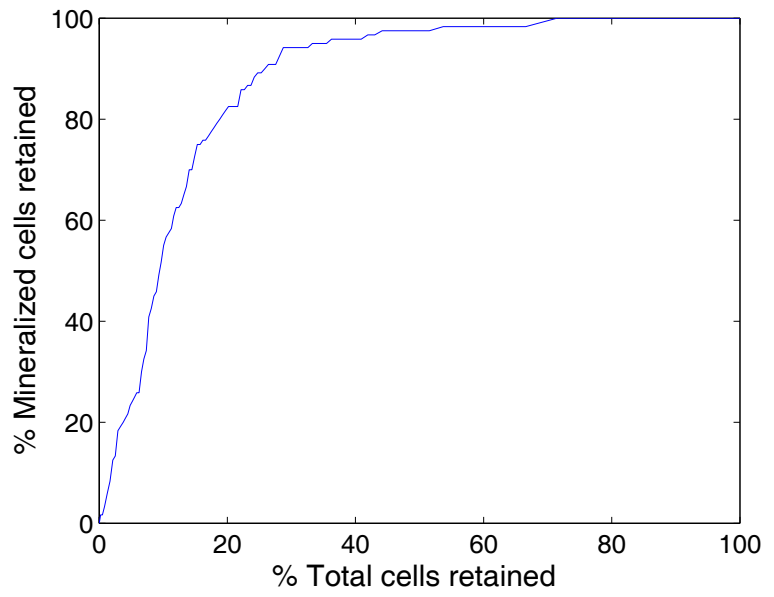

b) Percentage of deposit versus total cells captured for classification thresholds in the range $[0,1]$.

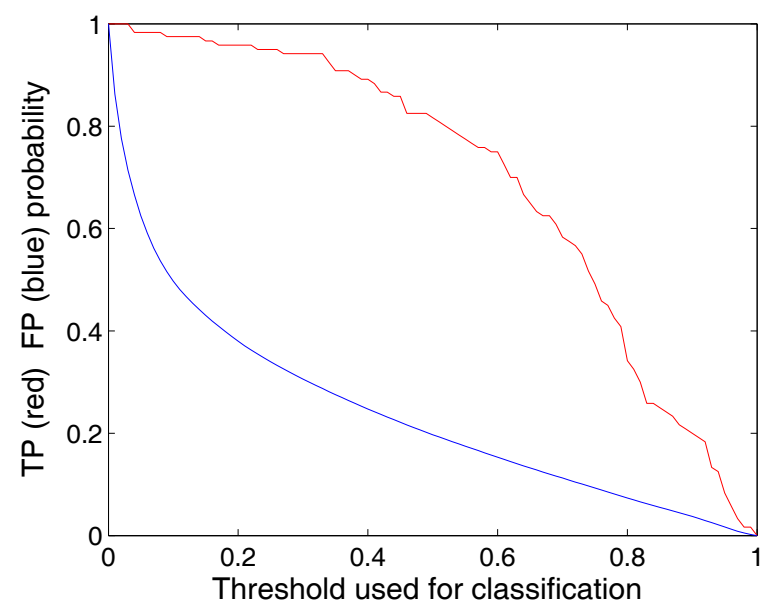

d) Proportion of correct and false deposit classification vs classification threshold. 


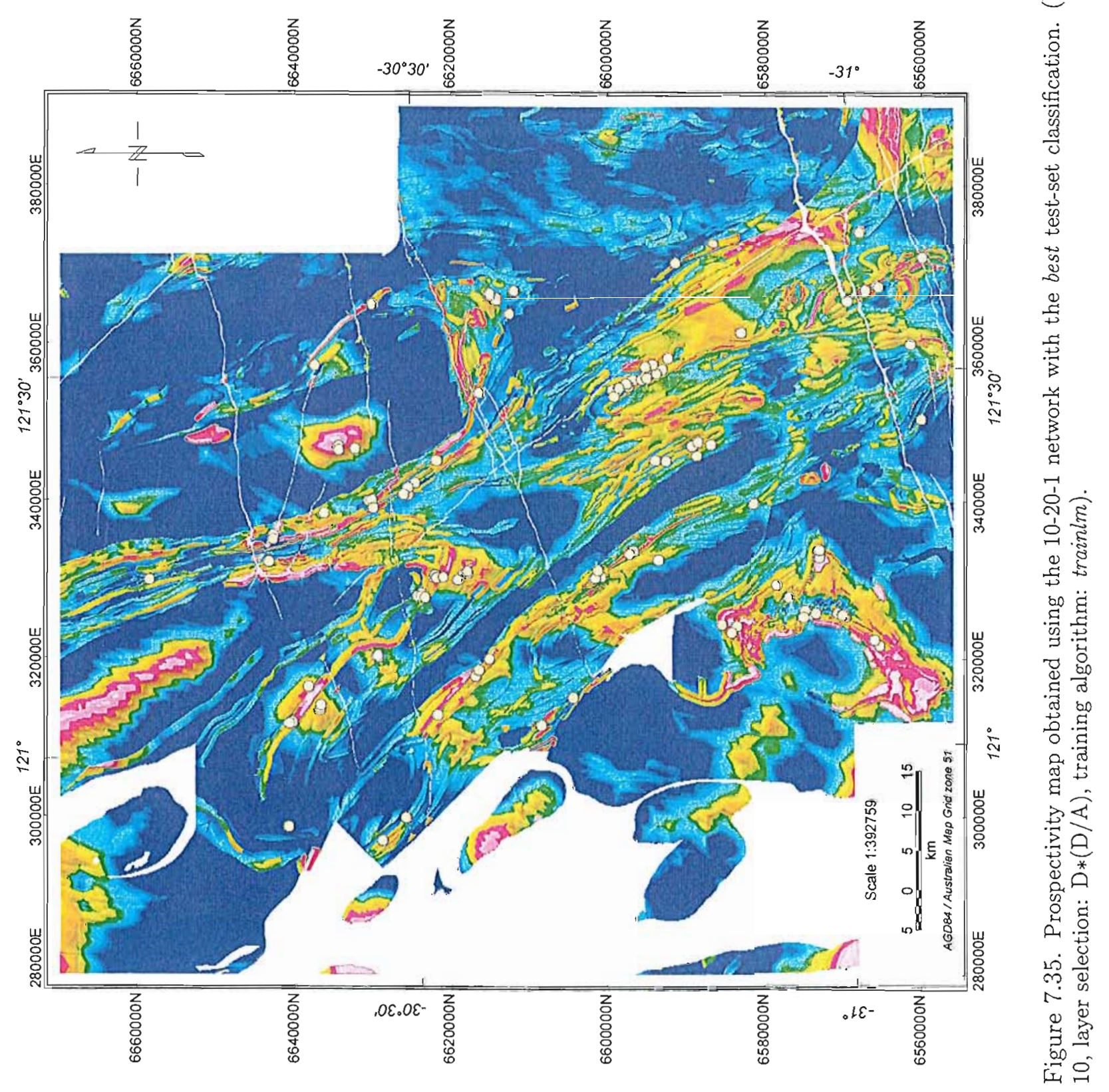




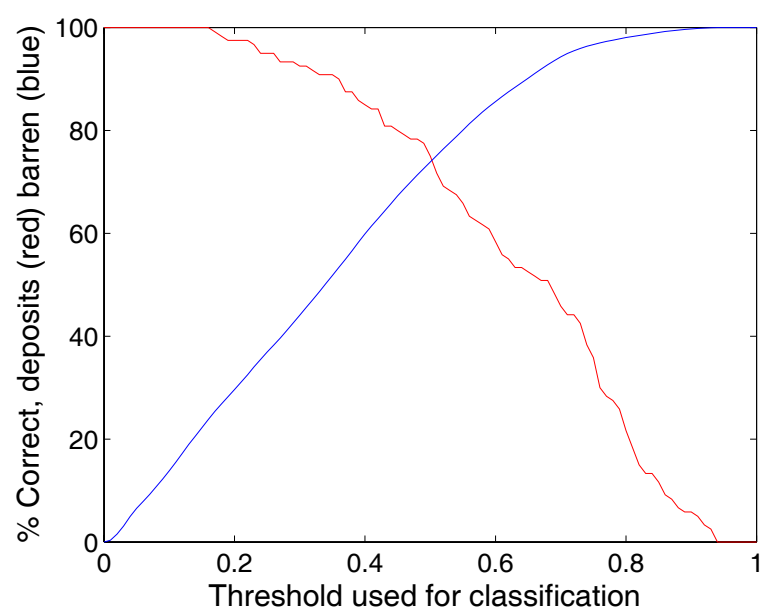

a) Classification accuracy versus threshold.

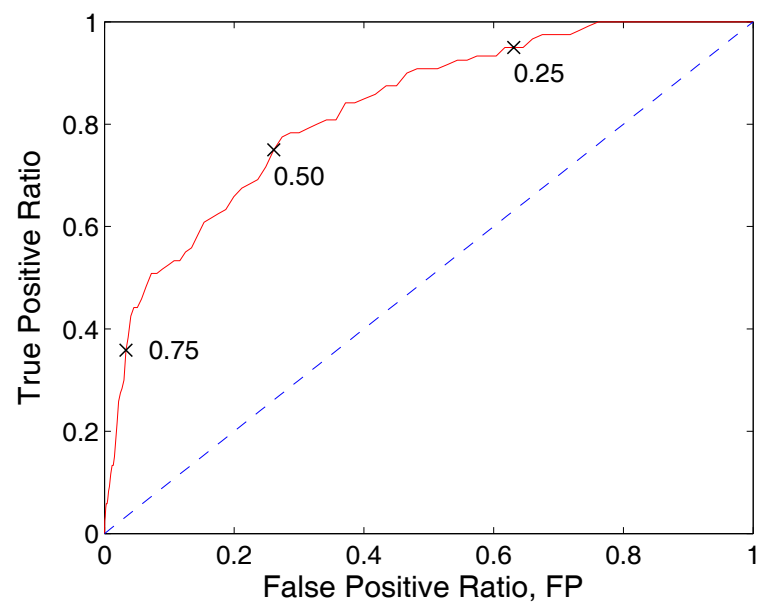

c) ROC curve showing threshold values.

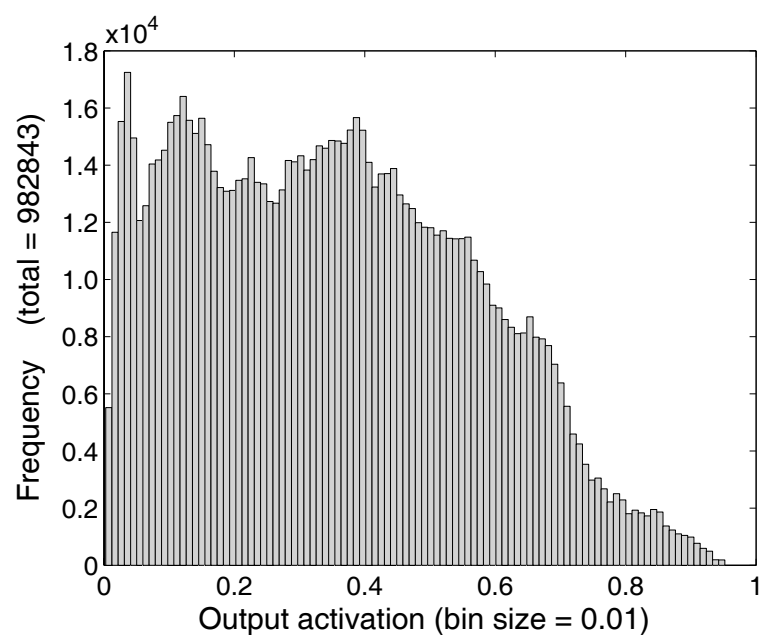

(e) Histogram of output activations of trained networks.

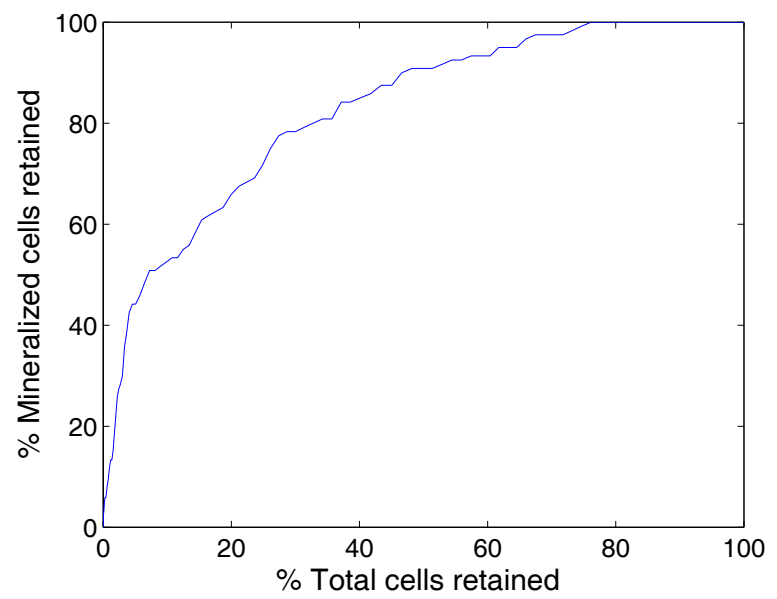

b) Percentage of deposit versus total cells captured for classification thresholds in the range $[0,1]$.

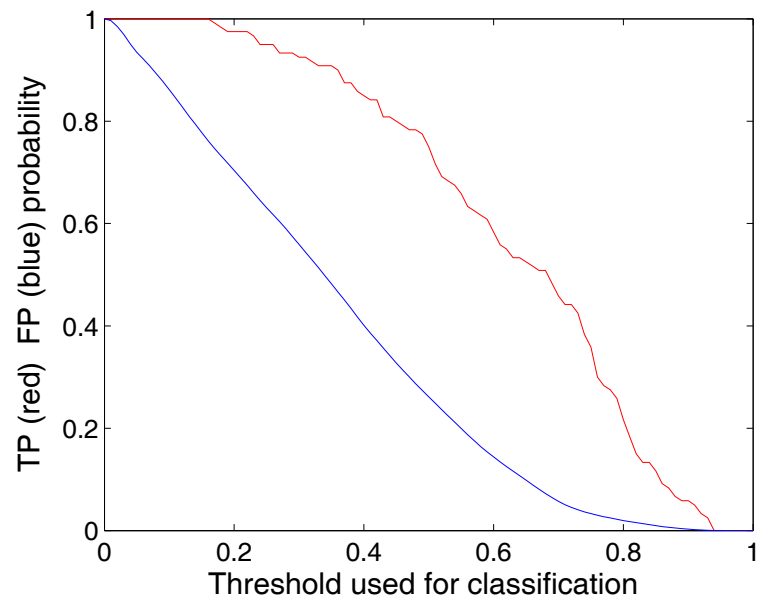

d) Proportion of correct and false deposit classification vs classification threshold.

Figure 7.36. Classification performance of the 10-20-1 neural network with the best test-set classification accuracy. Learning algorithm: trainlm. Input layer selection criteria: $\mathrm{D} *(\mathrm{D} / \mathrm{A}) A N D$ $\mathrm{D} \geq \sim 20 \% A N D \mathrm{D} / \mathrm{A} \geq \sim 3 \%$. 


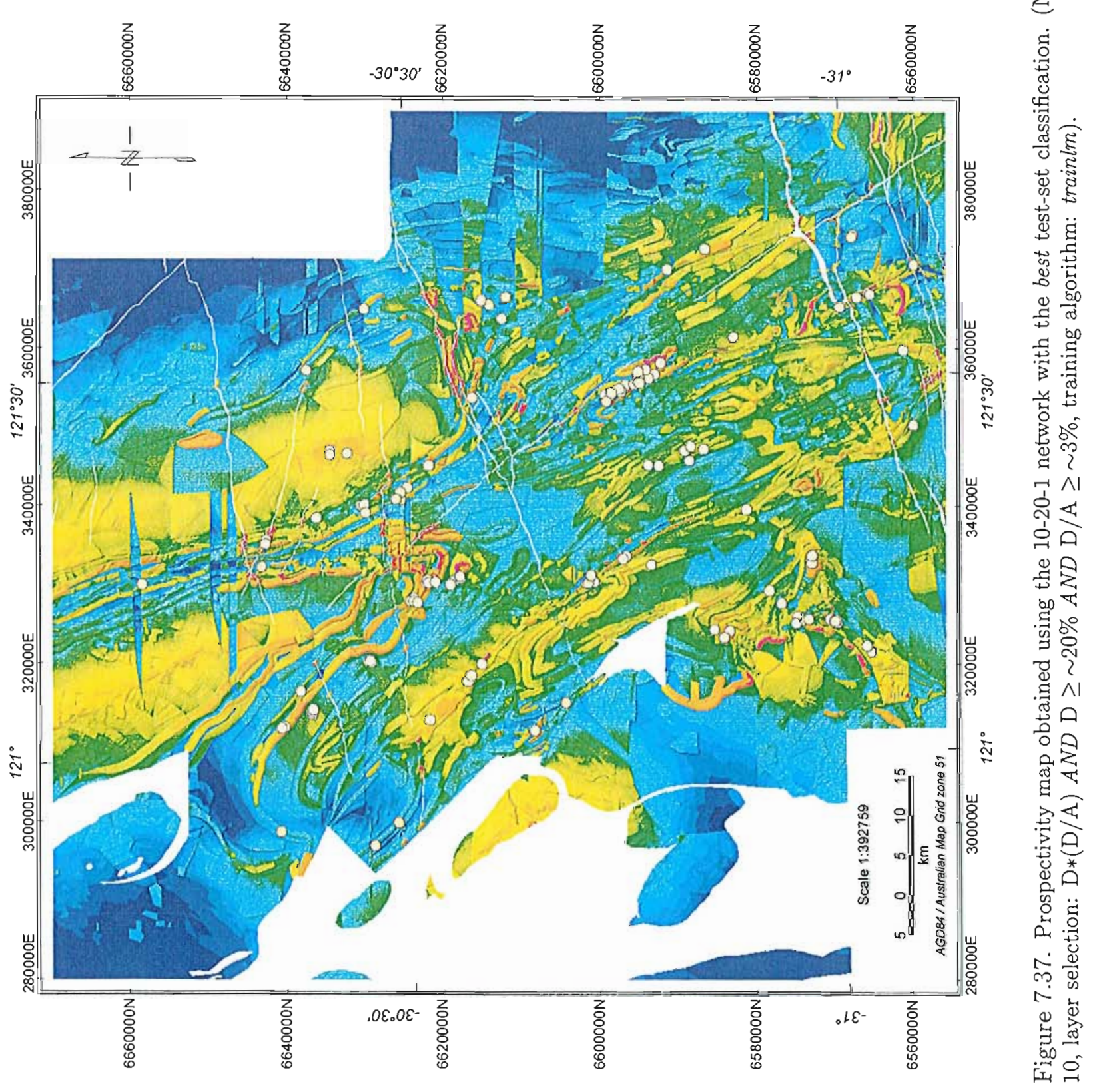




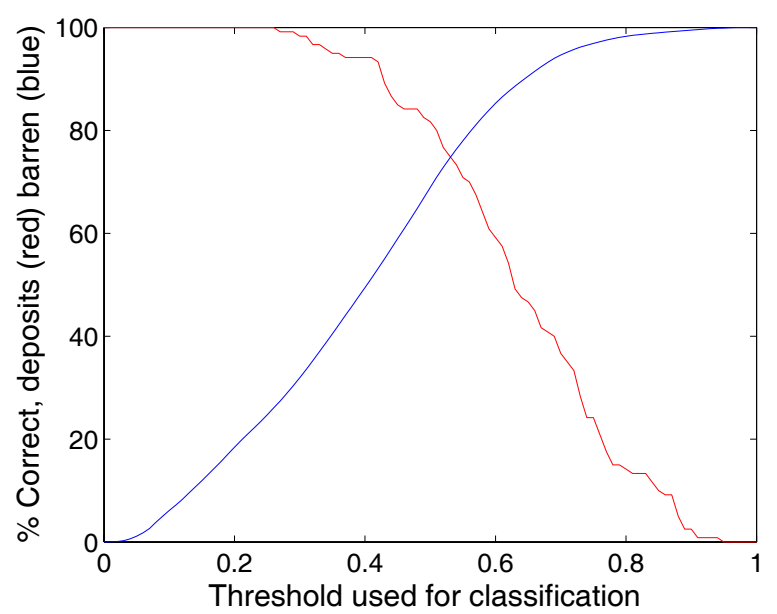

a) Classification accuracy versus threshold.

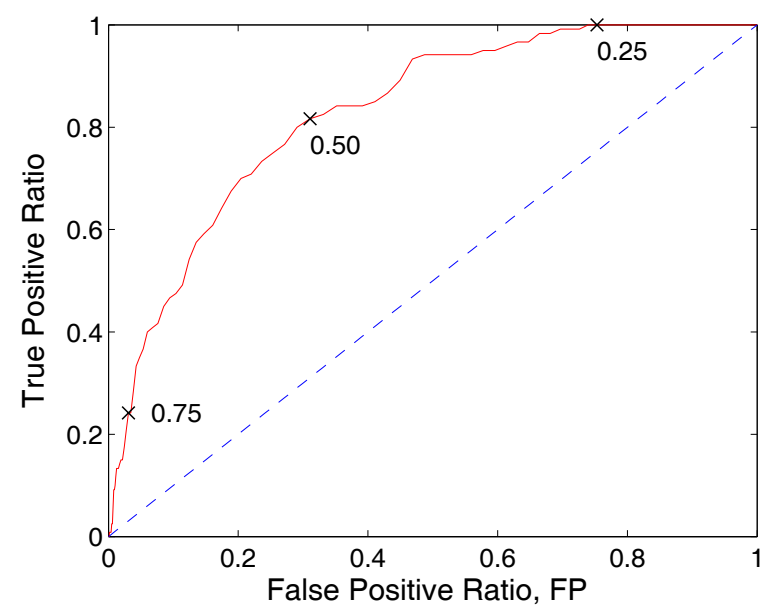

c) ROC curve showing threshold values.

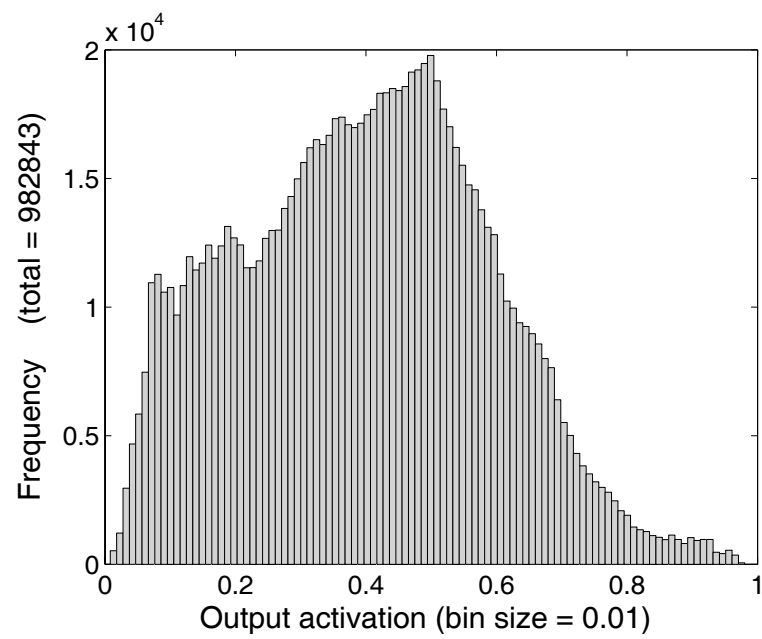

(e) Histogram of output activations of trained networks.

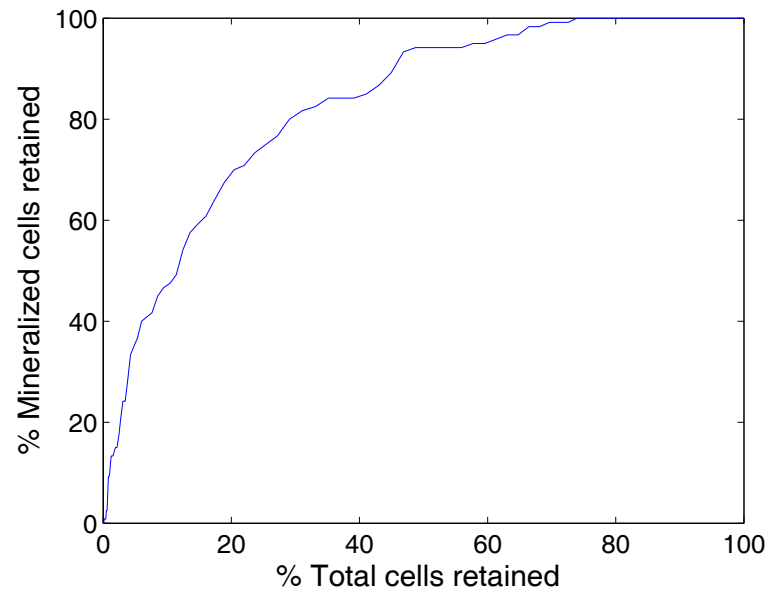

b) Percentage of deposit versus total cells captured for classification thresholds in the range $[0,1]$.

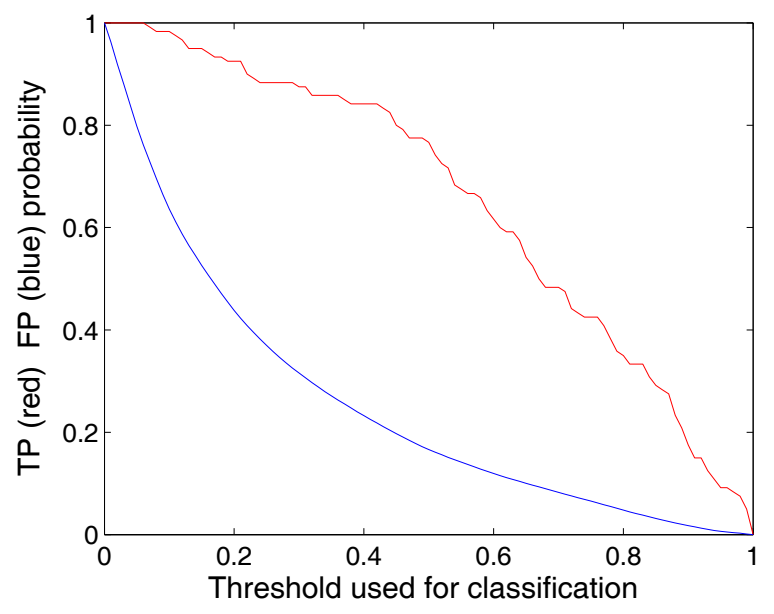

d) Proportion of correct and false deposit classification vs classification threshold.

Figure 7.38. Classification performance of the 17-20-1 neural network with the best test-set classification accuracy. Input layer selection: categories. Learning algorithm: trainlm. 


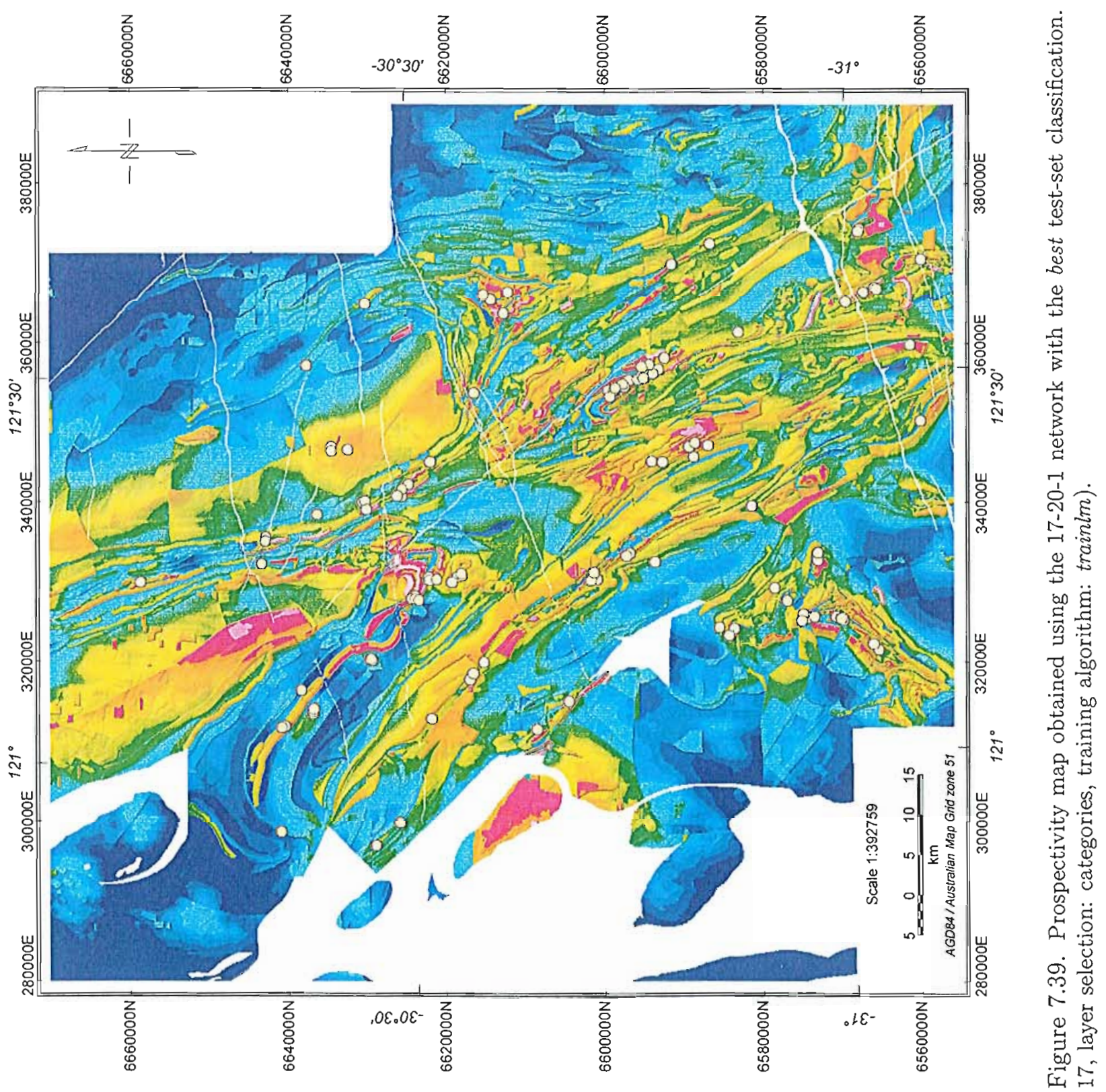




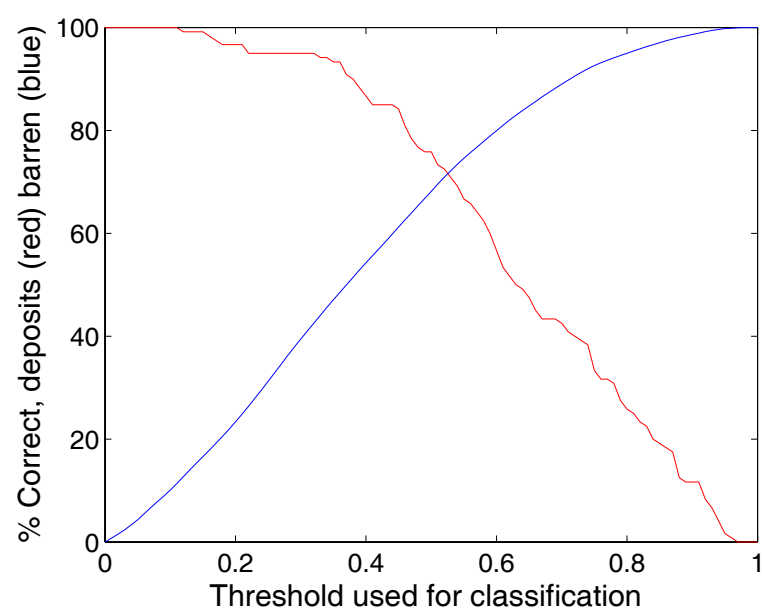

a) Classification accuracy versus threshold.

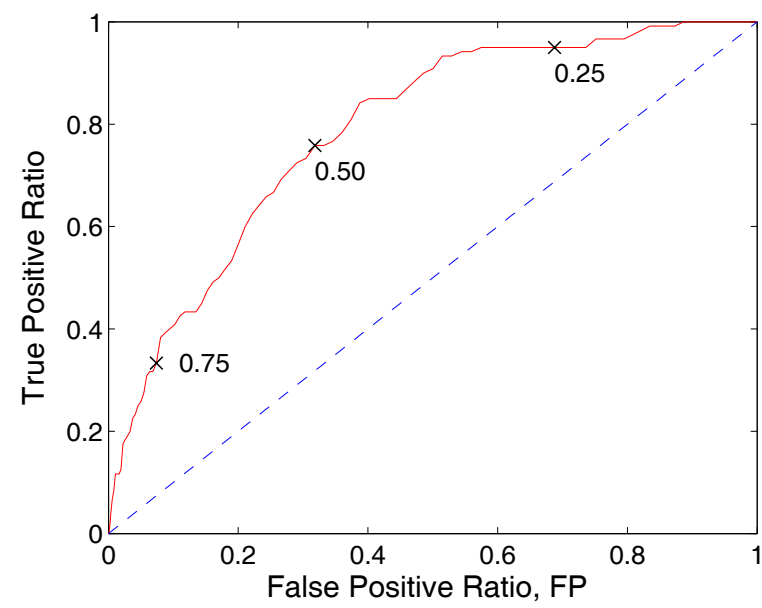

c) ROC curve showing threshold values.

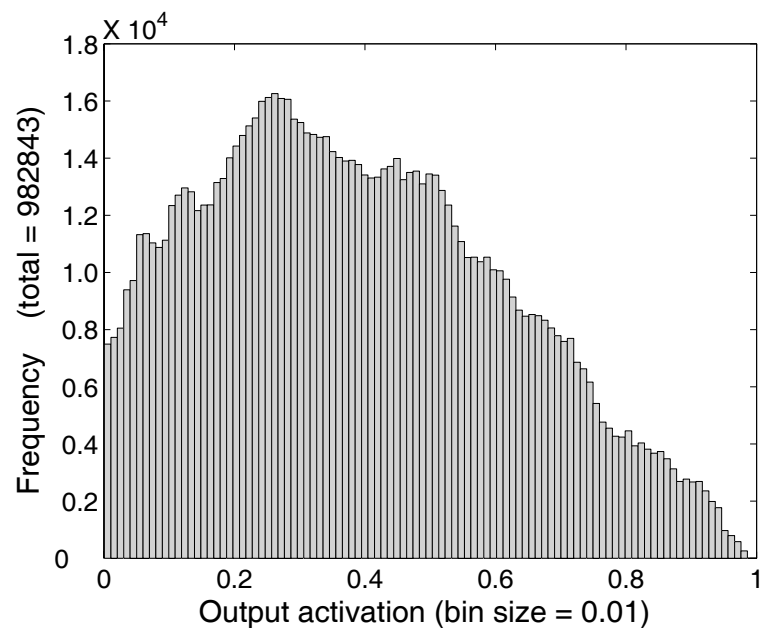

(e) Histogram of output activations of trained networks.

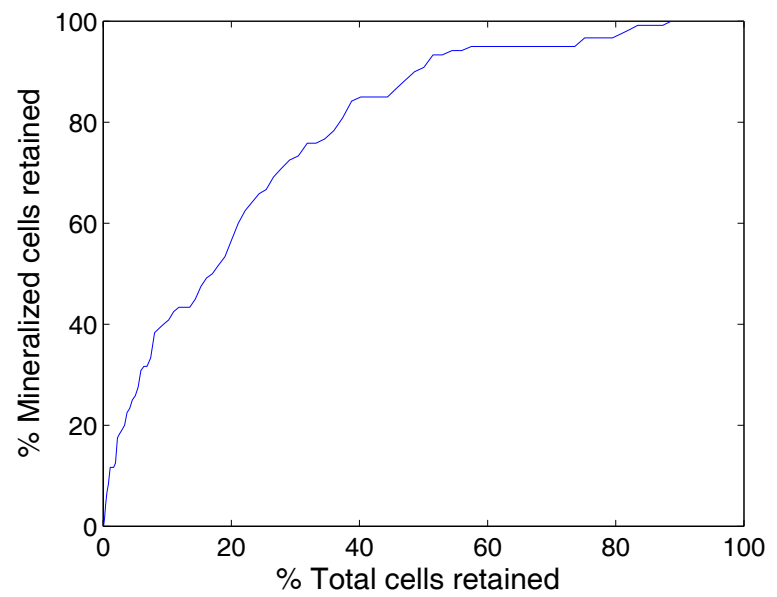

b) Percentage of deposit versus total cells captured for classification thresholds in the range $[0,1]$.

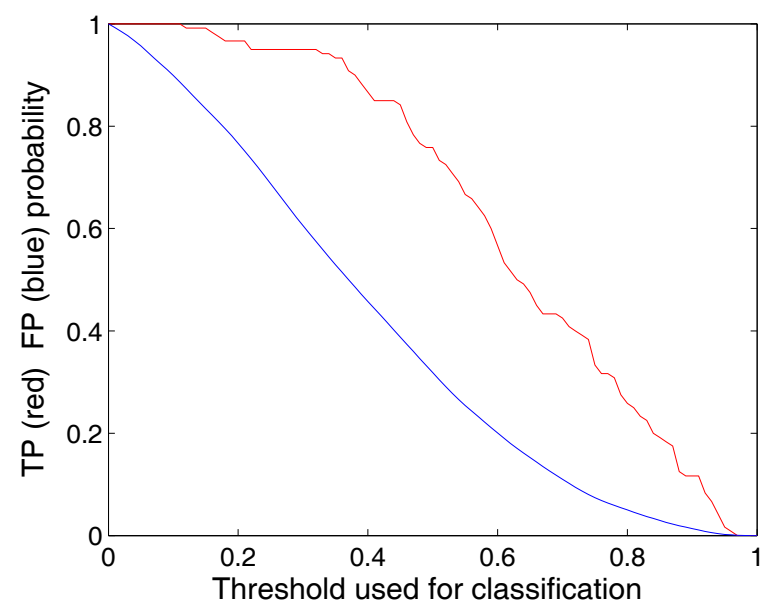

d) Proportion of correct and false deposit classification vs classification threshold.

Figure 7.40. Classification performance of the 17-20-1 neural network with the best test-set classification accuracy. Input layer selection: D/A. Learning algorithm: trainlm. 


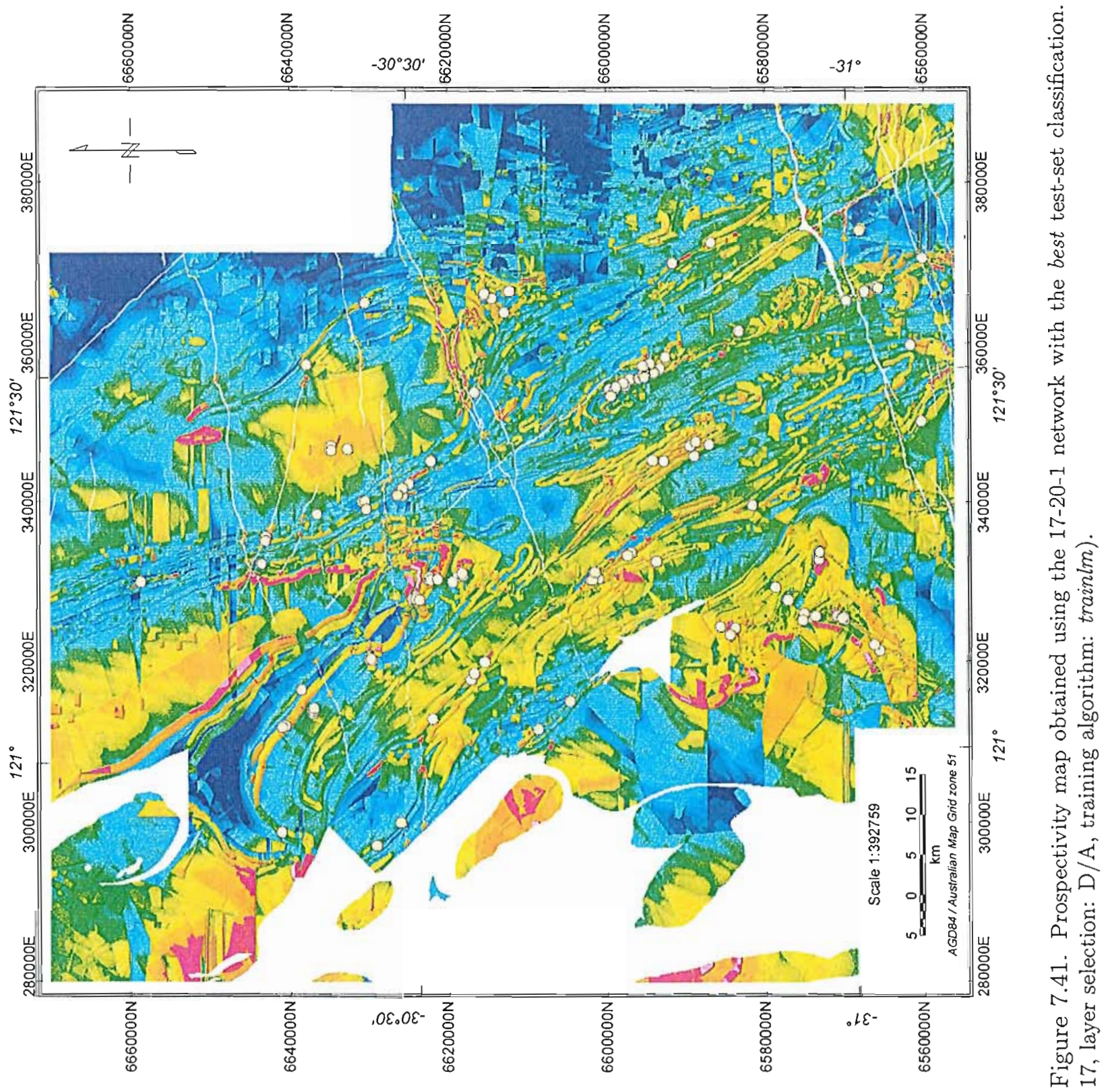




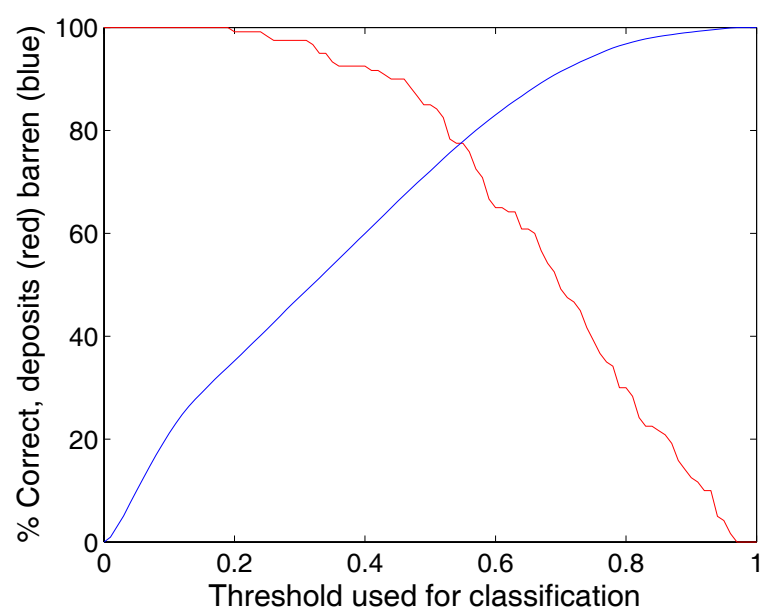

a) Classification accuracy versus threshold.

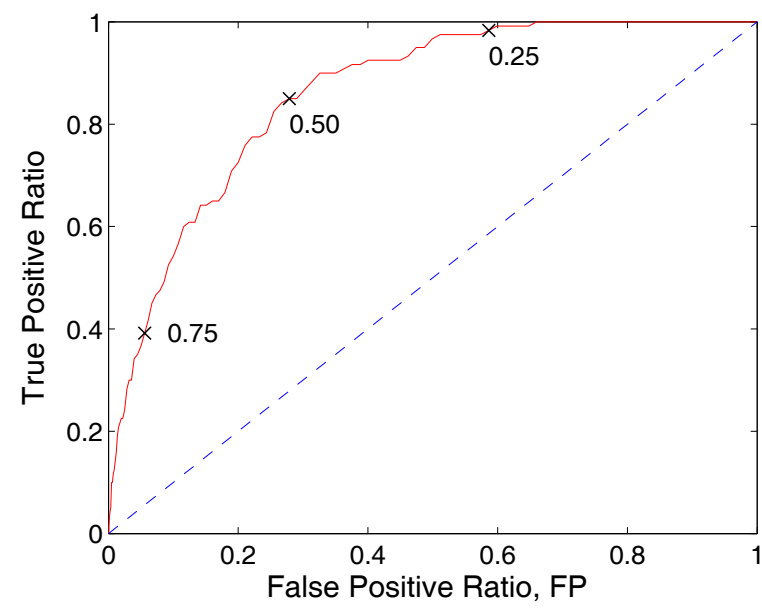

c) ROC curve showing threshold values.

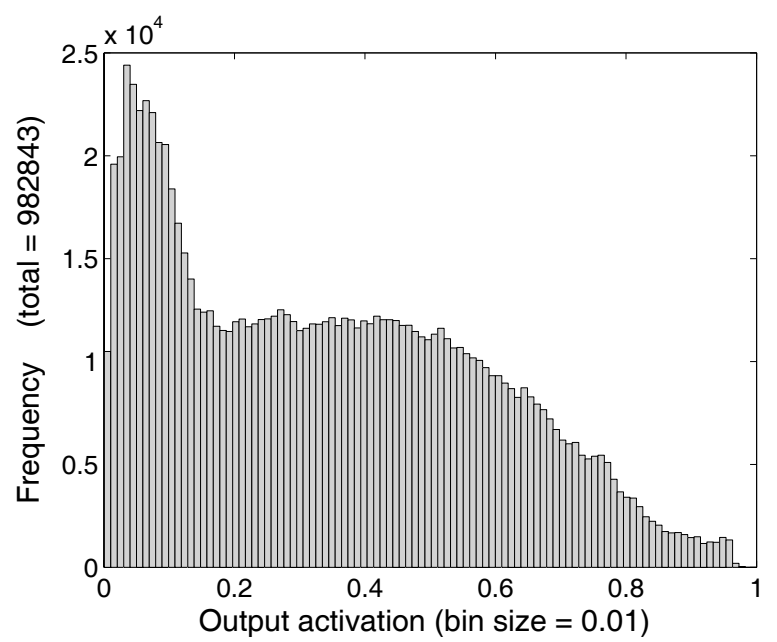

(e) Histogram of output activations of trained networks.

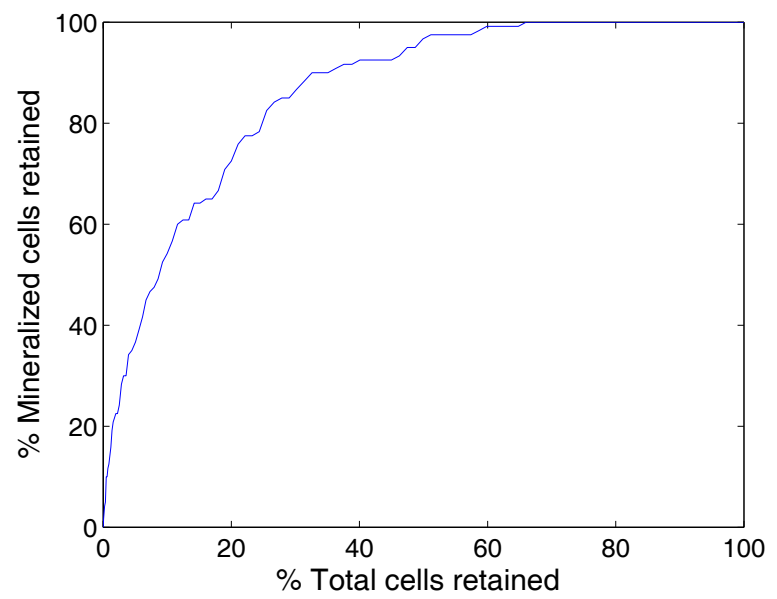

b) Percentage of deposit versus total cells captured for classification thresholds in the range $[0,1]$.

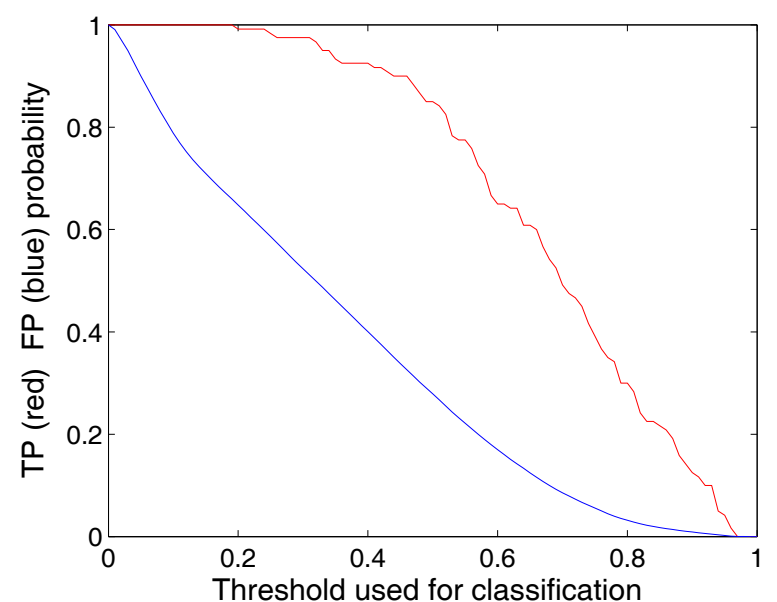

d) Proportion of correct and false deposit classification vs classification threshold.

Figure 7.42. Classification performance of the 17-20-1 neural network with the best test-set classification accuracy. Input layer selection: $\mathrm{D} *(\mathrm{D} / \mathrm{A})$. Learning algorithm: trainlm. 


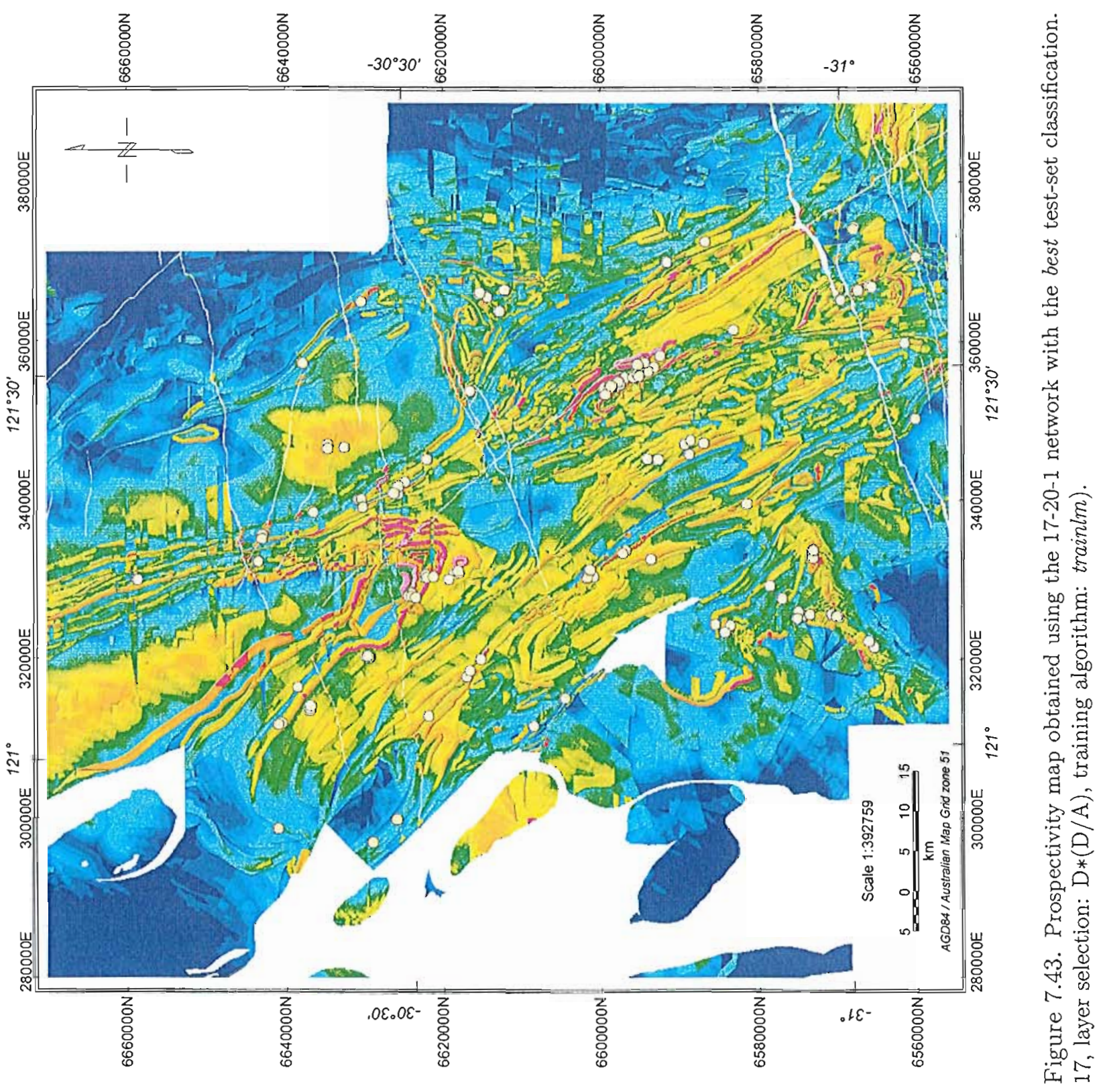




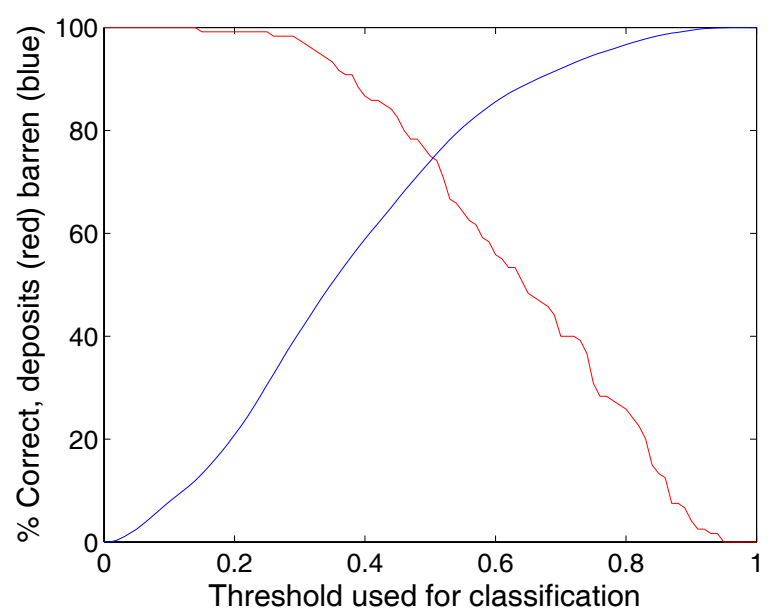

a) Classification accuracy versus threshold.

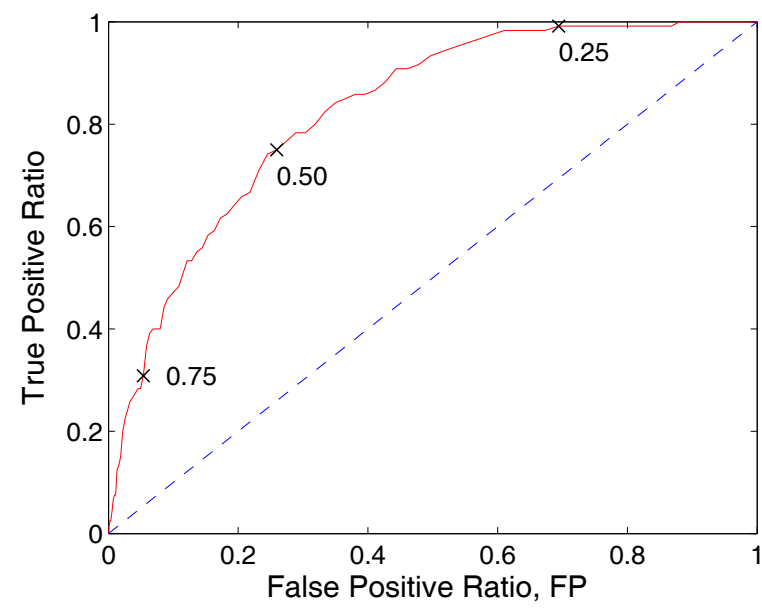

c) ROC curve showing threshold values.

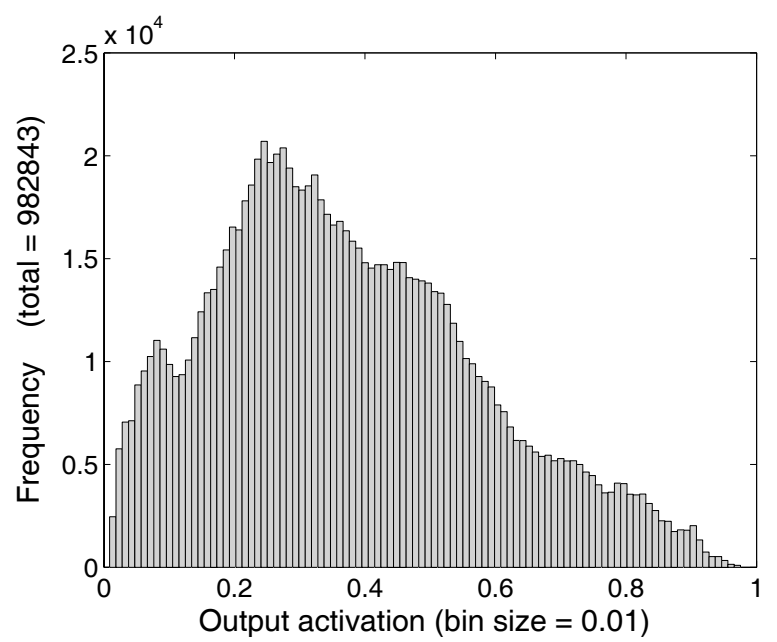

(e) Histogram of output activations of trained networks.

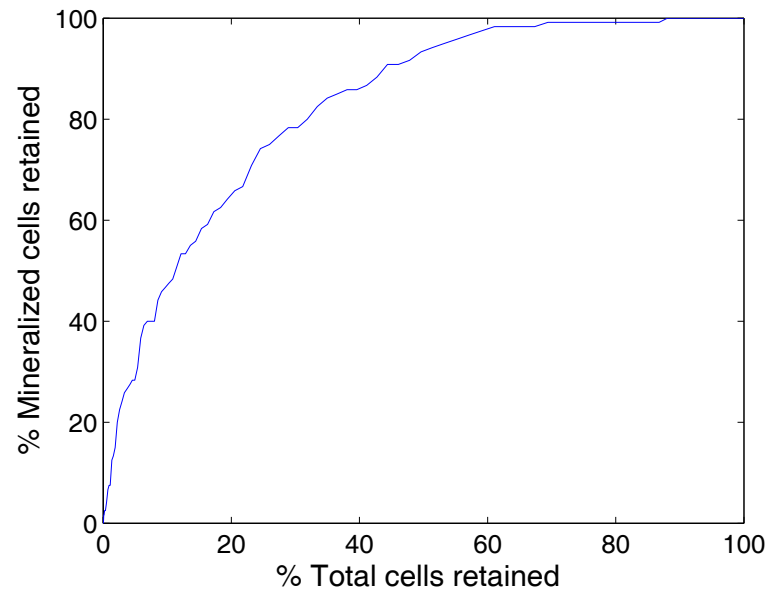

b) Percentage of deposit versus total cells captured for classification thresholds in the range $[0,1]$.

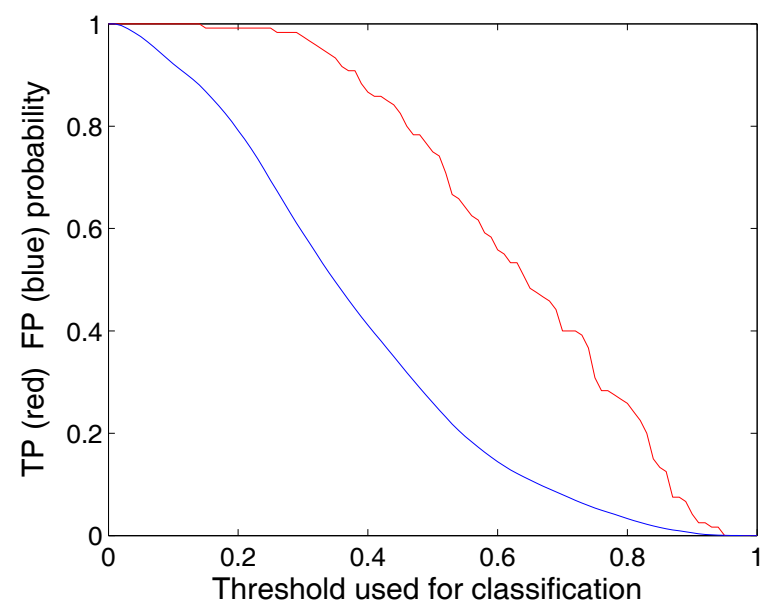

d) Proportion of correct and false deposit classification vs classification threshold.

Figure 7.44. Classification performance of the 17-20-1 neural network with the best test-set classification accuracy. Input layer selection: $\mathrm{D} *(\mathrm{D} / \mathrm{A}) A N D \mathrm{D} \geq \sim 20 \% A N D \mathrm{D} / \mathrm{A} \geq \sim 3 \%$. Learning algorithm: trainlm. 


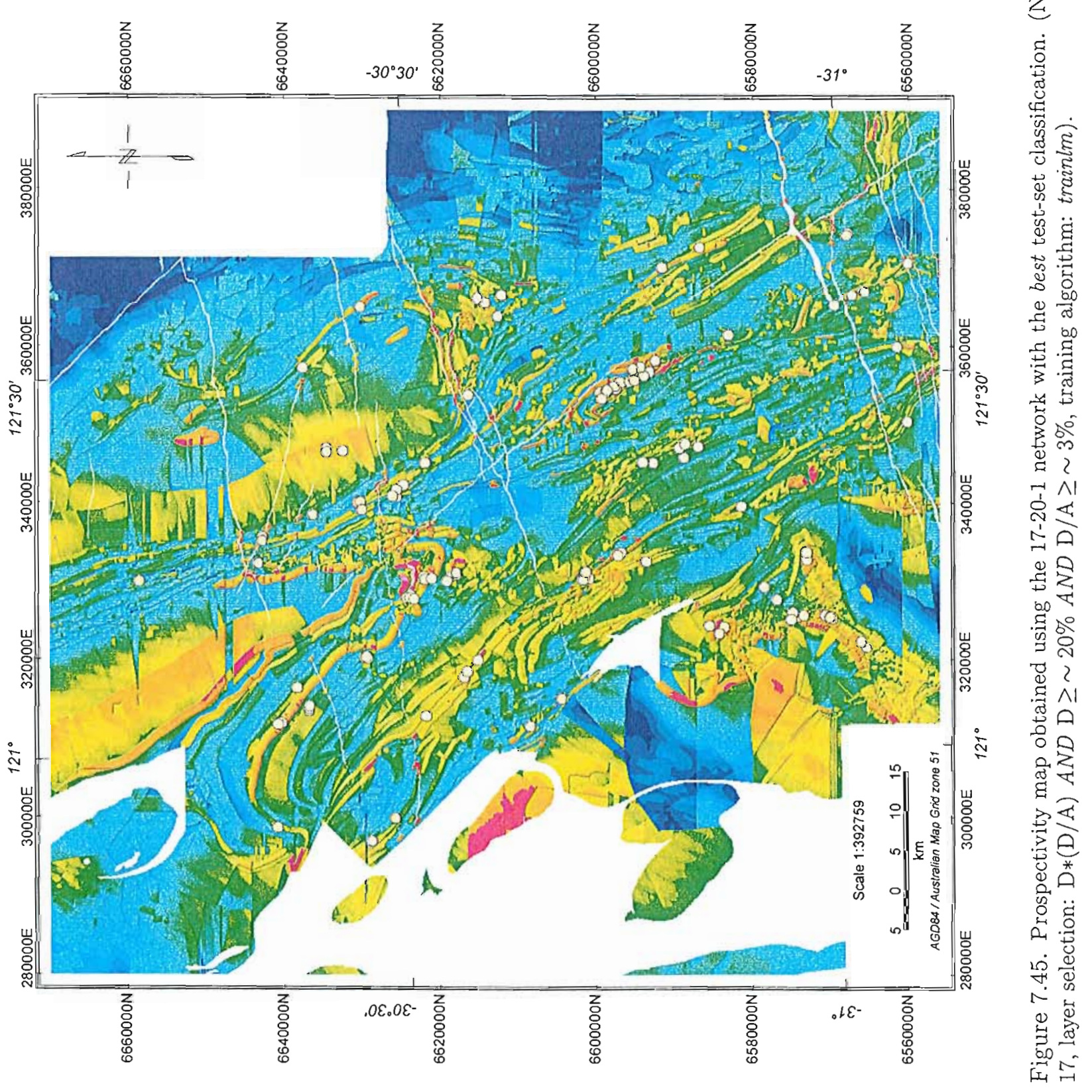




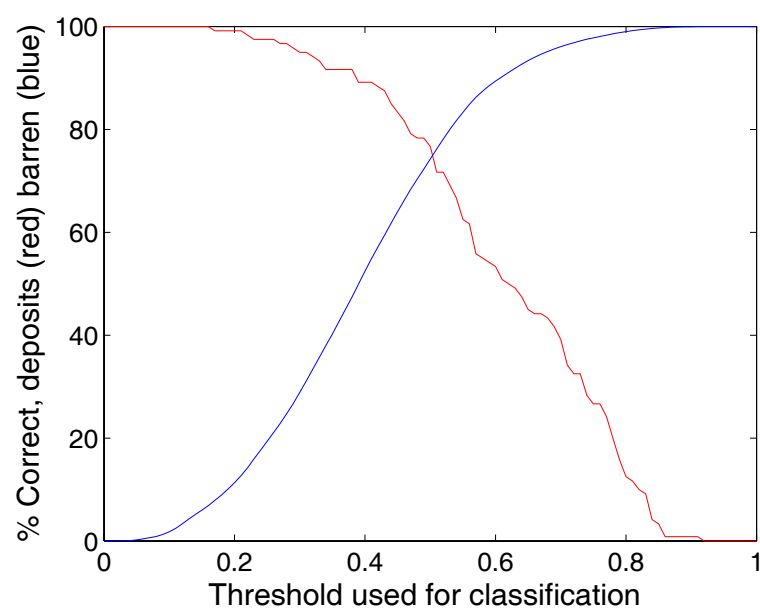

a) Classification accuracy versus threshold.

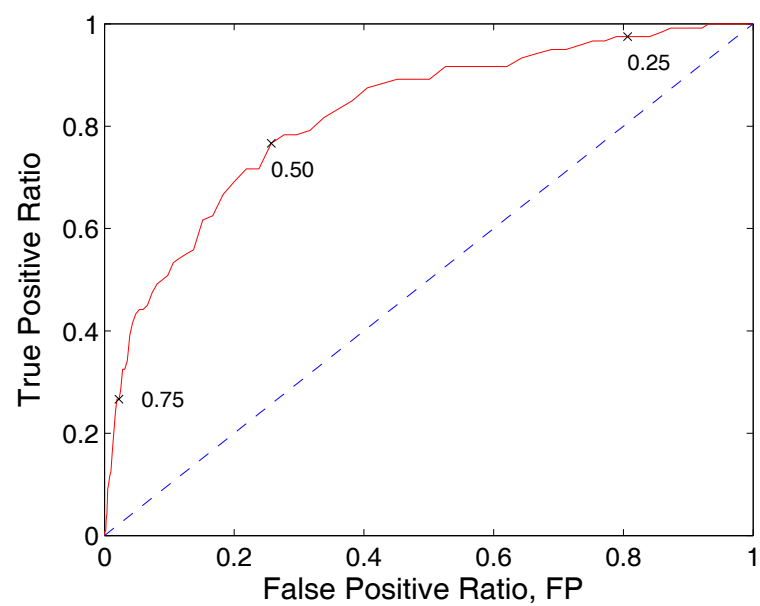

c) ROC curve showing threshold values.

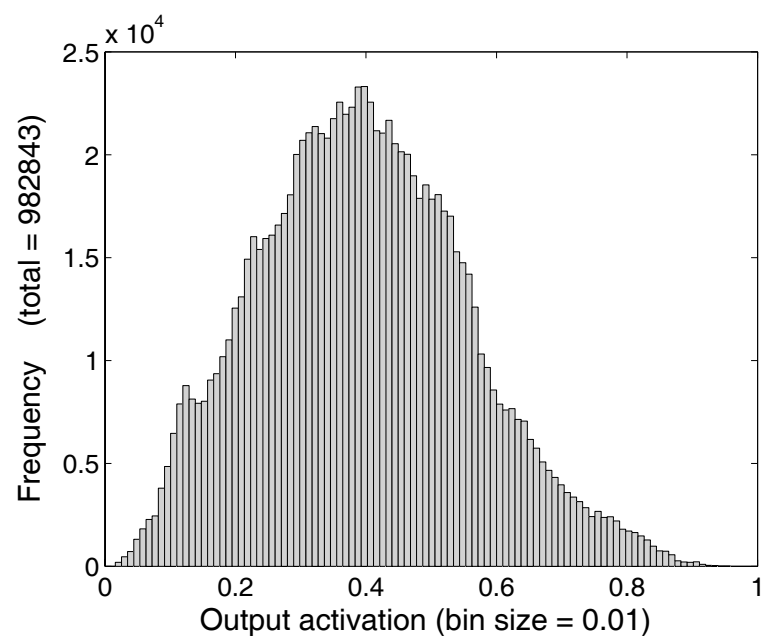

(e) Histogram of output activations of trained networks.

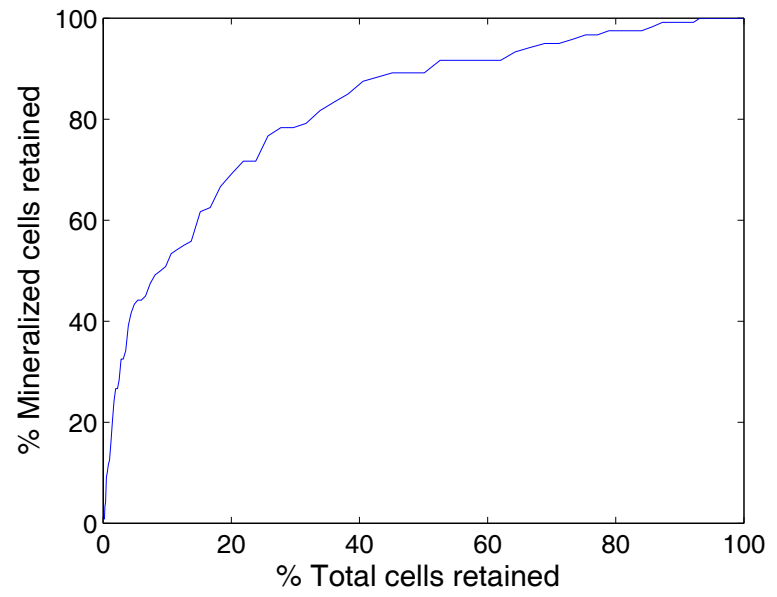

b) Percentage of deposit versus total cells captured for classification thresholds in the range $[0,1]$.

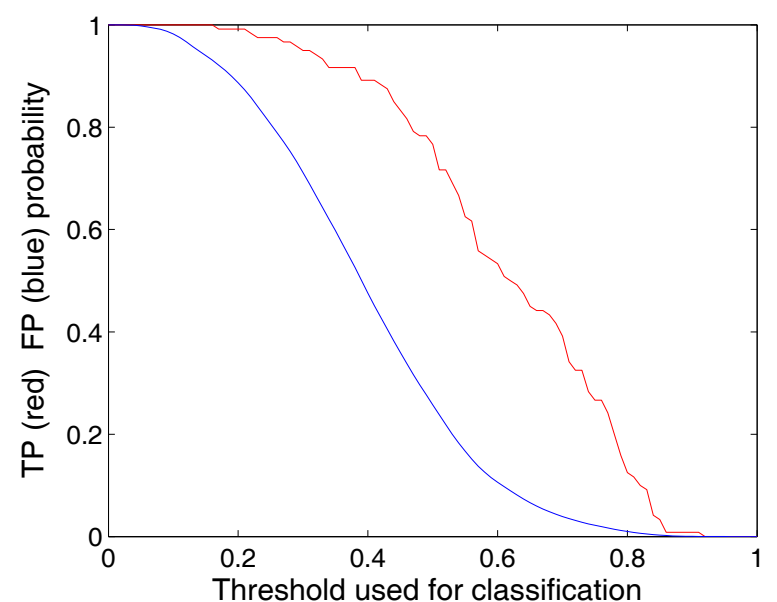

d) Proportion of correct and false deposit classification vs classification threshold.

Figure 7.46. Classification performance of the 25-20-1 neural network with the best test-set classification accuracy. Input layer selection: all layers used. Learning algorithm: trainlm. 


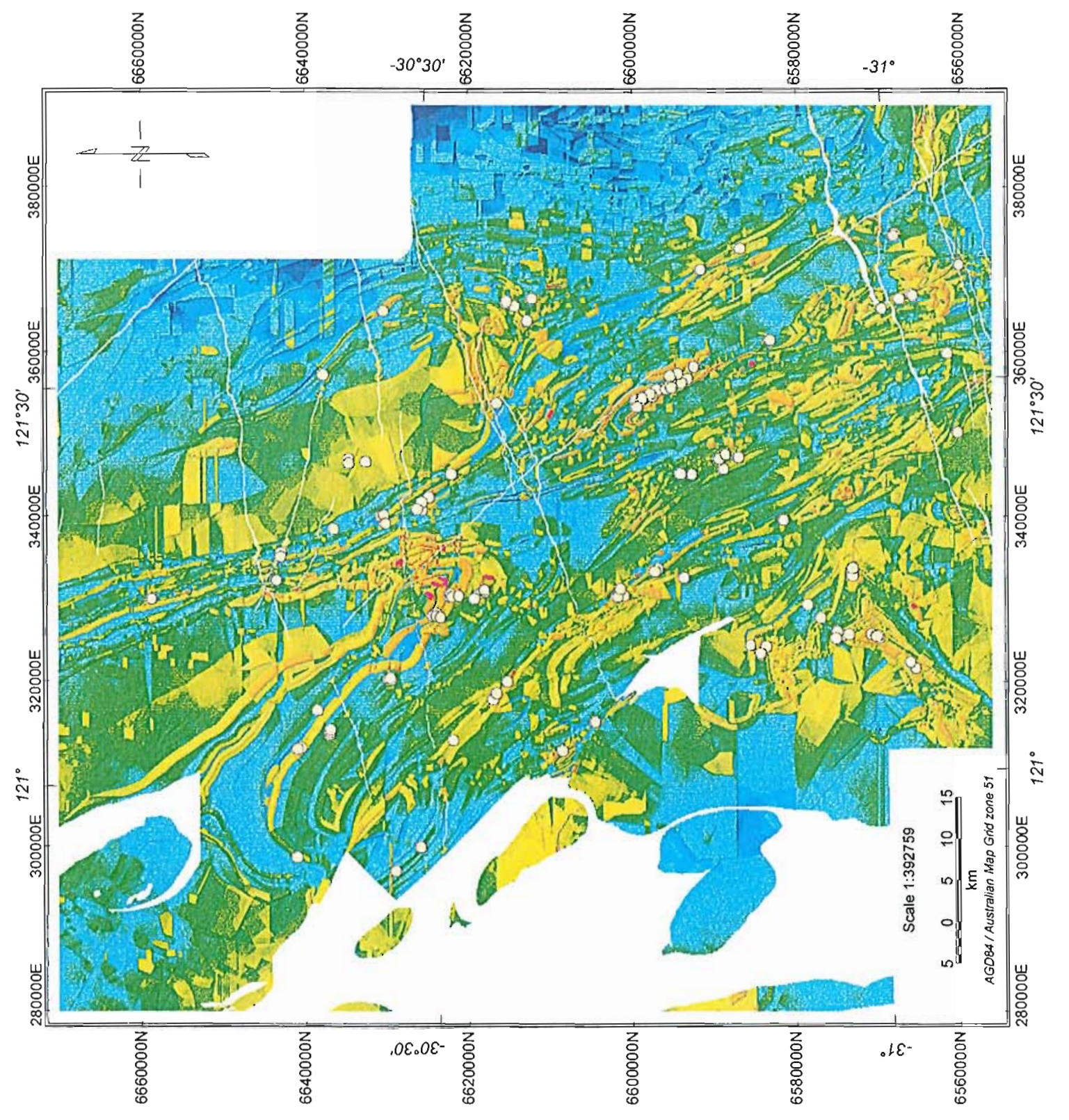

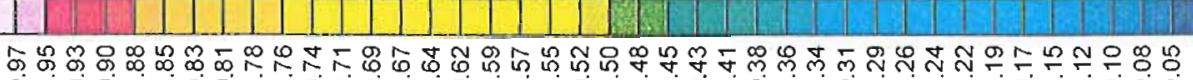

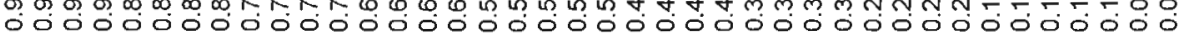

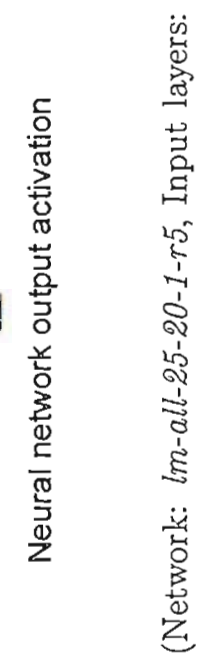

㤩

0
0
0
10
0
0

$\stackrel{+}{\mathscr{Q}}$

$\stackrel{0}{\square}$

兵

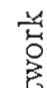

要

공.

เ่

언

,

牙

邑槑

营壱

응

畠

点怘

究焉

记

号

웜

N.

岕 잉 


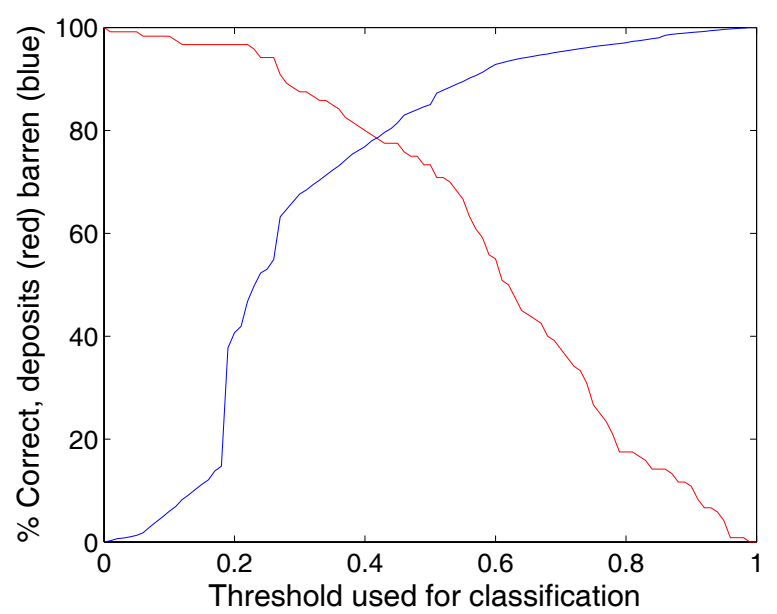

a) Classification accuracy versus threshold.

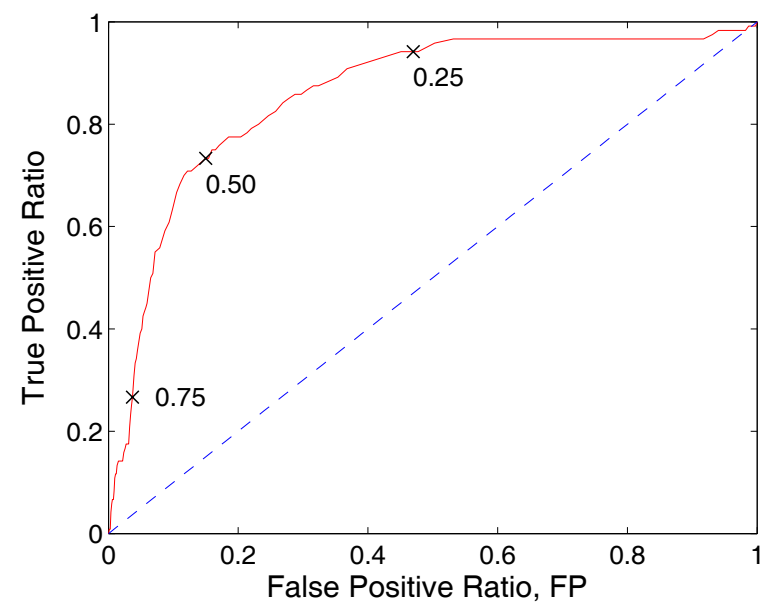

c) ROC curve showing threshold values.

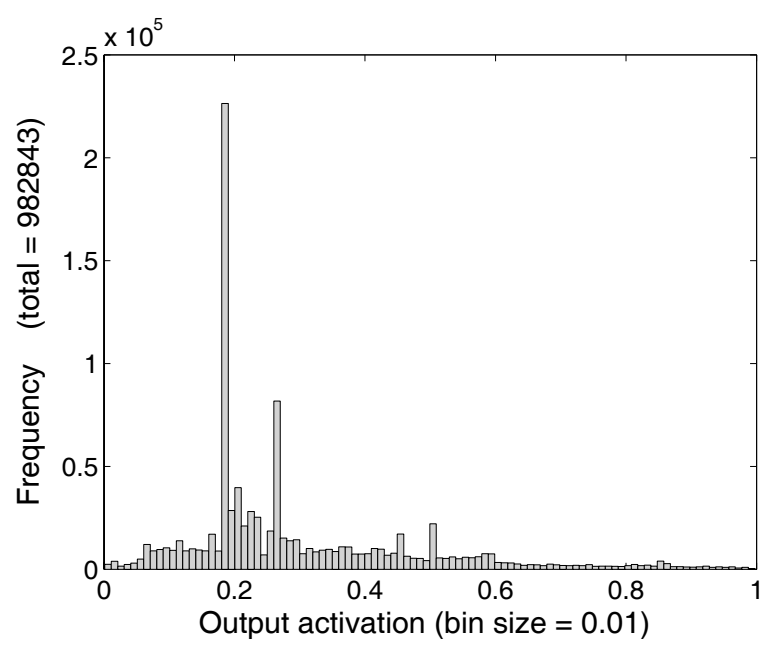

(e) Histogram of output activations of trained networks.

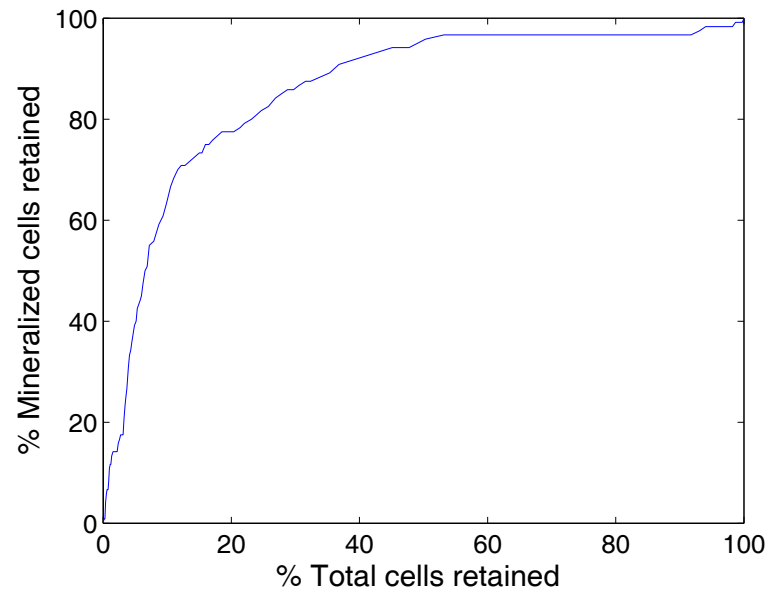

b) Percentage of deposit versus total cells captured for classification thresholds in the range $[0,1]$.

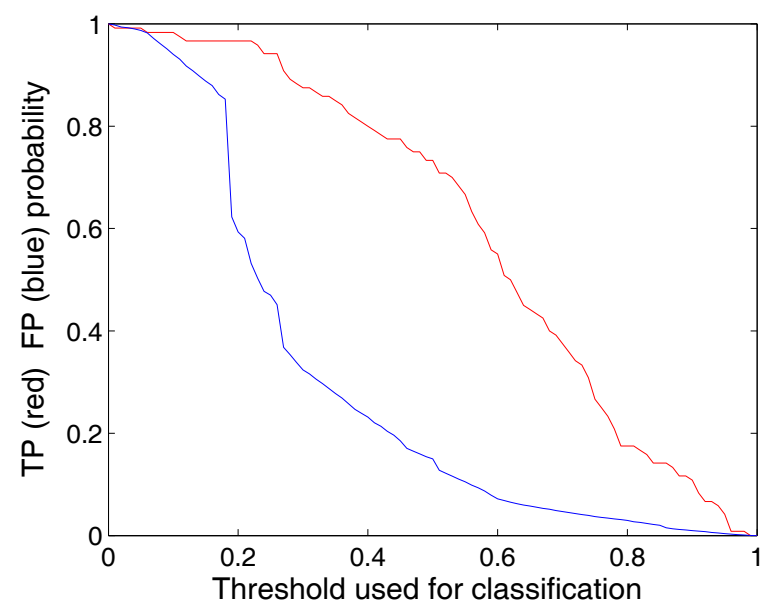

d) Proportion of correct and false deposit classification vs classification threshold.

Figure 7.48. Classification performance of the 10-20-1 hybrid fuzzy-membership-input network with the best test-set classification accuracy. All 10 inputs are fuzzy membership values. Input layer selection: categories. Learning algorithm: trainlm. 


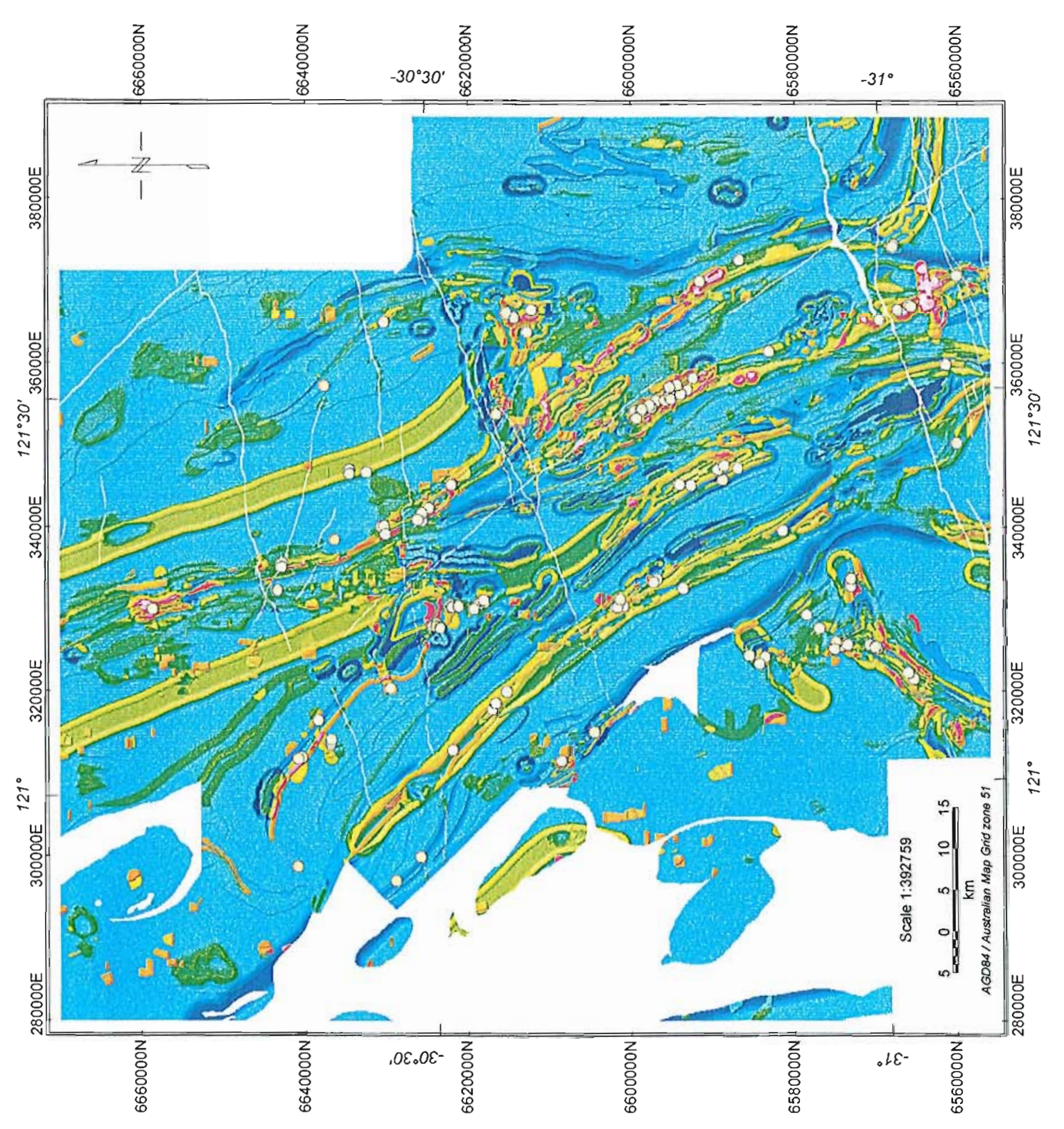

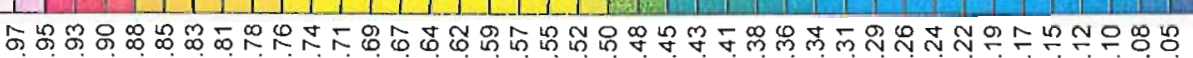

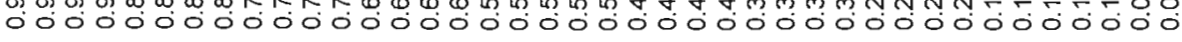

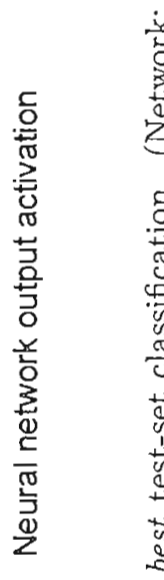

过

$\underset{\Xi}{ \pm}$

.

营 


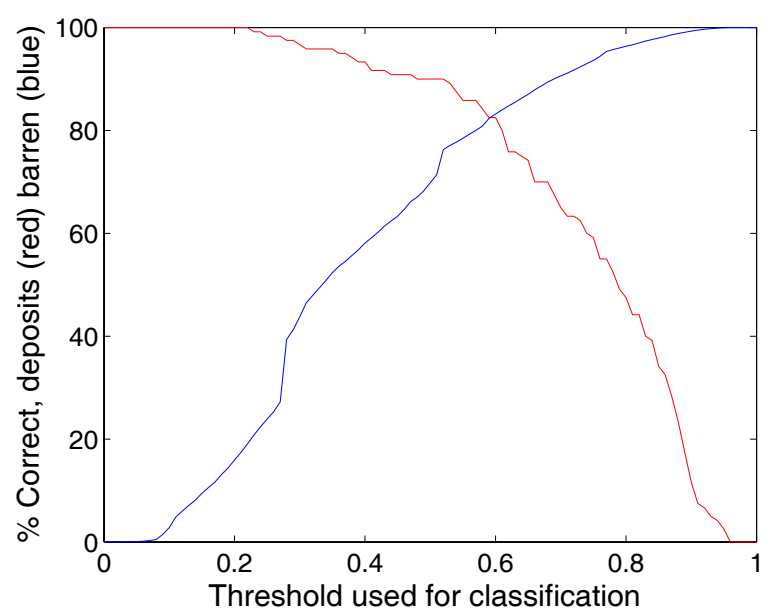

a) Classification accuracy versus threshold.

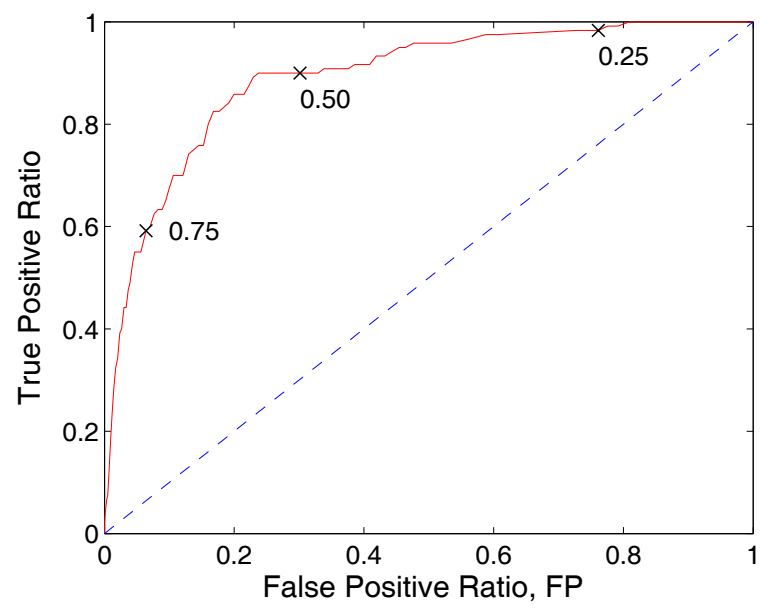

c) ROC curve showing threshold values.

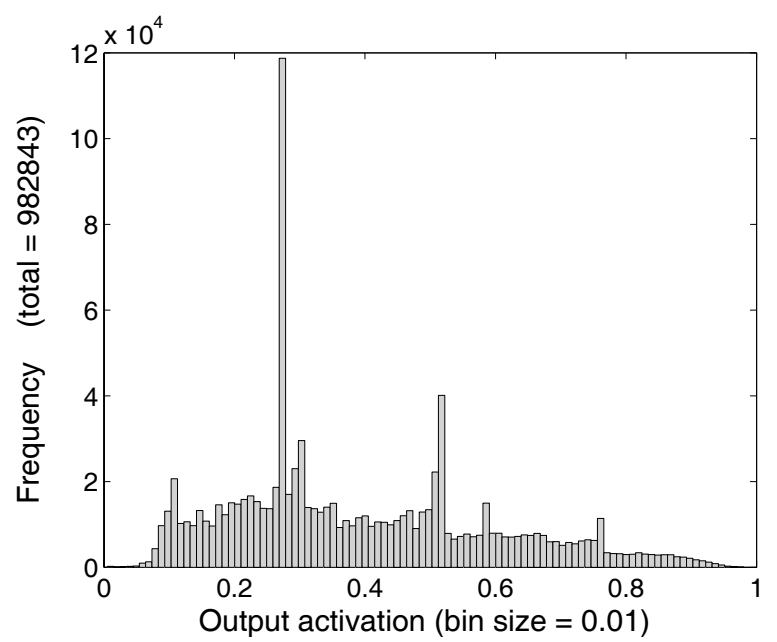

(e) Histogram of output activations of trained networks.

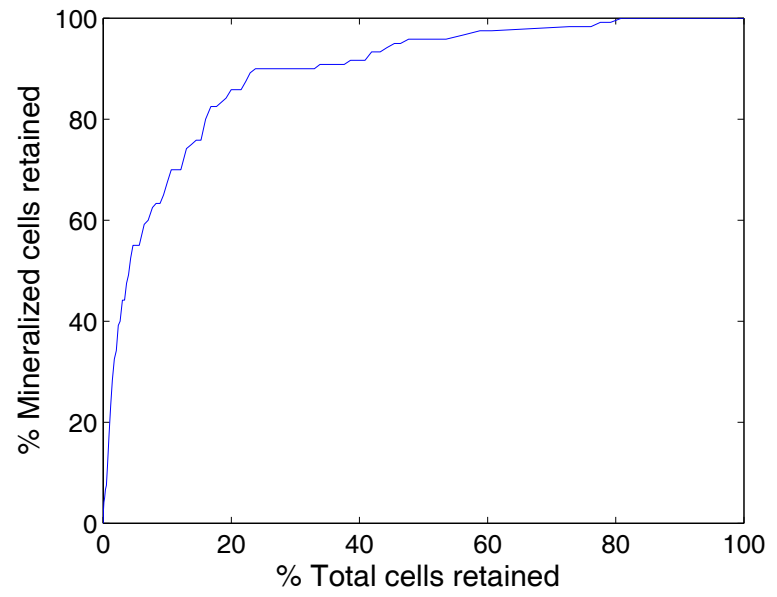

b) Percentage of deposit versus total cells captured for classification thresholds in the range $[0,1]$.

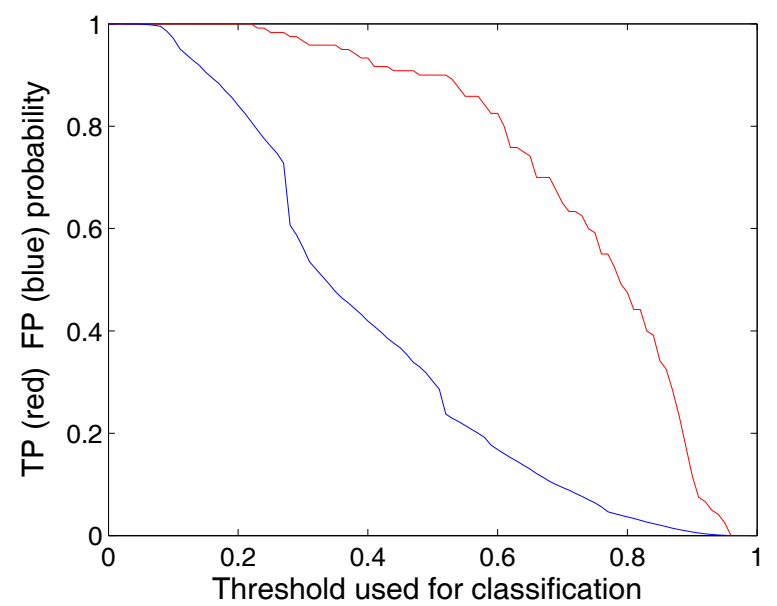

d) Proportion of correct and false deposit classification vs classification threshold.

Figure 7.50. Classification performance of the 10-20-1 hybrid fuzzy-membership-input network with the best test-set classification accuracy. All 10 inputs are fuzzy membership values. Input layer selection: $\mathrm{D} *(\mathrm{D} / \mathrm{A})$. Learning algorithm: trainlm. 


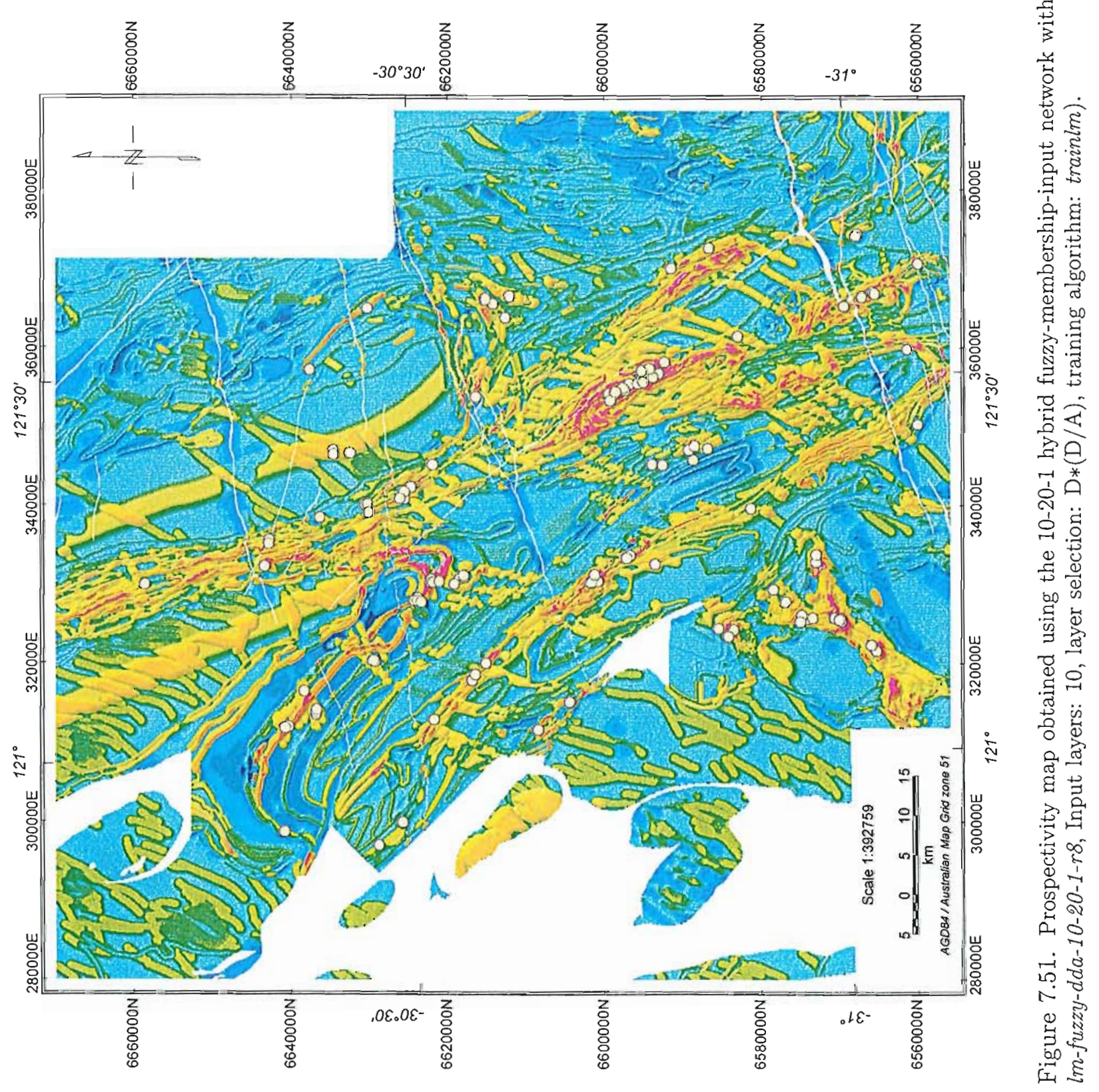




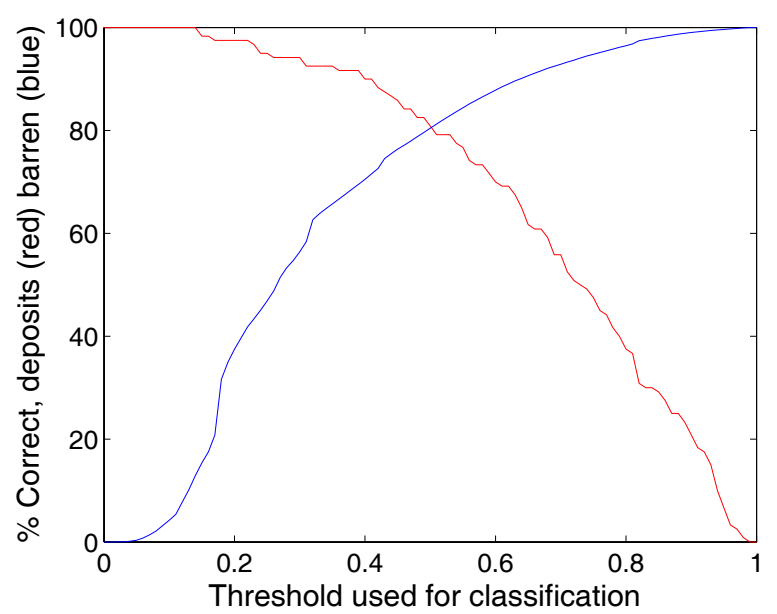

a) Classification accuracy versus threshold.

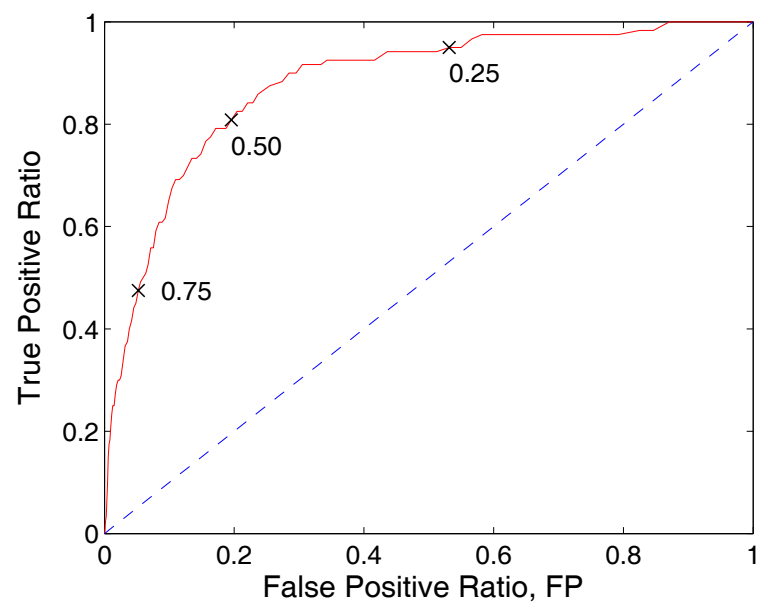

c) ROC curve showing threshold values.

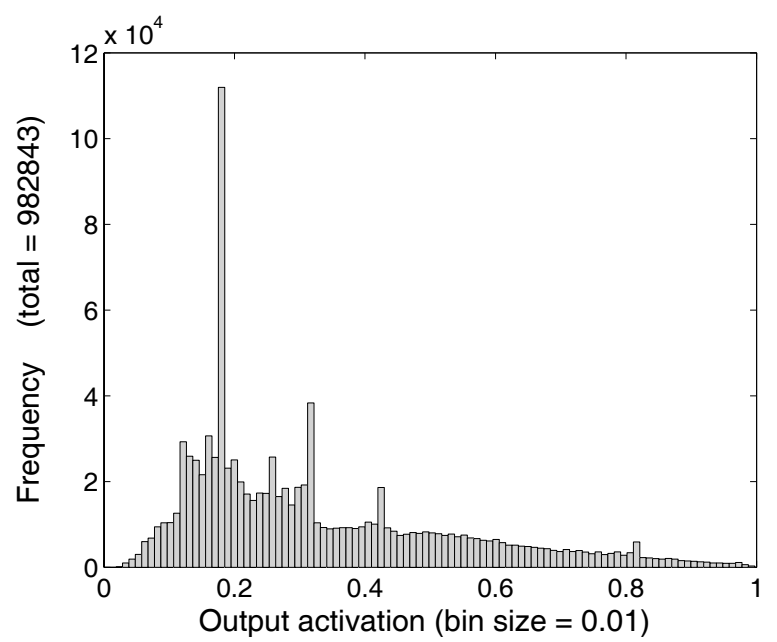

(e) Histogram of output activations of trained networks.

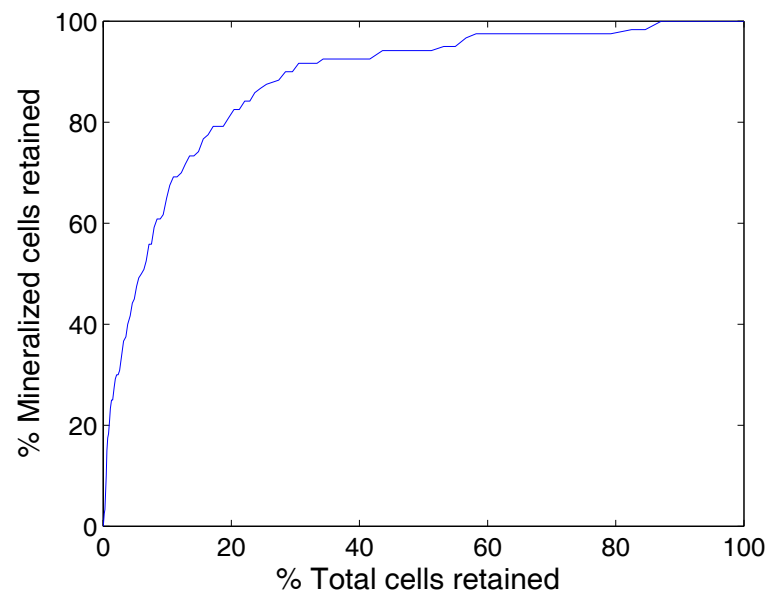

b) Percentage of deposit versus total cells captured for classification thresholds in the range $[0,1]$.

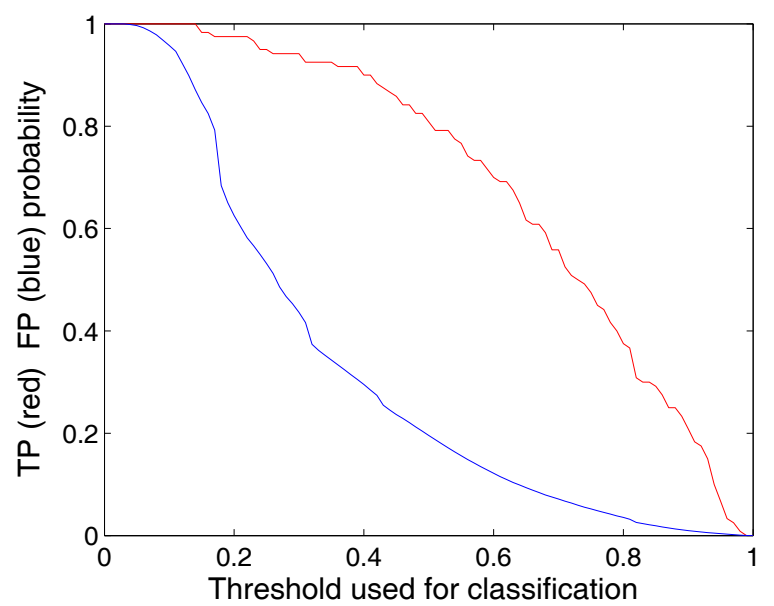

d) Proportion of correct and false deposit classification vs classification threshold.

Figure 7.52. Classification performance of the 17-20-1 hybrid fuzzy-membership-input network with the best test-set classification accuracy. All 17 inputs are fuzzy membership values. Input layer selection: categories. Learning algorithm: trainlm. 


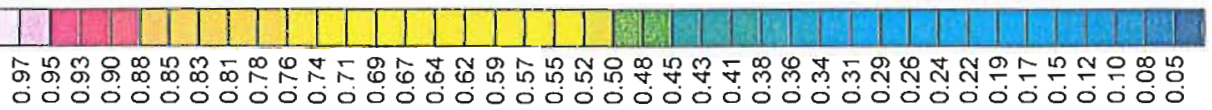

呈

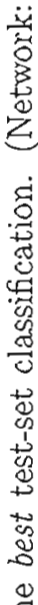

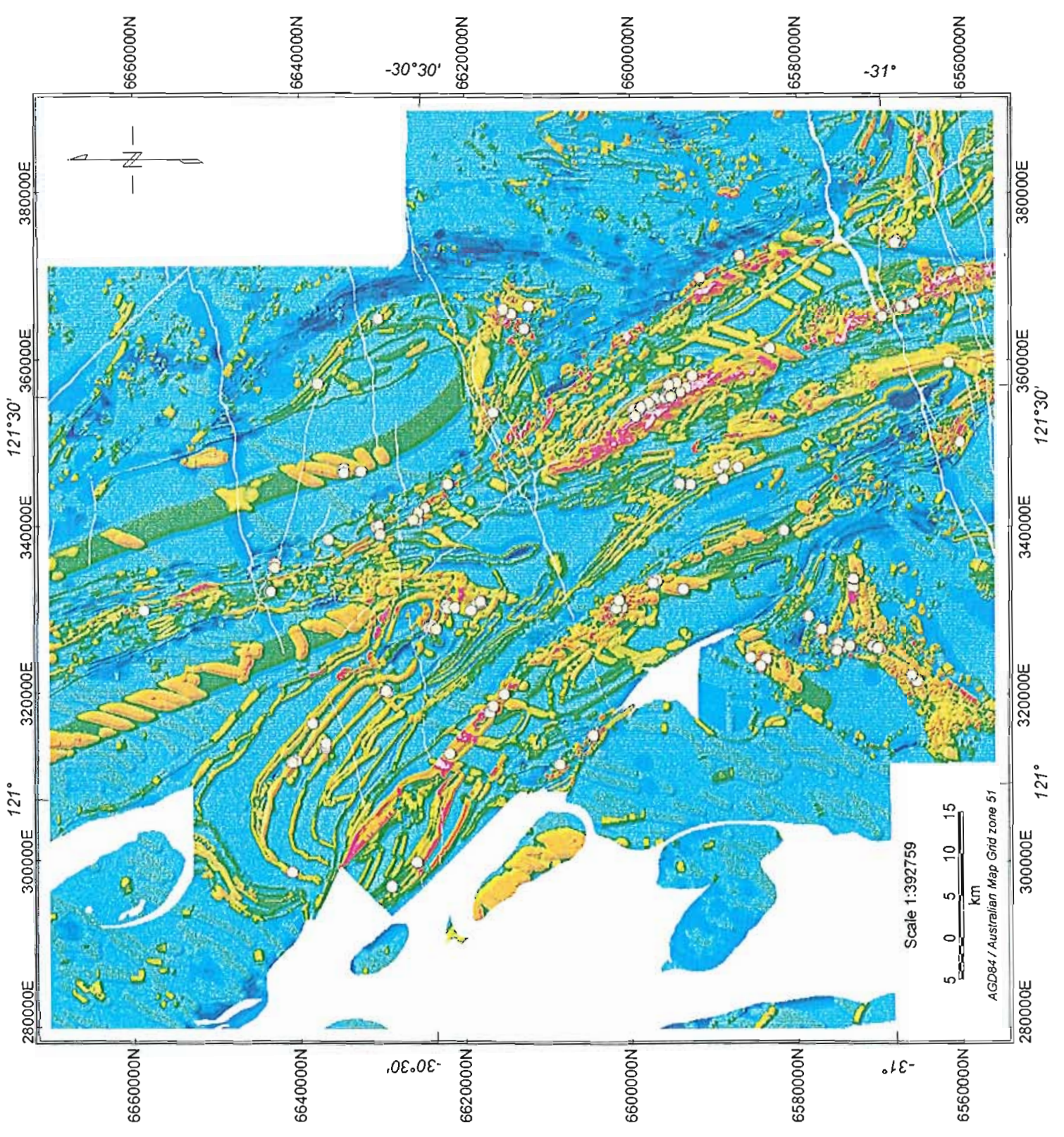

क

菅

承

胥

胥. 起

票

魚

है

更

a bo

근

疍

की

胥

호용

$\stackrel{1}{-1}$

要

so

氙

茎

요

䨔

空

है

영

背

苦

की

ㄴ.

ले

น. 8

돈

蛋

$\rightarrow \infty$ 


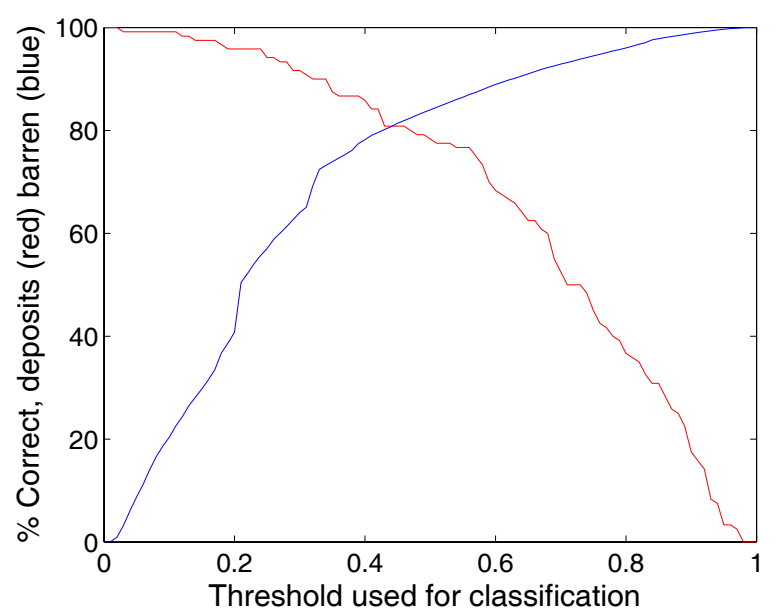

a) Classification accuracy versus threshold.

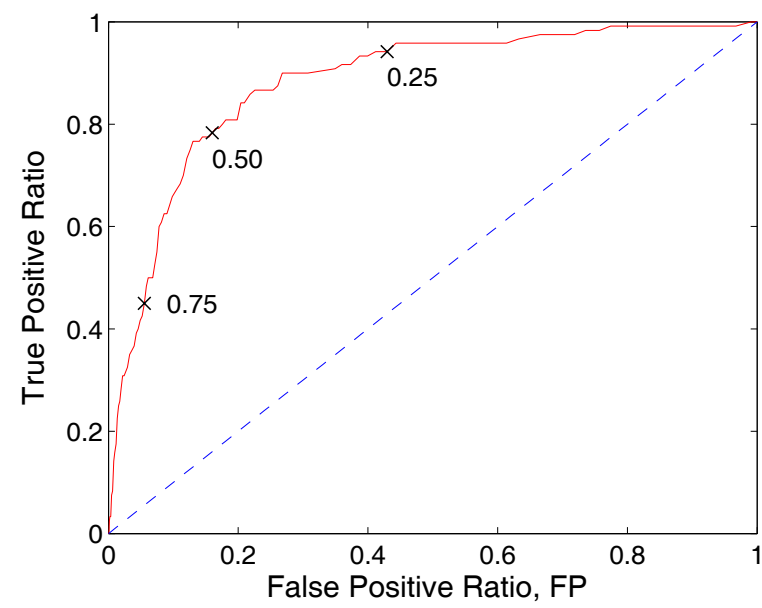

c) ROC curve showing threshold values.

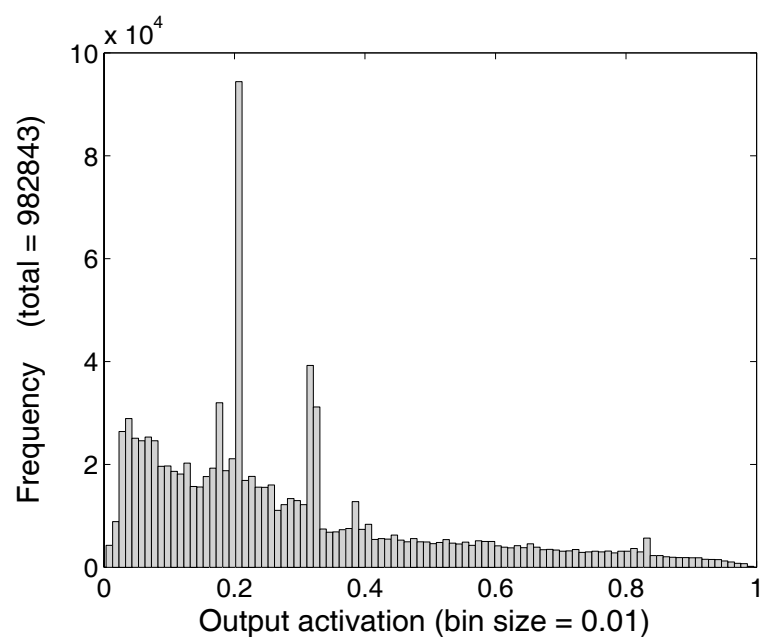

(e) Histogram of output activations of trained networks.

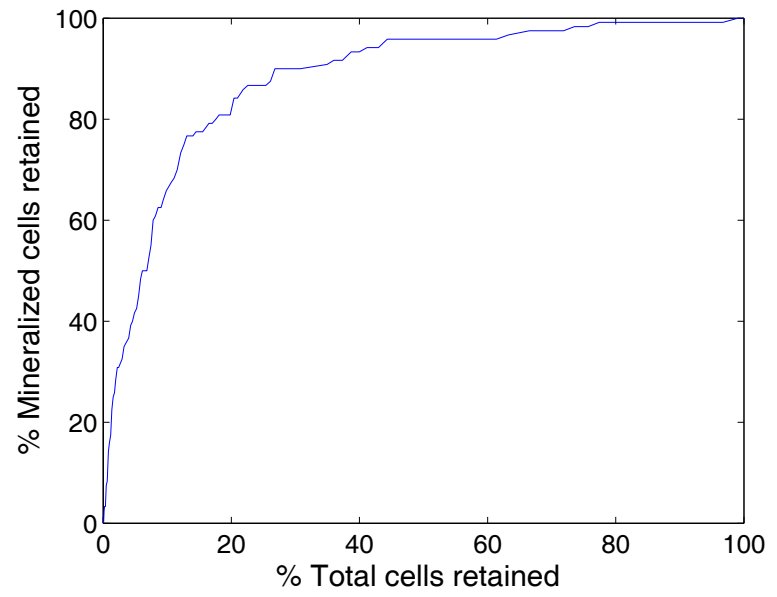

b) Percentage of deposit versus total cells captured for classification thresholds in the range $[0,1]$.

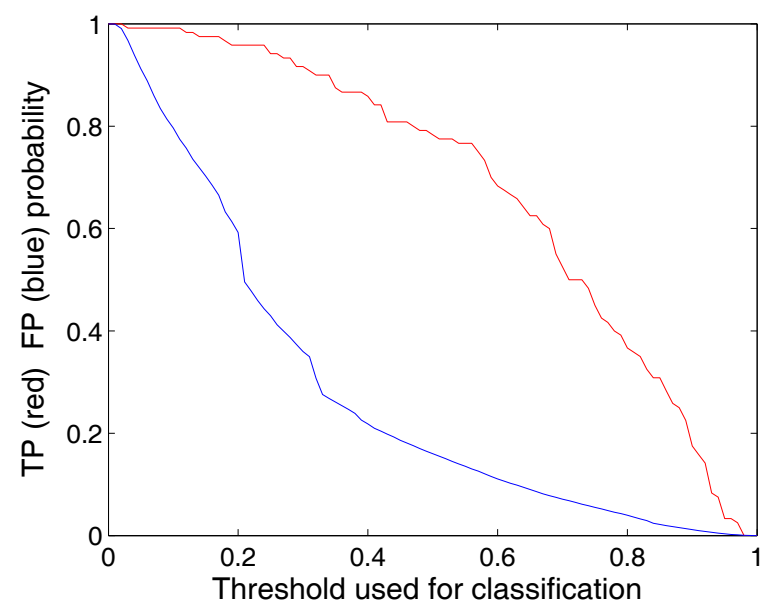

d) Proportion of correct and false deposit classification vs classification threshold.

Figure 7.54. Classification performance of the 17-20-1 hybrid fuzzy-membership-input network with the best test-set classification accuracy. All 17 inputs are fuzzy membership values. Input layer selection: $\mathrm{D} *(\mathrm{D} / \mathrm{A})$. Learning algorithm: trainlm. 


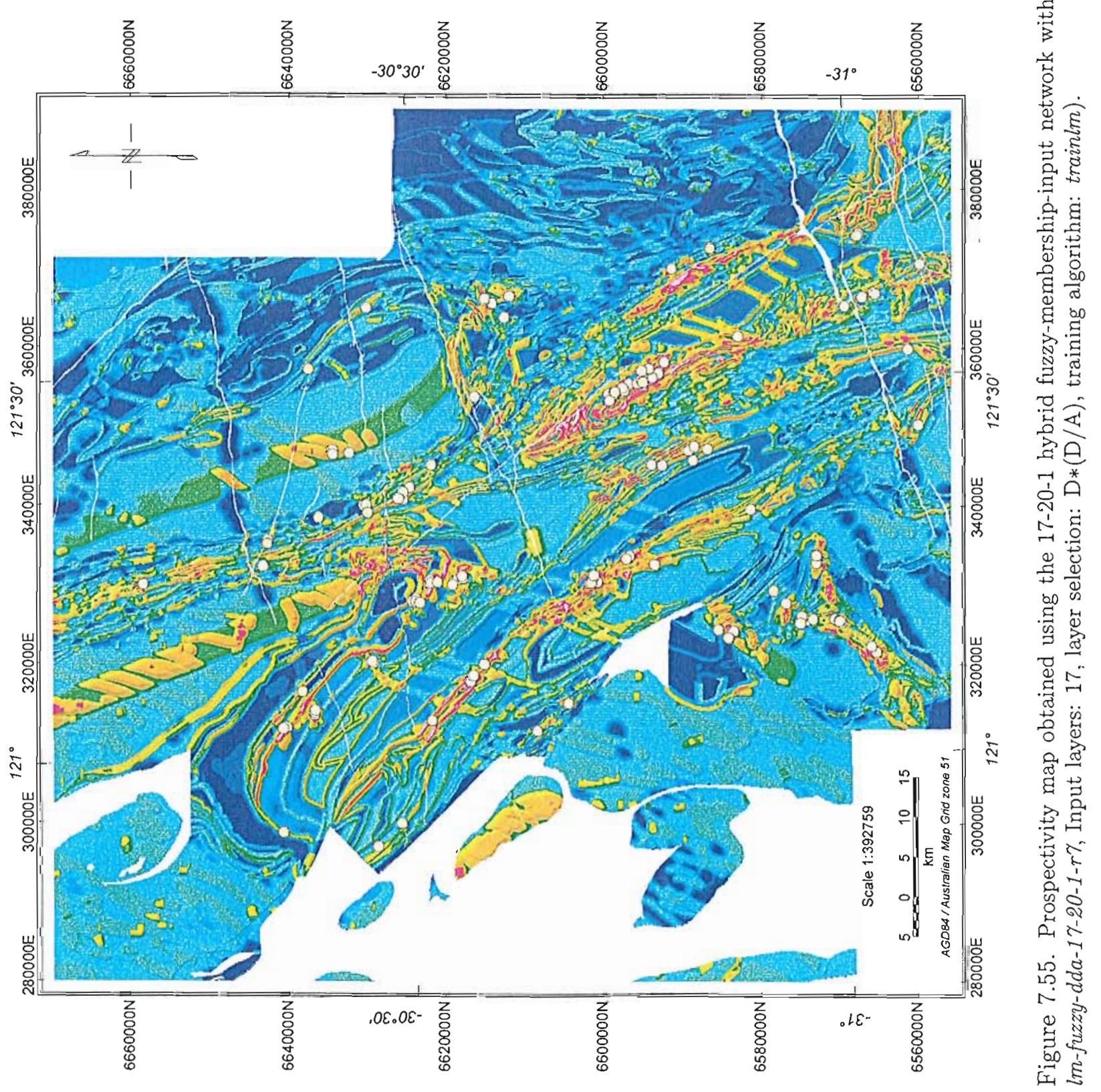




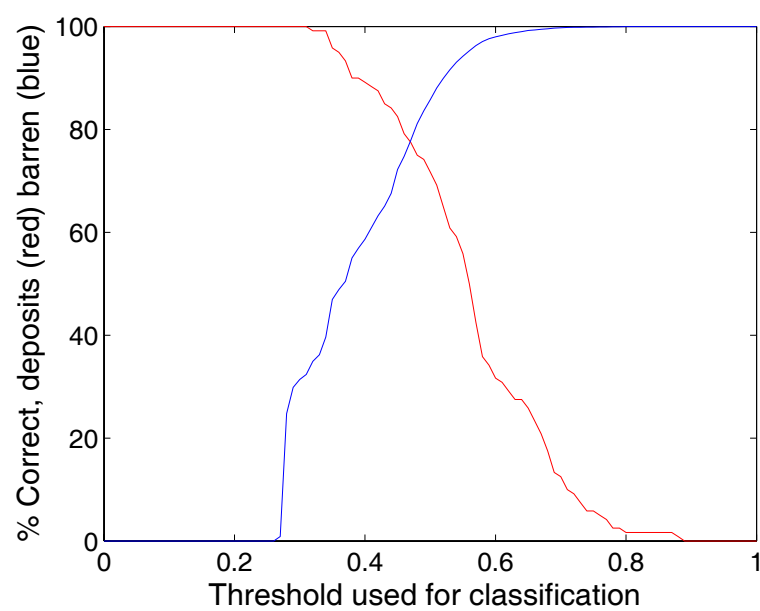

a) Classification accuracy versus threshold.

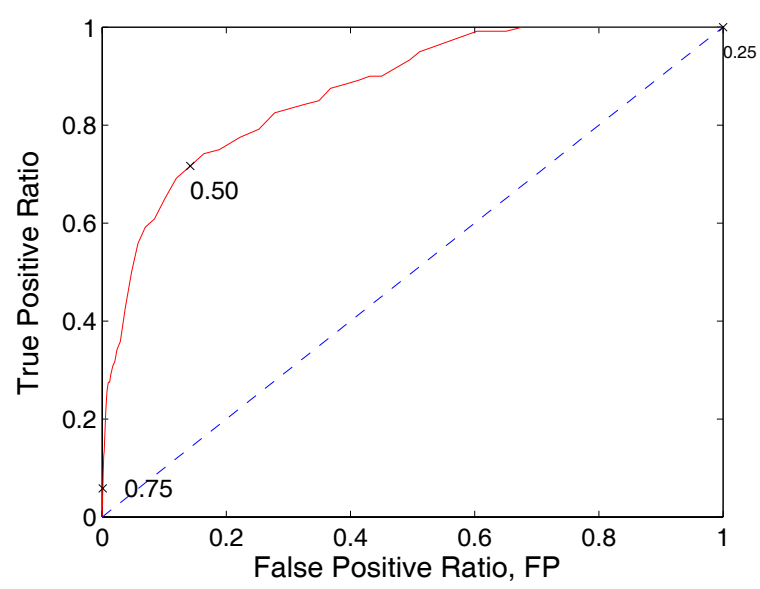

c) ROC curve showing threshold values.

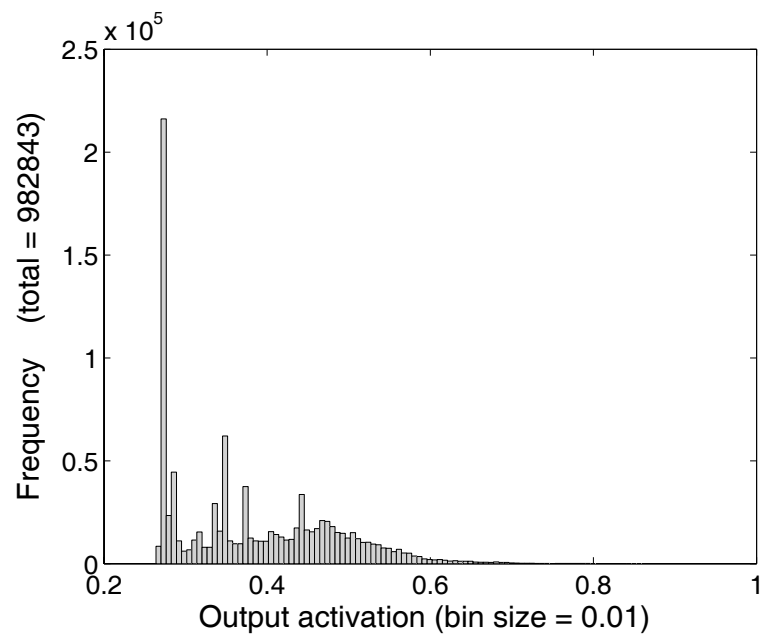

(e) Histogram of output activations of trained networks.

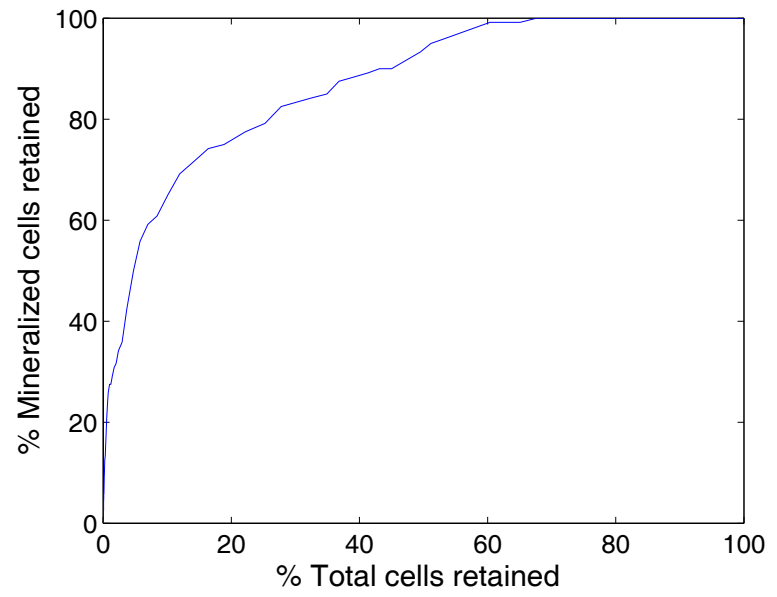

b) Percentage of deposit versus total cells captured for classification thresholds in the range $[0,1]$.

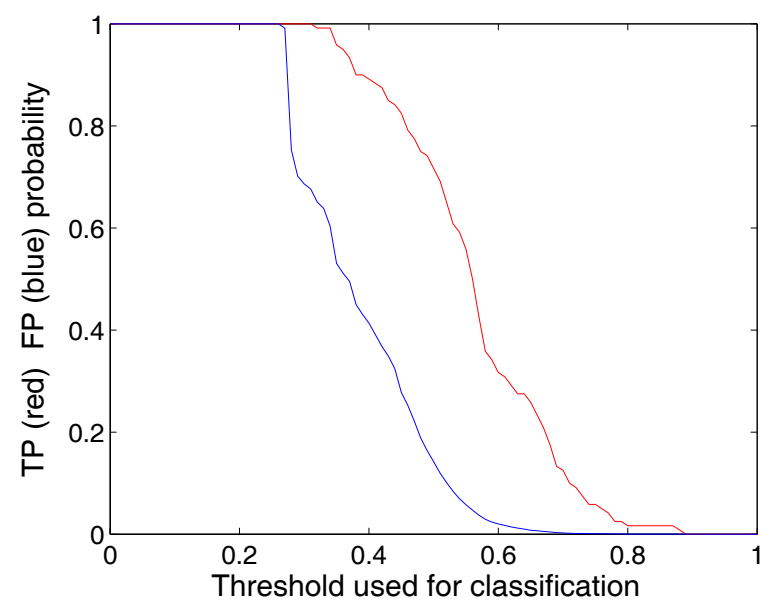

d) Proportion of correct and false deposit classification vs classification threshold.

Figure 7.56. Classification performance of the 10-input fuzzy system with the best test-set classification accuracy. Layers were combined using the gamma function $(\gamma=0.96)$. Layer selection: categories. 


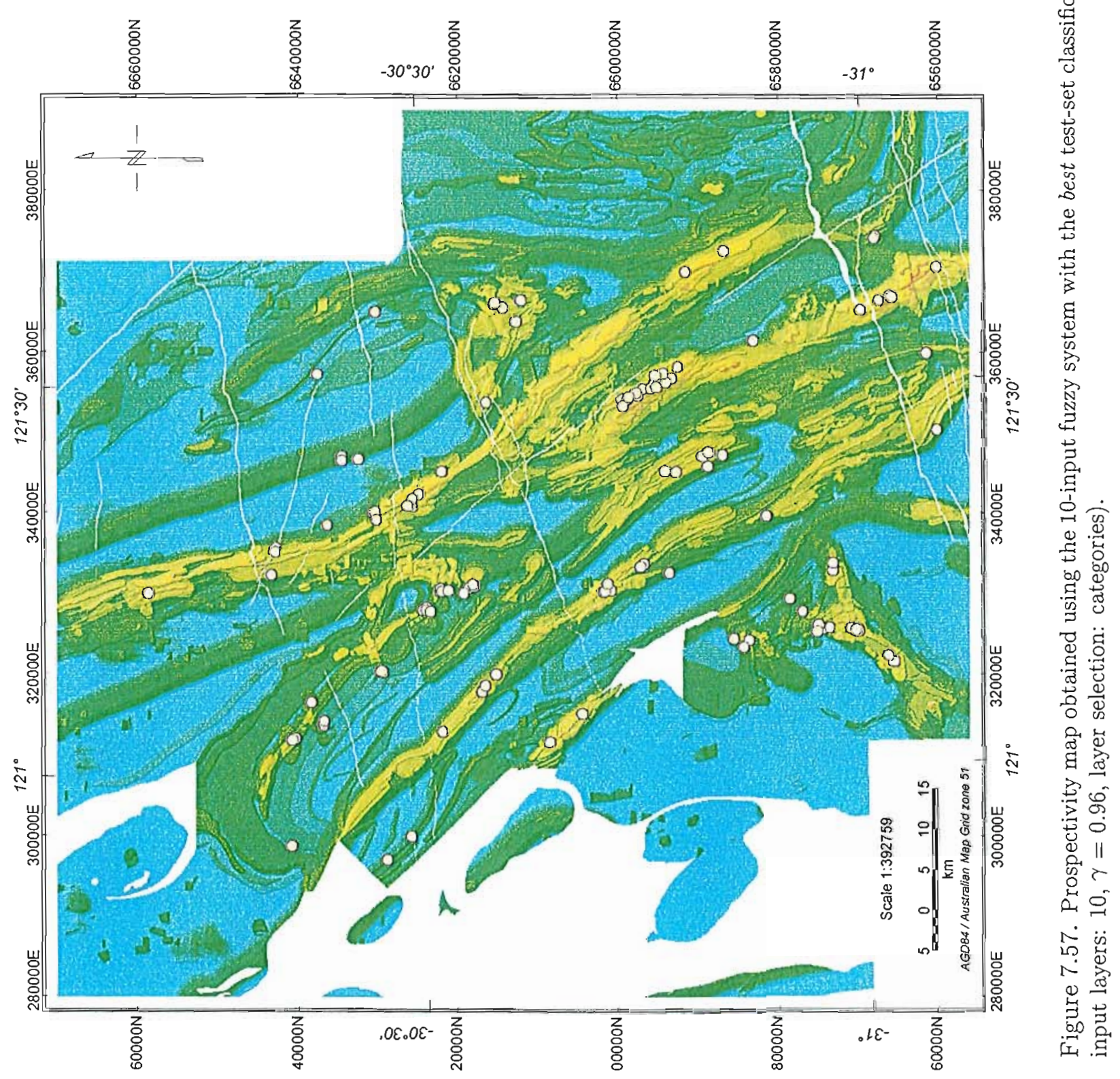




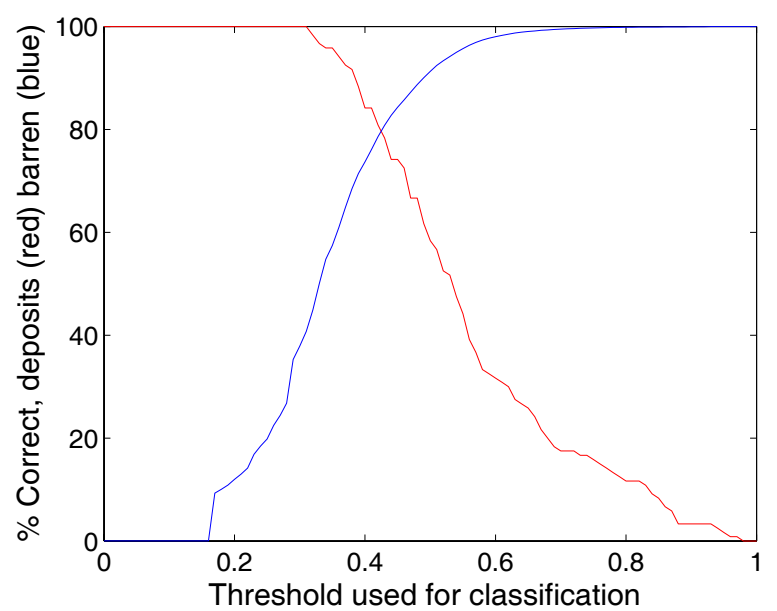

a) Classification accuracy versus threshold.

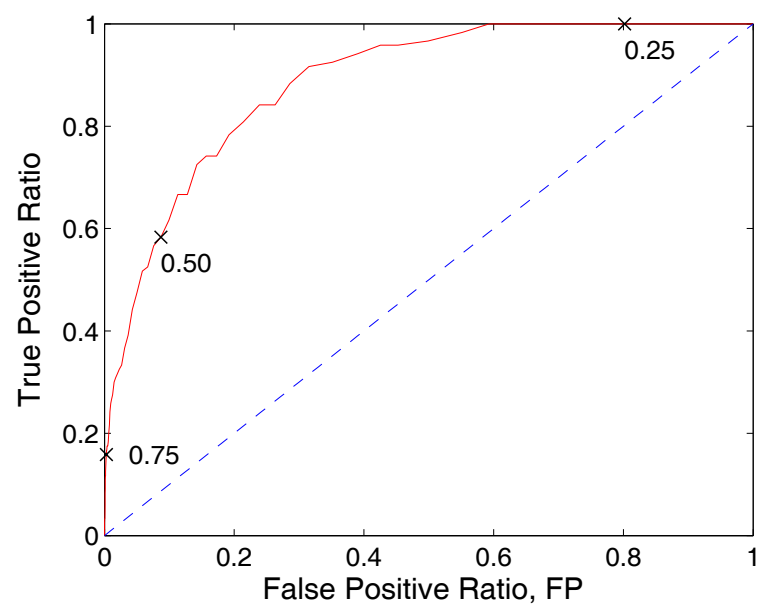

c) ROC curve showing threshold values.

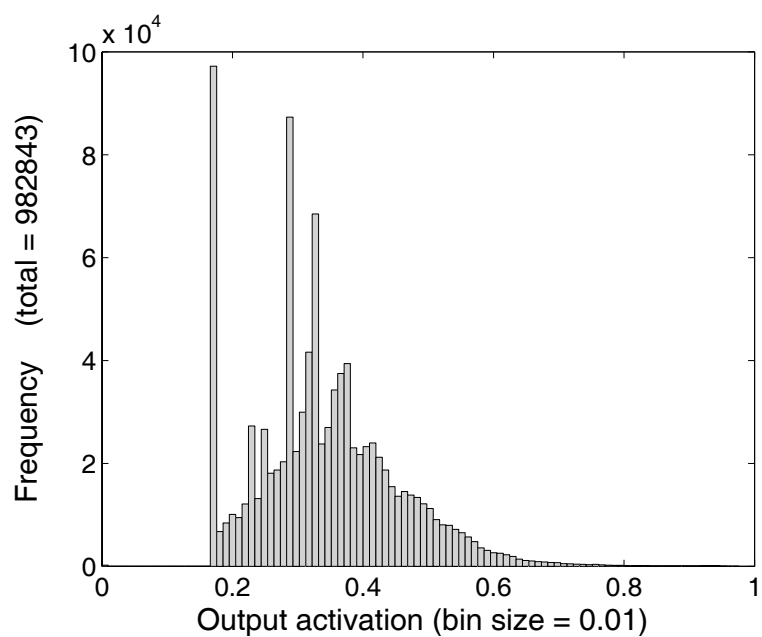

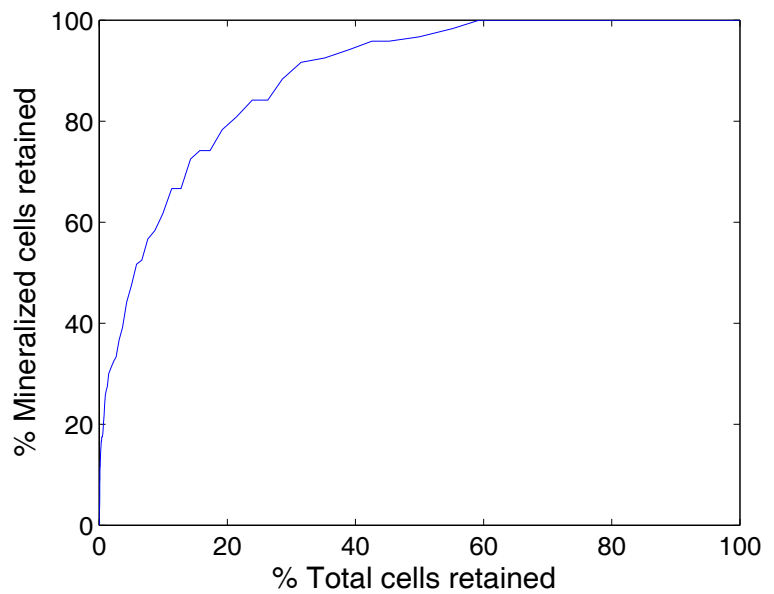

b) Percentage of deposit versus total cells captured for classification thresholds in the range $[0,1]$

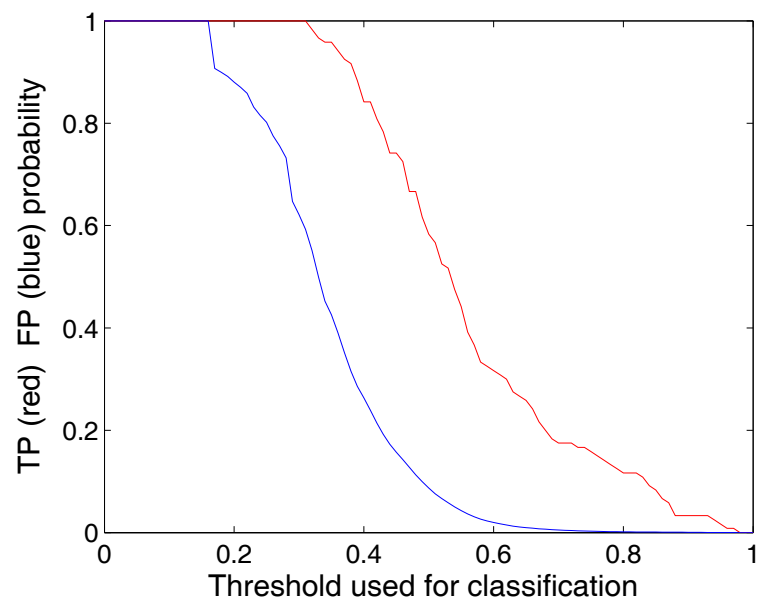

d) Proportion of correct and false deposit classification vs classification threshold.

e) Histogram of output activations of trained networks.

Figure 7.58. Classification performance of the 10-input fuzzy system with the best test-set classification accuracy. Layers were combined using the gamma function $(\gamma=0.94)$. Layer selection: $\mathrm{D} *(\mathrm{D} / \mathrm{A})$. 


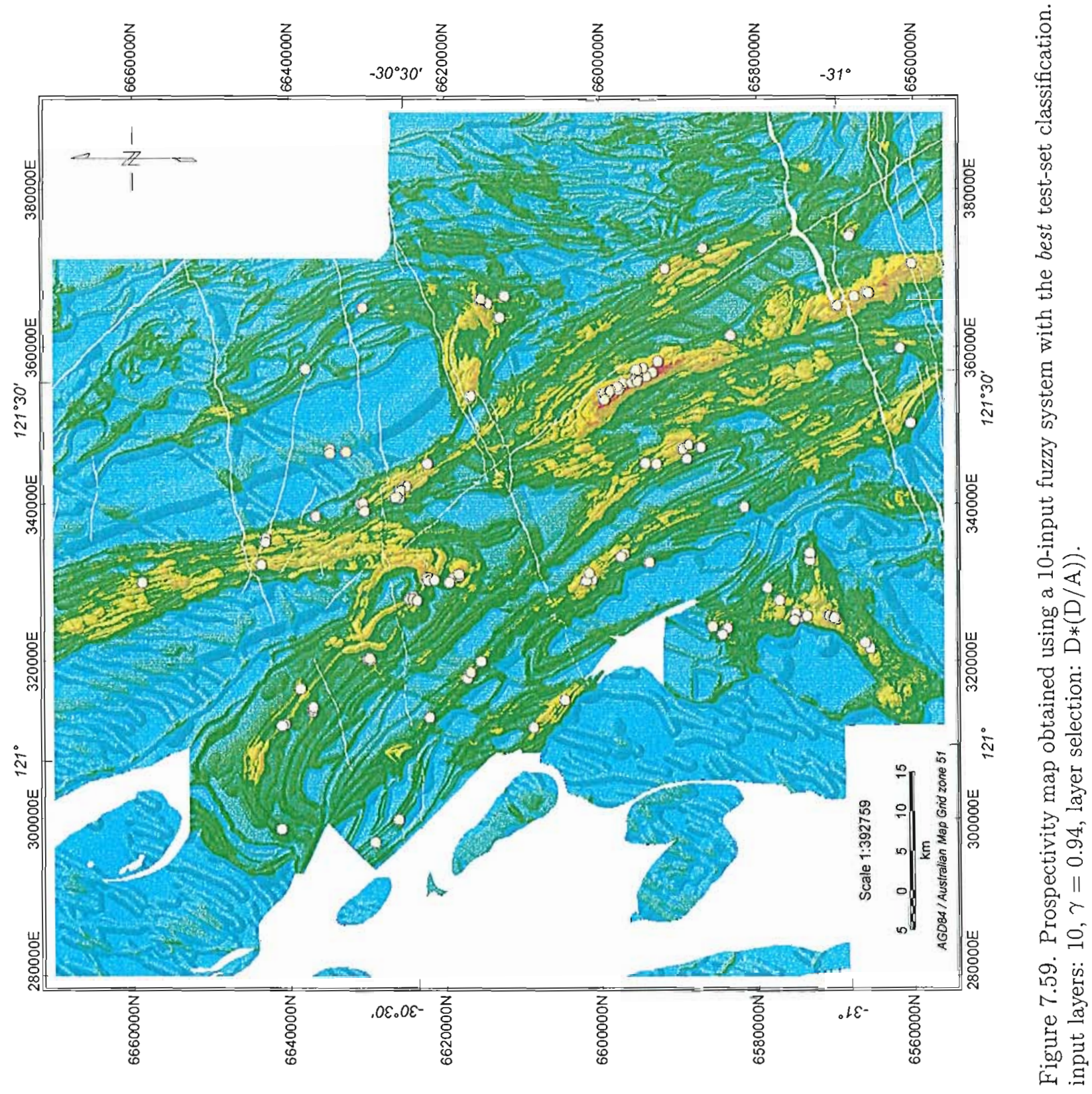

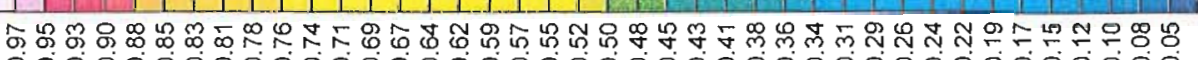

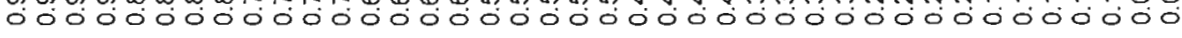




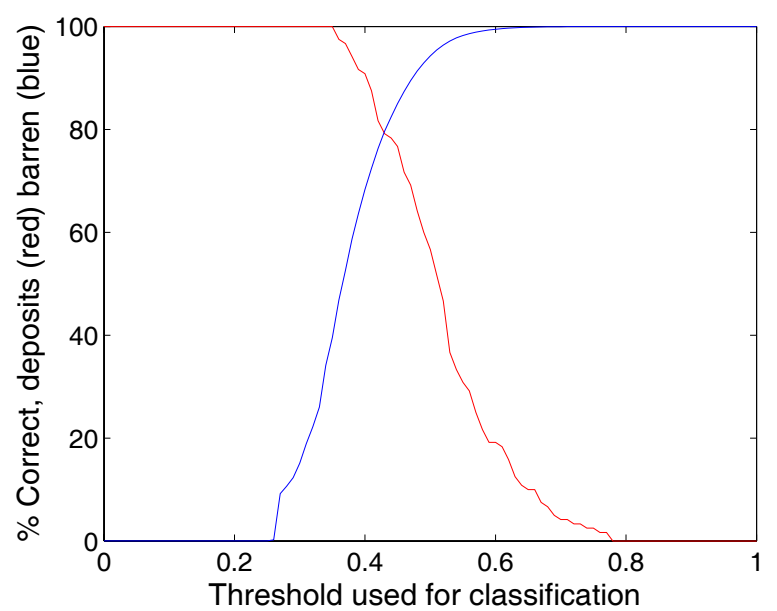

a) Classification accuracy versus threshold.

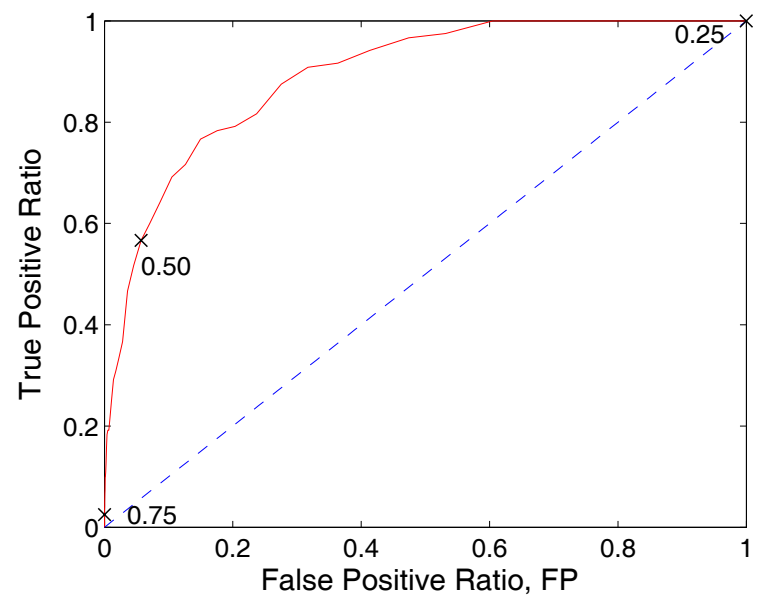

c) ROC curve showing threshold values.

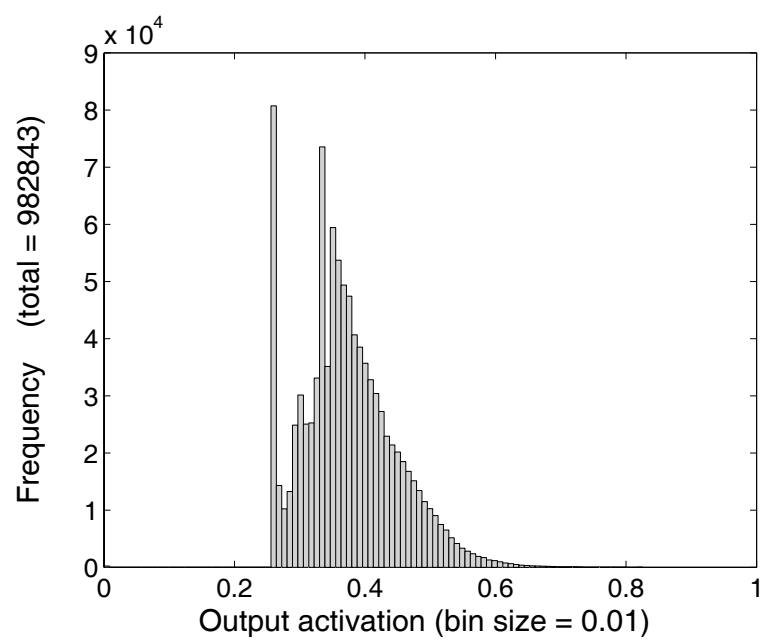

(e) Histogram of output activations of trained networks.

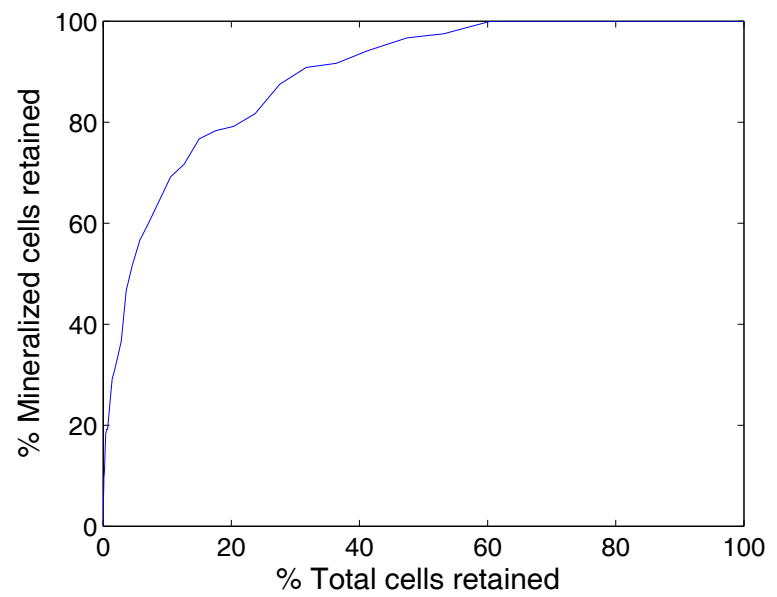

b) Percentage of deposit versus total cells captured for classification thresholds in the range $[0,1]$.

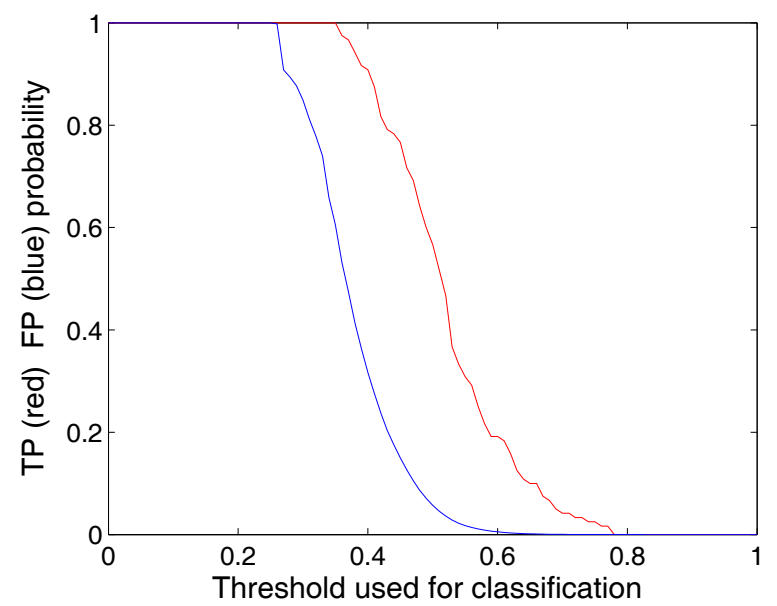

d) Proportion of correct and false deposit classification vs classification threshold.

Figure 7.60. Classification performance of the 17-input fuzzy system with the best test-set classification accuracy. Layers were combined using the gamma function $(\gamma=0.97)$. Layer selection: categories. 


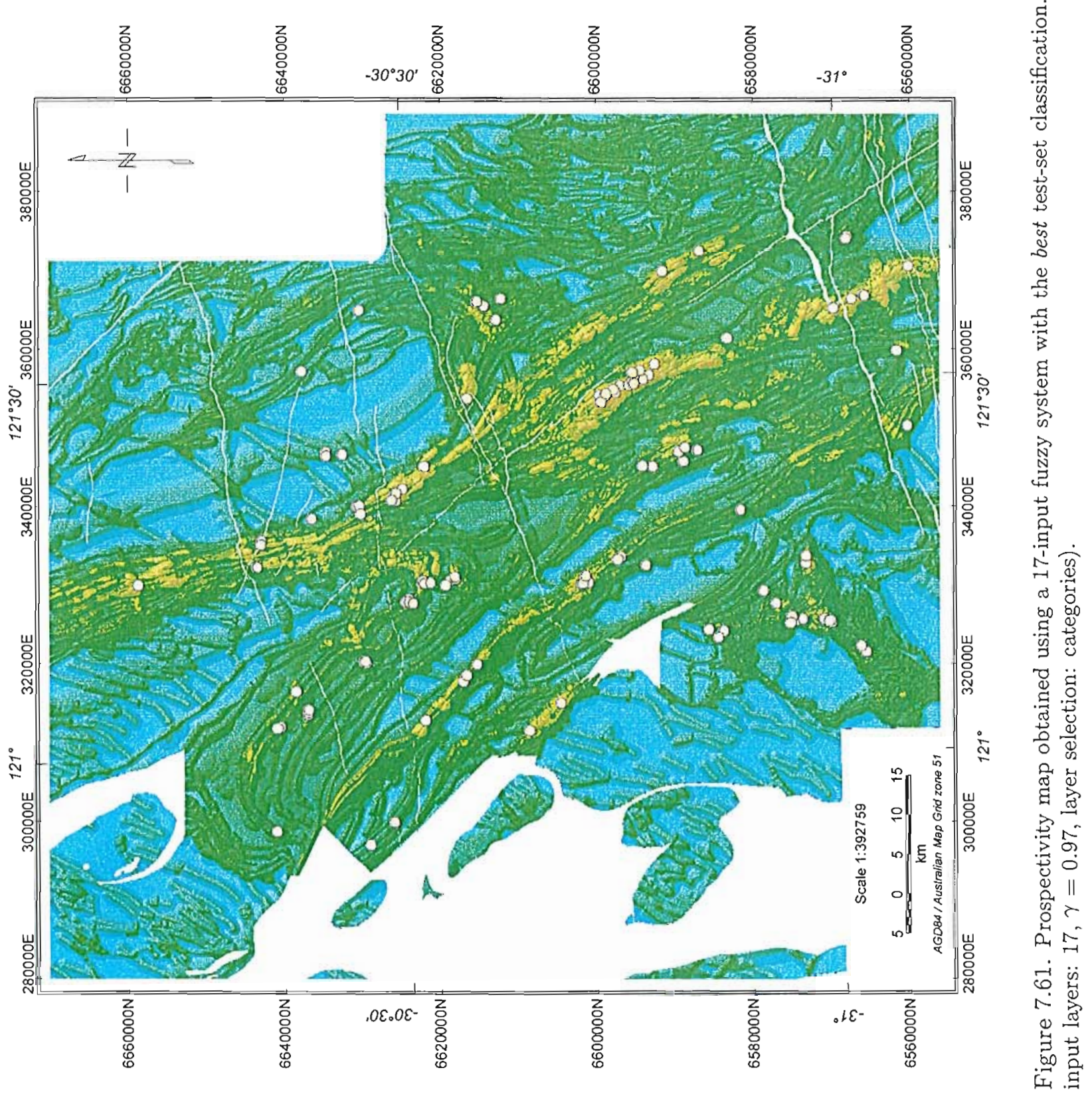




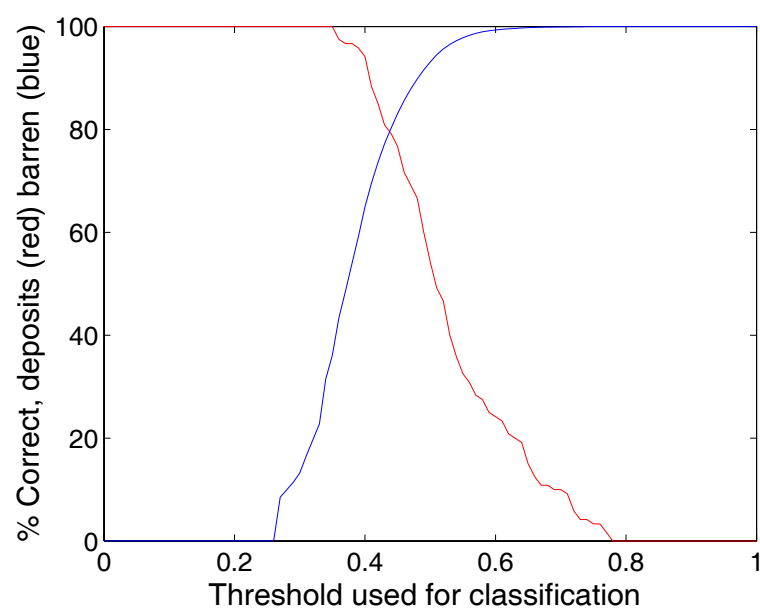

a) Classification accuracy versus threshold.

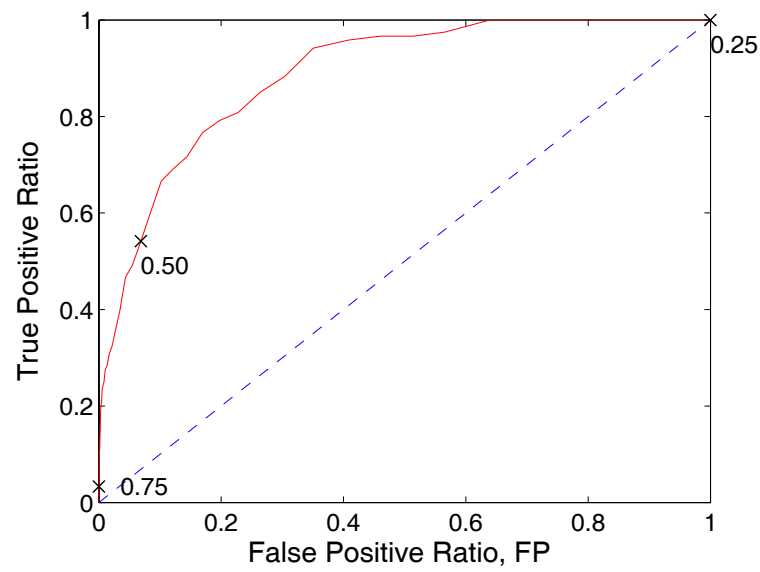

c) ROC curve showing threshold values.

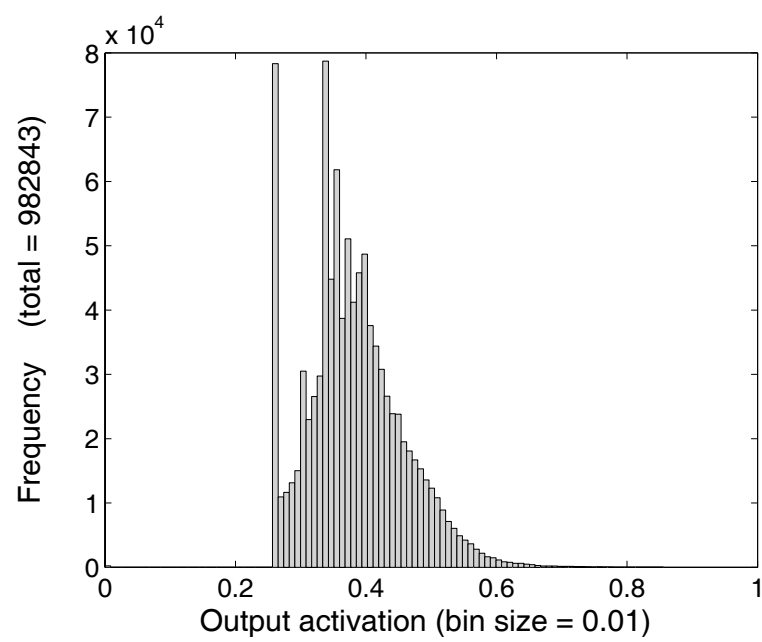

(e) Histogram of output activations of trained networks.

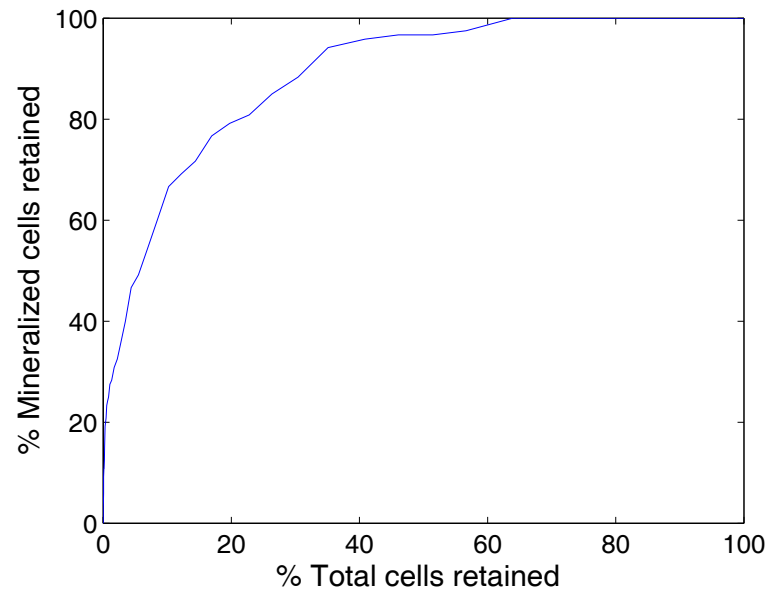

b) Percentage of deposit versus total cells captured for classification thresholds in the range $[0,1]$.

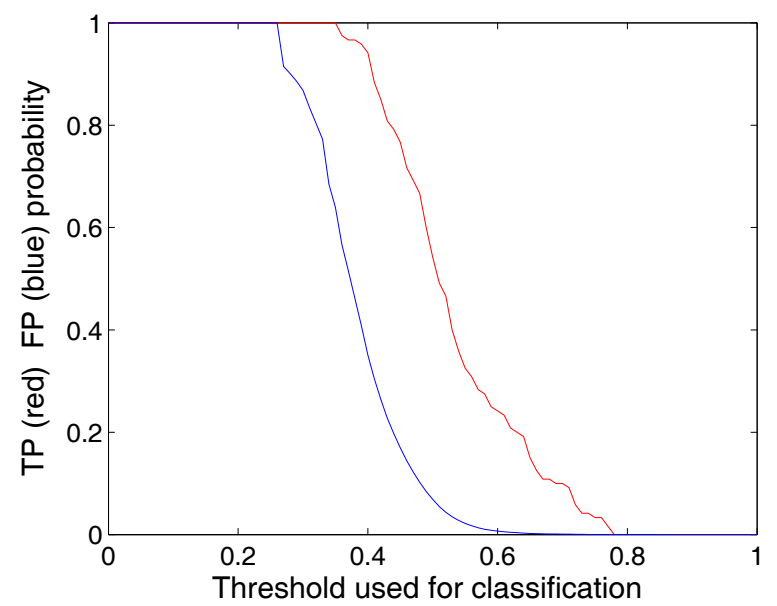

d) Proportion of correct and false deposit classification vs classification threshold.

Figure 7.62. Classification performance of the 17 input fuzzy system with the best test-set classification accuracy. Layers were combined using the gamma function $(\gamma=0.97)$. Layer selection: $\mathrm{D} *(\mathrm{D} / \mathrm{A})$. 


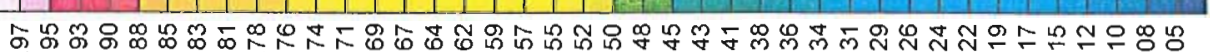

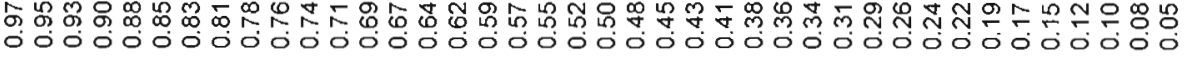



ผั

定

跑

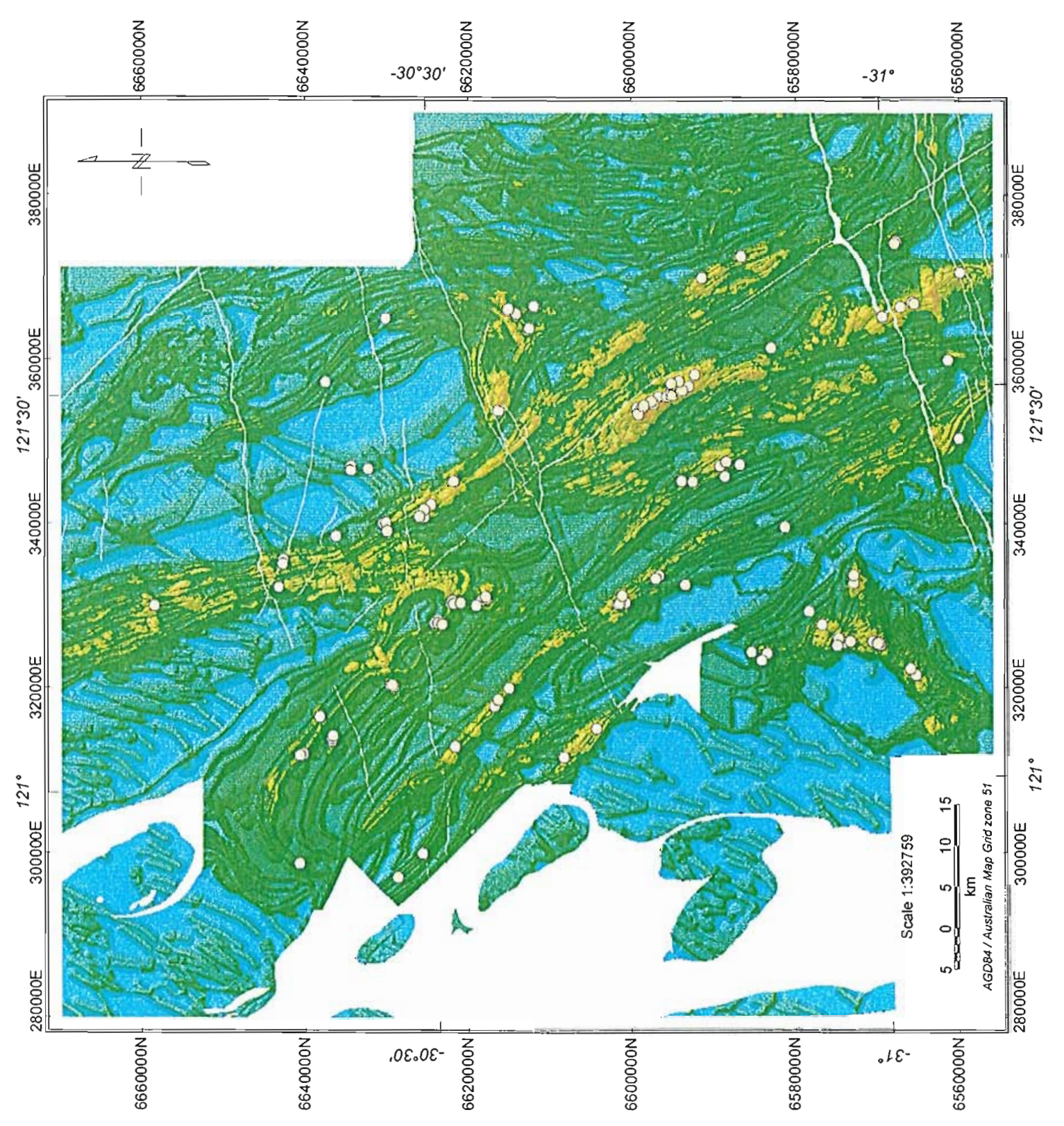

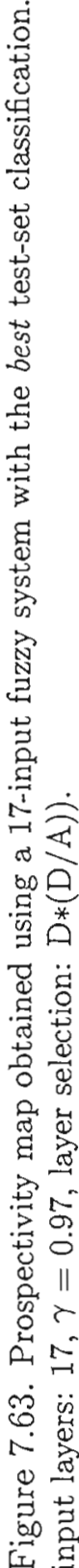




\section{Chapter 8}

\section{Summary and Conclusions}

\subsection{Contribution Demonstrated in this Thesis}

\subsubsection{Developed Neural Network Mineral Prospectivity Method}

The initial concept of this thesis was that neural networks have characteristics which make them well suited to the integration of mineral exploration data. These include their ability to learn the underlying patterns in large mixed data sets which: 1) are noisy or contain outliers, 2) involve non-linear relationships, 3) have high dimensionality, and 4) contain multiple and interacting variables. This leads to the hypothesis that digital geological, geophysical and geochemical maps can be superimposed in a GIS system to produce a set of feature vectors from which the signatures of ore deposits can be extracted and that neural networks can be trained to recognize these signatures, and hence predict the location of new deposits of similar type.

At the time this study commenced (1997), it was not clear that neural networks could be used to produce mineral prospectivity maps, in which favourability could be expressed as a continuous variable. Although a large body of literature describes the application of neural networks to pattern recognition and classification of remotely sensed data, only a few studies described the use of neural networks for mapping applications. Two studies described the use of neural networks to produce multiclass maps in which bedrock geology (Schau et al., 1994) and hydrothermal alteration type (An et al., 1994) were depicted as categorical variables. Bonham-Carter (1994) mentioned neural networks as a possible data-driven method for mineral potential mapping. However, only two studies existed in the literature that referred to the use of neural networks to produce prospectivity maps 
(Clare et al., 1997; Singer \& Kouda, 1996). The first of these described a proprietary system for which few details were available and, in the other study, the output was simply in the form of the distance to the nearest deposit.

Most of the early work in which neural networks were combined with GIS focused on automated pattern recognition and the classification of remotely sensed imagery. A neural network, used as a classifier in a mineral prospectivity problem, would produce a binary map since there are only two output classes. On the other hand, it seemed likely that a neural network trained using binary target variables (i.e. $1=$ deposit $\& 0=$ barren cells) might also produce outputs that closely approximate binary values, as do networks that learn Boolean functions.

Consequently, the first and most important problem in this study was to test the feasibility of the method using a simple GIS. A pilot study was a necessary step, since a fuller investigation of the use of neural networks for prospectivity mapping could only be justified if the networks performed reasonably well compared to the two main existing methods of prospectivity mapping, weights of evidence and fuzzy systems. Therefore, a second aspect of the pilot study was to establish methods suitable for comparing the quality of the prospectivity maps. As well as being used to assess the effectiveness of the neural network method, the empirical weights-of-evidence and the conceptual fuzzy-logic methods mineral prospectivity maps provide a means of validating the neural-network maps.

The main contribution of this study is that it demonstrates that neural networks can produce prospectivity maps that are at least as predictive as those produced using the weights-of-evidence and fuzzy-logic methods, but also offer many significant advantages compared to existing methods.

\subsubsection{Developed Methods for Quantitatively Comparing Prospectivity Maps}

Chapter 3 introduces three new methods for comparing the quality of prospectivity maps. A further two measures are introduced in Chapter 7. These are the use of:

1. Spearman's and Kendall's rank correlation coefficients, which show the degree to which the conditional probability of occurrence of known deposits $P\left(D / C_{k}\right)$, increases with increasing prospectivity map class $C_{k}$,

2. $P\left(D / C_{k}\right)$, the posterior conditional probability of a deposit given that a cell occurs in map class $C_{k}$, where $P\left(D / C_{k}\right)$ values are listed for each prospectivity map class, 
3. $P\left(D / C_{k}\right) / P(D)$, the ratio of posterior conditional probability to the prior probability of a deposit,

4. $D \times(D / A)$, and

5. area under a receiver operating characteristic (ROC) curve.

The third measure of map quality, $P\left(D / C_{k}\right) / P(D)$, shows how much being located in the area corresponding to map class $C_{k}$ upgrades or downgrades the probability of finding a known deposit relative to selecting a grid cell at random. The ratio is equivalent to $D / A$ where $\mathrm{D}=\%$ total known deposits and $\mathrm{A}=\%$ of total map area. The map class quoted is always the highest prospectivity class where the network or fuzzy system output is divided into nine classes.

A problem with the $D / A$ ratio is that the value can be high (e.g. $>300$ ) when only a few deposits are located in the highest prospectivity class, provided the area is very small. In order to account for this, a second ratio is quoted, in which $D / A$ is multiplied by the percentage of known deposits that occur in the favourable region of the binary map, giving the statistic, $D \times(D / A)$.

Although the fourth measure is a standard one for showing how classification performance varies with the value of the decision threshold, an examination of the literature indicates that it has not previously been used in mineral prospectivity studies.

\subsubsection{Demonstrated Effectiveness of Neural-Network Approach}

The pilot study, based on primary and secondary intrusion-related granite-hosted disseminated gold deposits in the Tenterfield area of New South Wales, (Chapter 4), shows that neural networks produce mineral prospectivity maps similar to those produced using the fuzzy systems and weights-of-evidence methods (Brown et al., 1999, 2000b,a). This similarity validates the neural network results in two ways. First, the similarity of neural-network maps to those produced with the conceptually-based, fuzzy-systems method indicates that the neural network result conforms to a current understanding of geological factors important for gold mineralization in the Tenterfield area. Second, the broad similarity between the neural network and empirically-derived weights-of-evidence maps indicates that the neural-network result accounts for observed spatial relationships between known mineral deposits and the variables of the GIS database.

Statistical measures of map quality indicate that the neural network method performs as 
well as, or better than, existing methods. Values of Spearman's correlation coefficient $\rho$, $P(D)$, and $P\left(D / C_{k}\right) / P(D)$ are all higher for the the neural-network maps than for maps produced with the other methods. The value of $\chi^{2}$ statistic indicates greater differences between the observed numbers of deposits and the numbers expected due to chance.

The network output is interpreted to represent an approximation to the posterior probability that the input vector $\mathbf{x}$ belongs to the class of deposits $C_{\text {deposit }}$; i.e. $P\left(C_{\text {deposit }} \mid \mathbf{x}\right)$, (White, 1989; Richard \& Lippmann, 1991; Bishop, 1995; Duda et al., 2001). This value can also be interpreted as a measure of how similar the input vector is to the deposit patterns in the training set. This interpretation of the network output fits with the intuitive notion that a prospective area has characteristics that closely resemble areas known to contain mineral deposits.

\subsubsection{Compared Performance with Weights-of-Evidence and Fuzzy Sys- tems}

An interpretation of the differences between the prospectivity estimates generated by the neural network, fuzzy-systems, and weights-of-evidence methods indicates that the neural network method has several advantages over existing methods. These include the ability to:

1. respond to critical combinations of variables, rather than automatically increasing the prospectivity due to all favourable variables,

2. combine data sets without the loss of information inherent in existing methods, and

3. produce results that are relatively unaffected by redundant data, spurious data and data containing multiple populations.

The Kalgoorlie case study is based on a large database used in an active exploration program for Archaean orogenic lode-gold deposits and therefore provides a more realistic test of the neural-network approach to prospectivity mapping than in the pilot study. Gold deposits in the Kalgoorlie Terrane formed at a wide range of crustal depths, resulting in a range of metamorphic grades, structural styles, host rocks and mineral assemblage. As a result, there are wide variations in the attributes that characterize deposits, making this a difficult pattern recognition problem. 


\subsubsection{Developed Novel GIS Layers}

Pre-processing and feature extraction can often greatly improve a pattern classification system, particularly if prior knowledge can be incorporated into the inputs (Kohavi \& Frasca, 1994; Bishop, 1995). Consequently, much work in Chapter 5 is devoted to the preparation of derived GIS layers in order to represent the essential ingredients of the mineral systems in terms of the regional-scale GIS data that were available. A regional approach rather than one focusing on ore deposit models was adopted, since mineral systems present a much larger target than deposits themselves, and because this is more appropriate to the scale at which the data were captured and compiled in a regional GIS (Wyborn et al., 1994c, 1995b). An important contribution of this work is the development of novel layers that help predict the location of Archaean orogenic gold mineralization, such as;

1. distance to felsic porphyritic intrusions,

2. distance to nearest magnetic anomaly (defined for each lithology using z-score thresholds),

3. rheological contrast at lithological boundaries (using both subjective fuzzy membership layers and a range of rock strength properties),

4. chemical contrast at lithological boundaries (sulphidation and oxidation ratios),

5. lithological diversity based on a fault density convolution,

6. strike of nearest fault (for specific types),

7. favourability of host rock (fuzzy membership values), and

8. favourability of nearest lithological contact (fuzzy membership values).

\subsubsection{Developed and Tested New Methods for Measuring Spatial Asso- ciations}

The selection of layers to be used as inputs for neural networks is the central theme of Chapter 5. Sixty potential input GIS layers were generated as a result of the work described in Chapter 6. In order to select the most useful layers for predicting deposits, a variety of statistical methods to identify positive spatial associations between known deposit points and spatial features were applied. Besides contrast of weights (BonhamCarter, 1994, $\left.C_{w}\right)$, chi-squared $\left(\chi^{2}\right)$, and Kolmogorov-Smirnov cumulative distribution function, three new methods are also introduced. These are;

1. cumulative bivariate J-function, 
2. interval-based bivariate J-function, and

3. plots of $(O-E) / E$ (where $\mathrm{O}$ is the observed and $\mathrm{E}$ is expected number of deposits in a buffer).

The latter two methods were developed in this study.

The advantage of the J-function methods is that a Monte Carlo test involving simulated J-estimates provide information about the significance of spatial relationships. Although Foxall \& Baddeley (2002) developed the bivariate J-function as a non-parametric measure of association between a point process, $X$, and any random set, $Y$, they restricted their tests to a comparison with Berman's analysis of a data set involving deposit points and lineaments (Berman, 1986). In this study, the bivariate J-function is compared to other statistical methods (listed above) and applied to a variety of types of spatial associations. As well as confirming the usefulness of the bivariate J-function for measuring spatial associations between deposit points and features represented by line segments (e.g. faults and lithological contacts), this work also shows that the bivariate J-function:

1. produces results on the identification of positive and negative associations which agree with the other methods,

2. is also useful for analysing spatial associations between deposit points and regions (e.g. magnetic anomalies) and point data (e.g. intersection of faults; Brown et al. 2002c), and

3. can be used to estimate the variable ranges in which the spatial association is maximized by determining the $x$-value corresponding to the maximum difference between the observed and simulated curves (e.g. Fig. 6.5).

The cumulative bivariate J-function is not well suited to measuring spatial associations for variables that do not involve distances (e.g. density of faults) or where positive or negative associations occur within discrete ranges of variable values (e.g. strike orientation of faults). It should be noted that none of the cumulative plots (contrast of weights, chi-squared, and the Kolmogorov-Smirnov cumulative distribution function) handle this latter type of spatial relationships well. In order to overcome this problem, a procedure for calculating the bivariate J-curve was developed in which the J-function is estimated for successive distance intervals rather than for cumulative distances. This interval-based J-function, represented as a bar graph, overcomes the problems referred to above. 


\subsubsection{Quantitatively Assessed Exploration Criteria}

An important result of the development of derived GIS layers and statistical methods to measure the spatial associations is that local empirical exploration criteria were quantitatively tested to determine:

1. the most favourable ranges of the variable values, and

2. whether the observed spatial associations are statistically significant.

Methods for measuring the spatial association between deposit points and GIS-layer variables have helped to confirm that proximity to felsic porphyritic intrusions ( $\geq 400 \mathrm{~m}$ and possibly up to $1400 \mathrm{~m}$ ) is a useful exploration criterion on a regional scale. Proximity to anticlinal axes, although well-established as an important control on the location of turbidite-hosted gold deposits (e.g. Meguma and Bendigo), has not been widely recognized as a control in the Archaean Yilgarn Block. This work provides evidence for their significance in the Kalgoorlie Terrane. Estimates of the favourable ranges of variables are useful for further exploration and contribute to our understanding of the regional metallogeny of the Yilgarn. The Yilgarn is one of Australia's most richly endowed areas, and improvements in our understanding of factors favourable for gold mineralization should improve the effectiveness of future exploration programs.

\subsubsection{Applied Neural Network Method to a Data Set Representative of those Used in Exploration}

In Chapter 7, neural networks are used to estimate the potential for Archaean orogenic lode gold deposits in the Kalgoorlie case study area. This work addresses the question of whether a neural network is an effective method for mineral prospectivity mapping. The results (in particular, percentage correct classification rates) show that the neural networks do learn the underlying patterns in the data and predict the locations of deposits for new patterns different to those in the training set. The mineral prospectivity maps produced using the trained networks also show that neural networks predict the locations of the known deposits in a geologically meaningful way. These results are obtained using a database that is typical, in terms of its size and complexity, of geoscience data sets used by mining companies for exploration, and therefore provides a realistic test of the neural network method.

Applying a simple approach, similar to that used in the pilot study, it is possible to 
produce a prospectivity map in which $27 \%$ of the known deposits ( $\geq 1000 \mathrm{~kg}$ TCG) are contained in $2.8 \%$ of the total area, which corresponds to the top two prospectivity classes in a nine-class prospectivity map (see the mapstats file on the attached CD-ROM, vol.1). This result is obtained with the 10-20-1 network which gives the best overall test set classification results (overall, $76.5 \%$; deposits, $77.1 \%$ ). The neural network that produced the best classification for all the test-set patterns then served as a benchmark against which other networks can be tested.

Using training sets augmented with the addition of random noise or using fuzzy membership values significantly improves this ability to define a small proportion of the area which contains a large proportion of the deposits. For example, maps produced with a network trained using \pm 10 normally-distributed noise and a hybrid fuzzy-neural network captured $25 \%$ of the deposits in $1.3 \%$ of the area and $27 \%$ of the deposits in $1.4 \%$ of the area, respectively (lm_cat_10_20_1_normal5pcnt_r7_log and $l m_{-} f u z z y_{-} d d a_{-} 10_{-} 20 \_1 \_r 8 \_l o g$ in file mapstats, CD-ROM vol. 1). These methods used to improve the neural-network performance are discussed in more detail below. It should be noted that although Knox-Robinson \& Groves (1997a) reported much better results in a study of the potential for Archaean orogenic gold deposits using fuzzy systems (i.e. in terms of percentage of deposits in percentage of the area), lithologies other than greenstone were excluded from the analysis.

\subsubsection{Investigated Factors Affecting the Performance of a Neural Net- work Mineral Prospectivity Method}

Factors affecting the performance of a neural-network approach to prospectivity mapping are the main subject of Chapter 7. An unexpected result of this work is that many of the neural network parameters, such as the number of hidden layers and units, and training algorithm, have only a relatively small effect on generalization performance and map quality. Other factors, such as transformation of the inputs representing distance (inverse and $\log$ ) and the use of buffer limits for layers describing the nearest fault or lithological boundary (e.g. strike or chemical contrast, respectively), also have relatively minor (but negative) effects on the results. Only capture efficiency statistic $D / A$, and possibly $D \times(D / A)$ increase if narrow buffers are used. However, the results for the hybrid fuzzy-neural networks (discussed below) suggest that buffer limits may be implemented more effectively using fuzzy membership functions.

Factors affecting the type and form of the inputs to the neural networks have the most significant effect on the results. In increasing order of effect on the test set classification 
performance, these factors are:

1. number of inputs,

2. criteria used to select inputs,

3. encoding of inputs; i.e. raw, scaled inputs versus fuzzy membership values, and

4. size of training sets.

It should be noted that the main effect of the use of fuzzy membership inputs is on the quality of the mineral prospectivity maps produced and on the capture efficiency statistics.

Each of these factors is investigated in Chapter 7, and the results are only summarized here. A comparison of networks trained using 10 and 17 inputs, selected using the same criteria, shows that 17 layers produce significantly better performance than that obtained by using 10 inputs or 25 (i.e. all available) input variables. Of the following criteria used to rank and select input GIS layers:

1. layer from each evidence category with highest $D / A$ value,

2. $D / A$ and $D \geq \sim 20 \%$,

3. $D \times(D / A)$, and

4. $D \times(D / A)$ and $D \geq \sim 20 \%$ and $D / A \geq \sim 3$,

the $D \times(D / A)$ criterion gives the best results. Networks trained with 10 layers selected using the highest $D \times(D / A)$ values produce the highest (average and best) results for classification of the test set (overall and deposit patterns), the capture efficiency statistic, $D / A$, and area under the ROC curve. For networks with 17 inputs, this criterion also gives the highest (average and best) values for overall test-set classification and $D / A$, and area under the ROC curve.

The last two factors affecting performance, shown above, highlight major limitations of the neural network method; that is,

1. train set size is limited by number of known deposits, and

2. it is a data-driven rather than a knowledge-driven method.

Both these limit or exclude neural networks from being applied to exploration in new areas where few known deposits exist. The most significant improvements in generalization and map quality in this study are achieved by attempting to address these limitations by improving the amount and type of training data; that is, through the augmentation of the training set with random noise and use of fuzzy membership inputs. These two topics are discussed in separate sections below. 


\subsubsection{Augmented Limited Training Data Using Random Noise}

The need for large amounts of training data potentially limits the use of the neural network to well-explored areas where there are abundant previously-discovered mineral occurrences and deposits. This is also the main factor affecting the performance of MLP neural networks trained using the backpropagation algorithm in mineral potential mapping. Data used to train neural networks in mineral prospectivity applications contain just two classes; a number of rare deposits and a vast number of non-deposit examples. Due to the need to represent classes within the data population in roughly equal proportions in the training data, the overall size of the training data sets is limited by the small number of known deposits.

To overcome this problem, random noise was added to the original training patterns in order to create additional synthetic deposit training data. Through the addition of noise to the original deposit training data, the number of deposit training patterns was increased from approximately 50 to 1000. Experiments in Chapter 7 on the effect of the number of deposit patterns available for training in the Kalgoorlie case study area show that both the classification performance of a trained network, and quality of the resultant prospectivity map, increase significantly with increased numbers of deposit patterns.

The use of random noise to create additional synthetic deposit-patterns helps overcome the lack of deposit patterns that limits the training set sizes. Training with the larger noise-augmented training data sets results in significantly increased:

1. Classification performance. The percentage of correct classifications significantly improves for the independent test set (overall) as well as for deposit patterns in the test set. For example, using $\pm 40 \%$ uniform random noise, the test-set classification performance increases from $67.9 \%$ and $68.0 \%$ to $72.8 \%$ and $77.1 \%$ for the average of 10 networks for test data set overall and test-set deposit patterns, respectively.

2. Measures of map quality. For example, for $\pm 40 \%$ uniform random noise, the statistics $D / A$ increases from 8.2 to $17.9, D \times(D / A)$ increases from 105 to 226 , and the area under the ROC curve increases from 0.79 to 0.87 . Generally, these improvements in performance apply to both the average and best results for $D / A$ and area under the ROC curve, although, in the case of $D \times(D / A)$, only the average values increase.

Increasing the size of the training-stop data set in addition to the training set results in a further increase in average classification performance. 
For $\pm 40 \%$ uniform random noise, the average percent classification of the training set increases from $72.8 \%$ to $73.5 \%$ (overall) and from $77.1 \%$ to $77.4 \%$ for deposit patterns, $D / A$ decreases from 17.9 to $14.7, D \times(D / A)$ increases from 226 to 296 , and the area under the ROC curve remains unchanged.

More work is needed to determine whether uniform or normally-distributed noise is more effective. Although $\pm 40 \%$ uniform random noise (network topology: 10-20-1) gives the best average results $(72.8 \%, 77.1 \%, 17.9,226,0.87$ for the percentage correct classification of the test set overall, test-set deposit patterns, $D / A, D \times(D / A)$, and area under the ROC curve, respectively), $\pm 5 \%$ normally-distributed noise gives the best results (i.e. $79.0 \%$, $85.1 \%, 19.0,269,0.87)$.

The method described here is not limited to MLP networks and could be applied to increase the number of patterns available for training for other types of neural networks or in other applications in which training data are rare or difficult or expensive to obtain.

\subsubsection{Used Fuzzy Membership Values as Neural Network Inputs}

The results of experiments in Chapter 7 demonstrate that using input GIS layers, in which the cell values represent fuzzy membership values, is an effective method of combining subjective geological knowledge with empirical data in a neural-network approach to mineral prospectivity mapping.

Two types of fuzzy membership layers are used:

1. Objective fuzzy membership layers. Statistical relationships between known gold deposits and the GIS-layer variable are used to determine the fuzzy membership values (e.g. favourable host-rock type and favourable contact type). This type of fuzzy-membership input is a useful alternative to the 1-of-n coding commonly used for categorical inputs, particularly if there are a large number of classes (e.g. hostrock type). It has the advantage of reducing the size of the input feature vectors, thus saving computer memory, and reduces the likelihood that the network will overfit the data because there are too many weights in relation to the size of the training data.

2. Subjective fuzzy membership layers. The assignment of fuzzy membership value, although based on geological field data, is purely subjective (e.g. favourable rheological contrast). It is shown in Chapter 6 that, in the case of rheological 
contrast, a subjectively estimated input can be superior to objective data if those data are inconsistent or not extensive enough to be representative at a regional scale.

The average performance statistics are generally similar for both methods. However, a comparison of the single networks that produced the best overall test-set performance shows that using fuzzy membership values to represent the host-rock geology input results in significantly improved values for the overall test-set performance and capture efficiency statistic $D \times(D / A)$, and higher values for $D / A$ and the area under the ROC curve.

\subsubsection{Extended Use of Fuzzy Inputs to Create a Hybrid Fuzzy-Neural Network}

Traditionally, mineral prospectivity methods are either entirely data-driven or entirely knowledge-driven. However, there is a need, particularly in new exploration areas, to combine very limited quantitative data with conceptual models or exploration criteria derived from mature areas. An extension of the concept of using fuzzy membership values in order to represent categorical data types as inputs is to use fuzzy membership values for all the network inputs. Such a system effectively represents a simple modular form of hybrid fuzzy-neural network system.

The main advantage of fuzzy membership inputs is that they enable a large range of subjective data (e.g. favourability of tectonic environment, host stratigraphy, or reactivation along major faults) which are currently used in regional exploration programs but which would not normally be included in an empirical neural-network approach.

\subsubsection{Compared Neural Network, Fuzzy and Hybrid Fuzzy-Neural Net- work Systems}

Hybrid fuzzy-neural systems produced slightly poorer and fuzzy systems produced significantly poorer generalization performance than neural networks, but significantly better map quality statistics (i.e. $D / A$ and $D \times(D / A)$ and area under ROC curve) . The highest overall test-set classification accuracies though, were obtained with networks using only fuzzy inputs (i.e. $79.0 \%$ for the best 10 input network with layers selected with the category criteria, Table 7.38 , and $79.0 \%$ for the best 17 input network with layers selected using the $D \times(D / A)$ criteria, Table 7.40).

The very high values for capture efficiency statistics $(D / A, D \times(D / A))$ for systems with 
fuzzy inputs are probably due to the very small proportion of cells which are assigned high prospectivity values (see histograms of the output values for the three types of systems, Fig. 7.14). In the case of fuzzy systems, this is most likely a result of the increasive nature of the gamma function at high gamma values. High output values would be rare since they would then depend on the relatively rare conjunction of a high number of favourable inputs at a single location. Hybrid systems also have high capture efficiency ratio values but for slightly different reasons. It is likely that the use of fuzzy membership functions used to transform the raw input values causes the trained hybrid fuzzy-neural network to combine the inputs in a more additive way.

The poorer generalization performance of the fuzzy, and to a lesser extent, the fuzzy hybrid systems is interpreted as the result of the way the fuzzy membership functions, which define the favourable variables, simplify and smooth out spatial relationships and ultimately narrow the definition of what constitutes highly favourable locations for gold deposits. Perhaps two thirds of the deposits fit the model implicit in the fuzzy membership functions and this, together with the small number of cells, leads to high $D / A$ and $D \times(D / A)$ ratios. Other deposits that do not fit this simple model are not recognized and contribute to the poorer generalization results.

Prospectivity maps presented in Chapter 7, using hybrid fuzzy-neural networks, clearly reflect the geology and structure in the input GIS maps, but, at the same time, appear to smooth some of the noise and artifacts, which are apparent in neural-network maps, without the loss of information that is apparent in maps produced using the fuzzy systems.

Thus, the hybrid fuzzy-neural systems have several advantages compared with either the neural network or fuzzy systems, that is,

1. ability to incorporate prior knowledge,

2. good generalization as reflected in best test-set classification performance,

3. very high values for map quality statistics, and

4. mineral prospectivity maps with low noise, no artifacts and high level of geological detail. 


\subsection{Achievable Improvements to Existing Study}

\subsubsection{Combine Factors that Improved Performance - Augmented Train- ing Data with Fuzzy Inputs}

As noted above the most significant increases in generalization ability and map quality result from modifying the quantity and type of training data; i.e. the augmentation of the training data with random noise, use of fuzzy membership values as inputs, and the use of input variables selected with the $D \times(D / A)$ criterion. Combining these factors is likely to lead to a further increase in performance.

None of these factors depends on the type of neural network. Recently, other types of networks have been tested in mineral prospectivity applications. In particular, the work of Harris \& Pan (1999) and Singer \& Kouda (1999a) suggests that it may be possible to achieve better results with probabilistic and general regression neural networks than with MLP networks.

\subsubsection{Maximize Use of Limited Training Data with a Leave-One-Out Training Strategy}

Increasing the number of deposit patterns through the addition of random noise is one way of training a neural network with limited known deposit examples. Other procedures for training a network when data are scarce include cross-validation (Stone, 1974) and the leave-one-out method. Cross-validation allows a high proportion of the data to be used to train the network by splitting the data into, for example, 10 segments and withholding one of the portions as a test set and training the network using the remaining nine tenths of the data. In this case the network would be trained 10 times. Each time a different segment is reserved for testing. This is referred to as 10-fold cross-validation. The validation error is calculated by averaging all the results. The leave-one-out procedure (sometimes referred to as the jackknife) refers to the case where only a single pattern is set aside in each training run for testing. An advantage of the leave-one-out procedure is that it can provide measures of confidence for comparing the classification performance of two networks (Duda et al., 2001).

Another method designed to improve the generalization ability, when patterns for a particular class of patterns are scarce, is interactive reinforcement training (Wong et al., 1997b). This method reinforces the effect of classes of patterns which are represented in 
the training set in low numbers by duplicating them.

\subsubsection{Use of Fuzzy Membership Functions to Implement Narrow Dis- tance Buffers}

It is shown in Chapter 7 (Tables 7.19-7.22) that, although narrow buffers in distance and strike and contrast layers, result in poorer generalization performance, the values of the capture efficiency statistics, $D / A$ and $D \times(D / A)$ are significantly improved. Setting the value for cells where the distance exceeds the buffer limits to the maximum distance for distance layers or to a contrast of zero for contrast layers is analogous to using a simple step function with a threshold. It would probably be more effective to implement these buffers using inputs in the form of fuzzy membership values and modifying the form of the fuzzy membership functions to reflect these limits.

\subsubsection{Input Standardization, Target Values and Sigmoid Function}

In this study, inputs are scaled to the range [0,1], target values to 0.1 and 0.9 , and a sigmoid function of the form:

$$
f(n e t)=\frac{1}{1+e^{-n e t}}
$$

(where net is the weighted, summed input to the processing unit) is used. Duda et al. (2001) recommend that inputs be standardized so that the average and variance for each feature component over the entire data set is set to zero and 1.0, respectively. They also suggest using a hyperbolic tangent sigmoid of the form:

$$
f(\text { net })=a \tanh (\text { bnet })=a\left[\frac{e^{+b n e t}-e^{-b n e t}}{e^{+b n e t}+e^{-b n e t}}\right]
$$

and target values of +1 and -1 for target category and non-target categories, respectively.

\subsubsection{Use of a Continuous Rather than Binary Target Variable}

A difficulty with the present method of training the networks using binary target values is that deposits are effectively treated as a single class. This is despite the fact that the total gold resource for the 120 known deposits ranges from 1050 to $1,246,500 \mathrm{~kg}$ tons of contained gold for the giant Kalgoorlie Golden Mile deposit. An alternative to using binary values to predict the similarity of patterns to deposit patterns in the training data set is to train the network to predict the total contained gold in each grid cell (Knox-Robinson, 
1994; Knox-Robinson et al., 1996). This may facilitate learning since the target values are likely to be less often contradictory. For example, due to the regional scale of the GIS data, there may be little evidence present in the GIS input layers for some small deposits. Therefore, input-variable values associated with small deposits may actually be more similar to those of barren cells than large deposits, yet, with binary target values, the network must produce the same output value for both cases.

\subsubsection{Elimination of Redundant Inputs}

Although considerable effort was put into selecting relevant input variables, redundant attributes were not identified and eliminated. In Chapter 4, it is demonstrated that neural networks are more robust than fuzzy systems with respect to redundant and irrelevant input variables. However, network performance is likely to be improved if redundant attributes can be removed (Langley \& Sage, 1994). Training a network, which has a large number of input variables, with a small number of training patterns, may cause the network to over-fit the data. Various techniques are available to do this. For example, Bishop (1995) and Duda et al. (2001) suggest the use of principal component analysis to reduce the dimensionality of the inputs, and Milne (1995b), Gedeon (1996) and Milne (1999) developed methods for analysing the trained weights matrix in a trained network to determine which inputs are significant.

\subsubsection{Use of Self Organizing Maps for Improved Split of Training Data}

In this study, the training data were randomly split ten times between training, trainingstop and test data and the performance of the trained network for each split was compared. The characteristics of the resultant data sets are important because the performance of the trained network on the test data set can only be a reliable guide to the performance on completely new data if the training sets are statistically similar. In addition, training with the early-stopping method also requires that the training and test sets are statistically similar. A more efficient means of achieving an optimal split has been suggested by Wong et al. (1996a), Fung et al. (1997) and Fung \& Wong (1999). They used a self organizing map to classify training-data vectors into 6 to 8 classes. Training sets based on a selection of patterns with low or mean quantization errors (i.e. difference between each pattern and the centroid of a class) gave the best results. The remaining data were used for the test set. 


\subsection{Extensions to This Work}

\subsubsection{Development of Rule-based Hybrid Fuzzy Systems}

The good results obtained with hybrid fuzzy-neural network systems in this study suggest that the application of hybrid systems to mineral prospectivity mapping should be further investigated. Fuzzy and neuro-fuzzy inference systems have been successfully used in engineering systems such as automatic control and computer vision (Jang et al., 1997; Kasabov \& Kozma, 1999). In contrast to the fuzzy systems used in mineral prospectivity systems, these engineering systems use if-then rules, whereby: 1) rules are evaluated by fuzzifying crisp input values using fuzzy membership functions, 2) multiple anticedents are combined using an implication method (Mamdani \& Assilian, 1975; Sugeno, 1985), 3) rules may be weighted, 4) rules are combined using an aggregation method, and 5) a defuzzification method is used to convert the fuzzy membership function representing the aggregated rules to a crisp output value (Jang \& Sun, 1995; Jang \& Gulley, 1995). In neuro-fuzzy inference systems, the fuzzy inference system is trained using a backpropagation algorithm, and input and output pairs. Hybrid neuro-fuzzy systems are discussed in Appendix A (Section A.2.5). Systems of this sort could have advantages for geoscience applications like mineral prospectivity mapping, in which there is often a mix of conceptual knowledge and data, but the rules can only be expressed in vague or imprecise terms.

\subsubsection{Estimates for Accuracy of Prediction}

An increasingly important problem for mining companies in assessing alterative exploration opportunities is that of risk management (Lerche, 2002). At present, it is difficult in the case of neural network and fuzzy systems to answer questions about how accurate the predictions are or perhaps how wrong they could be.

\subsubsection{Investigate Factors Affecting the Approximation of Network Out- puts to Probabilities}

Following on from the problem of estimating the accuracy of prospectivity estimates is the need to determine the conditions under which the outputs most closely approximate probabilities. For classification problems, this probability is the posterior probability of class membership $p\left(C_{k} \mid \mathbf{x}\right)$, where $C_{k}$ is the $k$ th class, and $\mathbf{x}$ is the input feature vector. Bishop (1995) and Duda et al. (2001) suggest using; 1) one output neuron per class, 
2) target values of 0 and 1, and 3) the sum of squares error function. Bishop (1995) notes that techniques such as re-training the network on patterns which are incorrectly classified alters the class-conditional density functions and are therefore not applicable if the network is to generate probabilities as outputs. Duda et al. (2001) also suggest using an exponential rather than a sigmoidal activation function, that is:

$$
f(n e t) \propto \exp ^{\text {net }}
$$

Bishop (1995) also describes methods for checking how closely the outputs approximate posterior probabilities and a method for re-scaling the network outputs if the prior probabilities, $P\left(C_{k}\right)$ for the classes in the data population are different from those of the training data.

Although, theoretically, outputs represent probabilities, this is based on the assumption of an infinite amount of training data. In practice, where data are finite, outputs approximate probabilities. Therefore, further work is needed to determine how closely the network outputs approximate probabilities in mineral prospectivity and the factors which affect the approximations in mineral prospectivity applications.

\subsubsection{Rule Extraction from Trained Neural Networks}

To discover the reasons why a neural network correctly produces high prospectivity estimates for particular patterns could be useful in two ways. First, an understanding of input variable values associated with large deposits could help test and refine current exploration criteria and second, the combination of input variable values required to produce particular responses could be reformulated as if-then rules and used in fuzzy or neuro-fuzzy rule-based systems.

Preliminary work by Gedeon \& Turner (1993) and Gedeon \& Treadgold (1997) suggests that one way of extracting rules from a neural network could be based on a causal index method. A causal index is the correlation between the neural network inputs and outputs. For example, if an input is always on, or has a high value when a particular output is on, or has a high value, then the correlation between the input and the output is high, and the input can be said to have caused the output. In a modification of this simple approach, the rate of change of the output with respect to the input is measured, in order to eliminate correlations that are due to chance. This form of causal index is determined by changing the value of a single input from 0 to 1 while the other inputs are held constant. This is repeated for all the inputs and then the resultant curves can be interpreted to yield rules involving $A N D$ and $O R$. 


\subsubsection{Uncertainty of Mineral Prospectivity Estimates}

One important aspect of a neural network method for mineral prospectivity mapping that has not been investigated in this study is the uncertainty of prediction. Whilst various methods for the estimating uncertainty are available for the weights-of-evidence method (Bonham-Carter et al., 1989; Bonham-Carter \& Agterberg, 1990; see discussion in Section 4.6.7, page 134), these methods can not be applied to a neural-network method. However, an estimate of uncertainty associated with the prospectivity map could be obtained using a Monte Carlo simulation.

For the neural network method uncertainty in the prediction arises from error in the input variables and error associated with network training. Sources of error in neural-networkbased prospectivity estimates are due to; 1 ) random selection of patterns corresponding to barren cells for training sets, 2) randomly splitting training data into training, trainingstop and test sets random initial weights, and 3) random initial weights. Random errors drawn from distributions appropriate for each input variable could be added to the network inputs to obtain a range of prospectivity estimates for each grid cell. Processing of the inputs, to which errors are added, could be repeated for different random initial weights and selections of training sets so that error associated with training of the neural networks could be simulated. Uncertainties based on the variance and standard deviation of the predictions for each cell could then be represented as additional maps.

\subsection{Concluding Statement}

Neural networks are shown to be an effective and promising new method for integrating regional exploration data sets to estimate the potential for mineral deposits in a given district. The pilot study establishes that neural networks produce geologically reasonable results. In addition, neural networks are shown to perform at least as well as both the most commonly used statistical methods, weights of evidence, and the conceptual fuzzy systems method, while offering several advantages over these techniques. The methodology established in this study is not restricted to use on a regional scale or in 2-D, and could potentially be used at a mine scale in 3-D.

Statistical methods developed as part of the Kalgoorlie Terrane case study can be applied independently of a neural network approach to mineral prospectivity mapping. These graphical statistical methods are useful for quantitatively assessing exploration criteria, which can be expressed in terms of features in a regional-scale GIS database within the 
framework of a mineral-systems approach. A mineral-systems, rather than a depositmodel, approach is important because it also enables prior geological knowledge to be incorporated into a neural network analysis in the form of novel derived layers using features which are appropriate to the regional scale of the GIS database.

This study demonstrates the effectiveness of a neural network approach in established exploration areas where large amounts of data are available and there are numerous deposits. However, experiments on the effect of deposit numbers available for training highlight the fact that neural networks are not well suited to greenfields areas where there are few data. Methods for extending the applicability of the neural network methods to new exploration areas are investigated in this study, for example, through the augmentation of the deposit training patterns using the addition of random noise, and the inclusion of prior knowledge through the use of fuzzy membership values as inputs. The preliminary results for this latter approach are promising and point the way for future research in which knowledge- and data-driven systems can be combined in the form of hybrid fuzzy-neural network systems.

Neural networks are capable of recognizing the underlying patterns in multisource geoscience data sets. Whilst the results of this study demonstrate that neural networks are effective in identifying areas containing known mineralization, it is their ability to generalize and to predict prospective areas for new deposits that makes them a useful tool for mineral exploration. 


\section{References}

Aaron, R. L., Aurali, F. B. \& Margarita, C. G. 1993. Genetic algorithm-like learning rule for neural networks. In: IEEE Systems, Man and Cybernetics Conference Proceedings. Los Alamitos, volume 4, pp. 137-142.

ADDY, S. K. 1998. Seismic facies maps: a quick exploration tool. AAPG Bulletin 82, 522.

AGSO 1992. Geophysical Mapping Section: Formatted Sequential File Structure for Airborne Geophysics Data. Technical report, Australian Geological Survey Organisation, Canberra (unpubl.).

Agterberg, F. P. 1974. Automatic contouring of geological maps to detect target areas for mineral exploration. Mathematical Geology 6, 373-395.

Agterberg, F. P. 1981. Application of image analysis and multivariate analysis to mineral resource appraisal. Economic Geology 76, 1016-1031.

Agterberg, F. P. 1989a. Computer programs for mineral exploration. Science 245, 76-81.

Agterberg, F. P. 1989b. Logdia-FORTRAN 77 program for logistic regression with diagnostics. Computers and Geosciences 15, 599-614.

Agterberg, F. P. 1989c. Systematic approach to dealing with uncertainty of geoscience information in mineral exploration. In: Proceedings of the 21st International Symposium on the Application of Computers and Operations Research in the Mineral Industry (APCOM) Symposium. pp. 165-178. Society for Mining, Metallurgy, and Exploration (SME), Littleton, Colorado.

Agterberg, F. P. 1992a. Combining indicator patterns in weights of evidence modelling for resource evaluation. Nonrenewable Resources 1, 35-50.

Agterberg, F. P. 1992b. Estimating the probability of occurrence of mineral deposits from multiple map patterns. In: Merriam, D. F. \& KurzL, H., eds., The Use of Microcomputers in Geology. pp. 73-92. Plenum Press, New York.

Agterberg, F. P., Bonham-Carter, G. F., Cheng, Q. \& Wright, D. F. 1993. Weights of evidence modelling and weighted logistic regression for mineral potential mapping. In: Davis, J. C. \& Herzfeld, U. C., eds., Computers in Geology -25 Years of Progress. pp. 13-32. Oxford University Press, Oxford.

Agterberg, F. P., Bonham-Carter, G. F. \& Wright, D. F. 1990. Statistical pattern integration for mineral exploration. In: GaÁL, G. \& Merriam, D. F., eds., Computer Applications in Resource Estimation Prediction and Assessment for Metals and Petroleum. pp. 1-21. Pergamon Press, Oxford.

Agterberg, F. P. \& Cheng, Q. 2002. Conditional independence test for weights-of-evidence modelling. Natural Resources Research 11, 249-255. 
Agterberg, F. P., Chung, C. F., Eade, K. E. \& Fabbri, A. G. 1981. Preliminary geomathematical analysis of geological, mineral occurrences and geophysical data, southern district of Keewatin, Northwest Territories. Geological Survey of Canada Open File 718, 1-31.

Agterberg, F. P., Chung, C. F., Fabbri, A. G., Kelly, A. H. \& Springer, J. S. 1972. Geomathematical evaluation of copper and zinc potential of the Abitibi area, Ontario and Quebec. Geological Survey Of Canada Paper 71-41, 1-55.

Allais, M. 1957. Method for appraising economic prospects of mining exploration over large territories. Management Science 3, 285-347.

AN, G. 1996. The effects of adding noise during backpropagation training on a generalization performance. Neural Computation 8, 643-647.

AN, P. 1991. Spatial reasoning and integration techniques for geophysical and geological exploration data. PhD thesis, University of Manitoba, Winnipeg (unpubl.).

An, P., Chung, C. J. F. \& Rencz, A. N. 1994. A preliminary study of alteration mapping from airborne geophysical and remote sensing data using feed-forward neural networks. In: Society of Exploration Geophysicists 64th Annual International Meeting; Technical Program Abstracts. pp. 837-840. Society of Exploration Geophysicists, Tulsa, Oklahoma 64.

An, P. \& Moon, W. M. 1993. An evidential reasoning system for integrating geophysical, geological and remote-sensing data. In: International Geoscience and Remote-Sensing Symposium (IGARSS'93). IEEE Comp. Soc. Press, volume 3, pp. 1359-1361.

An, P., Moon, W. M. \& Bonham-Carter, G. F. 1992. On knowledge-based approach of integrating remote-sensing, geophysical and geological information. In: Proceedings of IEEE International Geoscience and Remote Sensing Symposium (IGARSS'92). pp. 34-38. Institute of Electrical and Electronics Engineers, Piscataway.

An, P., Moon, W. M. \& Rencz, A. 1991. Application of fuzzy set theory to integrated mineral exploration. Canadian Journal of Exploration Geophysics 27, 1-11.

ANZLIC 1996. ANZLIC Guidelines: Core Metadata Elements Version $1 . \quad$ ANZLIC Working Group on Metadata. Digital version available at WWW page: http://www.anzlic.org.au/asdi/metaelem.htm.

Armstrong, M. P. \& Bennet, D. A. 1990. A bit-mapped classifier for groundwater quality assessment. Computers and Geosciences 16, 811-832.

Aronson, P. \& Moorehouse, S. 1983. The ARC/INFO map library: the design for a digital database. In: Wells, B. S., ed., Proceedings of the 6th International Symposium on Automated Cartography. pp. 327-382. American Congress Surveying and Mapping, Ottawa 1.

ASEG 1997. GXF exchange file format. Technical report, Australian Society of Exploration Geophysicists.

Aspinall, P. J.And Hill, A. R. 1983. Clinical inferences and decsions-I. Diagnosis and Bayes' theorum. Opthalmic and Physiological Optics 3, 295-304.

Aspinall, P. J. 1992. An inductive modelling procedure based on Bayes' theorum for analysis of patterns in spatial data. International Journal of Geographical Information Systems $\mathbf{6}$, $105-121$.

Baddeley, A., J. Kerscher, M., Schladitz, K. \& Scott, B. T. 2000. Estimating the J function without edge correction. Statistica Neerlandica 54, 999-999. 
BAHRAmi, M. 1993. Integration of knowledge acquired by different neural networks. In: KASABOV, N. K., ed., Proceedings 1993 The First New Zealand International Two-Stream Conference on Artificial Neural Networks and Expert Systems. IEEE Comp. Soc. Press, Los Alamitos, pp. $73-74$.

Baldwin, J., Bateman, R. \& Wheatley, C. L. 1990. Application of a neural network to the problem of mineral identification from well logs. The Log Analyst 31, 279-293.

Bampton, M. D. 1988. Alteration and mineralization of the southern part of the Stanthorpe Adamellite, near Tenterfield, New South Wales. BSc (Hons) thesis, University of Sydney, Sydney (unpubl.).

Bandy, S. 1997. Mineral Occurrences and Exploration Potential of the Bangemall Basin 1:500,000. Gis mineral occurrence and exploration potential package, Geological Survey of Western Australia, Perth (unpubl.).

Barnes, R., Jaieth, S. \& Miezitis, Y.and Suppel, D. W. 1999. Mineral potential assessment of parts of the southern New England Orogen. In: Flood, P. G., ed., Proceedings of the New England Orogen - Regional Geology, Tectonics and Metallogenis Conference. Earth Sciences, University of New England, University of New England, Armidale, pp. 373-382.

BARNES, R. G. 1993. Use of GIS and image processing for ehancing geological investigations. Case study: Tenterfield 1:100,000 sheet area, northern NSW. Geological Survey of New South Wales Report GS1993/290 (unpubl.).

BARNES, R. G. 1995. Data quality statment overview report for uner_geol-Arc/Info geological coverage-Upper North East Region comprising the NSW portions of the Warwick, Tweed Heads, Grafton, Maclean, Dorrigo and Coffs Harbour 1:250,000 sheet areas including contribution from NRAC project $S 1(C)$ - geological studies. Geological Survey of New South Wales Report (unpubl.).

BARnes, R. G. 1997a. Data quality statement overview report for metallic and industrial mineral deposits coverage - New England and north east NSW region comprising the NSW portions of the Warwick, Tweed Heads, Inverell, Grafton, Maclean, Manilla, Dorrigo, Coffs Harbour, Tamworth-Hastings, Singleton and Newcastle 1:250,000 sheet areas. Geological Survey of New South Wales Report (unpubl.).

Barnes, R. G. 1997b. Metadata for Tenterfield 1:100,000 data. Geological Survey of New South Wales Report (unpubl.).

Barnes, R. G., Brown, R. E., Brownlow, J. W., Gilligan, L. G., Krynen, J. \& Willis, I. L. 1988. A review of the mineral deposits of the New England Orogen in New South Wales. In: Kleeman, J. D., ed., New England Orogen - Tectonics and Metallogenesis. pp. 212-227. Department of Geology and Geophysics, University of New England, Armidale.

Barnes, R. G., Brown, R. E., Brownlow, J. W., Henley, H. F. \& Stroud, W. J. 1997. Structural control of metahydrothermal mineralisation in the Southern New England Orogen. In: 14th Australian Geological Convention Abstracts: Geoscience for the New Millenium. Geological Society of Australia, volume 49, p. 22.

Barnes, R. G., Henley, H. F. \& Henley, J. E. 1995. Exploration data package for the Tenterfield and Coaldale 1:100,000 sheet area. Geological Survey of New South Wales Report No. GS1995/004 (unpubl.).

BARnes, R. G. \& Suppel, D. W. 1995. Data quality overview report for NRAC project $S 1(A)-$ geochemical atlas. Geological Survey of New South Wales Report (unpubl.). 
Barnes, R. G. \& Willis, I. L. 1989. Geology of the Grafton and Maclean 1:250,000 areas. Geological Survey of New South Wales. Geological Survey of New South Wales Report GS1989/117 (unpubl.).

Barrett, T. J. \& MacLean, W. H. 1994. Mass changes in hydrothermal alteration zones associated with VMS deposits of the Noranda area. Exploration and Mining Geology 3, 131160.

Barton, Jr., P. B. 1986. User-friendly deposit models. In: Cargill, S. M. \& Green, S. B., eds., Prospects for Mineral Resource Assessments on Public Lands: Proceedings of the Leesburg Workshop. U.S. Geological Survey, volume 980, pp. 94-110. U.S. Geological Survey Circular.

BAtes, R. C. 1959. An application of statistical analysis to exploration for uranium on the Colorado Plateau. Economic Geology 54, 449-466.

Beale, R. \& Jackson, T. 1990. Neural Computing: An Introduction. Adam Hilger, Bristol.

Beams, S. 1995. Exploration data management on a mineral province scale. In: Second National Forum on GIS in the Geosciences - Forum Proceedings. Australian Geological Survey Organisation, pp. 98-107. Record 1995/46.

Bebis, G. \& Georgiopoulos, M. 1992. Increasing classification accuracy using multiple neural network schemes. In: Proceedings of the SPIE Conference on Application of Neural Networks III. International Society for Optical Engineering (SPIE). Part 1.

Benediktsson, J. A., Sveinsson, J. R. \& Arnason, K. 1998. Classification and integration of multitype data. In: IGARSS '98: Geoscience and Remote Sensing Symposium Proceedings. IEEE Comp. Soc. Press, Los Alamitos, volume 1, pp. 177-179.

Benediktsson, J. A., Swain, P. H. \& Ersoy, O. K. 1990a. Neural network approaches versus statistical methods in classification of multisource remote sensing data. IEEE Transactions on Geoscience and Remote Sensing 28, 540-551.

Benediktsson, J. A., Swain, P. H. \& Ersoy, O. K. 1993. Conjugate-gradient neural networks in classification of multisource and very-high dimensional remote sensing data. International Journal of Remote Sensing 14, 2883-2903.

Benediktsson, J. A., Swain, P. H., Ersoy, O. K. \& Hong, D. 1990b. Classification of very high dimensional data using neural networks. In: International Geoscience and Remote Sensing Symposium. pp. 1269-1272. IEEE Comp. Soc. Press, Los Alamitos 2.

Berenson, M. L. \& Levine, D. M. 1996. Basic Business Statistics: Concepts and Application (6th Edition). Prentice Hall, Englewood Cliffs.

Berkman, D. A. 1995. Field Geologists' Manual (3rd edition). Australasian Institute of Mining and Metallurgy. Monograph 9.

Berman, M. 1977. Distance distributions associated with Poisson processes of geometric figures. Journal of Applied Probability 14, 195-199.

Berman, M. 1986. Testing for spatial association between a point process and another stochastic process. Applied Statistics 35, 54-62.

BezDEK, J. C. 1993. A review of probabilistic, fuzzy, and neural models for pattern recognition. Journal of Intelligent and Fuzzy Systems 1, 1-25.

Bhaskara, R. D. \& Ramesh, B. N. 1991. A FORTRAN-77 program for three dimensional analysis of gravity anomalies with variable density contrast. Computers and Geosciences $\mathbf{1 7}$, 655-667. 
Binns, R. A., Gunthorpe, R. J. \& Groves, D. I. 1976. Metamorphic patterns and development of greenstone belts in eastern Yilgarn Block, Western Australia. In: WindLey, B. F., ed., The Early History of the Earth, John Wiley \& Sons, New York, pp. 303-313.

Bischof, H., Scheider, W. \& Pinz, A. J. 1992. Multispectral classification of Landsat-images using neural networks. IEEE Transactions on Geoscience and Remote Sensing 30, 482-490.

Bishop, C. M. 1995. Neural Networks for Pattern Recognition. Oxford University Press, Oxford.

Bishop, M. M., Fienberg, S. E. \& Holland, P. W. 1975. Discrete Multivariate Analysis: Theory and Practice. MIT Press, Cambridge, MA.

Biswas, G. \& LeE, G. 1994. Knowledge reorganization. A rule model scheme for efficient reasoning. In: Proceedings of the Tenth Conference on Artificial Intelligence for Applications. IEEE Comp. Soc. Press, Los Alamitos, pp. 312-318.

Blake, Jnr, M. C. \& Murchey, B. L. 1988. A California model for the New England Fold Belt. Geological Survey of Queensland Quarterly Notes 72, 1-9.

Blasgen, M. W. 1981. System R: An Architectural Overview. IBM Systems Journal 20, 41-62.

Blevin, P. L. \& Chappell, B. W. 1992. The role of magma sources, oxidation states and fractionation in determining the granite metallogeny of eastern Australia. In: Brown, P. E. \& Chappell, B. W., eds., The Second Hutton Symposium on the Origin of Granites and Related Rocks; Proceedings. pp. 305-316. Geological Society of America (GSA), Boulder, Special Paper 272.

Blevin, P. L. \& Chappell, B. W. 1996a. Internal evolution and metallogeny of Permo-Triassic high-K granites in the Tenterfield-Stanthorpe region, southern New England orogen, Australia. In: Mesozoic Geology of Eastern Australia Plate Conference-Extended Abstracts. pp. 94-100. Geological Society of Australia, Brisbane, 43.

Blevin, P. L. \& Chappell, B. W. 1996b. Permo-Triassic granite metallogeny of the New England Orogen. In: Mesozoic Geology of the Eastern Australia Plate Conference-Extended Abstracts. pp. 101-103. Geological Society of Australia, Sydney 43.

Bliss, J. D. 1992. Developments in mineral deposit modelling. United States Geological Survey. Bulletin 2004

Bloch, G., Sirou, F. \& Fatrez, P. 1997. Neural intellignet control for a stell plant. IEEE Transactions on Neural Networks 8, 910-918.

BoIs, P. 1987. Fuzzy sets and non-seismic applications: Improvement of seismic prospecting by using fuzzy subsets theory. In: AminzadeH, F., ed., Handbook of Geophysical Exploration, Section 1: Seismic Exploration, Geophysical Press, London, volume 20 of Pattern Recognition and Image Processing, pp. 473-503.

Bolt, G. \& Daneel, G. 1996. Prospect Explorer: a geophysical mineral exploration neural analysis tool. In: Ramani, R. V., ed., 26th Proceedings of the Application of Computers and Operations Research in the Mineral Industry. Pennsylvania State University, University Park.

Bonham-Carter, G. F. 1984. Quantitative relationships between Landsat-derived linears and occurrence of gold in the Timmins-Kirkland Lake area, Ontario. In: Cook, J. J., ed., Proceedings of the 18th International Symposium on Remote Sensing of Environment. pp. 1643-1644. (Paris, 1-5 Oct.) Environmental Resources Institute, Ann Arbor, Michigan 3.

Bonham-CARTER, G. F. 1985. Statistical association of gold occurrences with Landsat-derived lineaments, Timmins-Kirkland Lake area, Ontario. Canadian Journal of Remote Sensing 11, $195-210$. 
Bonham-Carter, G. F. 1994. Geographic Information Systems for Geoscientists: Modelling with GIS. Pergamon Press, New York.

Bonham-Carter, G. F. \& Agterberg, F. P. 1990. Application of a microcomputer-based geographic information system to mineral potential mapping. In: HANLEY, J. T. \& MERIAM, D. F., eds., Microcomputer Applications in Geology. Pergamon Press, Oxford, pp. 49-74.

Bonham-Carter, G. F. \& Agterberg, F. P. 1999. Arc-WofE: a GIS tool for statistical integration of mineral exploration datasets. In: Proceedings of the 52nd Session of the International Statisitical Institute (ISI). International Association of Mathematical Geology, volume 58, pp. 497-500. (10-18 August, Helsinki) International Statisitical Institute.

Bonham-Carter, G. F., Agterberg, F. P. \& Wright, D. F. 1988. Integration of geological datasets for gold exploration in Nova Scotia. Photogrammetric Engineering and Remote Sensing 54, 1585-1592.

Bonham-Carter, G. F., Agterberg, F. P. \& Wright, D. F. 1989. Weights of evidence modelling: a new approach to mapping mineral potential. In: Agterberg, F. \& BonhamCarter, G. F., eds., Statistical Applications in the Earth Sciences. pp. 171-183. Geological Survey of Canada Paper, Ottawa 89-9.

Bonham-Carter, G. F. \& Chung, C. F. 1983. Integration of mineral resource data for Kasmere Lake area, Northwest Manitoba, with emphasis on uranium. Computers and Geosciences 15, $25-45$.

Bonham-Carter, G. F., Kerswill, J. A. \& Franklin, J. 1998a. Mineral potential maps for Slave Province NWT using weights of evidence models. In: Abstracts with Programs, Geological Society of America 1998 annual meeting. p. 21. Geological Society of America (GSA), Boulder, Colorado 30.

Bonham-Carter, G. F., Kerswill, J. A., Shaw, J. \& Chorlton, L. 1998b. Iron-formation gold and volcanic-associated massive-sulphide base-metal potential in Slave Province by weights of evidence; a preliminary appraisal. In: Quebec 1998, Crossroads of Earth sciences: Program with Abstracts, Geological Association of Canada; Mineralogical Association of Canada; Canadian Geophysical Union, Joint Annual Meeting. p. A20. Quebec 23.

Bonham-Carter, G. F., Rencz, A. N. \& Harris, J. R. 1985. Spatial relationships of gold occurrences with lineaments derived from Landsat and Seasat imagery, Meguma Group, Nova Scotia. In: Cook, J. J. \& Morris-Jones, D. R., eds., Proceedings of the International Symposium on Remote Sensing of Environment, Fourth Thematic Conference: Remote Sensing for Exploration Geology. pp. 768-775. (San Francisco, 1-4 April) Environmental Resources Institute Ann Arbor Michigan.

Bотвоц, J. M. 1971. An application of characteristic analysis to mineral exploration. In: Decision Making in the Mineral Industry, International Symposium on Techniques for Decision Making in the Mineral Industry, 9th Montreal 1970 Proceedings. volume Canadian Institute of Mining and Metallurgy Special Volume, pp. 92-99. Vol. 12.

Botbol, J. M., Sinding-Larsen, R., McCammon, R. B. \& Gott, G. B. 1978. A regionalized multivariate approach to target selection in geochemical exploration. Economic Geology 73, $534-546$.

BoyD, D. M. 1993. Host-rock trace element geochemistry of the Roadmaster polymetallic volcanogenic massive sulphide (VMS) prospect, eastern Pilbara Craton, Western Australia. Bsc (hons) thesis, University of Western Australia, Perth (unpubl.). 
Brauhart, C. W. 2000. Regional Alteration Systems Associated with Archaean Volcanogenic Massive Sulphide Deposits at Panorama, Pilbara, Western Australia: a Digital GIS Data Package. Technical report, Centre for Global Metallogeny, University of Western Australia and the University Extension, Perth. CD-ROM.

Brauhart, C. W., Groves, D. I. \& Morant, P. 1998. Regional alteration systems associated with Archaean volcanogenic massive sulfide mineralization at Panorama, Pilbara, Western Australia. Economic Geology 93, 292-302.

Bridle, J. S. 1990. Probabilistic interpretation of feedforward classification network outputs, with relationships to statisitical pattern recognition. In: Fogelman Soulié, F. \& HÉrault, J., eds., Neurocomputing: Algorithms, Architectures and Applications, Springer-Verlag, Berlin, volume F68 of NATO ASI Series, pp. 227-236.

Briggs, P. L. \& Press, F. 1977. Pattern recognition applied to uranium prospecting. Nature 268, $125-127$.

Brodeur, G., El-Hihi, S. \& Jebrak, M. 1992. Applications of neural network computing to mining exploration in the Southern Abitibi greenstone belt. In: Program with Abstracts Geological Association of Canada, Mineralogical Association of Canada, Canadian Geophysical Union Joint Annual Meeting. p. 12. Geological Association of Canada, Acadia University, Wolfville 17.

Broomhead, D. S. \& Lowe, D. 1988. Multivariable functional interpolation and adaptive networks. Complex Systems 2, 321-355.

Brown, W., Gedeon, T. \& Groves, D. I. 2002a. Use of noise to augment training data to compensate for lack of deposit examples in training a neural network for mineral potential mapping. In: Kouda, R., Koike, K., Singer, D. \& Couglan, J., eds., Abstracts and Programs for Seventh International Symposium on Mineral Exploration: Application of Neural networks to the Earth Sciences. pp. 10-11. 20-21 August, NASA Moffett Field, Mountain View, California.

Brown, W., Groves, D. I. \& Gedeon, T. D. 2002b. Use of fuzzy membership input layers to combine subjective geological knowledge and empirical data in a neural network method for mineral potential mapping. In: KoudA, R., Kolke, K., Singer, D. \& Couglan, J., eds., Abstracts and Programs for Seventh International Symposium on Mineral Exploration: Application of Neural networks to the Earth Sciences. p. 9. 20-21 August, NASA Moffett Field, Mountain View, California.

Brown, W. M., Gedeon, T. D., Baddeley, A. J. \& Groves, D. I. 2002c. Bivariate J-function and other graphical statistical methods help select the best predictor variables as inputs for a neural network method of mineral prospectivity mapping. In: BAYER, U., BURGER, H. \& Skala, W., eds., IAMG 2002 8th Annual Conference of the International Association for Mathematical Geology. pp. 263-268. International Association of Mathematical Geology 1.

Brown, W. M., Gedeon, T. D. \& Barnes, R. G. 1999. The use of a multilayer feedforward neural network for mineral prospectivity mapping. In: Gedeon, T., Wong, P., Halgamude, Kasabov, N., Nauck, D. \& Fukushima, K., eds., Proceedings of ICONIP'99: $6^{\text {th }}$ International Conference on Neural Information Processing. pp. 160-165. IEEE, Piscataway 1.

Brown, W. M., Gedeon, T. D., Groves, D. I. \& Barnes, R. G. 2000a. Artificial neural networks: a new method for mineral prospectivity mapping. Australian Journal of Earth Sciences 47, 757-770. 
Brown, W. M., Gedeon, T. D., Groves, D. I. \& Barnes, R. G. 2000b. Mineral prospectivity mapping: a comparison of MLP neural network, fuzzy logic and weights of evidence methods. In: 15th Australian Geological Convention Abstracts. p. 63. Geological Society of Australia 59.

Brown, W. M., Taylor, G. R. T., Jusmady, Groves, D. I. \& Knox-Robinson, C. M. 1997. Application of artificial neural networks to prospectivity analysis in a GIS environment: a comparison with statistical and fuzzy logic methods for Au and Sn deposits of the Tenterfield area, NSW. In: 14th Australian Geological Convention Abstracts. p. 57. Geological Society of Australia 49.

Brunker, R. L. \& Chesnut, W. S. 1976. Grafton 1:250,000 geological sheet. Geological Survey of New South Wales, Sydney.

Bruzzone, L., Conese, C., Maselli, F. \& Roll, F. 1997. Multisource classification of complex rural areas by statistical and neural-network approaches. Photogrammetric Engineering and Remote Sensing 63, 523-533.

Bryson, A. E. \& Ho, Y.-C. 1969. Applied Optimal Control. Blaisdell, New York.

Buckley, J. J. \& Hayashi, Y. 1994. Fuzzy neural networks: a survey. Fuzzy Sets and Systems 66, $1-13$.

Bui, E., Moran, C. \& Simon, D. 1997. Facies prediction by mining environmental data space. In: 14th Australian Geological Convention Abstracts: Geoscience for the New Millenium. p. 93. Geological Society of Australia 49.

Bui, E. N., Moran, C. J. \& Simon, D. A. P. 1998. New geotechnical maps for the MurrayDarling Basin. Commonwealth Scientific and Industrial Research Organisation (CSIRO) Report 42/98 (unpubl.).

Burgess, L. A. 1997. Recent applications and research into mineral prospectivity mapping using GIS. In: Third National Forum on GIS in the Geosciences - Forum Proceedings. pp. 121-129. Australian Geological Survey Organisation Record 1997/36.

Burrough, P. A. 1986. Principles of Geographic Information Systems for Land Resources Assessment. Oxford University Press, Oxford.

Burrough, P. A. \& McDonnell, R. A. 1998. Spatial Information Systems and Geostatisitcs. Claredon Press, Oxford.

Cacoullos, T. 1966. Estimation of a multivariate density. Annals of the Institute of Statistical Mathematics, Tokyo 18, 178-189.

CAI, Y. 1994a. Application of artificial neural network on prediction of multimetallic deposits. In: Geological Society of America 1994 Annual Meeting - Program with Abstracts. Geological Society of America, Boulder, volume 26, pp. A-482.

CAI, Y. 1994b. On mineral resource prognostication by self-organisation artificial neural network. Hunan Geology 13, 53-56.

Campbell, A. H., Hollister, V. F. \& Duda, R. O. 1982. Recognition of a hidden mineral deposit by an artificial intelligence program. Science 217, 927-929.

Cardon, H. R. A. \& van Hoogstraten, R. 1995. Key issues for successful industrial neuralnetworks applications: an application in geology. In: Braspenning, P. J., ed., Artificial Neural Networks; An Introduction to ANN Theory and Practice. pp. 235-245. Springer Verlag, Berlin. 
Carr, H. W. \& Groves, D. I. 1994. Controls on the distribution of Merensky Reef potholes at the Western Platinum Mine, Bushveld Complex, South Africa: implications for disruptions of the layering and pothole formation in the Complex. South African Journal of Geology $\mathbf{9 7}$, $431-441$.

Carranza, E. J. M. \& Hale, M. 1999. Geologically constrained fuzzy mapping of gold mineralization potential, Baguio District, Phillipines. Natural Resources Research 10, 125-136.

Carranza, E. J. M. \& Hale, M. 2002. Where are copper deposits spatially localized? A case study in Berguet Province, Philippines. Natural Resources Research 11, 45-60.

Carranza, E. J. M., Hale, M. \& Mangaoang, J. C. 1999. Application of mineral exploration models and GIS to generate mineral potential maps as input for optimum land-use planning in the Philippines. Natural Resources Research 8, 165-173.

Cawood, P. A. \& Leitch, E. C. 1985. Accretion and dispersal tectonics of the southern New England Fold Belt, Eastern Australia. In: Howell, D. G., ed., Tectonostratigraphic Terranes of the Circum-Pacific Region. pp. 481-492. Earth Science Series, Circum-Pacific Council for Energy and Mineral Resources, Houston 1.

Cawsey, A. 1998. The Essence of Artificial Intelligence. Prentice Hall, London.

Chang, J., Valverde, J. M., Griswold, N. C., Duque-Carillo, J, F. \& Sánchez-Sinencio, E. 1997. Cork quality classification system using a unified image processing and fuzzy-neural network methodology. IEEE Transactions on Neural Networks 8, 964-974.

Chapman, S. J. 1999. Matlab 5.0 Programming For Engineers. Brooks/Cole, Stamford.

Chappell, B. W. \& Bryant, C. J. 1994. New England Batholith granites. Department of Geology, Australian National University, Canberra (unpubl.).

Chen, K. S., Tzeng, Y. C., Chen, C. F. \& Kao, W. L. 1995. Land-cover classification of multispectral imagery using a dynamic learning neural network. Photogrammetric Engineering and Remote Sensing 61, 403-408.

Chen, S., Cowan, C. F. N. \& Grant, P. M. 1991. Orthogonal least squares learning in radial basis function networks. IEEE Transactions on Neural Networks 2, 302-309.

Cheng, Q. \& Agterberg, F. P. 1998. Fuzzy weights of evidence method and its application in mineral potential mapping. Natural Resources Research 8, 27-35.

Cheong, D.-K., Strobel, J., Biswas, G., Lee, G., Kendall, C., Cannon, R. \& Bezdek, J. 1993. Playmaker, a knowledge-based expert system. Geobyte 7, 28-41.

Chisnall, A. W. 1994. Stress mapping applications to the large structurally-controlled lode-gold deposits at Kalgoorlie (Western Australia) and to Mississippi Valley-type lead-zinc mineralization on the Lennard Shelf (Western Australia). MSc thesis, University of Western Australia, Perth (unpubl.).

Cho, S., Cho, Y. \& Yoon, S. 1997. Reliable roll force prediction in cold mill using multiple neural networks. IEEE Transactions on Neural Networks 8, 874-882.

Cho, S., Wong, P., Kwon, Y.\& Gedeon, T. 1998. Estimation of rock permiability in petroleum reservoirs. In: Bulsari, A. B., Fernandez-De Canete, J. \& Kallio, S., eds., Proceedings of the International Conference on Engineering Applications of Neural Networks (EANN'98). pp. 161-164. Systems Engineering Association, Turku, Finland.

Chung, C. F. \& Agterberg, F. P. 1980. Regression models for estimating mineral resources from geological map data. Mathematical Geology 12, 473-488. 
Chung, C. F. \& FabBri, A. G. 1993. The representation of geoscience information for data integration. Nonrenewable Resources 2, 122-139.

Chung, C. F., Jefferson, C. W. \& Singer, D. A. 1992. A qualitative link among mineral deposit modelling, geoscience mapping, and exploration-resource assessment. Economic Geology 87, 194-197.

Chung, C. F. \& Moon, W. M. 1991. Combination rules of spatial geoscience data for mineral exploration. Geoinformatics 2, 159-169.

Chung, C.-J. F. 1983. SIMSAG: integrated computer system for use in evaluation of mineral and energy resources. Mathematical Geology 15, 47-58.

Cisar, D., Dickerson, J. \& Novotny, T. 1993. Electromagnetic data evaluation using a neural network. In: Proceedings of the Symposium on the Application of Geophysics to Engineering and Environmental Problems. pp. 599-612.

Crvco, D. L. 1993. Artificial neural networks for land-cover classification and mapping. International Journal of Geographic Information Systems 7, 173-186.

Clare, A., Bolt, G. \& Daneel, G. 1997. The use of neural analysis as an exploration tool. In: Proceedings of the 12th International Conference on Applied Geologic Sensing. pp. 199-205. Environmental Research Institute of Michigan (ERIM) 2.

Clare, A. P. \& Cohen, D. R. 2001. A comparison of unsupervised neural networks and kmeans clustering in the analysis of multi-element stream sediment data. In: GeochemistryExploration, Environment, Analysis 1, Part 2, Geological Society Publishing House, London, pp. 119-134.

Clarici, E., Owen, D. B., Duncan, S. \& Ravenscroft, P. J. 1993. Recoverable reserve estimation using a neural network. In: Elbrond, J. \& TANG, X., eds., 24th International Symposium on the Application of Computers and Operations Research in the Mineral Industries (APCOM). pp. 145-152. Society for Mining, Metallurgy, and Exploration (SME), Littleton, Colorado.

Claudhill, M. 1988a. Neural networks primer. AI Expert pp. 46-52. Part 1.

Claudhill, M. 1988b. Neural networks primer. AI Expert pp. 55-59. Part 2.

Claudhill, M. 1988c. Neural networks primer. AI Expert pp. 53-59. Part 3.

Clay, R. D. \& Sequin, C. H. 1992. Fault tolerance training improves generalization and robustness. In: International Joint Conference on Neural Network (IJCNN 1992). IEEE, New York, volume 4, pp. 769-774.

CoDD, E. F. 1970. A relational model of data for large shared data banks. Communications of the ACM 13, 378-387.

Cohen, D., Rutherford, N. \& Garnett, D. 1995. A geochemical survey of the Upper North East region, New South Wales, for the New South Wales Department of Mineral Resources. Report Unisearch Ltd, University of New South Wales, Kensington, NSW.

Cooke, D. R. \& Pongratz, J., eds. 2002. Giant Ore Deposits: Characteristics, Genesis and Exploration, volume 4. Codes Special Publications, University of Tasmania, Tasmania.

Coveney, P. V., Fletcher, P. \& Hughes, T. L. 1996. Using artificial neural networks to predict the quality and performance of oil field cements. AI Magazine 17, 41-53. 
Cover, T. M. 1974. The best two independent measurements are not the two best. IEEE Transactions on Systems, Man, and Cybernetics SMC-4, 116-117.

Cover, T. M. \& Van Campenhout, J. M. 1977. On the possible orderings in the measuremnet selection problem. IEEE Transactions on Systems, Man and Cybernetics SMC-7, 657-61.

Cox, D. P. 1990. Development and use of deposit models in the U.S. Geological Survey. In: Eight Symposium of the International Association on the Geology of Ore Deposits (IAGOD) Program with Abstracts. p. A99.

Cox, D. P., Berger, B. R., Ludingtion, S., Moring, B., Sherlock, M. G., Singer, D. A. \& Tingley, J. V. 1996. Delineation of mineral resource assessment tracts and estimation of number of undiscovered deposits in Nevada. In: An Analysis of Nevada's Metal-Bearing Mineral Resources, Nevada Bureau of Mines and Geology, volume 96-2, pp. 12-1-12.25.

Cox, D. P. \& Singer, D. A., eds. 1986. Mineral Deposit Models. United States Geological Survey. Bulletin 1693.

Craig, M. A. 1993. Regolith-landform mapping in the Yilgarn Craton, Western Australia; towards a standardized approach. CSIRO Division of Exploration Geoscience 338R (unpubl.).

Cross, K., Fergusson, C. \& Flood, P. 1987. Contrasting structural styles in the Paleozoic subduction complex of the southern New England orogen, eastern Australia. In: LEITCH, E. \& Scheibner, E., eds., Terrane Accretion and Orogenic Belts. pp. 83-92. American Geophysical Union, Geodynamics Series 19.

Crowe, R. W. A. \& Towner, R. R. 1981. 1:250 000 Geological series - Explanatory notes, Noonkanbah, Western Australia, sheet SE/51-12. Bureau of Mineral Resources, Geology and Geophysics. Australian Government Publishing Service, Canberra.

Cruikshank, B. L. 1994. Stream sediment geochemistry of the Ebagoola 1:250,000 sheet area, Cape York Peninsula, north Queensland. Australian Geological Survey Organisation 1994/8.

Cucuzza, J. \& Goode, A. D. T. 1998. Australian mineral exploration research. In: BerkMAN, D. A. \& MacKenzie, D. H., eds., Geology of Australian and Papua New Guinean Mineral Deposits. pp. 53-60. Australasian Institute of Mining and Metallurgy, Melbourne, Victoria.

Cybenko, G. 1989. Approximation by superpositions of a sigmoidal function. Mathematical Control, Signals and Systems 2, 303-314.

Daneshfar, B. \& Benn, K. 2002. Spatial relationships between natural seismicity and faults, S.E. Ontario and New York State. Tectonophysics 353, 31-44.

Date, C. J. 1999. An Introduction to Database Systems (7th Edition). Addison-Wesley.

Davidson, D. A., Theocharopoulos, S. P. \& Bloksma, R. L. 1994. A land evaluation project in Greece using GIS and based on Boolean and fuzzy set methodologies. International Journal of Geographic Information Systems 8, 369-384.

Davies, P. 1995. The Indispensable Guide to $C$ with Engineering Applications. Addison-Wesley, Harlow.

Dawson, C. K., O’Reilly, R. C. \& McClelland, J. L. 1997. The PDP++ Software Users Manual Version 1.2. Carnegie Mellon University.

Dayhoff, J. E. 1990. Neural Network Architectures: An Introduction. Van Nostrand Reinhold, New York. 
De Angulo, V. R. \& Torras, C. 1997. Self-calibration of a space robot. IEEE Transactions on Neural Networks 8, 951-963.

De Geoffroy, J. \& Wignall, T. K. 1970. Statistical decision in regional exploration: application of regression and Bayesian classification analysis in the southwest Wisconsin zinc area. Economic Geology 65, 769-777.

De Geoffroy, J. \& Wignall, T. K. 1971. A probabilistic appraisal of mineral resources in a portion of the Grenville Province of the Canadian Shield. Economic Geology 66, 466-479.

De Graff, L. 1999. Ross Mining N.L. Annual Report (year ending 30/6/99) — Operational Review. Milton, Qld. http://www.rossmining.com.au/AnnReport99.html.

Decateur, S. E. 1989. Application of neural networks to terrain classification. In: IEEE INNS International Joint Conference on Neural Networks. pp. 283-288. 1.

Deere, D. U. \& Miller, R. P. 1966. Engineering classifiaction and index properties for intact rock. Technical report, U.S. Air Force Weapons Laboratory, Kirtland Air Force Base, New Mexico. Report No. AFWL-TR-65-116.

DeMers, M. N. 1990. SEDRULE: a rule-based system for interpreting some major sedimentary environments. Computers and Geosciences 16, 833-846.

Denby, B. \& Burnett, C. C. H. 1993. A neural network based tool for grade estimation. In: Elbrond, J. \& TAng, X., eds., 24th International Symposium on the Application of Computers and Operations Research in the Mineral Industries (APCOM). pp. 153-160. Society for Mining, Metallurgy, and Exploration (SME), Littleton, Colorado.

D'Ercole, C. 1998. An integrated model for lead-zinc mineralisation on the southeastern Lennard Shelf based on geographic information systems (GIS). Phd, University of Western Australia, Perth (unpubl.).

D'Ercole, C., Groves, D. I. \& Knox-Robinson, C. M. 2000. Using fuzzy logic in a Geographic Information System environment to enhance conceptually based prospectivity analysis of Mississippi Valley-type mineralisation. Australian Journal of Earth Sciences 47, 913-927.

Derek, H., Johns, R. \& PAsternack, E. 1990. Comparative study of backpropagation neural network and statistical pattern recognition techniques in identifying sandstone lithofacies. In: Proceedings: Conference on Artificial Intelligence in Petroleum Exploration and Production. Texas A\&M University, College Station, pp. 41-49.

Derrick, G. M. \& Playford, P. E. 1973. 1:250 000 Geological series - Explanatory notes, Lennard River, Western Australia, sheet SE/51-8 International Index (2nd Edition). Bureau of Mineral Resources, Geology and Geophysics. Australian Government Publishing Service, Canberra.

Dillon, T., Payman, A. \& Marks, I. R. J. 1997. Guest editorial-everyday applications of neural networks. IEEE Trsansactions on Neural Networks 8, 825.

Dimitrakopoulos, R., McCammon, R. B. \& Shoji, T. 1994. Expert systems and artificial intelligence in the applied geosciences: 26th International Geological Congress. Mathematical Geology 26, 877-994.

DinG, Z. \& KAInZ, W. 1998. An approach using fuzzy set theory in natural resource analysis. In: Zhou, Q., LI, H. \& SHI, W., eds., Spatial Information Technology Towards 2000 and Beyond; The Proceedings of Geoinformatics'98 Conference. Association of Chinese Professionals in GIS, Beijing, China, pp. 299-307. 
Donaldson, W. S. 1996. The Etheridge goldfield, Queensland: an application of weights of evidence and fuzzy logic in a geographic information system to estimate the probability of gold mineralisation. MSc thesis, University of New South Wales, Sydney (unpubl.).

Dörling, S. L. 1996. Structural evolution of the southeast Fitzroy Trough and Lennard Shelf, Western Australia during the Devonian-Early Carboniferous Pilara tectonic episode: Implications for facies distribution and Mississippi Valley-type $\mathrm{Pb}-\mathrm{Zn}$ mineralisation. PhD thesis, University of Western Australia, Perth (unpubl.).

Dorronsoro, J. R., Ginel, F., Sánchez, C. \& Cruz Santa, C. 1997. Neural fraud detection in credit card operations. IEEE Transactions on Neural Networks 8, 827-834.

Dowla, F. U. \& Rogers, L. L. 1995. Solving Problems in Environmental Engineering and Geosciences with Artificial Neural Networks. MIT Press, Cambridge.

Dowla, F. U., Taylor, S. \& Anderson, R. W. 1990. Seismic discrimination with artificial neural networks; preliminary results with regional spectral data. Bulletin of the Seismological Society of America 80, 1346-1373.

Dreyer, P. 1993. Classification of land cover using optimized neural nets on SPOT data. Photogrammetric Engineering and Remote Sensing 59, 617-621.

DudA, R. O. 1980. The PROSPECTOR system for mineral exploration. Final report contract 1408-001-17296, Stanford Research Institute International, Artificial Intelligence Centre, Menlo Park, California.

Duda, R. O., Hart, P. E., Barrett, P., Gaschnig, J. C., Konolige, K., Reboh, R. \& SLOCUM, J. 1978. Development of the PROSPECTOR consultation system for mineral exploration. Final report, Stanford Research Institute International, SRI International, Artificial Intelligence Centre, Menlo Park, California.

Duda, R. O., Hart, P. E. \& Stork, D. G. 2001. Pattern Classification. John Wiley \& Sons, New York.

Eddy, B. G., Bonham-Carter, G. F. \& Jefferson, C. W. 1995. Mineral resource assessment of the Parry Islands, high Arctic, Canada: a GIS-based fuzzy logic model. In: Proceedings Canadian Conference on GIS. CD ROM Session C3, Paper 4.

Eisenlohr, B. N., Groves, D. I. \& Partington, G. A. 1989. Crustal-scale shear zones and their significance to Archaean gold mineralization in Western Australia. Mineralium Deposita 24, 1-8.

EPSG 1999. European Petroleum Survey Group geodesy parameters data set version 4.4. http://www.petroconsultants.com/products/geodetic.html.

FabBri, A. G. \& Kasvand, T. 1988. Automated integration of mineral resource data by image processing and artificial intelligence. In: Chung, C. F., Fabbri, A. G. \& Sinding-Larsen, eds., Proceedings of the NATO Advanced Study Institute on Quantitative Analysis of Mineral and Energy Resouces. pp. 215-236. D. Reidel Publishing Co., Dordrecht.

Fardanesh, M. T. \& Ersoy, O. K. 1998. Classification accuracy improvement of neural network clssifiers by using unlabeled data. IEEE Transactions on Geoscience and Remote Sensing $\mathbf{3 6}$, 1020-1025.

FAusett, L. 1994. Fundamentals of Neural Networks: Architectures, Algorithms, and Applications. Prentice Hall, Englewood Cliffs. 
Faust, N. L., Anderson, W. H. \& Star, J. L. 1991. Geographic Information Systems and remote sensing future computing environment. Photgrammetric Engineering and Remote Sensing 57, 655-668.

FGDC 1998. Content Standard for Digital Geospatial Metadata (FGDC-STD-001-1998). Metadata Ad Hoc Working Group, Federal Geographic Data Committee (FGDC), Washington, D.C. Digital version available at WWW page: http//www.fdgc.gov.

Fisher, P. F. 1990a. Introduction. Computers and Geosciences 16, 751-752.

Fisher, P. F. 1990b. A primer of geographic search using artificial intelligence. Computers and Geosciences 16, 753-776.

FLACK, J. C. 1995. Interpretation of remotely sensed data using guided techniques for land cover analysis. PhD thesis, School of Computing, Curtin University of Technology, Perth (unpubl.).

Flemming, G. \& Zhang, W. 1999. Geological Digital Data Package for the Discovery 2000 Cobar Project Area, Version 1. CD-ROM. Geological Survey of New South Wales.

Flood, P. \& Aitchison, J. 1988. Tectonostratigraphic terranes of the southern part of the New England Orogen. In: KleEman, J. D., ed., New England Orogen-Tectonics and Metallogenesis. pp. 7-10. Department of Geology and Geophysics, University of New England, Armidale, New South Wales.

Foody, G. M. 1993. Land cover classification by an artificial neural network with ancillary information. International Journal of Geographic Information Systems 9, 527-542.

Foody, G. M. 1996. Relating the land-cover composition of mixed pixels to artificial neural netowrk classification output. Photogrammetric Engineering and Remote Sensing 62, 491499.

Foody, G. M. 1998. Issues in Training set selection and refinement for classification by a feedforward neural network. In: Proceedings of International Geoscience and Remote Sensing Symposium (IGARSS'98). IEEE International, volume 1, pp. 409-411.

Foody, G. M., McCulloch, M. B. \& Yates, W. B. 1995. Classification of remotely sensed data by an artificial neural network: issues related to training data characteristics. Photogrammetric Engineering and Remote Sensing 61, 391-401.

Foschi, P. G. \& Smith, D. K. 1997. Detecting subpixel woody vegetation in digital imagery using two artificial intellignece approaches. Photogrammetric Engineering and Remote Sensing 63, 493-500.

Foxall, R. \& Baddeley, A. 2002. Non-parametric measures of association between a spatial point process and a random set. Journal of the Royal Statistical Society, Series C 51, 165-182.

Fuessel, D.; Isermann, R. 2000. Hierarchical motor diagnosis utilizing structural knowledge and a self-learning neuro-fuzzy scheme. IEEE Transactions on Industrial Electronics 47, 1070-1077.

Funabashi, M., Maeda, A., Morooka, Y. \& Mori, K. 1995. Fuzzy and neural hybrid expert systems: synergetic AI. IEEE Expert 10, 32-40.

FUnAHASHI, K. I. 1989. On the approximate realisation of continuous mappings by neural networks. Neural Networks 2, 183-192.

Fung, C. C. \& Wong, K. W. 1999. Petrophysical properties interpretation modelling: an integrated artificial neural network approach. Systems Research and Information Systems $\mathbf{8}$, 203-220. 
Fung, C. C., Wong, K. W. \& Eren, H. 1997. Modular artifical neural network for prediction of petrophysical properties from well log data. IEEE Transactions on Instrumentation and Measurement 46, 1295-1299.

Fung, C. C., Wong, K. W., Eren, H. \& Charlebois, R. 1995. Lithology classification using self-organising map. In: Proceedings of ICNN'95 International Conference on Neural Networks. pp. 526-31. IEEE, Perth 1.

Fung, C. C., Wong, K. W., Eren, H., Charlebois, R. \& Crocker, H. 1996. Modular artificial neural network for prediction of petrophysical properties from well log data. In: Proceedings of IEEE Instrumentation and Measurement Technology Conference. IEEE Comp. Soc. Press, pp. 1010-1014.

Gahegan, M. 1997. 422/EM/JC New computing technologies to support mineral exploration activity. In: AMIRA Quarterly Report. pp. 15-16. Australian Minerals Industry Reseach Association, Parkville.

Gallagher, R. 1993. The Mount Isa Inlier. ARC/INFO World Book 34.

Gallagher, R. 1995. Geographic information systems - an essential toolbox for exploration (ASEG Preview feature - GIS in Exploration). Preview pp. 19-27.

Gallagher, R. 1996. GIS in 3D. Preview pp. 19-27.

Gallant, S. I. 1988. Connectionist Expert systems. Communications of the ACM 31.

Gan, Q. \& Suen, C. Y. 1996. Neural networks for handwritten character recognition. In: CHen, C. H., ed., Fuzzy Logic and Neural Network Handbook, McGraw-Hill, pp. 15.29-16.16.

GAnhdi, S. S. \& Bell, R. T. 1990. Metallogenic concepts to aid exploration for the giant Olympic Dam-type deposits and their possible derivatives. In: Proceedings Eighth IAGOD Symposium.

Gardoll, S., Groves, D. I., Knox-Robinson, C. M., Yun, G. Y. \& Elliott, N. 2000. Developing the tools for geological shape analysis, with regional- to local-scale examples from the Kalgoorlie Terrane of Western Australia. Australian Journal of Earth Sciences 47, 943953.

Gedeon, T. D. 1996. Indicators of input contributions: analysing the weight matrix. In: Narasimhan, V. L. \& Jain, L. C., eds., Proceedings of ANZIIS-96: Australian New Zealand Conference on Intelligent Information Systems. IEEE, New York.

Gedeon, T. D. \& Slade, P. 1994. Reducing training set size to improve learning. In: Proceedings 2nd European Congress on Intelligent Techniques and Soft Computing (EUFIT'94). pp. 12321236.

Gedeon, T. D. \& Treadgold, N. K. 1997. Extracting Meaning from Cascade Networks. In: Proceedings of SMC'97: IEEE International Conference on Systems, Man and Cybernetics. pp. 3019-3024. IEEE, New York 4.

Gedeon, T. D. \& Turner, H. 1993. Explaining student grades predicted by a neural network. In: Proceedings of IJCNN'93 - Nagoya: International Joint Conference on Neural Networks. pp. 609-612. IEEE, New York 1.

Gedeon, T. D. \& Turner, H. 1994. Extracting contextual if-then rules from a feedforward neura lnetwork. In: Proceedings Brazil-Japan Joint Symposium on Fuzzy Systems. pp. ??-?? 
Gellanty, D. C. \& Derrick, G. M. 1967. 1:250 000 Geological series - Explanatory notes, Lansdowne, Western Australia, sheet SE/52-5 International Index. Bureau of Mineral Resources, Geology and Geophysics. Australian Government Publishing Service, Canberra.

George, H. \& Bonham-Carter, G. F. 1989. Spatial modelling of geological data for gold exploration Star Lake area, Saskatchewan. In: Agterberg, F. P. \& Bonham-Carter, G. F., eds., Statistical Applications in the Earth Sciences. pp. 157-169. Geological Survey of Canada, Paper 89-9.

GEOSOFT 1999. GXF Grid Exchange File Revision 3.0 Draft 9.1. On-line documentation accompanying Oasis Montaj version 4.3 data processing and analysis software package, Toronto, Canada.

German, G. 1996a. Automatic classification of GIS data. In: Proceedings of the 1st International Conference in Geocomputation. ???, ???, pp. ??-??

German, G. 1996b. Discrete Output Neural Network GIS Classifier. World Wide Web URL: www.s.curtin.edu.au/ gordon/donnet/topics/htl. DONNET Version 1.33.

German, G., Gahagen, M. \& West, G. 1997. Predictive assessment of neural network classifiers for applications in GIS. In: PAscoe, R. T., ed., Proceedings of the Second International Conference GeoComputation 97, Incorporating the Spatial Information Research Centre's 9th Annual Colloquium. Spatial Information Research Centre (SIRC), University of Otago, Dunedin, pp. $41-50$.

German, G. W. H. \& Gagehan, M. H. 1996. Neural network architectures for the classification of temporal image sequences. In: LEEs, B. G., ed., Neural Network Applications in the Geosciences, Elsevier, pp. 969-979. Computers \& Geosciences 22 (Special Issue).

Gilligan, L. B. \& Barnes, R. G. 1990. New England Fold Belt, New South Wales - regional geology and mineralization. In: Hughes, F. E., ed., Geology of the Mineral Deposits of Australia and Papua New Guinea. pp. 1417-1423. Australasian Institute of Mining and Metallurgy, Monograph 14, Melbourne 2.

Gilligan, L. B. \& Byrnes, J. G. 1995. Cobar Metallogenic Map 1:250 000 SH/55-14: Metallogenic Study and Mineral Deposit Data Sheets. Geological Survey of New South Wales, Sydney.

Gilligan, L. B., Byrnes, J. G., Watkins, J. J. \& Podgson, D. J. 1994. Cobar Metallogenic Map 1:250 $000 \mathrm{SH} / 55-14$. Geological Survey of New South Wales, Sydney.

Glasson, K. R. \& Rattigan, J. H., eds. 1990. Geological Aspects of the Discovery of Some Important Mineral Deposits in Australia. Monograph Series No. 17. Australian Institute of Mining and Metallurgy, Parkville.

Goldfarb, R. J., Groves, D. I. \& Gardoll, S. 2001. Rotund versus skinny orogens; wellnourished or malnourished gold? Geology 29, 539-542.

Goldfarb, R. J., Miller, L. D., Leach, D. L. \& Snee, L. W. 1997. Gold deposits of metamorphic rocks of Alaska. In: Goldfarb, R. J. \& Miller, L. D., eds., Mineral Deposits of Alaska. pp. 151-190. Economic Geology Monograph 9.

Gole, M. J. 1981. Archean banded iron-formations, Yilgarn Block, Western Australia. Economic Geology 76, 1954-1974.

GonG, P. 1996. Integrated analysis of spatial data from multiple sources: using evidential reasoning and artificial neural network techniques for geological mapping. Photogrammetric Engineering and Remote Sensing 62, 513-523. 
Goodacre, A. K., Bonham-Carter, G. F., Agterberg, F. P. \& Wright, D. F. 1993. A statistical analysis of the spatial association of seismicity with drainage patterns and magnetic anomalies in western Quebec. Tectonophysics 217, 285-305.

Gow, P. A. 1997. The application of digital terrain and satellite gravity data to structural mapping of Australia's northern margin. In: 14th Australian Geological Convention Abstracts: Geoscience for the New Millenium. Geological Society of Australia, volume 49, p. 173.

Grant, J. A. 1986. The isocon diagram - a simple solution to Gresen's equation for metasomatic alteration. Economic Geology 81, 1976-1982.

Green, A. \& Munday, T. 1998. P476: Image processing and interpretation of airborne EM data for regolith/geological mapping. In: Australian Mineral Industries Research Association (AMIRA) Annual Report 1997-8. p. 20. AMIRA, Melbourne.

Griffin, T. J., Tyler, I. M. \& Playford, P. E. 1993. 1:250 000 Geological series - Explanatory notes on the Lennard River, Western Australia, sheet SE/51-8. Geological Survey of Western Australia.

Griffiths, C. M. 1987. An example of the use of fuzzy-set based pattern recognition approach to the problem of strata recognition from drilling response. In: Aminzadeh, F., ed., handbook of Geophysical Exploration, Section I: Seismic Prospecting, Geophysical Press, London, volume 20 of Pattern Recognition and Image Processing, pp. 504-538.

Groves, D. I. 1993. The crustal continuum model for Late-Archaean lode-gold deposits of the Yilgran Block, Western Australia. Mineralum Deposita 28, 366-374.

Groves, D. I. 1999. Short Course: Orogenic Gold Deposits: Nature and Exploration. Centre for Teaching and Research in Strategic Mineral Deposits, University of Western Australia, Perth.

Groves, D. I., Barley, M. E., Barnicoat, A. C., Cassidy, K. F., Fare, R. J., Hagemann, S. G., Hronsky, J. M. A., Mikucki, E. J., Mueller, A. G., McNaughton, N. J., Perring, C. S., Ridley, J. R. \& Vearncombe, J. R. 1992. Sub-greenschist to granulitehosted Archaean lode-gold deposits of the Yilgarn Craton: A depositional continuum from deep sourced hydrothermal fluids in crustal-scale plumbing systems. In: The Archaean; Terrains, Processes and Metallogeny; Proceedings Volume for the Third International Archaean Symposium., Geology Department and Extension Service, University of Western Australia, Perth, pp. 325-338. Publ. 22.

Groves, D. I., Goldfarb, R. \& Hagemann, S. 2001. Orogenic Gold Deposits. Centre for Global Metallogeny, University of Western Australia, Perth. MSc Short Course.

Groves, D. I., Goldfarb, R. J., Gebre-Mariam, M., Hagemann, S. G. \& Robert, F. 1998. Orogenic gold deposits; a proposed classification in the context of their crustal distribution and relationship to other gold deposit types. Ore Geology Reviews 13, 7-27.

Groves, D. I., Goldfarb, R. J., Knox-Robinson, C., Ojala, J., Gardoll, S., Yun, G. Y. \& Holyland, P. 2000. Late-kinematic timing of oreogenic gold deposits and significance for computer-based exploration techniques with emphasis on the Yilgarn Block, Western Australia. Ore Geology Reviews 17, 1-38.

Groves, D. I., Ojala, V. J. \& Holyland, P. W. 1997. Use of geometric parameters of greenstone belts in conceptual exploration for orogenic lode-gold deposits. AGSO Record $1997 / 41,103-108$.

Groves, D. I., Ridley, J. R., Bloem, E. J. M., Gebre-Mariam, M., Hronsky, J. M. A., Knight, J. T., McCuaig, T. C., McNaughton, N. J. \& Ojala, J. 1995. Lode-gold 
deposits of the Yilgarn Block: products of late-Archaean crustal-scale over-pressured hydrothermal systems. In: Coward, R. P. \& Ries, A. C., eds., Early Precambrian Processes. pp. 152-172. Geol. Soc. London, Special Publ. 95.

Groves, D. I. \& Solomon, M. New York. Geology and Origin of Australia's Mineral Deposits. Oxford University Press.

Guo, Y. \& Hansen, R. O. 1992. Feature recognition from potential fields using neural networks. In: SEG 62nd Annual International Meeting an Exposition Technical Program and Expanded Abstract with Authors Biographis. pp. 1-5. Society of Exploration Geophysicists, Tulsa Section AI 1.1.

Gupta, M. M. \& RaO, D. H. 1994. On the principles of fuzzy neural networks. Fuzzy Sets and Systems 61, 1-18.

Gust, D., Blevin, P., Chappell, B. \& Stephens, C. 1996. Early Mesozoic magmatism of the New England Orogen; space, time, and compositional relationships. In: Mesozoic Geology of the Eastern Australia Plate Conference. Geological Society of Australia, Sydney, volume Abstracts - Geological Society of Australia of 43, pp. 224-227.

Hagan, M. T. \& MenhaJ, M. 1994. Training feedforward networks with the Marquardt algorithm. IEEE Transactions on Neural Networks 5, 989-993.

Hagemann, S. G., Groves, D. I., Ridley, J. R. \& Vearncombe, J. R. 1992. The Archean lode gold deposits at Wiluna, Western Australia; high-level brittle-style mineralization in a strike-slip regime. Economic Geology 87, 1022-1053.

Hair, Jr, J. F., Anderson, R. E., Tatham, R. L. \& Black, W. C. 1998. Multivariate Data Analysis (5th Edition). Prentice Hall.

Halgamuge, S. K. \& Glesner, M. 1994. Neural networks in designing fuzzy systems for real world applications. International Jouranl of Fuzzy Sets and Systems 65, 1-12.

HANnAFord, P. \& Opat, G. I. 1998. P444: A novel gravity gradiometer for the resource industry. In: Australian Mineral Industries Research Association (AMIRA) Annual Report 1997-8. p. 26. AMIRA, Melbourne.

Hansen, J. V. \& Nelson, R. D. 1997. Neural networks and traditional time series methods: a synergistic combination in state economic forecasts. IEEE Transactions on Neural Networks 8, 863-873.

Harnett, D. L. 1982. Statistical Methods. Addison-Wesley, Reading, 3rd edition.

HARRIS, D. P. 1973. A subjective probability appraisal of metal endowment of northern Sonora, Mexico. Economic Geology 68, 222-242.

Harris, D. P. 1984. Mineral Resource Appraisal. Clarendon Press, Oxford.

Harris, D. P. \& Carrigan, F. J. 1981. Estimation of uranium endowment by subjective geological analysis - a comparison of methods and estimates for the San Juan Basin, New Mexico. Economic Geology 76, 1032-1055.

HARRIS, D. P. \& PAN, G. 1991. Consistent geologic areas for epithermal gold-silver deposits in the Walker Lake quadrangle of Nevada and California: delineated by quantitative methods. Economic Geology 86, 142-165.

HARRis, D. P. \& PAN, G. 1999. Mineral favourability mapping: a comparison of artificial neural networks, logistic regression, and discriminant analysis. Natural Resources Research 8, 93109. 
Harris, J. R., Rencz, A. N., Ballantyne, B. \& Sheridon, C. 1998. Mapping altered rocks using Landsat TM and lithogechemical data: sulphurets-Brucejack Lake District, British Columbia, Canada. Photogrammetric Engineering and Remote Sensing 64, 309-322.

Harris, J. R., Wilkinson, L., Heather, K., Fumerton, S., Bernier, M. A., Ayer, J. \& DAHN, R. 2001. Application of GIS processing techniques for producing mineral prospectivity maps-A case study : mesothermal Au in the Swayze Greenstone Belt, Ontario, Canada. Natural Resources Research 10, 91-124.

Hashiguchi, H. \& Usui, H. 1975. An approach to delimiting targets for prospecting of the Kuroko ore deposit: on the sulfur and magnetic susceptibility haloes. Mining Geology 25, 293-301.

Hassoun, M. H. 1995. Fundamentals of Artificial Neural Networks. MIT Press, Cambridge Massachusetts.

Hatheway, A. W. \& Kiersch, G. A. 1989. Engineering Properties of Rock. In: Carmichael, R. S., ed., CRC Practical handbook of physical properties of rocks and minerals, CRC Press, Boca Raton, chapter Section X.

Haynes, D. 2000. Area selection in minerals exploration: A global perspective. In: 15th Australian Geological Convention Abstracts: Understanding Planet Earth. p. 214. Geological Society of Australia 59.

Hazell, M. \& Wyborn, L. A. I. 1995. The rigorous steps required in data capure for creating a useable GIS and the need for national data standards. In: Proceedings of the Third National Conference and Trade Exhibition on the Management of Geoscience Information and Data. pp. 22.1-22.10. Australian Mineral Foundation, Adelaide.

Hecht-Nielsen 1991. Neurocomputing. Addison-Wesley, Reading, MA.

Hecht-NiELSEN, R. 1989. Theory of the backpropagation neural network. In: IEEE INNS International Joint Conference on Neural Networks. volume 1, pp. 593-605.

Heermann, P. \& Khazenie, N. 1992. Classification of multispoectral remote sensing data using a back-propagation neural network. IEEE Transactions on Geoscience and Remote Sensing 30, 81-88.

Hepner, G. F., Logan, T., Ritter, N. \& Bryant, N. 1990. Artificial neural network classification using a minimal traing set: comparison to conventional supervised classification. Photogrammetric engineering and remote sensing 56, 469-473.

Hergert, F., Finnoff, W. \& Zimmermann, H. G. 1992. A comparison of weight elimination methods for reducing complexity in neural networks. In: Proceedings of the International Joint Conference on Neural Networks. IEEE, New York, volume III, pp. 980-987.

Herrires, G. M. \& Selige, T. 1998. Application of supervised neural network approaches to remotely sensed optical imagery. In: IGARSS '98: Geoscience and Remote Sensing Symposium Proceedings. IEEE International, volume 1, pp. 403-405.

Hickman, A. H. \& Lipple, S. L. 1978. Explanatory notes, Marble Bar, 1:250000 geology map: Western Australian Geological Survey map SF50-8. Technical report, Geological Survey of Western Australia.

Hill, R. G. M. 1997. Stratigraphy, structure and alteration of hanging wall sedimentary rocks at the Sulphur Springs volcanogenic massive sulphide (VMS) prospect, east Pilbara Craton, Western Australia. BSc (Hons) thesis, University of Western Australia, Perth (unpubl.). 
Hinman, M. C. \& Scott, A. K. 1990. The Peak gold deposit, Cobar. In: Hughes, F. E., ed., Geology of the mineral deposits of Australia and Papua New Guinea Volume 2, Australasian Institute of Miniing and Metallurgy, Melbourne, Monograph 14, pp. 1345-1351.

Hinton, G. \& Le Cun, Y. 1989. 12 tricks to improve backprop performance. Unknown .

Ho, Y.-C. 1997. Book Review: Neuro-fuzzy and soft computing - a computational approach to learning and machine intelligence by C. T. Sun and E. Mizutani. IEEE Transactions on Automatic Control 42, 1482-1484.

Hodgson, C. J. 1990. Uses (and abuses) of ore deposit models in mineral deposit exploration. Geoscience Canada pp. 79-89.

Hodgson, C. J. \& Troop, D. G. 1988. A new computer-aided methodology for area selection in gold exploration: a case study from the Abitibi Greenstone Belt. Economic Geology 83, 952-977.

Hoel, P. G. 1984. Introduction to mathematical statistics (5th edition). John Wiley \& Sons, New York.

Hollingum, J. 1990. Expert Systems - Commercial Exploitation of Artificial Intelligence. IFS/Springer, Kempston.

Hollister, V. F. 1992. On a proposed plutonic porphyry gold deposit model. Nonrenewable Resources 1, 293-302.

Holmstrom, L. \& Koistinen, P. 1992. Using additive noise in back-propagation training. IEEE Transactions on Neural Networks 3, 24-38.

Holyland, P. W. \& OJala, V. J. 1997. Computer-aided structural targeting in mineral exploration: two and three-dimensional stress mapping. Australian Journal of Earth Sciences 44, 421-432.

Holyland, P. W., Ridley, J. R. \& Vearncombe, J. R. 1993. Stress mapping technology (SMT $\left.{ }^{\mathrm{TM}}\right)$. In: Parnell, J., H., R. A. \& Moles, N. R., eds., Geofluids '93, Contributions to an International Conference on fluid evolution, migration and interaction in rocks (May 4-7, Torquay, England). Geological Society of London, Institution of Mining \& Metallurgy, The Institute of Petroleum, Geological Society London Publishing House, Bath, pp. 272-273.

Hone, I. G., Milligan, P. R., Mitchell, J. N. \& Horsfall, K. 1997. Australian national airborne geophysical databases. In: GunN, P., ed., Thematic Issue: Airborne Magnetic and Radiometric Surveys. pp. 11-21. AGSO Journal of Australian Geology and Geophysics 17.

Hornik, K. 1991. Approximation capabilites of multilayer feedforward networks. Neural Networks 4, 251-257.

Hornik, K., Stinchcombe, M. \& White, H. 1989. Multilayer feedforward networks are universal approximators. Neural Networks 2, 359-366.

Howarth, R. J., Koch, G. S., Chork, C. Y., Carpenter, R. H. \& Schuenemeyer, J. H. 1980. Statistical map analysis techniques applied to regional distribution of uranium in stream sediment from the southeastern United States for the National Uranium Resource Evaluation program. Mathematical Geology 12, 339-366.

Hronsky, J. M. A., Ridley, J. R. \& Cathcart, J. C. 1991. Structural controls on the distribution of ore shoots in a shear-zone hosted gold deposit: the Lancefield example. In: Structural Geology in Mining and Exploration. pp. 147-149. Geology and Geophysics Department (Key Centre) and University Extension Publication 25. 
Hronsky, J., M. A., Cassidy, K. F., Grigson, M. W., Groves, D. I., Hagemann, S. G., Mueller, A. G., Ridley, J. R., Skwarnecki, M. S. \& Vearncombe, J. R. 1990. Alteration patterns; Deposit- and mine-scale structural controls. In: Ho, S. E., Groves, D. I. \& Bennett, J. M., eds., Gold deposits of the Archaean Yilgarn Block, Western Australia; Nature, Genesis and Exploration Guides, Geology Department and Extension Service, University of Western Australia, Publication 20, pp. 38-54.

Huang, W. Y. \& Lippmann, R. P. 1987. Comparisons between neural net and conventional classifiers. In: IEEE International Conference on Neural Networks. IEEE Comp. Soc. Press, Los Alamitos, volume 4, pp. 485-493.

Huang, Y., Wong, P. M. \& Gedeon, T. D. 1996. An improved fuzzy neural network for permiability estimation from wireline logs in a petroleum reservoir. In: Preceedings of the IEEE International Conference on Digital signal Processing (TENCON '96). pp. 912-917. Los Alamitos.

Huang, Z. \& Williamson, M. A. 1994. Geological pattern recognition and modelling with a general regression neural network. Canadian Journal of Exploration Geophysics 30, 60-68.

Hudson, P. T. W. \& Postma, E. O. 1995. Choosing and using a neural net. In: Braspenning, P. J., ed., Artificial Neural Networks.An Introduction to ANN Theory and Practice, Springer, Berlin, pp. 273-287.

Huntington, J. F. \& Green, A. A. 1978. Relationships between lineaments and mineral deposits. In: RIDGE, J. D., ed., Proceedings of the Fifth Quadrennial IAGOD Symposium. Snowbird, Utah, 17 August, International Association on the Genesis of Ore Deposits (IAGOD), Vol. 1.

Ishikawa, Y., Sawaguchi, T., Iwaya, S. \& Horiuchi, M. 1976. Delineation of prospecting targets for Kuroko deposits based on modes of volcanism of underlying dacite and alteration haloes. Mining Geology 26, 105-117.

Jagodinsky, E. A., Wyborn, L. A. I. \& Gallagher, R. 1993a. Mount Isa Metallogenic Atlas, volume 1 of Geology. Australian Geological Survey Organisation, Canberra. Metallogenic Atlas Series.

Jagodinsky, E. A., Wyborn, L. A. I. \& Gallagher, R. 1993b. Mount Isa Metallogenic Atlas, volume 2 of Geochemistry. Australian Geological Survey Organisation, Canberra. Metallogenic Atlas Series.

Jagodinsky, E. A., Wyborn, L. A. I., Gallagher, R., Whitaker, A., Morse, M. P., TArlowski, C. \& Simonis, F. 1993c. Mount Isa Metallogenic Atlas, volume 3 of Geolphysics. Australian Geological Survey Organisation, Canberra. Metallogenic Atlas Series.

JANG, J.-S. R. 1991. Fuzzy modeling using generalized neural networks and Kalman filter algorithm. In: Proceedings of the 9th National Conference on Artificial Intelligence (AAAI-91). pp. 762-767. AAAI Press, Menlo Park, California 2.

JANG, J.-S. R. 1993. ANFIS: Adaptive-network-based fuzzy inference systems. IEEE Transactions on Systems, Man, and Cybernetics 23, 665-685.

JAng, J.-S. R. \& Gulley, R. 1995. Fuzzy logic toolbox for use with Matlab. The Mathworks Inc., Natick.

JANG, J.-S. R. \& Sun, C. T. 1995. Neuro-fuzzy modeling and control. Proceedings of the IEEE 83, 378-406.

Jang, J.-S. R., Sun, T.-C. \& Mitsutani, E. 1997. Neuro-Fuzzy and Soft Computing: A Computational Approach to Learning and Machine Intelligence. Prentice Hall, New York. 
Jeffrey, W. \& Rossner, R. 1986. Optimization algorithms: simulated annealing and neural network processing. Astrophysical Journal 310, 473-481.

Jusmady \& TAYlor, G. R. 1997. Geological Data Integration and Processing System (GEODIPS) Software Package Version 1.03. Technical report, Department of Applied Geology, University of New South Wales, Sydney.

KAPAGERIDIS, I. K. \& DenBy, B. 1997. Ore grade estimation with mdoular neural network systems - a case study. In: MineIT'97 First International Conference on Information Technologies in the Minerals IndustryviatheInternet. A.A. Balkema Publishers, Rotterdam.

Karmeli, A., Meisels, A., Fisher, L. \& Arkin, Y. 1996. Automatic Extraction of geological linear features from digital remote sensing data using a Hough transform. Photogrammetric Engineering and Remote Sensing 62, 525-531.

KARRI, V. \& Frost, F. 1999. Effect of altering the Gaussian function receptive field width in RBF neural networks on aluminium fluoride prediction in industrial reduction cells. In: Gedeon, T., Wong, P., Halgamude, Kasabov, N., Nauck, D. \& Fukushima, K., eds., Proceedings of the $6^{\text {th }}$ International Conference on Neural Information Processing ICONIP'99. pp. 101-106. IEEE, Perth 1.

Kartalopoulos, S. V. 1996. Understanding Neural Networks and Fuzzy Logic: Basic Concepts and Applications. I.E.E.E. Press, New York.

Kasabov, N. \& Kozma, R., eds. 1999. Neuro-Fuzzy Techniques for Intelligent Information Systems. Springer Verlag, Berlin.

Kasabov, N. \& Trifonov, R. 1993. Using hybrid connectionist systems for spatial information processing. In: Proceedings of the Fifth Colloquium of the Spatial Information Research Centre. pp. 85-95. University of Otago, Dunedin.

Kasabov, N., Watson, C., Sinclair, S. \& Kilgour, R. 1994. Integrating neural networks and fuzzy systems for speech recognition. In: Proceedings of the Speech Science and Technology Conference SST-94. pp. 462-467. University of Western Australia, Perth.

Kasabov, N. K. 1996. Foundations of Neural Networks, Fuzzy Logic, and Knowledge Engineering. MIT Press, Cambridge.

Kasabov, N. K. \& Jain, L. C. 1990. Hybrid connectionist rule-based systems. In: JorRAnd, P. \& Sgurev, V., eds., Artificial Intelligence-Methodology, Systems, Applications. pp. 227235. North-Holland, Amsterdam.

Kasabov, N. K. \& Jain, L. C. 1993. Connectionist expert systems. In: Kasabov, N. K., ed., Proceedings 1993 The First New Zealand International Two-Stream Conference on Artificial Neural Networks and Expert Systems. pp. 220-221. IEEE Comp. Soc. Press, Los Alamitos.

Kasvand, T. 1983. Computerized vision for the geologist. Mathematical Geology 15, 2-23.

KAtz, S. S. 1991. Emulating the Prospector expert system with a raster GIS. Computers and Geosciences 17, 1033-1050.

Kelly, A. M. \& Sherriff, W. J. 1969. A statistical examination of the metallic mineral resources of British Columbia. In: Proceedings of a Symposium on Decision-Maling in Mineral Exploration. pp. 221-243. Extension Department, University of British Columbia, Vancouver II.

Kemp, L., Bonham-Carter, G., Raines, G. \& Looney, C. 2001. Arc-SDM: Arcview extension for spatial data modelling using weights of evidence, logistic regression, fuzzy logic and neural network analysis. Technical report, Geological Survey of Canada, Otawa. Http://ntserv.gis.nrcan.gc.ca/sdm/. 
Këpuska, V. Z. \& Mason, S. O. 1995. A hierachical neural network system for signalised point recognition in aerial photographs. Photogrammetric Engineering and Remote Sensing 61, $917-925$.

Key, J., Maslanik, J. A. \& Schweiger, A. J. 1989. Classification of merged AVHRR and SMMR Arctic data with neural networks. Photogrammetric Engineering and Remote Sensing 55, 1331-1338.

Key, J., Maslanik, J. A. \& Schweiger, A. J. 1990. Neural network vs. maximum likelihood classifications of spectral and textural features in visible, thermal, and passive microwave data. In: IGARSS'90, International Geoscience and Remote Sensing Symposium. pp. 1277-1280. IEEE, New York 2.

Kilgour, B. \& HAZELl, M. 1998. Spatial representation of AGSO's OZCHEM whole-rock geochemical database. Digital data set in EXCEL format.

KinAG, R. K. 1992. Classification of remotely sensed data using OCR-inspired neural network techniques. In: IGARSS'92 International Geoscience and Remotel Sensing Symposium. IEEE Geoscience and Remote Sensing Society, New York, volume 2, pp. 1081-1083. Outputs as probabilities.

Klir, G. J. \& Folger, T. A. 1988. Fuzzy Sets, Uncertainty, and Information. Prentice Hall, Englewood Cliffs.

Knight, J. T., Groves, D. I. \& Ridley, J. R. 1993. The Coolgardie Goldfield, Western Australia; district-scale controls on an Archaean gold camp in an amphibolite facies terrane. Mineralium Deposita 28, 436-456.

Knox-Robinson, C. M. 1994. Archean lode-gold mineralisation potential of portions of the Yilgarn Block, Western Australia: development and implementation of methodology for the creation of regional-scale prospectivity maps using conventional geological map data and a geographic information system (GIS). PhD thesis, University of Western Australia, Perth (unpubl.).

Knox-Robinson, C. M. 2000. Vectorial fuzzy logic: a novel technique for enhanced mineral prospectivity mapping, with reference to the orogenic gold mineralization potential of the Kalgoorlie Terrane, Western Australia. Australian Journal of Earth Sciences 47, 929-242.

Knox-Robinson, C. M. \& Groves, D. I. 1997a. Gold prospectivity mapping using a Geographic Information System (GIS) with examples from the Yilgarn Block of Western Australia. Chronique de la Recherche Minière. 529, 127-138.

Knox-Robinson, C. M. \& Groves, D. I. 1997b. Present state of GIS as an exploration tool with implications for future development. In: Third National Forum on GIS in the GeosciencesForum Proceedings. pp. 155-162. Australian Geological Survey Organisation Record 1997/36.

Knox-Robinson, C. M. \& Groves, D. I. 1997c. Present state of prospectivity mapping in the Yilgarn Block, Western Australia. In: Kalgoorlie '9\%: An International Conference on Crustal Evolution, Metallogeny and Exploration fo the Yilgarn-an update.

Knox-Robinson, C. M., Groves, D. I., Robinson, D. C. \& Wheatley, M. R. 1996. Improved resource evaluation using geoscientific information systems (GIS): a pilot study. Final Report AMIRA project P383/MERIWA project M194.

Knox-Robinson, C. M. \& Robinson, D. C. 1993. Application of geographic information systems (GIS) to regional-scale gold prospectivity mapping. In: Williams, P. R. \& Haldane, J. L., eds., KALGOORLIE '93. An International Conference on Crustal evoltuion, metallogeny and exploration of the Eastern Goldfields. Australian Geological Survey Organisation. AGSO Record 1993/54. 
Knox-Robinson, C. M., Robinson, D. C. \& Groves, D. I. 1995. Use of a Geographic Information System (GIS) to quantify gold prospectivity for the Southern Cross greenstone belt, Western Australia. In: Southern Cross Greenstone Belt: Geology and Gold Mines. Abstract Volume. pp. 116-117.

Knox-Robinson, C. M. \& Wyborn, L. A. I. 1997. Towards a holistic exploration strategy: using Geographic Information Systems as a tool to enhance exploration. Australian Journal of Earth Sciences 44, 453-463.

Kobayashi, T., Semba, T., Yamada, Y., Williams, S. \& Kato, Y. 1994. A case study of the application of a microcomputer-based favourability evaluation system: uranium exploration in the Alligator Rivers Uranium Field, Northern Territory, Australia. Mathematical Geology 26, 937-960.

Koch, G. S., Howarth, R. J., Scheunemeyer, J. H. \& Lowry, R. K. 1981. Uranium resource assessment through statistical analysis of exploration geochemical and other data. Economic Geology 76, 1056-1066.

KoHAVI, R. 1995. A study of cross-validation and bootstrap for accuracy estimation and model selection. In: Mellish, C. S., ed., IJCAI-95 Proceedings of the 14 th International Joint Conference on Artificial Intelligence. pp. 1137-1143. Morgan Kaufmann Publishers, San Mateo 2.

Kohavi, R. \& Frasca, B. 1994. Useful feature subsets and rough set reducts. In: Lin, T. Y., ed., RSSC-'94 The Third International Workshop on Rough Sets and Soft Computing: Conference Proceedings. San Jose State Univ, San Jose, pp. 310-317.

Kohonen, T. 1982. Self-organized formation of topologically correct feature maps. Biological Cybernetics 43, 59-69.

Kohonen, T. 1988. Self-Organization and Associative Memory (2nd Edition). Springer-Verlag, Berlin.

Kohonen, T. 1990. The self-organizing map. Proceedings of the IEEE 78, 1464-1480.

Kolmogorov, A. N. 1956. Foundations of the Theory of Probability (1933). Chelsea Publishing, New York.

Kosko, B. 1992. Neural Networks and Fuzzy Systems: a Dynamical Approach to Machine Intelligence. Prentice Hall, Englewood Cliffs.

Krauskopf, K. B. 1979. Introduction to Geochemistry (2nd Edition). McGraw Hill, New York.

Krogh, A. \& Hertz, J. A. 1992. Generalization in a linear perceptron in the presence of noise. Journal of Physics A (Mathematical \&S General) 25, 1135-1147.

Kurzyn, M. S. 1993. Expert systems and neural networks: a comparison. In: KASABov, N. K., ed., Proceedings 1993 The First New Zealand International Two-Stream Conference on Artificial Neural Networks and Expert Systems. IEEE Comp. Soc. Press, Los Alamitos, pp. $222-223$.

Lang, J. R., Baker, T., Hart, C. J. R. \& Moretenson, J. K. 2000. An exploration model for intrusion-related gold systems. SEG Newsletter 40, 1, 6-15.

Lang, J. R., Thompson, J. F. H., Mortensen, J. K. \& Baker, T. 1997. Intrusion-related Au mineralization associated with lithophile elements: An under-recognised metallogenic association. In: Geological Society of America Program with Abstracts. p. 358. Geological Society of America (GSA), Boulder, Colorado 29. 
LANG, K. J. \& Witbrock, M. J. 1988. Learning to tell two spirals apart. In: Proceedings of the 1988 Connectionist Models Summer School. pp. 52-59. Morgan Kaufmann, San Mateo.

Langley, P. \& Sage, S. 1994. Induction of selective Bayesian classifiers. In: Proceedings of Uncertainty in AI. pp. 123-126.

LARGE, R. R. 1992. Australian volcanic-hosted massive sulfide deposits: features, styles and geneticmodels. Economic Geology 87, 471-510.

LARsen, J. N. 2000. Analysis of aeromagnetic data with neural network. Digital version available on WWW page: http://www.gfy.ku.dk.

Lawrance, L. 1995. Supergene Ore Deposit Geochemistry. Key Centre for Teaching and Research in Strategic Mineral Deposits, Department of Geology and Geophysics, University of Western Australia, Perth.

Lawrie, K. C. \& Hinman, M. C. 1998. Cobar-style polymetallic Au-Cu-Ag-Pb-Zn deposits. AGSO Journal of Australian Geology and Geophysics 17, 169-187.

Lazzerini, B., Reyneri, L. M. \& Chiaberge, M. 1999. A neuro-fuzzy approach to hybrid intelligent control. IEEE Transactions on Industry Applications 35, 413-425.

LE Cun, Y. 1988. A theoretical framework for back-propagation. Technical report crg-tr-88-6, Connectionist Research Group, University of Toronto.

Le Cun, Y., Jackel, L. D., Boser, B., Denker, J. S. Graf, H. P., Guyon, I., Henderson, D., Howard, R. E. \& Hubbard, W. 1989. Handwritten digit recognition: applications of neural network chips and automatic learning. IEEE Communications Magazine pp. 41-46.

Le Cun, Y., Matan, O., Boser, B., Denker, J. S., Graf, H. P., Henderson, D., Howard, R. E., Hubbard, W., Jackel, L. D. \& Baird, H. S. 1990a. Handwritten zip code recognition with multilayer networks. In: Proceedings 10th International conference on pattern recognition. pp. 35-40. IEEE Computer Society, Los Alamitos 2.

Le Cun, Y., Poujaud, L., Personnaz, L. \& Dryfus, G. 1990b. Comparing different neural network architectures for classifying handwritten digits. In: International joint conference on neural networks (IJCNN). pp. 127-132. IEEE Computer Society, Los Alamitos 2.

Leach, D. L. \& Sangster, D. F. 1993. Mississippi Valley-type lead-zinc deposits. In: Kirkam, R. V., Sinclair, W. D., Thorpe, R. I. \& Duke, J. M., eds., Mineral Deposit Modelling, Geological Association of Canada, pp. 289-314. Special Paper 40.

LEE, M. 2000. Unpublished rock strength parameters for rocks from mines in the Yilgarn Block. Australian Mining Consultants Pty Ltd.

Lee, T., Richards, J. A. \& Swain, P. H. 1987. Probabilistic and evidential approaches for multisource data analysis. IEEE Transactions on Geoscience and Remote Sensing 25, 283293.

Leeming, P., Sattel, D. \& Massey, S. 1997. Airborne EM to focus reconaissance drilling for gold exploartion. In: 14th Australian Geological Convention Abstracts: Geoscience for the New Millenium. Geological Society of Australia, volume 49, p. 266.

LEEs, B. G. 1994. Decision trees, artificial neural networks and genetic algorithms for classification of remotely-sensed and ancillary data. In: Proceedings of the 7th Australasian Remote Sensing Conference. Remote Sensing and Photogrammetry Association Australia, pp. 51-60.

LeEs, B. G. 1996. Neural network applications in the geosciences: an introduction. Computers and Geosciences 22, 955-957. 
LEGGE, P. J. 1995. Geoscience 1994 and beyond: thoughts on geology and exploration for worldclass deposits. Australian Journal of Earth Sciences 42, 1-10.

Leonard, J. A. \& Kramer, M. A. 1991. Radial basis function networks for classifying process faults. IEEE Control Systems Magazine 11, 31-38.

Lerche, I. 2002. "Don't tell me how right you you want to be, tell me how wrong you could be". In: BAyer, U., Burger, H. \& Skala, W., eds., IAMG 2002 8th Annual Conference of the International Association for Mathematical Geology. pp. 15-30. International Association of Mathematical Geology 1.

Leveille, R. C. A., Newberry, R. J. \& Bull, K. F. 1988. An oxidation state-alkalinity diagram for discriminating some gold-favourable plutons: an empirical and phonomenological approach. In: Geological Society of America Abstracts with Program. p. 142. 20.

Lin, C.-T. \& LEe, C. S. G. 1991. Neural-network-based fuzzy logic control and decision system. IEEE Transactions on Computers 40, 1320-1336.

Lin, Y. H. \& Cunningham, III, G. A. 1995. A new approach to fuzzy-neural system modelling. IEEE transactions on Fuzzy Systems 3, 190-197.

Linton, P. 1997. The application of airborne hyperspectral imagery to geological exploration. In: 14th Australian Geological Convention Abstracts: Geoscience for the New Millenium. Geological Society of Australia, volume 49, p. 275.

LipPmann, R. P. 1987. An introduction to computing with neural nets. IEEE ASSP Magazine 4, 4-22.

Lippmann, R. P. 1989. Pattern classification using neural networks. IEEE Communications Magazine 27, 47-64.

LiU, J. N. K. \& Sin, K. Y. 1997. Fuzzy neural networks for machine maintenance in mass transit railway system. IEEE Transactions on Neural Networks 8, 932-941.

LiU, Z. K. \& XiaO, J. Y. 1991. Classification of remotely-sensed image data using artificial neural networks. International Journal of Remote Sensing 12, 2433-2438.

Luo, X. \& Dimitrakopoulos, R. 2003. Data driven fuzzy analysis in quantitative mineral resource assessment. Computers and Geosciences 29, 3-13.

Lusted, L. B. 1968. Introduction to Medical Decision Making. Charles Thomas, Springfield.

MacEachren, A. M. AD KraAk, M.-J. 1997. Exploratory cartographic visualization: advancing the agenda. Computers and Geosciences 23, 335-343.

Macfarlane, P. A. 1998. Statistical methods for delineating water quality. Kansas Geological Survey, Dakota Aquifer Program, Open-File Report 93-1B,; URL=http://www.kgs.ukans.edu/Dakota/vol3/ofr93_1b/rep02.htm.

MacLean, W. H. \& Barrett, T. J. 1993. Lithogeochemical techniques using immobile elements. Journal of Geochmical Exploration 48, 109-133.

Mah, A., Taylor, G. R. \& Balia, L. 1995. Lineament analysis of Landsat Thematic Mapper images, Northern Territory, Australia. Photogrammetric Engineering and Remote Sensing 61, 761-773.

Mamdani, E. H. 1977. Application of fuzzy logic to approximate reasoning using linguistic synthesis. IEEE Transactions on Computers 26, 1182-1191. 
Mamdani, E. H. \& Assilian, S. 1975. An experiment in linguistic synthesis with a fuzzy logic controller. International Journal of Man-Machine Studies 7, 1-13.

Marston, R. J. 1978. The geochemistry of the Archaean clastic metasediments in relation to crustal evolution, northeastern Yilgarn Block, Western Australia. In: Trendall, A. F., ed., Evolution of Archaean Terrane, Elsevier, Amsterdam.

Masters, T. 1993a. Neural, Novel and Hybrid Algorithms for Time Series prediction. John Wiley $\&$ Sons.

Masters, T. 1993b. Practical Neural Network Recipes in $C++$. Academic Press, San Diego.

Masters, T. 1995. Advanced Algorithms for Neural Networks: a C++ Sourcebook. John Wiley $\&$ Sons.

MatsuOKA, K. 1992. Noise injection into inputs in back-propagation learning. IEEE Transactions on Systems, Man and Cybernetics 22, 436-440.

Maurer, W. J., Dowla, F. U. \& Jarpe, S. P. 1992. Seismic event interpretation using selforganizing neural networks. In: Proceedings of SPIE Conference: Applications of Artificial Neural Networks III. pp. 950-958. International Society for Optical Engineering, Orlando 1709.

McCammon, R. B. 1988. Prospector II. In: Proceedings of the Annual AI systems in Government Conference. IEEE Comp. Soc. Press, pp. 88-92.

McCammon, R. B. 1990. Prospector-age 15-an expert system for mineral deposit models. In: Eight Symposium of the International Association on the Geology of Ore Deposits (IAGOD) Program with Abstracts. pp. A227-A228. Ottawa, Canada.

McCammon, R. B. 1993. Recent experiences with Prospector II. In: Davis, J. C. \& Herzfeld, U. C., eds., Computers in Geology - 25 Years of Progress, Oxford University Press, Oxford, pp. $45-54$.

McCammon, R. B., Botbol, J. M., Sinding-Larson, R. \& Bowen, R. W. 1983. Characteristic analysis-1981: Final program and a possible discovery. Mathematical Geology 15, 59-83.

McCammon, R. B., Finch, W. I., Kork, J. O. \& Bridges, N. J. 1986. Estimation of uranium endowment in the Westwater Canyon member, Morrison Formation, San Juan Basin, using a data-directed numerical method. In: A Basin Analysis Case Study, the Morrison Formation Grants Uranium Region, New Mexico, American Association of Petroleum Geologists, volume 22 of AAPG Studies in Geology, pp. 331-354.

McClellan, G. E., N., D. R., Hemmer, T. H., Matheson, L. N. \& Moe, G. O. 1989. Multispectral image-processing with a three-layer backpropagation network. In: IEEE INNS International Joint Conference on Neural Networks. volume 1, pp. 151-152.

McClelland, J. J. \& Rumellhart, D. E. 1988. Explorations in Parallel Distributed Processing: A Handbook of Models, Programs and Exercises. MIT Press, Cambridge.

McCoy, D., Newberry, R. J., Layer, P., Dimarchi, J. J., Bakke, A., Masterman, S. \& Minehane, D. L. 1997. Plutonic-related gold deposits of Interior Alaska. In: GoldFARB, R. J. \& Miller, L. D., eds., Mineral Deposits of Alaska. pp. 191-241. Economic Geology, Monograph 9.

McCullagh, J. \& Bluff, K. 1991. Genetic Modification of a neural networks training data. In: Artificial Neural Networks and Fuzzy Systems - New Zealand Conference?? ??, pp. 58-59. 
McDermotт, J. 1982. R1: A rule-based configurer of computer systems. Artificial Intelligence 19, 39-88.

Meisel, W. 1972. Computer-Oriented Approaches to Pattern Recognition. Academic Press, New York.

Minalasky, M. J. 1999. Mineral potential modelling of gold and silver mineralization in the Nevada Great Basin - a GIS-based analysis in using weights of evidence. PhD thesis, University of Ottawa, Ottawa (unpubl.).

Minalasky, M. J. \& Bonham-Carter, G. F. 2001. Lithodiversity and its spatial association with metallic mineral sites, Great Basin of Nevada. Natural Resources Research 10, 209-226.

MikUCKI, E. J. 1998. Hydrothermal transport and depositional processes in Archean lode-gold systems; a review. Ore Geology Reviews 13, 307-321.

Milne, L. 1995a. Classifying dry schlerophyll forest from augmented satellite data: comparing neuralnetwork, decision tree and maximum likelihood. In: Proceedings of 6th Australian Conference on Neural Networks. Sydney, pp. 160-163.

MiLnE, L. 1995b. Feature selection using neural networks with contribution measures. In: YAO, X., ed., AI'95: Eighth Australian Joint Conferernce on Artificial Intelligence : Poster Proceedings. World Scientific, Singapore, pp. 49-56.

Milne, L. 1999. Machine learning for automatic classification of remotely sensed data. PhD thesis, University of New South Wales, Sydney (unpubl.).

Minsky, M. \& Papert, S. 1969. Perceptrons. MiT Press, Cambridge.

Mohnaty, K. K. \& Majumdar, T. J. 1996. An artificial neural network (ANN) based software package for classification of remotely sensed data. Computers and Geosciences 22, 81-87.

Moody, J. \& Darken, C. J. 1989. Fast learning in networks of locally-tuned processing units. Neural Computation 1, 281-294.

Moon, W, M. 1990. Integration of geophysical and geological data uisng evidential belief function. IEEE Transactions, Geoscience and Remote Sensing 28, 711-720.

Moon, W. M. \& So, C. S. 1995. Information representation and integration of multiple sets of spatial geoscience data. In: International Geoscience and Remote Sensing Symposium, IGARSS '95. pp. 2141-2144. IEEE Comp. Soc. Press, Los Alamitos.

Moore, H. D. \& Gregory, A. F. 1989. Weighting of geophysical data with SPANS for digital geological mapping. In: Agterberg, F. P. \& Bonham-Carter, G. F., eds., Statistical Applications in the Earth Sciences. pp. 193-200. Geological Survey of Canada, Paper 89-9.

Moore, I. 1994. Modelled concept learning for databse knowledge discovery. In: Proceedings The Second Australian and New Zealand Conference on Intellignet Information Systems (ANZIIS'94). IEEE Comp. Soc. Press, Los Alamitos, pp. 372-376.

Moore, I. \& Sattar, A. 1993. A mineral deposit modelling and assessment expert system. In: Proceedings The First New Zealand International Two-Stream Conference on Artificial Neural Networks and Expert Systems. IEEE Comp. Soc. Press, Los Alamitos, pp. 270-273.

Morgan, N. \& Bourland, H. 1990. Generalization and parameter estimation in feedforward nets: some experiments. In: Touretzsky, D. S., ed., Advances in Neural Information Processing Systems (Denver 1989). Morgan Kaufmann, San Mateo, volume 2, pp. 630-637. 
Mulder, N. J. \& Spreeuwers, L. 1991. Neural networks applied to the classification of remotely sensed data. In: International Geoscience and Remote Sensing Symposium. IEEE Comp. Soc. Press, Los Alamitos, volume 4, pp. 2211-2213.

Müller, R. D., Overkov, N. C., Dutkiewicz, A. \& Keene, J. B. 1998. Seabed classification of the South Tasman Rise from SIMRAD EM12 backscatter data using artificial neural networks. Australian Journal of Earth Sciences 44, 689-700.

Mustard, R. 1999. Disseminated granite hosted gold deposits at Timbarra northern New South Wales. Australian Institute of Geoscientists. Digital version available at WWW page http://www.aig.asn.au/timbarra.htm.

Mustard, R., Partington, G., Nielsen, R. \& Mitchell, D. S. 1997. Disseminated granite hosted gold deposits at Timbarra, Northern New South Wales. In: 14th Australian Geological Convention Abstracts: Geoscience for the New Millenium. Geological Society of Australia, volume 49, p. 333.

Mutschler, F. E., Griffen, M. E., Stevens, D. S. \& Shannon, Jnr, S. S. 1985. Precious metal deposits related to alkaline rocks in the North American cordillera: an interpretative view. Transactions of the Geological Society of South Africa 88, 355-377.

NADARAYA, E. A. 1964. On estimating regression. Theory of Probability and its Applications 9, $141-142$.

NATMAP 1986. The Australian geodetic datum technical manual (10th Edition). National Mapping Council of Australia. National Mapping Council Special Publication.

Neall, F. B. \& Phillips, G. N. 1987. Fluid-wall rock interaction in an Archean hydrothermal gold deposit; a thermodynamic model for the Hunt Mine, Kambalda. Economic Geology 82, 1679-1694.

NERI, P. 1999. Stratimagic: a revolutionary software for seismic analysis. CGG Petrosystems, http://www.flagshipgeo.com/reservoir5a.html.

Nikolenko, V. N. 1980. Construction of a learning system of complex interpretation of geophysical fields for the recognition of geological objects. Izvestiya Earth Physics 16, 193-197.

Ojala, J. V., Ridley, J. R., Groves, D. I. \& Hall, G. C. 1993. The Granny Smith gold deposit: the role of heterogeneous stress distribution at an irregular granitoid contact in a greenschist facies terrane. Mineralium Deposita 28.

Openshaw, S., Blake, M. \& Wymer, C. 1995. Using neurocomputing methods to classify Britain's residential areas. In: FISCHER, P., ed., Innovations in GIS 2: Selected Papers from the Second National Conference on GIS Research UK. Taylor \& Francis, London, pp. 97-110.

Orlando, J., Mann, R. \& Haykin, S. 1990. Radar classification of sea-ice using traditional and neural classifiers. In: International Joint Conference on Neural Networks. pp. II-263-II-266. IEEE Computer Society, Los Alamitos 2.

Osborne, D. 1992. Neural networks provide more accurate reservoir permeability. Oil and Gas Journal 90, 80-83.

P., K. H., Gedeon, T. D. \& Wong, P. M. 1999. A Clustering Assisted Method for Fuzzy Rule Extraction and Pattern Classification. In: Gedeon, T., Wong, P., Halgamude, Kasabov, N., Nauck, D. \& Fukushima, K., eds., Proceedings of ICONIP'99: $6^{\text {th }}$ International Conference on Neural Information Processing. pp. 679-684. IEEE, Piscataway 2. 
PAN, G. 1993. Canonical favorability model for data integration and mineral potential mapping. Mathematical Geology 19, 1077-1100.

PAN, G. \& Harris, D. P. 1992. Estimating a favorability equation for the integration of geodata and selection of mineral exploration targets. Mathematical Geology 24, 177-201.

Pan, G. \& Harris, D. P. 2000. Information Synthesis for Mineral Exploration. OUP, New York.

Paola, J. D. \& Schowengerdt, R. A. 1997. The effect of neural-network structure on a multispectral land-use/land-cover classification. Photogrammetric Engineering and Remote Sensing 63, 535-544.

Parikh, C., Pont, M. \& Jones, N. 1999. Improving the performance of multi-layer perceptrons where limited training data are available for some classes. In: ICANN 99 Ninth International Conference on Artificial Neural Networks. pp. 227-232. (Conf. Publ. No. 470), 1.

PARK, J. \& SANDBERG, I. W. 1991. Universal approximation using radial-basis-function networks. Neural Computation 3, 246-257.

PARK, J. D. \& Jones, W. L. 1998. Comparison of neural network classifiers for NSCAT sea ice flag. In: 1998 IEEE International Geoscience and Remote Sensing Symposium (IGARSS '98). IEEE, New York, volume 4, pp. 2237-2239.

PARkeR, D. B. 1985a. Learning Logic. Technical Report TR-47, MIT Center for Research in Computational Economics and Management Science, Cambridge.

Parker, D. B. 1985b. Learning logic. Technical report tr-47, Centre for Computational Research in Economics and Management Science, Massachusetts Institute of Technology.

PARzen, E. 1962. On estimation of a probability density function and mode. Annals of mathematical Statistics 33, 1065-1076.

Patterson, D. W. 1996. Artificial Neural Networks. Prentice Hall, Singapore.

Pavlopoulos, S., Kyriacou, E., Koutsouris, D., Blekas, K., Stafylopatis, A. \& Zoumpoulis, P. 2000. Fuzzy neural network-based texture analysis of ultrasonic images. IEEE Engineering in Medicine and Biology Magazine 1, 39-47.

Peddle, D. 1995. Knowledge formulation for supervised evidential classification. Photogrammetric Engineering and Remote Sensing 57, 409-417.

Peddle, D. \& Franklin, S. E. 1992. Multisource evidential classification of surface cover and frozen ground. International Journal of Remote Sensing 13, 3375-80.

Penn, B. S., Gordon, A. J. \& Wendlandt, R. F. 1993. Using neural networks to locate edges and linear features in satellite images. Computers and Geosciences 19, 1545-1565.

PhilliPs, G. N. 1986. Geology and alteration in the Golden Mile, Kalgoorlie. Economic Geology 81, 779-808.

Phillips, G. N. \& Groves, D. I. 1983. The nature of Archaean gold-bearing fluids as deduced from gold deposits of Western Australia. Journal of the Geological Society of Australia 30, $25-39$.

Phillips, G. N., Groves, D. I. \& Kerrich, R. 1996. Factors in the formation of the giant Kalgoorlie gold deposit. Ore Geology Reviews 10, 295-317.

PickARD, A. L. 1993. Textural, mineralogical, and geochemical evaluation of gossans developed over a volcanic-hosted massive sulphide deposit at Sulphur Springs, eastern Pilbara Craton, Western Australia. BSc (Hons) thesis, University of Western Australia, Perth (unpubl.). 
Piramuthu, S. 1996. Effects of feature selection with 'blurring' on neurofuzzy systems. In: ArikaWa, S. \& Sharma, A. K., eds., ALT-'96 7th International Workshop on Algorithmic Learning Theory: Proceedings. Springer-Verlag, Berlin, pp. 135-142.

Pommereau? 1990. ALVIN etc?? IEEE? 99, 99-99.

Porwal, A., Carranza, E. J. M. \& Hale, M. 2003a. Knowledge-driven and data-driven fuzzy models for predictive mineral potential mapping. Natural Resources Research 12, 1-25.

Porwal, A., Carranza, E. J. M. \& Hale, M. 2003b. Knowledge-driven and data-driven fuzzy models for predictive mineral potential mapping. Natural Resources Research 12, 1-25.

Poulton, M. M., Sternberg, B. K. \& Glass, C. E. 1992. Location of subsurface targets in geophysical data using neural networks. Geophysics 57, 1534-1544.

Prelat, A. E. 1977. Discriminant analysis as a method of predicting mineral occurrence potentials in central Norway. Mathematical Geology 9, 343-367.

Press, W. H. \& Vetterling, W. T. 1992. Numerical Recipes in $C$ : the Art of Scientific Computing. Cambridge University Press.

Pugmire, R. H., Hodgson, R. M. \& Chaplin, R. I. 1993. Educating a neural network for image processing. In: Kasabov, N. K., ed., Proceedings 1993 The First New Zealand International Two-Stream Conference on Artificial Neural Networks and Expert Systems. IEEE Comp. Soc. Press, Los Alamitos, pp. 86-91.

QIN, S. J. \& RAJAGOPAL, B. 1993. Combining statistics and expert systems with neural networks for empirical process modelling. In: Proceedings of Instrument Society of America (ISA'93). volume 48, pp. 1711-1720.

Qui, Y. \& McNaughton, N. J. 1999. Source of $\mathrm{Pb}$ in orogenic lode-gold mineralisation; $\mathrm{Pb}$ isotope constraints from deep rock from the southwestern Archean Yilgarn Craton, Australia. Mineralium Deposita 34, 366-381. Springer Verlag, Berlin.

Quinlan, J. R. 1993. C4.5: Programs for Machine Learning. Morgan Kaufmann, San Mateo.

Raines, G. L. 1999. Evaluation of weights of evidence to predict epithermal gold deposits in the Great Basin of Western United States. Natural Resources Research 8, 257-276.

Raines, G. L. \& Minalasky, M. J. 2002. A reconaissance method for delineation of tracts for regional-scale mineral-resource assessment based on geologic-map data. Natural Resources Research 11, 241-248.

Ramsayer, G. R. \& Bonham-Carter, G. F. 1974. Numerical classification of geological patterns characterized by binary variables. Mathematical Geology 6, 59-71.

RAU, Y. C. \& Lure, Y. M. F. 1993. Classification of remote sensing data using partially trained neural network. In: IGARSS '93: Geoscience and Remote Sensing Symposium Proceedings. IEEE Comp. Soc. Press, volume 2, pp. 728-730.

RaYmond, O. 1992. Mount Isa Inlier and environs mineral deposit database version 1. Bureau of Mineral Resources, Geology and Geophysics, Australia Record 1992/66.

Reddy, R. K. T. Agterberg, F. P. \& Bonham-Carter, G. F. 1991a. Application of GiSbased logistic models to base-metal potential mapping in Snow Lake area. In: Proceedings of Canadian Conference on GIS. Ottawa, pp. 607-618.

Reddy, R. K. T. \& Bonham-Carter, G. F. 1991b. A decision-tree approach to mineral potential mapping in Snow Lake area, Manitoba. Canadian Journal of Remote Sensing 17, 191-200. 
Reddy, R. K. T., Bonham-Carter, G. F. \& Galley, A. G. 1992. Developing a geographic expert system for regional mapping of Volcanogenic Massive Sulphide (VMS) deposit potential. Nonrenewable Resources 1, 112-124.

Reddy, R. K. T. \& Koch, JnR, G. S. 1988. A case study of silver exploration in parts of Idaho and Montana using mathematical modelling techniques. Economic Geology 83, 1008-1014.

Redfearn, J. C. B. 1948. Transverse Mercator Formulae. Empire Survey Review 69.

Reed, R., Marks II, R. J. \& OH, S. 1992. An equivalence between sigmoidal gain scaling and training with noisy (jittered) input data. Neuroinformatics and Neurocomputers 1, 120-127.

Reed, R., Marks II, R. J. \& OH, S. 1995. Similarities of error regularization, sigmoid gain scaling, target smoothing, and training with jitter. IEEE Transactions on Neural Networks 6, 529-538.

Reid, A. B., Allsop, J. M., Grauser, H., Millett, A. J. \& Somerton, I. W. 1990. Magnetic interpretation in three dimensions using Euler deconvolution. Geophysics 55, 80-91.

Rhind, D. \& Connolly, T., eds. 1995. Understanding GIS: the ARC/INFO Method (3rd Edition). Environmental Systems Research Institute, Redlands.

Richard, M. D. \& Lippmann, R. P. 1991. Neural network classifiers estimate Bayesian aposteriori probabilities. Neural Computation 3, 461-483.

RidLEY, J. R. 1993. The relations between mean rock stress and fluid flow in the crust: with reference to vein-and lode-style deposits. Ore Geology Reviews 8, 23-37.

Ridley, J. R. \& Mengler, F. 2000. Lithological and structural controls on the form and setting of vein stockwork orebodies at the Mount Charlotte gold deposit, Kalgoorlie. Economic Geology 95, 85-98.

RIPley, B. D. 1996. Pattern Recognition and Neural Networks. Cambridge University Press, Cambridge.

Ritter, N. D. \& Hepner, G. F. 1990. Application of an artificial neural network to land-cover classification of thematic mapper imagery. Computers and Geosciences 16, 873-880.

Ritter, N. D., Logan, T. L. \& Bryant, N. A. 1988. Integration of neural network technologies with geographic information systems. In: Thomas, H. F., ed., GIS: Integrating Technology and Geoscience Applications. pp. 102-103. National Academy of Sciences, Washington, DC.

RoAch, M. J. 1994. Design and implementation of geological and geochemical databases in ArcInfo. In: Proceedings of OZRI8-The 8th Australian ESRI/ERDAS Users' Conference, Hobart. (unpaginated). ESRI Australia, Perth.

Roadknight, C. M., Balls, G. R., Mills, G. E. \& Palmer-Brown, D. 1997. Modelling comlex environmental data. IEEE Transactions on Neural Networks 8, 852-862.

Robert, F. 1997. A preliminary geological model for syenite-associated disseminated gold deposits in the Abitibi Belt, Ontario and Quebec. Current Research - Geological Survey of Canada pp. 201-210.

Robinove, C. J. 1989. Principles of logic and the use of digital geographic information systemss. In: Ripple, W. J., ed., Fundamentals of Geographic Information Ssytems: a Compendium, American Society for Photogrammetry and Remote Sensing, pp. 61-80.

Robinson, D. C. 1990a. Gold resource potential of the Southern Cross Greenstone Belt, Western Australia: mineral resource assessment using deposit models and a geographic information system. Bsc (hons) thesis, University of Western Australia, Perth (unpubl.). 
Robinson, V. B. 1990b. Interactive machine acquisition of a fuzzy spatial relation. Computers and Geosciences 16, 857-872.

Rock, N. M. S. 1988. Numerical Geology: a Source Guide, Glossary, and Selected Bibliography to Geological Issues of Computers and Statistics. Springer Verlag, New York.

Roethe, G. \& Tarantola, A. 1991. Use of neural networks for inversion of seismic data. In: Society of Exploration Geophysicists 61st Annual International Meeting and Exposition. pp. 302-305. Society of Exploration Geophysicists, Tulsa 61.

Roethe, G. \& Tarantola, A. 1995. Neural networks and inversion of seismic data. Journal of Geophysical Research, B, Solid Earth Physics 99, 6753-6768.

Rogers, S. J., FAng, J. H., Karr, C. L. \& Stanley, D. A. 1992. Determination of lithology from well logs using a neural network. American Association of Petroleum Geologists Bulletin 76, 731-739.

Rogers, S. K. \& Kabrisky, M. 1991. An Introduction to Biological and Artificial Neural Networks for Pattern Recognition, volume /textbfTT4. SPIE Optical Engineering Press, Bellingham.

Roli, F., Serpico, S. B. \& Vernazza 1996. Neural networks for classification of remotely sensed images. In: Chen, C. H., ed., Fuzzy Logic and Neural Network Handbook, McGraw-Hill, pp. $15.1-15.27$.

Rosenblatt, F. 1958. The perceptron: a probabilistic model for information storage and organization in the brain. Psychology Review 65, 386-408.

Rowan, L. C. \& Bowers, T. L. 1995. Analysis of linear features mapped in Landsat Thematic Mapper and side-looking airborne radar images of the Reno 1deg by 2 deg quadrangle, Nevada and California: implications for mineral resource studies. Photogrammetric Engineering and Remote Sensing 61, 749-759.

Rumelhart, D., Durbin, R., Golden, R. \& Chauvin, Y. 1995. Backpropagation: the basic theory. In: Chauvin, Y. \& Rummelhart, D. E., eds., Backpropagation: theoy, architectures, and applications, Lawrence Erlbaum, Hillsdale, N. J., pp. 1-34.

Rumelhart, D. E., Hinton, G. E. \& Williams, R. J. 1986a. Learning internal representations by error propagation. In: Rummelhart, D. E. \& McClelland, J. L., eds., Parallel Distributed Processing: Explorations in the Microstructure of Cognition. Volume 1 Foundations. pp. 318-362. MIT Press, Cambridge.

Rumelhart, D. E., Hinton, G. E. \& Williams, R. J. 1986b. Learning representations by back-propagating errors. Nature 323, 533-536.

SAEKI, Y. \& DATE, J. 1980. Computer application to the alteration data of the footwall dacite at the Ezuri Kuroko deposits, Aikta prefecture. Mining Geology 30, 241-250.

SAngster, D. F. 1986. Application of mineral deposit models to regionall assesments: discussion. In: Cargill, S. M. \& Green, S. B., eds., Prospects for Mineral Resource Assessments on Public Lands: Proceedings of the Leesburg Workshop. U.S. Geological Survey, volume 980, pp. 287-292. U.S. Geological Survey Circular.

SAWkins, F. J. 1990. Integrated tectonic-genetic model for volcanic-hosted massive sulfide deposits. Geology 18, 1061-1064.

SAWKINS, F. J. 1994. Integrated tectonic-genetic model for volcanic-hosted massive sulfide deposits. Geology 18, 1061-1064. 
Schalkoff, R. J. 1997. Artificial Neural Networks. McGraw-Hill, New York.

Schau, M., An, P., Chung, G. F. \& Rencz, A. N. 1994. Reconnaissance bedrock mapping by neural network integration of remotely sensed data with lithologic units on northern Melville Peninsula, Nunavut, Canada. In: Geological Association of Canada, Mineralogical Association of Canada Annual Meeting - Program with Abstracts. p. 99. Geological Association of Canada, Waterloo 19.

Schmidt, B. L. 1990. Elura zinc-lead-silver deposit, Cobar. In: Hughes, F. E., ed., Geology of the mineral deposits of Australia and Papua New Guinea Volume 2, Australasian Institute of Miniing and Metallurgy, Melbourne, Monograph 14, pp. 1329-1336.

Schmidt, P. W. 1998. P444: A novel gravity gradiometer for the resource industry. In: Australian Mineral Industries Research Association (AMIRA) Annual Report 1996-\%. AMIRA 26.

Schramm, U. \& Spinnler, K. 1993. An evaluation of different network models in machine vision applications. In: Albrecht, R., Reeves, C. R. \& Steele, N. C., eds., Proceedings of the International Conference on Artificial Neural Nets and Genetic Algorithms. pp. 265-268. Springer Verlag, Berlin.

Schum, D. A. 1994. Evidential Foundation of Probabilistic Reasoning. John Wiley \& Sons, New York.

Scott, A. K. \& Phillips, K. G. 1990. C.S.A. copper-lead-zinc deposit. In: Hughes, F. E., ed., Geology of the mineral deposits of Australia and Papua New Guinea Volume 2, Australasian Institute of Miniing and Metallurgy, Melbourne, Monograph 14, pp. 1337-1343.

Scotr, M. 1997. A mineral potential study of porphyry-copper-type deposits in the Yarrol province, Central Queensland. In: 14th Australian Geological Convention Abstracts: Geoscience for the New Millenium. Geological Society of Australia, volume 49, p. 396.

Scott, M. \& Dimitrakopoulos, R. 2001. Quantitative analysis of mineral resources for strategic planning: implications for Australian geological surveys. Natural Resources Research 10, 159177.

Scott, R. L., Whiting, T. \& Turner, R. 1994. Role of geophysics in exploration for MVT leadzinc deposits on the Lennard Shelf, Western Australia. In: Dentith, M. C., Frankcombe, K. F., Ho, S. E., Shepard, J. M., Groves, D. I. \& Trench, A., eds., Geophysical Signatures of West Australian Mineral Deposits. pp. 107-117. Geology and Geophysics Department (Key Centre) and University Extension Publication 26.

Serpico, S. \& Vernazza, G. 1996. A hybrid system for two-dimensional image recognition. Proceedings of the IEEE 84, 1659-81.

Shafer, G. 1976. A Mathematical Theory of Evidence. Princeton University Press, Princeton.

Sharma, S. 1995. Applied Multivariate Techniques. John Wiley \& Sons, New York.

Shaw, S. E. \& Flood, R. H. 1981. The New England batholith, eastern Australia: geochemical variations in time and space. Journal of Geophysical Research 86, 10530-10544.

Shaw, S. E. \& Flood, R. H. 1993. A compilation of Late Permian and Triassic biotite Rb-Sr data from the New England batholith and areas to the southeast. In: CARR, P. F., ed., Centre for Isotope Studies, Research Report. pp. 151-155. CSIRO Mineral Research Laboratories, North Ryde, Sydney.

Shen, S. S. \& Horblit, B. D. 1992. Application of neural networks to hyper-spectral image analysis and interpretation. In: Proceedings of the SPIE Conference on Application of Neural Networks III. International Society for Optical Engineering (SPIE). Part 1. 
Short, JR., N. 1991. A real-time expert system and neural network for the classification of remotely sensed data. In: Proceedings of ASPRS/ACSM Annual Convention. volume 3, pp. $406-418$.

Shortcliffe, E. H. 1976. Computer-based Medical Consultations. Elsevier, New York.

SiBson, R. H. 1987. Earthquake rupturing as a hydrothermal mineralising agent in hydrothermal systems. Geology 15, 701-704.

Sietsma, J. \& Dow, R. J. F. 1991. Creating artificial neural networks that generalize. Neural Networks 4, 67-79.

Sillitoe, R. H. 1991. Intrusion-related gold deposits. In: Foster, R. P., ed., Gold Metallogeny and Exploration. pp. 165-209. Blackie, Glasgow.

SimaAn, M. A. \& Zhang, Z. 1995. SEISIS: a rule-based system for segmentation of a seismic section based on texture. In: International Geoscience and Remote-Sensing Symposium (IGARSS'95). IEEE Comp. Soc. Press, Los Alamitos, volume 1, pp. 461-463.

Simmons, H. W., Pollard, P. J., Stewart, J. L., Taylor, L. A. \& G., T. R. 1996. Granitehosted, disseminated gold mineralisation at Timbarra, New South Wales. In: Mesozoic Geology of Eastern Australia Plate Conference. pp. 507-509. Geological Society of Australia, Extended Abstracts 43.

Simpson, C., Huntington, J. F., Leishman, J. \& Green, A. A. 1980. A study of the Pine Creek Geosyncline using integrated Landsat and aeromagnetic data. In: FERGuson, J. \& Goleby, A. B., eds., Uranium in the Pine Creek Geosyncline; Proceedings of the International Uranium Symposium on the Pine Creek Geosyncline. pp. 141-155. Sydney, 4-8 June 1979, International Atomic Energy Agency (IAEA), Vienna.

Sinclair, A. J. \& Woodsworth, G. J. 1970. Multiple regression as a method of estimating exploration potential in an area near Terrace, B. C. Economic Geology 65, 998-1003.

Singer, D. A. 1998. Combining Variables with Neural Networks. In: Unknown, ed., Unknown. University of Queensland, pp. ??-??

Singer, D. A. \& Cox, D. P. 1988. Applications of mineral deposit models to resource assessments. U.S. Geological Survey Yearbook Fiscal Year 1988 pp. 55-57.

Singer, D. A. \& KoudA, R. 1988. Integrating spatial and frequency information in the search for Kuroko deposits of the Hokuroku district, Japan. Economic Geology 83, 18-29.

Singer, D. A. \& KoudA, R. 1996. Application of a feedforward neural network in the search for Kuroko deposits in the Hokuroku District, Japan. Mathematical Geology 28, 1017-1023.

Singer, D. A. \& KoudA, R. 1997a. Classification of mineral deposits into types using mineralogy with a probabilistic neural network. Nonrenewable Resources 6, 27-32.

Singer, D. A. \& KoudA, R. 1997b. Use of a neural network to integrate geoscience information in the classification of mineral deposits and occurences. In: GuBIns, A., ed., Proceedings of Exploration 9\%: Fourth Decennial International Conference on Mineral Exploration. pp. 127-134. Prospectors and Developers Association of Canada, Toronto.

Singer, D. A. \& KoudA, R. 1999a. A comparison of the weights-of-evidence method and probabilistic neural networks. Natural Resources Research 8, 287-298.

Singer, D. A. \& Kouda, R. 1999b. Examining risk in mineral exploration. Natural Resources Research 8, 111-122. 
Singer, D. A. \& Mosier, D. L. 1981. A review of regional mineral resource assessment methods. Economic Geology 76, 1006-1015.

Singer, D. A. \& Mosier, D. L. 1988. A review of regional mineral resource assessment methods. Economic Geology 76, 1006-1015.

Skabar, A., Biswas, K., Pham, B. \& Maeder, A. 1999a. Contextual classification of multisource geoscientific data using a fuzzy/genetic learner. Research Report 13/99, School of Information Technology and Mathematical Sciences, University of Ballarat, Ballarat.

Skabar, A., Biswas, K., Pham, B. \& Maeder, A. 1999b. Inductive concept learning in the absence of labeled counter-examples. Research Report 27/99, School of Information Technology and Mathematical Sciences, University of Ballarat, Ballarat.

Skabar, A., Biswas, K., Pham, B. \& Maeder, A. 2000. Inductive concept learning in the absence of labeled counter-examples. In: Proceedings 23rd Australasian Computer Science Conference, ACSC 2000. pp. 220-226. IEEE Computer Society, Los Alamitos.

Skabar, A., Pham, B., Biswas, K. \& Maeder, A. 1999c. Inductive concept learning based on limited class information using evolutionary search. Research Report 14/99, School of Information Technology and Mathematical Sciences, University of Ballarat, Ballarat.

Skala, W. \& Heynisch, S. 1993. Expert systems in environmental geology. In: Davis, J. C. \& Herzfeld, U. C., eds., Computers in Geology -25 Years of Progress, Oxford University Press, Oxford, pp. 255-264.

Skidmore, A., BaAng, J. \& Luckananurug, P. 199? Knowledge based methods in remote sensing and GIS. In: 6th Australasian Remote Sensing Conference. pp. 2-394-2-403.

Skidmore, A., Watford, F., Luckananurug, P. \& Ryan, P. J. 1996. An operational GIS expert system for mapping forest soils. Photogrammetric Engineering and Remote Sensing 62, 501-511.

Skidmore, A. K., Brinkhof, W., Delaney, J., Turner, B. J. \& Turner, P. 1994. Using neural networks to analyse spatial data. In: Proceedings 7th Australasian Remote Sensing Conference. volume 3, pp. 235-246.

Skidmore, A. K. \& Gauld, A. 1996. Classification of Kangaroo habitat distribution using three GIS models. International Journal of Geographic Information Systems 10, 441-454.

Skidmore, A. K., Turner, B. J., Brinkhof, W. \& Knowles, E. 1997. Performance of a neural network: mapping forests using GIS and remotely sensed data. Photogrammetric Engineering and Remote Sensing 63.

Smith, A. B. \& Gardoll, S. J. 1997. Structural analysis in mineral exploration using a Geographic Information Systems-adapted stereographic-projection plotting system. Australian Journal of Earth Sciences 44, 445-452.

Smith, T. R., Zhan, C. \& Gao, P. 1990. A knowledge-based, two-step procedure for extracting channel networks from noisy DEM data. Computers and Geosciences 16, 777-786.

Somarin, A. K. \& Ashley, P. M. 1997. The Glen Eden Mo-Sn-W deposit, New England Orogen, NSW. In: 14th Australian Geological Convention Abstracts: Geoscience for the New Millenium. Geological Society of Australia, volume 49, p. 232.

Specht, D. 1990. Probabilistic neural networks. Neural Networks 3, 109-118.

SpeCHT, D. F. 1991. A general regression neural network. IEEE Transactions on Neural Networks 2, 568-576. 
Spiegelhalter, D. J. \& Knill-Jones, R. P. 1984. Statistical and knowledge-based approaches to clinical decision-support systems with an application to gastroenterology. Journal of the Royal Statistical Society A Part 1, 35-77.

Spitz, G. \& DARLing, R. 1978. Major and minor element lithogeochemical anomalies surrounding the Louvem copper deposit, Val d'Or, Quebec. Canadian Journal of Earth Sciences 15, 11611169 .

Stackhouse, C. \& Boyd, R. 1985. POLOG: a mineral exploration expert system. In: Proceedings of the International Conference on Cybernetics and Society. pp. 116-120. IEEE Systems, Man and Cybernetics Society, Tuscon.

Stoessel, e., Johnson, V., Rogers, L. \& Scheevel, J. 1998. Deeplookresearches a new strategy: neural networks. World Oil 219, 83-91.

Stone, M. 1974. Cross-validation: a review. Mathematische Operationsforschung Statisitischen 9, 127-140.

Stoodley, K. D. C., Lewis, T. \& Stainton, C. L. S. 1980. Applied Statistical Techniques. E. Horwood, New York.

Strang, G. 1993. Introduction to Linear Algebra. Wellesley-Cambridge Press, Wellesley.

Stroud, W. J. 1994. An explanantion of the letter symbols used on the North Eastern Section's 1:250,000, 1:50,000, 1:100,000 and 1:250,000 geological and metallogenic maps. Geological Survey of New South Wales. Geological Survey of New South Wales Report GS1990/083 (unpubl.).

Suewatanakul, W. \& Himmelblau, D. M. 199? Comparison of artificial neural networks and traditional classifiers via the two-spiral problem. Neural Networks pp. 275-281.

Sugeno, M. 1985. Industrial Applications of Fuzzy Control. Elsevier Science Ltd, London.

Sun, C.-T. \& JAng, J.-S. R. 1993. A neuro-fuzzy classifier and its applications. In: Proceedings of the Second IEEE International Conference on Fuzzy Systems. pp. 94-98. San Francisco 1.

Suppel, D. W. \& Gilligan, L. B. 1993. Cobar Metallogenic Map 1:250 000 SI/55-2: Metallogenic Study and Mineral Deposit Data Sheets. Geological Survey of New South Wales, Sydney.

Suppel, D. W. \& Scheibner, E. 1990. Lachlan Fold Belt in New South Wales - regional geology and mineral deposits. In: Hughes, F. E., ed., Geology of the mineral deposits of Australia and Papua New Guinea Volume 2, Australasian Institute of Miniing and Metallurgy, Melbourne, Monograph 14, pp. 1321-1327.

SwAGER, C. 1989. Structure of Kalgoorlie greenstones - regional deformation history and implications for the structural setting of the Golden Mile gold deposits. Technical report, Geological Survey of Western Australia. Report 25.

Swager, C. \& Griffin, T. 1990. Geology of the Archaean Kalgoorlie Terrane 1:250,000 scale geological map. Technical report, Geological Survey of Western Australia.

Swager, C., Griffin, T. J., Witt, W. K., Wyche, S., Ahmat, A. L., Hunter, W. M. \& MCGoldrick, P. J. 1990. Geology of the Archaean Kalgoorlie Terrane-an explanatory note. Technical report, Geological Survey of Western Australia. Record 1990/12.

Szwedzicki, T. \& Jiang, J. 1994. Report on rock testing, Pancontinental Goldmining Pty Ltd Areas, Western Australia. Technical report, Western Australian School of Mines. 
TABesh, E. 1992. Map integration model applied in site selection. In: KüzL, H. \& MerriAm, D. F., eds., Use of Microcomputers in Geology. pp. 241-266. Plenum Press.

Taylor, G. R., ed. 1997. Geoscience GIS: Desktop and Prospectivity Mapping. Short Course at the Key Centre for Mines. University of New South Wales, Sydney.

Taylor, R. B. \& Steven, T. A. 1983. Definition of mineral resource potential. Economic Geology 78, 1268-1270.

Thesen, A. 2001. IE 642 Simulation of manufacturing systems. University of Wisconsin at Madison, http://www.cae.wisc.edu/,ie642/content/Techniques/K-S-Test.htm.

Thinrt, C., Bonham-Carter, G. \& Agterberg, F. 2003a. Conditional independence in weights-of-evidence: Application of an Improved test. In: IAMG 2003 9th Annual Conference of the International Association for Mathematical Geology. International Association of Mathematical Geology 1.

Thinrt, C., Bonham-Carter, G., Agterberg, F., Cheng, Q. \& Panah, A. 2003b. Application of the new omnibus test for conditional independence in weights-of-evidence modeling. In: Harris, J. R. \& Wright, D. F., eds., GAC Special Volume, Geological Association of Canada. (unpublished).

Thompson, J. F. H., Sillitoe, R. H., Baker, T., Lang, J. R. \& Mortenson, J. K. 1999. Intrusion-related gold deposits associated with tungsten-tin provinces. Mineralium Deposita 34, 323-334.

Thorpe, M. H. 1993. A semi-conformable alteration zone associated with the Sulphur Springs volcanogenic massive sulphide (VMS) deposit, east Pilbara, Western Australia. BSc (Hons) thesis, University of Western Australia, Perth (unpubl.).

Tomlin, C. D. 1983. Digital cartographic modelling in environmental planning. PhD thesis, Yale University, Connecticut (unpubl.).

Tomlin, C. D. 1990. Geographic Information Systems and Cartographic Modelling. Prentice Hall, Englewood Cliffs.

Townsend, D. B., Mai, G. \& Morgan, W. R. 2000. Mines and mineral deposits of Western Australia: digital extract from MINEDEX - an explanatory note. Technical report, Geological Survey of Western Australia, Department of Minerals and Energy. Record textbf2000/13.

Uncini, A., Marchesi, M., Orlandi, G. \& Piazza, F. 1990. Improved evoked potential estimation using neural networks. In: IEEE Conference on Neural Networks, San Diego, 1990. pp. II-143-II-148.

Van Kranendonk, M. 1999. North Shaw W.A. Sheet 2755. Technical report, West Australian Geological Survey. 1:100000 Geological Series.

van Lieshout, M. N. N. \& Baddeley, A. J. 1997. A nonparametric measure of spatial interaction in point patterns. Statistica Neerlandica 50, 344-361.

VARnES, D. J. 1974. The logic of maps with reference to their interpretation and use for engineering purposes. United States Geological Survey Professional Paper 837. 48p.

Vearncombe, S. E. 1995. Volcanogenic massive sulphide-sulphate mineralization at Strelley, Pilbara Craton, Western Australia. PhD thesis, University of Western Australia, Perth (unpubl.). 
Vemuri, V. R. 1993. Main problems and issues in neural networks application. In: KASABOV, N. K., ed., Proceedings 1993 The First New Zealand International Two-Stream Conference on Artificial Neural Networks and Expert Systems. IEEE Comp. Soc. Press, Los Alamitos, p. 226.

Venkataraman, G., Madhayan, B. B., Ratha, D. S., Antony, J. P., Goyal, R. S., Banglani, S. \& Roy, S. S. 2000. Spatial modelling for base-metal mineral exploration through integration of geological data sets. Natural Resources Research 9, 27-42.

Viega, M. M. \& Meech, J. A. 1997. Fuzzy-neural systems for adaptive reasoning on environmental risk analysis. In: MineIT'97 First International Conference on Information Technologies in the Minerals IndustryviatheInternet. A.A. Balkema Publishers, Rotterdam.

Wagner, D. E., Ehlers, J. N. \& Velzhinathan, J. 1990. First break picking using neural networks. In: Society of Exploration Geophysicists Sixtieth Annual International Meeting and Exposition. pp. 370-373. Tulsa 60.

Walker, P. A. \& Moore, D. M. 1988a. An inductive modelling and mapping tool for spatiallyoriented data. International Geographical Information Systems 2, 347-363.

Walker, P. A. \& Moore, D. M. 1988b. SIMPLE-an inductive modelling and mapping tool for spatially-oriented data. International Journal of Geographical Information Systems 2, $347-363$.

Walker, P. A. \& Moore, D. M. 1988c. SIMPLE: an inductive modelling and mapping tool for spatially-oriented data. International Journal of Geographical Information Systems 2, $347-63$.

WAN, E. A. 1990. Neural network classification: a bayesian interpretation. IEEE Transactions on Neural Networks 1, 303-305. Outputs as probabilities.

WAng, C. \& PRincipe, J. C. 1999. Training neural networks with additive noise in the desired signal. IEEE Transactions on Neural Networks 10, 1511-1517.

Wang, C., Venkatesh, S. S. \& Judd, J. S. 1994. Optimal stopping and effective machine complexity in learning. In: Cowan, J. D., Tesauro, G. \& Alspector, J., eds., Advances in Neural Information Processing Systems (Denver, 1993). Morgan Kaufmann, San Francisco, pp. 303-310.

Wang, L., Wong, P. M. \& Shibli, S. A. R. 1998. Modelling porosity distribution in the A'nan oilfield: use of geological quantification, neural networks and geostatisitics. In: SPE International COnference and Exhibition, Beijing, China. Society of Petroleum Engineers, volume SPE 48884, pp. 509-515.

WAnG, L.-X. \& Mendel, J. M. 1992. Back-propagation fuzzy systems as non-linear dynamic system identifiers. In: Proceedings of the IEEE International Conference on Fuzzy Systems. pp. 1409 -1418. San Diego.

Wanstedt, S., Huang, Y. \& Malstrom, L. 1997. Using neural networks to interpret geophysical logs in the Zinkgruvan Mine. A case study. In: MineIT'97 First International Conference on Information Technologies in the Minerals IndustryviatheInternet. A.A. Balkema Publishers, Rotterdam.

Watson, G. P. \& Rencz, A. N. 1989. Data integration studies in northern New Brunswick. In: Agterberg, F. P. \& Bonham-Carter, G. F., eds., Statistical Applications in the Earth Sciences. pp. 185-191. Geological Survey of Canada Paper 89-9.

Watson, G. P., Rencz, A. N. \& Bonham-Carter, G. 1989. Computers assist prospecting. Geos 18, 8-15. 
Watson, G. S. 1964. Smooth regression analysis. Sankhyā: The Indian Jorunal of Statistics. Series A 26, 359-372.

Waugh, S. 1995. TasCas - A Cascade-Correlation Simulator. Department of Computer Science, University of Tasmania, Hobart. Report R95-9.

Weigend, A. S., Rummelhart, D. E. \& Huberman, B. A. 1991. Generalization by weightelimination with application to forecasting. In: Lippmann, R. P., Moody, J. E. \& TouretZKY, D. S., eds., Advances in Neural Information Processing Systems 3 (Denver, 1990). Morgan Kaufmann, San Mateo.

Wellman, P. \& Blewett, R. S. 1997. Mapping of granites using remotely sensed data. In: 14th Australian Geological Convention Abstracts: Geoscience for the New Millenium. Geological Society of Australia, volume 49, p. 463.

Werbos, P. 1974. Beyond regression; new tools for prediction and analysis in the behavioural sciences. PhD thesis, Applied Mathematics, Harvard University, Cambridge (unpubl.).

White, H. 1989. Learning in artificial neural networks: a statisitical perspective. Neural Computation 1, 425-464.

Widrow, B. \& Hoff, M. E. 1960. Adaptive switching circuits. In: IRE WESCON Convention Record. pp. 96-1-4. New York 4.

Widrow, B. \& Winter, R. 1988. Neural nets for adaptive filtering and adaptive pattern recogition. Computer 21, 25-39.

WiLKInson, G. G. 1996. A review of current issues in the integration of GIS and remote sensing data. International Journal of Geographic Information Systems 10, 85-101.

Wilkinson, G. G., Kanellopoulos, I., Kontoes \& MÉgier 1992. A comparison of neural network and expert system methods for analysis of remotely-sensed imagery. In: Proceedings of International Geoscience and Remote Sensing Symposium (IGARSS'92). IEEE Comp. Soc. Press, Los Alamitos, volume 1, pp. 62-64.

Winston, P. H. 1992. Artificial Intelligence. Addison-Wesley, Reading, 3rd edition.

WitT, W. K. 1993. Gold mineralization in the Menzies-Kambalda region, Eastern Goldfields, Western Australia. Technical report, Geological Survey of Western Australia. Report 39.

Wolf, K. H. 1994. Artificial intelligence (AI) and expert systems (ES). Ore Geology Reviews 9, $241-243$.

Wong, K. K., Fung, C. C. \& Eren, H. 1996a. A study of the use of self-organising map for splitting training and validation sets for backpropagation neural network. In: Proceedings of TENCON '96, IEEE Conference on Digital Signal Processing Applications. pp. 157 -162. IEEE, Piscataway, 1.

Wong, K. K., Fung, C. C. \& Eren, H. 1997a. Modifying the generalisation characteristics of a neural network with interactive reinforcement training. In: Proceedings of ICIPS'97: International Conference on Intelligent Processing System. pp. 472-476. IEEE, Piscataway, 1.

Wong, P. M., Gedeon, T. D., Huang, Y. \& Shibli, S. A. R. 1998. AI-based multi-dimensional spatial interpolators. Systems Research and Information Systems 8, 81-95.

Wong, P. M., Gedeon, T. D. \& Taggart, I. J. 1995a. An improved technique in porosity prediction: a neural network approach. IEEE Transactions on Geoscience and Remote Sensing 33, 971-980. 
Wong, P. M., Gedeon, T. D. \& Taggart, I. J. 1996b. Fuzzy ARTMAP: a new tool for Lithofacies Classification. AI Applications 10, 29-39.

Wong, P. M., Taggart, I. J. \& Gedeon, T. D. 1995b. Use of neural network methods to predict porosity and permiability of a petroleum reservoir. AI Applications 9, 27-37.

Wong, P. M., TAmhane, D. \& Wang, L. 1997b. A neural-network approach to knowledge-based well interpolation: a case study of a fluvial sandstone reservoir. Journal of Petroleum Geology 20, 363-372.

WoODALL, R. 1985. Limited Vision: a personal experience of mining geology and scientific mineral exploration. Australian Journal of Earth Sciences 32, 231-237.

WoOdAlL, R. 1994. Empiricism and concept in successful mineral exploration. Australian Journal of Earth Sciences 41, 1-10.

Worrall, L., Munday, T. \& Green, A. 1997. Airborne electromagnetics-mapping geology through cover. In: 14th Australian Geological Convention Abstracts: Geoscience for the New Millenium. Geological Society of Australia, volume 49, p. 478.

Wright, D. F. 1988. Data integration and geochemical evaluation of Meguma Terrane, Nova Scotia, for gold mineralization. MSc thesis, University of Ottawa, Ottawa (unpubl.).

Wright, D. F. \& Bonham-CARTER, G. F. 1996. VHMS favourability mapping with GISbased integration models, ChiselLake-Anderson Lake area. In: Bonham-Cartter, G. F., Galley, A. G. \& Hall, G. E. M., eds., Extech I: A multidisciplinary Approach to Massive Sulphide Research in Rusty Lake-Snow Lake Greenstone Belts, Manitoba, Geological Survey of Canada, volume 426 of Bulletin, pp. 339-376, 387-401.

Wu, X. \& Zhou, Y. 1993. Reserve estimation using neural network techniques. Computers and Geosciences 19, 567-575.

Wyborn, L., Gallagher, R. \& Jagodzinski, E. 1994a. A conceptual approach to metallogenic modelling using GIS: examples from the Pine Creek Inlier. In: Proceedings of a Symposium on Australian Research in Ore Genesis. Australian Mineral Foundation, Adelaide, South Australia, pp. 15.1-15.5.

Wyborn, L. A. I. 1990. BMR completes a pilot Geographic Information System, the Kakadu GIS. BMR Research Newsletter 2.

Wyborn, L. A. I., Bastrakova, I. V. \& Budd, A. R. 1997. Using GIS in a data-driven, minerals systems approach to assess the mineral potential of Australian Proterozoic granites. In: 14th Australian Geological Convention Abstracts: Geoscience for the New Millenium. Geological Society of Australia, volume 49, p. 480.

Wyborn, L. A. I., Gallager, R., Jaques, A. L., Jagodinski, E. A., Thost, D. \& Ahmad, M. 1994b. Developing metallogenic geographic information systems: examples from Mount Isa, Kakadu and Pine Creek. In: Australian Institute of Mining and Metallurgy Annual Conference. Australian Institute of Mining and Metallurgy, volume 5/94, pp. 129-133.

Wyborn, L. A. I. \& Gallagher, R. 1994. Regional analysis of the mineral potential of geological provinces. In: OZRI8 - The 8th Australian ESRI/ERDAS Users' Conference Proceedings. ??

Wyborn, L. A. I., Gallagher, R., Goleby, B. R. \& Kigour, B. 1996a. Metallogenic applications of 3D GIS in Mount Isa. In: MIC'96: New Developments in Metallogenic ResearchThe McArthur, Mt ISa, Cloncurry Minerals Province. pp. 153-156. James Cook University, Townsville. 
Wyborn, L. A. I., Gallagher, R. \& Raymond, O. 1995a. Creating mineral province GIS packages - issues, problems, solutions, and opportunities. In: Second National Forum on GIS in the Geosciences - Forum Proceedings. pp. 6-24. Australian Geological Survey Organisation Record 1995/46.

Wyborn, L. A. I., Gallagher, R. \& Raymond, O. 1995b. Using GIS for mineral potential evaluation in areas with few known mineral occurrences. In: Second National Forum on GIS in the Geosciences - Forum Proceedings. pp. 199-211. Australian Geological Survey Organisation Record 1995/46.

Wyborn, L. A. I., Hazell, M., Brooke, G. \& Gallagher, J. 1996b. Successful implementation of GIS in mineral exploration. In: unknown??

Wyborn, L. A. I., Heinrich, C. A. \& Jaques, A. L. 1994c. Australian Proterozoic mineral systems: essential ingredients and mappable criteria. In: Australian Institute of Mining and Metallurgy Annual Conference. pp. 109-115. Australian Institute of Mining and Metallurgy, Melbourne.

Wyborn, L. A. I., Jagodinsky, E. A. \& Ratajkoski, M. 1995c. The Pine Creek metallogenic GIS - turning 45 years of geoscientific paper products into a more accessible and applicable digital package. In: Proceedings of the 2nd North Australian Remote Sensing GIS Forum (NARGIS). Australasian Urban and Regional Information Systems Association Inc., pp. 128135. Monograph 11.

Wyborn, L. A. I., Jagodzinski, E., Morse, M., Wellman, P. \& Valenta, R. K. 1989. Integration of multiple geoscience data sets in the Coronation Hill region, NT, as an aid to delineating areas of potential mineralisation. In: Geoscience mapping towards the 21st century: 1989 BMR Research Symposium Papers. Bureau of Mineral Resources, Geology and Geophysics, pp. ?-? 7-9 November, 1989, Canberra.

Yamakawa, T. 1990. Pattern recognition hardware system employing a fuzzy neuron. In: Proceedings of the International Conference on Fuzzy Logic and Neural Networks. pp. 943-948. Iizuka, Japan.

Yatabe, S. M. \& FAbBri, A. G. 1988. Artificial intelligence in the geosciences: a review. In: Namyslowska, B. \& Royer, J. J., eds., Sciences de la Terre Part 1: Geomathematics and Geostatistics Analysis applied to Space and Time Dependent Data. pp. 37-67. Nancy, Sér. Inf. 27.

Yatabe, S. M. \& Fabbri, A. G. 1989. Putting AI to work in geoscience. Episodes 12, 10-16.

Yeats, C. J. \& McNaughton, N. J. 1997. Significance of SHRIMP II U-Pb geochronology on lode-gold deposits of the Yilgarn Craton. Australian Geological Survey Organisation. AGSO Record pp. 125-130. $1997 / 41$.

Young, C. 1997. Footwall alteration at the Sulphur Springs Zn-Cu volcanic-hosted massive sulphide (VHMS) prospect, east Pilbara, Western Australia. BSc (Hons) thesis, University of Western Australia, Perth (unpubl.).

Yudong, C. 1994. Application of artificial neural network on prediction of multimetallic deposits. In: Geological Society of America, 1994 Annual Meeting: Abstracts with Programs. pp. A-482. Geological Society of America, Boulder 26.

Yudong, C., Bin, Y. \& Hong, S. 1994. Discussion on mineral resource prognostication by self-organization artificial neural network. Hunan Geology 13, 53-56. 
Yun, G. J., Groves, D. I., Knox-Robinson, C. M. \& Gardoll, S. J. 1998. GIS-based methodology for prospectivity analysis of orogenic lode-gold deposits: a preliminary study of the Kalgoorlie terrane as an example. In: ZHou, Q., LI, H. \& SHI, W., eds., Spatial Information Technology Towards 2000 and Beyond; The Proceedings of Geoinformatics'98 Conference. Association of Chinese Professionals in GIS, Beijing, China, pp. 288-298.

Yun, G. Y. 1998. Craton-scale controls on mesothermal gold deposits: a global study within a GIS environment - an initial prospectivity analysis report. Technical report, Centre for Strategic Mineral Deposits, University of Western Australia.

Yun, Y. G. 2000. Controls on orogenic (mesothermal) gold deposits: a craton to province scale study within a GIS environment. Phd, University of Western Australia. Perth (unpubl.).

ZADEH, L. 1965. Fuzzy sets. Information and Control 8, 338-353.

ZADEH, L. A. 1973. Outline of a new approach to the analysis of complex systems and decision processes. IEEE Transactions on Systems, Man and Cybernetics 3, 28-44.

Zahlmann, G., Scherf, M., Wegner, A., Obermaier, M. \& Mertz, M. 2000. Situation assessment of glaucoma using a hybrid fuzzy neural network. IEEE Engineering in Medicine and Biology Magazine 1, 84-91.

ZAKNICH, A. 1995. Detection of sodium oxalate needles in optical images using neural networks. In: Proceedings of 1995 IEEE International Conference on Neural Networks. pp. 1699-1702. Perth 4.

ZAKNICH, A. 1997. Characterization of aluminium hydroxide particles fron the Bayer process using neural network and Bayesian classifiers. IEEE Transactions on Neural Networks 8, 919-931.

Zaknich, A. 1998. 620.450 - Artificial Neural Networks 450 Course(4 points). Department of Electrical and Electronic Engineering, University of Western Australia, Perth.

Zaknich, A. 1999. Artificial Neural Networks: a research dominated engineering discipline. Department of Electrical and Electronic Engineering, University of Western Australia, Perth (unpubl.).

Zaknich, A. \& Attikiouzel, Y. 1993. Automatic optimization of the modified probabilistic neural network for pattern recognition and time series analysis. In: Proceedings of the First Australian and New Zealand Conference on Intelligent Informations Systems. pp. 152-156. Perth.

Zaknich, A. \& Cornell, J. 1994. Application of neural networks to image analysis in the alumina industry. In: Image Analysis Seminar. Fremantle, pp. 27-37.

Zell, A., Mamier, G., Vogt, M., Mache, N., Hübner, R., Döring, S., Herrmann, K.-U., Soyez, T., Schmalzl, M., Sommer, T., Hatzigeorgiou, A., Posselt, D., Schreiner, T., Kett, B., Clemente, G. \& Wieland, J. 1995. SNNS Stuttgart Neural Network Simulator User Manual, Version 4.1. Institute for Parallel and distributed High Performance Systems (IPVR), University of Stuttgart, Stuttgart. Report No. 6/95.

ZhANG, D. \& Lutz, T. M. 1989. Structural control of igneous complexes and kimberlites: a new statistical method. Tectonophysics 159, 137-148.

Zhоu, Q. 1994. Principles of Geographic Information Systems. Course notes for Principles of Geographic Informations Systems GEOG9240, School of Geography, University of New South Wales, Sydney. 
Zhou, Q., Evans, A. \& Kumar, L. 1997. GIS advanced topics and applications. Course notes for Advanced Geographic Informations Systems GEOG9241, School of Geography, University of New South Wales.

Zimmermann, H.-J. 1984. Fuzzy Set Theory and its Applications. Kluwer Academic Publishing, Boston. 


\section{Appendix A}

\section{Additional Mineral Prospectivity Mapping Methods: Review of Theory and Literature}

This appendix contains two sections. The first part contains a review of the theory and literature describing mineral prospectivity methods in addition to those which are applied in this thesis. In the second part, a number of neural network architectures that represent alternatives to the multilayer perceptron and which are potentially useful for the estimation of mineral prospectivity are examined. These are the probabilistic (PNN), general regression (GRNN), radial basis function (RBF) and self-organizing feature map (SOFM) or Kohonen neural networks and a hybrid neuro-fuzzy system. These networks have been chosen because they have are been shown to be useful for a wide range of applications, and secondly these architectures span a range of neural network paradigms.

\section{A.1 Additional mineral prospectivity methods}

\section{A.1.1 Boolean Algebra}

Boolean algebra and index-overlay are the simplest procedures for combining geoscience data sets. In Boolean algebra, features in each layer are treated a binary variables. For raster data, if a feature is present, the cell value is set to one otherwise the cell value set to zero. Boolean AND and OR operators are then used to combine the different binary 
layers. The binary favourability value of a particular grid location is given by:

$$
S=\left\{\begin{array}{lll}
1 & : & \text { if }(\text { condition }) \text { TRUE } \\
0 & : & \text { otherwise }
\end{array}\right.
$$

where condition is a Boolean expression containing AND, OR and NOT operators and a binary variable representing the grid cell value for each thematic layer. Bonham-Carter (1994) has suggested the use of the Boolean OR operator in cases where favourable evidence for mineralization is rare.

The Boolean AND operator was used by Bates (1959) in a study to determine the factors controlling the location of uranium-vanadium deposits in the Colorado Plateau area of Colorado and Utah, USA. Bates (1959) selected predictor variables by testing the strength of the correlation between ranked values of candidate predictor variables and the ranked production of known deposits in tons.

George \& Bonham-Carter (1989) used the Boolean AND operator to combine layers containing principal components scores for biogeochemical data, buffered faults, and bedrock geology to predict the favourability for gold deposits in the Star Lake area of Saskatchewan, Canada. They argued that a simple approach using Boolean logic is appropriate when exploration is at an early stage and low numbers of known deposits preclude the use of statistical methods.

\section{A.1.2 Index-Overlay}

A disadvantage of the Boolean algebraic method is that all evidence maps are treated as being of equal importance. The index overlay method overcomes this problem by allowing a constant weight factor to be assigned to each map layer. For binary input maps the overall favourability index, $F$, for an individual grid cell is given by:

$$
F=\left(w_{1} x_{1}+w_{2} x_{2}+w_{3} x_{3}+\ldots+w_{N} x_{N}\right) / \sum_{i}^{N} w_{i}
$$

where $x_{i}$ is the binary value of the $i_{t h}$ predictor variable, $\mathrm{N}$ is the number of binary input maps and $w_{i}$ is the subjectively chosen weight assigned to the $i_{t h}$ map (Bonham-Carter, 1994). The features represented by binary variables could be simple ones such as the presence of a particular rock type or they could be represented by the logical union of 
more than one feature; e.g. anomalous $A u$ OR anomalous Ag. The score is normalized to the range $[0,1]$ by dividing the weighted sum (numerator) by the maximum possible score represented by the sum of the weights (denominator).

Koch et al. (1981) used an index overlay method to calculate scores for uranium prospectivity for two degree quadrangles in Colorado based on point located data and subjectively chosen weights and a mixture of binary and metric predictor variables.

Bonham-Carter (1994) described an index overlay method for multiclass maps in which weights are assigned to each class within each map in addition to the weights for each map type. the Index overlay method has also been used in a recent study by Carranza \& Hale (1999) to classify the nickeliferous-laterite potential of an area in the north-eastern part of the Phillipines.

The favourability index is given by equation A.3:

$$
F=\left(w_{1} c_{1 i}+w_{2} c_{2 i}+w_{3} c_{3 i}+\ldots+w_{N} c_{N i}\right) / \sum_{i}^{N} w_{i}
$$

where

$w_{i}=$ the weight for the $i^{\text {th }}$ map layer,

$c_{j i}=\quad$ is the weight for the $i^{t h}$ class of the $j^{t h}$ map

$\mathrm{N}=$ the number of map layers.

A modelling procedure similar to the one described above was employed by Reddy \& Koch (1988) to combine 13 geophysical, geological, and geochemical map layers to predict the favourability for silver deposits in the Butte, Dillon, Hamilton and Elk City two degree quadrangles in Idaho and Montana, USA. A variety of statistical procedures were used to select a set of control cells. The value of weighting factors for each class within a map were set equal to the fraction of control cells having that attribute value. Weights were assigned to classes within map layers but weights were not assigned to the map layers themselves.

Moore \& Gregory (1989) applied an index overlay method to produce a geological map for an area with poor outcrop near Halifax, Nova Scotia. They used a statistical measure of correlation between the input maps to define weighting factors for each class within each input map.

The advantage of the binary and index overlay methods lies in their simplicity. However, 
disadvantages of these methods include the following points.

- The methods are subjective. In the Boolean method the decision whether to use the $A N D$ or $O R$ operator and the choice of cutoff values used to convert multiclass input maps to binary format are subjective. The choice of weighting factors in both binary and multiclass index overlay methods is also subjective.

- Both Boolean and binary index overlay methods limit the information in the input maps to just the presence and absence of a feature.

- The methods are based on a linear additive model and therefore cannot model complex non-linear relationships. Also due their linear nature, the methods are likely to over-estimate the favourability index in cases where some input layers are correlated or contain redundant information.

\section{A.1.3 Multiple Linear Regression}

Multiple linear regression analysis is a statistical technique which is used to predict and explain the relationship between a single independent variable and several independent variables (also referred to as explanatory variables). The regression equation has the form:

$$
Y_{i}=b_{0}+b_{1} X_{1 i}+b_{2} X_{2 i}+\ldots+b_{n} X_{n i}
$$

where $Y_{i}$ is the predicted value of the dependent variable for the $i^{\text {th }}$ observation, $b_{0}$ is the Y-axis intercept, $b_{1} \ldots b_{n}$ are regression coefficients, and $X_{1} \ldots X_{n}$ are the independent (predictor) variables. Multiple regression analysis uses a mathematical procedure known as least squares to estimate the values of $b_{0} \ldots b_{n}$ such that the sum of the squared errors is minimized, that is:

$$
\sum_{i-1}^{n}\left(Y_{i}-\hat{Y}_{i}\right)^{2}
$$

where $Y_{i}$ and $\hat{Y}_{i}$ are the actual and predicted values of the $i_{t h}$ observation, respectively. The weights denote the relative contribution of the individual predictor variables to the overall result.

In the form of multiple regression shown in equation A.4, the independent and dependent 
variables can only represent interval or ratio data (rather than ordinal or categorical variables; Hair et al., 1998).

In order to build a multiple linear regression model, an approach known as stepwise regression is used. New variables are added to the model in steps and only retained if a test (partial F-test criterion) shows that the new variable significantly improves the model. At each stage, previously entered explanatory variables are re-tested to see if they are still significant. If not, they can be eliminated from the model. In this way a minimal model incorporating the most effective explanatory variables is produced.

Multiple linear regression was one of the earliest and most popular methods used in mineral-prospectivity mapping (e.g. Kelly \& Sherriff, 1969; Sinclair \& Woodsworth, 1970; De Geoffroy \& Wignall, 1970, 1971). For example, Sinclair \& Woodsworth (1970) used multiple regression to combine 12 geological data sets to produce separate contoured favourability maps of total ore tonnage, average ore grade, and total dollar value for an area near Terrace, British Columbia, Canada, in which $\mathrm{Cu}-\mathrm{Pb}-\mathrm{Zn}$-precious metal vein deposits occur. Independent variables included the percentage of a cell occupied by rocks belonging to a particular formation and distance from the centre of a cell to the nearest plutonic rock. A control area of contiguous cells covering the best explored part of the study area was used to develop the regression model. The resulting model was then used to provide estimates for all the cells in the area.

Assumptions required in order to apply multiple regression analysis are:

- linearity: the relationship between the dependent and independent variables is linear

- normality: the values of $\mathrm{Y}$ are normally distributed at each value of $\mathrm{X}$

- homoscedasticity: that the variation around the line of regression does not change over the range of $\mathrm{X}$ values

- that there is no significant correlation between predictor variables

A difficulty with the use of multiple regression in mineral prospectivity studies is that geological variables are often not normally distributed and few studies present scatter plots or residual plots to demonstrate that the normality assumption is satisfied. Where variables are not normally distributed, variables can be transformed so that the frequency distribution becomes as near-normal as possible. For example De Geoffroy \& Wignall (1971) used $\ln (Y)$ instead of $\mathrm{Y}$ for the dependent variable and $\ln (1+x)$ in place of $\mathrm{X}$ for all the independent variables. Sinclair \& Woodsworth (1970) used other transformations; for example $\arcsin (\sqrt{X / 100})$ in place of $\mathrm{X}$, the dependent variable representing the percentage of plutonic rocks. 
Multiple regression analysis cannot be used where the dependent variable is binary (e.g. deposit present or absent). To overcome this restriction, some researchers have used: Multiple regression analysis cannot be used where the dependent variable is binary (e.g. deposit present or absent). To overcome this restriction, some researchers have used:

- very large grid cells (in order to guarantee that every cell contains a deposit or at least a percentage of all the rock types in the study, or

- total metal value per cell (thus in some cases effectively grouping several mineralization types together).

Some examples include Sinclair \& Woodsworth (1970) who estimated grade, tonnage, and total dollars for $4 \times 4$ mile cells, Hodgson \& Troop (1988) who used $\log$ (Au oz./cell), Agterberg et al. (1972) used $\log (\mathrm{Cu}$ tonnage/cell $)$ for $10 \times 10 \mathrm{~km}$, and De Geoffroy \& Wignall (1971) who used the total ore value in $10 \times 10$ mile cells. Some examples include Sinclair \& Woodsworth (1970) who estimated grade, tonnage, and total dollars for $4 \times 4$ mile cells, Hodgson \& Troop (1988) who used $\log (\mathrm{Au}$ oz./cell), Agterberg et al. (1972) used $\log (\mathrm{Cu}$ tonnage/cell $)$ for $10 \times 10 \mathrm{~km}$, and De Geoffroy \& Wignall (1971) who used the total ore value in $10 \times 10$ mile cells.

\section{A.1.4 Multiple Discriminant Analysis}

Multiple Discriminant Analysis (MDA) is used when the dependent variable is categorical (as with logistic regression) and independent variables represent metric data. In contrast to logistic regression, MDA is capable of handling dependent variables with more than two classes. Similar to multiple regression, discrimination is achieved by using a linear combination of independent variables multiplied by weights. The analysis procedure is based on a sample of the data and involves an attempt to find the combination of independent variables and appropriate values of the weight for each variable that maximizes the variance between groups relative to the variance within groups. The discrimination function has the following form:

$$
Z_{j i}=a+w_{1} X_{1 i}+w_{2} X_{2 i}+\ldots+w_{n} X_{n i}
$$

where

$$
\begin{aligned}
& Z_{j i}=\text { discriminant } \mathrm{Z} \text { score for the } i^{\text {th }} \text { observation of the } j^{\text {th }} \text { predictor variable } \\
& a=\mathrm{Y} \text {-intercept }
\end{aligned}
$$


$w_{k}=$ discriminant weight for independent variable $k$

$x_{k i}=$ independent variable $\mathrm{k}$ for the $i^{\text {th }}$ observation

In order to classify observations belonging to two groups for example, the discriminant scores are calculated for each observation and a cut-off score (called the critical $Z$ score) is selected to divide the results into two groups. The approach can be extended to more than two groups.

Prelat (1977) applied MDA to VMS deposits in central Norway to produce a contoured map for gridded data showing the probability that the grid cell belongs to the group of cells containing VMS mineralization. Predictor variables were selected from a group of 36 geological and structural features, and aeromagnetic data. Prelat (1977) transformed the raw data to bring the distributions closer to normality using the following functions: $\sqrt{x+k}, \log (x+k)$, and $\arcsin (x+k)$, where $x$ is the value of the raw variable and $\mathrm{k}$ is a constant. Probabilities were calculated from discriminant $Z$ scores. The probability that a cell belongs to one of the two groups is a function of the squared Euclidean distance (in multidimensional input space) between the cell and the mean of the group being considered.

The discriminant analysis method is dependent on the following assumptions:

- multivariate normality of independent variables,

- equal variance and covariance for the classes, and

- absence of significant multicollinearity between variables (Prelat, 1977; Hair et al., 1998).

Ramsayer \& Bonham-Carter (1974) described an adaptive pattern recognition algorithm based on MDA to class sediment formations on the basis of binary input data on the presence or absence of fossils and other characteristics. De Geoffroy \& Wignall (1971) compared a form of MDA, which they refer to as Bayesian classification analysis, with multiple regression. The result of this MDA method is a raster-based map showing cells coloured according to 4 classes of probability of belonging to a group for which ore values exceeded $\$ 2 \mathrm{M}$ (Canadian). 


\section{A.1.5 Cluster Analysis}

Cluster Analysis is a multivariate technique that has been applied to problems of mineral prospectivity (Briggs \& Press, 1977; Nikolenko, 1980). The purpose of the technique is to group objects (e.g. grid cells in a GIS) according to the similarity of their characteristics. In the study by Briggs:1977, input variables are continuous and the output variable is binary (mineralized or barren). Unlike multiple regression and multiple discrimination analysis where the problem of which predictor variables to use is determined as part of the method, in cluster analysis, the researcher must specify which independent variables are to be used. Cluster analysis is very sensitive to the inclusion of irrelevant variables and to outliers (Hair et al., 1998).

An example of the use of cluster analysis is the study by Briggs \& Press (1977). They applied a simple form of cluster analysis to predict uranium-bearing areas on the Colorado Plateau, USA. Geological structural, and topographic data sets were converted to binary form. Variables (or features in GIS layers) were then selected to characterize mineralized areas on the basis of whether the features satisfy equation A.7:

$$
\mathrm{IF}\left(K_{\text {deposits }}<N_{\text {feature }} / N_{\text {cells }}\right) \text { AND }\left(N_{\text {feature }} / N_{\text {cells }}<K_{\text {barren }}\right) \text { THEN select feature }
$$

where $K_{\text {deposit }}$ and $K_{\text {barren }}$ are cutoff percentages, which are different for each data set, $N_{\text {feature }}$ is the number of cells in which the feature occurs and $N_{\text {cells }}$ is the number of cells. The same procedure was applied to barren areas within the control area. The classification criterion for a particular cell is given by equation A.8;

$$
\begin{gathered}
\text { IF }\left(N_{\text {deposit traits }}>N_{\text {barrentraits }}\right) \quad \text { THEN classification }=\text { Uranium-bearing } \\
\text { ELSE classification }=\text { barren }
\end{gathered}
$$

\section{A.1.6 Factor Analysis}

Characteristic analysis is a multivariate statistical technique that has been applied by Botbol (1971); Botbol et al. (1978); McCammon et al. (1983, 1986) to the identification of exploration targets. The method is similar to factor analysis. Agterberg (1989a) has described it as a simplified form of principal component analysis. A set of mineralized cells (together with some prospective non-mineralized cells to make the model more general) are 
selected to represent a particular type of deposit. The parameters in various raster-based input layers containing both these model cells and the regional-scale area to be explored are transformed to binary form.

Locations that are favourable for mineralization are assigned a value of 1 and other areas are assigned 0. Favourable areas were defined as anomalous areas for the purpose of the study of part of the Ag- $\mathrm{Pb}$ Coeur D'Alene district by Botbol et al. (1978). The choice of model cells and the selection of input layers requires subjective judgement and Botbol et al. (1978) notes that these choices have a critical effect on the output.

The favourability for a cell is calculated using Equation A.9:

$$
f=a_{1} x_{1}+a_{2} x_{2}+\ldots+a_{n} x_{i}
$$

where $a_{i}$ represents the weights for each of the $n$ binary (or ternary) variables $x_{i}$. Once the model cells and parameters have been chosen, the weights for each variable shown in Equation A.9 are determined by solving the matrix equation:

$$
\left(X^{\prime} X\right) \mathbf{a}=\lambda \mathbf{a}
$$

where $\lambda$ is the largest eigenvalue of $\left(X^{\prime} X\right)$ and $X$ is the $m \times n$ matrix of $n$ binary or ternary transformed parameter values for the $m$ selected model cells. The weights $a-i$ are scaled so that the values of the favourability function are in the range $[-1,+1]$. The favourability value is interpreted as a measure of similarity of the values in Equation A.9 for a particular cell to those selected to represent the deposit (i.e. the model cells).

In a further development of the method, ternary variables were introduced (where $1=$ favourable, 0 = unevaluated, and $-1=$ unfavourable) along with truth tables for combining the ternary variables with logical AND, OR, and NOT (McCammon et al., 1983, 1986). Kobayashi et al. (1994) has used this ternary form of characteristic analysis to estimate the potential for unconformity-type uranium deposits in the Alligator Rivers region of the Pine Creek Geosyncline, Northern Territory, Australia. 


\section{A.1.7 Expert Systems}

Expert systems are computer systems that attempt to automate the sort of tasks that normally require a human expert (Cawsey, 1998) ${ }^{1}$. Expert systems have often been applied to diagnostic tasks, which include medical diagnosis (e.g. the MYCIN system, Shortcliffe, 1976), fault-finding, configuring computer systems (e.g. McDermott, 1982), and mineral exploration (e.g. Duda et al., 1978).

The basic components of an expert system are a set of facts and some interpreter controlling the application of the rules (Cawsey, 1998). Facts are represented as a set of IF-THEN rules that lead to a conclusion that is true if the evidence is true. A simple rule can be represented as a single piece of evidence $E$ implying a single hypothesis $H$; i.e. IF evidence is true THEN hypothesis is true (Fig. A.1a). In more complex rules, the IF condition contains multiple pieces of evidence (Fig. A.1b).

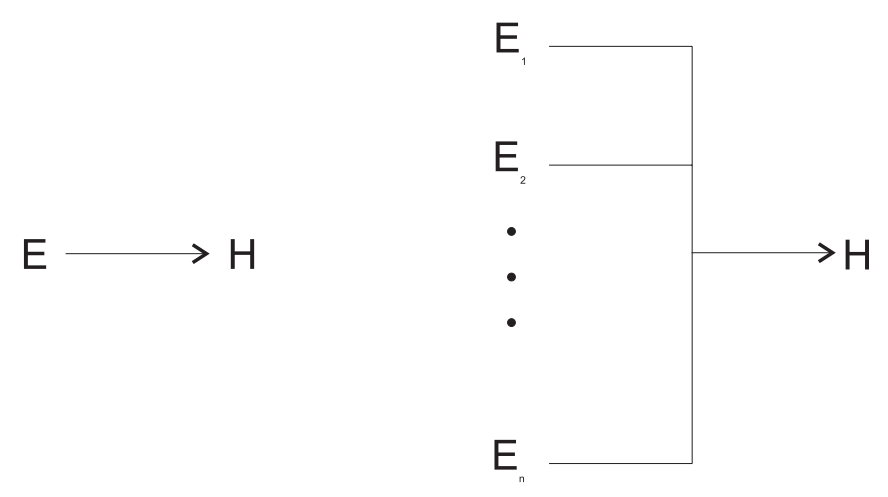

Figure A.1. Form of simple IF-THEN rules in an expert system; a) single piece of evidence implying a single hypothesis and b) multiple pieces of evidence.

The type of expert systems used in two important geological applications (Duda et al., 1978; Reddy et al., 1992) is a rule-based inference systems in which the main inference mechanism used is forward chaining. Forward chaining systems are primarily data-driven (Cawsey, 1998). Rules are organized into a hierarchical network in which the rules (statements about evidence and hypotheses) are linked as shown in Figure A.2. In a forward-

\footnotetext{
${ }^{1}$ Expert systems are also referred to as knowledge-based systems.
} 


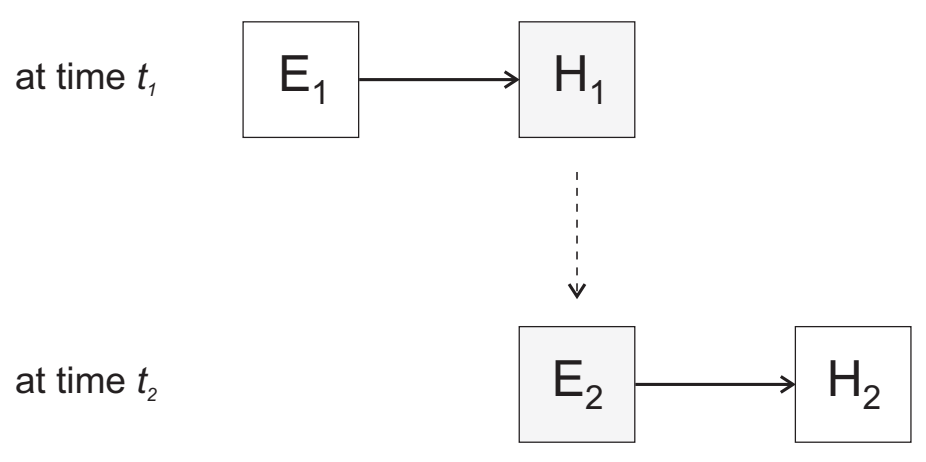

Figure A.2. Linking of rules in an expert system through forward chaining.

chaining system, rules are linked so that the hypothesis from one rule becomes the evidence for the next rule and so on. Rules are invoked under the control of the interpreter only when evidence contained in the IF part of the rule is available.

Using the example of Kuroko-type VMS deposits, the nodes represent items of field evidence and/or geological hypotheses. For example, $D_{1}=$ limonite casts near the intrusive contact, $C=$ sulphide mineralization, $\mathrm{B}=$ economic mineralization, and $\mathrm{A}=\mathrm{VMS}$ mineralization.

A key feature of expert systems used in mineral exploration is the ability to deal with uncertainty or incomplete evidence. A procedure called Bayesian updating is used. The prior probability of the geological statement at the end of the arrows in Figure A.2 is updated depending on whether field evidence is available. In a simple Bayesian system, the evidence is assumed to be either definitely present (i.e. true) or definitely absent (i.e. false).

The following discussion is based on a description of an expert system designed for mineral exploration by Stackhouse \& Boyd (1985). An odds-likelihood formulation of Bayes' rule is used to update the probabilities according to the type of evidence available. It is derived as follows. The prior probability, $P(H)$, of a hypothesis $H$ (e.g. the area represented by 
a grid cell is favourable for gold mineralization) can be updated if there is some evidence $E$ available using the odd-likelihood formulation of Bayes' rule:

$$
P(H \mid E)=\frac{P(E \mid H)}{P(E)} \cdot P(H)
$$

where

$$
\begin{array}{ll}
P(E \mid H)= & \text { the conditional probability of evidence } E \text { given } H \\
P(H \mid E)= & \text { the updated or posterior probability of } H \text { given } E
\end{array}
$$

An equivalent expression for the probability that $\mathrm{H}$ is not true given evidence $E$ is shown in equation A.12:

$$
P(\bar{H} \mid E)=\frac{P(E \mid \bar{H})}{P(E)} \cdot P(\bar{H})
$$

where $\bar{H}$ means "not $H$ ".

For example, the prior probability of a porphyry copper deposit, $\mathrm{P}(\mathrm{H})$, could be determined as the number of grid cells containing deposits divided by the total number of cells in the study area. This represents the probability that any cell selected at random contains a deposit. Evidence $E$ in the form of the presence of argillic alteration could be used to update this prior probability. $P(E)$ could be calculated from the number of cells in the study area in which argillic alteration is present, and $P(E \mid H)$ represents the number of cases where argillic alteration $(E)$ is present given the location corresponds to a deposit (hypothesis, $H$ ).

The odds, $O(H)$, of an event $H$ is equal to the ratio of the probability that the event occurs to the probability that the event does not occur and is given by:

$$
O(H)=\frac{P(H)}{1-P(H)}
$$

From this definition, it follows that:

$$
O(H \mid E)=\frac{P(H \mid E)}{P(\bar{H} \mid E)}
$$

$$
O(H)=\frac{P(H)}{P(\bar{H})}
$$


The odds-likelihood form of Bayes' rule is obtained by dividing equation A.11 by equation A.12 and using equations A.14 and equation A.15 to replace the probability ratios with odds:

$$
O(H \mid E)=\frac{P(E \mid H)}{P(E \mid \bar{H})} \times O(H)
$$

A similar expression is obtained for the case where the evidence is absent:

$$
O(H \mid \bar{E})=\frac{P(\bar{E} \mid H)}{P(\bar{E} \mid \bar{H})} \times O(H)
$$

If the evidence $E$ is definitely present, the strength of a rule is represented by the ratio $L S^{1}$. This is obtained by rearranging equation A.16:

$$
L S=\frac{P(E \mid H)}{P(E \mid \bar{H})}=\frac{O(H \mid E)}{O(H)}
$$

If the evidence $E$ is definitely absent, the rule strength is represented by the ratio $^{2} L N$ (obtained by rearranging equation A.17 as follows:

$$
L N=\frac{P(\bar{E} \mid H)}{P(\bar{E} \mid \bar{H})}=\frac{O(H \mid \bar{E})}{O(H)}
$$

Rearranging equations A.18 and A.19 gives the equations used of for updating probabilities:

$$
O(H \mid E)=L S \times O(H) \quad(\text { A.20) } O(H \mid \bar{E})=L N \times O(H)
$$

These formulas are only useful if there is just one item of evidence. $L S, L N$, and $P(H)$ (from which $O(H)$ is obtained) are estimated subjectively by an expert.

In order to extend this basic approach to updating prior probabilities to cases where two or more pieces of evidence are combined, the assumption that evidence is conditionally independent is made (Bonham-Carter, 1994). For $n$ items of evidence, Bayes rule (equation A.11) becomes:

\footnotetext{
${ }^{1}$ The ratio LS is known as the likelihood ratio for sufficiency or sufficiency ratio.

${ }^{2}$ The ratio LN is known as the likelihood ratio for necessity or necessity ratio.
} 


$$
P\left(H \mid E_{1} \cap E_{2} \cap \ldots \cap E_{n}\right)=\frac{p\left(E_{1} \cap E_{2} \cap \ldots E_{n} \mid H\right)}{P\left(E_{1} \cap E_{2} \cap \ldots \cap E_{n}\right)} \times P(H)
$$

Applying equation A.13 to convert the left-hand side and the term $P(H)$ to odds gives:

$$
O\left(H \mid E_{1} \cap E_{2} \cap \ldots \cap E_{n}\right)=\frac{p\left(E_{1} \cap E_{2} \cap \ldots E_{n} \mid H\right)}{p\left(E_{1} \cap E_{2} \cap \ldots E_{n} \mid \bar{H}\right)} \times O(H)
$$

If events $E_{1} \ldots E_{n}$ are assumed to be conditionally independent then:

$$
P\left(E_{1} \cap E_{2} \cap \ldots \cap E_{n} \mid H\right)=P\left(E_{1} \mid H\right) \times P\left(E_{2} \mid H\right) \times \ldots \times P\left(E_{n} \mid H\right)
$$

Substituting the right-hand of equation A.24 into the numerator of equation A.23 and using a similar expression for the denominator gives:

$$
\begin{aligned}
O\left(H \mid E_{1} \cap E_{2} \cap \ldots \cap E_{n}\right) & =\frac{P\left(E_{1} \mid H\right)}{P\left(E_{1} \mid \bar{H}\right)} \times \frac{P\left(E_{2} \mid H\right)}{P\left(E_{2} \mid \bar{H}\right)} \times \ldots \times \frac{P\left(E_{n} \mid H\right)}{P\left(E_{n} \mid \bar{H}\right)} \times O(H) \\
& =\prod_{i=1}^{N} L S_{i} \times O(H)
\end{aligned}
$$

Similarly for the case where all the evidence is absent:

$$
O\left(H \mid \bar{E}_{1} \cap \bar{E}_{2} \cap \ldots \cap \bar{E}_{n}\right)=\prod_{i=1}^{N} L N_{i} \times O(H)
$$

Equations A.25 and A.25 are only applicable if the evidence is definitely true (present) or definitely false (absent). The rule strengths, $L S$ and $L N$, and the prior probabilities (which are converted to odds using equation A.13 for use in the equations shown above) represent the facts that are stored in the expert system. These are estimated subjectively.

Expert systems constructed for mineral prospectivity by Duda et al. (1978), Reddy et al. (1992) and Stackhouse \& Boyd (1985) take the degree of certainty to which the user believes the evidence exists into account. The probability that an item of evidence exists (or is true) given a particular observation $O b$ concerning that evidence is denoted by $P(E \mid O b)$. In this case $L S_{i}$ and $L N_{i}$ are replaced by a quantities that Reddy et al. (1992) refer to as the effective likelihood ratios:

$$
L E=\frac{O\left(H \mid O b_{i}\right)}{O(H)} \quad(\mathrm{A} .27) \quad \overline{L E}=\frac{O\left(H \mid \bar{O} b_{i}\right)}{O(H)}
$$


Consequently, in the case of uncertainty about evidence, instead of equations A.25 and A.26, we have:

$$
\begin{aligned}
& O\left(H \mid O b_{1} \cap O b_{2} \cap \ldots \cap O b_{n}\right)=\prod_{i=1}^{N} L E_{i} \times O(H) \\
& O\left(H \mid \bar{O} b_{1} \cap \bar{O} b_{2} \cap \ldots \cap \bar{O} b_{n}\right)=\prod_{i=1}^{N} \bar{L} E_{i} \times O(H)
\end{aligned}
$$

For example, $P(E \mid O b)$ could indicate the probability that an argillic alteration zone exists given a degree of alteration or proportion of certain minerals. $P(E \mid O b)$ can vary between two extreme cases. The probabilities for the following three conditions can be calculated:

1. if the evidence $E$ is true then $P(E \mid O b)=1$ and $P(H \mid O b)=P(H \mid E)$,

2. if the evidence $E$ is false then $P(E \mid O b)=0$ and $P(H \mid O b)=P(H \mid \bar{E})$, and

3. if no observations are available concerning the evidence for a deposit, e.g. argillic alteration, then the probability of the evidence is just the prior probability $P(E)$ so that $P(E \mid O b)=P(E)$ and $P(H \mid O b)=P(H)$.

These conditions define the three end points of the segments in the piecewise linear function illustrated in in Figure A.3.

The values of these three points are defined in the following way. As noted previously prior probabilities of evidence $P(E)$ and deposits $P(H)$, and rule strengths (in equations A.18 \& A.19) are estimated by an expert based on statistics of deposit examples and stored in the system. The right-hand side of equations A.18 \& A.19 together with the prior probabilities yield the quantities $O\left(H \mid O b_{i}\right)$ and $O\left(H \mid \bar{O} b_{i}\right)$.

The user of the system then estimates $P(E \mid O b)$ subjectively based on some field observation. The value of $P(H \mid O b)$ is obtained by interpolating the piecewise linear function in Figure A.3 and then used to update the rule strengths $L E_{i}=O\left(H \mid O b_{i}\right) / O(H)$. Given the rule strengths and the prior probability, the posterior probability of a deposit can then be calculated using equation A.25.

$P(H \mid O b)$ can also be estimated using the following formula (Reddy et al., 1992): 


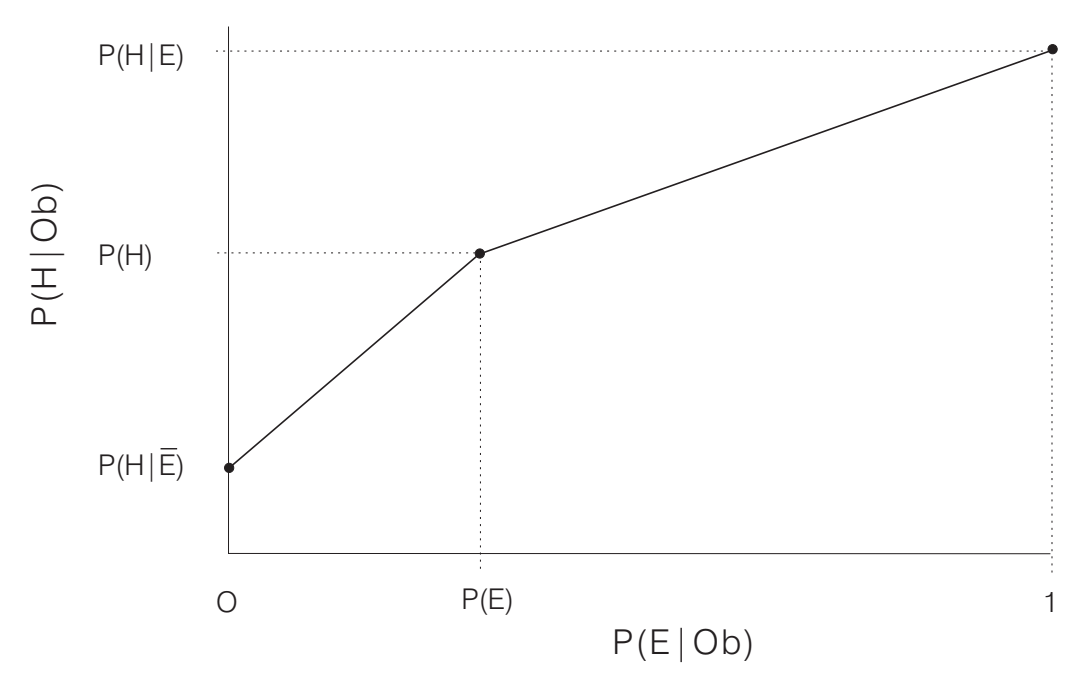

Figure A.3. Interpolation of a piecewise function in the PROSPECTOR expert (after (Stackhouse \& Boyd, 1985)). system

$$
P(H \mid O b)= \begin{cases}P(H \mid \bar{E})+\frac{P(H)-P(H \mid \bar{E})}{P(E)} P(E \mid O b) & \text { if } 0 \leq P(E \mid O b)<P(E) \\ P(H)+\frac{P(H \mid E)-P(H)}{1-P(E)}[P(E \mid O b-P(E)] & \text { if } P(E) \leq P(E \mid O b) \leq 1\end{cases}
$$

The system described by Stackhouse \& Boyd (1985) applies the following processing steps.

1. The system asks the user in an interactive session to provide an estimate of the certainty of the evidence; e.g. -5 indicates "definitely not", 0 indicates "don't know", and +5 indicates "definitely yes".

2. The system converts this rating to a probability $P(E \mid O b)$. In a map-based processing system, the value of $P(E \mid O b)$ would be stored as the grid cell values of a map.

3. The piecewise linear function (Fig. A.3) is interpolated to obtain an estimate of $P(H \mid O b)$

4. The the rule strengths (also referred to as the effective likelihood ratio; equations A.27 \& A.28 are calculated)

5. Multiple pieces of evidence are combined using equations A.29 and A.30.

Systems like PROSPECTOR (Duda et al., 1978), its GIS-based successor (Katz, 1991), POLOG (Stackhouse \& Boyd, 1985), and the MAPS system developed by Reddy et al. (1992) use fuzzy logic in addition to Bayesian updating to combine pieces of uncertain 
evidence. The following operators, min and $\max$ (analogous to the Boolean AND and $O R$ ) are used;

$$
\begin{aligned}
P(E \mid O b) & =\min _{i} P\left(E_{i} \mid O b\right) \\
P(E \mid O b) & =\max _{i} P\left(E_{i} \mid O b\right)
\end{aligned}
$$

Before attempting to evaluate the advantages and disadvantages of expert systems in mineral prospectivity analysis it is necessary to note that the type of expert system examined here is a rule-based system that uses mainly forward chaining as the inference mechanism similar to that used in many mineral prospectivity studies (e.g. PROSPECTOR, Duda et al., 1978).

Many geoscience applications of expert systems have been documented in the literature. Review articles include Fabbri \& Kasvand (1988) and Yatabe \& Fabbri (1988, 1989). Yatabe \& Fabbri $(1988,1989)$ examined 37 geological expert systems. More recent examples of the application of expert systems in the geosciences are given by Dimitrakopoulos et al. (1994)

The best known expert system that has been applied to mineral exploration is called PROSPECTOR. This is a rule-based system that mainly uses the forward chaining and the type of Bayesian updating described above as the inference mechanism (Duda et al., 1978)). Although its main mode of operation is non-spatial and involves matching field observations to ore deposit models, PROSPECTOR also has a map-based mode used for drill-site selection. In this latter mode, input in the form of digital geological, structural, geochemical and geophysical maps can be combined to produce a raster-based favourability map for porphyry system mineralization. Duda et al. (1978) has produced a favourability map for the Island Copper deposit in British Columbia, Canada. In later work, a favourability map was produced, that predicted extensions to the Mt Tolman porphyry molybdenum deposit. The extensions were later confirmed by drilling (Duda, 1980; Campbell et al., 1982). Katz (1991) adapted a public domain GIS package (MAPS) to emulate the map-based functionality of the PROSPECTOR system. In order to validate the new system, Katz combined 26 input maps consisting of geological, geophysical and geochemical data to duplicate the original Island Copper deposit analysis by Duda et al. (1978). Reddy et al. (1992) developed an expert system in conjunction with a commercial GIS and applied the system to a regional scale evaluation of the favourablilty for VMS deposits in the Snow Lake area, Manitoba. In this study, geological evidence (e.g. lithology, alteration and proximity to intrusive contacts) was converted to a series of binary input maps.

Although expert systems that have been discussed contain knowledge about the association 
of field evidence and geological hypothesis and deal with uncertainty, the systems are difficult and time-consuming to construct. A new deposit model and set of rules must be developed for each deposit type and this requires both a domain expert and someone skilled in eliciting and converting expert knowledge into a form that can be used by the system. There is also the assumption that the deposit models correctly represent all deposits both known and unknown (Reddy et al., 1992). Despite the formalism of the Bayesian updating and inference networks, the prior probabilities and likelihood ratios, which form the basis of all predictions, are subjectively estimated (Duda et al., 1978; Duda, 1980). In addition, in systems like PROSPECTOR, the user is required to subjectively estimate the certainty of the evidence. Finally, all the systems use Bayesian updating as the main inference method require the assumption of conditional independence between the evidence maps in order to combine multiple items of evidence for a single hypothesis (Agterberg, 1989a).

\section{A.1.8 Logistic Regression}

Logistic regression is a type of multivariate analysis that is used to predict a dependent variable that can take only one of two values (i.e. a binary variable). It is a similar technique to multiple regression and also performs a similar function to two-group discriminant analysis but differs in that it directly predicts the probability of an event occurring and assumes a non-linear relationship between the independent variables and the dependent variable. The regression equation for the logistic model has the following form:

$$
\ln [\operatorname{Odds}(D)]=b_{0}+b_{1}+b_{1} X_{1 i}+b_{2} X_{2 i}+\ldots+b_{n} X_{n i}
$$

where $b_{0}$ to $b_{n}$ are coefficients whose values are determined by using a set of control data for which both the dependent and independent variables are known, $\mathrm{n}$ is the number of independent variables, $X_{j i}$ is the value of the $i^{\text {th }}$ observation of the $j^{\text {th }}$ predictor variable, and $O(D)$ is the odds of event $\mathrm{D}$. The relationship between the probability and odds of an event is given by equation A.13:

The odds is equivalent to the ratio of the probability that an event will occur to the probability that the event will not occur. Event $D$ could represent the occurrence of a deposit in a grid cell for which data for many variables are known.

A mathematical procedure known as the maximum likelihood procedure is used in an iterative manner to determine the coefficients $b_{0}$ to $b_{n}$. Once the coefficients have been determined, the equation can be used to predict the values of the log of the odds ratio, 
known as the logit, which corresponds to the left-hand side of equation A.34. The logit can be converted to a probability estimate in two steps. First the estimated odds ration is determined using equation A.35:

$$
O(D)]=e^{\ln [O(D)]}
$$

In the second step, the odds ratio is calculated to probability using equation A.36:

$$
P(D)=\frac{O(D)}{1+O(D)}
$$

Logistic regression was first applied to the analysis of mineral prospectivity by Agterberg (1974) who compared the use of multiple linear and logistic regression to predict the probability of occurrence of Archaean Ni-Cu sulphide and $\mathrm{Cu}-\mathrm{Zn}$ deposits. The percentages of 8 rock types and combinations of rock types in $10 \times 10 \mathrm{~km}$ cells were used as the predictor variables (giving a total of 37 variables). The dependent variable was a categorical variable with binary values representing the presence and absence of a deposit in a cell. Agterberg (1974) attempted to overcome the problem of the control area not being sufficiently representative of the study area by using deposit cells together with the 8 neighbouring cells for 87 deposits over a very large area $\left(850000 \mathrm{~km}^{2}\right)$ as the control cells to develop the logistic model. The model was then used to estimate the probability of deposit occurrence in a much smaller area $\left(254000 \mathrm{~km}^{2}\right)$. Agterberg (1974) concluded that the logistic model produces better results than multiple regression if there are few deposit occurrences

Chung \& Agterberg (1980) also used logistic regression and the proportion of 5 rock types to produce a map depicting the probability of occurrence of VMS deposits in part of Newfoundland, Canada. Three classes of probability were displayed as a grey-scale image.

In another study, Chung (1983) used an interactive graphic system to analyze multivariate geoscience data called SIMSAG. They compared logistic regression and multiple linear regression methods for prospectivity mapping. The logistic model was based on 16 independent variables representing geology, lake sediment geochemistry, airborne magnetic and radiometric survey data, gravity and topography. The result of the analysis was a contoured probability map for uranium occurrences in the Kasmere Lake area, Manitoba, Canada.

The advantage of logistic regression compared with multiple regression is that the method is less affected when assumptions that independent variables follow a normal distribution 


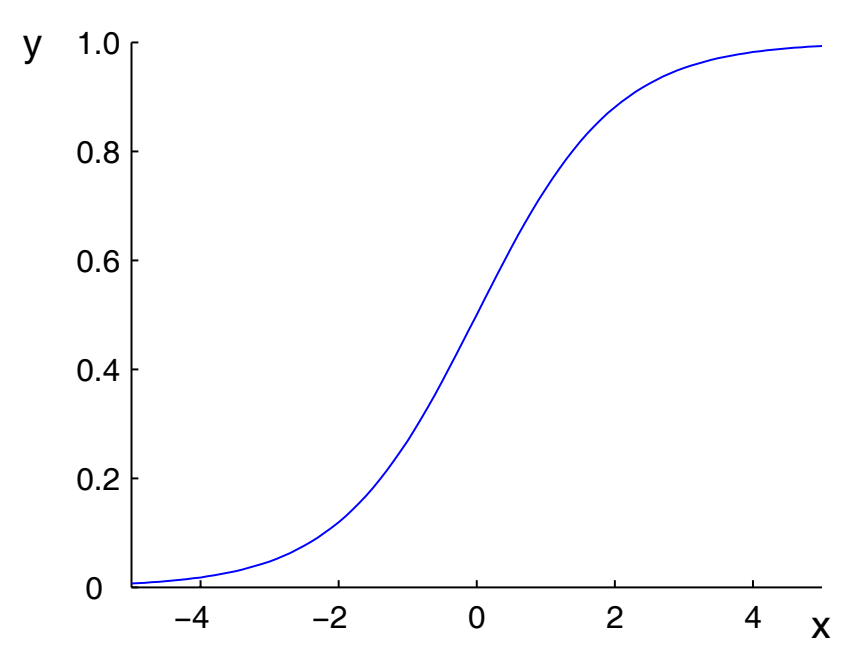

Figure A.4. Form of the logistic function showing the relationship between the dependent and independent variables. The dependent variable shown on the $y$-axis represents the probability of an event. This curve was produced using the function, $f(x)=1 /\left(1+e^{-x}\right)$.

and that there is no correlation between independent variables (Hair et al., 1998).

Agterberg (1974) and Chung \& Agterberg (1980) have noted that the logistic regression method gives better results than multiple linear regression in situations where there are few known deposits. The logistic model has been used by Agterberg et al. (1981) in preference to the Poisson model when events consisting of one or more deposits have been defined to avoid local clustering effects.

Use of the logistic model assumes a very specific type of relationship. Whereas the multiple linear regression model assumes the relationship between the dependent and independent variable is linear so that the dependent variable can be predicted by a linear combination of predictor variables, the logistic regression assumes the relationship between the dependent and independent variables is a logistic one of the form shown in Figure A.4. The latter relationship is shown in equation A.37:

$$
P(D)=\frac{e^{b_{0}+b_{1}+b_{1} X_{1 i}+b_{2} X_{2 i}+\ldots+b_{n} X_{n i}}}{1+e^{b_{0}+b_{1}+b_{1} X_{1 i}+b_{2} X_{2 i}+\ldots+b_{n} X_{n i}}}
$$

\section{A.1.9 Dempster-Shafer Evidential Belief Theory}

Dempster-Shafer evidential belief theory is the basis of a method of probabilistic reasoning that involves subjective judgement about the credibility of evidence that is used to support a proposition. One of the reasons for interest in the method as a means of integrating spatial geoscience data sets is that it deals with uncertainty in evidence explicitly and 
allows an estimate to be made for each probability estimate.

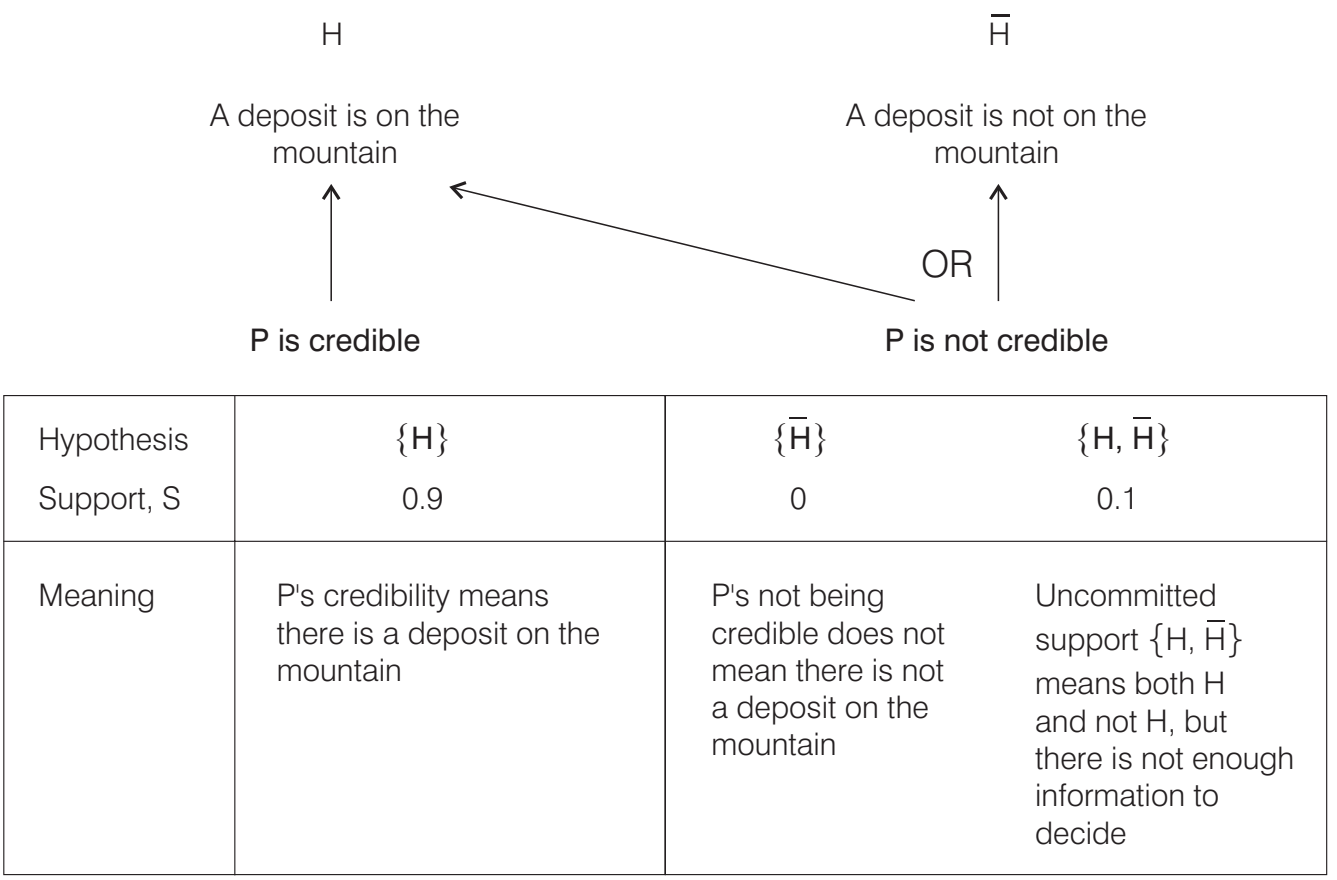

Figure A.5. An example illustrating partial belief in evidence and support

The key difference between ordinary probability and Shafer's theory can be illustrated by the following example adapted from Schum (1994). Let us suppose a geologist, P, reports that there is a deposit at the top of a mountain (referred to as hypothesis, $\mathrm{H}$, in Fig. A.5), without any further evidence, we might judge that there is a $90 \%$ chance that $\mathrm{P}$ is credible and a $10 \%$ chance that $\mathrm{P}$ isn't. This means that the evidence together with what is believed about $\mathrm{P}$ supports $\mathrm{H}$ to degree 0.9, i.e. evidential support is 0.9 .

There are three main rules in Shafer's scheme for allocating evidential support. These are:

- $\operatorname{support}(\mathrm{S})$ is assigned a number such that $0 \leq S \leq 1$,

- the values of S always sum to 1.0 across the power set. The power set represents the collection of all possible subsets of the set of hypotheses. For example, in Figure A.5 above, the power sets is $\{\{H\},\{\bar{H}\},\{H, \bar{H}\}, \emptyset\}$

- for the empty set $\emptyset, S=0$

Despite the fact that these rules look similar to those for ordinary probability, there is a major difference in regard to how the remaining 0.1 evidential support in Figure A.5 is 
interpreted and what is implied if $\mathrm{P}$ is not credible. In the Bayesian model, it is assumed that there is a Boolean phenomena, which either does or does not exist; i.e. the only possibilities are, $H$, (a deposit exists) and $\bar{H}$, (a deposit does not exist). Consequently, if $\mathrm{P}$ is not credible then $H$ is not true so $\bar{H}$ is true and the remaining 0.1 of support must be assigned to hypothesis, $\bar{H}$.

Evidential belief theory differs from ordinary probability by allowing for uncertainty in the following way. If geologist $\mathrm{P}$ is not credible, it does not mean there is no deposit. Although $\mathrm{P}$ being credible is compatible with $H, \mathrm{P}$ not being credible is compatible with both $H$ and $\bar{H}$ and so 0.1 of evidential support remains uncommitted; i.e. on the basis of the present evidence, it might go to $H$ or to $\bar{H}$.

Similarly, while $P(H)=0$ in ordinary probability means that event $H$ is impossible, evidential support $S\{H\}=0$ means that the evidence available is incompatible with $H$. Conversely, $S\left\{H_{1}, H_{2}, H_{3}\right\}=1.0$ means the evidence could mean $H 1, H 2$, or $H 3$ are true but not in any discernible proportions among these.

\section{Concept of the Belief Functions}

Central to evidential belief theory is the possibility of allocating evidential support to combinations of hypothesis. For example, for multiple hypotheses, $H_{1}, H_{2}, H_{3}$, where $H_{1}$ = epithermal gold deposit, $H_{2}=$ porphyry copper deposit, and $H_{3}=$ no deposit. The collection of possible combinations or subsets (known as the power set) is $\left\{H_{1}\right\},\left\{H_{2}\right\}$, $\left\{H_{3}\right\},\left\{H_{1}, H_{2}\right\},\left\{H_{1} H_{3}\right\},\left\{H_{2}, H_{3}\right\},\left\{H_{1}, H_{2}, H_{3}\right\}$ and empty set $\emptyset$. Table A.1 shows a set of example values used in the following discussion of evidential belief theory concepts from Schum (1994). In this example, $\left\{H_{1} H_{2}\right\}=0.1$ means that evidential support for the hypothesis that there is either an epithermal gold or porphyry copper deposit is equal to 0.1. If $S\left\{H_{1}\right\}=0.5$ and $S\left\{H_{2}\right\}=0.2$ then the support committed to the idea "that there is an epithermal deposit" also supports $\left\{H_{1}, H_{2}\right\}$; i.e. there is some kind of deposit. The same applies to $S\left\{H_{2}\right\}$.

The combination of two different sets of evidence is performed using Dempster's rule. Applying the rule gives a new basic probability number which is equivalent to degree of support for the hypothesis provided by the combined evidence. This discussion follows Schum (1994) in referring to the support, $S$, provided by evidence. Instead of $S$, Shafer (1976) uses the term, basic probability assignment (bpa). S(C) is the combined evidential support (or bpa) for two independent bodies of evidence, 1 and 2 for hypothesis $C$ in some frame of discernment. (The frame of discernment is the set of hypotheses being 
Table A.1. Values of variables used to illustrate evidential belief theory concepts for the power set of hypothesis in $F=\left\{H_{1}, H_{2}, H_{3}\right\}$ after Schum (1994). The belief functions $S\{\}, \operatorname{Bel}\{\}, \operatorname{Dou}\{\}$, $P l\{\}$ are explained in the text.

\begin{tabular}{|c|c|c|c|c|c|c|c|c|}
\hline \multirow{2}{*}{ Belief } & \multicolumn{7}{|c|}{ Power set of hypotheses in $F=\left\{H_{1}, H_{2}, H_{3}\right\}$} \\
\cline { 2 - 9 } Function & $\left\{H_{1}\right\}$ & $\left\{H_{2}\right\}$ & $\left\{H_{3}\right\}$ & $\left\{H_{1} H_{2}\right\}$ & $\left\{H_{1} H_{3}\right\}$ & $\left\{H_{2} H_{3}\right\}$ & $\left\{H_{1} H_{2} H_{3}\right\}$ & $\emptyset$ \\
\hline$S\{\}$ & 0.5 & 0.2 & 0.0 & 0.1 & 0.0 & 0.1 & 0.1 & 0.0 \\
Bel \{\} & 0.5 & 0.2 & 0.0 & 0.8 & 0.5 & 0.3 & 1.0 & 0.0 \\
Dou \{\} & 0.3 & 0.5 & 0.8 & 0.0 & 0.2 & 0.5 & 0.0 & 1.0 \\
$P l\{\}$ & 0.7 & 0.5 & 0.2 & 1.0 & 0.8 & 0.5 & 1.0 & 0.0 \\
\hline
\end{tabular}

considered.) Focal elements are subsets in a frame of discernment that have non-zero support. Dempster's rule is given by:

$$
S\{C\}=\frac{\sum_{A_{i} \cap B_{j}=C} S_{1}\left(A_{i}\right) S_{2}\left(B_{j}\right)}{1-\sum_{A_{i} \cap B_{j}=\emptyset} S_{1}\left(A_{i}\right) S_{2}\left(B_{j}\right)}
$$

where the numerator is the product of all the focal element pairs with intersections equal to subset $\mathrm{C}$, the denominator represents all focal element intersections, which equal an empty set, $S_{1}$ and $S_{2}$ refer to the support assignments made for items of evidence 1 and 2 and $A_{i}$ and $B_{j}$ refer to hypotheses. The meaning of equation A.38 as it applies to the combination of two gridded geoscience data sets used to estimate prospectivity is illustrated in Figure A.6 below. The frame of discernment is $F=D, \bar{D}$, where the two hypotheses are $D$ : the grid cell contains mineralization and $\bar{D}$ : the grid cell is barren. Evidence, $e_{1}$ could represent airborne magnetic data in GIS layer 1 and $e_{2}$ could represent solid geology in GIS layer 2.

Belief denoted BelA, for any group of hypotheses indicates the total amount of evidential support committed to A or any hypotheses that are subsets of A. In the example discussed above, if $A=\left\{H_{1}, H_{2}\right\}$ and nonempty subsets of $A$ are $\left\{H_{1}\right\},\left\{H_{2}\right\}$ and $\left\{H_{1}, H_{2}\right\}$, then:

$$
\operatorname{Bel}\left\{H_{1}, H_{2}\right\}=S\left\{H_{1}\right\}+S\left\{H_{2}\right\}+S\left\{H_{1}, H_{2}\right\}=0.5+0.2+0.1=0.8
$$

This indicates that the belief that the evidence means one of the hypotheses in $\left\{H_{1}, H_{2}\right\}$ is true. For $H_{1}$ there are no subsets so $\operatorname{Bel}\left\{H_{1}\right\}=S\left\{H_{1}\right\}$. 


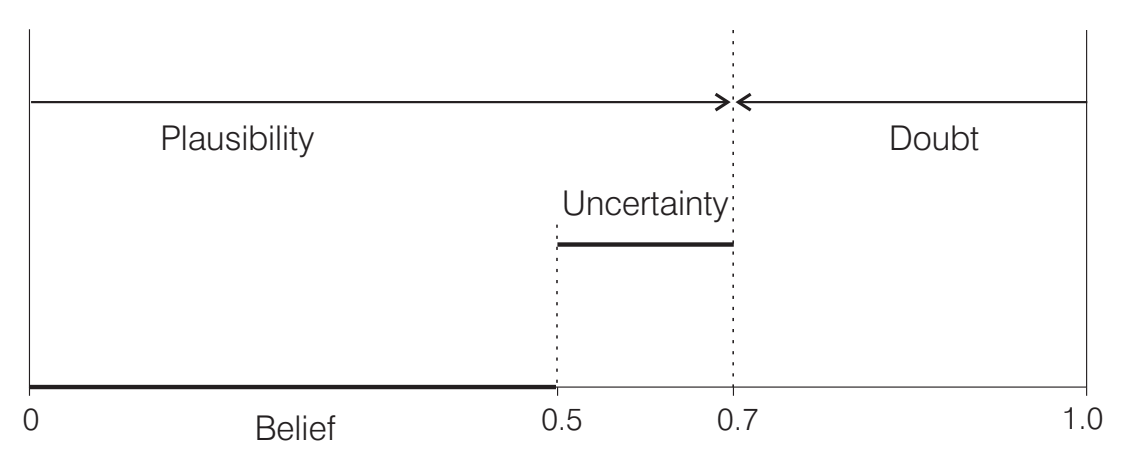

Figure A.6. An illustration of the relationship between belief, uncertainty, doubt and plausibility in Dempster-Shafer belief theory (modified from Flack, 1995).

Doubt that the evidence means one of the hypotheses in $A$ is true is defined by equations A.40 and A.41:

$$
\operatorname{Dou}\{A\}=\operatorname{Bel}\{\bar{A}\}
$$

where $A=\left\{H_{1}\right\}$ and $\bar{A}$ represents hypotheses incompatible with $A$; i.e. $\bar{A}=\left\{H_{2}, H_{3}\right\}$ :

$$
\operatorname{Dou}\left\{H_{1}\right\}=\operatorname{Bel}\left\{H_{2}, H_{3}\right\}=S\left\{H_{2}\right\}+S\left\{H_{3}\right\}+S\left\{H_{2}, H_{3}\right\}=0.3
$$

Plausibility of an hypothesis $A$ is the total amount of support that is, or might possibly be, committed to $A$ given by:

$$
P l\left\{H_{1}\right\}=S\left\{H_{1}\right\}+S\left\{H_{1}, H_{2}\right\}+S\left\{H_{1}, H_{3}\right\}+S\left\{H_{1}, H_{2}, H_{3}\right\}
$$

The concept is that for hypothesis $H_{1}$, each of the subsets $\left\{H_{1}, H_{2}\right\},\left\{H_{2}, H_{3}\right\},\left\{H_{1}, H_{2}, H_{3}\right\}$ may contain uncommitted support specifically for $H_{1}$ that could, if additional evidence were available, be assigned to $H_{1}$. So in an extreme case all of the uncommitted support assigned to sets in which $H_{1}$ appears could be allocated to $H_{1}$. The total is the upper probability or plausibility of $\left\{H_{1}\right\}$.

Uncertainty indicates how uncertain we are about the evidence and is represented by the interval, $[\operatorname{Bel}\{A\}, P l\{A\}]=[0.5,0.7]$.

These quantities are represented graphically in Figure A.6 (Flack, 1995). In the cross tabulation of hypotheses in Figure A.7, the elements represent the intersections of the sets shown at the left and bottom margins of the table. Note that the intersection of $D$ and $\bar{D}$ is an empty set $\emptyset$.

$S_{1}(D)$ refers to the degree of support from the value at a particular grid cell in the data 


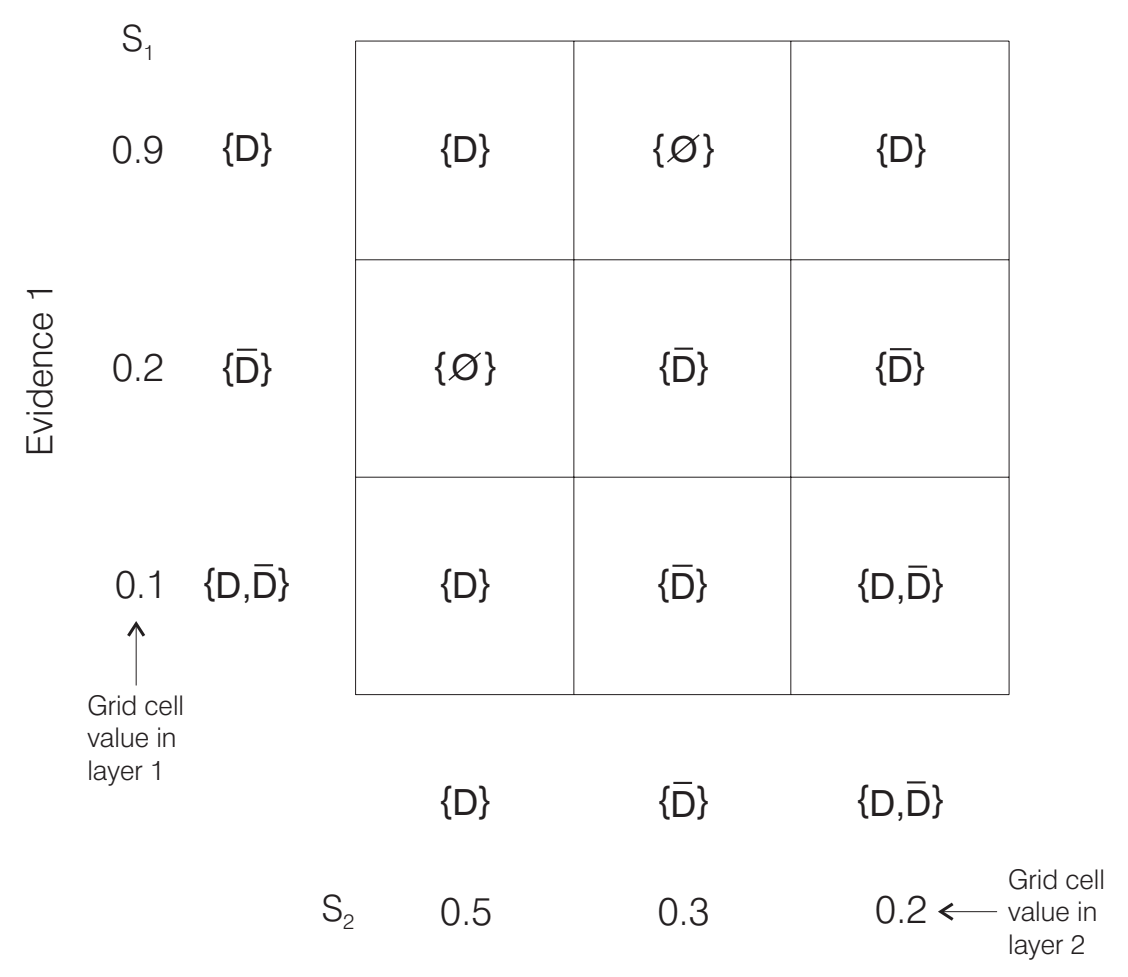

Evidence 2

Figure A.7. Values of support used to illustrate Dempster's rule (after Schum, 1994).

set 1 from the proposition; "deposit in grid cell" and $S_{2}(\bar{D})$ refers to support assignments based on the cell values for data set 2 .

Pairs of support assignments (shown in the column labelled $S_{1}$ and in the row labelled $S_{2}$ ) corresponding to the hypothesis $D$ are multiplied and summed to give the numerator in equation A.38. Pairs for which the intersection corresponds to the empty set $\emptyset$ are used to determine the denominator. Applying Dempster's rule to the example shown in Table above gives:

$$
S\left(D \mid e_{1}, e_{2}\right)=\frac{S_{1}\left(D \mid e_{1}\right) S_{2}\left(D \mid e_{2}\right)+S_{1}\left(D \mid e_{1}\right) S_{2}\left(D, \bar{D} \mid e_{2}\right)+S_{2}\left(D \mid e_{2}\right) S_{1}\left(D, \bar{D} \mid e_{1}\right)}{1-\left[S_{1}\left(D \mid e_{1}\right) S\left(\bar{D} \mid e_{2}\right)+S\left(\bar{D} \mid e_{2}\right)+S\left(\bar{D} \mid e_{1}\right) S\left(D \mid e_{2}\right)\right]}
$$

where $S\left(D \mid e_{1}, e_{2}\right)$ is the support assignment for hypothesis $D$ on the basis of combined evidence, $e_{1}, e_{2}$.

In an application of Dempster-Shafer evidential belief theory to combining data sets for mineral exploration, Moon \& So (1995) simplified the equation for the support assignment for an hypothesis (equation A.43). The sum of the support assignment over the frame of 
discernment must equal 1.0, that is:

$$
S\left(D \mid e_{1}\right)+S\left(\bar{D} \mid e_{1}\right)+S\left(D, \bar{D} \mid e_{1}\right)=1
$$

Re-arranging equation A.44, gives the following expression for $S\left(D, \bar{D} \mid e_{1}\right)$ :

$$
S\left(D, \bar{D} \mid e_{1}\right)=1-S\left(D \mid e_{1}\right)-S\left(\bar{D} \mid e_{1}\right)
$$

Moon \& So (1995) further simplified Equation A.43 by making the following substitutions:

$$
\begin{aligned}
a & =S\left(D \mid e_{1}\right) \\
a^{\prime} & =S\left(\bar{D} \mid e_{1}\right) \\
b & =S\left(D \mid e_{2}\right) \\
b^{\prime} & =S\left(\bar{D} \mid e_{2}\right)
\end{aligned}
$$

and from A.45:

$$
\begin{aligned}
1-a-a^{\prime} & =S\left(D, \bar{D} \mid e_{1}\right) \\
1-b-b^{\prime} & =S\left(D, \bar{D} \mid e_{2}\right)
\end{aligned}
$$

The resultant simplified form of equation A.43 is given by:

$$
S\left(D \mid e_{1}, e_{2}\right)=\frac{a b+a\left(1-b-b^{\prime}\right)+b\left(1-a-a^{\prime}\right)}{1-a b^{\prime}-a^{\prime} b}
$$

Since we are dealing with a hypothesis that has no subsets; i.e. $D$, the belief function value is the same as the support assignment:

$$
\operatorname{Bel}\left(D \mid e_{1}, e_{2}\right)=\frac{a b+a\left(1-b-b^{\prime}\right)+b\left(1-a-a^{\prime}\right)}{1-a b^{\prime}-a^{\prime} b}
$$

The uncertainty, $U(A)=P L(A)-\operatorname{Bel}(A)$, is calculated by determining the plausibility. The plausibility is defined as:

$$
\operatorname{Pl}(A)=1-\operatorname{Bel}(\bar{A})
$$


where $\bar{A}$ is the subset of hypotheses incompatible with $A$. In this case, the plausibility is calculated with equation A.51:

$$
P l\left(D \mid e_{1}, e_{2}\right)=1-\frac{a^{\prime} b^{\prime}+a^{\prime}\left(1-b^{\prime}-b\right)+b^{\prime}\left(1-a^{\prime}-a\right)}{1-a^{\prime} b-a b^{\prime}}
$$

where quantities on the right hand side are defined in equation A.48. To combine $n$ data sets, Dempster's rule must be applied $n-1$ times. Each time the result of the previous combination becomes one of two input data sets that is combined in the next step.

The benefits of the Dempster-Shafer approach are

- that it offers an approach to handling uncertainty that is similar to the way people think about uncertainty

- it allows a map to be produced that shows the degree of uncertainty about how the evidence supports the proposition; "area represented in the cell is favourable for mineralization".

- the method handles missing data well; an estimate of favourability can be made as long as the cell is covered by at least one data set.

There are some problems with the Dempster-Shafer method. It is unclear what effect a the lack of independence of some of the sources of evidence has on the results. Shafer states that the pieces of evidence to be combined with Dempster's rule must be distinct bodies of evidence (Shafer, 1976) and the multiplication of focal terms in the numerator in equation A.43 is based on the product rule for independent events used in ordinary probability. However, it is common for geoscience data sets to be correlated with one another (Bonham-Carter, 1994; Singer \& Kouda, 1999a).

An et al. (1992) suggest that evidential independence depends on the frame of discernment that is chosen. They cite an example involving two airborne magnetic surveys. They interpreted the surveys to be evidentially dependent if the surveys are used to prove a hypothesis that a deposit is located at a location but evidentially independent if the surveys are used to prove the hypothesis that a magnetic anomaly exists at the location. The problem here is that, although many intermediate hypotheses are used complex inference networks in expert systems, in most mineral prospectivity studies there is generally just one relevant hypothesis namely; "mineralization is present" so according to An et al. (1992), all the layers would be evidentially dependent. 
There are several further potential problems in the application of the Dempster-Shafer approach. Moon (1990) highlights the need to assign the degrees of support to values in the various evidence map layers according to the relative importance of the data layers. The support assignment or basic probability assignments are subjective and unless a logical framework exists for the assignment of the values such as an expert system, then it may be difficult for different geologist to produce similar results.

Evidential belief theory has been applied to the classification of satellite imagery (e.g. Lee et al., 1987; Peddle \& Franklin, 1992; Peddle, 1995). Lee et al. (1987) compared Bayesian and Dempster-Shafer approaches to the integration of multiple data sources and found that both methods produced similar results. A Dempster-Shafer approach has also been applied to the integration of geoscience data sets in mineral prospectivity studies. Moon (1990) subjectively assigned degrees of support to classes within airborne magnetic, EM, Ground EM, and solid geology maps to produce support (i.e. favourability), plausibility, and uncertainty maps for iron and base metal deposits in the Farley Lake area of Manitoba, Canada.

In a later study of the same area, An et al. (1992) produced a rule-based expert system that combined Boolean AND and OR operators with the Dempster-Shafer method. In this way, they combined 12 data sets to produce favourability and uncertainty maps for basemetal deposits. Belief functions were also used to quantify the uncertainty associated with each rule. An \& Moon (1993) applied an expert system incorporating a Dempster-Shafer method to base-metal exploration in the Snow Lake area. Seven geology and geophysical map layers were combined to produce favourability and uncertainty maps.

The Dempster-Shafer method is applied in the PLAYMAKER expert system which was designed to assist geologists estimate the potential of new prospects in a hydrocarbon field (Cheong et al., 1993).

\section{A.1.10 Decision Trees}

A decision tree is a method in the field of artificial intelligence that is used to perform classification tasks. A classification task is one where things are assigned to an enumerable set of possible categories of classes based on a set of properties or input features (Quinlan, 1993). For example, in medical diagnosis, the input features are patient symptoms and the output categories are possible diseases. A set of example classifications (both positive and negative) are required. Decision tree systems represent a type of machine learning because the model is constructed inductively by generalizing from specific examples (Cawsey, 
1998). This is known in machine learning terminology as supervised learning.

Decision-tree induction systems try to construct the simplest tree that correctly classifies all the training data. A decision tree consists of leaves which represent output classes and nodes at which the input features are used in questions designed to split the examples into groups. Figure A.8 shows an example of a decision tree for determining whether someone coming to a doctor with chest pains has had a heart attack Cawsey (1998). The diagnosis is performed by answering the yes/no questions posed by the system.

The system selects the features that are the best predictors of the outcomes and places these at the root of the tree. The tests are constructed in order to split the examples into as homogeneous groups as possible. The best split is one that reduces the overall misclassification of the examples. Once the tree is constructed using training data, it can be used to classify new cases. In the inference system C4.5 developed by Quinlan (1993), the tree is converted to a set of rules. The rules have the form:

$$
L \longrightarrow R
$$

where the left-hand side consists of a set of attribute tests joined by Boolean AND operators and the right-hand side is a class that the objects belong to. The system examines the list of rules and applies the first one for which the left-hand side condition is true. The classification is set to the class on the right-hand side.

Decision trees have been used in geoscience applications by Bui et al. $(1997,1998)$ and Reddy \& Bonham-Carter (1991b). Bui et al. (1998) used features based on lithology, digital elevation, terrain attributes (slope \& aspect) and LANDSAT MSS (range, mean and variance of each band) as inputs to C5.0 (a decision tree classification system) to predict the soil types in the upper Darling Basin area, NSW. Correct classification rates of $76 \%$ and $83 \%$ for two different training data sets were achieved. Reddy \& BonhamCarter (1991b) used a decision-tree system to classify the potential for VMS mineralization in the Snow Lake area, Manitoba, Canada using lithology, alteration type and intensity, and proximity to lithological contacts.

The most important limitation of induction systems like C4.5 is that they require that classes are sharply defined. Clearly, in a mineral prospectivity application, classes like "favourable host rock for mineralization present" and "favourable host rock for mineralization absent" are gross simplifications which ignore the range between the extremes. An end-member of one class may be very similar to extreme members of an other class yet they are treated as being different. 


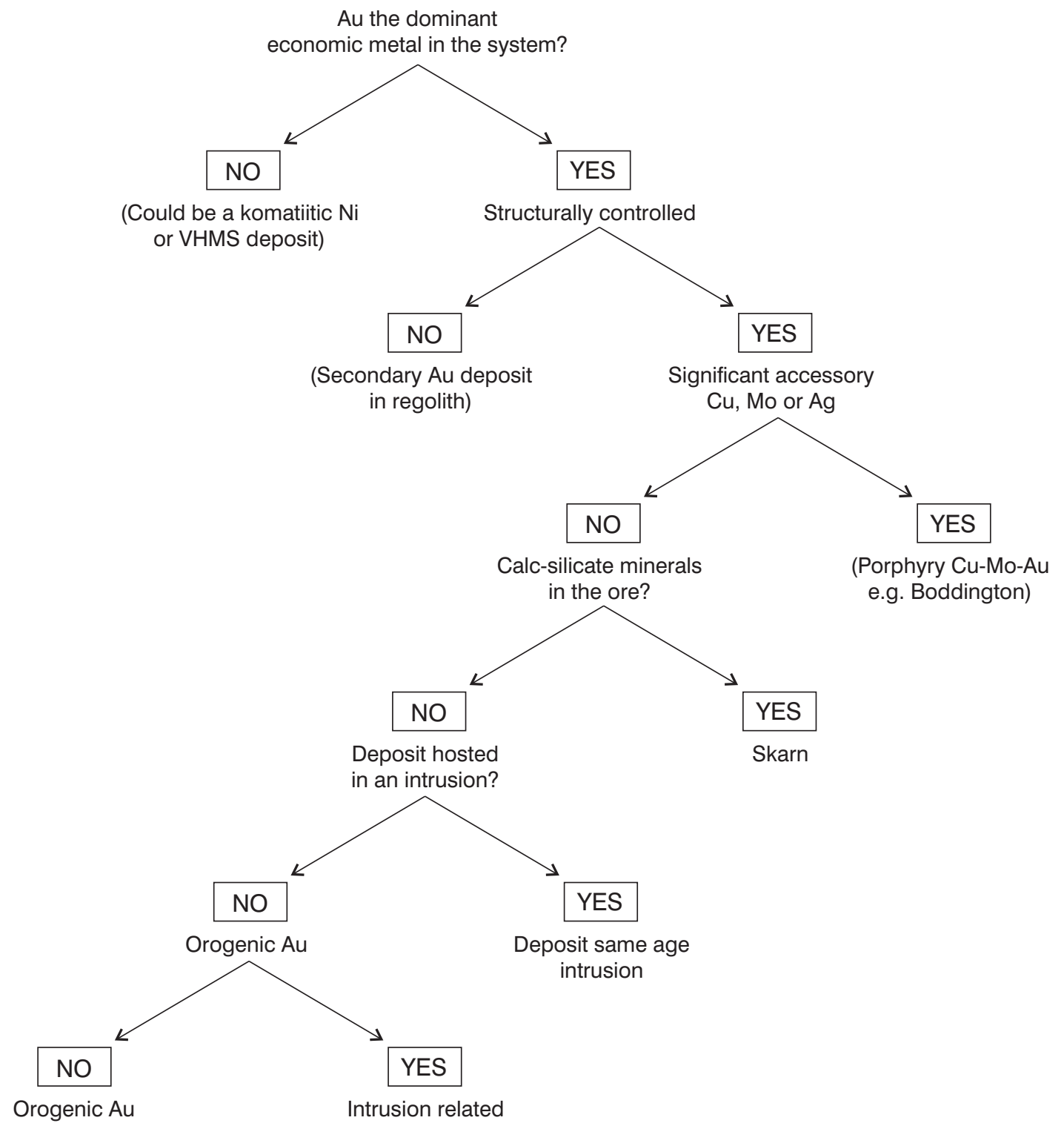

Figure A.8. Illustration of a decision tree (after Cawsey, 1998) 
The prediction of continuous values is not possible in C4.5 and tasks in which a continuousvalued class is broken up into vague categories (as is done in fuzzy logic) are problematical (Quinlan, 1993).

Quinlan (1993) has noted that decision-tree systems do not perform well when;

- irrelevant attributes exist,

- the data are noisy ,

- concept complexity is high (equated with fragmented regions in feature space),

- the classification task is probabilistic, and

- the number of training examples close to some of the decision boundaries in hyperdimensional feature space are low.

The first and second points suggest that decision-tree systems are quite sensitive to noise. Walker \& Moore (1988c) observed that noisy training data caused the CART decision-tree system to introduce unnecessary nodes and sub-trees to account for the data.

Decision-tree systems appear to require very large training sets. For example, Bui et al. (1998) reported that in order to achieve good precision for soil classification maps, 20-25\% of the cells in 1:250000 maps and 35\% of cells in 1:100000 maps were used as training data.

\section{A.1.11 Canonical Correlation}

Canonical Correlation is a multivariate statistical technique that is similar to multiple regression in that the general form of the model is linear. Whereas multiple regression predicts a single dependent variable from a set of multiple independent variables, canonical correlation simultaneously predicts multiple dependent variables (Hair et al., 1998). The form of the canonical correlation equation is:

$$
b_{1} Y_{1}+b_{2} Y_{2}+b_{3} Y_{3}+\ldots+b_{n} Y_{n}=a_{1} X_{1}+a_{2} X_{2}+a_{3} X_{3}+\ldots+a_{n} X_{n}
$$

$X$ and $Y$ can be either metric or categorical data type. The left-hand and right-hand sides of equation A.53 are linear composites of variables called canonical variates. The result of the analysis is to find the set of $a$ and $b$ that maximize the correlation between the linear composites (Sharma, 1995)

Pan \& Harris (1992) used the canonical correlation method to produce a favourability map 
for epithermal Au-Ag vein deposits in the Walker Lake quadrangle of Nevada and California. For the purposes of the study, Pan \& Harris (1992) distinguished between explanatory and target variables. Explanatory variables are those that can be measured throughout the study area and only provide indirect evidence for mineralization; e.g. airborne magnetic anomalies. Target variables provide direct evidence of mineralization and are likely to be available in only very well explored areas; e.g. mineral occurrences or gold anomalies in stream sediment geochemical samples. In the canonical correlation method is capable of predicting more than a single dependent or target variable. In addition to favourability for a deposit, Pan \& Harris (1992) also used hydrothermal alteration, Tertiary intrusives and $\mathrm{Au}-\mathrm{Ag}$ occurrences as dependent variables (i.e. $Y_{1}, Y_{2}$, and $Y_{3}$ on the left-hand side of equation A.53 above). The independent or explanatory variables consisted of a set of eight geological, structural, geophysical and geochemical variables.

The method requires a well explored training area where the target and explanatory variables ( $Y$ and $X$, respectively) are known in order to develop the model. Once the values of $a_{1} \ldots a_{n}$ and $b_{1} \ldots b_{n}$ were established, the favourability was determined using the right-hand side of equation A.53 as:

$$
\hat{F}=a_{1} X_{1}+a_{2} X_{2}+a_{3} X_{3}+\ldots+a_{8} X_{8}
$$

Pan \& Harris (1992) argue that defining favourability in terms of multiple target variables avoids ambiguities in the meaning attached to favourability and helps to capture important genetic ingredients of the ore formation process in the model. Yet the information captured by their use of Tertiary intrusives as a target variable (to define the presence of a heat source) could also be achieved by making it one of the explanatory variables and using a standard multiple linear regression model.

The major assumption underlying canonical correlation is that the relationships between independent and dependent variables (e.g. equation A.53) are assumed to be linear. Deviations from linearity can sometimes be corrected by transforming one of the variables, however if after the transformation the assumption is not satisfied then the relationship can not be modelled by canonical correlation (Hair et al., 1998). 


\section{A.2 Additional Neural Network Architectures}

\section{A.2.1 Probabilistic Neural Networks}

The probabilistic neural network (PNN) is a three layer multilayer feedforward network, which is based on a statistical algorithm developed by Meisel (1972). Specht (1990) reformulated the algorithm as a neural network. MLP networks rely on heuristic approaches to determine the network topology (i.e. the number of hidden nodes) and optimal values of system parameters (e.g. learning rate and momentum). MLP networks also require long training times and training may lead to a local rather than a global minimum. In contrast, the PNN directly implements Bayes' decision strategy and is guaranteed to approach an optimal solution (Bayes' optimal decision surface) as the number of training samples increase (Masters, 1993b; Zaknich, 1999).

PNNs are best suited to pattern classification problems. The following summary of the theory of PNNs is drawn from Masters (1993b), Bishop (1995), Fausett (1994), and Zaknich (1999). In pattern classification, patterns are represented by a feature vector; $\mathbf{x}^{n}=\left(x_{1}, x_{2}, x_{3}, \ldots, x_{n}\right)$ and training vectors are used to estimate the probability of an unknown vector belonging to a particular class.

The optimum decision rule is the one which minimizes the probability of misclassification. This can be achieved if the unknown feature vector $\mathbf{x}$ is assigned to the class $C_{k}$ for which the posterior probability $P\left(C_{k} \mid \mathbf{x}\right)$ is the largest. This decision strategy is referred to as Bayes' decision rule. According to this rule vectors belong to a class $C_{k}$ if:

$$
P\left(C_{i} \mid \mathbf{x}\right)>P\left(C_{j} \mid \mathbf{x}\right) \text { for all } i=1,2,3 \ldots, N \text { and } i \neq j
$$

The cost associated with misclassifying vectors from the different classes is assumed in equation A.55 to be equal. Bayes' theorem can be used to restate equation A.55 as;

$$
\begin{aligned}
P\left(C_{i} \mid \mathbf{x}\right) & =\frac{p\left(\mathbf{x} \mid C_{i}\right) P\left(C_{i} \mid \mathbf{x}\right)}{p(\mathbf{x})} \\
\text { where } P(\mathbf{x}) & =\sum_{j=1}^{N}\left(P \mathbf{x} \mid C_{j}\right) P\left(c_{j}\right) \text { for all } i=1,2,3 \ldots, N \text { and } i \neq j
\end{aligned}
$$

Substituting the right hand side of equation A.56 and a similar expression for class $j$ and discarding the term $p(\mathbf{x})$ which is common to both sides in equation A.55 gives: 


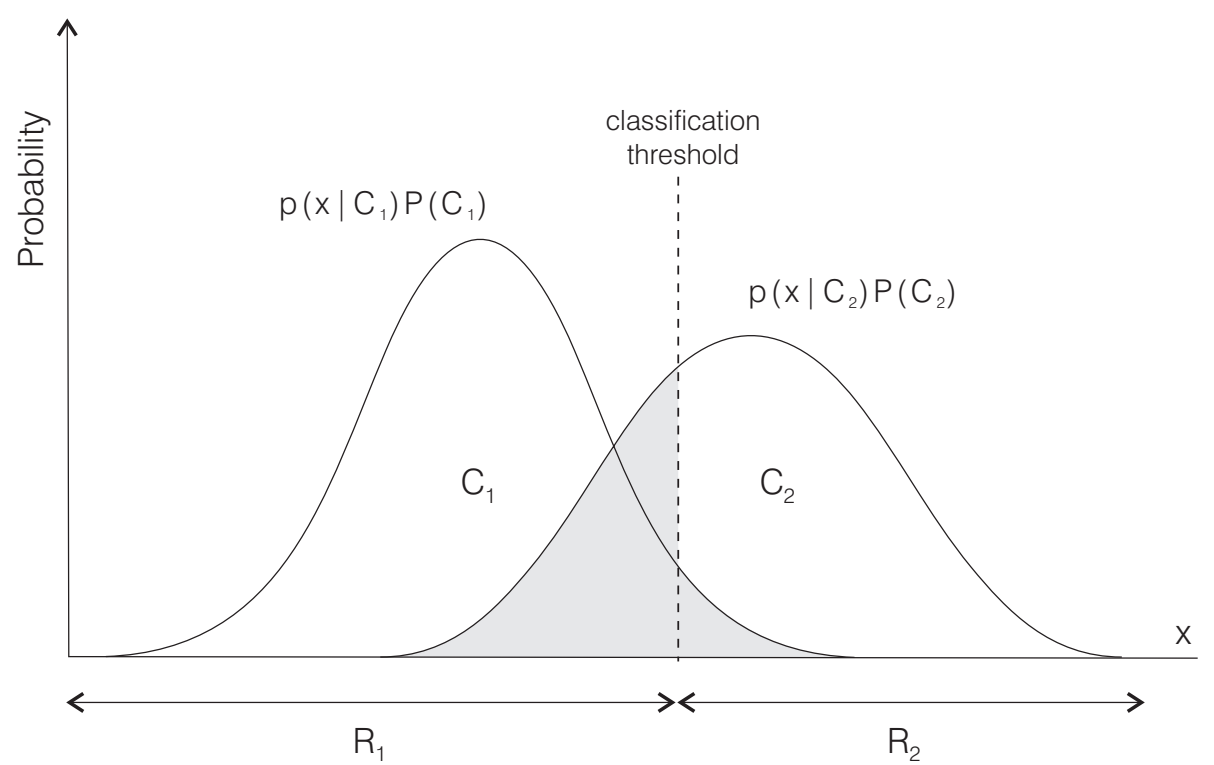

Figure A.9. Example of Bayes' decision rule classification (after Bishop, 1995).

$$
P\left(C_{i}\right) P\left(\mathbf{x} \mid C_{i}\right)>p\left(\mathbf{x} \mid C_{j}\right) P\left(C_{j}\right) \text { for all } i=1,2,3 \ldots, N \text { and } i \neq j
$$

where

$$
\begin{aligned}
& P\left(C_{i}\right)=\text { the prior probability that an unknown vector belongs to class } C_{i} \\
& p\left(\mathbf{x} \mid C_{i}\right)=\text { the class conditional probability density function that an unknown } \\
& \text { vector is } \mathrm{x} \text { given that it belongs to class } C_{i} \\
& P\left(C_{i} \mid \mathbf{x}\right)=\text { the posterior conditional probability that an unknown vector } \\
& \text { belongs to class } C_{i} \text { given that the vector is } \mathbf{x} \\
& p(\mathbf{x})=\text { the probability density that a vector is } \mathbf{x} \text { irrespective of its class } \\
& \text { membership }
\end{aligned}
$$

The decision rule is illustrated with a simple case shown in Figure A.9. (after Bishop (1995)). The probability of misclassification is minimized if the decision boundary between the two regions $R_{1}$ and $R_{2}$ representing classes $C_{1}$ and $C_{2}$ is located where the two probability density curves cross. Consequently, the unknown pattern for which the value of feature $x=a$ is assigned to the class with the highest posterior probability $(\mathrm{g}(\mathrm{x})=$ $\left.p\left(x \mid C_{k}\right) P\left(C_{k}\right)\right)$. In Figure A.9, $g_{2}(x)>g_{1}(x)$ so the vector is assigned to class 2.

The Bayes' optimal decision rule (equation A.57 above) is often written as (Zaknich, 1999):

$$
h_{i} f_{i}(\mathbf{x})>h_{j} f_{j}(\mathbf{x}) \text { where } i \neq j
$$

where 


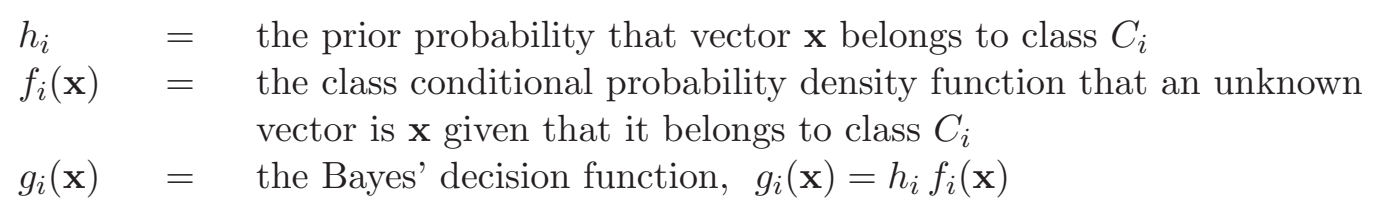

and where the cost of misclassification is assumed to be equal for all classes and is therefore discarded because it does not contribute to the decision.

In order to apply equation A.58, both the prior probability $h_{i}$ and the class probability density function $f_{i}(\mathbf{x})$ must be estimated. The probability density function or PDF is estimated from the training sample using a method developed for univariate data by Parzen (1962) and later extended by Cacoullos (1966) to multivariate data.

The Parzen PDF estimator uses the sum of small multivariate spherical Gaussian basis functions centred on each training sample vector (Zaknich, 1999). Masters (1995) has illustrated the concept in terms of a classification problem shown in Figure A.10 below. Figure A.10 shows an unknown sample (symbol "?") representing a vector with just two components which must be classified as either belonging to population $X$ or $O$. A nearestneighbour method would assign class $O$ to the unknown sample. The method based on the Parzen PDF estimator assigns a sphere of influence to each training sample. The function representing the sphere of influence has its largest value at zero distance and diminishes to very small values for large distances. The method involves finding the sum of this function for all training set members of each population. The class of the unknown sample is then set to the class having the largest sum (i.e. greatest combined influence).

The Parzen PDF estimator used to implement the PNN is given by Zaknich (1999) as:

$$
f_{i}(\mathbf{x})=\frac{1}{(2 \pi)^{p / 2} \sigma^{p} M_{i}} \sum_{j=1}^{M_{i}} \exp \frac{\frac{-\left\|\mathbf{x}-\mathbf{x}_{\mathbf{i}} \mathbf{j}\right\|^{2}}{2 \sigma^{2}}}{{ }^{2}}
$$

where

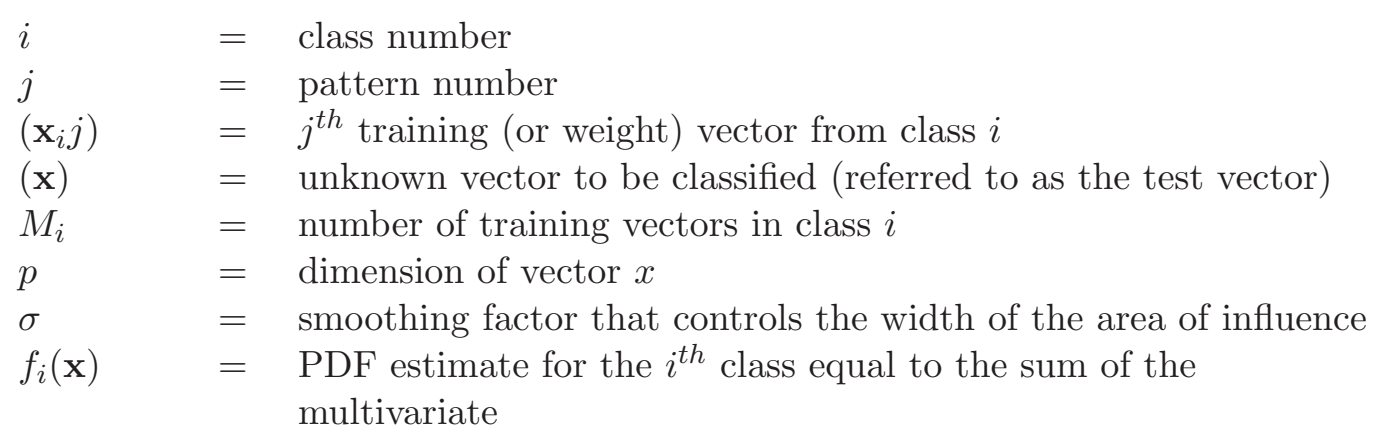




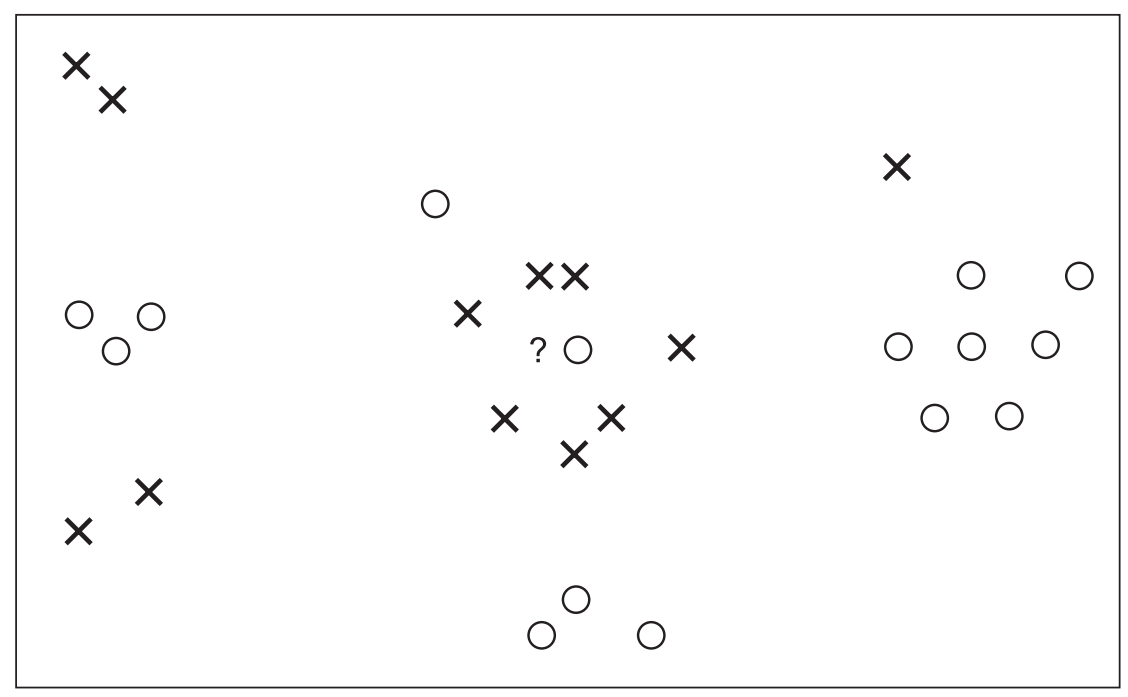

Figure A.10. An example of a classification problem involving two classes; shown as $X$ or $O$ (after Masters, 1993b). The feature vectors have two components and can therefore be plotted in a plane. The unknown sample to be classified is shown as "?"). A nearest-neighbour method would assign class $O$ to the unknown sample. In contrast, the Parzen PDF estimator which assigns a sphere of influence to each training sample, would assign the unknown sample to class " $\mathrm{X}$ ".

$$
\begin{aligned}
& \text { spherical Gaussian distribution centred at each of the training } \\
& \text { vectors } \mathbf{x}_{i j} \\
& \text { the length of the vector representing the difference between the } \\
& \text { unknown vector } \mathbf{x} \text { and the training vector } \mathbf{x}_{i j} j . \text { This is equivalent } \\
& \text { to the squared Euclidean distance measure. }
\end{aligned}
$$

The value of the PDF function $f_{i}(\mathbf{x})$ must be evaluated separately for each class. In equation A.59, the function actually evaluates the average of the multivariate Gaussian (normal) distributions for all the training vectors belonging to the same class. The computation of equation A.59 is sometimes simplified in the following ways (Zaknich, 1999; Masters, 1993b):

- the factor $1 / M_{i}$ is eliminated when $g_{i}(\mathbf{x})=h_{i} f_{i}(\mathbf{x})$ is calculated because it is assumed that $h_{i} \propto M_{i}$ i.e. the proportion of vectors of class $i$ in the training set $M_{i}$ is equal to the prior probability of class $i$ vectors in the population. Note that this assumption cannot hold for mineral prospectivity applications where the two classes of vectors represent deposit and non-deposit cells. For example, in training neural networks it is standard practice to set the numbers of training examples in each class to be approximately equal (this was done in the pilot study in Chapter 4) but the ratio of non-deposit to deposit vectors in actual data population is likely to be 
more than 1000 to 1.

- if vectors $\mathbf{x}$ and $\mathbf{x}_{i j}$ are normalized to unit length then the expression $\frac{-\left\|\mathbf{x}-\mathbf{x}_{i j}\right\|^{2}}{2 \sigma^{2}}$ reduces to $\left(Z_{i j}-1\right) / \sigma^{2}$ where $Z_{i j}=\mathbf{x} \cdot \mathbf{x}_{\mathbf{i j}}$

- the factor $\frac{1}{\left.(2 \pi)^{p / 2} \sigma^{p}\right)}$ is the same for all classes and is therefore discarded because it does not contribute to the classification.

Thus $g_{i}(\mathbf{x})=h_{i} f_{i}(\mathbf{x})$ is reduced to:

$$
g_{i}(\mathbf{x})=\sum_{j=1}^{M_{i}} \exp \frac{Z_{i j}-1}{\sigma^{2}} \text { where } Z_{i j}-\mathbf{x} \cdot \mathbf{x}_{\mathbf{i j}}
$$

As a result of the simplification, the calculation required has been reduced to a dot product $\left(\mathbf{x} \cdot \mathbf{x}_{\mathbf{i j}}\right)$ and an exponential function.

The architecture of the PNN is shown in Figure A.11. The input units represent the components of a test or unknown vector and distribute the values to the pattern units. There is a pattern unit for each vector in the training data set. There are $N$ classes denoted by $M_{1}, M_{2}, \ldots, M_{N}$. The links from each of the inputs $x_{1}, x_{2}, \ldots, x_{p}$ to the pattern unit within class 1 for example indicate that the difference between the input vector components and the stored training data set vector (represented by the pattern unit) is to be calculated and stored. These squared Euclidean distances are summed for each class to give $g_{i}(\mathbf{x})$ (refer to equation A.58) and the class of the input vector is set to the class with the largest value of $g(\mathbf{x})$.

The smoothing parameter is equivalent to the standard deviation of the spherical Gaussian functions. The effect of different choices of smoothing parameter, $\sigma$ on the form of the probability density functions for two classes is shown in Figure A.12a-c (based on Masters, 1993b, 1995 and Zaknich, 1999). Patterns in Figure A.12a-c are represented as points in the horizontal plane, corresponding to the tip of the vectors. Only two features are shown and therefore the vectors have only two components. At very small values of $\sigma$, the system is equivalent to a nearest neighbour classifier. At larger values, the classifier is a maximum likelihood function.

Training in a PNN network consists of comparing a set of stored training vectors $\mathbf{x}$ with an independent set of test vectors $\mathbf{x}_{\mathbf{i j}}$ for which the classification is known, in order to find a value for the smoothing parameter, $\sigma$ that gives the best classification accuracy. A single value of sigma is used for all classes. In contrast to the back-propagation algorithm, 


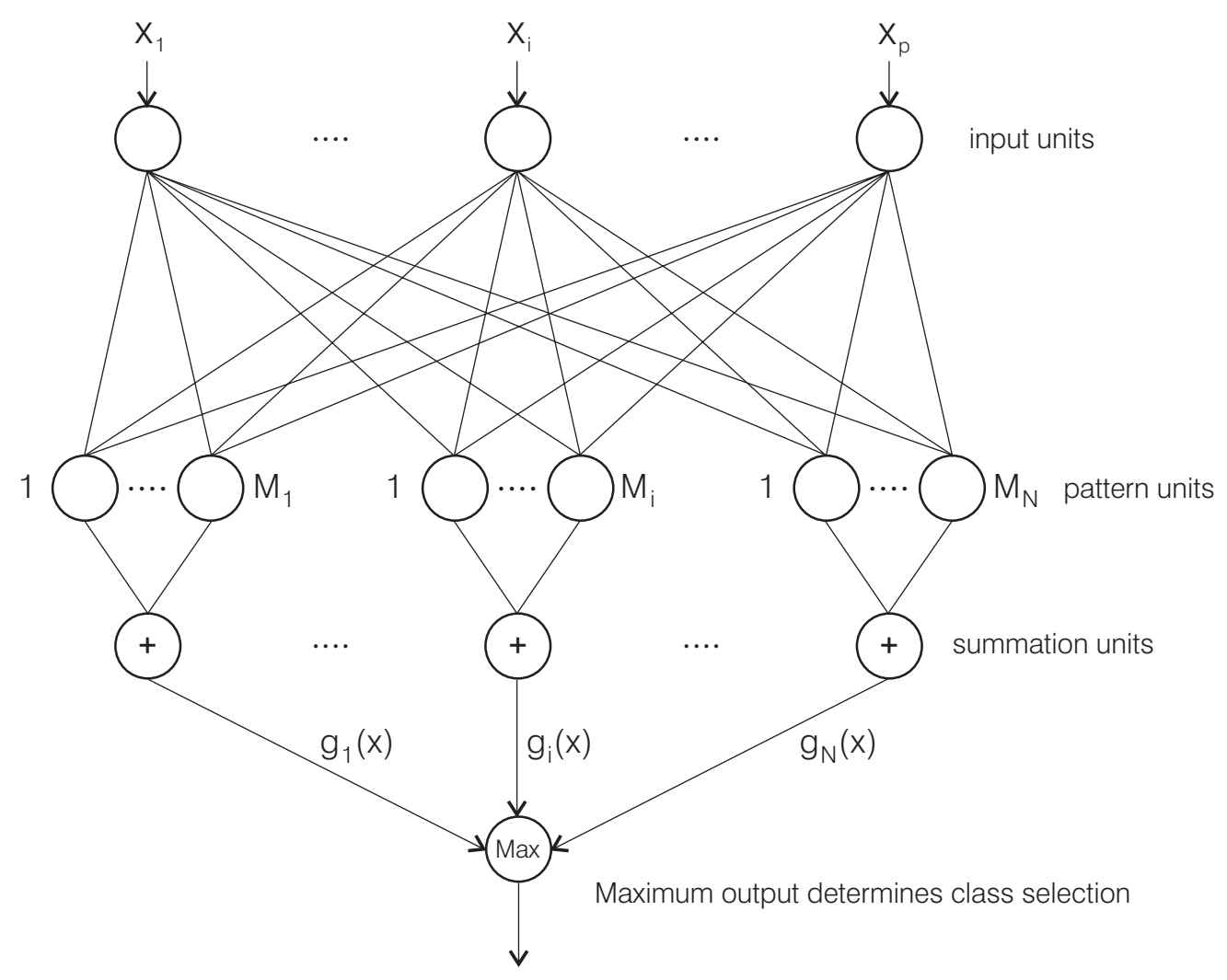

Figure A.11. Architecture of a probabilistic neural network (PNN) adapted from Zaknich (1999). See text for explanation.

this is a one-pass process. If there is not enough data for both a training and test set then a procedure called the hold-one-out method can be used for testing (Bishop, 1995).

Practical descriptions of the use of PNNs have been given by Masters (1993b, 1995) and Zaknich (1999). In a study on the classification of crop types using PNNs, Serpico \& Vernazza (1996) found that PNNs were faster to train and easier to configure than MLP networks but training time for the PNN grew proportionally to the size of the training set.

Masters (1993b) warns that the PNN is more sensitive that most types of neural network to training sets that do not cover all possible classes in the data population and states that an MLP network is likely to give better generalization performance in cases where only a few training samples are available. In a study in which a PNN was used to classify deposits, Singer \& Kouda (1997b) noted that unknown samples which do not belong to any of the classes represented in the training set are likely to be incorrectly classified by the network. On the other hand, Zaknich (1999) has stated that sparse samples are 


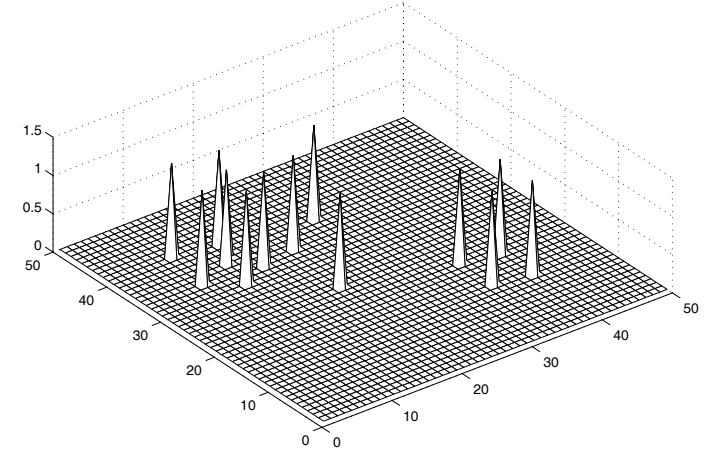

(a)

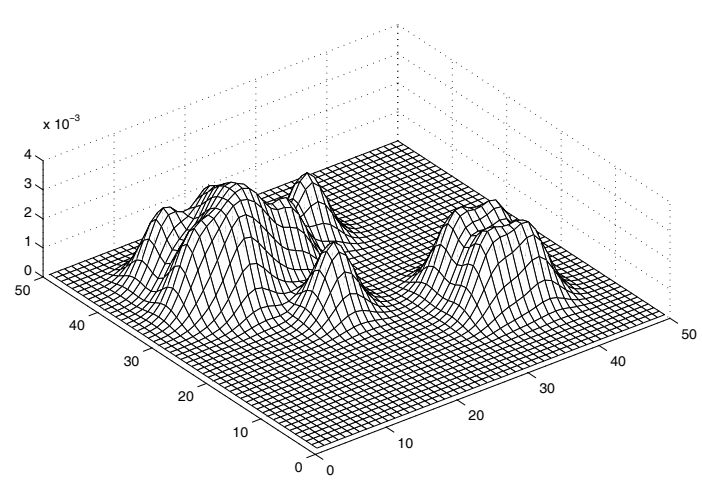

(d)

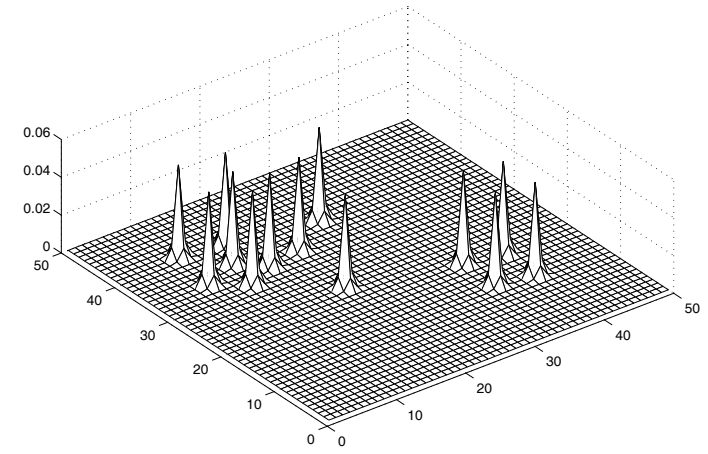

(b)

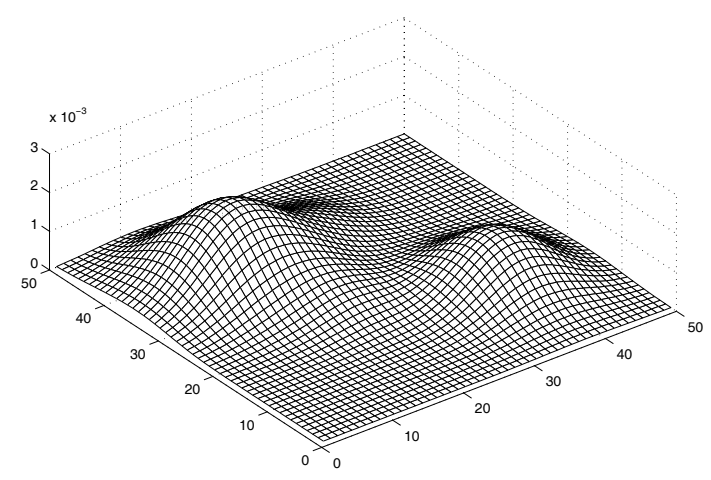

(e)

Figure A.12. The effect of different choices of smoothing parameter, $\sigma$ on classification in a PNN; a) $\sigma=0.1$, b) $\sigma=0.5$, c) $\sigma=0.2 .25$, and d) $\sigma=5.0$. Patterns are represented as points in the horizontal plane, corresponding to the tip of the vectors. At very small values of $\sigma$, the system is equivalent to a nearest neighbour classifier. At larger values, the classifier is a maximum likelihood function. 
adequate for good performance from PNN networks. Although this appears to contradict Master's observations, it is possible that a PNN could perform well if the few training samples that are available happen to fall very close to the modal values for the population and the variance in the vector component values is low.

PNNs have been used or a variety of classification problems. Zaknich (1997) compared the use of PNN and MLP networks for edge detection in order to characterize aluminium hydroxide particles produced in the Bayer process for aluminium refining. He reported that a simple MLP, PNN and a more complex MLP resulted in classification accuracies of $85 \%, 91 \%$, and $95 \%$, respectively.

Singer \& Kouda (1997b) used a PNN to classify deposits into 8 deposit types based on the presence or absence of 58 ore and alteration minerals. They reported better performance for the PNN (88\% classification accuracy) than the PROSPECTOR II expert system (83\%) in which a range of lithological, geochemical and other attributes were used in addition to mineralogy. Another of their results illustrates the importance of selecting the most useful features as inputs. They found that the addition of an input feature indicating whether the marine volcanic rocks in the vicinity of the deposit were mafic or felsic improved the classification rate (for the training-stop set) from $88 \%$ to $98 \%$.

In a related study by Singer \& Kouda (1997a), deposits were classified into 28 categories instead of 8 deposit as described above. The agreement between classes assigned by the neural network and those assigned by experts was only 58\% Singer \& Kouda (1997a) explained this poorer than expected performance as follows; 1) the training data for the first study consisted of deposits that had been described in scientific studies whereas those in the second study were from regional mineral databases, and 2) experts used data which were not available to the network. These results highlight the importance of ancillary and expert knowledge.

In a recent study, Singer \& Kouda (1999a) have compared the performance of the PNN and weights-of-evidence methods for the prediction of mineral potential for VMS deposits in the Chisel Lake-Anderson Lake area, Manitoba, Canada. Twenty-three variables were used to classify areas into deposit and non-deposit groups. The correct classification rates of $99.3 \%$ for PNN and $83 \%$ for the weights-of-evidence method were obtained for an independent training-stop data set containing 7 deposit and 3223 non-deposit cells. Large differences between correct classification rates were obtained using; 1) a combination of training and test data sets consisting of all the available data, and 2) a training-stop set consisting of half the data (where the other half was used for training and test sets). The differences 
were interpreted to indicate over-fitting (learning of noise rather than general patterns in the data) caused by the large number of variables (23) in relation to the small size of the training data set representing deposits (8). Singer \& Kouda (1999a) stated that the PNN handled correlated input variables well compared to the weights-of-evidence method, which was adversely affected by small to moderate correlations leading to upward biased probabilities.

Harris \& Pan (1999) also examined the use of a PNN for mineral prospectivity analysis. They compared a PNN to general regression (GRNN) and MLP neural networks, logistic regression and discriminant analysis and found that the PNN gave the best results. A feature of their study was an analysis of how the percentages of correctly classified mineralized and barren cells varied with the choice of cutoff probability used to make the classification. The PNN performed better than the other methods because it produced the highest percentages of correct classification of both mineralized and barren cells over a wide range of cutoff probabilities $(86.7 \%$ and $93.8 \%$, respectively for the combined training, test and training-stop data sets). An interesting aspect of their study was that Harris \& Pan (1999) used software that calculated the value of the smoothing factor $\sigma$ for each input variable (rather than a single value for all classes). This enabled Harris \& Pan (1999) to interpret the relative contribution of the input variables to the classification. Sigma values in the range $0.01-0.1$ were interpreted to have been practically ignored by the network, whereas inputs with sigma values in the range 1.80-2.95 were regarded as being moderately to very important.

Some of the advantages of PNNs include;

- the output can be used to calculate Bayesian posterior probabilities

- the decision surfaces are guaranteed to approach Bayes' optimal boundaries unlike MLP networks in which training can result in a local rather than global minimum

- training is fast and easy

- outliers in the data are handled well

Disadvantages of PNNs include;

- performance depends strongly on having a training set that accurately represents the data (i.e. it important that all classes and variation in the classes are represented, (Masters, 1993b, 1995), and

- memory requirements are large because all training vectors are stored and all vectors are processed each time an unknown vector is classified. 


\section{A.2.2 General Regression Neural Networks}

The General Regression neural network (GRNN) is another network developed by Specht (1991) which is very similar to the PNN. The GRNN is suitable for both function approximation and pattern classification. Like the PNN, the GRNN uses radial basis functions in a Parzen kernel estimator and stores each training vector in its structure. The GRNN is based on methods for estimating regression functions from noisy data (Bishop, 1995). Bishop (1995) explains that the goal of learning in a network is to find a smooth mapping from input vectors $\mathbf{x}$ to an output vector $\mathbf{y}$, which captures the underlying structures of the data without fitting the noise in the training data as part of the model. In a GRNN, this optimal mapping is achieved by performing a regression with the target data as a dependent variable and the input data as the independent variable. The regression value is equivalent to the conditional average, $\langle t \mid \mathbf{x}\rangle$, of the target data, where $t$ is the target output, and $\mathbf{x}$ is the input to the network; this is illustrated in Figure A.13 below.

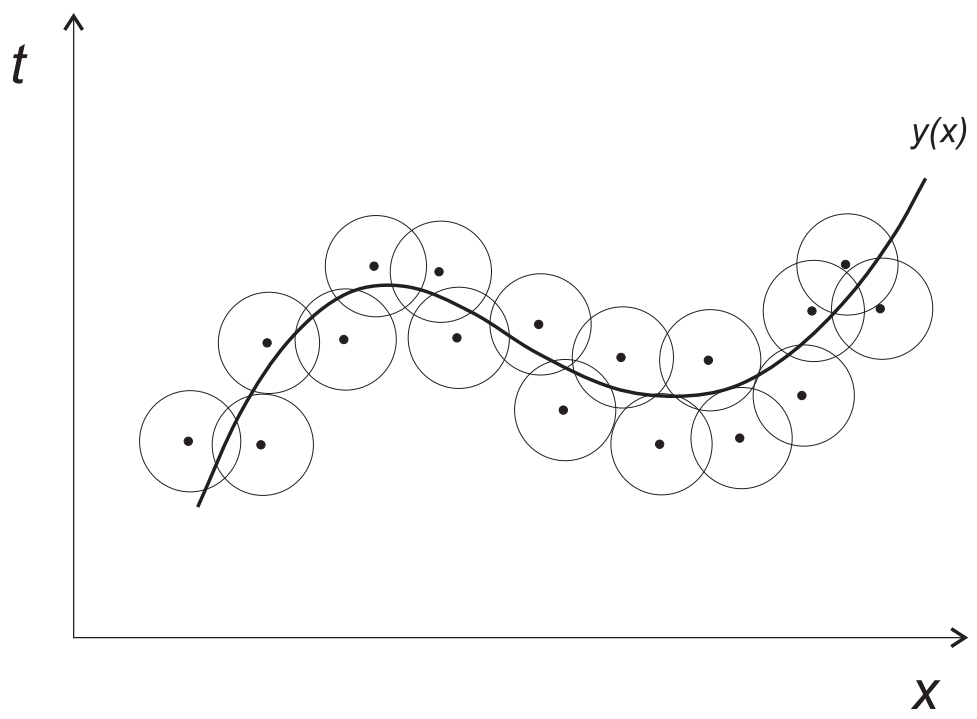

Figure A.13. Schematic illustration of the concept behind the GRNN network (after Bishop, 1995. Dots represent the data points and circles represent the Gaussian kernel functions. The regression curve is given by conditional average, $\langle t \mid \mathbf{x}\rangle$, of the target data, where $t$ is the target output and $\mathbf{x}$ is the input to the network.

The network mapping which minimizes a sum-of-squares error function is given by the conditional average of the target data. Here we consider a mapping from a single input variable $x$ to a single target variable $t$. At any given value $x_{0}$ of the input variable, the network output $y\left(x_{0}\right)$ is given by the average of $t$ with respect to the distribution $p\left(t \mid x_{0}\right)$ of the target variable, for that value of $\mathrm{x}$. 
The concept behind the general regression neural network has been explained by Bishop (1995) who states that under certain circumstances the optimal mapping of the inputs to the output for a network that minimizes a sum-of-squares error function is given by the conditional average of the target data. That is, the minimum of the error function occurs when;

$$
\begin{aligned}
y(\mathbf{x}) & =<t \mid \mathbf{x}> \\
& =\int_{-\infty}^{\infty} t p(t \mid \mathbf{x}) d t \\
& =\frac{\int_{-\infty}^{\infty} t p(\mathbf{x} \mid t) d t}{\int_{-\infty}^{\infty} p(\mathbf{x} \mid t) d t}
\end{aligned}
$$

where the independent variable is assumed to be a vector, $\mathbf{x}$, and the dependent variable $t$ is a scalar, $y(\mathbf{x})$ is the network output, $p(t \mid \mathbf{x})$ is the conditional density and $p(\mathbf{x} \mid, t)$ is the joint probability density function. This result is based on the assumptions that; 1 ) the data set is sufficiently large, and 2) the number of adaptive weights is sufficiently large (Bishop, 1995). Equation A.62 is the standard formula for the expected value of a continuous random variable $<t \mid \mathbf{x}>$ (Berenson \& Levine, 1996).

For a set of training data $\left\{\mathbf{x}_{i}, t\right\}$ where $i=1,2, \ldots, N$, the probability density $p(\mathbf{x}, t)$, can be modelled by using a Parzen kernel estimator based on the data set. If a Gaussian kernel function is used the estimator takes the form:

$$
\hat{p}(\mathbf{x}, t)=\frac{1}{N} \sum_{n=1}^{N} \frac{1}{\left(2 \pi \sigma^{2}\right)^{(d+c) / 2}} \exp \left\{-\frac{\left\|\mathbf{x}-\mathbf{x}_{\mathbf{i}}\right\|^{2}}{2 \sigma^{2}}-\frac{\left\|t-t_{i}\right\|^{2}}{2 \sigma^{2}}\right\}
$$

where;

$$
\begin{array}{lll}
\hat{p}(\mathbf{x}, t) & = & \text { the estimated value of the joint probability density } \\
d & = & \text { dimensionality of the input space } \\
c & = & \text { dimensionality of the output space } \\
N & = & \text { number of training pairs } \\
(\mathbf{x}) & = & \text { unknown or test vector } \\
\left(\mathbf{x}_{i}\right) & =i^{t h} \text { training vector (stored in network) } \\
t_{i} & =\text { target value for the } i^{t h} \text { training example } \\
t_{i} & =\text { target value for the test vector } \\
\sigma & =\text { smoothing parameter determined during network training } \\
\left\|\mathbf{x}-\mathbf{x}_{\mathbf{i}}\right\|^{2}= & \text { the square of the Euclidean distance between the test } \\
& & \text { and training vectors (equivalent to their dot product, } \mathbf{x} \cdot \mathbf{x}_{\mathbf{i}} \text { ) }
\end{array}
$$


Substituting equation A.64 into A.63 and some manipulation gives estimated value of the target data (Masters, 1995):

$$
\hat{y}(\mathbf{x})=\frac{\sum_{n=1}^{N} t_{i} \exp \left\{-\left\|\mathbf{x}-\mathbf{x}_{\mathbf{i}}\right\|^{2} / 2 \sigma^{2}\right\}}{\sum_{n=1}^{N} \exp \left\{-\left\|\mathbf{x}-\mathbf{x}_{\mathbf{i}}\right\|^{2} / 2 \sigma^{2}\right\}}
$$

where $\hat{y}(\mathbf{x})$ is the value of the target variable; predicted by the network (i.e. network output). Equation A.65 is known as the Nadaraya-Watson estimator (Nadaraya, 1964; Watson, 1964) and it is this procedure that was re-discovered and formulated as a neural network by Specht (1991).

Although, the GRNN can be extended to handle multidimensional output (Zaknich, 1999) in the network proposed by Specht (1991), the target output is a scalar value. Equation A.65 shows that the regression value $y(\mathbf{x})$ is the weighted average of all the values $t_{i}$. Huang \& Williamson (1994) noted that when the smoothing factor $\sigma$ approaches zero, $\hat{y}(\mathbf{x})$ assumes the value $t_{i}$ associated with the value $x_{i}$ and when $\sigma$ becomes very large the value of $\hat{y}(\mathbf{x})$ becomes the mean of all $t_{i}$ values. For intermediate values of $\sigma$ just the values of $t_{i}$ in a small neighbourhood contribute to the estimate of the target value.

The architecture of the GRNN is shown in Figure A.14 (Zaknich, 1999). It is very similar to that of the PNN. All the components of the input vector (three are shown) are presented to the network and the input units calculate the Euclidean distance measure (small input layer circles); then the Parzen window function (similar to the activation function) is performed (large circles in input layer). At the output units, the product of $y_{i}$ with the output of the activation function is calculated (corresponding to the numerator in equation A.65). The signal that is not modified corresponds to the denominator in equation A.65. The two output units perform the summations in numerator and denominator of equation A.65. The output unit performs the division in equation A.65.

Training in a GRNN network consists of finding a single value of the smoothing parameter $\sigma$ which minimizes the mean square error (MSE) between the network output and the desired output for the test data set. This is done by trial and error. The vector pairs $\{\mathbf{x}, t\}$ of the training data set are incorporated into the network structure (i.e. pattern units contain $\mathbf{x}_{i}$ and output units store $t$ in Fig. A.14). The mean square error (MSE) for a GRNN with a single output unit is given by:

$$
M S E=\frac{\sum_{p=1}^{N}\left(\hat{y}_{p}-u_{p}\right)^{2}}{N}
$$




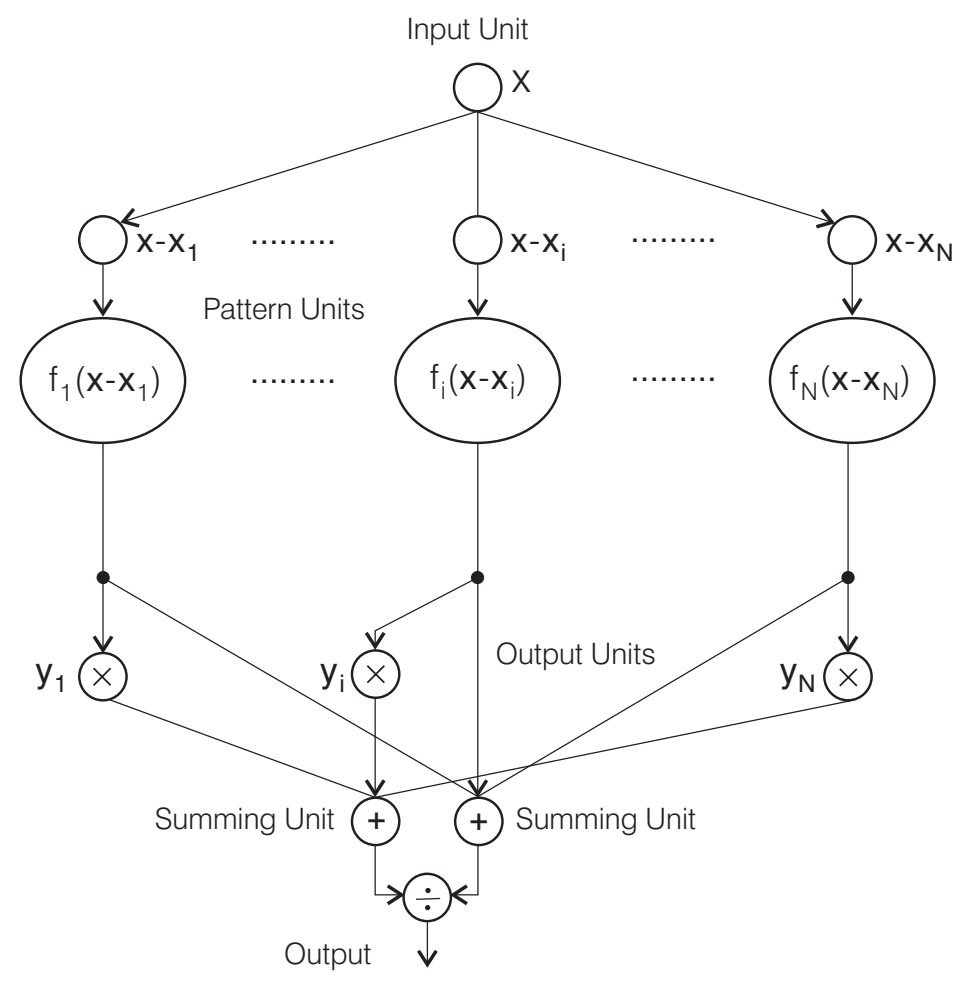

Figure A.14. Architecture of a general regression neural network (Zaknich, 1999).

A plot of MSE versus the smoothing parameter $\sigma$ shows that the variation of MSE with $\sigma$ is smooth and has a broad minimum (Zaknich, 1999). Although trial and error can yield a suitable value of $\sigma$, a convergent optimization algorithm based on recurrent parabolic curve fitting has also been used (Zaknich \& Attikiouzel, 1993).

The strengths and weaknesses of the GRNN are similar to those of the PNN discussed previously (refer to page 482). The main advantages of the GRNN are;

- the faster training time compared to the MLP network,

- the fact that there is just one parameter to adjust compared to many in the MLP (learning rate, momentum, batch or pattern update, and the number of hidden nodes) and

- the problem of training in MLP networks leading to a local instead of a global minimum does not exist.

Zaknich (1999) has noted that the fact that the centres of the radial basis functions are determined by the training data vectors means that the network can not be overtrained. 
Huang \& Williamson (1994) compared the performance of GRNN and MLP networks for two tasks related to oil exploration. For the task of predicting the total organic carbon content of rocks in exploration wells based on wire-line log data. Although a 4-4-1 MLP network performed slightly better than the GRNN, Huang \& Williamson (1994) noted that much more time was required to determine the topology and to train the MLP network. In addition, they found that the GRNN required fewer patterns in order to be trained than the MLP network. A second task was the identification of the dominant source rocks (carbonate, deltaic, and marine shale oil) based on the carbon isotopic profiles (i.e. carbon isotopic ratios for 11 crude oil distillation fractions). Only the GRNN was applied to this task. A 95\% correct classification rate was achieved including the identification of 6 out of 7 incomplete patterns.

In a comparative study of different mineral prospectivity methods, Harris \& Pan (1999) found that a GRNN performed very well but was slightly poorer than the PNN. Using a cut-off value of 0.5 at the network output to categorize input vectors as mineralized $(\geq 0.5)$, the PNN classed $13.6 \%$ of the total cells in the study area as mineralized $(68.2 \%$ correct) versus $13.5 \%(59.5 \%)$ for the GRNN.

\section{A.2.3 Radial Basis Neural Networks}

The radial basis neural network (RBFNN)is a type of kernel classifier that uses radial basis activation functions in the hidden layer that depend on a measure of distance between the input vector and the training vector associated with the pattern unit. As in the PNN and GRNN, a smoothing factor controls the diameter of the units receptive field (Broomhead \& Lowe, 1988; Moody \& Darken, 1989). In contrast with PNN and GRNN networks though, the weights associated with hidden units are not determined by a single training vector and the smoothing parameter $\sigma$ can be set to different values for each hidden unit (Schalkoff, 1997). Like the GRNN, the RBFNN can be used as a universal function approximator as well as a classifier (Park \& Sandberg, 1991).

The concept behind the RBFNN is closely related to distance-weighted regression (Zaknich, 1999). Figure A.15 shows the architecture of an RBFNN. The value computed be each output unit represents the weighted sum of radial basis functions (e.g. Gaussian) that depends on the distance between the input vector to the centroid in input space corresponding to the hidden unit as follows: 


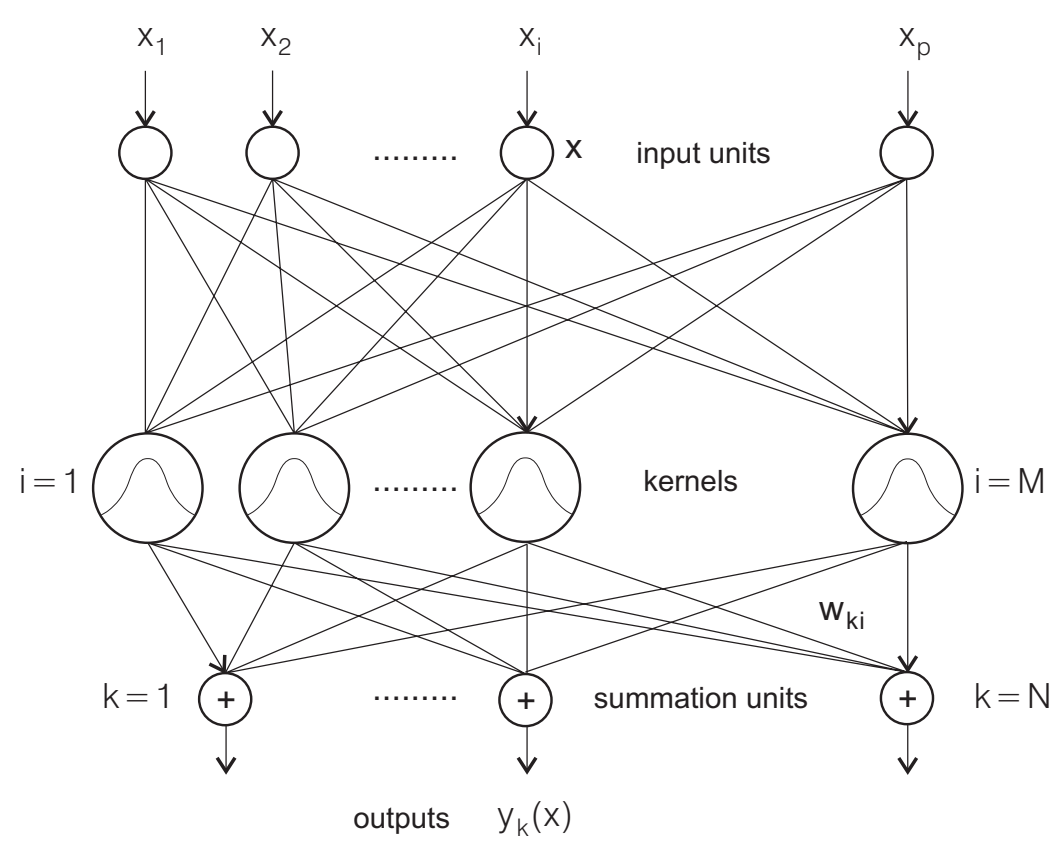

Figure A.15. Architecture of a radial basis function neural network (adapted from Zaknich, 1999)

$$
y_{k}(\mathbf{x})=\sum_{i=1}^{M} w_{k i} f_{i}\left(\left\|\mathbf{c}_{\mathbf{i}}-\mathbf{x}\right\|, \sigma_{i}\right)
$$

where;

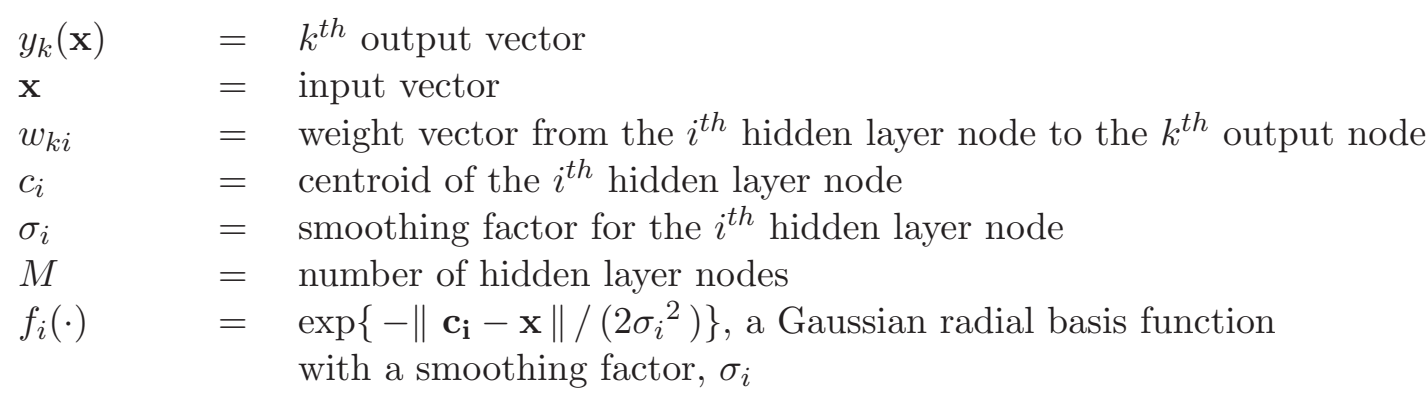

Training involves finding suitable values for the position of the centroids (i.e. the position in multidimensional space occupied by the input vectors) represented by the hidden units $c_{i}$; weights from hidden units to output units, $w_{k i}$; and the smoothing factors $\sigma_{i}$, for the radial basis functions associated with each hidden layer unit. Although many procedures to do this have been developed, training generally consists of two stages; 1) determination of RBF centroids, and 2) determination of $\sigma_{i}$ and the weights from the hidden to output 
layer (Schalkoff, 1997).

Various methods exist for determining the centroids, weights and smoothing factors $\mathbf{c}_{\mathbf{i}}$, ( $w_{k i}$ and $\sigma_{i}$, respectively) are described by Zaknich (1999);

1. selecting $\mathbf{c}_{\mathbf{i}}$ uniformly or randomly and using Singular Value Decomposition to solve for $w_{k i}$ and $\sigma_{i}$. Each cluster centre or centroid is then represented by a hidden layer unit. The weights are optimized by minimizing an error function (e.g. sum of squares) representing the error between the actual network output and the desired output over the vectors in the training set.

2. orthogonal least squares learning (Chen et al., 1991)

3. use of k-means clustering to determine $\mathbf{c}_{\mathbf{i}}$, a k-nearest neighbour heuristic to determine $\sigma_{i}$, and multiple linear regression to determine $w_{k i}$ (Leonard \& Kramer, 1991). The k-means clustering algorithm groups vectors according to a Euclidean-distance measure of similarity.

4. Karri \& Frost (1999) compared estimates of $\sigma_{i}$ based on the standard deviation of the output vector and by plotting the root-mean-square (RMS) error against $\sigma_{i}$ for the training and test data and choosing the value of $\sigma$ that gave the minimum RMS error.

The value of $\sigma$ determines how smoothly the network output fits the training data and therefore how well the underlying function is represented and how well the network is able to generalize to new data (Bishop, 1995).

Advantages of the RBFNN include;

1. the fact that the number of basis functions (and therefore hidden layer units) is determined by the complexity of the mapping between the inputs and outputs. This contrasts with the PNN and GRNN, in which the training data set size determines the number of pattern units.

2. there is no local minima problem as in the MLP network (Kasabov, 1996)

3. training is an order of magnitude faster than a comparable MLP network (Kasabov, 1996)

Kasabov (1996) has pointed out that a disadvantage of the RBFNN is that it may not be clear how many hidden units to use and that the choice may adversely affect the performance of the network. 
RBFNN have been applied to control applications. One example in mineral processing is the work of Karri \& Frost (1999). They used a RBFNN to predict the $\mathrm{AlF}_{3}$ concentration in electrolyte in electrolysis cells in the aluminium smelting process.

\section{A.2.4 Kohonen Neural Networks}

The self-organizing feature map (SOFM), also known as a Kohonen network, uses an unsupervised training scheme (Kohonen, 1982, 1988, 1990). Instead of presenting both inputs together with the correct outputs to the network during training (as in supervised training for MLP, PNN and GRNN networks), just the inputs are used and the network develops its own clustering of the data. Another difference to the networks architectures previously discussed is that the SOFM is almost always used as classifier rather than a universal function approximator like the MLP network, GRNN and RBFNN.

The way in which multidimensional feature space is divided into complex decision regions in order to perform a classification differs between neural network architectures (Lippmann, 1989). MLP networks divide feature space using hyperplanes. In RBFNN and GRNN and PNNs, decision regions are formed by radial basis functions or kernels. The SOFM is an exemplar classifier, in which classification of an unknown feature vector is based on the identity of the training examples that are nearest to the input in multidimensional feature space (Lippmann, 1989). These differences are summarized in Figure A.16

The architecture of the SOFM is shown in Figure A.17. The SOFM is usually arranged into two layers. There is an input unit for each component of the input feature vector. Each of these input units is connected to every unit in the output layer. The output layer is usually arranged as a two-dimensional grid. After the presentation of each input pattern during training, units in the output layer compete with one another to learn. Just the output unit which is closest to the input vector (in terms of distance between the vectors in multidimensional feature space) together with a group of units in the neighbourhood adjacent to the winning cell have their weights adjusted (i.e. learn).

Prior to training, raw data must be pre-processed. Masters (1993b) recommends performing a linear scaling to convert raw inputs to a symmetrical range; e.g. $[-1,+1]$ and normalizing training and test vectors to unit length. This latter step is performed as follows (Strang, 1993);

$$
\hat{\mathbf{x}}=\frac{x_{i}}{l} \quad \text { where } i=1,2, \ldots, N
$$




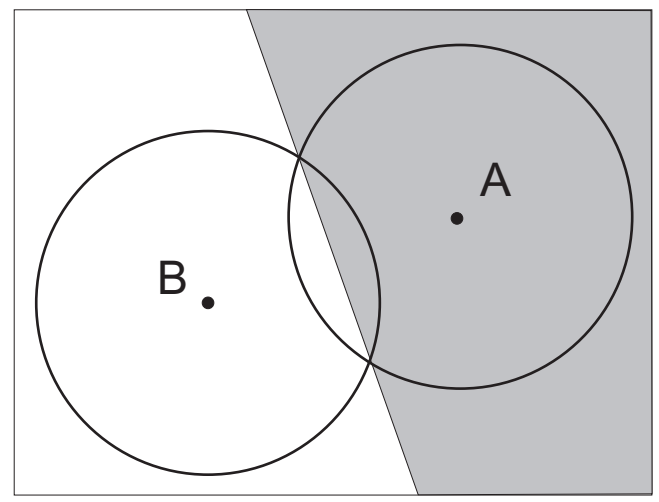

a) Probabilistic; e.g. PNN

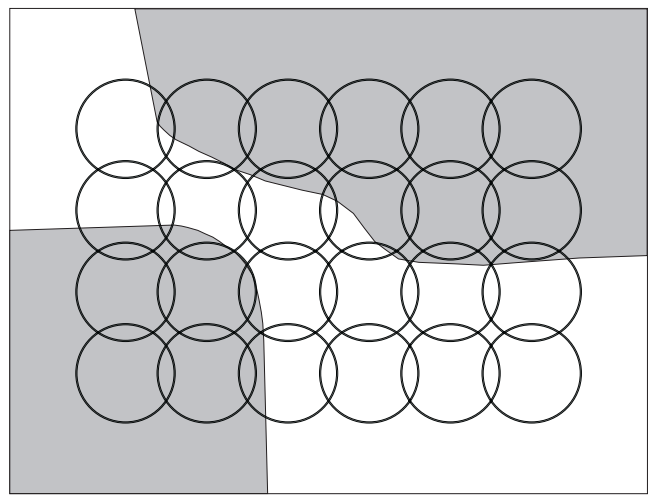

c) Receptive fields (kernels); e.g. Radial Basis Function network

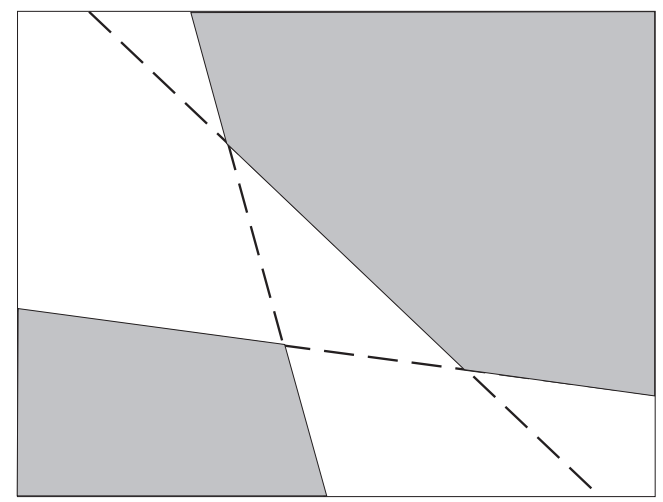

b) Hyperplane; e.g. MLP neural network

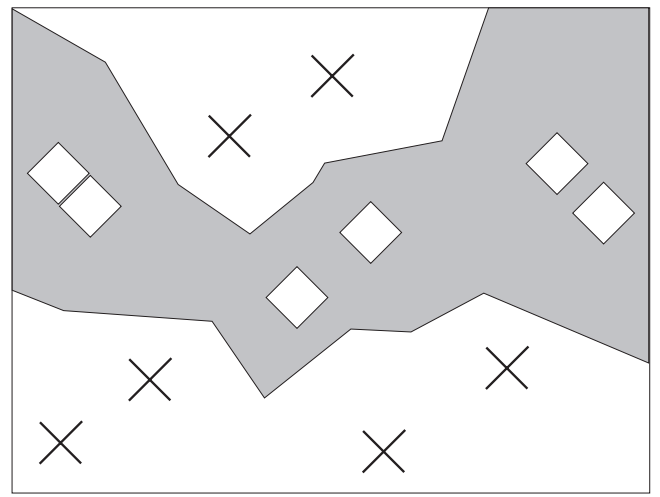

d) Exemplar; e.g. Kohonen network

Figure A.16. Range of methods used by neural network types to divide multidimensional feature space into decision regions in order to classify patterns (after Lippmann, 1989).

A. Probabilistic neural network: class probability density functions are estimated using the sum of small multivariate spherical Gaussian distributions centred at each training sample vector. Bayes' decision rule is implemented to assign an unknown vector to the class that it has a maximum likelihood of belonging to.

B. Multilayer perceptron: class regions within multidimensional feature space are divided by decision surfaces known as hyperplanes. MLP networks contain nodes which calculate the weighted sum of the inputs and transform this with a non-linear function. The position of the hyperplanes depend on the connection weights linking the nodes and the threshold or bias input to the nodes.

C. Radial basis function neural network: this type of network contains a hidden layer with radial basis activation (spherical Gaussian). functions that are connected in parallel. These radial basis functions (also referred to as kernel functions) are defined by parameters which control their centroid and spread and are designed to overlap in feature space. Training involves adjusting the parameters and optimizing the output-layer weights which control the linear combination of the kernel functions. Classification of an unknown vector is made according to the class of the output unit with the highest activation.

D. Kohonen network or self-organizing feature map: unknown input vectors are assigned to the same class as the output node that the unknown vector is closest to (in terms of the Euclidean distance in feature space). Output nodes are trained to represent the centroid of clusters of vectors using labelled training examples. 


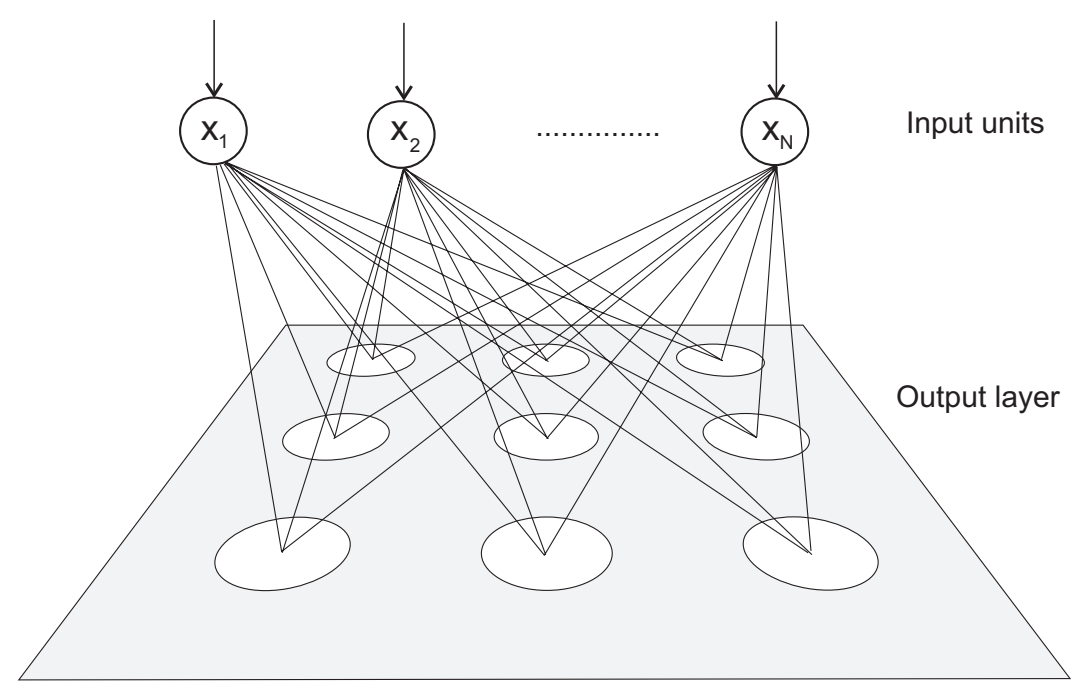

Figure A.17. Architecture of a Kohonen neural network (modified from Dowla \& Rogers, 1995; Dayhoff, 1990

$$
l=\|\mathbf{x}\|=\sqrt{\sum_{i=1}^{N} x_{i}^{2}}
$$

where;

$$
\begin{aligned}
& \hat{x}_{i}=\text { new value of the } i^{\text {th }} \text { component of input vector } \mathbf{x} \\
& x_{i}=\text { raw value of the } i^{\text {th }} \text { component of } \mathbf{x} \\
& \|\mathbf{x}\|=\text { length of } \mathbf{x} \\
& N=\text { dimensionality of } \mathbf{x}
\end{aligned}
$$

According to Masters (1993b) a disadvantage of normalization is that it discards absolute magnitude information. Masters (1993b) proposed using a procedure called z-axis normalization to create an additional synthetic input to overcome the problem.

In order to train the network, weights are initialized to small random values. Then the following two steps are applied;

- determine the output unit with a weight vector that best matches the training input vector; this output node is referenced as the winner,

- update the weight vector of the winner and a neighbourhood of surrounding cells to increase their alignment (i.e. similarity) to the input vector.

The effect of this form of training is to create regions in the output layer that are sensitive to clusters or classes of input patterns. The use of neighbourhoods ensures that once the 
learning algorithm has converged, vectors corresponding to output units that are close on the output layer grid respond to similar input patterns; i.e. vectors that are close in input feature space. This means that the patterns are topologically ordered in the output layer (also called the feature map).

The best matching unit is the output unit $c$ with the minimum Euclidean distance between its weight vector and the input vector. This distance is given by;

$$
d_{c}=\min _{j}\left\|\mathbf{x}-\mathbf{w}_{j}\right\|
$$

where;

$d_{c}=$ distance for the best matching unit $c$, i.e. the minimum distance of all the output-unit vectors $j$

$\mathbf{x}=$ input vector for a single training pattern

$\mathbf{w}_{\mathbf{j}}=$ weight vector associated with the output unit $j$

Weights for the best matching unit and its neighbours are updated according to the rule;

$$
\mathbf{w}_{j}(k+1)=\mathbf{w}_{j}(k)+h_{c j}(k)\left(\mathbf{x}(k)-\mathbf{w}_{j}(k)\right)
$$

where;

$$
\begin{aligned}
& \mathbf{x}=\text { input vector for a single training pattern } \\
& \mathbf{w}_{\mathbf{j}}=\text { weight vector associated with the output unit } j \\
& \mathrm{k}=\text { time expressed as the training iteration count } k \\
& h_{c j}(k)=\text { neighbourhood kernel function at time } k
\end{aligned}
$$

The neighbourhood kernel is defined as the area around the best matching output unit $c$. Usually, the neighbourhood kernel is decreased linearly with time until it reaches a limit of one. At this point the fine tuning phase of training continues until some limit is reached. The neighbourhood kernel function at iteration $k$ is given by;

$$
h_{c j}(t)= \begin{cases}\alpha(k) & \text { if the unit } j \text { is within the neighbourhood } N_{c}(k) \\ 0 & \text { otherwise }\end{cases}
$$

where 
$h_{c j}(k)=$ neighbourhood kernel function

$\alpha(k)=$ learning rate; monotonically decreasing function of time

$N_{c}(k)=$ current neighbourhood around best matching output unit

Both the learning rate $\alpha$ and the neighbourhood radius $N_{c j}$ should decrease according to some monotonically decreasing function of time as training progresses until some minimum is reached. Masters (1993b) has recommended a learning rate of 0.5 and Kohonen (1988) recommended that the neighbourhood $N_{c j}$ should be initially set to half the diameter of the output grid. In order that local areas of the output layer specialize their response to certain classes of input patterns, it is important that the learning rate decreases rapidly with distance (Masters, 1993b). This can be achieved by applying a Gaussian function to decrease the learning rate according to the distance measured on the output grid between best matching unit and its neighbours. (Zaknich, 1999):

$$
h_{c j}(k+1)=\alpha(k) \exp \left\{-\left\|\mathbf{r}_{c}-\mathbf{r}_{j}\right\|^{2} / 2 \sigma^{2}(k)\right\}
$$

$h_{c j}(t)=$ neighbourhood kernel function

$\alpha(k)=$ learning rate; monotonically decreasing function of time

$\sigma(k)=$ spread parameter for Gaussian function centred around best matching unit $c$

$\mathbf{r}_{c}=$ vector representing the position of the best matching unit $c$ on output grid

$\mathbf{r}_{j}=$ vector representing the position of unit $j$ on output grid

Training is halted when the weight changes become insignificant. Masters (1993b) has suggested a number of heuristics to determine; 1) if the learning rate is sufficient, 2) if more output units to represent outlier patterns are required, 3) to optimize specialization by identifying units that represent too wide a variety of patterns, and 4) when to stop training. Hecht-Nielsen (1991) has proposed a conscience algorithm to deal with output units that do not represent any patterns during training.

Once the network has been trained, clusters on the output grid must be labelled to indicate the classes so unknown input patterns can be classified (Beale \& Jackson, 1990). This is done using training patterns for which the class is known.

The SOFM network is useful as a clustering algorithm since the number of features in the input feature vector is reduced to clusters represented graphically on the two dimensional output grid. However, Rogers \& Kabrisky (1991) have likened the attempt to identify patterns, which span a multidimensional feature space, on the basis of their associations 
with units on a 2-D grid to trying to identify animals after they have been squashed flat on the side of a road. Also Masters (1993b) has noted that the SOFM is a weak classifier since it has no hidden units and is therefore strictly linear in its response.

A number of applications of SOFMs have been reported in the geosciences. Maurer et al. (1992) have used an SOFM in combination with a fuzzy-logic inference system to identify classes of seismic events as part of an attempt to automate monitoring networks used to verify nuclear test ban treaties. Fung et al. (1995) was able to classify six lithologies from wire-line log data using a combination of an SOFM and a Learning Vector Quantization algorithm. Yudong et al. (1994) applied an SOFM to predict the presence and absence of mineralization at 25 sample locations. Twenty input variables consisting of assay values of 8 elements and the presence or absence of various structural features (e.g. proximity to certain types of lithological contacts) and rock types were used. Output classes were mineralized and not mineralized. Yudong (1994) also applied an SOFM to the classification of gossan types using geochemical and mineralogical inputs.

Clare \& Cohen (2001) compared an SOFM and a K-means clustering algorithm to perform a cluster analysis on regional stream sediment analyses (comprising 33 elements and 1677 sub-catchments). They found that although the two methods produced similar results, the SOFM was relatively unaffected by the presence of outliers and non-normal populations. Clare \& Cohen (2001) produced a series of maps for each cluster identified by the network by colouring catchment areas according to their degree of similarity between the feature vector for that catchment and the vector associated with the particular cluster represented by the output layer of the trained network. A mineral prospectivity map based on this principle was constructed using the PROSPECT EXPLORER software package by Clare et al. (1997). In this case the map is coloured according to the similarity of vectors associated with grid cells and the cluster type representing anomalous characteristics associated with mineralization.

STRATIMAGIC is another commercial system that incorporates an unsupervised neural network classifier (Addy, 1998; Neri, 1999). The system produces seismic facies maps based on the trace shapes (due to phase, frequency and amplitude) in seismic reflection data. Textural classes can be labelled using patterns associated with wells where the lithologies are known. 


\section{A.2.5 Hybrid Fuzzy-Neural Network Systems}

Since the early 1990's there has been considerable interest in methods for combining neural networks and fuzzy systems. Much of the research has been directed towards solving complex non-linear control problems in engineering (Jang \& Sun, 1995; Kasabov, 1996). The reason for combining the two methods is that they appear to complement one another. Whilst both methods can deal with uncertain and imprecise inputs, neural networks represent a data-driven approach, whereas fuzzy systems represent a conceptual approach. Neural networks can detect very complex patterns in data but fuzzy systems are able to incorporate expert knowledge, which neural networks cannot do directly. It has been shown that combining the two approaches can increase performance and speed up system development (Lazzerini et al., 1999). Huang et al. (1996) found that a modified version of the fuzzy neural network proposed by Lin \& Cunningham (1995) resulted in faster convergence and a lower test set error than an MLP network, which was trained using the backpropagation algorithm.

Three main ways of combining neural networks and fuzzy systems have been described in the literature (Gupta \& Rao, 1994; Jang \& Sun, 1995; Kasabov, 1996)

1. fuzzy neurons,

2. multi-modular hybrid neural network-fuzzy systems, and

3. adaptive network-based fuzzy inference systems (ANFIS).

In fuzzy neurons, the computations are based on fuzzy membership functions (Yamakawa, 1990). Modular hybrid neural network-fuzzy systems contain fuzzy inference and neural network systems which act together in a sequential modular way. The neural network inputs and outputs are crisp whereas the fuzzy system uses fuzzy linguistic variables. Consequently, conversions to and from fuzzy variables (referred to as fuzzification and defuzzification,) must be performed. This type of system is described by Kasabov \& Jain (1990, 1993), Kasabov et al. (1994), and Masters (1993b). Kohonen (1988) describes a speech recognition system in which a self-organizing map is used for the low-level task of phoneme recognition and a fuzzy inference system is used to apply rules about word context to improve the accuracy of the system.

A class of systems called adaptive fuzzy inference systems (ANFIS) has been developed independently by Jang (1991, 1993), Lin \& Lee (1991), and Wang \& Mendel (1992). In these systems, fuzzy if-then rules and membership functions are represented as nodes in a feedforward network structure. The membership functions can be adapted through super- 
vised training and a hybrid backpropagation-least mean squares learning algorithm (Jang \& Sun, 1995). The advantage of ANFIS's are that they; 1) achieve better performance than either fuzzy systems or neural networks alone and 2) they can incorporate linguistic knowledge from experts (in the form of stored rules) and adapt to numerical training data. ANFIS's have been applied to control a steam engine-boiler system (Mamdani \& Assilian, 1975), control part of a steel rolling mill (Funabashi et al., 1995), analyze of ultrasonic images, (Pavlopoulos et al., 2000), assist in medical diagnosis (Zahlmann et al., 2000), and diagnose faults in motors (Fuessel, 2000).

\section{Comparison of Boolean and Fuzzy Inference}

Both the modular and adaptive fuzzy systems depend on a fuzzy inference procedure. An inference procedure is used to derive conclusions from a set of fuzzy if-then rules and one or more conditions. In two-valued Boolean logic, the fundamental rule of inference is called modus ponens (Cawsey, 1998), which is summarized as:

$$
A \wedge(A \rightarrow B) \rightarrow B
$$

It states that the truth of a proposition $B$ can be inferred from the truth of a condition $A$ and the implication $A \rightarrow B$. For example, a rule such as "if distance to fold axis is low then favourability for gold deposits is high" can be represented in the following syllogism.

\begin{tabular}{lll} 
premise 1 & (fact) & $x$ is $A$ \\
premise 2 & (rule) & If $x$ is $A$ then $y$ is $B$ \\
\hline conclusion & $y$ is $B$
\end{tabular}

In Boolean logic, the premise and conclusion can either belong to the set true completely or not at all. In contrast, in fuzzy set theory, a variable can belong to a set partially and the degree of membership is expressed as a real number in the range [0,1] (Zadeh, 1965).

Fuzzy membership functions are used to quantify the $x$ is $A$ and $y$ is $B$ parts of premises 1 and 2 in the syllogism above. For example, if crisp sets in the if-then rule; "if distance to fold axis is high" are replaced by fuzzy sets then the rule becomes; "if distance to fold axis is low then favourability for gold deposits is more or less high". The rule can be represented as a syllogism as follows; 


\begin{tabular}{lll} 
premise 1 & (fact) & $x$ is $A^{\prime}$ \\
premise 2 & (rule) & If $x$ is $A$ then $y$ is $B$ \\
\hline conclusion & $y$ is $B^{\prime}$
\end{tabular}

where $A^{\prime}$ is close to $A$ and $B^{\prime}$ is close to $B$ (Jang \& Sun, 1995).

\section{Processing Steps in a Fuzzy Inference System}

Processing in a fuzzy inference system consists of the following steps;

1. convert input variables to membership degree (fuzzification) using fuzzy membership functions,

2. combine antecedents to determine the firing strength of rules using fuzzy operators (e.g. fuzzy $A N D$ and $O R$ ),

3. calculate consequent membership functions (MFs) based on the firing strength using an implication operator,

4. combine the consequent MFs (i.e. output of rules) using an aggregate operator, and

5. conversion of fuzzy membership degree to crisp values (defuzzification),

In a single fuzzy if-then rule of the form

If $x$ is $A$ then $y$ is $B$,

the first two steps are required to evaluate the premise or antecedent part of a fuzzy ifthen rule. The if-part of the rule " $x$ is $\mathrm{A}$ " is called the premise or antecedent while the then-part of the rule is called the " $y$ is $\mathrm{B}$ " is called the consequent or conclusion.

The first five steps are illustrated in a example (Fig A.18; adapted from Jang et al., 1997). All of the processing steps listed above are discussed in the following sections. The system only contains two rules in which the parameters are the distance to the nearest fault or shear zone and the rheological contrast at the nearest lithological boundary. Two fuzzy variables for each rule, low and high, express the degree of favourability of the conditions in map cells for gold deposits. The discussion focusses on the rule 1 in Figure A.18.

The first step is to convert crisp values of distance ( $=1000 \mathrm{~m}$, in left-hand column) to a membership degree for the fuzzy set low. From the fuzzy membership function for distance is low, it can be seen that the degree is approximately 0.9 . The rheological contrast (= 
8, in column 2) belongs to the fuzzy set rheological contrast moderate to a degree 0.2 (Fig. A.18).

In the second step, the two input conditions in rule 1 are combined using a fuzzy AND operator. In the example shown in Figure. A.18, AND operator is be implemented by taking the minimum of the fuzzy membership degrees for the two antecedent parts of the rule (indicated by the horizontal dashed line which is continued across to the right-hand column).

The third step is to determine the output of the rule. The output of each rule is a fuzzy membership function (shown the right-hand column, Fig. A.18). This membership function describes the degree to which crisp values of "favourability for gold deposits" belong to the fuzzy set low. This output fuzzy membership function is modified according to the degree to which the input conditions of the rule are satisfied. The approach is as follows; if the input conditions to a rule are only partially fulfilled, then the result of the rule can only be partially fulfilled. Consequently, the minimum value of the input conditions (horizontal dashed line at $y=0.2$ ) is used to truncate the output fuzzy membership function for rule 1 .

The fourth step is to combine the results of the rules. In this example, the max is used to take the maximum value of the output membership functions for rules 1 and 2, giving an aggregate membership function. The fifth step is to convert this aggregate membership function to a single crisp value. This is done by taking the $x$-value corresponding to the centroid (i.e. centre of mass) of the shape.

\section{Conversion of Input Variables to Membership Degree using Fuzzy Membership Functions}

A fuzzy set $A=\left\{\left(x, \mu_{A}(x)\right) \mid x \in X\right\}$ can be represented as a sequence of value/membership pairs; for example, $\{(1 / 0),(2 / 0),(3 / 0.5),(4 / 1),(5 / 0.5),(6 / 0)\}$. Another way of representing a fuzzy set is with a membership function such as the ones shown in Figure A.18 above and in Figure 2.1 on page 24). In Figure 2.1, the linguistic variable amount of gold has three fuzzy values; i.e. background, weakly anomalous, and strongly anomalous, each of which is represented by a fuzzy set.

As noted in Section 2.2.3 on page 21, the definition of a membership function is often subjective. In real-time control applications, membership functions composed of straightline segments are often chosen because of their simplicity and computational efficiency 
1.fuzzy $\mathrm{m} /$ ship degree

2. rule firing strength
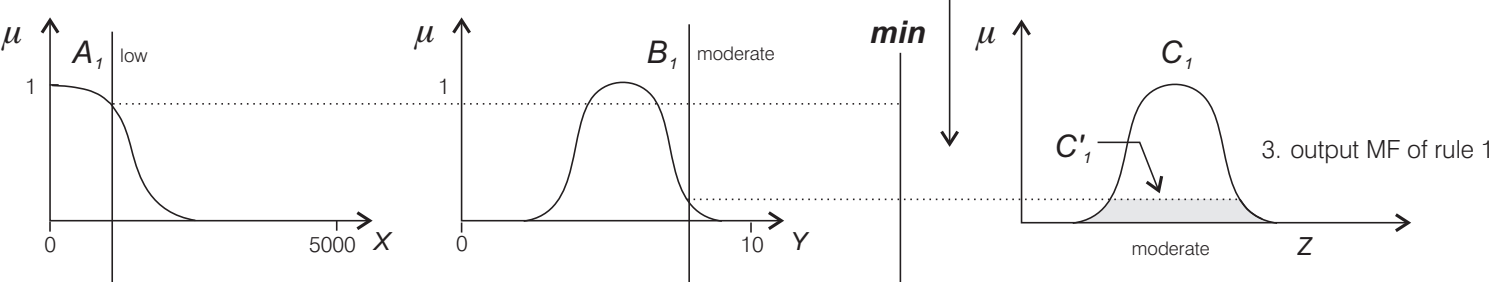

Rule 1: If distance is low AND contrast is moderate then favourability is moderate
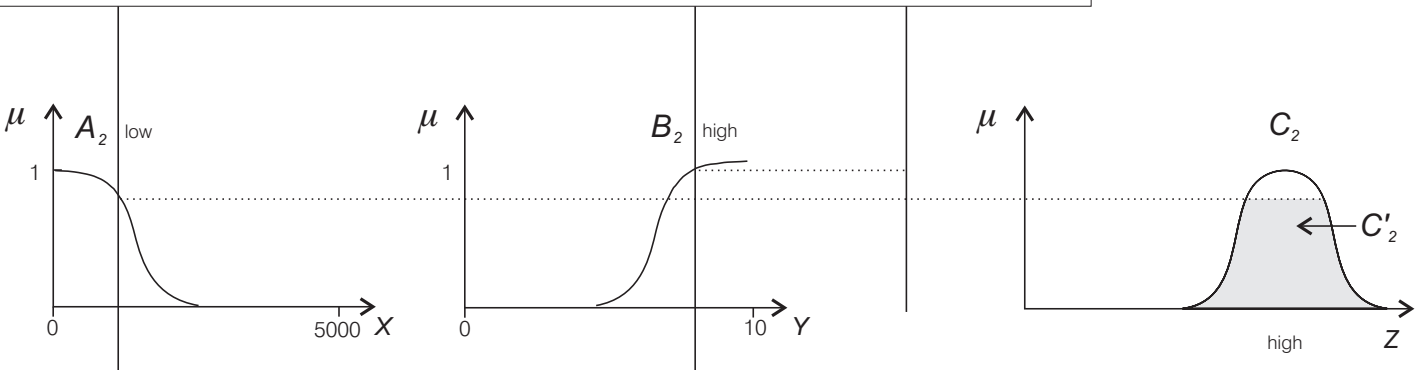

Rule 2: If distance is low AND contrast is high then favourability is high

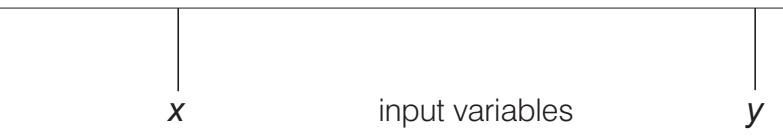

distance to nearest fault $=1000$ rheological contrast at nearest fault $=8$

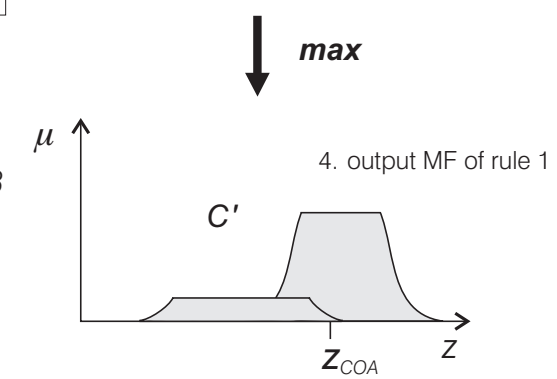

5. overall output (crisp) $Z=$ centroid of area

Figure A.18. An example of a Mamdani fuzzy inference procedure for two rules and two premises. The Mamdani inference system uses the max-min composition, and min and max for the for the T-norm $(A N D)$ and T-conorm $(O R)$ operators, respectively (modified from Jang \& Gulley, 1995). 
(Kosko, 1992). An example is the triangular membership function. It is specified by three parameters $a, b$ and $c$, which determine the $x$ coordinates of the three corners. The membership value is given by Schalkoff (1997):

$$
\mu_{\text {triangle }(a, b, c)}=\max \left[\min \left(\frac{x-a}{b-a}, \frac{c-x}{c-b}\right)\right]
$$

In applications where real-time response is not critical and an neural network is used to simulate the behaviour of a fuzzy inference system, smooth non-linear membership functions such as the Gaussian or generalized bell (sigmoidal) (Halgamuge \& Glesner, 1994) function can be used. Equations A.75 and A.76 shown the bell and Gaussian membership functions, respectively (Schalkoff, 1997);

$$
\begin{aligned}
\mu_{b e l l(x, a, b, c)} & =\frac{1}{1+\left\|\frac{x-c}{a}\right\|^{2} b}, \text { where } b>0 \\
\mu_{\text {Gaussian }(x, c, \sigma)} & =\exp ^{-[(x-c) / \sigma]^{2}}
\end{aligned}
$$

In equation A.76, $c$ and $\sigma$ represent the centre and width of the membership function, respectively. Both the form of the fuzzy membership function (Masters, 1993b) and the number of fuzzy values used to represent the fuzzy variable is crucial for a fuzzy system (Kasabov, 1996). For example, the variable temperature could be represented by linguistic variables and corresponding fuzzy sets, very cold, cold, medium, hot, and very hot. Kosko (1992) used seven fuzzy values and trapezoidal membership functions with a 25 functions are discussed in detail by Schalkoff (1997) and Kasabov (1996).

\section{Using Fuzzy Operators to Evaluating the Firing Strength of Rules}

Fuzzy membership values express the degree to which each part of the antecedent of a rule is satisfied. Where the antecedent is composed of multiple parts as in the rule;

If $x$ is $A$ and $y$ is $B$ then $z$ is $C$,

fuzzy operators are used to combine the two fuzzy membership values in the antecedent to obtain a single number which describes the firing strength of the rule. 
Fuzzy intersection is applied to antecedents linked with $A N D$ and a fuzzy union is applied to antecedents linked with $O R$ (step 2 in Fig A.18). The most common method for combining antecedents linked with AND is to use the min (minimum) operator. The intersection of two fuzzy sets $A$ and $B$ is a fuzzy set $C$ whose membership function (MF) is related to the MFs for $A$ and $B$ by;

$$
\mu_{C}(x)=\min \left(\mu_{A}(x), \mu_{B}(x)\right)=\mu_{A}(x) \wedge \mu_{B}(x)
$$

The union of two fuzzy sets (i.e. those combined with OR) is implemented using the maximum method;

$$
\mu_{C}(x)=\max \left(\mu_{A}(x), \mu_{B}(x)\right)=\mu_{A}(x) \vee \mu_{B}(x)
$$

A variety of other fuzzy operators are available. For example, a general class of operators known as T-norm operators that include the prod (product) operator are used to perform fuzzy intersection. Similarly, there are also a variety of fuzzy union operators called Tconorm operator (e.g. the probabilistic OR operator, $\operatorname{prob}(a, b)=a+b-a b)$. In addition a range of parameterized T-norm and T-conorm operators have been proposed (Jang et al., 1997) to perform fuzzy $A N D$ and $O R$ functions, respectively.

\section{Using Implication Operators to Calculate Consequent Membership Functions}

In fuzzy implication, the antecedent part of the if-then rule is represented by a single number (referred to as the firing strength of the rule). The output of a rule or consequent is an entire fuzzy set represented as an MF. Fuzzy implication involves modifying the consequent MF according to the degree to which the antecedent part of the rule is satisfied.

Two main implication methods are used in the Mamdani fuzzy model. These are the min, which truncates the output fuzzy set, and the algebraic product function, which scales the output fuzzy set. The consequent membership is modified using the min function in step 3 in Fig A.18). 


\section{Using Aggregate Operators to Combine the Output of Rules}

In the Mamdani inference system (Mamdani \& Assilian, 1975), the truncated output fuzzy membership functions obtained for each rule are combined with the fuzzy OR function (implemented using the max operator; step 4 in Fig A.18). This procedure is applicable if the conclusions of the rules have the same domain for the fuzzy set (e.g. all the rules represent degrees of membership of the fuzzy set favourable for gold deposits; Masters, 1993b). Other methods, depending on the inference model used (e.g. Sugeno or Tsukamoto models) include the sum (the sum of the output of each rule) and the probabilistic OR function (Jang \& Sun, 1995). Kosko (1992) has suggested that arithmetic addition of membership functions gives better results although this option is only appropriate where max-product composition is used.

\section{Conversion of Fuzzy Membership Degree to Crisp Values (Defuzzification)}

In the Mamdani inference system the result of combining the outputs of each rule is a single fuzzy membership function. Defuzzification is the process of converting this aggregate output fuzzy membership function to a single crisp value. The crisp value can be obtained from the following characteristics of the aggregate fuzzy membership function (Fig. A.19);

1. centroid of area (the most widely used method),

2. bisector of area,

3. mean of the range of the crisp variable over which the membership degree is at a maximum,

4. smallest of maximum (the smallest value of the crisp variable over which the membership degree is at a maximum), and

5. largest of maximum (the largest value of the crisp variable over which the membership degree is at a maximum).

In practice the need to perform defuzzification in the Mamdani fuzzy inference system has two disadvantages. First, it is time consuming to compute and second, the process it not easily subject to rigourous mathematical analysis (Jang \& Sun, 1995). In the Sugeno fuzzy model, which overcomes this problem, fuzzy rules have the form;

If $x$ is $A$ and $y$ is $B$ then $z=f(x, y)$ 


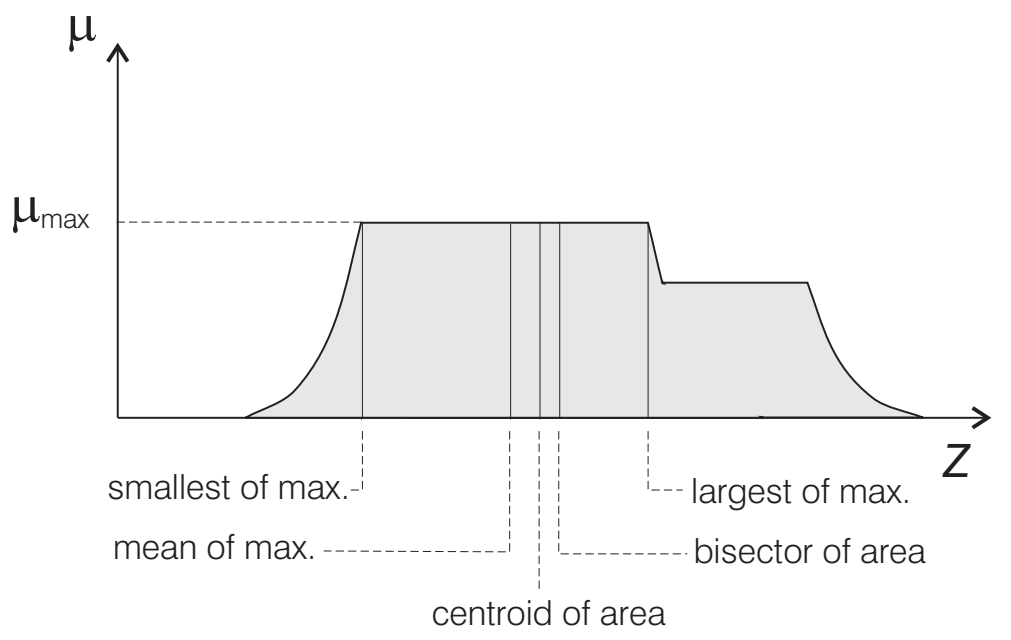

Figure A.19. Methods for converting the aggregate output fuzzy membership function to a single crisp value. This process is known as defuzzification (see text for explanation; from Jang et al., 1997).

where $\mathrm{A}$ and $\mathrm{B}$ are fuzzy sets in the antecedent, and $z=f(x, y)$ is a crisp function in the consequent. The output $f(x, y)$ is either a constant (zero-order Sugeno model) or a first-order polynomial (first-order-Sugeno model).

\section{How Rules are Represented in a Neuro-Fuzzy Inference System}

Figure A.20 shows the ANFIS architecture used to implement a simple fuzzy system consisting of two input variables $x$ and $y$ and two rules. This structure corresponds to a first-order Sugeno model with the following rules;

$$
\begin{aligned}
& \text { If } x \text { is } A_{1} \text { and } y \text { is } B_{1} \text { then } f=p_{1} x+q_{1} y+r_{1} \\
& \text { If } x \text { is } A_{2} \text { and } y \text { is } B_{2} \text { then } f=p_{2} x+q_{2} y+r_{2} .
\end{aligned}
$$

These rules are represented within the ANFIS by the links between layer 1 and layer 2 . In order to illustrate this, the rules above have are applied to a geological example giving;

If Distance is low and Contrast is low then favourability is high

If Distance is high and Contrast is high then favourability is moderate

where distance is the distance to the nearest fault or shear zone, contrast is the rheological contrast between adjacent rock units at the nearest lithological boundary, and favourability is the favourability for gold deposits. 
The output of nodes in layer 1 are fuzzy membership values which express the degree to which the input variables belong to the fuzzy set represented by a particular node. In Figure A.20, the top node in layer 2 receives input from node 1 indicating the degree to which distance) is low and an input from the node 4 , indicating the degree to which contrast is low. Links corresponding to rules 1 and 2 are shown in red and blue, respectively.

The example has been simplified so that the rules for this example illustrating the Sugeno system in Figure. A.20 are the same as those used in the example of a Mamdani fuzzy model shown in Figure A.18. Normally, the rules would cover a complete range of fuzzy sets in the antecedent (i.e. low, moderate and high for both input variables and the output MF's of the rules would comprise low, moderate and high favourability). Thus, for two variables (distance and contrast, both of which can belong to three fuzzy sets (low, moderate and high), nine rules would be required to cover all combinations.

Although, the general procedure for the Mamdani and Sugeno fuzzy models illustrated in Figures A.18 and A.20 is the same, the processing steps is are performed differently. In the Mamdani model shown, the rule firing strength is determined using the min function, the rule output MF is truncated using min function, the aggregate output of all rules is determined with the max function, and defuzzification is performed using the centroid of area of the aggregate MF. In contrast, in the Sugeno model (Fig. A.18), the rule firing strength is determined using the prod function, the rule output MF is determined using a first-order polynomial expression involving the input variables and the overall output is calculated as a crisp value, without defuzzification, by calculating the weighted average of the rule outputs (calculated using the normalized firing strengths).

\section{Architecture of an Adaptive Neuro-Fuzzy Inference System (ANFIS)}

The functionality of the layers in the ANFIS (Fig. A.20) are summarized from Jang \& Gulley (1995), Jang \& Sun (1995) and Jang et al. (1997) below.

Layer 1 Inputs are converted to fuzzy membership values by applying fuzzy membership functions as follows;

$$
\begin{aligned}
& O_{1, i}=\mu_{A_{i}}(x) \text { for } i=1,2 \\
& O_{1, i}=\mu_{B_{i}}(y) \text { for } i=3,4
\end{aligned}
$$

where $x$ and $y$ are input variables, $\mu_{A_{i}}$ and $\mu_{B_{i}}$ are membership functions, and $O_{1, i}$ represents the output of the $i^{\text {th }}$ node in layer 1 . Input variables are converted to 


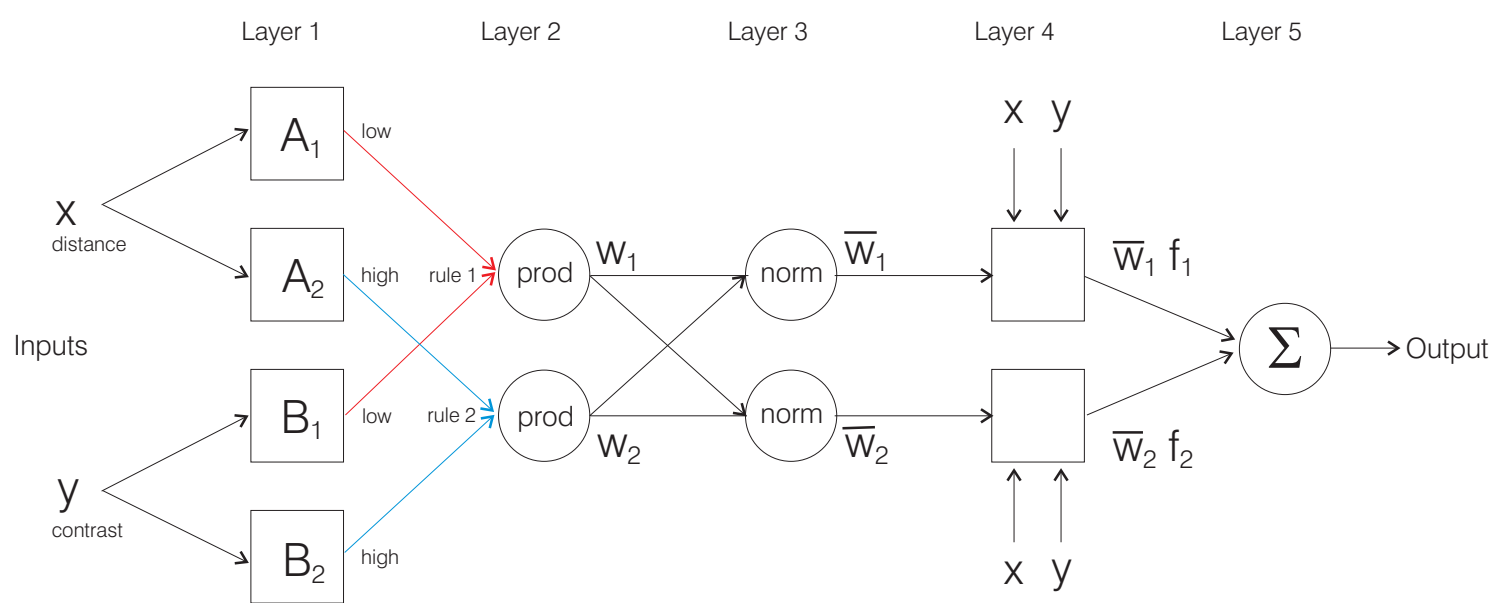

Figure A.20. ANIFS architecture for two rules and two variables using a Sugeno fuzzy inference model. Layer 1 converts inputs to fuzzy membership values using fuzzy membership functions that are specified by three parameters $(a, b$ and $c)$. In layer 4 , the output of each rule is given by a first-order polynomial containing three parameters $(p, q$ and $r)$. These parameters are adapted during training. Links corresponding to rules 1 and 2 are shown in red and blue, respectively (modified from Jang \& Gulley, 1995).

fuzzy membership values according to parameterized fuzzy membership functions, such as the bell function;

$$
\mu_{b e l l(x, a, b, c)}=\frac{1}{1+\left\|\frac{x-c}{a}\right\|^{2} b}, \quad \text { where } \quad b>0
$$

The parameters $a, b$, and $c$ are different for each node and are adapted during training.

Layer 2 The output of each node in this layer represents the firing strength of the rules which are obtained by calculating the product of the inputs;

$$
O_{2, i}=w_{1}=\mu_{A_{i}}(x) \mu_{B_{i}}(y)
$$

where $i=1,2$ (rule number). The fuzzy AND operator is implemented using the prod function. The number of nodes in layer 2 is equal to the number of rules in the fuzzy system.

Layer 3 Nodes in this layer calculate the normalized firing strengths of the $i^{\text {th }}$ rule;

$$
O_{3, i}=\bar{w}_{1}=\frac{w_{i}}{w_{1}+w_{2}},
$$

where $i=1,2$ (rule number) and $\bar{w}_{1}$ is the normalized firing strength. 
Layer 4 Each node in this layer takes the normalized firing strength of a single rule from the previous layer as its input and determines the shape of the fuzzy set that represents the consequent or output of the rule. Using the first-order Sugeno fuzzy implication model, the output of the $i^{\text {th }}$ node of layer 4 is given by;

$$
O_{4, i}=\bar{w}_{1} f_{i}=\bar{w}_{i}\left(p_{i} x+q_{i} y+r_{i}\right)
$$

where $i=1,2$ (rule number), $\bar{w}_{i}$ is the normalized firing strength and $p_{i}, q_{i}$ and $r_{i}$ are are adaptable consequent parameters.

Layer 5 The node in this layer aggregates the outputs of the two rules by summing them;

$$
O_{5, i}=\sum_{i} \bar{w}_{i} f_{i}
$$

where $i=1,2$ (rule number), and $\bar{w}_{i}$ is the normalized firing strength, and $f_{i}$ is the first-order polynomial $f_{i}=\left(p_{i} x+q_{i} y+r_{i}\right.$ representing the output of rule $i$.

As noted previously, the Mamdani fuzzy inference system shown in Figure A.18 uses the max-min composition (i.e. minimum, and $\min$ and $\max$ for the for the T-norm $(A N D)$ and T-conorm $(O R)$ operators, respectively. The corresponding ANFIS network to implement this system would be slightly different to the Sugeno fuzzy model shown in Figure A.20. First, in the layer 2, the min function would be used to combine the precedent parts of the rules (i.e. firing strength). Second, the calculation of the normalized firing strengths in layer 3 is not required. Fourth, the polynomial functions in layer 4 nodes would be replaced by a single node that uses the max function to aggregate the output fuzzy membership function from all the rules. Finally, the function of layer 5 would be to apply a discrete approximation algorithm to calculate the centroid of the aggregate output fuzzy set to give a single crisp output value.

\section{Training to Adapt Fuzzy Membership Parameters and Weights in an ANFIS}

Two layers in the ANFIS contain adaptable parameters that are modified during training. Each node in layer 2 contains three premise parameters that determine the shape of the fuzzy membership functions used to convert inputs to fuzzy membership degrees. There is a separate membership function for each fuzzy variable (e.g. low and high distance, and low and high rheological contrast). In layer 4, each node contains three consequent parameters. These are the coefficients in the polynomial functions used to calculate the rule outputs. There is a node for each rule in layer 4 . 
Hybrid learning is performed in two steps. In a forward pass, the premise (membership function) parameters are fixed, and the signals are calculated up to layer 4 . This is done for all the training input and output pairs. In layer 4, the consequent parameters are estimated by the least squares method using the formula for the Least Squares Estimator (LSE) (equation A.85; see discussion below) or the recursive least squares procedure (Jang et al., 1997). Once the consequent parameters have been determined, the overall output and therefore the errors for each training pattern can be calculated. In the backward pass, the consequent signals are fixed and the error signals are propagated backwards to layer 2. The premise parameters are then updated using a gradient descent method similar to that described in Section 2.4.5. The update formula for a generic parameter $\alpha$ is given by;

$$
\Delta \alpha=-\eta \frac{\partial^{+} E_{p}}{\partial \alpha}
$$

where $\eta$ is the learning rate, $E_{p}$ is the error measure for pattern $p$, and $\partial^{+}$is the ordered partial derivative (Jang et al., 1997).

Expanding the formula given in step 4 above, the overall output $f$ of the ANFIS can be re-written as;

$$
\begin{aligned}
y & =\bar{w}_{1}\left(p_{1} x+q_{1} y+r_{1}\right)+\bar{w}_{2}\left(p_{2} x+q_{2} y+r_{2}\right) \\
y & =\left(\bar{w}_{1} x\right) p_{1}+\left(\bar{w}_{1} y\right) q_{1}+\left(\bar{w}_{1}\right) r_{1}+\left(\bar{w}_{2} x\right) p_{2}+\left(\bar{w}_{2} y\right) q_{2}+\left(\bar{w}_{2}\right) r_{2}
\end{aligned}
$$

This can be considered as a linear expression where the consequent parameters are the coefficients. In order to represent this linear equation in more general terms, the consequent parameters $p_{1}, q_{1}, r_{1}, p_{2}, q_{2}$ and $r_{2}$ are replaced with $\theta_{1}, \cdots, \theta_{n}, x$ and $y$ are replaced with input vector $\mathbf{u}=\left[u_{1}, \cdots u_{p}\right]^{T}$, and $\bar{w}_{1} x, \bar{w}_{1} y, \bar{w}_{1} r_{1}, \bar{w}_{2} x, \bar{w}_{2} y$ and $\bar{w}_{2} r_{2}$, are replaced with $f_{1} \cdots f_{n}$ which are functions of $\mathbf{u}$, giving;

$$
y=\theta_{1} f_{1}(\mathbf{u})+\theta_{2} f_{2}(\mathbf{u})+\cdots+\theta_{n} f_{n}(\mathbf{u})
$$

This equation is a linear regression function. An equation can be written relating each pair of input feature vectors and target output values in the training data set. For $m$ training patterns and $n$ parameters this gives a set of $m$ linear equations; 


$$
\left\{\begin{array}{ccc}
f_{1}\left(\mathbf{u}_{\mathbf{1}}\right) \theta_{1}+f_{2}\left(\mathbf{u}_{\mathbf{1}}\right) \theta_{2}+f_{n}\left(\mathbf{u}_{\mathbf{1}}\right) \theta_{n}= & y_{1} \\
f_{1}\left(\mathbf{u}_{\mathbf{2}}\right) \theta_{1}+f_{2}\left(\mathbf{u}_{\mathbf{2}}\right) \theta_{2}+f_{n}\left(\mathbf{u}_{\mathbf{2}}\right) \theta_{n}= & y_{2} \\
\vdots & \vdots & \vdots \\
f_{1}\left(\mathbf{u}_{\mathbf{m}}\right) \theta_{1}+f_{2}\left(\mathbf{u}_{\mathbf{m}}\right) \theta_{2}+f_{n}\left(\mathbf{u}_{\mathbf{m}}\right) \theta_{n}= & y_{m}
\end{array}\right.
$$

This can be written in matrix notation as:

$$
\mathbf{A} \theta=\mathbf{y}
$$

where; $\mathbf{A}=\left[\begin{array}{ccc}f_{1}\left(\mathbf{u}_{\mathbf{1}}\right) & \cdots & f_{n}\left(\mathbf{u}_{\mathbf{1}}\right) \\ \vdots & \vdots & \vdots \\ f_{1}\left(\mathbf{u}_{\mathbf{m}}\right) & \vdots & f_{n}\left(\mathbf{u}_{\mathbf{m}}\right)\end{array}\right], \quad \theta=\left[\begin{array}{c}\theta_{1} \\ \vdots \\ \theta_{n}\end{array}\right], \quad \mathbf{y}=\left[\begin{array}{c}y_{1} \\ \vdots \\ y_{n}\end{array}\right]$

The least squares estimator (LSE) $\hat{\theta}$ is the best solution of the system of linear equations which minimizes the sum of squared error $\|\mathbf{A} \theta-y\|^{2}$ and satisfies the normal equation;

$$
\mathbf{A}^{T} \mathbf{A} \theta=\mathbf{A}^{T} y,
$$

and provided that $\mathbf{A}^{T} \mathbf{A}$ is singular, $\hat{\theta}$ is unique (Jang et al., 1997). The LSE $\hat{\theta}$ is given by;

$$
\hat{\theta}=\left(\mathbf{A}^{T} \mathbf{A}\right)^{-1} \mathbf{A}^{T} y
$$

Jang et al. (1997) also describes a recursive method for calculating the least squares estimator.

\section{Advantages of Hybrid Neuro-Fuzzy Inference Systems}

At present there are no applications of hybrid neuro-fuzzy systems in the geosciences. Sun \& Jang (1993) have applied an adaptive network-based neuro-fuzzy inference procedure to data classification. A hybrid modular fuzzy neural network system for selecting optimal sites for land development using geographic data has been described by Kasabov \& Trifonov (1993).

Although neuro-fuzzy systems are varied in their architecture and mode of operation, general advantages of a combined approach include;

1. a combined approach allows both expert knowledge and training data to be incorporated into the system 
2. performance of hybrid systems is often superior to that of the component systems used individually, and

3. modular hybrid and adaptive fuzzy inference systems are often easier to develop than alternative neural network or static fuzzy systems. 


\section{Appendix B}

\section{Documentation of GIS Databases}

\section{B.1 Directory Structure of GIS Databases on CD-ROM}

CD volume 1 contains a copy of all the primary and derived ARCVIEw GIS thematic layers and associated database tables used in the Kalgoorlie case study. In addition, CD volume 1 contains the code for $\mathrm{C}++$ programs used in the Tenterfield pilot study and the MAtLAB scripts and functions used in the Kalgoorlie case study, and a text file which explains the meaning of the abbreviated ARCVIEW layer names.

CD volume 2 contains mineral prospectivity maps and input GIS layers in GeOsofT format, and software to install a GEOSOFT viewer.

\section{B.2 Metadata for the GIS Data Sets}

\section{B.2.1 What are Metadata?}

Metadata provides information about the characteristics of a data set. For example, metadata at a summary level of detail describe the content, geographic extent, date of collection or compilation, positional accuracy, source and contact details for the custodian of the data set. This enables data sets to be indexed and allows potential users to determine which data exist for a particular geographic location, assess the usefulness of the data for a specific purpose, and acquire the data set or obtain further information from the custodian. 
Table B.1: Directory structure of GIS databases on the CD-ROM

\begin{tabular}{|l|l|}
\hline \multicolumn{2}{|l|}{ CD-ROM 1} \\
\hline Directory name & Description \\
\hline polygon_layers & Vector (point, line \& polygon) layers; e.g. deposits, faults and solid geology \\
\hline raster_layers & Raster layers (cell size $=100 \mathrm{~m}$ ); i.e. all derived GIS layers \\
\hline info & ARCVIEW attribute tables for vector layers; e.g. MINDEX deposit DB \\
\hline arcview.apr & ARCVIEW project file for Kalgoorlie GIS database \\
\hline Matlab programs & $\begin{array}{l}\text { MATLAB scripts and functions for pre- \& post-processing, neural networks, } \\
\text { and fuzzy logic }\end{array}$ \\
\hline
\end{tabular}

\section{CD-ROM 2}

\begin{tabular}{|l|l|}
\hline Directory name & Description \\
\hline geosoft maps & Top-level directory containing input GIS layers and prospectivity maps \\
\hline maps neural & Neural-network mineral prospectivity maps; entire study area \\
\hline maps noise & NN-prospectivity maps created with noise-augmented training sets \\
\hline maps fuzzy systems & Fuzzy logic mineral prospectivity maps \\
\hline maps fuzzy neural & Fuzzy-neural-network mineral prospectivity maps \\
\hline maps detail neural & Neural-network mineral prospectivity maps; area around Coolgardie \\
\hline maps detail noise & Noise-augmented, NN mineral prospectivity maps; area around Coolgardie \\
\hline maps detail fuzzy & Fuzzy logic mineral prospectivity maps; area around Coolgardie \\
\hline $\begin{array}{l}\text { maps detail fuzzy } \\
\text { neural }\end{array}$ & Fuzzy-neural-network mineral prospectivity maps; area around Coolgardie \\
\hline maps base map & Base map with geographic and AMG coordinates and scale \\
\hline maps location & Maps of Western Australia showing location of study area \\
\hline grids & Raster layers used to create input GIS and prospectivity maps \\
\hline input gis layers & Input layers and prospectivity maps with geographic and AMG coordinates \\
\hline tbl & Tables required by GEOsofT for legend colour schemes \\
\hline
\end{tabular}

Detailed metadata provides information that may be needed by users to access, process and interpret the data supplied in digital format by transfer or purchase from another organization. For example a thematic layer in a GIS package may be stored in Arc/Info Export Format or MAPINFo Interchange Format (MIF) or the scheme used to encode an image may be Band Interleaved by Line (BIL), Band Interleaved by Pixel (BIP) or Band Interleaved Sequential (BSQ). Entities, attributes and domains for information stored in database tables must be documented in order for users to correctly interpret and maintain the data. 
Metadata are particularly important for digital geospatial data sets because metadata is not normally a part of the data set itself. In contrast, a paper map usually contains printed information about the date of compilation, projection, grid system and details of the individuals and organization responsible for producing it. Further, the content and method used to encode digital data may not be clear as it is for a paper map with a legend.

\section{B.2.2 Metadata Standards}

The metadata used to document the data sets used in this study follow the recommendations of the Australia New Zealand Land Information Council (ANZLIC) and the United States Federal Geographic Data Committee (FGDC).

\section{Australia New Zealand Land Information Council (ANZLIC) Guidelines}

ANZLIC is the main body responsible for coordinating the management of geospatial information in Australia. One of the main tasks of ANZLIC is to develop and implement a nationally consistent geographic data directory system using summary-level metadata. ANZLIC has published a guide which defines a minimum number of characteristics, which should be described at the summary level, called core metadata elements ANZLIC (1996). This is the mandatory information required for the national metadata directory system and is general enough to be independent of the type of data involved.

ANZLIC only provides a guide to the content and format for a minimum amount of summary metadata. These core metadata items are also referred to as Page 0 within the hierarchical metadata framework proposed by ANZLIC. In addition to Page 0, state or theme-based directories contain more detailed metadata (referred to as Page 1). These types of metadata contain general descriptions of the data sets and information about the custodian of the data. Agency-based directories collect and maintain more detailed metadata (referred to as Page 2 metadata) in additional to the information prescribed by the standards for the first two categories. The Australian National Geoscience Information System (NGIS)is an example of a theme-based or page-0-type directory and the Minerals and Energy Airborne Geophysics Information Exchange (MAGIX), a Geographic Information System-based register and index of airborne geophysical surveys reported to the Department of Minerals and Energy Western Australia, is an example of an agency-based directory.

Unfortunately, ANZLIC does not provide details on the type and format of metadata to 
be included in Page 1 and Page 2 categories. Instead, it is only suggested that additional information be consistent with the corresponding metadata elements in the U.S. Federal Government Data Committee (FGDC) content standard FGDC (1998).

\section{Federal Government Data Committee(FGDC)Guidelines}

The U.S. Federal Government Data Committee (FGDC) provides very detailed information about how to document spatial data stored in electronic form. The FGDC standards FGDC (1998) specifies the names, format and type of content for 220 items which describe digital geospatial data sets. The FGDC metadata standard was used as a basis for the draft International Organization for Standardization's metadata standard (ISO Metadata Standard 14056 Part 15).

\section{B.2.3 Metadata Format used in This Study}

Both the ANZLIC metadata guidelines and the FGDC metadata standard are used to document the data sets which were used in this study. The ANZLIC guidelines are used for summary information about each data set (e.g. Kalgoorlie data set, Appendix D) or category of data, where a data set has multiple sources (e.g. the Tenterfield intrusionhosted gold deposit). Additional items from the FGDC metadata standard, which are relevant to the data set being described, are used to provide more detailed information. The format of the metadata presented in this study is the same as that used by the Department of Minerals and Energy, Western Australia, and is based on a sample metadata file for the Bangemall Basin data set provided by Steve Bandy (Manager, Geoscience Data Management, Geological Survey of Western Australia; Bandy, 1997).

\section{B.3 Data Dictionaries for GIS Database Layers}

\section{B.3.1 Data Dictionaries for Spatial Attributes}

A data dictionary is provided for each thematic layer in the GIS databases. A data dictionary describes the attributes stored in a database table associated with a thematic layer. The attributes are equivalent to the column headings in the table and are also referred to as fields or items. Each data dictionary contains the following information: name of the thematic layer (also referred to as coverage), coverage class (point, line, polygon), and for each attribute; the attribute name, attribute type and a brief description. 
In the ARCVIEw GIS package, different attribute types are described by the following codes;

$\mathrm{B}=$ binary integers,

$\mathrm{C}=$ a character of ASCII text,

$\mathrm{D}=$ data in format YYYYMMDD,

$\mathrm{F}=$ decimal number stored in internal floating point format,

$\mathrm{I}=$ integer, and

$\mathrm{N}=$ decimal number.

Database tables associated with thematic layers contain two types of attributes; spatial and non-spatial. In ARC/INFo and ARCView, the spatial attributes are generated automatically and appear in the left-most columns of the database table. The internal spatial attributes differ according to whether thematic layer contains point, line or polygon data. The internal spatial attributes generated by Arc/Info for point and polygon coverages are shown in Table B.2 below.

Table B.2: Spatial attributes generated automatically by ARC/InFo for point and polygon coverages

\begin{tabular}{|l|c|l|}
\hline Item names & Type & Item description \\
\hline AREA & $\mathrm{F}$ & $\begin{array}{l}\text { Area of polygon calculated by ARC/InFO. Set to zero for point cov- } \\
\text { erages. }\end{array}$ \\
\hline PERIMETER & $\mathrm{F}$ & $\begin{array}{l}\text { Perimeter of polygon calculated by Arc/Info. Set to zero to point } \\
\text { coverage. }\end{array}$ \\
\hline$<$ coverage_name>\# & $\mathrm{B}$ & $\begin{array}{l}\text { Internal number of point/polygon assigned and maintained by } \\
\text { Arc/Info. }\end{array}$ \\
\hline$<$ coverage_name>-ID & $\mathrm{B}$ & $\begin{array}{l}\text { Unique user-assigned point/polygon identifier. If not assigned by } \\
\text { user, defaults to <coverage_name>\#. }\end{array}$ \\
\hline
\end{tabular}

The internal spatial attributes generated by ARC/INFO for line coverages are shown in Table B.3 below. In the ARCVIEw GIS package, there is a single file type for all vector coverages (i.e. all data stored as points, lines and polygons) called a shape file. The spatial attributes generated automatically for shape files are shown in Table B.4 below. 
Table B.3: Spatial attributes generated automatically by ARC/Info for line coverages

\begin{tabular}{|l|c|l|}
\hline Item names & Type & Item description \\
\hline FNODE\# & F & Internal number of the from-node. Arc starts at From-node. \\
\hline TNODE\# & F & Internal number of the to-node. Arc ends at to-node. \\
\hline LPOLY\# & B & Left polygon number assigned and maintained by ARC/INFO. \\
\hline RPOLY\# & B & Right polygon number assigned and maintained by ARC/INFO. \\
\hline LENGTH & F & Length of arc measured in coverage units. \\
\hline$<$ coverage_name>\# & B & Internal number of arc assigned and maintained by ARC/INFO. \\
\hline$<$ coverage_name>-ID & B & $\begin{array}{l}\text { Unique user-assigned line identifier. If not assigned by user, defaults } \\
\text { to }<\text { coverage_name }>\# .\end{array}$ \\
\hline
\end{tabular}

Table B.4: Spatial attributes generated automatically by ArCVIEW for point, line and polygon coverages

\begin{tabular}{|l|c|l|}
\hline Item names & Type & Item description \\
\hline SHAPE & B? & Type of coverage: point, line or polygon \\
\hline AREA & F & $\begin{array}{l}\text { Area of polygon calculated by ARC/INFO. Set to zero for point cov- } \\
\text { erages. }\end{array}$ \\
\hline PERIMETER & F & $\begin{array}{l}\text { Perimeter of polygon calculated by ARC/INFO. Set to zero to point } \\
\text { coverage. }\end{array}$ \\
\hline$<$ coverage_name>\# & B & $\begin{array}{l}\text { Internal number of point/polygon assigned and maintained by } \\
\text { ARC/INFO. }\end{array}$ \\
\hline$<$ coverage_name>-ID & B & $\begin{array}{l}\text { Unique user-assigned point/polygon identifier. If not assigned by } \\
\text { user, defaults to <coverage_name }>\# . ~\end{array}$ \\
\hline
\end{tabular}




\section{Appendix C}

\section{Metadata for Tenterfield GIS Database}

\section{C.1 Location of the Tenterfield Data Set}

The Tenterfield data set used for the pilot study described in Chapter 4 corresponds to the area of the Tenterfield 1:100000 sheet (map 9339). The area is located in north eastern New South Wales and represents part of the southern portion of the New England Orogen (Fig. C.1 below).

The boundary of the Tenterfield 1:100000 map sheet is defined in geographic coordinates. Coordinates of the map corners in both geographic coordinates and Australian Map Grid (AMG) coordinates are given in Table C.1. AMG coordinates must be used in conjunction with the zone number, 56. Geographic coordinates based on the Australian Geodetic Datum (AGD66) were converted to the cartesian coordinate system of the Australian Map Grid (AMG66) by applying a Universal Transverse Mercator projection using Redfearn's formulae (Redfearn, 1948; NATMAP, 1986). Ellipsoid parameters used for the conversion are those of the Australian National Spheroid (ANS). All standards used (ANS, AGD66 and AMG66) are standards current at the time of the publication of the Grafton 1:250000 geological map (Brunker \& Chesnut, 1976). 


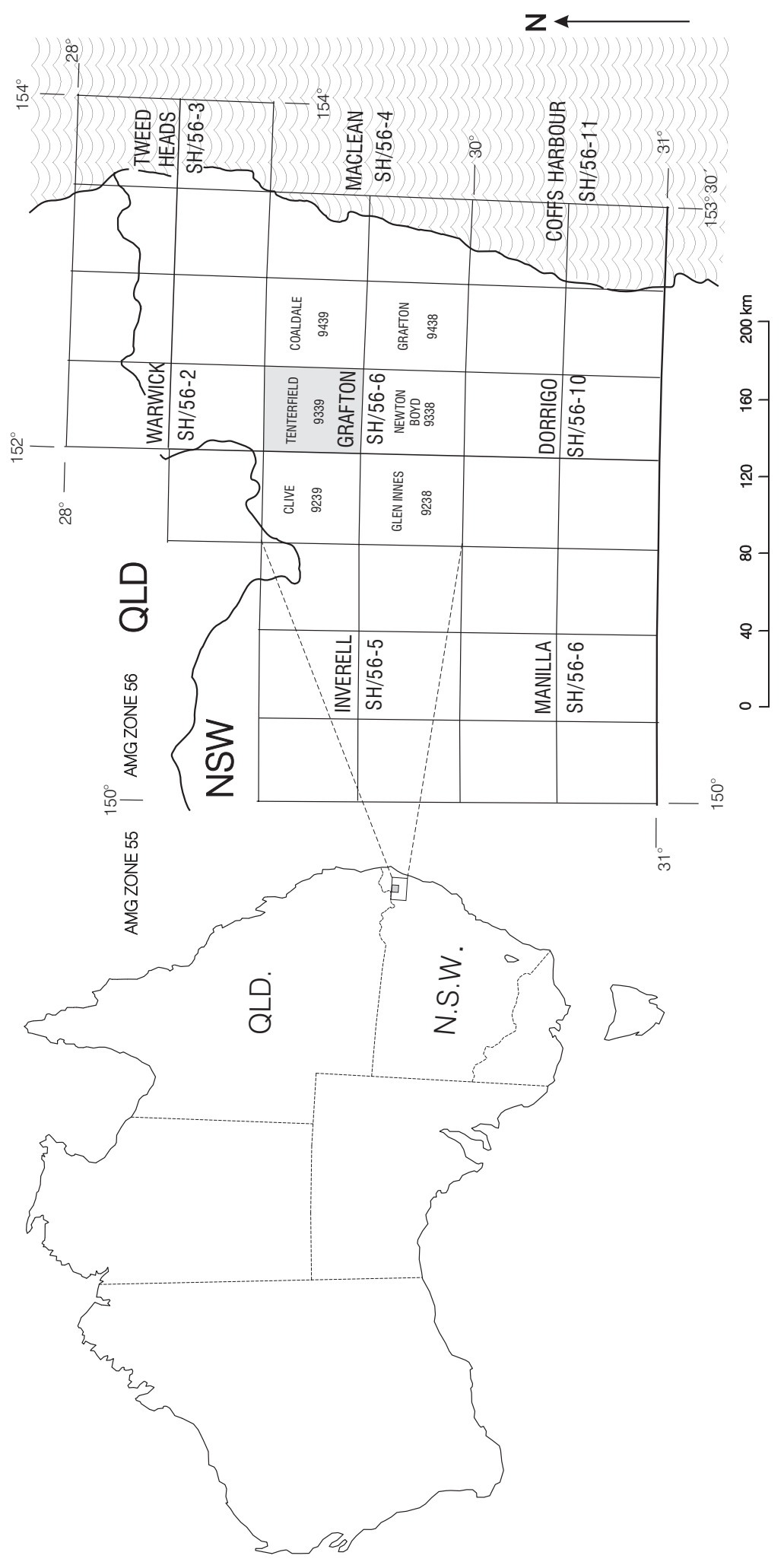

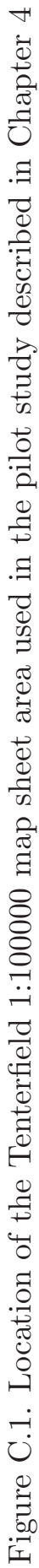


Table C.1: Location of the Tenterfield 1:100000 map sheet in latitude and longitude and AMG coordinates. The Tenterfield area is located in AMG zone 56.

\begin{tabular}{|l|l|l|l|l|}
\hline Corner & Longitude & Latitude & Easting & Northing \\
\hline \multirow{2}{*}{ Northwest } & Deg. Min. & Deg. Min. & Metres & Metres \\
\cline { 2 - 5 } & $152^{\circ} \mathrm{E}$ & $29^{\circ} \mathrm{S}$ & 402597.1 & 6791591.2 \\
\hline Northeast & $152^{\circ} 30^{\prime} \mathrm{E}$ & $29^{\circ} \mathrm{S}$ & 451299.5 & 6791900.3 \\
\hline Southeast & $152^{\circ} 30^{\prime} \mathrm{E}$ & $29^{\circ} 30^{\prime} \mathrm{S}$ & 451535.8 & 6736501.2 \\
\hline Southwest & $152^{\circ} \mathrm{E}$ & $29^{\circ} 30^{\prime} \mathrm{S}$ & 403069.6 & 6736188.7 \\
\hline \hline Standards & $\begin{array}{l}\text { Ellipsoid } \\
\text { Datum } \\
\text { Geographic coordinate set } \\
\text { Grid coordinates (UTM) }\end{array}$ & $\begin{array}{l}\text { Aust. National Spheroid (ANS) } \\
\text { Aust. Geodetic Datum (AGD) } \\
\text { AGD66 } \\
\text { Australian Map Grid (AMG66) }\end{array}$ \\
\hline $\begin{array}{l}\text { Ellipsoid } \\
\text { parameters }\end{array}$ & $\begin{array}{l}\text { Semi-major axis } \\
\text { Inverse flattening }\end{array}$ & $\begin{array}{l}\text { a }=6,378,160.0 \mathrm{~m} \\
1 / \mathrm{f}=298.25\end{array}$ \\
\hline $\begin{array}{l}\text { AGD } \\
\text { parameters }\end{array}$ & $\begin{array}{l}\text { Initial zone number } \\
\text { Initial central meridian longitude } \\
\text { Zone width } \\
\text { Central scale factor } \\
\text { False easting } \\
\text { False northing }\end{array}$ & $\begin{array}{l}31 \\
3 \text { degrees } \\
6 \text { degrees } \\
0.9996 \\
500,000 \mathrm{~m} \\
10,000,000 \mathrm{~m}\end{array}$ \\
\hline
\end{tabular}

In some cases, the Tenterfield data were extracted from larger regional data sets. The airborne geophysical data were acquired over the whole Inverell and Grafton 1:250000 map sheets (Fig. C.1) below) and the main stream sediment geochemistry data set, the NRAC survey of the Upper North East covers portions of the Warwick, Tweed Heads, Grafton, Maclean, Dorrigo, and Coffs Harbour 1:250000 sheet areas.

\section{C.2 Overview of Primary Thematic Layers}

Table C.2 lists all the data sets in the Tenterfield GIS package, which were supplied by the Geological Survey of NSW. The coverages shown below are in ARCVIEW shape file format. All image files were supplied in IDRISI image format (designated by the suffix, $i m g$ ) except for the igrrad image which is in BIL format. The tables are in ARCVIEW tables format. The term Upper North East Region is used by the Geological Survey of NSW and includes portions of the Warwick, Tweed Heads, Grafton, Maclean, Dorrigo, and Coffs Harbour 1:250000 sheet areas. File names of layers in the GIS database are shown in typewriter font. 
Table C.2: Primary thematic layers in the Tenterfield GIS database

\begin{tabular}{|c|c|}
\hline Layer & Description \\
\hline uner5ge & Solid geology of the Upper North East region, NSW. \\
\hline t_geology & Solid geology of the Tenterfield 1:100000 map sheet area (Map 9339). \\
\hline unew5fa & Regional scale faults and shear zones in the Upper North East region, NSW. \\
\hline t_struct & Regional scale faults and shear zones in the Tenterfield 1:100000 map sheet area. \\
\hline t_deps & Mineral deposits and occurrences in the Tenterfield 1:100000 sheet area. \\
\hline t_nracstrmgc & $\begin{array}{l}\text { Tenterfield 1:100000 map sheet subset of Upper North East region stream sediment } \\
\text { geochemistry data set. }\end{array}$ \\
\hline t_strmgc & Stream geochemistry (mainly $\mathrm{Cu}, \mathrm{Pb} \& \mathrm{Zn}$ ) over Tenterfield area. \\
\hline t_whrkgc & Whole-rock geochemistry over the Tenterfield area. \\
\hline Image Name & Description \\
\hline igu & U channel radiometric image covering Tenterfield 1:100,000 sheet area. \\
\hline igth & Th channel radiometric image covering Tenterfield 1:100,000 sheet area. \\
\hline igk & K channel radiometric image covering Tenterfield 1:100,000 sheet area. \\
\hline igtc & Total count radiometric image covering Tenterfield 1:100,000 sheet area. \\
\hline ingrrad & $\begin{array}{l}\text { Composite radiometric image over parts of Inverell, and Grafton 1:250000 map } \\
\text { sheets. Channels: red }=\mathrm{U} \text {, green }=\mathrm{Th} \text {, blue }=\mathrm{K} \text { ). ARCVIEW BIL image format } \\
\text { (file suffixes: .bil, .hdr, \& .stx). }\end{array}$ \\
\hline igmag & total intensity magnetic image covering Tenterfield 1:100,000 sheet area. \\
\hline Table Name & Description \\
\hline unew5gel2 & $\begin{array}{l}\text { Details on Quaternary, Tertiary and basin sedimentary units for the Upper North } \\
\text { East Region geology coverage, oldage_my. }\end{array}$ \\
\hline geolage & Explanation of oldage_my \& yngage_my attributes codes in $t_{-} g e o l o g y$ cover. \\
\hline geolprov & Explanation of geol_province attribute codes in $t_{-}$geology coverage. \\
\hline geolsys & $\begin{array}{l}\text { Links tectonostratigraphic division attribute (geolsystem) to } t_{-} \text {geology coverage } \\
\text { table. via common symbol attribute. }\end{array}$ \\
\hline gransuit & $\begin{array}{l}\text { Links gransuit and sfsuite and description attributes to the } t_{-} \text {geology coverage } \\
\text { table via common symbol attribute. }\end{array}$ \\
\hline domlith & $\begin{array}{l}\text { Links description and colour attributes to } t_{-} \text {geology coverage table via the domlith } \\
\text { attribute (same as the lithology attribute in } t_{-} g e o l o g y \text { coverage). }\end{array}$ \\
\hline depsize & $\begin{array}{l}\text { Describes equivalence between the size, size } 2 \text { and value } 1967 a \text { attribute values in } \\
\text { the } t_{-} \text {deps coverage. }\end{array}$ \\
\hline depcodeb & $\begin{array}{l}\text { Links label, code, add_commod and examples fields to the mineral deposits cover- } \\
\text { age, t_deps via code attribute. }\end{array}$ \\
\hline
\end{tabular}




\section{C.3 Metadata for Tenterfield GIS Database}

The Tenterfield GIS package consists of a number of different unpublished digital geology, geophysics, geochemistry, and deposit location data sets, which were compiled by Robert Barnes at the Armidale Office of the Geological Survey, Department of Minerals and Energy, NSW (Barnes, 1993; Barnes et al., 1995). Since the data sets were collected by different groups at different times and have different geographic extents, there are separate sets of metadata for each of these categories. The data in Section C.4.1 are summarized from unpublished reports held at the Armidale office of the Geological Survey of NSW (Barnes, 1995; Barnes \& Suppel, 1995; Barnes, 1997a,b).

\section{C.4 Geological Data}

\section{C.4.1 Core Metadata Elements for Geological Data}

The core metadata items (Page 0) described in the Australia New Zealand Land Information Council (ANZLIC) metadata guidelines (ANZLIC, 1996) are given in Table C.3 below.

Table C.3: ANZLIC core metadata elements for Tenterfield geological data

\begin{tabular}{|l|l|l|}
\hline \multirow{5}{*}{ Dataset } & Title & $\begin{array}{l}\text { unew5ge - regional geology layer: Upper } \\
\text { North East region, NSW }\end{array}$ \\
\cline { 2 - 3 } Description & Custodian & NSW Department of Mineral Resources \\
\cline { 2 - 3 } & Jurisdiction & New South Wales \\
\hline \multirow{5}{*}{ Abstract } & $\begin{array}{l}\text { Geology of the Upper North East region comprises por- } \\
\text { tions of the Warwick, Tweed Heads, Grafton, Maclean, } \\
\text { Dorrigo, and Coffs Harbour 1:250,000 sheet areas in- } \\
\text { cluding a contribution from NRAC Project S1(C) }\end{array}$ \\
\cline { 2 - 3 } & Search Words & $\begin{array}{l}\text { GEOSCIENCES, } \\
\text { GEOSCIENCES Geochemistry }\end{array}$ \\
\cline { 2 - 3 } & $\begin{array}{l}\text { Geographic } \\
\text { Extent Name(s) }\end{array}$ & $\begin{array}{l}\text { Warwick 1:250000 Map Sheet, Tweed Heads 1:250,00 } \\
\text { Map Sheet, Grafton 1:250000 Map Sheet, Maclean } \\
1: 250000 \text { Map Sheet, Dorrigo 1:250000 Map Sheet, } \\
\text { Coffs Harbour 1:250000 Map Sheet }\end{array}$ \\
\hline \multirow{2}{*}{ Data Currency } & Beginning Date & 01JAN1971 \\
\cline { 2 - 3 } & Ending Date & 01JAN1994 \\
\hline
\end{tabular}


Table C.3: continued

\begin{tabular}{|c|c|c|}
\hline \multirow[t]{2}{*}{ Dataset Status } & Progress & Complete \\
\hline & $\begin{array}{l}\text { Maintenance and } \\
\text { Update } \\
\text { Frequency }\end{array}$ & Not Known \\
\hline \multirow[t]{3}{*}{ Access } & $\begin{array}{l}\text { Stored Data } \\
\text { Format }\end{array}$ & $\begin{array}{l}\text { DIGITAL ARC/INFO } 7.0 .3 \text { under SUNOS, vector cov- } \\
\text { erage, AMG } 66\end{array}$ \\
\hline & $\begin{array}{l}\text { Available Format } \\
\text { Type }\end{array}$ & ARC/InFo coverage, ARCVIEW shape file \\
\hline & Access Constraint & Available for research, subject to license agreement. \\
\hline \multirow[t]{3}{*}{ Data Quality } & Lineage & $\begin{array}{l}\text { The upper North East Region includes the Grafton } \\
\text { 1:250000 sheet, which contains the Tenterfield 1:100000 } \\
\text { sheet. Published and unpublished geological maps were } \\
\text { compiled onto 1:25,000 topographic maps and then re- } \\
\text { compiled at 1:100000 scale and further simplified and } \\
\text { recompiled at 1:250000 scale. } \\
\text { The map was then digitized using INFOMAP and PC } \\
\text { ARC/INFO software. The Upper North East region } \\
\text { geology (stored as digital coverages: uner_geol and } \\
\text { unew5ge) was compiled from both the } 1: 100000 \text { and } \\
\text { 1:250000 scale maps in } 1995 \text { (Barnes \& Willis, } 1989 \text {; } \\
\text { Barnes, 1997b). }\end{array}$ \\
\hline & $\begin{array}{l}\text { Positional } \\
\text { Accuracy }\end{array}$ & $\begin{array}{l}\text { Most geological boundaries and features have a hori- } \\
\text { zontal accuracy of } 10-500 \mathrm{~m} \text {. Geological reliability is } \\
\text { moderate to good. In many parts of the map the ter- } \\
\text { rain is extremely rugged and little detailed geological } \\
\text { mapping has been done. }\end{array}$ \\
\hline & $\begin{array}{l}\text { Attribute } \\
\text { Accuracy }\end{array}$ & $\begin{array}{l}\text { Each unit on the geological map is given a letter symbol } \\
\text { using a convention described by Stroud (1994). The } \\
\text { main attribute in the geology coverage is the litho- } \\
\text { stratigraphic unit. } \\
\text { The map shows stratigraphic units, which contain sev- } \\
\text { eral rock types, any one of which may be present at } \\
\text { a particular location. Areas were mapped at different } \\
\text { levels of detail. } \\
\text { Approximately two field days per } 1: 25,000 \text { map area } \\
\text { were spent on reconnaissance checking over the Grafton } \\
\text { 1:250000 sheet area. }\end{array}$ \\
\hline
\end{tabular}


Table C.3: continued

\begin{tabular}{|c|c|c|}
\hline & Logical Accuracy & $\begin{array}{l}\text { The data were checked during the initial map compila- } \\
\text { tions but the new compilation has not been subject } \\
\text { to rigourous checking. Some polygons are not fully } \\
\text { labelled and some lines overshoot or undershoot and } \\
\text { sliver polygons exist. }\end{array}$ \\
\hline & Completeness & Attribute data are available for the entire area. \\
\hline \multirow[t]{11}{*}{ Contact Information } & $\begin{array}{l}\text { Contact } \\
\text { Organization }\end{array}$ & Geological Survey of NSW \\
\hline & Contact Position & Senior Geologist \\
\hline & Mail Address 1 & P.O. Box 65 \\
\hline & Mail Address 2 & \\
\hline & Suburb/Locality & Armidale \\
\hline & State/Locality 2 & NSW \\
\hline & Country & Australia \\
\hline & Postcode & 2350 \\
\hline & Telephone & $\begin{array}{l}\text { Australia Fixed Network Number } \\
0267702118 \text { (Armidale) } \\
0299018888 \text { (St Leonards, Sydney) }\end{array}$ \\
\hline & Facsimile & 0267702121 \\
\hline & Email & barnesr@minerals.nsw.gov.au \\
\hline Metadata Date & Metadata Date & 01SEP1995 \\
\hline Additional Metadata & & $\begin{array}{l}\text { See Barnes (1995); Barnes \& Suppel (1995); Barnes } \\
(1997 a, b)\end{array}$ \\
\hline
\end{tabular}

\section{C.4.2 Data Dictionary for Regional Geology Layer}

The data dictionaries that describe the non-spatial attributes for each thematic layer are presented as separate tables below. The internal spatial attributes are not shown. There is no data dictionary for the structural coverage $t$ struct since it does not contain any non-spatial attributes. Table C.4 shows the data dictionary for the Upper North East Region geology, corresponding to the layer; uner_geol. 
Table C.4: Data dictionary for the Tenterfield regional geology coverage (Upper North East Region, NSW)

\begin{tabular}{|l|c|l|}
\hline \multicolumn{2}{|l|}{ Coverage name: uner_geol $^{\text {a }}$} \\
\hline \multicolumn{2}{|l|}{ Coverage type: Polygon } \\
\hline Item names & Type & Item description \\
\hline sym_arc & $\mathrm{C}$ & $\begin{array}{l}\text { Unique code for each polygon used to link data in the sym_geol LUT to the } \\
\text { geology coverage. }\end{array}$ \\
\hline lpoly & $\mathrm{I}$ & Identifier for polygon on the left side of current polygon. \\
\hline rpoly & $\mathrm{I}$ & Identifier for polygon on the right side of current polygon. \\
\hline
\end{tabular}

\section{C.4.3 Data Dictionary for Tenterfield Geology Layer}

The data dictionary for the Tenterfield geology (layer: $t_{-} g e o l$ ) is shown in Table C.5 below.

Table C.5: Data dictionary for the Tenterfield 1:100000 map sheet geology coverage

\begin{tabular}{|l|c|l|}
\hline \multicolumn{2}{|l|}{ Coverage name: $t$ geol } \\
\hline Coverage type: Polygon \\
\hline Item names & Type & Item description \\
\hline mapno & $\mathrm{C}$ & Number of NSW Geological Survey 1:100000 map sheet for area. \\
\hline sym_arc & $\mathrm{C}$ & $\begin{array}{l}\text { Unique code for each polygon used to link data in the sym_geol LUT to the } \\
\text { geology coverage. }\end{array}$ \\
\hline mapnum & $\mathrm{C}$ & Number of NSW Geological Survey 1:100000 map sheet for area. \\
\hline geolprov & $\mathrm{C}$ & Major geological province in which the unit occurs. \\
\hline supergp & $\mathrm{C}$ & Tectonostratigraphic terranes after Flood \& Aitchison (1988). \\
\hline gpsuite & $\mathrm{C}$ & Group or plutonic suite. \\
\hline subgp & $\mathrm{C}$ & Subgroup. \\
\hline frm_plut & $\mathrm{C}$ & Formation or pluton. \\
\hline bath_no & $\mathrm{I}$ & Pluton number listed in confidential report by Chappell \& Bryant (1994). \\
\hline mem_body & $\mathrm{C}$ & Member or granite body. \\
\hline all_strat2 & $\mathrm{C}$ & $\begin{array}{l}\text { Stratigraphic name components for each unit; i.e. supergroup/group or plu- } \\
\text { tonic suite/subgroup/formation or pluton/member of body. }\end{array}$ \\
\hline lithology & $\mathrm{C}$ & $\begin{array}{l}\text { Dominant lithology within a unit (see explanation of codes in C.8 below. Litho- } \\
\text { logical units are generalized and do not follow strict definitions; e.g granite } \\
\text { includes a range of felsic plutonic rocks and not just granite senso stricto. }\end{array}$ \\
\hline
\end{tabular}


Table C.5: continued

\begin{tabular}{|l|c|l|}
\hline lith_descr & C & Range of rock types that can be found in mapped units. \\
\hline oldage_my & I & $\begin{array}{l}\text { Code for the oldest age of unit in terms of period or epoch in the geological } \\
\text { time scale. below. }\end{array}$ \\
\hline yngage_my & I & $\begin{array}{l}\text { Code for the youngest age of unit in terms of period or epoch in the geological } \\
\text { time scale. below. }\end{array}$ \\
\hline oldage_ab & I & $\begin{array}{l}\text { Old end of range of absolute ages quoted for unit (Ma) or old end of error } \\
\text { range (mainly from Shaw \& Flood, 1993). }\end{array}$ \\
\hline yngage_ab & I & $\begin{array}{l}\text { Young end of range of absolute ages quoted for unit (Ma) or young end of } \\
\text { error range. }\end{array}$ \\
\hline symbol & I & Colour code for use with the AGSO shade set AGSO.shd. \\
\hline screen & I & $\begin{array}{l}\text { Texture code for use with shadeset CARTO.shd; applies an overprint on some } \\
\text { units. }\end{array}$ \\
\hline
\end{tabular}

The geol_prov attribute is restricted to the following values;

$\begin{array}{ll}\text { NEW ENGLAND = } & \text { all rocks in the basement sedimentary and granite units of } \\ & \text { the New England Orogen, } \\ \text { CLARENCE } & \text { rocks of the Clarence-Moreton basement sedimentary and } \\ & \text { granite units, which form most of the northeastern part } \\ & \text { of the coverage, } \\ = & \text { all Tertiary volcanics, intrusives and sediments, } \\ = & \text { all Quaternary rocks consisting mainly of alluvium and } \\ \text { TERTIARY coastal sands. }\end{array}$

\section{C.4.4 Data Dictionary for Geological Province Table}

Table C.6 shows the data dictionary for the table, geolprov.

Table C.6: Data dictionary for database table, geolprov

\begin{tabular}{|l|c|l|}
\hline \multicolumn{2}{|l|}{ Table name: geolprov } \\
\hline Item names & Type & Item description \\
\hline geolprov & $\mathrm{C}$ & $\begin{array}{l}\text { Geological province; e.g. NEWENGLAND, CLARENCE, AND COVER. } \\
\text { This field is used as a foreign key to link this table to the main Tenterfield } \\
\text { geology table, } t_{-} \text {geol. }\end{array}$ \\
\hline description & $\mathrm{C}$ & Free form textural description of the packages of rocks found in the province. \\
\hline
\end{tabular}




\section{C.4.5 Data Dictionary for Tenterfield Granite Suite Table}

Table C.7 shows the data dictionary for the Tenterfield granite suite table, gransuit.

Table C.7: Data dictionary for database table, gransuit

\begin{tabular}{|c|c|c|}
\hline Item names & Туре & Item description \\
\hline gransuit & I & Code for plutonic suite assigned according to Chappell \& Bryant (1994). \\
\hline sfgsuite & I & $\begin{array}{l}\text { Code for plutonic suite interpreted according to Shaw \& Flood (1981). The } \\
\text { codes are the same as those for gransuite but interpretations of individual } \\
\text { units is different. }\end{array}$ \\
\hline $\operatorname{lgd} \_$pos & I & Legend position. Units with higher numbers are younger. \\
\hline desc & $\mathrm{C}$ & Name of plutonic suite from Chappell \& Bryant (1994). \\
\hline symbol & I & $\begin{array}{l}\text { Number code for colour of unit using the } A G S O S H D \text { shade set obtained from } \\
\text { the Australian Geological Survey Organisation. This field is used as a foreign } \\
\text { key to link this table to the main Tenterfield geology table, } t_{-} \text {geol. }\end{array}$ \\
\hline
\end{tabular}

Values of the lithology attribute are codes defined in the Mineral Resources Land Information System (MRLIS) of the New South Wales Department of Mineral Resources. An explanation of the MRLIS dominant lithology codes is given in Table C.8.

Table C.8: Explanation of MRLIS dominant lithology codes used in t_geol attribute table

\begin{tabular}{|l|l|}
\hline Lithology code & Description \\
\hline AMPHIBOL & Amphibolite \\
\hline ANDESITE & Andesite \\
\hline BASALT & Basalt \\
\hline CARBONSED & Carbonaceous sediment \\
\hline CHERT & Chert \\
\hline CLAY & Clay \\
\hline COLLUVIUM & Colluvial/eluvial deposits \\
\hline CONGLOM & Conglomerate, sedimentary breccia \\
\hline DIORITE & Diorite \\
\hline DUNE & Dune and sandplain-unconsolidated sand and silt \\
\hline ECLOGITE & Eclogite \\
\hline FERRICRETE & Ferricrete \\
\hline
\end{tabular}


Table C.8: continued

\begin{tabular}{|c|c|}
\hline GABBRO & Gabbroic rocks \\
\hline GNEISS & Gneiss \\
\hline GRANITE & Granitoids \\
\hline GRANULITE & Granulite \\
\hline GRAVEL & Gravel \\
\hline HORNFELS & Hornfels \\
\hline IRONSTONE & Oolitic and other ironstones \\
\hline LATERITE & Laterite \\
\hline LATITE & Latite \\
\hline LIMESTONE & Limestone \\
\hline LUTITE & Mudstone \\
\hline MONZONITE & Monzonite \\
\hline MUD & Mud \\
\hline OTHERMET & Metamorphic rock \\
\hline OTHERPLUT & Plutonic rock \\
\hline OTHERSED & Sedimentary rock \\
\hline OTHERVOLC & Volcanic rock \\
\hline PEGMATITE & Pegmatite \\
\hline PHYLITE & Phylite \\
\hline QTZMAG & Quartz magnetite/BIF-type rocks \\
\hline QUARTZITE & Quartzite \\
\hline RHYOLITE & Rhyolite-dacite \\
\hline SAND & Sand \\
\hline SANDSTONE & Sandstone \\
\hline SCHIST & Schist \\
\hline SEDMIX & Interbedded clastic rocks, no single dominant lithology \\
\hline SILCRETE & Silcrete \\
\hline SLATE & Slate \\
\hline SYENITE & Syenite \\
\hline TRACHYTE & Trachyte \\
\hline ULTRAMAFIC & Ultramafic rock \\
\hline UMAFICVOLC & Ultramafic volcanic rock \\
\hline UNCONSED & Unconsolidated sedimentary rock \\
\hline
\end{tabular}


Table C.8: continued

\begin{tabular}{|l|l|}
\hline USATPLUT & Undersaturated plutonic rocks $(<10 \%$ undersaturated minerals \\
\hline USATVOLC & Undersaturated volcanic rocks \\
\hline VOLCSED & Volcaniclastics \\
\hline
\end{tabular}

\section{C.4.6 Data Dictionary for Tenterfield Dominant Lithology Table}

Table C.9 shows the data dictionary for the dominant lithology table, domlith.

Table C.9: Data dictionary for database table domlith

\begin{tabular}{|l|c|l|}
\hline \multicolumn{2}{|l|}{ Table name: domlith } \\
\hline Item names & Type & Item description \\
\hline domlith & $\mathrm{C}$ & $\begin{array}{l}\text { Name of dominant rock type in the geological unit. This field is identical to } \\
\text { the lithology field in the main geology table } t_{-} g \text { eology and is used as a foreign } \\
\text { key to link the domlith table to the } t_{-} \text {geology table. }\end{array}$ \\
\hline desc & $\mathrm{C}$ & $\begin{array}{l}\text { Explanation of the lithology code. Generally this field is identical to the lithol- } \\
\text { ogy code. }\end{array}$ \\
\hline colornames & I & Code for the colour in the ARC/InFO shade set used in the map legend. \\
\hline
\end{tabular}

\section{C.4.7 Data Dictionary for Tenterfield Geological Age Table}

Table C.10 shows the data dictionary for the Tenterfield geological age table, geolage.

Table C.10: Data dictionary for database table, geolage

\begin{tabular}{|l|c|l|}
\hline \multicolumn{2}{|l|}{ Table name: geolage } \\
\hline Item names & Type & Item description \\
\hline class & I & $\begin{array}{l}\text { Number code for the geological eras and periods. This field is used as a foreign } \\
\text { key to link this table to the main Tenterfield geology table, } t_{-} g e o l .\end{array}$ \\
\hline descriptio & $\mathrm{C}$ & $\begin{array}{l}\text { The qualifier, undifferentiated, may be used in conjunction with the era or } \\
\text { period name-otherwise the description is the same as the label field below. }\end{array}$ \\
\hline label & $\mathrm{C}$ & Name of geological era or period. \\
\hline
\end{tabular}

An explanation for the codes used for the ages of the geological units in the Tenterfield geology data attribute table $\left(t_{-} g e o l\right)$ is given in Table C.11. The age codes are defined 
in the Mineral Resources Land Information System (MRLIS) of the New South Wales Department of Mineral Resources

Table C.11: Explanation of MRLIS age codes used in $t_{-} g e o l$ attribute table

\begin{tabular}{|c|c|c|c|}
\hline Age code & Description & Age code & Description \\
\hline 100 & Cainozoic, undifferentiated & 317 & Early Permian \\
\hline 109 & Cainozoic, undifferentiated? & 318 & Early Permian? \\
\hline 110 & Quaternary, undifferentiated & 319 & Permian, undifferentiated? \\
\hline 111 & Holocene (Recent) & 320 & Carboniferous, undifferentiated \\
\hline 112 & Holocene? (Recent?) & 311 & Late Carboniferous \\
\hline 115 & Pleistocene & 312 & Late Carboniferous? \\
\hline 116 & Pleistocene? & 317 & Early Carboniferous \\
\hline 119 & Quaternary, undifferentiated? & 318 & Early Carboniferous? \\
\hline 120 & Tertiary, undifferentiated & 329 & Carboniferous, undifferentiated? \\
\hline 121 & Pliocene & 330 & Devonian, undifferentiated \\
\hline 122 & Pliocene? & 331 & Late Devonian \\
\hline 123 & Miocene & 332 & Late Devonian? \\
\hline 124 & Miocene? & 334 & Middle Devonian \\
\hline 125 & Oligocene & 335 & Middle Devonian? \\
\hline 126 & Oligocene? & 337 & Early Devonian \\
\hline 127 & Eocene & 338 & Early Devonian? \\
\hline 127 & Eocene? & 339 & Devonian, undifferentiated? \\
\hline 129 & Palaeocene & 340 & Silurian, undifferentiated \\
\hline 130 & Palaeocene? & 341 & Late Silurian \\
\hline 131 & Tertiary, undifferentiated? & 342 & Late Silurian? \\
\hline 200 & Mesozoic, undifferentiated & 344 & Middle Silurian \\
\hline 209 & Mesozoic, undifferentiated? & 345 & Middle Silurian? \\
\hline 210 & Cretaceous, undifferentiated & 347 & Early Silurian \\
\hline 211 & Late Cretaceous & 348 & Early Silurian? \\
\hline 212 & Late Cretaceous? & 349 & Silurian, undifferentiated? \\
\hline 217 & Early Cretaceous & 350 & Ordovician, undifferentiated \\
\hline 218 & Early Cretaceous? & 351 & Late Ordivician \\
\hline 219 & Cretaceous, undifferentiated? & 352 & Late Ordovician? \\
\hline 220 & Jurassic, undifferentiated & 357 & Early Ordivician \\
\hline 221 & Late Jurassic & 358 & Early Ordovician? \\
\hline 222 & Late Jurassic? & 359 & Ordovician, undifferentiated? \\
\hline
\end{tabular}

continued on next page 
Table C.11: continued

\begin{tabular}{|c|c|c|c|}
\hline Age code & Description & Age code & Description \\
\hline 224 & Middle Jurassic & 360 & Cambrian, undifferentiated \\
\hline 225 & Middle Jurassic? & 361 & Late Cambrian \\
\hline 226 & Early Jurassic & 362 & Late Cambrian? \\
\hline 227 & Early Jurassic? & 364 & Middle Cambrian \\
\hline 229 & Jurassic, undifferentiated? & 365 & Middle Cambrian? \\
\hline 230 & Triassic, undifferentiated & 367 & Early Cambrian \\
\hline 231 & Late Triassic & 368 & Early Cambrian? \\
\hline 232 & Late Triassic? & 369 & Cambrian, undifferentiated? \\
\hline 234 & Middle Triassic & 400 & Precambrian, undifferentiated \\
\hline 235 & Middle Triassic? & 409 & Precambrian, undifferentiated? \\
\hline 237 & Early Triassic & 500 & Proterozoic, undifferentiated \\
\hline 238 & Early Triassic? & 510 & Late Proterozoic \\
\hline 239 & Triassic, undifferentiated? & 511 & Late Proterozoic? \\
\hline 300 & Paleozoic, undifferentiated & 540 & Middle Proterozoic \\
\hline 309 & Paleozoic, undifferentiated? & 541 & Middle Proterozoic? \\
\hline 310 & Permian, undifferentiated & 570 & Early Proterozoic \\
\hline 311 & Late Permian & 571 & Early Proterozoic? \\
\hline 312 & Late Permian? & 579 & Proterozoic, undifferentiated? \\
\hline 314 & Middle Permian & 600 & Archaean \\
\hline 315 & Middle Permian? & 610 & Late Archaean \\
\hline
\end{tabular}

\section{C.4.8 Data Dictionary for Tenterfield Geological System Table}

Table C.7 shows the data dictionary for the Tenterfield geological system table, geolsys.

Table C.12: Data dictionary for database table, geolsys

\begin{tabular}{|l|c|l|}
\hline \multicolumn{2}{|l|}{ Table name: geolsys } \\
\hline Item names & Type & Item description \\
\hline geolsystem & C & Tectonostratigraphic group; e.g. Accretionary Complex. \\
\hline symbol & I & $\begin{array}{l}\text { Number code for colour of unit using the AGSOSHD shade set obtained from } \\
\text { the Australian Geological Survey Organisation. This field is used as a foreign } \\
\text { key to link this table to the main Tenterfield geology table, } t_{-} g e o l .\end{array}$ \\
\hline
\end{tabular}




\section{C.5 Geophysical Data}

\section{C.5.1 Core Metadata Elements for Geophysical Data}

The geophysical data used in the Tenterfield pilot study consists of an aeromagnetic and gamma-ray survey, which were flown simultaneously. The core metadata items (Page 0) described in the Australia New Zealand Land Information Council (ANZLIC) metadata guidelines (ANZLIC, 1996) are given in Table C.13 below.

Table C.13: ANZLIC core metadata elements for Tenterfield geophysical data

\begin{tabular}{|c|c|c|}
\hline \multirow[t]{3}{*}{ Dataset } & Title & $\begin{array}{l}\text { igmag, igu, igth, igk, igtc-ARC/INFO BIL images } \\
\text { of the Inverell and Grafton 1:250000 map sheet areas }\end{array}$ \\
\hline & Custodian & NSW Department of Mineral Resources \\
\hline & Jurisdiction & New South Wales \\
\hline \multirow[t]{3}{*}{ Description } & Abstract & $\begin{array}{l}\text { Airborne magnetic and gamma-ray survey of the In- } \\
\text { verell and Grafton 1:250000 sheet areas as gridded grey } \\
\text { scale (0-255 levels) images in IDRISI BIL format (file } \\
\text { suffix: img). Each file is accompanied by a Word } 97 \text { file } \\
\text { containing header information. }\end{array}$ \\
\hline & Search Words & GEOSCIENCES, GEOSCIENCES Geophysics \\
\hline & $\begin{array}{l}\text { Geographic } \\
\text { Extent Name(s) }\end{array}$ & Inverell and Grafton 1:250000 Map Sheets \\
\hline \multirow[t]{2}{*}{ Data Currency } & Beginning Date & $01 \mathrm{FEB} 1982$ \\
\hline & Ending Date & 01APR1982 \\
\hline \multirow[t]{2}{*}{ Dataset Status } & Progress & Complete \\
\hline & $\begin{array}{l}\text { Maintenance and } \\
\text { Update } \\
\text { Frequency }\end{array}$ & Not Known \\
\hline \multirow[t]{3}{*}{ Access } & $\begin{array}{l}\text { Stored Data } \\
\text { Format }\end{array}$ & $\begin{array}{l}\text { IDRISI image format. This is a single channel BIL file } \\
\text { (file suffix: img) with an 8-bit per value binary coding. } \\
\text { Accompanying each file is a Word } 97 \text { file (file suffix: } \\
\text {.doc) that provides header information }\end{array}$ \\
\hline & $\begin{array}{l}\text { Available Format } \\
\text { Type }\end{array}$ & ARC/INFO image format \\
\hline & Access Constraint & Available for research, subject to license agreement. \\
\hline
\end{tabular}


Table C.13: continued

\begin{tabular}{|c|c|c|}
\hline \multirow[t]{5}{*}{ Data Quality } & Lineage & $\begin{array}{l}\text { The magnetic and gamma-ray data were collected si- } \\
\text { multaneously by EG \& G Geometrics International } \\
\text { Corporation. These data were originally presented to } \\
\text { the NSW Department of Mineral Resources as a se- } \\
\text { ries of coloured contour maps. The data were later } \\
\text { re-gridded and contoured by Ross Spencer, Geophysics } \\
\text { Section, NSW Geological Survey. The floating point } \\
\text { digital data were converted by Ross Spencer to a series } \\
\text { of } 8 \text {-bit single channel BIL files. } \\
\text { A UTM projection giving AMG } 66 \text { coordinates was } \\
\text { used (Barnes, 1993). Barnes (1993) has pointed out } \\
\text { that because the highly fractionated, K-, Th- and U- } \\
\text { rich Mole granite was included in the data set prior } \\
\text { to classification, the variability of the data set in the } \\
\text { adjacent Tenterfield area was significantly reduced. }\end{array}$ \\
\hline & $\begin{array}{l}\text { Positional } \\
\text { Accuracy }\end{array}$ & $\begin{array}{l}\text { A rough estimate based on the equipment used is in the } \\
\text { range } \pm 10 \text { to } \pm 50 \mathrm{~m} .\end{array}$ \\
\hline & $\begin{array}{l}\text { Attribute } \\
\text { Accuracy }\end{array}$ & $\begin{array}{l}\text { Magnetic data were collected with a Geometrics G- } 803 \\
\text { proton procession magnetometer with a nominal sensi- } \\
\text { tivity of } 0.5 \text { nanoteslas. } \\
\text { Radiometric data were collected with a Geometrics GR- } \\
800 \text { spectrometer with a } 2048 \mathrm{cu} \text {. in. crystal. The ac- } \\
\text { curacy of the radiometric data are not known, however } \\
\text { it is likely to be approximately } \pm 10 \% \text {. }\end{array}$ \\
\hline & Logical Accuracy & Unknown. \\
\hline & Completeness & $\begin{array}{l}\text { Coverage is complete over the Inverell and Grafton } \\
1: 250000 \text { map sheet areas. }\end{array}$ \\
\hline \multirow[t]{9}{*}{ Contact Information } & $\begin{array}{l}\text { Contact } \\
\text { Organization }\end{array}$ & Geological Survey of NSW \\
\hline & Contact Position & Senior Geolophysicist \\
\hline & Mail Address 1 & P.O. Box 536 \\
\hline & Mail Address 2 & \\
\hline & Suburb/Locality & St Leonards \\
\hline & State/Locality 2 & NSW \\
\hline & Country & Australia \\
\hline & Postcode & 1590 \\
\hline & Telephone & $\begin{array}{l}\text { Australia Fixed Network Number } \\
0899018364\end{array}$ \\
\hline
\end{tabular}


Table C.13: continued

\begin{tabular}{|l|l|l|}
\hline & Facsimile & 0899018777 \\
\cline { 2 - 3 } & Email & spencerr@minerals.nsw.gov.au \\
\hline Metadata Date & Metadata Date & 01NOV1993 \\
\hline Additional Metadata & & See Barnes (1993) \\
\hline
\end{tabular}

Table C.14: Survey specifications for the Tenterfield geophysical data set

\begin{tabular}{|l|l|}
\hline Magnetometer & Geometrics G-803 proton procession \\
\hline Sensitivity & 0.5 nanoteslas \\
\hline Spectrometer & Geometrics GR-800 $(2048 \mathrm{cu}$. in. $)$ \\
\hline Digital acquisition system & Geometrics G-714 \\
\hline Flight path record & Geocam $35 \mathrm{~mm}$ continuous strip film \\
\hline Time base & 0.8 seconds \\
\hline Average sample interval & 50 metres \\
\hline Flight line direction & East-west \\
\hline Tie line direction & North-south \\
\hline Nominal flight line spacing & 1.5 kilometres \\
\hline Survey altitude & 150 metres terrain clearance \\
\hline
\end{tabular}

\section{C.6 Geochemical Data}

\section{C.6.1 Core Metadata Elements for Geochemical Data}

The most important geochemical data set covering the Tenterfield area is the stream sediment geochemical survey carried out for the NRAC survey (referred to as the NRAC S1(A) Geochemical Atlas project). No metadata are available for the other geochemical data, which include a small amount of stream sediment sampling over the eastern portion of the Tenterfield 1:100,000 map sheet area (file: $t_{-}$stmgc) and whole-rock geochemistry (file: t_whrkgc).

The core metadata items (Page 0) described in the Australia New Zealand Land Information Council (ANZLIC) metadata guidelines (ANZLIC, 1996) given for the NRAC stream sediment geochemical data in Table C.15 below was obtained from Barnes \& Suppel (1995). 
Table C.15: ANZLIC core metadata elements for Tenterfield Geochemical Data

\begin{tabular}{|c|c|c|}
\hline \multirow[t]{3}{*}{ Dataset } & Title & $\begin{array}{l}\text { t_nracstrmgc - NRAC Stream Sediment Geo- } \\
\text { chemistry,Upper North East Region, NSW }\end{array}$ \\
\hline & Custodian & NSW Department of Mineral Resources \\
\hline & Jurisdiction & New South Wales \\
\hline \multirow[t]{3}{*}{ Description } & Abstract & $\begin{array}{l}\text { NRAC geochemistry for the Upper North East Re- } \\
\text { gion. The area comprises portions of the Warwick, } \\
\text { Tweed Heads, Grafton, Maclean, Dorrigo, and Coffs } \\
\text { Harbour 1:250000 sheet areas in NSW. The data com- } \\
\text { prise drainage basin polygons, sample points, and at- } \\
\text { tributes. There is one sample analysis for each polygon. } \\
\text { Active stream channels were sampled with } 3 \text { to } 5 \mathrm{~kg} \\
\text { of material which was analysed for } 39 \text { elements. Each } \\
\text { sample locality represents a drainage basin of } 8 \text { to } 12 \\
\text { square kilometres. }\end{array}$ \\
\hline & Search Words & GEOSCIENCES, GEOSCIENCES Geochemistry \\
\hline & $\begin{array}{l}\text { Geographic } \\
\text { Extent Name(s) }\end{array}$ & $\begin{array}{l}\text { Warwick 1:250000 Map Sheet, Tweed Heads 1:250,000 } \\
\text { Map Sheet, Grafton 1:250000 Map Sheet, Maclean } \\
\text { 1:250000 Map Sheet, Dorrigo 1:250000 Map Sheet, } \\
\text { Coffs Harbour 1:250000 Map Sheet }\end{array}$ \\
\hline \multirow[t]{2}{*}{ Data Currency } & Beginning Date & 01JAN1995 \\
\hline & Ending Date & \\
\hline \multirow[t]{2}{*}{ Dataset Status } & Progress & Complete \\
\hline & $\begin{array}{l}\text { Maintenance and } \\
\text { Update } \\
\text { Frequency }\end{array}$ & Not Planned \\
\hline \multirow[t]{3}{*}{ Access } & $\begin{array}{l}\text { Stored Data } \\
\text { Format }\end{array}$ & $\begin{array}{l}\text { DIGITAL ARC/INFO } 7.0 .3 \text { under SunOS, vector cover- } \\
\text { age, AMG 88, records }\end{array}$ \\
\hline & $\begin{array}{l}\text { Available Format } \\
\text { Type }\end{array}$ & ArC/INFO coverage, ARCVIEW shape file \\
\hline & Access Constraint & Available for research, subject to license agreement. \\
\hline Data Quality & Lineage & $\begin{array}{l}\text { The data were compiled by Dr David Cohen, Dr Neil } \\
\text { Rutherford, and David Garnett (Cohen et al., 1995; } \\
\text { Barnes \& Suppel, 1995). Drainage polygons were } \\
\text { marked on topographic sheets and digitized using Au- } \\
\text { tocad. Autocad vectors were converted to a ARC/INFO } \\
\text { polygon coverage using the sample points as polygon la- } \\
\text { bel points and the sample ID to identify each polygon. } \\
\text { The regional coverage was clipped to the Tenterfield } \\
\text { 1:100000 map sheet area in ARC/INFO. }\end{array}$ \\
\hline
\end{tabular}


Table C.15: continued

\begin{tabular}{|c|c|c|}
\hline & $\begin{array}{l}\text { Positional } \\
\text { Accuracy }\end{array}$ & $\begin{array}{l}\text { Most sample sites located to an accuracy of } 50 \mathrm{~m} \text {. All } \\
\text { sites are located to an accuracy of better than } 100 \mathrm{~m} \text {, } \\
\text { which corresponds to the accuracy with which localities } \\
\text { can be plotted on a 1:25,000 scale topographic map. } \\
\text { Some sites were located with GPS. }\end{array}$ \\
\hline & $\begin{array}{l}\text { Attribute } \\
\text { Accuracy }\end{array}$ & $\begin{array}{l}\text { For concentrations below the detection limit, a value } \\
\text { of half the detection is shown. This method avoids ap- } \\
\text { parent gaps in the data and statistical distortions as- } \\
\text { sociated with using negative values to represent values } \\
\text { below the detection limit (Cruikshank, 1994). Checking } \\
\text { for errors was done by duplicating: 1) samples at one } \\
\text { in fifty sites and analyzing those samples separately, } 2 \text { ) } \\
\text { duplicating approximately one in fifty analyses by re- } \\
\text { analyzing a separate split, and } 3 \text { ) performing analyses } \\
\text { on up to four size fractions on some samples to check } \\
\text { for possible sampling problems. }\end{array}$ \\
\hline & Logical Accuracy & $\begin{array}{l}\text { A high degree of internal consistency is expected as } \\
\text { all samples were collected and analysed using the same } \\
\text { laboratories in a short period of time. The data set was } \\
\text { visually and statistically checked. }\end{array}$ \\
\hline & Completeness & $\begin{array}{l}\text { Approximately two percent of the planned sample sites } \\
\text { were not sampled due to access problems. All sam- } \\
\text { ples were analysed for the full range of elements. } \\
\text { Sites were logged using electronic notebooks with pre- } \\
\text { programmed question-and-answer prompts. }\end{array}$ \\
\hline \multirow[t]{10}{*}{ Contact Information } & $\begin{array}{l}\text { Contact } \\
\text { Organization }\end{array}$ & Geological Survey of NSW \\
\hline & Contact Position & Senior Geologist \\
\hline & Mail Address 1 & P.O. Box 65 \\
\hline & Mail Address 2 & \\
\hline & Suburb/Locality & Armidale \\
\hline & State/Locality 2 & NSW \\
\hline & Country & Australia \\
\hline & Postcode & 2350 \\
\hline & Telephone & $\begin{array}{l}\text { Australia Fixed Network Number } \\
0267702118 \text { (Armidale) } \\
0299018888 \text { (St Leonards, Sydney) }\end{array}$ \\
\hline & Facsimile & 0267702121 \\
\hline
\end{tabular}

continued on next page 
Table C.15: continued

\begin{tabular}{|l|l|l|}
\hline & Email & barnesr@minerals.nsw.gov.au \\
\hline Metadata Date & Metadata Date & 01MAY1995 \\
\hline Additional Metadata & & See Barnes \& Suppel (1995); Cohen et al. (1995) \\
\hline
\end{tabular}

\section{C.6.2 Data Dictionary for Regional Geochemistry Layer}

The data dictionary for the NRAC regional stream sediment geochemistry layer, $t$ _nracstrmgc is shown in Table C.16 below.

Table C.16: Data dictionary for the regional stream sediment geochemistry layer, Upper North East region, NSW, t_nracstrmgc

\begin{tabular}{|c|c|c|}
\hline \multicolumn{3}{|c|}{ Coverage name: $t_{-}$nracstrmgc } \\
\hline \multicolumn{3}{|c|}{ Coverage type: Point } \\
\hline Item names & Type & Item description \\
\hline X_coord & $\mathrm{F}$ & GPS location in AMG Easting \\
\hline Y_coord & $\mathrm{F}$ & GPS location in AMG Northing \\
\hline Major & I & Not used (set to constant value of 1 ) \\
\hline Minor & I & unique identifier for point (same as ArCVIEw _id attribute) \\
\hline East & $\mathrm{F}$ & Planned AMG Easting \\
\hline North & $\mathrm{F}$ & Planned AMG Northing \\
\hline X_log & $\mathrm{F}$ & $\begin{array}{l}\text { Logarithm to the base } 10 \text { of the element } X \text { concentration in ppm. This current } \\
\text { item and the one below are repeated for } 36 \text { elements; } \mathrm{Ag}, \mathrm{Au}, \mathrm{Ba}, \mathrm{Br}, \mathrm{Cd}, \mathrm{Ce} \text {, } \\
\mathrm{Co}, \mathrm{Cr}, \mathrm{Cu}, \mathrm{Eu}, \mathrm{Fe}, \mathrm{Ga}, \mathrm{Hf}, \mathrm{K}, \mathrm{La}, \mathrm{Lu}, \mathrm{Mo}, \mathrm{Na}, \mathrm{Nb}, \mathrm{Ni}, \mathrm{Pb}, \mathrm{Rb}, \mathrm{Sb}, \mathrm{Sc}, \mathrm{Sm} \text {, } \\
\mathrm{Sn}, \mathrm{Sr}, \mathrm{Ta}, \mathrm{Th}, \mathrm{U}, \mathrm{W}, \mathrm{Y}, \mathrm{Yb}, \mathrm{Z}, \mathrm{Zn} \text {, and } \mathrm{Zr} \text {. }\end{array}$ \\
\hline X_1 & $\mathrm{F}$ & Element $X$ concentration in ppm \\
\hline Site_no & I & Site number (same as minor 1 and ARCVIEW _ $i d$ attributes) \\
\hline Geol & I & Geology code \\
\hline Majbld & I & Usage unknown \\
\hline Minbld & I & Usage unknown \\
\hline Size & I & Usage unknown \\
\hline Geogrp & $\mathrm{I}$ & Usage unknown \\
\hline Bldgp & I & Usage unknown \\
\hline Slope & $\mathrm{C}$ & $\begin{array}{l}\text { Slope of stream banks: } \mathrm{F}=\text { flat, } \mathrm{M}=\text { moderate, } \mathrm{S}=\text { steep, } \mathrm{N} / \mathrm{R}=\text { not } \\
\text { recorded }\end{array}$ \\
\hline
\end{tabular}


Table C.16: continued

\begin{tabular}{|l|c|l|}
\hline Item names & Type & Item description \\
\hline Landu & $\mathrm{C}$ & Land use code \\
\hline Ckwid & $\mathrm{F}$ & Creek width $(\mathrm{m}),-999=$ no data \\
\hline Ckdpth & $\mathrm{F}$ & Creek depth $(\mathrm{m})$ \\
\hline Treecov & $\mathrm{C}$ & $\begin{array}{l}\text { Density of tree cover: } \mathrm{S}=\text { sparse, } \mathrm{M}=\text { moderate, } \mathrm{H}=\text { heavy, N/R }=\text { not } \\
\text { recorded }\end{array}$ \\
\hline Treesamp & $\mathrm{C}$ & Species of trees; e.g. Casuarina, Acacia, Eucalypt, N/R = not recorded \\
\hline Ckflow & $\mathrm{C}$ & Creek flow: $\mathrm{S}=$ small, $\mathrm{M}=$ moderate, $\mathrm{N}=$ normal, N/R = not recorded \\
\hline Waterq & $\mathrm{C}$ & Water quality: $\mathrm{C}=$ clear, $\mathrm{M}=$ muddy, N/R = not recorded \\
\hline Weather & $\mathrm{C}$ & Weather: $\mathrm{S}=\mathrm{sunny}, \mathrm{O}=$ overcast \\
\hline Ph & $\mathrm{C}$ & Ph value or $\mathrm{N} / \mathrm{R}=$ not recorded \\
\hline Cond & $\mathrm{F}$ & Usage unknown \\
\hline
\end{tabular}

\section{C.7 Deposit Data}

\section{C.7.1 Core Metadata Elements for Tenterfield Deposit Location Data}

Core metadata elements (Page 0) described in the Australia New Zealand Land Information Council (ANZLIC) metadata guidelines (ANZLIC, 1996) for the deposit locations used in the Tenterfield GIS database are listed in Table C.17 below. The information summarizes that given in Barnes (1997a)

Table C.17: ANZLIC core metadata elements for Tenterfield Deposit Location Data

\begin{tabular}{|l|l|l|}
\hline \multirow{2}{*}{ Dataset } & Title & $\begin{array}{l}\text { t_deps - Mineral deposit locations for New } \\
\text { England and North East Region, NSW }\end{array}$ \\
\cline { 2 - 3 } & Custodian & NSW Department of Mineral Resources \\
\cline { 2 - 3 } & Jurisdiction & New South Wales \\
\hline Description & $\begin{array}{l}\text { The mineral deposit locations data set covers the } \\
\text { NSW portions of the Warwick, Tweed Heads, In- } \\
\text { verell, Grafton, Maclean, Manilla, Dorrigo, Coffs Har- } \\
\text { bour, Tamworth, Hastings, Singleton, and Newcastle } \\
1: 250000 \text { sheet areas. The data set includes metallic } \\
\text { and non-metallic industrial minerals. }\end{array}$ \\
\hline
\end{tabular}


Table C.17: continued

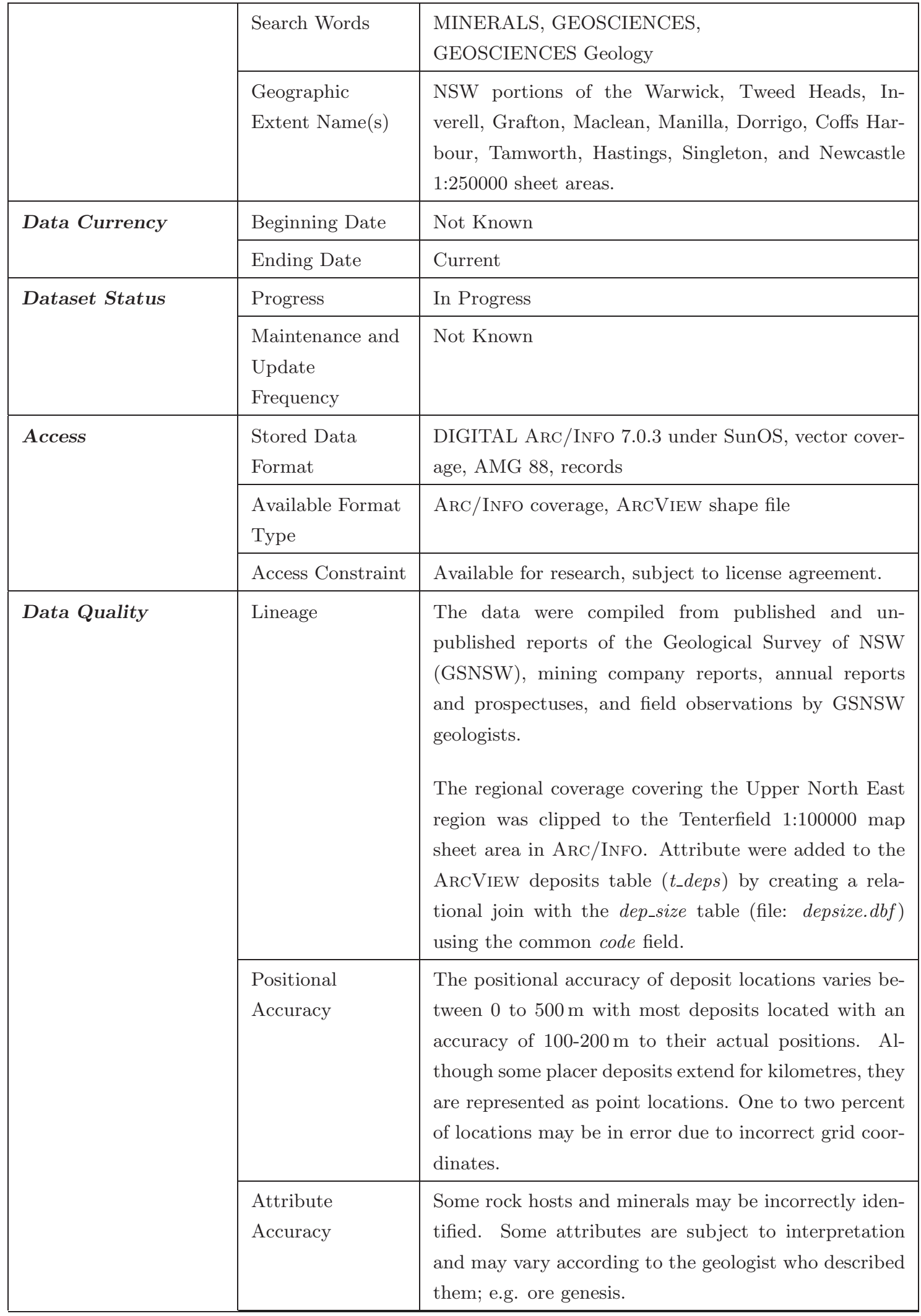

continued on next page 
Table C.17: continued

\begin{tabular}{|c|c|c|}
\hline & Logical Accuracy & $\begin{array}{l}\text { There may be a slight mismatch between the actual } \\
\text { host rock for a mineral deposit and the host rock } \\
\text { recorded in the data set due to the amount of detail } \\
\text { recorded for a particular locality. }\end{array}$ \\
\hline & Completeness & $\begin{array}{l}\text { Coverage: Barnes (1997a) estimates that over } 95 \% \text { of } \\
\text { the deposits in the Tenterfield 1:100000 map sheet area } \\
\text { have been identified and that } 95 \% \text { of the identified de- } \\
\text { posits have been checked in the field. } \\
\text { Classification: Over } 75 \% \text { of the attributes for each de- } \\
\text { posit are complete. }\end{array}$ \\
\hline \multirow[t]{11}{*}{ Contact Information } & $\begin{array}{l}\text { Contact } \\
\text { Organization }\end{array}$ & Geological Survey of NSW \\
\hline & Contact Position & Senior Geologist \\
\hline & Mail Address 1 & P.O. Box 65 \\
\hline & Mail Address 2 & \\
\hline & Suburb/Locality & Armidale \\
\hline & State/Locality 2 & NSW \\
\hline & Country & Australia \\
\hline & Postcode & 2350 \\
\hline & Telephone & $\begin{array}{l}\text { Australia Fixed Network Number } \\
0267702118 \text { (Armidale) } \\
0299018888 \text { (St Leonards, Sydney) }\end{array}$ \\
\hline & Facsimile & 0267702121 \\
\hline & Email & barnesr@minerals.nsw.gov.au \\
\hline Metadata Date & Metadata Date & 01JUL1997 \\
\hline Additional Metadata & & See Barnes (1997a) \\
\hline
\end{tabular}

\section{C.7.2 Data Dictionary for Tenterfield Mineral Deposits Layer}

Table C.18 shows the data dictionary for the Tenterfield geology, corresponding to the layer; t_deps. 
Table C.18: Data dictionary for the Tenterfield mineral deposits layer

\begin{tabular}{|c|c|c|}
\hline \multicolumn{3}{|c|}{ Coverage name: $t_{-} d e p s$} \\
\hline \multicolumn{3}{|c|}{ Coverage type: Point } \\
\hline Item names & Type & Item description \\
\hline pubno & $\mathrm{C}$ & Cross-reference to Geological Survey of NSW reports and publications. \\
\hline fieldno & $\mathrm{C}$ & Cross-reference to geologist's field notes. \\
\hline code & $\mathrm{C}$ & $\begin{array}{l}\text { Code for deposit style (GSNSW nomenclature). Item is used to link descrip- } \\
\text { tions of deposit types from the table depcode to the main table } t_{-} \text {deps. }\end{array}$ \\
\hline order & $\mathrm{C}$ & Use unknown \\
\hline refno & $\mathrm{C}$ & Field is composed of the previous two items concatenated; $<$ code $><$ order $>$. \\
\hline $\mathrm{cl}$ & $\mathrm{C}$ & Commodity class; $\mathrm{M}=$ metallic and $\mathrm{I}=$ industrial. \\
\hline abbrevname & $\mathrm{C}$ & Abbreviated name of deposit. \\
\hline names & $\mathrm{C}$ & Name of deposit. \\
\hline map100k & $\mathrm{C}$ & Number of 1:100000 map sheet in which deposit is located. \\
\hline map250k & $\mathrm{C}$ & Number of 1:250000 map sheet in which deposit is located. \\
\hline amge & $\mathrm{C}$ & AMG easting for deposit location. \\
\hline amgn & $\mathrm{C}$ & AMG northing for deposit location. \\
\hline majcom & $\mathrm{C}$ & Major commodities. \\
\hline mincom & $\mathrm{C}$ & Minor commodities. \\
\hline size & $\mathrm{C}$ & Size of deposit expressed in classes; occ, sml, med, lge, vlge, and N/A. \\
\hline size 2 & I & Size expressed in terms of numbers in the range $[0-5]$. \\
\hline N16 & I & tonnages and grades or total metal produced. \\
\hline $1967 \mathrm{a}$ & I & Dollar value of total contained metal in 1967 Australian dollars. \\
\hline
\end{tabular}

The genesis attribute has the following values and meanings:

$\begin{array}{ll}\text { MAGVEIN }= & \text { magmatic vein in granitoid host, } \\ \text { PIPE } & \text { granite-hosted breccia pipes e.g. Bungulla Si pipe, } \\ \text { GREISEN } & \text { greisen, } \\ \text { METAHYDRO }= & \text { structurally controlled Au-Sb veins occurring along granite; } \\ & \text { contacts e.g. Lionsville-Solferino, } \\ \text { MAGDIS } & \text { disseminated Au in granite; e.g. Timbarra-Poverty Point, } \\ \text { APLITE }= & \text { mineralization spatially associated with aplite dykes, } \\ \text { ALLUVIAL }= & \text { Au placer; e.g. Timbarra, } \\ \text { COLLUVIAL }= & \text { residual Au deposits, } \\ \text { VOLOTHER }= & \text { epithermal Au-(Ag-Te) in volcanic complex; e.g. Drake. }\end{array}$




\section{C.7.3 Data Dictionary for Deposit Size Database Table}

Deposits are classed into categories according to the value of the total contained metal expressed in 1967 Australian dollars (attribute: value1967a). The attributes, size and size2, are alternative names for the same deposit sizes. The equivalence between values of these three attributes is given by the GIS database table, depsize.dbf, shown in Table C.19 below.

Table C.19: Data dictionary for database table, depsize

\begin{tabular}{|l|c|l|}
\hline \multicolumn{2}{|l|}{ Table name: $t$ _depsize } \\
\hline Item names & Type & Item description \\
\hline size & $\mathrm{C}$ & Size of the deposit in classes; Occ, Sml, Med, Lge, and Vlge. \\
\hline size2 & $\mathrm{I}$ & Size of deposit in five classes labelled 1 to 5. \\
\hline value1967a & $\mathrm{C}$ & Size of deposit in value of contained metal (1967 Australian dollars). \\
\hline
\end{tabular}

The equivalence between the attributes size, size2 and value1967a in table depsize is described in table C.20 below.

Table C.20: Explanation of deposit size attributes used in Tenterfield depsize table

\begin{tabular}{|l|c|l|}
\hline size & size2 & value1967a \\
\hline NA & 0 & Not applicable \\
\hline Occ & 1 & $<\$ 10,000^{\mathrm{a}}$ \\
\hline Sml & 2 & $\$ 10,000-\$ 1,000,000$ \\
\hline Med & 3 & $\$ 1,000,000-\$ 10,000,000$ \\
\hline Lge & 4 & $\$ 10,000,000-\$ 100,000,000$ \\
\hline Vlge & 5 & $>\$ 100,000,000$ \\
\hline
\end{tabular}

\footnotetext{
${ }^{a}$ Value of total contained metal in 1967 Australian dollars
}

\section{C.7.4 Data Dictionary for Tenterfield Deposit Code Table}

Table C.21 shows the data dictionary for the dominant lithology table, depcode. 
Table C.21: Data dictionary for database table, depcode

\begin{tabular}{|l|c|l|}
\hline \multicolumn{2}{|c|}{ Table name: depcode } \\
\hline Item names & Type & Item description \\
\hline code & I & $\begin{array}{l}\text { Deposit code used as a foreign key to link this table to the deposit table, } \\
\text { t_deps. }\end{array}$ \\
\hline symbol & I & $\begin{array}{l}\text { Code for colour of the symbol representing the unit using an ArC/INFO shade } \\
\text { set. }\end{array}$ \\
\hline label & $\mathrm{C}$ & Name for the deposit type. \\
\hline desc & $\mathrm{C}$ & Description of deposit. \\
\hline add_commod & $\mathrm{C}$ & A list of commodities in order of significance (for metallic deposits only). \\
\hline examples & $\mathrm{C}$ & Name of a representative example for the deposit type. \\
\hline
\end{tabular}

\section{C.8 Derived Thematic Layers}

The primary thematic layers shown in Table C.22 below were derived from the data sets originally supplied by the Geological Survey of New South Wales.

Table C.22: Primary thematic layers in the Tenterfield GIS database

\begin{tabular}{|l|l|}
\hline $\begin{array}{l}\text { Coverage } \\
\text { Name }\end{array}$ & Description \\
\hline trglmap & Simplified solid geology for Tenterfield 1:100000 map sheet area (Map 9339). \\
\hline trstruct & Regional scale faults and shear zones with post-mineralization faults removed. \\
\hline trminocc & All mineral deposits and occurrences in the Tenterfield 1:100000 sheet area. \\
\hline magslc & Classed total magnetic intensity (RTP). Image data were converted to 10 classes. \\
\hline uslc & Classed Uranium channel gamma-ray survey data. Converted to 10 classes. \\
\hline thslc & Classed Thorium channel gamma-ray survey data. Converted to 10 classes. \\
\hline kslc & Classed Potassium channel gamma-ray survey data. Converted to 10 classes. \\
\hline tcslc & Classed total count gamma-ray survey data. Converted to 10 classes. \\
\hline
\end{tabular}




\section{C.9 Derived Thematic Layers}

The thematic layers shown in Table C.23 below were derived from the data sets originally supplied by the Geological Survey of New South Wales. The derived thematic layers were used as inputs for the neural network, fuzzy logic and weights of evidence prospectivity analysis methods.

Table C.23: Derived thematic layers in the Tenterfield GIS database

\begin{tabular}{|c|c|}
\hline $\begin{array}{l}\text { Coverage } \\
\text { Name }\end{array}$ & Description \\
\hline dauplgru & $\begin{array}{l}\text { Granite-related, placer, \& unknown-type gold deposits (probably granite-hosted) } \\
\text { in Tenterfield area. }\end{array}$ \\
\hline dauplace & Placer gold deposits and occurrences in the Tenterfield 1:100000 sheet area. \\
\hline daugran & $\begin{array}{l}\text { Granite-related mineral deposits and occurrences in the Tenterfield 1:100000 sheet } \\
\text { area. }\end{array}$ \\
\hline d_unkn & $\begin{array}{l}\text { Gold deposits and occurrences of unknown type in the Tenterfield 1:100000 sheet } \\
\text { area. }\end{array}$ \\
\hline buf_flt & Distance to nearest major fault (post-mineralization faults removed). \\
\hline uplusth & $\begin{array}{l}\text { Index equal to unclassed U plus Th gamma-ray channel values derived from image } \\
\text { containing } 256 \text { discrete grey-scale levels. }\end{array}$ \\
\hline k_dv_mag & $\begin{array}{l}\text { Index equal to unclassed } \mathrm{K} \text { gamma-ray channel values divided by classed magnetic } \\
\text { value. }\end{array}$ \\
\hline th_dv_mag & $\begin{array}{l}\text { Index equal to unclassed Th gamma-ray channel values divided by classed mag- } \\
\text { netic value. }\end{array}$ \\
\hline u_dv_mag & $\begin{array}{l}\text { Index equal to unclassed U gamma-ray channel values divided by classed magnetic } \\
\text { value. }\end{array}$ \\
\hline u_x_th & Index equal to product of unclassed $\mathrm{U} \times \mathrm{Th}$ gamma-ray channel values. \\
\hline u_xth_xk & Index equal to product of unclassed $\mathrm{U} \times \mathrm{Th} \times \mathrm{K}$ gamma-ray channel values. \\
\hline uth_d_m & $\begin{array}{l}\text { Index equal to unclassed } \mathrm{U} \times \mathrm{Th} \text { gamma-ray channel values divided by classed } \\
\text { magnetic value. }\end{array}$ \\
\hline uthk_d_m & $\begin{array}{l}\text { Index equal to unclassed } \mathrm{U} \times \mathrm{Th} \times \mathrm{K} \text { gamma-ray channel values divided by classed } \\
\text { magnetic value. }\end{array}$ \\
\hline uplusth & Index equal to unclassed $\mathrm{U}+\mathrm{Th}$ gamma-ray channel. \\
\hline
\end{tabular}




\section{Appendix D}

\section{Metadata for Kalgoorlie GIS Database}

\section{D.1 Location of the Kalgoorlie Data Set}

This appendix describes the data sets used in Chapter 5 for the case study on Archaean orogenic lode-gold deposits from the Kalgoorlie district in the Norseman-Wiluna Belt of the Yilgarn block, Western Australia. The data were collected and compiled by Dr Scott Halley, Dr Paul Kitto and other geologists at Goldfields Exploration Pty Ltd as part of a large gold exploration program.

The data set covers an approximately $110 \times 110 \mathrm{~km}$ area near Kalgoorlie, Western Australia. The northern margin of the area is about $25 \mathrm{~km}$ north of Bardoc and the southern margin is located approximately $12 \mathrm{~km}$ south of Coolgardie. The data set covers the eastern half of the Kalgoorlie 1:250000 geological map sheet (SH51-9), with a small proportion of the data set extending into the Kurnalpi, Boorabbin and Widgiemooltha 1:250000 sheet areas (SH51-10, SH51-13, \& SH51-14). The location of the case study area is shown in Figure 5.1 on page 139.

Coordinates of the corners of the Kalgoorlie data set together with parameters values used to convert the Australian Map Grid (AMG84) coordinates to geodetic coordinates, are shown in Table D.1 below. 
Table D.1: Location of the Kalgoorlie study area in relation to the 1:250000 geological map sheets of the Geological Survey of Western Australia

\begin{tabular}{|c|c|c|c|c|c|}
\hline \multirow[t]{2}{*}{ Corner } & Longitude & Latitude & Easting & Northing & Zone \\
\hline & Deg. Min. & Deg. Min. & Metres & Metres & \\
\hline \multicolumn{6}{|c|}{ Eastern Kalgoorlie 1:250000 sheet area } \\
\hline Northwest & $120^{\circ} 43^{\prime} 3^{\prime \prime} \mathrm{E}$ & $30^{\circ} 4^{\prime} 53^{\prime \prime} \mathrm{S}$ & $280,000.00$ & $6,670,000.00$ & 51 \\
\hline Northeast & $121^{\circ} 51^{\prime} 30^{\prime \prime} \mathrm{E}$ & $30^{\circ} 5^{\prime} 46^{\prime \prime} \mathrm{S}$ & $390,000.00$ & $6,670,000.00$ & 51 \\
\hline Southeast & $121^{\circ} 50^{\prime} 47^{\prime \prime} \mathrm{E}$ & $31^{\circ} 7^{\prime} 29^{\prime \prime} \mathrm{S}$ & $390,000.00$ & $6,556,000.00$ & 51 \\
\hline Southwest & $120^{\circ} 41^{\prime} 35^{\prime \prime} \mathrm{E}$ & $31^{\circ} 6^{\prime} 33^{\prime \prime} \mathrm{S}$ & $280,000.00$ & $6,556,000.00$ & 51 \\
\hline Standards & \multicolumn{2}{|c|}{$\begin{array}{l}\text { Ellipsoid } \\
\text { Datum } \\
\text { Geographic coordinate set } \\
\text { Grid coordinates (UTM) }\end{array}$} & \multicolumn{3}{|c|}{$\begin{array}{l}\text { Aust. National Spheroid (ANS) } \\
\text { Aust. Geodetic Datum (AGD) } \\
\text { AGD84 } \\
\text { Australian Map Grid (AMG84) }\end{array}$} \\
\hline $\begin{array}{l}\text { Ellipsoid } \\
\text { parameters }\end{array}$ & \multicolumn{2}{|l|}{$\begin{array}{l}\text { Semi-major axis } \\
\text { Inverse flattening }\end{array}$} & \multicolumn{3}{|c|}{$\begin{array}{l}\mathrm{a}=6,378,160.0 \mathrm{~m} \\
1 / \mathrm{f}=298.25\end{array}$} \\
\hline $\begin{array}{l}\text { AGD } \\
\text { parameters }\end{array}$ & \multicolumn{2}{|c|}{$\begin{array}{l}\text { Initial zone number } \\
\text { Initial central meridian longitude } \\
\text { Zone width } \\
\text { Central scale factor } \\
\text { False easting } \\
\text { False northing }\end{array}$} & \multicolumn{3}{|l|}{$\begin{array}{l}31 \\
3 \text { degrees } \\
6 \text { degrees } \\
0.9996 \\
500,000 \mathrm{~m} \\
10,000,000 \mathrm{~m}\end{array}$} \\
\hline
\end{tabular}

\section{D.2 Overview of Primary Thematic Layers}

Table D.2 lists all the GIS layers in the Kalgoorlie data set that were supplied by Goldfields Exploration. The area that forms the basis of the Kalgoorlie case study described in Chapters 5, 6, and 7 is a subset of Goldfields Exploration Pty Ltd's Bardoc Tectonic Zone (BTZ) data set (completed November 2000). Data for the Kalgoorlie area was extracted by Sean Centa, cartographer, Goldfields Exploration Pty Ltd. The solid geology and structural layers layer were prepared by Dr Scott Halley, Chief Geologist by; 1) combining an interpretation of aeromagnetic survey data with geological maps and 2) re-interpreting company mapping and drill cores. Derived GIS layers are described in a separate section below and in Chapter 5. 
Table D.2: Primary thematic layers in the Kalgoorlie GIS database

\begin{tabular}{|l|l|}
\hline Coverage & Description \\
\hline geol_btz & $\begin{array}{l}\text { Solid geology along part of the Bardoc Tectonic Zone; new interpretation current in } \\
\text { mid } 2000\end{array}$ \\
\hline geol_kal & $\begin{array}{l}\text { Solid geology over the eastern part of the Kalgoorlie 1:250000 map sheet; old inter- } \\
\text { pretation based on pre-2000 work }\end{array}$ \\
\hline struct_btz & $\begin{array}{l}\text { Structural geology along part of the Bardoc Tectonic Zone; new interpretation current } \\
\text { in mid } 2000\end{array}$ \\
\hline struct_kal & $\begin{array}{l}\text { Structural geology over the eastern part of the Kalgoorlie 1:250000 map sheet; old } \\
\text { interpretation based on pre-2000 work }\end{array}$ \\
\hline mag_trend & Trend lines interpreted from airborne magnetic images, Kalgoorlie area \\
\hline au_as & Location of drill holes with gold and arsenic assays (ppb), Kalgoorlie area \\
\hline reglth_au & Location of auger holes; max. gold assay in regolith (ppb), Kalgoorlie area \\
\hline grav_locn & Location of gravity stations for AGSO gravity survey, Kalgoorlie area \\
\hline Grid & Description \\
\hline magnetic & World Geoscience, airborne magnetic survey data; TMI, RTP, Kalgoorlie area \\
\hline gravity & AGSO Bouguer gravity anomaly grid, Kalgoorlie area \\
\hline maj_deps & Location of mineral deposits, MINDEX 2000, Geological Survey of Western Australia \\
\hline
\end{tabular}

\section{D.3 Core metadata elements for Kalgoorlie GIS data set}

Core metadata is presented for the Kalgoorlie GIS data set as a whole since all layers have been obtained from Goldfields Exploration Pty Ltd. The Australia New Zealand Land Information Council (ANZLIC) core metadata items (ANZLIC, 1996) for the Kalgoorlie data are given in Table D.3 below.

Table D.3: ANZLIC core metadata elements for the Kalgoorlie GIS database

\begin{tabular}{|l|l|l|}
\hline \multirow{2}{*}{ Data set } & Title & $\begin{array}{l}\text { Goldfields Exploration regional gold exploration } \\
\text { in the Kalgoorlie district, W.A. }\end{array}$ \\
\cline { 2 - 3 } & Custodian & Goldfields Exploration Pty Ltd \\
\cline { 2 - 3 } & Jurisdiction & Western Australia \\
\hline
\end{tabular}


Table D.3: continued

\begin{tabular}{|c|c|c|}
\hline \multirow[t]{3}{*}{ Description } & Abstract & $\begin{array}{l}\text { Regional exploration data set used by Goldfields Ex- } \\
\text { ploration Pty Ltd in the search for Archaean lode-gold } \\
\text { deposits in the Kalgoorlie area, Western Australia. The } \\
\text { GIS database includes the following layers; solid geol- } \\
\text { ogy and structural geology for each of the Kalgoorlie } \\
\text { and Bardoc Tectonic Zone areas, magnetic trend lines, } \\
\text { maximum gold in regolith, maximum gold and arsenic } \\
\text { in drill holes, gravity, total magnetic intensity, major } \\
\text { deposits, minor deposits, and deposit outlines. }\end{array}$ \\
\hline & Search Words & $\begin{array}{l}\text { GEOSCIENCES, GEOSCIENCES Geology, GEO- } \\
\text { SCIENCES Geochemistry, GEOSCIENCES Geo- } \\
\text { physics }\end{array}$ \\
\hline & $\begin{array}{l}\text { Geographic } \\
\text { Extent Name(s) }\end{array}$ & $\begin{array}{l}\text { Kalgoorlie (SH51-9), Kurnalpi (SH51-10), Boorabbin } \\
\text { (SH51-13), Widgiemooltha (SH51-14) 1:250000 map } \\
\text { sheets }\end{array}$ \\
\hline \multirow[t]{2}{*}{ Data Currency } & Beginning Date & 02FEB1998 \\
\hline & Ending Date & CURRENT \\
\hline \multirow[t]{2}{*}{ Data set Status } & Progress & IN PROGRESS \\
\hline & $\begin{array}{l}\text { Maintenance and } \\
\text { Update } \\
\text { Frequency }\end{array}$ & Not Planned \\
\hline \multirow[t]{3}{*}{ Access } & $\begin{array}{l}\text { Stored Data } \\
\text { Format }\end{array}$ & $\begin{array}{l}\text { DIGITAL MAPINFO } 5.0 \text { and ARCVIEW under Windows } \\
98, \text { raster \& vector, AMG } 84\end{array}$ \\
\hline & $\begin{array}{l}\text { Available Format } \\
\text { Type }\end{array}$ & MAPINFO tables and ARCVIEW shape files and grids \\
\hline & Access Constraint & $\begin{array}{l}\text { Access may be granted for research. Publication of re- } \\
\text { search based on data requires the approval of Goldfields } \\
\text { Exploration Pty Ltd }\end{array}$ \\
\hline Data Quality & Lineage & $\begin{array}{l}\text { The data were obtained from Goldfields Exploration } \\
\text { Pty Ltd. The maps were compiled from published } \\
\text { maps, and company maps. } \\
\text { Geological and geochemical data were compiled in the } \\
\text { MAPINFO GIS package. Data in MAPINFO format were } \\
\text { exported from MAPINFO in Mapinfo Interchange For- } \\
\text { mat (MIF) and converted to ARCVIEW shape files (vec- } \\
\text { tor format) using a DOS program, supplied with AR- } \\
\text { CVIEW, called miftoshape. } \\
\text { Geophysical data were stored as ASCII files and re- } \\
\text { gridded by Dr Mike House, using } 100 \text { metre cell size in } \\
\text { the ERMAPPER data analysis package. }\end{array}$ \\
\hline
\end{tabular}


Table D.3: continued

\begin{tabular}{|c|c|c|}
\hline \multirow[t]{4}{*}{ Data Quality } & $\begin{array}{l}\text { Positional } \\
\text { Accuracy }\end{array}$ & $\begin{array}{l}\text { No estimate of positional accuracy is given by Gold- } \\
\text { fields Exploration Pty Ltd. GPS devices were used; } \\
\text { therefore, the positional accuracy of geological bound- } \\
\text { aries in layer, geology, is likely to be in the range } \pm 50 \mathrm{~m} \text {. } \\
\text { The accuracy of older mapping is probably in the range } \\
\pm 100 \mathrm{~m} \text {. }\end{array}$ \\
\hline & $\begin{array}{l}\text { Attribute } \\
\text { Accuracy }\end{array}$ & $\begin{array}{l}\text { The attribute values are the result of several phases } \\
\text { of work by different geologists and are therefore likely } \\
\text { to contain some errors in terms of identification and } \\
\text { naming of some rock units and currency of data. }\end{array}$ \\
\hline & Logical Accuracy & Unknown. \\
\hline & Completeness & $\begin{array}{l}\text { Attribute data available for entire area of the Kalgoorlie } \\
\text { data set. }\end{array}$ \\
\hline \multirow[t]{11}{*}{ Contact Information } & $\begin{array}{l}\text { Contact } \\
\text { Organization }\end{array}$ & Goldfields Exploration Pty Ltd. \\
\hline & Contact Position & Chief Geologist \\
\hline & Mail Address 1 & 89 Burswood Road \\
\hline & Mail Address 2 & Victoria Park \\
\hline & Suburb/Locality & Perth \\
\hline & State/Locality 2 & WA \\
\hline & Country & Australia \\
\hline & Postcode & 6100 \\
\hline & Telephone & $\begin{array}{l}\text { Australia Fixed Network Number } \\
0894428110\end{array}$ \\
\hline & Facsimile & 0894428181 \\
\hline & Email & shalley@goldfields.com.au \\
\hline Metadata Date & Metadata Date & 22MAY2000 \\
\hline Additional Metadata & & none \\
\hline
\end{tabular}

\section{D.4 Geological Data}

\section{D.4.1 Data Dictionary for Solid Geology Layer, geology.shp}

The Kalgoorlie solid geology layer (vector format, ArcView shape file, geology.shp), is the result of combining a maps produced by Goldfields Exploration Pty Ltd for the Kalgoorlie area prior to mid-1999 with a more recent interpretation of the Bardoc Tectonic Zone 
(produced in the period, 2000-2001). Where the two areas overlap, the Bardoc Tectonic Zone map has been used to update the Kalgoorlie map. The work of combining the two maps was carried out by Sean Centa at Goldfields Exploration Pty Ltd. Table D.4 shows the data dictionary for the layer; geology.shp

A subset of the solid geology map of the Bardoc Tectonic Zone was selected for the study and all other GIS layers have these same boundaries (shown in Table D.1).

\section{Lineage}

The earlier solid geology map around the Kalgoorlie area is a compilation of published Geological Survey of Western Australia maps, mapping by Goldfields Exploration Pty Ltd, and information obtained from drilling and interpretation of aeromagnetic images. A geological interpretation at 1:100000 scale by Etheridge, Henley and Williams (now SRK Consulting, Australasia) forms the south-eastern part of the layer. All the layers for the Kalgoorlie area are subsets of more extensive data sets compiled at Goldfields Exploration Pty Ltd. GIS layers in MAPINFO version 4.5 format were initially clipped to the boundaries shown in Table D.1.

Much of the geology in the original Kalgoorlie area map had been updated resulting in many overlapping polygons, sliver polygons, and small holes in the coverage. Since the MAPINFO program is primarily used for map production and presentation, rather than spatial analysis, the topological correctness of GIS layers is not an issue, however it did present problems when importing data into ARCVIEW. All overlap areas are interpreted as new polygons in ARCVIEW. Consequently, these problems were corrected in MAPINFO prior to converting to ARCVIEW format. The layer was then exported from MAPINFO as MAPINFo Interchange Format (MIF) files and converted to ARCVIEW shape files (vector format) using a DOS program, supplied with ARCVIEW, called miftoshape.

The Geological mapping for Bardoc Tectonic Zone map was carried out at a 1:5000 scale by John Crossing (Principal Geologist, Goldfields Exploration Pty Ltd). Aerial photographs, which were registered using the surveyed positions of drill hole collars, were used as base maps. Drill collar positions were determined with differential GPS and are therefore accurate to within $\pm 10 \mathrm{~m}$. A significant proportion of this layer is the result of a geological interpretation of high-resolution aeromagnetic images by Dr Scott Halley and John Bayson. The layer also incorporates information from maps published by the Geological Survey of Western Australia, and drilling and mapping by Goldfields Exploration Pty Ltd. The layer was exported from MAPINFO as Mapinfo Interchange Format (MIF) files and converted 
to ArCVIEW shape files (vector format) using a DOS program, supplied with ARCVIEW, called miftoshape.

\section{Logical Accuracy}

The original map of the Kalgoorlie area was compiled from multiple sources and was updated by different geologists. Consequently, the number of logical errors are likely to be relatively high. All of the topological errors were corrected prior to importing the data into ArcVIEW, as explained above.

The Bardoc Tectonic Zone map was prepared over a period of approximately six months, primarily by Dr Scott Halley and John Bayson, and is therefore likely to be significantly more accurate and consistent than the older Kalgoorlie map.

Table D.4: Data dictionary for the Kalgoorlie geology coverage

\begin{tabular}{|l|c|l|}
\hline \multicolumn{2}{|l|}{ Coverage name: geology.shp } \\
\hline \multicolumn{2}{|l|}{ Coverage type: Polygon } \\
\hline Item names & Type & Item description \\
\hline feature_id & I & One to three digit code for rock type \\
\hline feature & C & Lithological code for use in a stratigraphic legend $(2-3$ characters $)$ \\
\hline description & C & Short description of lithology and textural features \\
\hline feature_na & C & Usage unknown - not used \\
\hline source_id & F & Usage unknown - not used \\
\hline
\end{tabular}

\section{D.5 Rock Strength Data and Rheological Contrast Calcu- lations}

\section{D.5.1 Data for Rheological Contrast Calculations, geology.shp}

Rock strength and deformational property data provided by Mark Lee (unpublished data, Australian Mining Consultants Pty Ltd, AMC). These data were used to calculate the rheological contrast at lithological boundaries. Data supplied by AMC consisted of Young's Modulus, uniaxial compressive and tensile strength and fracture toughness. Some of the data supplied by AMC originated from Szwedzicki \& Jiang (1994). In cases where the 
original compressive strength data were measured on $35 \mathrm{~mm}$ core, the following conversion was applied to give the values for a $50 \mathrm{~mm}$ diameter core size;

$$
\frac{U C S_{x}}{U C S_{50}}=\left(\frac{50}{x}\right)^{0.18}
$$

where $x$ is the diameter of the core in millimetres. Data for rheological properties are contained as attributes in the database table associated with the solid geology GIS layer (refer to Table D.4 above). Rock strength values shown in Table 5.5 in Chapter 5 represent the averages of rock types from different localities in the AMC data that are of the same type or are used to approximate those in the Kalgoorlie solid geology coverage. The AMC data for a given rock type represent the average of many tests (generally $>10$ ) at particular mine site.

Although many rock engineering measurements are routinely made, published data for specific areas and rock types are scarce. This, combined with the fact that the solid geology attribute table contains 108 rock types meant it was often necessary to use data for a generic rock type to approximate the more specific types appearing in the solid geology attribute table (e.g. granite data used for tonalite). The correspondence between the rock types for which engineering data were available and the rock types shown in the Kalgoorlie solid geology coverage (i.e. rock types in the detailed Goldfields Exploration geology legend) is shown in Table D.5 below.

Table D.5: Calculation of average rock strength and deformational properties for rocks in the Kalgoorlie study area. Unpublished data provided by M. Lee, Australian Mining Consultants Pty Ltd, 2000. Left rock type column shows rock types in legend of solid geology GIS layer (108 rock types). The second rock type column shows closest equivalent rock types from the AMC data set that were used to estimate rock strength properties. Abbreviations: Fract. Tough. = fracture toughness, UCS \& UTS = Uniaxial compressive \& tensile strength, respectively,

\begin{tabular}{|c|c|c|c|c|c|c|}
\hline $\begin{array}{l}\text { Rock Type } \\
\text { (GIS layer) }\end{array}$ & $\begin{array}{l}\text { Rock Type } \\
\text { (AMC data) }\end{array}$ & $\begin{array}{l}\text { Location } \\
\text { or mine }\end{array}$ & $\begin{array}{l}\text { Young's } \\
\text { Modulus } \\
\text { GPa }\end{array}$ & $\begin{array}{l}\mathrm{UCS} \\
\mathrm{MPa}\end{array}$ & $\begin{array}{l}\mathrm{UTS} \\
\mathrm{MPa}\end{array}$ & $\begin{array}{l}\text { Fract. } \\
\text { Tough. } \\
\text { MPa m }^{\frac{1}{2}}\end{array}$ \\
\hline $\begin{array}{l}\text { Chert } \\
\text { Quartzite }\end{array}$ & Vein quartz & Zarmitain, USSR & 85.0 & 156 & 8 & $-^{3}$ \\
\hline $\begin{array}{l}\text { Si-rich Hornfels } \\
\text { Fe-rich Ortho Hornfels } \\
\text { Mg-rich Ortho Hornfels }\end{array}$ & $\begin{array}{l}\text { Metasediments (h.w.) } \\
\text { Metasiltstone } \\
\text { Ave. }\end{array}$ & $\begin{array}{l}\text { Perseverance } \\
\text { Big Bell }\end{array}$ & $\begin{array}{l}74.9 \\
77.1 \\
76.0\end{array}$ & $\begin{array}{l}166 \\
268 \\
217\end{array}$ & $\begin{array}{l}19.4 \\
21.8 \\
20.6\end{array}$ & $\begin{array}{l}\overline{-} \\
3.12 \\
3.12\end{array}$ \\
\hline $\begin{array}{l}\text { Banded Iron Formation } \\
\text { Iron Formation }\end{array}$ & Banded Iron Formation & Bounty & 64.0 & 152 & 13.6 & 1.76 \\
\hline $\begin{array}{l}\text { Felds+Qtz Porphyry }{ }^{15} \\
\text { Felds+Qtz+Hbl Porph. } \\
\text { Felds+Qtz+Bio Porph. } \\
\text { Felds Porphyry }\end{array}$ & $\begin{array}{l}\text { Qtz felds. porphyry } \\
\text { Porphyry } \\
\text { Porphyry } \\
\text { Porphyry }\end{array}$ & $\begin{array}{l}\text { Bounty } \\
\text { Junction } \\
\text { Mount Charlotte } \\
\text { Macassa }\end{array}$ & $\begin{array}{l}65.7 \\
56.8 \\
66.7 \\
60.0\end{array}$ & $\begin{array}{l}258 \\
216 \\
118 \\
233\end{array}$ & $\begin{array}{l}12.2 \\
19.3 \\
15.3 \\
20.6\end{array}$ & $\begin{array}{l}2.50^{4} \\
2.66 \\
2.10 \\
-\end{array}$ \\
\hline
\end{tabular}


Table D.5: continued

\begin{tabular}{|c|c|c|c|c|c|c|}
\hline $\begin{array}{l}\text { Plag } \pm \text { Hbl Porph. } \\
\text { Felds }+ \text { Hbl+Bio Porph. }\end{array}$ & $\begin{array}{l}\text { Porphyry } \\
\text { Porphyry } \\
\text { Porphyry } \\
\text { Ave. }\end{array}$ & $\begin{array}{l}\text { Kanowna Belle } \\
\text { Kambalda-St Ives } \\
\text { Long Shaft }\end{array}$ & $\begin{array}{l}69.7 \\
61.7 \\
62.7 \\
63.3\end{array}$ & $\begin{array}{l}67 \\
266 \\
277 \\
250\end{array}$ & $\begin{array}{l}18.1 \\
17.6 \\
17.5 \\
17.2\end{array}$ & $\begin{array}{l}-\overline{2.3} \\
2.31 \\
2.37\end{array}$ \\
\hline $\begin{array}{l}\text { Granite } \\
\text { Quartz Monzonite } \\
\text { Granodiorite } \\
\text { Weebo Granodiorite } \\
\text { Tonalite } \\
\text { Monzonite } \\
\text { Syenodiorite } \\
\text { Granitoid }\end{array}$ & Granite & Zarmitain, USSR & 48.2 & 141 & 10.8 & 1.61 \\
\hline Syenite & $\begin{array}{l}\text { Basic syenite } \\
\text { Basic syenite (siliceous) } \\
\text { Ave. }\end{array}$ & $\begin{array}{l}\text { Macassa } \\
\text { Macassa }\end{array}$ & $\begin{array}{l}44.8 \\
57.2 \\
51.0\end{array}$ & $\begin{array}{l}135 \\
208 \\
172\end{array}$ & $\begin{array}{l}18.8 \\
14.5 \\
16.7\end{array}$ & $\begin{array}{l}\overline{-}^{5} \\
1.61\end{array}$ \\
\hline $\begin{array}{l}\text { Dolerite } \\
\text { Porphyritic Dolerite } \\
\text { Leucocratic Dolerite } \\
\text { Porphyritic Andesite } \\
\text { Dacite } \\
\text { Andesite } \\
\text { Magnetic Basalt }\end{array}$ & $\begin{array}{l}\text { Dolerite } \\
\text { Altered Dolerite } \\
\text { Junction Dolerite } \\
\text { Golden Mile Dolerite } \\
\text { (unit 6) } \\
\text { Golden Mile Dolerite } \\
\text { (unit 7) } \\
\text { Golden Mile Dolerite } \\
\text { (unit 8) carbonated } \\
\text { Golden Mile Dolerite } \\
\text { (unit 9) carbonated } \\
\text { Williamstown Dolerite } \\
\text { (fuxchite alt.) } \\
\text { Ave. }\end{array}$ & $\begin{array}{l}\text { Bounty } \\
\text { Bounty } \\
\text { Junction } \\
\text { Mount Charlotte } \\
\text { Mount Charlotte } \\
\text { Mount Charlotte } \\
\text { Mount Charlotte } \\
\text { Mount Charlotte }\end{array}$ & $\begin{array}{l}75.4 \\
71.7 \\
92.6 \\
87.6 \\
83.9 \\
64.8 \\
69.4 \\
88.5 \\
80.3\end{array}$ & $\begin{array}{l}153 \\
177 \\
167 \\
331 \\
215\end{array}$ & $\begin{array}{l}13.2 \\
12.1 \\
21.8 \\
- \\
- \\
14.1 \\
17.3 \\
16.0 \\
16.7\end{array}$ & $\begin{array}{l}2.97 \\
2.70 \\
3.20 \\
2.96\end{array}$ \\
\hline Quartz Dolerite & Ore (quartz dolerite) & $\begin{array}{l}\text { Centenary Lode, } \\
\text { Darlot }\end{array}$ & 76.5 & 223 & 17.9 & $2.96^{6}$ \\
\hline $\begin{array}{l}\text { Felsic Volc. Rock } \\
\text { M/m Felsic Tuff \& Lava } \\
\text { Felsic P/clastic Breccia } \\
\text { Felsic Tuff } \\
\text { (crystal,lithic) } \\
\text { Undiv. Felsic A/clastic } \\
\text { Undiv. Felsic P/clastic } \\
\text { Volc. Feldspathic Wacke } \\
\text { Fragmental (m.g.) } \\
\text { Fragmental (c.g.) }\end{array}$ & $\begin{array}{l}\text { Felsics } \\
\text { Tuff } \\
\text { Felsic (clastic) } \\
\text { Felsic (volcanic) } \\
\text { Felsic Volcanic } \\
\text { Felsic Volcanic }\end{array}$ & $\begin{array}{l}\text { Perseverance } \\
\text { Macassa } \\
\text { Black Swan } \\
\text { Black Swan } \\
\text { Kanowna Belle } \\
\text { Centenary Lode, } \\
\text { Darlot }\end{array}$ & $\begin{array}{l}80.5 \\
64.1 \\
54.0 \\
57.1 \\
\\
68.2 \\
81.5\end{array}$ & $\begin{array}{l}142 \\
218 \\
84 \\
99\end{array}$ & $\begin{array}{l}13.3 \\
16.2 \\
17.2 \\
15.1 \\
\\
16.3 \\
19.1\end{array}$ & $\begin{array}{l}- \\
- \\
1.40 \\
- \\
1.43 \\
-\end{array}$ \\
\hline $\begin{array}{l}\text { Undiv. Int. Volc. Rock } \\
\text { Undiv. Int. A/clastic } \\
\text { Int. P/clastic Breccia }\end{array}$ & $\begin{array}{l}\text { Int. Volcanics (IMVT) } \\
\text { Int. Volcanics (IMVT) } \\
\text { Int. Volcanics (IMVT) } \\
\text { Tuff (IVT) FW } \\
\text { Tuff (IVT) FW } \\
\text { Tuff (IVT) FW } \\
\text { Tuff (IVT) FW } \\
\text { Tuff (IVT) FW } \\
\text { Tuff (IVT) FW } \\
\text { Tuff (IVT) FW } \\
\text { Tuff (IVT) FW } \\
\text { Tuff (IVT) FW } \\
\text { Tuff (IVT) FW }\end{array}$ & $\begin{array}{l}\text { Kundana KD28 }{ }^{1} \\
\text { Kundana KD29 } \\
\text { Kundana KD21 } \\
\text { Kundana CD6-1 } \\
\text { Kundana CD4-14 } \\
\text { Kundana CD4-15 } \\
\text { Kundana CD6-2 } \\
\text { Kundana KC2D-14 } \\
\text { Kundana CD6-3 } \\
\text { Kundana CD5-11 } \\
\text { Kundana KC2D- } \\
\text { 16A } \\
\text { Kundana KC2D- } \\
\text { 16B } \\
\text { Kundana CD5-12 }\end{array}$ & $\begin{array}{l}- \\
- \\
- \\
- \\
- \\
- \\
59.7 \\
- \\
62.5 \\
51.2 \\
-\end{array}$ & $\begin{array}{l}150 \\
105 \\
171 \\
- \\
- \\
- \\
- \\
- \\
- \\
- \\
136\end{array}$ & $\begin{array}{l}- \\
- \\
- \\
10.6 \\
17.5 \\
25.1 \\
20.0 \\
- \\
23.9 \\
13.9 \\
-\end{array}$ & $\begin{array}{l}-^{7} \\
- \\
- \\
- \\
- \\
- \\
- \\
- \\
-\end{array}$ \\
\hline
\end{tabular}


Table D.5: continued

\begin{tabular}{|c|c|c|c|c|c|c|}
\hline & $\begin{array}{l}\text { Tuff (IVT) FW } \\
\text { Tuff (IVT) FW } \\
\text { Ave. }\end{array}$ & $\begin{array}{l}\text { Kundana CD5-13B } \\
\text { Kundana CD5-13A }\end{array}$ & $\begin{array}{l}- \\
57.8\end{array}$ & $\begin{array}{l}- \\
141\end{array}$ & $\begin{array}{l}26.2 \\
18.9 \\
20.6\end{array}$ & 2.29 \\
\hline $\begin{array}{l}\text { Pegmatite } \\
\text { Felsic Gneiss }\end{array}$ & $\begin{array}{l}\text { Pegmatite } \\
\text { Ave. }\end{array}$ & $\begin{array}{l}\text { Bounty } \\
\text { Perseverance }\end{array}$ & $\begin{array}{l}63.6 \\
- \\
63.6\end{array}$ & $\begin{array}{l}205 \\
179 \\
192\end{array}$ & $\begin{array}{l}7.5 \\
8.3 \\
7.9\end{array}$ & $\begin{array}{l}1.70 \\
- \\
1.70\end{array}$ \\
\hline $\begin{array}{l}\text { Basalt } \\
\text { Mount Goode Basalt } \\
\text { Porphyritic Basalt } \\
\text { Mafic P/clastic Breccia } \\
\text { Undiv. Mafic Rocks } \\
\text { Undiv.Basalt.A/clastic } \\
\text { Undiv. Mafic P/clastic }\end{array}$ & $\begin{array}{l}\text { Basalt } \\
\text { Devon Consols } \\
\text { Basalt } \\
\text { H.w. tholeitic basalt } \\
\text { Basalt } \\
\text { Basalt (MB) FW } \\
\text { Basalt (MB) FW } \\
\text { Basalt (MB) FW } \\
\text { Basalt (MB) FW } \\
\text { Basalt (MB) FW } \\
\text { Basalt (MB) FW } \\
\text { Basalt (MB) FW } \\
\text { Basalt (MB) FW } \\
\text { Basalt (MB) FW } \\
\text { Basalt (MB) FW } \\
\text { Basalt (MB) FW } \\
\text { Basalt (MB) FW } \\
\text { Basalt (MB) FW } \\
\text { Basalt (MB) FW } \\
\text { Basalt (MB) FW } \\
\text { Basalt (MB) FW } \\
\text { Basalt (MB) FW } \\
\text { Basalt (MB) FW } \\
\text { Basalt (MB) FW } \\
\text { Basalt (MB) FW } \\
\text { Basalt (MB) FW } \\
\text { Basalt (MB) FW } \\
\text { Basalt (MB) FW } \\
\text { Basalt (MB) FW } \\
\text { Ave } \\
\text { Ba } \\
\text { Ba } \\
\text { Ba }\end{array}$ & $\begin{array}{l}\text { Bounty } \\
\text { Mount Charlotte } \\
\text { Kambalda-St Ives } \\
\text { Lancefield } \\
\text { Centary Lode, } \\
\text { Darlot } \\
\text { Kambalda KCD2- } \\
\text { 19A } \\
\text { Kambalda KCD2- } \\
\text { 19C } \\
\text { Kambalda KCD2- } \\
\text { 19B } \\
\text { Kundana CD5-5B }{ }^{1} \\
\text { Kundana CD5-5C } \\
\text { Kundana CD5-5A } \\
\text { Kundana CD5-1 } \\
\text { Kundana KCD2-11 } \\
\text { Kundana KCD2- } \\
\text { 20C } \\
\text { Kundana KCD2- } \\
\text { 20A } \\
\text { Kundana KCD2- } \\
\text { 20B } \\
\text { Kundana CD5-6B } \\
\text { Kundana CD5-2 } \\
\text { Kundana CD5-6A } \\
\text { Kundana CD5-6C } \\
\text { Kundana CD3-8 } \\
\text { Kundana CD5-3A } \\
\text { Kundana CD5-3B } \\
\text { Kundana CD5-7B } \\
\text { Kundana CD5-4 } \\
\text { Kundana CD5-7A } \\
\text { Kundana CD5-7C } \\
\text { Kundana CD5-8A } \\
\text { Kundana CD5-8B }\end{array}$ & $\begin{array}{l}- \\
- \\
- \\
- \\
- \\
- \\
64.2 \\
74.9 \\
- \\
- \\
- \\
- \\
74.7 \\
67.0 \\
64.4 \\
58.9 \\
60.8 \\
- \\
- \\
720\end{array}$ & $\begin{array}{l}- \\
- \\
- \\
- \\
- \\
- \\
- \\
- \\
- \\
- \\
101\end{array}$ & $\begin{array}{l}13.0 \\
18.0 \\
22.0 \\
19.0 \\
21.0 \\
16.0 \\
- \\
- \\
21.0 \\
16.0 \\
30.0 \\
19.0 \\
22.0 \\
20.0 \\
- \\
25.0 \\
- \\
21.0 \\
23.0 \\
15.0 \\
17.0 \\
204\end{array}$ & $\begin{array}{l}- \\
- \\
- \\
- \\
- \\
- \\
-\end{array}$ \\
\hline $\begin{array}{l}\text { Magnesian Basalt } \\
\text { Mafic Ultramafic }\end{array}$ & $\begin{array}{l}\text { Ave. } \\
\text { High magnesium basalt }\end{array}$ & Bounty & $\begin{array}{l}72.0 \\
88.3\end{array}$ & $\begin{array}{l}191 \\
212\end{array}$ & $\begin{array}{l}20.4 \\
16.9\end{array}$ & $\begin{array}{l}3.31 \\
3.65\end{array}$ \\
\hline $\begin{array}{l}\text { Gabbro } \\
\text { Gabbro-Kundana unit } 1 \\
\text { Gabbro-Kundana unit } 2 \\
\text { Gabbro-Kundana unit } 3 \\
\text { Gabbro-Kundana } \\
\text { unit6A } \\
\text { Porphyritic Gabbro } \\
\text { Leucocratic Gabbro } \\
\text { Melanocratic Gabbro } \\
\text { Gabbro-Kundana unit } 5 \\
\text { Gabbro-Kundana unit } 6\end{array}$ & $\begin{array}{l}\text { Gabbro } \\
\text { Gabbro }\end{array}$ & $\begin{array}{l}\text { Bounty } \\
\text { Lancefield }\end{array}$ & $\begin{array}{l}72.5 \\
85.4\end{array}$ & $\begin{array}{l}216 \\
-\end{array}$ & $\begin{array}{l}11.7 \\
17.5\end{array}$ & $\begin{array}{l}2.67^{9} \\
-\end{array}$ \\
\hline Alkali-Ortho Amphib. & $\begin{array}{l}\text { Metasediments (h.w., } \\
\text { amphib.) }\end{array}$ & Perseverance & 74.9 & 166 & 19.4 & - \\
\hline
\end{tabular}


Table D.5: continued

\begin{tabular}{|c|c|c|c|c|c|c|}
\hline $\begin{array}{l}\text { Fe-Ortho Amphib. f.g. } \\
\text { Fe-Ortho Amphib. c.g. } \\
\text { Calcic Para Amphibolite } \\
\text { Para-Amphibolite, mafic } \\
\text { Mg-rich Ortho Amphib. } \\
\text { Mafic Amphibolite }\end{array}$ & Metasiltstone (amphib.) & Big Bell & 77.1 & 268 & 21.8 & 3.12 \\
\hline Undivided Arenite & $\begin{array}{l}\text { Black Flag Beds } \\
\text { (sed. rock) } \\
\text { Sandstone } \\
\text { Ave. }\end{array}$ & $\begin{array}{l}\text { Junction } \\
\text { Kanowna Belle }\end{array}$ & $\begin{array}{l}69.2 \\
79.2 \\
74.2\end{array}$ & - & - & - \\
\hline $\begin{array}{l}\text { Conglomerate } \\
\text { Monomict Conglomerate } \\
\text { Polymict Congl. (Kur- } \\
\text { rawang) } \\
\text { Jones Ck Conglomerate } \\
\text { Volc.-derived Conglom. } \\
\text { (mafic-u/m) }\end{array}$ & $\begin{array}{l}\text { Cemetery Conglomerate } \\
\text { Felsic conglomerate } \\
\text { Footwall conglomerate } \\
\text { Golden Valley Conglom. } \\
\text { Ave. }\end{array}$ & $\begin{array}{l}\text { Kanowna Belle } \\
\text { Kanowna Belle } \\
\text { Kanowna Belle } \\
\text { Kanowna Belle }\end{array}$ & $\begin{array}{l}72.2 \\
75.1\end{array}$ & $\begin{array}{l}144 \\
94 \\
-\end{array}$ & $\begin{array}{l}15.9 \\
14.4\end{array}$ & $\begin{array}{l}3.68 \\
2.87\end{array}$ \\
\hline $\begin{array}{l}\text { Int. Volcanogenic Sed. } \\
\text { Volcanogenic Sed. Rock } \\
\text { Feldspathic Wacke } \\
\text { Fine-grained Fragmental } \\
\text { Undivided Wacke } \\
\text { Volcanic Wacke }\end{array}$ & $\begin{array}{l}\text { Arkosic grit } \\
\text { Felsic grit } \\
\text { Felsic clastic }\end{array}$ & $\begin{array}{l}\text { Kanowna Belle } \\
\text { Kanowna Belle } \\
\text { Black Swan }\end{array}$ & $\begin{array}{l}61.4 \\
69.3 \\
54.0\end{array}$ & $\frac{161}{84}$ & $\begin{array}{l}15.2 \\
17.2\end{array}$ & $\begin{array}{l}0.80 \\
1.40\end{array}$ \\
\hline $\begin{array}{l}\text { Siltstone } \\
\text { Interbedded Slst-Shale }\end{array}$ & $\begin{array}{l}\text { Black Flag Beds } \\
\text { (sed. rock), carbonated } \\
\text { Black Flag Beds } \\
\text { (sed. rock) } \\
\text { Ave. }\end{array}$ & $\begin{array}{l}\text { Mount Charlotte } \\
\text { Junction }\end{array}$ & $\begin{array}{l}53.0 \\
69.2 \\
61.1\end{array}$ & $\begin{array}{l}116 \\
219 \\
168\end{array}$ & $\begin{array}{l}15.2 \\
21.7 \\
18.5\end{array}$ & $\begin{array}{l}2.30 \\
2.92 \\
2.61\end{array}$ \\
\hline Undiv. Sed. Rocks & $\begin{array}{l}\text { Contact sediments } \\
\text { Sediments } \\
\text { Contact sediments } \\
\text { Grit } \\
\text { Black Flag Beds } \\
\text { (sed. rock) } \\
\text { Arkosic grit } \\
\text { Felsic grit } \\
\text { Felsic clastic } \\
\text { Ave. }\end{array}$ & $\begin{array}{l}\text { Kambalda } \\
\text { Zamitain, USSR } \\
\text { Long, Kambalda } \\
\text { Kanowna Belle } \\
\text { Junction } \\
\text { Kanowna Belle } \\
\text { Kanowna Belle } \\
\text { Black Swan }\end{array}$ & $\begin{array}{l}48.5 \\
53.9 \\
48.5 \\
77.1 \\
69.2 \\
\\
61.4 \\
69.3 \\
54.0 \\
60.2\end{array}$ & $\begin{array}{l}141 \\
166 \\
144 \\
162 \\
219 \\
161 \\
84 \\
154\end{array}$ & $\begin{array}{l}\overline{12.2} \\
\overline{18.0} \\
21.7 \\
15.2 \\
- \\
17.2 \\
16.9\end{array}$ & $\begin{array}{l}- \\
- \\
1.33 \\
2.92 \\
0.80 \\
1.40 \\
1.28\end{array}$ \\
\hline $\begin{array}{l}\text { Komatiite } \\
\text { Ultramafic Autobreccia }\end{array}$ & $\begin{array}{l}\text { Komatiitic Basalt } \\
\text { Altered komatiitic basalt } \\
\text { F.w. komatiitic basalt } \\
\text { Ave. }\end{array}$ & $\begin{array}{l}\text { Bounty } \\
\text { Bounty } \\
\text { Lancefield }\end{array}$ & $\begin{array}{l}82.2 \\
70.2 \\
93.6 \\
82.0\end{array}$ & $\begin{array}{l}169 \\
180 \\
273 \\
207\end{array}$ & $\begin{array}{l}15.6 \\
16.4 \\
19.4 \\
17.1\end{array}$ & $\begin{array}{l}4.73 \\
3.07 \\
- \\
3.90\end{array}$ \\
\hline $\begin{array}{l}\text { Peridotite } \\
\text { Pyroxenite }\end{array}$ & $\begin{array}{l}\text { Peridotite, not } \\
\text { serp./ized }\end{array}$ & Bounty & 70.8 & 118 & 19.3 & 2.61 \\
\hline $\begin{array}{l}\text { Serpentinised Komatiite } \\
\text { Serpentinised Peridotite } \\
\text { Serpentinite }\end{array}$ & $\begin{array}{l}\text { U/mafic (serp.-talc) } \\
\text { U/mafic (ol.-serp.) } \\
\text { Hannans Lake Serpenti- } \\
\text { nite } \\
\text { Ave. }\end{array}$ & $\begin{array}{l}\text { Perseverance } \\
\text { Perseverance } \\
\text { Mount Charlotte }\end{array}$ & $\begin{array}{l}36.8 \\
79.7 \\
26.8 \\
47.8\end{array}$ & $\begin{array}{l}90 \\
128 \\
68 \\
95\end{array}$ & \begin{tabular}{l|}
7.1 \\
12.1 \\
5.7 \\
8.3
\end{tabular} & $\begin{array}{l}\overline{-} \\
1.76 \\
-\end{array}$ \\
\hline $\begin{array}{l}\text { Ultramafic Rock } \\
\text { Amph-Chl u/mafic Rock }\end{array}$ & $\begin{array}{l}\mathrm{U} / \text { mafic (talc-carb.) } \\
\mathrm{U} / \text { mafic (antigorite) } \\
\mathrm{U} / \text { mafic (talc-chl.) } \\
\mathrm{U} / \text { mafic (serp.-talc) }\end{array}$ & $\begin{array}{l}\text { Long Shaft } \\
\text { Long Shaft } \\
\text { Perseverance } \\
\text { Perseverance }\end{array}$ & $\begin{array}{l}49.3 \\
66.4 \\
33.7 \\
36.8\end{array}$ & $\begin{array}{l}64 \\
212 \\
46 \\
90\end{array}$ & \begin{tabular}{l|}
7 \\
25.3 \\
4.3 \\
7.1
\end{tabular} & $\begin{array}{l}1.22 \\
- \\
-\end{array}$ \\
\hline
\end{tabular}


Table D.5: continued

\begin{tabular}{|c|c|c|c|c|c|c|}
\hline & U/mafic (ol.-serp.) & Perseverance & 79.7 & 128 & 12.1 & 1.76 \\
\hline & $\begin{array}{l}\text { Talc-carbonate } \\
\text { (soft/weak) TA } 1\end{array}$ & Black Swan & 47.1 & 56 & 9.0 & - \\
\hline & $\begin{array}{l}\text { Talc-carbonate } \\
\text { (stiff/strong) TA } 2\end{array}$ & Black Swan & 87.2 & 177 & 18.3 & - \\
\hline & $\begin{array}{l}\text { U/mafic (talc-chl.) } \\
\text { serpentinized }\end{array}$ & Kambalda-St Ives & 16.3 & 27 & 4.4 & - \\
\hline & $\begin{array}{l}\text { U/mafic (talc-carb.) } \\
\text { serpentinized }\end{array}$ & Kambalda-St Ives & 44.0 & 44 & 6.3 & 1.4 \\
\hline & $\begin{array}{l}\mathrm{U} / \text { mafic (antigorite) } \\
\text { serpentinized }\end{array}$ & Kambalda-St Ives & 67.3 & 209 & 25.3 & - \\
\hline & $\mathrm{U} /$ mafic (talc-chl.) f.w. & Lancefield & 17.0 & 28 & 5.2 & - \\
\hline & Ave. & & 49.5 & 98 & 11.3 & 1.46 \\
\hline \multirow[t]{7}{*}{ Ultramafic Schist } & U/mafic (talc-chlorite) & Perseverance & 33.7 & 46 & 4.3 & $-{ }^{10}$ \\
\hline & $\mathrm{U} /$ mafic (serp.-talc) & Perseverance & 36.8 & 90 & 7.1 & - \\
\hline & Talc-carbonate & Black Swan & 47.1 & 56 & 9.0 & - \\
\hline & U/mafic (talc-chl.) & Kambalda-St Ives & 16.3 & 27 & 4.4 & - \\
\hline & $\begin{array}{l}\mathrm{U} / \text { mafic (talc-carb.) } \\
\text { serpentinized }\end{array}$ & Kambalda-St Ives & 44.0 & 44 & 6.3 & 1.4 \\
\hline & $\mathrm{U} /$ mafic (talc-chl.) f.w. & Lancefield & 17.0 & 28 & 5.2 & - \\
\hline & Ave. & & 32.5 & 49 & 6.1 & 1.31 \\
\hline Shale & \multirow[t]{20}{*}{ Shale } & $\begin{array}{ll}\text { Kundana } & \text { KD } 37 \\
\text { (SHAB) FW } & \end{array}$ & - & 31 & - & $-^{11}$ \\
\hline Shale (altered) & & $\begin{array}{l}\text { Kundana } \\
\text { (SHAB) FW }\end{array}$ & - & 34 & - & - \\
\hline Carbonaceous Shale & & 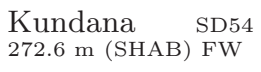 & - & 35 & - & - \\
\hline Actinolite Schist & & $\begin{array}{l}\text { Kundana } \\
273.8 \text { m (SHAB) } \\
\text { FW }\end{array}$ & - & 60 & - & - \\
\hline Fuchsite Schist & & $\begin{array}{l}\text { Kundana KD34 } \\
177.3 \mathrm{~m} \text { (SHAB) } \mathrm{FW}\end{array}$ & - & 98 & - & - \\
\hline Undiff. Felsic Schists & & $\begin{array}{l}\text { Kundana KD34 } \\
178.4 \mathrm{~m}(\mathrm{SHAB}) \mathrm{FW}\end{array}$ & - & 154 & - & - \\
\hline Sericite Schist & & $\begin{array}{l}\text { Kundana KD34 } \\
177.3 \mathrm{~m}(\mathrm{SHAB}) \mathrm{FW}\end{array}$ & - & 115 & - & - \\
\hline \multirow[t]{13}{*}{ Muscovite Schist } & & $\begin{array}{l}\text { Kundana KD34 } \\
182.5 \mathrm{~m} \text { (SHAB) } \mathrm{FW}\end{array}$ & 一 & 134 & - & - \\
\hline & & $\begin{array}{l}\text { Kundana KD34 } \\
194.6 \mathrm{~m}(\mathrm{SHAB}) \mathrm{FW}\end{array}$ & - & 118 & - & - \\
\hline & & $\begin{array}{l}\text { Kundana KD34 } \\
196.1 \mathrm{~m}(\mathrm{SHAB}) \mathrm{FW}\end{array}$ & - & 117 & - & - \\
\hline & & $\begin{array}{l}\text { Kundana KD34 } \\
199.5 \mathrm{~m} \text { (SHAB) FW }\end{array}$ & - & 138 & - & - \\
\hline & & $\begin{array}{l}\text { Kundana } \\
\text { (SHCC,ORE) }\end{array}$ & 72.3 & - & - & - \\
\hline & & $\begin{array}{l}\text { Kundana CD3-17A } \\
\text { (SHCC,ORE) }\end{array}$ & - & - & 29.0 & - \\
\hline & & $\begin{array}{l}\text { Kundana CD3-17C } \\
\text { (SHCC,ORE) }\end{array}$ & - & - & 17.0 & - \\
\hline & & $\underset{\text { (SHCC,ORE) }}{\text { Kundana }}$ CD5-9B & - & - & 14.0 & - \\
\hline & & $\underset{\text { (SHCC,ORE) }}{\text { Kundana CD5-9A }}$ & - & - & 10.0 & - \\
\hline & & $\underset{\text { (SHCC,ORE) }}{\text { Kundana }}$ & - & - & 7.0 & - \\
\hline & & $\underset{\text { (SHCC,ORE) }}{\text { Kundana }}$ & 75.8 & 113 & - & - \\
\hline & & $\begin{array}{l}\text { Kundana } \\
\text { (SHCC,ORE) }\end{array}$ & 69.3 & - & - & - \\
\hline & & & 72.5 & 96 & 15.4 & $1.1^{12}$ \\
\hline \multirow[t]{2}{*}{ Talc-Chl. U/mafic Rock } & U/mafic (talc-chl.) & Perseverance & 33.7 & 46 & 4.3 & $-^{13}$ \\
\hline & U/mafic (talc-chl.) & Kambalda-St Ives & 16.3 & 27 & 4.4 & - \\
\hline
\end{tabular}


Table D.5: continued

\begin{tabular}{|l|l|l|l|l|l|l|}
\hline & serpentinized & & & & \\
& U/mafic (talc-chl.), f.w. & Lancefield & 17.0 & 28 & 5.2 & - \\
& Ave. & & 28.5 & 39 & 5.7 & 1.22 \\
\hline Unknown $^{2}$ Mylonite $^{2}$ & Ave. all rock types used & & 66.0 & 166 & 15.6 & $2.33^{14}$ \\
& Ave. all rock types used & & 66.0 & 166 & 15.6 & $2.33^{14}$ \\
\hline
\end{tabular}

\footnotetext{
${ }^{1}$ Lee:2000 cites source of Kundana rock property database as Szwedzicki \& Jiang, 1994.

${ }^{2}$ Cells in the solid geology raster GIS layer, which are classified as "unknown "or as mylonite were assigned average values calculated using all available data.

${ }^{3}$ Fracture toughness set to granite value.

${ }^{4}$ Two very low UCS values were omitted from the average calculation.

${ }^{5}$ Fracture toughness $=$ granite value.

${ }^{6}$ Fracture toughness $=$ ave. of dolerite.

${ }^{7}$ Fracture toughness $=$ ave. of granite $\&$ dolerite.

${ }^{8}$ Lower 50 percentile of basalts, incl. altered \& amygdaloidal rocks.

${ }^{9}$ Brittle quartz-rich unit; used highest values of Young's Modulus \& UTS available.

${ }^{10}$ Used lower 50 percentile of ultramafic samples above.

${ }^{11}$ Omitted one sample from AMC data with extremely high Young's Modulus

${ }^{12}$ Fracture toughness $=$ average of arkosic grit (0.8) \& felsic clastic (1.4).

${ }^{13}$ Omitted extremely strong sample from average. Fracture toughness value from talc-carb. rock, Long shaft, Kambalda.

${ }^{14}$ Average for all rock types used.

${ }^{15}$ Abbreviations: alt. = altered, amphib. = amphibolite, a/clastic = autoclastic, bio. = biotite, carb. $=$ carbonated, chlorite, felds. = feldspar, f.w. = footwall, hbl = hornblende, h.w. = hanging wall, int. $=$ intermediate, $\mathrm{m} / \mathrm{m}=$ metamorphosed, $\mathrm{ol}=$ olivine, plag $=$ plagioclase, porph. $=$ porphyry, serp $=$ serpentinite, undiv. $=$ undivided, $\mathrm{u} /$ mafic $=$ ultramafic.
} 


\section{D.5.2 Data Dictionary for Structural Geology Layer}

The layer shows the structural geology for the Bardoc Tectonic Zone. The shears correspond to $\mathrm{D}_{2}$ structures and the faults are late brittle $\mathrm{D}_{3}$ structures. The data dictionary for the layer, faults, is shown in Table D.6 below. Lineage details are given in Section D.4.1 on page 548 above.

Table D.6: Data dictionary for the Kalgoorlie structural geology layer

\begin{tabular}{|l|c|l|}
\hline \multicolumn{2}{|l|}{ Coverage name: faults } \\
\hline Coverage type: Polygon \\
\hline Item names & Type & Item description \\
\hline feature_id & I & Code; 323 = fault, $365=$ shear, $671=$ magnetic trend line \\
\hline feature & C & Name of feature; i.e., fault or trend \\
\hline description & C & Description of feature; i.e., shear zone, fault inferred, trend line \\
\hline feature_na & C & Usage unknown - not used \\
\hline source_id & F & Usage unknown - not used \\
\hline
\end{tabular}

\section{D.5.3 Data Dictionary for Fold Axis Layer}

A GIS layer, which shows the location of major anticlinal and synclinal axes were obtained from Yun (2000). Both maps are based on interpretations of the geology and structural maps of the Kalgoorlie area (Swager \& Griffin, 1990; Swager et al., 1990) by Prof. David Groves. The data dictionary for the anticlinal-fold axis layer, faults, is shown in Table D.7 below.

Table D.7: Data dictionary for the anticlinal-fold axis; layer 145 _dist_anti.

\begin{tabular}{|l|c|l|}
\hline \multicolumn{2}{|l|}{ Coverage name: } \\
\hline Coverage type: Polygon \\
\hline Item names & Type & Item description \\
\hline syncline_i & I & Code; 1 = anticline, 2 = syncline \\
\hline Typefeature & C & Name of feature; i.e., fault or trend \\
\hline description & C & Description of feature; i.e., shear zone, fault inferred, trend line \\
\hline feature_na & C & Usage unknown - not used \\
\hline source_id & F & Usage unknown - not used \\
\hline
\end{tabular}

\section{D.6 Geochemical Data}

\section{D.6.1 Data for Chemical Contrast Calculations}

This section documents the whole-rock geochemical data used to calculate the chemical ratios as the first step in creating GIS layers, which depict the chemical contrast at lithological boundaries (refer to Section 5.5.12 in Chapter 5). The data used to calculate 
the chemical ratios represents a subset of the Australian Government Survey Organization's (AGSO) OZCHEM database for the Yilgarn Block. It contains 4473 analyses. The OZCHEM database was imported into ArcView as an ASCII text file and converted to a shape file (point coverage). ArcViews polygon tool was used select the sample points corresponding to the Kalgoorlie Terrane as defined by Swager et al. (1990). This reduced the total number of analyses to 2476 .

In Table D.8, the first set of rows show the rock code and rock type from the ArcView solid geology shape file (vector format) together with the calculated chemical ratios; $\mathrm{Fe}_{2} \mathrm{O}_{3}$ (total $\mathrm{wt} \%) \times \mathrm{Fe}_{t o t} /\left(\mathrm{Fe}_{t o t}+\mathrm{Mg}+\mathrm{Ca}\right), \mathrm{Fe}_{\text {tot }} /\left(\mathrm{Fe}_{\text {tot }}+\mathrm{Mg}+\mathrm{Ca}\right), \mathrm{Fe}_{2} \mathrm{O}_{3} /\left(\mathrm{Fe}_{2} \mathrm{O}_{3}+\mathrm{FeO}\right), \mathrm{Fe}_{2} \mathrm{O}_{3} / \mathrm{FeO}$, and $\mathrm{Fe}^{3+} / \mathrm{Fe}^{2+}$. The next set of rows in each group show selected fields from the corresponding OZCHEM database record; Rock Number, Total Decsription, $\mathrm{Fe}_{2} \mathrm{O}_{3}$, total iron as $\mathrm{Fe}_{2} \mathrm{O}_{3}, \mathrm{FeO}, \mathrm{MgO}$, $\mathrm{CaO}$, Easting, and Northing. 


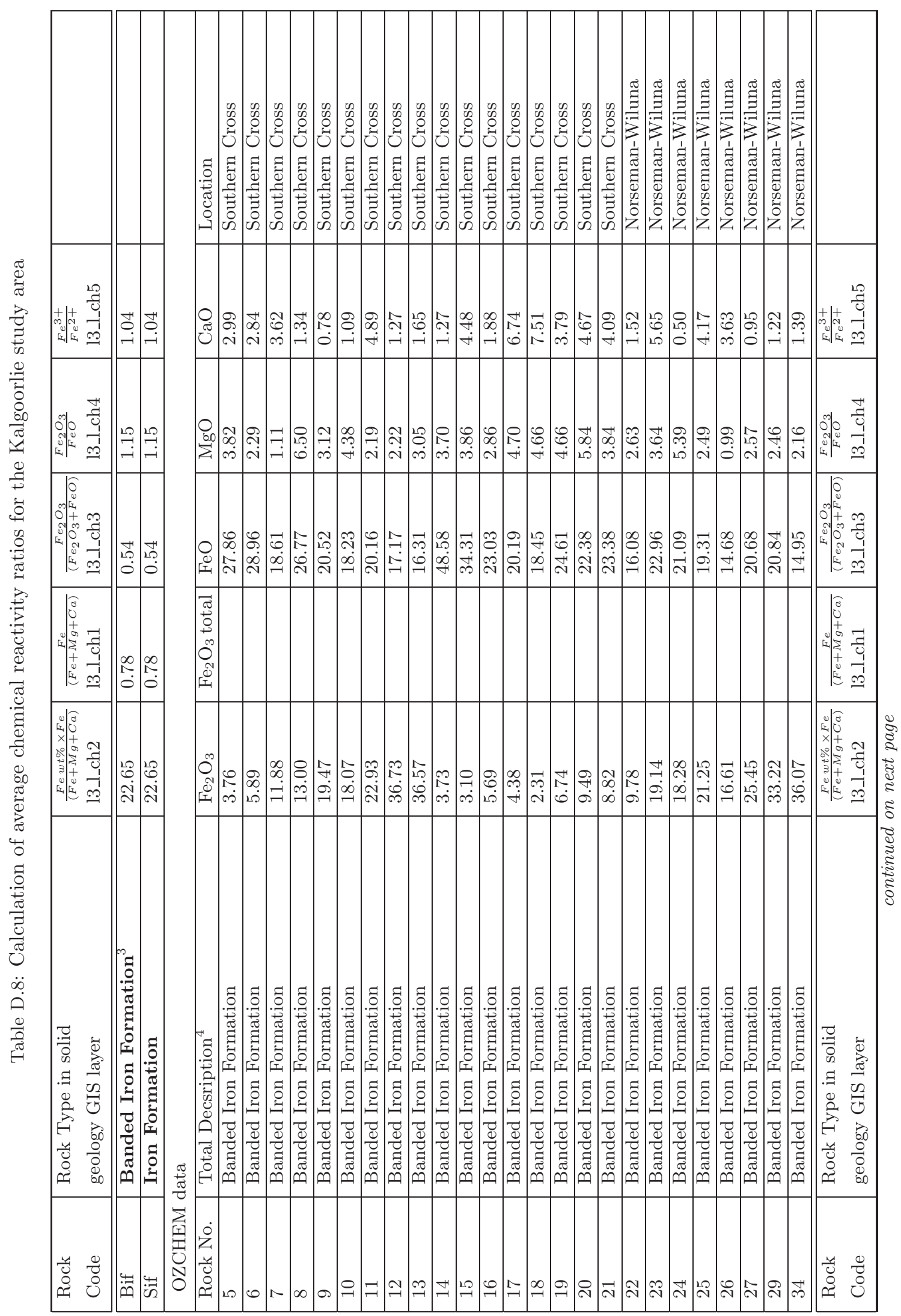




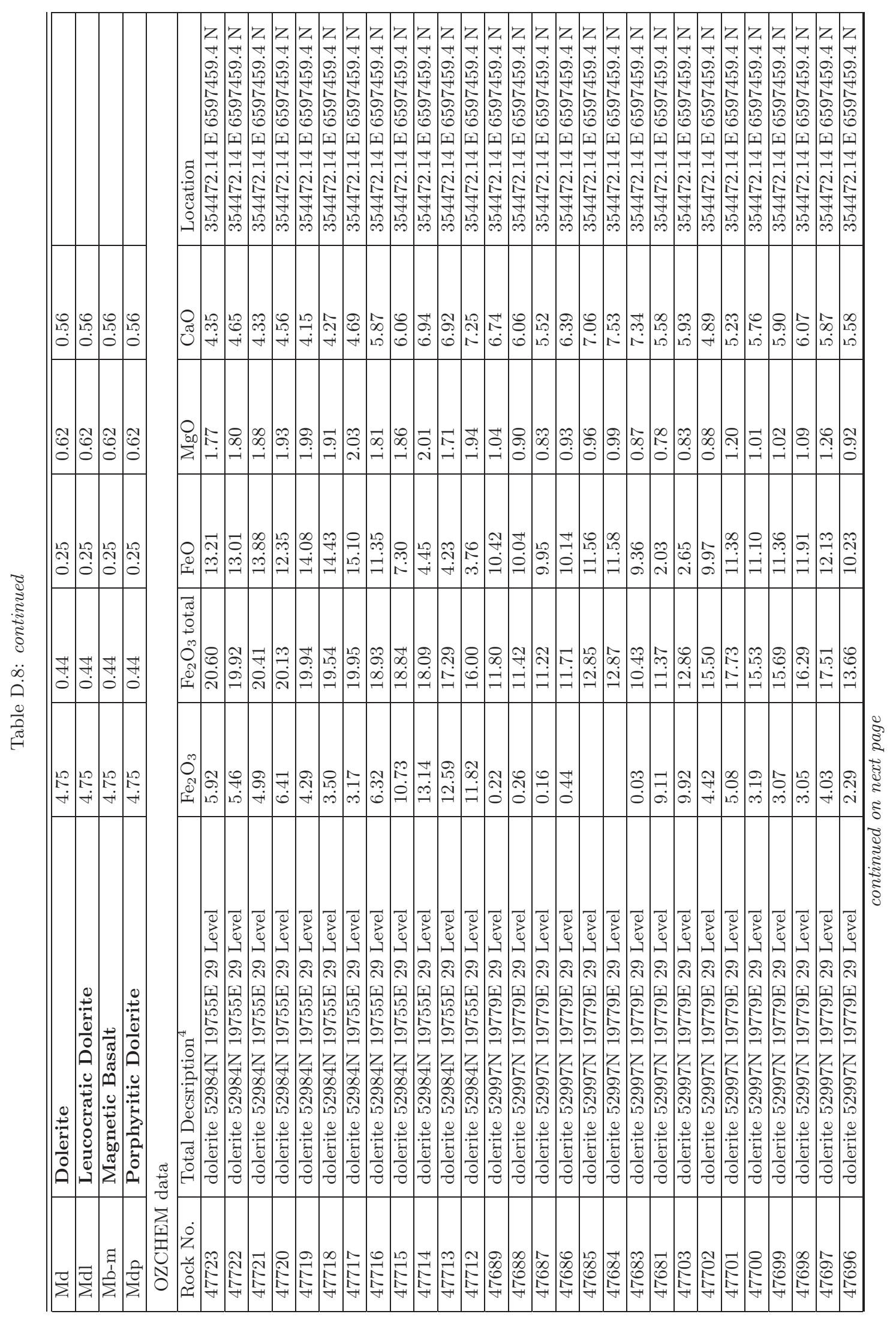




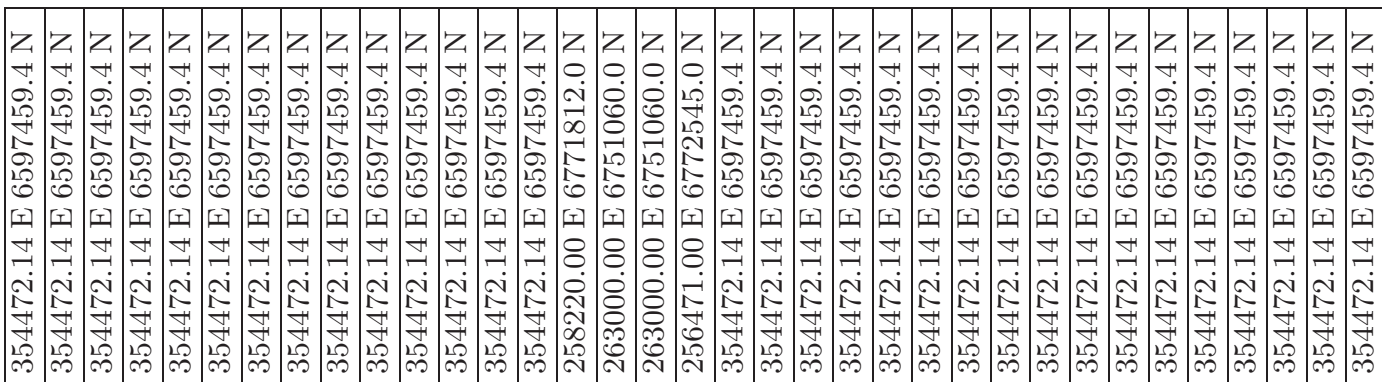

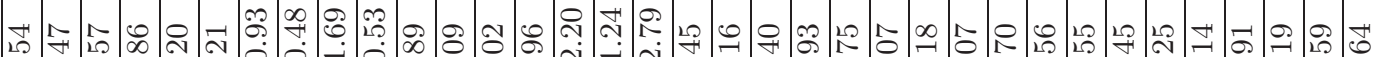

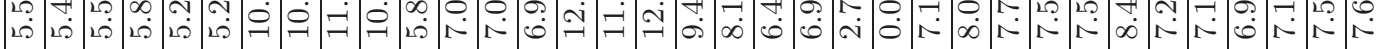

ஜூ:

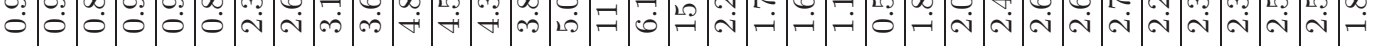

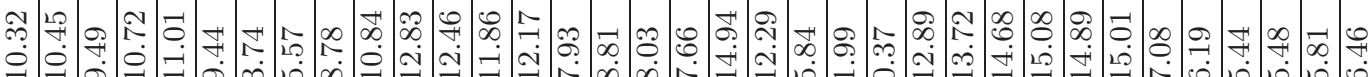

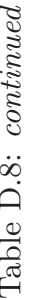

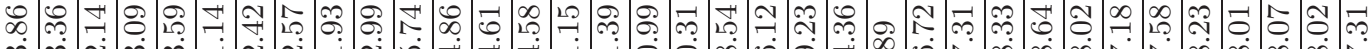

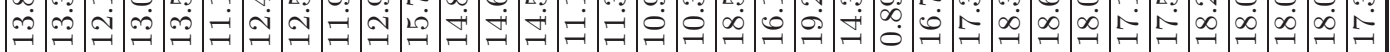

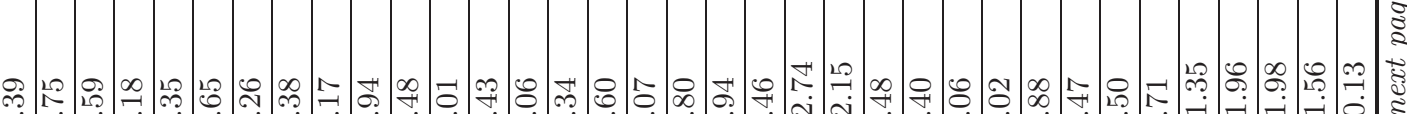

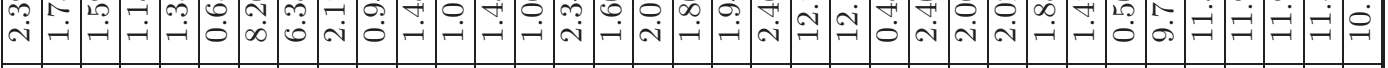

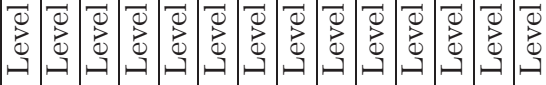

ลิ

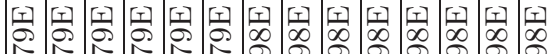

空

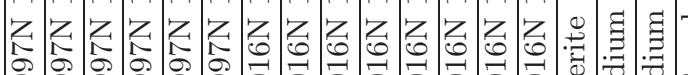

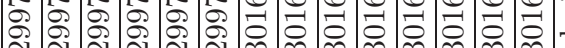

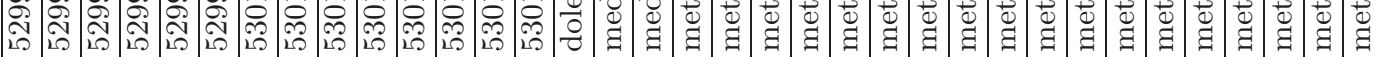

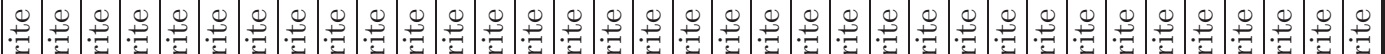

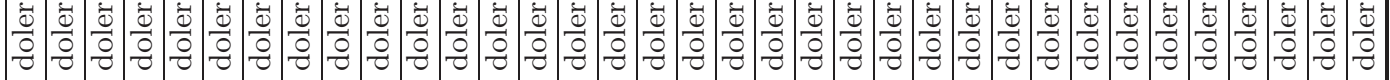

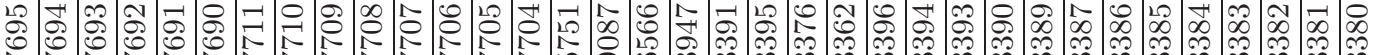

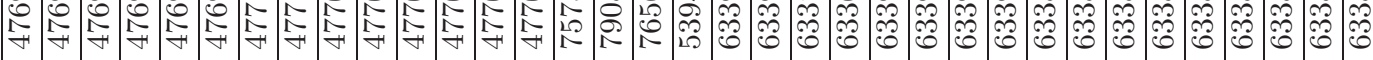




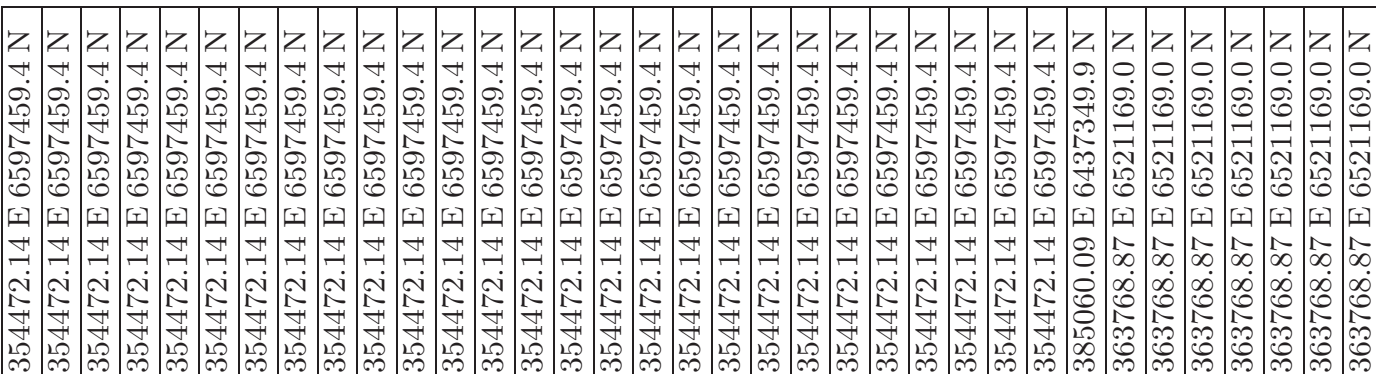

년

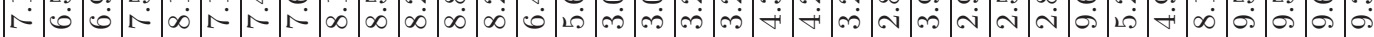

₹

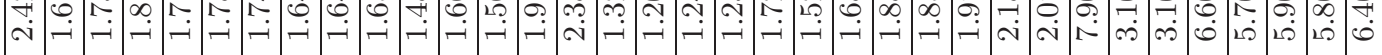

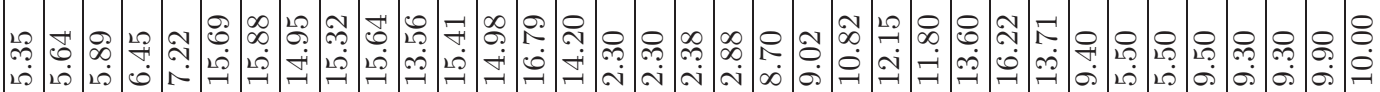

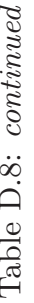

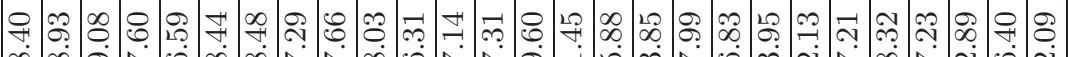

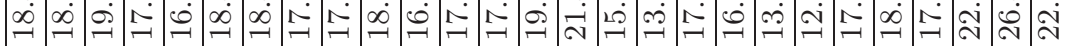

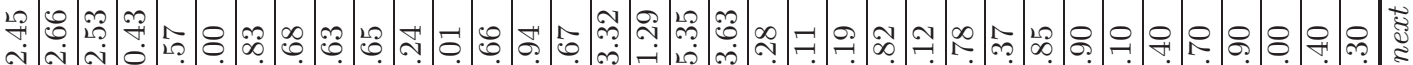

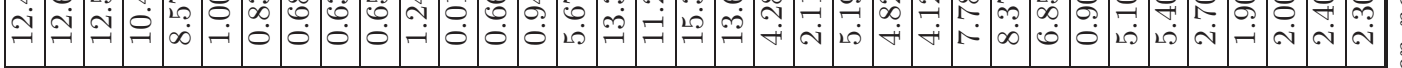

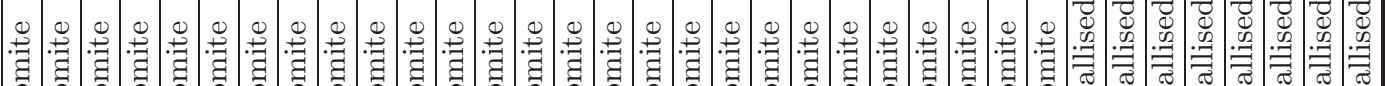

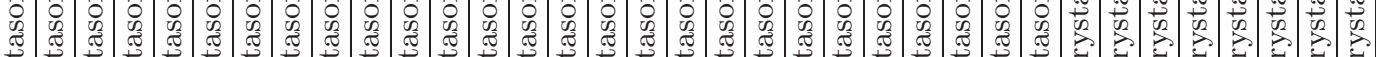
沗

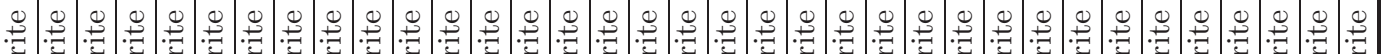

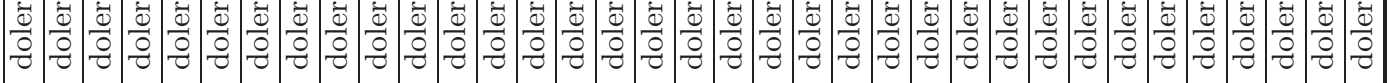

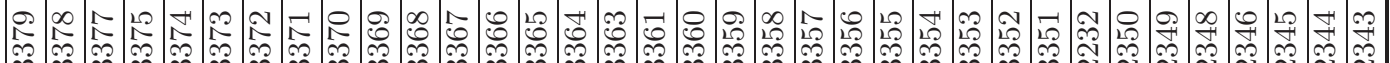

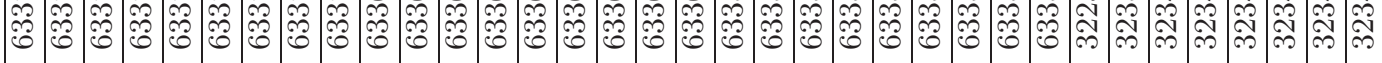




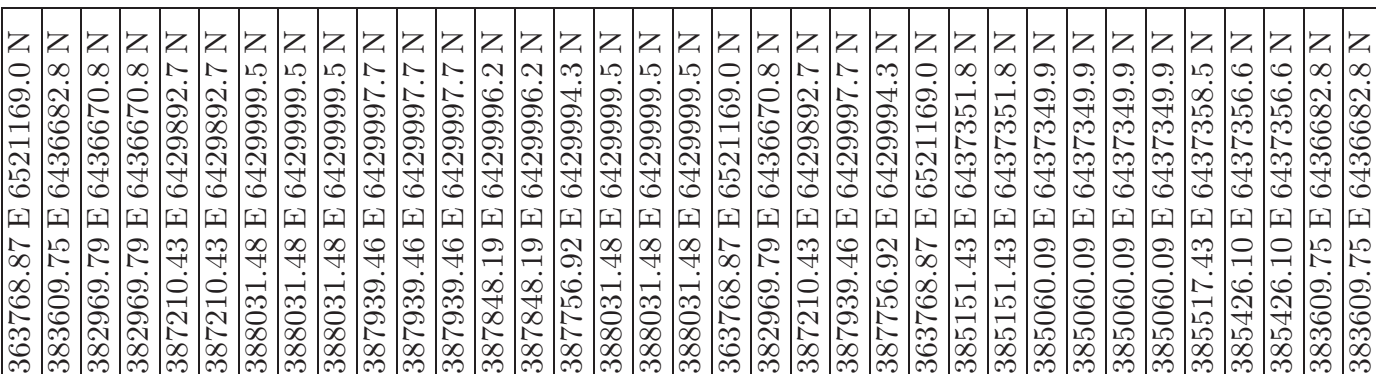

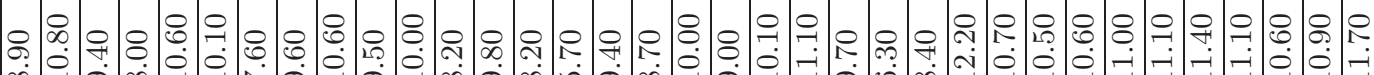

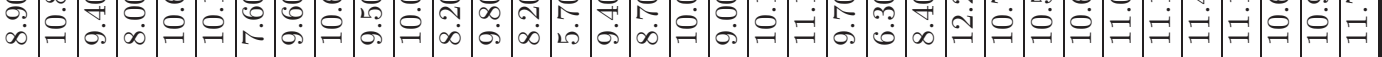

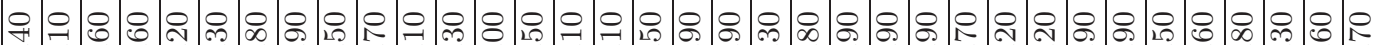

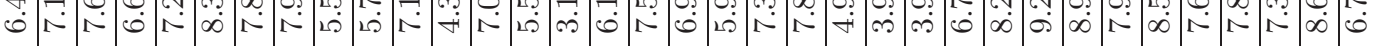

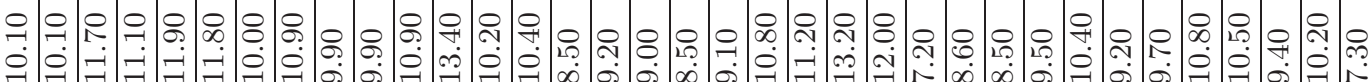

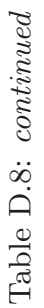

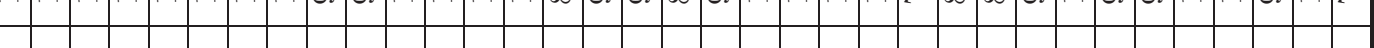

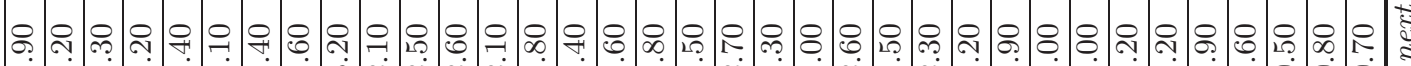

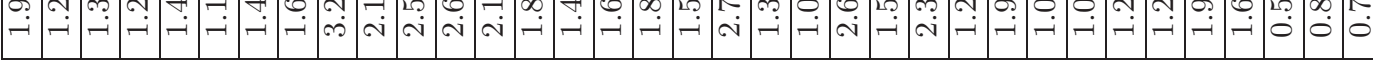

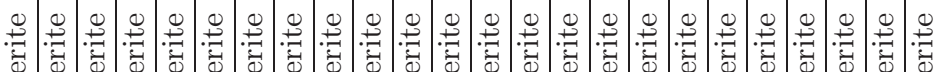

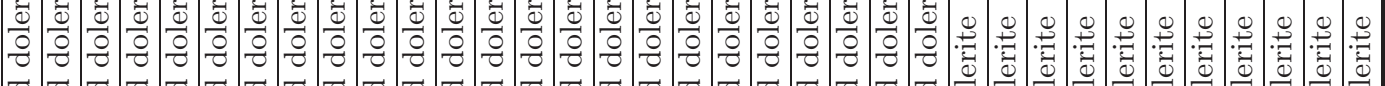

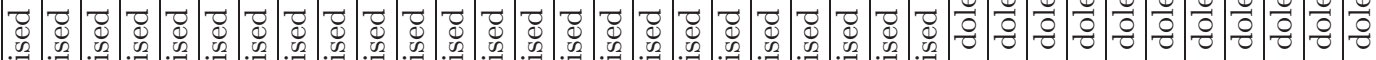

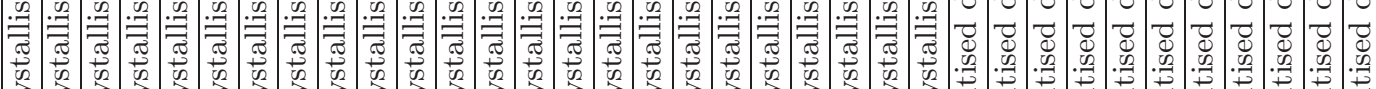

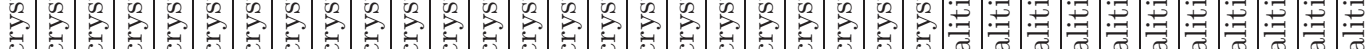

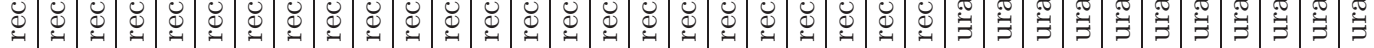

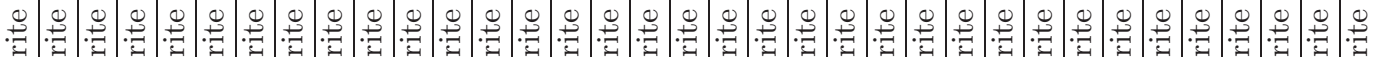

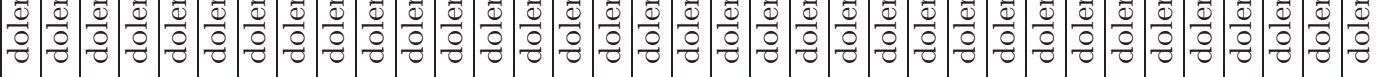

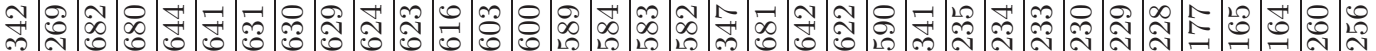

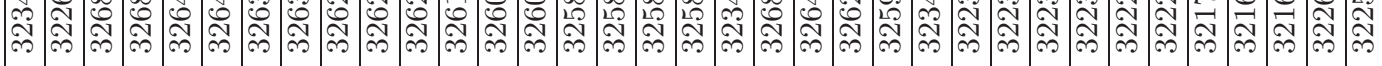




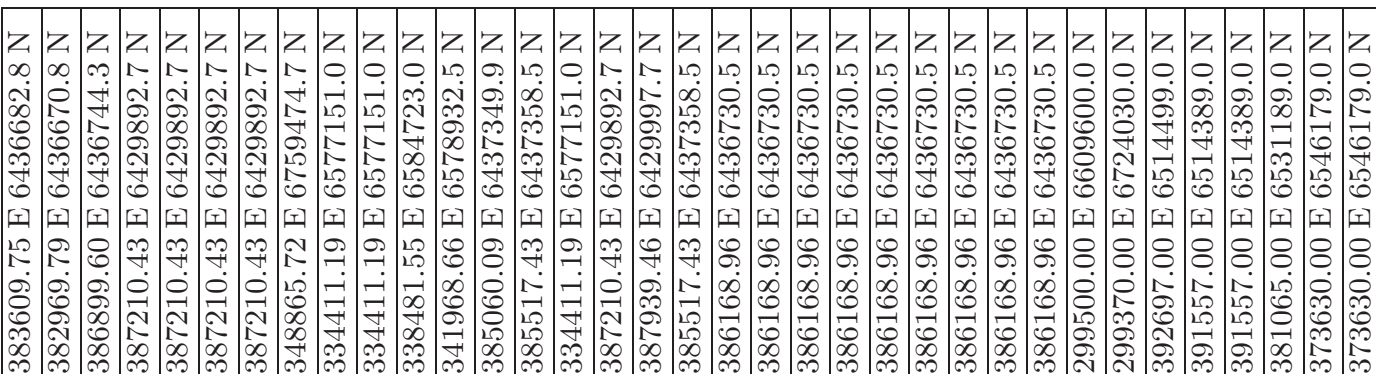

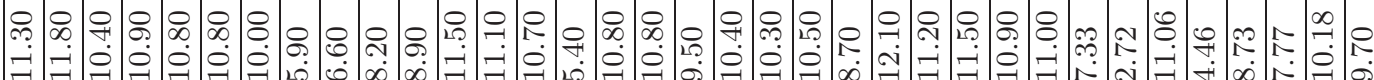

나유.

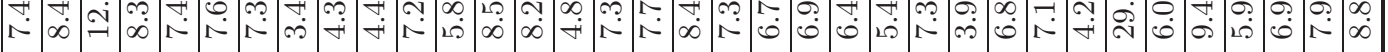

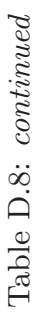

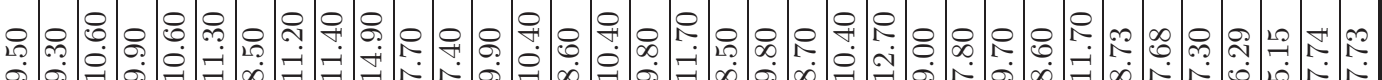

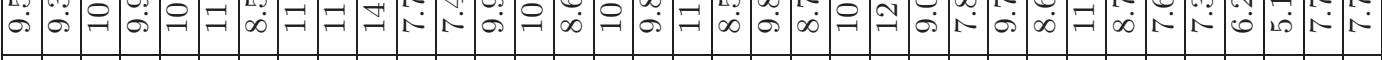

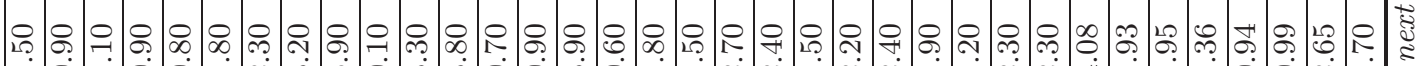

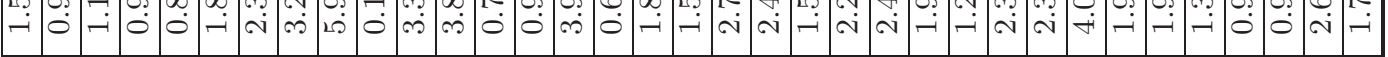

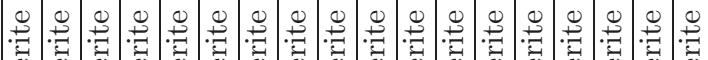

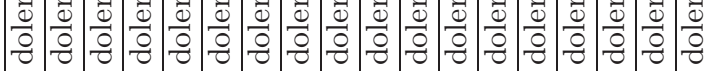

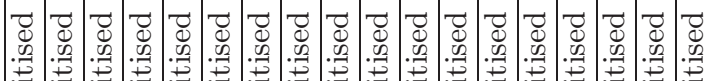

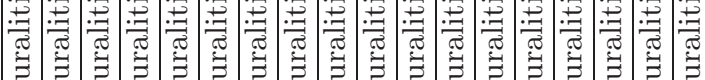

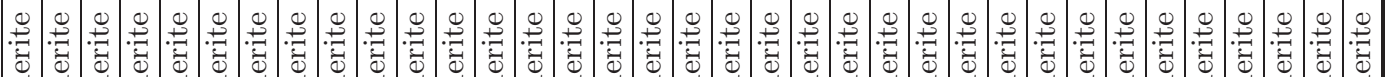

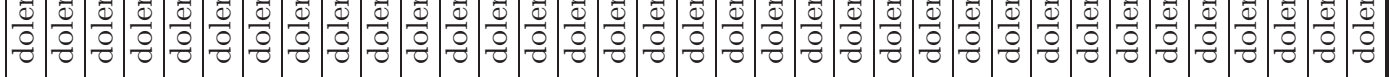

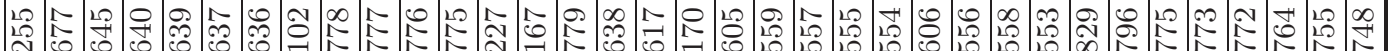

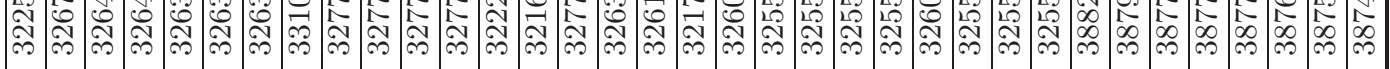




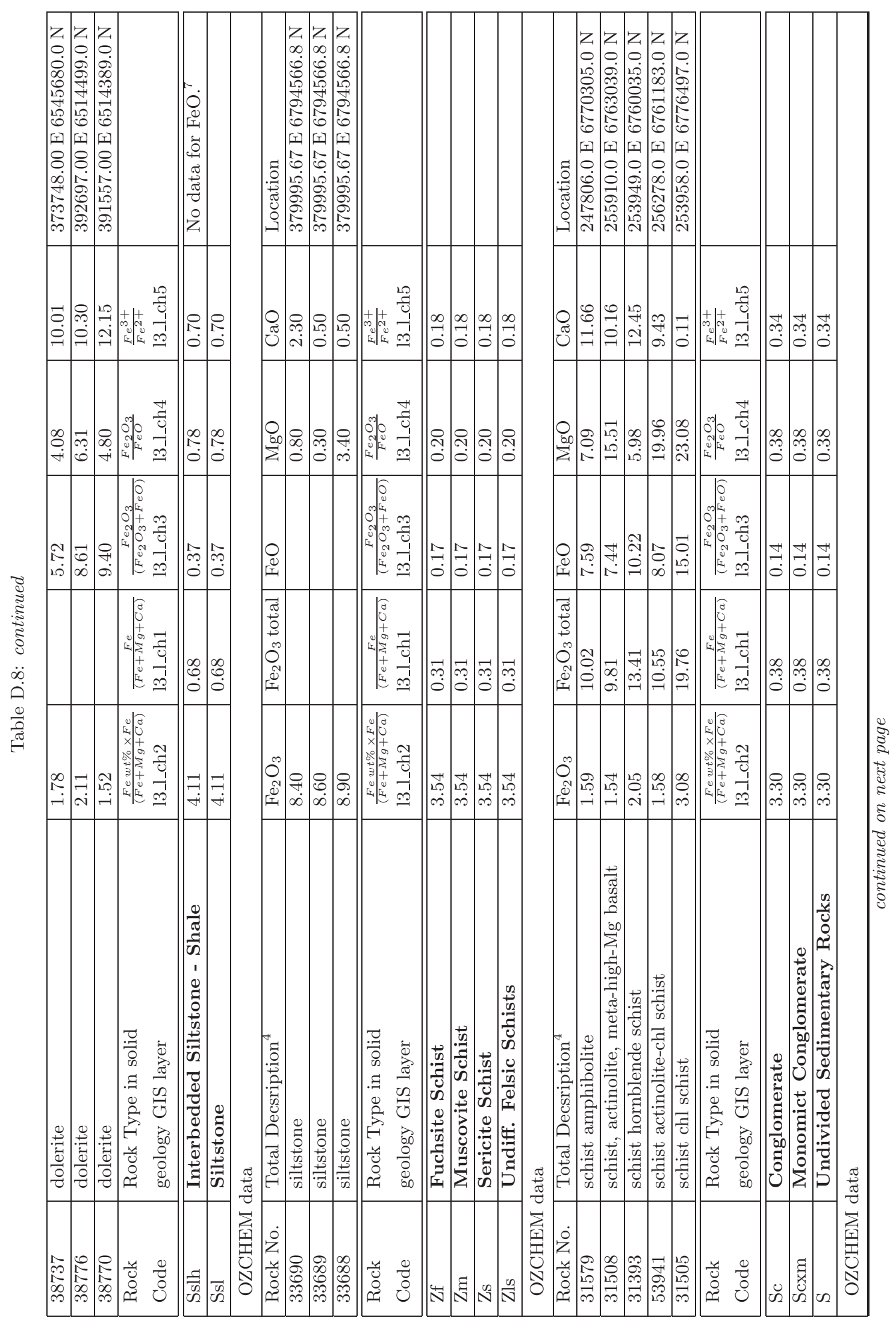




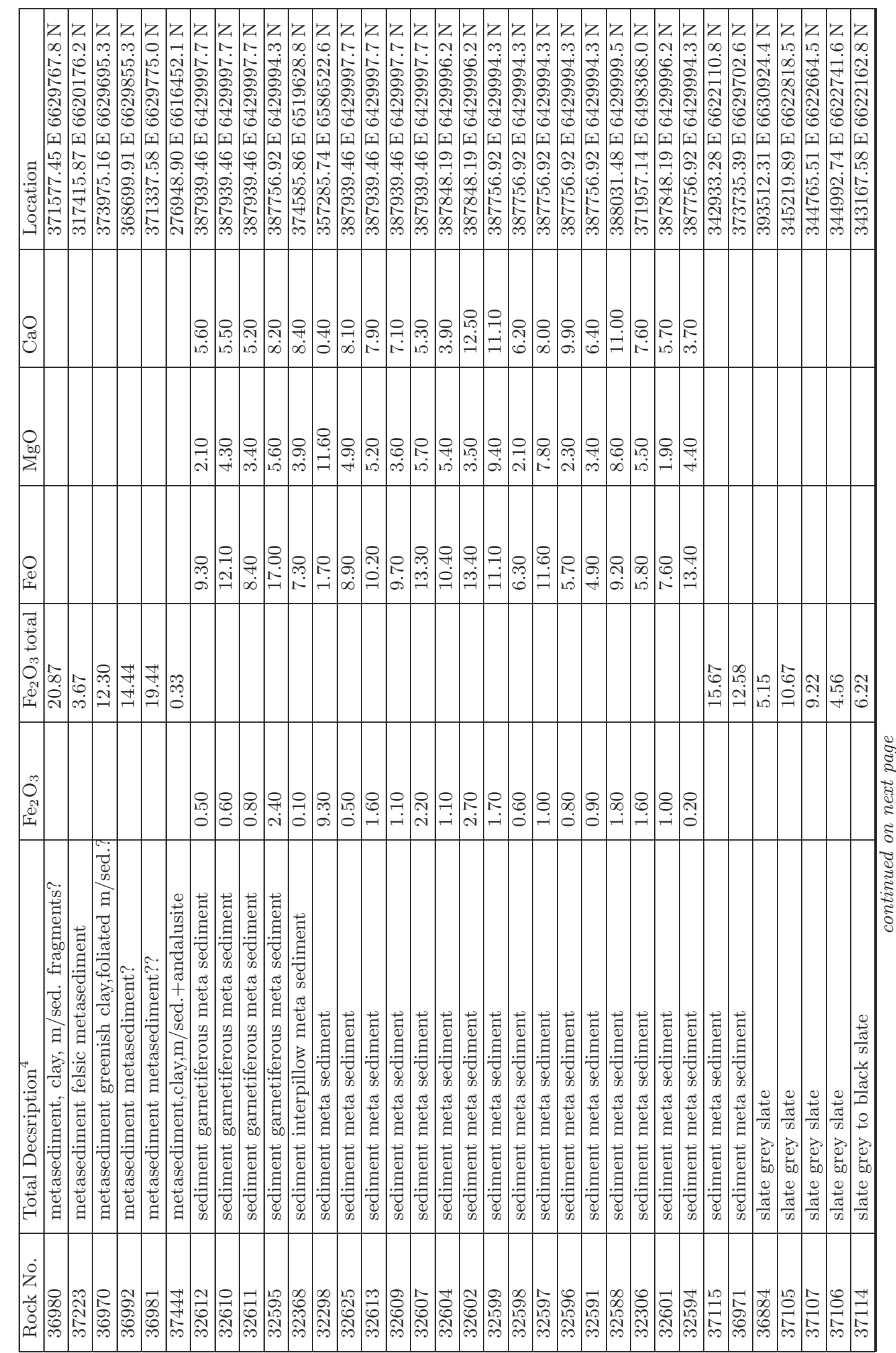




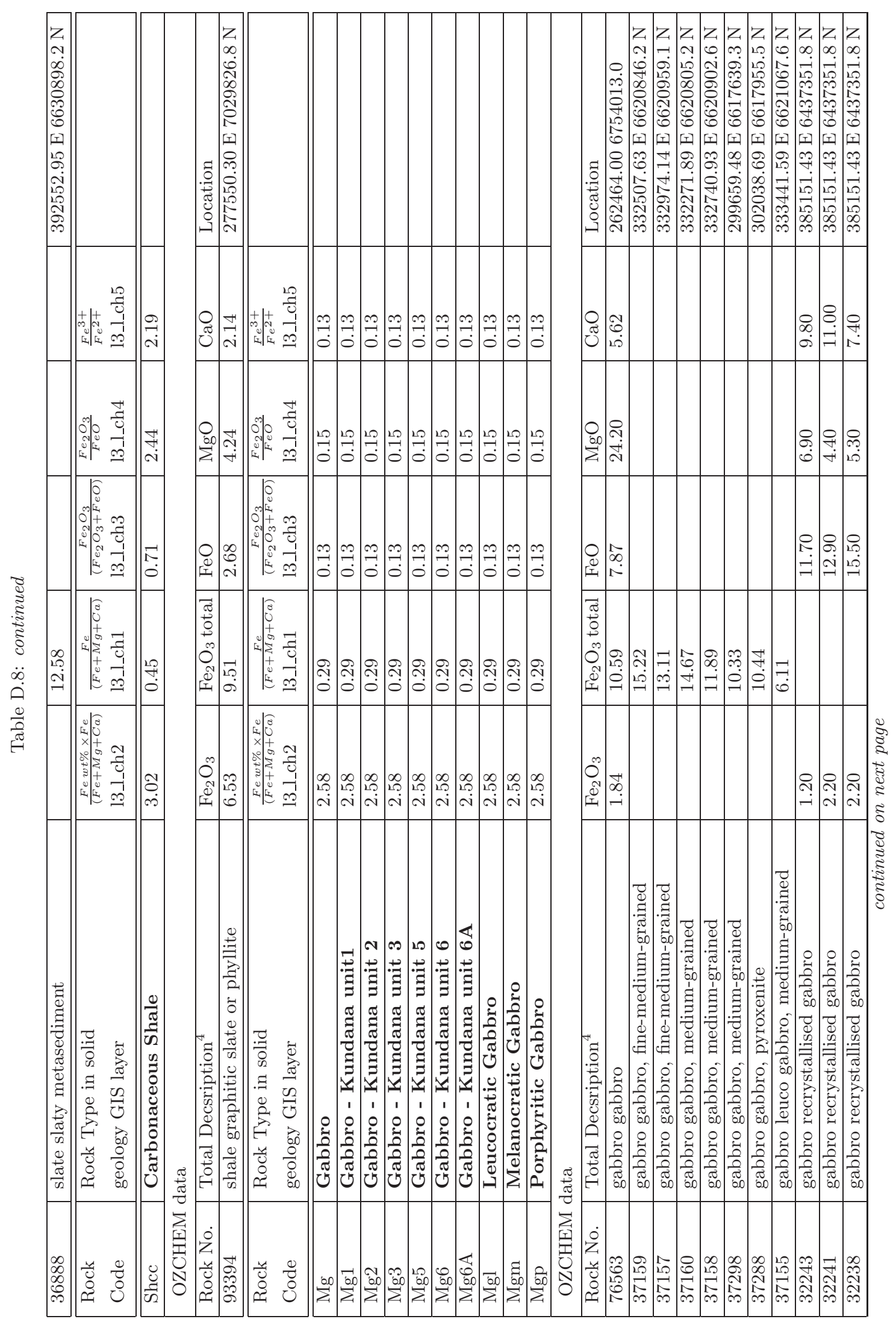




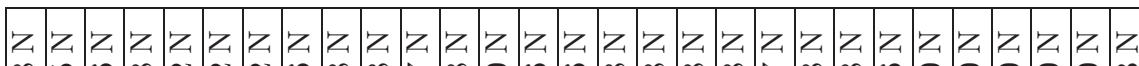

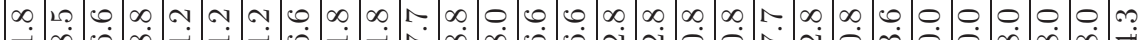

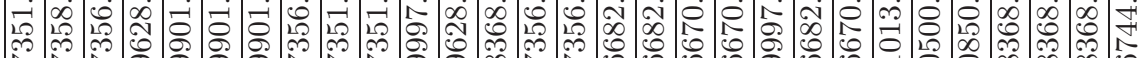

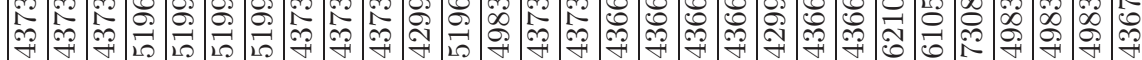

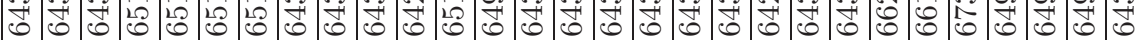

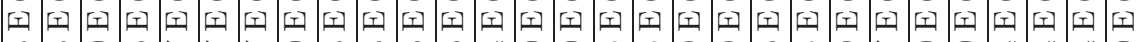

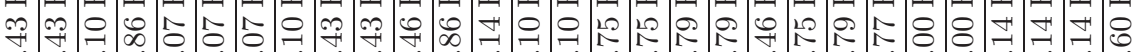

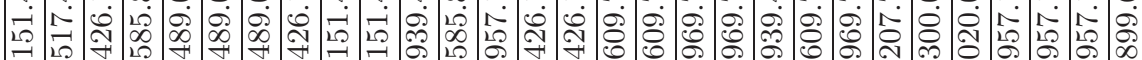

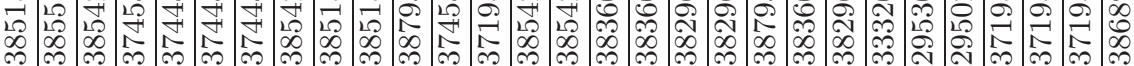

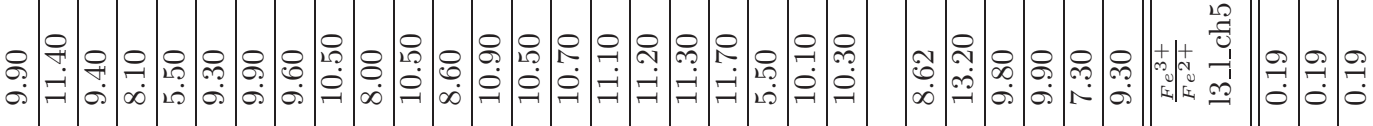

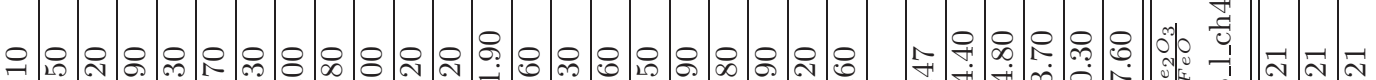

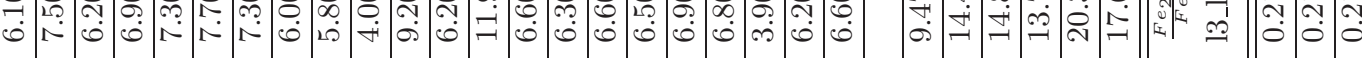

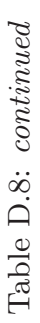

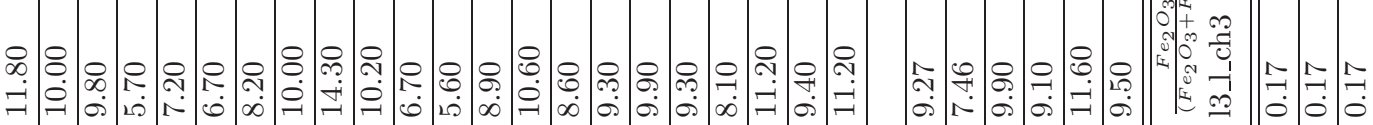

$$
\begin{array}{|}
\hline \\
\hline
\end{array}
$$

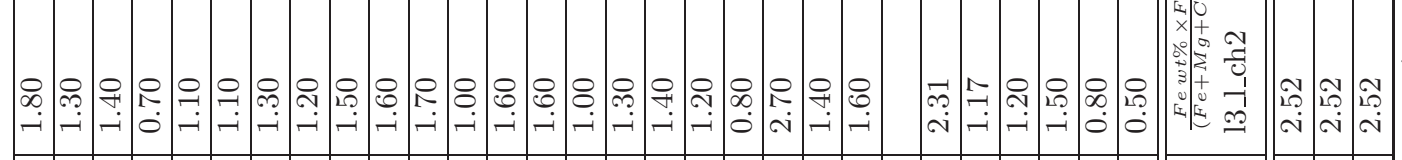

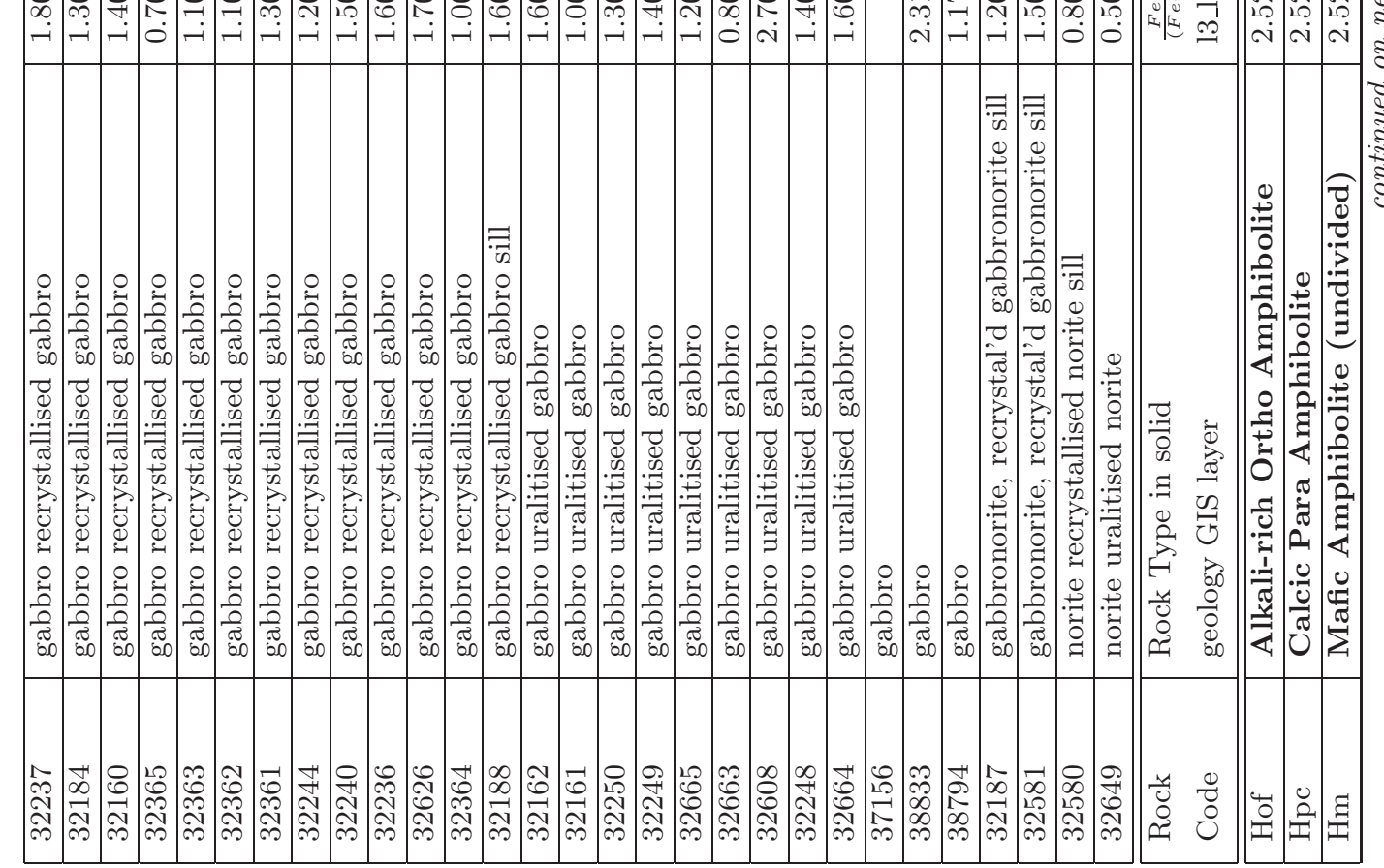




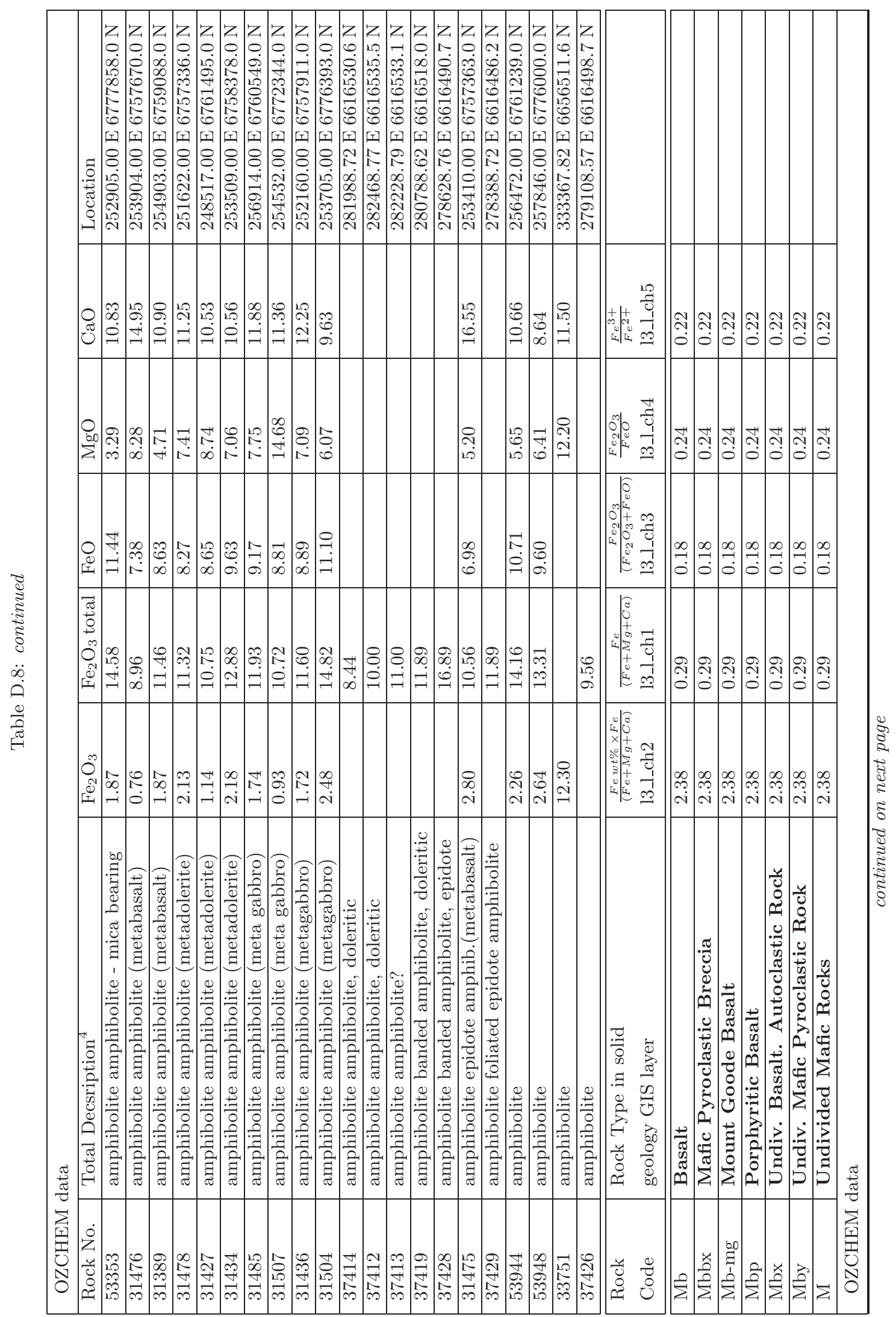




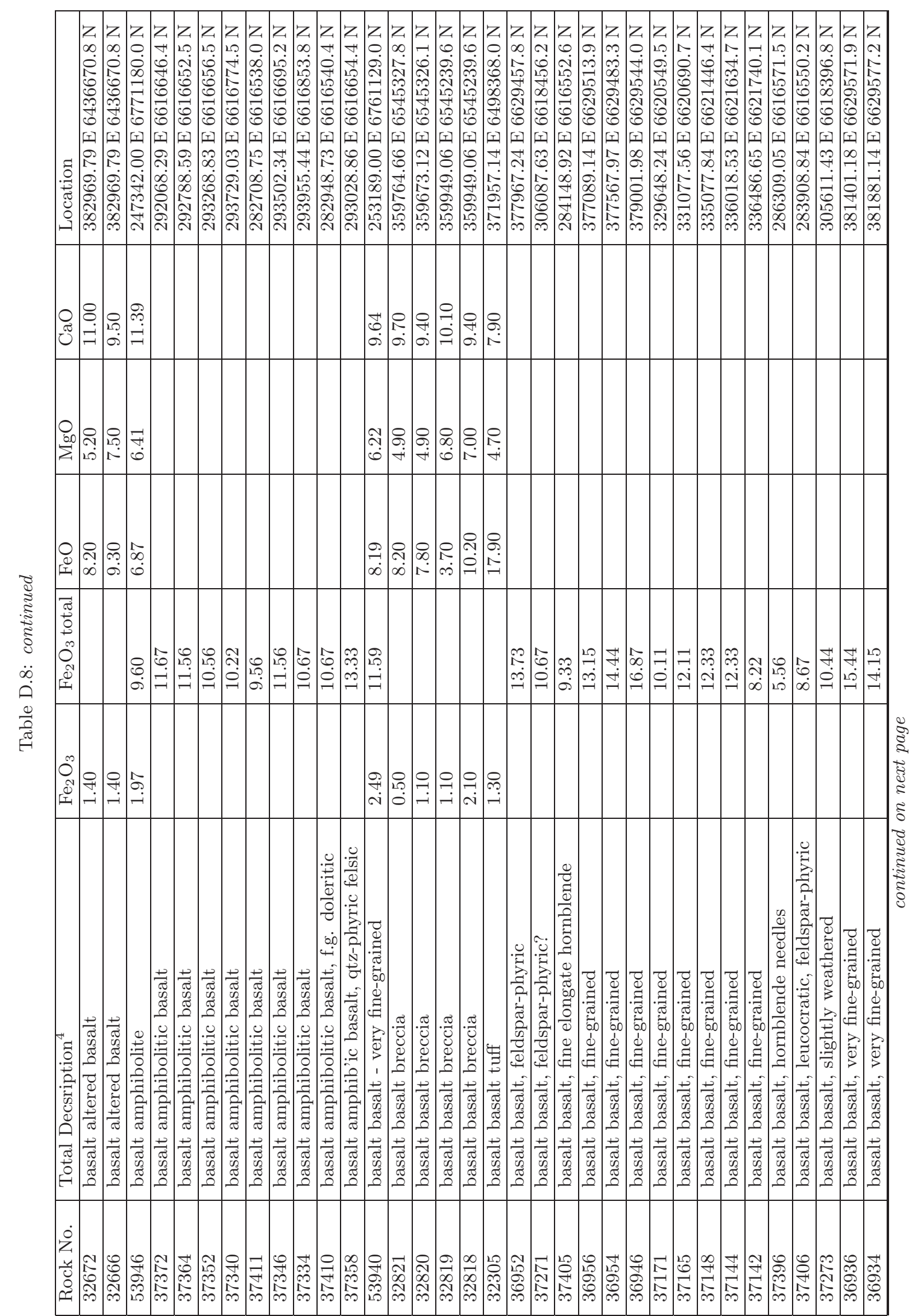




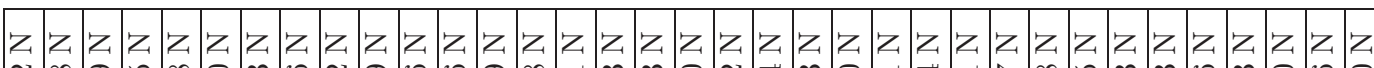

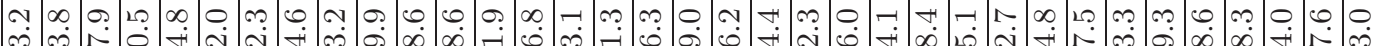

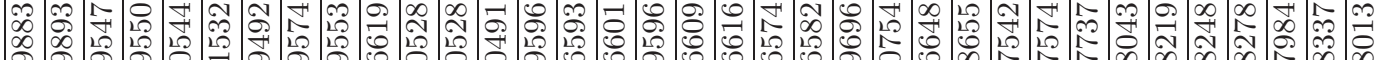

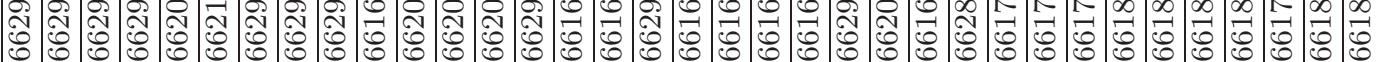

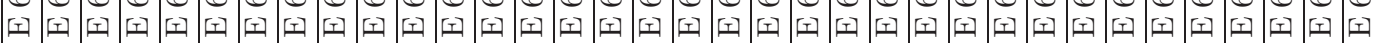
\& 象

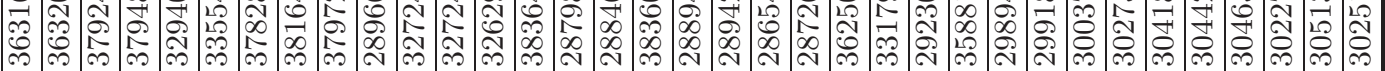

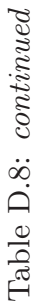

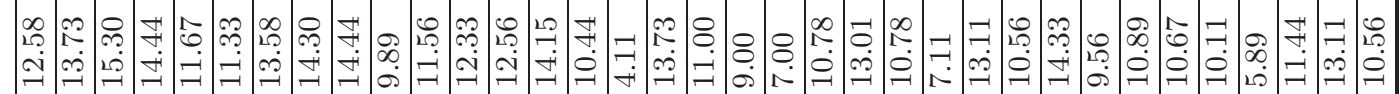

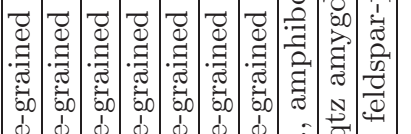

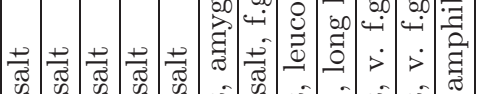

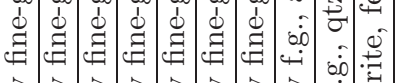

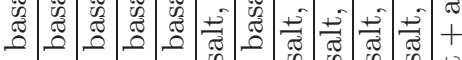

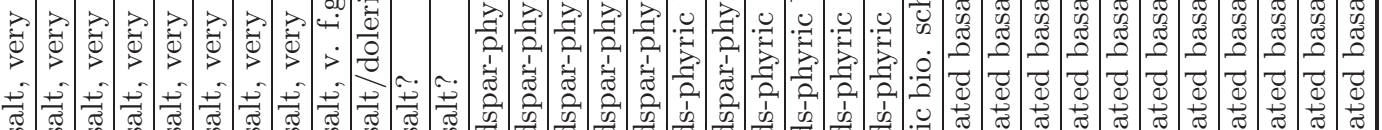

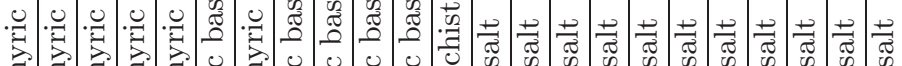

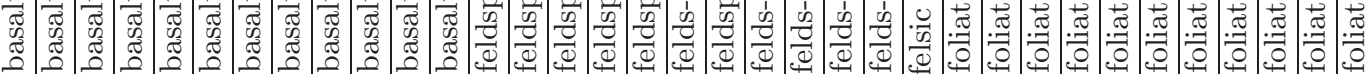

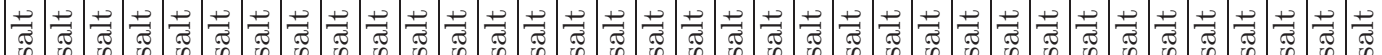
倠

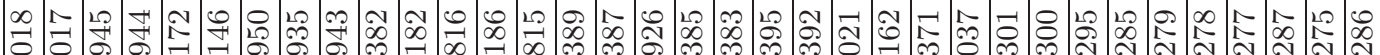
至 


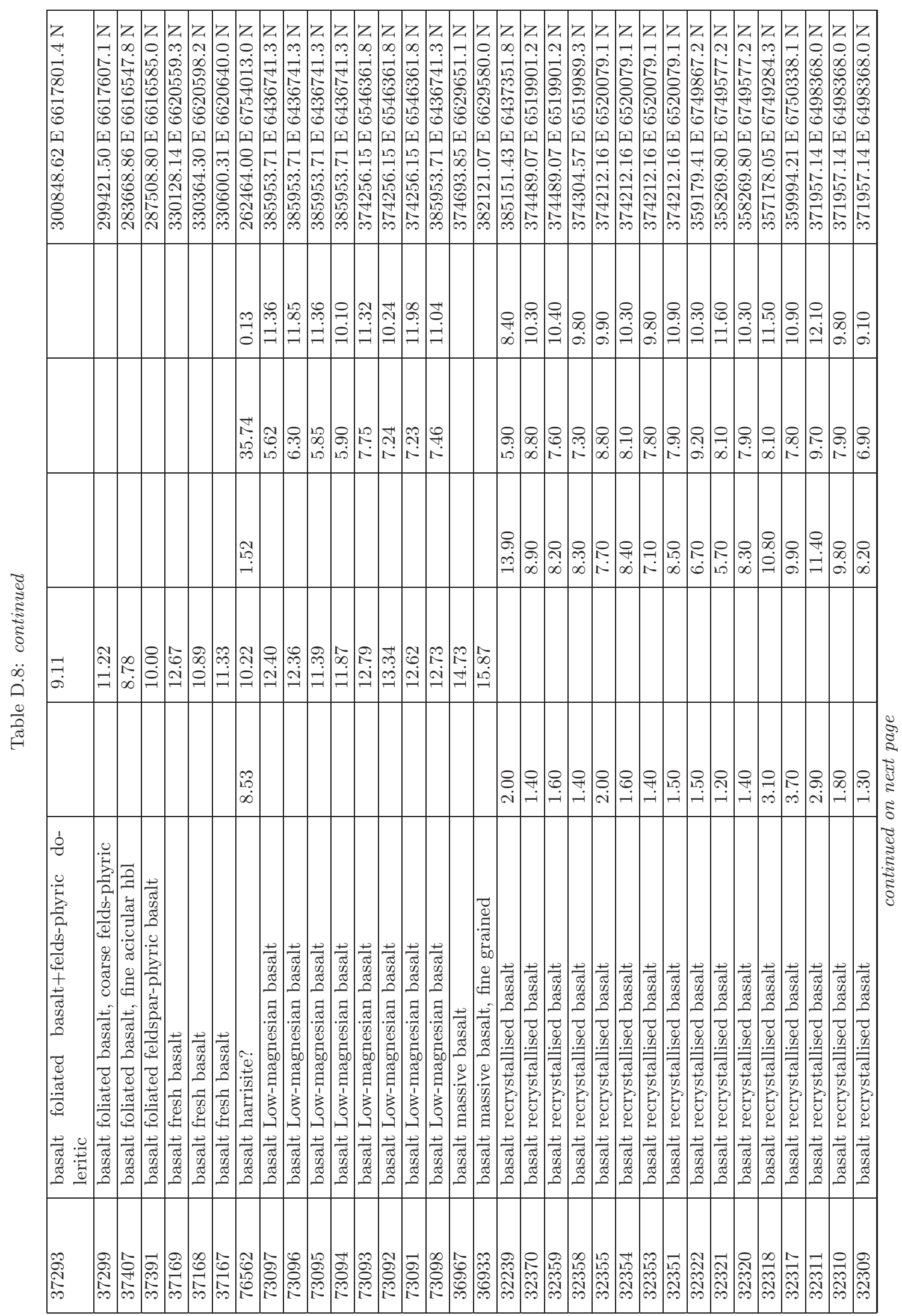




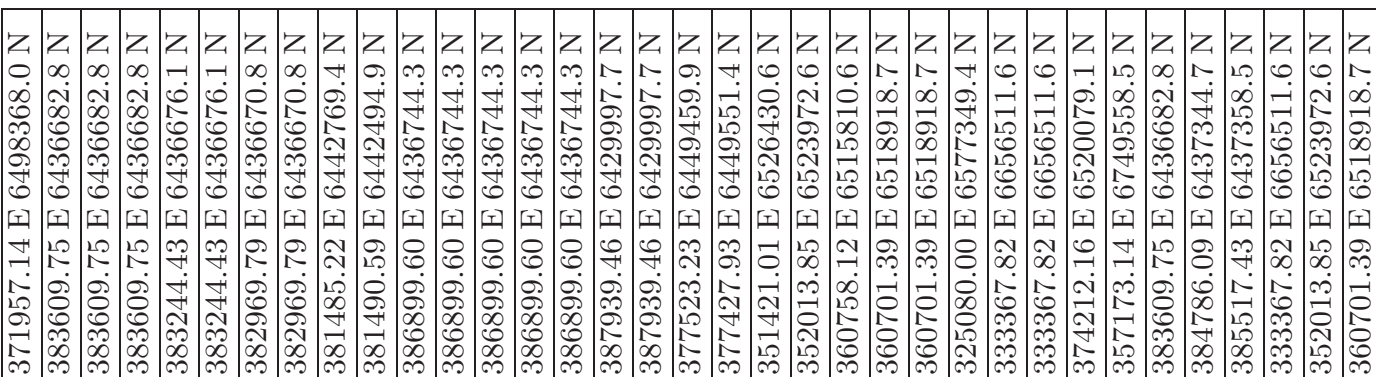

๑

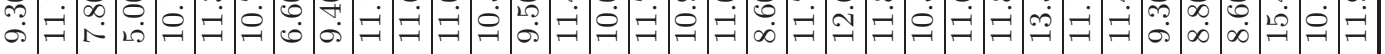

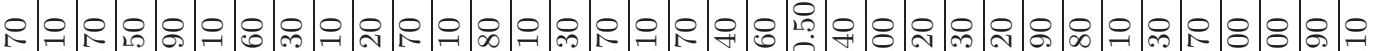

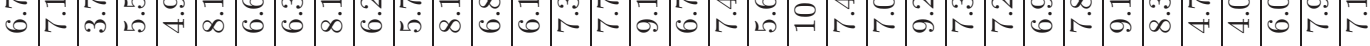

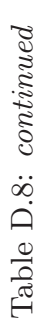

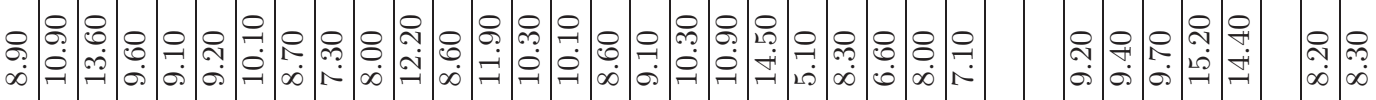

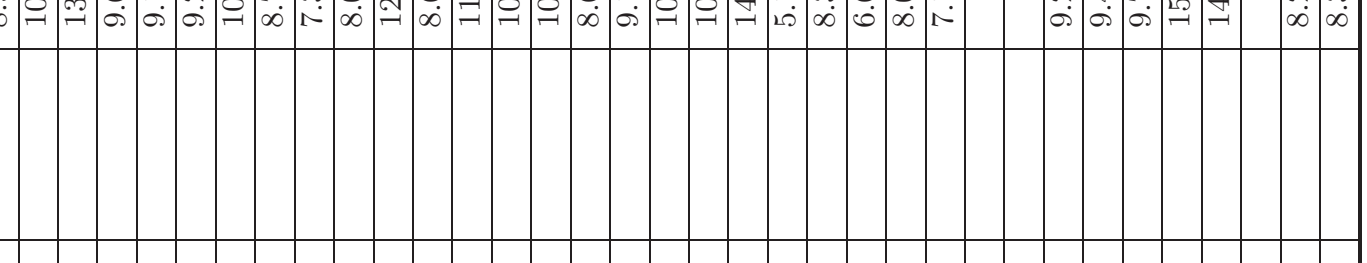

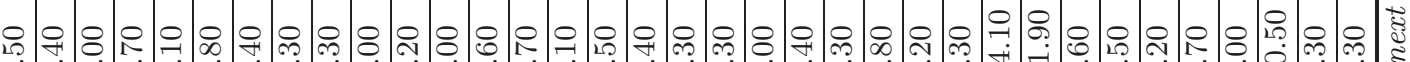

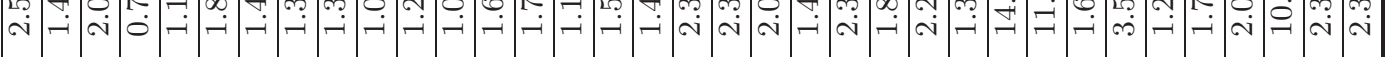

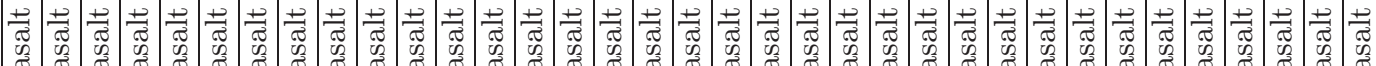
芯

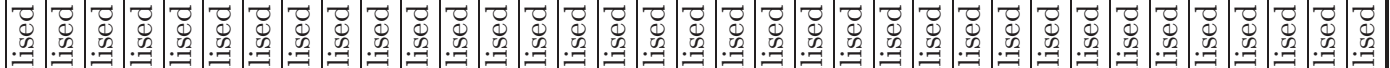

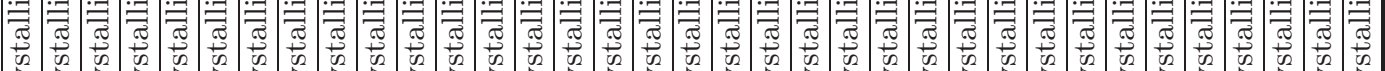

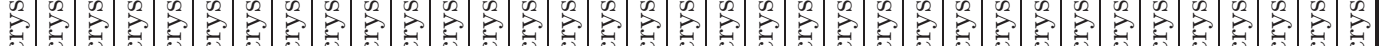

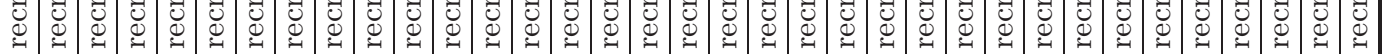

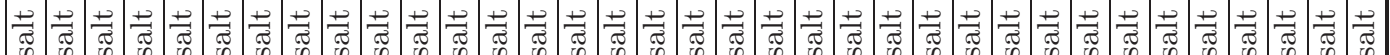

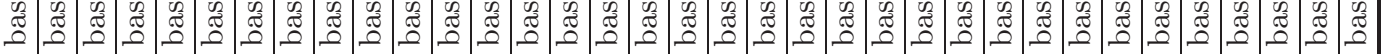

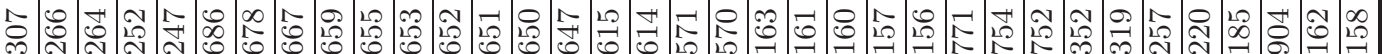

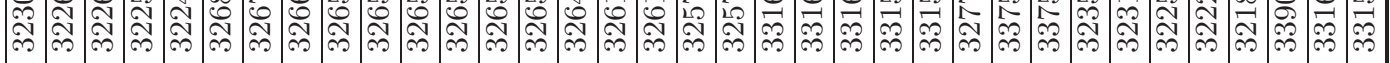




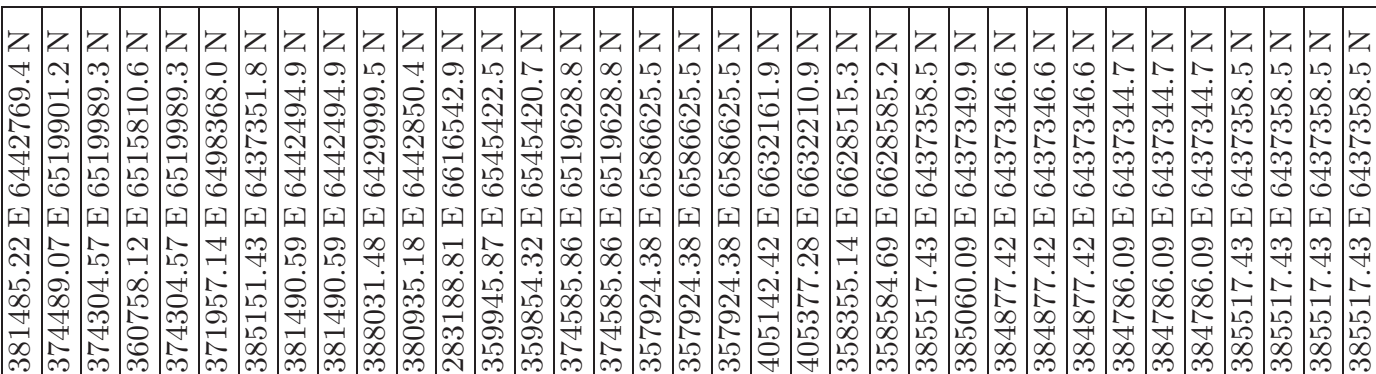

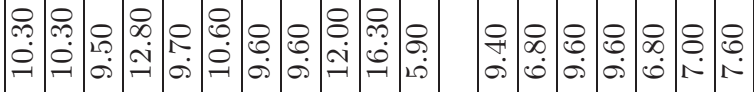

๑

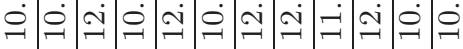

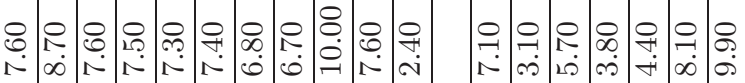

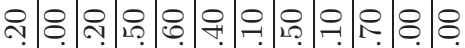

定

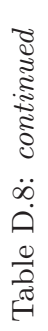

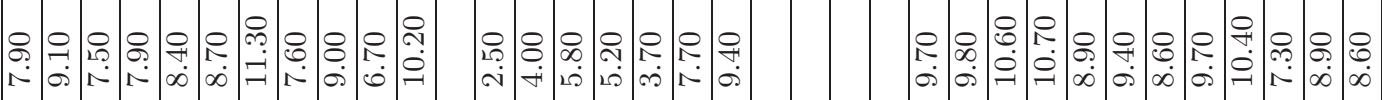

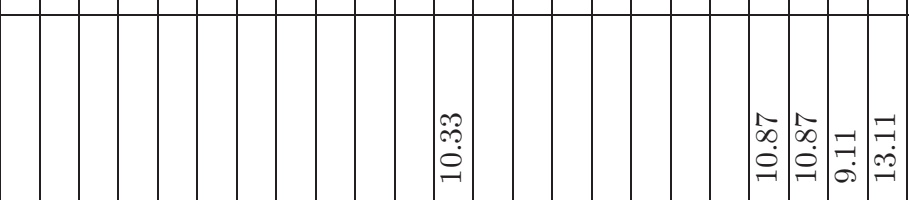

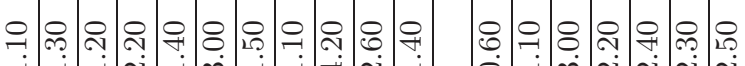

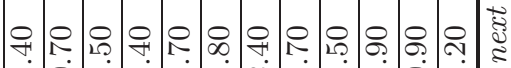

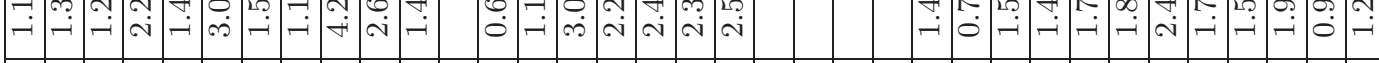

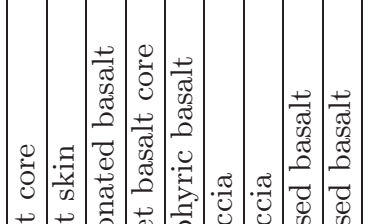

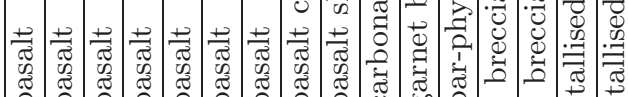

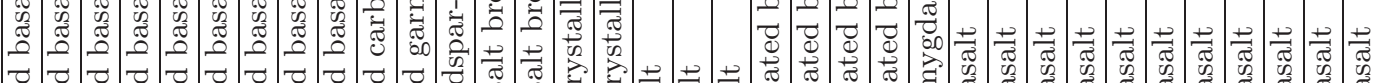

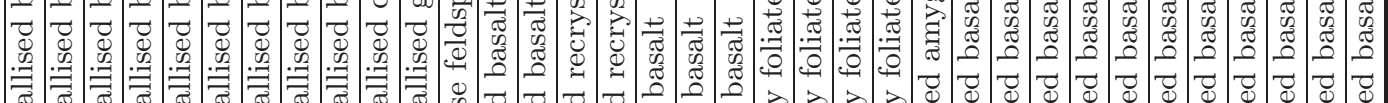

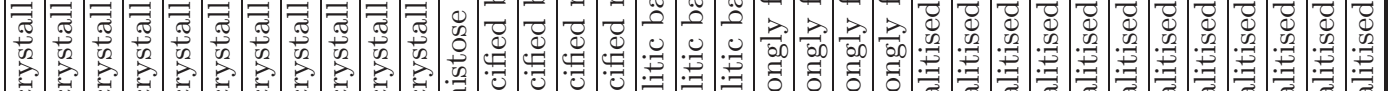

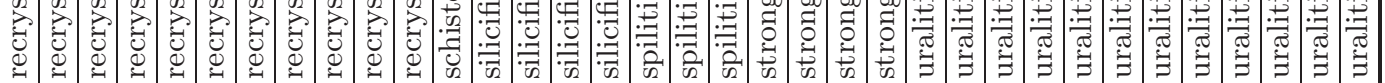

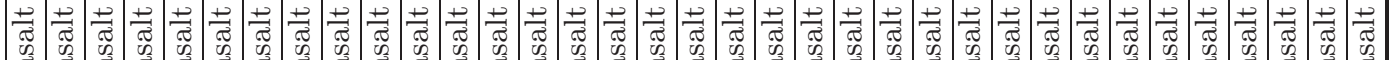

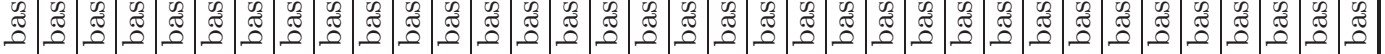

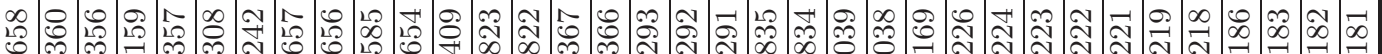

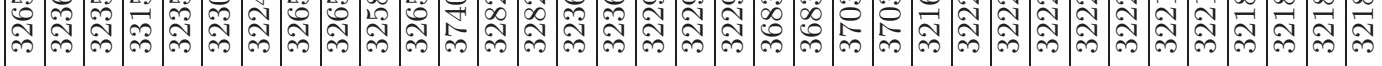




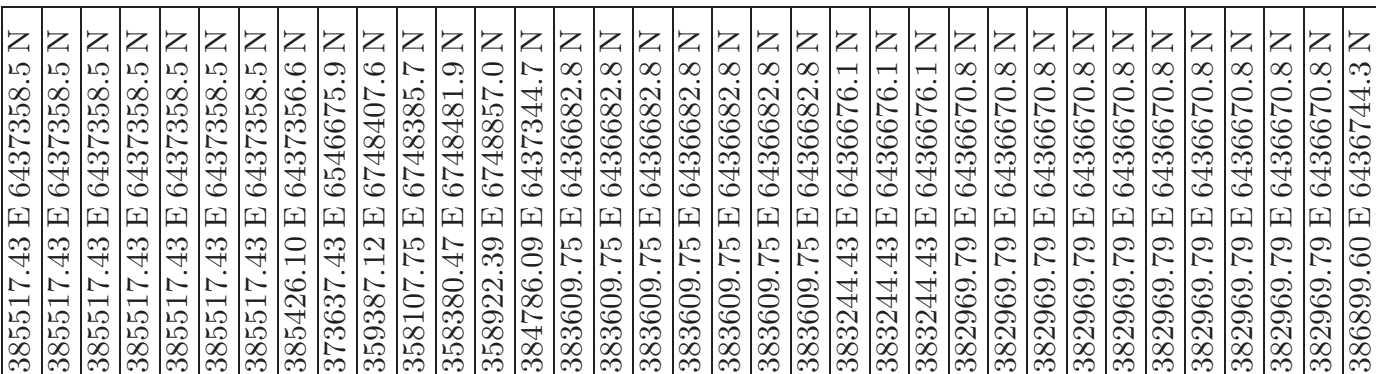

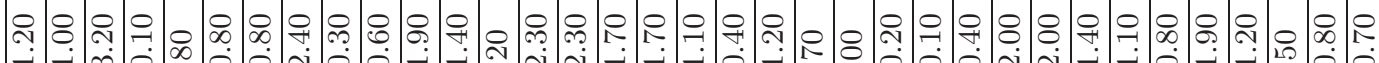

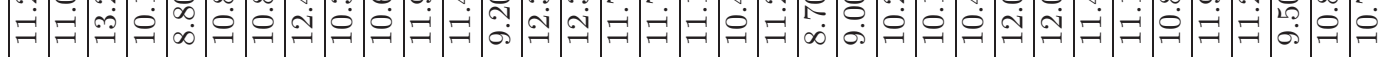

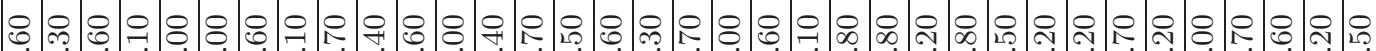

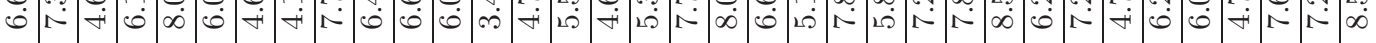

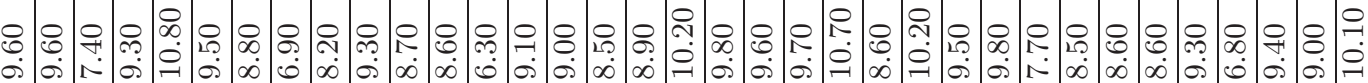

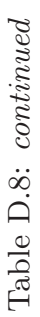

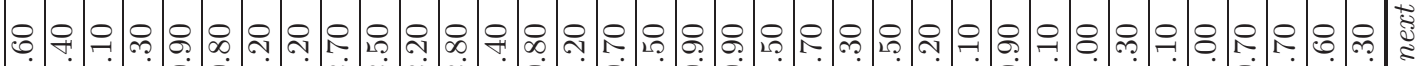

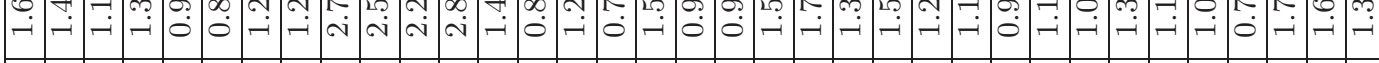

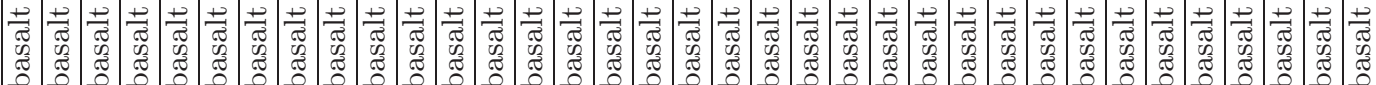

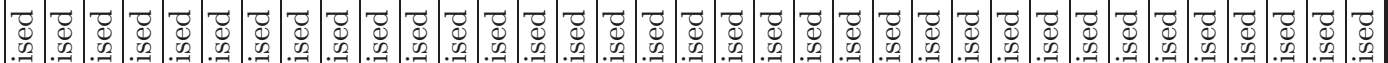

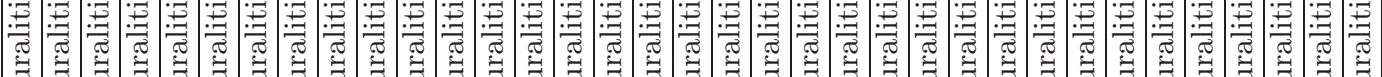

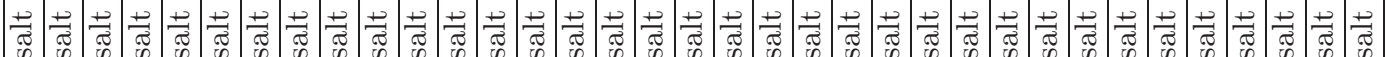

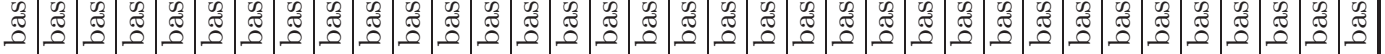

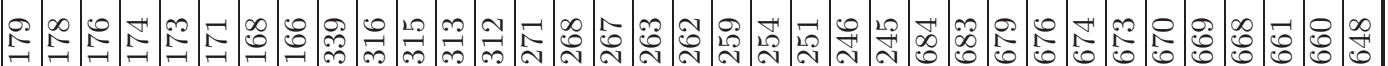

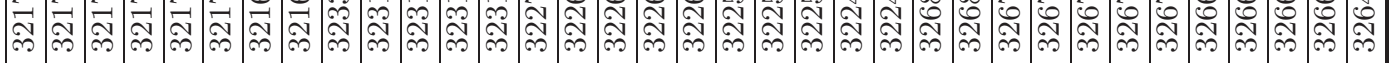




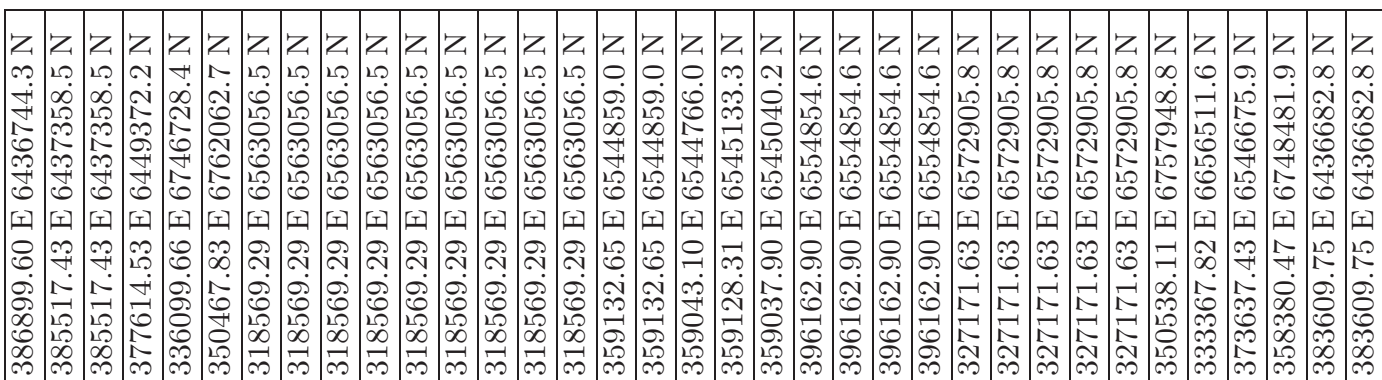

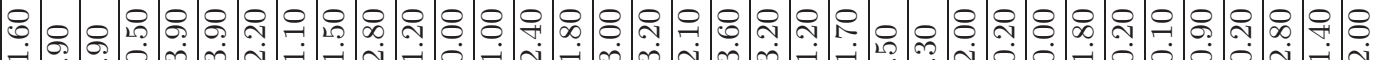

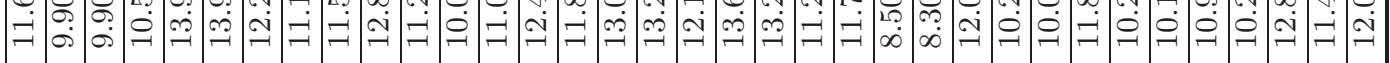

\&. 으.

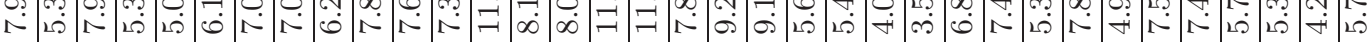

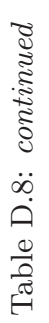

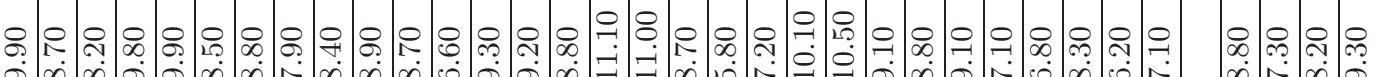

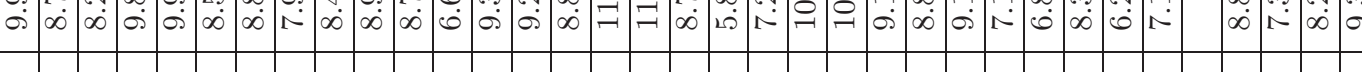

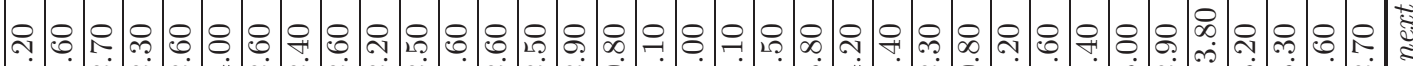

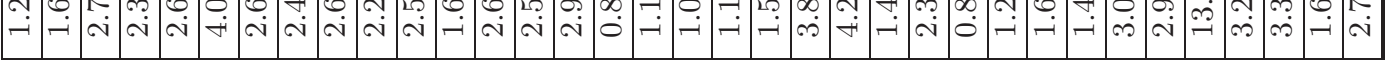

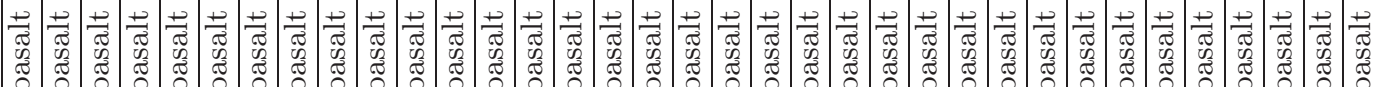

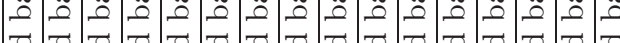

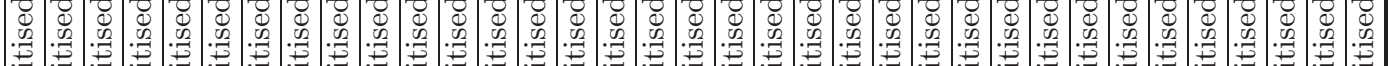

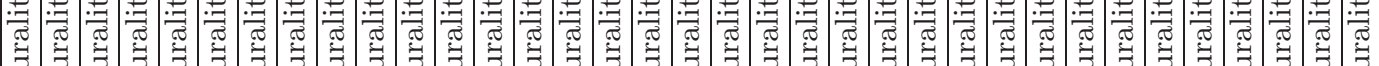

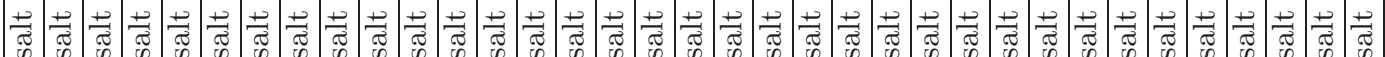

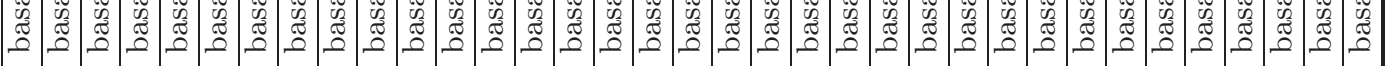

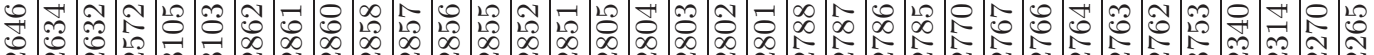

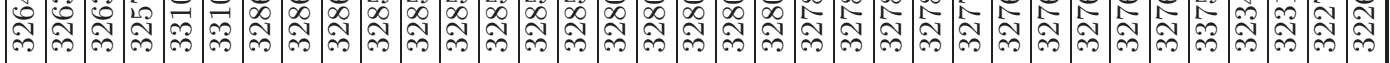




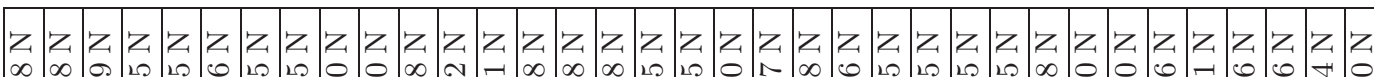

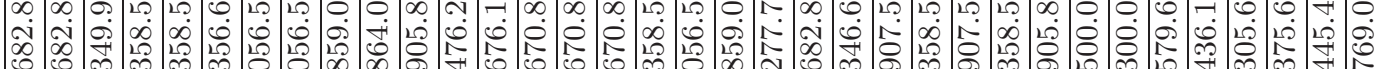

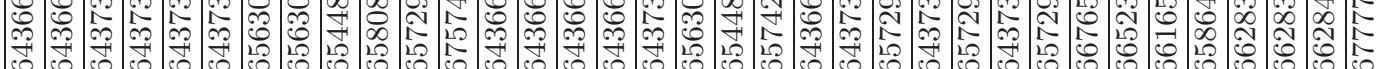

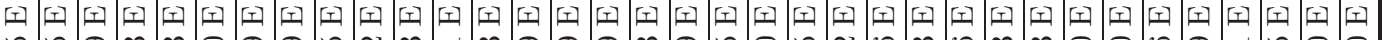

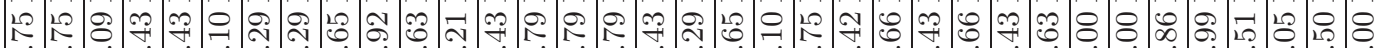

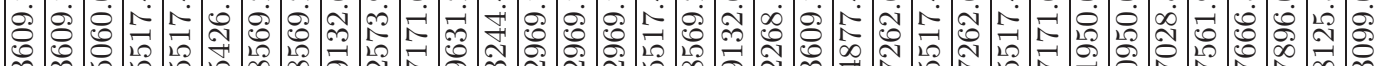

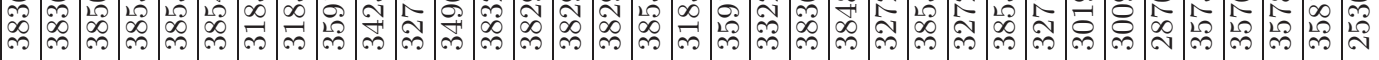

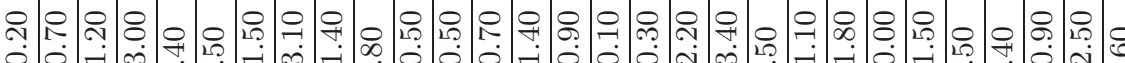

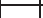




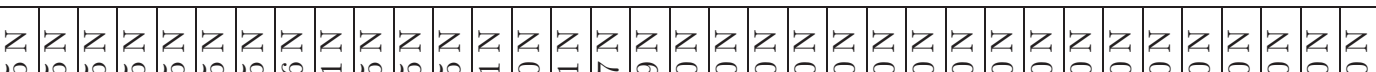

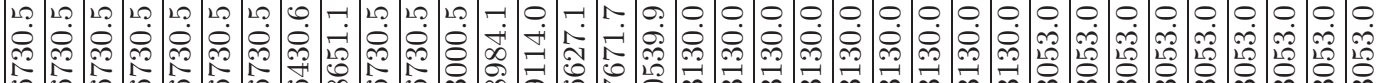
等

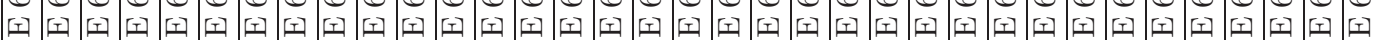

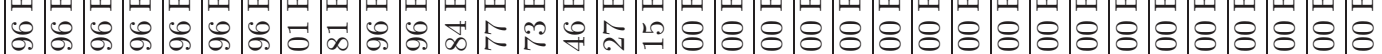

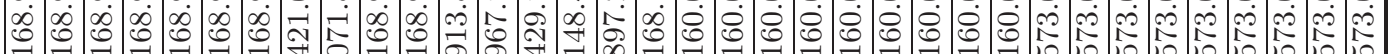

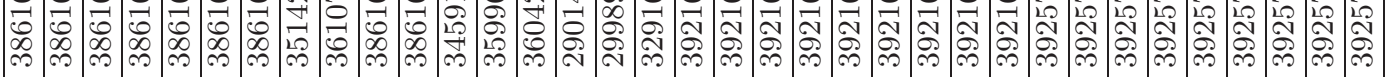

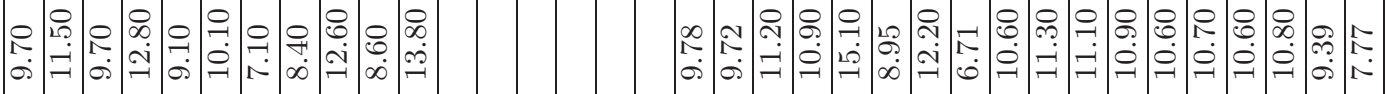

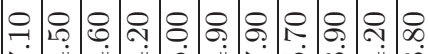
ก.

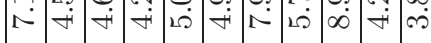

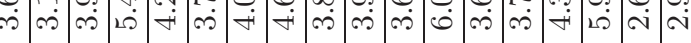

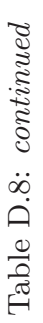

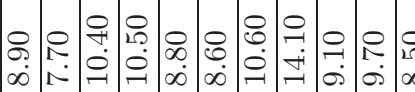

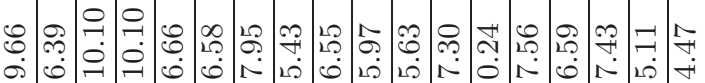

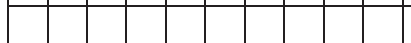
: $\because=\dot{=}=\dot{\sim}$

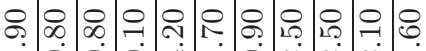

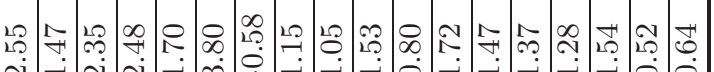
ङ

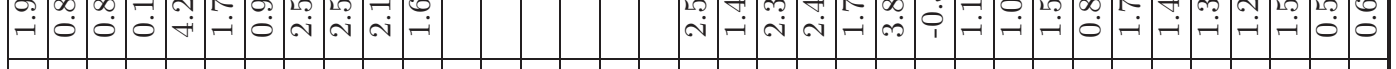
纺

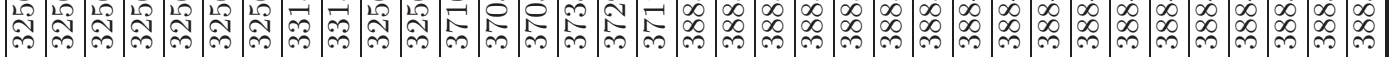




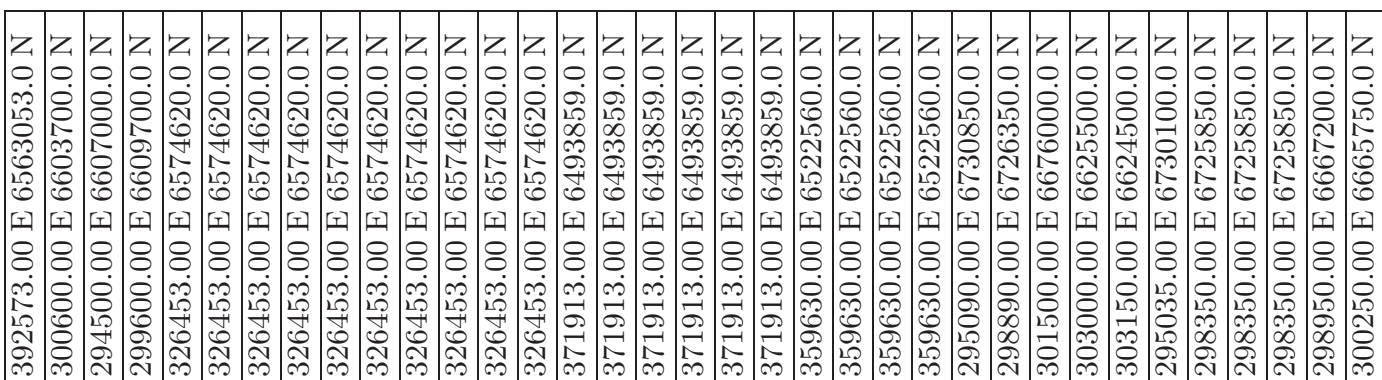

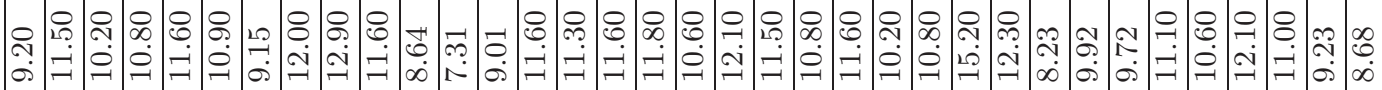

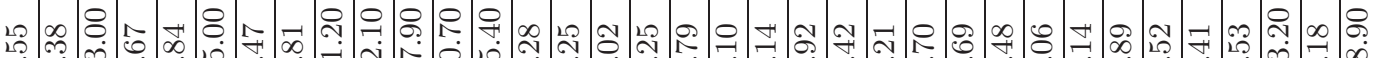
ஸे

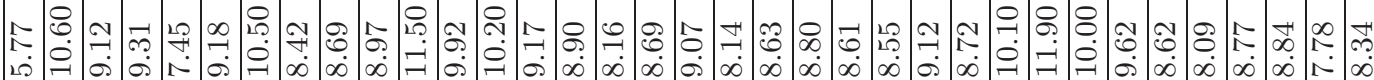

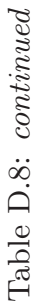

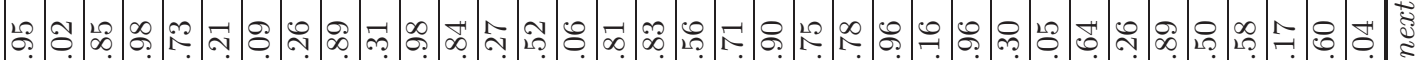

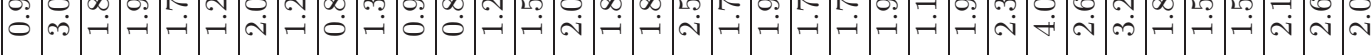

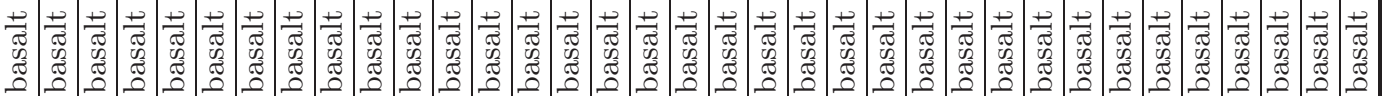

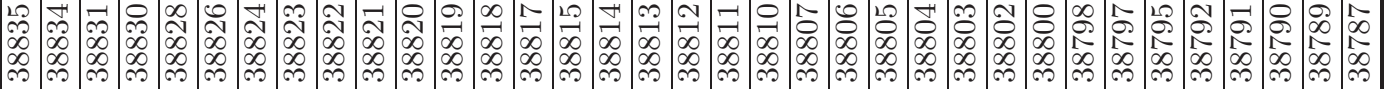




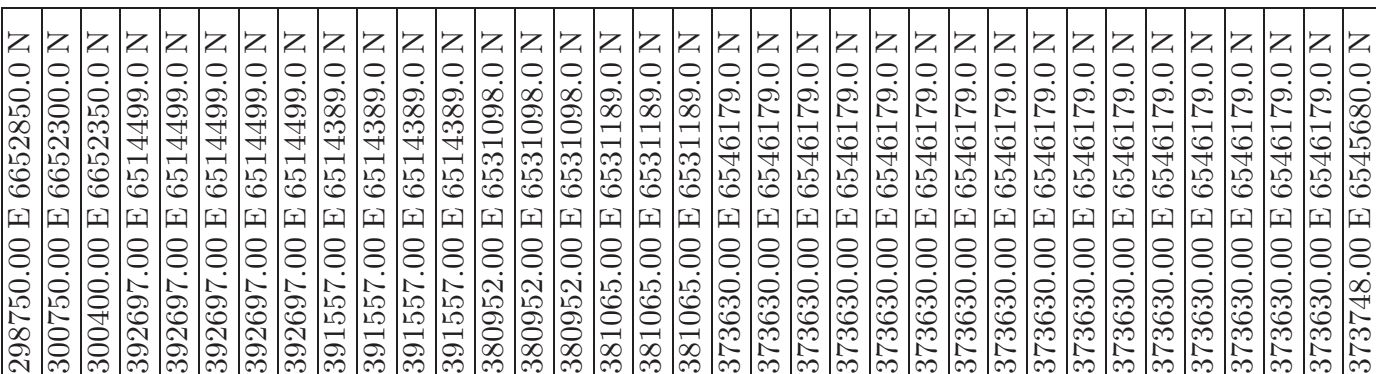

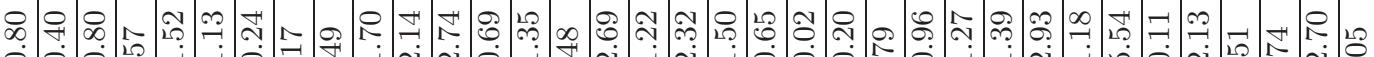

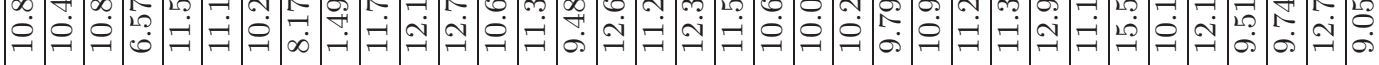

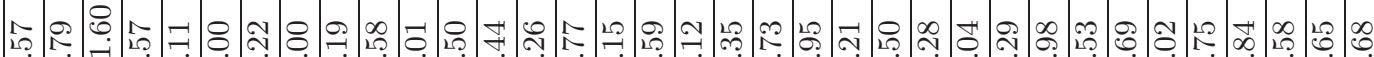

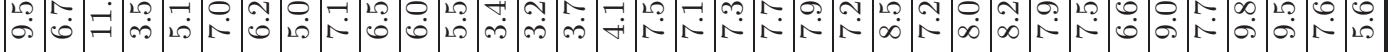

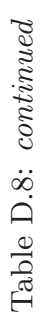

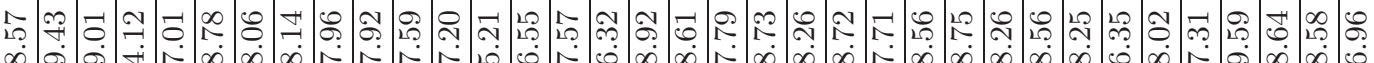

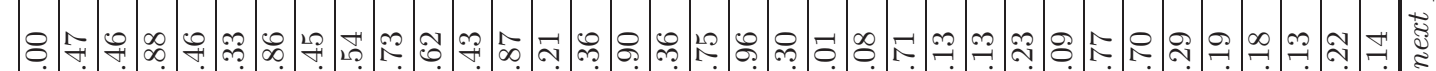

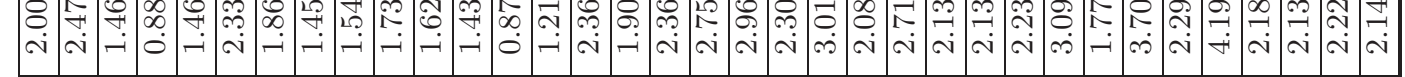

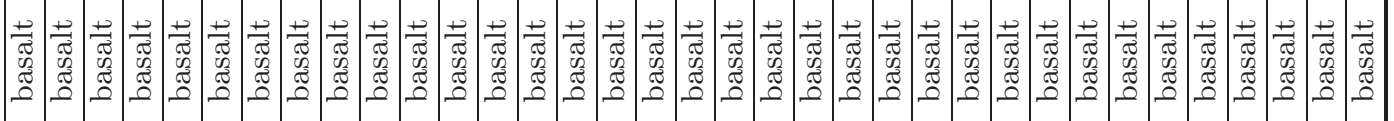

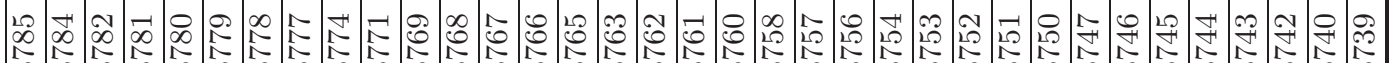

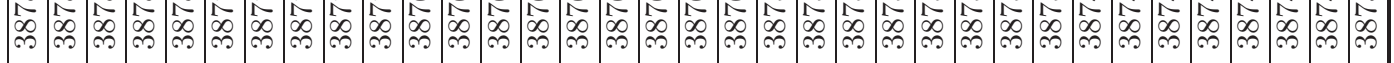




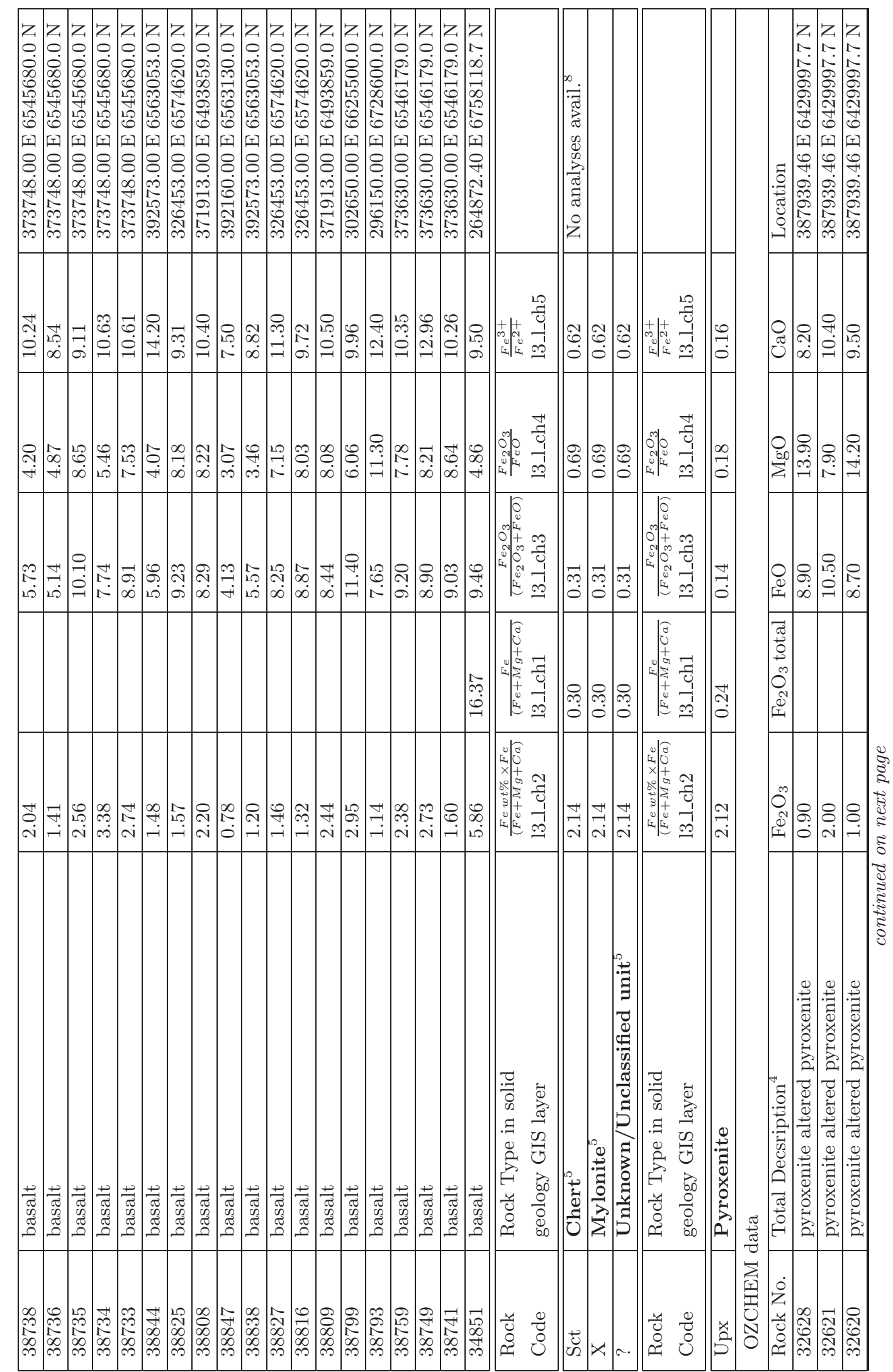




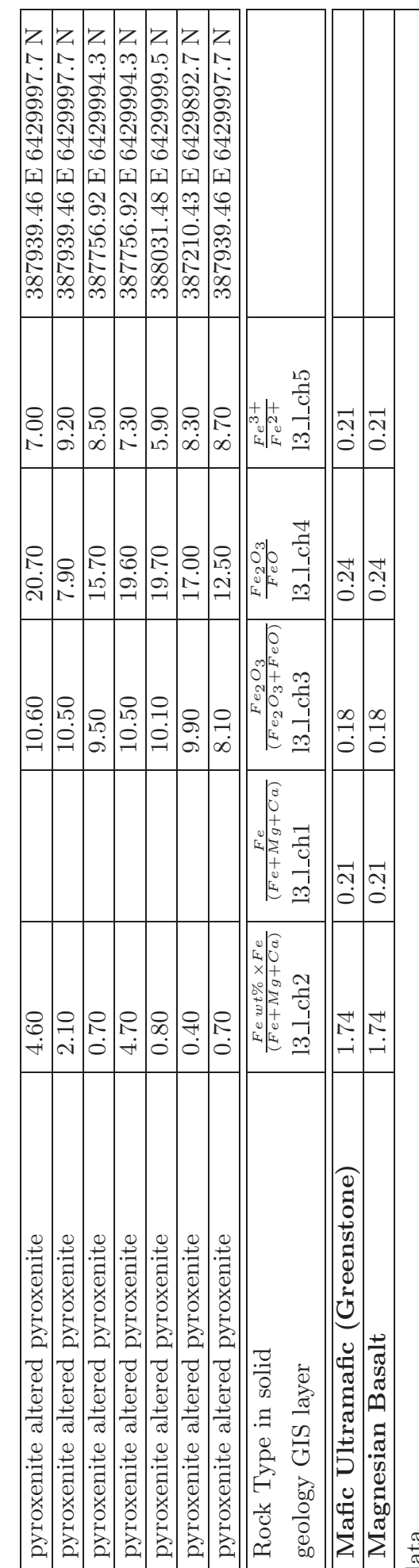

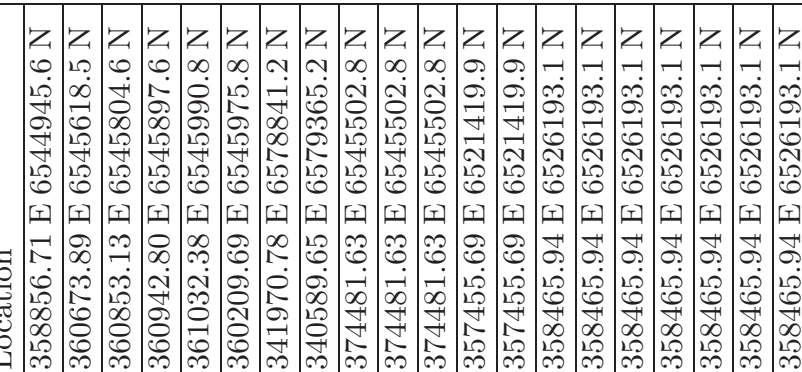

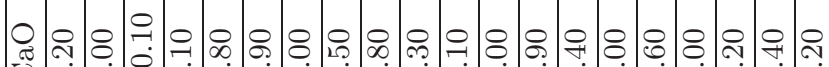

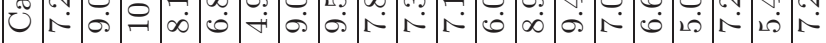

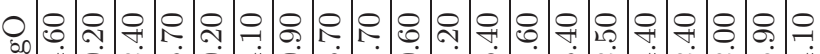

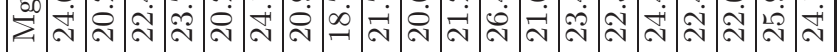

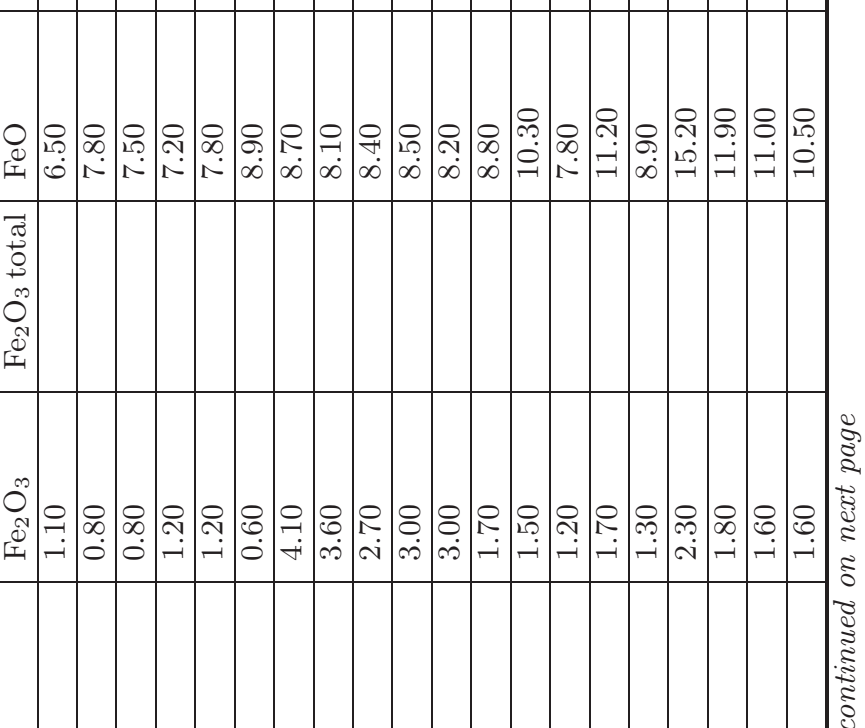

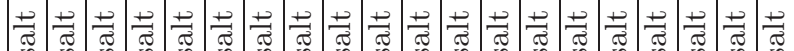

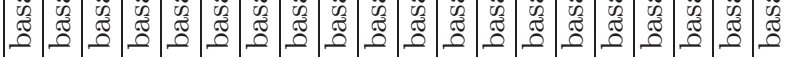

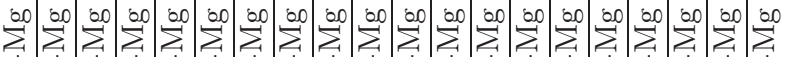

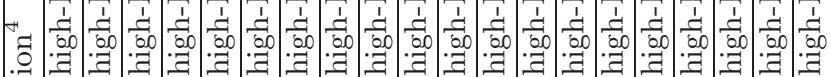
:

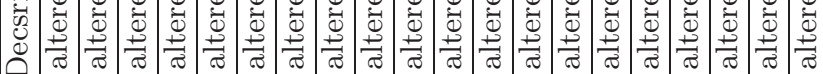

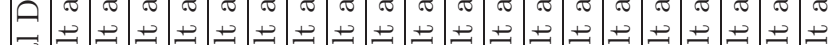

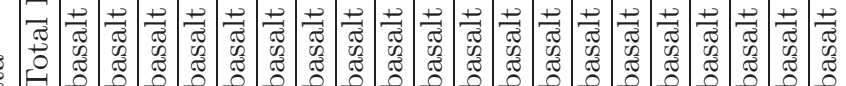
๘

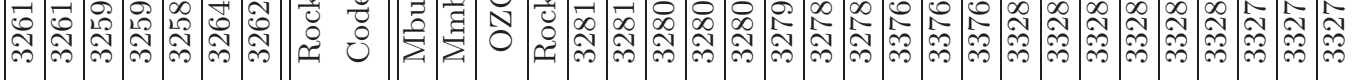




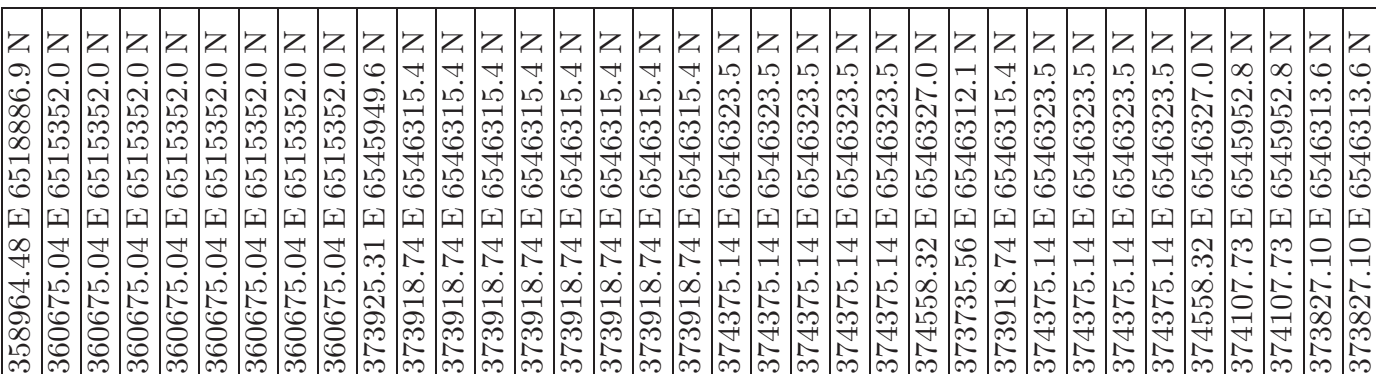

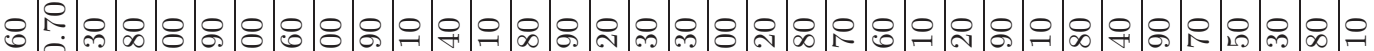

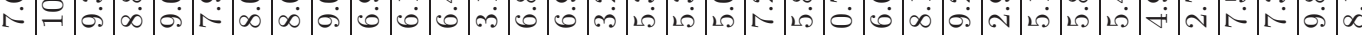

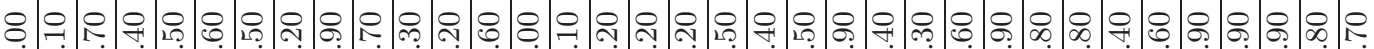
৯่

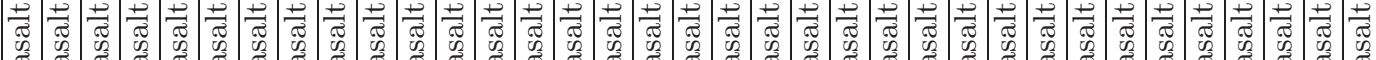

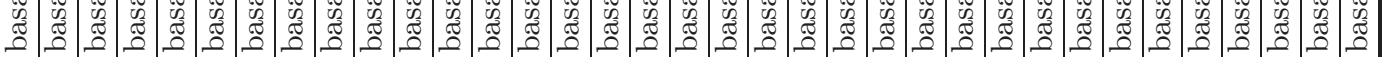

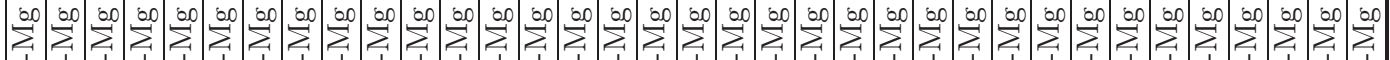

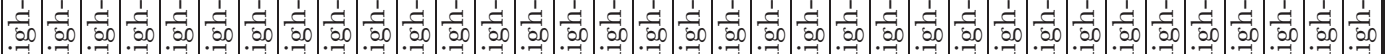

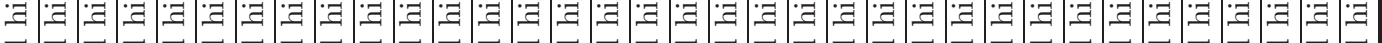

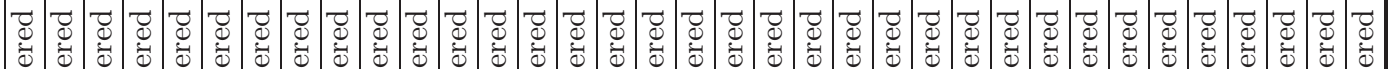

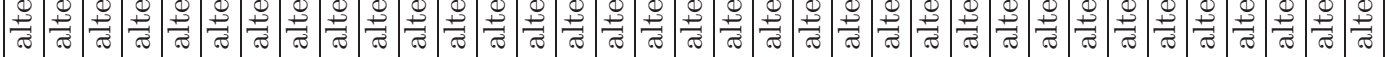

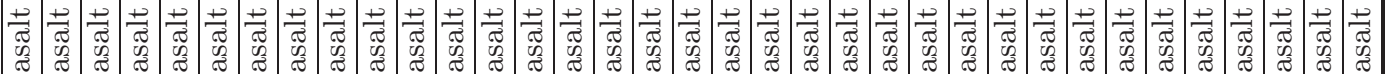

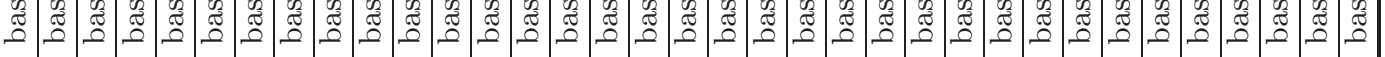

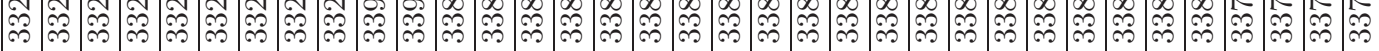




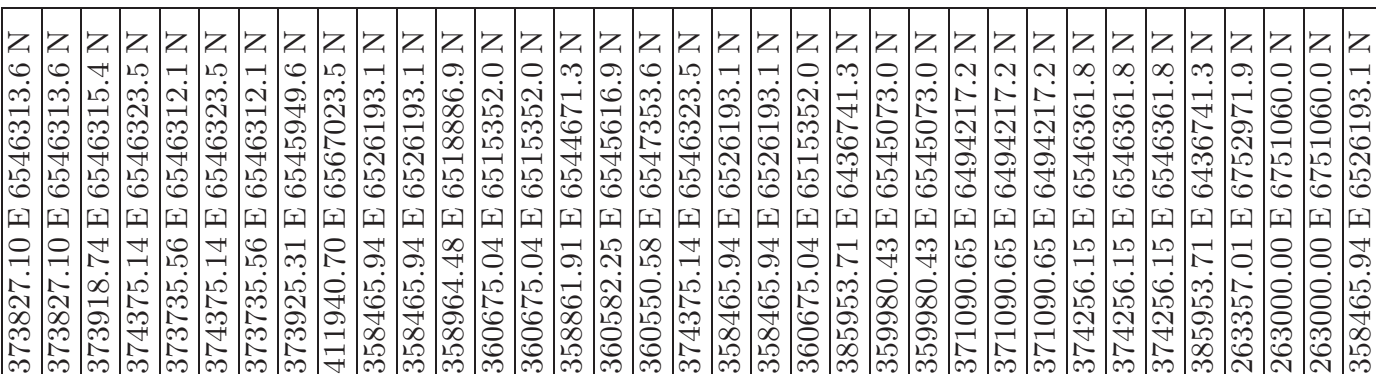

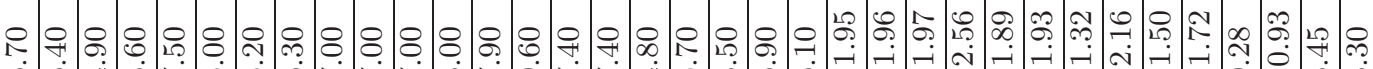

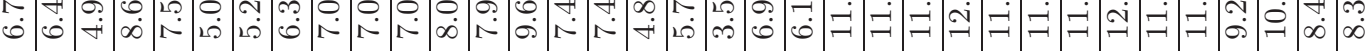

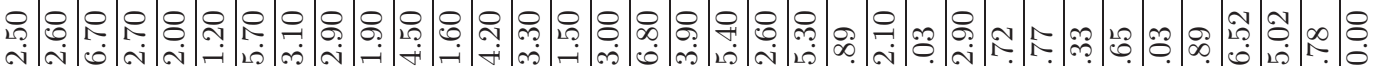
নं

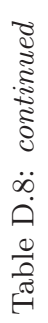

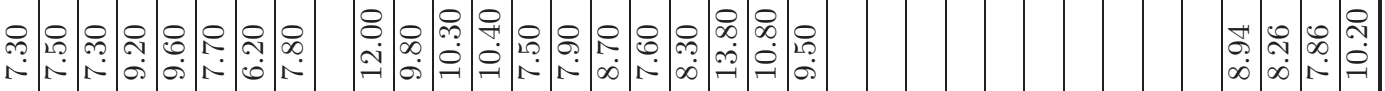

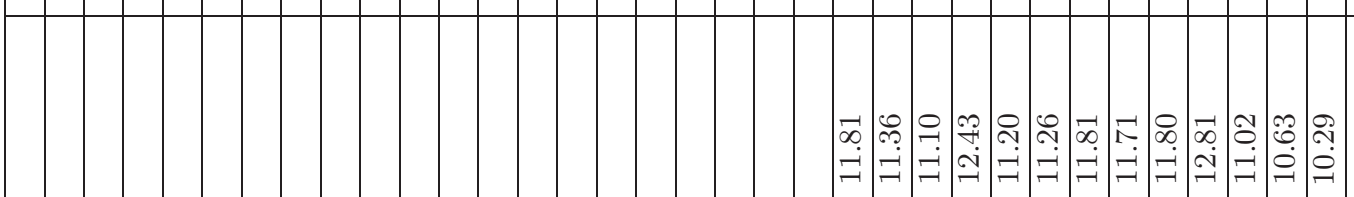

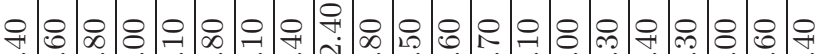

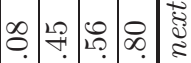

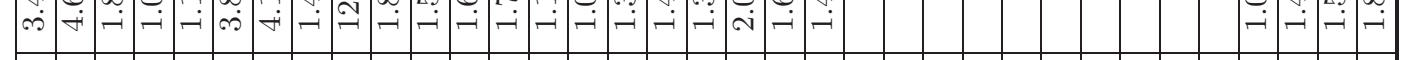

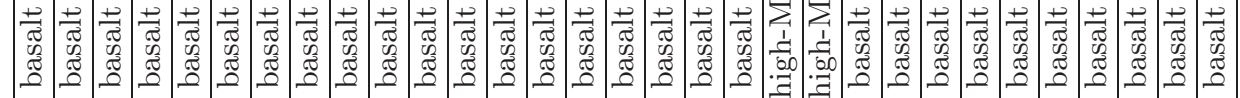

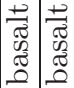

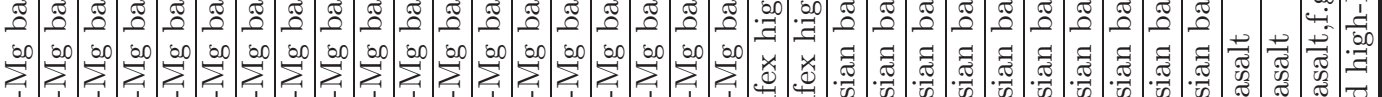

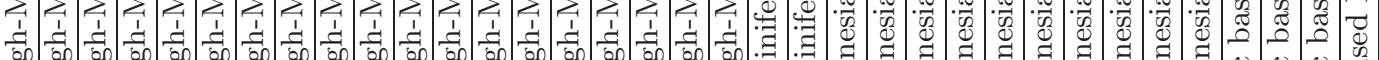

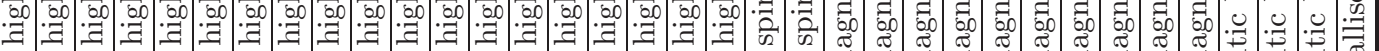

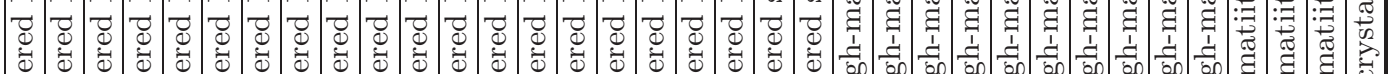

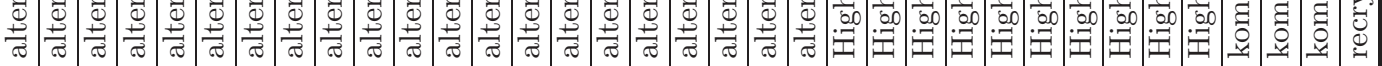

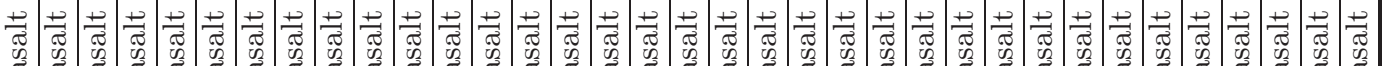

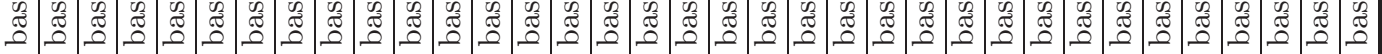

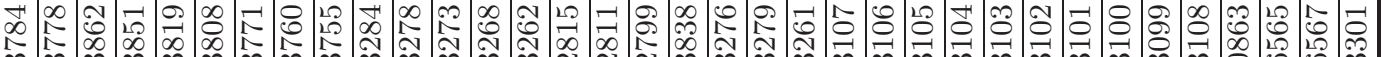

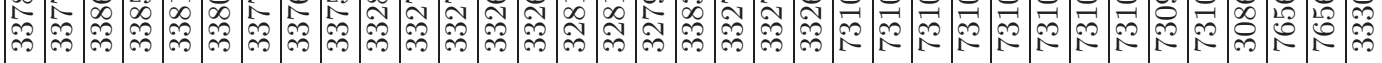




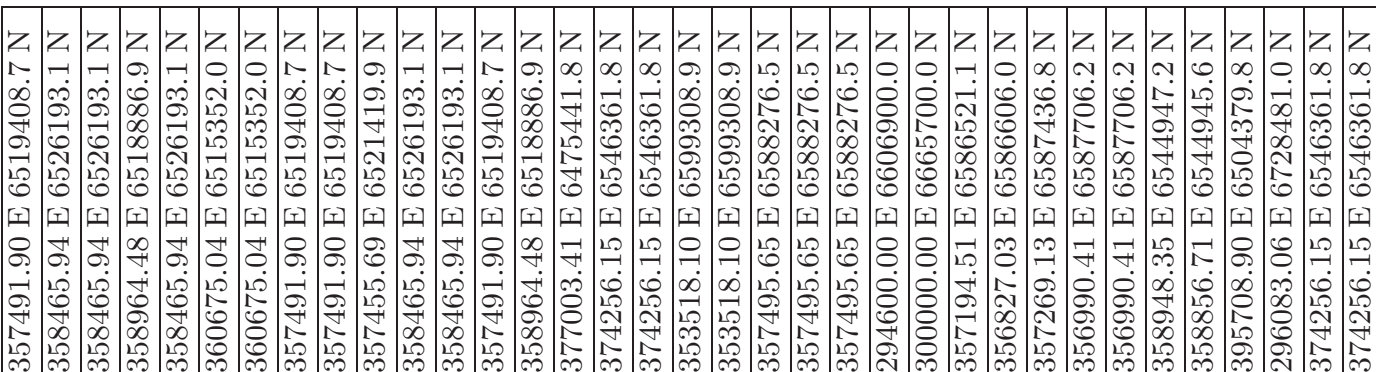

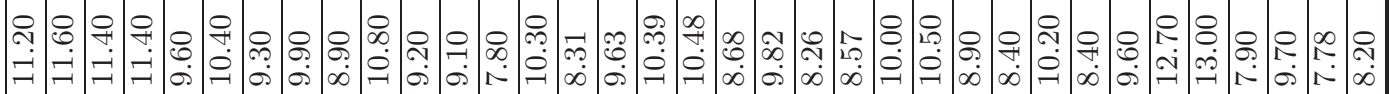

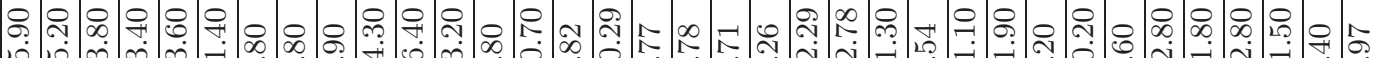

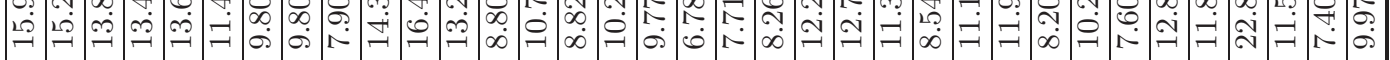

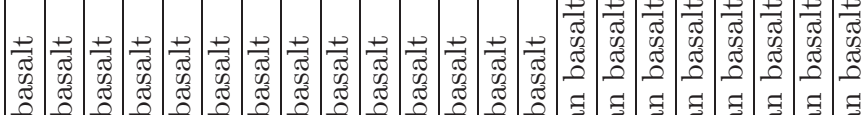

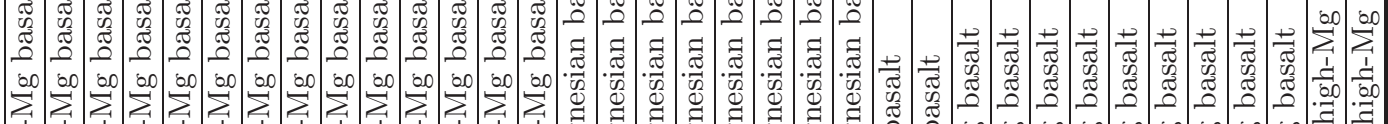

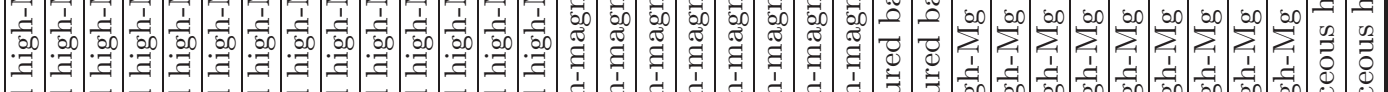

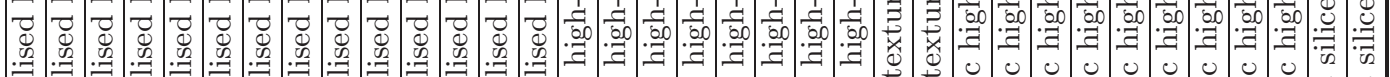

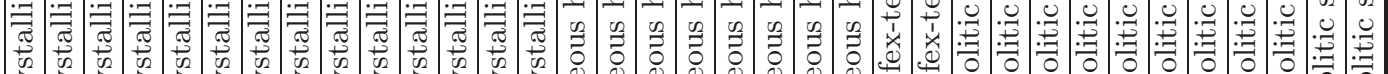

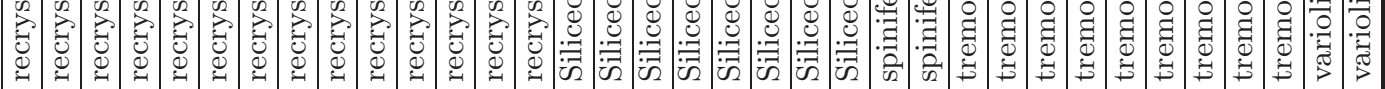

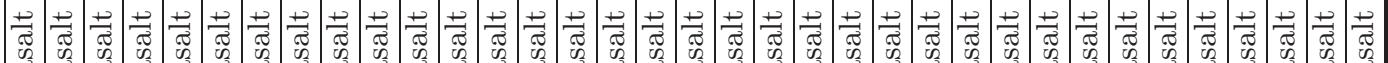

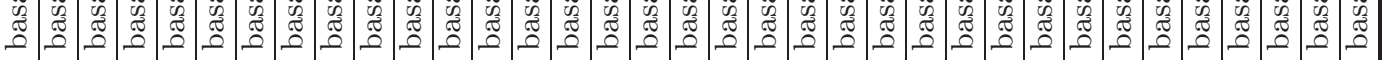

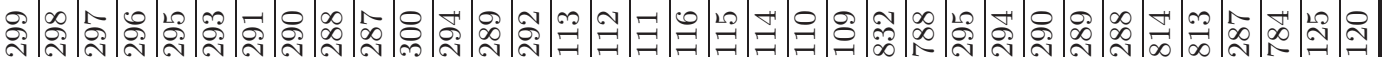

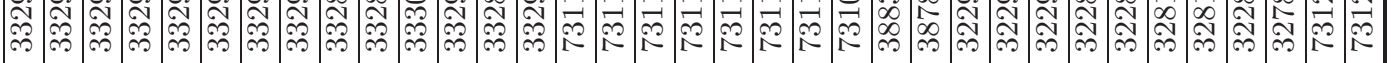




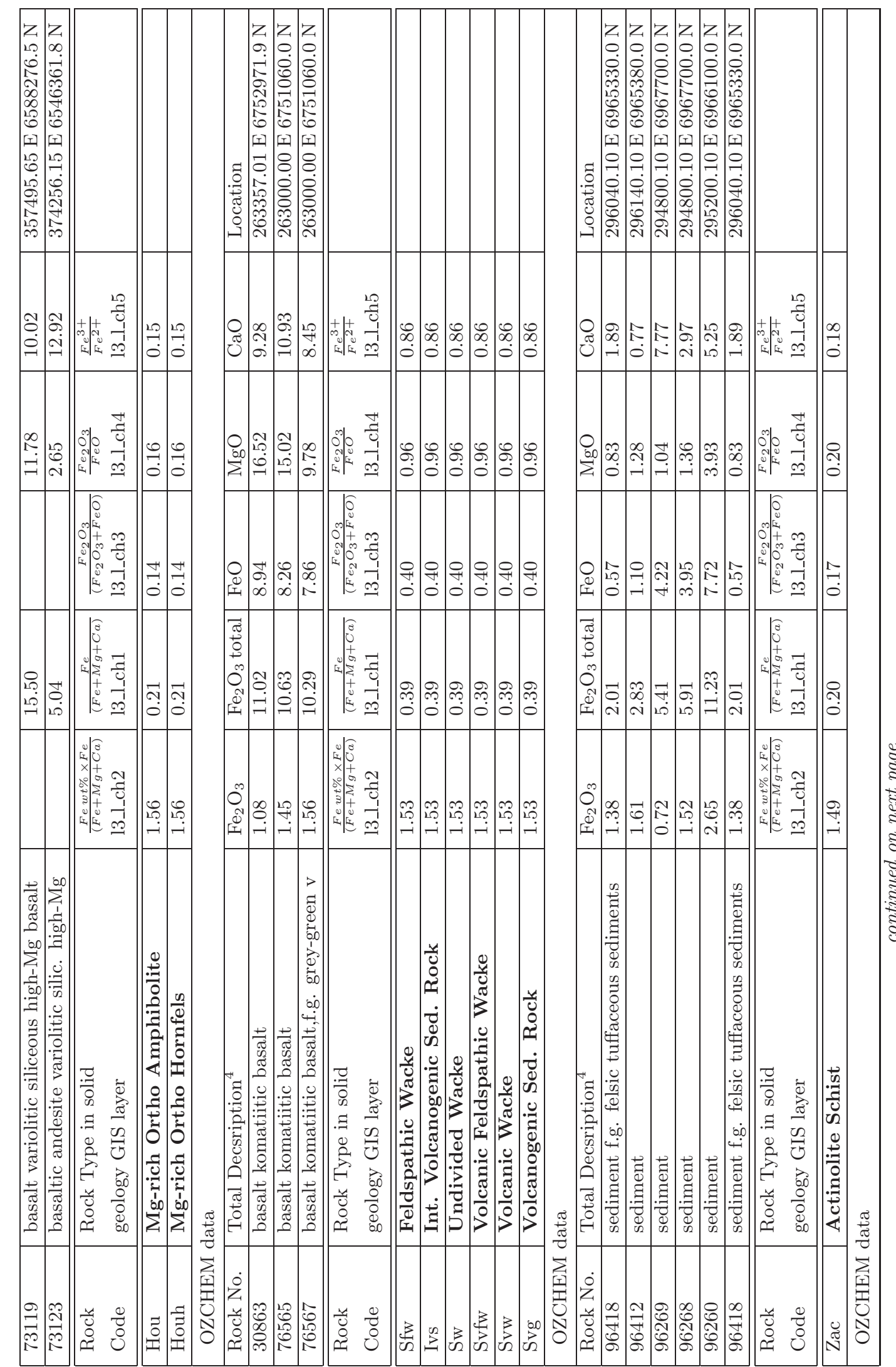




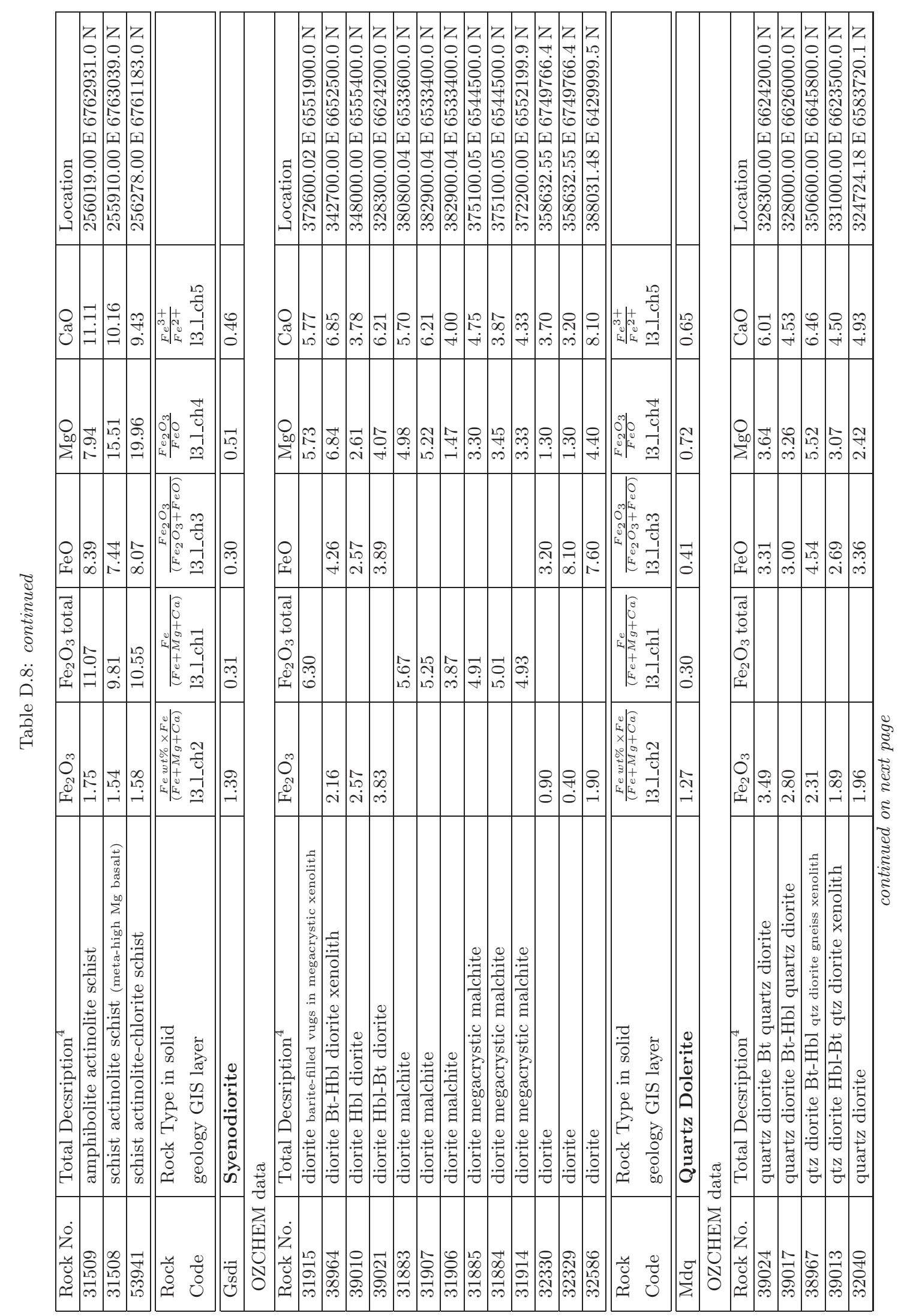




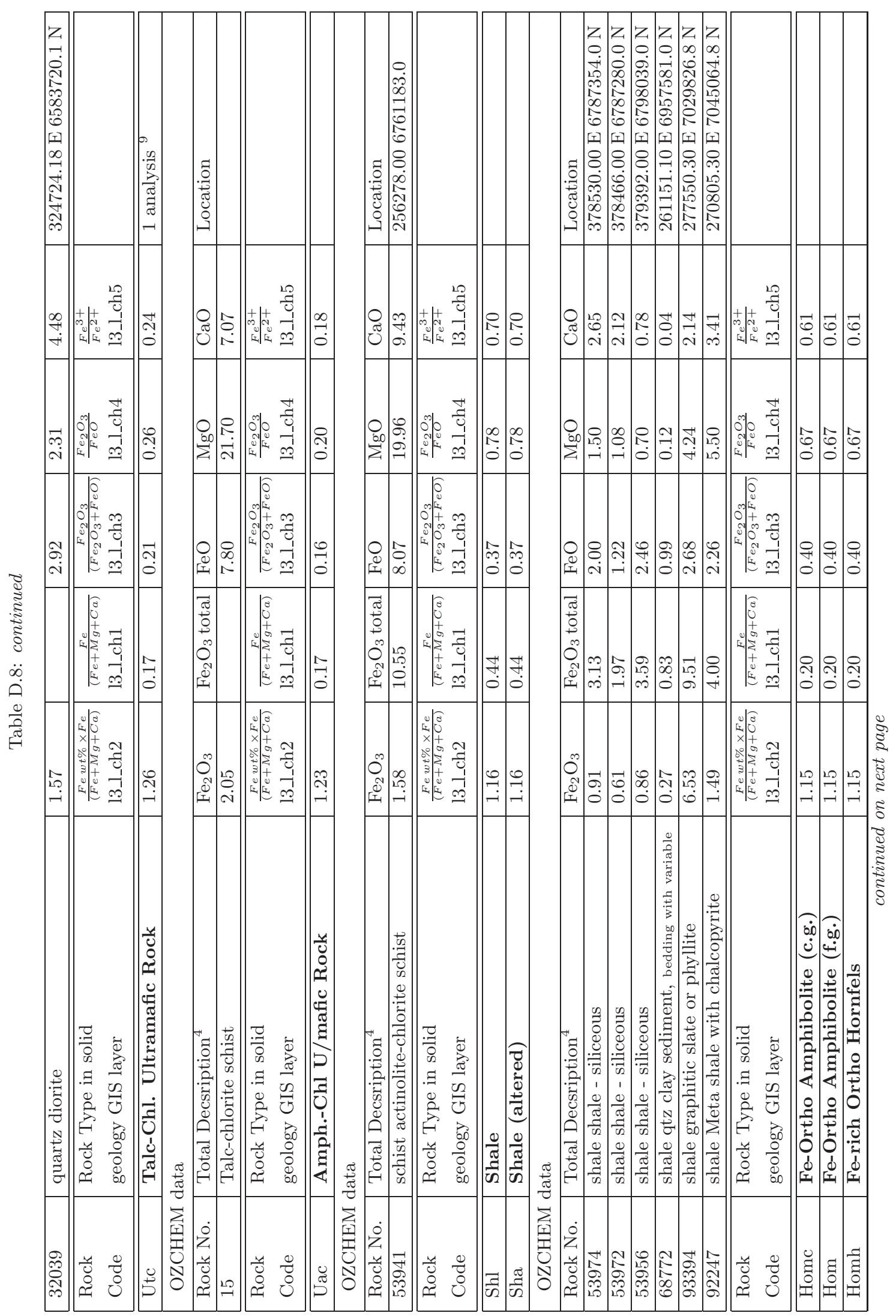




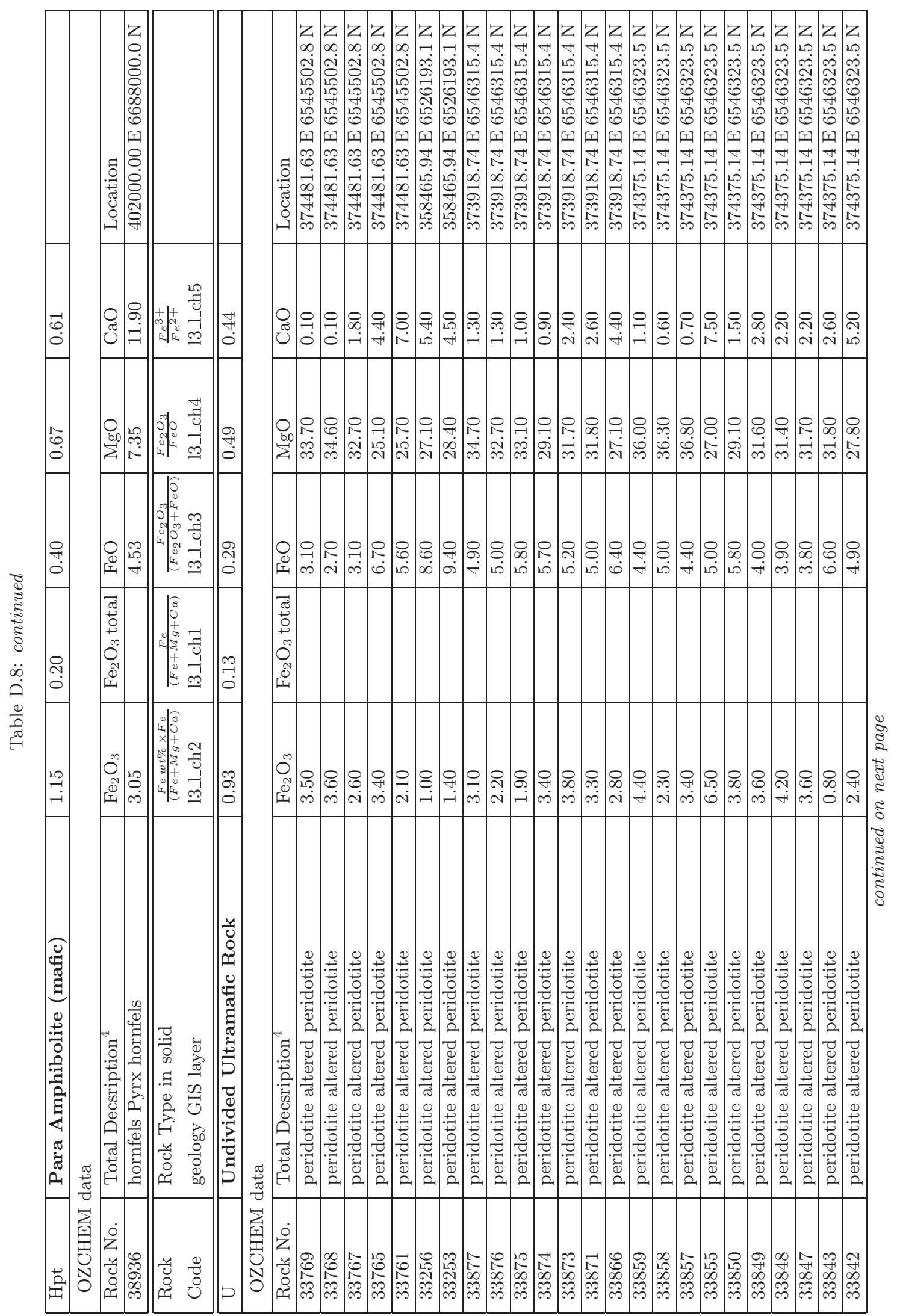




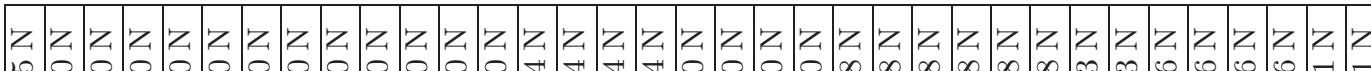

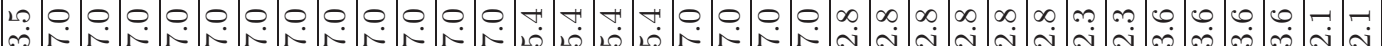
พื) N

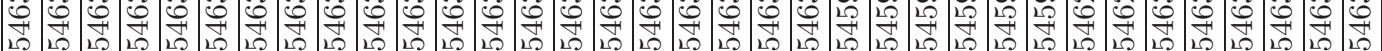

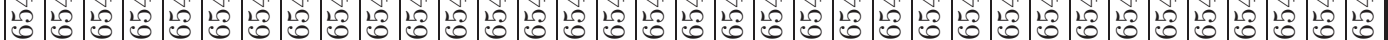

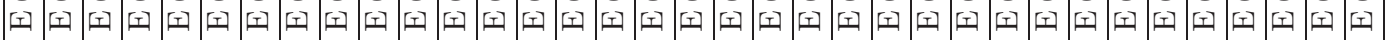

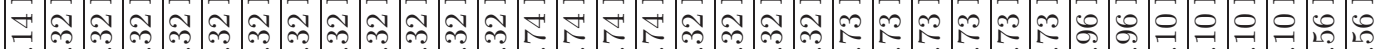

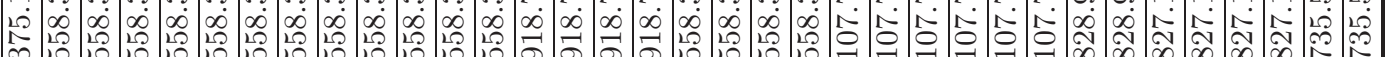

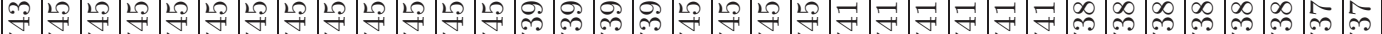

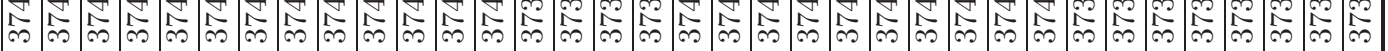

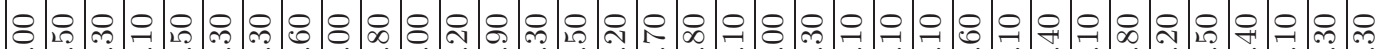

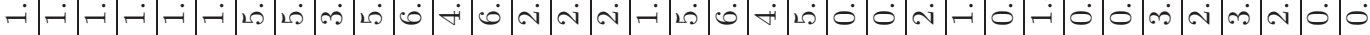

으응

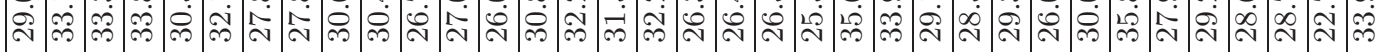

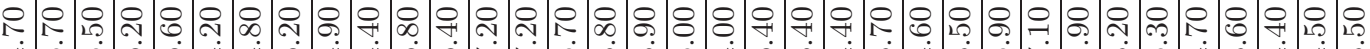

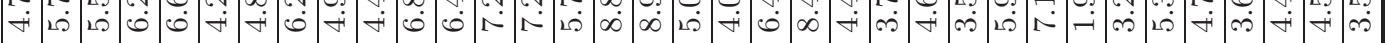

ه্ণ

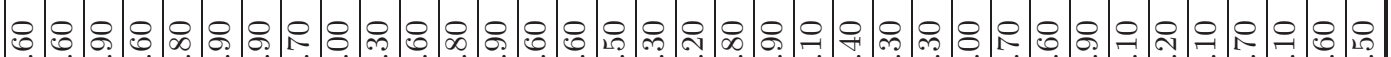

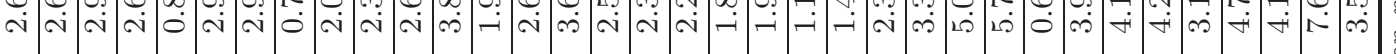

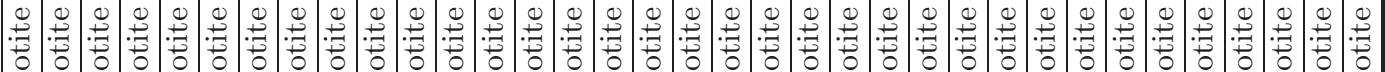

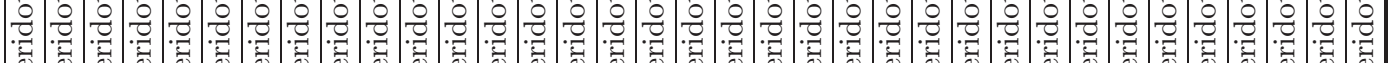

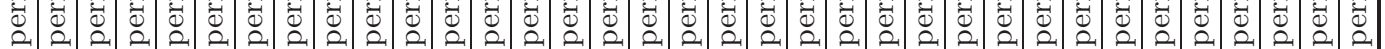

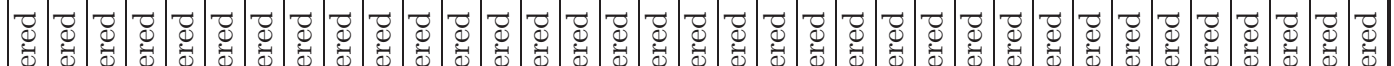

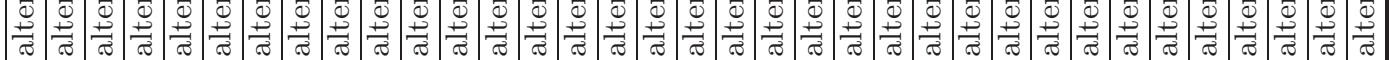

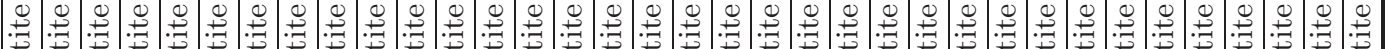

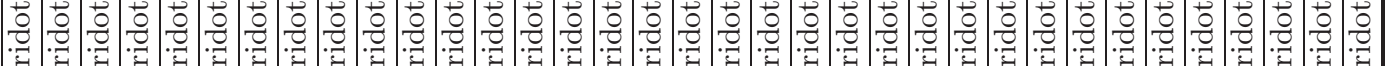

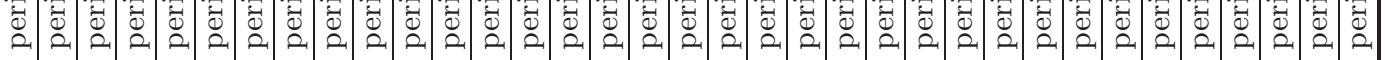




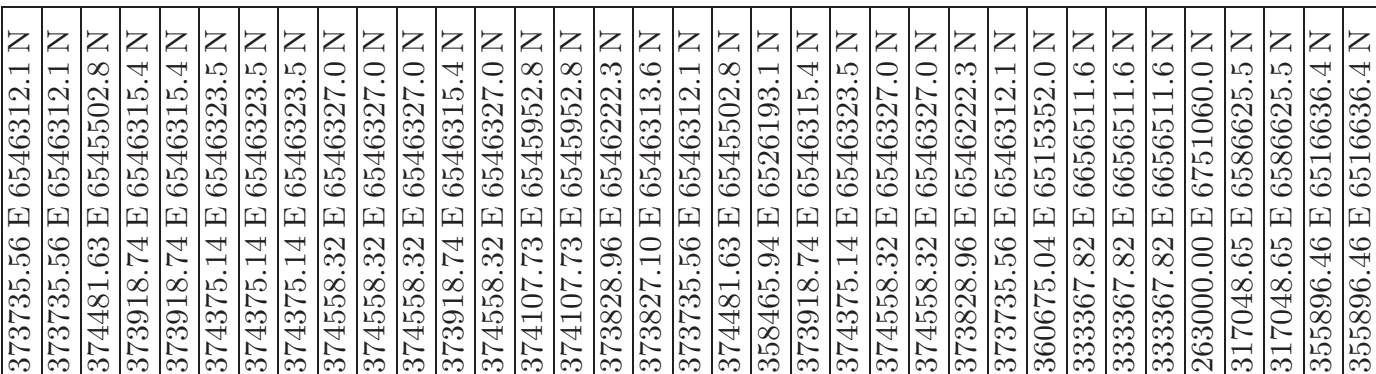

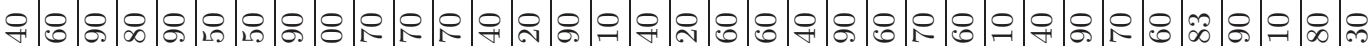
ó $\mathbf{r}$ -

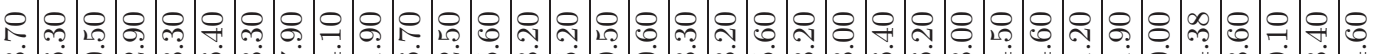
ண்

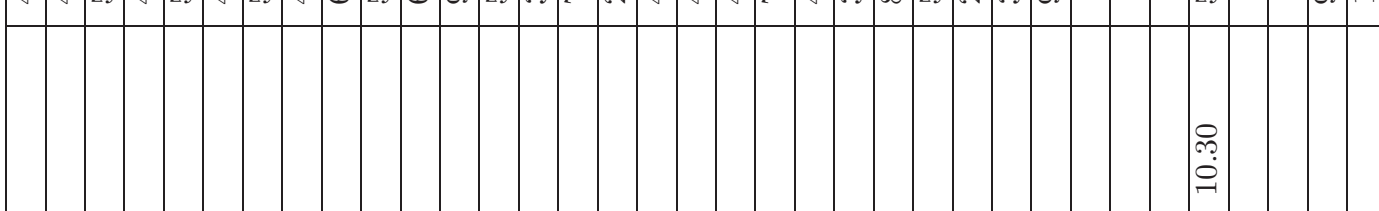

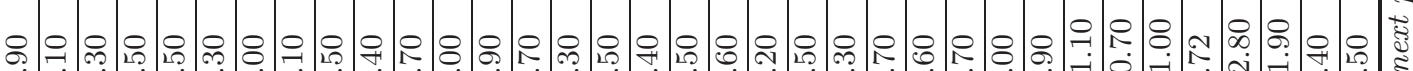

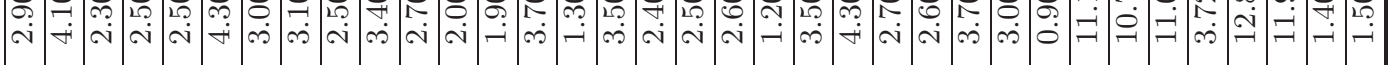

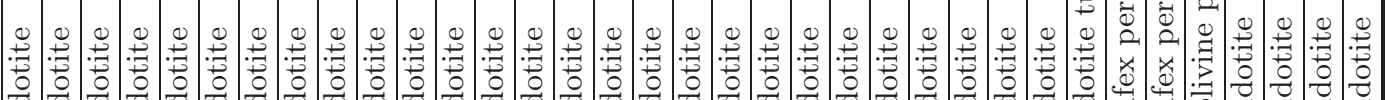

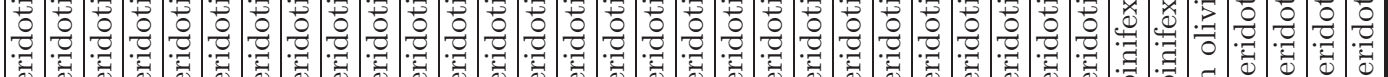
岕

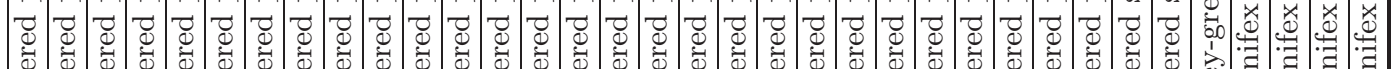

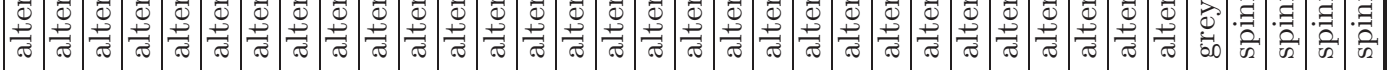

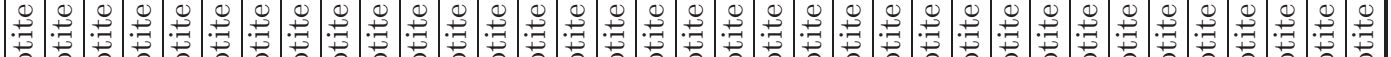
齐

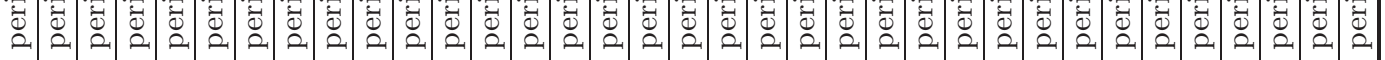

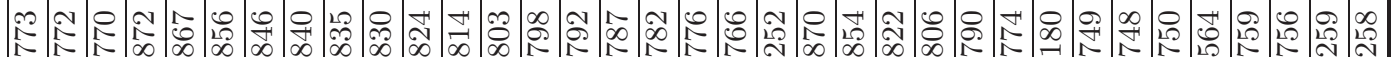

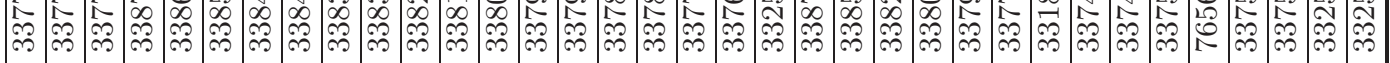




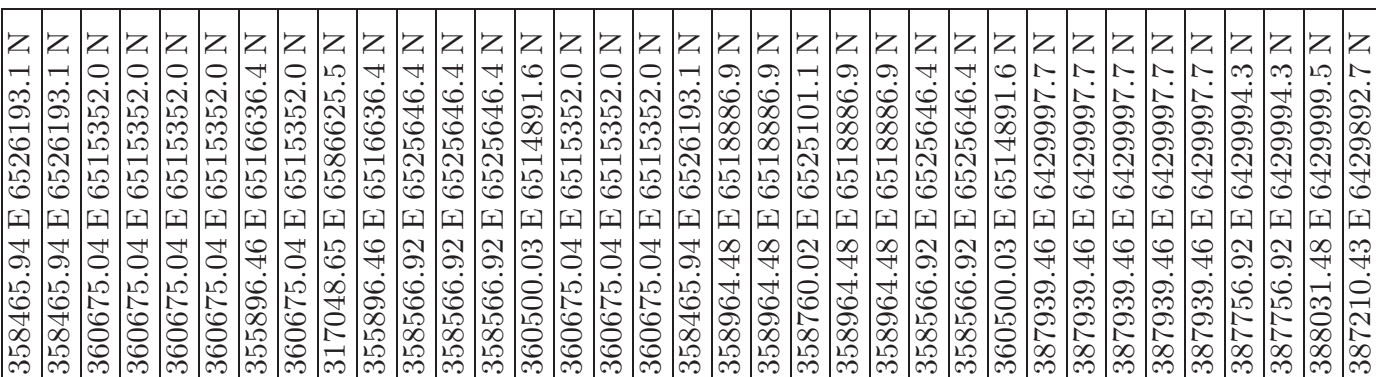

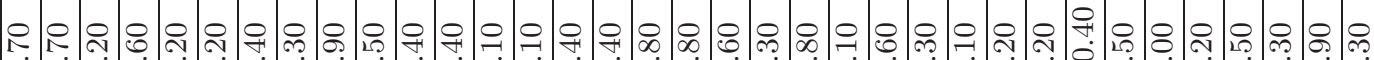

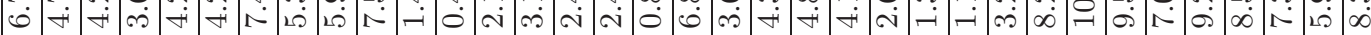

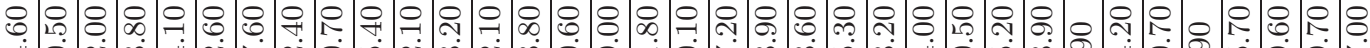

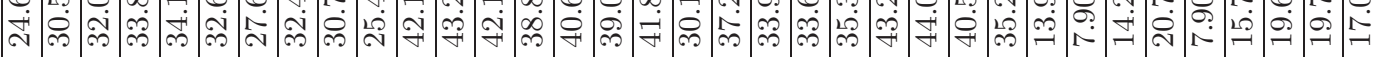

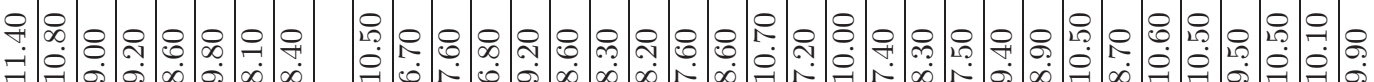

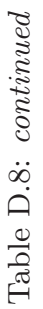

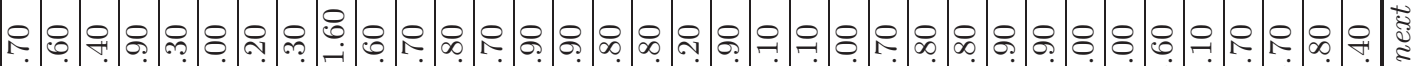

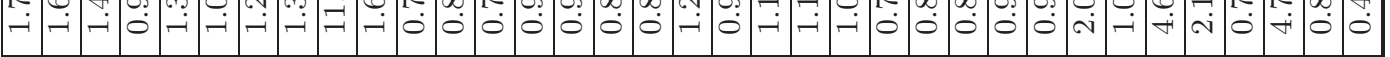

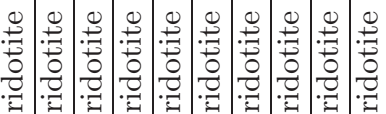

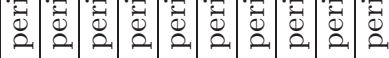

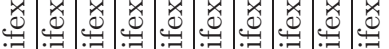

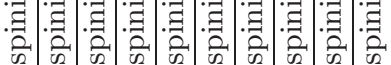

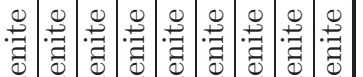

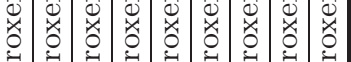

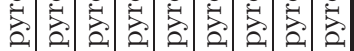

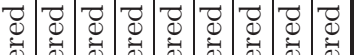

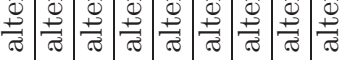

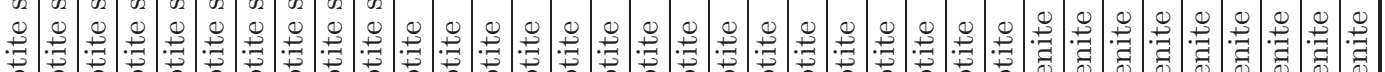

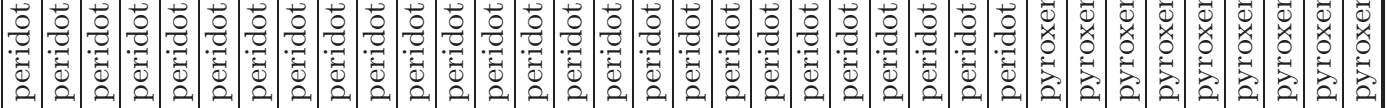

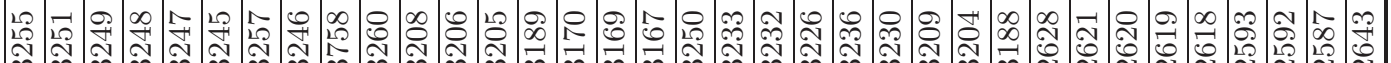

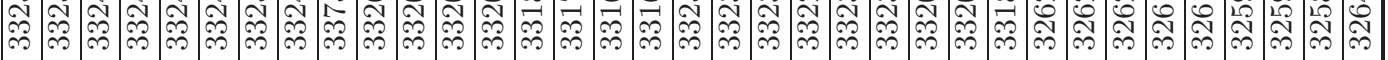




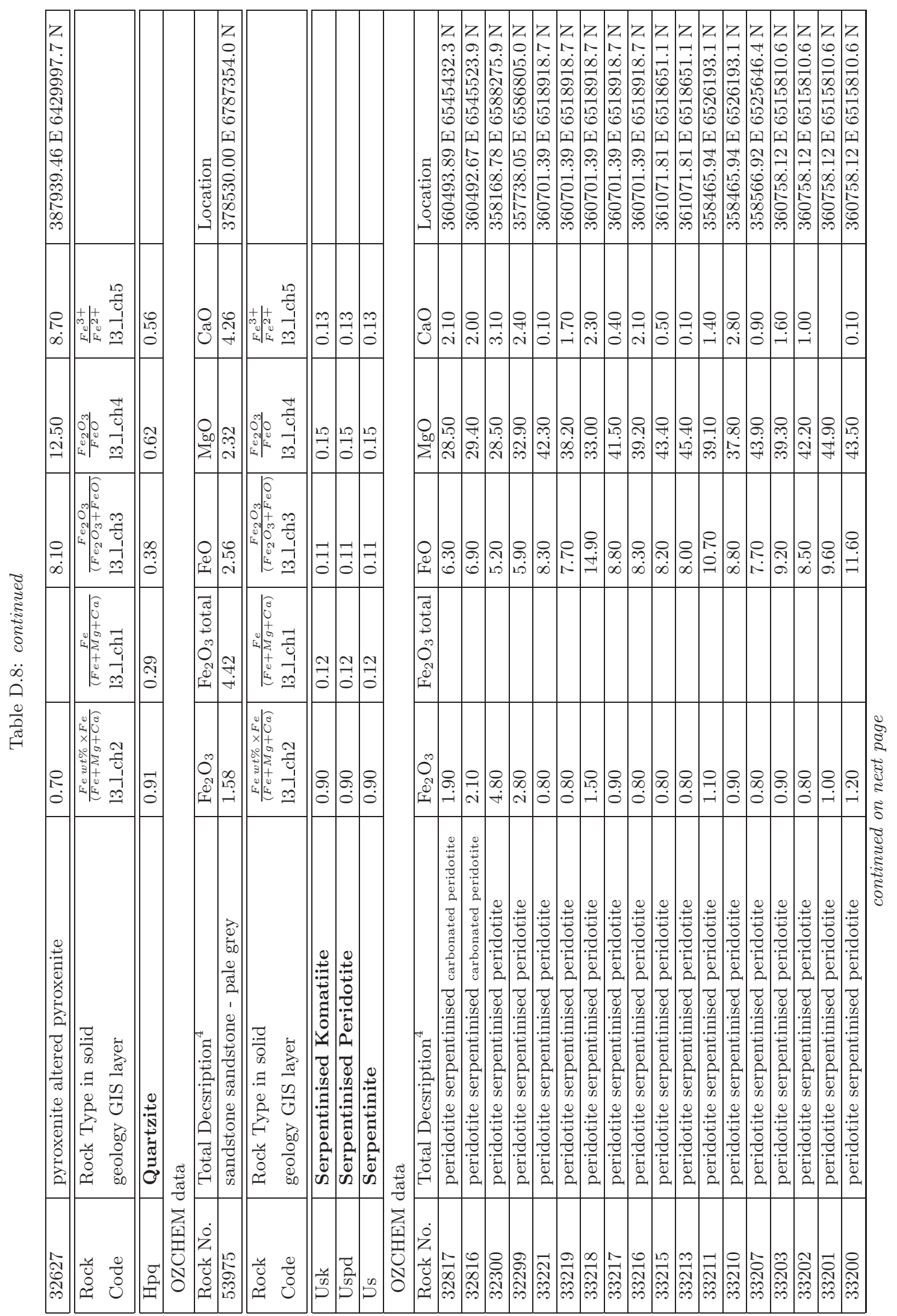




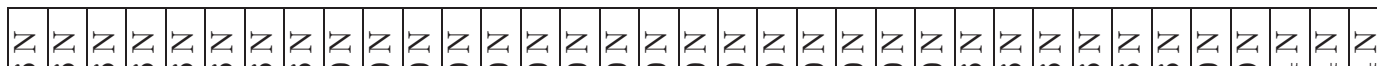

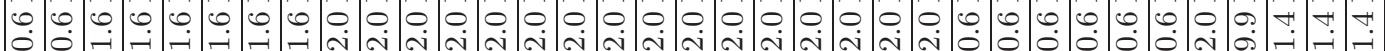

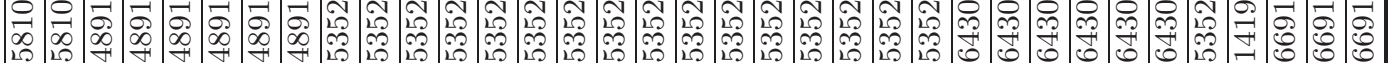

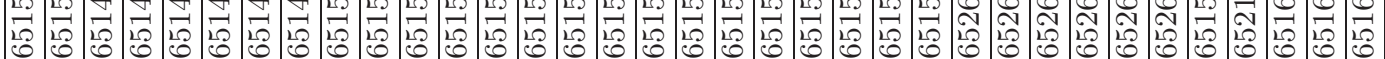

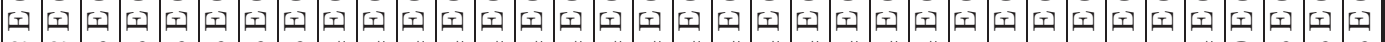
Э

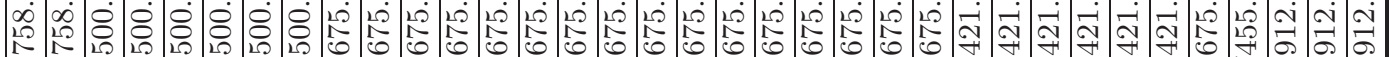

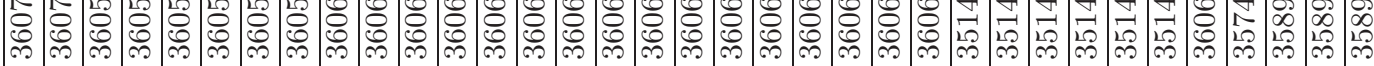

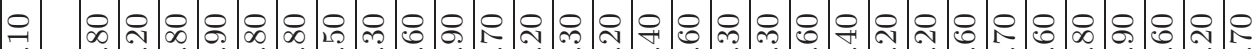

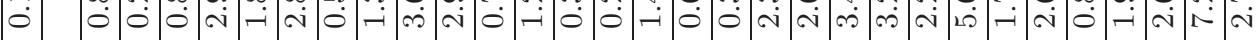

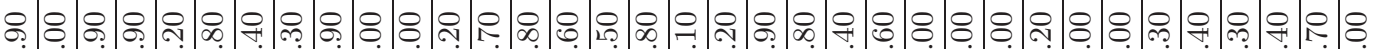

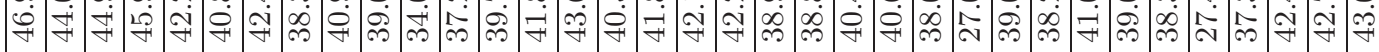

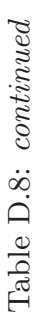

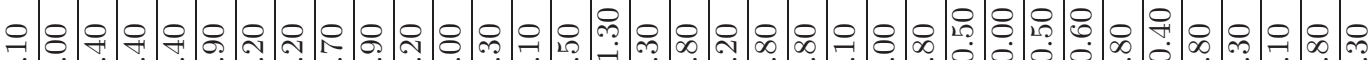

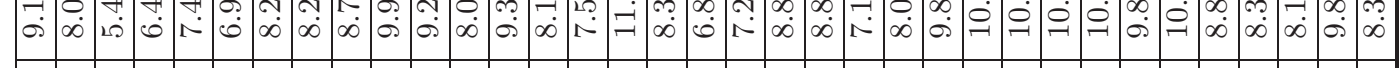

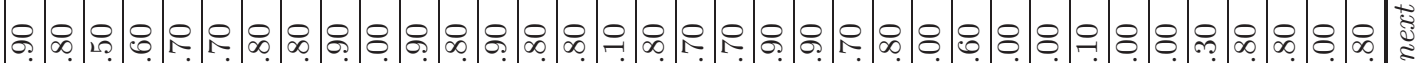

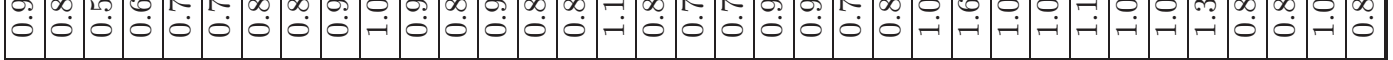

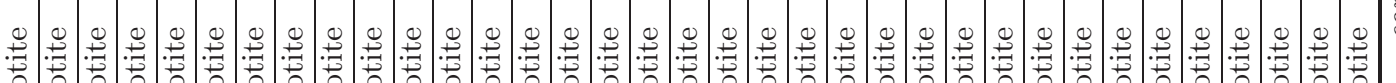

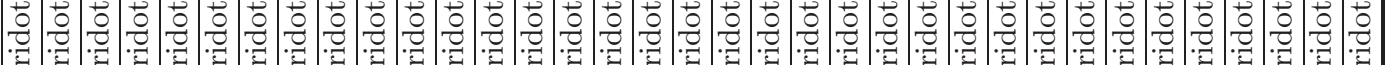

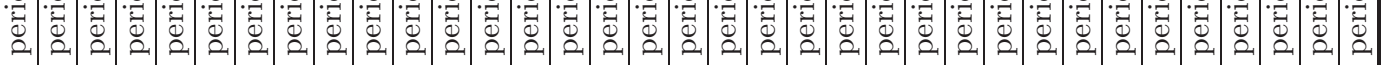

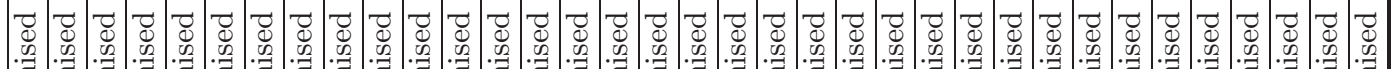

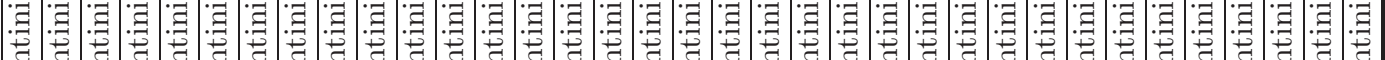

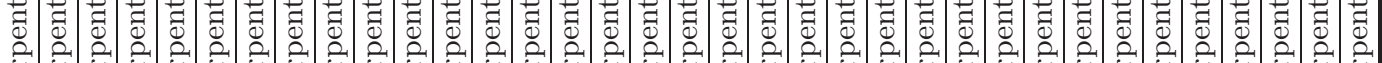
竞

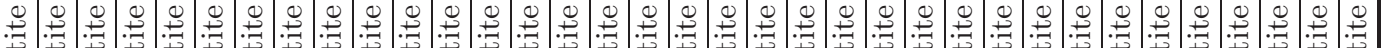
荡

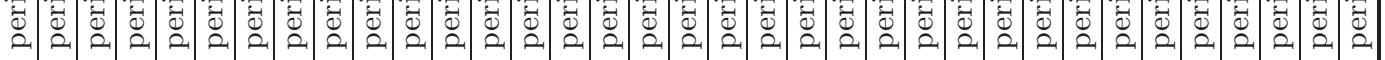

\&

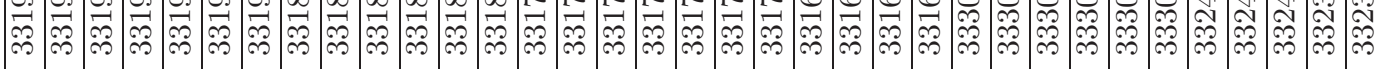



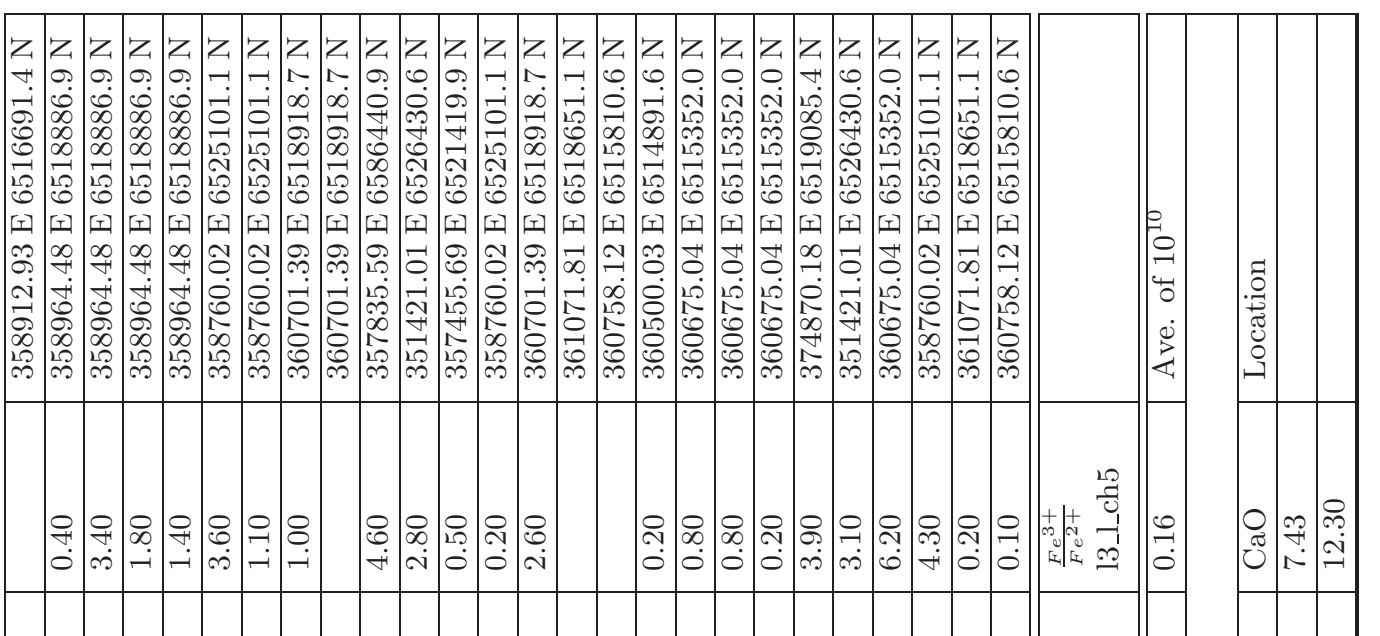

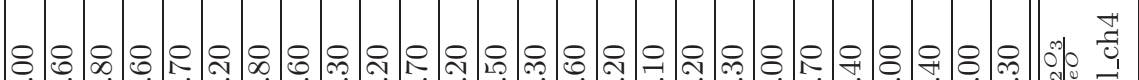

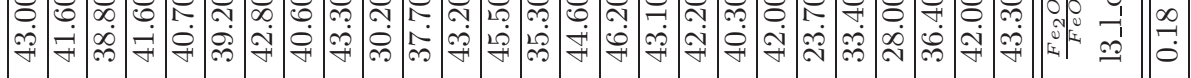

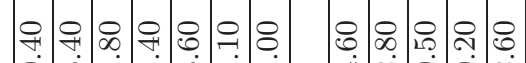

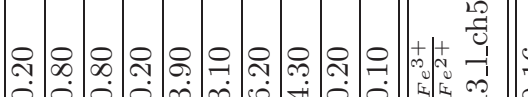

$\sum$ ลั

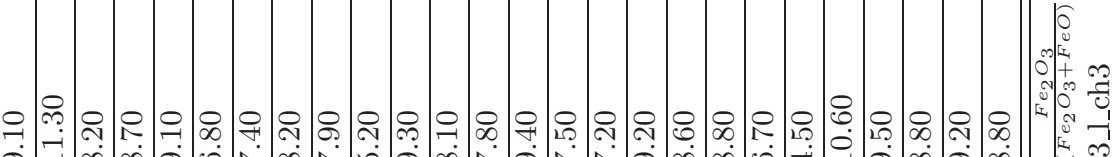

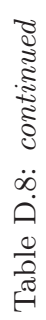

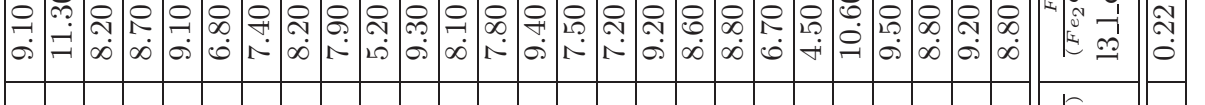

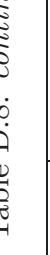

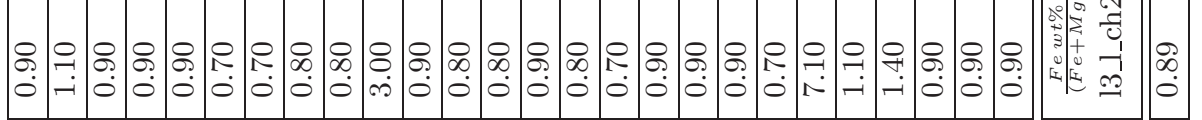




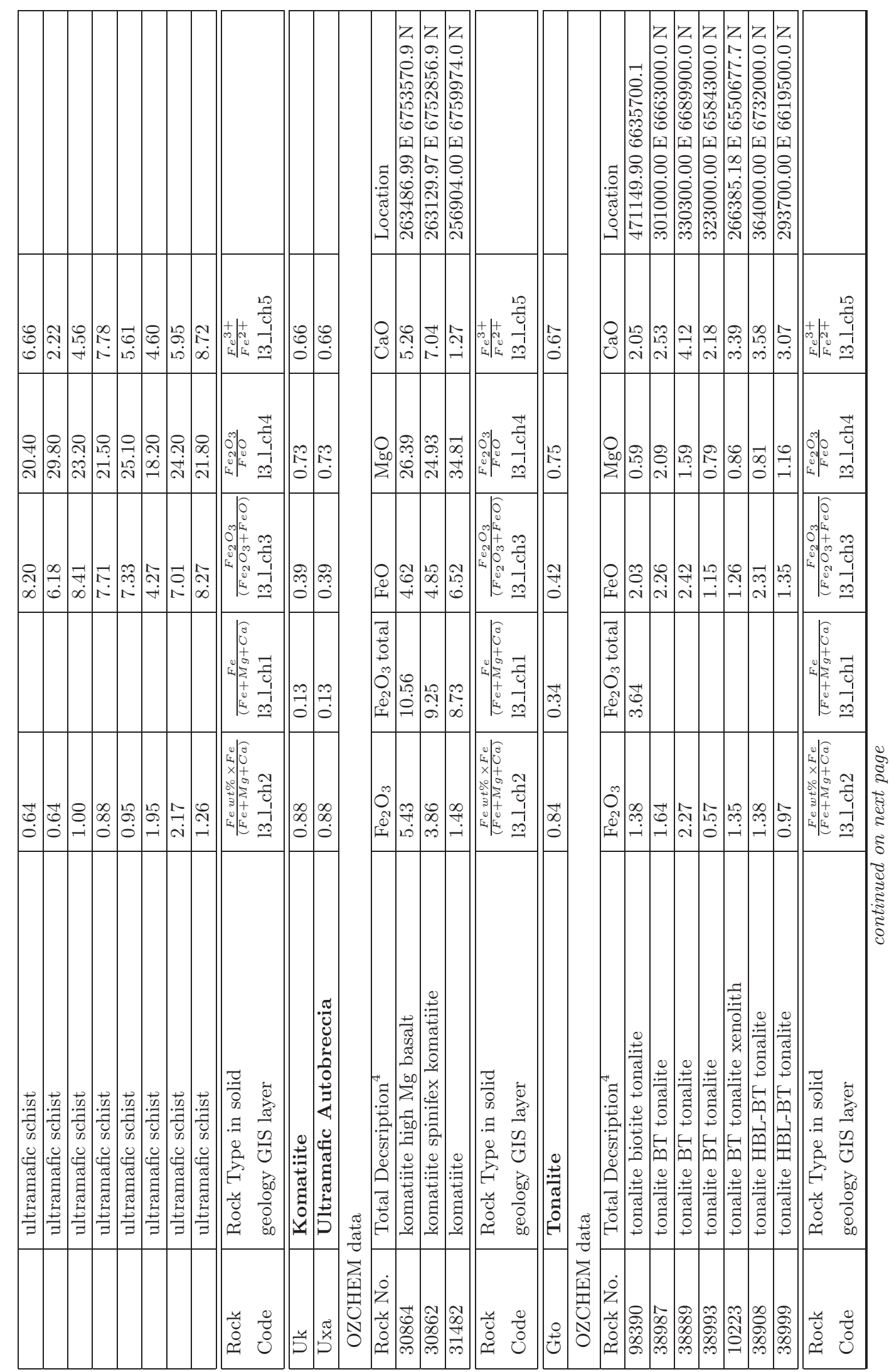




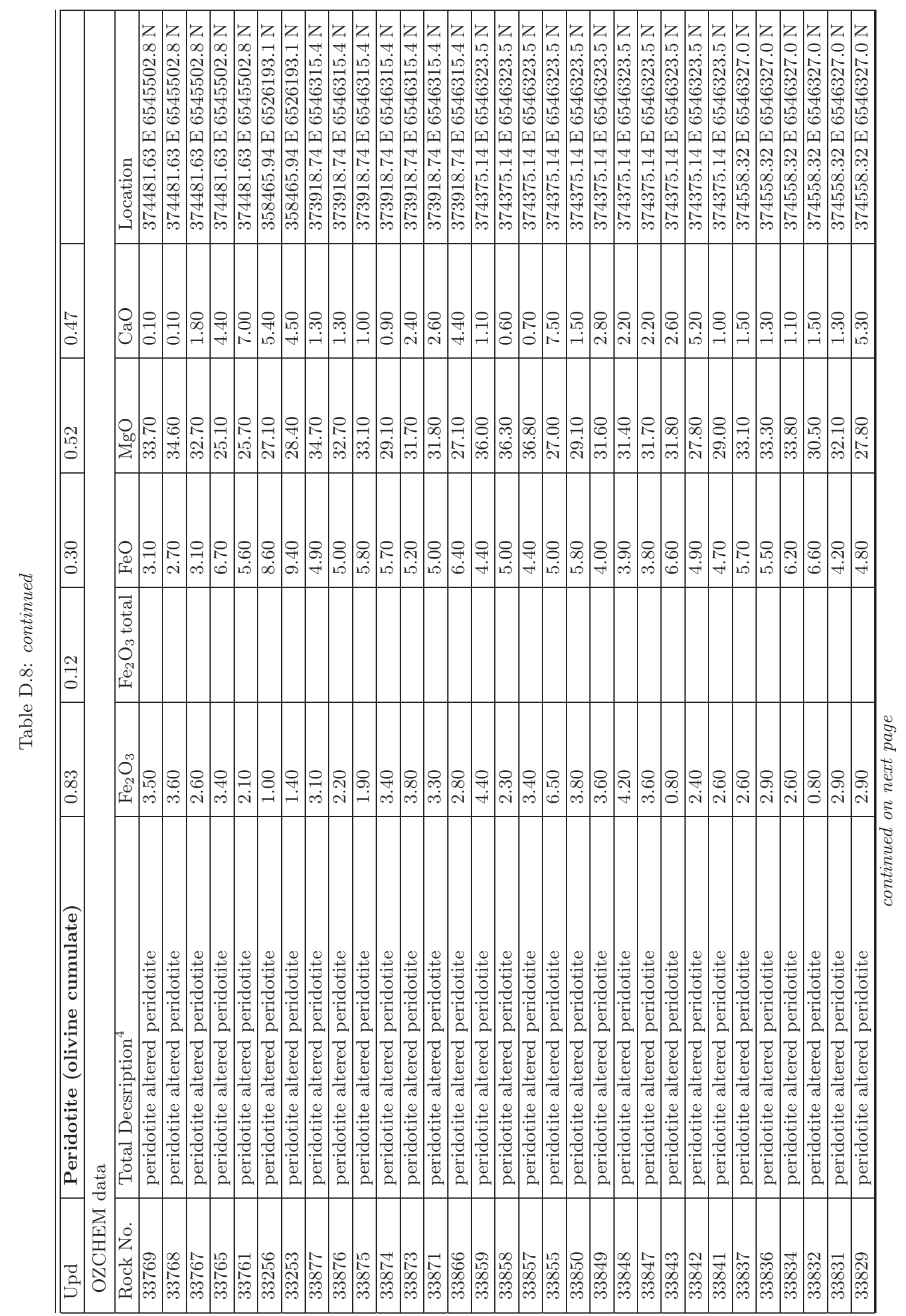




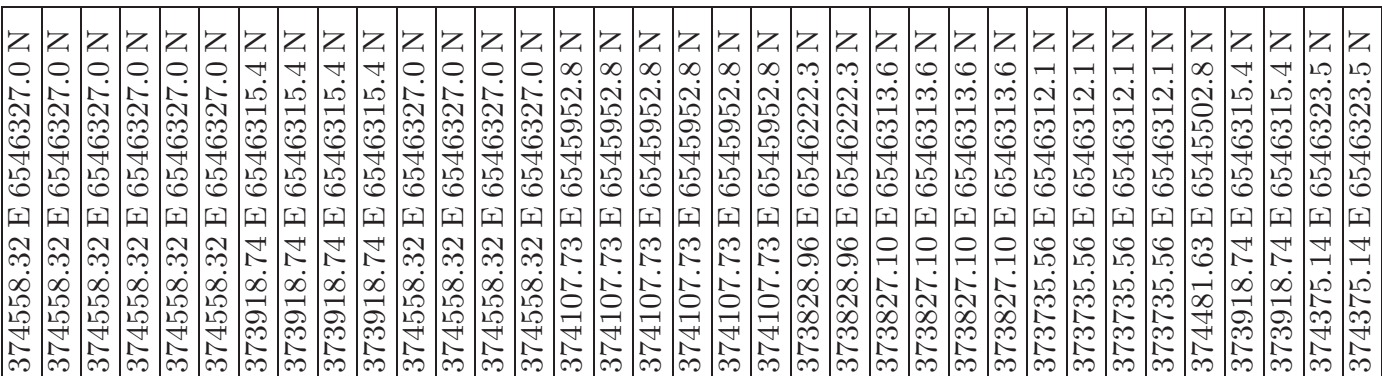

$\therefore 8$ \&

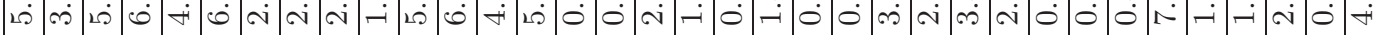

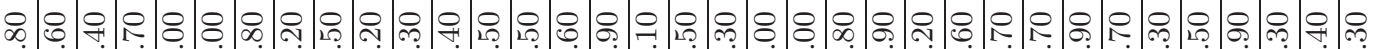
ล่

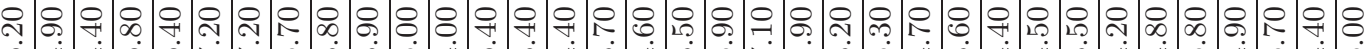

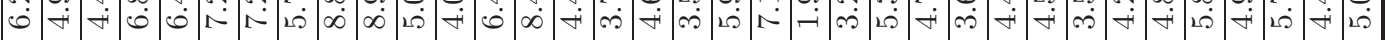

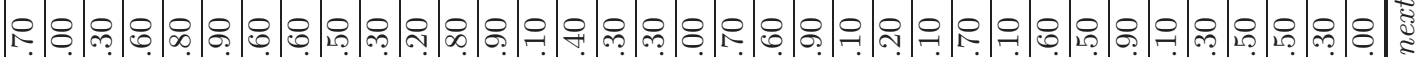

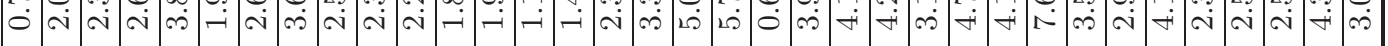

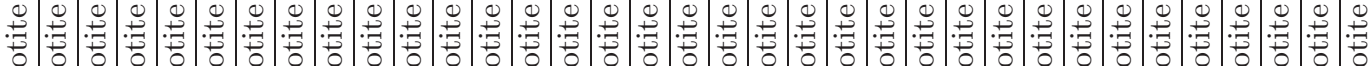

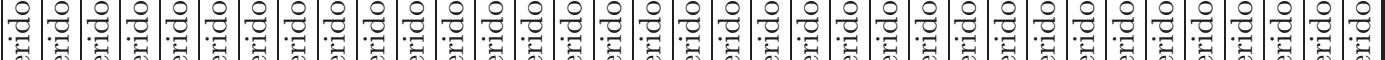

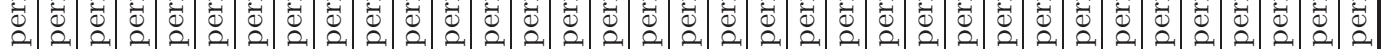

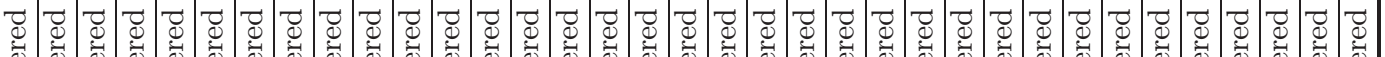

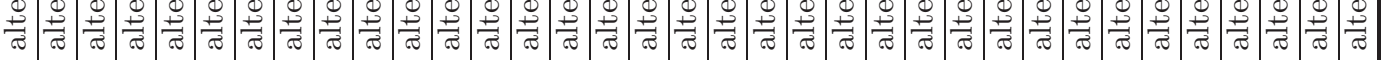

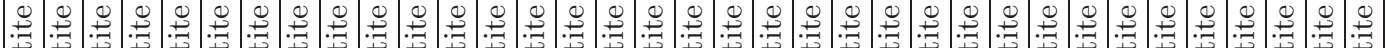
齐

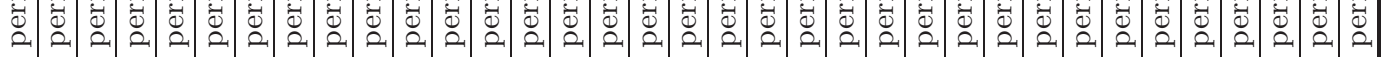

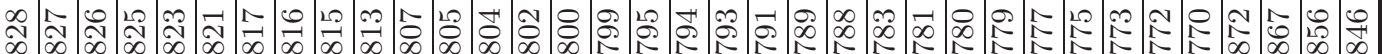

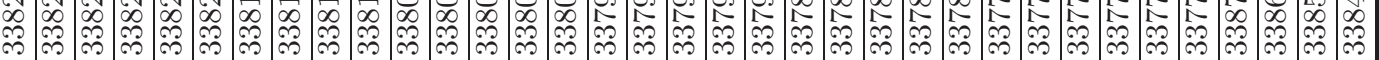




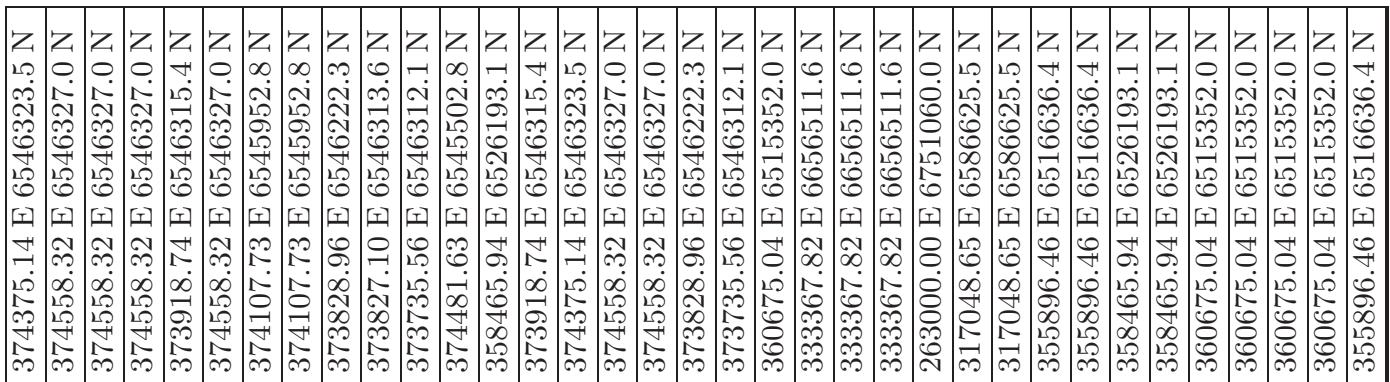

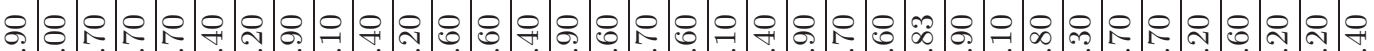

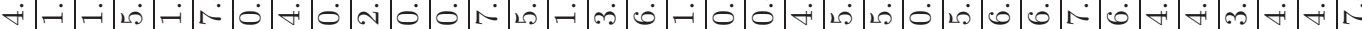

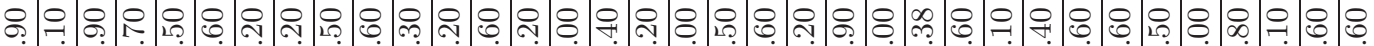
న̂

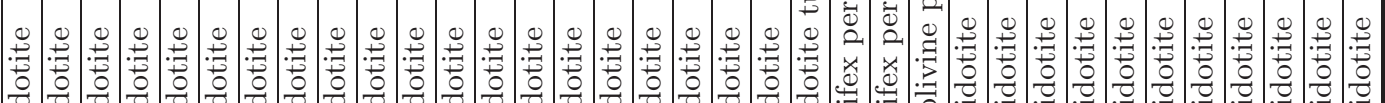

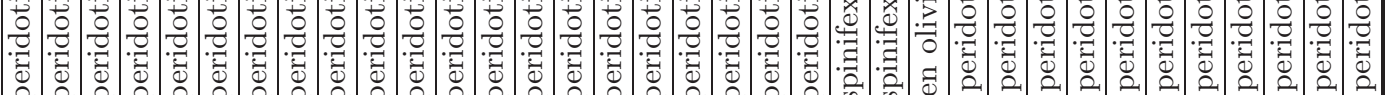

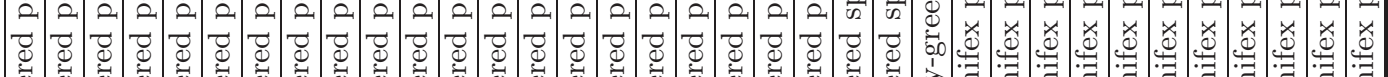

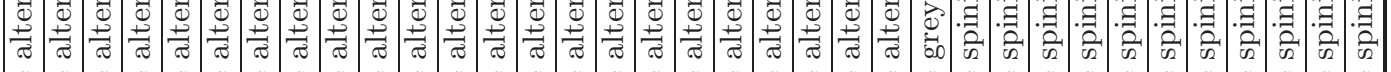

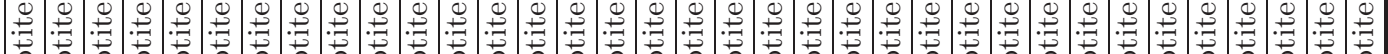
齐

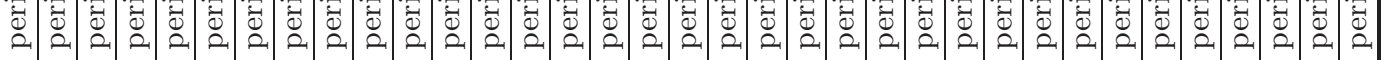




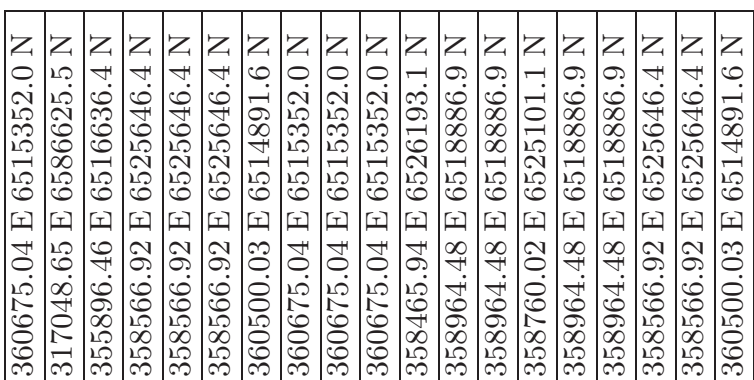

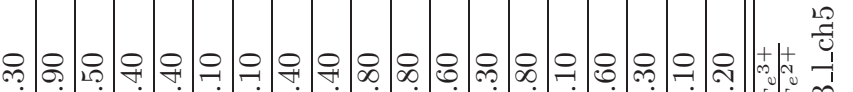

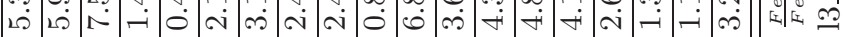

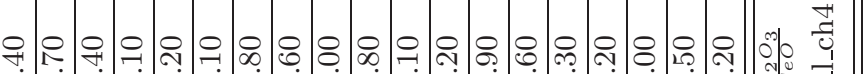

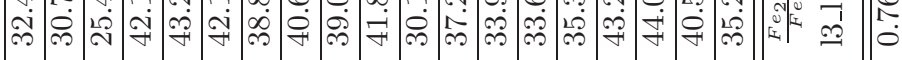

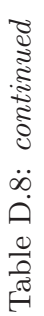

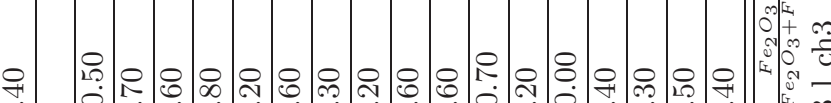

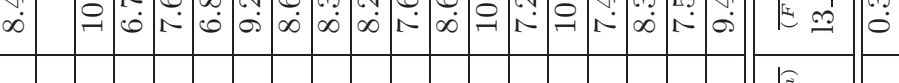

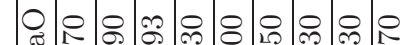
U O)

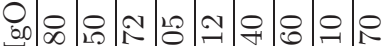

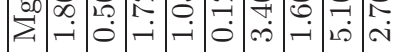

\begin{tabular}{l|l|l|l|l|l|}
\hline & & & & &
\end{tabular}

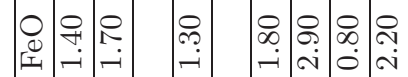

莣

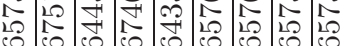

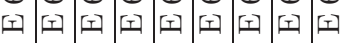
$=$ శ. 要 等

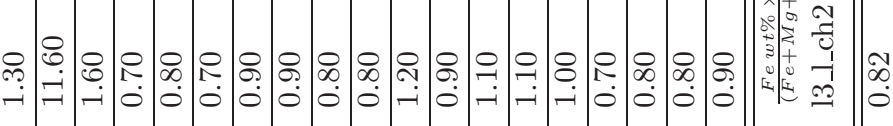

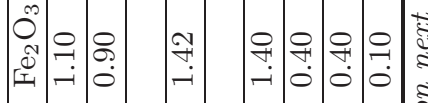

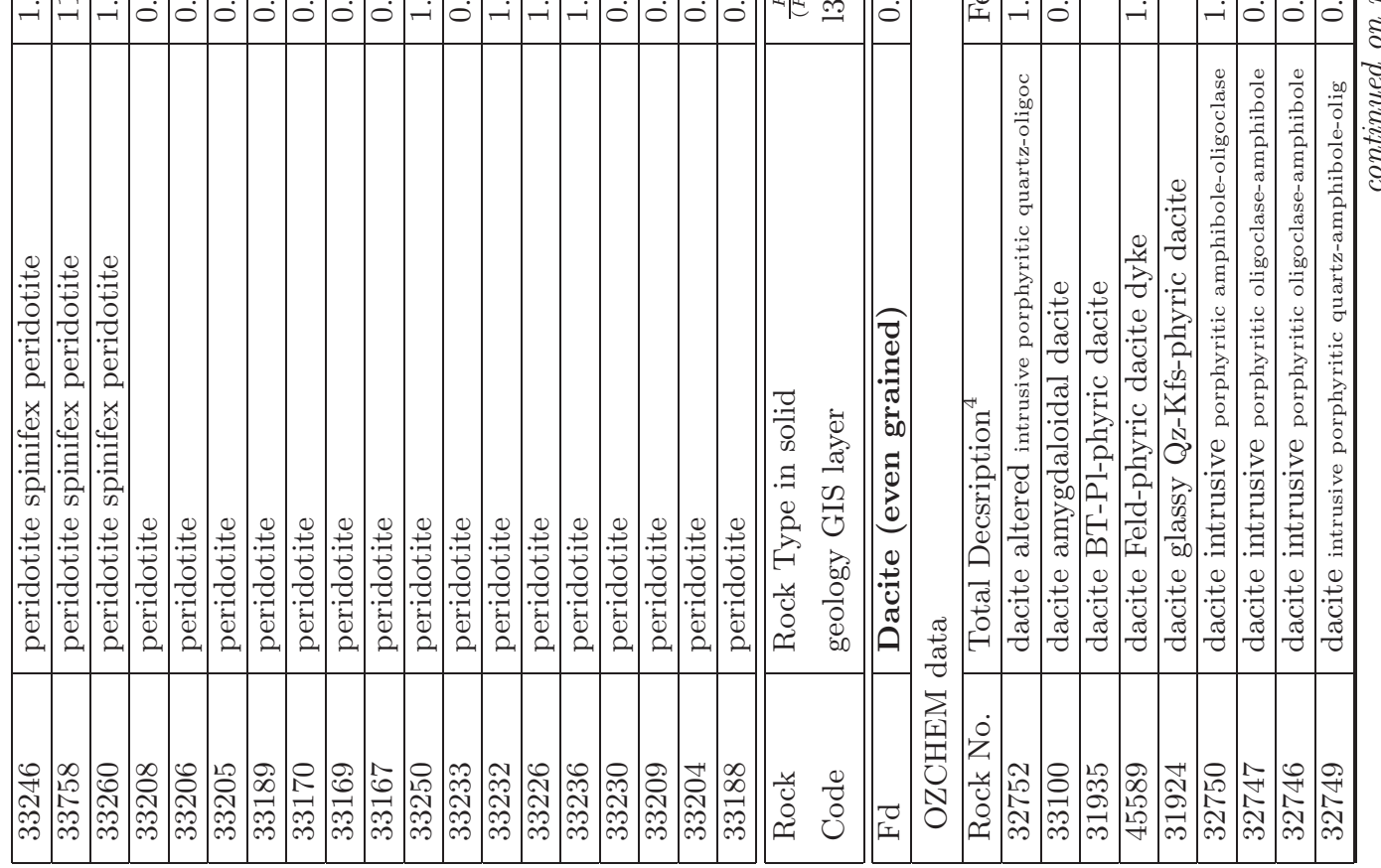




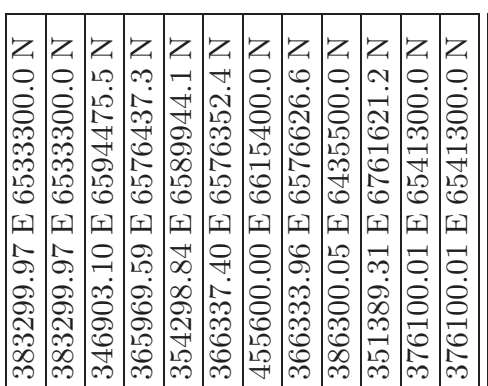

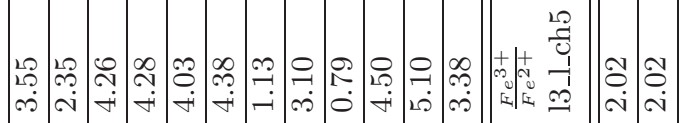
ช่

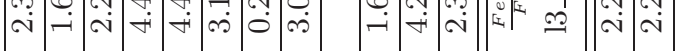

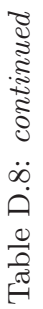

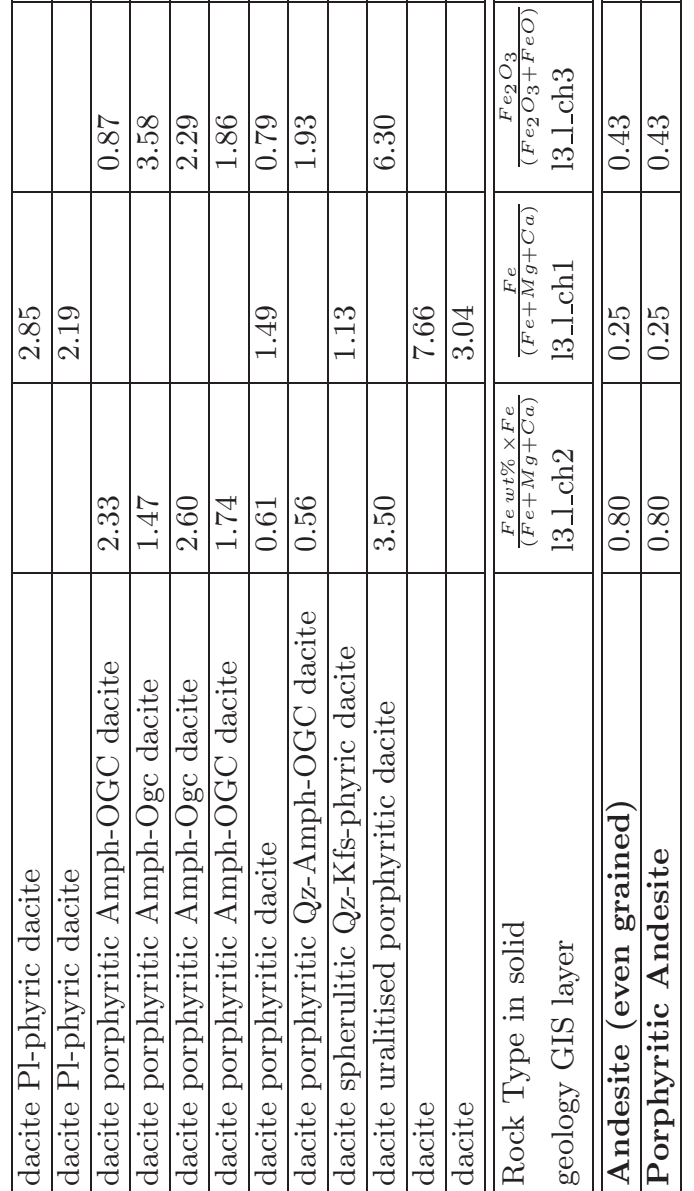

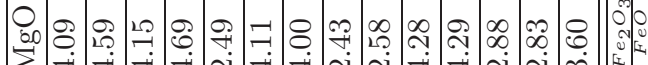

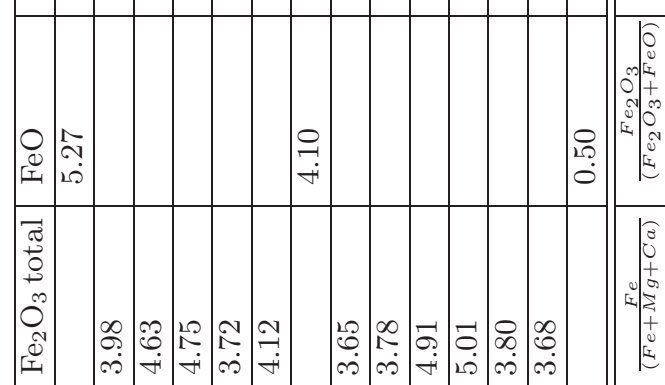

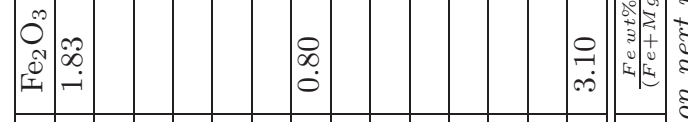

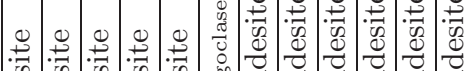

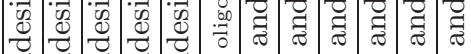
๑ สี

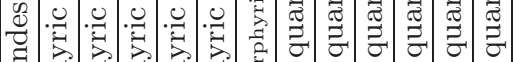
I $.2 \div \frac{1}{2} \frac{1}{2} \frac{1}{2} \frac{1}{2}=5$ .

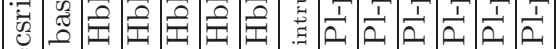

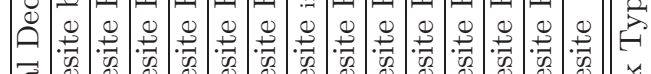

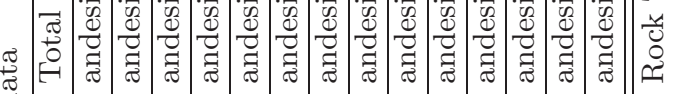

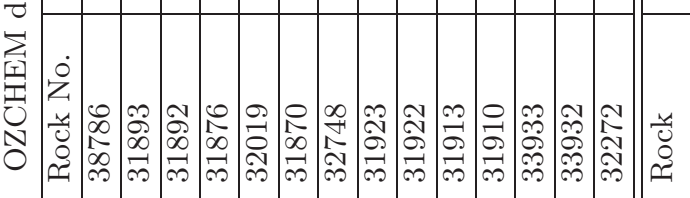




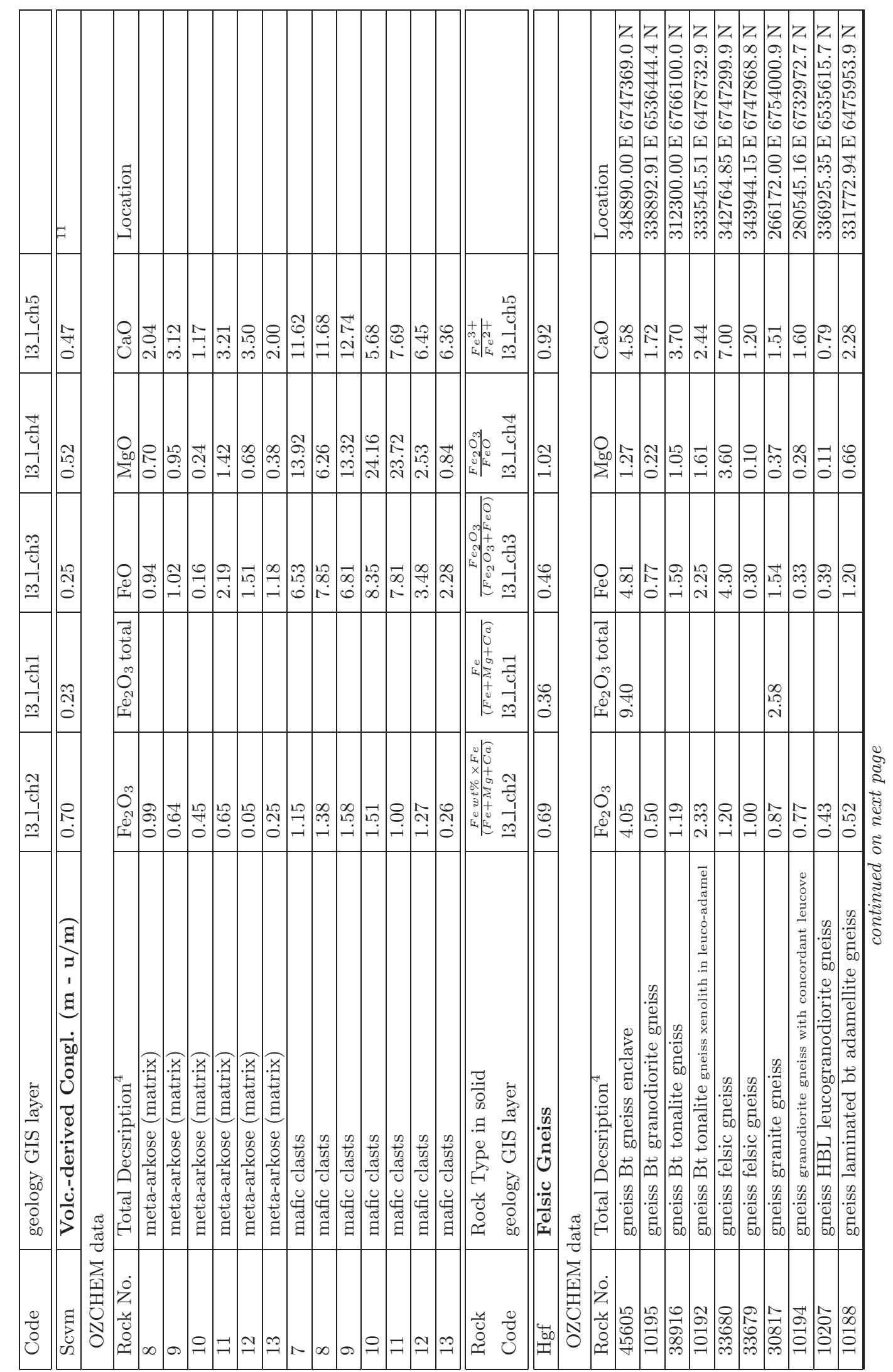




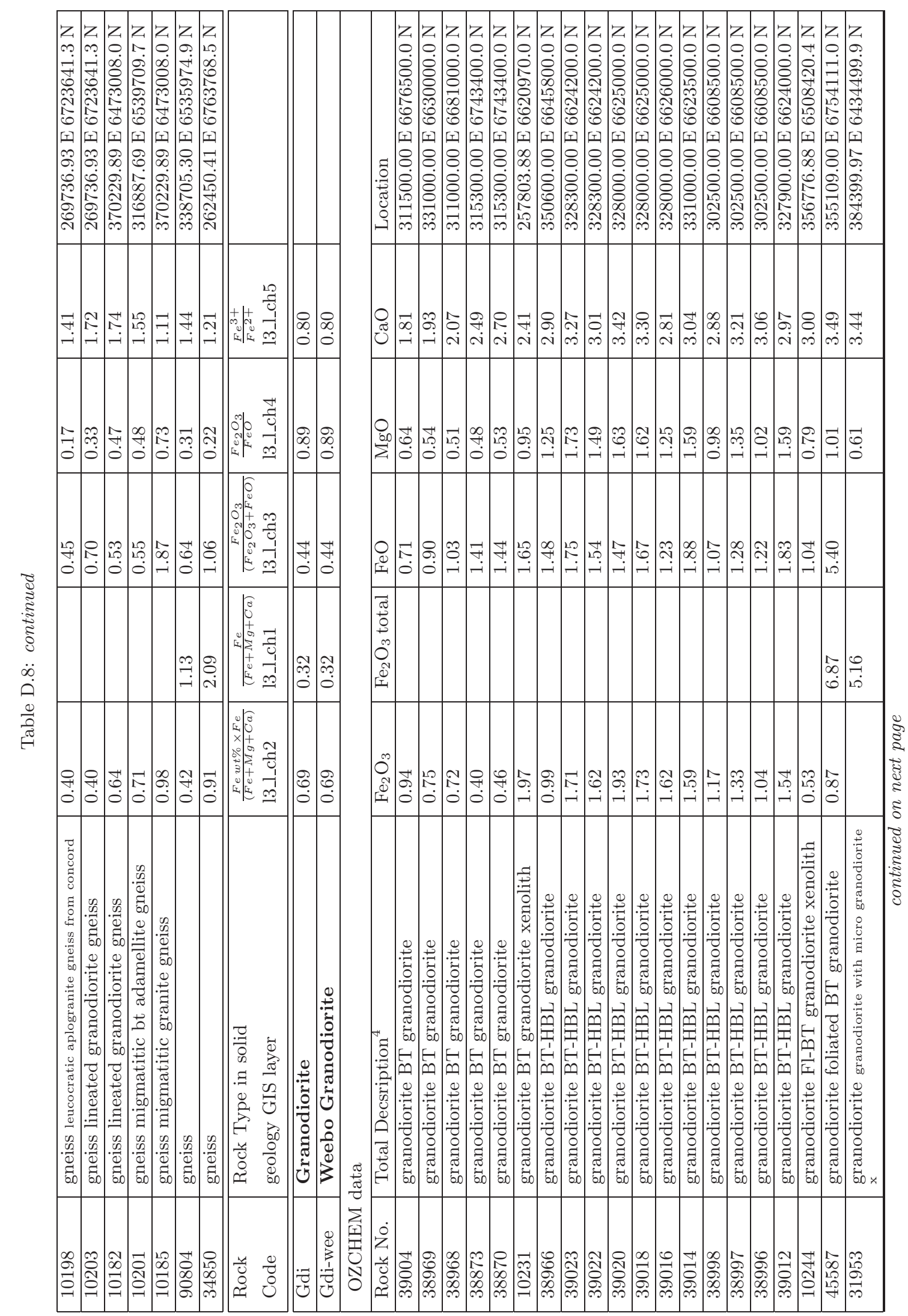




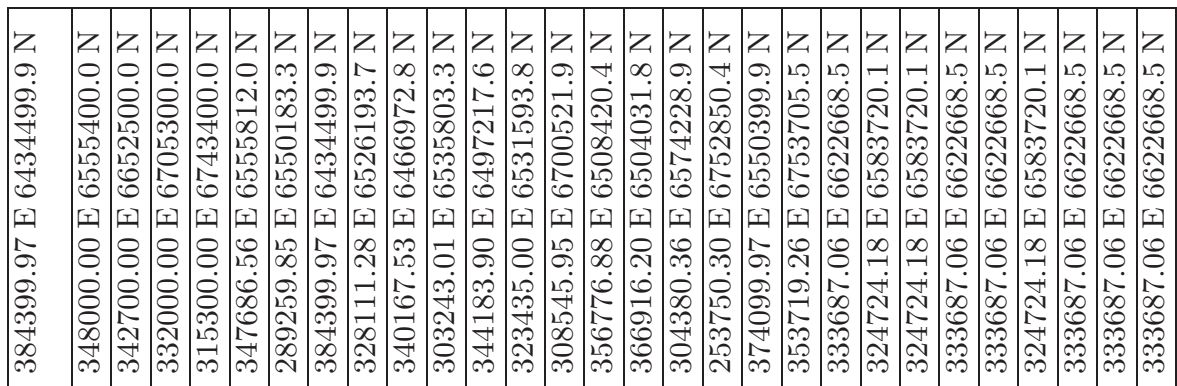

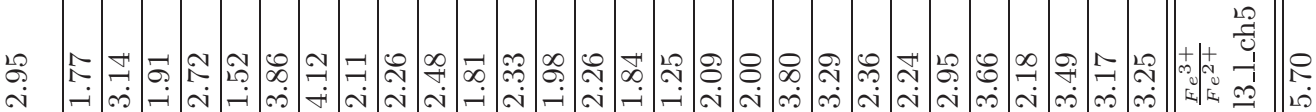

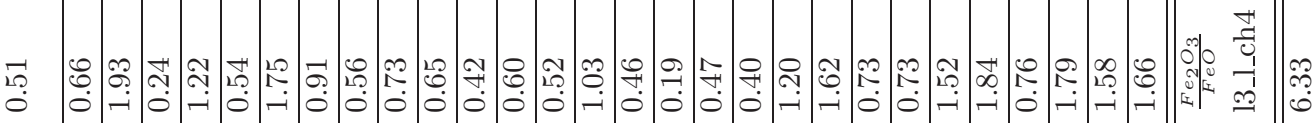

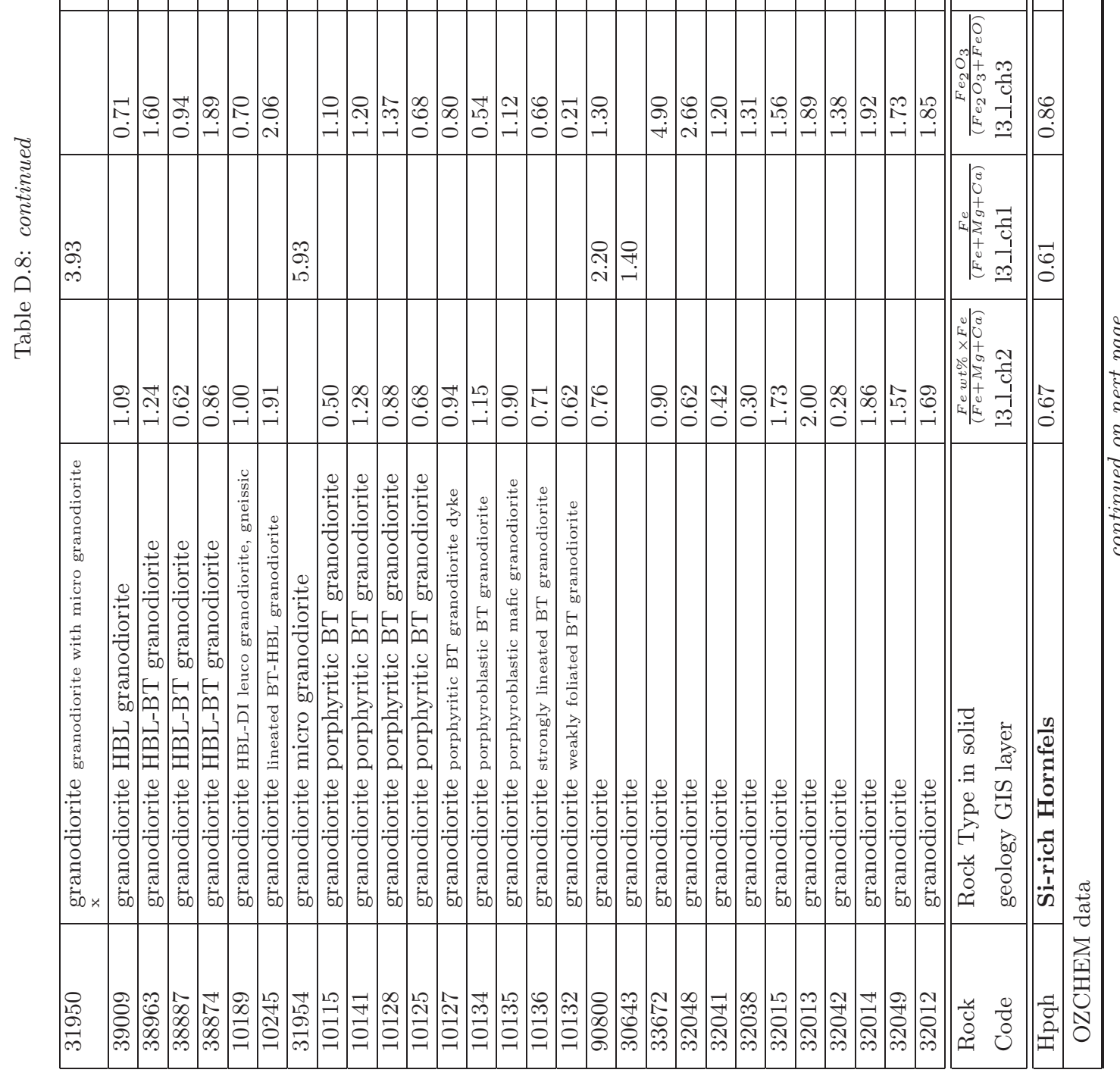




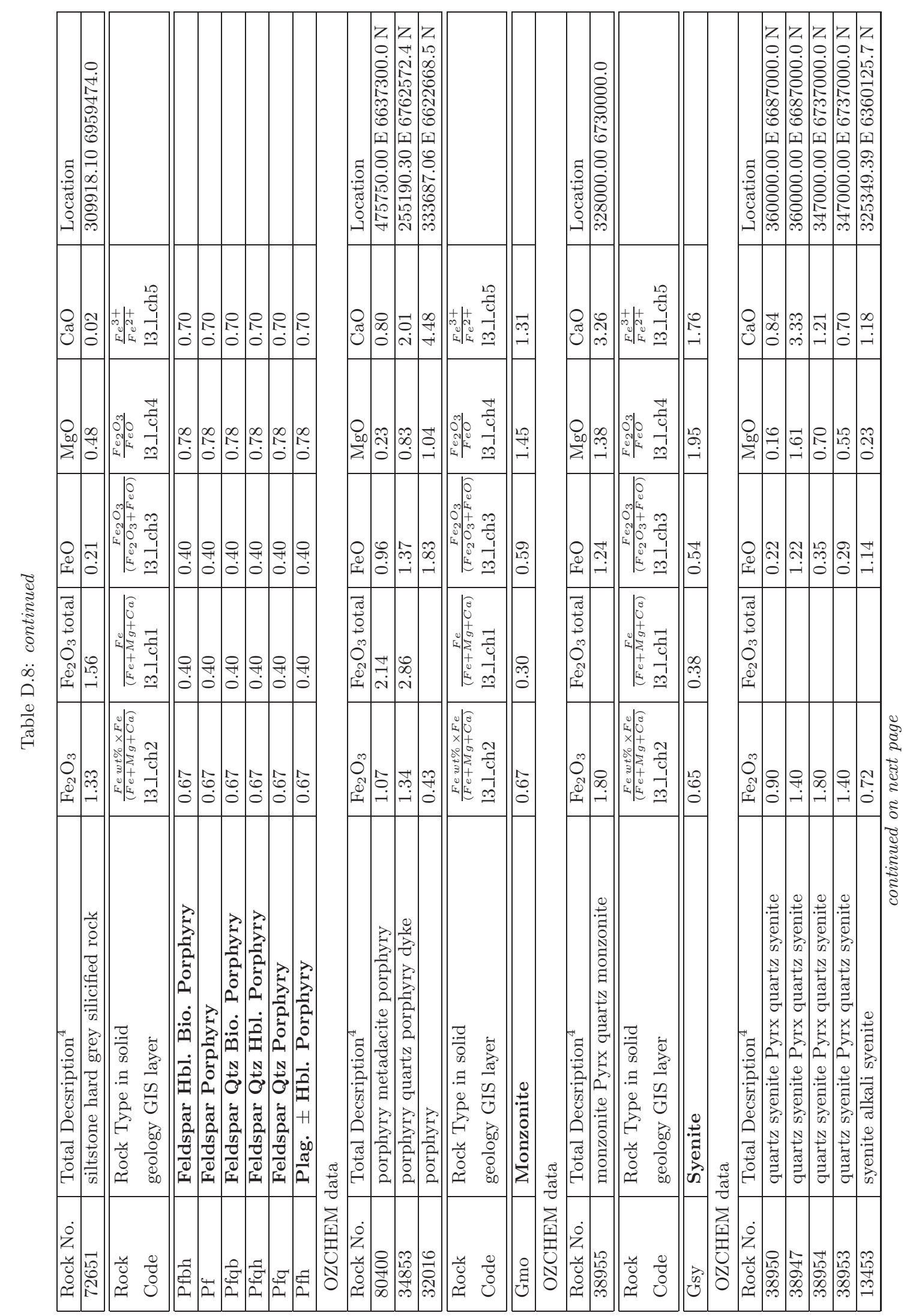




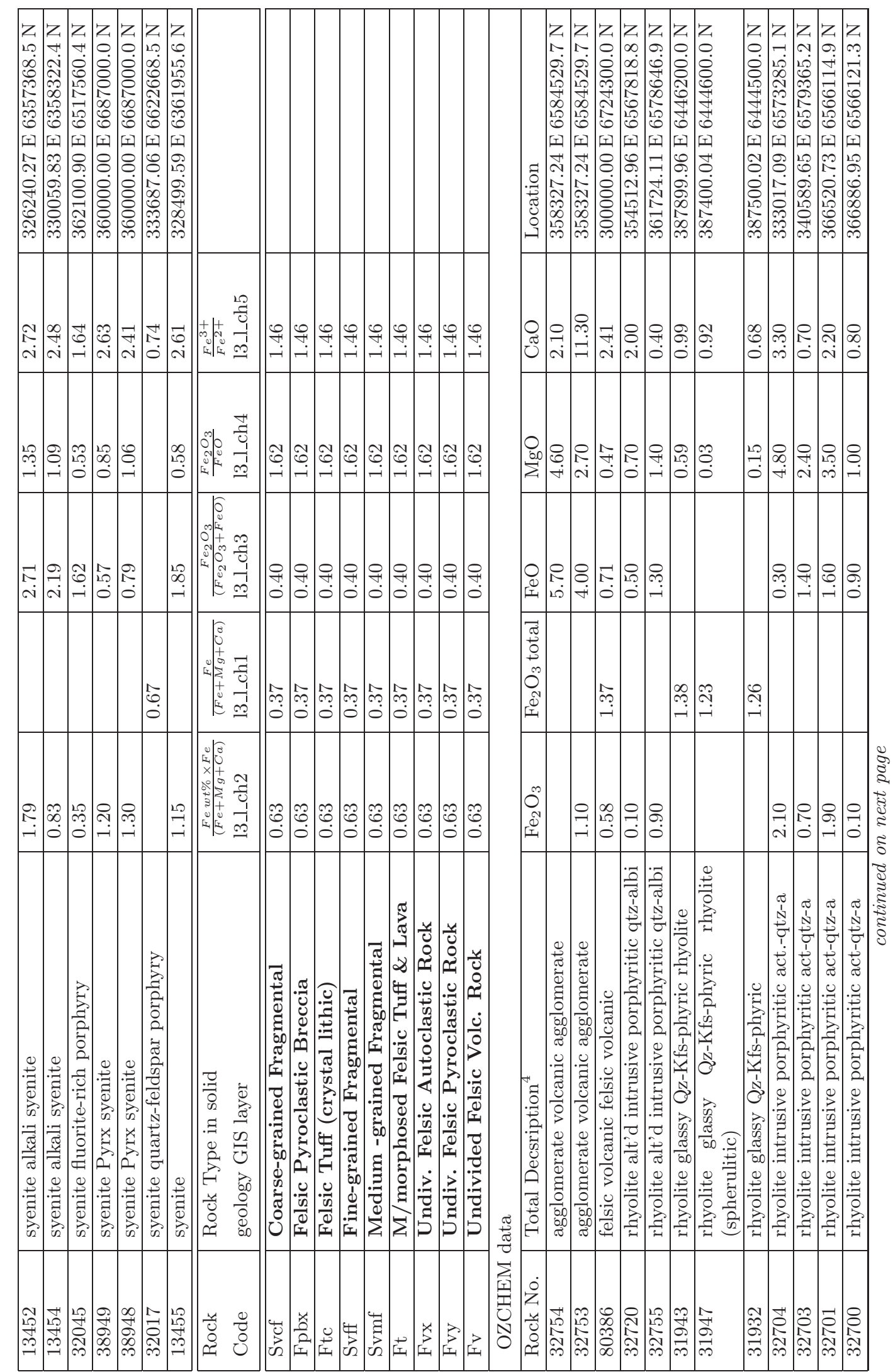



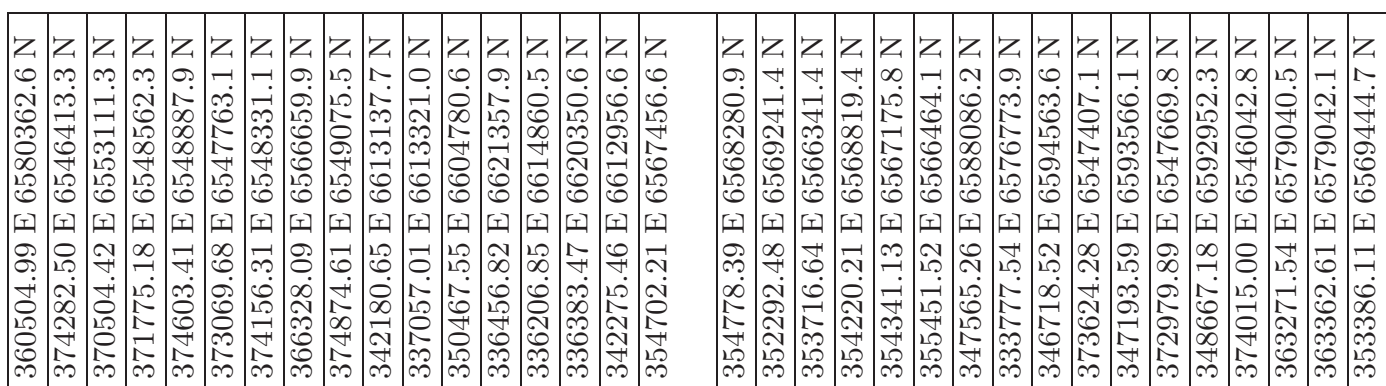

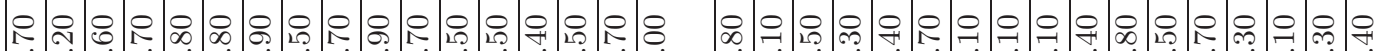

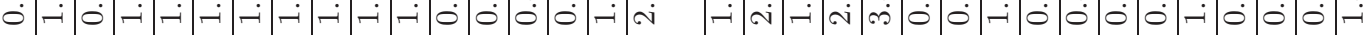

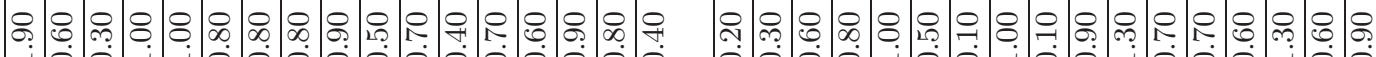

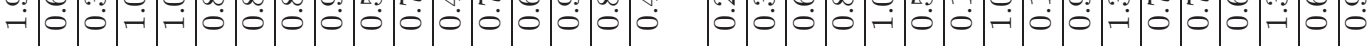

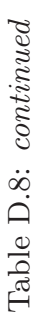

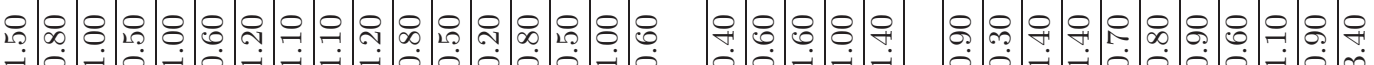

$$
\text { |. }
$$

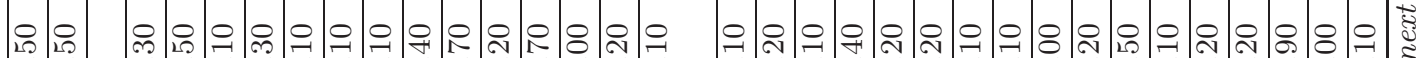

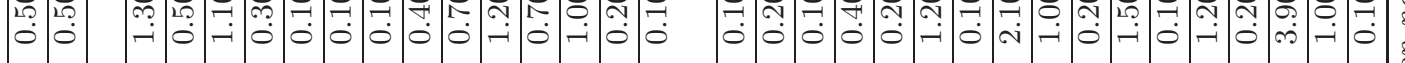

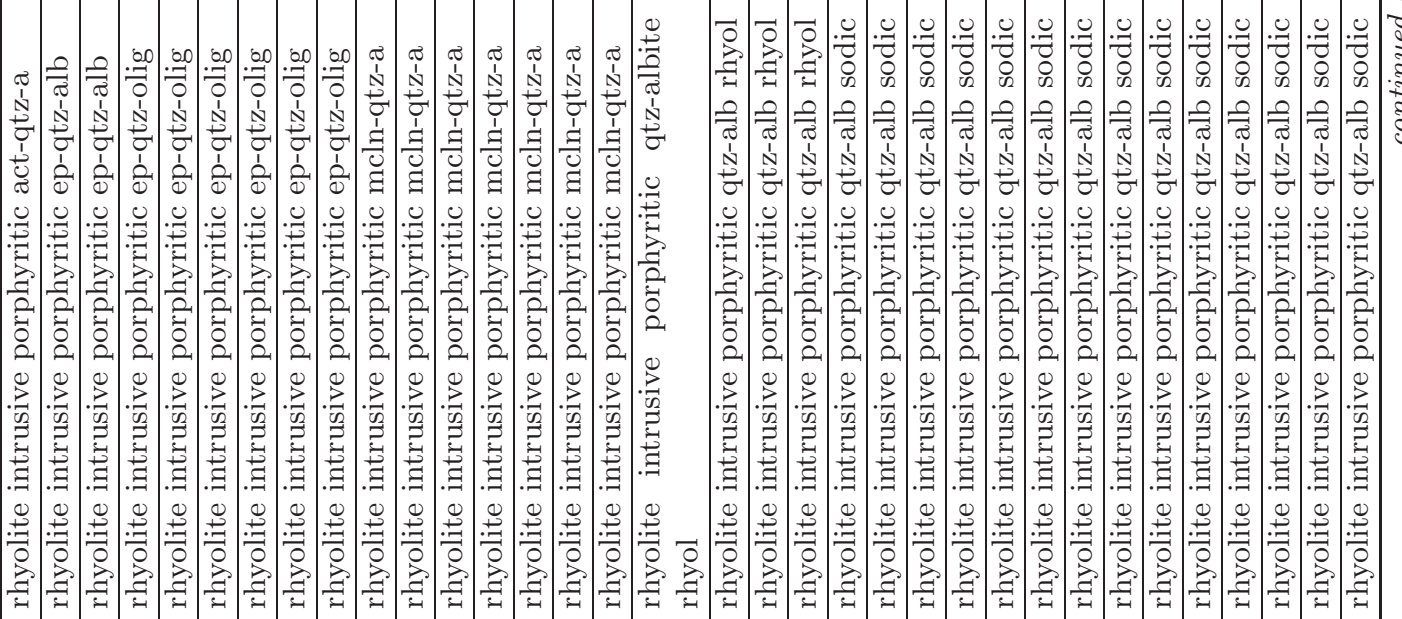



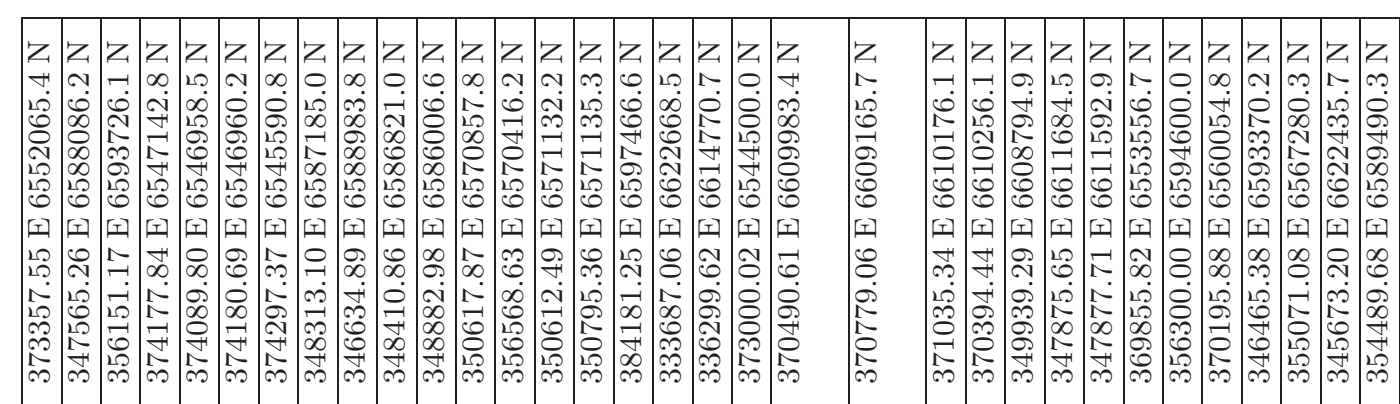

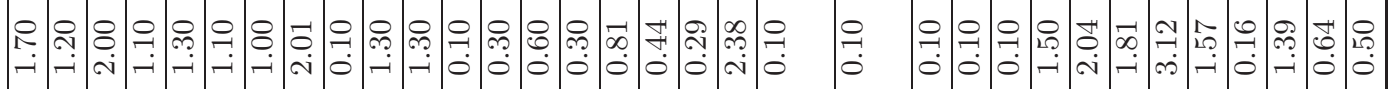

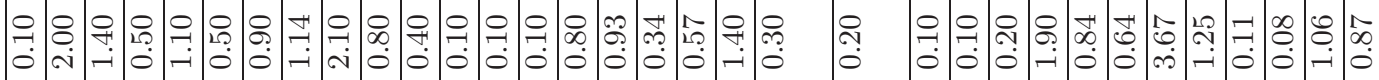

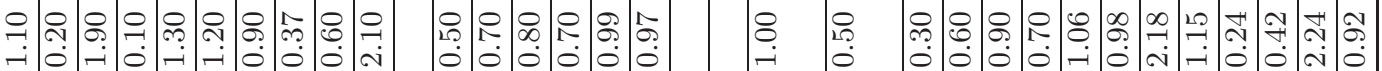

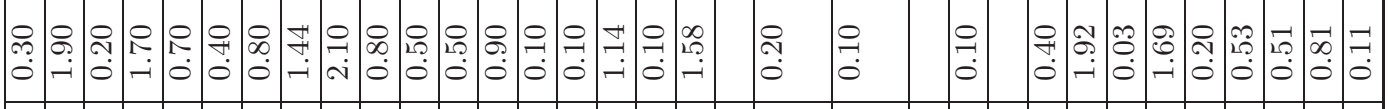

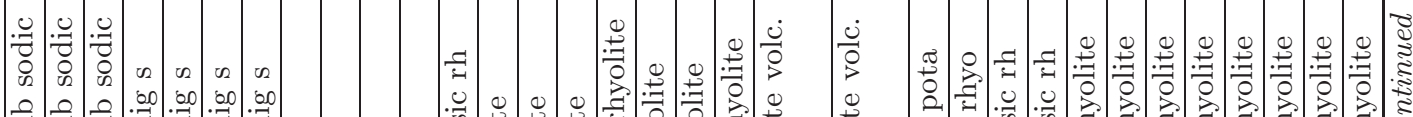
击

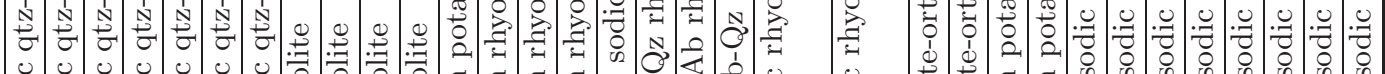

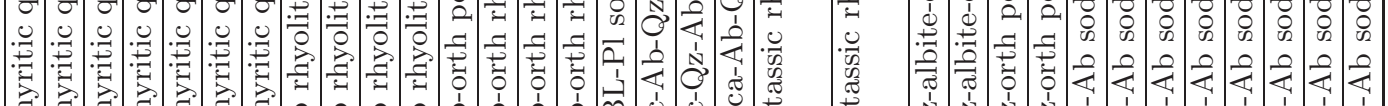

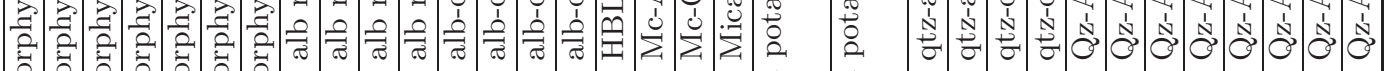

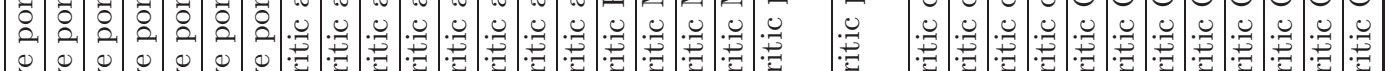

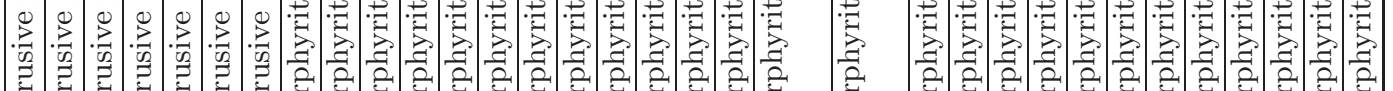

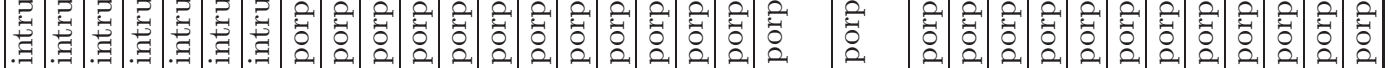

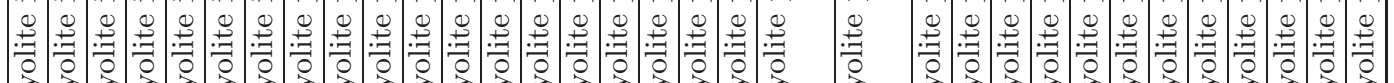

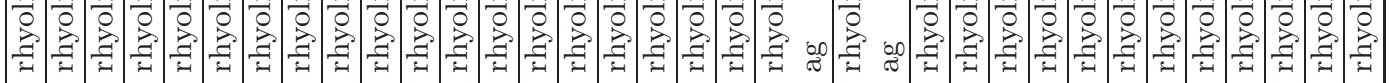

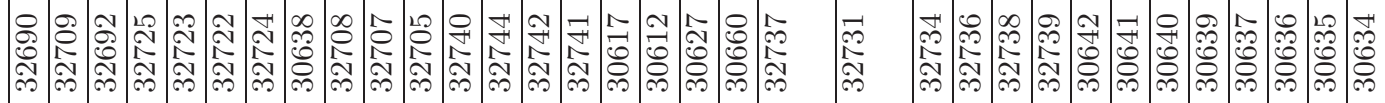



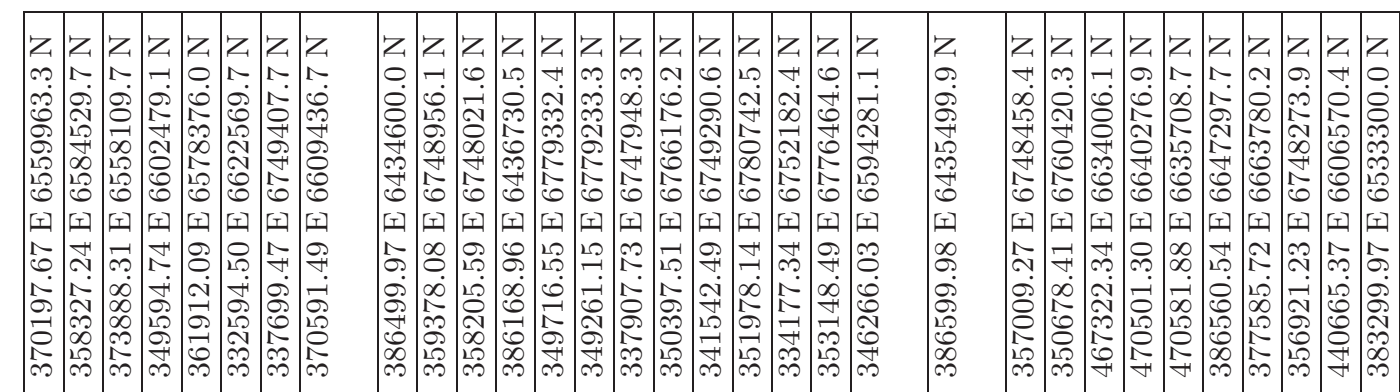

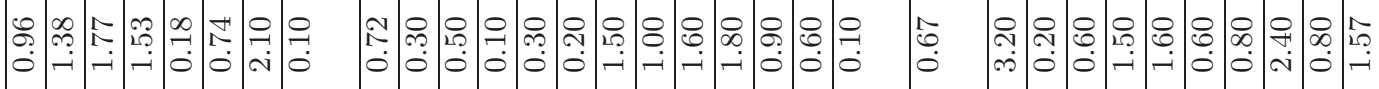

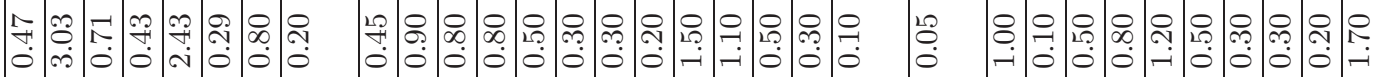

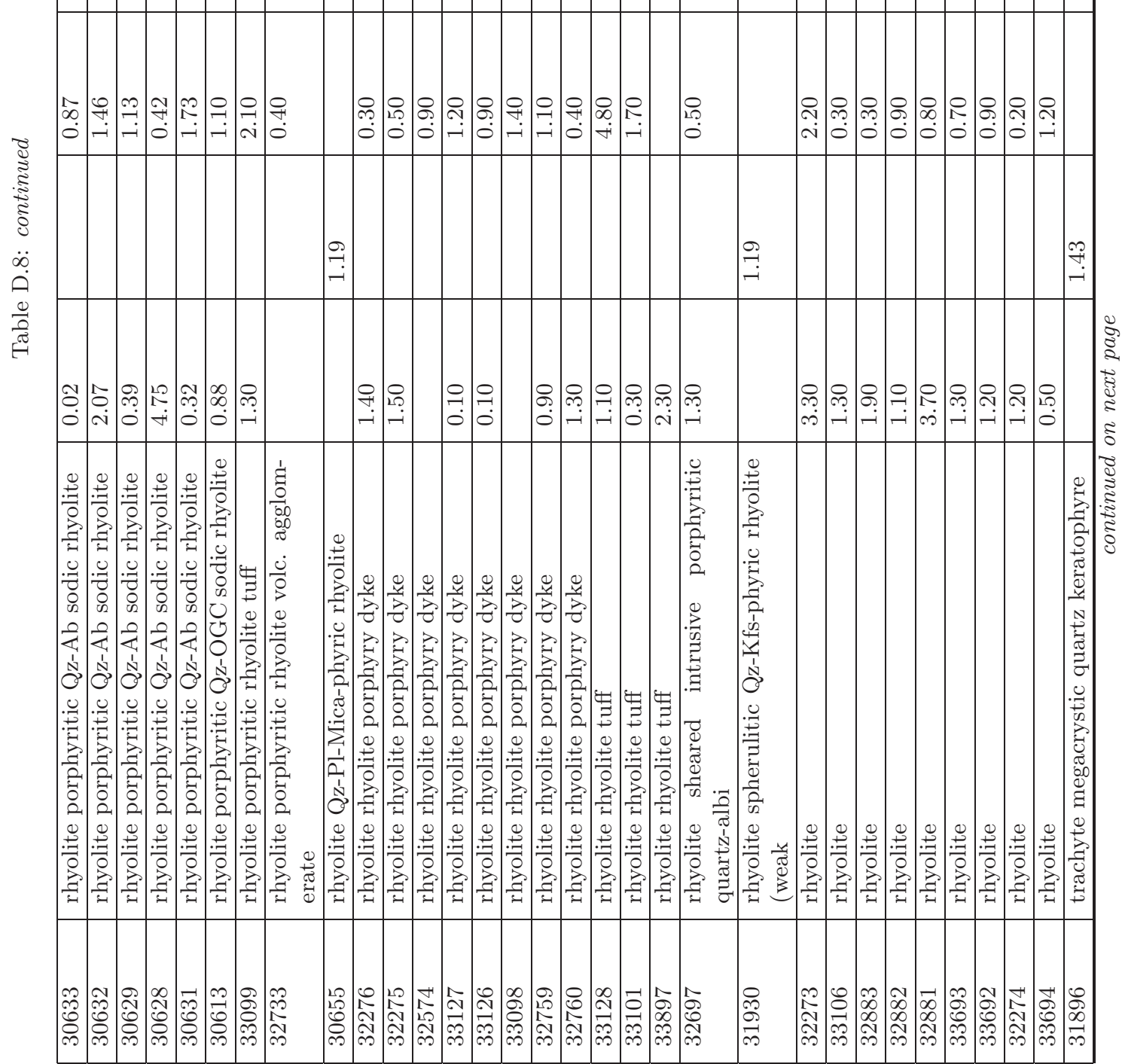




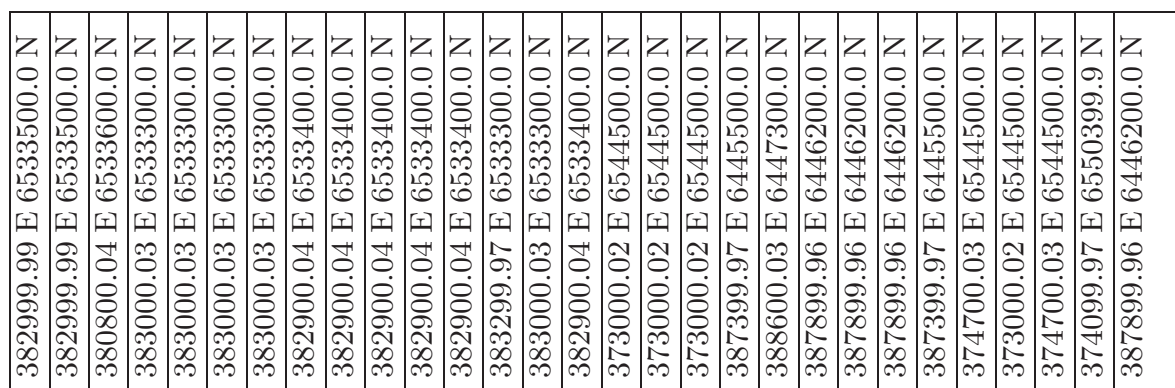

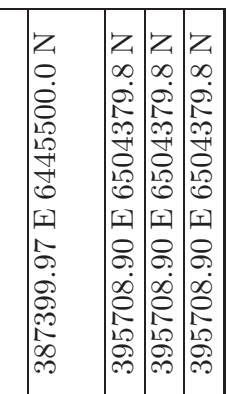

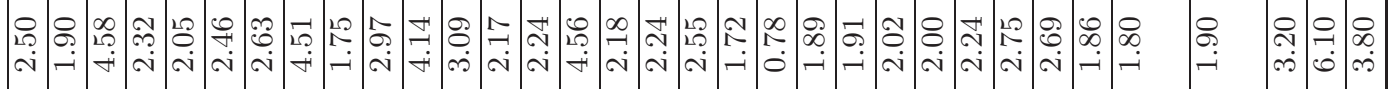

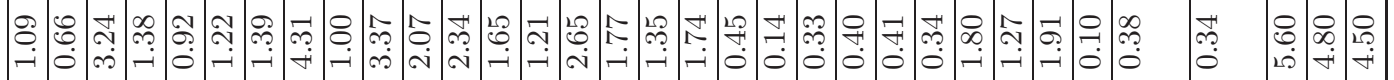

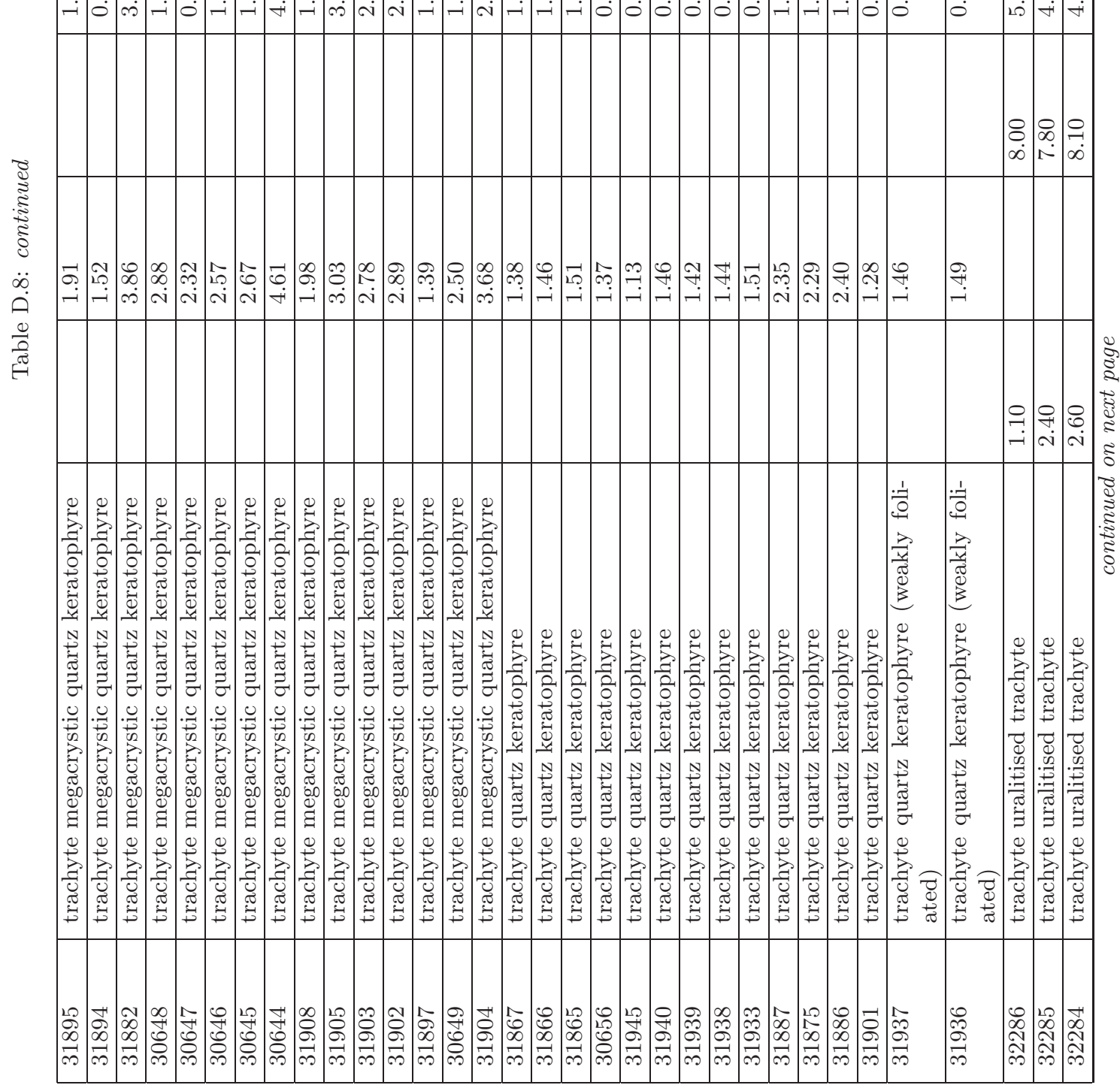




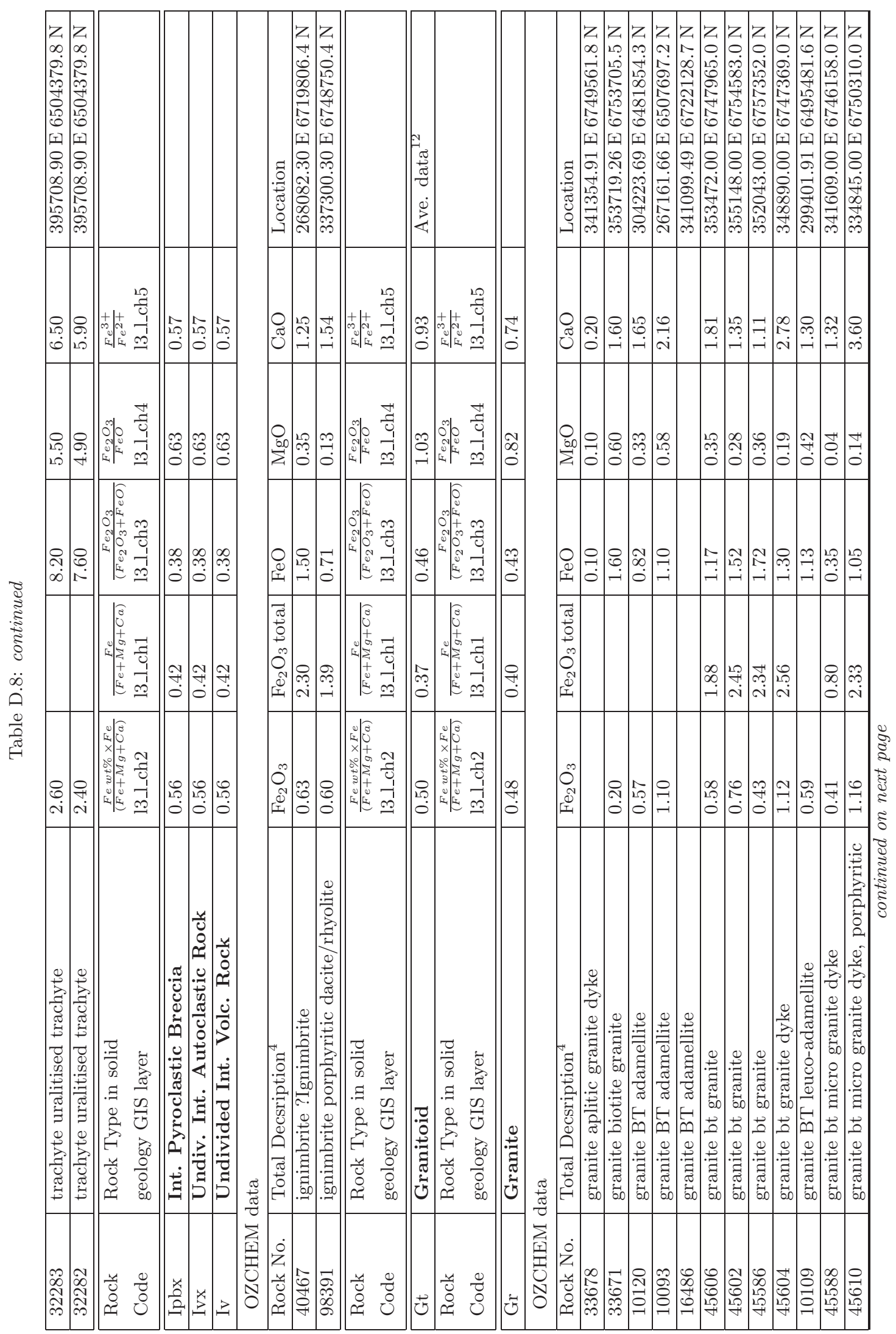




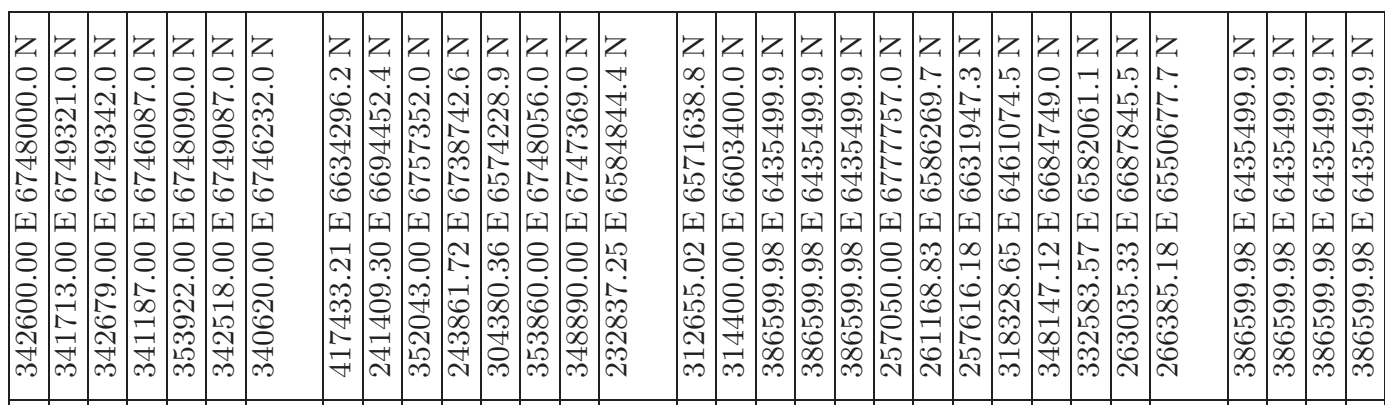

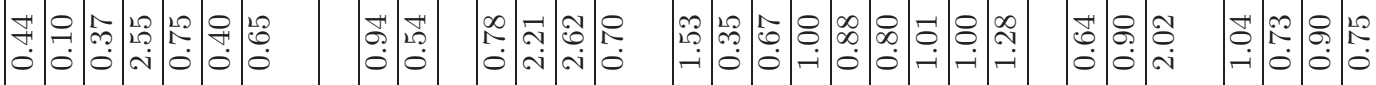

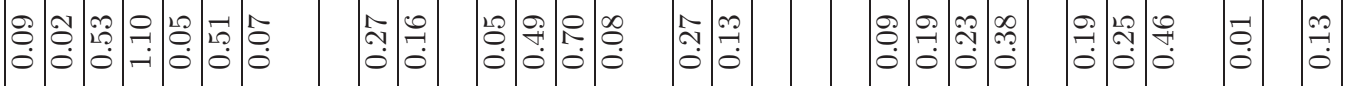

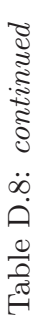

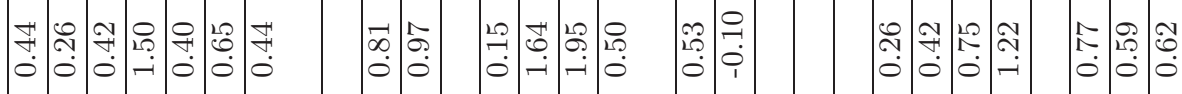

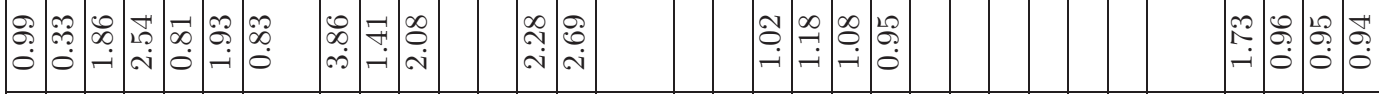

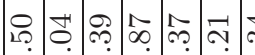

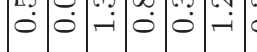

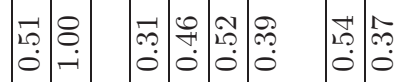

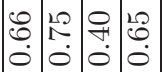

ลิ่า:

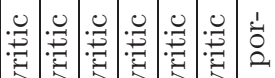

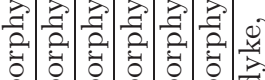

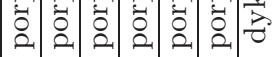

$\Leftrightarrow$ \&

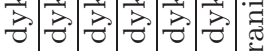

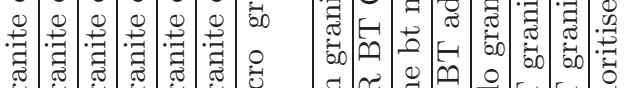

50000000

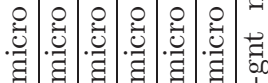

总究

党

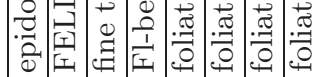

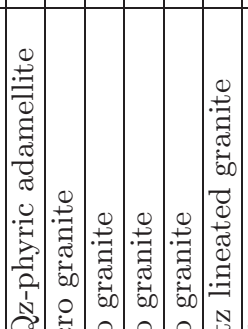

守.

先

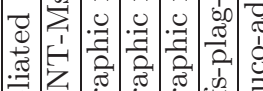

范

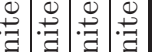

莺

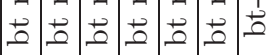

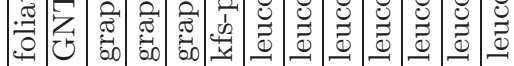

:

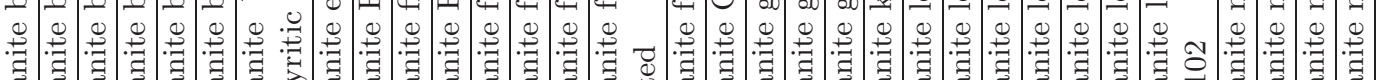

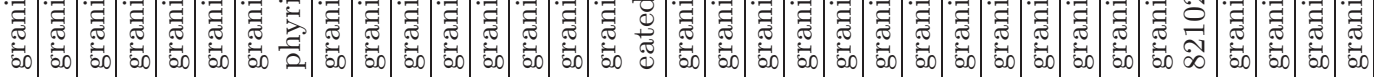

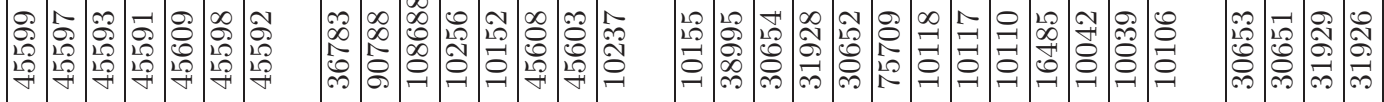




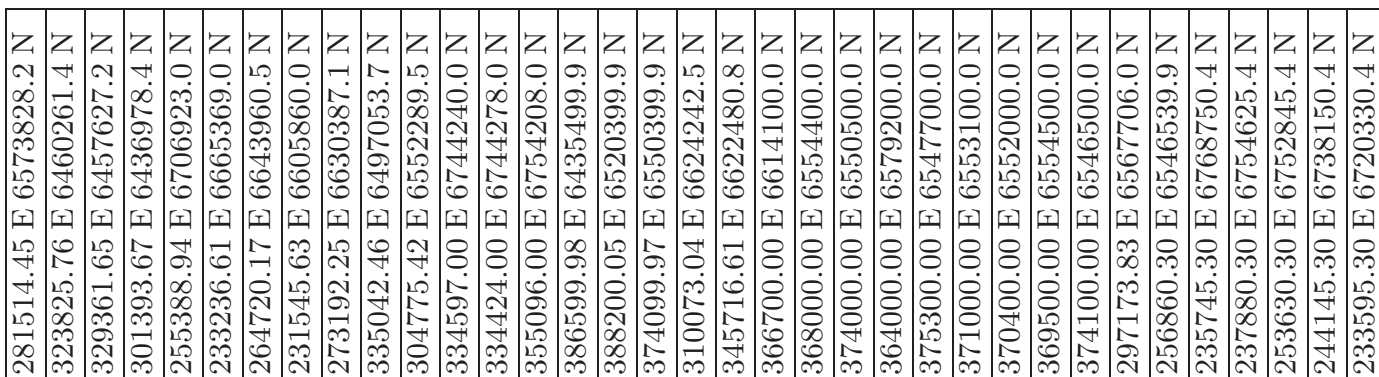

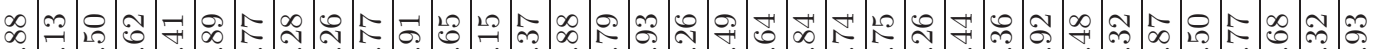
$\dot{0}$ ) ก

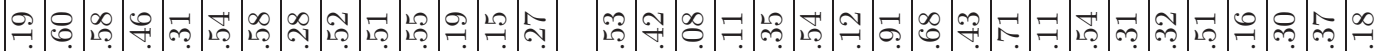

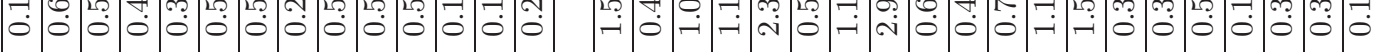

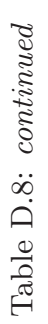

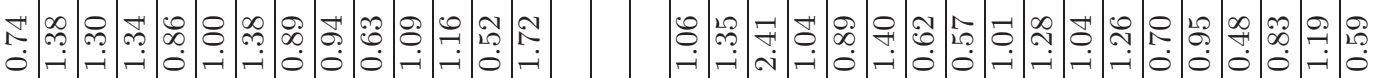

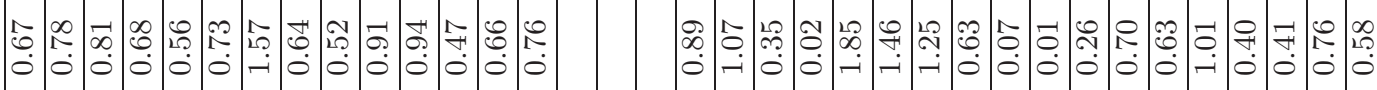

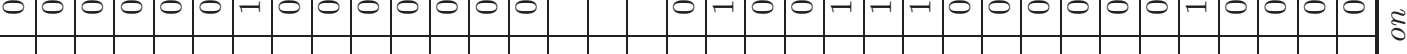

$\stackrel{\oplus}{*}$

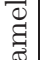

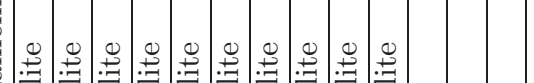

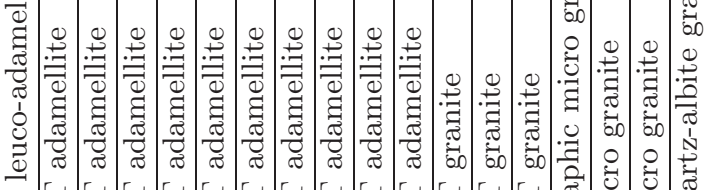

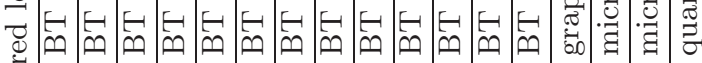

F⿻二丨凵口.

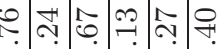

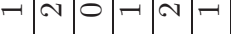

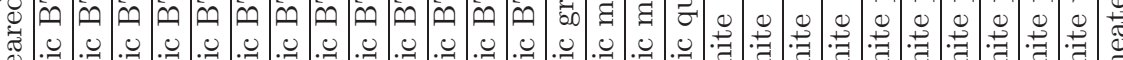

क

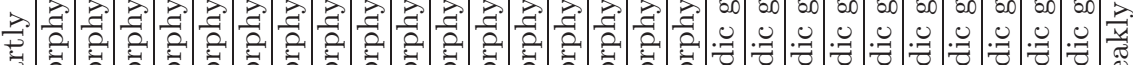

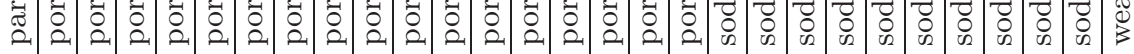

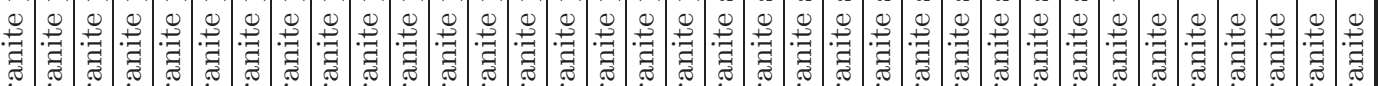

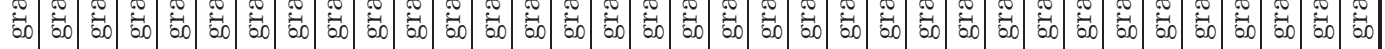

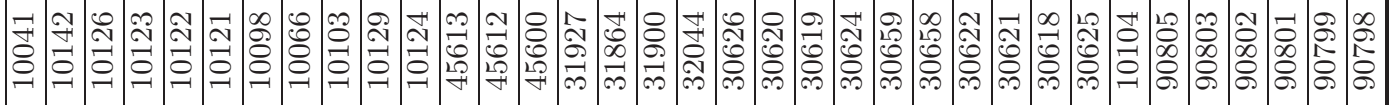




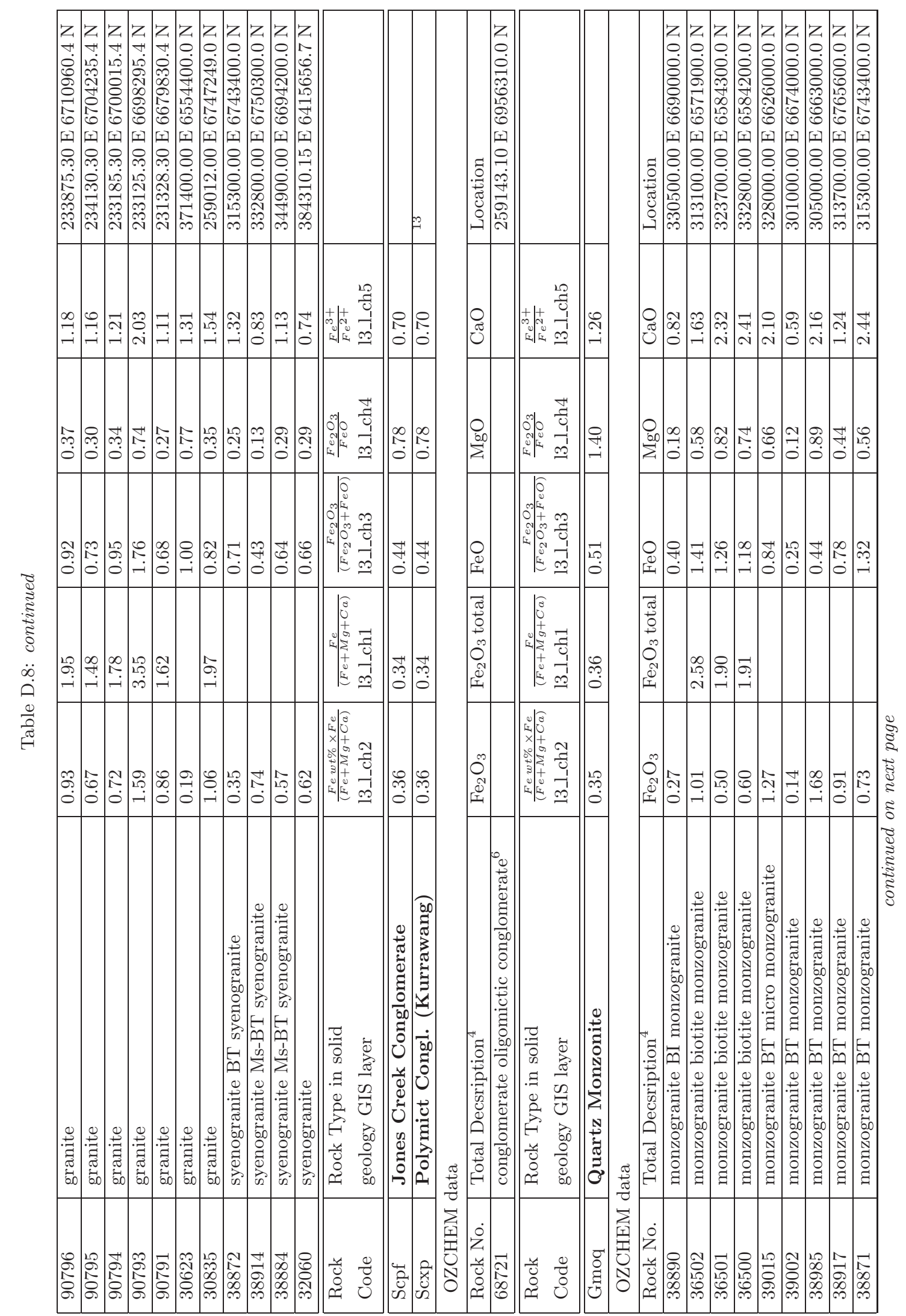




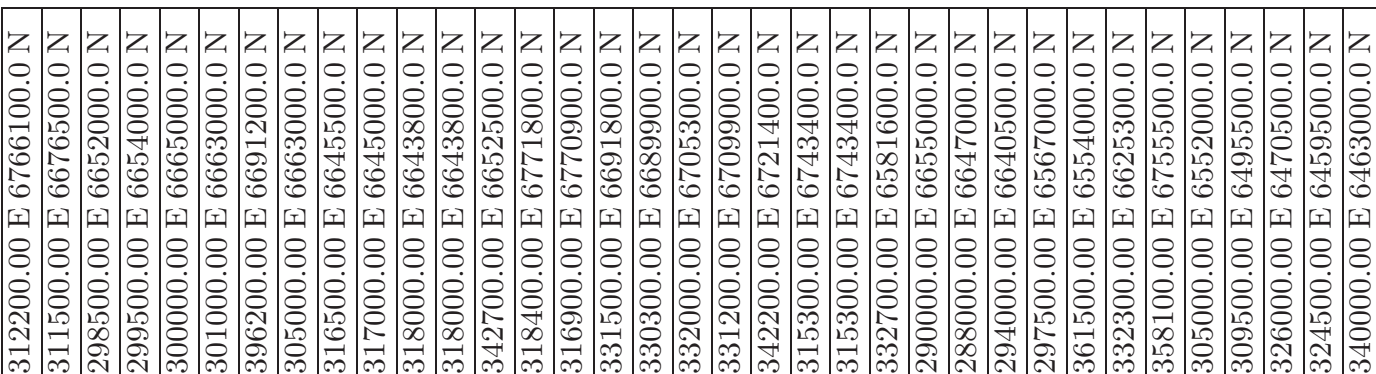

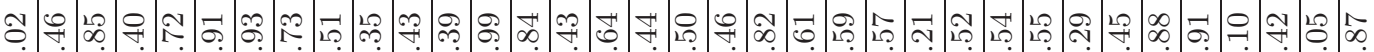

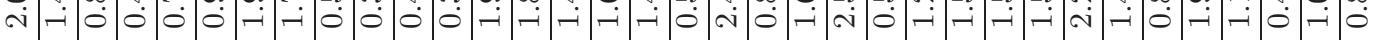

Ғ

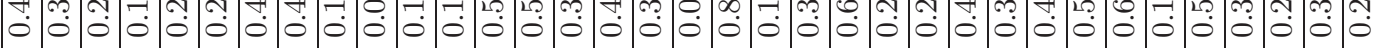

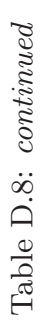

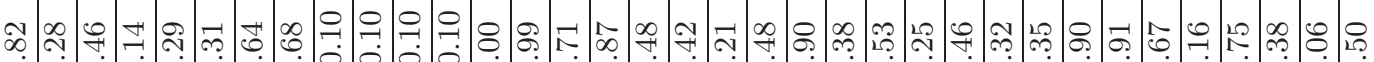

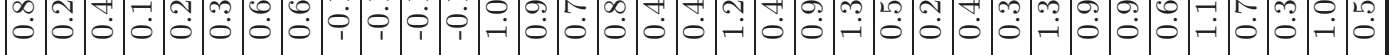

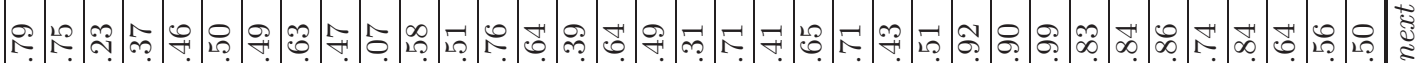

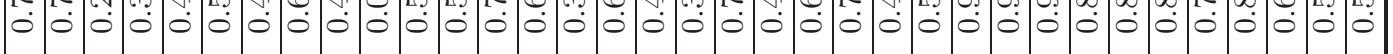

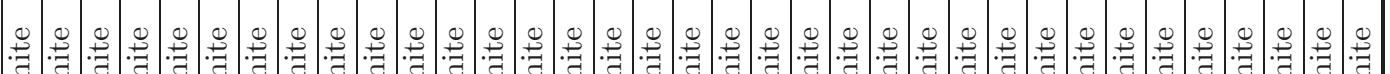

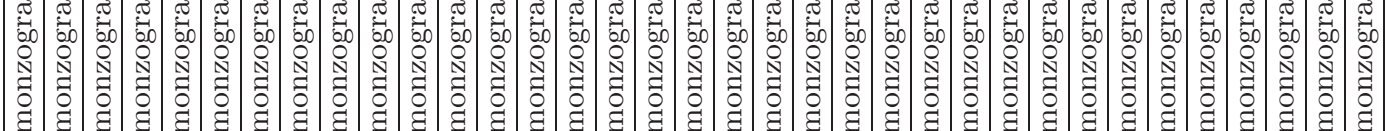

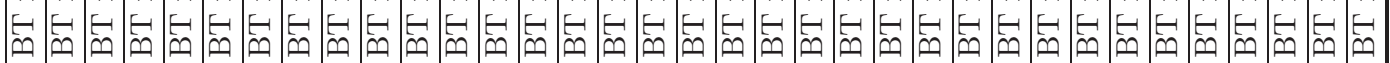

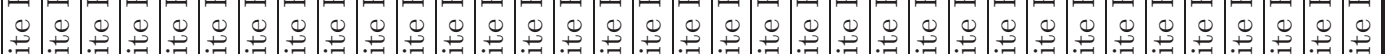

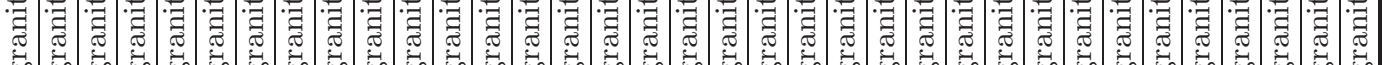

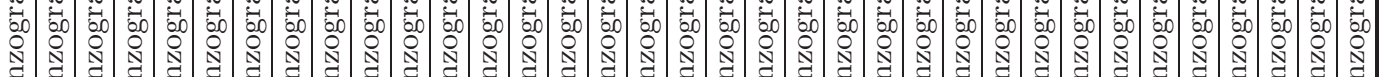

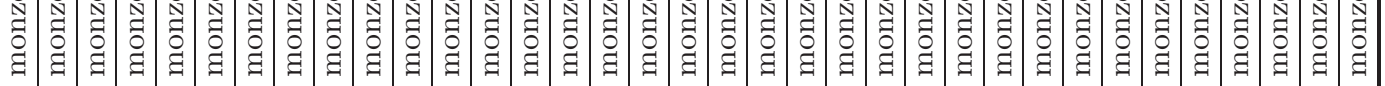

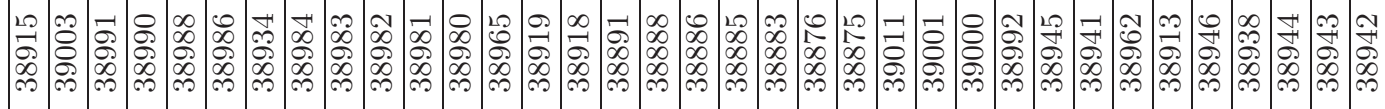




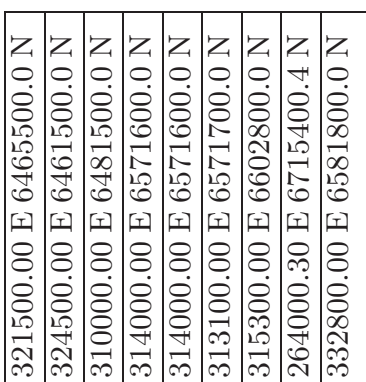

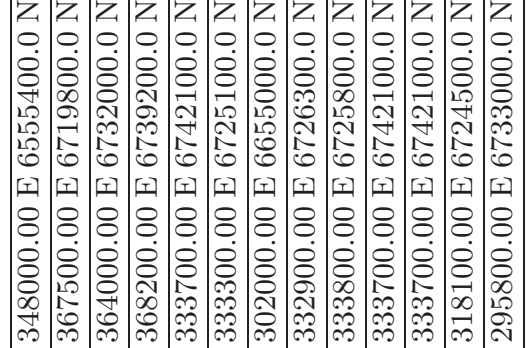

車

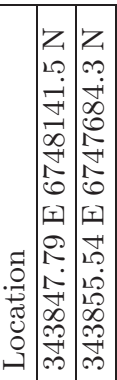

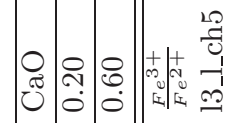

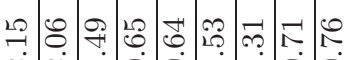

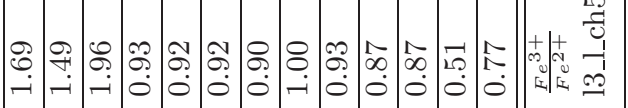

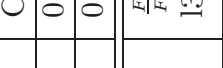

5.

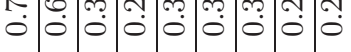

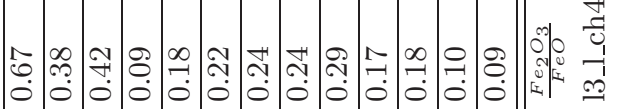

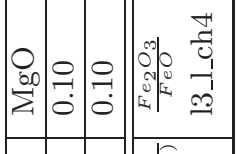

$0_{\substack{n+\infty \\+\infty}}^{2}$

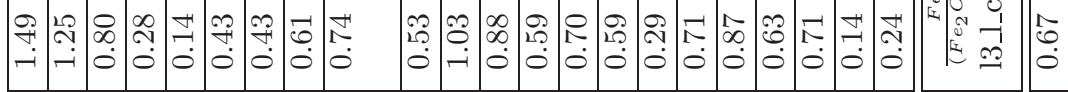

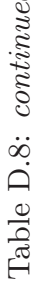

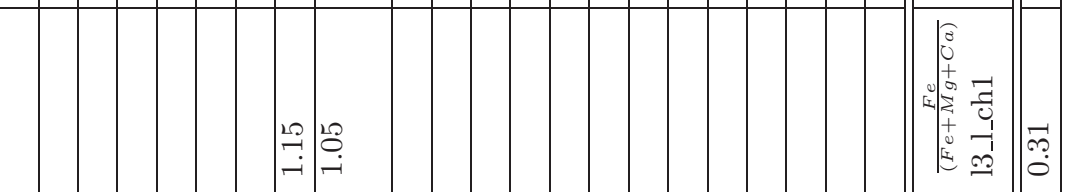

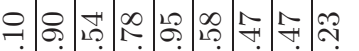

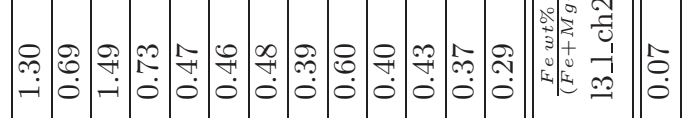

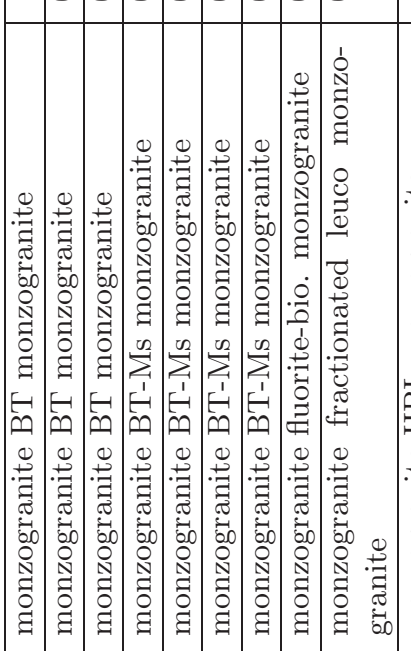

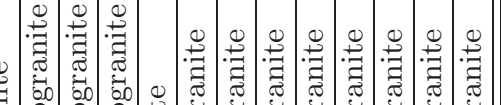

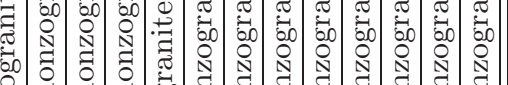

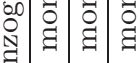

落

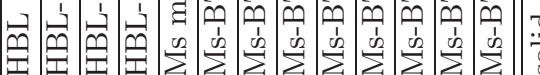

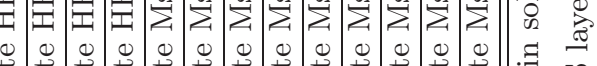

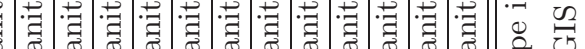

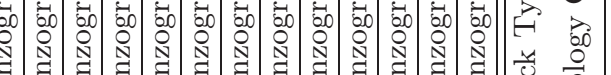

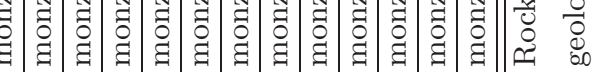

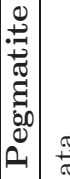

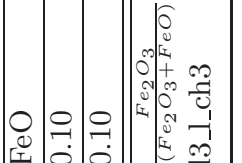

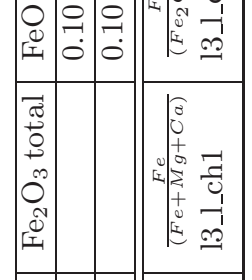

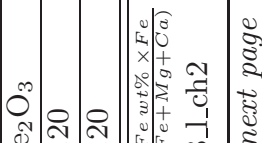

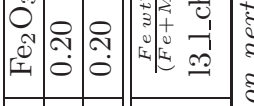

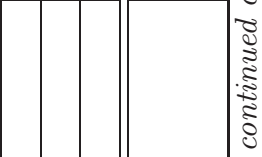

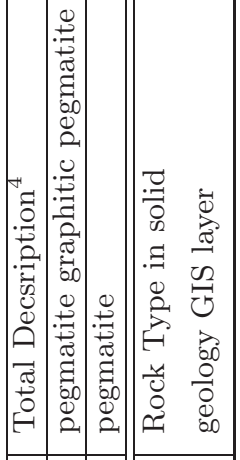

空

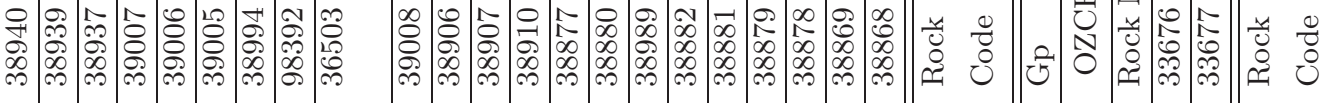



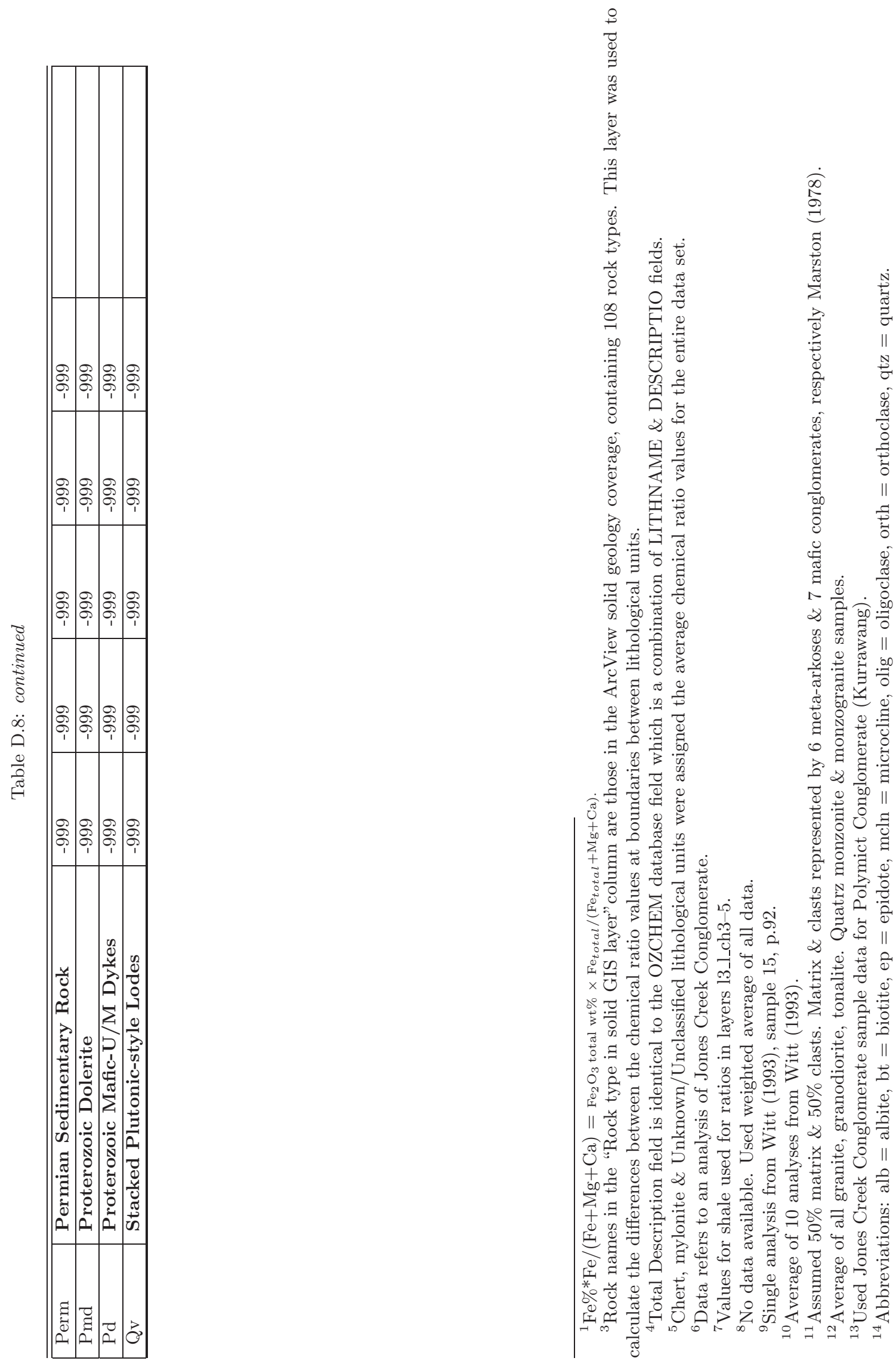


\section{D.7 Geophysical Data}

\section{D.7.1 Data dictionary for Gravity Layer}

The layer shows the station locations for gravity measurements in the Kalgoorlie area. The data set was used to produce the gridded Bouguer gravity anomaly layer described below. Table D.9 shows the data dictionary for the grav_pts layer.

\section{Lineage}

The layer was produced from point-located survey data in ASCII format which was supplied by AGSO. Different sets of data, which were acquired at different times have been combined by Goldfields Exploration Pty Ltd. The earliest data were acquired in 1971 and is based on an $11 \mathrm{~km}$ station spacing (AGSOs 1:1,000,000 scale gravity data set entitled, Kalgoorlie gravity digital data 1:1M, Catalog \#21302. A later data set (probably (AGSO's 1:250000 scale gravity data set entitled, Kalgoorlie gravity database digital, Catalog \#21535 has been added to the original data.

Table D.9: Data dictionary for gravity station Layer

\begin{tabular}{|l|c|l|}
\hline \multicolumn{2}{|l|}{ Coverage name: $g r a v \_p t s$} \\
\hline \multicolumn{2}{|l|}{ Coverage type: Point } \\
\hline Item names & Type & Item description \\
\hline amg_north & F & Australian Map Grid (AMG84) grid coordinate; northing \\
\hline amg_east & F & Australian Map Grid (AMG84) grid coordinate; easting \\
\hline rl & F & Relative level or elevation above datum (m) \\
\hline mgal_bouge & F & Bouguer gravity anomaly value in gravity units (g.u. $=0.1$ mgal) \\
\hline
\end{tabular}

\section{D.7.2 Metadata for Bouguer Gravity Anomaly Layer}

The layer shows the gridded Bouguer gravity anomaly data over the Kalgoorlie area. The data set was derived from the point-located data in the grav_pts layer described in Section D.7.1 above. Table D.10 shows the metadata for the gravity layer below. 


\section{Lineage}

Point-located data were re-gridded with a 100 metre cell size by Dr Mike House using the ERMAPPER data analysis package (refer to the lineage information for Section D.7.1 above).

Table D.10: Metadata for Bouguer gravity anomaly layer

\begin{tabular}{|l|l|}
\hline \multicolumn{2}{|l|}{ Grid name: gravity } \\
\hline Grid parameters: cell size $=100 \mathrm{~m}$, rows $=441$, columns $=441$, grid type $=$ float \\
\hline Cell value & Description \\
\hline 1 & Bouguer gravity anomaly data \\
\hline-999 & no data \\
\hline
\end{tabular}

\section{D.7.3 Metadata for Aeromagnetic Survey Layer}

The layer is an ARCVIEw grid showing the first vertical derivative of total magnetic intensity (RTP) from an airborne survey. Table D.11 shows the data dictionary for the magnetic layer.

\section{Lineage}

Goldfields Exploration obtained the aeromagnetic data as point-located and gridded data sets from World Geosciences (now Fugro Airborne Surveys Ltd). World Geoscience refers to the data set as Group B: Yilgarn 1989. It is a multiclient data set and was flown in multiple stages from 1986 to 1989. Reduction-to-pole corrections and the calculation of the first vertical derivative were performed by World Geoscience. The ASCII data were gridded with a $100 \mathrm{~m}$ cell size and clipped to the boundaries shown in Table D.1 by Dr Mike House (geophysicist, Goldfields Exploration Pty Ltd) using the ERMAPPER data processing and analysis software package.

The details for the aeromagnetic survey are as follows;

Traverse line spacing

Tie line spacing

Traverse line direction

Tie line direction

Sensor height
$200 \mathrm{~m}$

$2000 \mathrm{~m}$

east/west

north/south

$60 \mathrm{~m}$ 
Navigation

Cesium vapour magnetometer

Magnetometer sample interval

Geometrics 256 channel spectrometer

Spectrometer sample interval

Crystal volume radio positioning

$17 \mathrm{~m}$

$68 \mathrm{~m}$

16 litres

Table D.11: Metadata for the Kalgoorlie airborne magnetic survey layer

\begin{tabular}{|l|l|}
\hline \multicolumn{2}{|l|}{ Grid name: magnetic } \\
\hline Grid parameters: cell size $=100 \mathrm{~m}$, grid type $=$ float \\
\hline Cell value & Description \\
\hline 1 & airborne total magnetic intensity \\
\hline-999 & no data \\
\hline
\end{tabular}

\section{D.8 Deposit Data}

\section{D.8.1 Data Dictionary for Deposit Layer}

The layer, deps-23-11-01, shows point locations for 1437 gold deposits in the the Kalgoorlie study area (boundaries are shown at the top of Table D.1. The data dictionary for the layer is given in Table D.12 below.

\section{Lineage}

The layer is based on a subset the 2000 edition of the Mines and Mineral Deposits Database (MINEDEX) which was available as an Microsoft ACCESS 97 database from the Geological Survey of Western Australia (Townsend et al., 2000). MINEDEX is a textual database containing information on production and remaining resources from over 11,000 historic mines and 1,000 recent mines and deposits in the Yilgarn craton. Additional information includes the tectonic unit, commodity, current ownership, and development status.

Estimates of pre-mining resources were calculated by Paul Hodkiewcz at the Centre for Global Metallogeny, Department of Geology and Geophysics, University of Western Australia in the following way; tonnes of gold, corresponding to the largest resource category 
from the Australian Institute of Mining and Metallurgy's (AusIMM) JORC code (inferred, indicated, and measured) for which data were available was added to any historic and recent production. The database was also updated by Paul Hodkiewicz to include new deposit locations (using data from Australian Gold Annual) and some errors in deposit names and locations were corrected by An ArcView point coverage including just the gold deposits for the Kalgoorlie study area was created by Stephen Gardoll, from the GIS Laboratory (in the same department), Only those attributes shown in Table D.12 were retained. The gold endowment expressed as kilograms of total-contained-gold for each deposit was determined by taking the value of the largest resource estimate available (where values for more than one of the parameters; inferred, indicated, measure, probable and proven were given).

Table D.12: Data dictionary for layer showing gold deposits in the Kalgoorlie area, deps23-11-01.

\begin{tabular}{|l|c|l|}
\hline \multicolumn{2}{|l|}{ Coverage name: $d e p s$-23-11-01 } \\
\hline \multicolumn{2}{|l|}{ Coverage type: polygon } \\
\hline Item names & Type & Item description \\
\hline database & C & Name of database used as the source of the data 8008; mine, 8000 \\
\hline name & C & name of deposit or mine \\
\hline grade & F & grade of deposit (grams per tonne) \\
\hline tcg & F & total gold endowment as Tonnes Contained Gold (TCG) \\
\hline oreton & F & ore tonnage (tonnes) for largest resource quoted \\
\hline x_coord & F & deposit location; Easting (AMG84, metres) \\
\hline y_coord & F & deposit location; Northing (AMG84, metres) \\
\hline
\end{tabular}

\section{D.9 Metamorphic Grade}

A metamorphic-grade map was prepared from a combination of maps by Binns et al. (1976) and Witt (1993). Scanned images of the maps, which covered different parts of the Kalgoorlie study area, were digitized in ArcView. Boundaries of the metamorphic domains were adjusted slightly so that they matched the crustal-scale faults and shear zones of the solid geology map of the study area. Areas that were categorized as shear zone lithologies were reclassified by assigning the areas equally to the metamorphic facies on either side of the shear zone. The attributes for the metamorphic layer are shown in Table D.13 below. 
Table D.13: Data dictionary for metamorphic grade layer, $145_{-} l_{-}$meta for the Kalgoorlie study area.

\begin{tabular}{|l|c|l|}
\hline \multicolumn{2}{|l|}{ Grid name: $\quad$ l45_l_meta } \\
\hline Grid parameters: cell size $=100 \mathrm{~m}$, rows $=1140$, columns $=1100$, grid type $=$ float \\
\hline Item names & Type & Item description \\
\hline 0 & I & granitoid (no metamorphic facies assigned \\
\hline 1 & I & amphibolite \\
\hline 2 & I & upper greenschist \\
\hline 3 & I & lower greenschist \\
\hline
\end{tabular}

\section{D.10 Favourable Contact-Type Data}

Data and calculations used to create the favourable contact-type layers

l44_favcont25_pr0110_200.mat, 144_favcont25_pxtcg0110_200.mat) are shown in Table D.14 below. Fuzzy membership values indicating the degree of favourability are based on a statistic which is generated by multiplying the probability that cells contain a known deposit (for which TCG $\geq 1000 \mathrm{~kg}$ ) by the total gold resource per cell; i.e. $\mathrm{P}(D) \times \mathrm{TCG} /$ cell. 


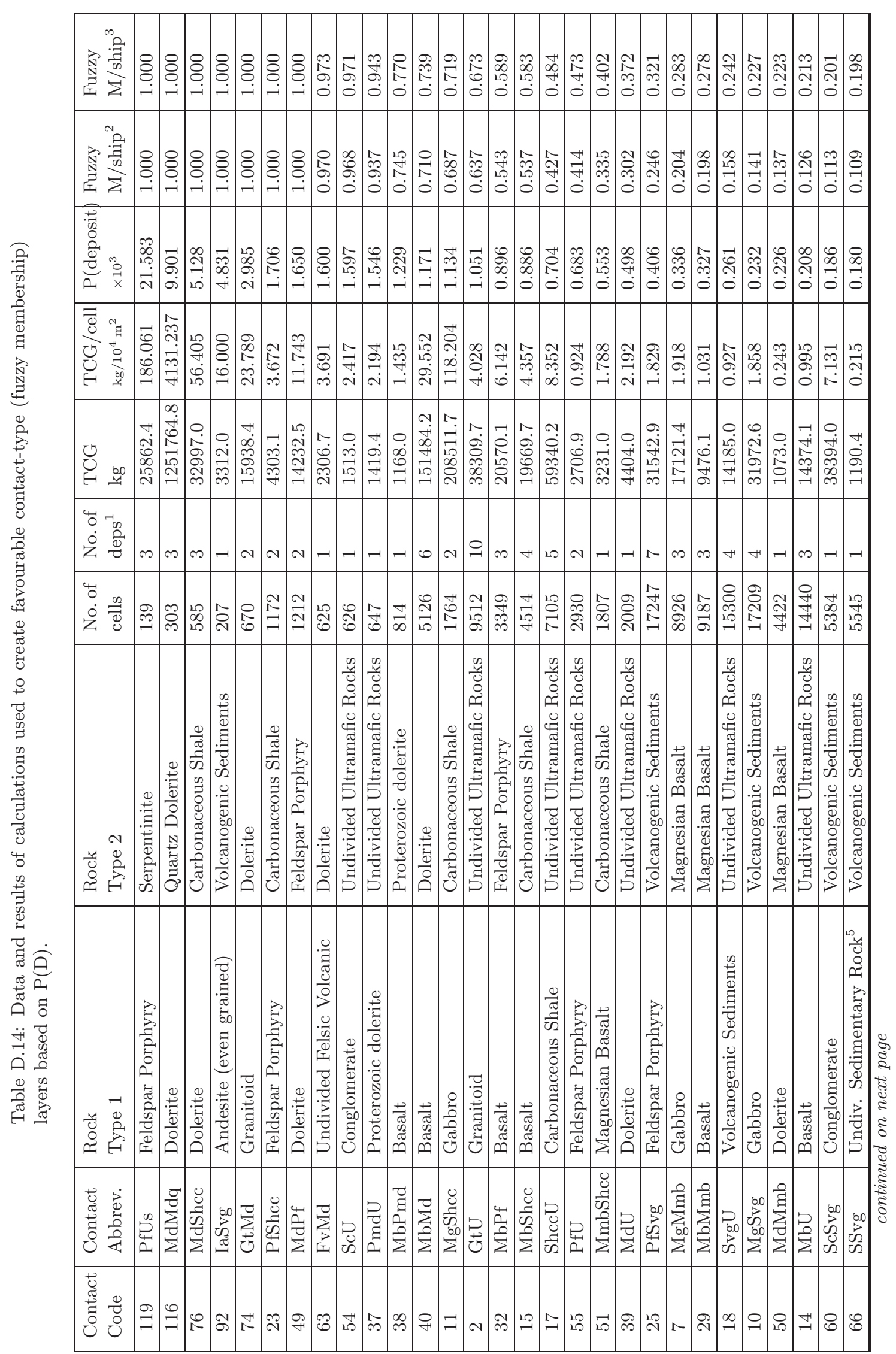




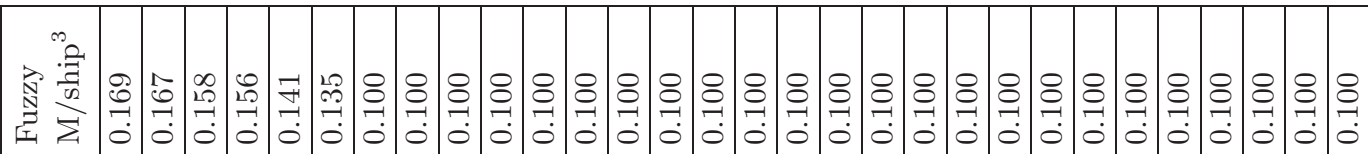

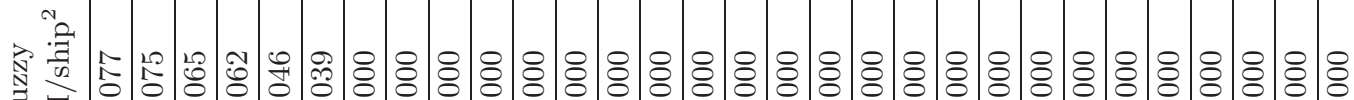

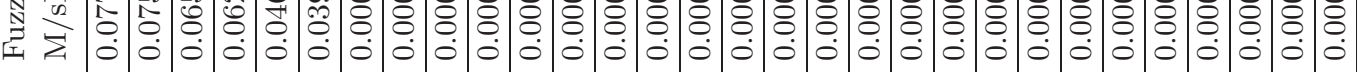
展

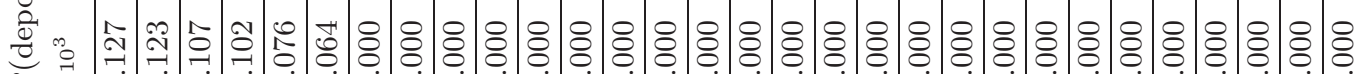

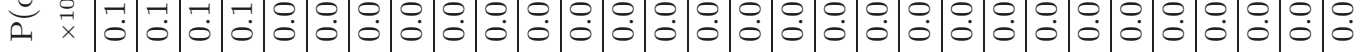
$\bar{\nabla}{ }^{2}$

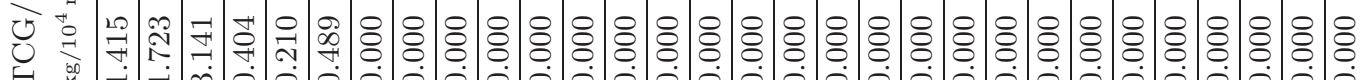

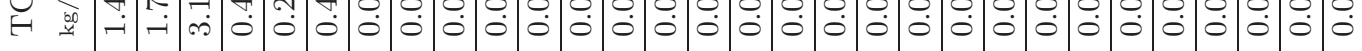

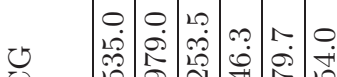

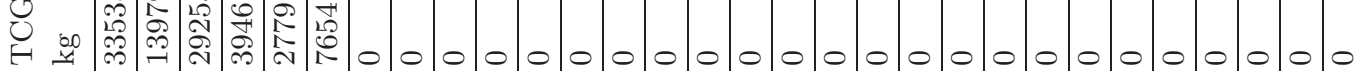
$\overrightarrow{0} \cdot r_{0}$

$\dot{8}$

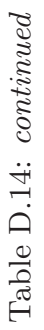

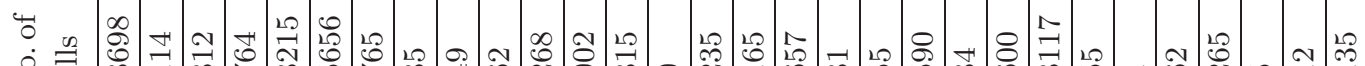

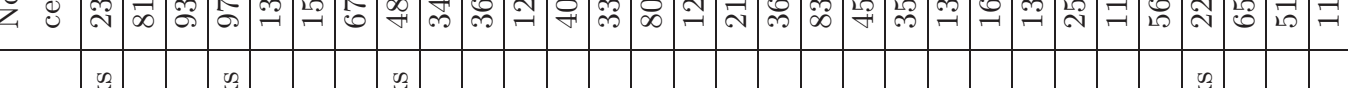

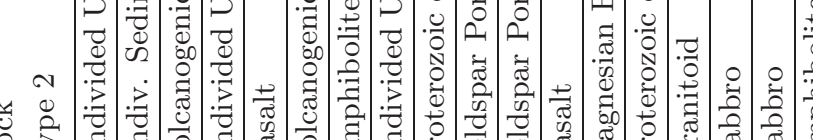

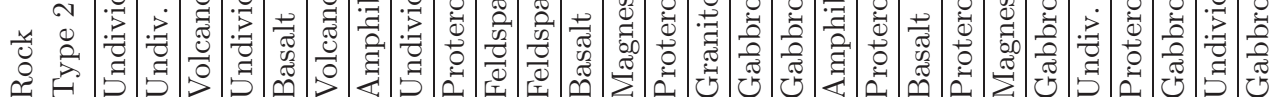

\begin{tabular}{|c|c|c|c|c|c|c|c|c|c|c|c|c|c|c|c|c|c|c|c|c|c|c|c|c|}
\hline 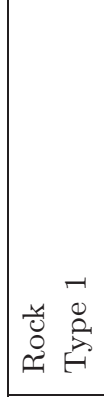 & 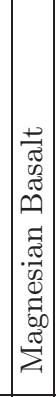 & & 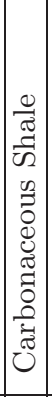 & & 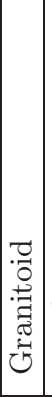 & & : & 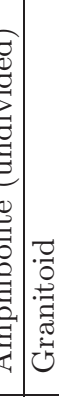 & 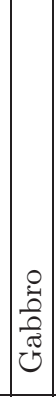 & 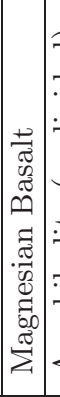 & 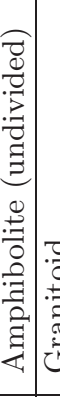 & 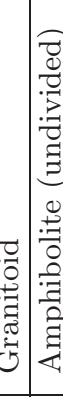 & 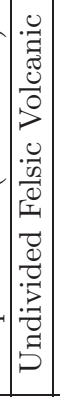 & & 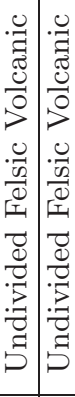 & $\mid$ & 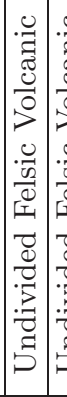 & 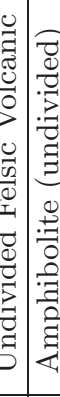 & 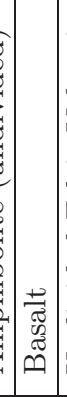 & 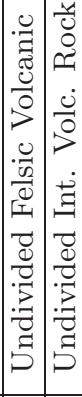 & 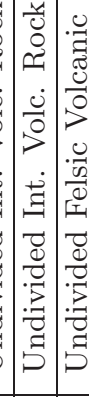 & 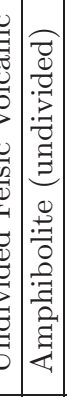 & 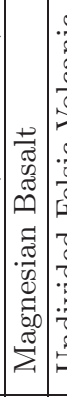 & \\
\hline 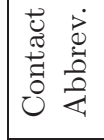 & $\begin{array}{l}\vec{D} \\
\overrightarrow{0} \\
\vec{z} \\
\vec{z}\end{array}$ & & $\mid$ & $\mid$ & $\sum_{\vec{U}}^{B}$ & $\begin{array}{l}\infty \\
\vdots \\
0 \\
0 \\
\vdots \\
\vdots\end{array}$ & & 思 & $\sum_{\vec{c}}^{\overrightarrow{b 0}}$ & & 党 & 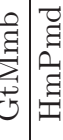 & \begin{tabular}{|l|}
$\overrightarrow{0}$ \\
$\vec{J}$ \\
$\vec{D}$ \\
$\vec{I}$ \\
\end{tabular} & $\sum_{0,0}^{\infty}$ & & $\mid \begin{array}{c}\vec{z} \\
\vec{g} \\
\vec{c} \\
\sum_{00} \\
\bar{z}\end{array}$ & & 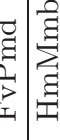 & 资 & 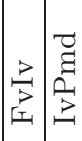 & $\sum_{i}^{\infty}$ & $\sum_{i}^{\infty}$ & 运 & $\sum_{5}^{5}$ \\
\hline 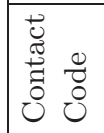 & & & & & 0 & $\stackrel{\infty}{f}$ & - & +120 & & & & & & & & & $\hat{N}$ & & & & $\begin{array}{ccc}0 \\
\infty\end{array}$ & $: F$ & & \\
\hline
\end{tabular}




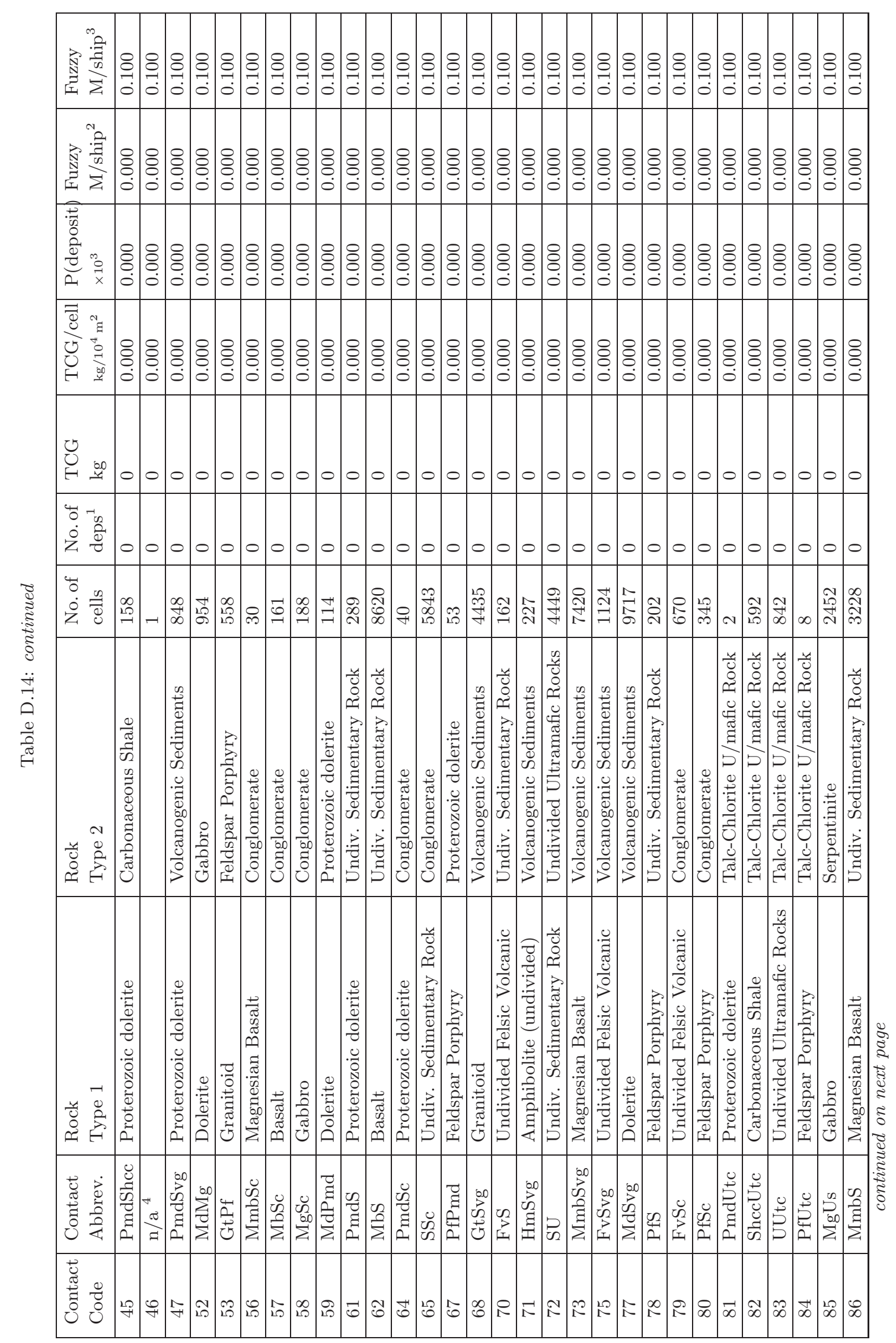




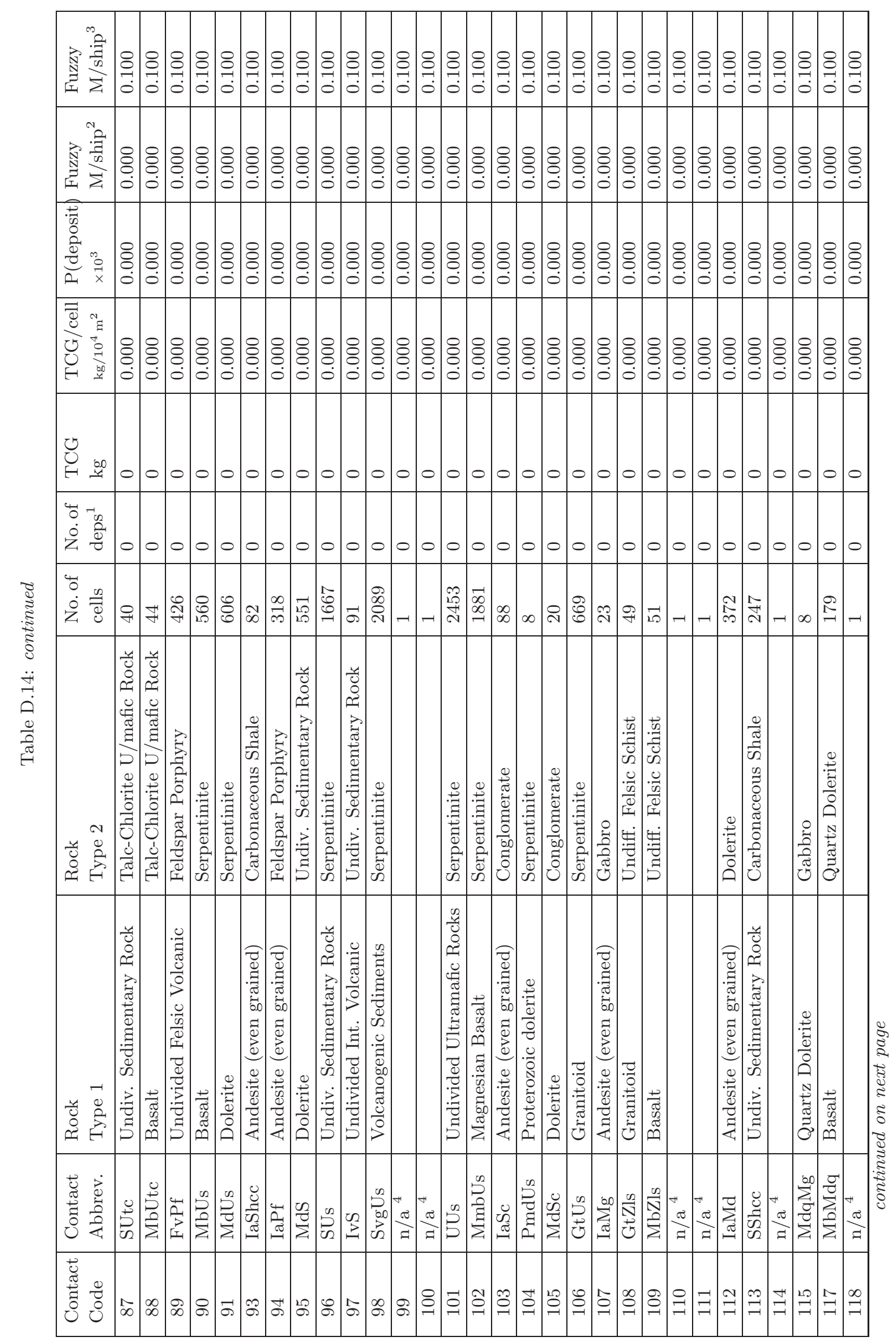




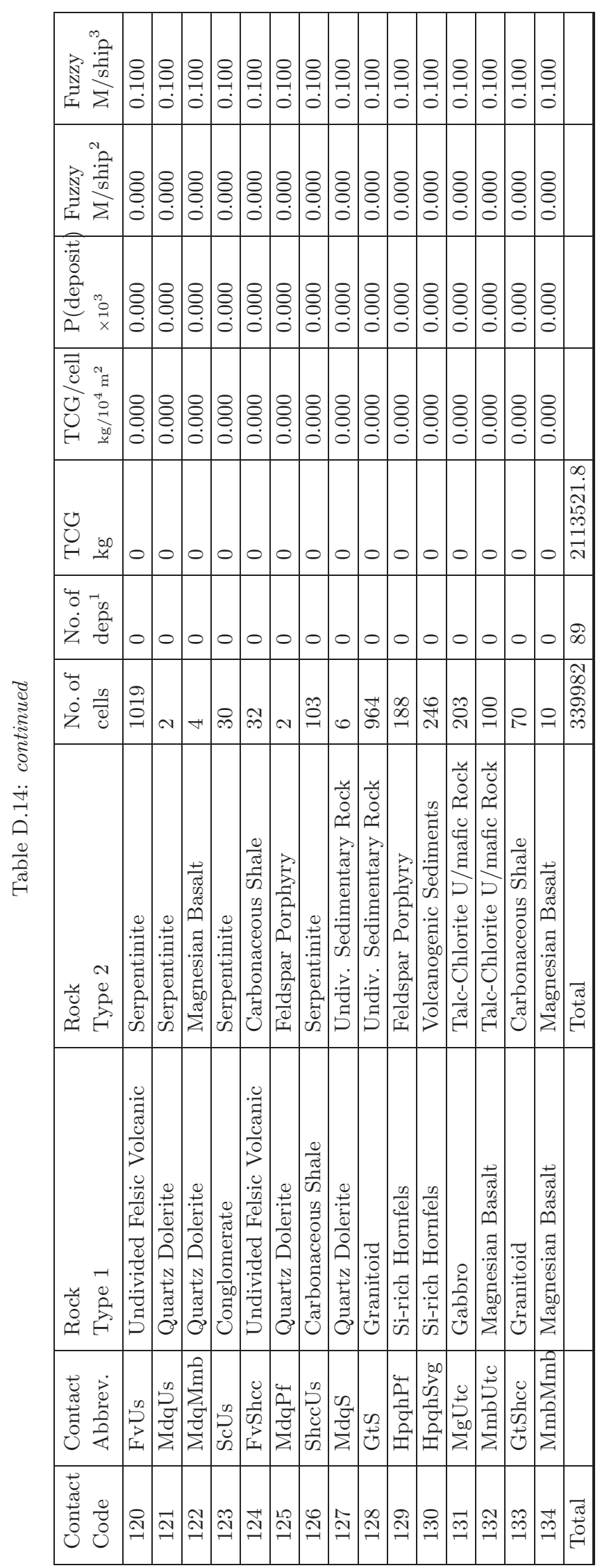

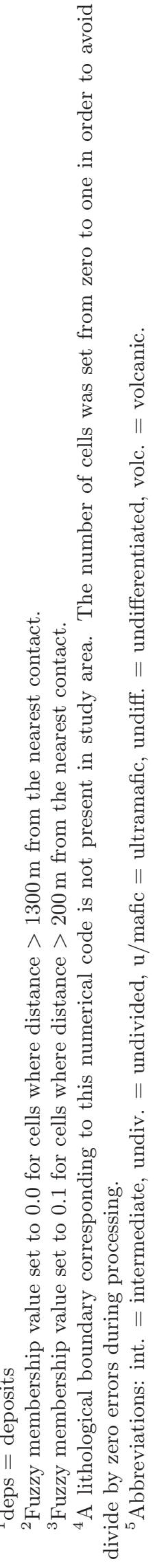


Data and calculations used to create the favourable contact-type layers 144 favcont25_pr_1300m.mat and 144 favcont25_pxtcg_1300m.mat) are shown in Table D.15 below. Fuzzy membership values indicating the degree of favourability are based on ranking the contact types in order of the probability that cells, for which the nearest contact-type has a certain value, contain one of the known deposits (TCG ge $1000 \mathrm{~kg}$ ) multiplied by the total contained gold per 100 metre cell (P(D)*TCG/cell). 


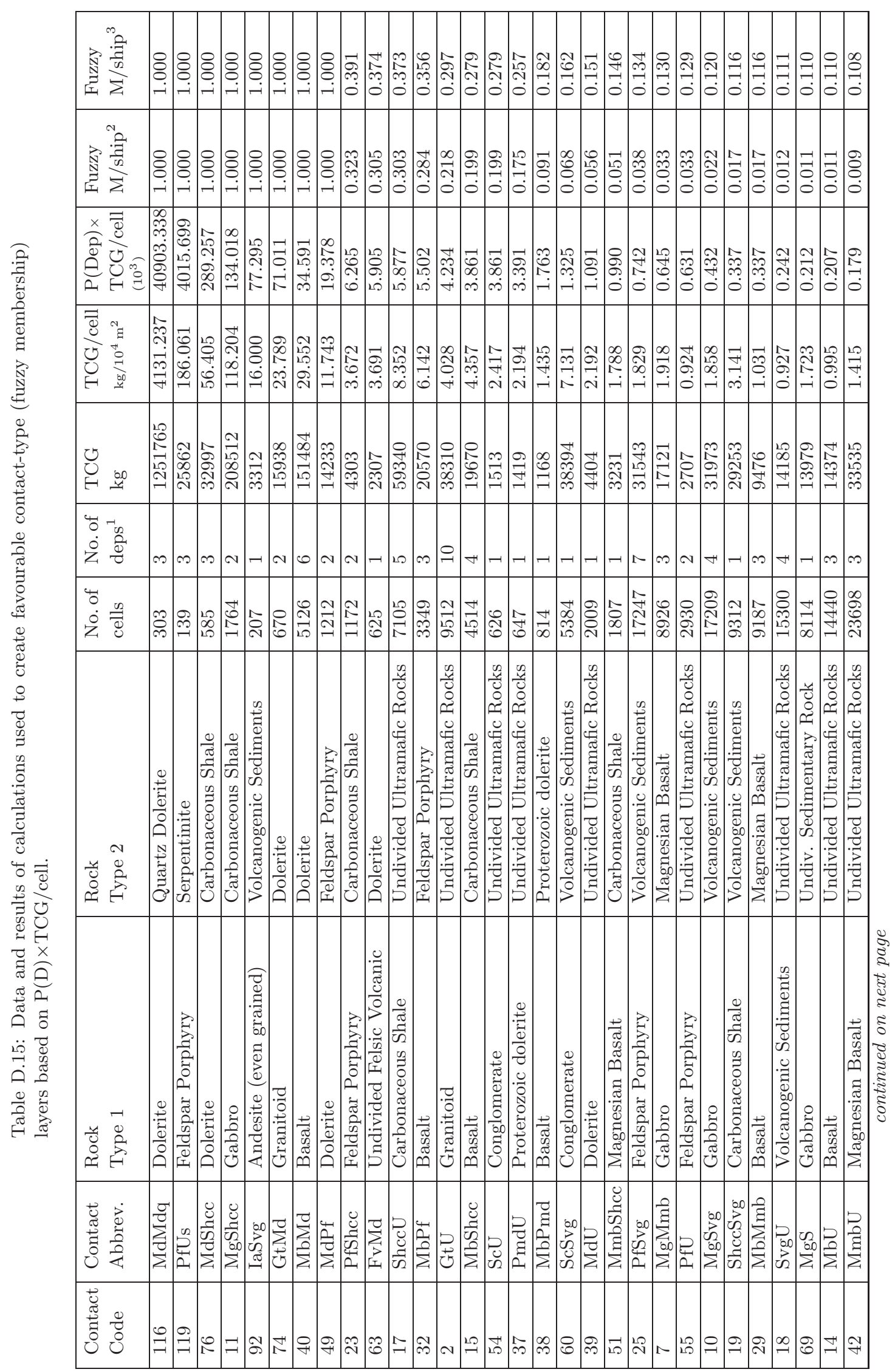




\begin{tabular}{|c|c|c|c|c|c|c|c|c|c|c|c|c|c|c|c|c|c|c|c|c|c|c|c|c|}
\hline$\frac{d}{i}$ & & $\left|\begin{array}{c}1 \\
0 \\
0 \\
0\end{array}\right|$ & & 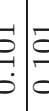 & & $\frac{8}{6}$ & & 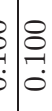 & & & \begin{tabular}{c|c}
8 & 8 \\
\hdashline & 0 \\
0 & 0
\end{tabular} & 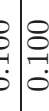 & & & \begin{tabular}{c}
8 \\
\hdashline \\
0 \\
0 \\
0
\end{tabular} & & & $\begin{array}{cc}8 & 8 \\
\\
& 0 \\
\end{array}$ & 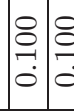 & 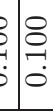 & & & & $\mid \begin{array}{l}8 \\
0 \\
0\end{array}$ \\
\hline$\stackrel{\sqrt{n}}{\Sigma}$ & 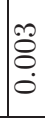 & 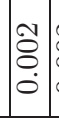 & & 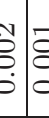 & 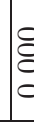 & ஜ & 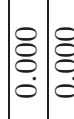 & 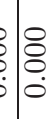 & 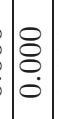 & & \begin{tabular}{c}
8 \\
\hdashline \\
\hdashline \\
\hdashline
\end{tabular} & & @ & $\begin{array}{l}8 \\
\vdots \\
0 \\
0\end{array}$ & 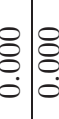 & 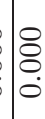 & $\begin{array}{l}8 \\
\varnothing \\
\vdots\end{array}$ & \begin{tabular}{c|c}
8 \\
8 \\
\hdashline \\
\end{tabular} & $\begin{array}{l}8 \\
\vdots \\
\vdots \\
0\end{array}$ & 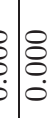 & @o & & & 递 \\
\hline 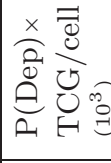 & 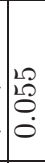 & & & $\vec{b}$ & & : & 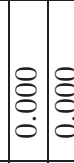 & 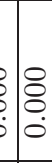 & 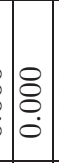 & & 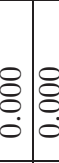 & & ¿ & $\begin{array}{l}8 \\
\vdots \\
\vdots \\
0\end{array}$ & 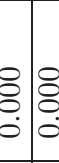 & 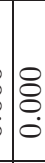 & $\begin{array}{l}8 \\
\vdots \\
\vdots\end{array}$ & \begin{tabular}{c|c}
8 \\
8 \\
\hdashline \\
\end{tabular} & 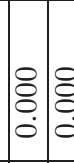 & 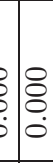 & $\stackrel{8}{8}$ & & & 递 \\
\hline 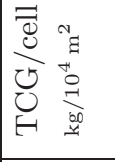 & đֶ: & : & & & & $\stackrel{8}{\square}$ & 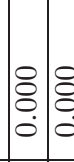 & 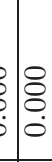 & & & 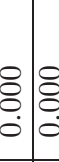 & & ஜ & $\begin{array}{l}8 \\
\vdots \\
\vdots \\
0\end{array}$ & 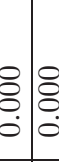 & & & \begin{tabular}{l}
8 \\
\hdashline \\
\\
\end{tabular} & & 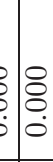 & 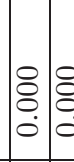 & & & : \\
\hline 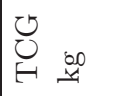 & & & & 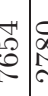 & 10 & 0 & 100 & 0 & 0 & 0 & 00 & 0 & 0 & 0 & 00 & 10 & 0 & 00 & 100 & 0 & 100 & 0 & 0 & 10 \\
\hline 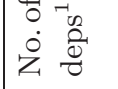 & & & & -1 & & 0 & 100 & 0 & 0 & 0 & 00 & 0 & 0 & 0 & 00 & 10 & 0 & 00 & 100 & 0 & 100 & 0 & & 0 \\
\hline $\begin{array}{l}\vec{b} \\
\dot{\circ} \\
\dot{z}\end{array}$ & & & & 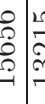 & & 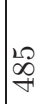 & & 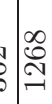 & & & & & 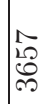 & & 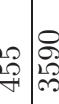 & & & & 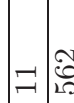 & 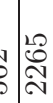 & $28 \frac{2}{2 .}$ & & $\mid \begin{array}{l}\infty \\
2 \\
-2 \\
-\rightarrow\end{array}$ & - \\
\hline 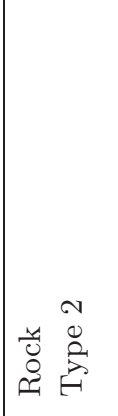 & 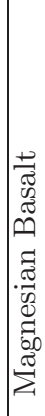 & 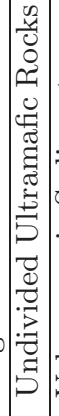 & & 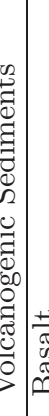 & & 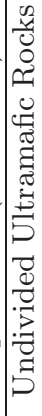 & 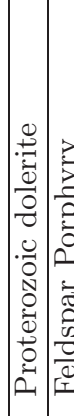 & 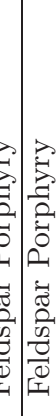 & 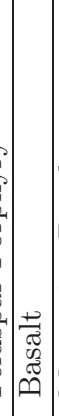 & 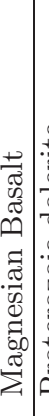 & 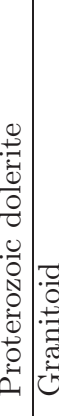 & 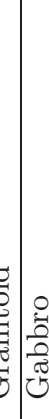 & 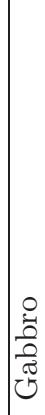 & 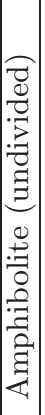 & 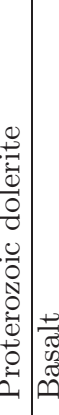 & & 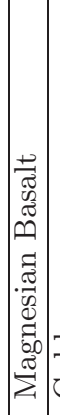 & 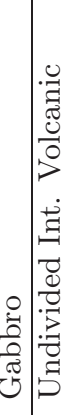 & 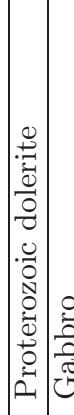 & 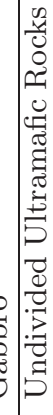 & 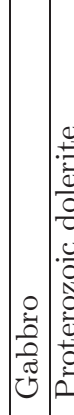 & & 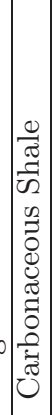 & \\
\hline 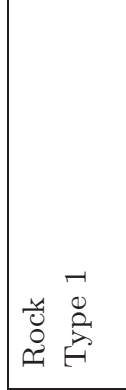 & & : & . & 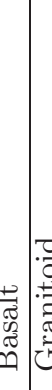 & & & | & 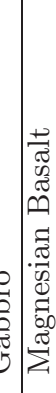 & 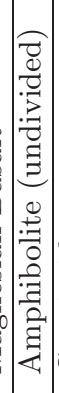 & 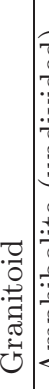 & 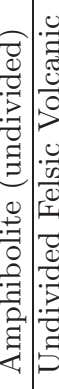 & 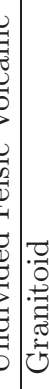 & 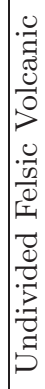 & 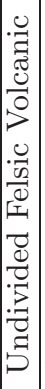 & 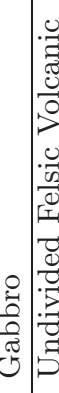 & 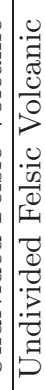 & 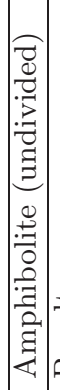 & 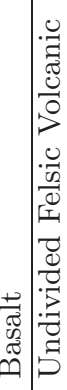 & 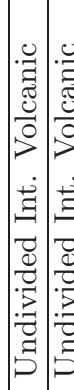 & 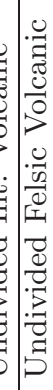 & 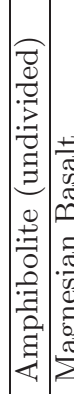 & & 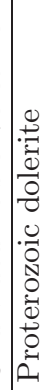 & \\
\hline 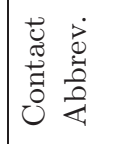 & 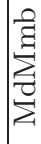 & $\mid \vec{b}$ & & $=$ & & & 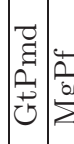 & $=$ & 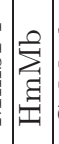 & & 焉 & & $\sum_{15}^{5}$ & 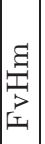 & $\overbrace{60}^{0}$ & D & 氞 & $\sum_{\substack{\infty \\
\sum^{2}}}^{\infty}$ & o & 象 & $\sum_{\substack{0 \\
0}}^{\infty}$ & 这 & है & 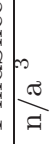 \\
\hline 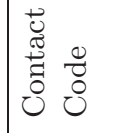 & & & & & & & & & & & 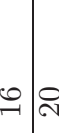 & & & & $\stackrel{\sim}{*}$ & & \& & $\vec{m}$ & Rু & 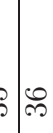 & $F$ & $\begin{array}{lll}P & \Rightarrow\end{array}$ & $\stackrel{12}{7}$ & 4 \\
\hline
\end{tabular}




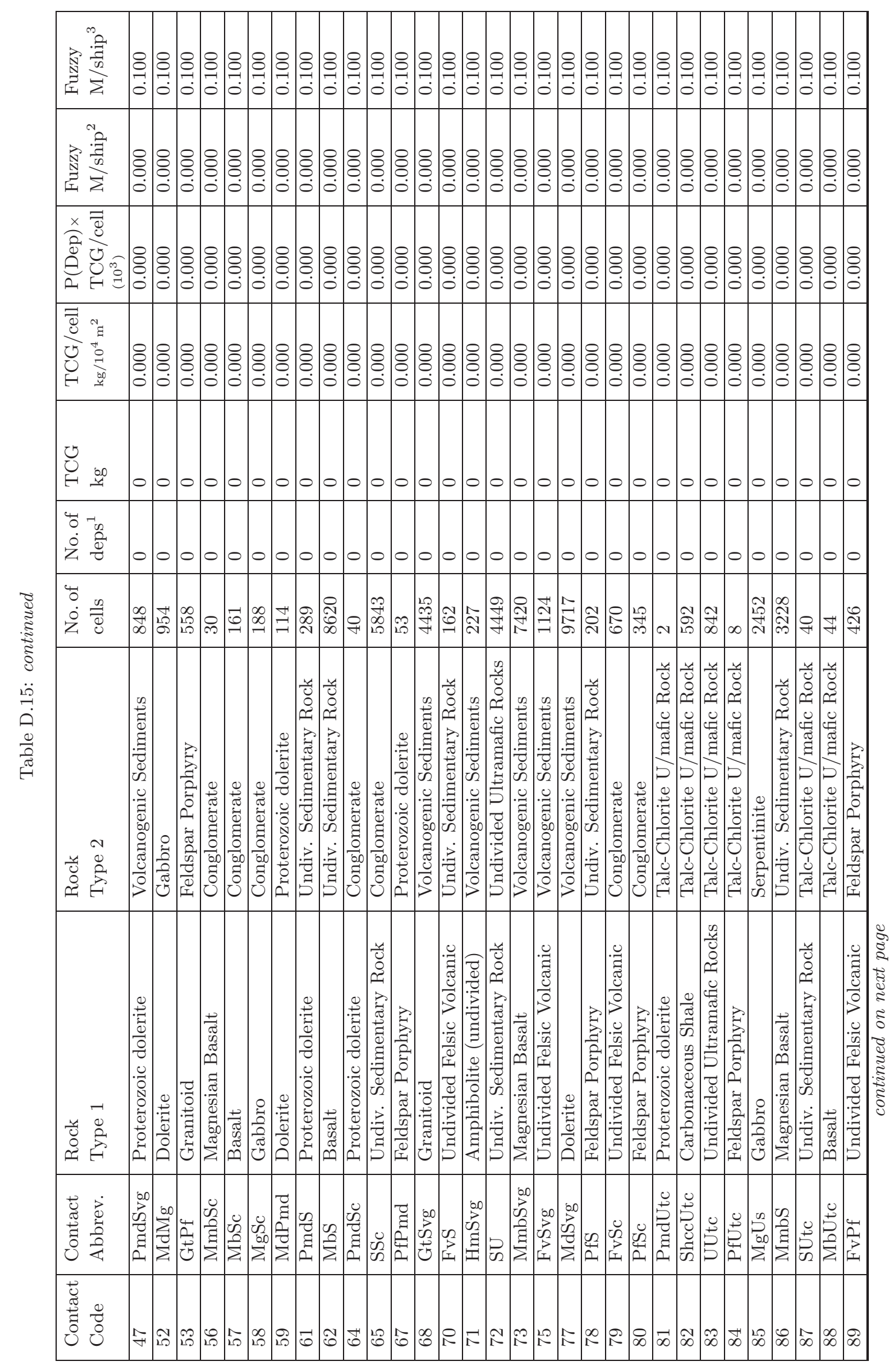




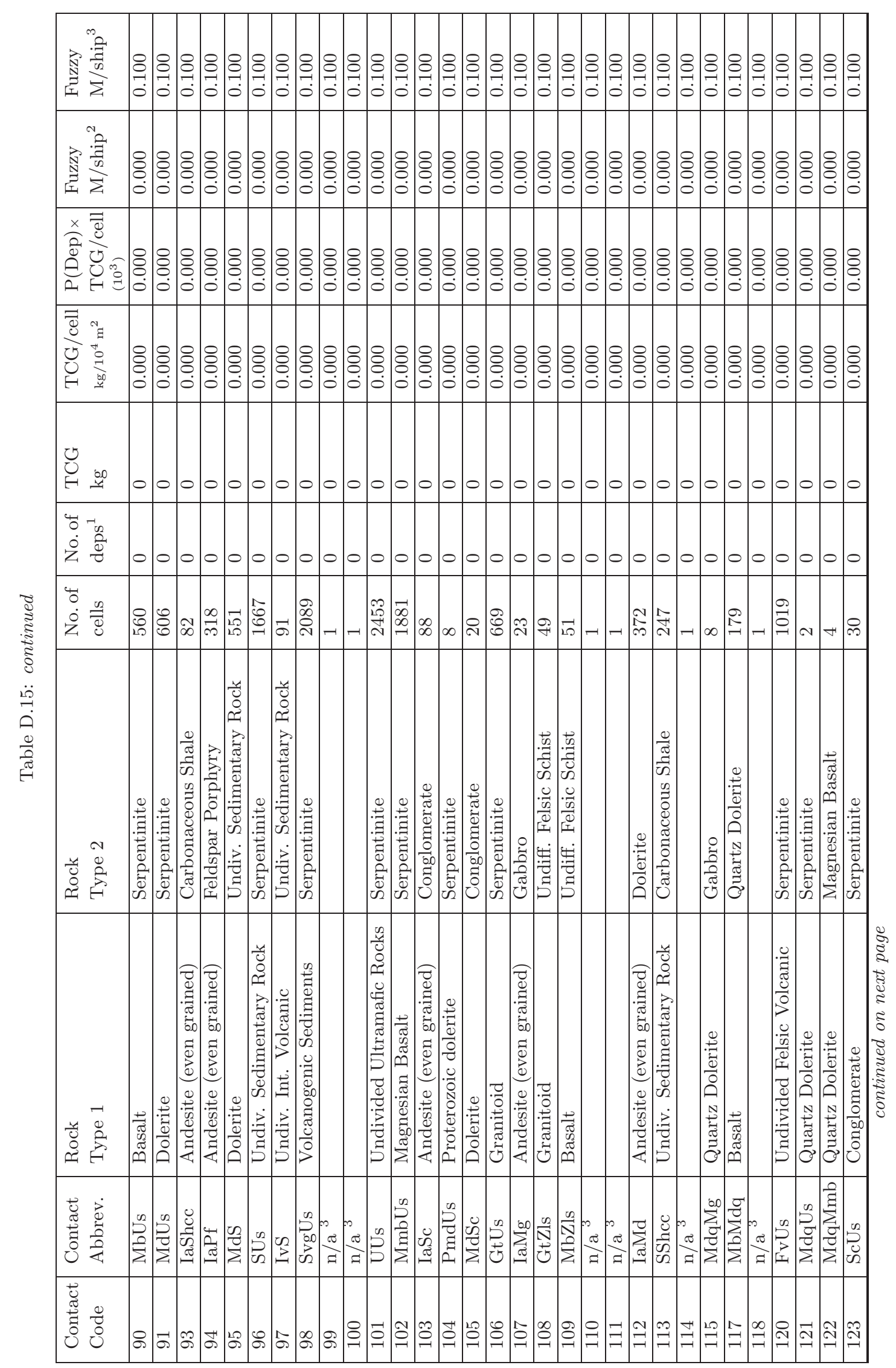



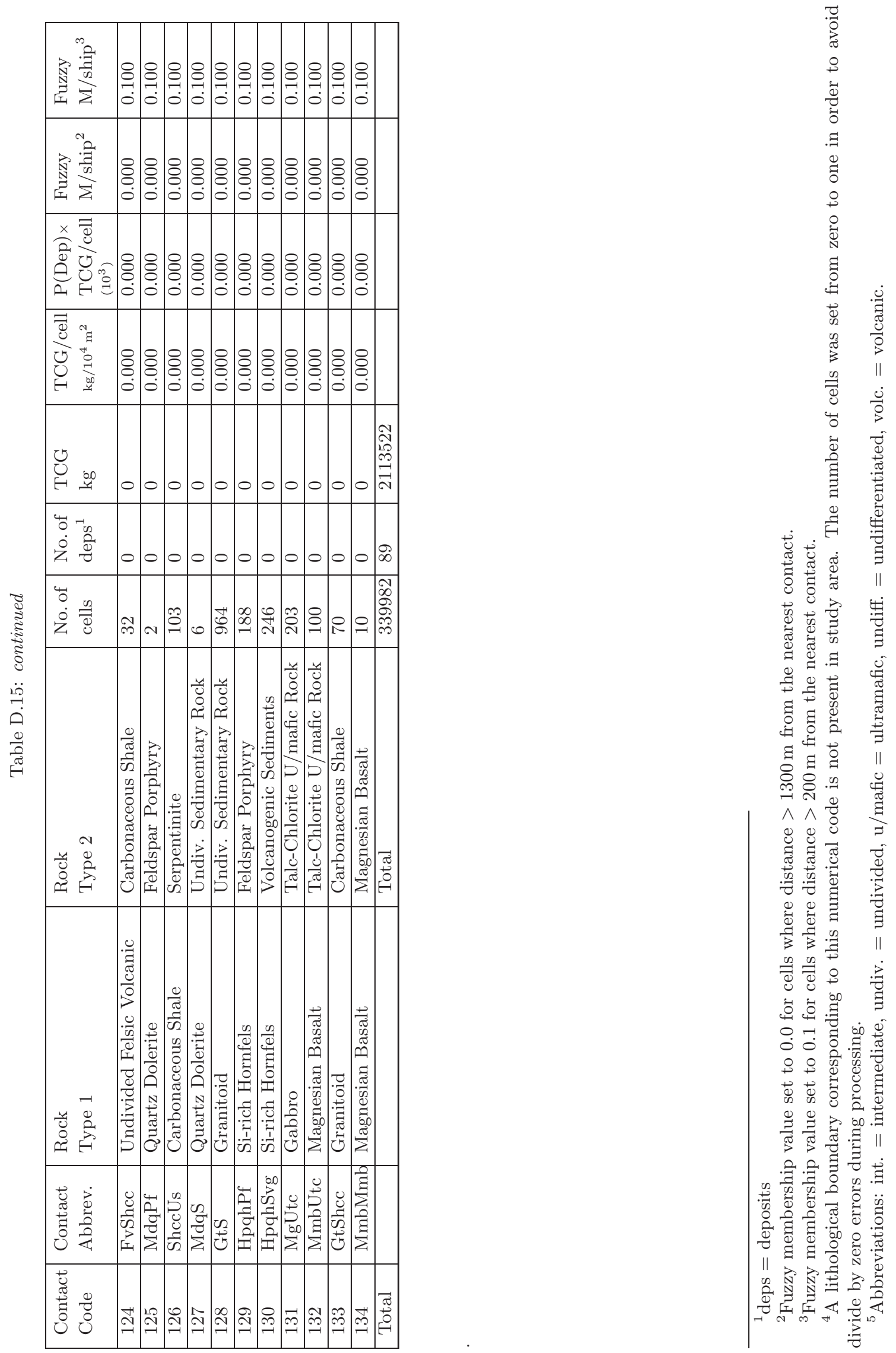


\section{D.11 Favourable Lithology Data}

Data and calculations used to create the favourable lithology layer (l1_favgeol25_pr0110.mat) is shown in Table D.16 below. Fuzzy membership values indicating the degree of favourability are based on the probability of a deposit (> $1000 \mathrm{~kg}$ TCG). 


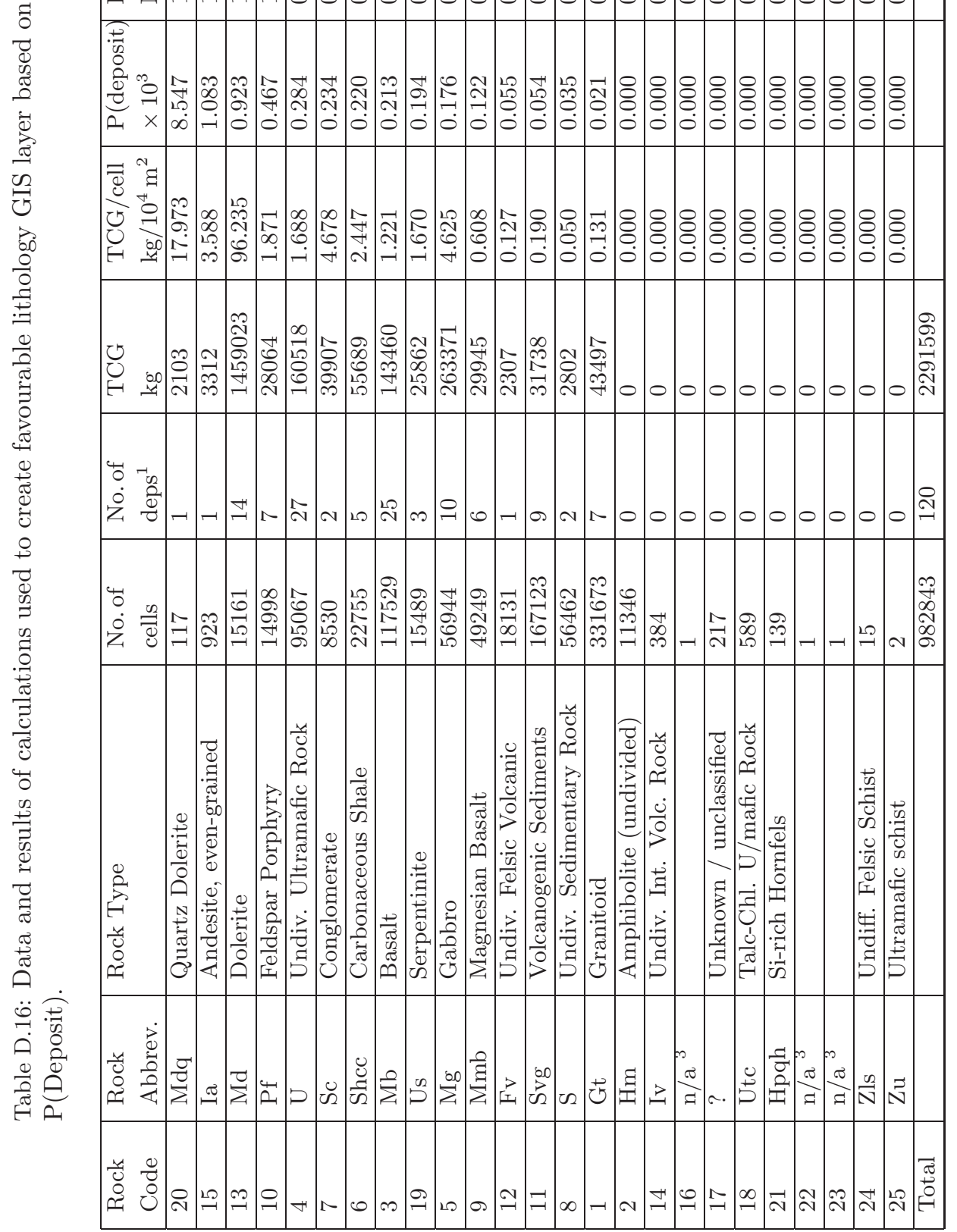

نூ

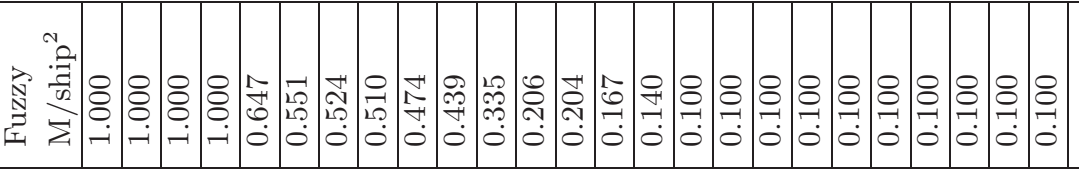

(0)

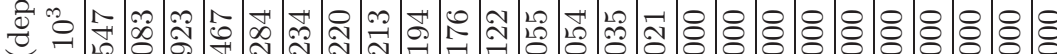

$\overbrace{}^{\mathrm{N}} \mathrm{冫}$

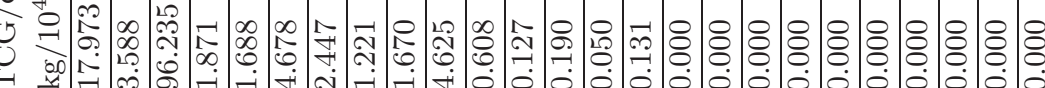

U

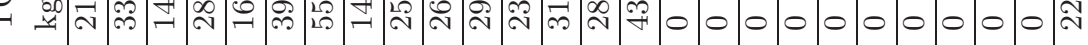

$+\frac{1}{0}+r^{-1}$

பـ

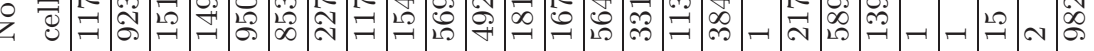

$\stackrel{0}{\circ}$

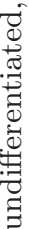


Data and calculations used to create the favourable lithology layer (l1_favgeol_pxtcg.mat is shown in Table D.17 below. Fuzzy membership values indicating the degree of favourability are based on ranking the lithologies according to the index calculated as the probability of the lithology containing one of the known deposits (TCG ge $1000 \mathrm{~kg}$ ) multiplied by the total contained gold per 100 metre cell $(\mathrm{P}(\mathrm{D}) \times \mathrm{TCG} /$ cell $)$. 


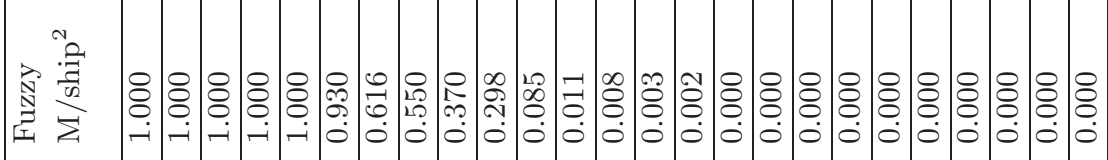

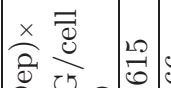

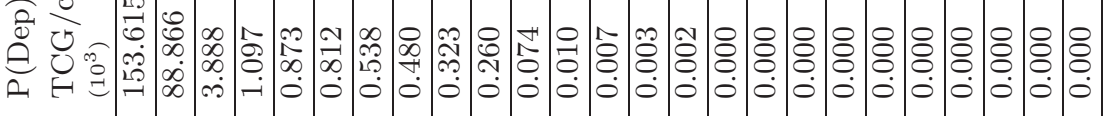

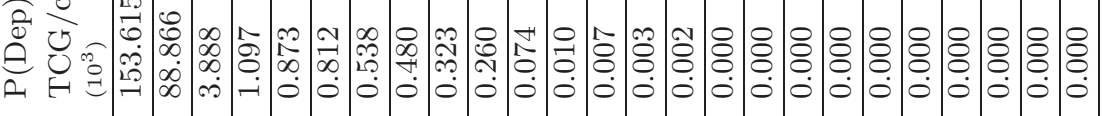

高

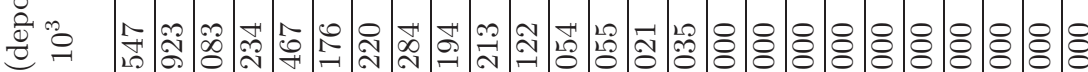

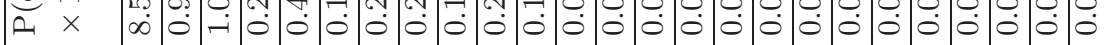

$\overline{\mathrm{d}}{ }^{\mathrm{N}}$ घ

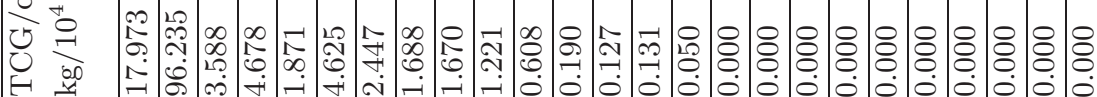

(n)

U

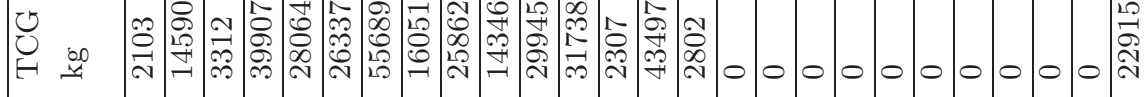

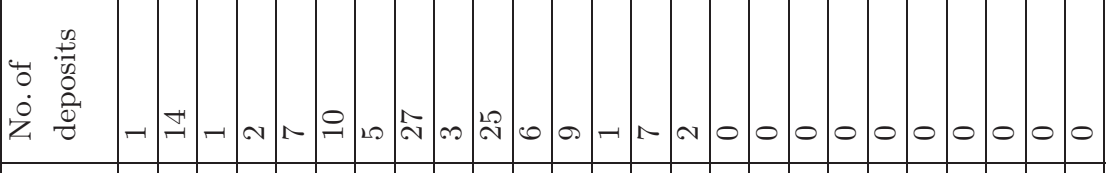

눙

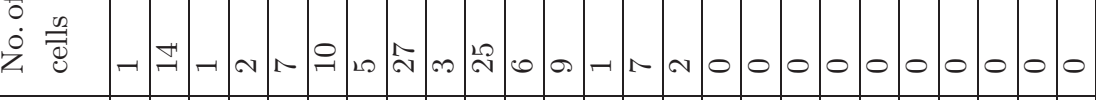

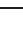

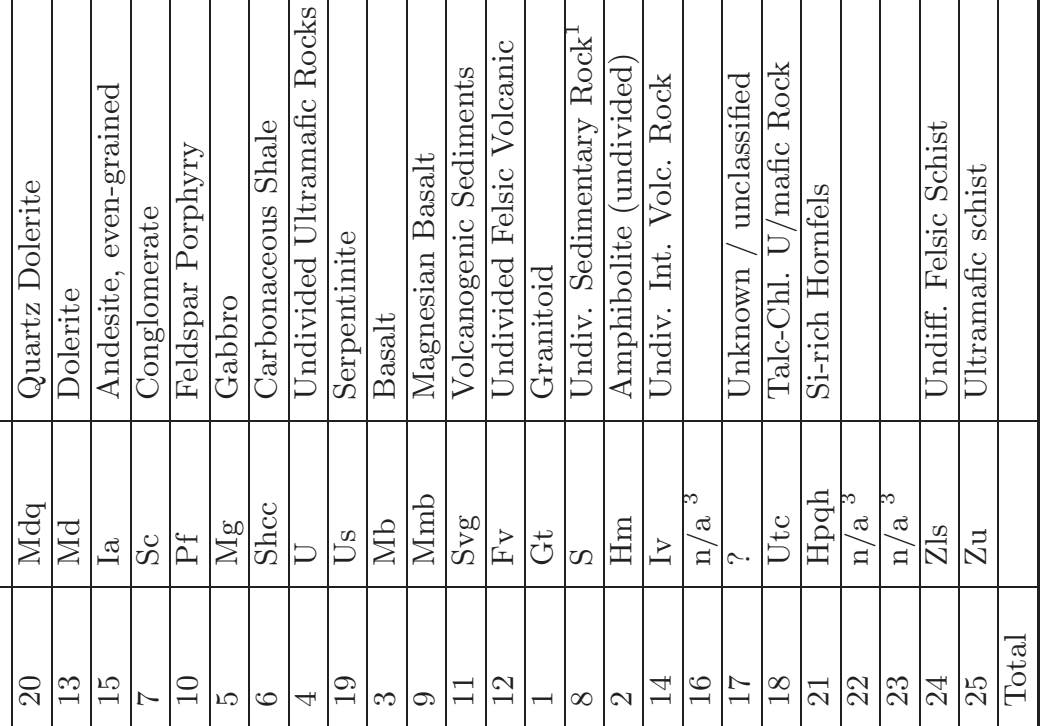




\section{Appendix E}

\section{Summary of Data Processing used in the Tenterfield Pilot Study}

\section{E.1 Data Processing Procedure used in Pilot Study, Chp. 4}

\section{E.1.1 Pre-processing}

Step 1. Convert all vector and image files to grid format.

GIS layers were prepared using the software package GEODIPS Jusmady \& Taylor (1997) and ARC/INFO. Depending on the initial format one of the following steps are taken;

1. Remove file header. This is necessary if, for example, file has been exported from GEODIPS.

2. Convert ARC/Info coverages to grids using polygrid command

3. Convert ARC/INFo images to grids using imagegrid command

4. Convert ArC/INFo grids to an ASCII grid using gridascii command

Step 2. Convert GIS layers to vectors

Run program, merge_sls, to merge GIS database layers into a list of vectors. Program also scales, labels and sorts output into multiple files. Table E.1 shows the input and outputs for program merge_sls below. 
Table E.1: Inputs and outputs for program, merge_sls

\begin{tabular}{|c|c|}
\hline \multicolumn{2}{|l|}{ Inputs: } \\
\hline File name & Description \\
\hline geology & Geology grid cell values ${ }^{\mathrm{a}}$ \\
\hline$<$ input\#2> & Airborne magnetic layer: grid cell values \\
\hline$<$ input\#3> & Uranium channel, gamma-ray survey data: grid cell values \\
\hline$<$ input\#4> & Thorium channel, gamma-ray survey data: grid cell values \\
\hline$<$ input\#5> & Potassium channel, gamma-ray survey data: grid cell values \\
\hline$<$ input\#6> & Total count channel, gamma-ray survey data: grid cell values \\
\hline$<$ input\# $\rangle^{\mathrm{b}}$ & Proximity to nearest fault: grid cell values \\
\hline deposits & Deposit occurrence data (binary): grid cell values ${ }^{\mathrm{c}}$ \\
\hline \multicolumn{2}{|l|}{ Outputs: } \\
\hline File name & Description \\
\hline log_merge & Count of vectors read and processed \\
\hline rawvec & Unscaled vectors without 1-of-n coding of geology \\
\hline rawvecd & Unscaled deposit vectors \\
\hline rawvecn & Unscaled non-deposit vectors \\
\hline vectorsall & Vectors (scaled, geology1-of-n coded) \\
\hline vectorsd & Deposit vectors (scaled, geology in 1-of-n code) \\
\hline vecn1 & Non-deposit vectors (scaled, geology in 1-of-n code) \\
\hline vecn 15 & Vectors with geology codes 1 to 15 \\
\hline
\end{tabular}

${ }^{a}$ First layer must be the geology layer

${ }^{b}$ With minor modifications, the program can handle up to 30 user-specified input files

${ }^{c}$ Last layer must be the deposit occurrence layer

\section{Step 3. Randomly select training data}

Run program, rand_select, to create training, training-stop and test data sets by randomly selecting feature vectors from an input file containing either all the deposit or all the non-deposit feature vectors from the GIS map grid. Deposit and non-deposit feature vectors are selected separately. Table E.2 shows the input and outputs for program rand_select below. 
Table E.2: Inputs and outputs for program,rand_select

\begin{tabular}{|l|l|}
\hline \multicolumn{2}{|l|}{ Inputs: } \\
\hline File name & Description \\
\hline$<$ file\#1> & Name of user specified file containing vectors; i.e., vectors or vectors ${ }^{\text {a }}$ from step two \\
\hline$<$ parameter\#1 $>$ & Number of vectors to randomly select $(\leq$ total no. of vectors in file) \\
\hline$<$ parameter\#2 $>$ & Number of output files to create; usually the 3 files listed below \\
\hline \hline Outputs: & Description \\
\hline File name & Name of 1 to 24 user specified files to store user specified number of vectors each \\
\hline$<$ file\#n $>$ & Count of vectors read and processed \\
\hline $\log _{-}$rand &
\end{tabular}

${ }^{a}$ The file containing all the deposit vectors, vectorsd, is processed in a separate run

\section{E.1.2 Neural Network Training}

\section{Step 4. Train neural network}

Run program, $b p$ Win using command, $b p<$ template file $><$ startup file $>$ to train a neural network using training data sets produced in steps above. The program is a multilayer feedforward neural network simulation and is a 32-bit version of the bp program created by by McClelland \& Rumellhart (1988). The program and ancillary input files listed below are contained in the network directory on the CD-ROM (vol. 1) submitted with the thesis (refer to Table B.1 on page 512). Table E.3 shows the input and outputs for program bp Win below.

Training involves alternately processing the input feature vector file (1.pat using the command ptrain, then changing the input file to the training-stop data set, (2.pat), using the command, get patterns 2.pat, and testing the partially trained network using the command, tall; i.e., "test all". Test runs were performed initially every 100 epochs and then more frequently, reducing from every ten to every two epochs, as the number of epochs approached the minimum Total Sum of Squares (TSS) error. 
Table E.3: Inputs and outputs for program, $b p$ Win

\begin{tabular}{|c|c|}
\hline \multicolumn{2}{|l|}{ Inputs: } \\
\hline File name & Description \\
\hline 1821.tem & Template file; specifies data displayed on screen during program execution \\
\hline \multirow[t]{9}{*}{ 1821.str } & Start file which specifies; \\
\hline & Network file name (1821.net) \\
\hline & Input file names (1.pat) \\
\hline & $\begin{array}{l}\text { Global display level ( } \text { llevel }=3 \text { then, when the display is updated, all templates with } \\
\text { display level } \leq 3 \text { are displayed }\end{array}$ \\
\hline & lflag $=1$. If flag variable $\neq 0$ learning occurs and weights are updated. \\
\hline & Learning rate $(0.50)$ \\
\hline & Momentum (0.00) \\
\hline & $\begin{array}{l}\text { Weight-update frequency. If set to pattern then weights updated after every input } \\
\text { pattern. }\end{array}$ \\
\hline & Initial mode of program operation ( tall $=$ test all patterns in input file) \\
\hline \multirow[t]{3}{*}{ 1821.net } & File defines network topology; i.e., 18-2-1 \\
\hline & ecrit $=0.04 ;$ If the total sum of squares $(T S S)$ falls below ecrit then training stops \\
\hline & $\begin{array}{l}\text { nepochs }=50 \text {; i.e., the no. of cycles of training performed when ptrain command is } \\
\text { issued }\end{array}$ \\
\hline 1.pat & Training pattern file (contains a list of input feature vectors) \\
\hline 2.pat & Training-stop pattern file (contains a list of input feature vectors) \\
\hline \multicolumn{2}{|l|}{ Outputs: } \\
\hline File name & Description \\
\hline 10a.wts & $\begin{array}{l}\text { Initial random connection weights. This file is created as the first step after starting } \\
b p \text { Win }\end{array}$ \\
\hline 10atrain.wts & Trained connection weights. The number and letter denote the training run \\
\hline \multicolumn{2}{|l|}{ Parameters: } \\
\hline Name & Description \\
\hline learning rate & 0.5 \\
\hline momentum & 0.0 \\
\hline pattern order & Patterns presented in permutated order if command ptrain is used \\
\hline weight update & $\begin{array}{l}\text { Stochastic (i.e. after each pattern). This is set using the commands set mode, lgrain } \\
=\text { epoch }\end{array}$ \\
\hline weight range & {$[-0.5,+0.5]$} \\
\hline topology & $\begin{array}{l}18 \text { input, } 2 \text { hidden and } 1 \text { output unit. The number of hidden units were varied from } \\
0 \text { to } 9 \text {. The .net and str files were modified accordingly. }\end{array}$ \\
\hline
\end{tabular}


In order to recognize the minimum TSS error, the training TSS error and the number of epochs of training were recorded before each test together with the TSS error for the training-stop data set. Training is stopped at the number of epochs corresponding to the minimum TSS error for the training data set. The trained weights are saved in a file; e.g., 10atrain.wts. The training procedure is repeated for up to ten sets of random initial weights in order to avoid the possibility of the network converging to a local minimum in weight space. The network with the best performance on the test data set was selected to process the entire map grid.

\section{Step 5. Test neural network}

Run program $b p$ Win using the test data set 3.pat as the input file. The TSS error for this data set gives an indication of the generalization ability of the network. Table E.4 shows the input and outputs for $b p$ Win, in test mode, below.

Table E.4: Inputs and outputs for program, $b p$ Win, for testing the generalization ability of the trained network

\begin{tabular}{|l|l|}
\hline \multicolumn{2}{|l|}{ Inputs: } \\
\hline File name & Description \\
\hline 1821.tem & Template file; specifies data displayed on screen during program execution \\
\hline 1821.str & Start file. See Table E.3 \\
\hline 1821.net & Defines network topology, ecrit $=0.04$, and nepochs $=50$ \\
\hline 3.pat & Test pattern file (contains a list of input feature vectors) \\
\hline \hline Parameters: & \multicolumn{2}{|l|}{} \\
\hline Name & Description \\
\hline TSS & Total Sum of Squares (TSS) error for the test data set \\
\hline
\end{tabular}

\section{Step 6. Process map grid using the trained network}

Run program, $b p$ Win using the file containing the feature vectors corresponding to the entire map grid as input. Connection weights that result from training are saved in a file in the previous step. In production mode, this trained-weights file becomes an input file. Table E.5 shows the input and outputs for $b p$ Win, in production mode, below. 
Table E.5: Inputs and outputs for program, $b p$ Win, in production mode. The weights that were modified as a result of training are used to process vectors for the entire GIS map grid.

\begin{tabular}{|l|l|}
\hline \multicolumn{2}{|l|}{ Inputs: } \\
\hline File name & Description \\
\hline prodn.tem & $\begin{array}{l}\text { Template file; to speed processing only minimal data are displayed on screen in pro- } \\
\text { duction mode }\end{array}$ \\
\hline prodn.str & Start file. The pattern file is set to 4.pat. See Table E.3 \\
\hline 1821.net & Defines network topology, ecrit $=0.04$, and nepochs $=50$ \\
\hline 4.pat & Feature vectors corresponding to the entire study area \\
\hline 10atrain.wts & $\begin{array}{l}\text { Trained connection weights, derived from training in step } 4 \text { above, are loaded into } \\
\text { the network }\end{array}$ \\
\hline \hline Outputs: & \multicolumn{2}{|l|}{} \\
\hline File Name & Description \\
\hline log_tnet & log file records the output activation values for the trained network \\
\hline
\end{tabular}

\section{E.1.3 Post-processing}

\section{Step 7. Convert continuous output of network to map classes}

Run program, map_class. This program is used to classify the raw output of a neural network into a set of prospectivity-map class values. Output activation values are in the approximate range $[0.05,0.95]$. Mineral prospectivity maps with 9 classes were prepared in the pilot study described in Chapter 4. Table E.6 shows the input and outputs for map_class, in production mode, below.

Table E.6: Inputs and outputs for program, map_class, which converts the raw output of a trained network into a user specified number of prospectivity map classes.

\begin{tabular}{|l|l|}
\hline \multicolumn{2}{|l|}{ Inputs: } \\
\hline File name & Description \\
\hline log_tnet & Output activation values for the trained network; user specified file name \\
\hline Parameter & Description \\
\hline new_max & New maximum value used to scale the data set \\
\hline new_min & New minimum value used to scale the data set \\
\hline n_rows & Number of rows in the output grid files: scaled and classed \\
\hline
\end{tabular}


Table E.6: continued

\begin{tabular}{|l|l|}
\hline \multicolumn{2}{|l|}{ Inputs: } \\
\hline File name & Description \\
\hline n_cols & Number of columns in the output grid files: scaled and classed \\
\hline \hline Outputs: \\
\hline File Name & Description \\
\hline log_class & Count of values processed \\
\hline scaled & File containing scaled data in grid format \\
\hline classed & File containing integers representing map classes \\
\hline
\end{tabular}

\section{Step 8. Calculate map quality statistics}

Run program map_qstats to calculate the chi-squared statistic and Spearman's and Kendall's rank correlation coefficients as measures of prospectivity map quality for a single map. Table E.7 shows the input and outputs for deposit_locns below.

Table E.7: Inputs and outputs for program map_qstats which calculates the chi-squared statistic and Spearman's and Kendall's rank correlation coefficients as measures of prospectivity map quality.

\begin{tabular}{|l|l|}
\hline \multicolumn{2}{|l|}{ Inputs: } \\
\hline File name & Description \\
\hline classed & File containing integers representing map classes; i.e. a prospectivity map file \\
\hline deposits & Deposit occurrence file (binary): grid cell values \\
\hline \hline Outputs: & \multicolumn{2}{|l|}{ Description } \\
\hline File Name & Count of the number of values read and total number of deposits \\
\hline log_qstats & $\begin{array}{l}\text { Map file name with stats.txt appended to it; contains map quality statistics and } \\
\text { intermediate results }\end{array}$ \\
\hline $\begin{array}{l}<m a p \\
\text { name> stats.txt }\end{array}$
\end{tabular}




\section{E.1.4 Miscellaneous}

\section{Step 9. Calculate number of grid cell and deposit for each rock unit}

Run program deposit_locns to counts the number of grid cells and deposits in each geological unit in an input ASCII grid file corresponding to the geology GIS database layer. The file $<$ map file name $>$ stats.txt obtained from step 8 is used to calculate the probability-based measures of map quality described in Chapter 4. Table E.8 shows the input and outputs for deposit_locns below.

Table E.8: Inputs and outputs for program deposit_locns which calculates statistics on the number of grid cells and deposits for each geological unit.

\begin{tabular}{|l|l|}
\hline \multicolumn{2}{|l|}{ Inputs: } \\
\hline File name & Description \\
\hline 4.pat & $\begin{array}{l}\text { File containing list of vectors for map grid covering the entire study area; user specified } \\
\text { file name }\end{array}$ \\
\hline \hline Outputs: & \multicolumn{2}{|l|}{} \\
\hline File Name & Description \\
\hline log_dep_stats & Count of vectors read and processed \\
\hline stats & Numbers of cells and deposits contained in each geology map class \\
\hline
\end{tabular}




\section{Appendix $\mathrm{F}$}

\section{Summary of Matlab Scripts used in Kalgoorlie Case Study}

The code for all functions and scripts shown in Table F.1 is contained on the CD-ROM (vol. 1) that accompanies the thesis.

Table F.1: Summary of MatLab functions and scripts.

\begin{tabular}{|c|c|}
\hline Script/Function Name & Application \\
\hline \multicolumn{2}{|c|}{ Creating Derived Input Layers } \\
\hline fault_density & $\begin{array}{l}\text { Calculate total length of faults in a } 5 \times 5 \mathrm{~km} \text { window and assign } \\
\text { that value to the central cell. Based on simplified geology legend } \\
\text { containing } 25 \text { rock types. }\end{array}$ \\
\hline favcontact25_pr0110 & $\begin{array}{l}\text { Create a fuzzy membership layer for favourable contact type based } \\
\text { on } \mathrm{P}(\mathrm{D}) \text { by reclassifying a contact type layer. }\end{array}$ \\
\hline favcontact25_pxtcg0110 & $\begin{array}{l}\text { Create a fuzzy membership layer for favourable contact type based } \\
\text { on } \mathrm{P}(\mathrm{D})^{*} \mathrm{TCG} / \text { cell by reclassifying a contact type layer. }\end{array}$ \\
\hline favgeol25_pr0110 & $\begin{array}{l}\text { Create a fuzzy membership layer for favourable rock type based on } \\
\mathrm{P}(\mathrm{D}) \text { by reclassifying a solid geology layer. }\end{array}$ \\
\hline litho_diversity & $\begin{array}{l}\text { Calculate the number of lithological contacts in a } 5 \times 5 \mathrm{~km} \text { window } \\
\text { surrounding cell. }\end{array}$ \\
\hline
\end{tabular}


Table F.1: continued

\begin{tabular}{|c|c|}
\hline Script/Function Name & Application \\
\hline geolcodes_kal & $\begin{array}{l}\text { Reclassify rock type codes in a geology input layer to form a contigu- } \\
\text { ous sequence (required as a preliminary step to create 1-of-n coded } \\
\text { solid geology input layer). }\end{array}$ \\
\hline \multicolumn{2}{|l|}{ Importing GIS Layers } \\
\hline strip_hdr & $\begin{array}{l}\text { Remove the header from an ArcView ASCII export file in order to } \\
\text { load the ASCII file into MATLAB. }\end{array}$ \\
\hline \multicolumn{2}{|c|}{ Formating Input GIS Layers } \\
\hline makearray & $\begin{array}{l}\text { Format an non-grid formatted ASCII file into rows of length for load- } \\
\text { ing into Matlab. Used to correct formatting errors in GEODIPS } \\
\text { ASCII export files for Tenterfield project area. }\end{array}$ \\
\hline \multicolumn{2}{|c|}{ Evaluation of Input Layers } \\
\hline chi_wts3 & $\begin{array}{l}\text { Plots, prints and saves cumulative chi-squared and weights contrast } \\
\text { versus distance plots using a vectorized algorithm to avoid for FOR } \\
\text { loops. }\end{array}$ \\
\hline j_function_dist & $\begin{array}{l}\text { Plot bivariate J-function for the distance relationship between deposit } \\
\text { points and the distance to the linear or point features represented in } \\
\text { the input grid. }\end{array}$ \\
\hline j_function_degrees & Plot bivariate J-function for input layers representing strike angles. \\
\hline j_function15_step_wise_dist & $\begin{array}{l}\text { Plot step-wise version of bivariate J-function for input layers repre- } \\
\text { senting distance. }\end{array}$ \\
\hline j_function_step_wise_10deg & $\begin{array}{l}\text { Plot step-wise version of bivariate J-function for input layers repre- } \\
\text { senting strike angles. }\end{array}$ \\
\hline ks3 & Plot and calculate D-statistic for Kologorov-Smirnov test. \\
\hline ave_vec_dist & $\begin{array}{l}\text { Calculate the average, maximum and minimum distance between vec- } \\
\text { tors in an input array in which the vectors correspond to columns } \\
\text { Used to determine the value of spread for a probabilistic neura } \\
\text { network. }\end{array}$ \\
\hline median_dist & $\begin{array}{l}\text { Calculate median distance to nearest fault or lithological contact. } \\
\text { The average distance between faults is } 2 \mathrm{x} \text { this value. }\end{array}$ \\
\hline
\end{tabular}


Table F.1: continued

\begin{tabular}{|ll|}
\hline Script/Function Name & Application \\
\hline \hline rescale_big_is_fav & Convert input array values to "distance" from maximum value. This \\
& function converts grids containing parameters like chemical reactivity, \\
& in which large values are favourable, into a grid in which small values \\
& are favourable by calculating the difference between each cell value \\
& and the maximum value in the grid. \\
& Plots a bar graph of the function, $y=(O-E) / E$ for the relationship \\
& between deposit points and some linear or point feature in an input \\
& grid at intervals of 200 metres (distance layers ) or for 25 equal inter- \\
& vals, where $O=$ observed number of deposits in interval and $E=$ ex- \\
& pected number of deposits (assuming a uniform random distribution). \\
& Version of function $x c h i$ above for input layers representing strikes of \\
& faults or shear zones.
\end{tabular}

\section{Pre-processing Input Layers}

add_geol1ofn

inv_layer

inv_scale_batch_vec2vec

log_invx10to5_layer
Convert the input pattern array (containing the rock type in the first column) to 1-of-n coded geology.

Convert distances values input to the inverse of distance (i.e. 1/x) whilst preserving Nodata $=-9999$ values.

Batch job to transform neural network input layers that represent distance using the function, inv_layer.

Convert distance input layers. Calculate $\log _{10}\left(1 / \mathrm{x} \times 10^{5}\right)$ of each cell value whilst preserving Nodata $=-9999$ values.

log_invx10to5_scale_bat_vec2vec Batch job to transform neural network input layers that represent distance using the function, log_invx10to5_layer.

reclass_dist_contrast_nrrow_buf Batch job to reclassify input layers containing rheological and chemical contrasts so that beyond a particular distance to the lithological contact, the contrast is set to zero.

reclass_dist_strike_wide_buf

Batch job, as above to reclassify input layers containing strike angles and distances. For distances to nearest e.g., fault exceeding a distance limit, the strike is set to the mean strike value and distance is set to the maximum value. Distance limits vary for each layer and represent upper limits based on $(O-E) / E$ bar graphs (see Section 6.2.6 in Chapter 5.

reclass_dist_strike_nrrow_buf
As above but distance limits represent lower limits. 
Table F.1: continued

\begin{tabular}{|c|c|}
\hline Script/Function Name & Application \\
\hline scalemat & $\begin{array}{l}\text { Scale elements of an input map array to user defined limits. Output } \\
\text { is an array of the same dimensions as the input array. }\end{array}$ \\
\hline scalemat_batch_vec2vec & $\begin{array}{l}\text { Batch job to scale neural network input layers to range }[0,1] \text { and } \\
\text { target deposit values to range }[0.1,0.9] \text {. }\end{array}$ \\
\hline \multicolumn{2}{|l|}{ Creating Training Sets } \\
\hline add_noise_equal & $\begin{array}{l}\text { Create a set of larger neural network training data sets by adding } \\
\text { random noise to training input pattern \& target sets. Equal num- } \\
\text { ber of patterns created for each rock type. Uniform and normally } \\
\text { distributed random noise can be selected by user. }\end{array}$ \\
\hline add_noise_propn & $\begin{array}{l}\text { Create a set of larger neural network training data sets by adding } \\
\text { random noise to training input pattern \& target sets. Numbers of } \\
\text { patterns from each rock type proportional to number of deposits in } \\
\text { each rock type. Uniform and normally distributed random noise can } \\
\text { be selected by user. }\end{array}$ \\
\hline bin2arr_selctdgeol_bat & $\begin{array}{l}\text { Batch job to convert the output binary files, r1_1 and r1_2, } \\
\text { r2_1 and r2_2 etc created by functions that randomly select non- } \\
\text { deposit 1-of-n coded patterns (selectgeol_1ofn_vecbinvec and select- } \\
\text { geol_1ofn_vecbinvec) into mat-arrays. }\end{array}$ \\
\hline join_selected_deps_batch & $\begin{array}{l}\text { Concatenate the separate deposit files } \mathrm{d} 1 \_1 \text { and d1_2, d2_1 and d2_2, } \\
\text { etc. These files were created because the } 1 \text {-of-n coded geology pattern } \\
\text { files were too large to process as mat-arrays. This problem was solved } \\
\text { by splitting the patterns into } 2 \text { halves. }\end{array}$ \\
\hline join_selected_geol_batch & $\begin{array}{l}\text { Concatenate the separate non-deposit files } r 1 \_1 \text { and } r 1 \_2, r 2 \_1 \text { and } \\
\text { r2_2, etc. }\end{array}$ \\
\hline make_train_batch & $\begin{array}{l}\text { Convert a set of input layers into neural network training sets; train, } \\
\text { valid, test. }\end{array}$ \\
\hline make_train_batch_1ofn & $\begin{array}{l}\text { Convert a set of input layers, including the 1-of-n coded geology, into } \\
\text { neural network training sets; train, valid, test. }\end{array}$ \\
\hline make_train_batch_som & Create training set for an SOFM neural network. \\
\hline make_train_batch_som_noise & Vreate a noise-augmented training set for an SOFM neural net \\
\hline
\end{tabular}


Table F.1: continued

\begin{tabular}{|c|c|}
\hline Script/Function Name & Application \\
\hline make_train_Ndeps & $\begin{array}{l}\text { Create train, training-stop and test data sets of patterns from deposit } \\
\text { and non-deposit Matlab arrays so that the train data set is based on } \\
\text { Ndeps deposits. For example, if Ndeps }=5 \text { then the train data set } \\
\text { is based on } 5 \text { deposits and the remaining } 120-5=115 \text { deposits are } \\
\text { used to create the valid and test data sets. }\end{array}$ \\
\hline make_som_selected_train_set & $\begin{array}{l}\text { Create training set based on non-deposit patterns that have been } \\
\text { classified into } 22 \text { classes by an SOFM neural network. }\end{array}$ \\
\hline merge_cat_10 & $\begin{array}{l}\text { Combine } 10 \text { grids (mat-files) to produce an array of pattern column } \\
\text { vectors excluding geology. The geology code in patterns is the } 22 \\
\text { rock-code contiguous code, which is converted to 1-of-n coding in a } \\
\text { separate step. }\end{array}$ \\
\hline merge_fuzzy_10_cat & $\begin{array}{l}\text { Combine } 10 \text { fuzzy membership input grids (mat-files) selected as the } \\
\text { best representatives of each evidence type to produce an array of } \\
\text { pattern column vectors. }\end{array}$ \\
\hline merge_fuzzy_17_cat & $\begin{array}{l}\text { Combine } 17 \text { fuzzy membership input grids (mat-files) selected as the } \\
\text { best representatives of each evidence type to produce an array of } \\
\text { pattern column vectors. }\end{array}$ \\
\hline merge_fuzzy_10_dda & $\begin{array}{l}\text { Combine } 10 \text { fuzzy membership input grids (mat-files) to produce an } \\
\text { array of pattern column vectors. Selection of layers: top } 10 \text { layers } \\
\text { ranked by } \mathrm{D} \times \mathrm{D} / \mathrm{A} \text {, where } \mathrm{D}=\% \text { of deposits } \& \mathrm{~A}=\% \text { of total area. }\end{array}$ \\
\hline merge_fuzzy_17_dda & $\begin{array}{l}\text { Combine } 17 \text { fuzzy membership input grids (mat-files) to produce an } \\
\text { array of pattern column vectors. Selection of layers: top } 10 \text { layers } \\
\text { ranked by } \mathrm{D} \times \mathrm{D} / \mathrm{A} \text {, where } \mathrm{D}=\% \text { of deposits } \& \mathrm{~A}=\% \text { of total area. }\end{array}$ \\
\hline merge_fuzzygeol_10_cat & $\begin{array}{l}\text { Combine } 10 \text { input grids (mat-files) selected as the best representatives } \\
\text { of each evidence type to produce an array of pattern column vectors. } \\
\text { Geology is represented as favourable geology using fuzzy membership } \\
\text { values. }\end{array}$ \\
\hline merge_fuzzygeol_10_DA & $\begin{array}{l}\text { Combine } 10 \text { input grids (mat-files) to produce an array of pattern } \\
\text { column vectors. Geology is represented as favourable geology using } \\
\text { fuzzy membership values. Selection of layers: top } 10 \text { layers ranked } \\
\text { by D/A } A N D D \geq \sim 20 \% \text {. }\end{array}$ \\
\hline
\end{tabular}


Table F.1: continued

\begin{tabular}{|c|c|}
\hline Script/Function Name & Application \\
\hline merge_fuzzygeol_10_DDA & $\begin{array}{l}\text { Combine } 10 \text { input grids (mat-files) to produce an array of pattern } \\
\text { column vectors. Geology is represented as favourable geology using } \\
\text { fuzzy membership values. Selection of layers: top } 10 \text { layers ranked } \\
\text { by } D \times(D / A) \text {. }\end{array}$ \\
\hline merge_fuzzygeol_10_DDA2 & $\begin{array}{l}\text { Combine } 10 \text { input grids (mat-files) to produce an array of pattern } \\
\text { column vectors. Geology is represented as favourable geology using } \\
\text { fuzzy membership values. Selection of layers: top } 10 \text { layers ranked } \\
\text { by } \mathrm{D} \times(\mathrm{D} / \mathrm{A}) A N D \mathrm{D} \geq \sim 20 \% A N D \mathrm{D} / \mathrm{A} \geq 3 \text {. }\end{array}$ \\
\hline merge_fuzzygeol_17_cat & $\begin{array}{l}\text { Combine } 17 \text { layers chosen using "category "criteria. (See } \\
\text { merge_fuzzygeol_10_cat above.) }\end{array}$ \\
\hline merge_fuzzygeol_17_DA & $\begin{array}{l}\text { Combine } 17 \text { layers chosen using "D/A "criteria. } \\
\text { merge_fuzzygeol_10_DA above.) }\end{array}$ \\
\hline merge_fuzzygeol_17_DDA & $\begin{array}{l}\text { Combine } 17 \text { layers chosen using "DDA "criteria. } \\
\text { merge_fuzzygeol_10_DDA above.) }\end{array}$ \\
\hline merge_fuzzygeol_17_DDA2 & $\begin{array}{l}\text { Combine } 17 \text { layers chosen using "DDA2 "criteria. } \\
\text { merge_fuzzygeol_10_DDA2 above.) }\end{array}$ \\
\hline merge_fuzzygeol_25 & Combine 25 layers included in all selections of layers used above. \\
\hline merge_geolsubs_14 & $\begin{array}{l}\text { Combine } 14 \text { layers including substitutes for solid geology. The follow- } \\
\text { ing layers are used in place of solid geology; rock mechanical prop- } \\
\text { erties L2_l_rh5 = uniaxial compressive strength, L2_l_rh7 = fracture } \\
\text { toughness, L2_l_rh4 = Young's Modulus, and sulphidation and oxida- } \\
\text { tion ratio layers; } \mathrm{L} 3 \_l \_c h 2=\mathrm{Fe}(\text { total wt\% }) \text { oxides } \times \mathrm{Fe} /(\mathrm{Fe}+\mathrm{Mg}+\mathrm{Ca}) \text {, } \\
\text { and L3_l_ch3 }=\mathrm{Fe}_{2} \mathrm{O}_{3} /\left(\mathrm{Fe}_{2} \mathrm{O}_{3}+\mathrm{FeO}\right) .\end{array}$ \\
\hline noiseNorm & $\begin{array}{l}\text { Randomly modify an input scalar by a normally distributed random } \\
\text { number with mean }=1 \text {, variance }=1 \text { and standard distribution }= \\
\text { 1. Used to augment neural network training data sets by randomly } \\
\text { adding or subtracting noise. }\end{array}$ \\
\hline noiseUni & $\begin{array}{l}\text { Randomly modify an input scalar by a percentage of its value spec- } \\
\text { ified by parameter "percent" (uniform random noise). Used to aug- } \\
\text { ment neural network training data sets by randomly adding or sub- } \\
\text { tracting noise. }\end{array}$ \\
\hline randselect_batch & $\begin{array}{l}\text { Calls function randselect_vec to create train, training-stop and test } \\
\text { data sets of patterns from deposit and non-deposit MATLAB arrays. }\end{array}$ \\
\hline
\end{tabular}


Table F.1: continued

\begin{tabular}{|c|c|}
\hline Script/Function Name & Application \\
\hline randselect_batch_som_all & $\begin{array}{l}\text { Version of } \text { randselect_batch to select patterns that have been classified } \\
\text { into } 22 \text { classes by an SOFM network. }\end{array}$ \\
\hline randselect_batch_som_cntr_edge & $\begin{array}{l}\text { Version of randselect_batch to select patterns that have been classified } \\
\text { into } 22 \text { classes by an SOFM network and which fall into the upper or } \\
\text { lower } 25 \text { percentile of distancetocentroidofdepositfeaturevectors. }\end{array}$ \\
\hline randselect_1ofn_batch & $\begin{array}{l}\text { Version of randselect_batch to select patterns in which the rock type } \\
\text { is coded in 1-of-n format. }\end{array}$ \\
\hline randselect_vec_1 & $\begin{array}{l}\text { Randomly select one set of } N \text { patterns from a input MATLAB array } \\
\text { consisting of rows of patterns. } N \text { is specified in the function call. }\end{array}$ \\
\hline randselect_vec & $\begin{array}{l}\text { Create } 3 \text { sets of deposits patterns from a input MATLAB array con- } \\
\text { sisting of rows of patterns. Used to create training sets with different } \\
\text { numbers of deposits. }\end{array}$ \\
\hline rowpats2colpt & $\begin{array}{l}\text { Split mat-arrays containing training, test, and training-stop patterns } \\
\text { into separate pattern and target arrays (and cell arrays), P, T, and } \\
\text { a structure containing the training-stop patterns and target values } \\
\text { (V.P, and V.P). The patterns are also converted from row = patterns } \\
\text { to columns = patterns. }\end{array}$ \\
\hline rowpats2colpt_train & $\begin{array}{l}\text { Version of the function above used to create } \mathrm{P}, \mathrm{T} \text { training sets for } \\
\text { SOFM } 1 \mathrm{x} 9 \text { selected training sets for patterns close to centre of the } \\
\text { class centroids (CENTRE) and, in a separate step, for patterns at } \\
\text { the edge of the cluster (EDGE). }\end{array}$ \\
\hline rowpats2colpt_valid_test & $\begin{array}{l}\text { Version of the function above used to create training-stop (VV) and } \\
\text { test (TV) sets for SOFM 1x9 selected training sets for patterns close } \\
\text { to centre of the class centroids (CENTRE) and, in a separate step, } \\
\text { for patterns at the edge of the cluster (EDGE). }\end{array}$ \\
\hline selectdeps_1ofn_vecbinvec & $\begin{array}{l}\text { Select deposit patterns from a mat-array containing all training pat- } \\
\text { terns and create separate files for patterns corresponding to each rock } \\
\text { type. Use for training patterns in which geology is represented by } 1 \text { - } \\
\text { of-n codes values. }\end{array}$ \\
\hline selectdeps_fuzzy_vecbinvec & $\begin{array}{l}\text { Split a mat-array containing training patterns into separate deposit } \\
\text { arrays per rock type or SOFM class resulting in files; d1, d2, ..d22. }\end{array}$ \\
\hline
\end{tabular}


Table F.1: continued

\begin{tabular}{|c|c|}
\hline Script/Function Name & Application \\
\hline selectdeps_fuzzy_som_pcntl2 & $\begin{array}{l}\text { Split deposit patterns from a mat-array containing all training pat- } \\
\text { terns into separate deposit arrays per SOFM class resulting in files; } \\
\mathrm{d} 1, \mathrm{~d} 2, \ldots \mathrm{d} 22 \text {. }\end{array}$ \\
\hline selectgeol_1ofn_vecbinvec & As selectdeps_1ofn_vecbinvec above but selects non-deposit patterns. \\
\hline selectgeol_fuzzy_vecbinvec & As selectdeps_fuzzy_vecbinvec above but selects non-deposit patterns. \\
\hline selectgeol_fuzzy_som_pcntl2 & $\begin{array}{l}\text { As selectdeps_fuzzy_som_pcntl2 above but selects non-deposit } \\
\text { patterns. }\end{array}$ \\
\hline select_trainset_10 & $\begin{array}{l}\text { Select the } 10 \text { th split of training patterns into training, training-stop, } \\
\text { and test data sets. }\end{array}$ \\
\hline select_trainset_10_1ofN & ript above for 1-of-n coded geology training patterns. \\
\hline
\end{tabular}

\section{Neural Network Training}

newpnn_purelin

pnn_find_best_sigma2

pnn_test3

som_class_batch1x9

som_class_batch1x22

som_train_batch1x9

som_classifn_test

som_make_train_pproc_batch
Modification of MATLAB function, newpnn, which creates a new probabilistic neural network. The modified version produces probability values as outputs instead of a classification of the inputs in the form of a class label; e.g. $1=$ deposit and $2=$ non-deposit.

Determine optimal value of spread (= sigma) for radial basis functions used in a PNN network by plotting the performance for the test data set versus spread over a large range of values.

Test a modified version of a PNN with linear outputs.

Run a trained SOFM neural network (with a $1 \times 9$ output layer to classify an input array consisting of rows of map pattern vectors.

Run a trained SOFM neural network (with a $1 \times 22$ output layer to classify an input array consisting of rows of map pattern vectors.

Train a SOFM neural network with an output layer with a $1 \times 9$ topology in order to classify an input array containing feature vectors.

Run a test example (hard-coded) showing how a set of feature vectors can be classified using a SOFM neural network using the training algorithm trainr.

Create training set, train, produce a 22-class prospectivity map and assess the quality of the map for 10 SOFM networks. 
Table F.1: continued

\begin{tabular}{|c|c|}
\hline Script/Function Name & Application \\
\hline som_train_batch1x22 & $\begin{array}{l}\text { Train a SOFM neural network with an output layer with a } 1 \times 22 \\
\text { topology in order to classify an input array containing feature vectors }\end{array}$ \\
\hline som_train_batch1x9 & $\begin{array}{l}\text { Train a SOFM neural network with an output layer with a } 1 \times 9 \\
\text { topology in order to classify an input array containing feature vectors. }\end{array}$ \\
\hline train_mlp_gd_10_20_1 & $\begin{array}{l}\text { Train an 10-20-1 MLP neural network with traingd algorithm; loads } \\
\text { input arrays, sets and prints parameter values and saves trained net- } \\
\text { work weights. Versions of this function exist for each network topol- } \\
\text { ogy used. }\end{array}$ \\
\hline train_mlp_gdx_10_20_1 & $\begin{array}{l}\text { Train an 10-20-1 MLP neural network with traingdx algorithm (see } \\
\text { above). }\end{array}$ \\
\hline train_mlp_lm_10_20_1 & $\begin{array}{l}\text { Train an 10-20-1 MLP neural network with trainlm algorithm (see } \\
\text { above). }\end{array}$ \\
\hline train_pnn & Train an PNN neural network (see above). \\
\hline train_post_proc_batch & $\begin{array}{l}\text { Train network and assess map quality of resultant mineral prospec- } \\
\text { tivity map for } 10 \text { networks per training data set. }\end{array}$ \\
\hline \multicolumn{2}{|l|}{ Post-processing } \\
\hline act2map & Create map from a row vector of neural network output activations. \\
\hline add_nodata & Add Nodata values from geology to prospectivity map. \\
\hline mapclass & $\begin{array}{l}\text { Convert input array (mat-file) containing fuzzy membership values } \\
\text { or network output activations to } 9 \text { classes of mineral prospectivity. } \\
\text { The classes represent equal intervals. }\end{array}$ \\
\hline mapclass_eucdist & $\begin{array}{l}\text { Convert input array (mat-file) containing Euclidean distances of } \\
\text { grid cell vectors to the centroid of the deposit vector class or most } \\
\text { deposit-like class. For example, the SOFM prospectivity map } \\
\text { som_dist_nclass9, contains cell values representing the distance to the } \\
\text { neuron representing class } 9 \text { (i.e. class containing the most deposits } \\
\text { or class with the highest P(D). This function converts the input map } \\
\text { to a conventional 9-class prospectivity map. }\end{array}$ \\
\hline add_depdata_2_pat & $\begin{array}{l}\text { Add deposit name, Easting, Northing, } \mathrm{X}, \mathrm{Y}, \mathrm{TCG} \text { to training pattern } \\
\text { vectors to produce an array structures. }\end{array}$ \\
\hline
\end{tabular}


Table F.1: continued

\begin{tabular}{|c|c|}
\hline Script/Function Name & Application \\
\hline post_proc_batch & $\begin{array}{l}\text { Batch job to convert a trained MLP network and an array containing } \\
\text { input patterns and target values into a prospectivity map. The script } \\
\text { also analyses the quality of the map. }\end{array}$ \\
\hline post_proc_batch_1ofn & $\begin{array}{l}\text { Version of batch job described above for network using a 1-of-n coded } \\
\text { lithology input. }\end{array}$ \\
\hline post_proc_batch_fuzzy & $\begin{array}{l}\text { Version of batch job described above for network using fuzzy mem- } \\
\text { bership values as inputs. }\end{array}$ \\
\hline post_proc_batch_pnn & $\begin{array}{l}\text { Batch job to convert a trained PNN network and an array containing } \\
\text { input patterns and target values into a prospectivity map. The script } \\
\text { also analyses the quality of the map. }\end{array}$ \\
\hline reclass_som_class_map & $\begin{array}{l}\text { Reclassify the values in a map that has been classified using a } \\
1 \times 22 \text { SOFM network so that the class numbers represent increasing } \\
\text { prospectivity. }\end{array}$ \\
\hline scalemat09vec & $\begin{array}{l}\text { Scale array containing neural network activations prior to producing } \\
\text { a 9-class prospectivity map. }\end{array}$ \\
\hline training_set_perf & $\begin{array}{l}\text { Calculate the performance of a trained network on the training, } \\
\text { training-stop and test data sets. Calculate overall percentage of pat- } \\
\text { terns correct, and percentages of deposits and non-deposits correct } \\
\text { based on an activation threshold of } 0.5 \text {. }\end{array}$ \\
\hline training_set_perf & $\begin{array}{l}\text { Version of function above for neural networks with fuzzy membership } \\
\text { inputs. }\end{array}$ \\
\hline \multicolumn{2}{|c|}{ Fuzzy Systems - Creating Input Layers } \\
\hline batch_layer2fav_0110_500 & $\begin{array}{l}\text { Batch job to reclassify input layers to fuzzy membership values using } \\
\text { trapezoidal fuzzy membership functions. Fuzzy membership value of } \\
0.1 \text { was assigned to all cells where distance to nearest lithological } \\
\text { contact } \geq 500 \text { metres. }\end{array}$ \\
\hline batch_layer2fav_0510_500 & $\begin{array}{l}\text { As above but fuzzy membership value of } 0.5 \text { was assigned to all cells } \\
\text { where distance to nearest lithological contact } \geq 500 \text { metres. }\end{array}$ \\
\hline check_maxmin_fuzzy_batch & $\begin{array}{l}\text { Batch job to check the result of converting input layers to fuzzy mem- } \\
\text { bership values by calculating and displaying the max. and min. value } \\
\text { for all layers used as inputs to a fuzzy logic prospectivity analysis. }\end{array}$ \\
\hline
\end{tabular}


Table F.1: continued

\begin{tabular}{|c|c|}
\hline Script/Function Name & Application \\
\hline check_contact_type_fuzzy_vals & $\begin{array}{l}\text { Check fuzzy } \mathrm{m} / \text { ship values assigned to each contact type in a fuzzy } \\
\text { contact-type layer. }\end{array}$ \\
\hline check_litho_fuzzy_vals & $\begin{array}{l}\text { Check fuzzy } \mathrm{m} / \mathrm{ship} \text { values assigned to each rock type in a fuzzy } \\
\text { lithology-type layer. }\end{array}$ \\
\hline deps_cont_type & $\begin{array}{l}\text { Calculate number of cells, deposits, TCG, D/A, TCG/A, TCG/cell, } \\
\text { and } \mathrm{P}\left(\mathrm{D} / \mathrm{C}_{k}\right) \times \mathrm{TCG} / \text { cell for each contact type within a mboxdis- } \\
\text { tance } \leq \text { LIMIT from the nearest lithological contact. }\end{array}$ \\
\hline deps_litho & $\begin{array}{l}\text { Calculate number of cells, deposits, TCG, D/A and } \mathrm{P}\left(\mathrm{D} / \mathrm{C}_{k}\right) \times \\
\text { TCG/cell for each lithology type (see above). }\end{array}$ \\
\hline favcontact25_pr0110 & $\begin{array}{l}\text { Create a fuzzy membership layer for favourable contact type based on } \\
T C G \text { by reclassifying a contact type layer. Fuzzy membership value } \\
\text { of } 0.1 \text { was assigned to all cells where distance to nearest lithological } \\
\text { contact } \geq 500 \mathrm{~m} \text {. }\end{array}$ \\
\hline favcontact25_pr0510 & $\begin{array}{l}\text { As above but fuzzy membership value of } 0.5 \text { was assigned to all cells } \\
\text { where distance to nearest lithological contact } \geq 500 \mathrm{~m} \text {. }\end{array}$ \\
\hline favcontact25_pxtcg0110 & $\begin{array}{l}\text { Create a fuzzy membership layer for favourable contact type based } \\
\text { on } \mathrm{P}\left(\mathrm{D} / \mathrm{C}_{k}\right) \times \mathrm{TCG} / \text { cell by reclassifying a contact type layer. Fuzzy } \\
\text { membership value of } 0.1 \text { was assigned to all cells where distance to } \\
\text { nearest lithological contact } \geq 500 \mathrm{~m} \text {. }\end{array}$ \\
\hline favcontact25_pxtcg0510 & $\begin{array}{l}\text { As above but fuzzy membership value of } 0.5 \text { was assigned to all cells } \\
\text { where distance to nearest lithological contact } \geq 500 \mathrm{~m} \text {. }\end{array}$ \\
\hline favgeol25_pr0110 & $\begin{array}{l}\text { Create a fuzzy membership layer for favourable geology (based on } \\
\mathrm{P}(\mathrm{D}) \text { ) by reclassifying a geology GIS layer. Rocks types that do not } \\
\text { contain known deposits were assigned a fuzzy membership value of } \\
0.1 \text {. }\end{array}$ \\
\hline favgeol25_pr0510 & $\begin{array}{l}\text { As above but rocks types that do not contain known deposits were } \\
\text { assigned a fuzzy membership value of } 0.5 \text {. }\end{array}$ \\
\hline layer2fav_batch & Batch job to Reclassify an input layer to fuzzy membership values. \\
\hline layer2fav & $\begin{array}{l}\text { Reclassify an input layer to fuzzy membership values using trape- } \\
\text { zoidal fuzzy membership functions. }\end{array}$ \\
\hline trap & $\begin{array}{l}\text { Convert values of an input vector into fuzzy membership values using } \\
\text { a trapezoidal fuzzy membership function. }\end{array}$ \\
\hline
\end{tabular}


Table F.1: continued

\begin{tabular}{|c|c|}
\hline Script/Function Name & Application \\
\hline traplhs & $\begin{array}{l}\text { Asymmetric trapezoidal fuzzy membership function-as above but } \\
\text { with fuzzy membership function starts with low values of } X \text { set to a } \\
\text { fuzzy membership }=1.0 \text { and at an inflection point decreases linearly } \\
\text { to a second inflection point where } Y=0.0 \text { or } 0.1 \text {. }\end{array}$ \\
\hline traprhs & $\begin{array}{l}\text { Asymmetric trapezoidal fuzzy membership function - as above but } \\
\text { with fuzzy membership function starts with low values of } X \text { set to a } \\
\text { fuzzy membership }=0.0 \text { or } 0.1 \text { and at an inflection point increases } \\
\text { linearly to a second inflection point where } Y=1.0 \text {. }\end{array}$ \\
\hline $\begin{array}{l}\text { Fuzzy Systems - Making } \\
\text { fuzzy_make_analyze_map_batch }\end{array}$ & $\begin{array}{l}\text { Prospectivity Maps } \\
\text { Batch job to create fuzzy systems prospectivity maps using calls to } \\
\text { function fuzzy_maps_gamma2 (see below). }\end{array}$ \\
\hline fuzzy_maps_gamma2 & $\begin{array}{l}\text { Creates a series of prospectivity maps for a particular set of input } \\
\text { layers (10 or } 17 \text { ) using a range of gamma values as well as AND, OR, } \\
\text { product and sum. Output are classed and map quality statistics are } \\
\text { calculated and written to an ASCII log file. }\end{array}$ \\
\hline
\end{tabular}

\section{Prospectivity Map Analysis}

act2histo_nodata

kendalls

map_quality

map_quality_som_class_map

mapstats

mapstats_binary

map2histo_nodata
Plot an histogram of output activations from a trained neural network (exclude Nodata map areas).

Calculate Kendall's rank correlation coefficient for data in two input vectors.

Calculate map quality statistics and produce plots used to assess the quality of a map.

Calculate map quality statistics and produce plots used to assess the quality of a the map, som_reclass_map, classified by an SOFM using a $1 \times 9$ output layer.

Calculate statistics used to compare input layers and output maps as predictors of the mineral deposits.

Version of mapstats used for binary input layers where the favourable zones $=1$ and the rest $=0$.

Plots histogram of raw neural network or fuzzy systems map cell values. 
Table F.1: continued

\begin{tabular}{|c|c|}
\hline Script/Function Name & Application \\
\hline maxmin_actmap & $\begin{array}{l}\text { Calculate the maximum and minimum output values for raw neural } \\
\text { network or fuzzy system prospectivity map. }\end{array}$ \\
\hline roc_curve & Plot Receiver Operating Characteristic (ROC) curves. \\
\hline roc_curve_pnn & $\begin{array}{l}\text { Version of function above for maps created with probabilistic } \\
\text { networks. }\end{array}$ \\
\hline roc_curve_som_class & Version of function above for maps created with SOFM networks. \\
\hline run_net_2_make_actmap & $\begin{array}{l}\text { Run a trained network to produce an output activation map and save } \\
\text { it. }\end{array}$ \\
\hline run_net_2_make_actmap_batch & $\begin{array}{l}\text { Batch job to run a trained network to produce an output activation } \\
\text { map (see above). }\end{array}$ \\
\hline spearmans & $\begin{array}{l}\text { Calculate Spearman's rank correlation coefficient for data in two in- } \\
\text { put vectors. Called by function, mapsstats to calculate statistic for } \\
\text { the relationship between the classes in a mineral prospectivity map } \\
\text { and the probability of a known deposit occurring in the area corre- } \\
\text { sponding to a particular map class. }\end{array}$ \\
\hline training_set_perf & $\begin{array}{l}\text { Calculate the performance of a trained network on the training, } \\
\text { training-stop and test data sets. Calculate overall percentage of pat- } \\
\text { terns correct, and percentages of deposits and non-deposits correct } \\
\text { based on an activation threshold of } 0.5 \text {. }\end{array}$ \\
\hline training_set_perf_fuzzy & Version of function training_set_perf for fuzzy systems maps. \\
\hline training_set_perf_pnn & Version of function training_set_perf for PNN maps. \\
\hline \multicolumn{2}{|l|}{ Figure Preparation } \\
\hline batch_add_nodata & $\begin{array}{l}\text { adds Nodata values to raw neural network output activation and } \\
\text { fuzzy systems fuzzy-membership maps and converts resultant maps } \\
\text { to ASCII format prior to importing maps into the software package, } \\
\text { GEOSOFT Oasis Montaj. }\end{array}$ \\
\hline batch_change_fig_props & $\begin{array}{l}\text { Changes Matlab figure properties; e.g. axis labels \& font size and } \\
\text { exports new file to Postscript format for a group of figures listed in } \\
\text { a file. }\end{array}$ \\
\hline fig_logistic & eate plot of sigmoidal logistic activation function. \\
\hline
\end{tabular}


Table F.1: continued

\begin{tabular}{|c|c|}
\hline Script/Function Name & Application \\
\hline fig_parzen & $\begin{array}{l}\text { Batch job to create a perspective plot of spherical Gaussian radial } \\
\text { basis functions used in Parzen window PDF. Calls functions parzen } \\
\text { and set_up_plot. }\end{array}$ \\
\hline parzen2 & $\begin{array}{l}\text { Calculate spherical Gaussian radial basis function used in the Parzen } \\
\text { PDF estimator. }\end{array}$ \\
\hline plot_run_traind_nn_roc_figs & $\begin{array}{l}\text { Batch job to run trained neural networks to create ROC curves, and } \\
\text { histogram of nn output activations. Raw output activation maps are } \\
\text { also created and saved. }\end{array}$ \\
\hline plot_som_reclass_deps_vs_class & $\begin{array}{l}\text { Plots a histogram of frequency of deposits for each of the } 9 \text { or } 22 \\
\text { classes of feature vectors defined by using an SOFM to classify the } \\
\text { patterns corresponding to all the grid locations in the map. SOFM } \\
\text { class labels are first reclassified so they are in order of in order of } \\
\text { increasing P(deposit - class). }\end{array}$ \\
\hline plot_som_reclass_PD_vs_class & $\begin{array}{l}\text { Plot histogram of } \operatorname{Pr}(\mathrm{D} \text { - class }) \text { vs SOFM class after reclassifying the } \\
\text { input map, som_class_map to give som_reclass_map. }\end{array}$ \\
\hline post_proc_batch_fuzzy_final_fig & $\begin{array}{l}\text { Creates fuzzy prospectivity maps, calculates map quality statistics } \\
\text { and plots ROC graphs. Called by plot_run_traind_nn_roc_figs. }\end{array}$ \\
\hline post_proc_batch_nn_final_fig & as above: for neural network maps. \\
\hline print_setup & Sets printing options prior to printing a figure. \\
\hline setup_parzen_plot & Set up Plot Aspect Ratio in function, fig_parzen. \\
\hline \multicolumn{2}{|l|}{ Utilities } \\
\hline array 2 bin & $\begin{array}{l}\text { Convert mat-file in memory to stored binary file. Data is written as } \\
\text { double precision floating point format (32-bit). }\end{array}$ \\
\hline array2bin_batch & $\begin{array}{l}\text { Batch job to save Matlab arrays as binary files. Use for } 10,17 \text { fuzzy } \\
\text { neural network inputs. }\end{array}$ \\
\hline array2bin_lge & $\begin{array}{l}\text { Convert .mat file in memory to stored binary file. Data is written as } \\
\text { double precision floating point format (32-bit) for very large arrays } \\
(>17 \times 1254000 \text { double precision floating point values). }\end{array}$ \\
\hline ascii2array & $\begin{array}{l}\text { Reads an ASCII file containing a single line header with } 2 \text { integers, } \\
\text { indicating the number of columns and the number of lines, into a } \\
\text { Matlab array. }\end{array}$ \\
\hline
\end{tabular}


Table F.1: continued

\begin{tabular}{|c|c|}
\hline Script/Function Name & Application \\
\hline batch_mat2ascii & $\begin{array}{l}\text { Batch job to convert a series of mat-files prospectivity maps) to ASCII } \\
\text { files with ARCVIEW headers suitable for importing into ArcView GIS. }\end{array}$ \\
\hline bin2array & Convert float32 binary file to a Matlab array file. \\
\hline bin2array_lines & $\begin{array}{l}\text { Convert float } 32 \text { very large binary files ( }>17 \times 1254000 \text { double preci- } \\
\text { sion floating point values) to a Matlab array file. }\end{array}$ \\
\hline count_data & Count data cells in a map grid (mat-array). \\
\hline histo & Plot a histogram of elements stored in a row vector. \\
\hline mat_flt_x_10002arc & $\begin{array}{l}\text { Convert mat-array file containing floating point values to an ASCII } \\
\text { file containing a header suitable for importing into ARCVIEW. Values } \\
\text { in the MATLAB file are multiplied by } 1000 \text { prior to rounding because } \\
\text { ArcView can only import integer ASCII grid files. }\end{array}$ \\
\hline mat_int2arc & $\begin{array}{l}\text { Convert mat-array containing integer values to file suitable for im- } \\
\text { porting into ARCVIEW. }\end{array}$ \\
\hline $\operatorname{maxmin}$ & $\begin{array}{l}\text { Calculate maximum and minimum value of an array with and without } \\
N o d a t a=-9999 \text { values. }\end{array}$ \\
\hline maxmin_noprint & $\begin{array}{l}\text { Version of maxmin that does not print results to screen; called from } \\
\text { within other functions. }\end{array}$ \\
\hline readbin_1stline & $\begin{array}{l}\text { Read first pattern in a binary file containing float } 32 \text { data and display } \\
\text { it on the screen. }\end{array}$ \\
\hline reclass & Reclassify the values in an input grid. \\
\hline
\end{tabular}


Appendix G

Evaluation of GIS Input Layers:

Kalgoorlie Case Study

G.1 Graphs used to evaluate spatial relationships in GIS layers 


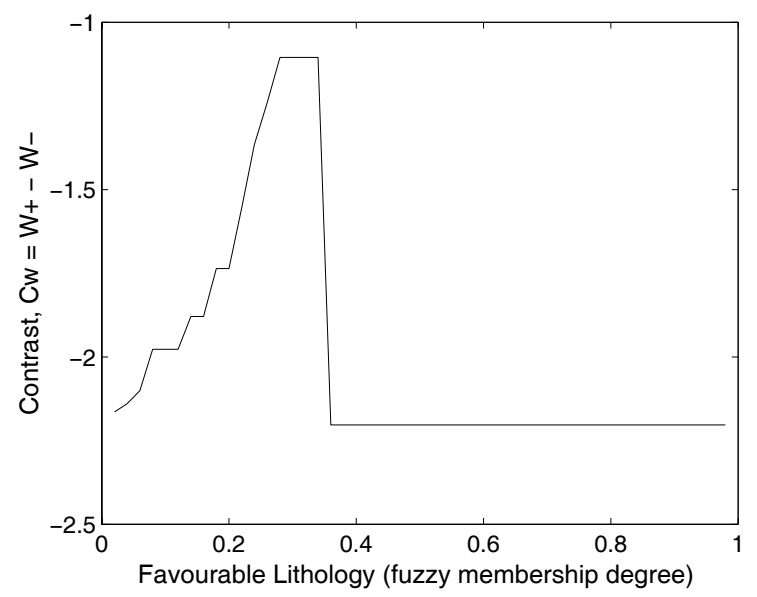

(a) Contrast, $C_{W}$

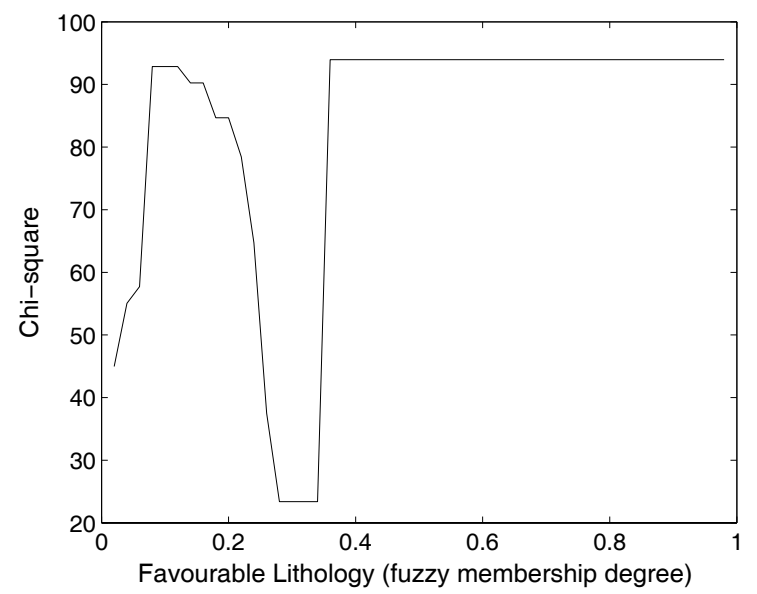

(c) Chi-squared

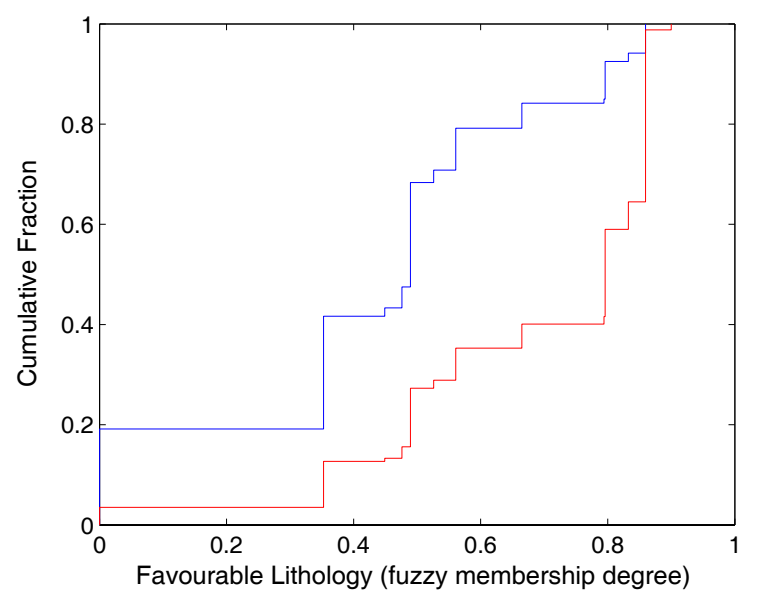

(e) Kolmogorov-Smirnov test

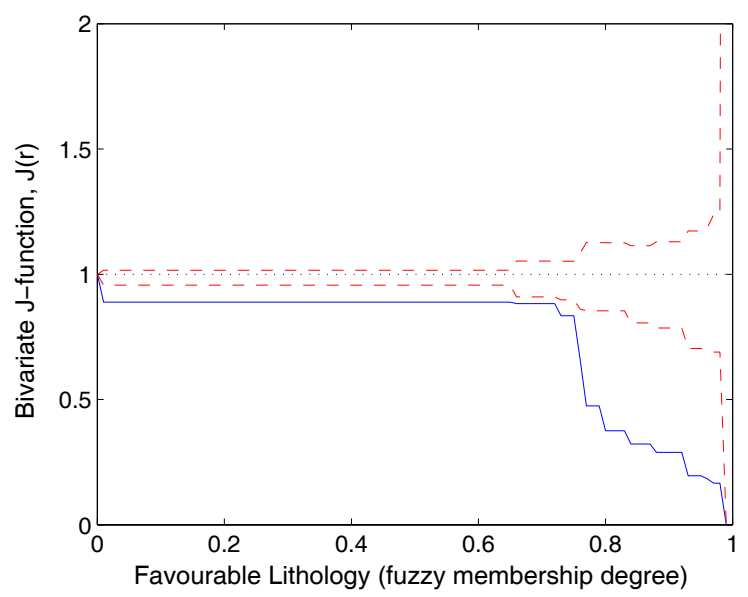

(b) Bivariate J-function (cumulative)

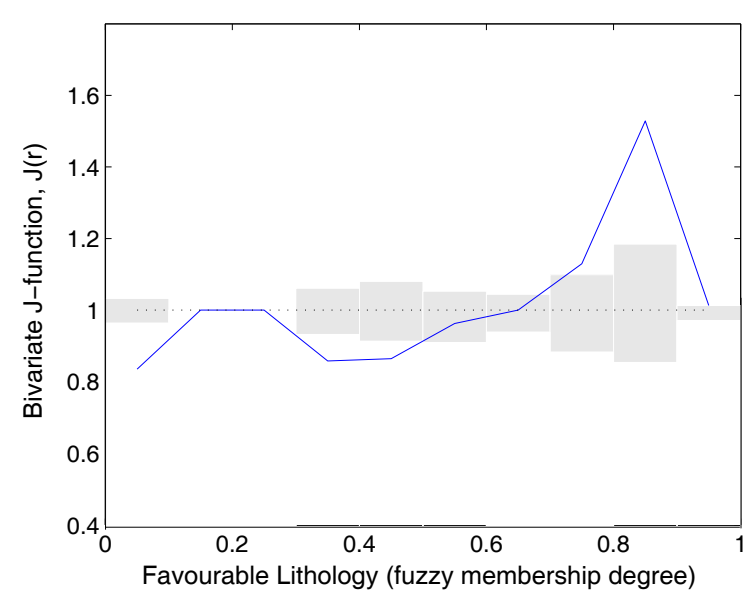

(d) Bivariate J-function (interval-based)

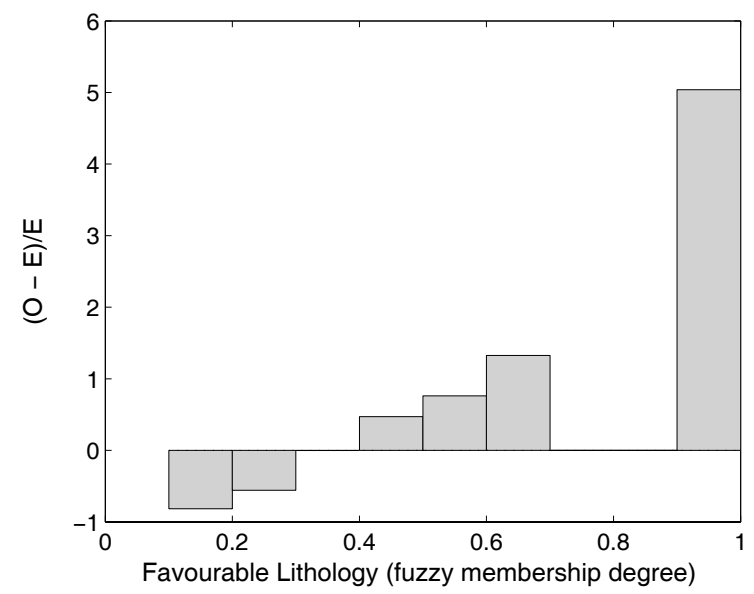

(f) $(O-E) / E$

Figure G.1. Plots used to examine the relationship between deposits and favourable lithology in layer l1favgeol25pr. The layer contains fuzzy membership values. The empirical curve is shown in blue in b), d) \& e). The theoretical curve is shown in red in b) \& e) and as grey bars in d). The $\mathrm{X}$-axis parameter in graphs a), b), c) \& d) was transformed to create a new parameter representing the difference between the maximum and the original parameter value; i.e., $x=X_{\max }-x$. 


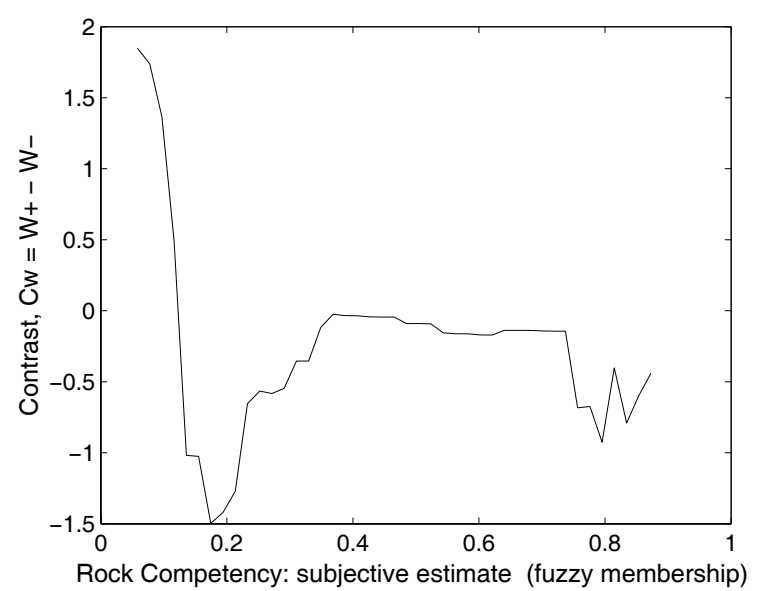

(a) Contrast, $C_{W}$

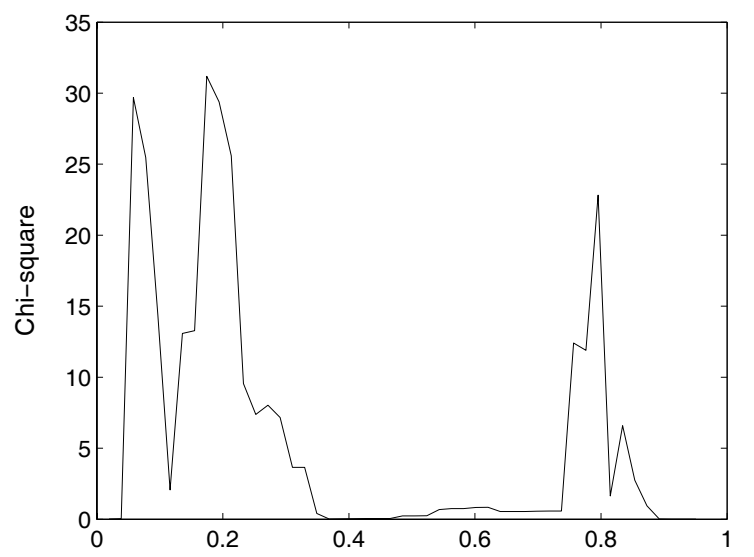

Rock Competency: subjective estimate (fuzzy membership)

(c) Chi-squared

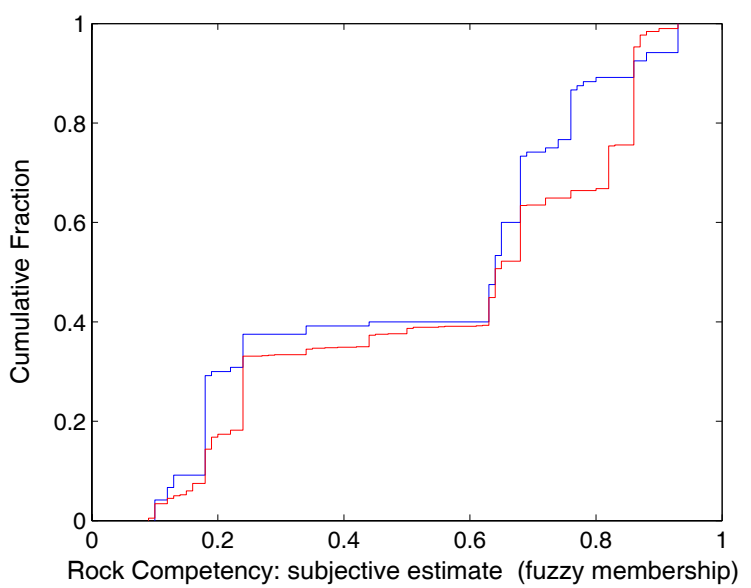

(e) Kolmogorov-Smirnov test

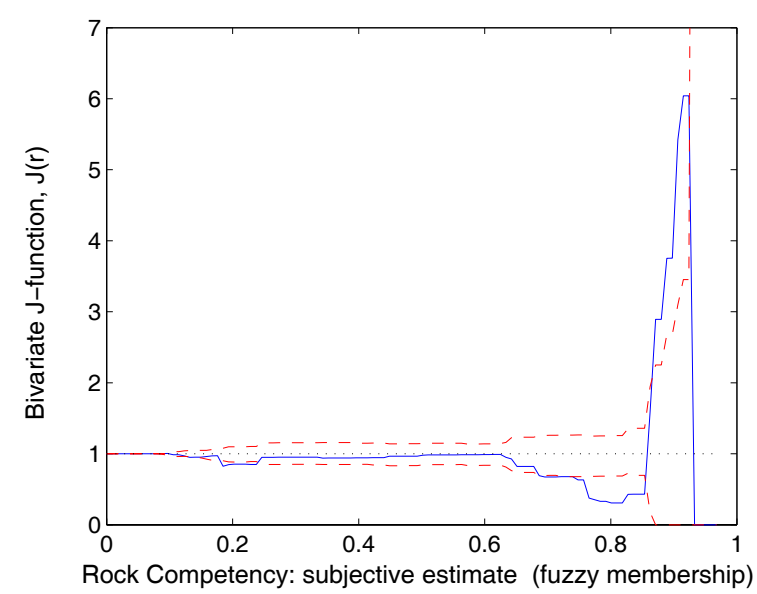

(b) Bivariate J-function (cumulative)

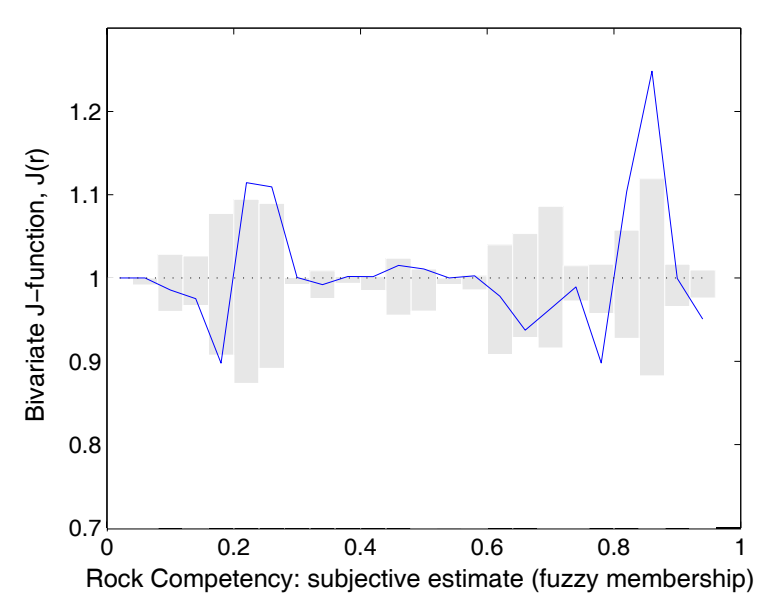

(d) Bivariate J-function (interval-based)

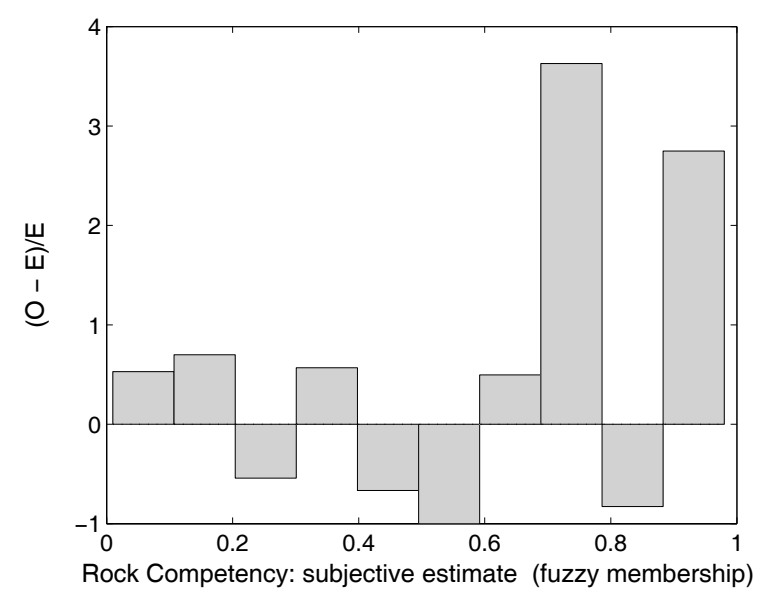

(f) $(O-E) / E$

Figure G.2. Plots used to examine the relationship between deposits and subjective (fuzzy) rock competency in the layer, l2_l_rh1b. The empirical curve is shown in blue in b), d) \& e). The theoretical curve is shown in red in b) \& e) and as grey bars in d). The X-axis parameter in graphs a), b) \& c) was transformed to create a new parameter representing the difference between the maximum and the original parameter value; i.e., $x=X_{\max .}-x$. 


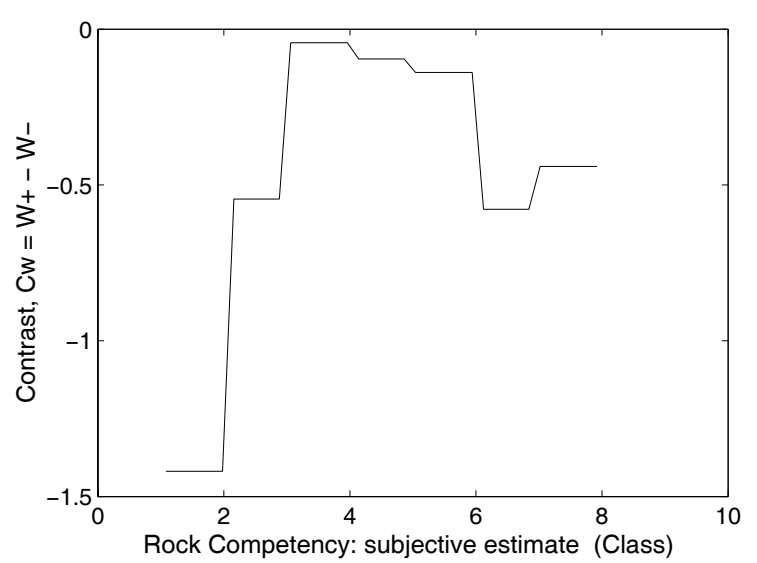

(a) Contrast, $C_{W}$

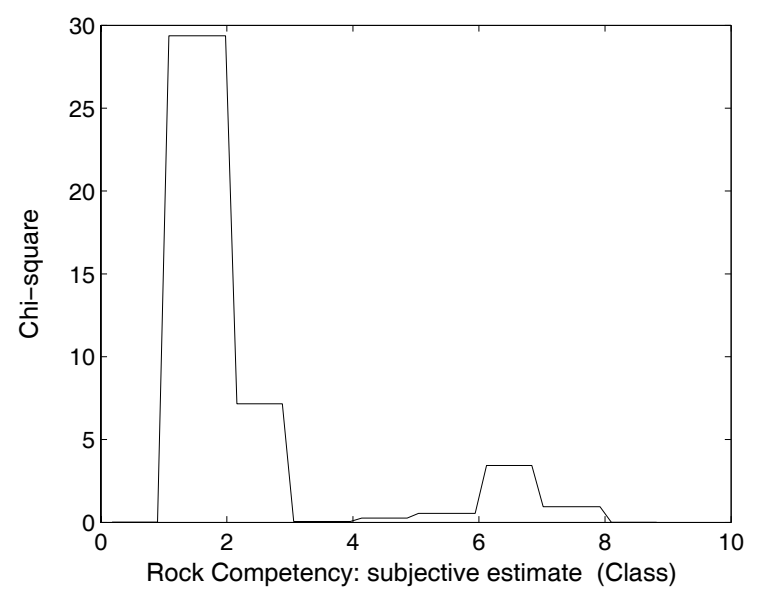

(c) Chi-squared

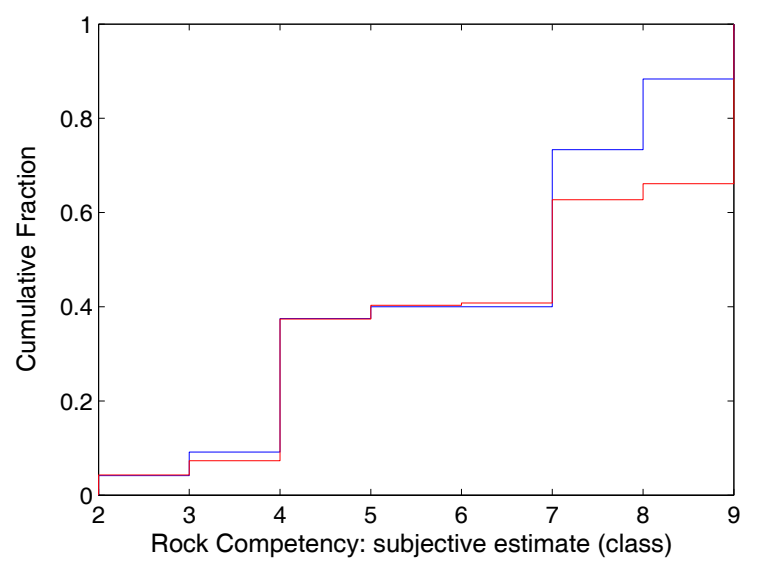

(e) Kolmogorov-Smirnov test

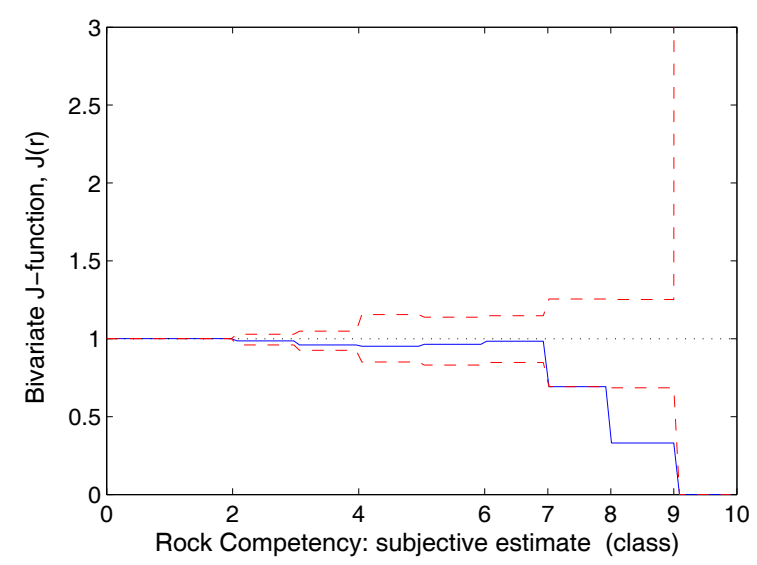

(b) Bivariate J-function (cumulative)

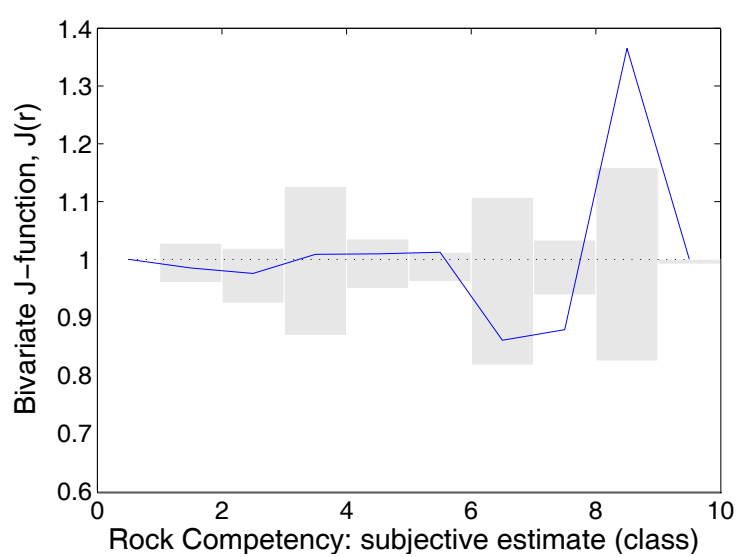

(d) Bivariate J-function (interval-based)

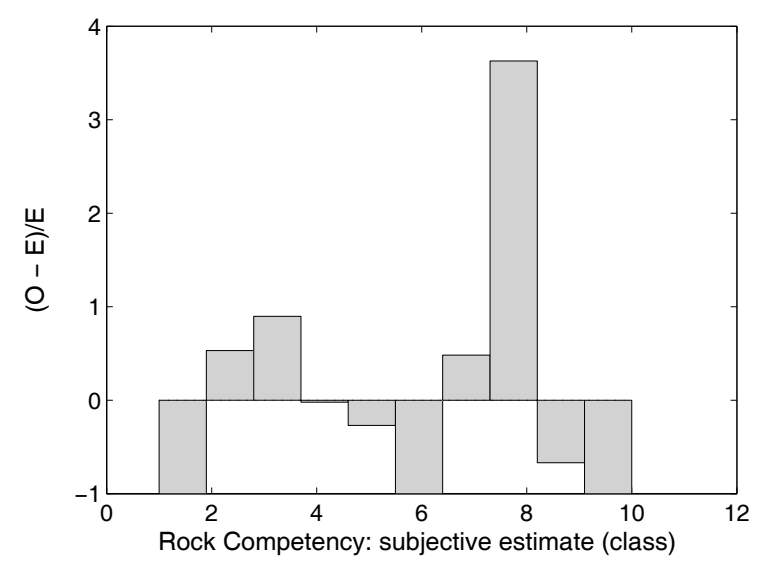

(f) $(O-E) / E$

Figure G.3. Plots used to examine the relationship between deposits and subjective (classes) rock competency in the layer, $12 \_l_{-} r h 2 b$. The empirical curve is shown in blue in b), d) \& e). The theoretical curve is shown in red in b) \& e) and as grey bars in d). The X-axis parameter in graphs a), b) \& c) was transformed to create a new parameter representing the difference between the maximum and the original parameter value; i.e., $x=X_{\max .}-x$. 


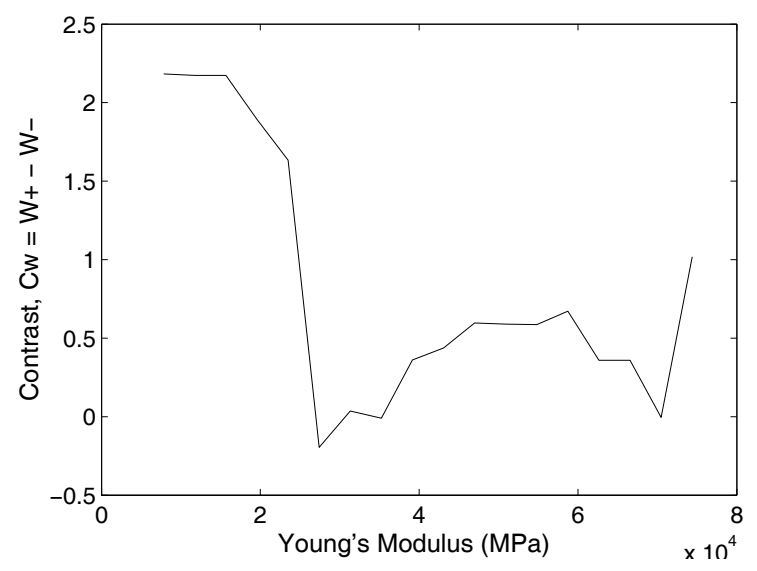

(a) Contrast, $C_{W}$

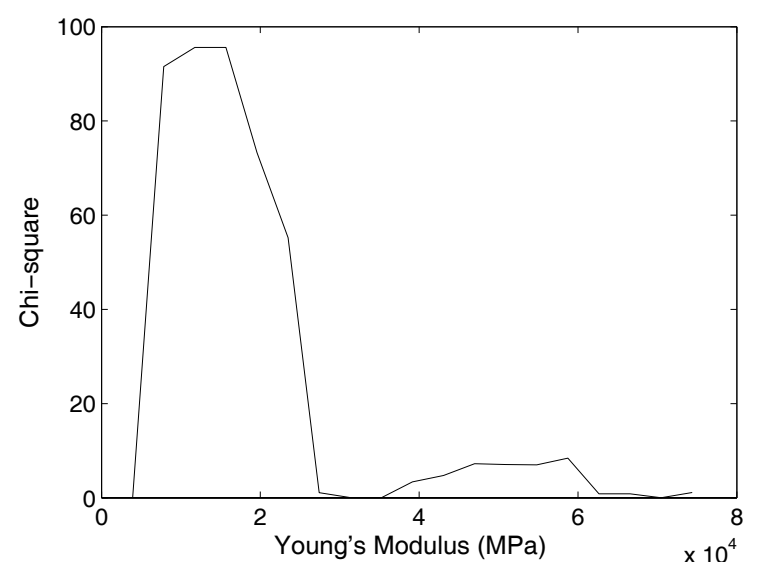

(c) Chi-squared

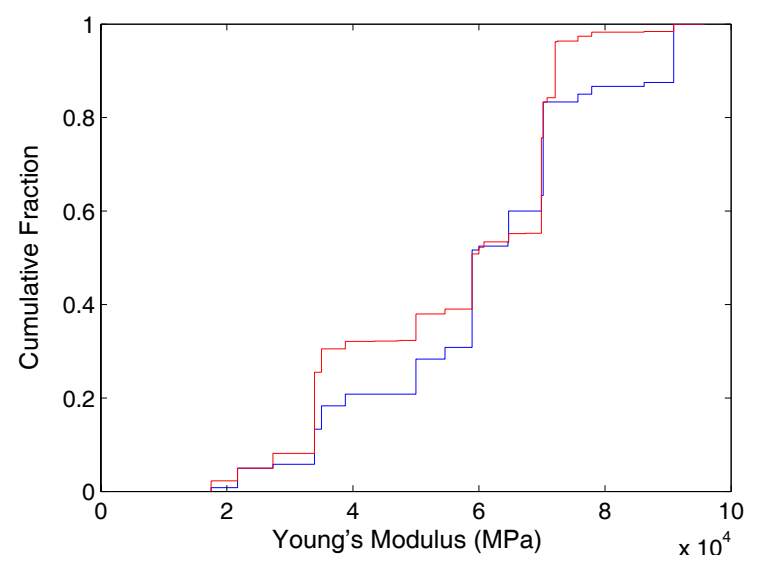

(e) Kolmogorov-Smirnov test

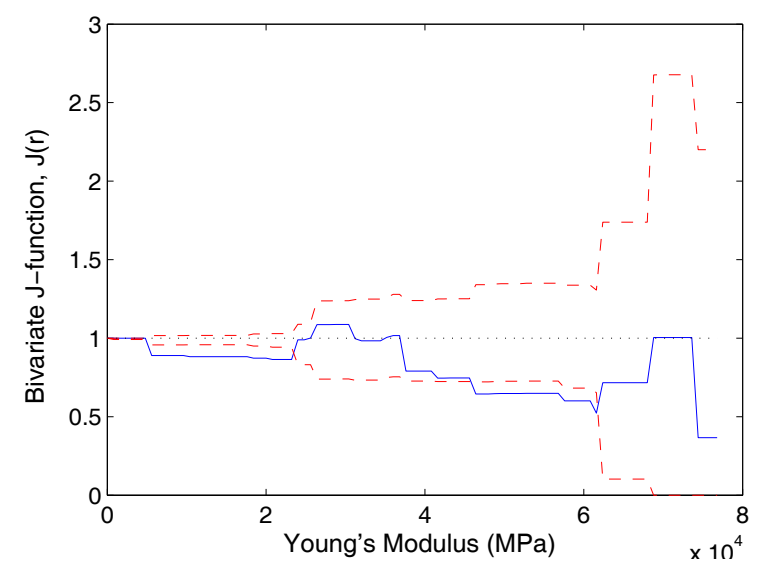

(b) Bivariate J-function (cumulative)

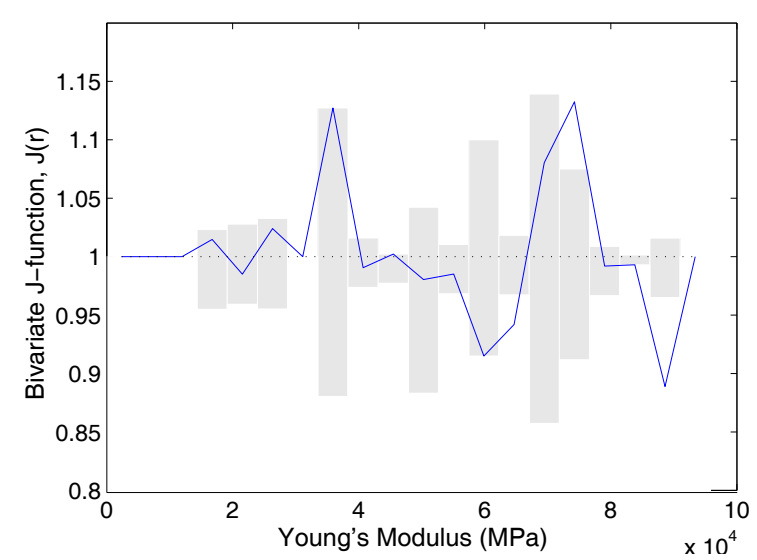

(d) Bivariate J-function (interval-based)

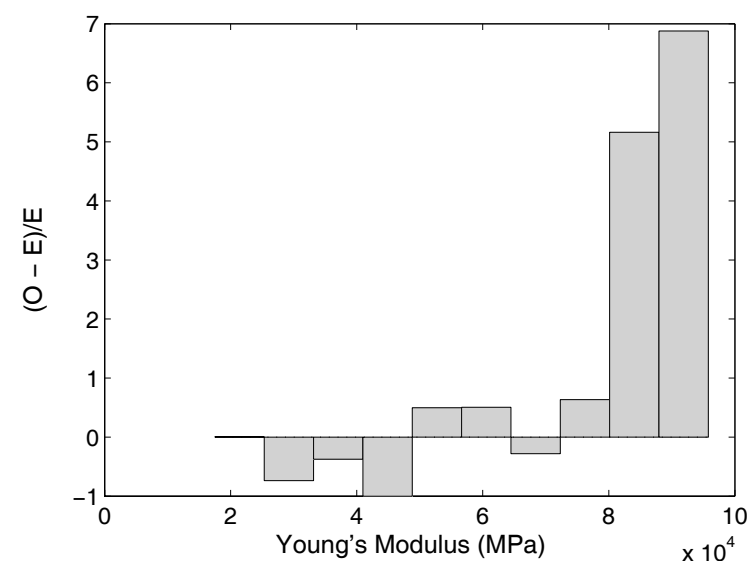

(f) $(O-E) / E$

Figure G.4. Plots used to examine the relationship between deposits and rock strength using Young's Modulus compiled from Hatheway \& Kiersch (1989) in the layer, l2_l_rh3. The empirical curve is shown in blue in b), d) \& e). The theoretical curve is shown in red in b) \& e) and as grey bars in $\mathrm{d}$ ). The $\mathrm{X}$-axis parameter in graphs a), b) \& c) was transformed to create a new parameter representing the difference between the maximum and the original parameter value; i.e., $x=X_{\max }-x$. 


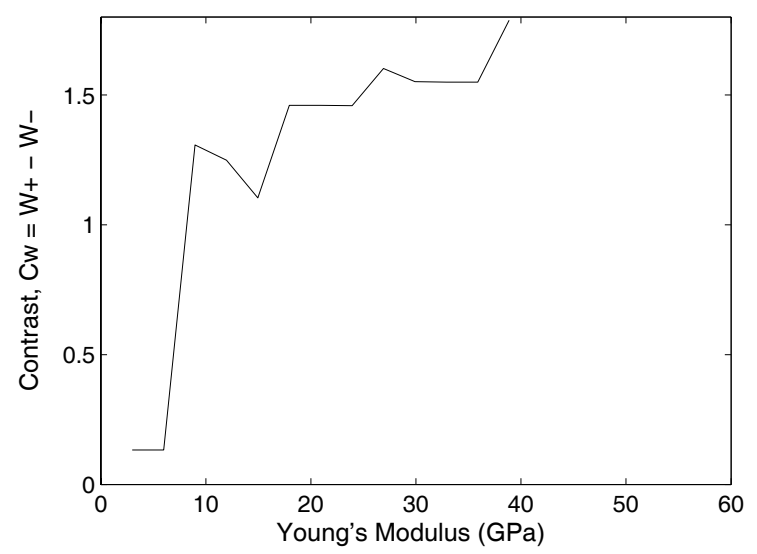

(a) Contrast, $C_{W}$

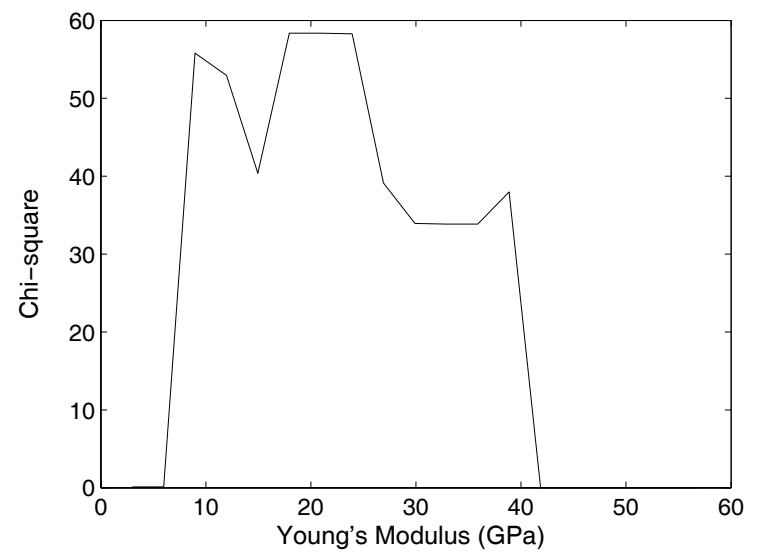

(c) Chi-squared

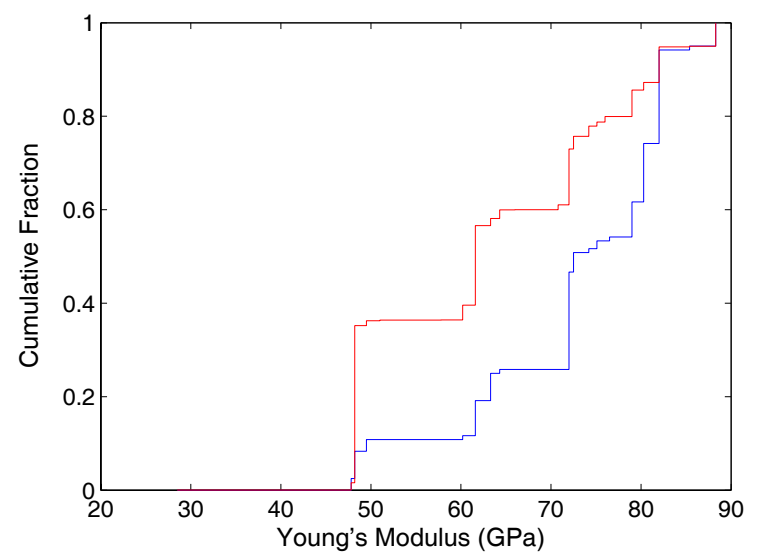

(e) Kolmogorov-Smirnov test

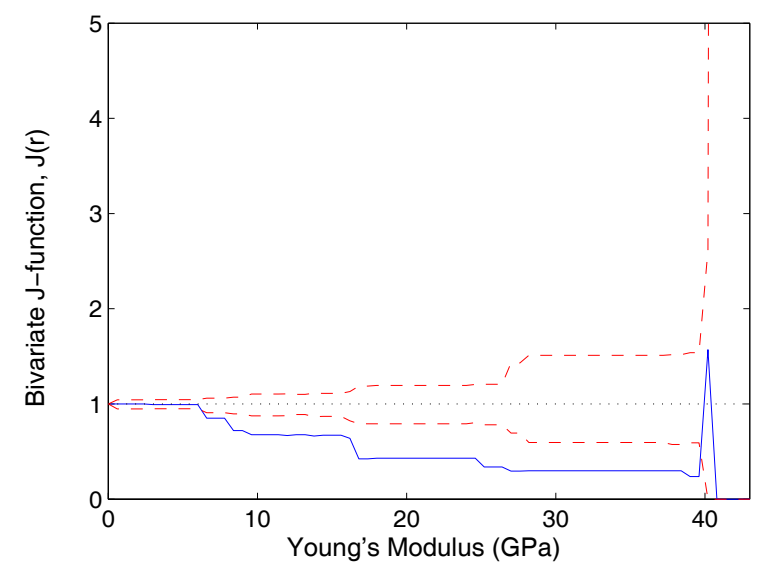

(b) Bivariate J-function (cumulative)

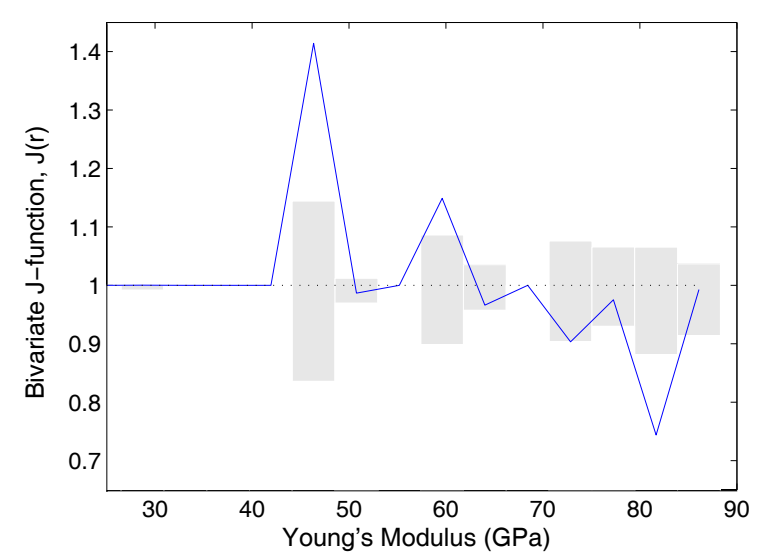

(d) Bivariate J-function (interval-based)

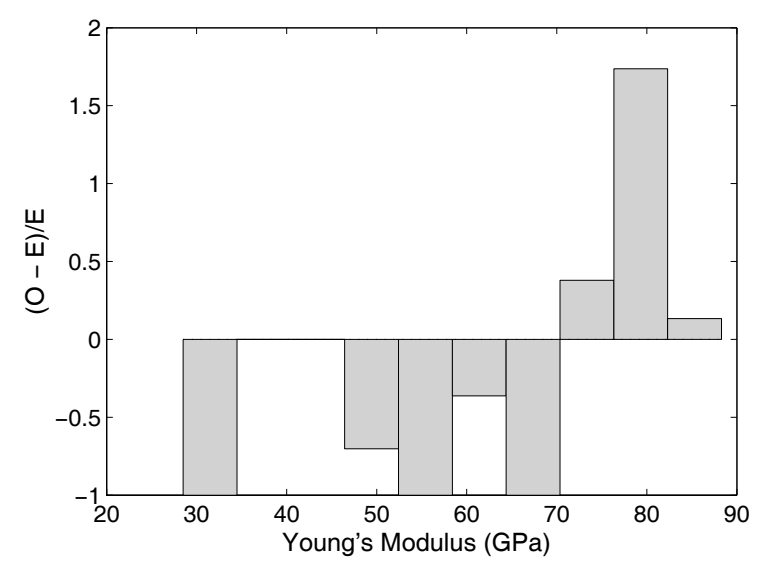

(f) $(O-E) / E$

Figure G.5. Plots used to examine the relationship between deposits and rock strength using Young's Modulus compiled from Lee (2000) in the layer, l2_l_rh4. The empirical curve is shown in blue in b), d) \& e). The theoretical curve is shown in red in b) \& e) and as grey bars in d). The $\mathrm{X}$-axis parameter in graphs a), b) \& c) was transformed to create a new parameter representing the difference between the maximum and the original parameter value; i.e., $x=X_{\max }-x$. 


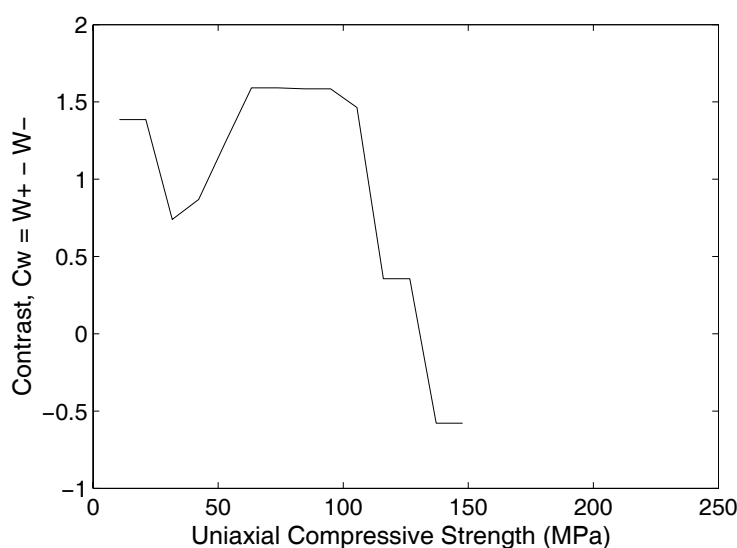

(a) Contrast, $C_{W}$

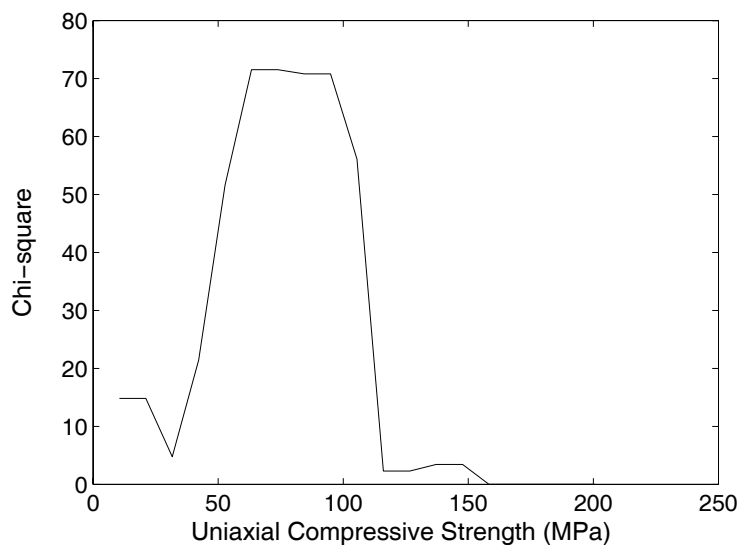

(c) Chi-squared

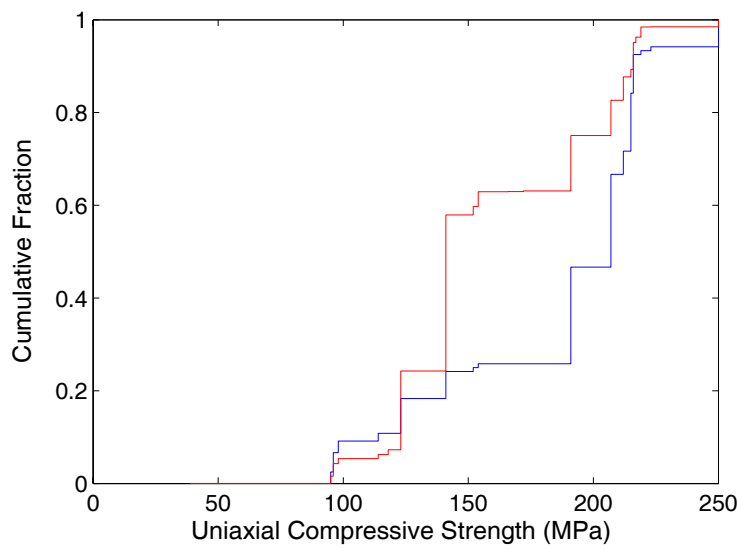

(e) Kolmogorov-Smirnov test

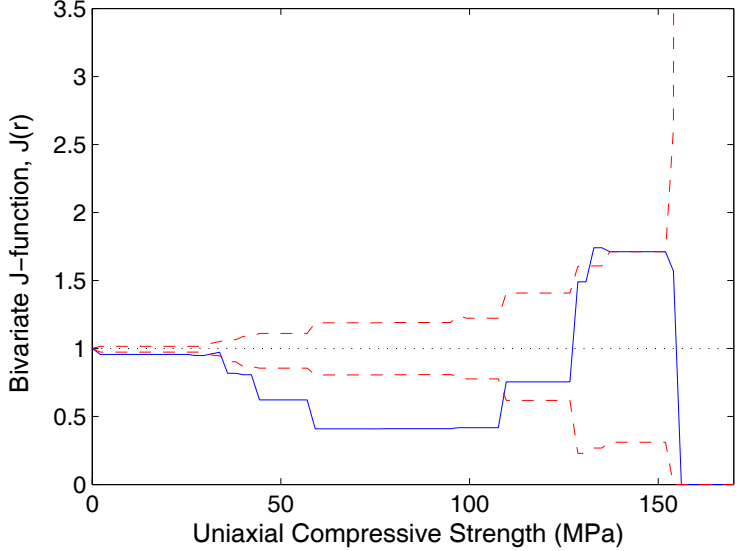

(b) Bivariate J-function (cumulative)

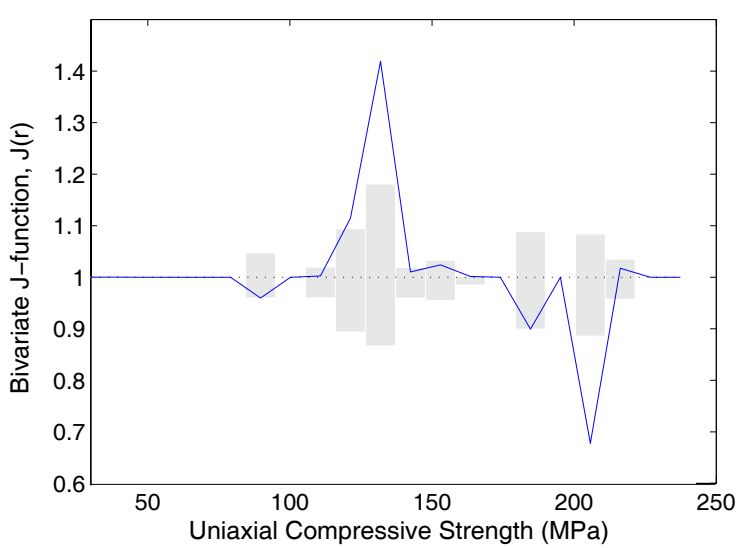

(d) Bivariate J-function (interval-based)

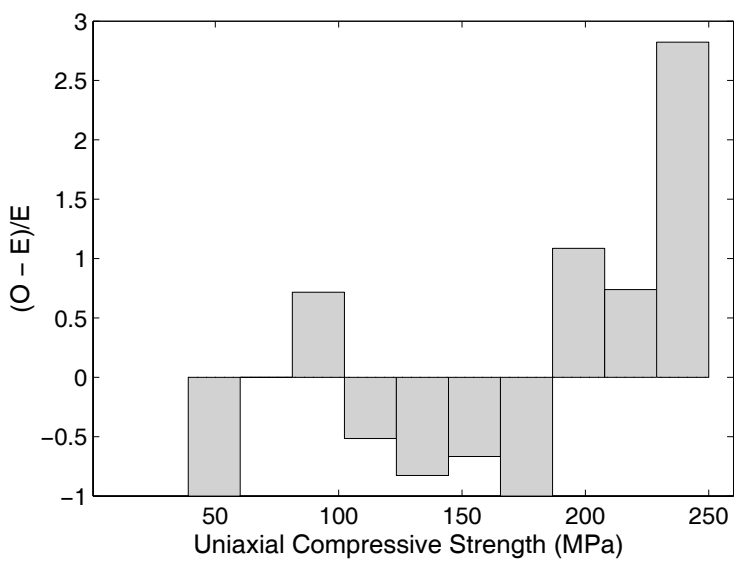

(f) $(O-E) / E$

Figure G.6. Plots used to examine the relationship between deposits and rock strength using uniaxial compressive strength in the layer, l2_l_rh5. The empirical curve is shown in blue in b), d) \& e). The theoretical curve is shown in red in b) \& e) and as grey bars in d). The X-axis parameter in graphs a), b) \& c) was transformed to create a new parameter representing the difference between the maximum and the original parameter value; i.e., $x=X_{\max }-x$. 


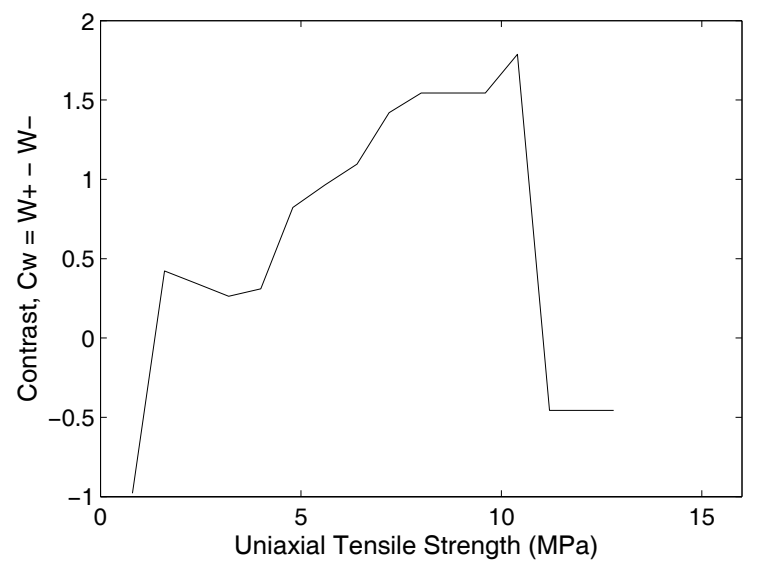

(a) Contrast, $C_{W}$

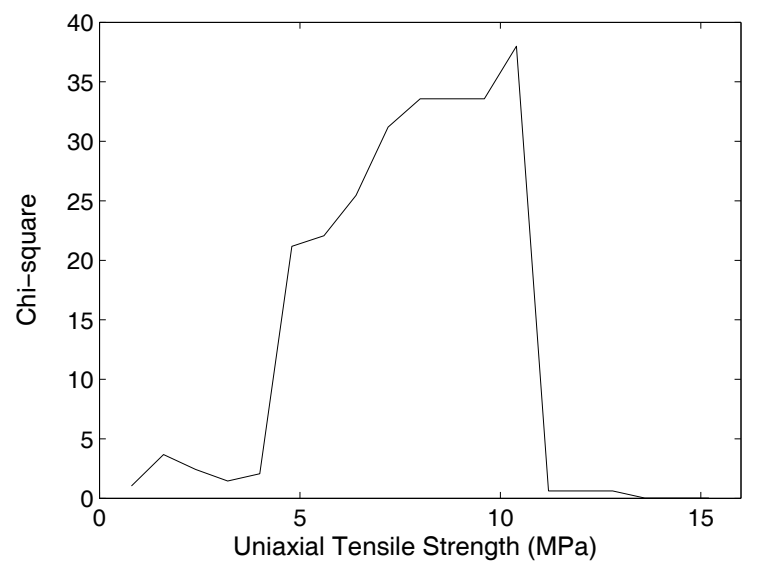

(c) Chi-squared

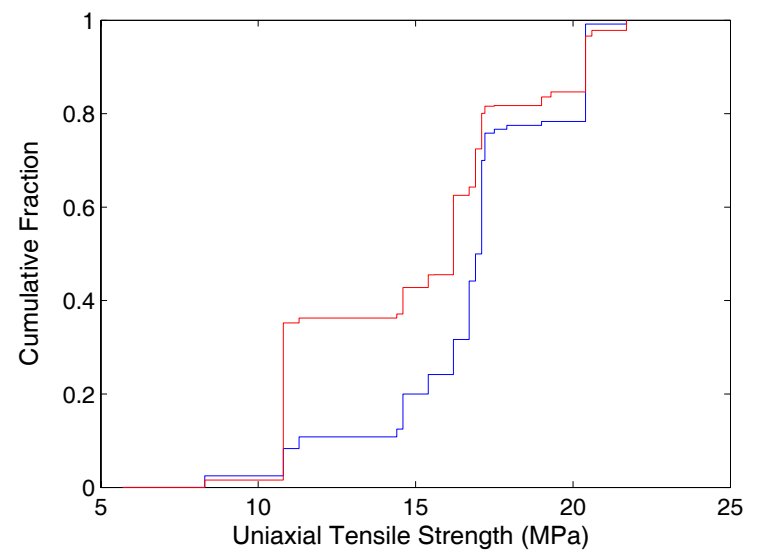

(e) Kolmogorov-Smirnov test

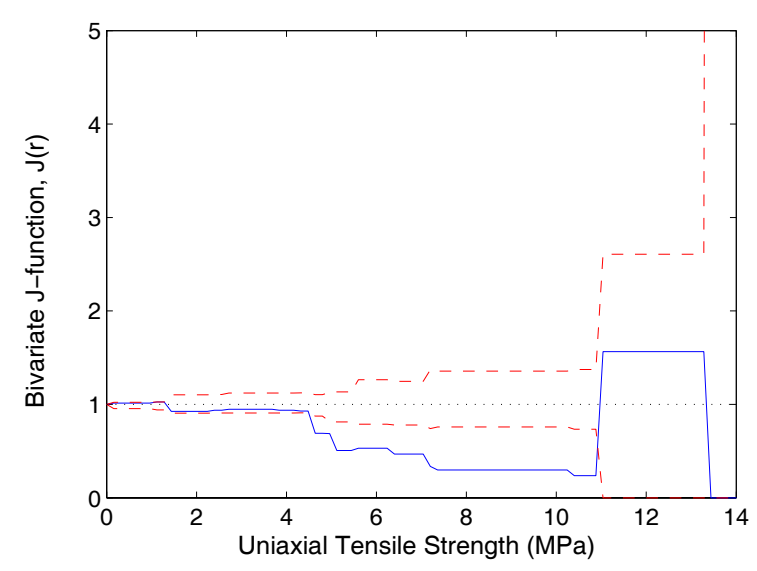

(b) Bivariate J-function (cumulative)

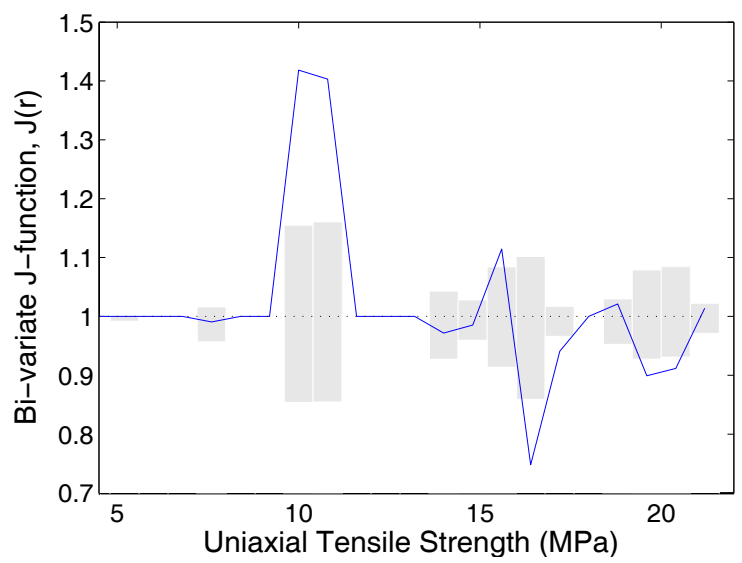

(d) Bivariate J-function (interval-based)

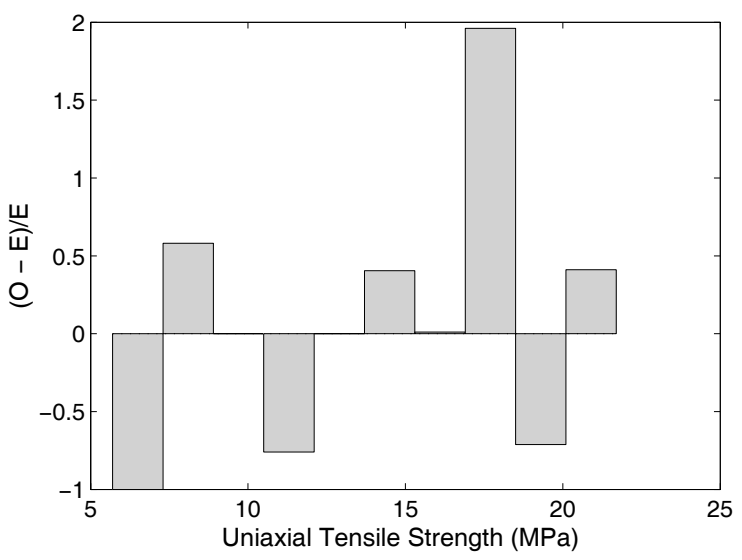

(f) $(O-E) / E$

Figure G.7. Plots used to examine the relationship between deposits and rock strength using uniaxial compressive strength in the layer, l2_l_rh6. The empirical curve is shown in blue in b), d) \& e). The theoretical curve is shown in red in b) \& e) and as grey bars in d). The X-axis parameter in graphs a), b) \& c) was transformed to create a new parameter representing the difference between the maximum and the original parameter value; i.e., $x=x_{\max }-x$. 


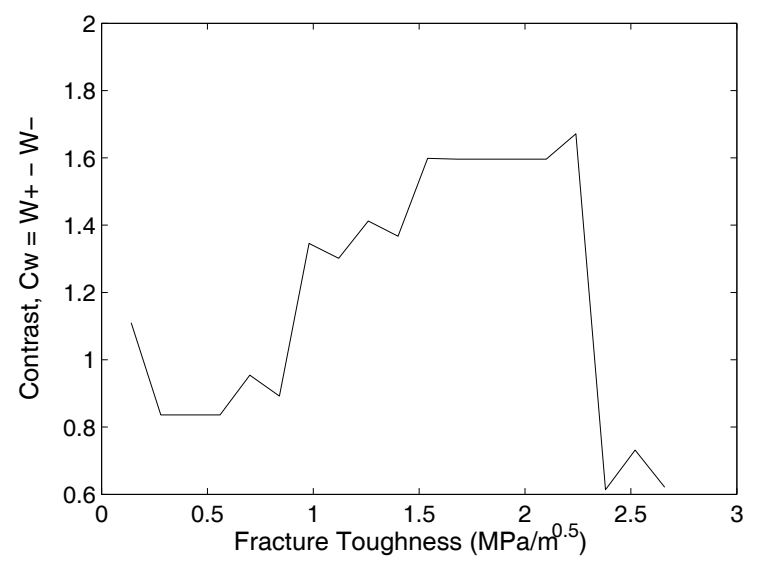

(a) Contrast, $C_{W}$

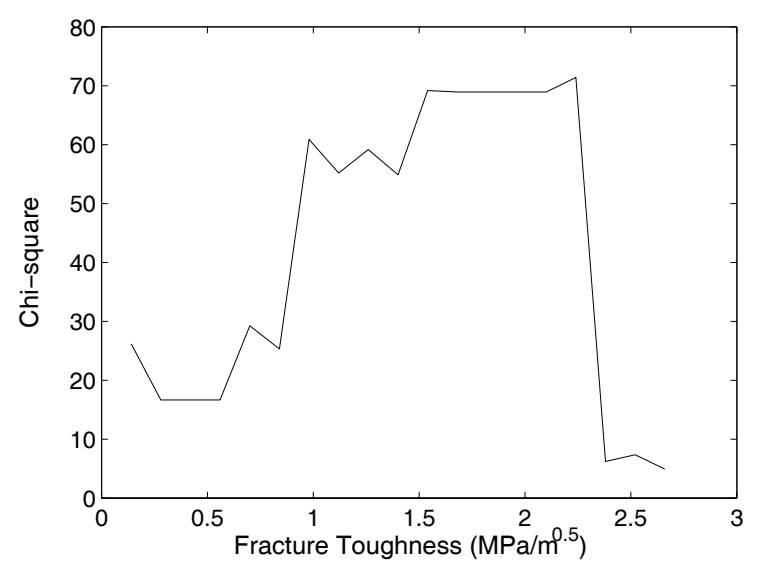

(c) Chi-squared

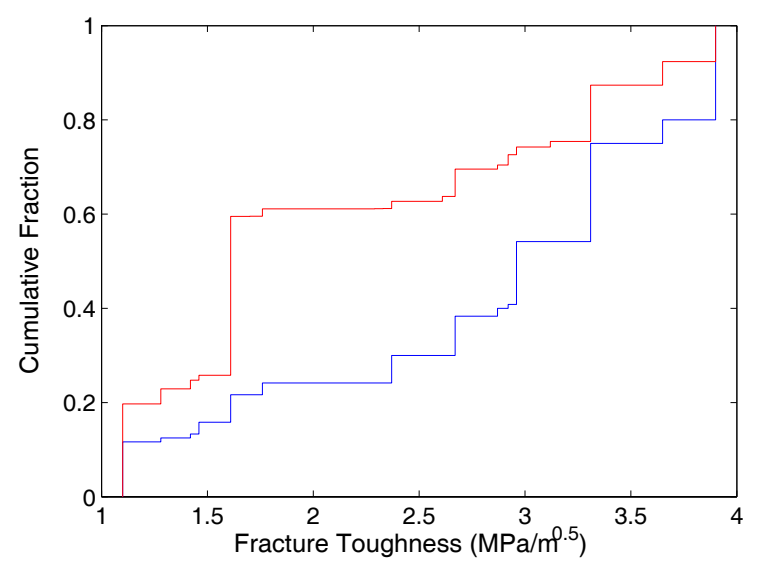

(e) Kolmogorov-Smirnov test

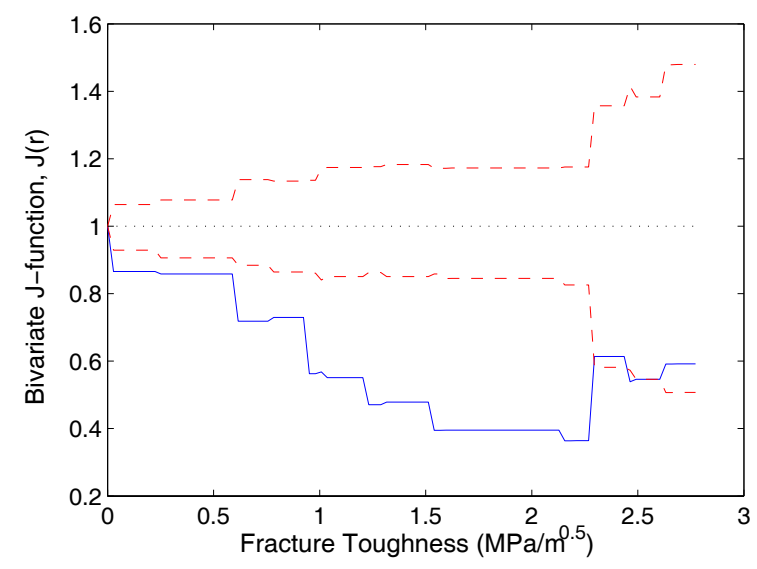

(b) Bivariate J-function (cumulative)

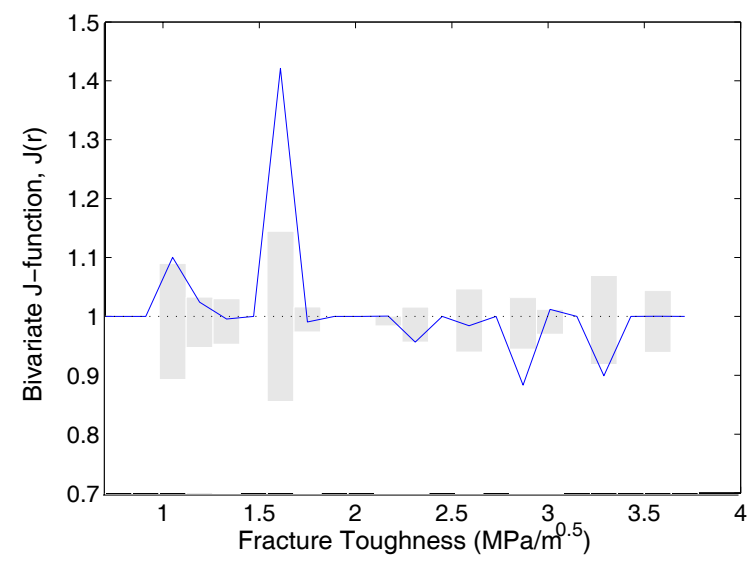

(d) Bivariate J-function (interval-based)

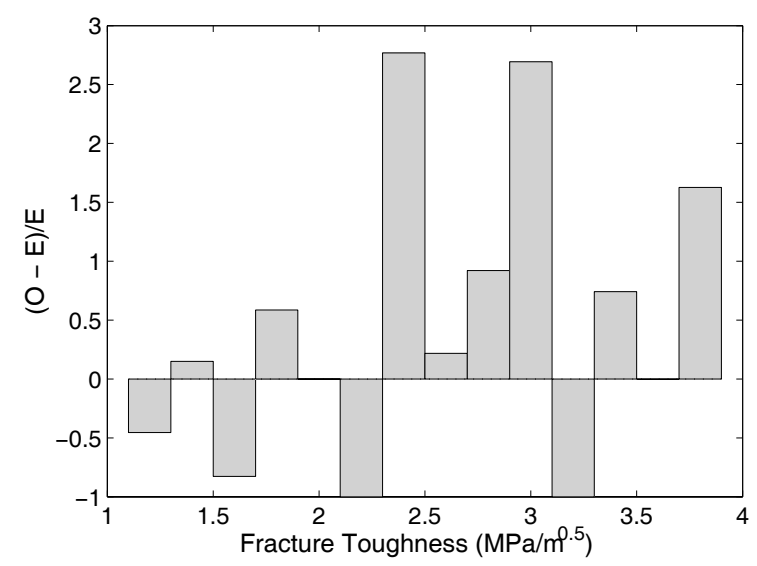

(f) $(O-E) / E$

Figure G.8. Plots used to examine the relationship between deposits and rock strength using fracture toughness in the layer, l2_l_$_{-} r h \%$. The empirical curve is shown in blue in b), d) \& e). The theoretical curve is shown in red in b) \& e) and as grey bars in d). The X-axis parameter in graphs a), b) \& c) was transformed to create a new parameter representing the difference between the maximum and the original parameter value; i.e., $x=X_{\max }-x$. 


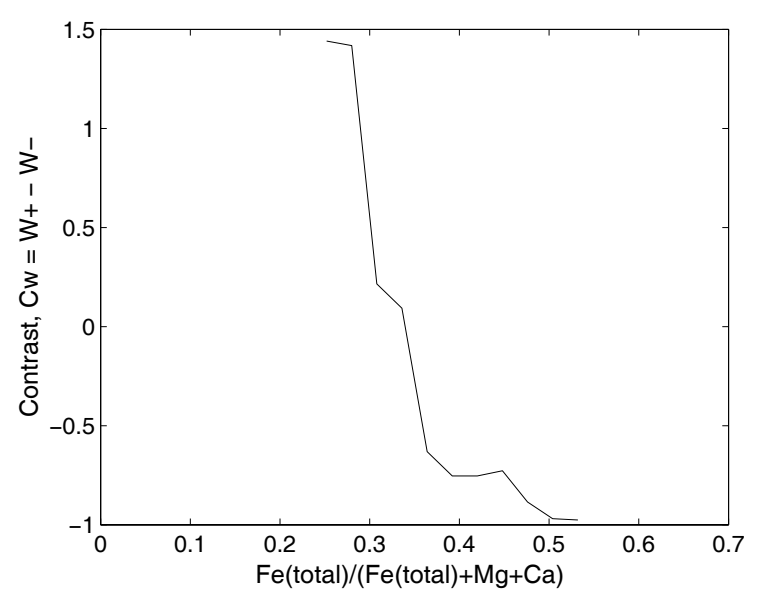

(a) Contrast, $C_{W}$

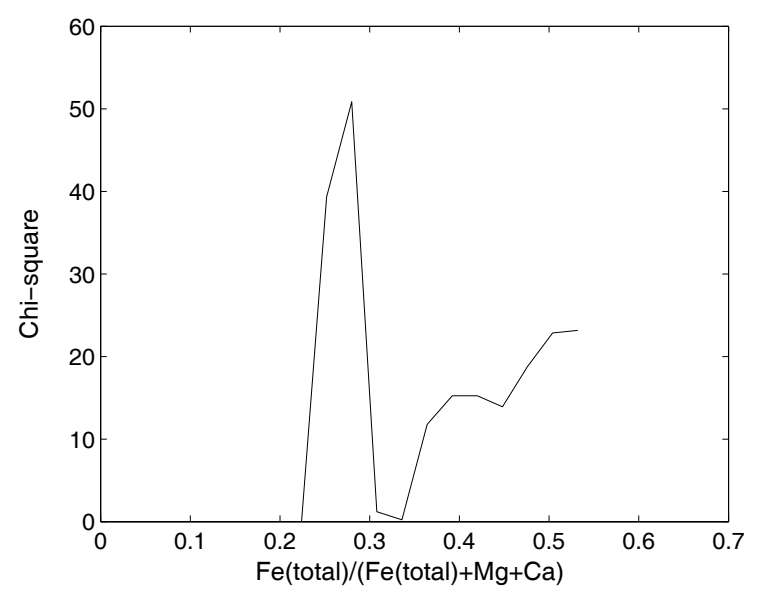

(c) Chi-squared

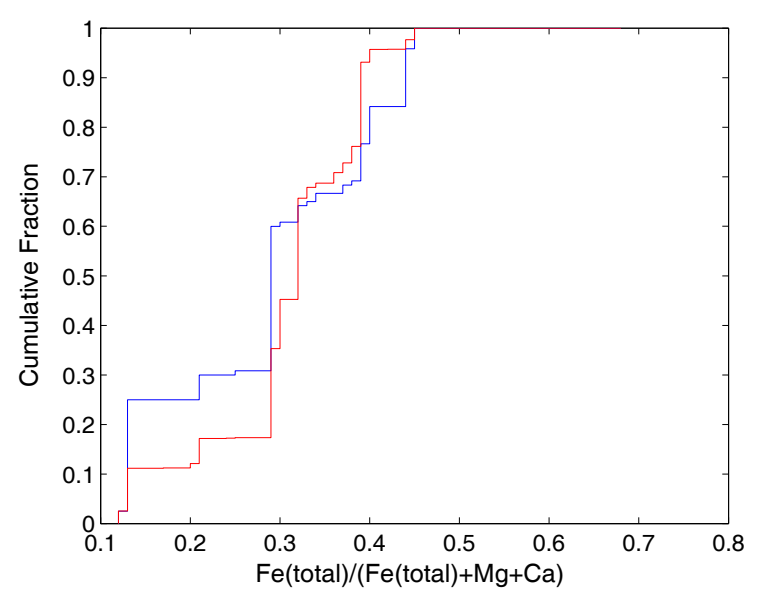

(e) Kolmogorov-Smirnov test

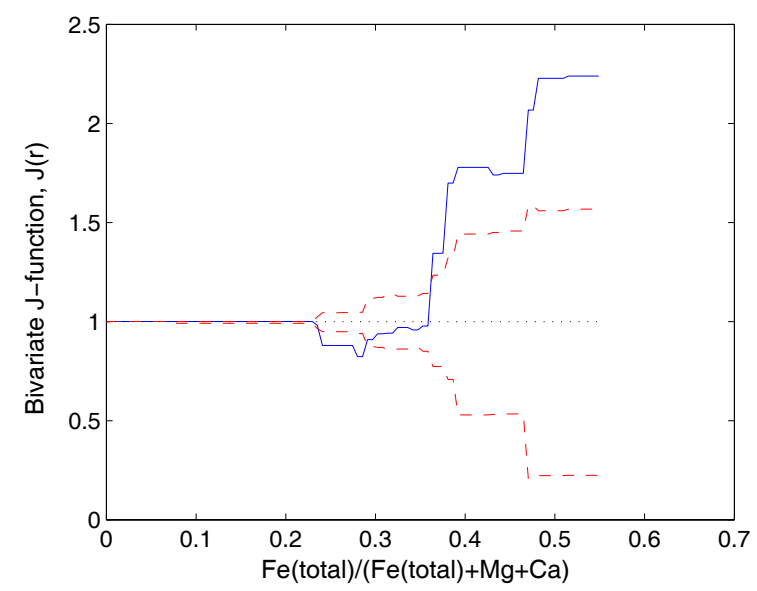

(b) Bivariate J-function (cumulative)

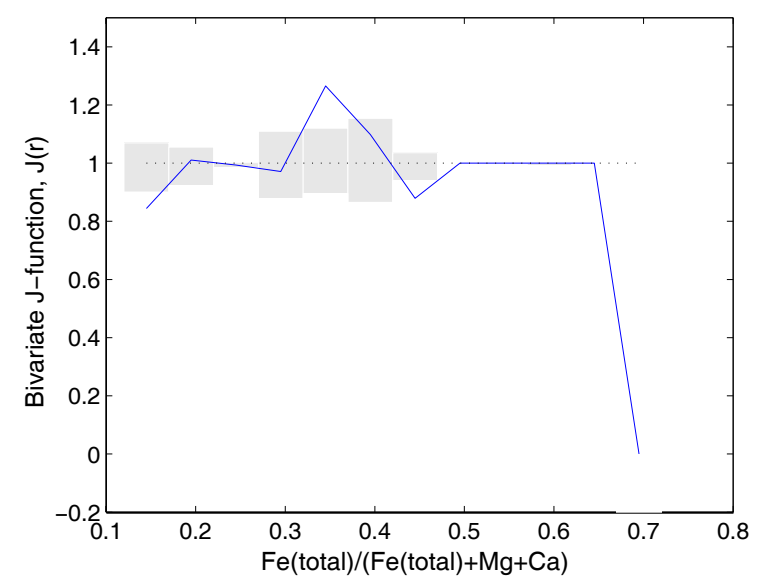

(d) Bivariate J-function (interval-based)

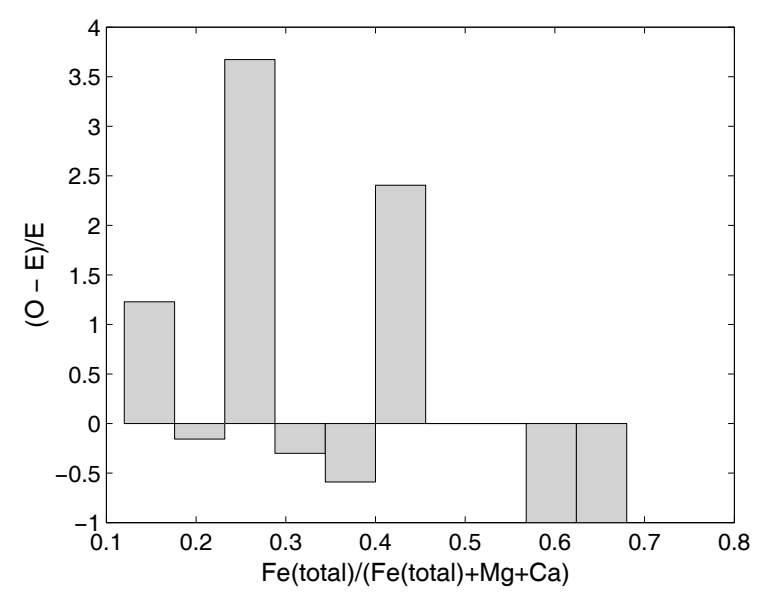

(f) $(O-E) / E$

Figure G.9. Plots used to examine the relationship between deposits and sulphidation index, $\mathrm{Fe}_{\text {tot }} /\left(\mathrm{Fe}_{\text {tot }}+\mathrm{Mg}+\mathrm{Ca}\right)$ in the layer, l3_l_ch1. The empirical curve is shown in blue in b), d) \& e). The theoretical curve is shown in red in b) \& e) and as grey bars in d). The X-axis parameter in graphs a), b) \& c) was transformed to create a new parameter representing the difference between the maximum and the original parameter value; i.e., $x=X_{\max .}-x$. 


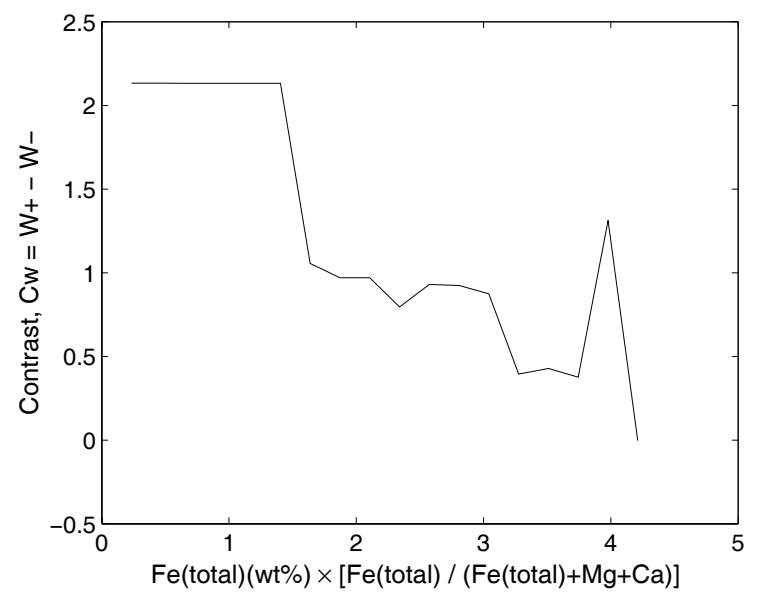

(a) Contrast, $C_{W}$

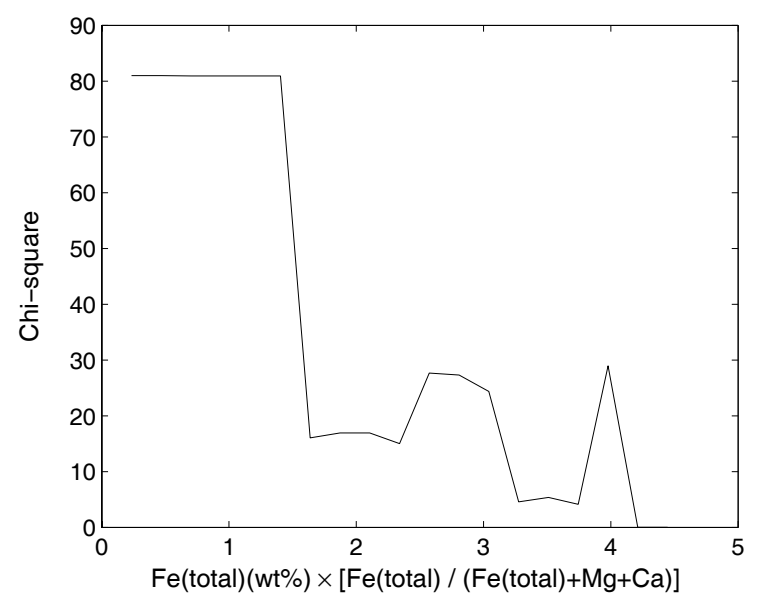

(c) Chi-squared

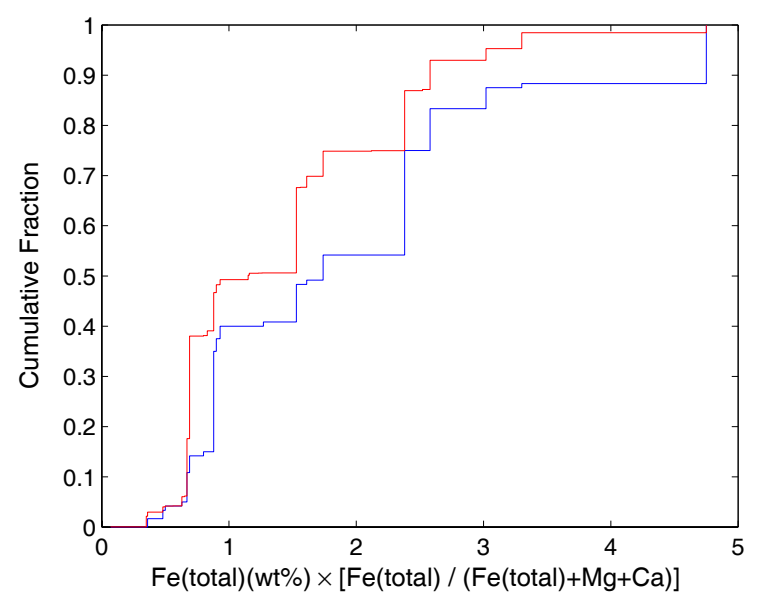

(e) Kolmogorov-Smirnov test

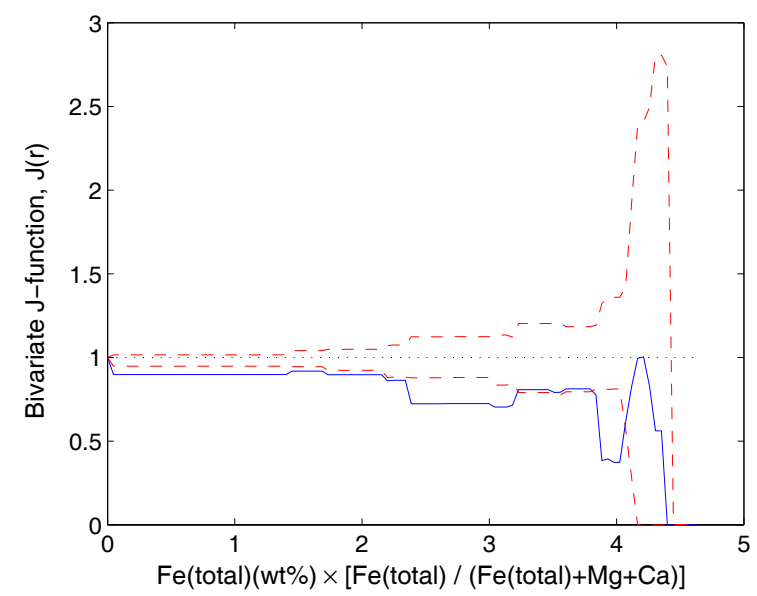

(b) Bivariate J-function (cumulative)

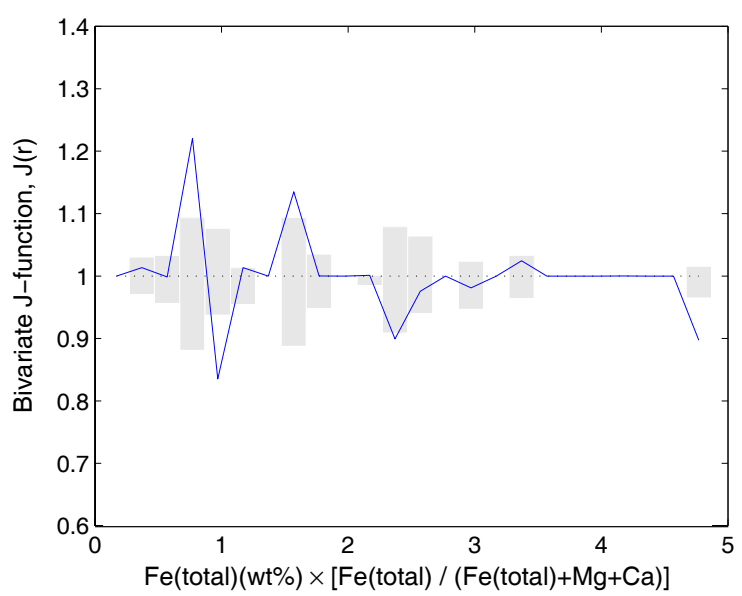

(d) Bivariate J-function (interval-based)

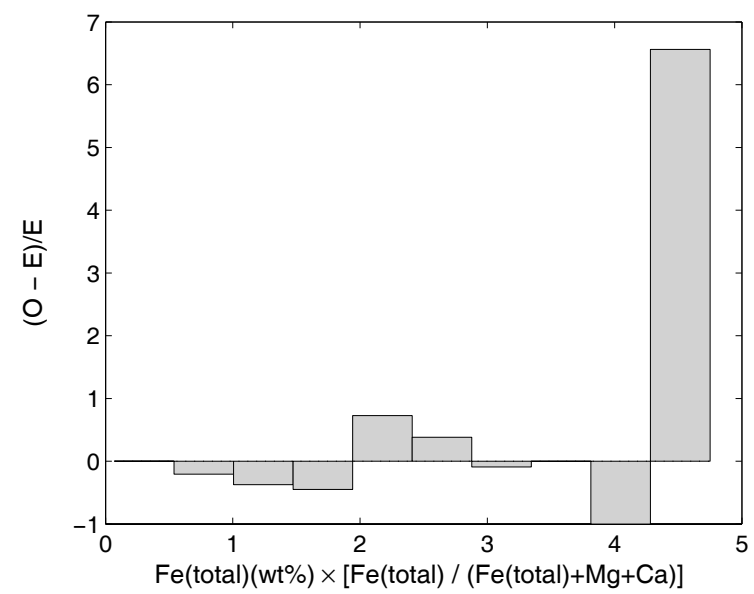

(f) $(O-E) / E$

Figure G.10. Plots used to examine the relationship between deposits and sulphidation index, $\mathrm{Fe}_{t o t}(\mathrm{wt} \%) * \mathrm{Fe}_{\text {tot }} /\left(\mathrm{Fe}_{\text {tot }}+\mathrm{Mg}+\mathrm{Ca}\right)$ in the layer, 13_l_ch2. The empirical curve is shown in blue in b), d) \& e). The theoretical curve is shown in red in b) \& e) and as grey bars in d). The $\mathrm{X}$-axis parameter in graphs a), b) \& c) was transformed to create a new parameter representing the difference between the maximum and the original parameter value; i.e., $x=X_{m a x}-x$. 


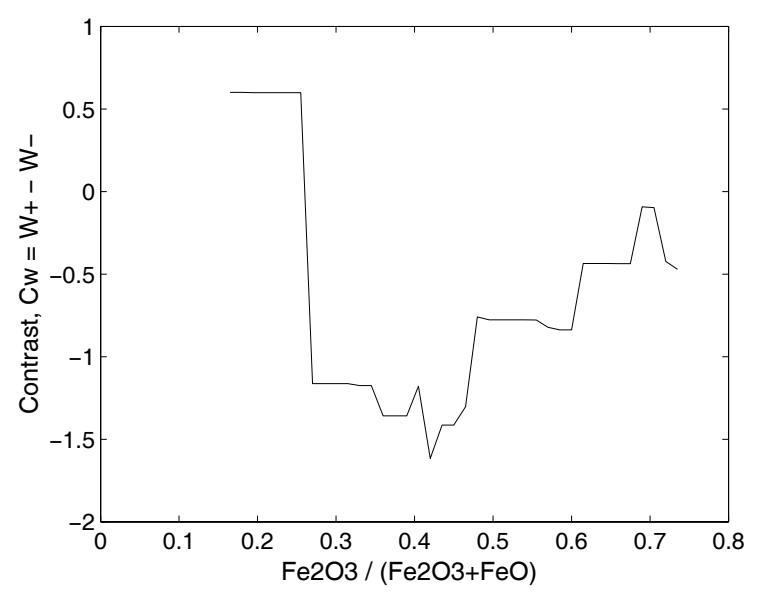

(a) Contrast, $C_{W}$

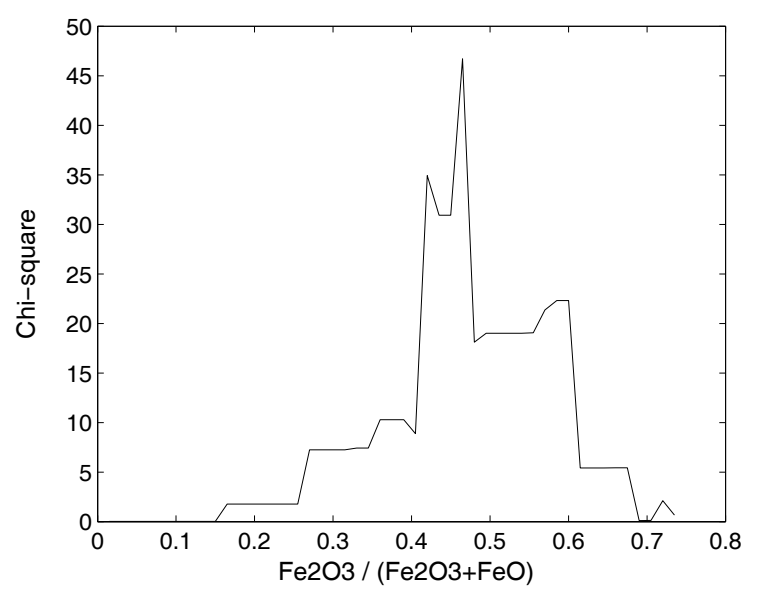

(c) Chi-squared

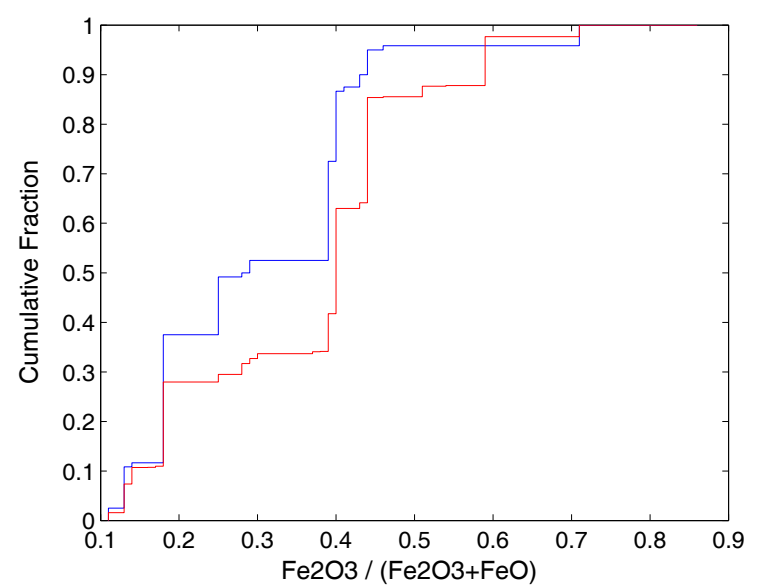

(e) Kolmogorov-Smirnov test

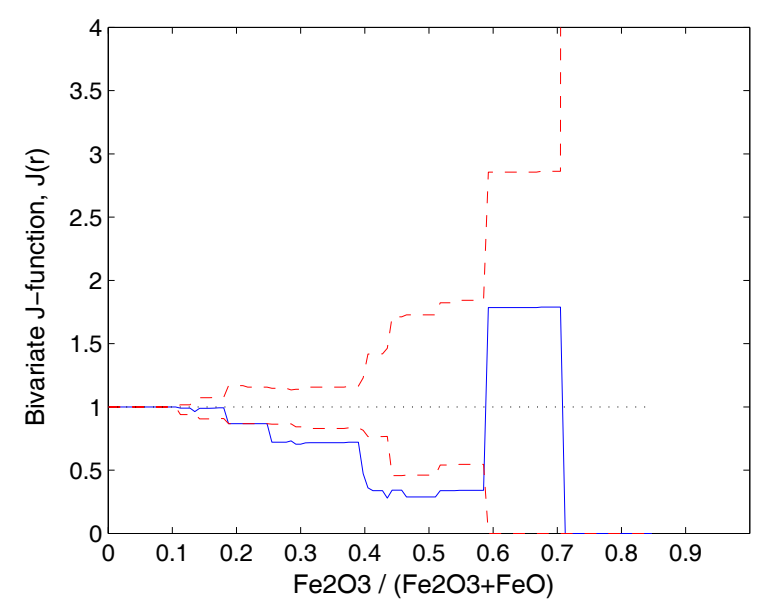

(b) Bivariate J-function (cumulative)

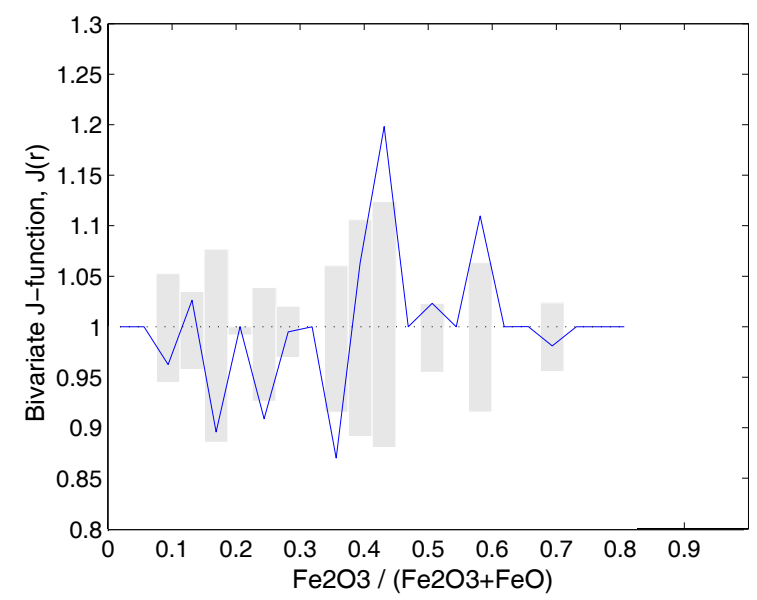

(d) Bivariate J-function (interval-based)

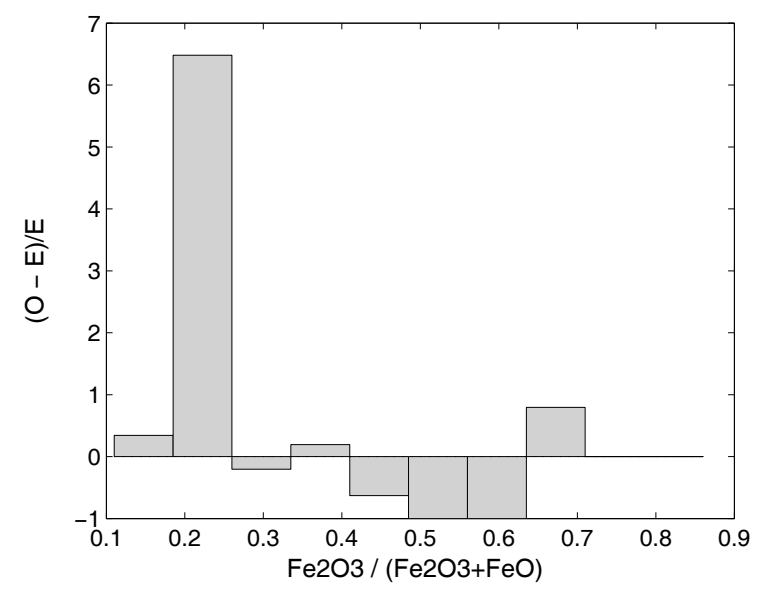

(f) $(O-E) / E$

Figure G.11. Plots used to examine the relationship between deposits and oxidation index, $\mathrm{Fe}_{2} \mathrm{O}_{3} /\left(\mathrm{Fe}_{2} \mathrm{O}_{3}+\mathrm{FeO}\right)$ in the layer, 13_l_ch3. The empirical curve is shown in blue in b), d) \& e). The theoretical curve is shown in red in b) \& e) and as grey bars in d). The X-axis parameter in graphs a), b) \& c) was transformed to create a new parameter representing the difference between the maximum and the original parameter value; i.e., $x=X_{\max .}-x$. 


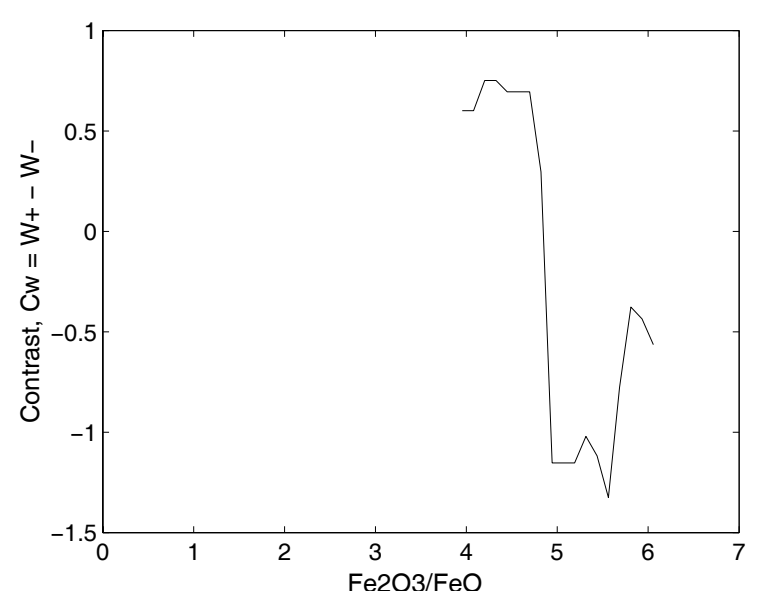

(a) Contrast, $C_{W}$

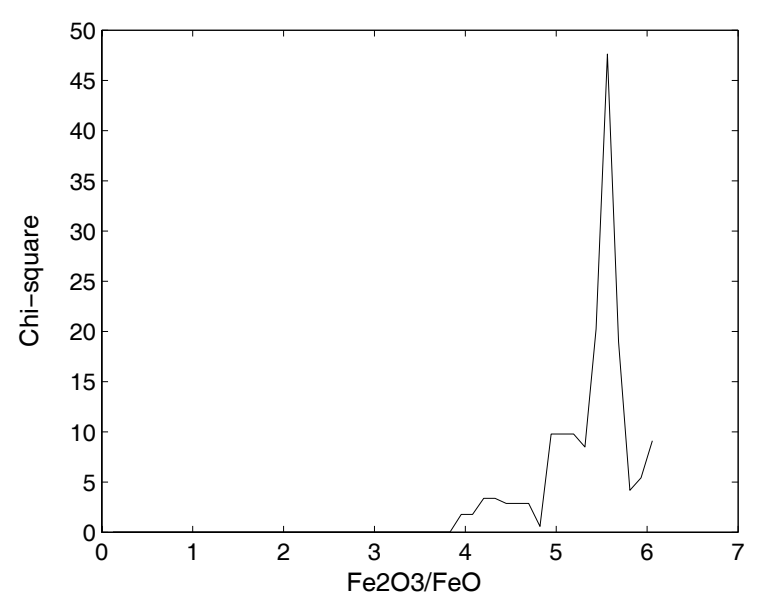

(c) Chi-squared

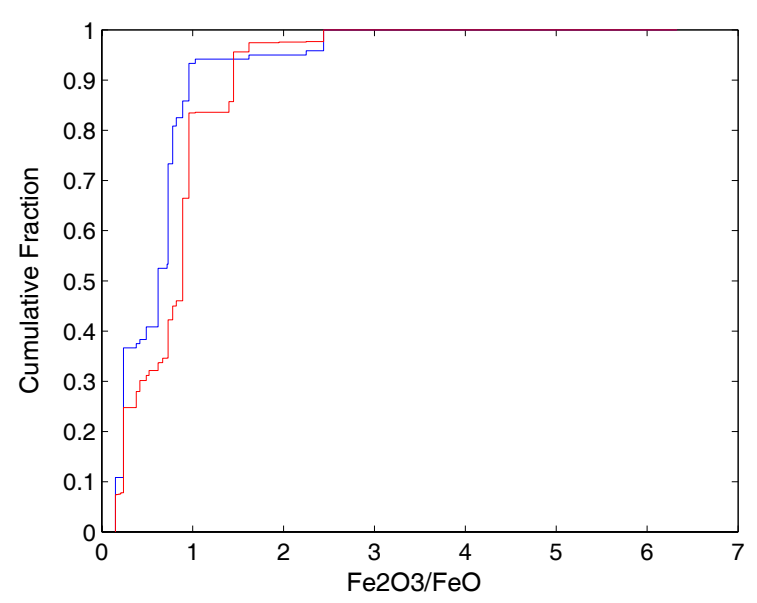

(e) Kolmogorov-Smirnov test

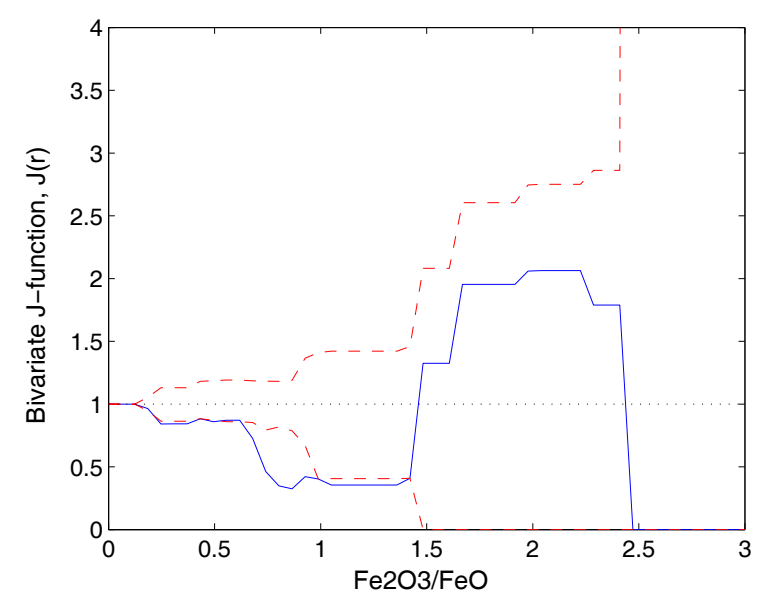

(b) Bivariate J-function (cumulative)

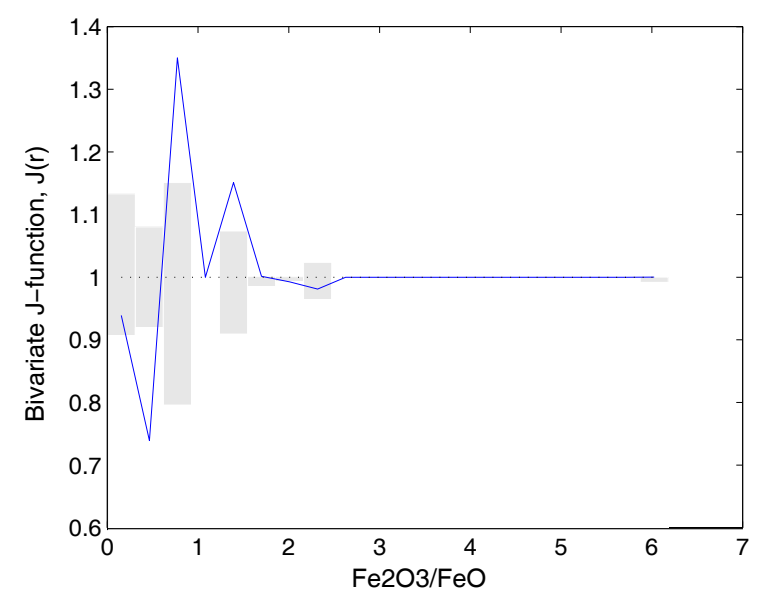

(d) Bivariate J-function (interval-based)

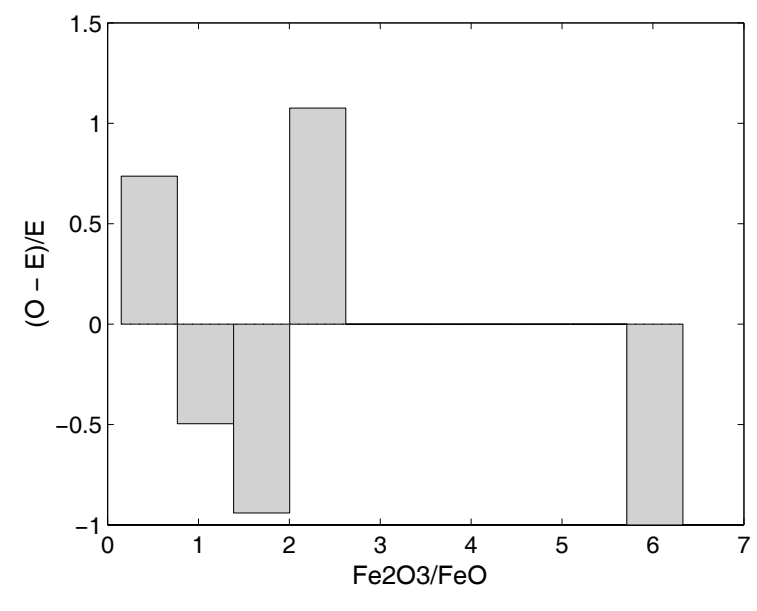

(f) $(O-E) / E$

Figure G.12. Plots used to examine the relationship between deposits and oxidation index, $\mathrm{Fe}_{2} \mathrm{O}_{3} /(\mathrm{FeO})$ in the layer, $133_{-} l_{-}$ch4. The empirical curve is shown in blue in b), d) \& e). The theoretical curve is shown in red in b) \& e) and as grey bars in d). 


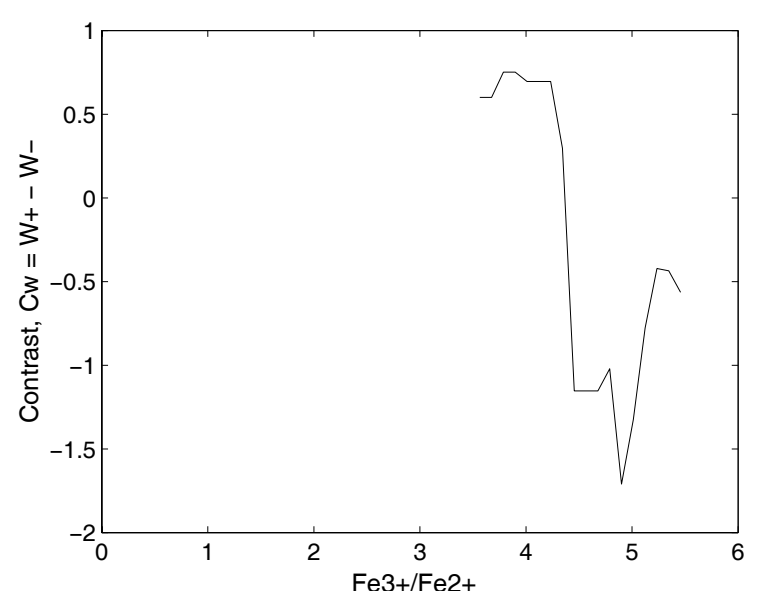

(a) Contrast, $C_{W}$

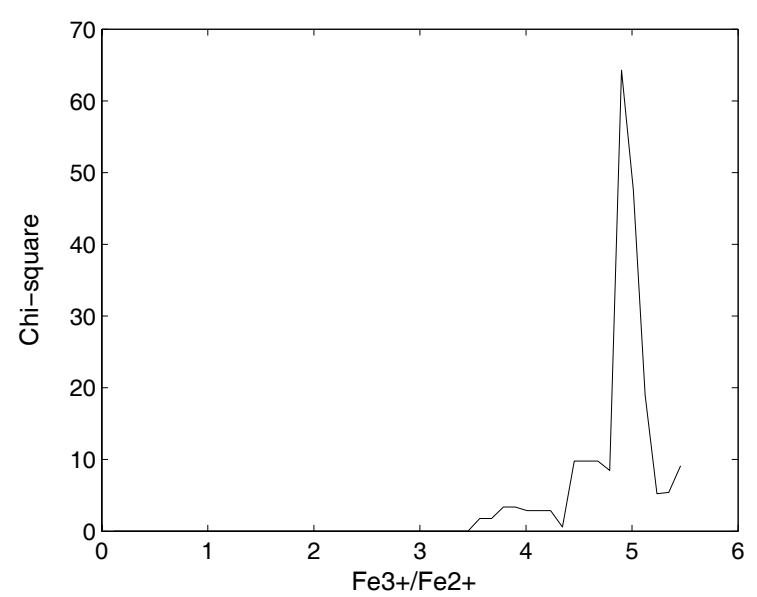

(c) Chi-squared

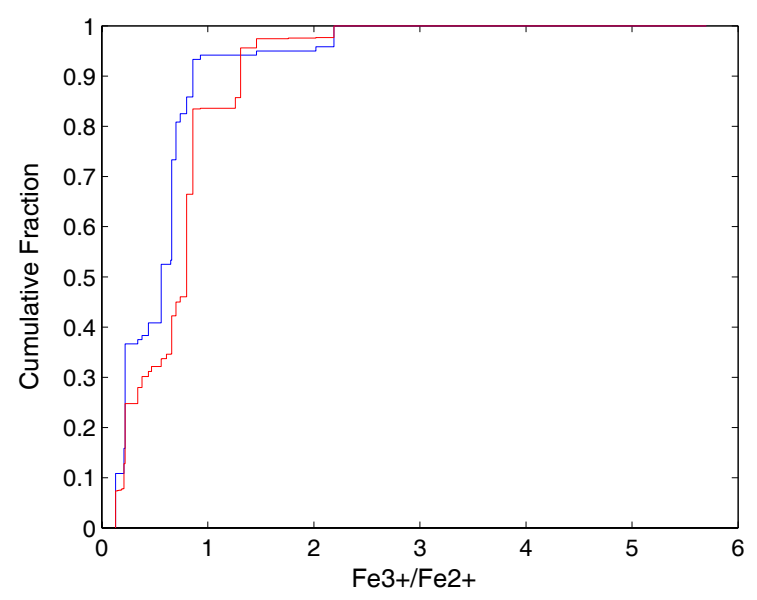

(e) Kolmogorov-Smirnov test

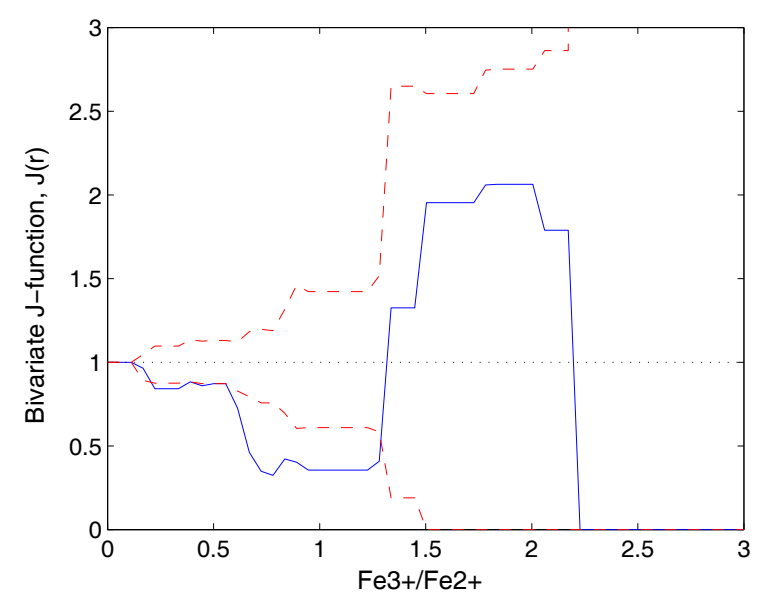

(b) Bivariate J-function (cumulative)

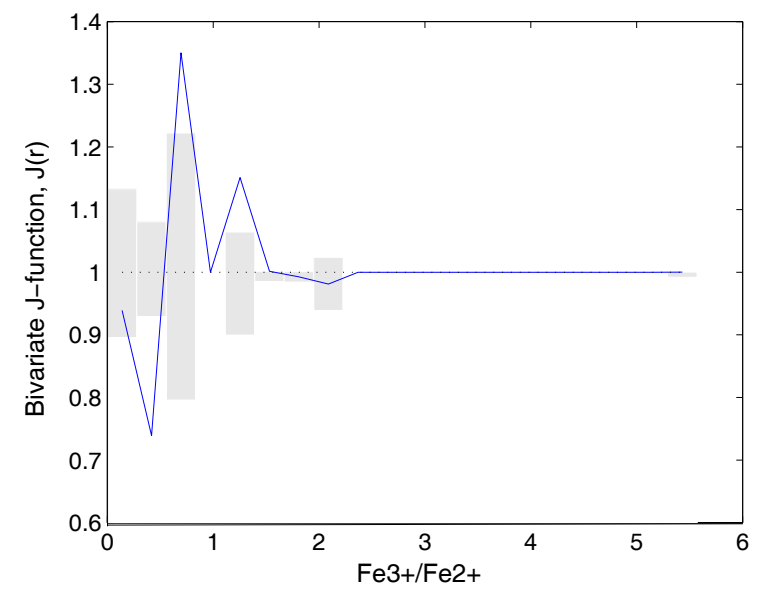

(d) Bivariate J-function (interval-based)

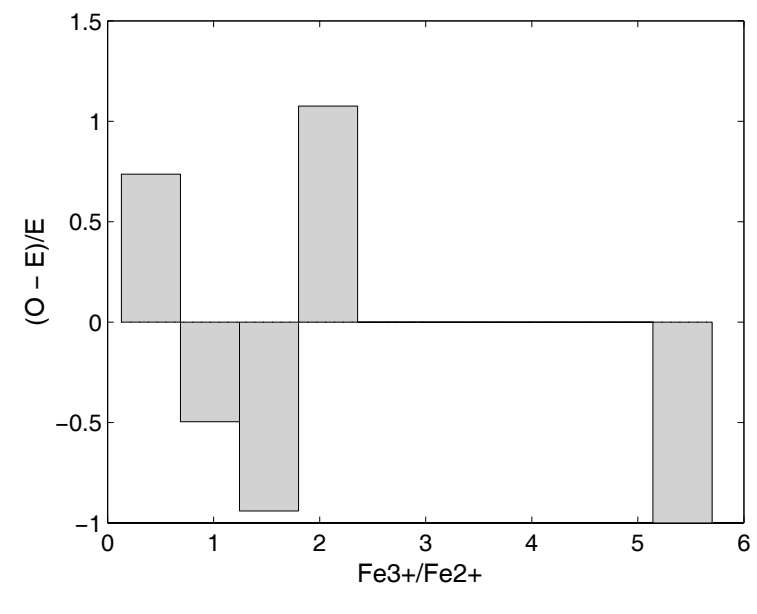

(f) $(O-E) / E$

Figure G.13. Plots used to examine the relationship between deposits and oxidation index, $\mathrm{Fe}^{2+} / \mathrm{Fe}^{3+}$ in the layer, 13_l_ch5. The empirical curve is shown in blue in b), d) \& e). The theoretical curve is shown in red in b) \& e) and as grey bars in d). 


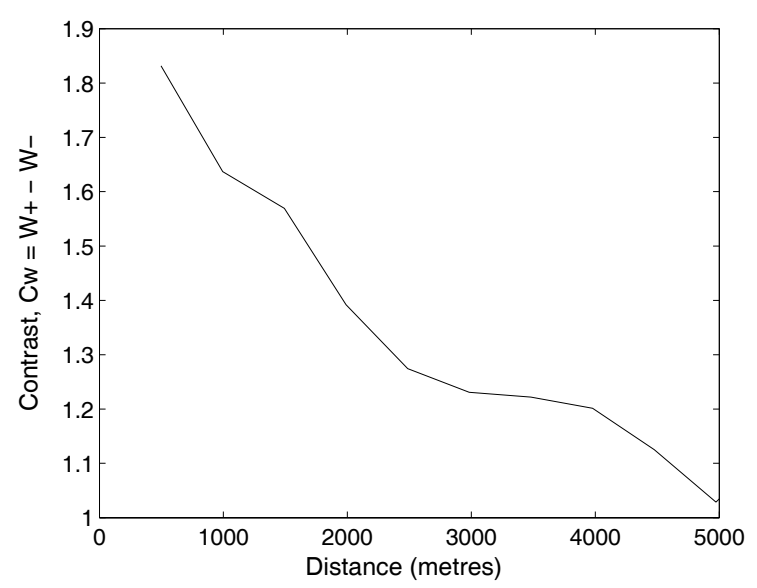

(a) Contrast, $C_{W}$

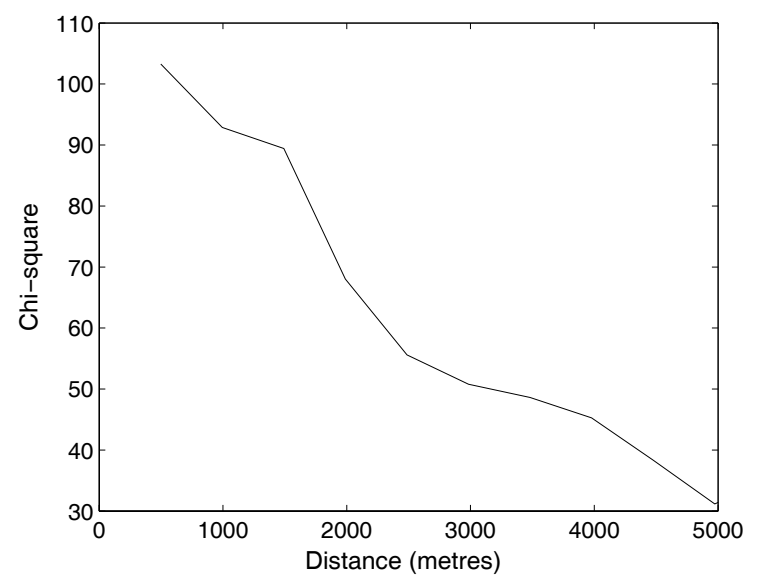

(c) Chi-squared

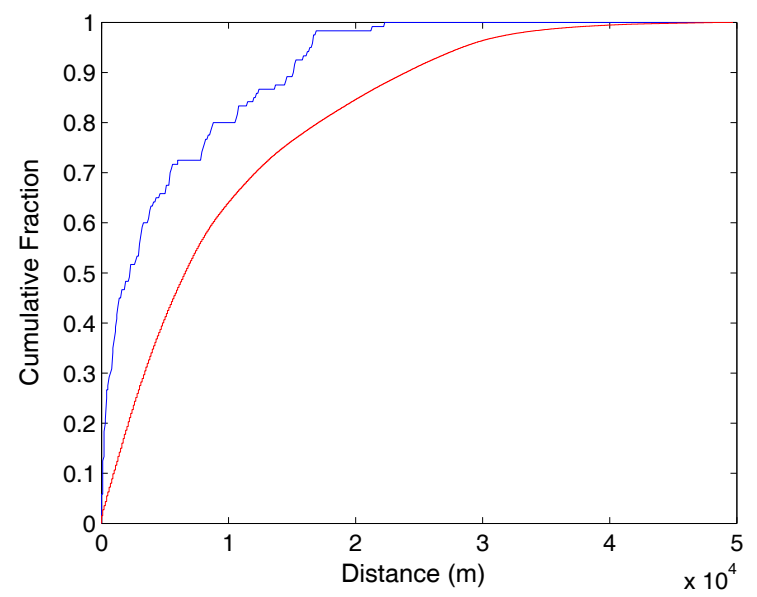

(e) Kolmogorov-Smirnov test

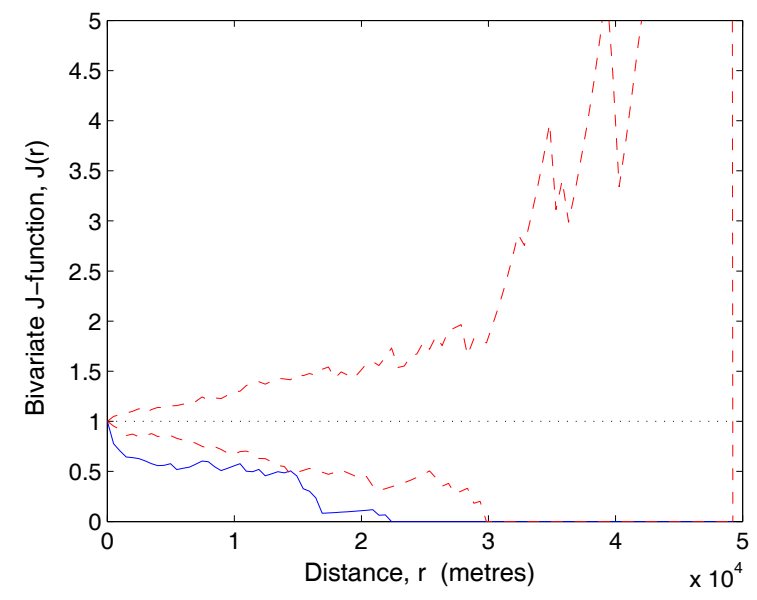

(b) Bivariate J-function (cumulative)

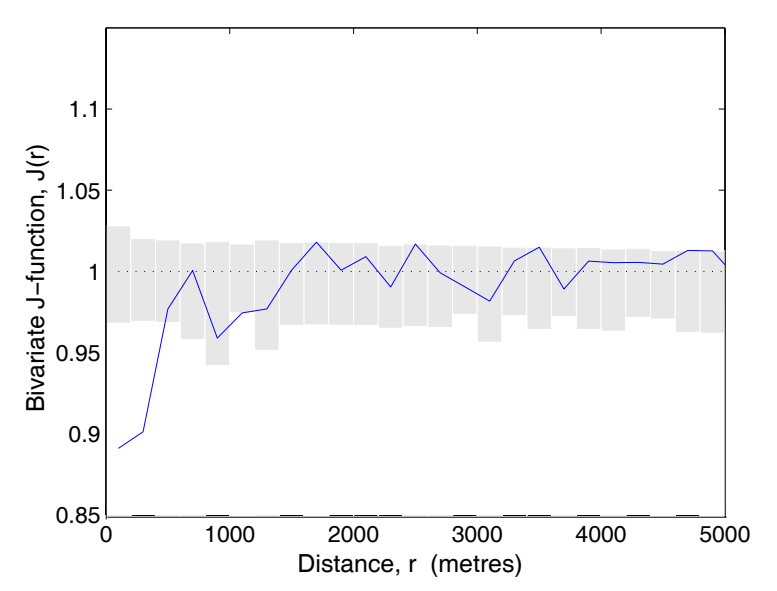

(d) Bivariate J-function (interval-based)

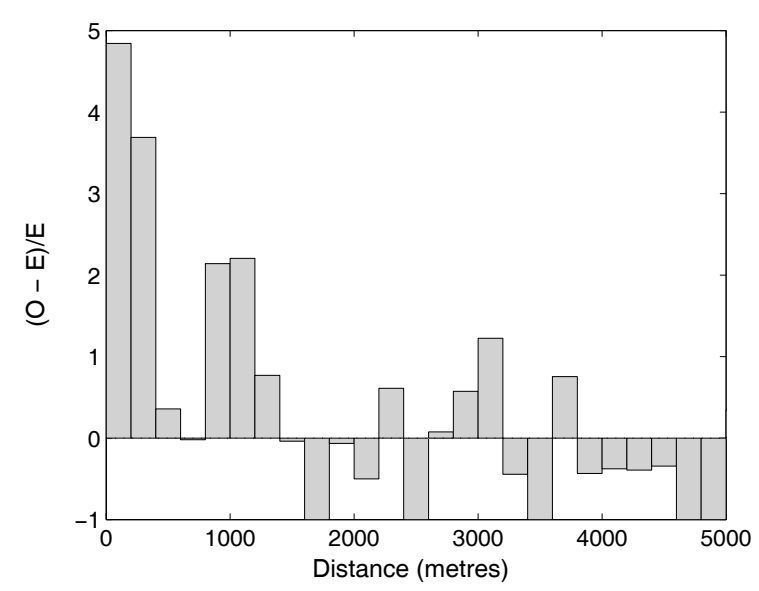

(f) $(O-E) / E$

Figure G.14. Plots used to examine the relationship between deposits and distance to felsic porphyritic intrusions in the layer, $l_{4}$ l_ p f_dist. The empirical curve is shown in blue in b), d) \& e). The theoretical curve is shown in red in b) \& e) and as grey bars in d). 


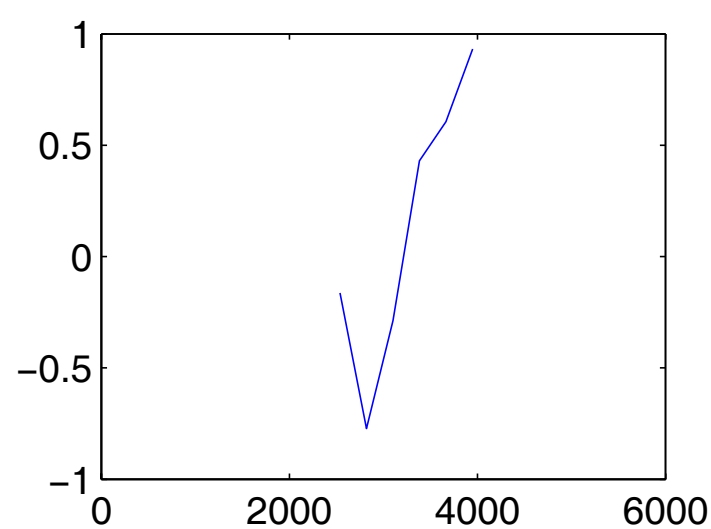

(a) Contrast, $C_{W}$

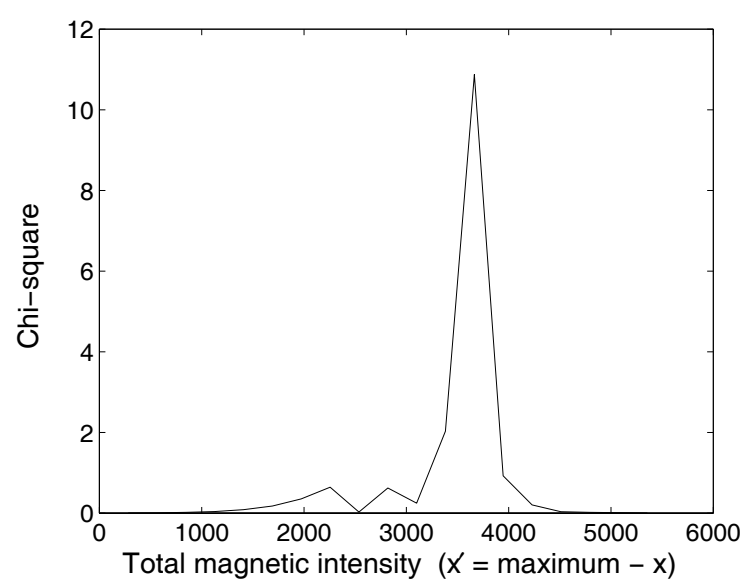

(c) Chi-squared

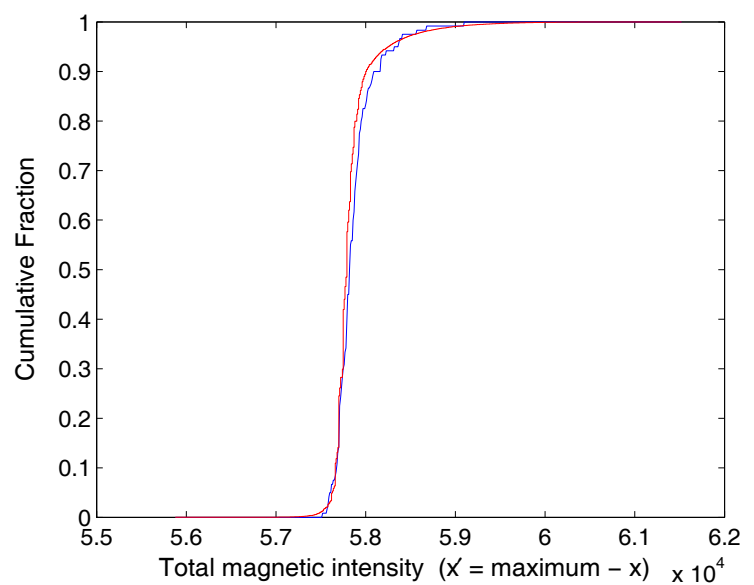

(e) Kolmogorov-Smirnov test

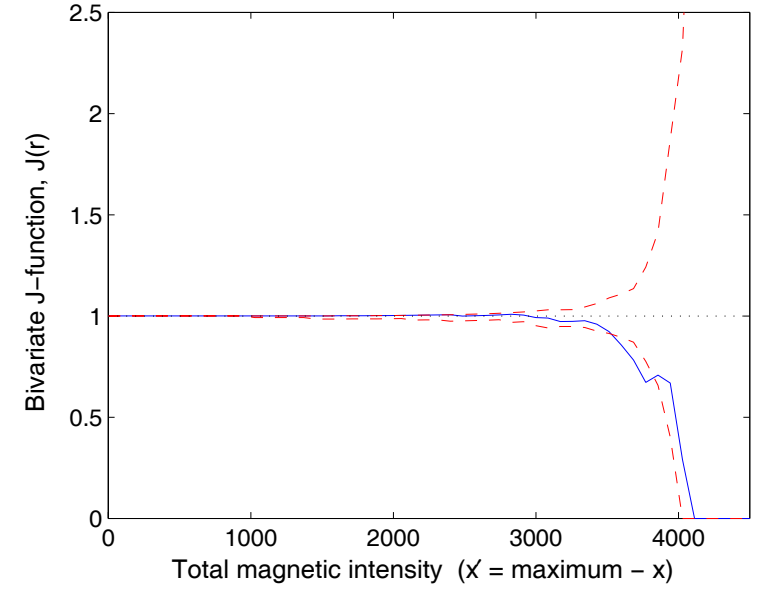

(b) Bivariate J-function (cumulative)

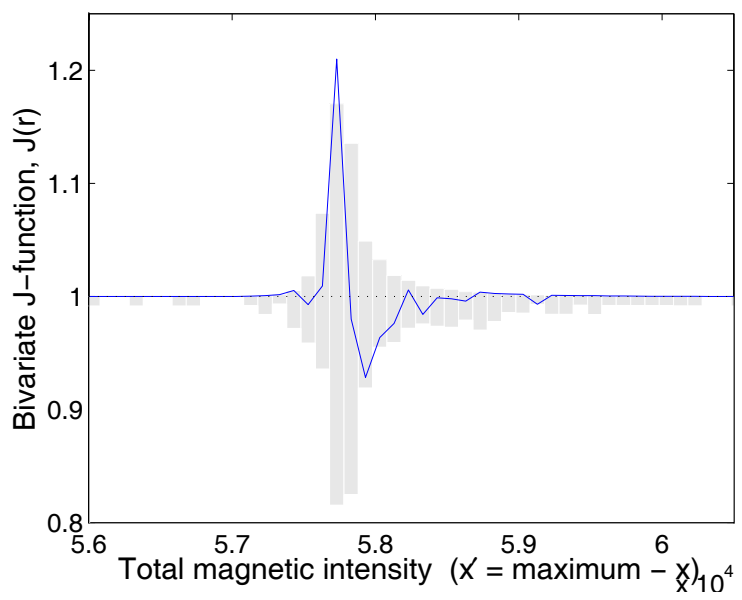

(d) Bivariate J-function (interval-based)

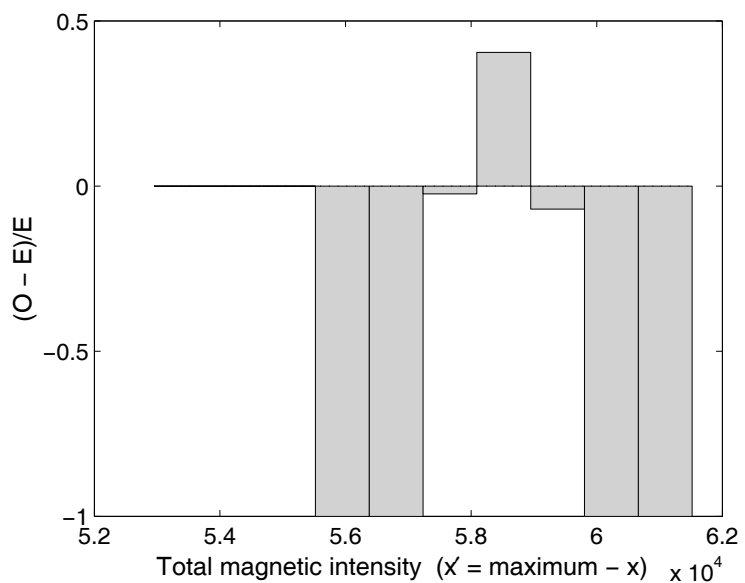

(f) $(O-E) / E$

Figure G.15. Plots used to examine the relationship between deposits and airborne total magnetic intensity in the layer, $15 \_m_{-} r t p$. The empirical curve is shown in blue in b), d) \& e). The theoretical curve is shown in red in b) \& e) and as grey bars in d). 


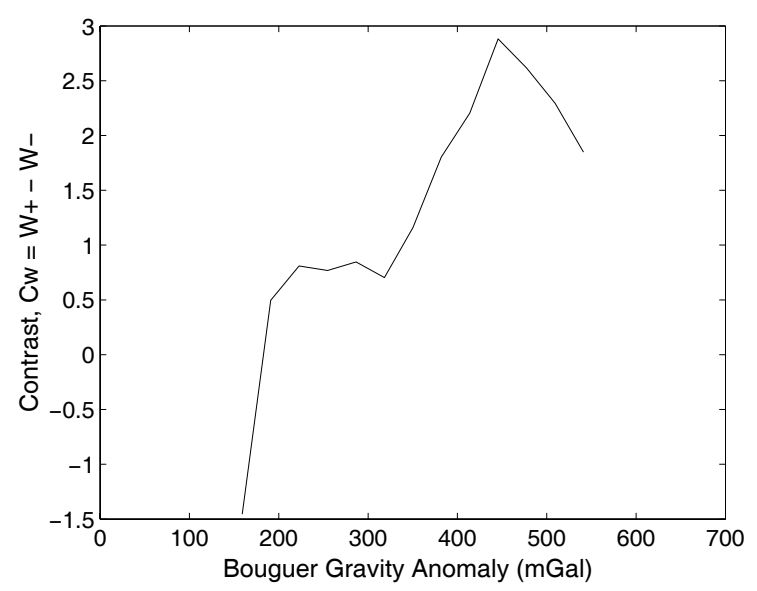

(a) Contrast, $C_{W}$

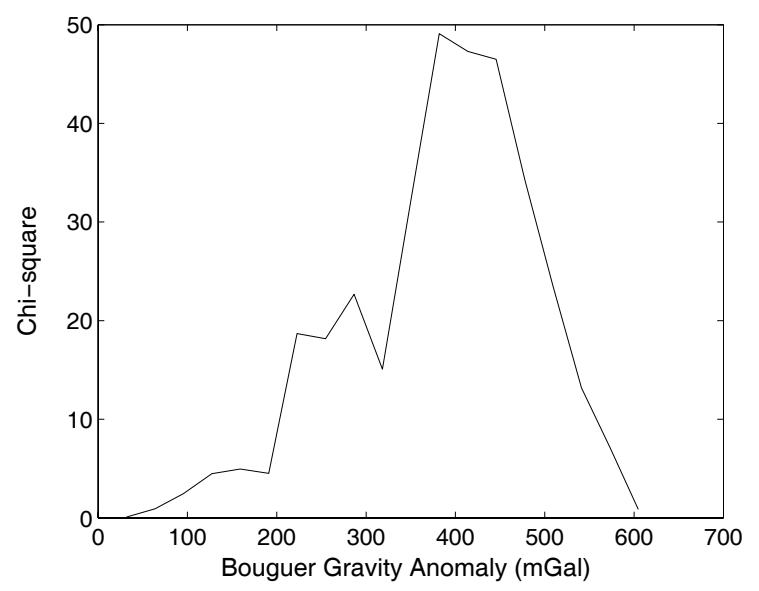

(c) Chi-squared

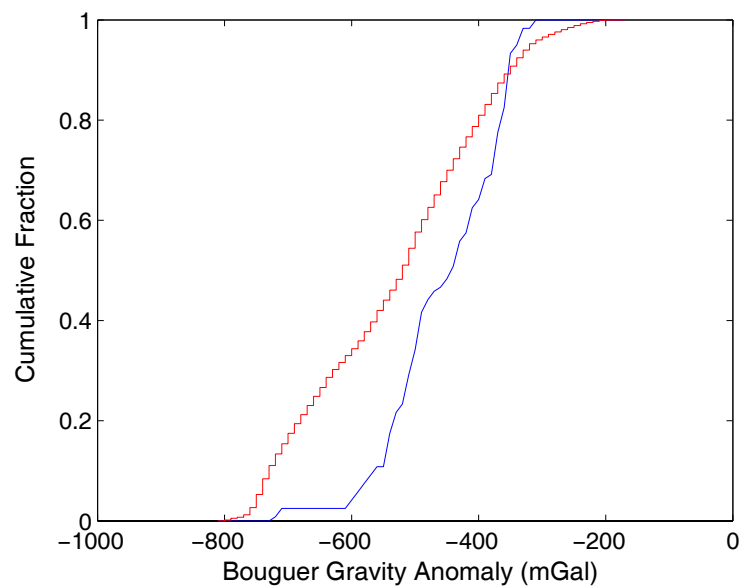

(e) Kolmogorov-Smirnov test

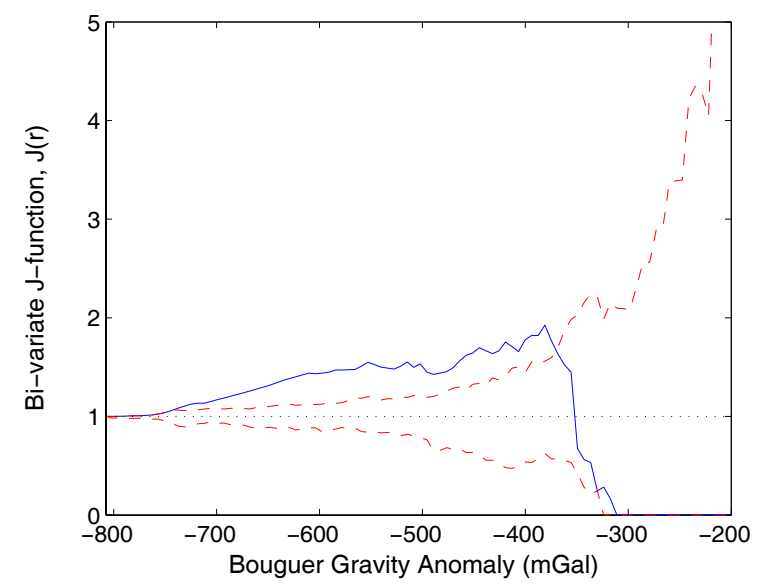

(b) Bivariate J-function (cumulative)

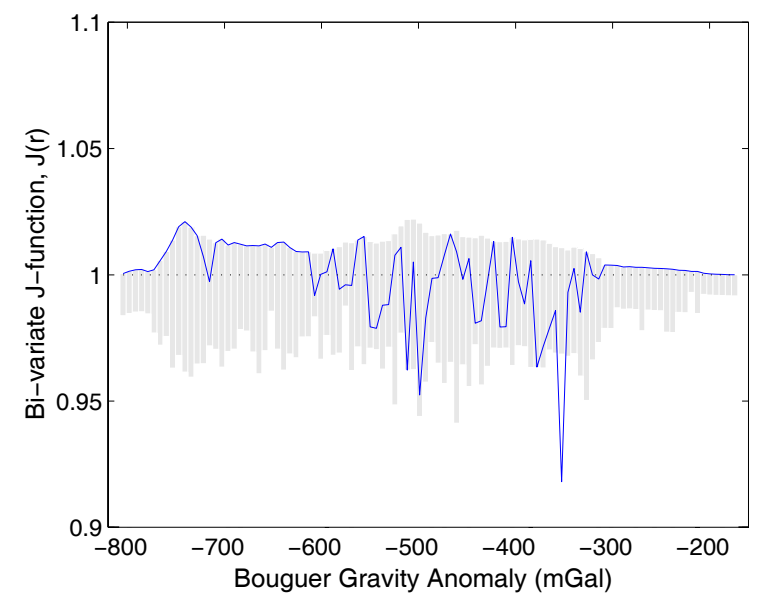

(d) Bivariate J-function (interval-based)

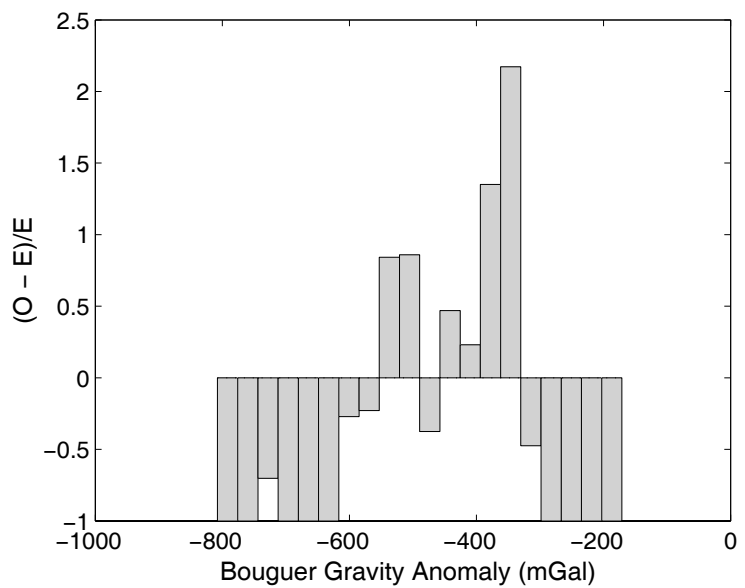

(f) $(O-E) / E$

Figure G.16. Plots used to examine the relationship between deposits and Bouguer gravity anomalies in the layer, l12_grav. The $\mathrm{X}$-axis parameter in graphs a), b), c) \& d) was transformed to create a new parameter representing the difference between the maximum and the original parameter value; i.e., $x=X_{\max }-x$. The empirical curve is shown in blue in b), d) \& e). The theoretical curve is shown in red in b) \& e) and as grey bars in d). 


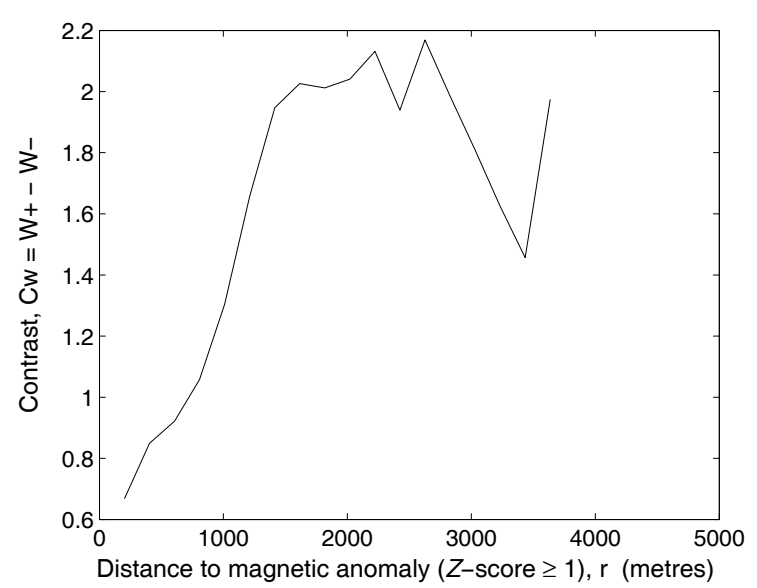

(a) Contrast, $C_{W}$

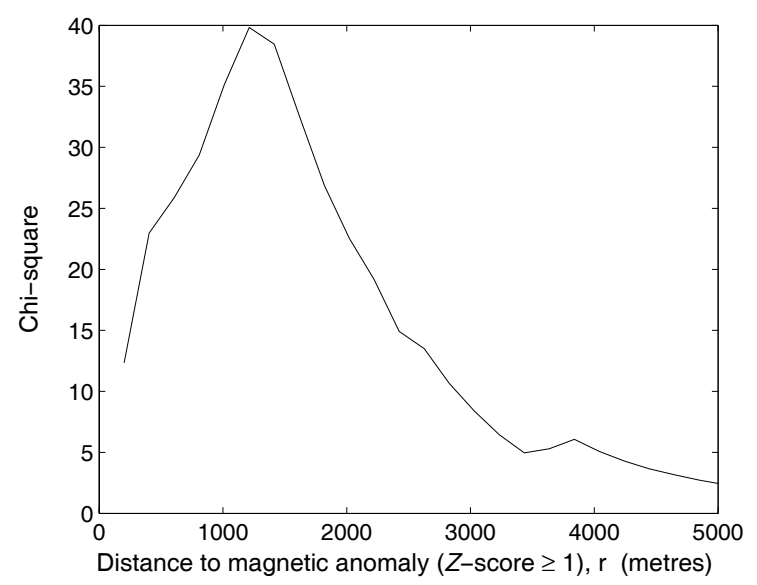

(c) Chi-squared

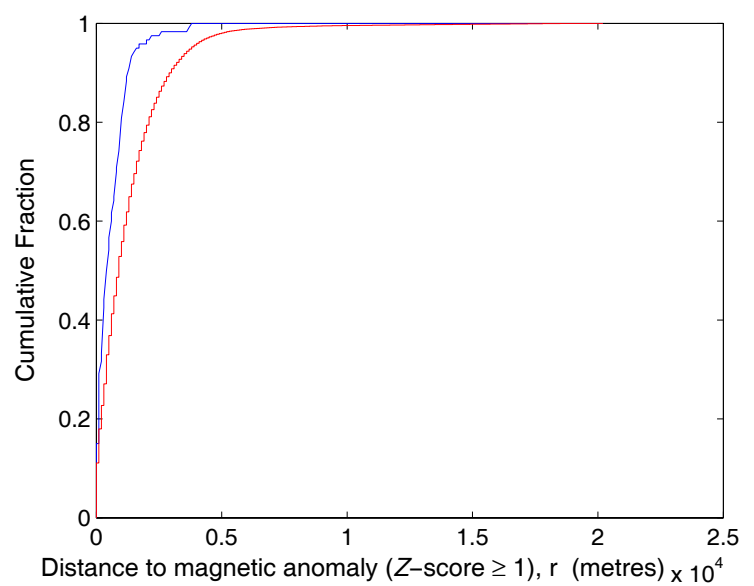

(e) Kolmogorov-Smirnov test

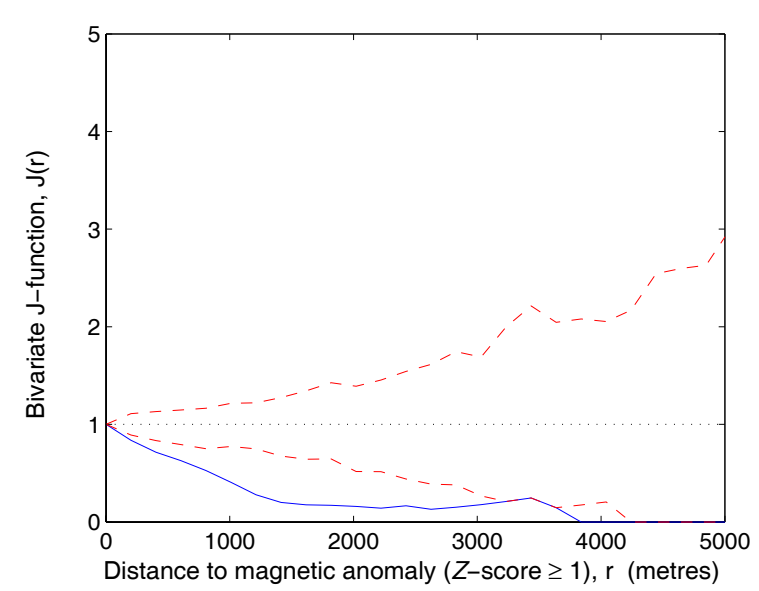

(b) Bivariate J-function (cumulative)

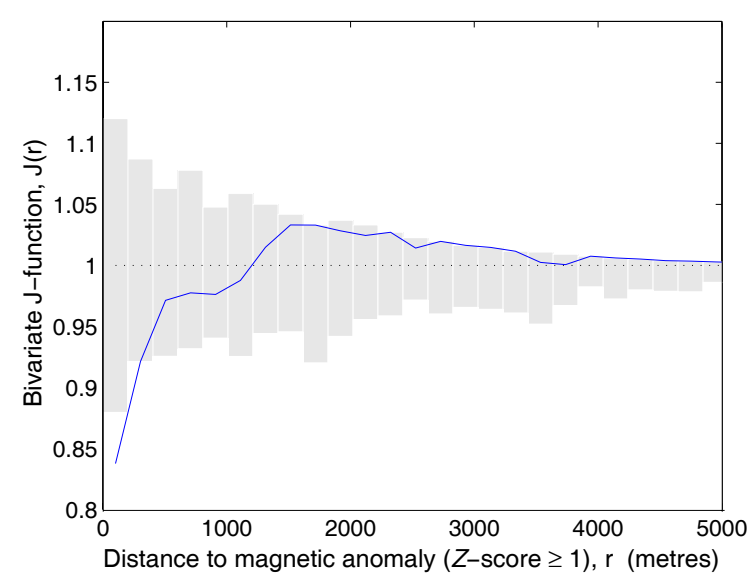

(d) Bivariate J-function (interval-based)

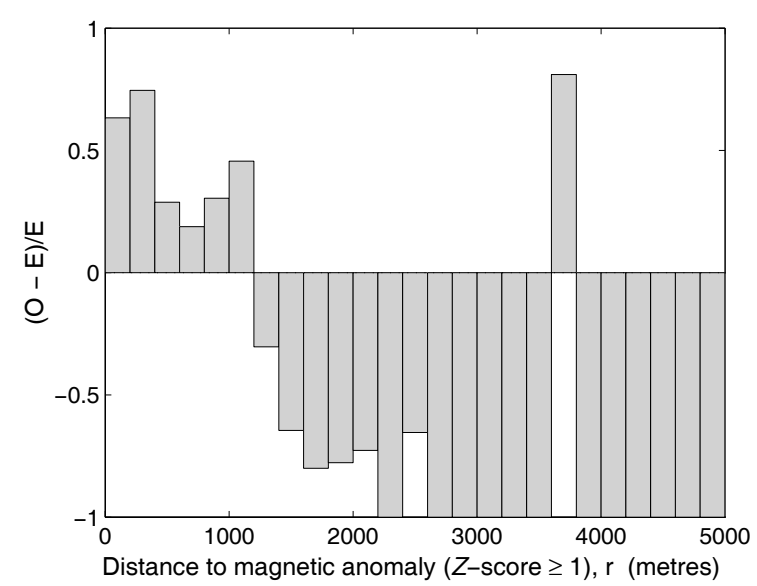

(f) $(O-E) / E$

Figure G.17. Plots used to examine the relationship between deposits and distance to positive magnetic anomalies, where the z-score, $Z \geq 1$, in the layer, l14_mz1_dist. In b), d) \& e), the empirical curve is blue and the theoretical curve is red. 


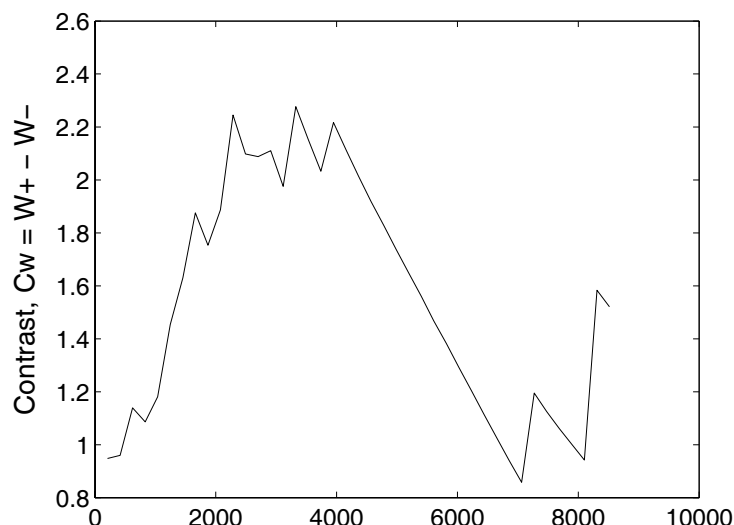

Distance to magnetic anomaly $(Z$-score $\geq 2), r$ (metres)

(a) Contrast, $C_{W}$

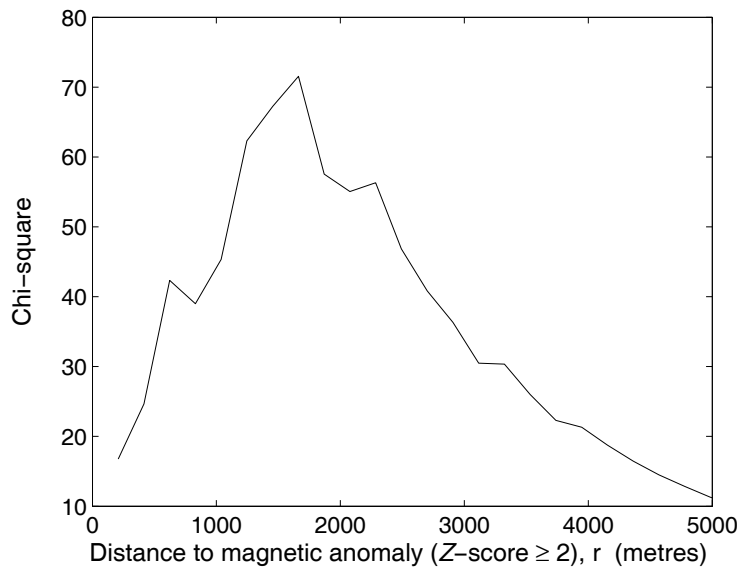

(c) Chi-squared

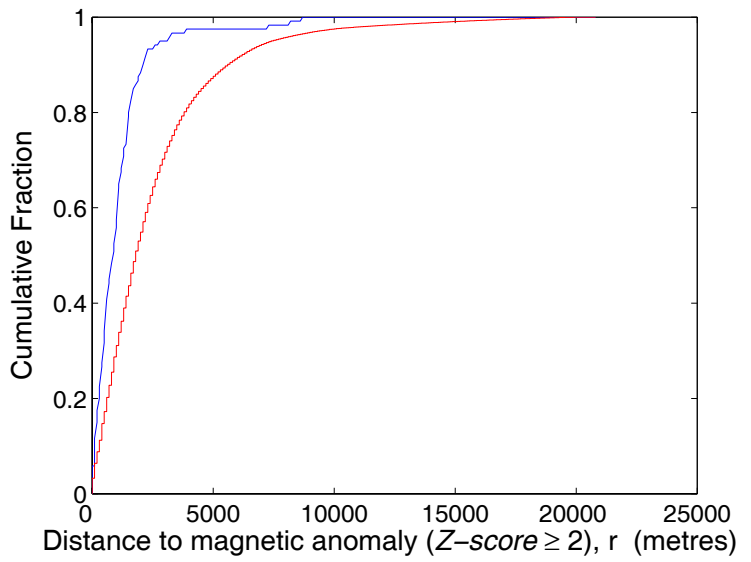

(e) Kolmogorov-Smirnov test

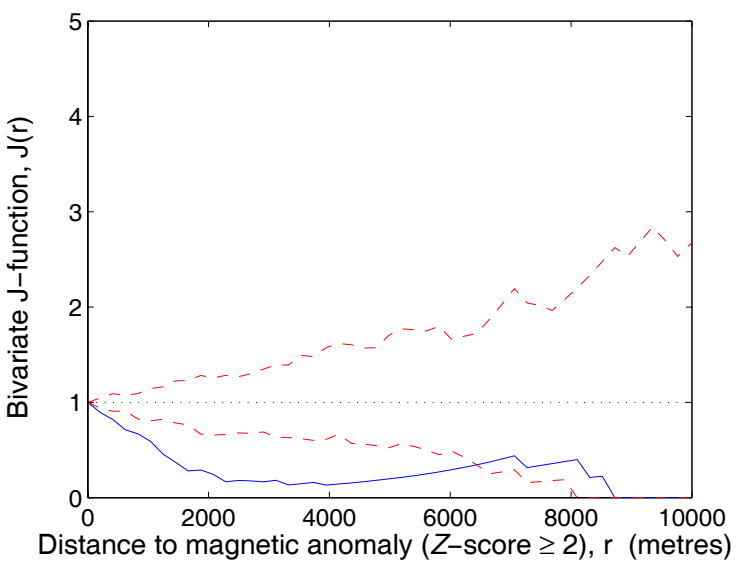

(b) Bivariate J-function (cumulative)

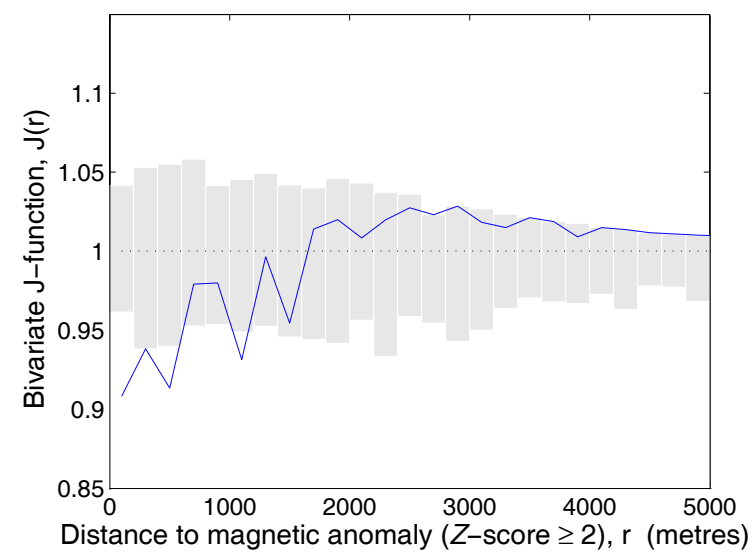

(d) Bivariate J-function (interval-based)

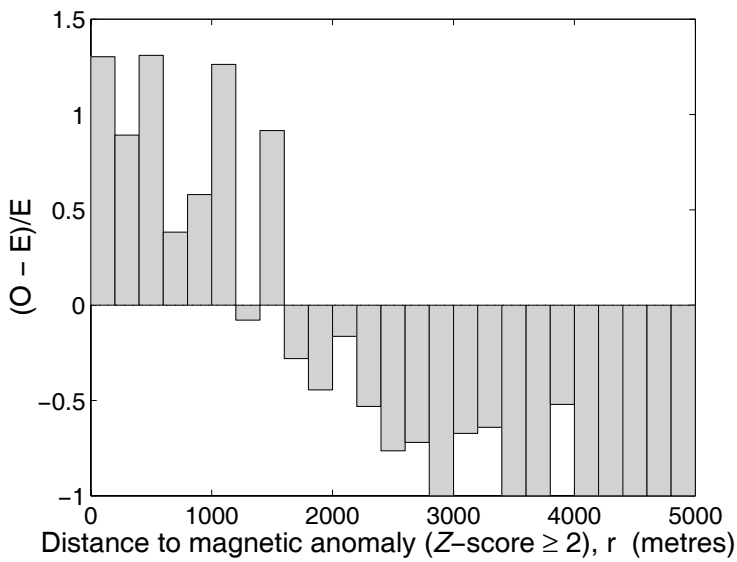

(f) $(O-E) / E$

Figure G.18. Plots used to examine the relationship between deposits and distance to positive magnetic anomalies, where the z-score, $Z \geq 2$, in the layer, l14_mz2_dist. In b), d) \& e), the empirical curve is blue and the theoretical curve is red. 


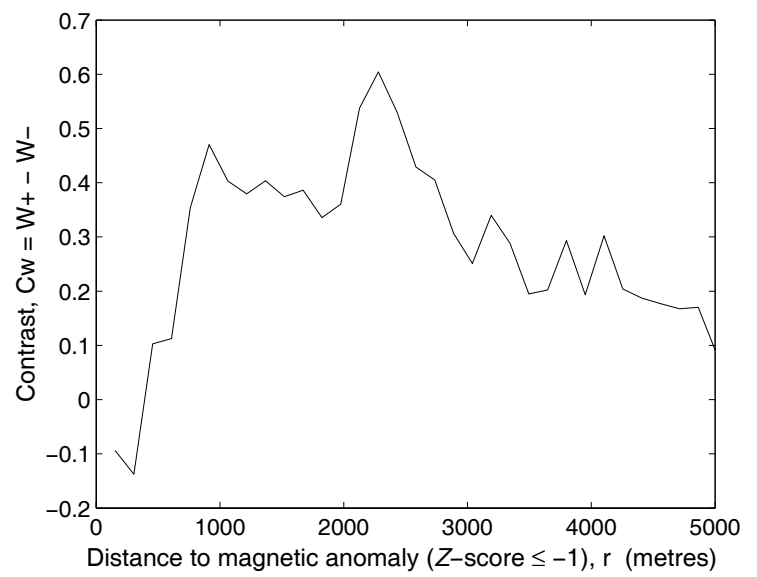

(a) Contrast, $C_{W}$

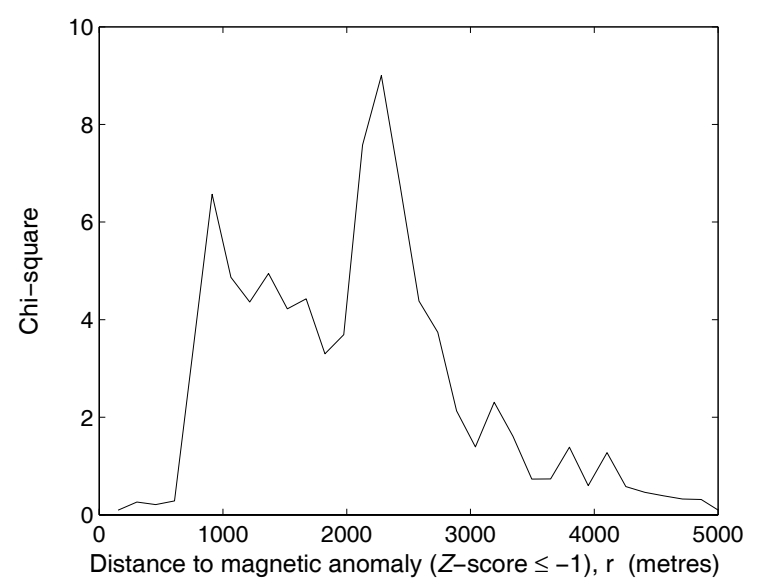

(c) Chi-squared

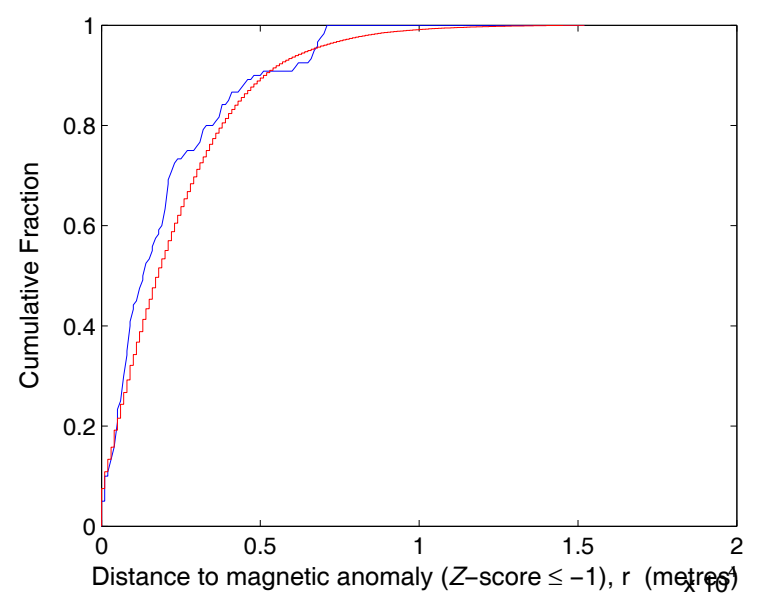

(e) Kolmogorov-Smirnov test

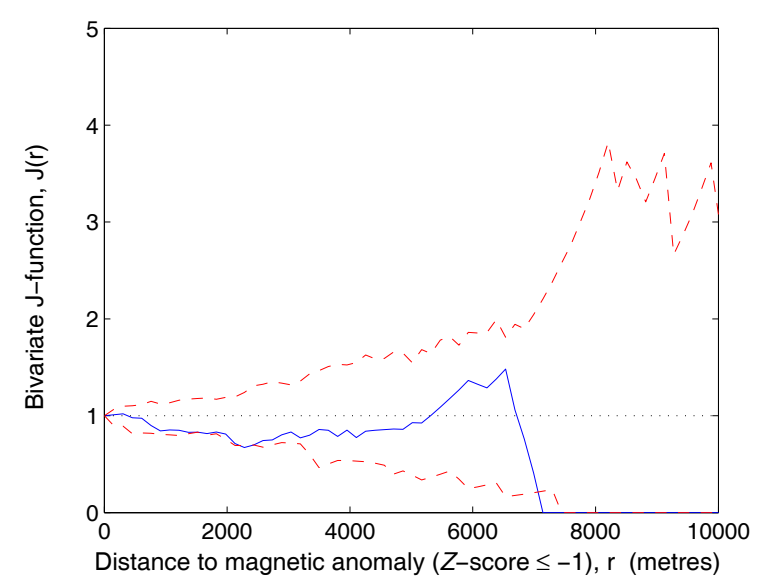

(b) Bivariate J-function (cumulative)

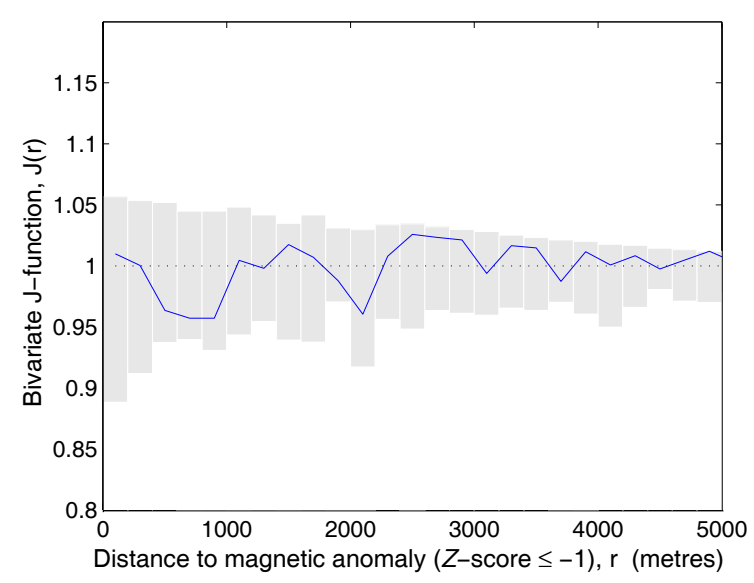

(d) Bivariate J-function (interval-based)

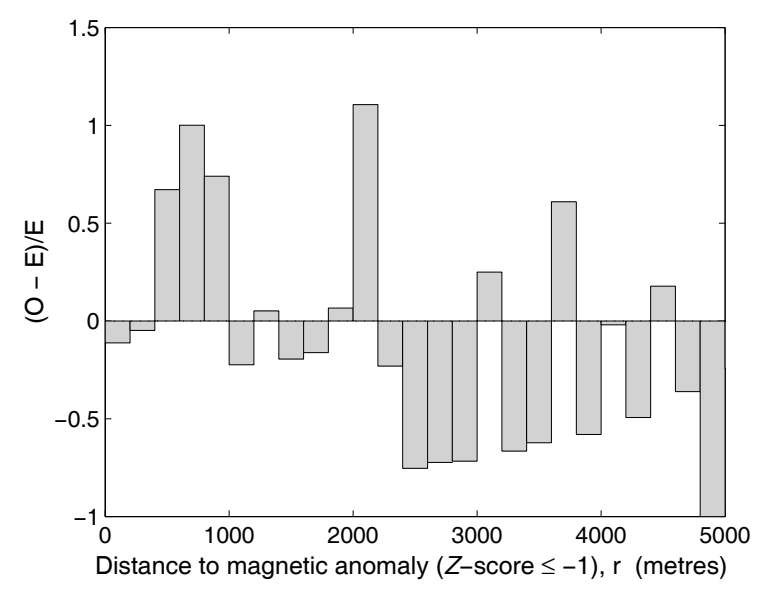

(f) $(O-E) / E$

Figure G.19. Plots used to examine the relationship between deposits and distance to negative magnetic anomalies, where the z-score, $Z \leq 1$, in the layer, l14_mz-1_dist. In b), d) \& e), the empirical curve is blue and the theoretical curve is red. 


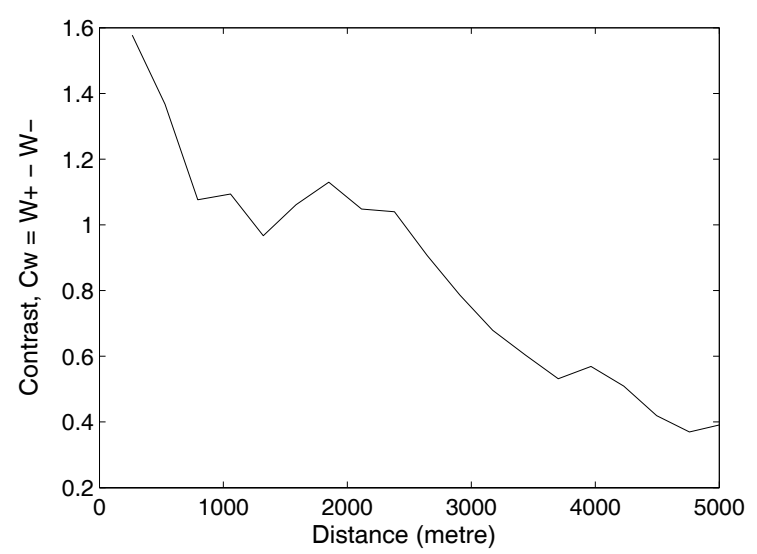

(a) Contrast, $C_{W}$

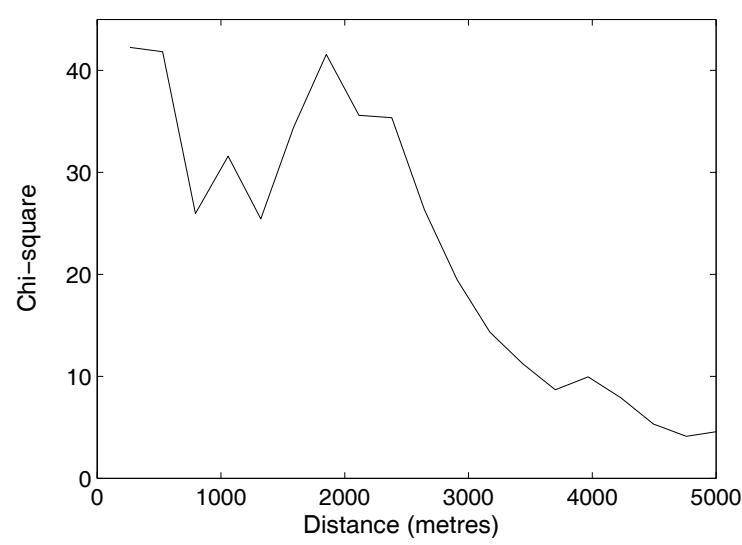

(c) Chi-squared

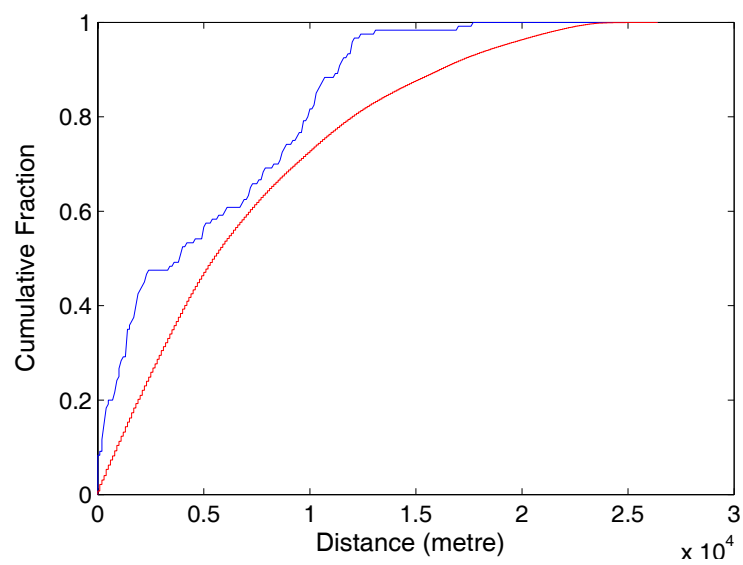

(e) Kolmogorov-Smirnov test

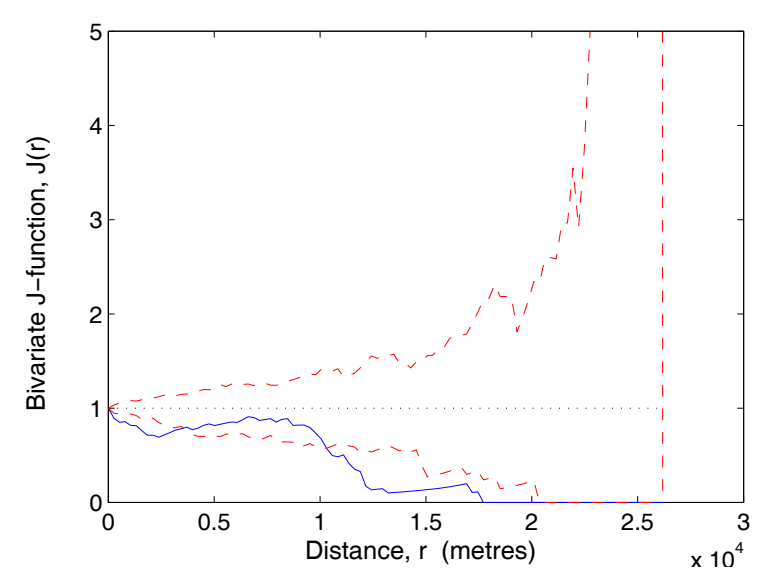

(b) Bivariate J-function (cumulative)

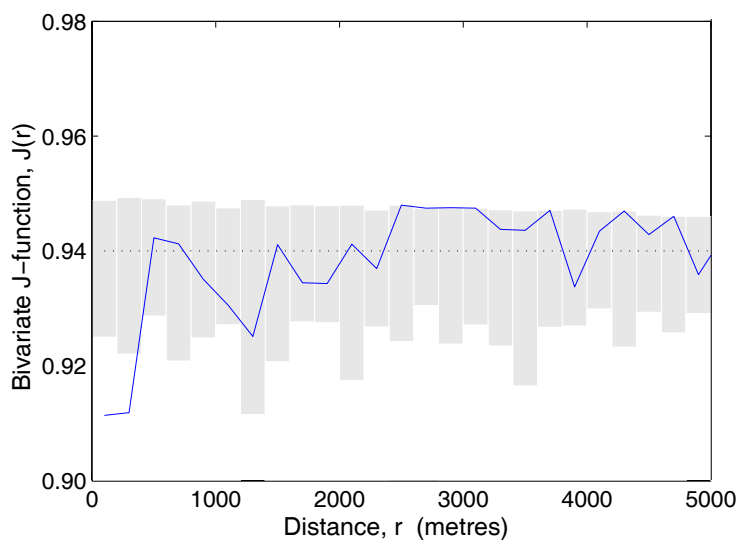

(d) Bivariate J-function (interval-based)

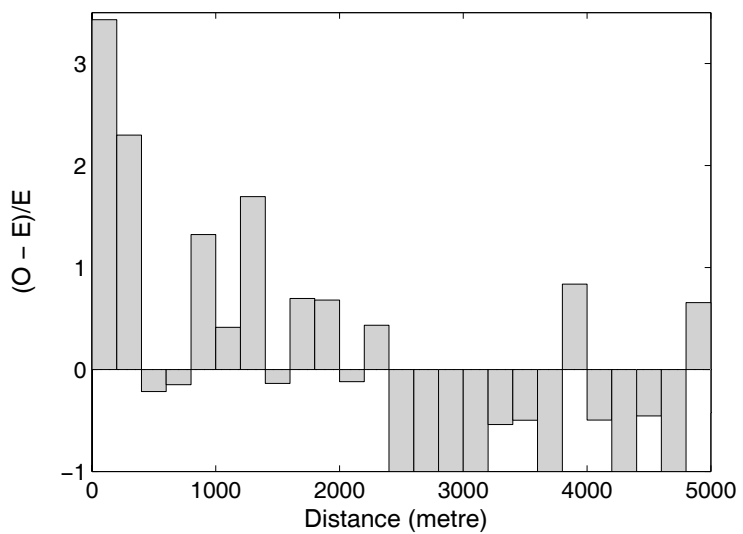

(f) $(O-E) / E$

Figure G.20. Plots used to examine the relationship between deposits and distance to crustalscale faults and shear zones in the layer, l20_f1_dist. The empirical curve is shown in blue in b), d) \& e). The theoretical curve is shown in red in b) \& e) and as grey bars in d). 


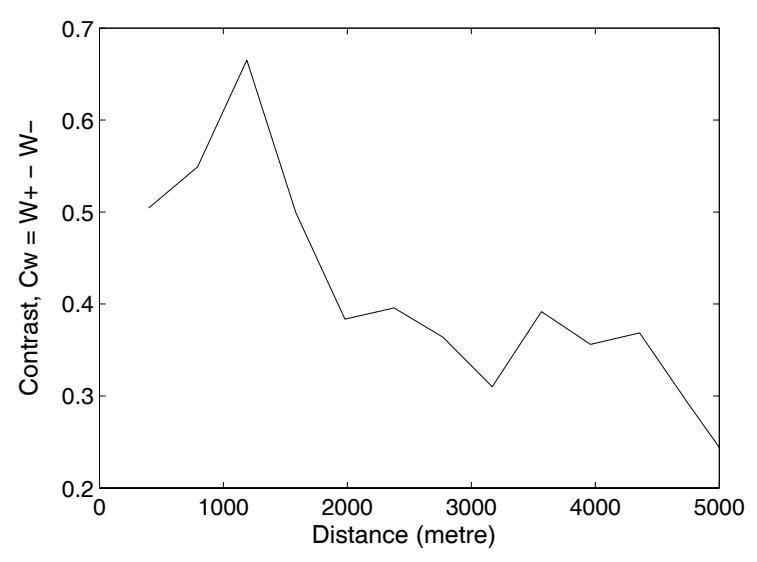

(a) Contrast, $C_{W}$

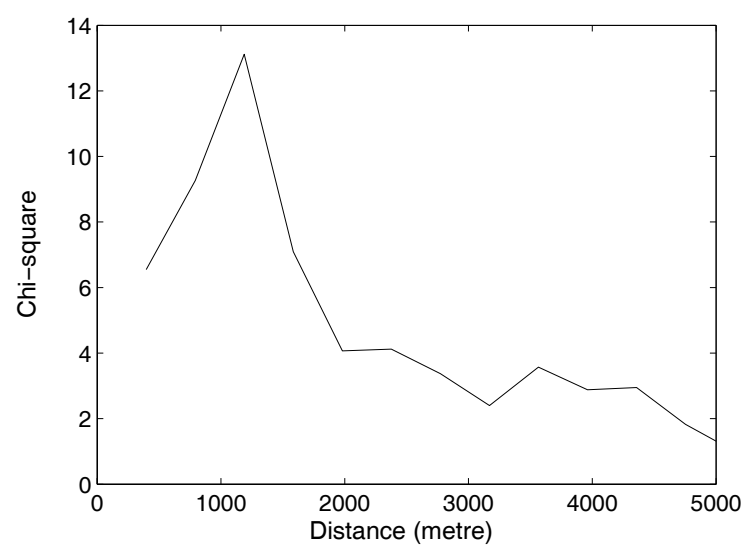

(c) Chi-squared

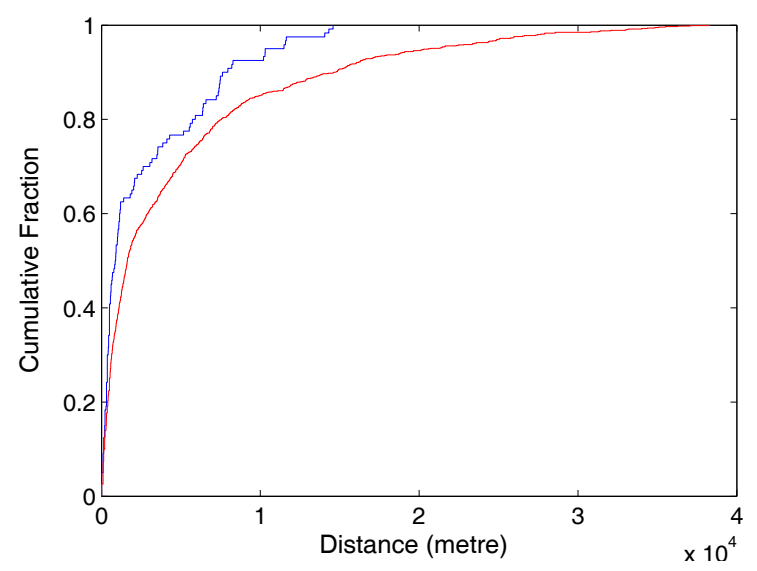

(e) Kolmogorov-Smirnov test

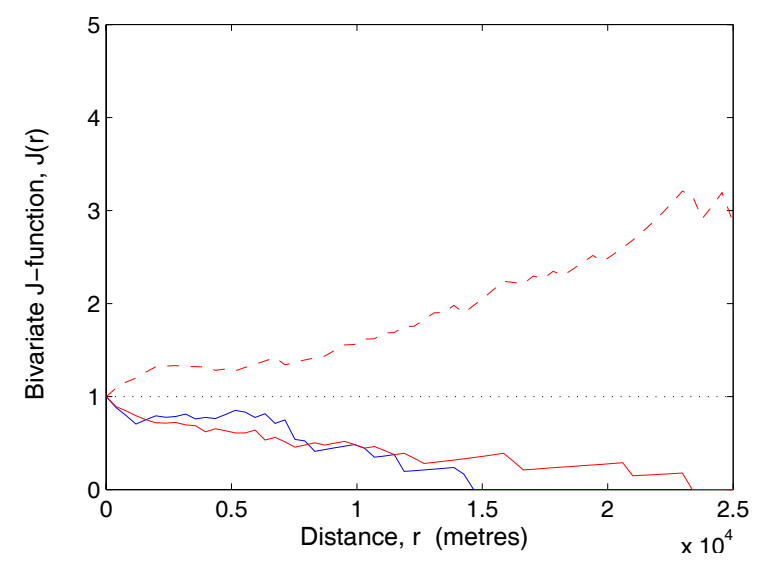

(b) Bivariate J-function (cumulative)

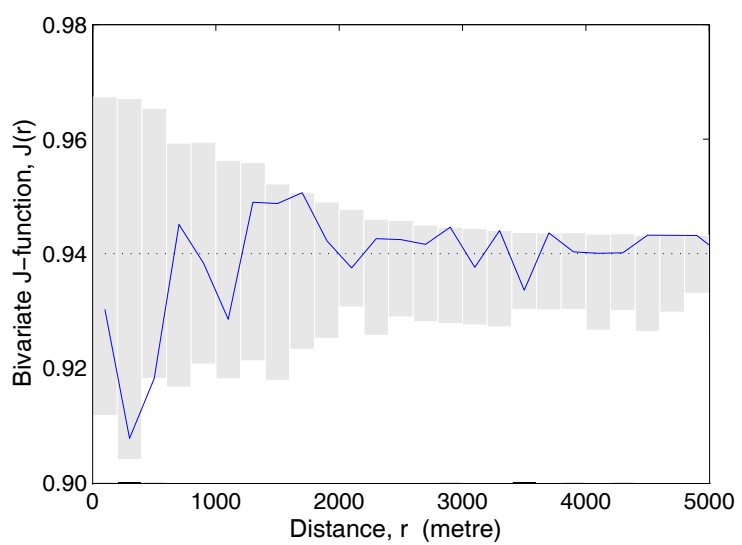

(d) Bivariate J-function (interval-based)

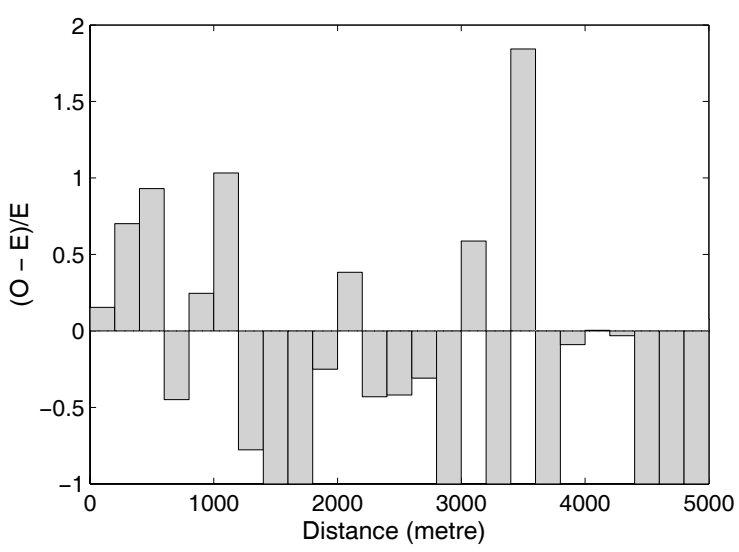

(f) $(O-E) / E$

Figure G.21. Plots used to examine the relationship between deposits and distance to NNWtrending regional-scale faults and shear zones in the layer, l21_f2_dist. The empirical curve is shown in blue in b), d) \& e). The theoretical curve is shown in red in b) \& e) and as grey bars in d). 


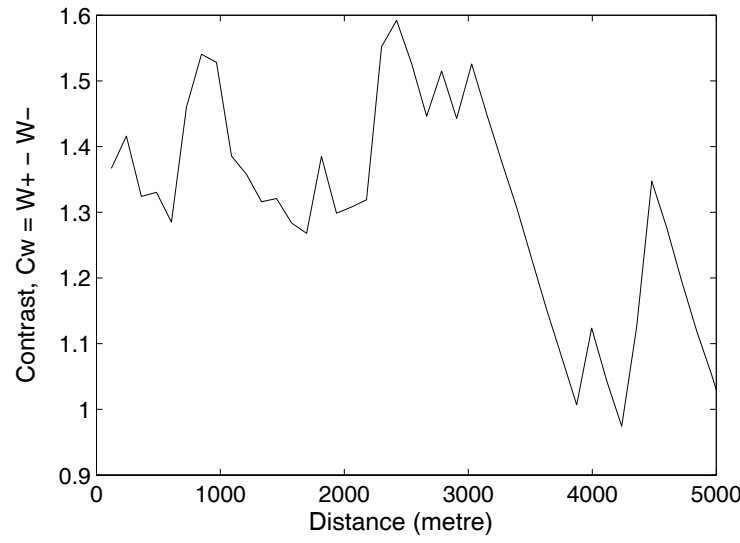

(a) Contrast, $C_{W}$

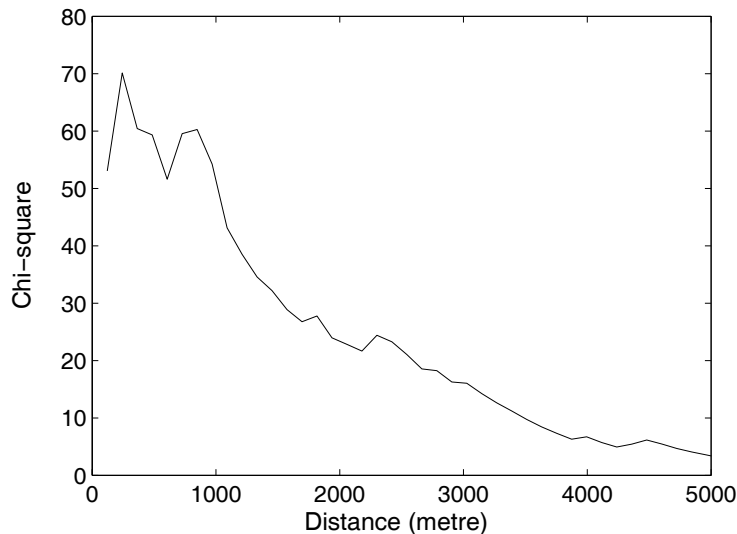

(c) Chi-squared

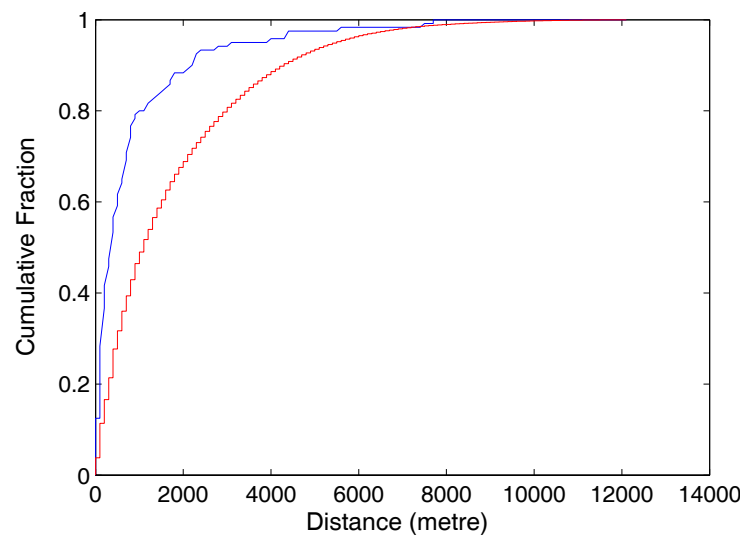

(e) Kolmogorov-Smirnov test

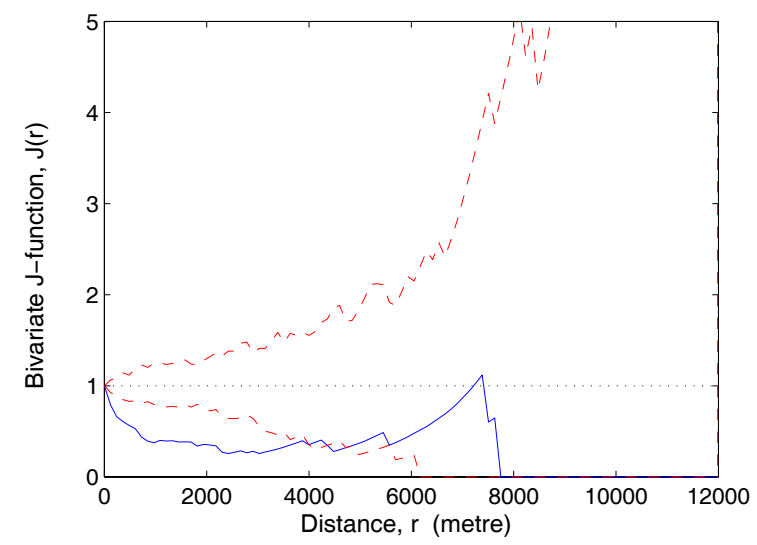

(b) Bivariate J-function (cumulative)

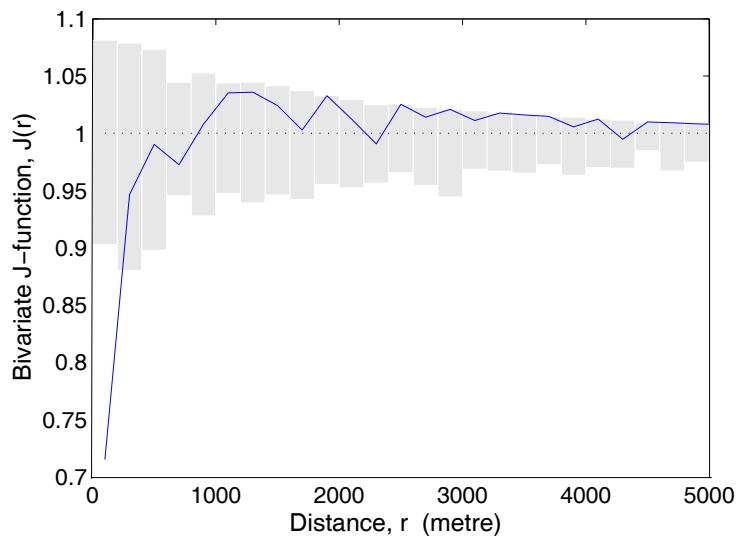

(d) Bivariate J-function (interval-based)

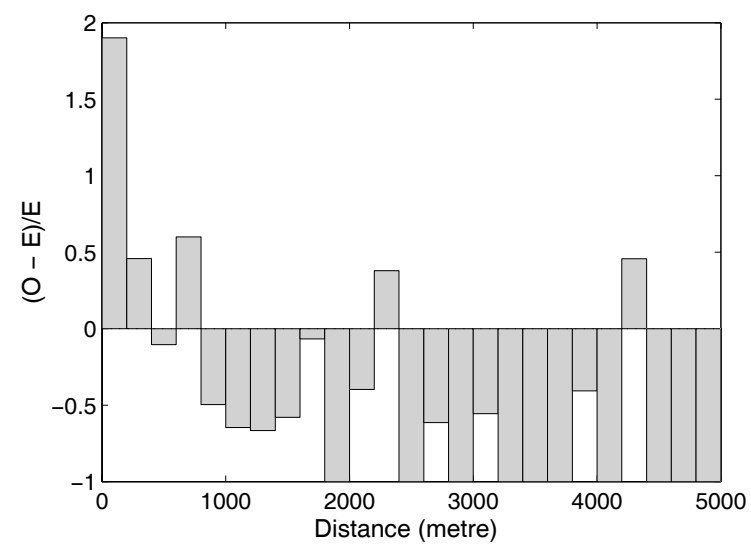

(f) $(O-E) / E$

Figure G.22. Plots used to examine the relationship between deposits and distance to NE-trending regional faults and shear zones in the layer, l22_d3_dist. The empirical curve is shown in blue in b), d) \& e). The theoretical curve is shown in red in b) \& e) and as grey bars in d). 


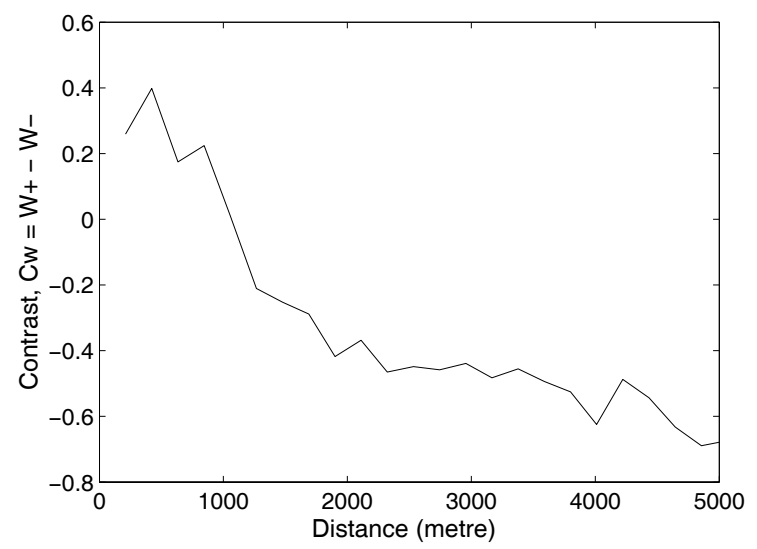

(a) Contrast, $C_{W}$

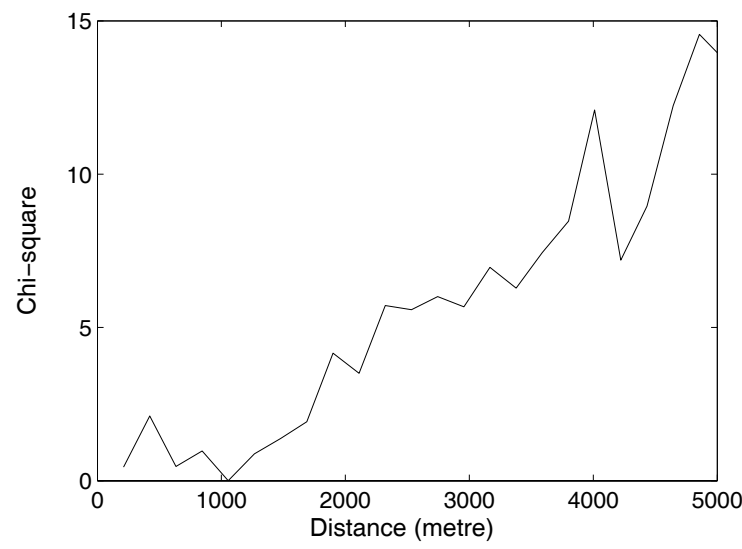

(c) Chi-squared

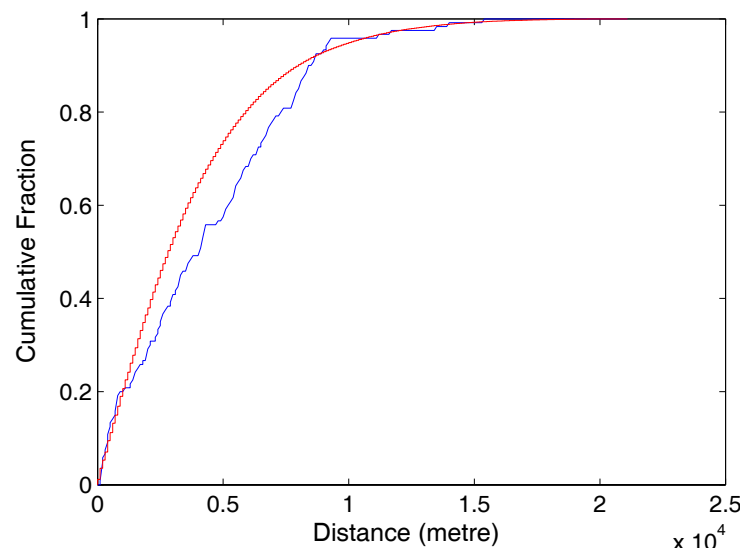

(e) Kolmogorov-Smirnov test

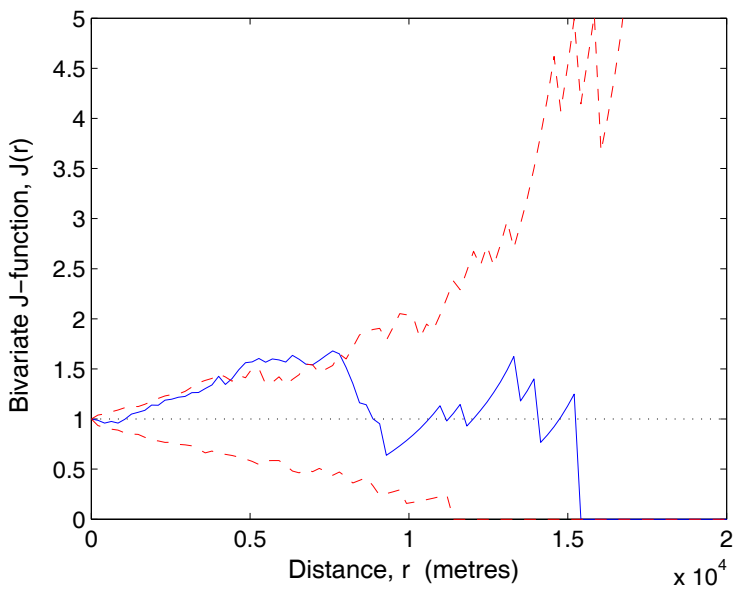

(b) Bivariate J-function (cumulative)

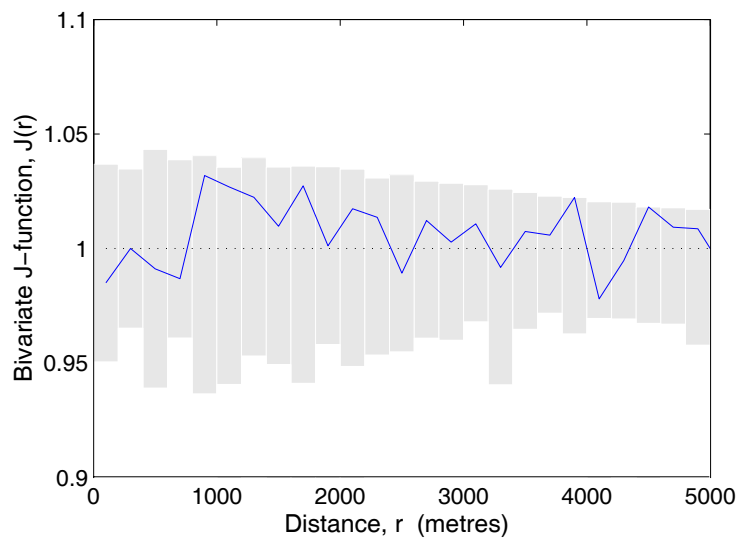

(d) Bivariate J-function (interval-based)

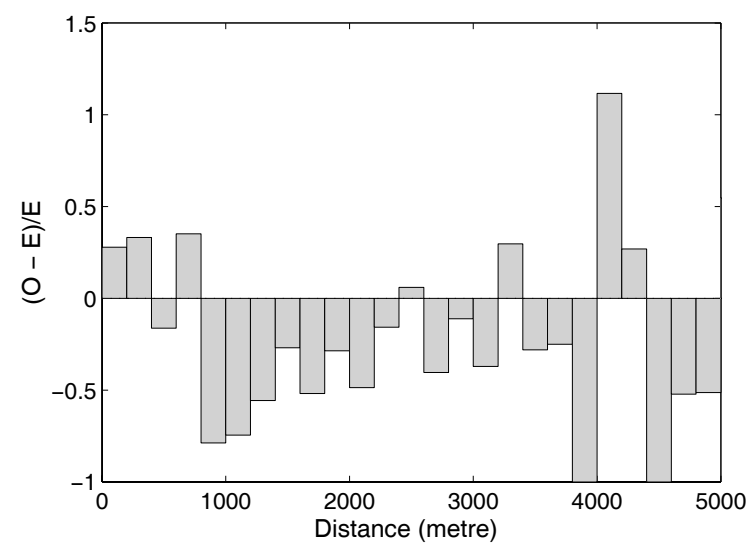

(f) $(O-E) / E$

Figure G.23. Plots used to examine the relationship between deposits and distance to E-trending regional faults and shear zones in the layer, 123_d4_dist. The empirical curve is shown in blue in b), d) \& e). The theoretical curve is shown in red in b) \& e) and as grey bars in d). 


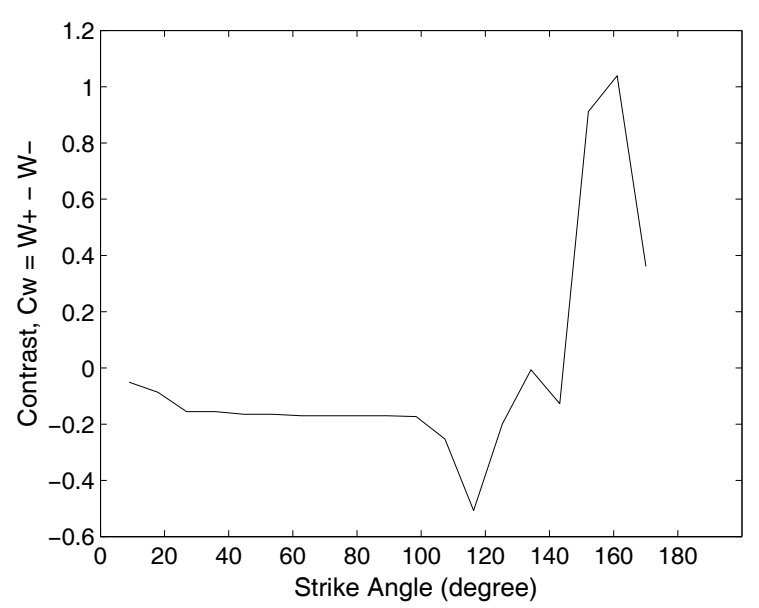

(a) Contrast, $C_{W}$

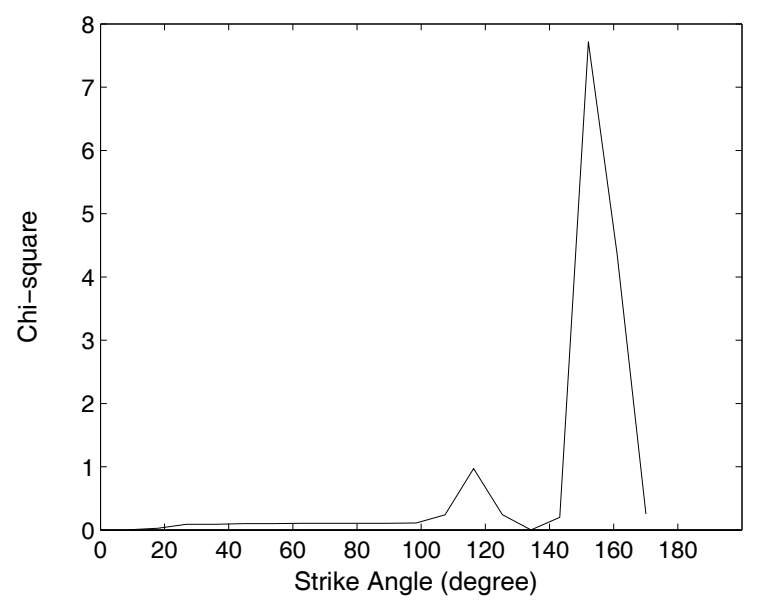

(c) Chi-squared

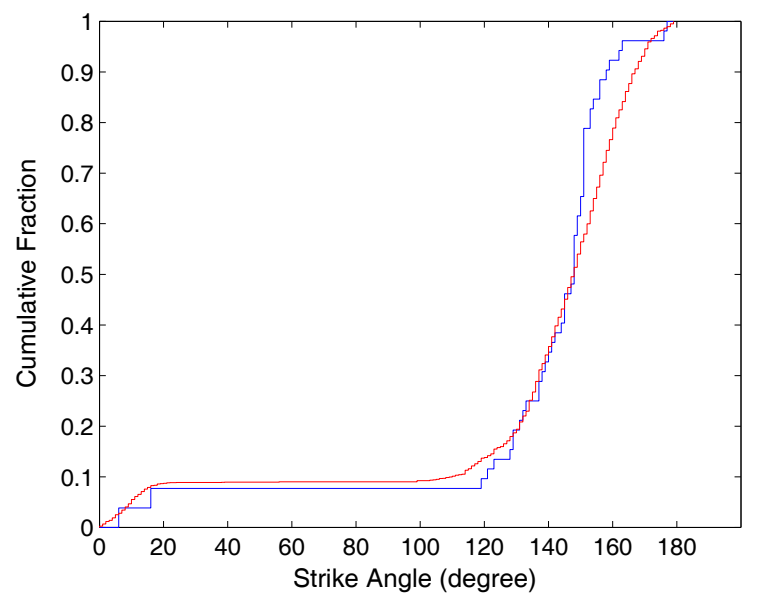

(e) Kolmogorov-Smirnov test

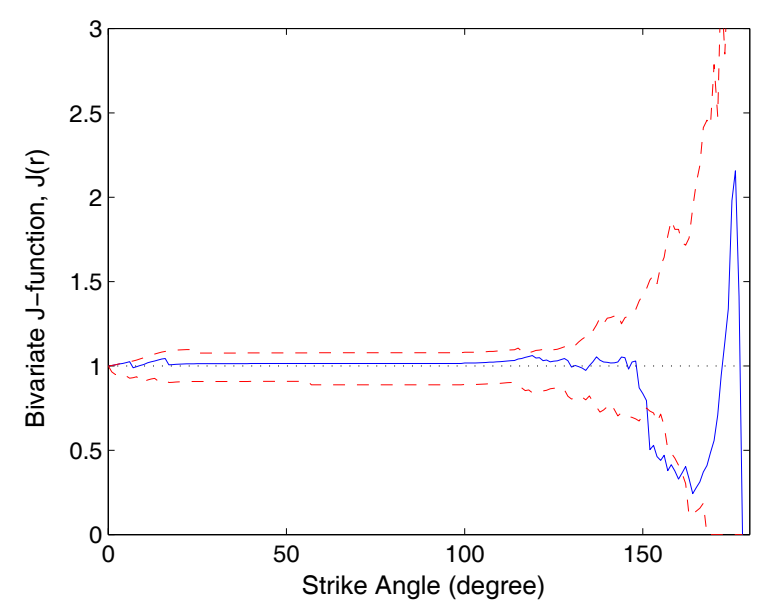

(b) Bivariate J-function (cumulative)

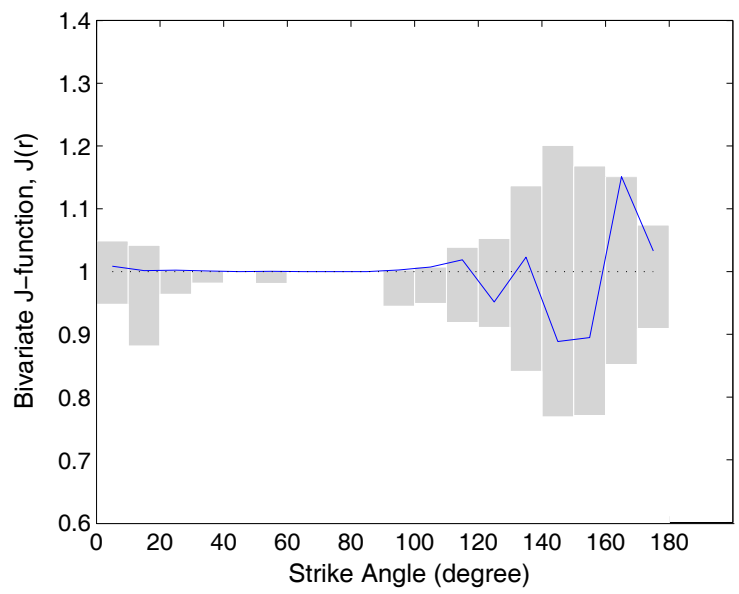

(d) Bivariate J-function (interval-based)

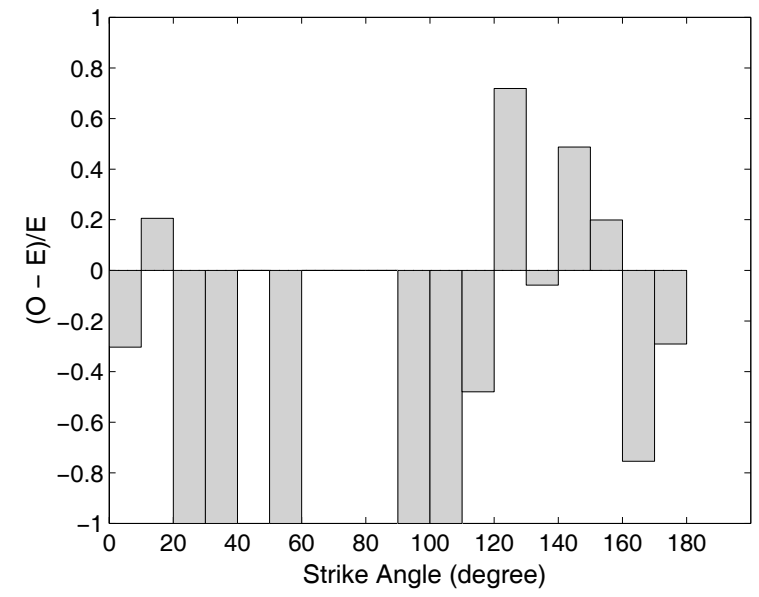

(f) $(O-E) / E$

Figure G.24. Plots used to examine the relationship between deposits and the strike of crustalscale shear zones in the layer $128 \_$s_strf1. The strike angles have been reduced to a range $0-179^{\circ}$. The empirical curve is shown in blue in b), d) \& e). The theoretical curve is shown in red in b) \& e) and as grey bars in d). 


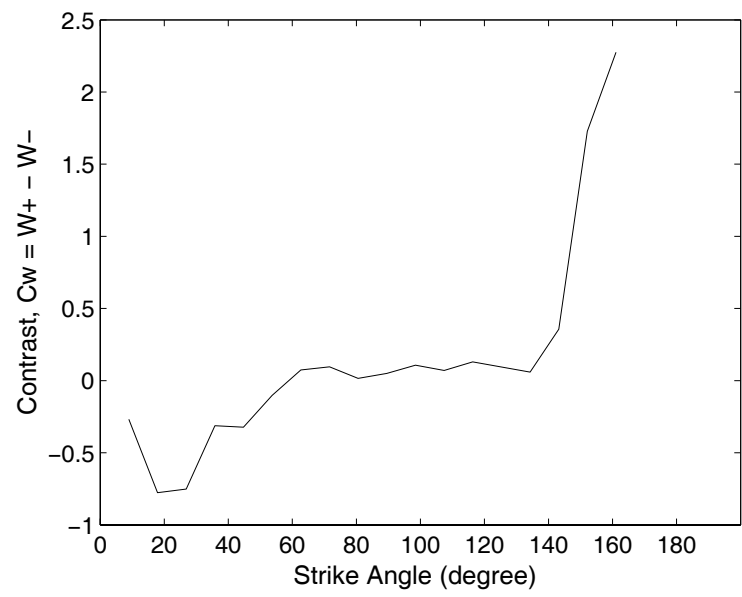

(a) Contrast, $C_{W}$

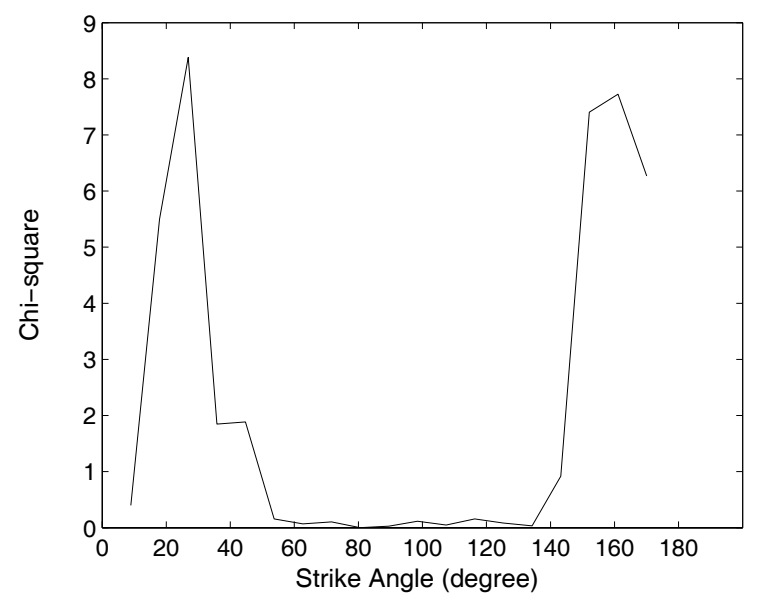

(c) Chi-squared

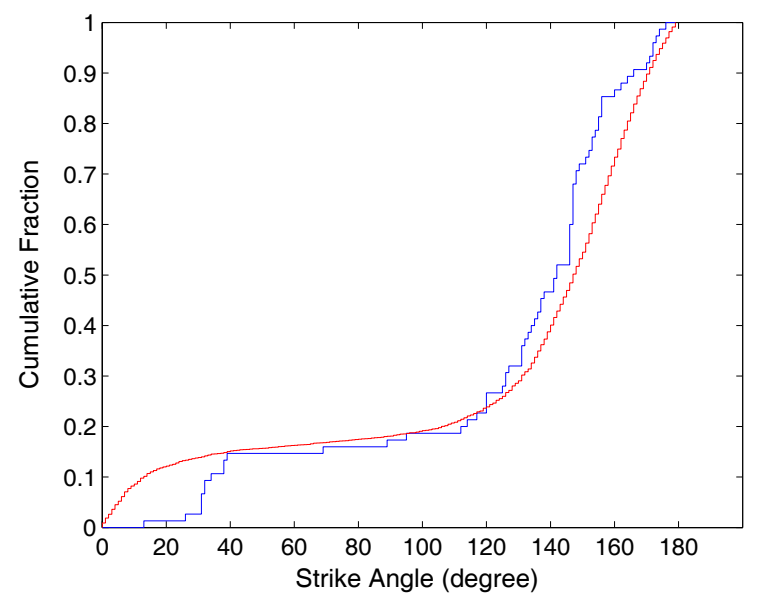

(e) Kolmogorov-Smirnov test

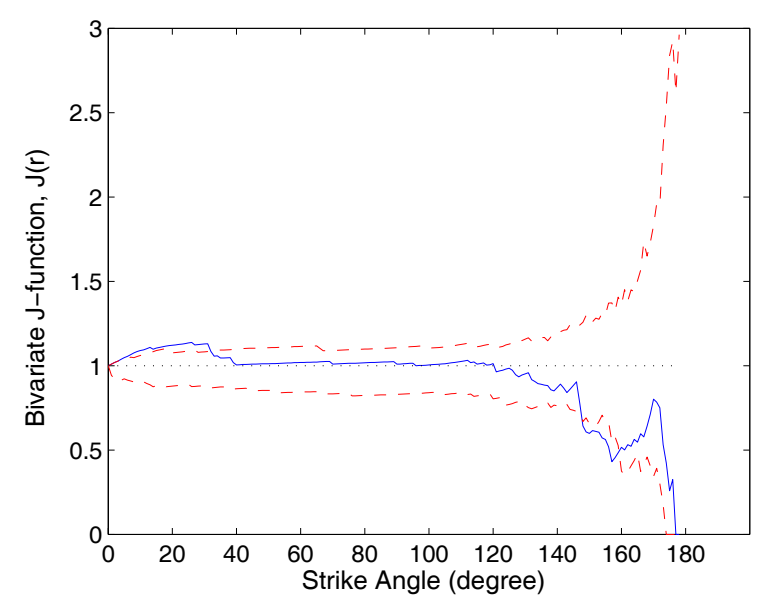

(b) Bivariate J-function (cumulative)

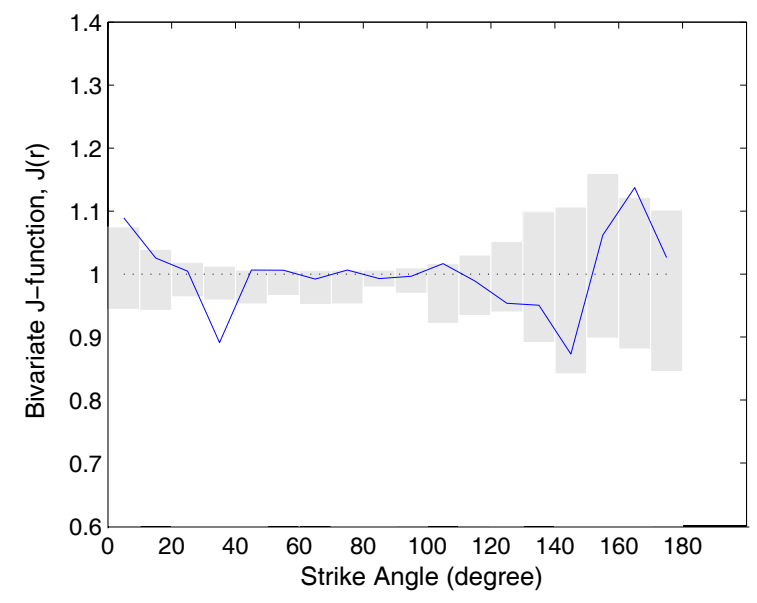

(d) Bivariate J-function (interval-based)

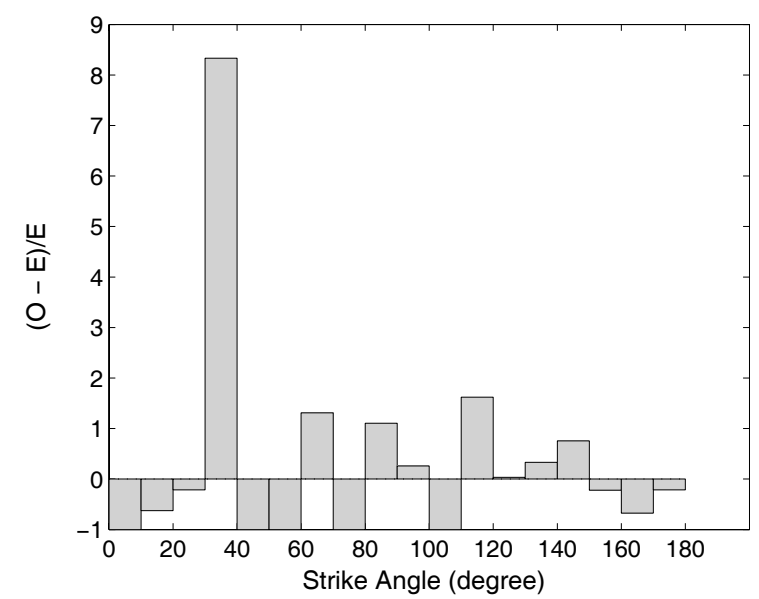

(f) $(O-E) / E$

Figure G.25. Plots used to examine the relationship between deposits and strike of NNW-trending regional-scale faults and shear zones in the layer, 129_s_f2. The strike angles have been reduced to a range $0-179^{\circ}$. The empirical curve is shown in blue in b), d) \& e). The theoretical curve is shown in red in b) \& e) and as grey bars in d). 


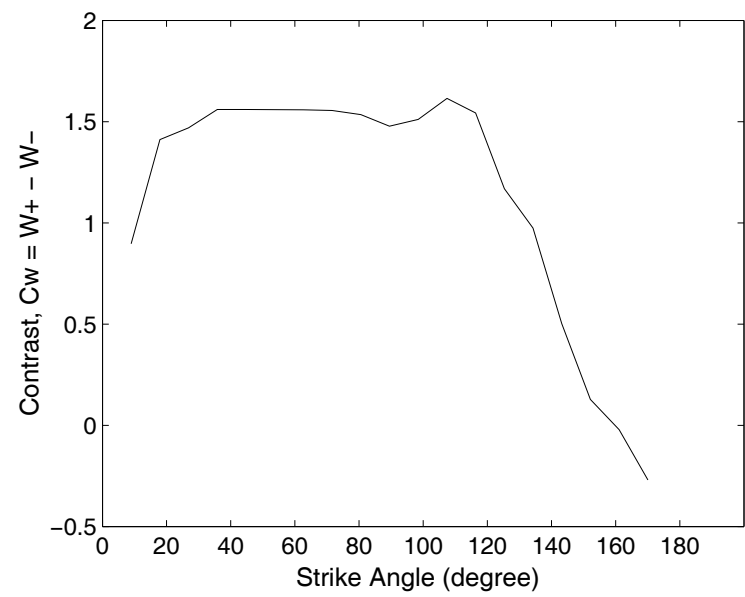

(a) Contrast, $C_{W}$

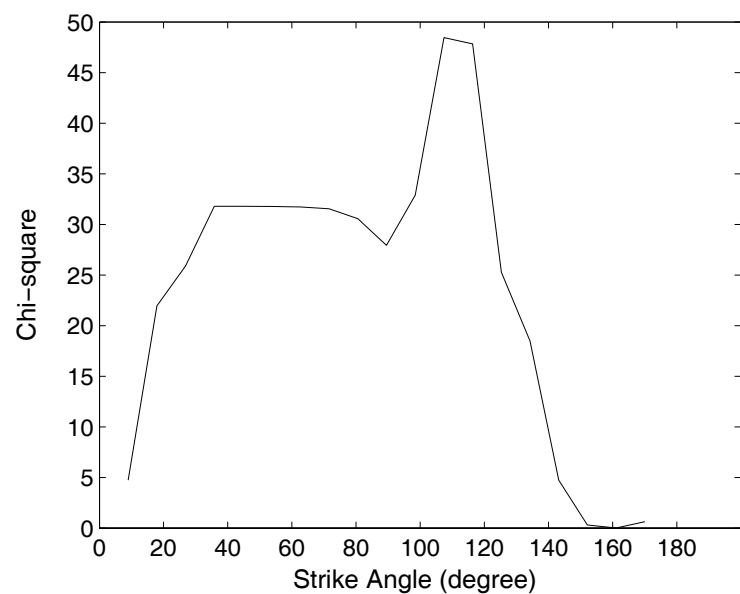

(c) Chi-squared

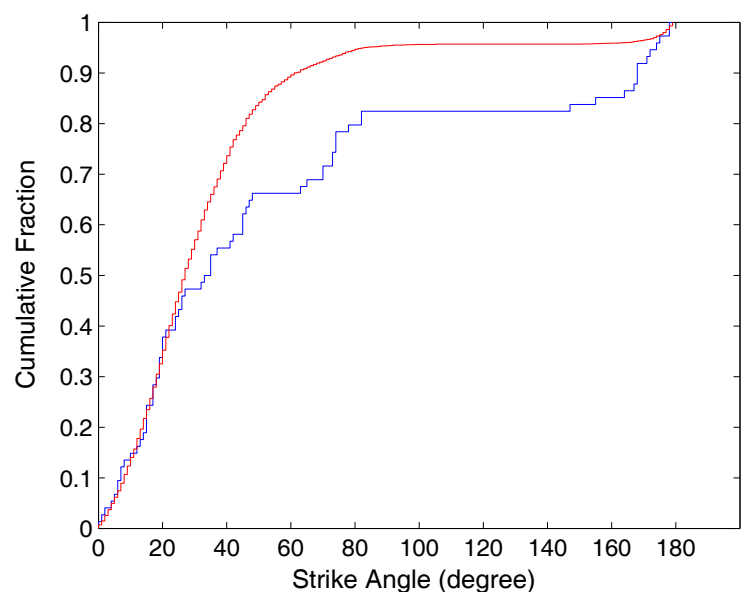

(e) Kolmogorov-Smirnov test

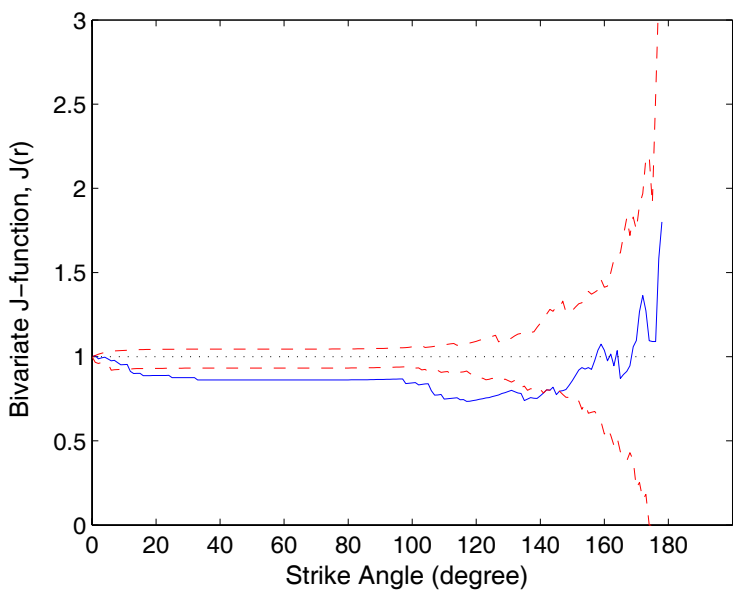

(b) Bivariate J-function (cumulative)

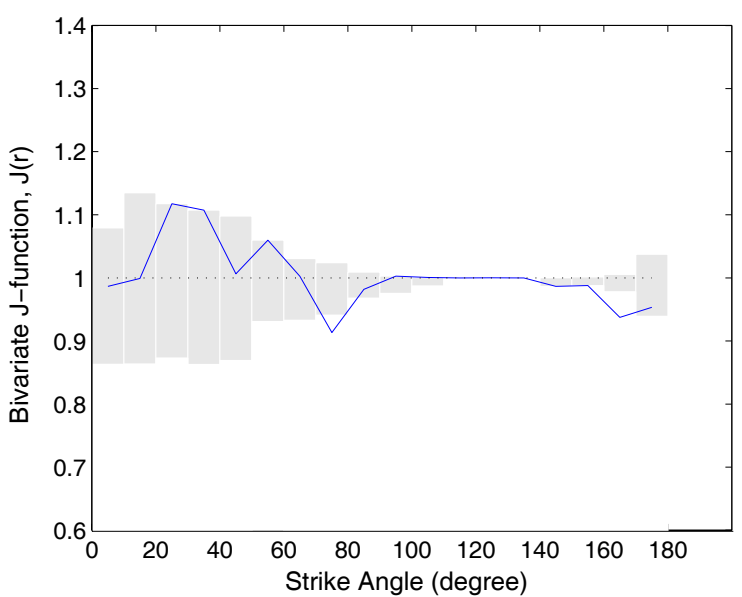

(d) Bivariate J-function (interval-based)

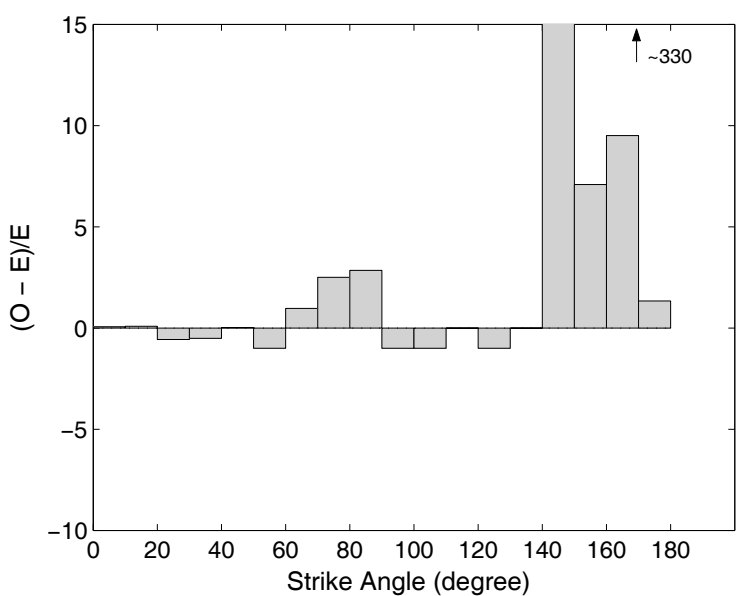

(f) $(O-E) / E$

Figure G.26. Plots used to examine the relationship between deposits and strike of NE-trending regional-scale faults and shear zones in the layer, 130_s_f3. The strike angles have been reduced to a range $0-179^{\circ}$. The empirical curve is shown in blue in b), d) \& e). The theoretical curve is shown in red in b) \& e) and as grey bars in d). 


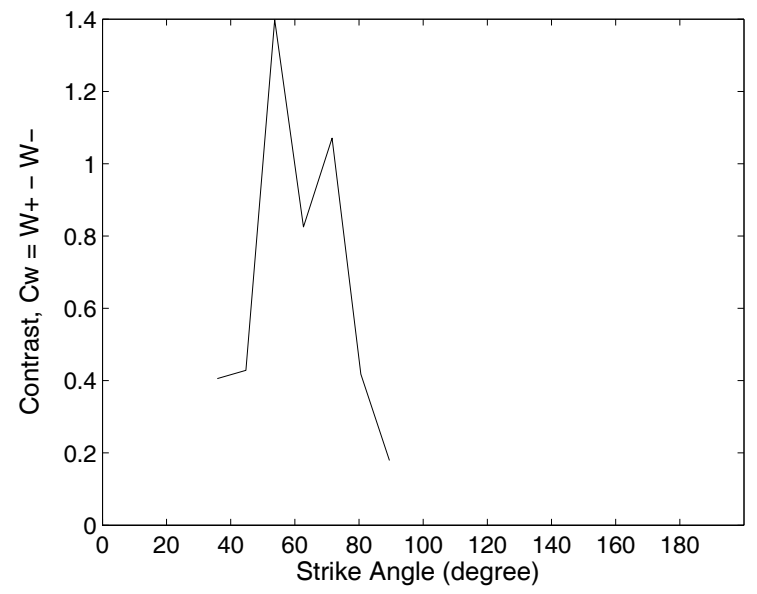

(a) Contrast, $C_{W}$

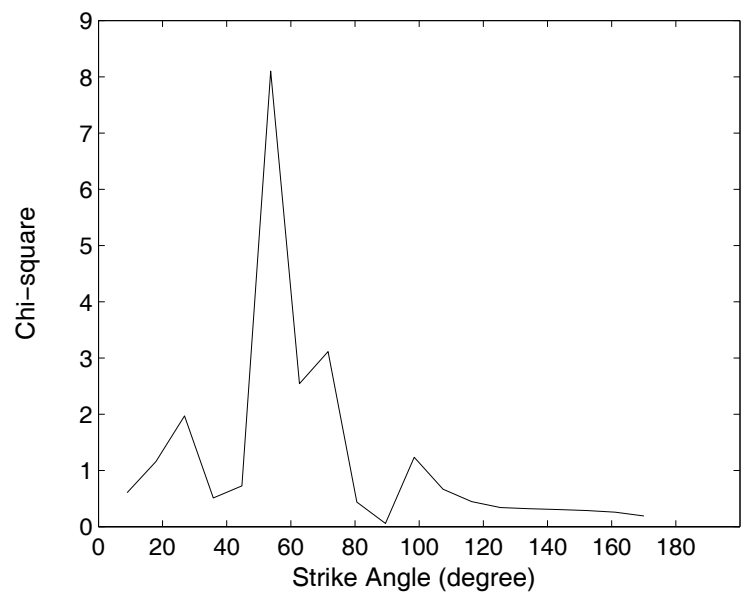

(c) Chi-squared

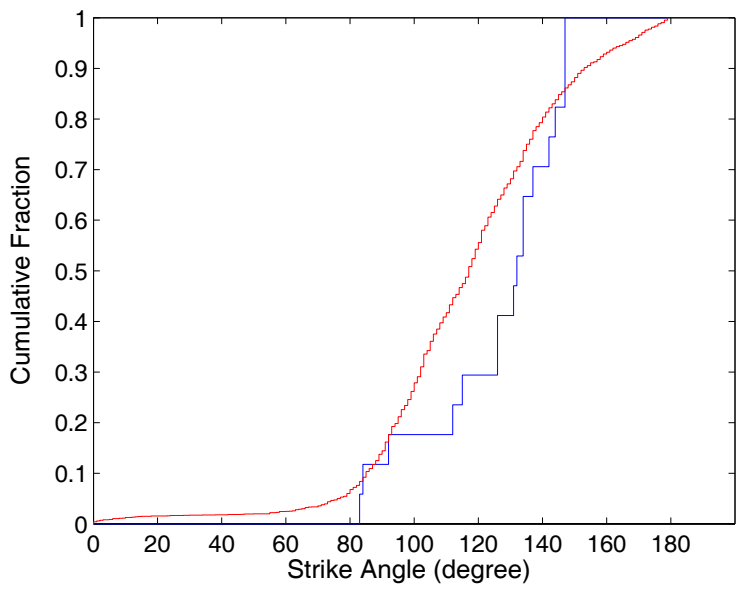

(e) Kolmogorov-Smirnov test

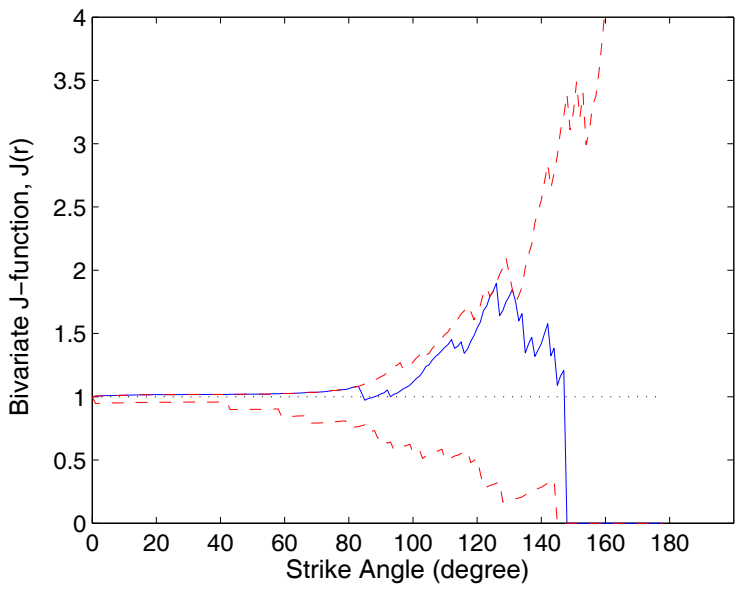

(b) Bivariate J-function (cumulative)

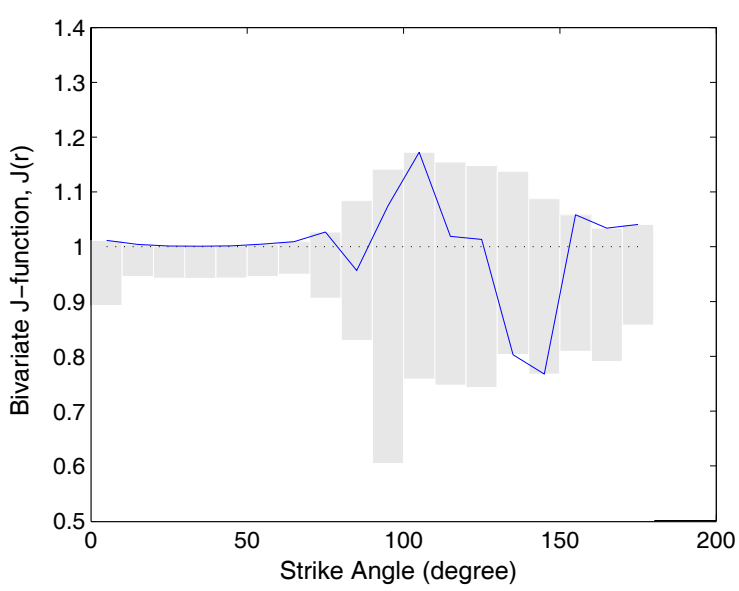

(d) Bivariate J-function (interval-based)

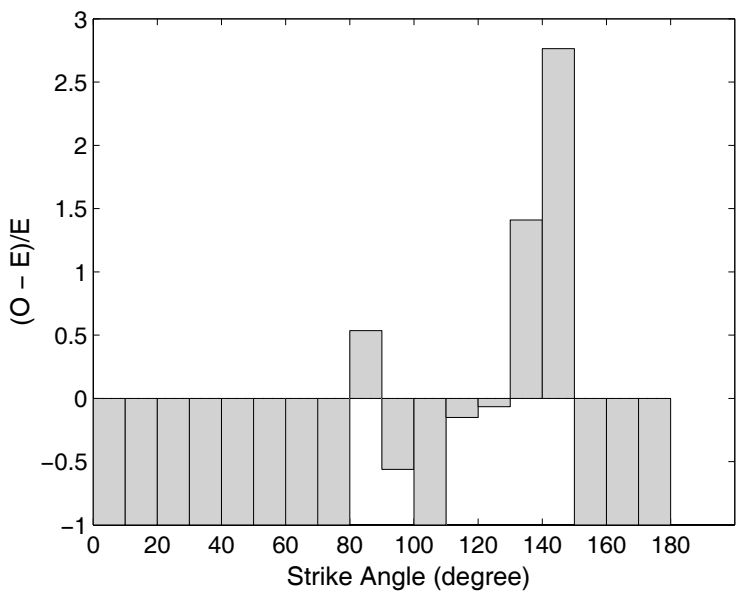

(f) $(O-E) / E$

Figure G.27. Plots used to examine the relationship between deposits and strike of E-trending regional-scale faults and shear zones in the layer, l31_s_f4. The strike angles have been reduced to a range $0-179^{\circ}$. The empirical curve is shown in blue in b), d) \& e). The theoretical curve is shown in red in b) \& e) and as grey bars in d). 


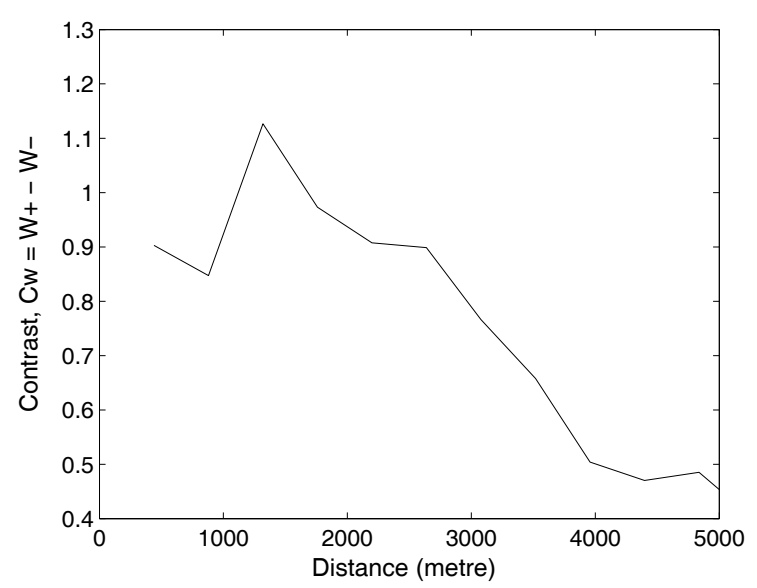

(a) Contrast, $C_{W}$

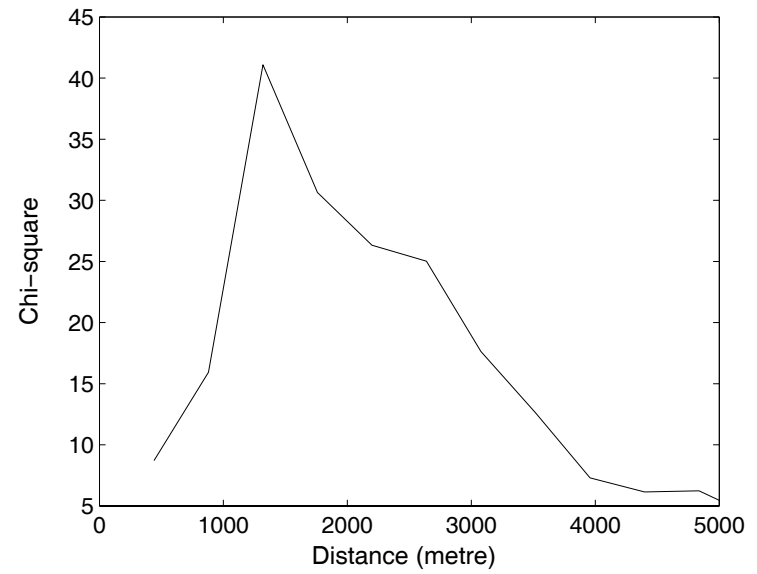

(c) Chi-squared

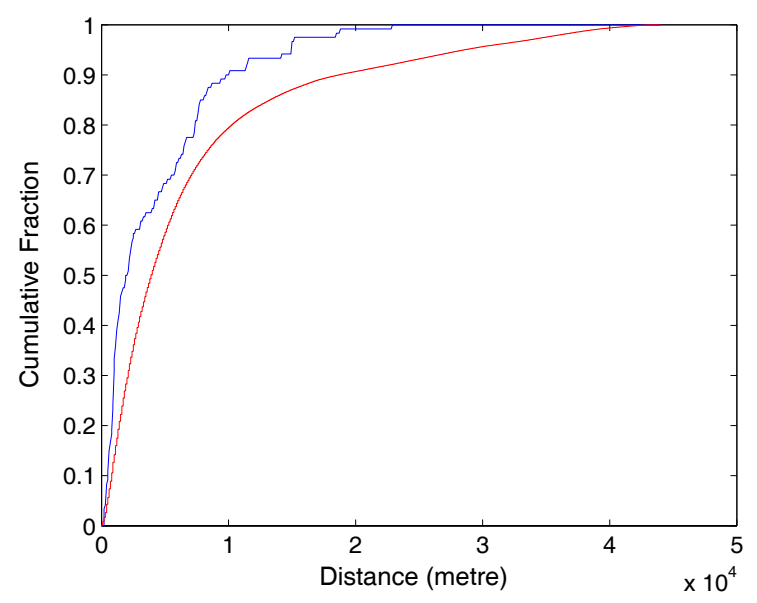

(e) Kolmogorov-Smirnov test

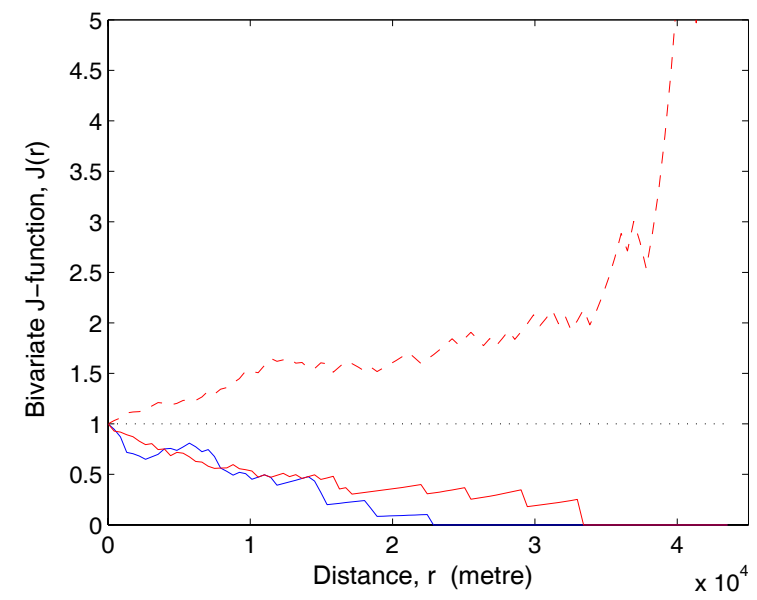

(b) Bivariate J-function (cumulative)

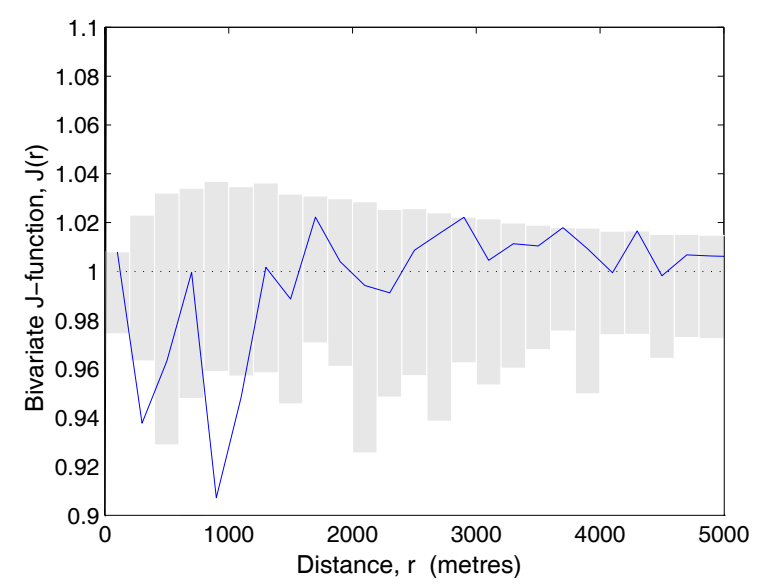

(d) Bivariate J-function (interval-based)

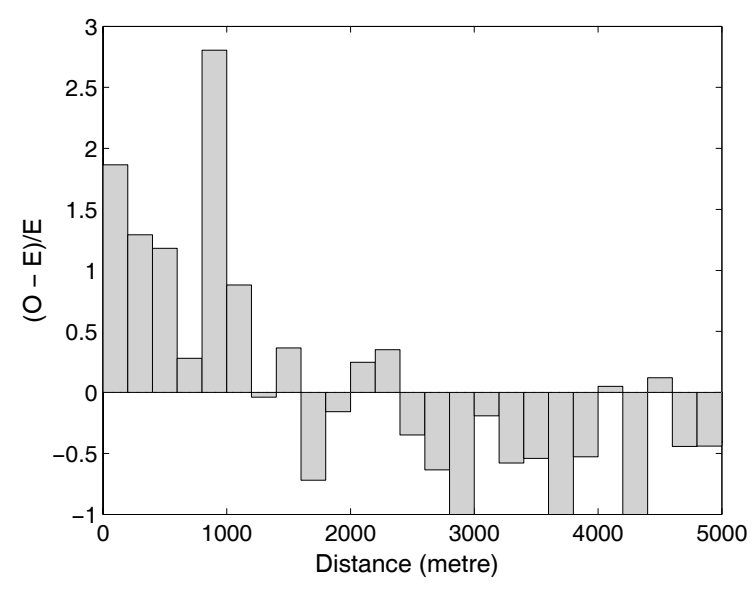

(f) $(O-E) / E$

Figure G.28. Plots used to examine the relationship between deposits and distance to the intersection of the nearest regional-scale faults and shear zones in the layer, 134_s_f23pdst. The empirical curve is shown in blue in b), d) \& e). The theoretical curve is shown in red in b) \& e) and as grey bars in $\mathrm{d}$ ). 


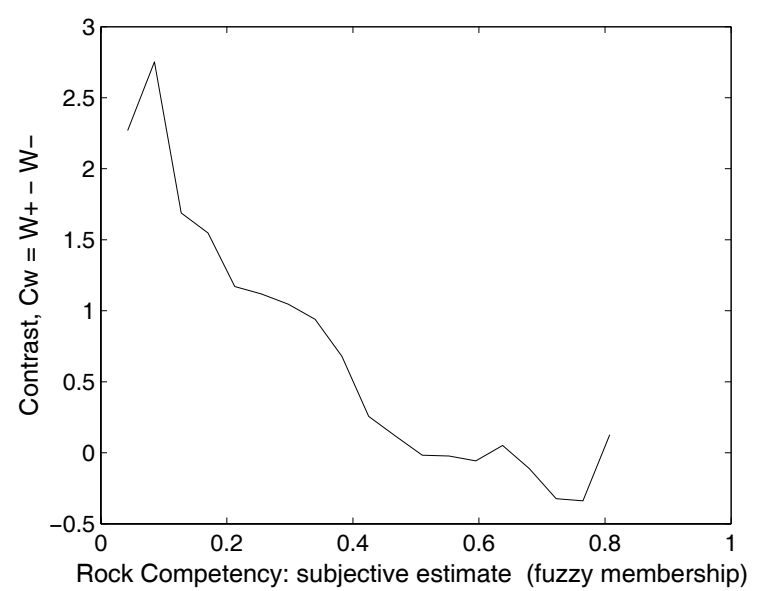

(a) Contrast, $C_{W}$

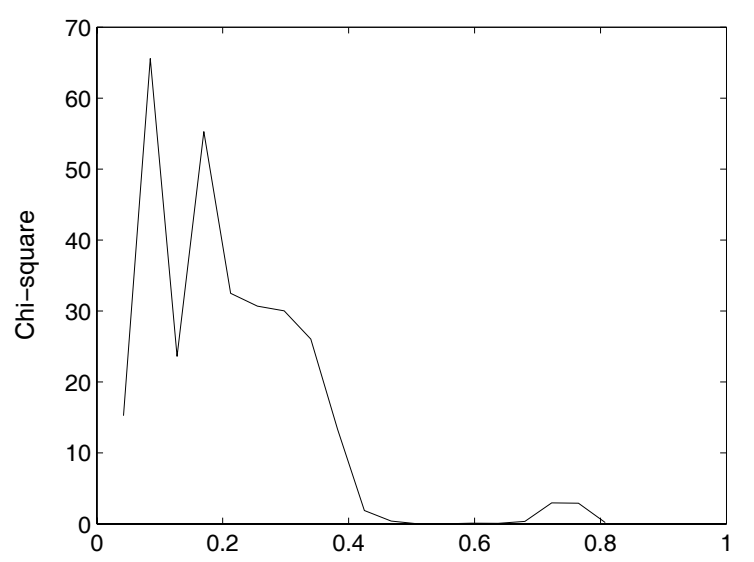

Rock Competency: subjective estimate (fuzzy membership)

(c) Chi-squared

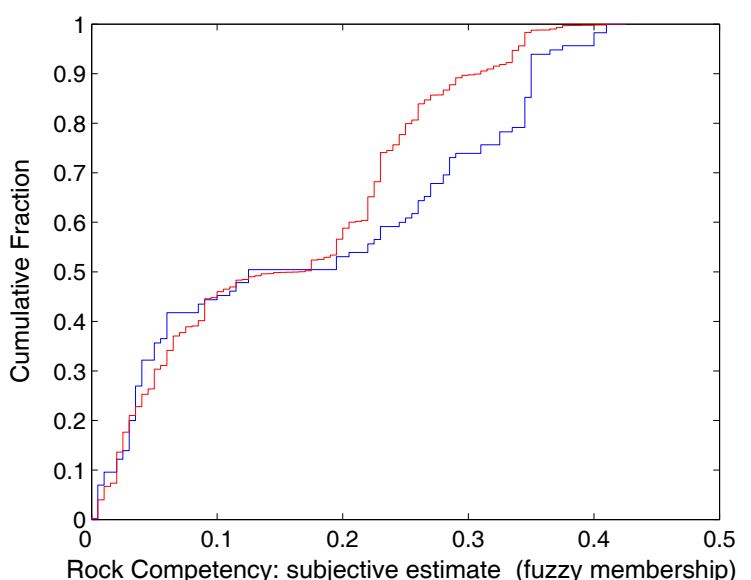

(e) Kolmogorov-Smirnov test

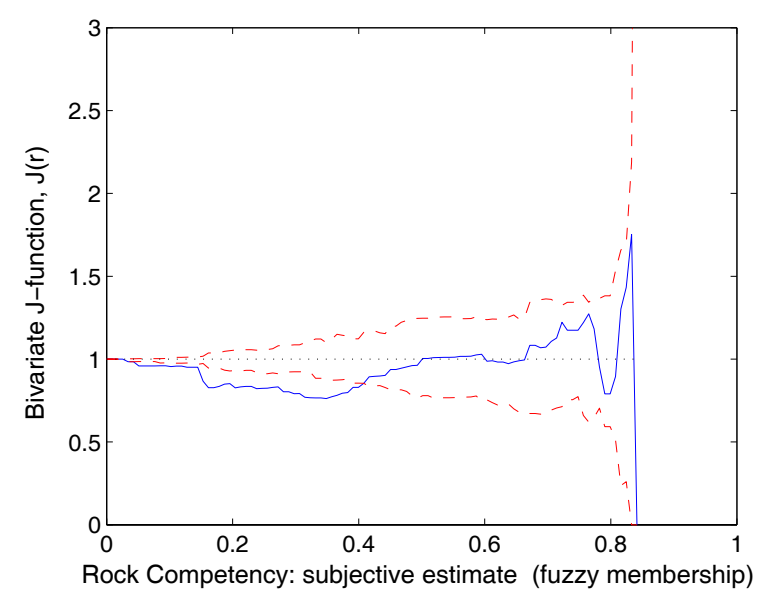

(b) Bivariate J-function (cumulative)

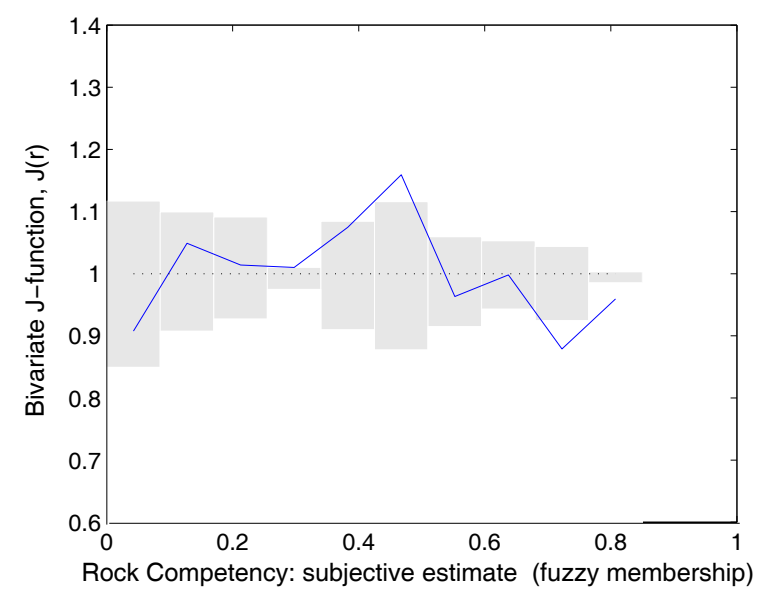

(d) Bivariate J-function (interval-based)

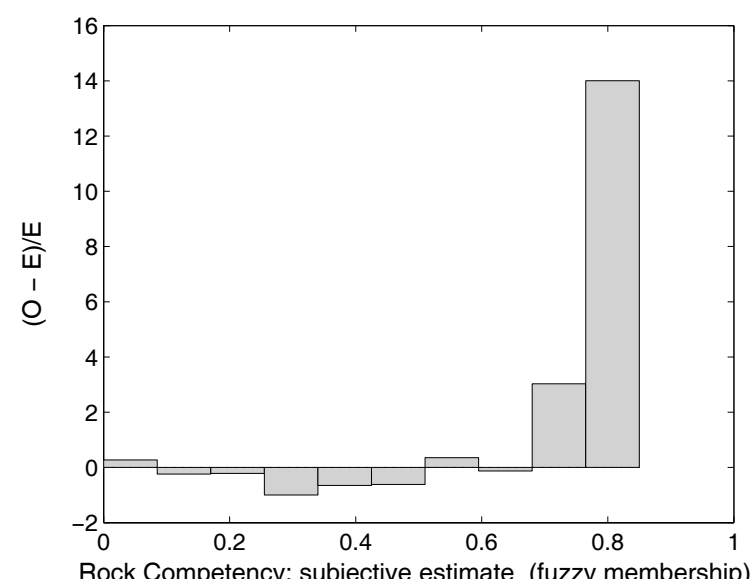

(f) $(O-E) / E$

Figure G.29. Plots used to examine the relationship between deposits and subjective (fuzzy) rheological contrast in the layer, l36_l_rh11k. The empirical curve is shown in blue in b), d) \& e). The theoretical curve is shown in red in b) \& e) and as grey bars in d). The X-axis parameter in graphs a), b) \& c) was transformed to create a new parameter representing the difference between the maximum and the original parameter value; i.e., $x=X_{\max .}-x$. 


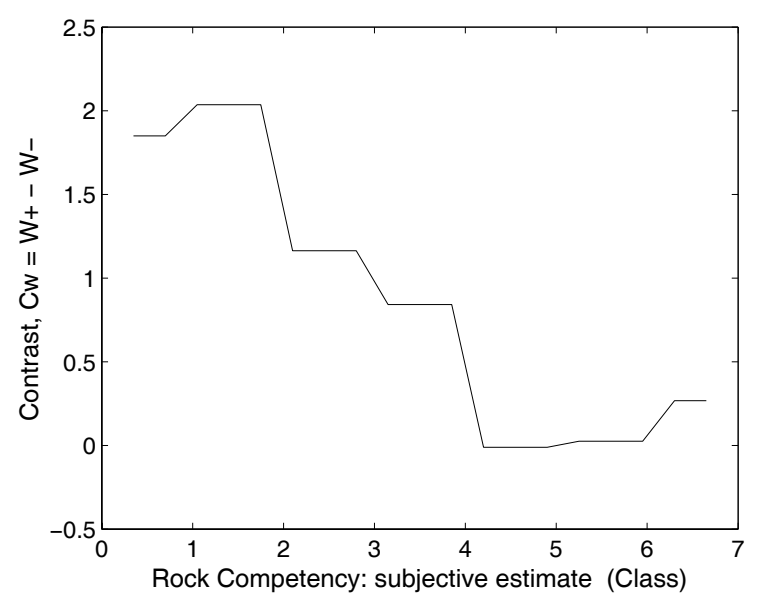

(a) Contrast, $C_{W}$

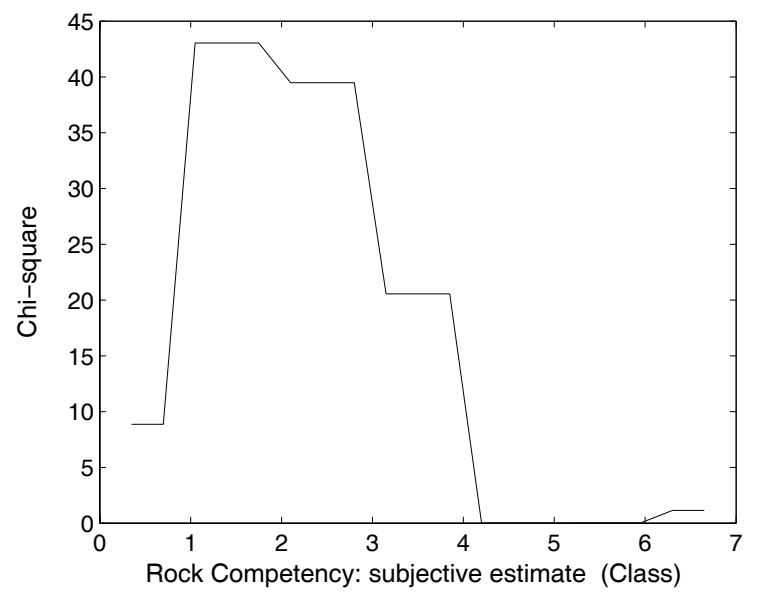

(c) Chi-squared

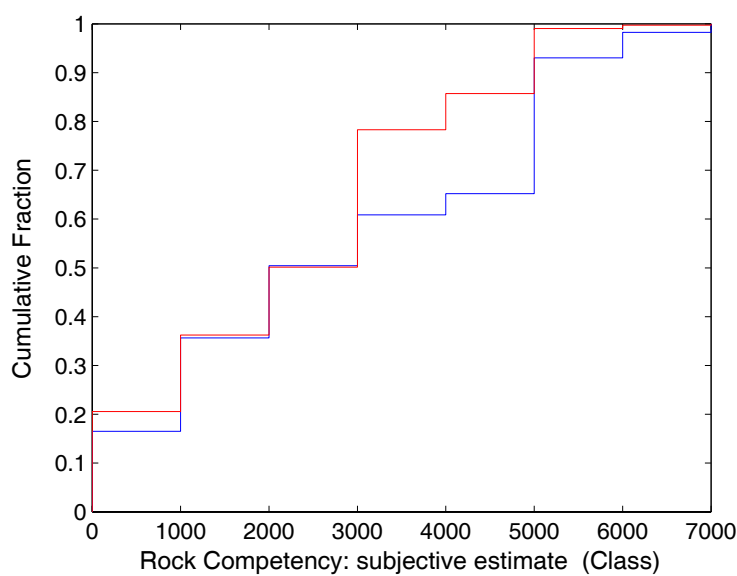

(e) Kolmogorov-Smirnov test

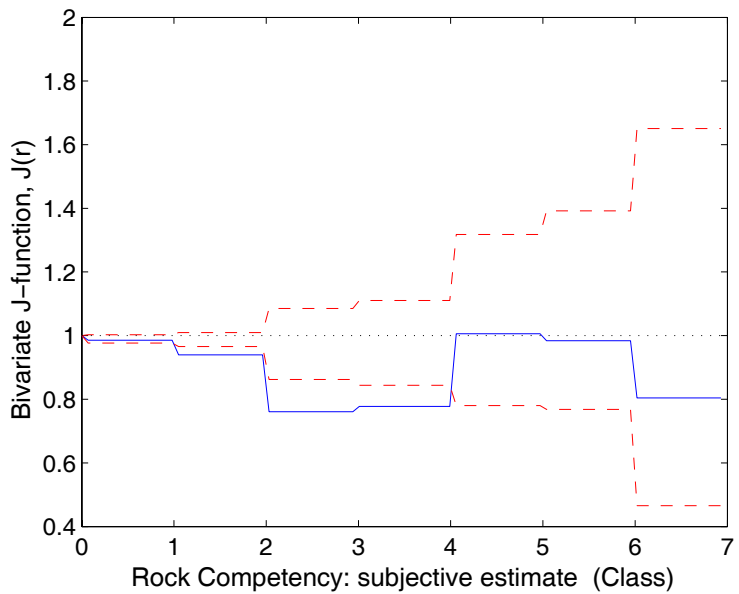

(b) Bivariate J-function (cumulative)

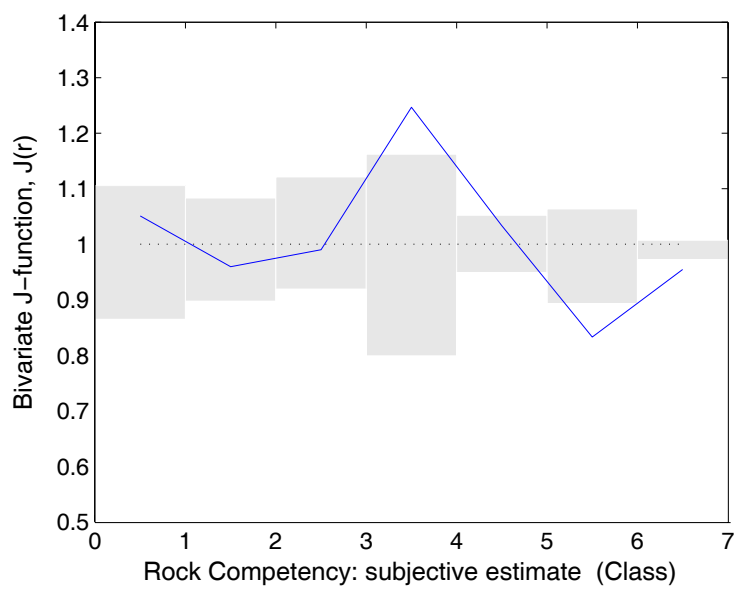

(d) Bivariate J-function (interval-based)

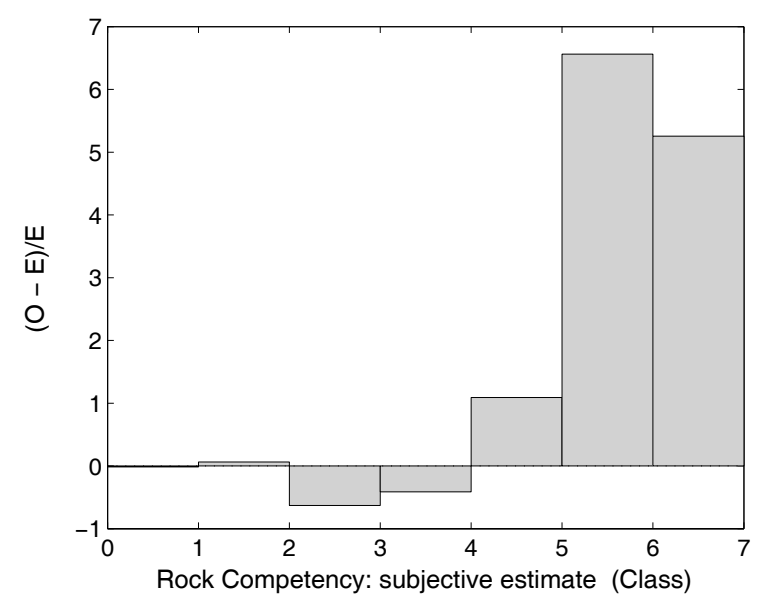

(f) $(O-E) / E$

Figure G.30. Plots used to examine the relationship between deposits and subjective (classes) rheological contrast in the layer, l36_l_rh21k. The empirical curve is shown in blue in b), d) \& e). The theoretical curve is shown in red in b) \& e) and as grey bars in d). The $\mathrm{X}$-axis parameter in graphs a), b) \& c) was transformed to create a new parameter representing the difference between the maximum and the original parameter value; i.e., $x=X_{\max }-x$. 


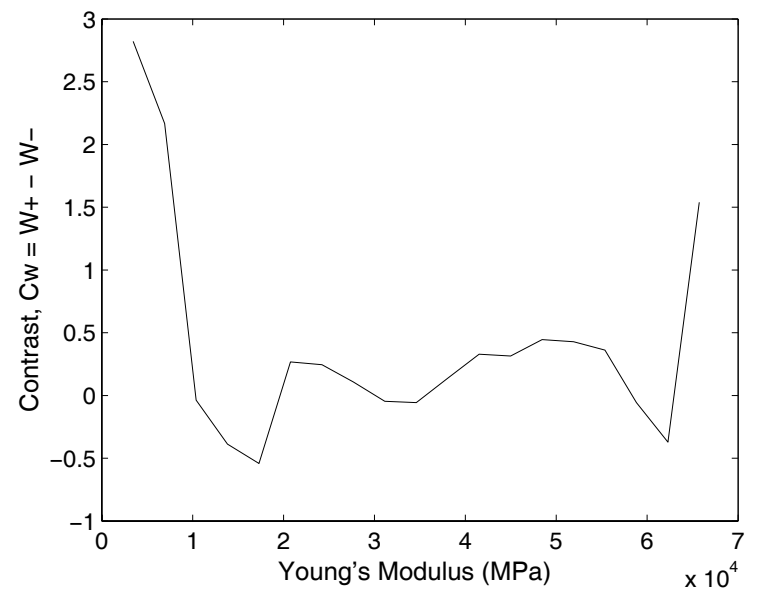

(a) Contrast, $C_{W}$

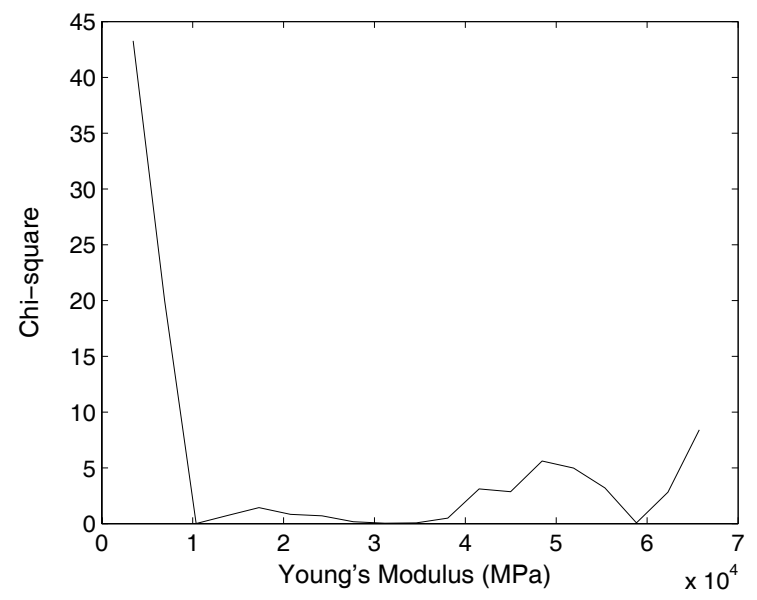

(c) Chi-squared

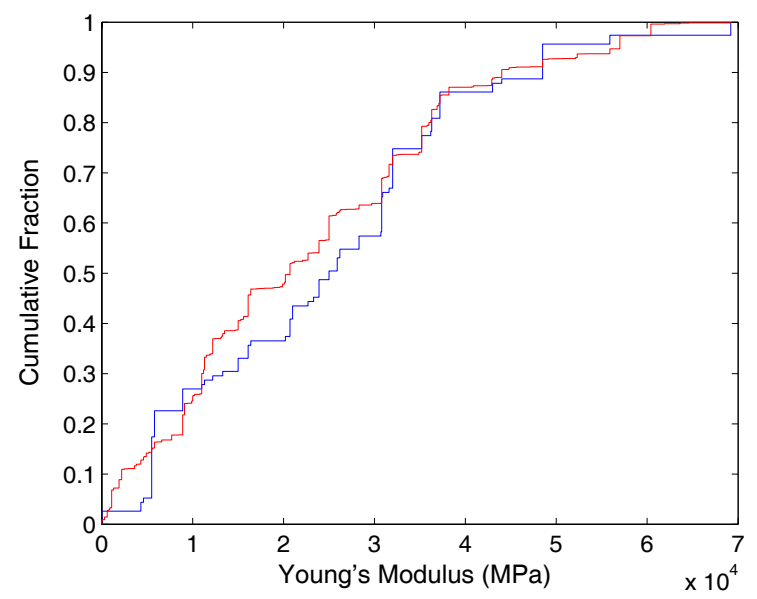

(e) Kolmogorov-Smirnov test

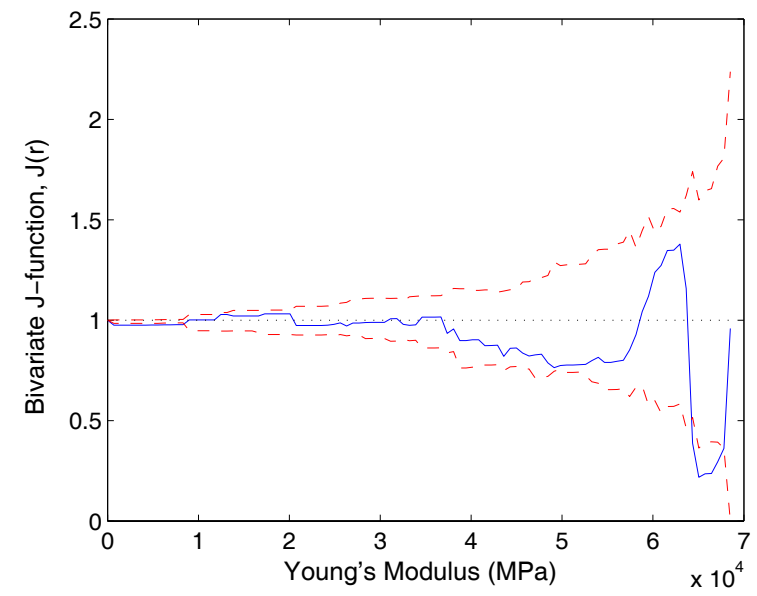

(b) Bivariate J-function (cumulative)

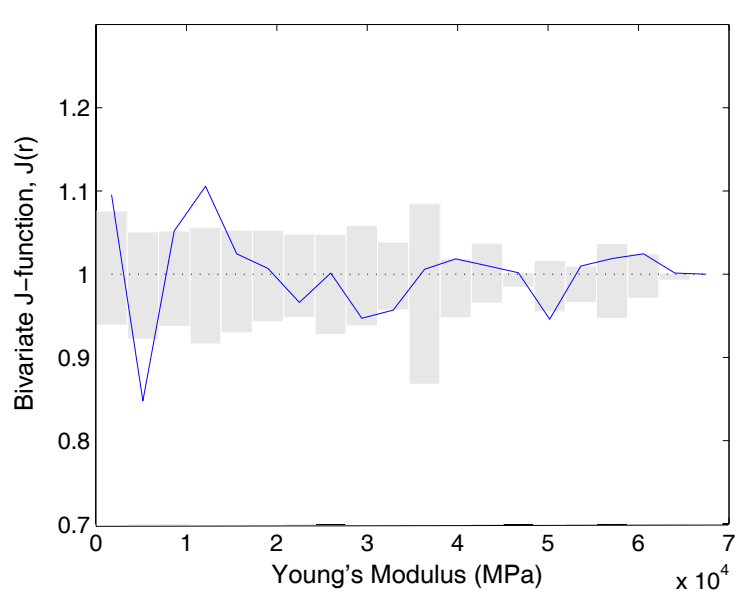

(d) Bivariate J-function (interval-based)

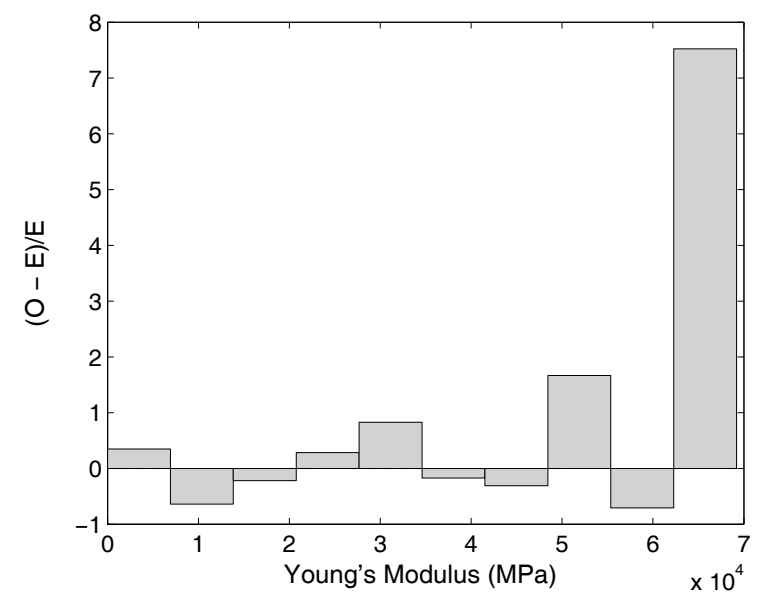

(f) $(O-E) / E$

Figure G.31. Plots used to examine the relationship between deposits and rheological contrast using Young's Modulus compiled from Hatheway \& Kiersch (1989) in the layer, l36_l_rh31k. The empirical curve is shown in blue in b), d) \& e). The theoretical curve is shown in red in b) \& e) and as grey bars in $\mathrm{d}$ ). The $\mathrm{X}$-axis parameter in graphs a), b) \& c) was transformed to create a new parameter representing the difference between the maximum and the original parameter value; i.e., $x=X_{\max }-x$. 


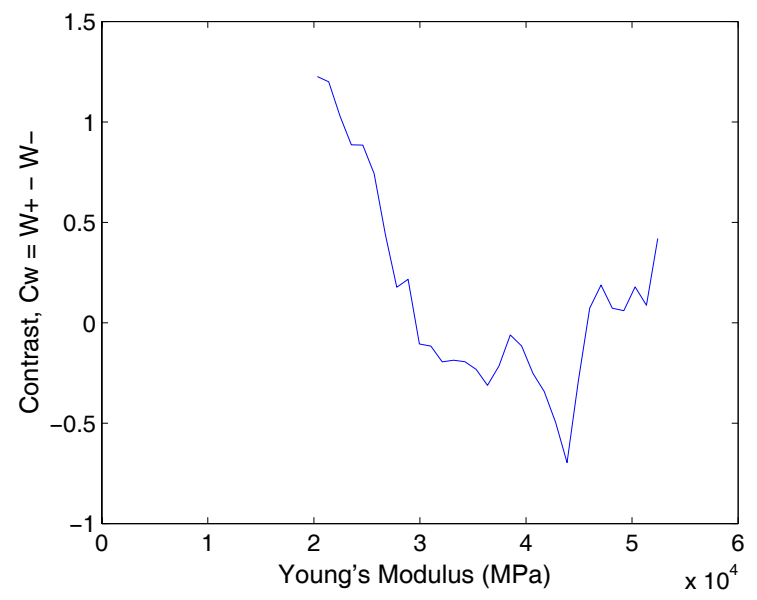

(a) Contrast, $C_{W}$

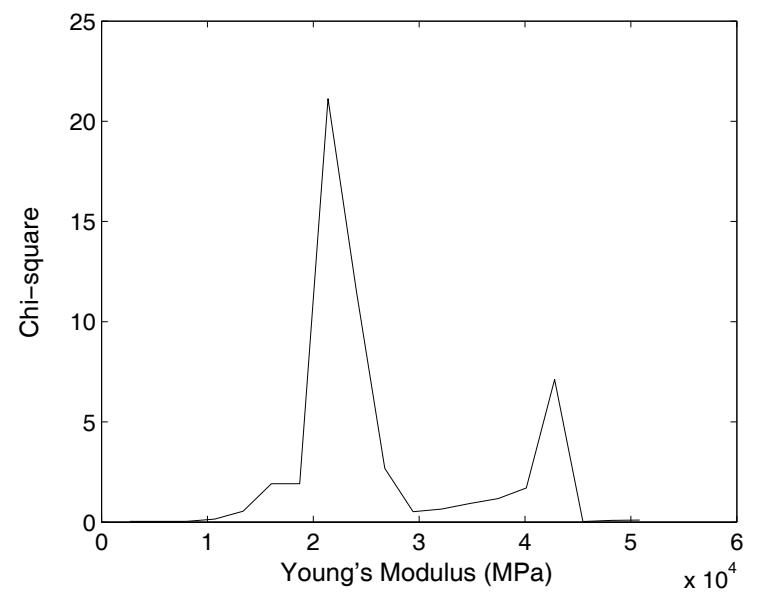

(c) Chi-squared

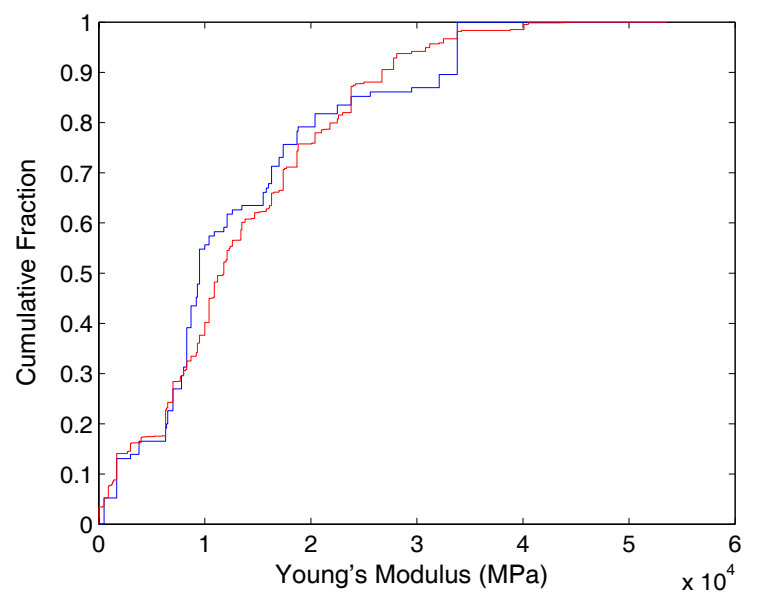

(e) Kolmogorov-Smirnov test

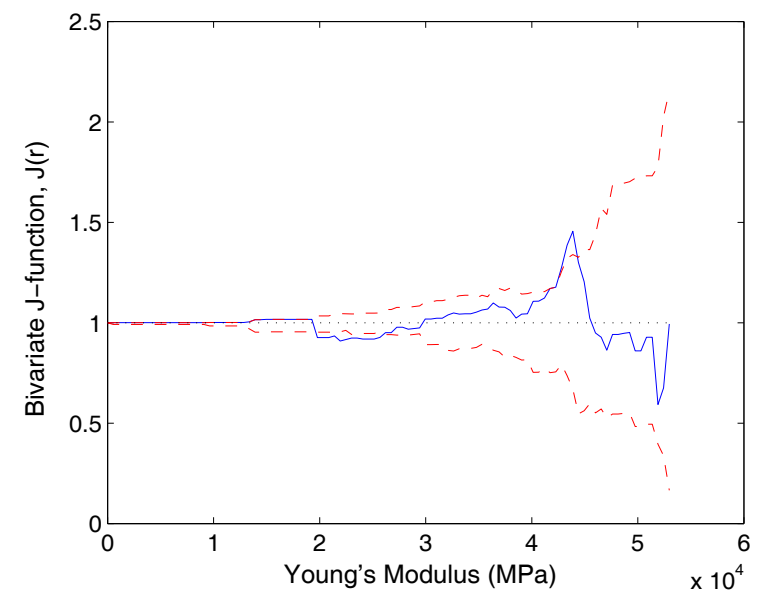

(b) Bivariate J-function (cumulative)

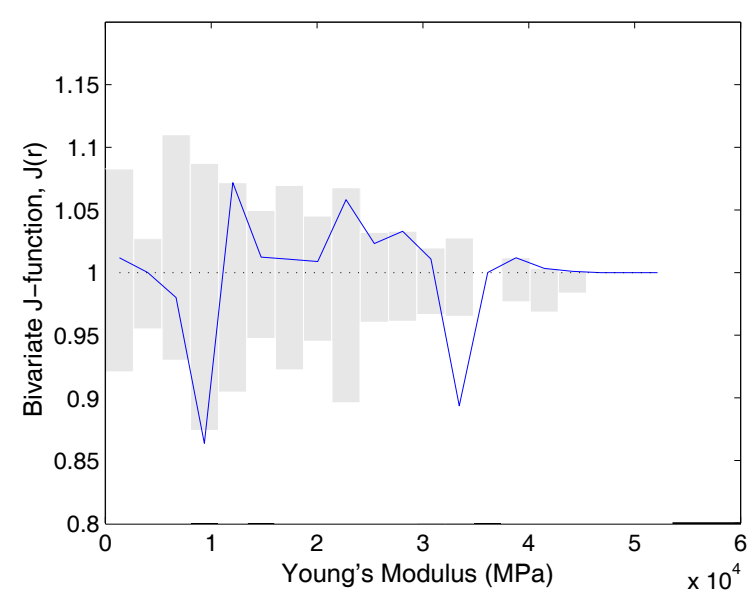

(d) Bivariate J-function (interval-based)

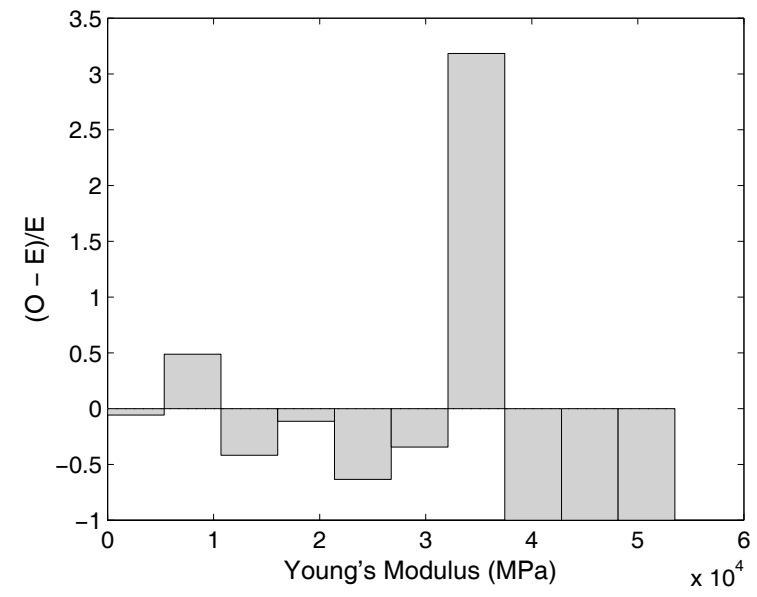

(f) $(O-E) / E$

Figure G.32. Plots used to examine the relationship between deposits and rheological contrast using Young's Modulus compiled from Lee (2000) in the layer, l36_l_rh41k. The empirical curve is shown in blue in b), d) \& e). The theoretical curve is shown in red in b) \& e) and as grey bars in d). The $\mathrm{X}$-axis parameter in graphs a), b) \& c) was transformed to create a new parameter representing the difference between the maximum and the original parameter value; i.e., $x=X_{\max }-x$. 


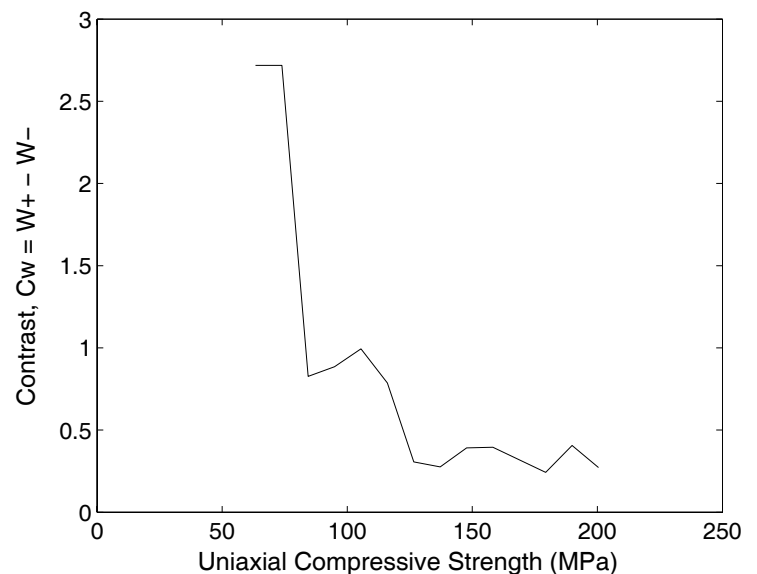

(a) Contrast, $C_{W}$

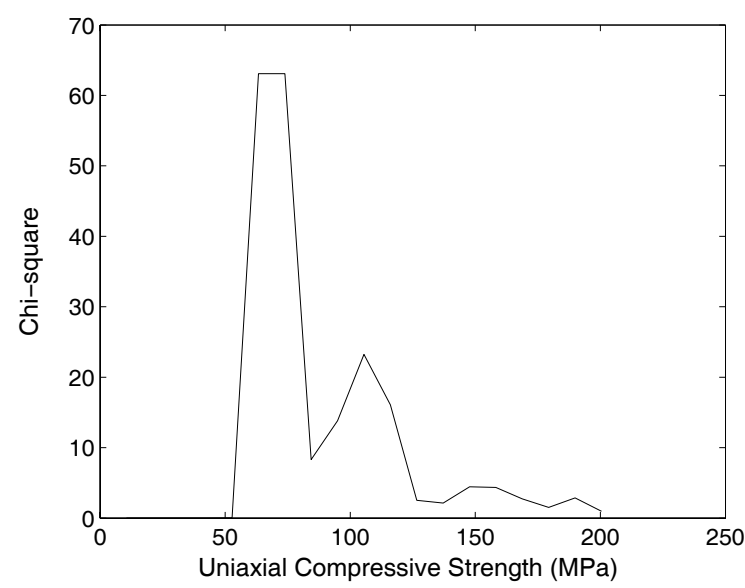

(c) Chi-squared

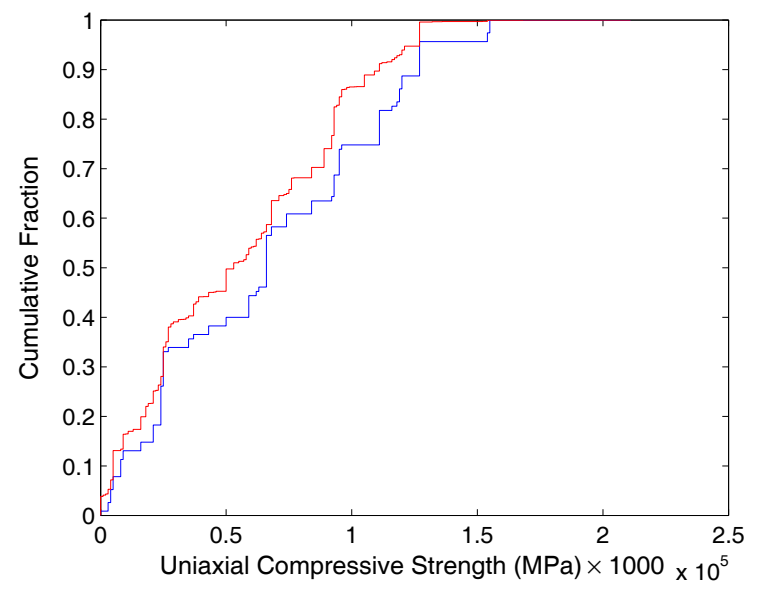

(e) Kolmogorov-Smirnov test

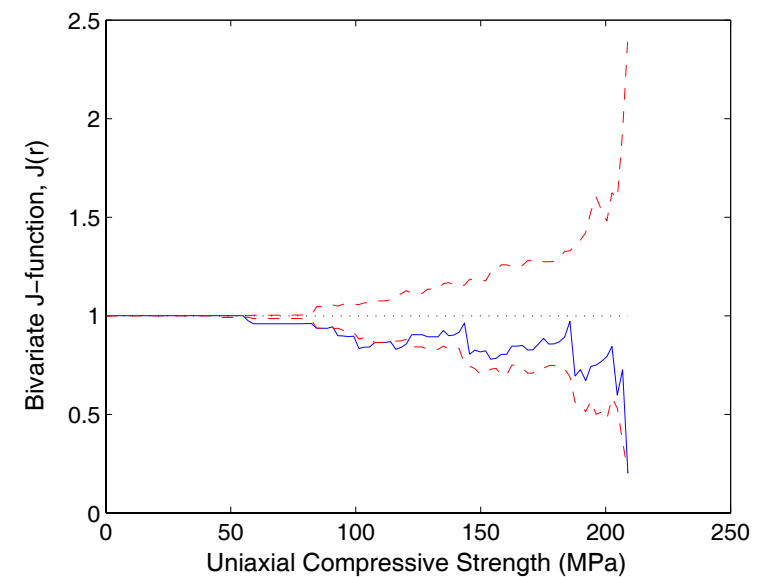

(b) Bivariate J-function (cumulative)

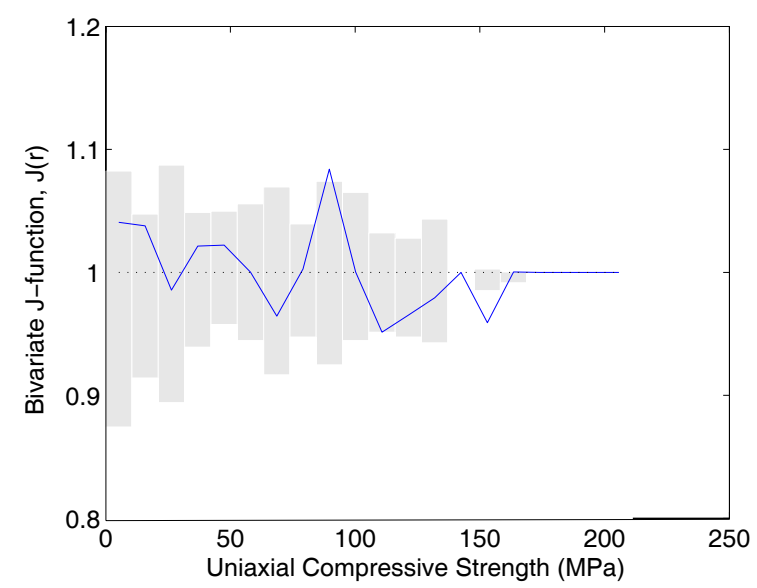

(d) Bivariate J-function (interval-based)

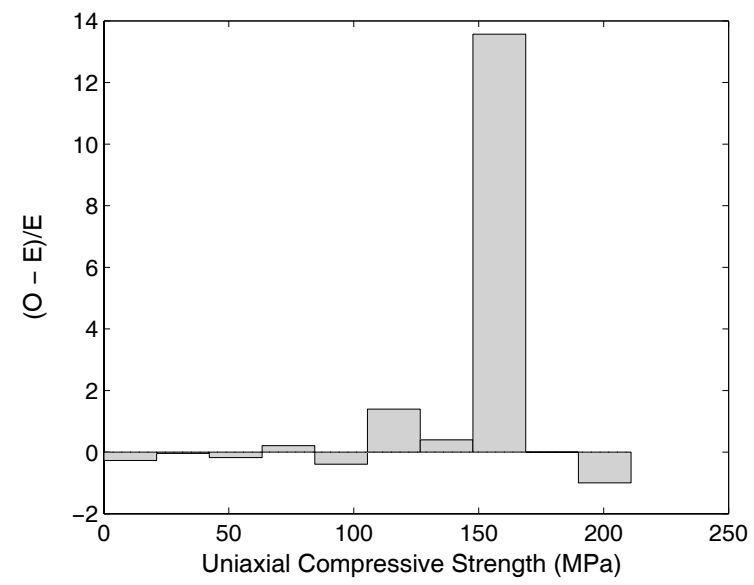

(f) $(O-E) / E$

Figure G.33. Plots used to examine the relationship between deposits and rheological contrast using uniaxial compressive strength in the layer, $136 \_l \_r h 51 k$. The empirical curve is shown in blue in b), d) \& e). The theoretical curve is shown in red in b) \& e) and as grey bars in d). The $\mathrm{X}$-axis parameter in graphs a), b) \& c) was transformed to create a new parameter representing the difference between the maximum and the original parameter value; i.e., $x=X_{\max .}-x$. 


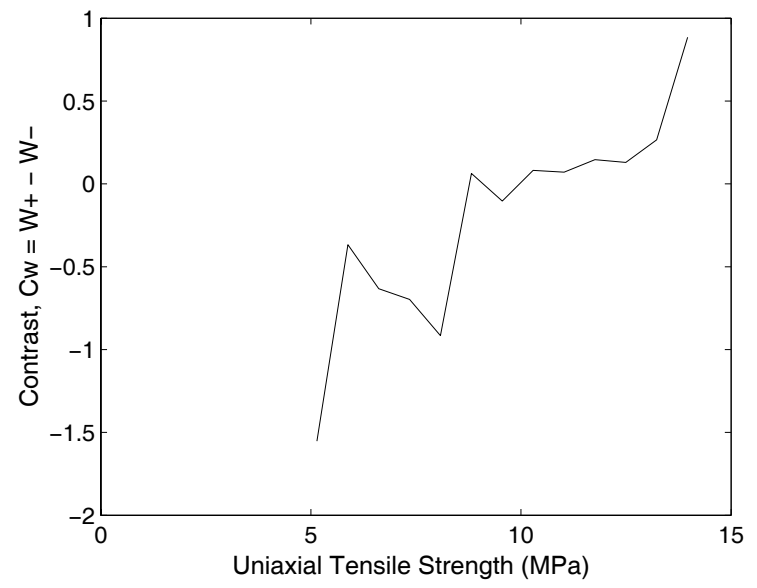

(a) Contrast, $C_{W}$

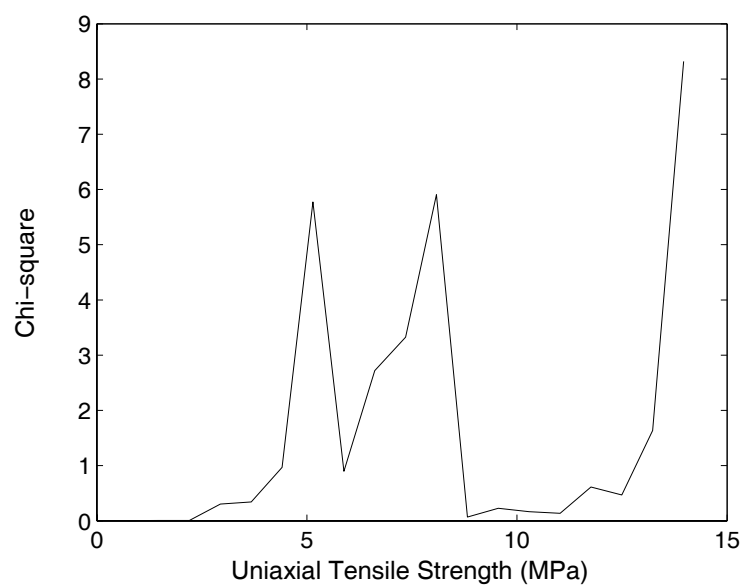

(c) Chi-squared

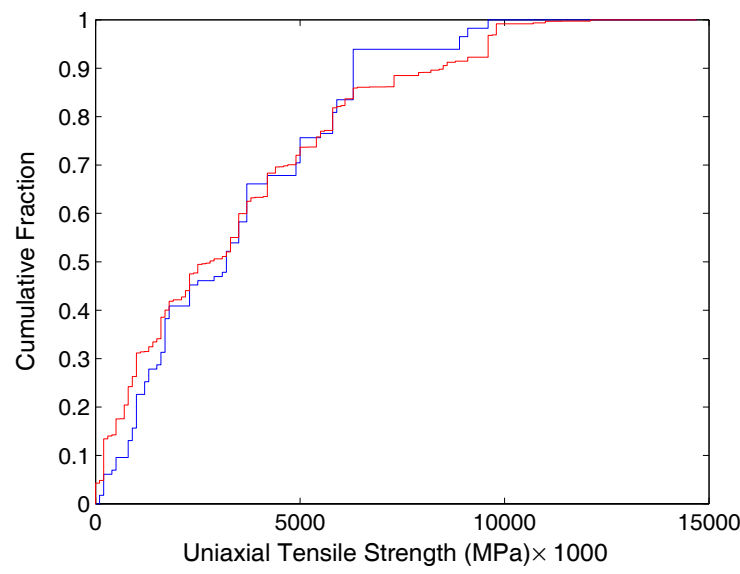

(e) Kolmogorov-Smirnov test

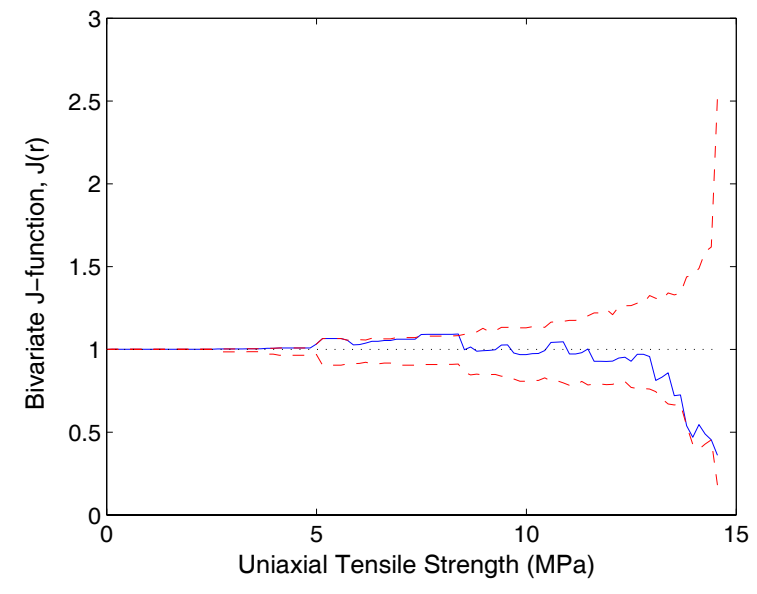

(b) Bivariate J-function (cumulative)

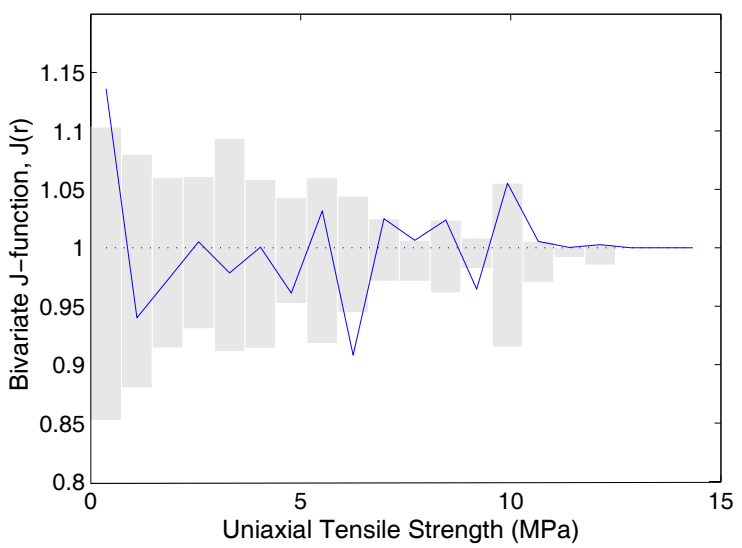

(d) Bivariate J-function (interval-based)

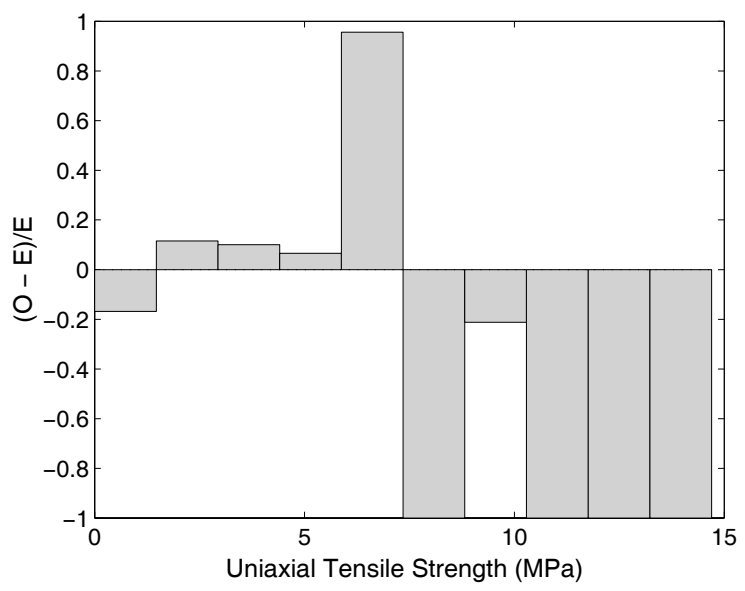

(f) $(O-E) / E$

Figure G.34. Plots used to examine the relationship between deposits and rheological contrast using uniaxial compressive strength in the layer, l36_l_rh61k. The empirical curve is shown in blue in b), d) \& e). The theoretical curve is shown in red in b) \& e) and as grey bars in d). The $\mathrm{X}$-axis parameter in graphs a), b) \& c) was transformed to create a new parameter representing the difference between the maximum and the original parameter value; i.e., $x=X_{\max }-x$. 


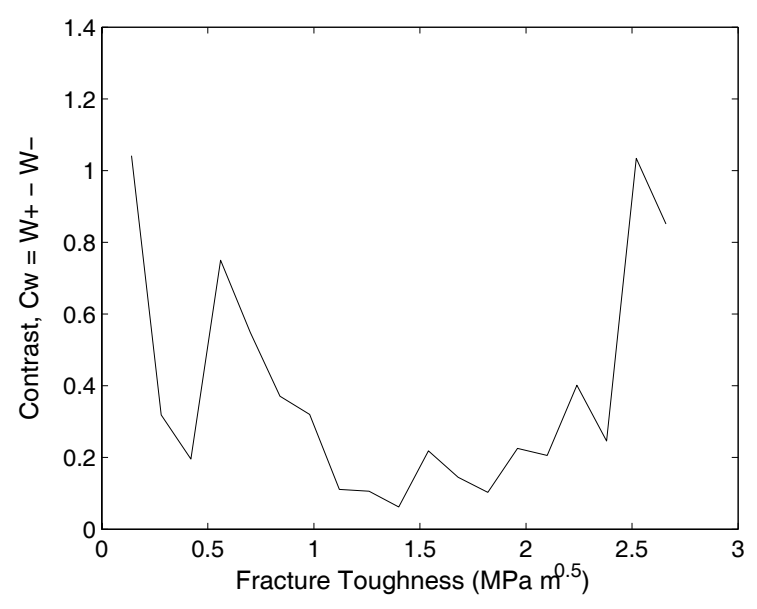

(a) Contrast, $C_{W}$

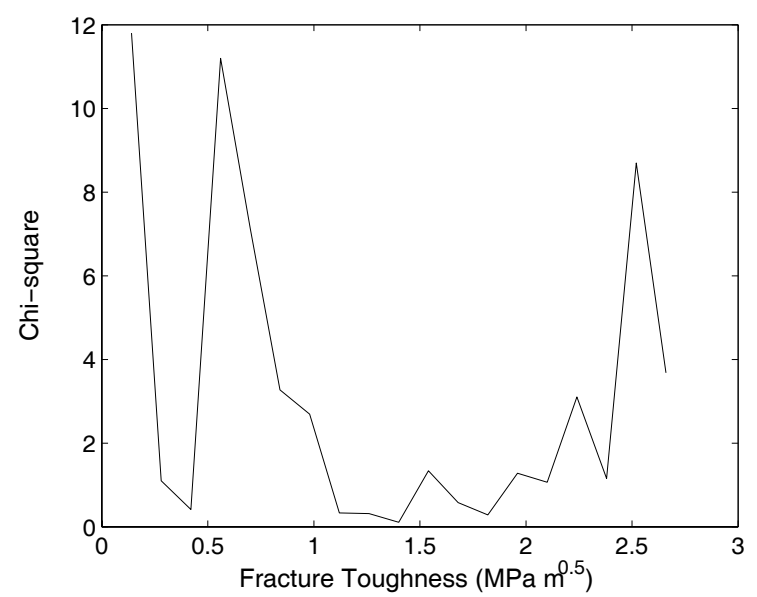

(c) Chi-squared

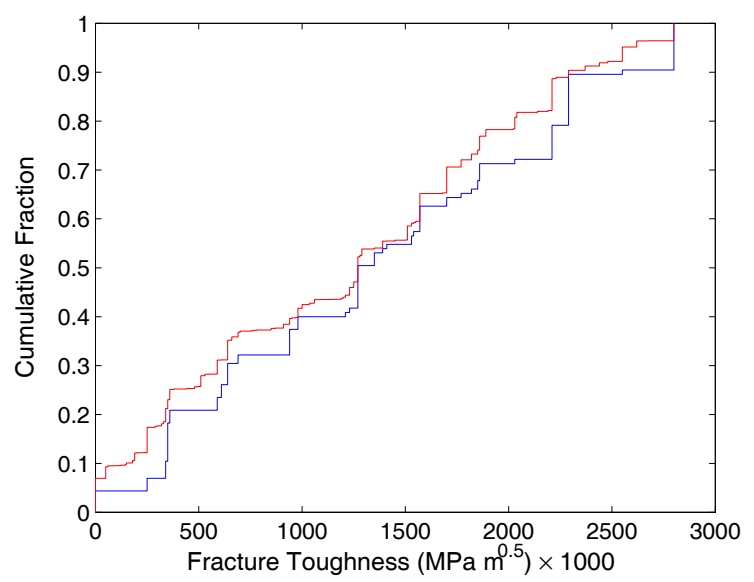

(e) Kolmogorov-Smirnov test

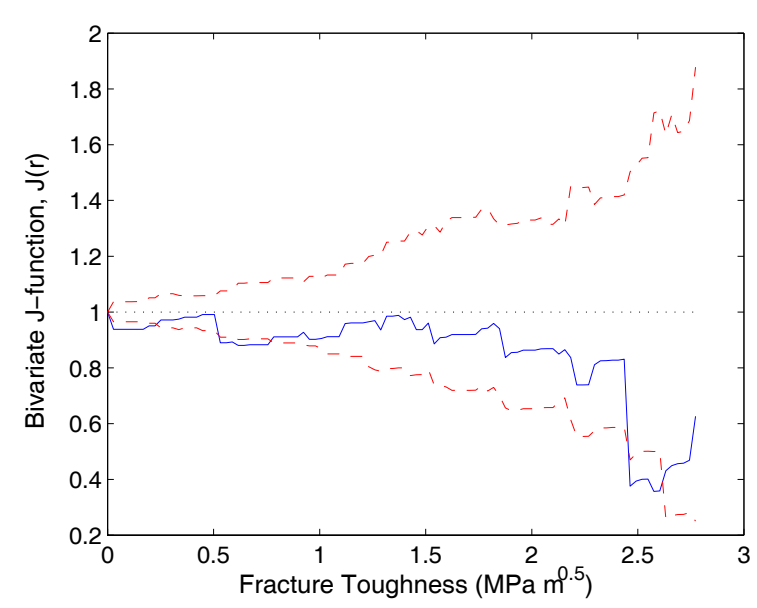

(b) Bivariate J-function (cumulative)

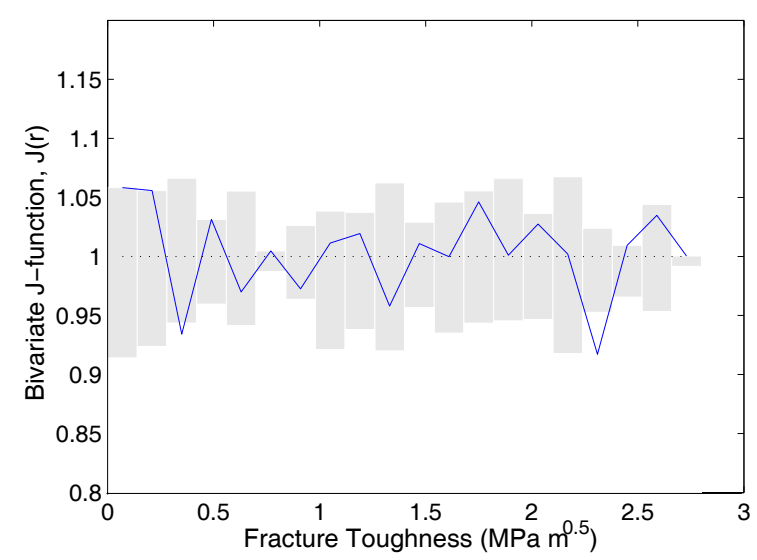

(d) Bivariate J-function (interval-based)

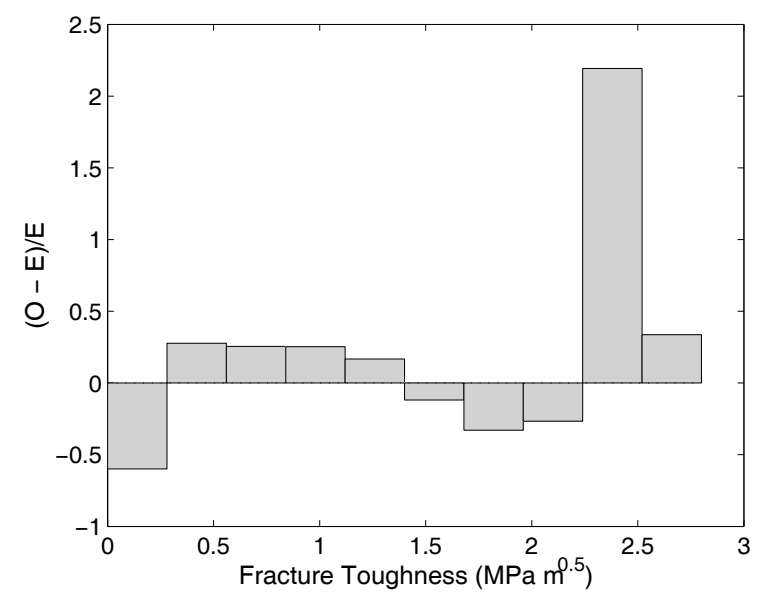

(f) $(O-E) / E$

Figure G.35. Plots used to examine the relationship between deposits and rheological contrast using fracture toughness in the layer, $136 \_l \_r h 71 k$. The empirical curve is shown in blue in b), d) \& e). The theoretical curve is shown in red in b) \& e) and as grey bars in d). The X-axis parameter in graphs a), b) \& c) was transformed to create a new parameter representing the difference between the maximum and the original parameter value; i.e., $x=X_{\max .}-x$. 


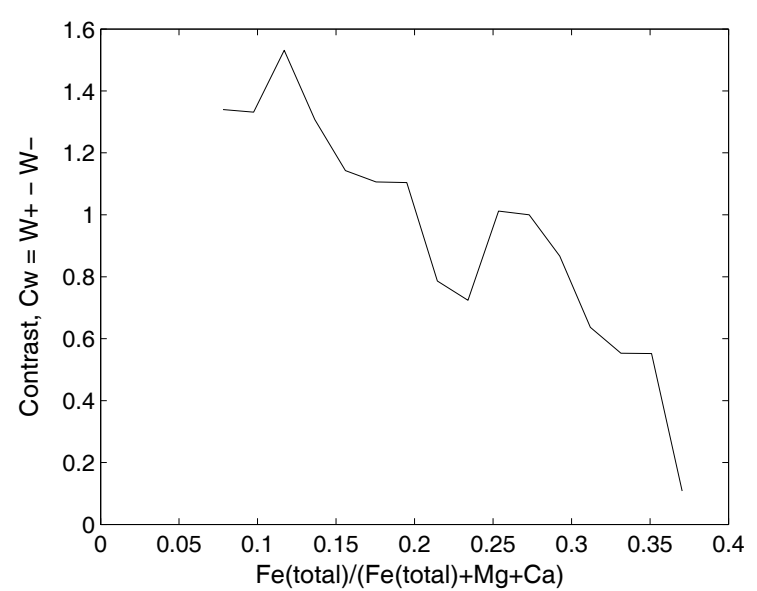

(a) Contrast, $C_{W}$

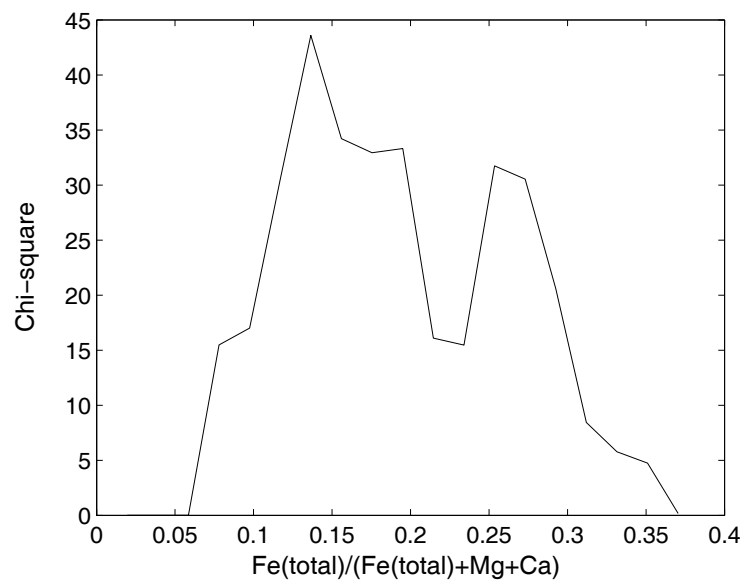

(c) Chi-squared

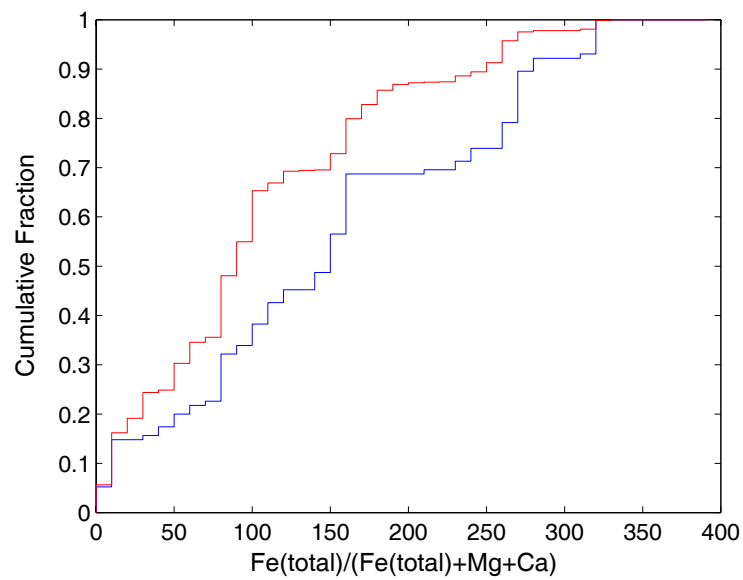

(e) Kolmogorov-Smirnov test

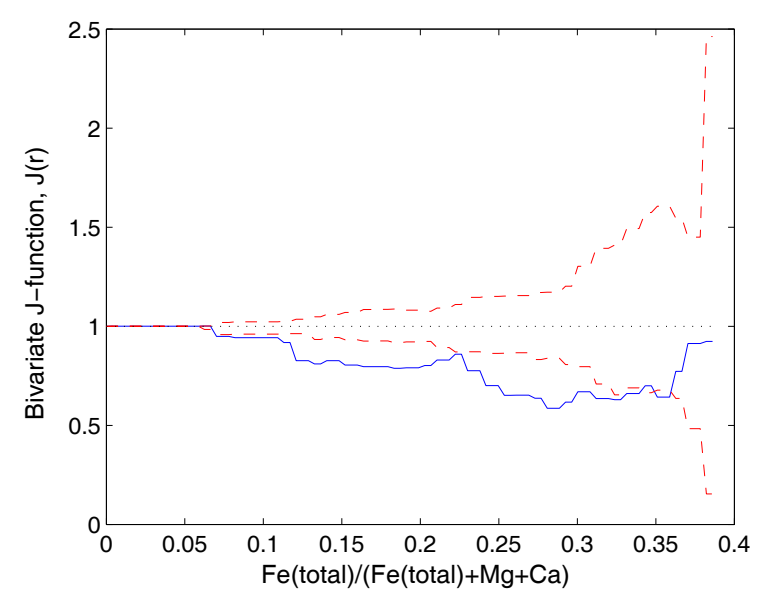

(b) Bivariate J-function (cumulative)

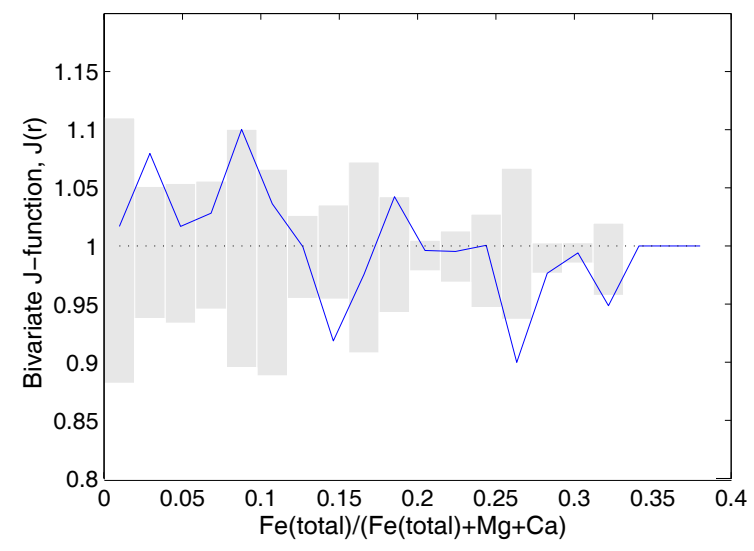

(d) Bivariate J-function (interval-based)

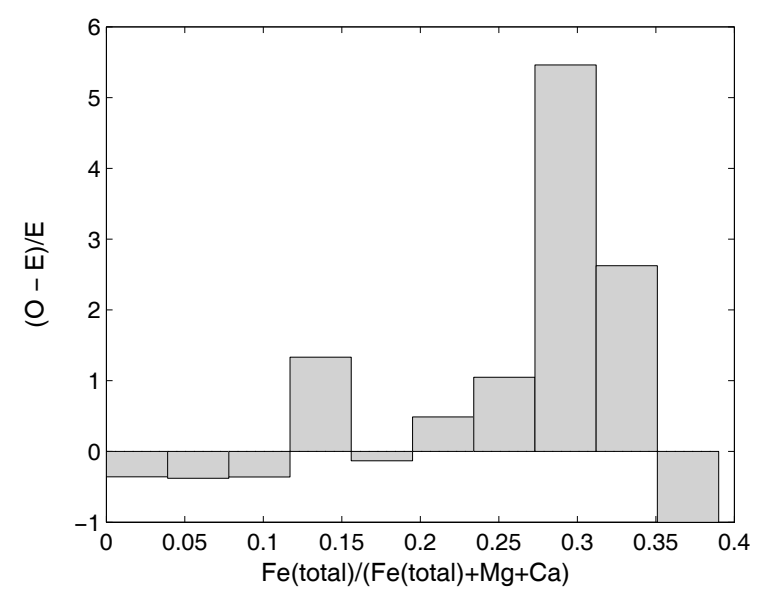

(f) $(O-E) / E$

Figure G.36. Plots used to examine the relationship between deposits and chemical contrast, $\mathrm{Fe}_{\text {tot }} /\left(\mathrm{Fe}_{\text {tot }}+\mathrm{Mg}+\mathrm{Ca}\right.$ ) (sulphidation index) in the layer, l37_l_ch11k. The empirical curve is shown in blue in b), d) \& e). The theoretical curve is shown in red in b) \& e) and as grey bars in d). The $\mathrm{X}$-axis parameter in graphs a), b) \& c) was transformed to create a new parameter representing the difference between the maximum and the original parameter value; i.e., $x=X_{\max }-x$. 


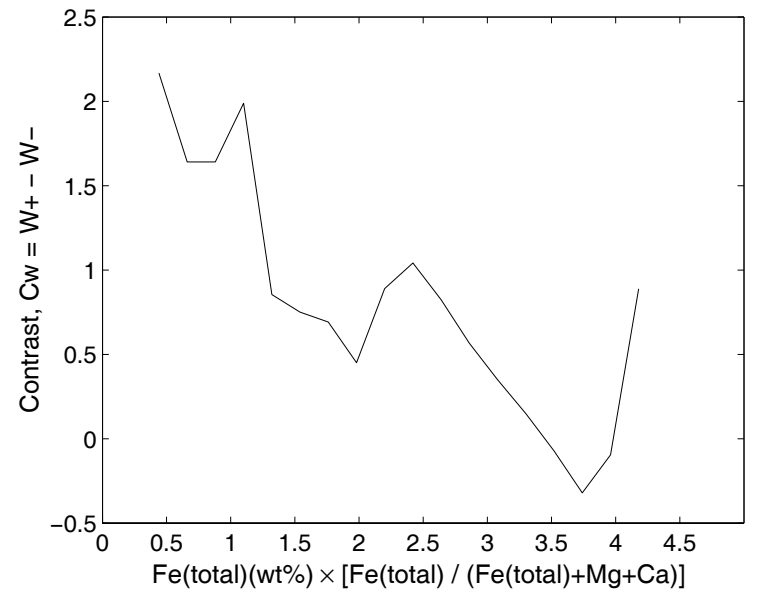

(a) Contrast, $C_{W}$

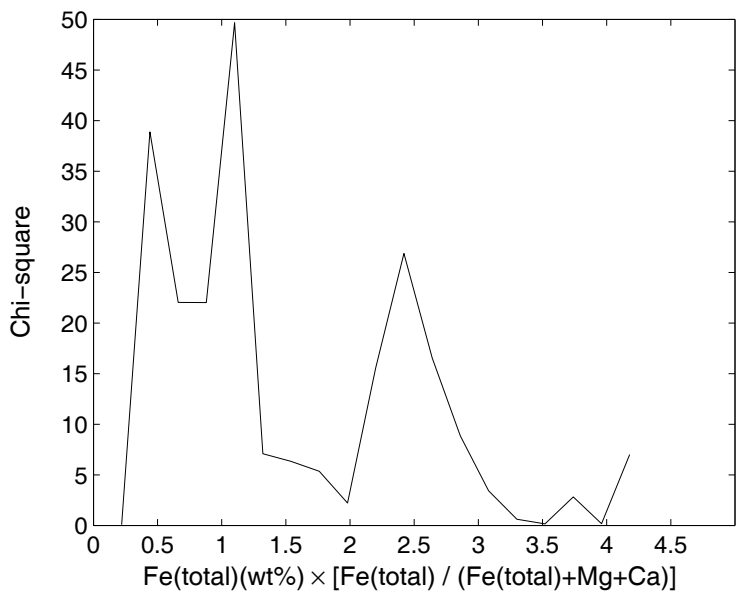

(c) Chi-squared

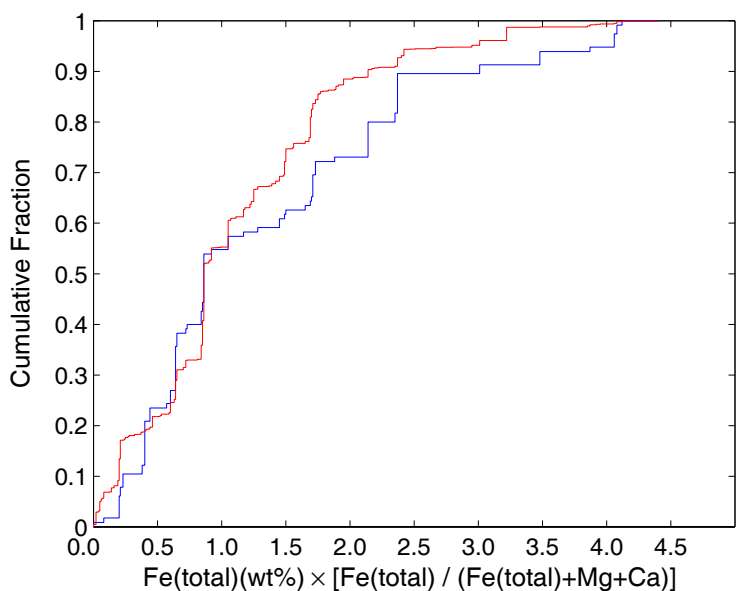

(e) Kolmogorov-Smirnov test

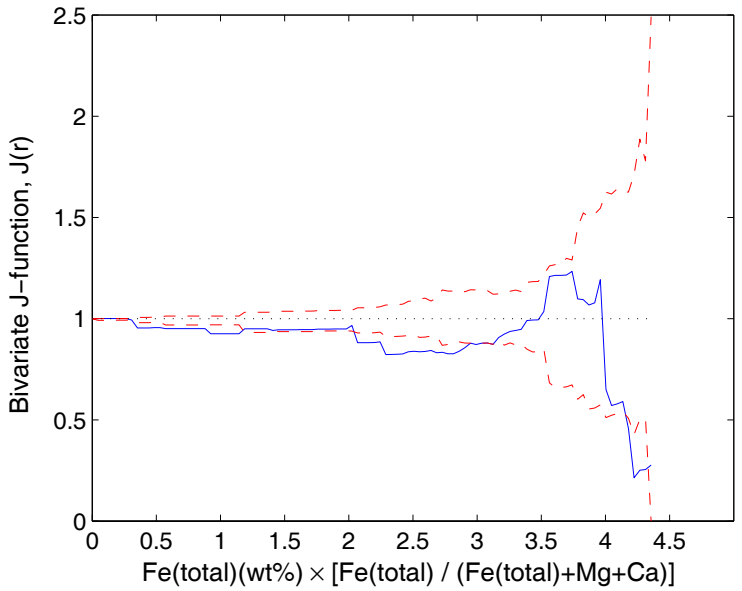

(b) Bivariate J-function (cumulative)

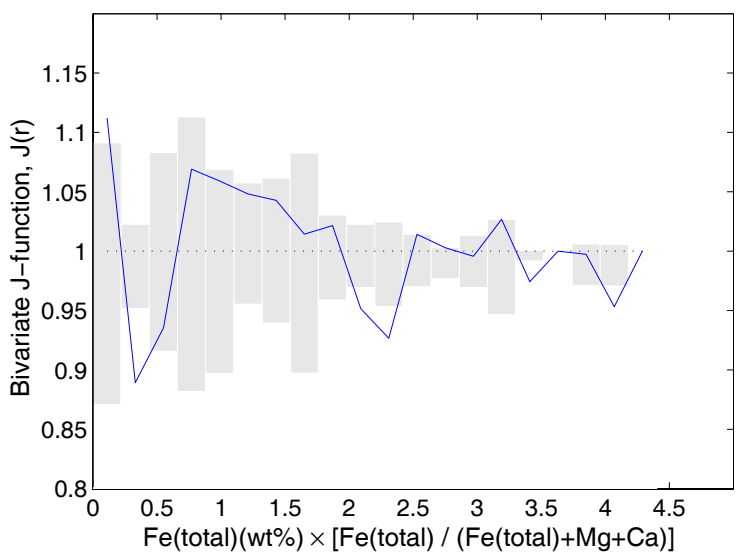

(d) Bivariate J-function (interval-based)

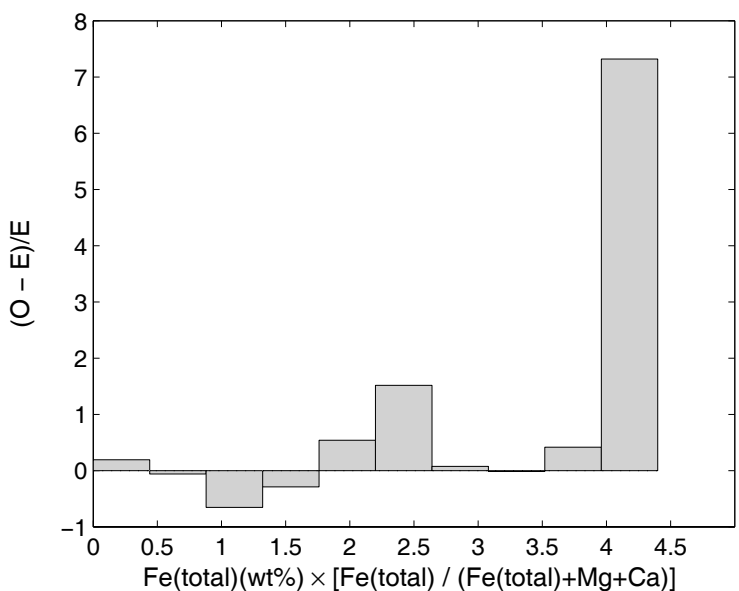

(f) $(O-E) / E$

Figure G.37. Plots used to examine the relationship between deposits and chemical contrast, $\mathrm{Fe}_{\text {tot }}(\mathrm{wt} \%) * \mathrm{Fe}_{\text {tot }} /\left(\mathrm{Fe}_{\text {tot }}+\mathrm{Mg}+\mathrm{Ca}\right)$ (sulphidation index) in the layer, l37_l_ch21k. The empirical curve is shown in blue in b), d) \& e). The theoretical curve is shown in red in b) \& e) and as grey bars in $\mathrm{d}$ ). The $\mathrm{X}$-axis parameter in graphs a), b) \& c) was transformed to create a new parameter representing the difference between the maximum and the original parameter value; i.e., $x=X_{\max .}-x$. 


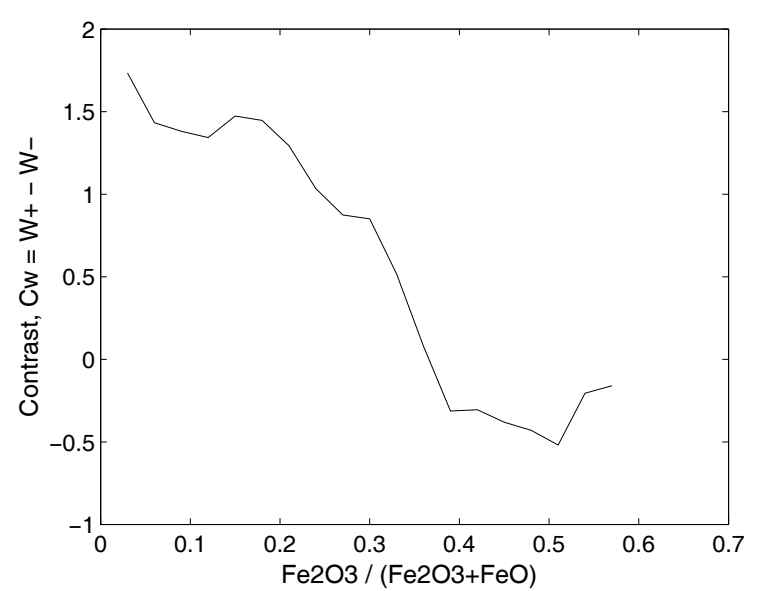

(a) Contrast, $C_{W}$

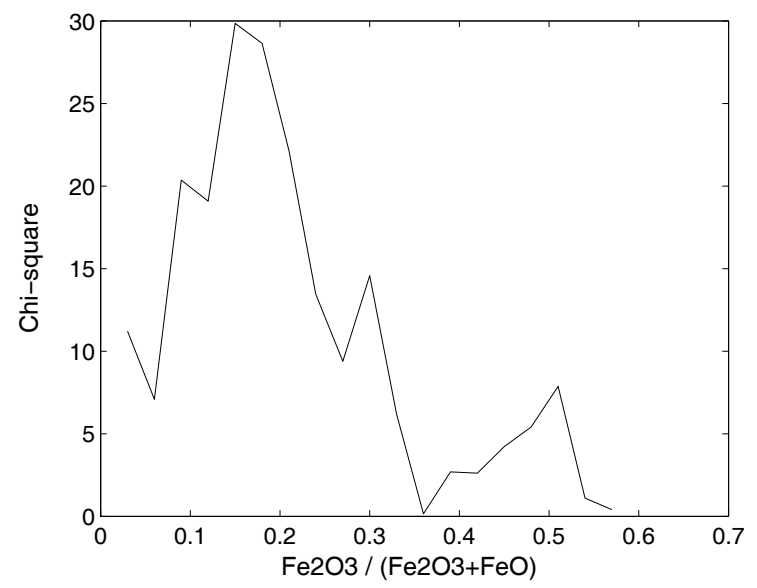

(c) Chi-squared

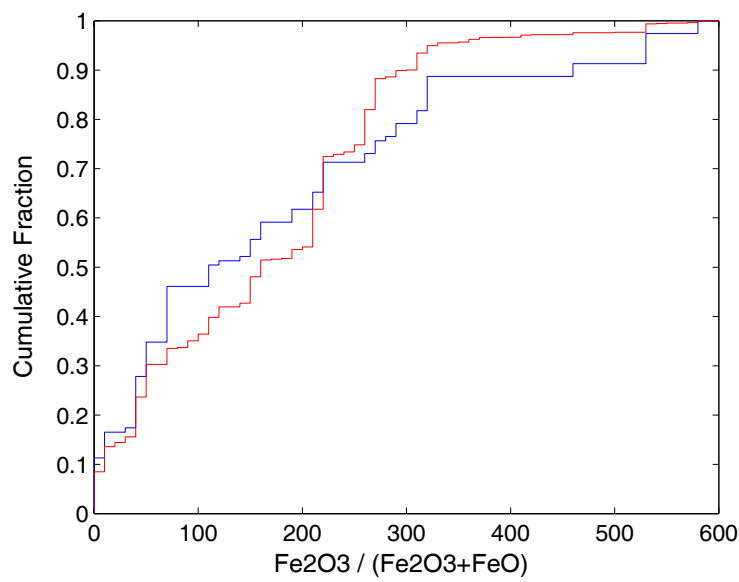

(e) Kolmogorov-Smirnov test

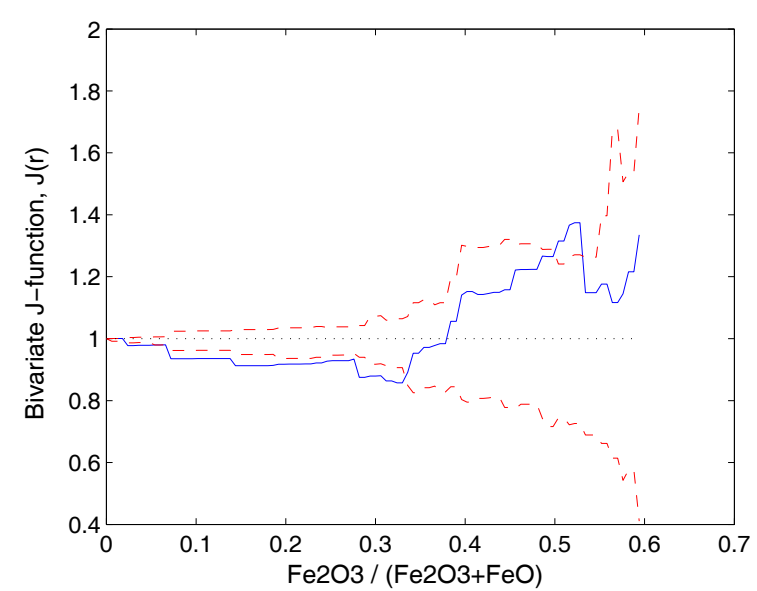

(b) Bivariate J-function (cumulative)

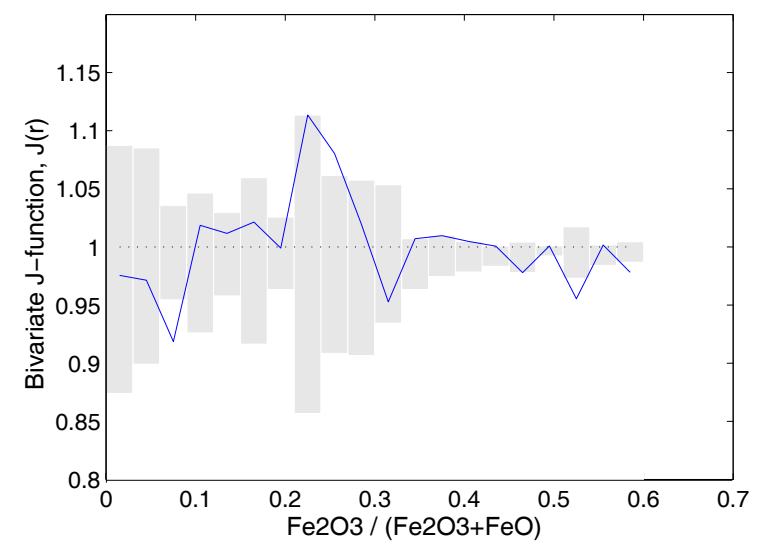

(d) Bivariate J-function (interval-based)

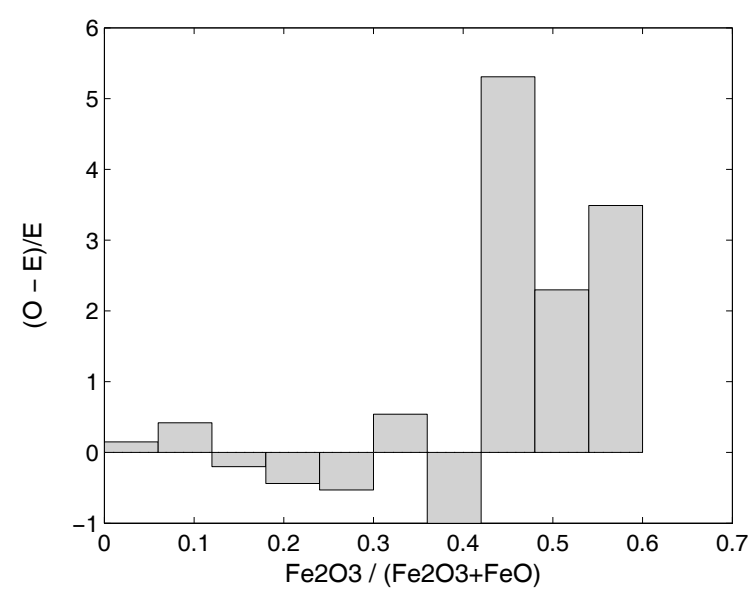

(f) $(O-E) / E$

Figure G.38. Plots used to examine the relationship between deposits and chemical contrast, $\mathrm{Fe}_{2} \mathrm{O} /\left(\mathrm{Fe}_{2} \mathrm{O}_{3}+\mathrm{FeO}\right)$ (oxididation index) in the layer, l37_l_ch31k. The empirical curve is shown in blue in b), d) \& e). The theoretical curve is shown in red in b) \& e) and as grey bars in d). The $\mathrm{X}$-axis parameter in graphs a), b) \& c) was transformed to create a new parameter representing the difference between the maximum and the original parameter value; i.e., $x=X_{\max }-x$. 


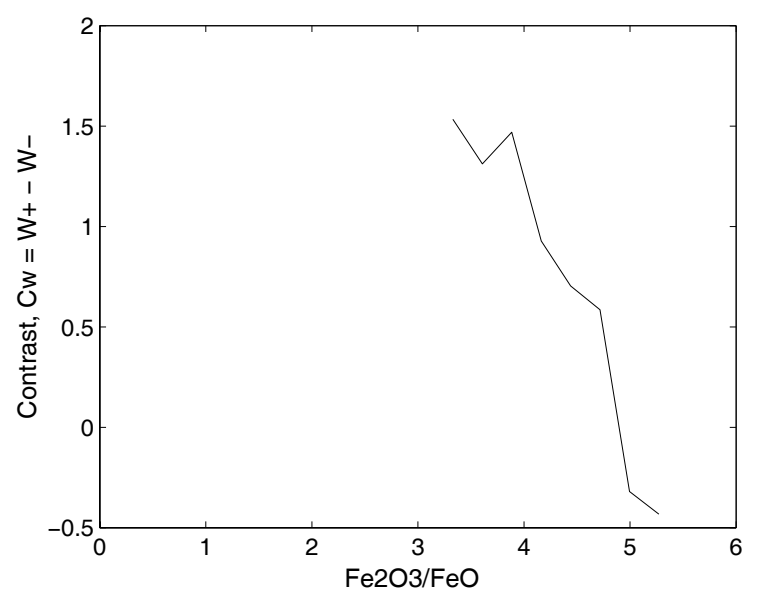

(a) Contrast, $C_{W}$

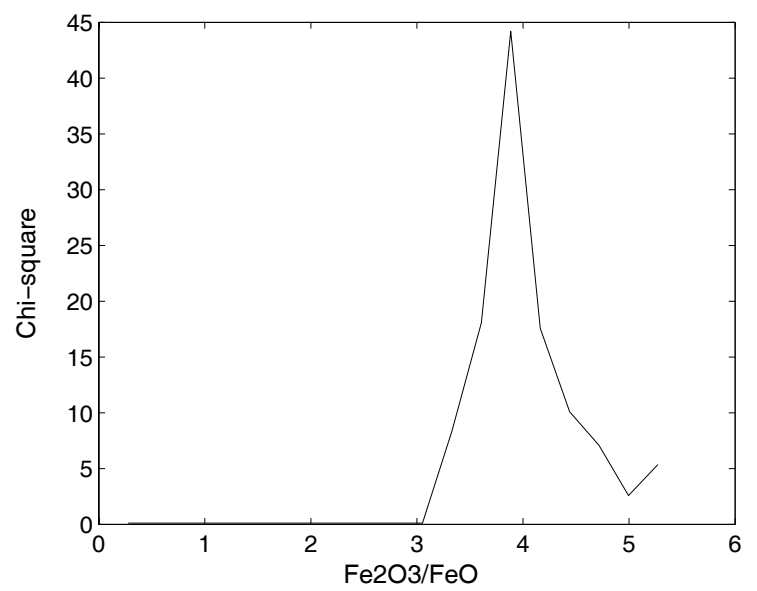

(c) Chi-squared

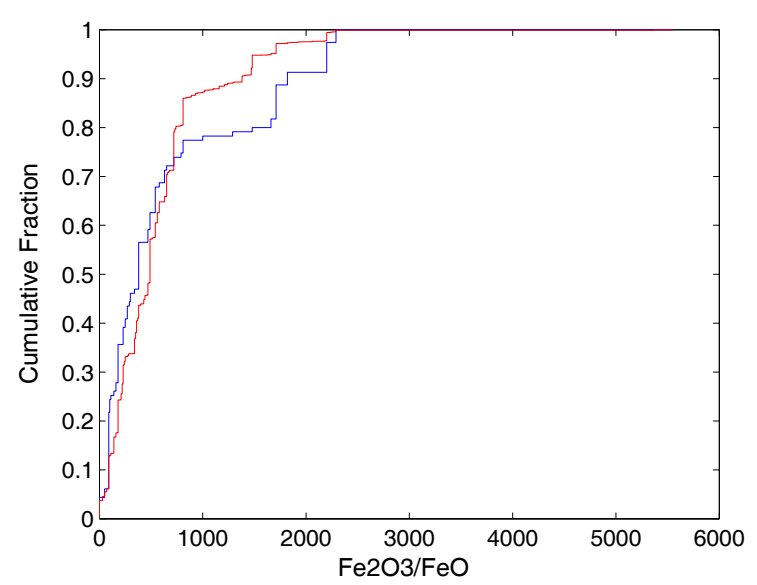

(e) Kolmogorov-Smirnov test

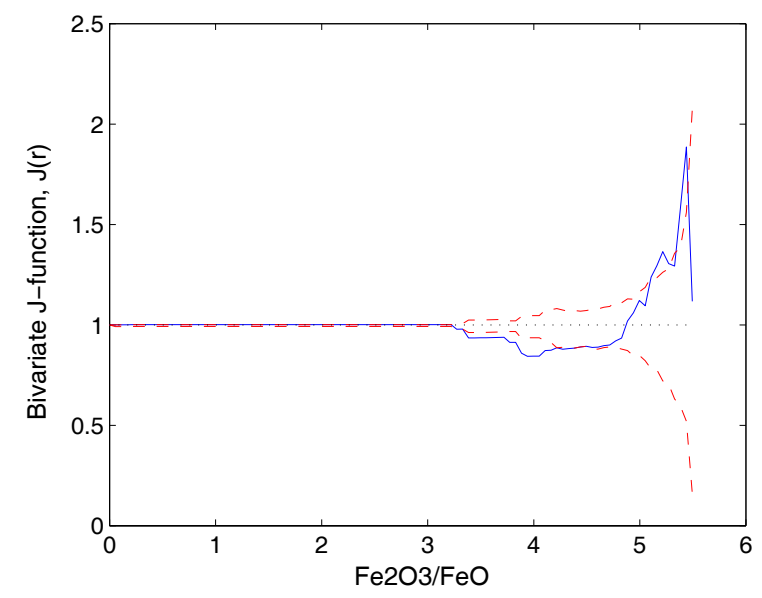

(b) Bivariate J-function (cumulative)

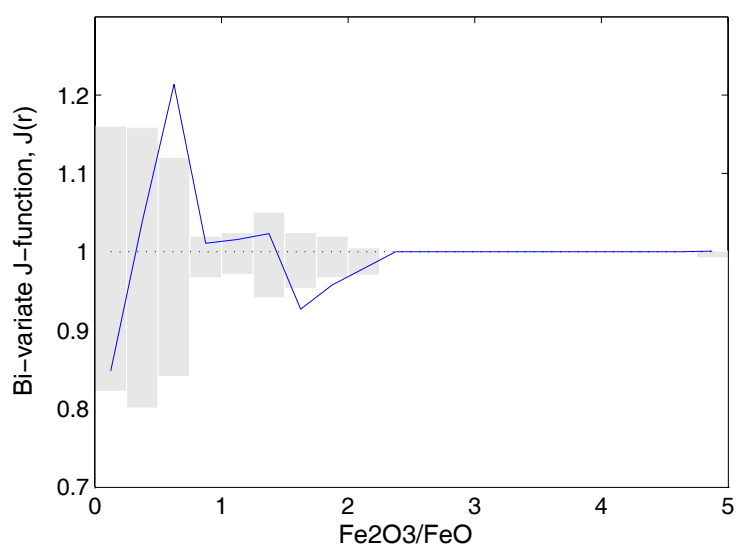

(d) Bivariate J-function (interval-based)

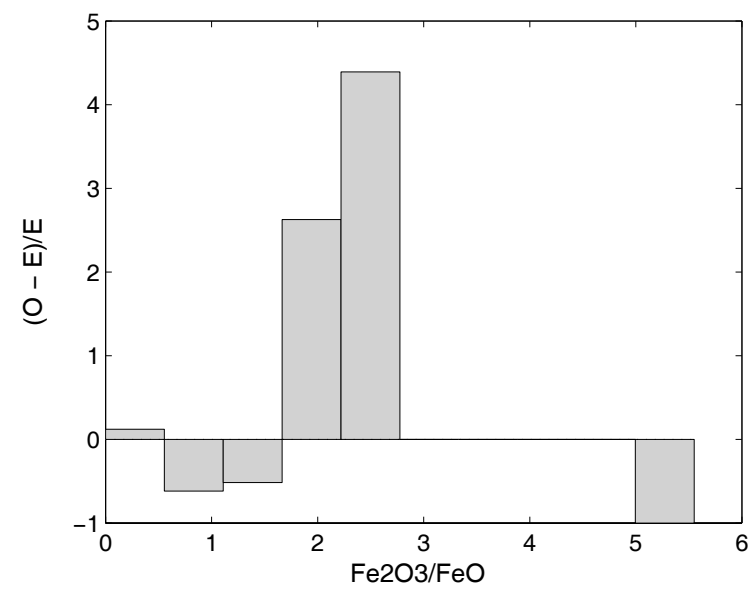

(f) $(O-E) / E$

Figure G.39. Plots used to examine the relationship between deposits and chemical contrast, $\mathrm{Fe}_{2} \mathrm{O}_{3} /(\mathrm{FeO})$ (oxididation index) in the layer, 137 _l_ch $41 k$. The empirical curve is shown in blue in b), d) \& e). The theoretical curve is shown in red in b) \& e) and as grey bars in d). The $\mathrm{X}$-axis parameter in graphs a), b) \& c) was transformed to create a new parameter representing the difference between the maximum and the original parameter value; i.e., $x=X_{\max }-x$. 


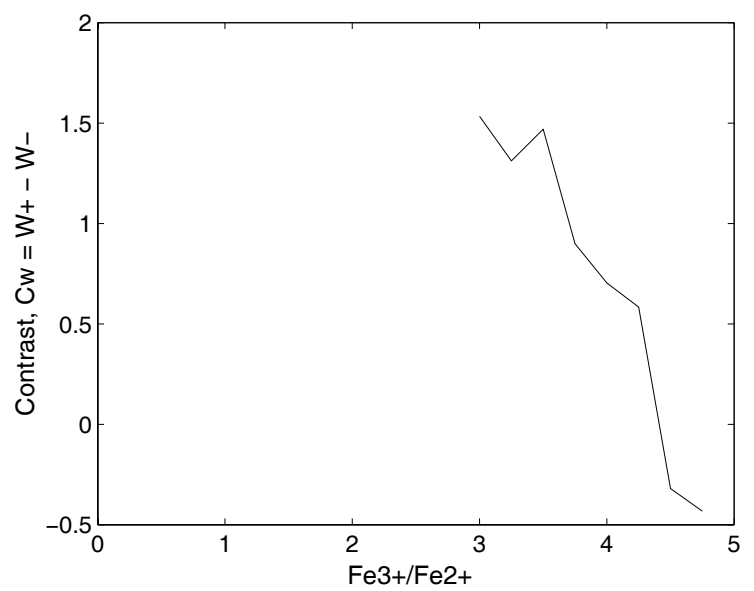

(a) Contrast, $C_{W}$

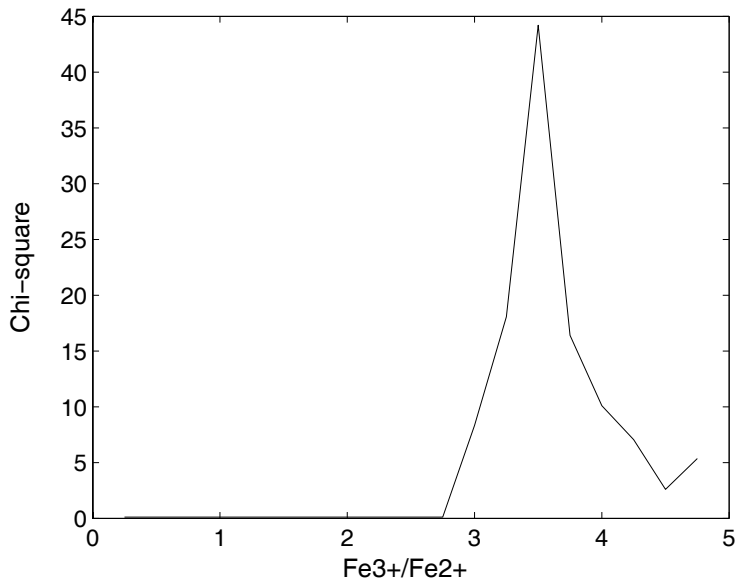

(c) Chi-squared

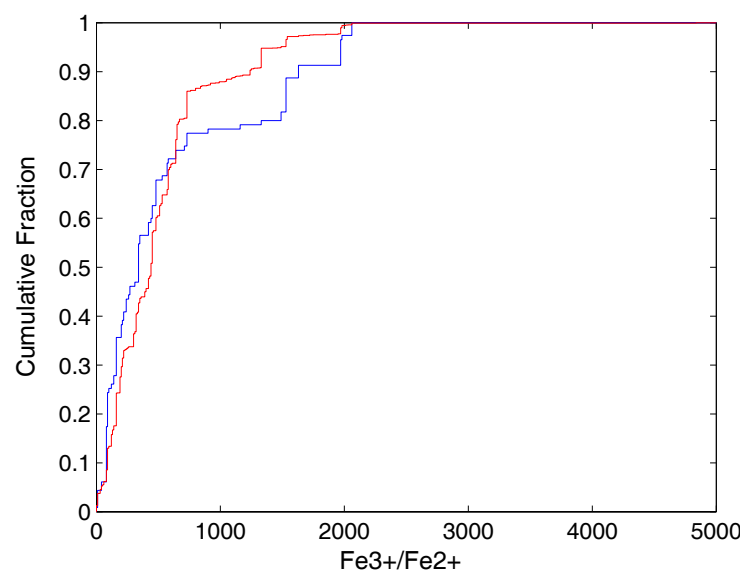

(e) Kolmogorov-Smirnov test

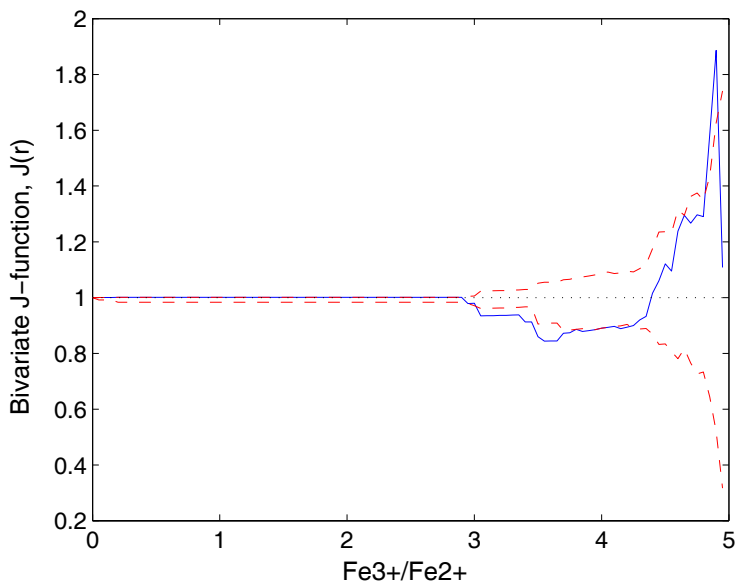

(b) Bivariate J-function (cumulative)

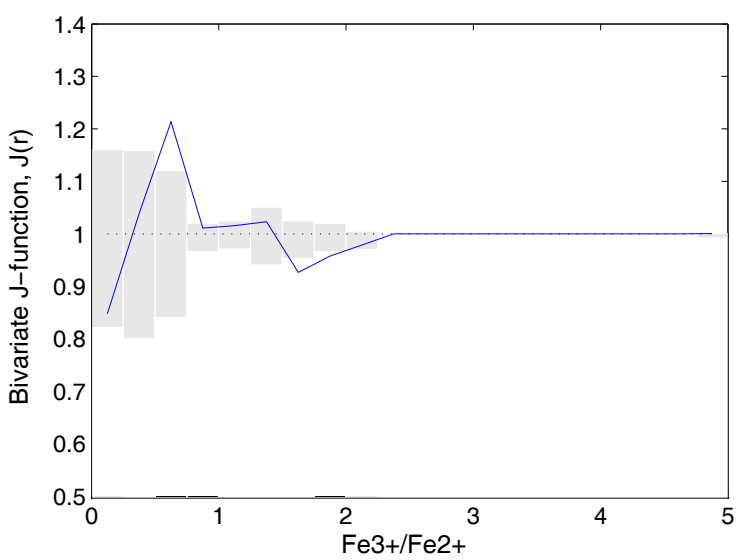

(d) Bivariate J-function (interval-based)

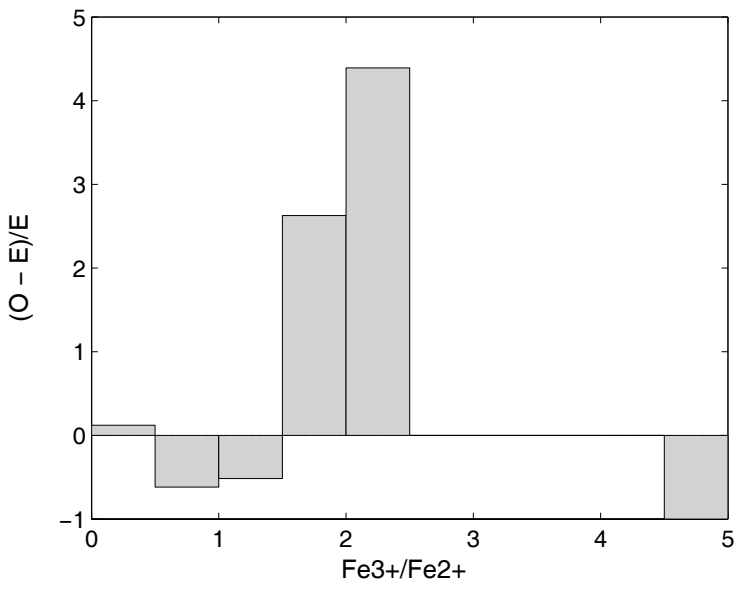

(f) $(O-E) / E$

Figure G.40. Plots used to examine the relationship between deposits and chemical contrast, $\mathrm{Fe}_{2} \mathrm{O} /\left(\mathrm{Fe}_{2} \mathrm{O}_{3}\right)$ (oxidation index) in the layer, l37_l_ch51k. The empirical curve is shown in blue in b), d) \& e). The theoretical curve is shown in red in b) \& e) and as grey bars in d). The $\mathrm{X}$-axis parameter in graphs a), b) \& c) was transformed to create a new parameter representing the difference between the maximum and the original parameter value; i.e., $x=X_{\max }-x$. 


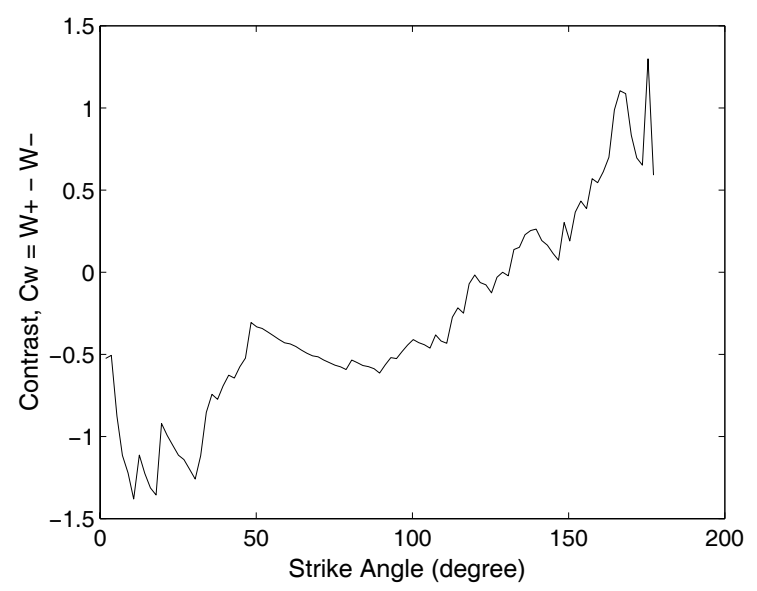

(a) Contrast, $C_{W}$

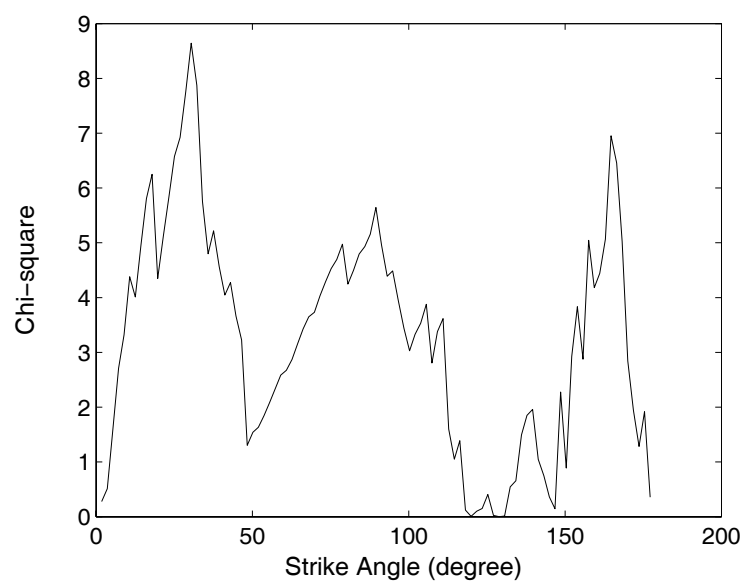

(c) Chi-squared

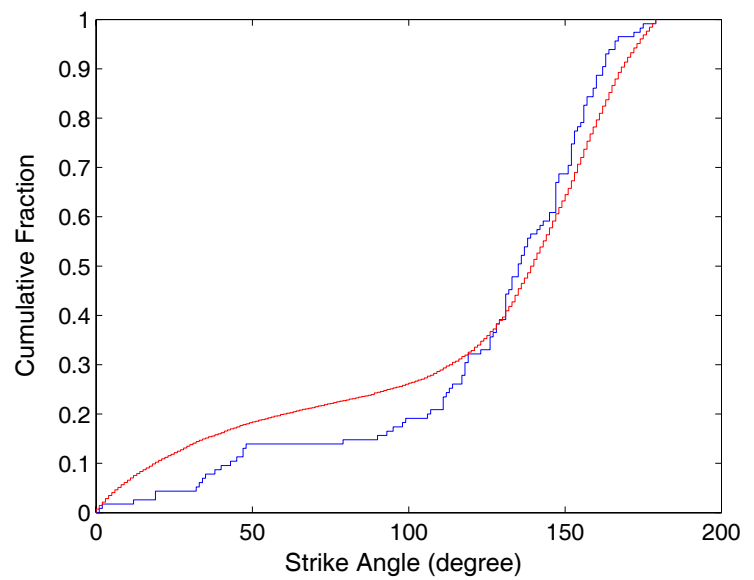

(e) Kolmogorov-Smirnov test

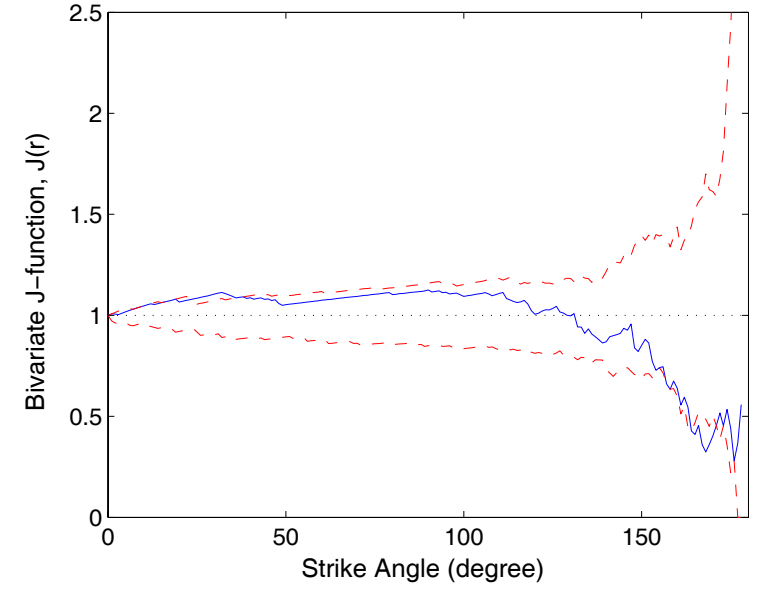

(b) Bivariate J-function (cumulative)

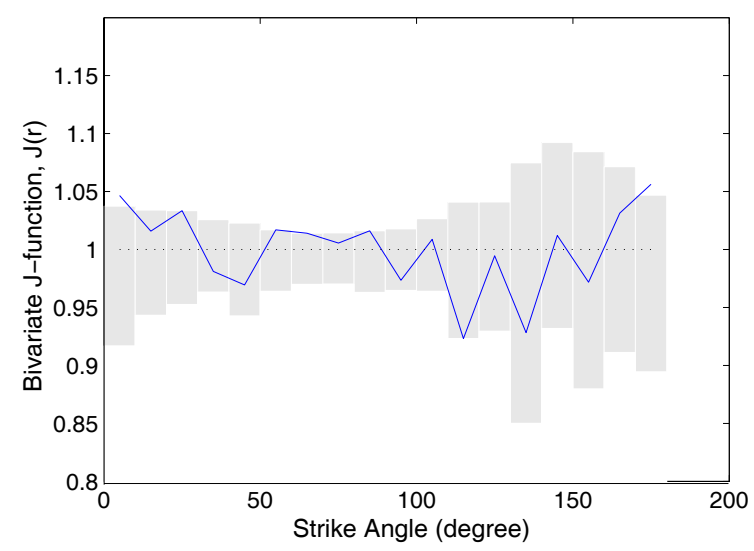

(d) Bivariate J-function (interval-based)

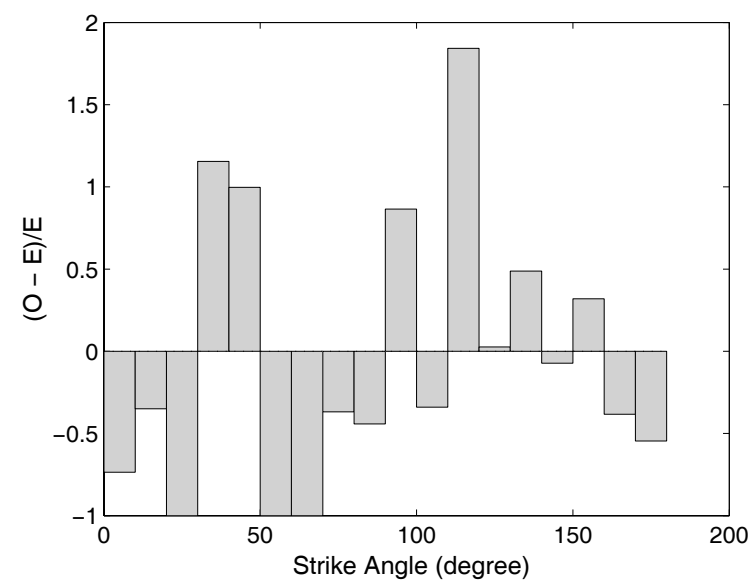

(f) $(O-E) / E$

Figure G.41. Plots used to examine the relationship between deposits and strike of lithological contacts in the layer $138 \_c \_s t r 1 k$. The strike angles have been reduced to a range $0-179^{\circ}$. The empirical curve is shown in blue in b), d) \& e). The theoretical curve is shown in red in b) \& e) and as grey bars in d). 


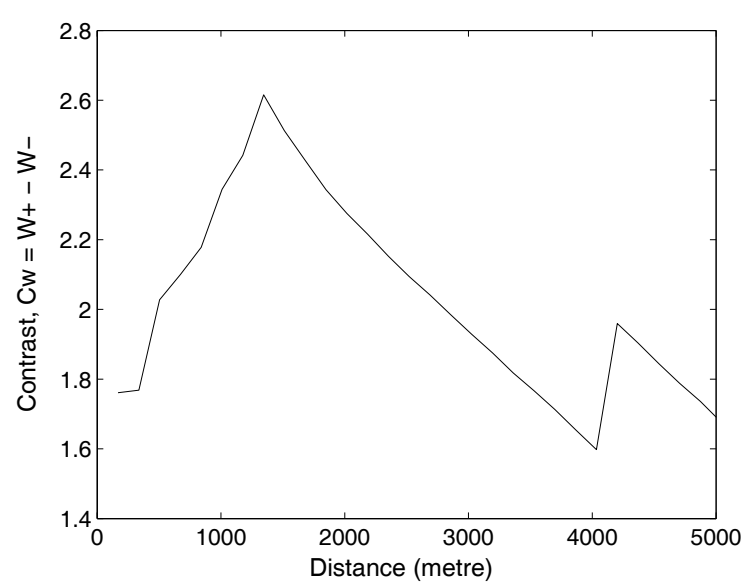

(a) Contrast, $C_{W}$

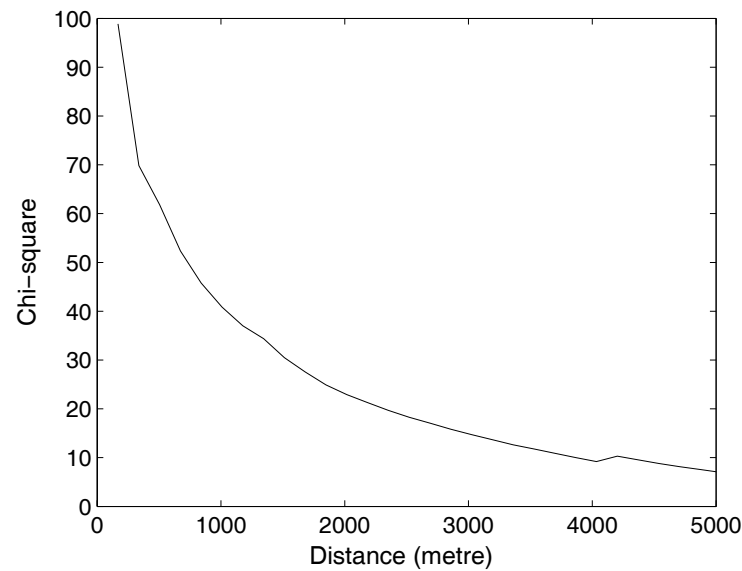

(c) Chi-squared

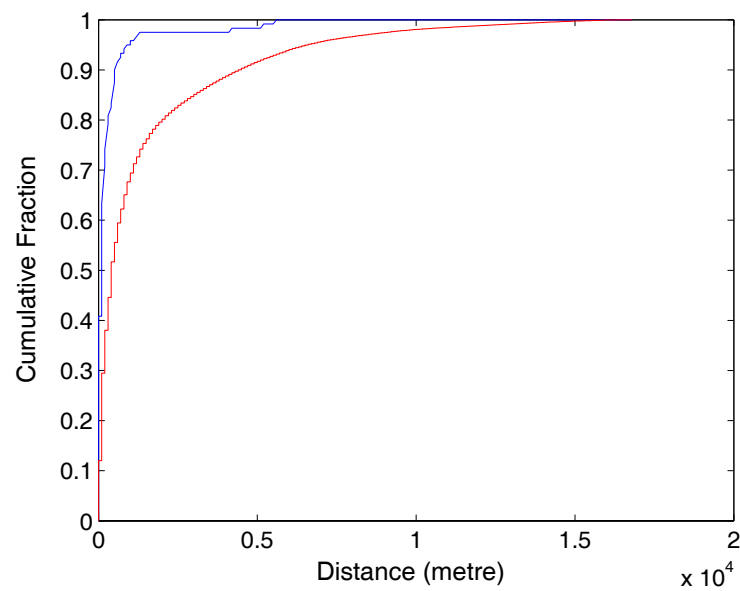

(e) Kolmogorov-Smirnov test

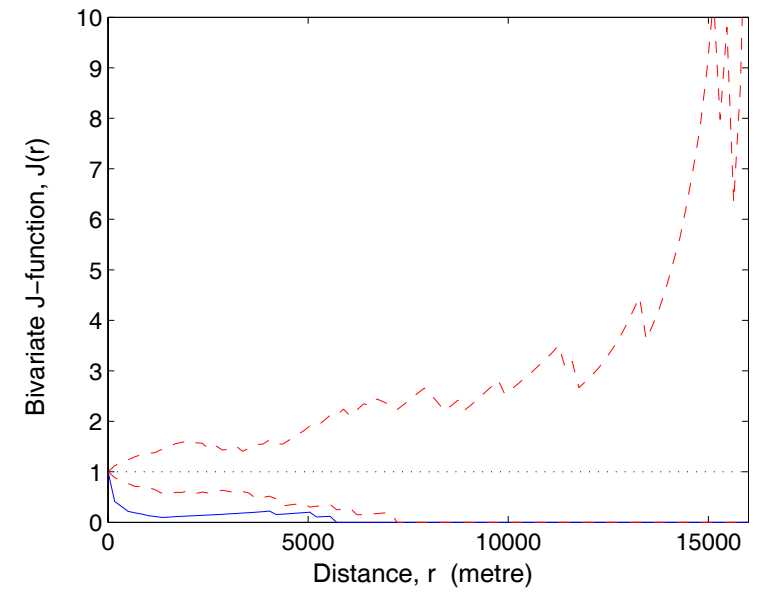

(b) Bivariate J-function (cumulative)

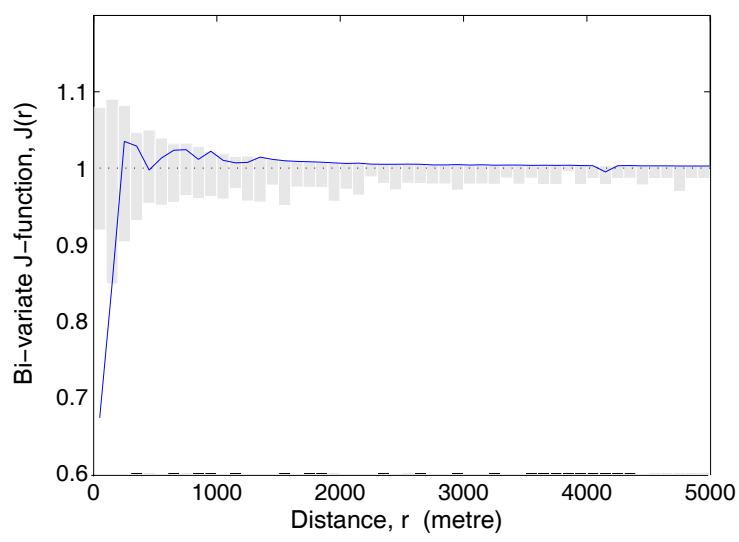

(d) Bivariate J-function (interval-based)

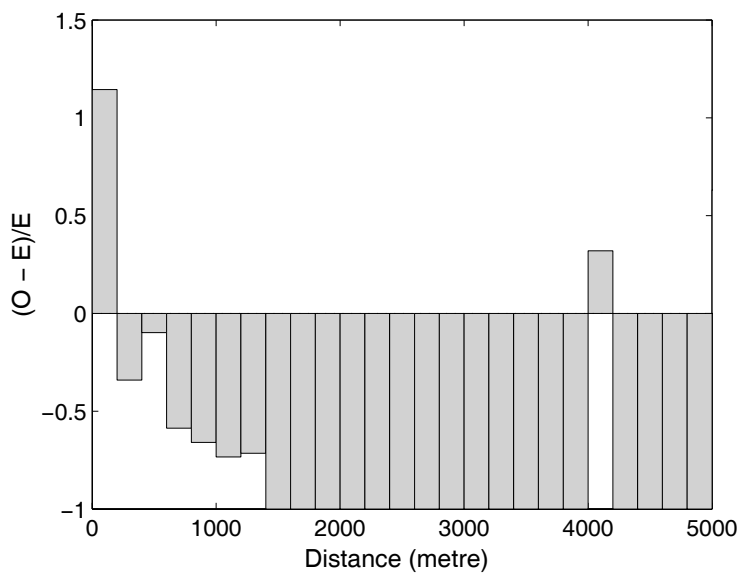

(f) $(O-E) / E$

Figure G.42. Plots used to examine the relationship between deposits and distance to nearest lithological contact in the layer l39_c_dist. The empirical curve is shown in blue in b), d) \& e). The theoretical curve is shown in red in b) \& e) and as grey bars in d). 


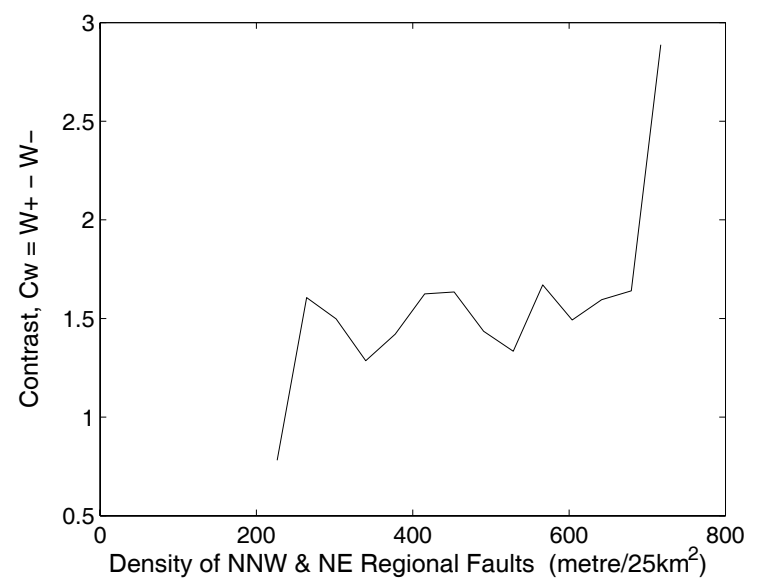

(a) Contrast, $C_{W}$

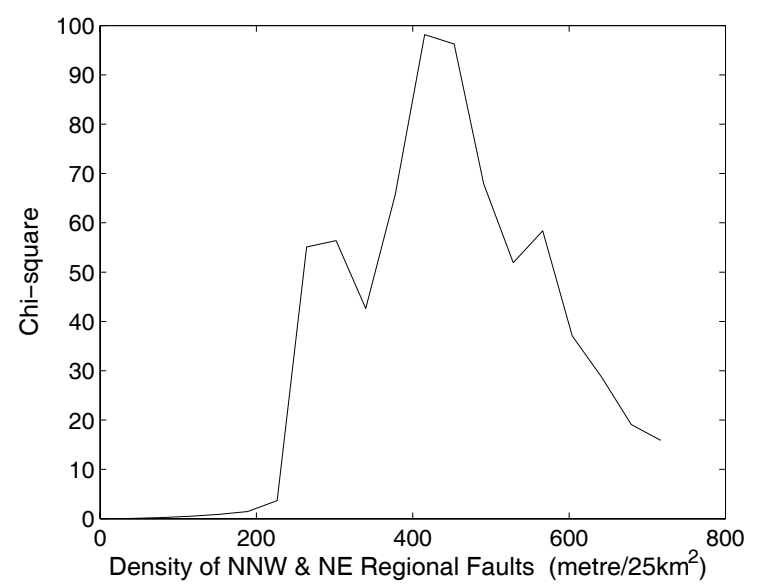

(c) Chi-squared

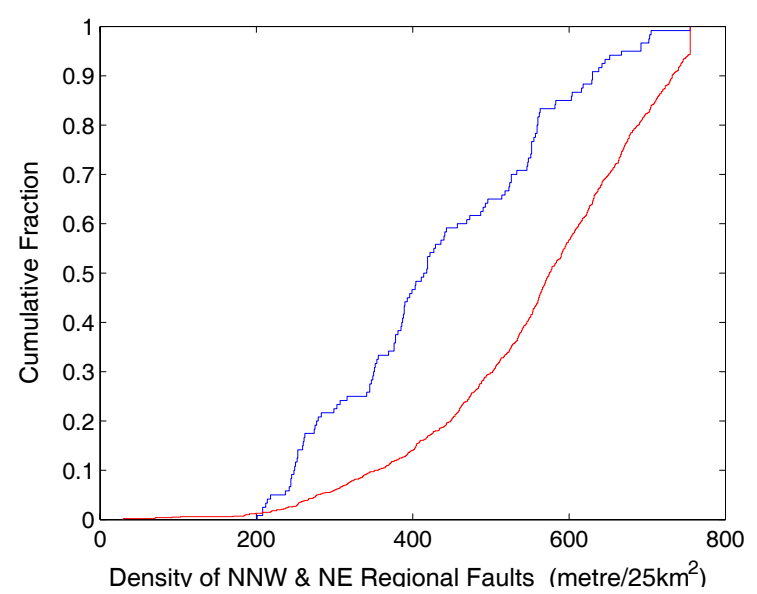

(e) Kolmogorov-Smirnov test

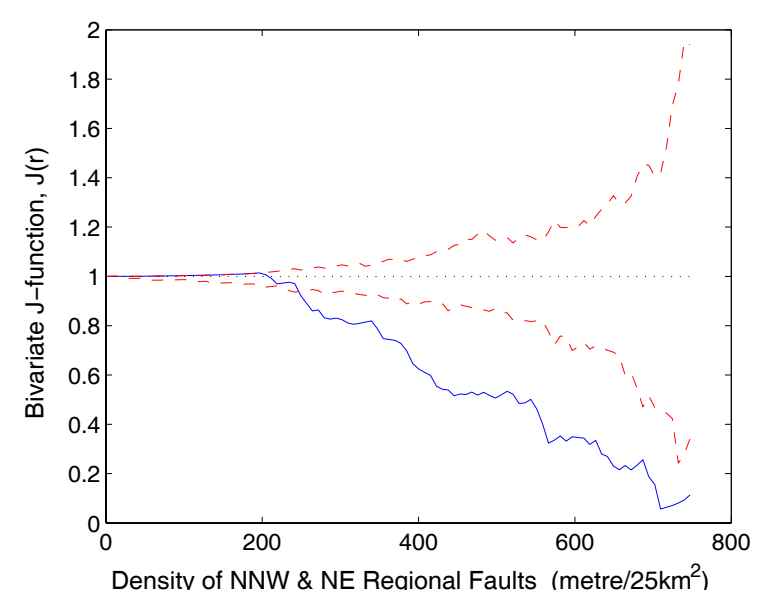

(b) Bivariate J-function (cumulative)

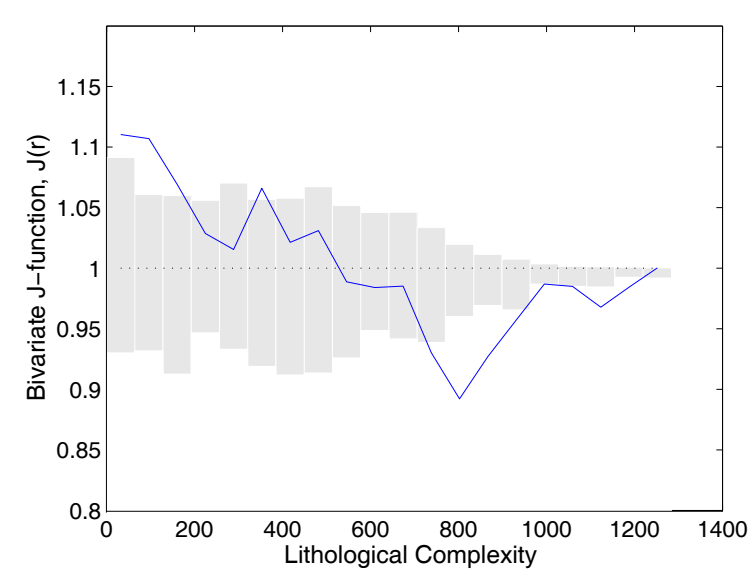

(d) Bivariate J-function (interval-based)

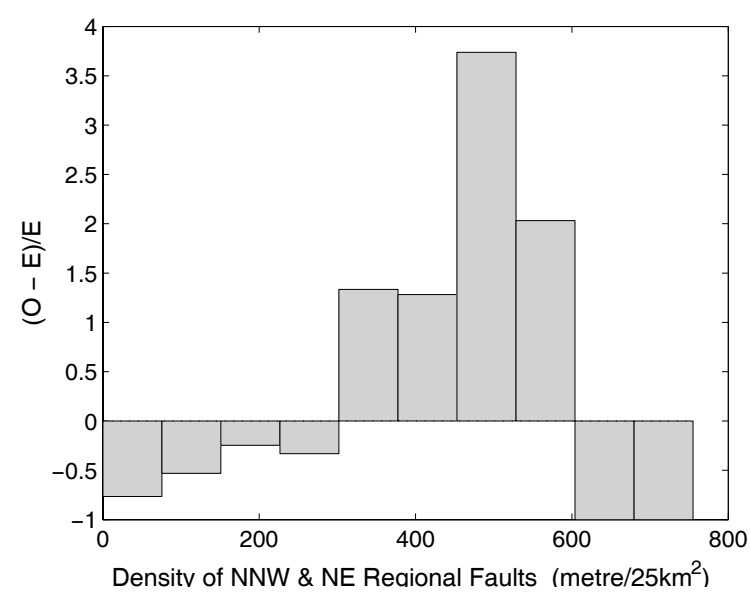

(f) $(O-E) / E$

Figure G.43. Plots used to examine the relationship between deposits and density of regional NNW- and NE- trending faults in layer l42_f123_density. The length of faults in a $5 \times 5 \mathrm{~km}$ window was assigned to the central cell. The empirical curve is shown in blue in b), d) \& e). The theoretical curve is shown in red in b) \& e) and as grey bars in d). The X-axis parameter in graphs a), b) \& c) was transformed to create a new parameter representing the difference between the maximum and the original parameter value; i.e., $x=X_{\max .}-x$. 


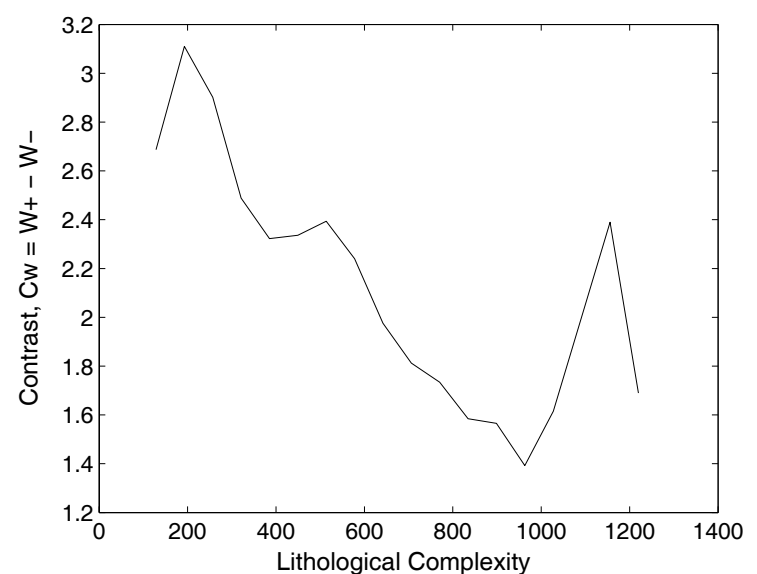

(a) Contrast, $C_{W}$

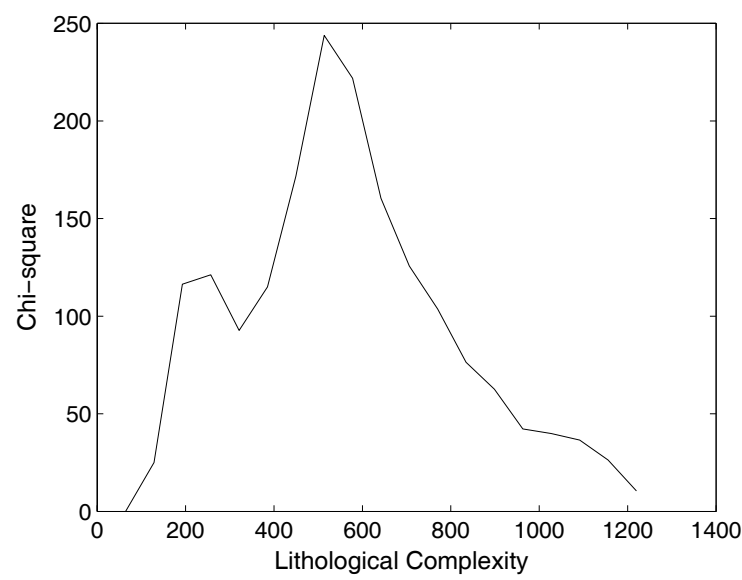

(c) Chi-squared

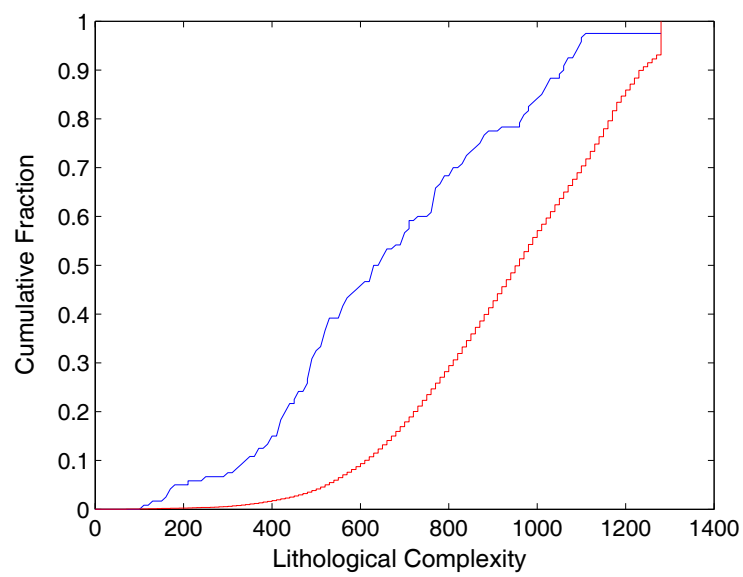

(e) Kolmogorov-Smirnov test

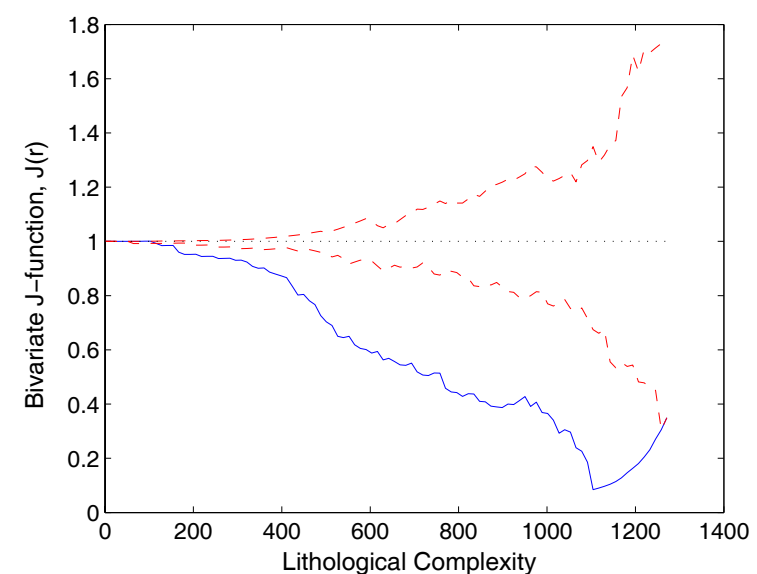

(b) Bivariate J-function (cumulative)

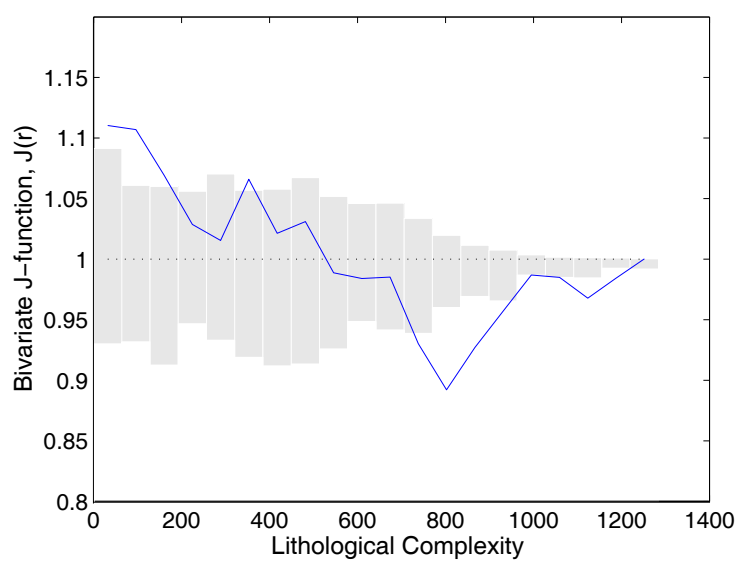

(d) Bivariate J-function (interval-based)

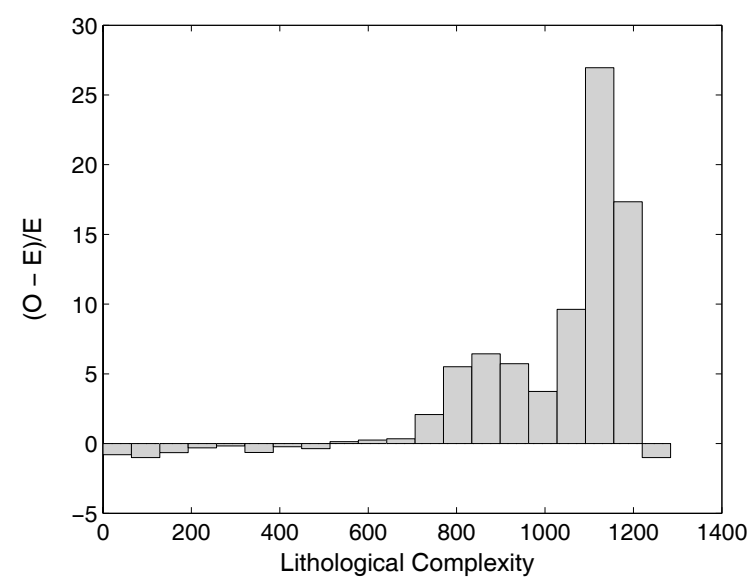

(f) $(O-E) / E$

Figure G.44. Plots used to examine the relationship between deposits and density of lithological contacts in layer 143 _lith_diversity. The number of lithological boundaries in horizontal and vertical directions in a $5 \times 5 \mathrm{~km}$ window is used as a relative measure of lithological complexity. The empirical curve is shown in blue in b), d) \& e). The theoretical curve is shown in red in b) \& e) and as grey bars in d). In graphs a), b) \& c), a new variable $x^{\prime}$ was created to represent the difference between the maximum and the original variable; i.e. $x^{\prime}=X_{\max }-x$. 


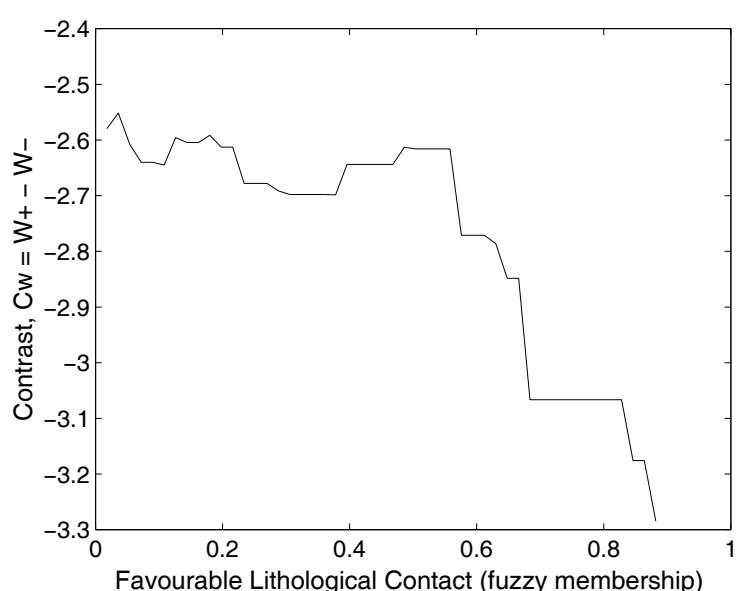

(a) Contrast, $C_{W}$

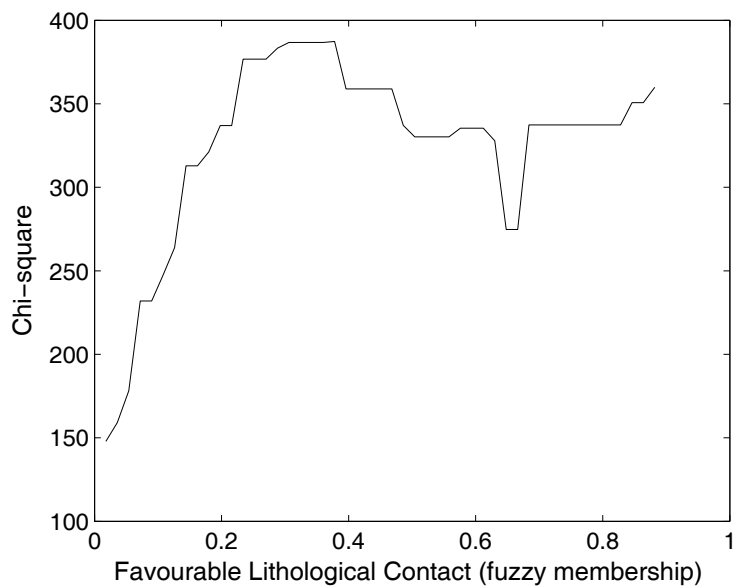

(c) Chi-squared

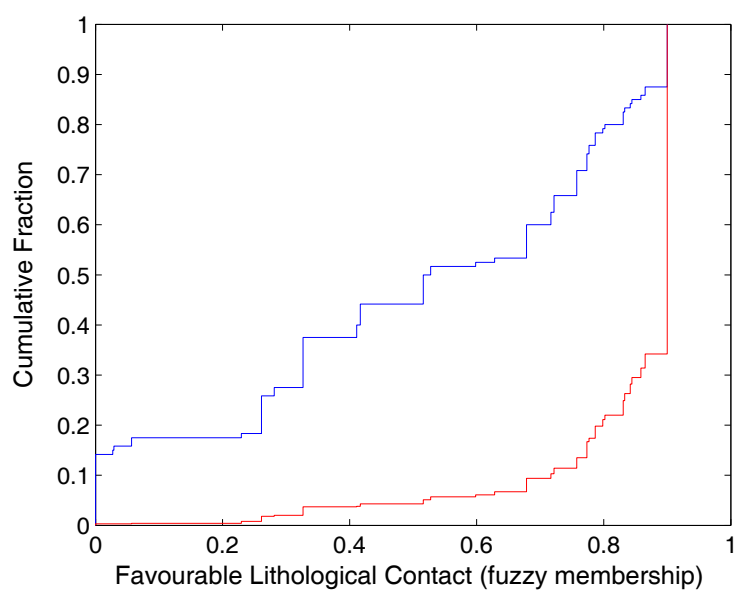

(e) Kolmogorov-Smirnov test

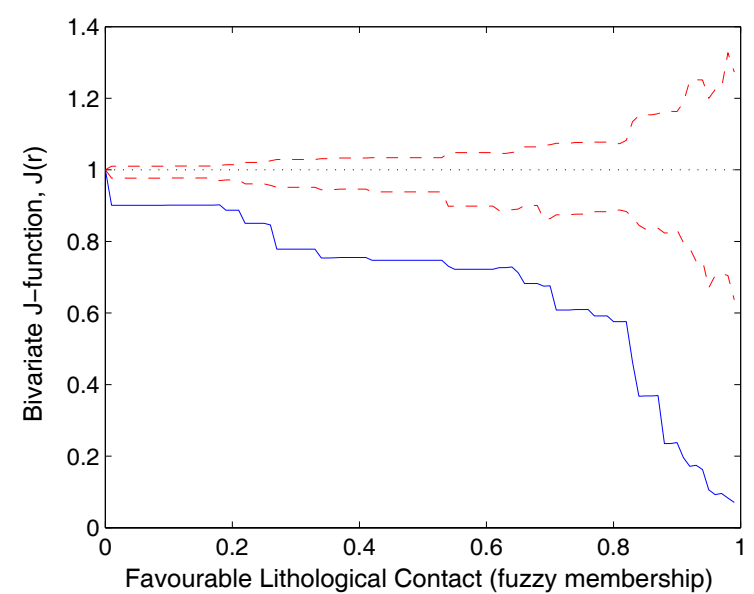

(b) Bivariate J-function (cumulative)

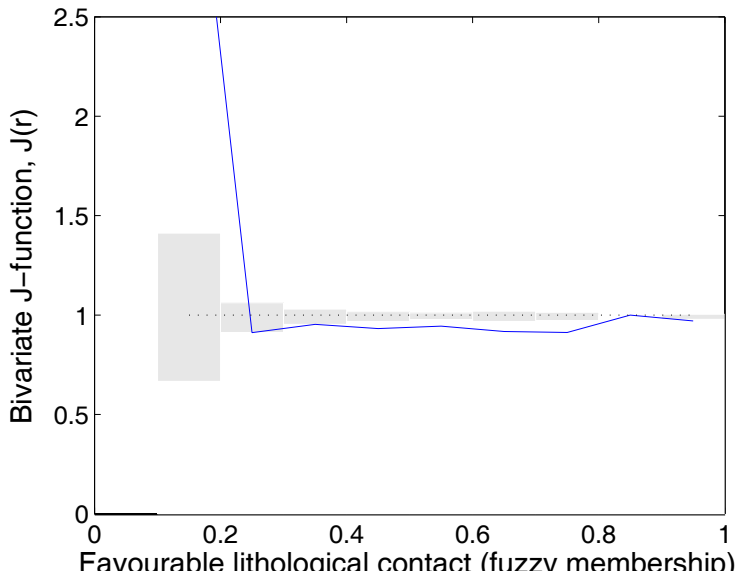

(d) Bivariate J-function (interval-based)

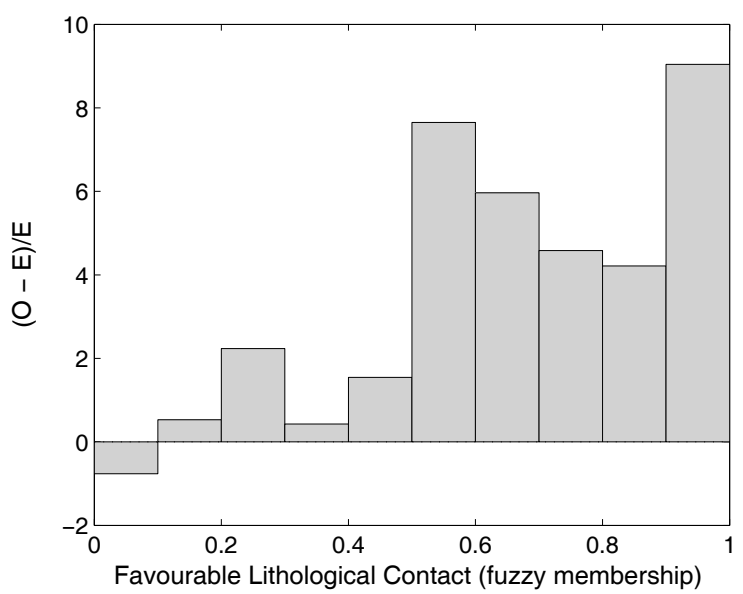

(f) $(O-E) / E$

Figure G.45. Plots used to examine the relationship between deposits and favourable lithological contacts in layer 144 favcnt25pr. The layer contains fuzzy membership values. The empirical curve is shown in blue in b), d) \& e). The theoretical curve is shown in red in b) \& e) and as grey bars in d). In graphs a), b), c) \& d) a new variable $x^{\prime}$ was created to represent the difference between the maximum and the original variable; i.e. $x^{\prime}=X_{\max }-x$. 


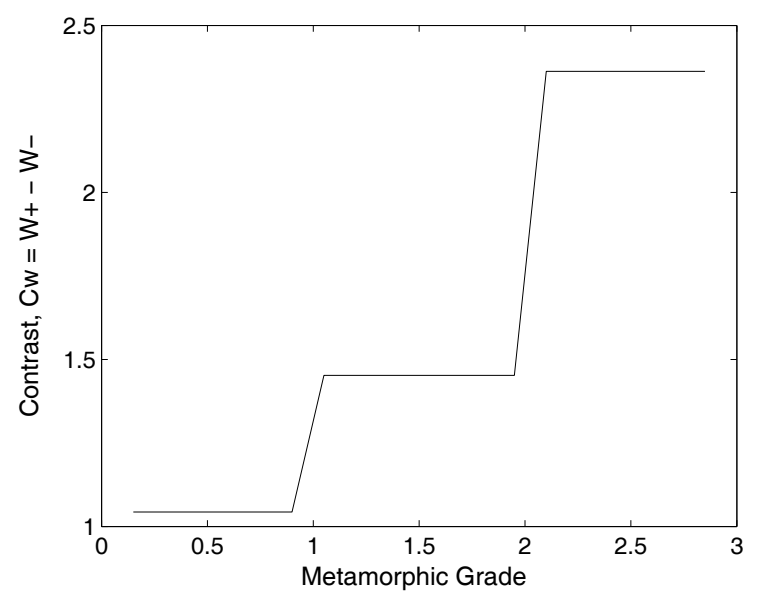

(a) Contrast, $C_{W}$

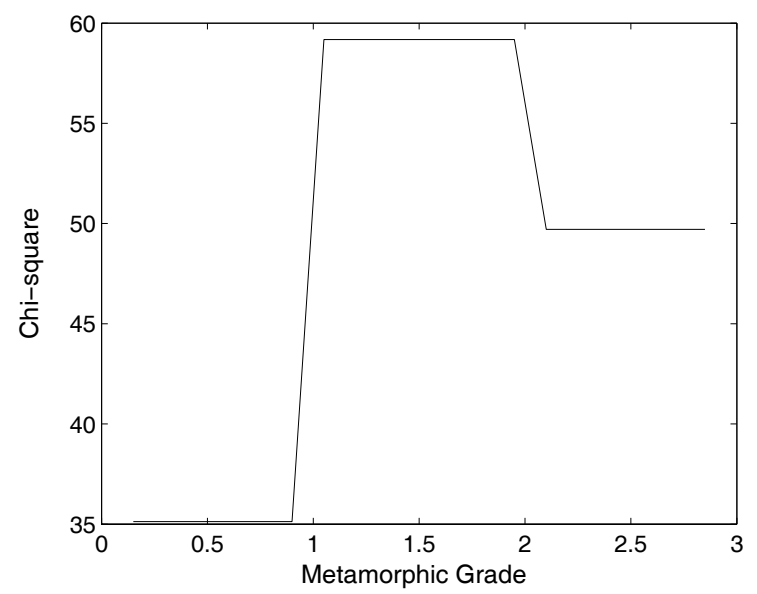

(c) Chi-squared

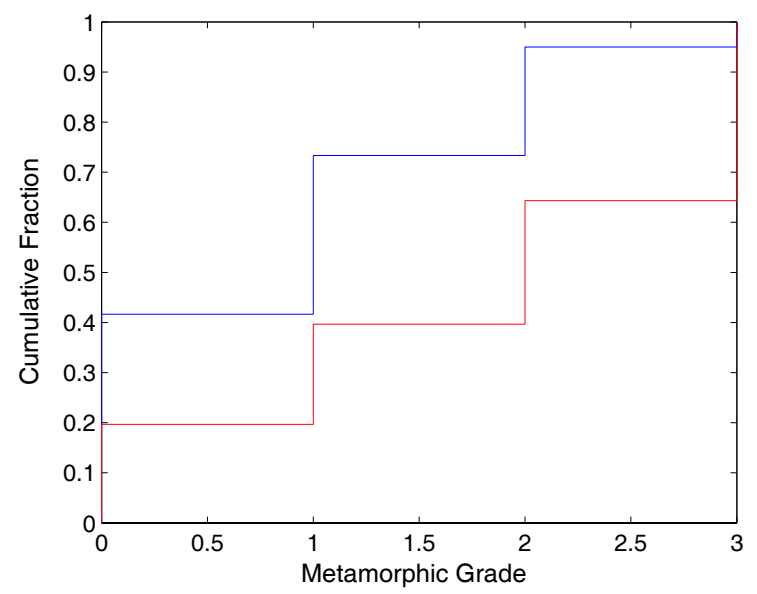

(e) Kolmogorov-Smirnov test

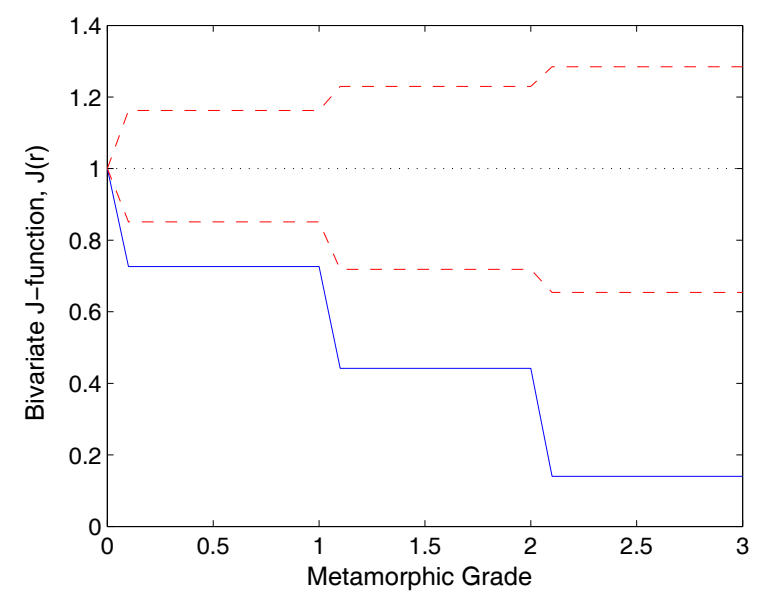

(b) Bivariate J-function (cumulative)

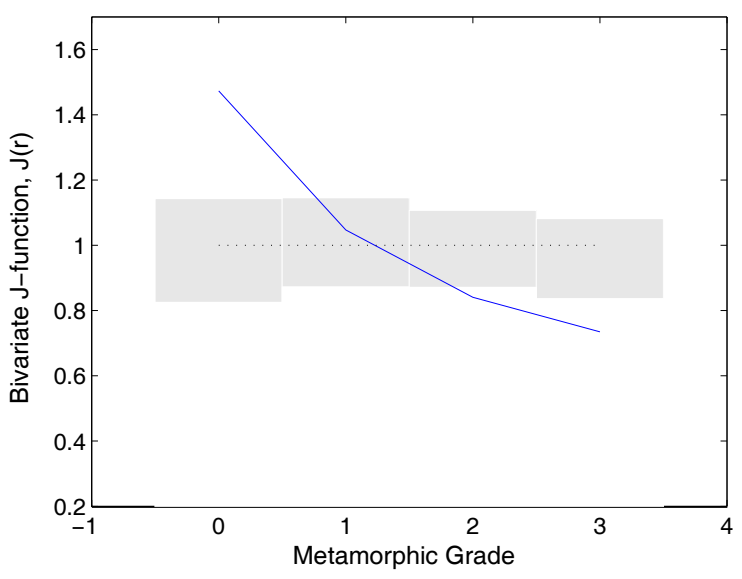

(d) Bivariate J-function (interval-based)

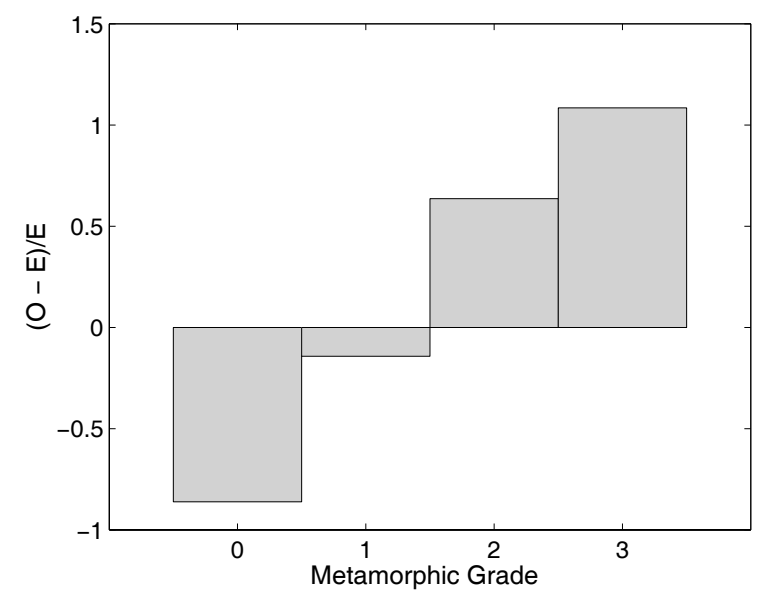

(f) $(O-E) / E$

Figure G.46. Plots used to examine the relationship between deposits and metamorphic grade in layer 145 _l_meta. There are four classes of rock in the metamorphic-grade map, which were assigned the numeric codes shown in brackets; granite (0), amphibolite (1), upper greenschist (2), and lower greenschist facies (3). The numeric codes were assigned so that the code increases with increasing favourability for gold deposits. The empirical curve is shown in blue in b), d) \& e). The theoretical curve is shown in red in b) \& e) and as grey bars in d). In graphs a), b) \& c) a new variable $x^{\prime}$ was created to represent the difference between the maximum and the original variable; i.e. $x^{\prime}=X_{\max }-x$. 


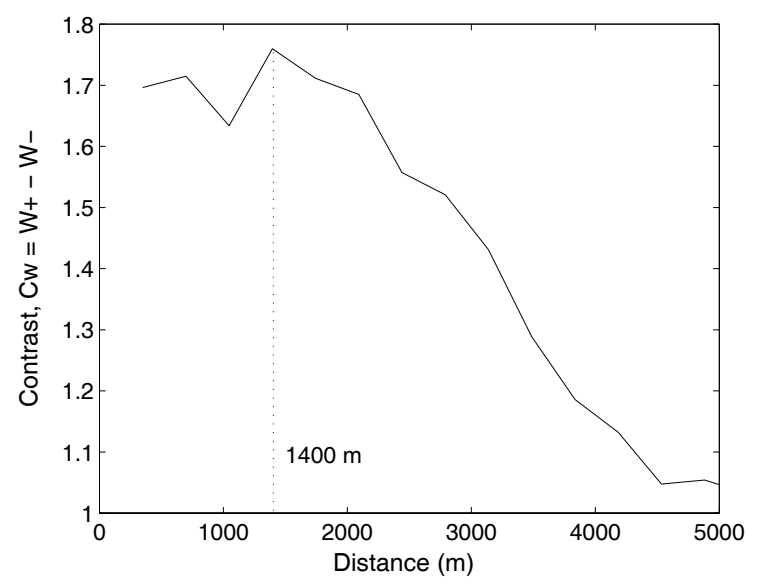

(a) Contrast, $C_{W}$

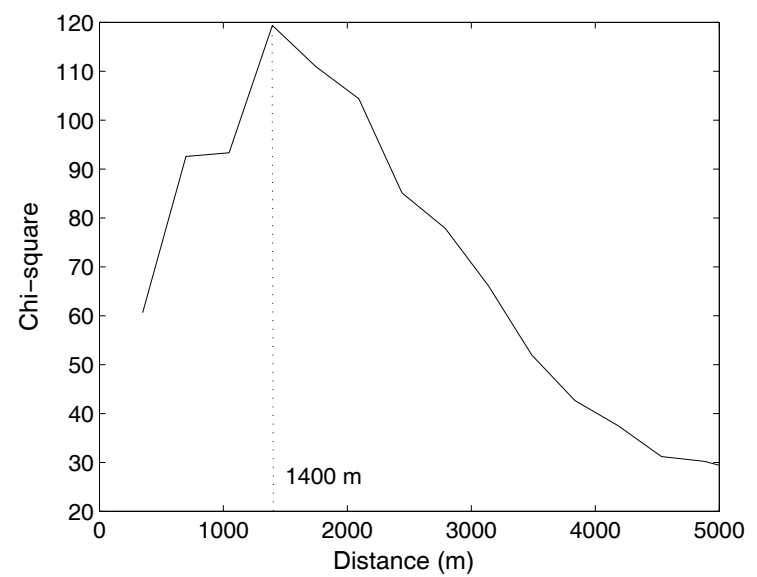

(c) Chi-squared

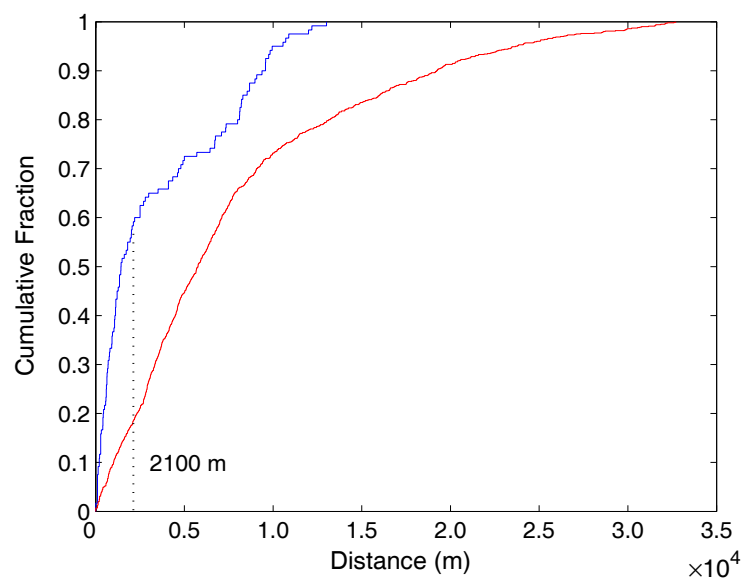

(e) Kolmogorov-Smirnov test

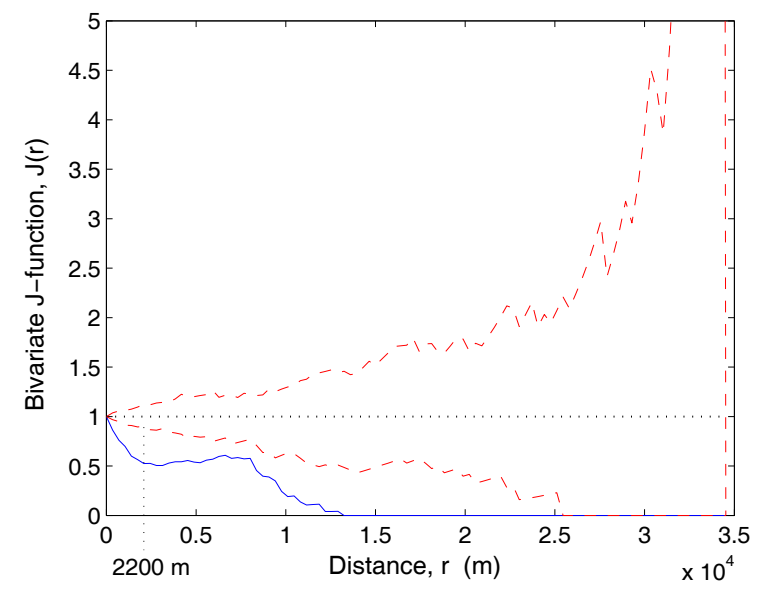

(b) Bivariate J-function (cumulative)

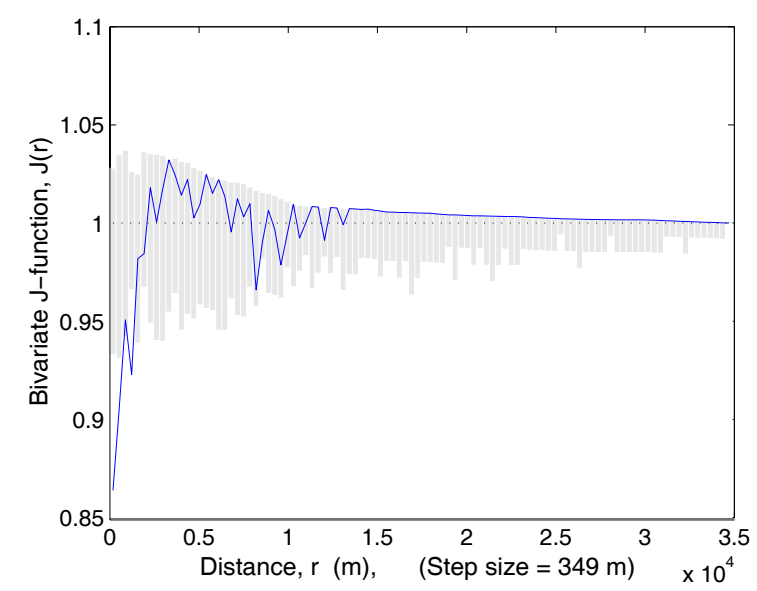

(d) Bivariate J-function (interval-based)

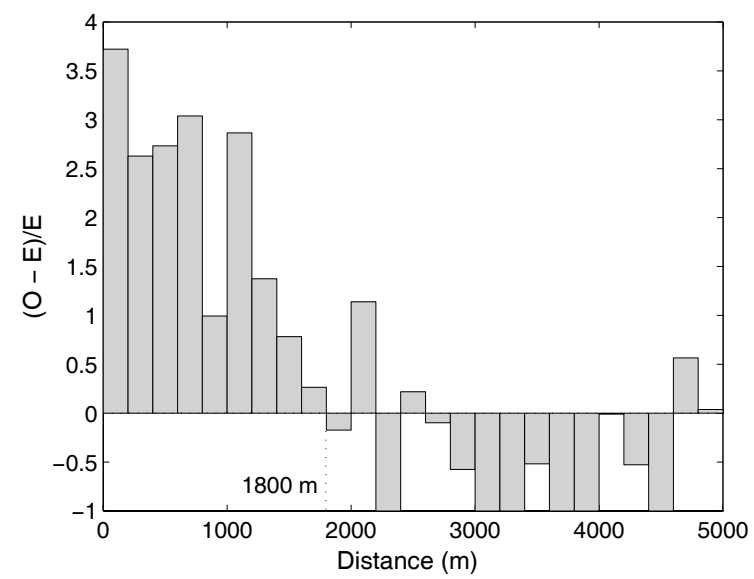

(f) $(O-E) / E$

Figure G.47. Plots used to examine the relationship between deposits and the distance to the ground trace of the nearest anticlinal axis in the layer, $l_{4} 6_{-}$dist_anti. The empirical curve is shown in blue in b), d) \& e). The theoretical curve is shown in red in b) \& e) and as grey bars in d). 


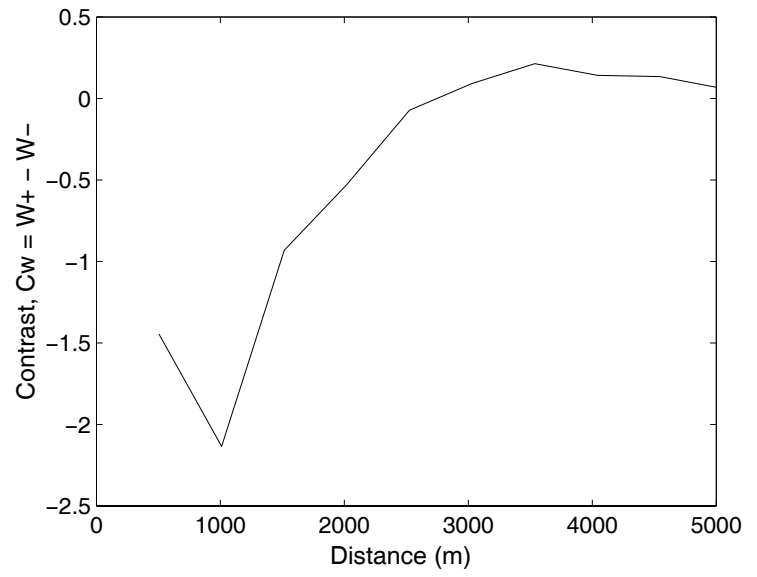

(a) Contrast, $C_{W}$

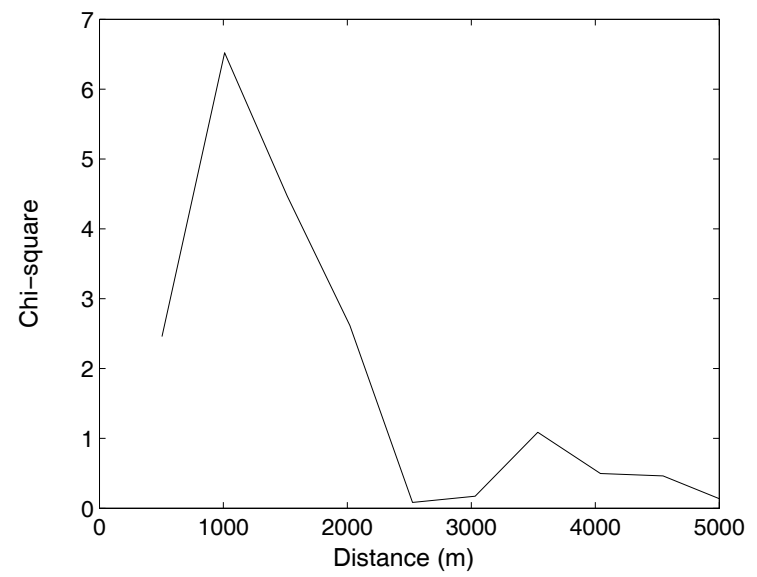

(c) Chi-squared

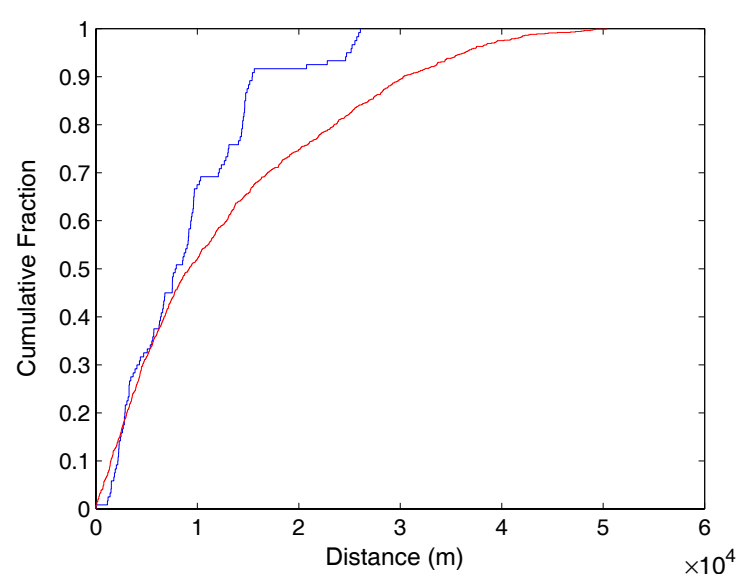

(e) Kolmogorov-Smirnov test

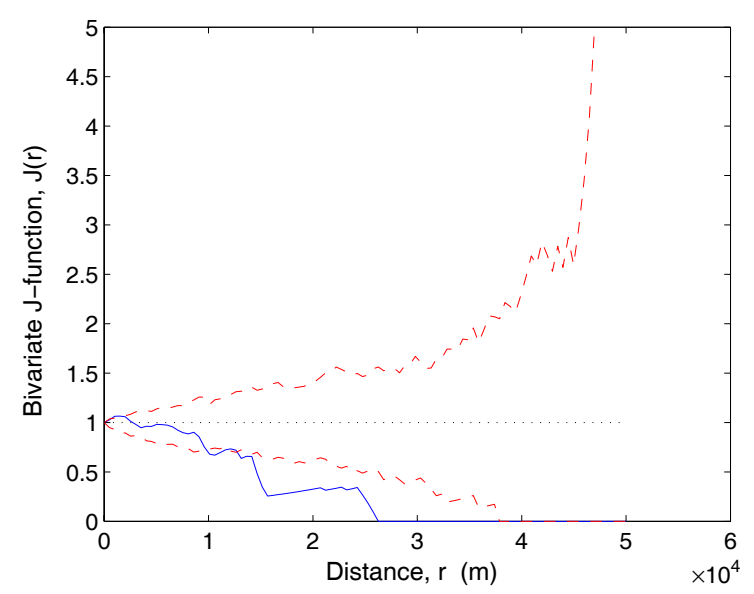

(b) Bivariate J-function (cumulative)

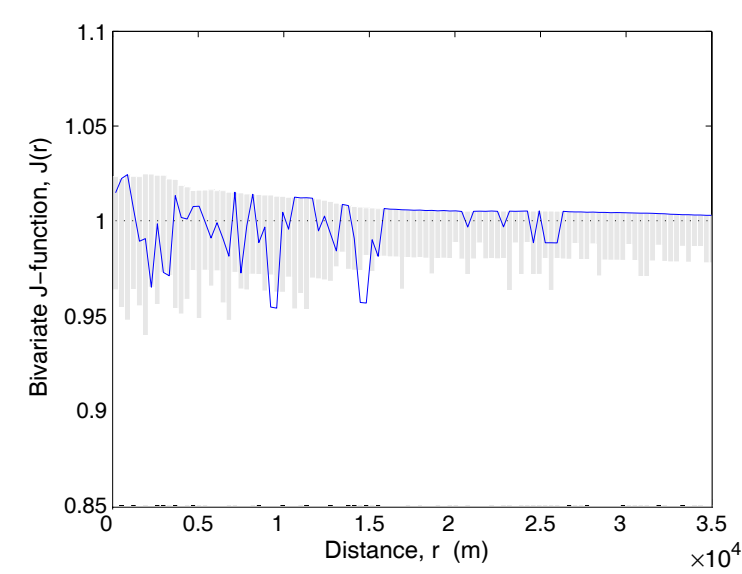

(d) Bivariate J-function (interval-based)

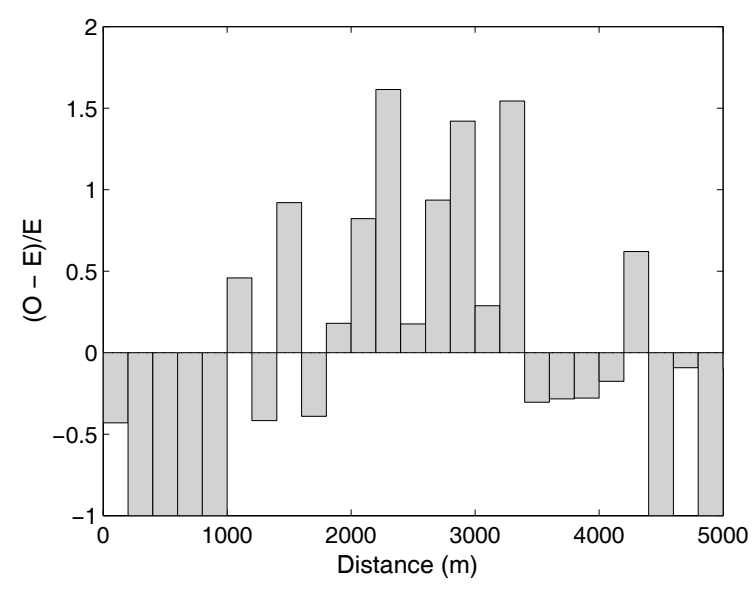

(f) $(O-E) / E$

Figure G.48. Plots used to examine the relationship between deposits and the distance to the ground trace of the nearest synclinal axis in the layer, 146 _dist_sync. The empirical curve is shown in blue in b), d) \& e). The theoretical curve is shown in red in b) \& e) and as grey bars in d). 


\section{G.2 Ranking of potential GIS input layers}

Fifty-nine potential input GIS layers were ranked according to their ability to predict the known deposits in the study area. The best 10 and best 17 of these were selected as inputs to the neural networks used in experiments in Chapter 7. Four methods of ranking the GIS layers are presented in the following tables; Several criteria were used used to rank the layers;

1. best representatives of each evidence category (Table G.1)

2. D/A $A N D D \geq \sim 20 \%$ (Table G.2)

3. D*D/A (Table G.3)

4. $\mathrm{D}^{*} \mathrm{D} / \mathrm{A} A N D \mathrm{D} \geq \sim 20 \% A N D \mathrm{D} / \mathrm{A} \geq \sim 3$, (Table G.4)

where $D$ is the percentage of the deposits and $A$ is the percentage of the map area. In each of the tables, the names of layers that belong to the 10-layer set are shown in dark grey and those belonging to 17-layer set are shown in light grey. 


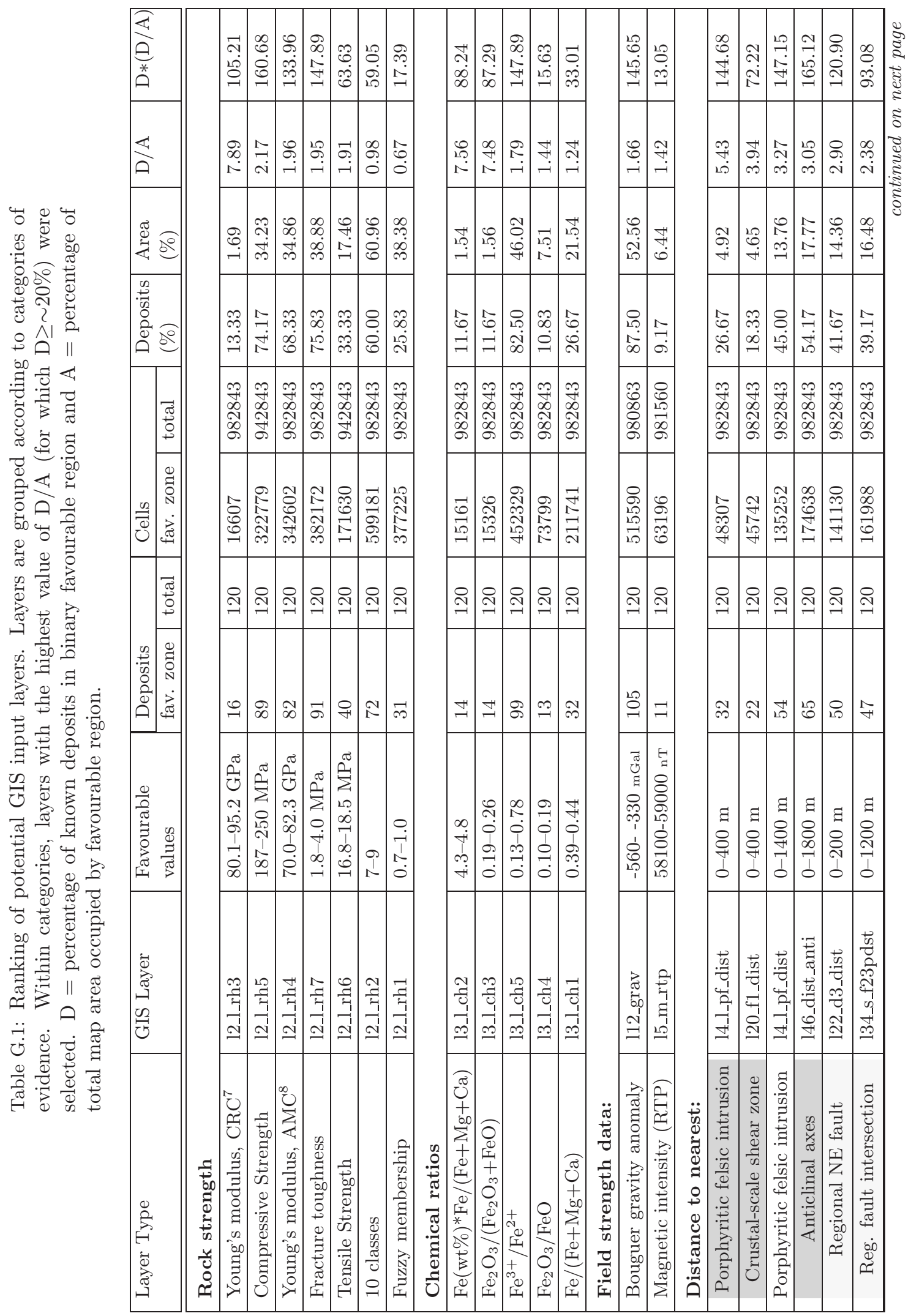




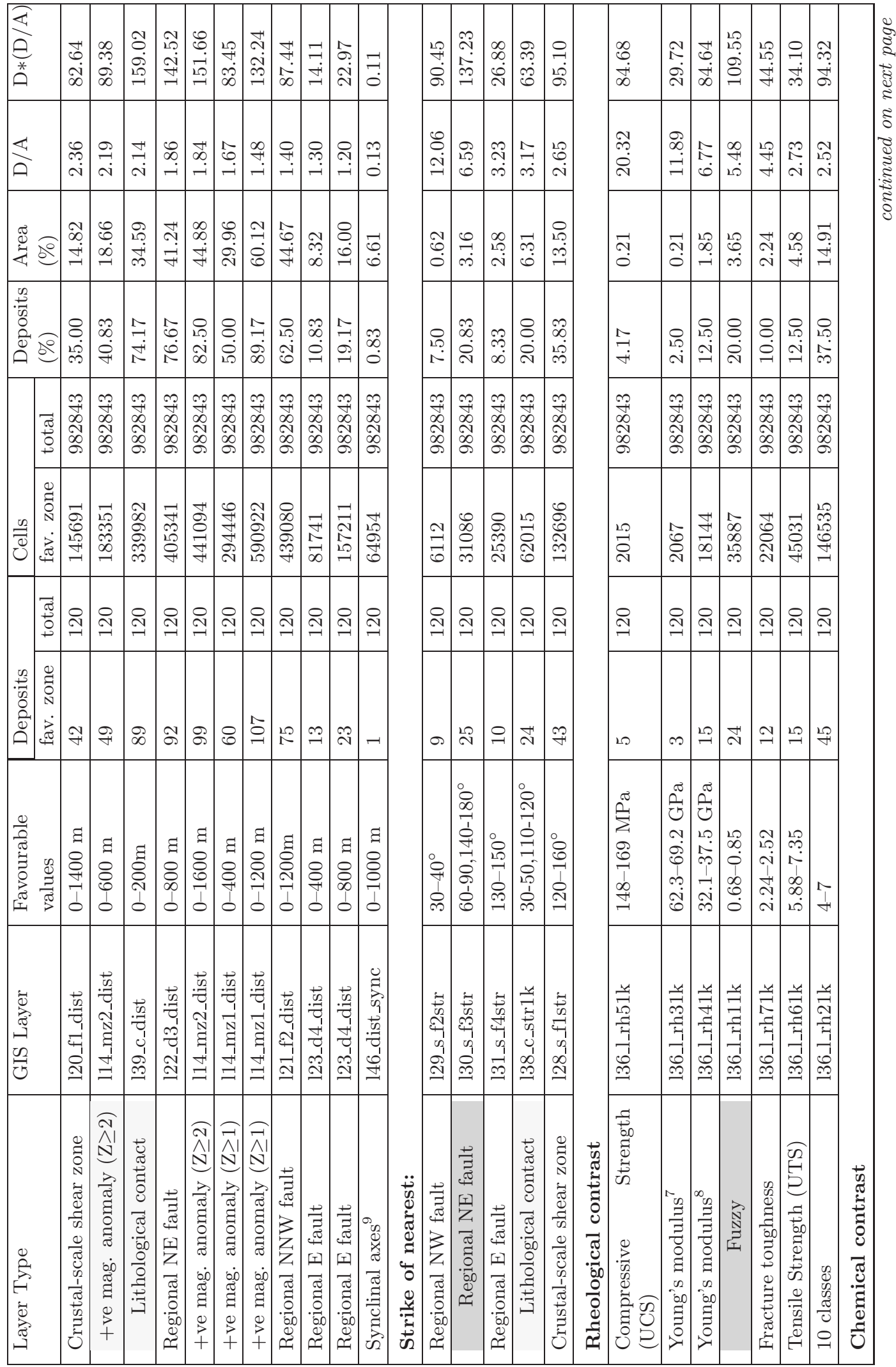




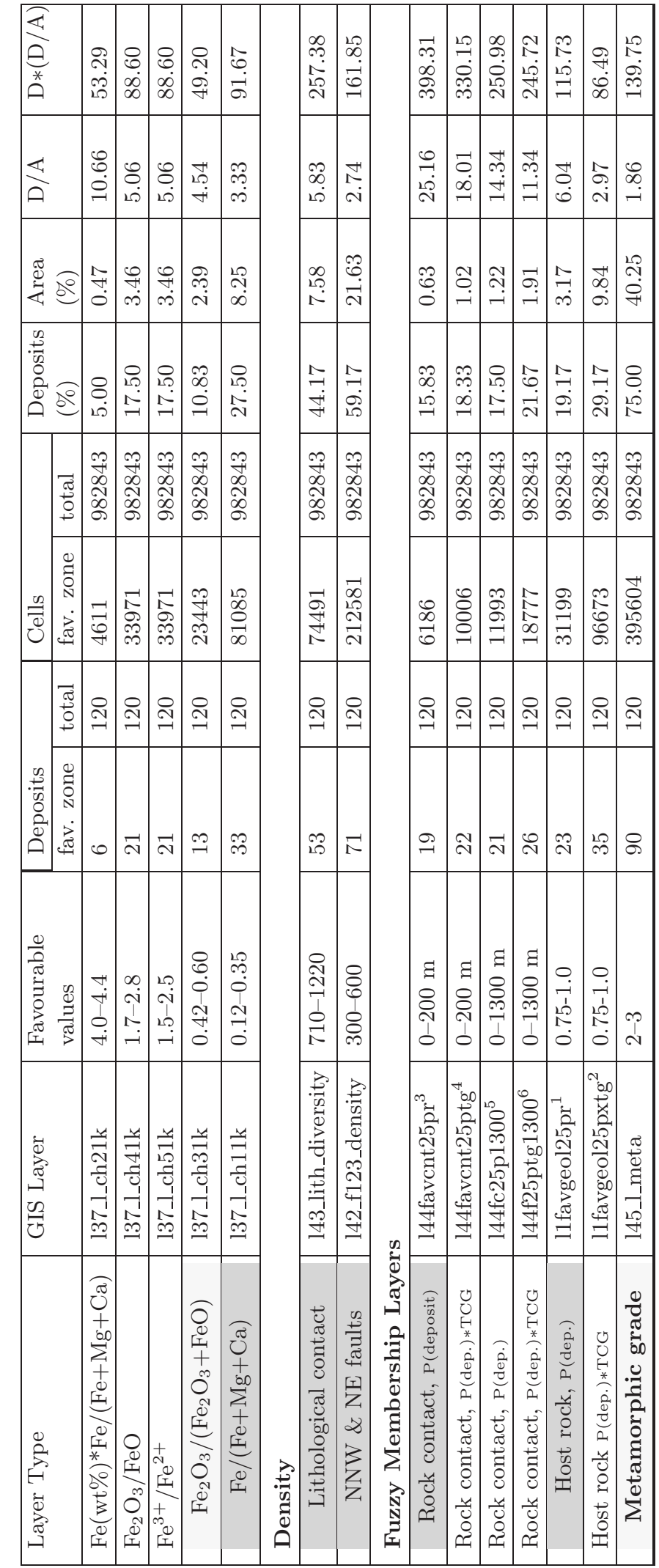

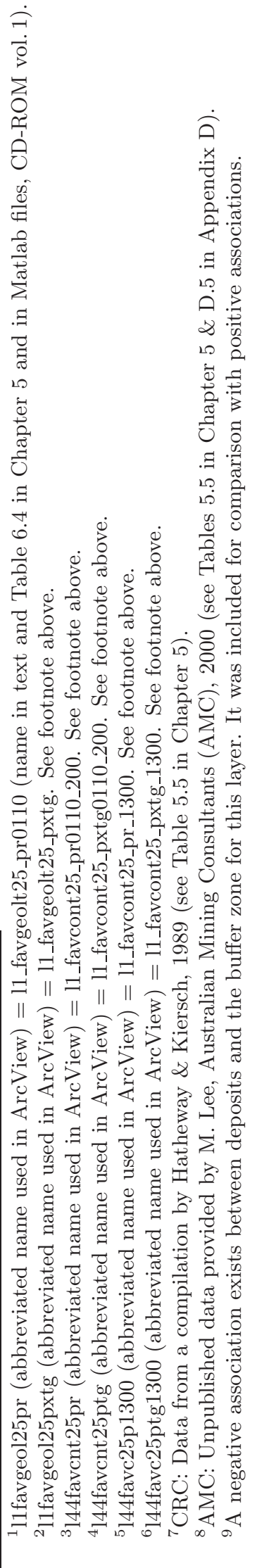




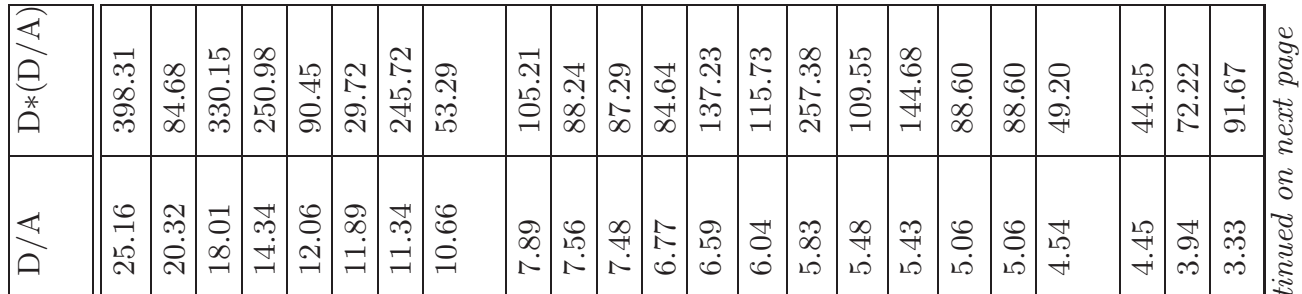

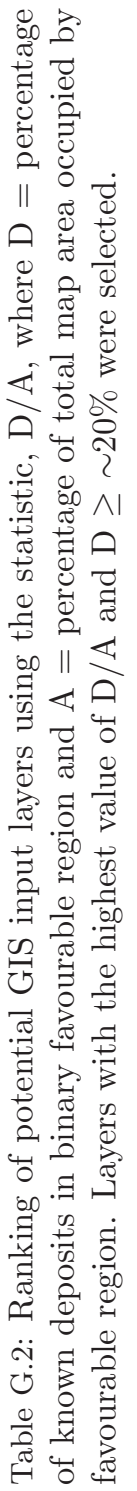

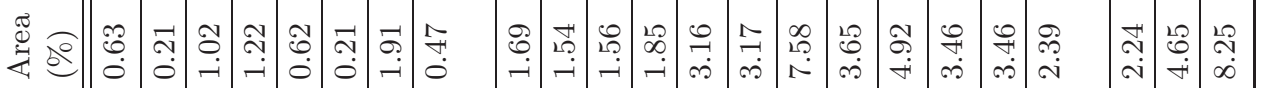

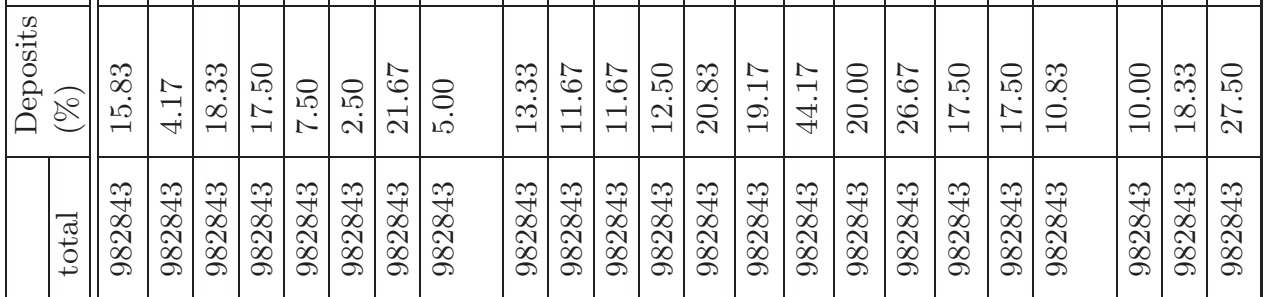

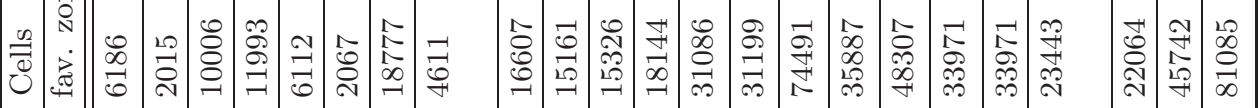

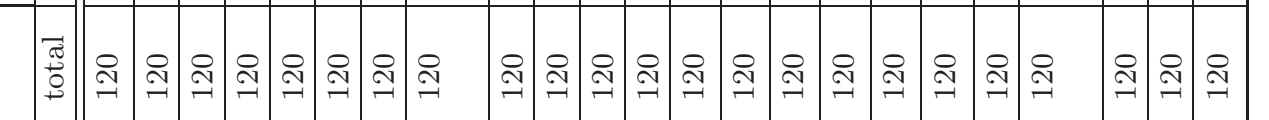

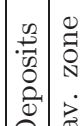

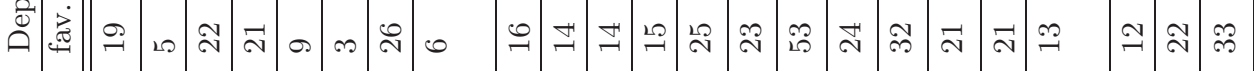

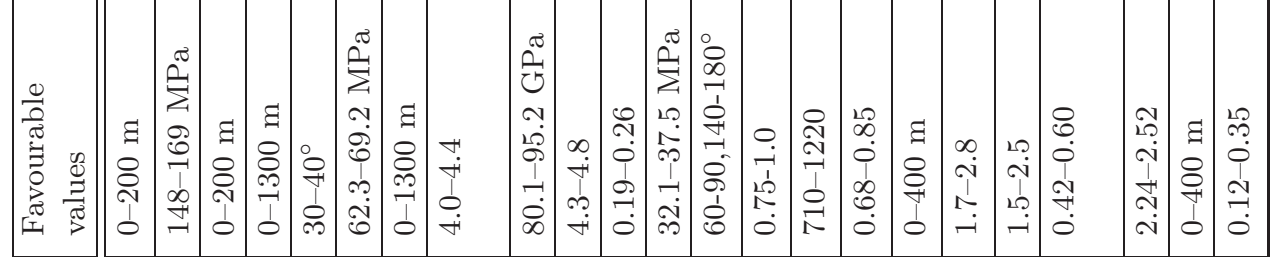

\begin{tabular}{|c|c|c|c|c|c|c|c|c|c|c|c|c|c|c|c|c|c|c|c|c|c|}
\hline & 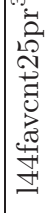 & 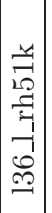 & 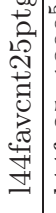 & 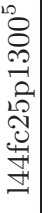 & 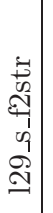 & 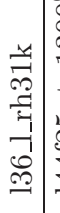 & 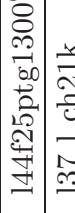 & 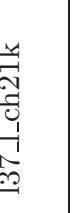 & 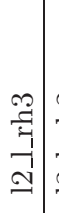 & & 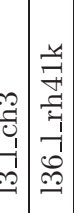 & 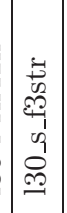 & 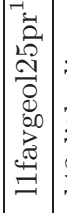 & 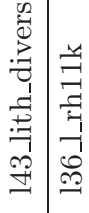 & 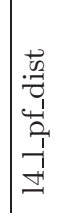 & 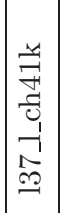 & 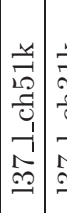 & 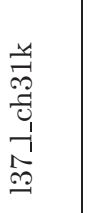 & & 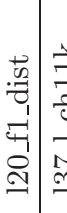 & \\
\hline & 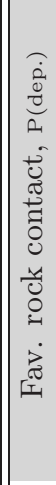 & 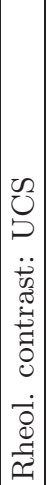 & 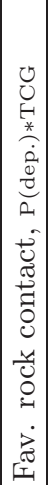 & $\begin{array}{c}0 \\
0 \\
0 \\
0 \\
0 \\
0 \\
0 \\
0 \\
0 \\
0 \\
0 \\
0 \\
0 \\
0\end{array}$ & 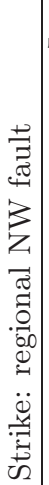 & 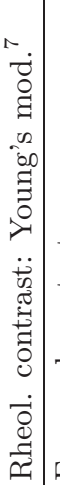 & 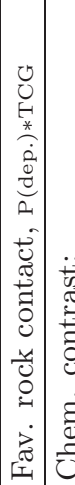 & 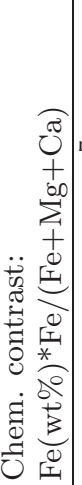 & 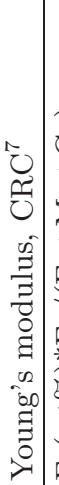 & 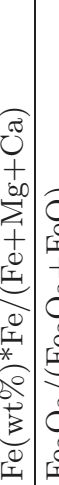 & 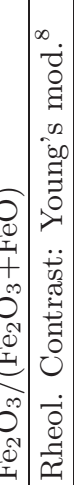 & 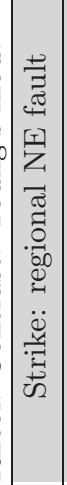 & 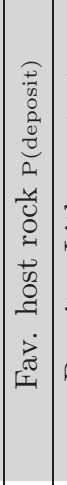 & 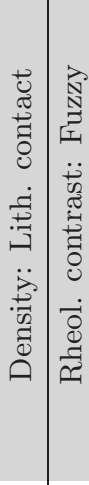 & 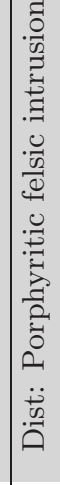 & 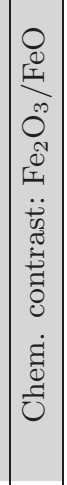 & 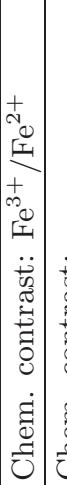 & 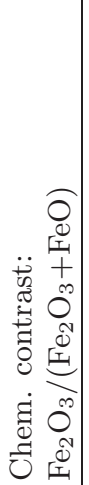 & 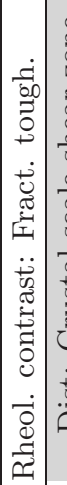 & 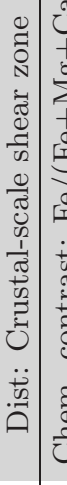 & \\
\hline
\end{tabular}




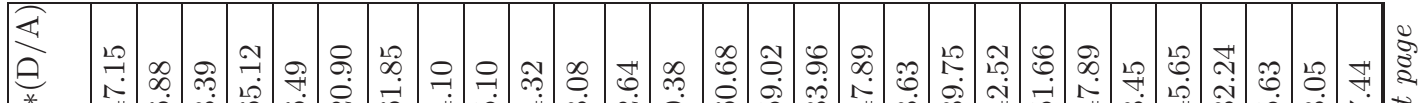

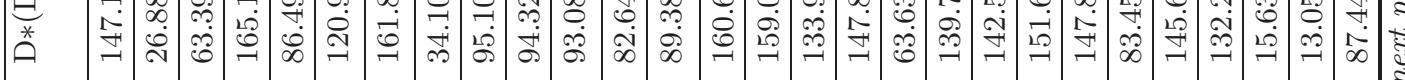

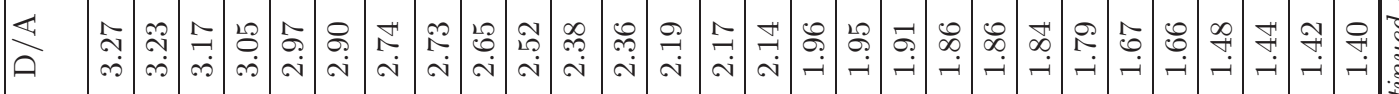

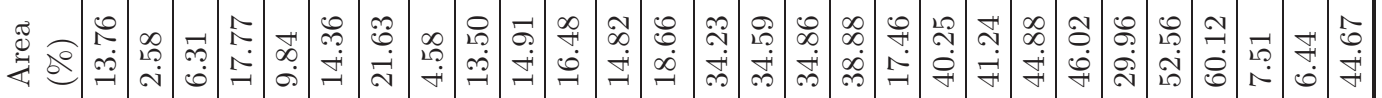

药

嵌

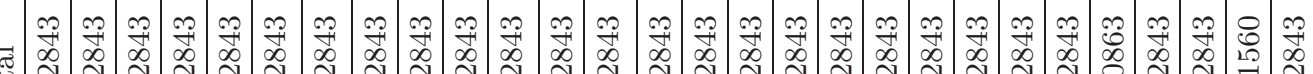

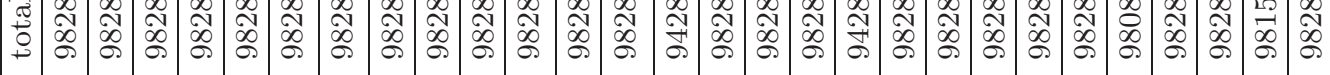

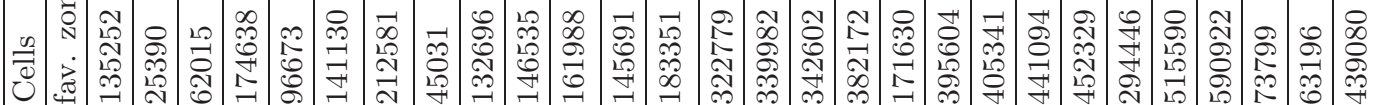

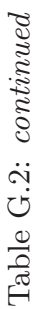

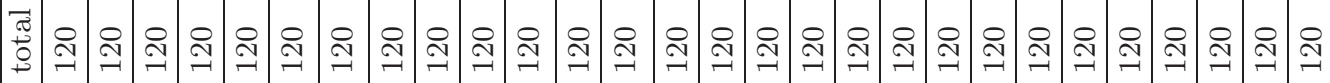

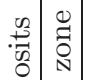

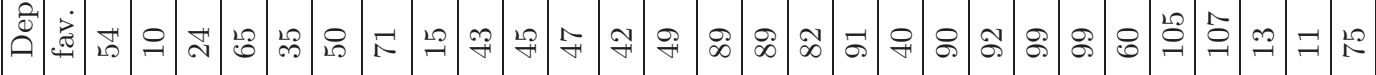

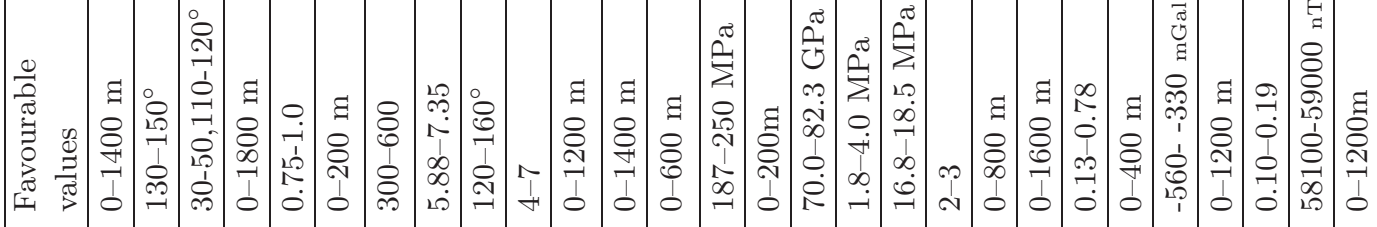

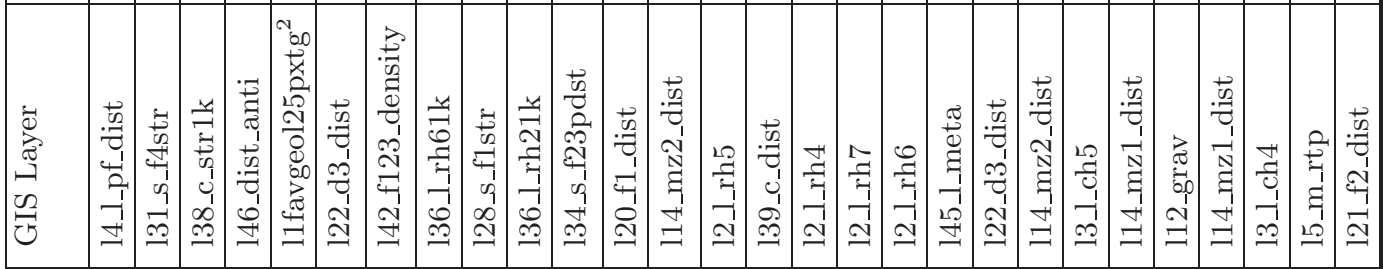

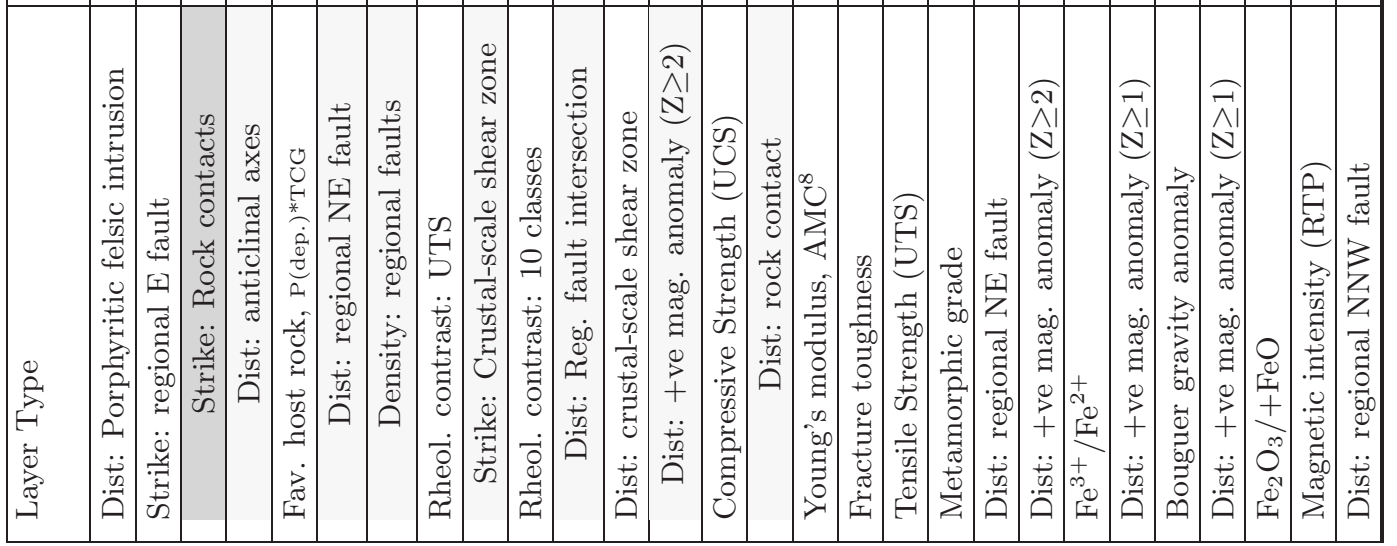



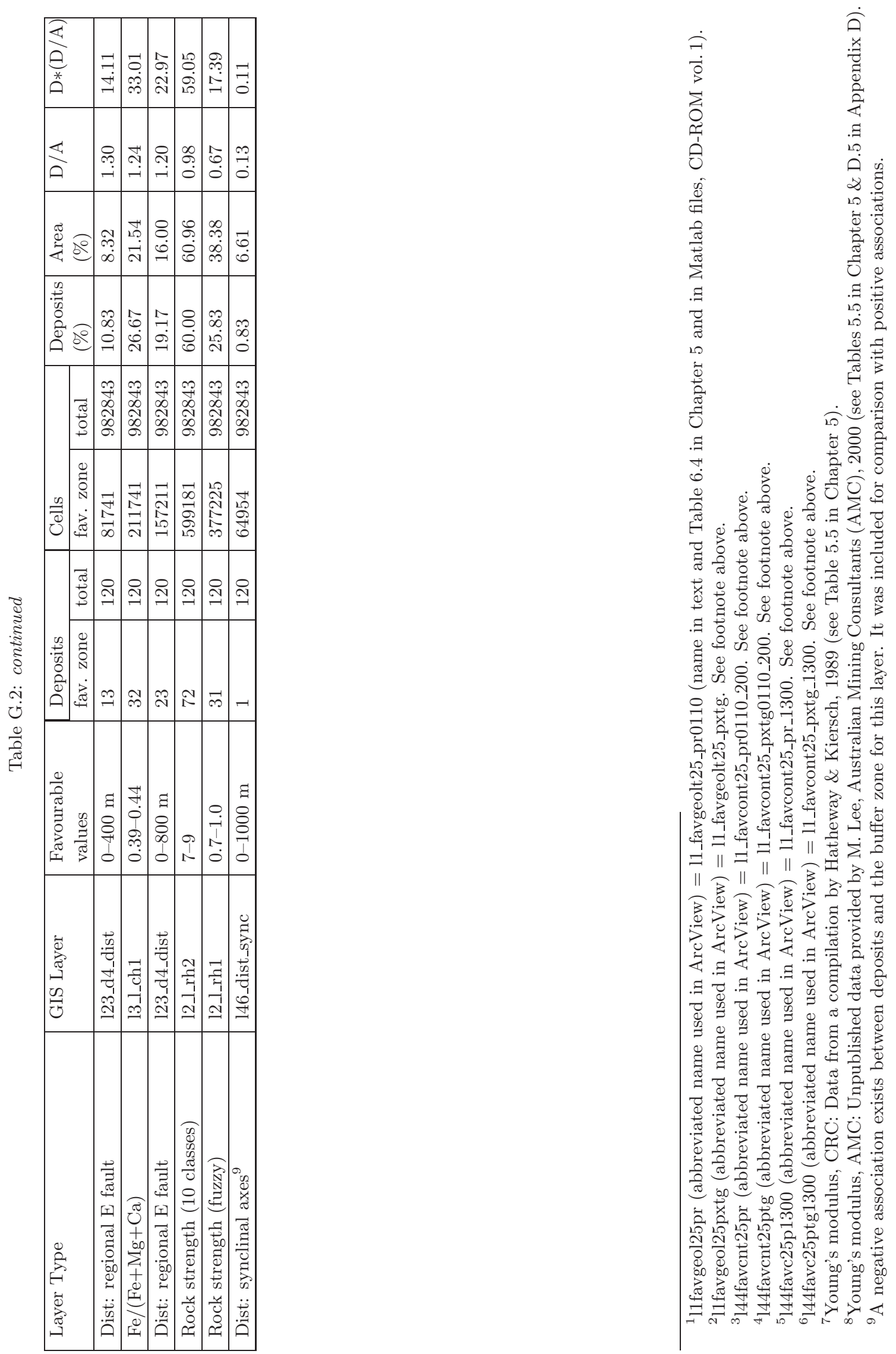


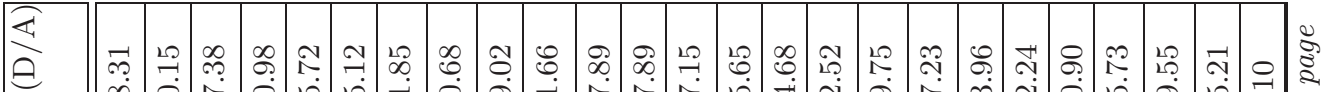

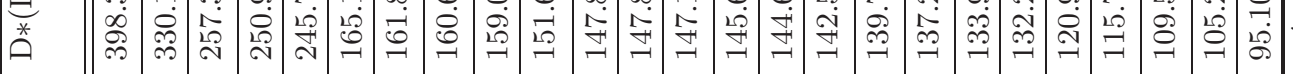

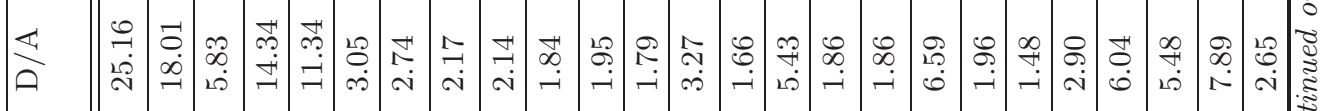

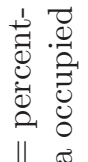

○

离

至要焉

䑸

至

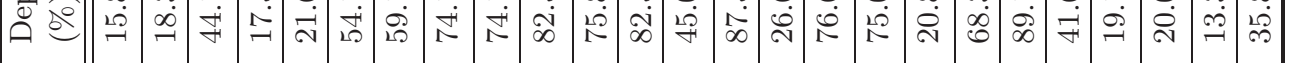

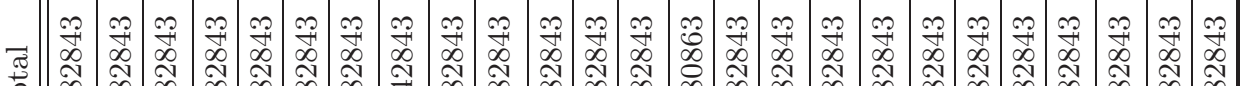

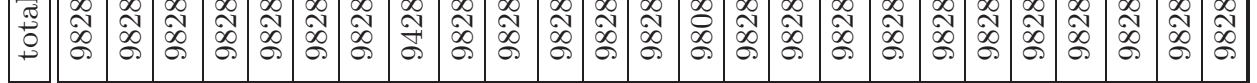

$\hat{\theta} \underset{0}{m}$

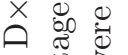

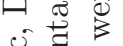

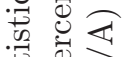

焉

$\exists \leftrightarrow \stackrel{x}{\square}$

bo

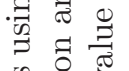

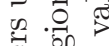

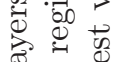

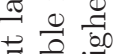

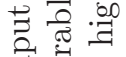

.$\exists \Xi$

忿密

西窟

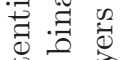

离空

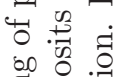

$\because 0$

苟过

范

$\ddot{0}$

نे

용

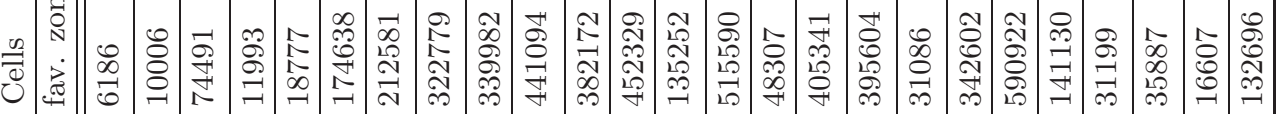

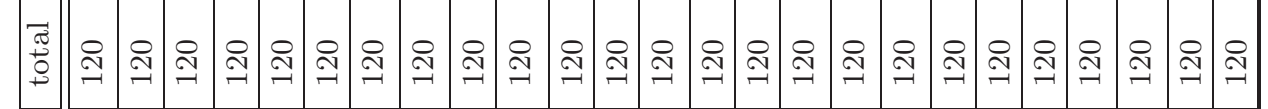

:

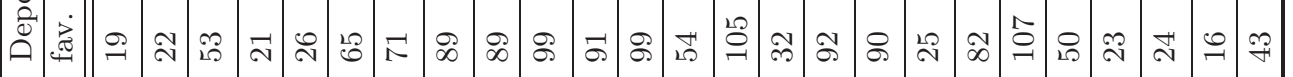

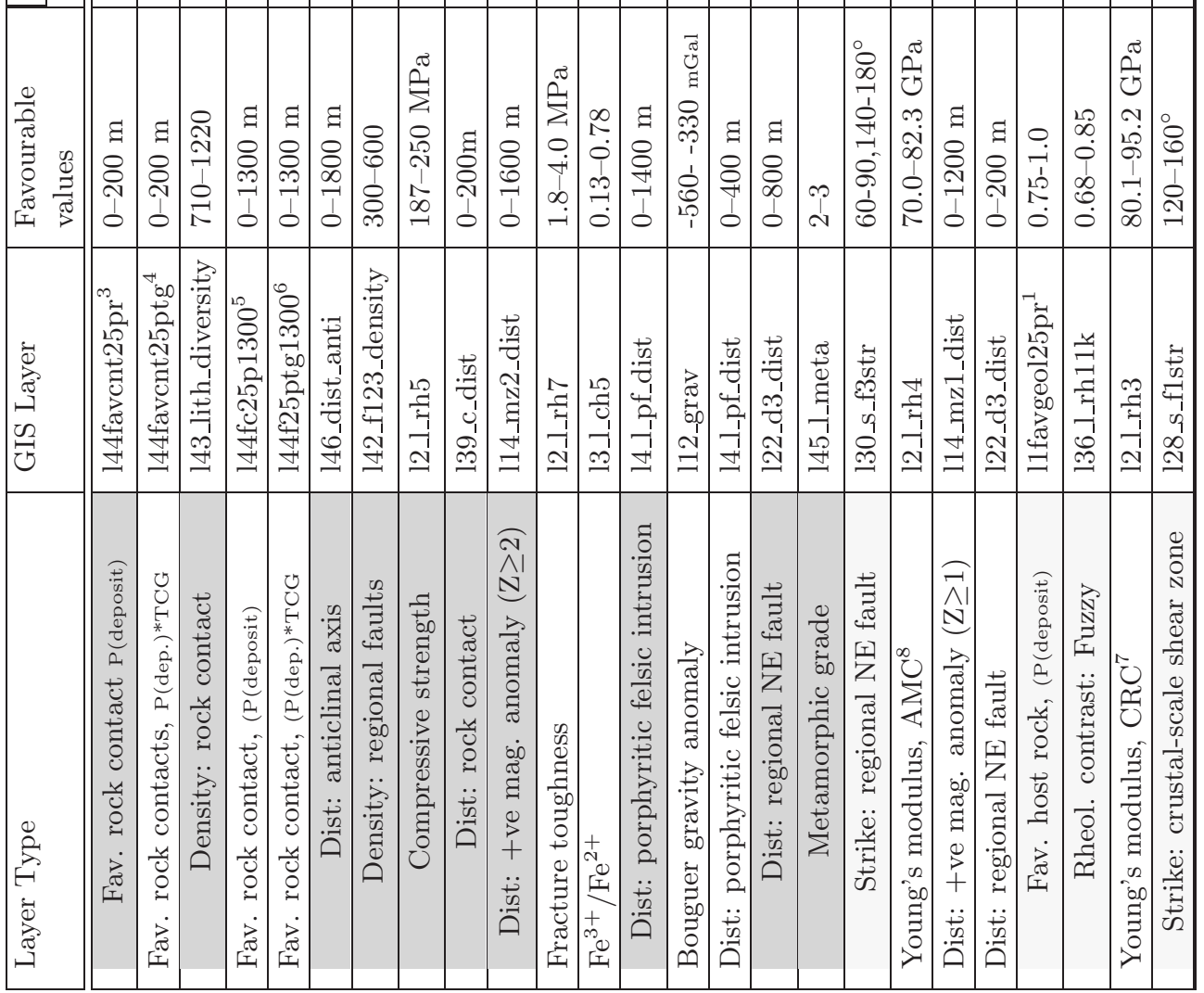




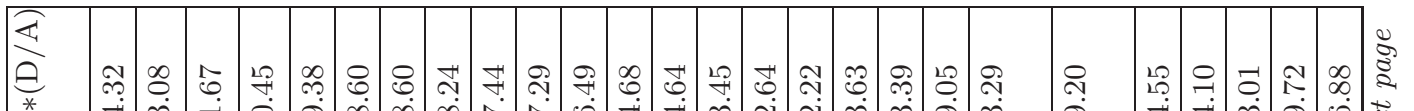

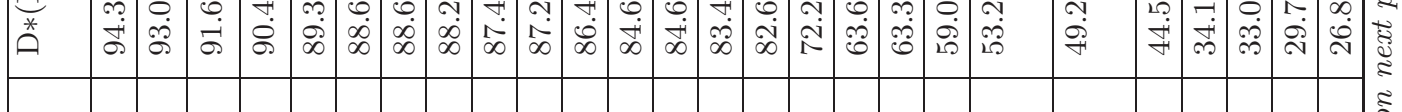

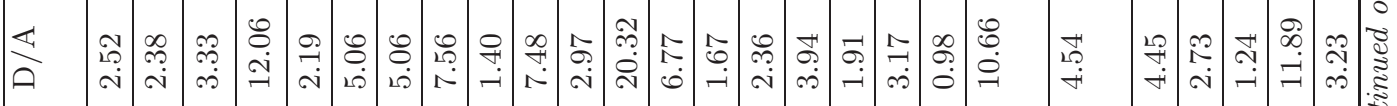

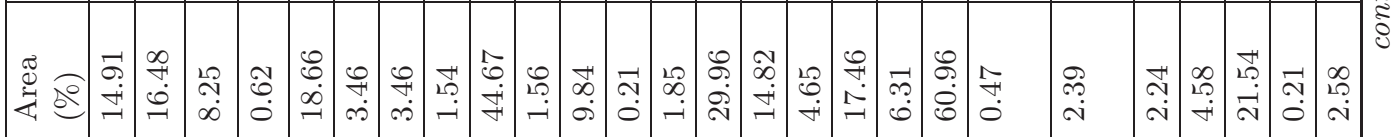

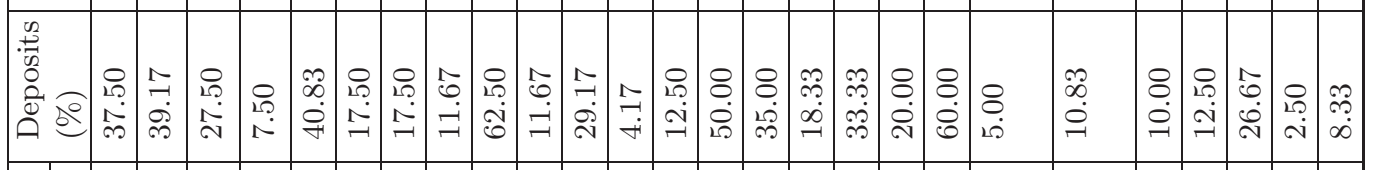

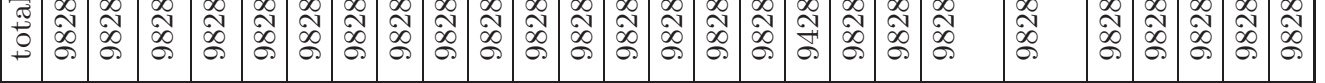

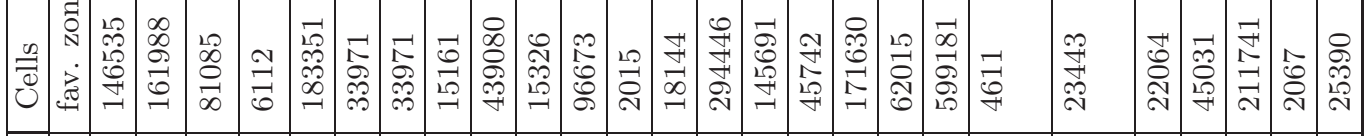

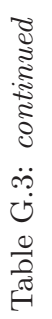

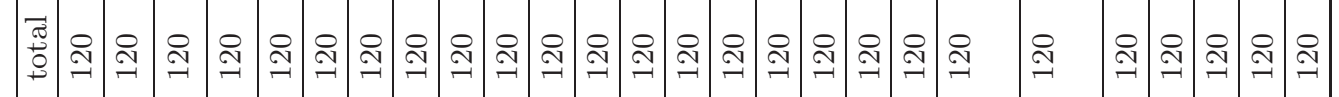

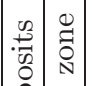

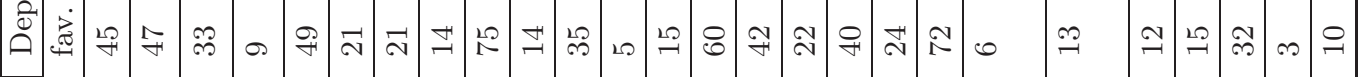

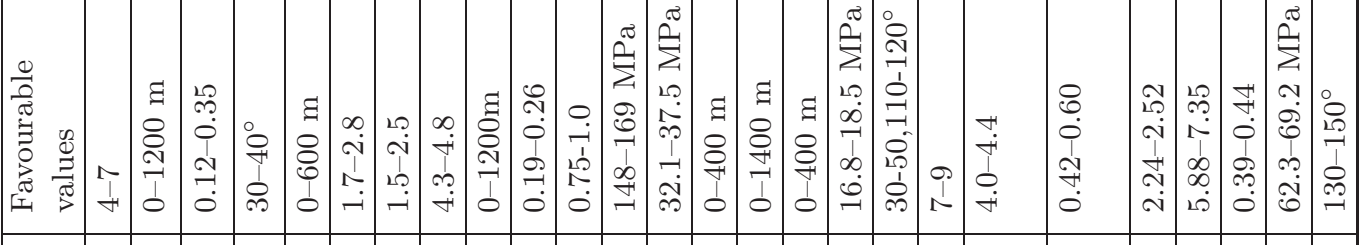

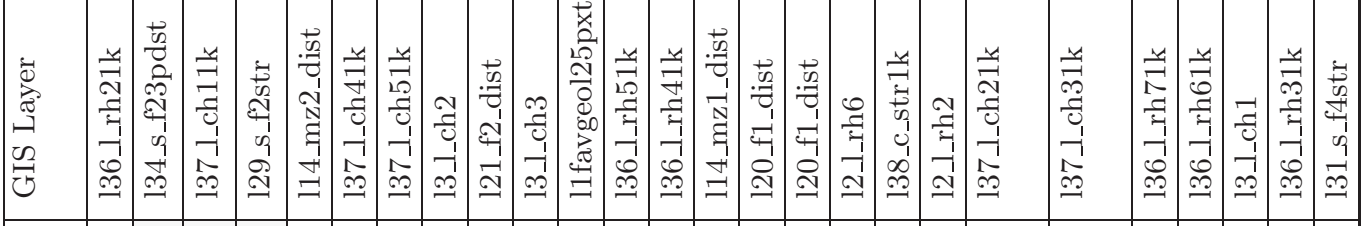

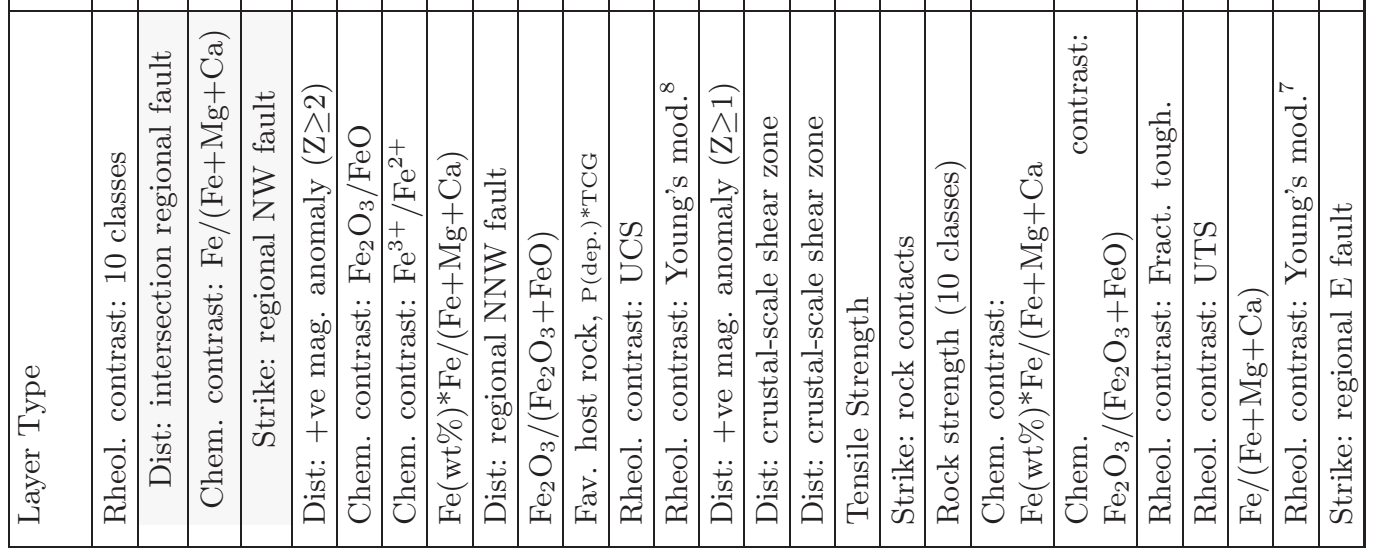



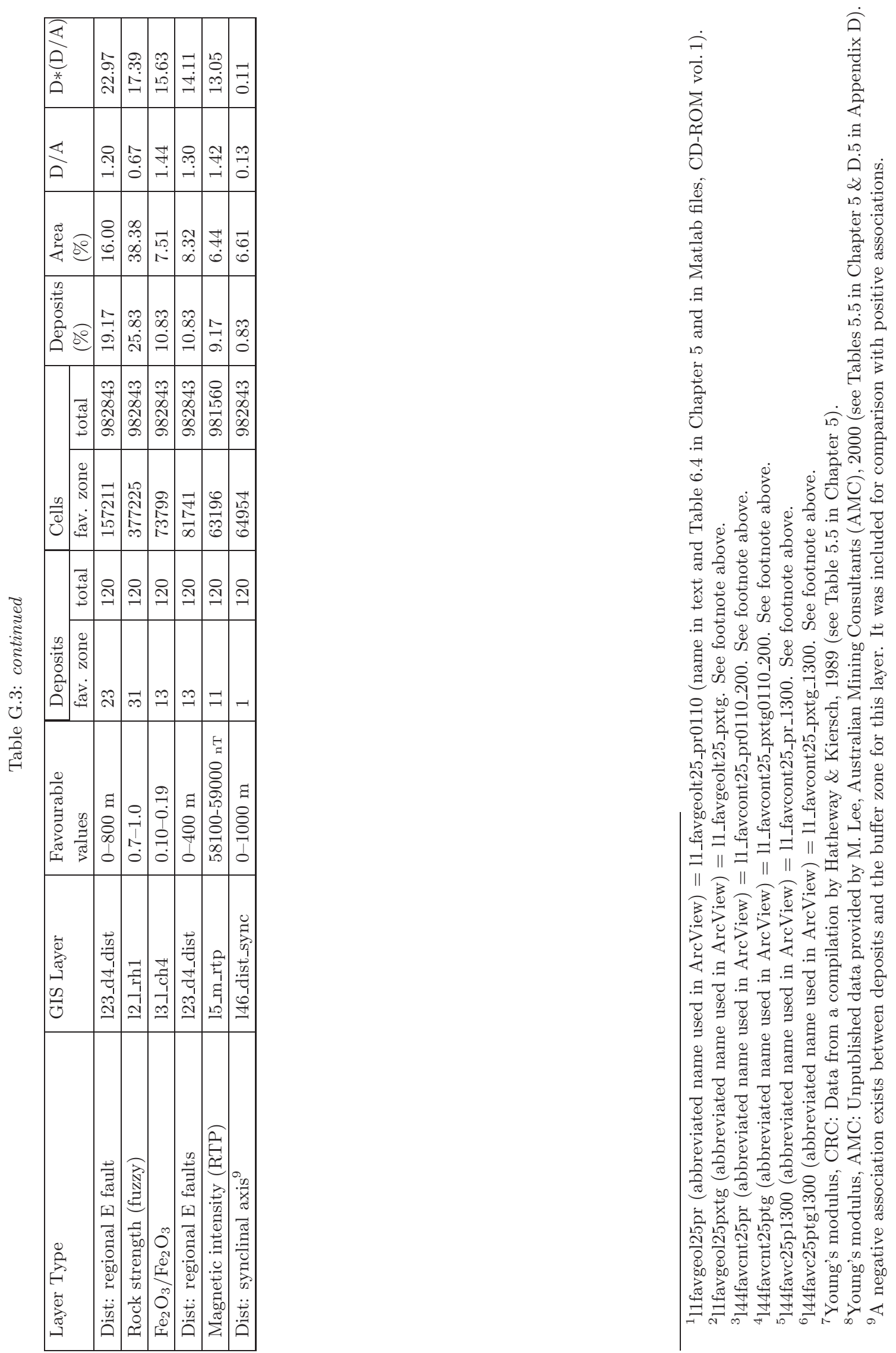


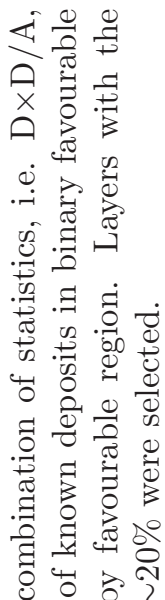
$\approx+0$. : का

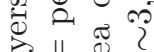
छ $\|$ 号羟 : पू 3 है Uु ज्ञ ते चै 莒里焉文 넝월 <o

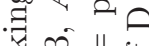
दे

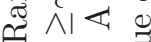
팡 ن் 过

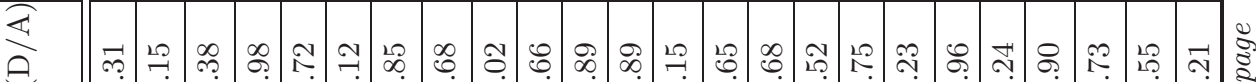

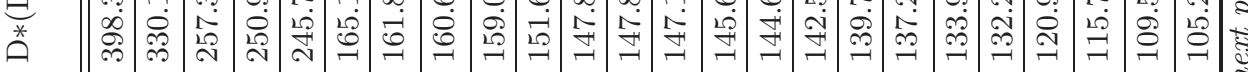

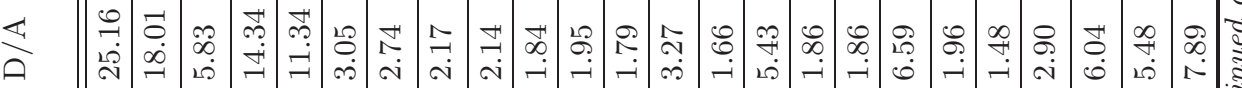

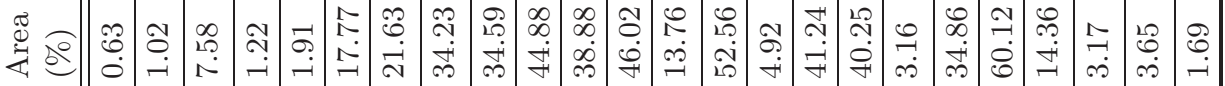

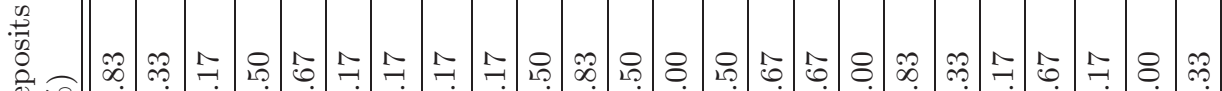

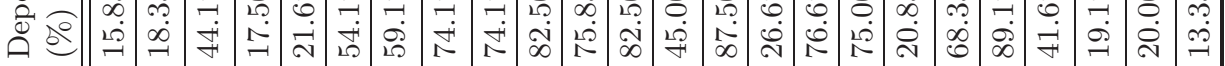

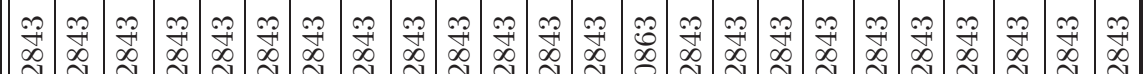

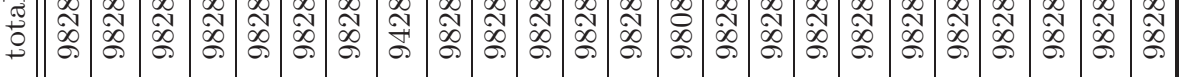

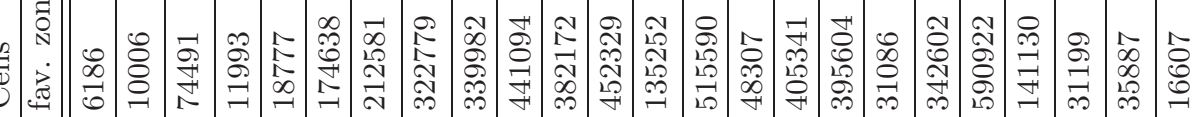

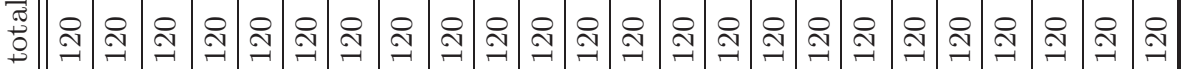

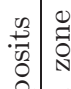

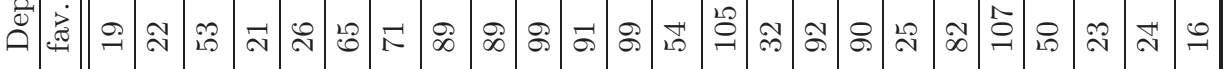

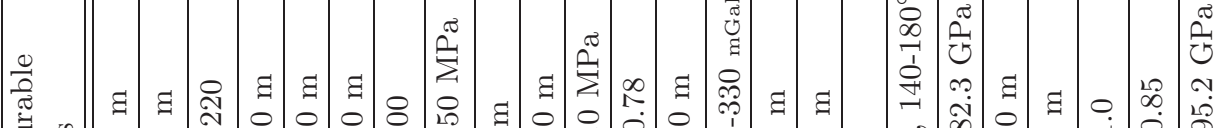

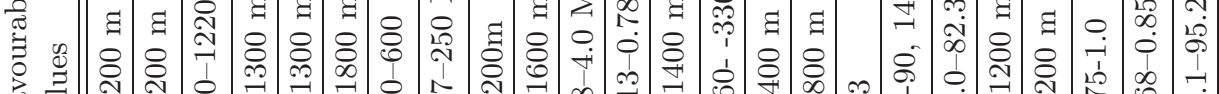

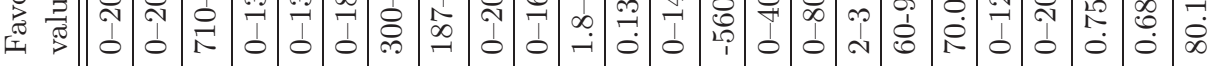

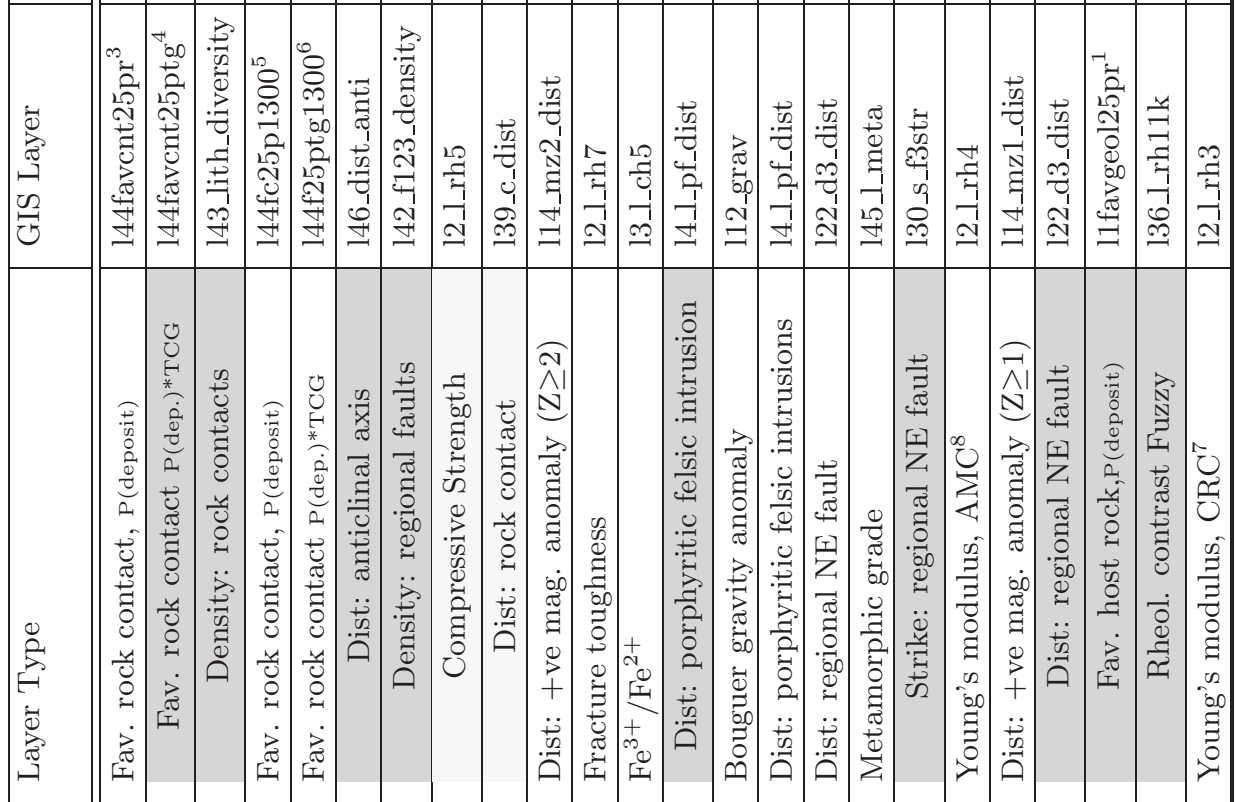




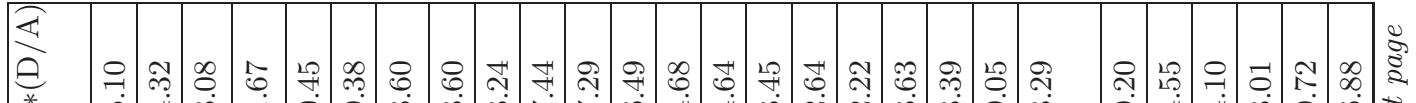

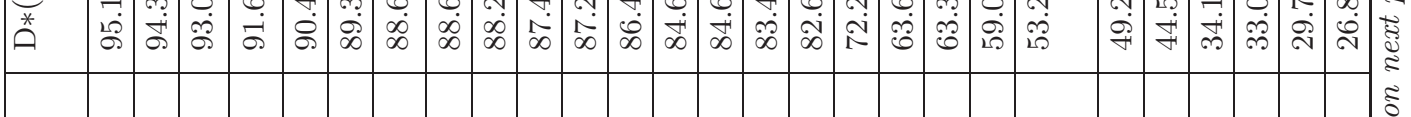

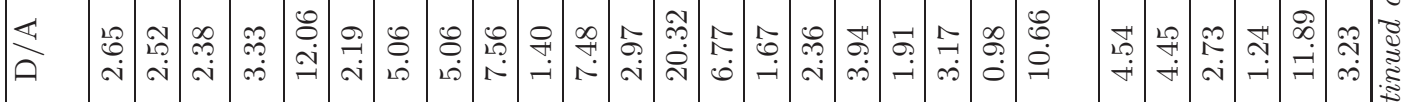

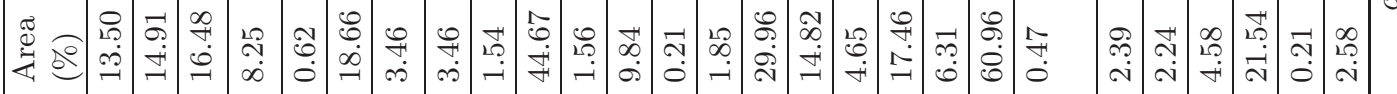

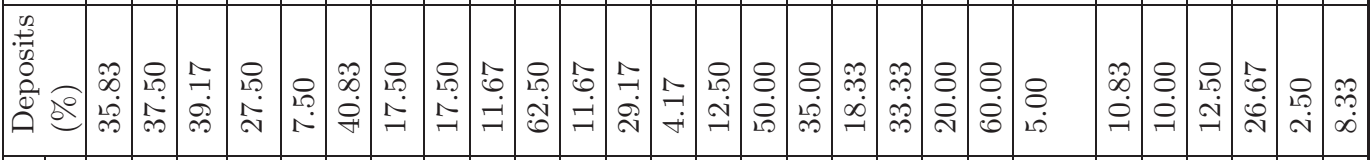

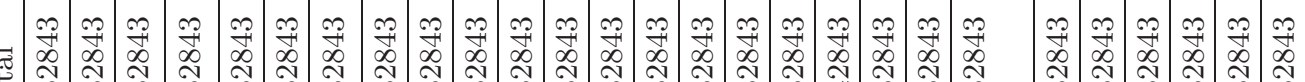

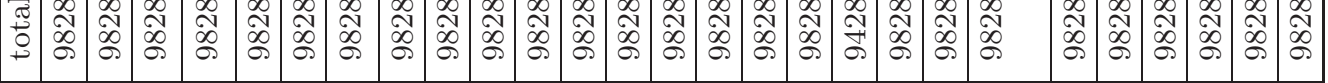

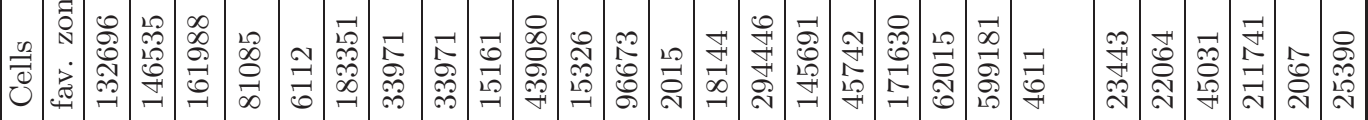

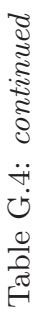

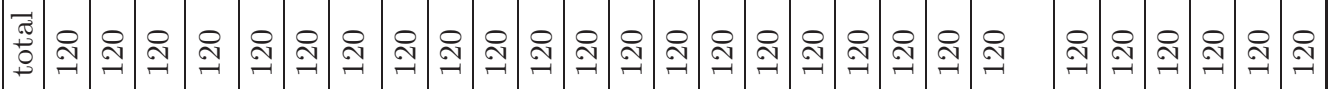

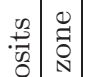

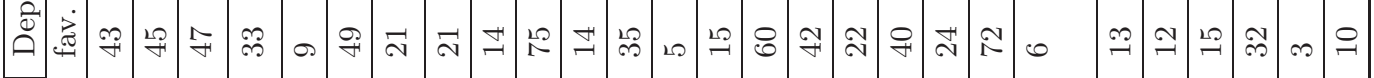

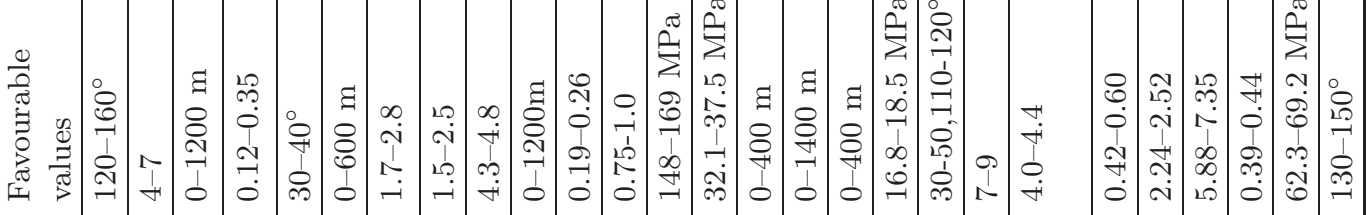

\begin{tabular}{|c|c|c|c|c|c|c|c|c|c|c|c|c|c|c|c|c|c|c|c|c|c|}
\hline & 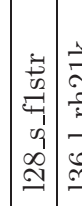 & 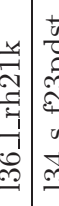 & 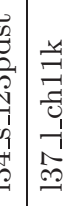 & 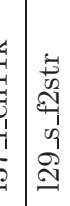 & 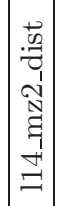 & 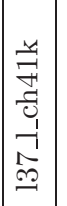 & 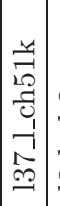 & 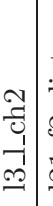 & 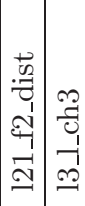 & 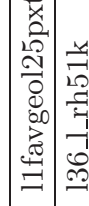 & 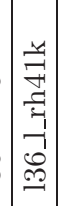 & 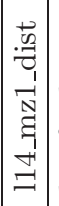 & 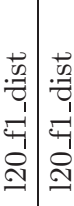 & 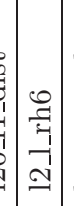 & 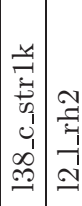 & 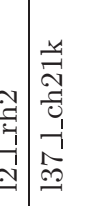 & 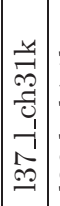 & 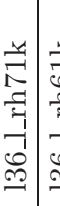 & 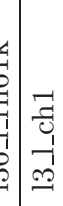 & & \\
\hline & 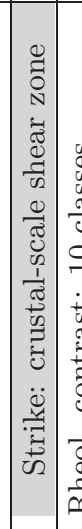 & 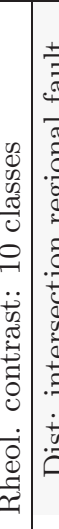 & 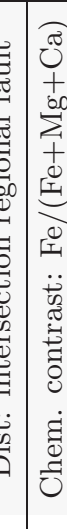 & 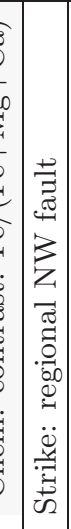 & 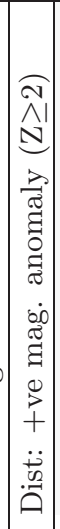 & 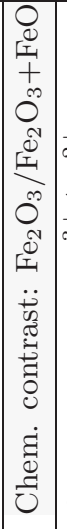 & 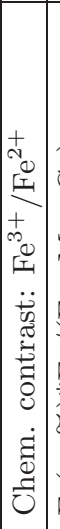 & 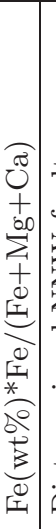 & 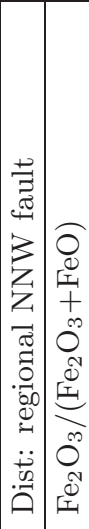 & 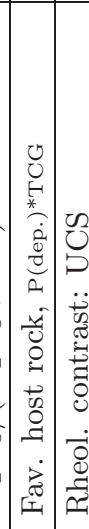 & 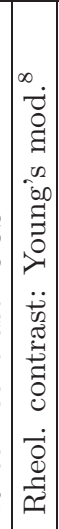 & 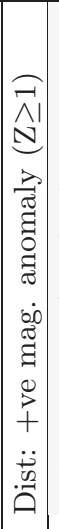 & 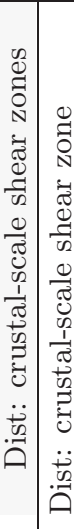 & 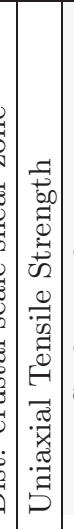 & 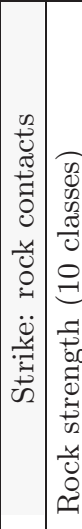 & 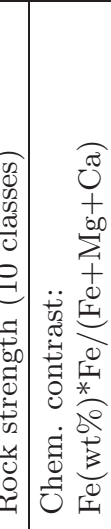 & 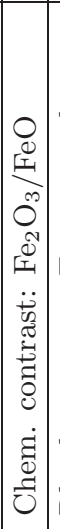 & 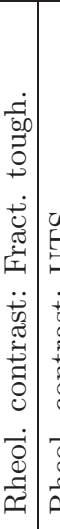 & 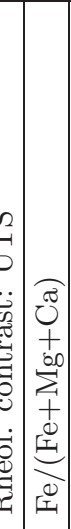 & 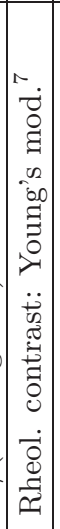 & \\
\hline
\end{tabular}



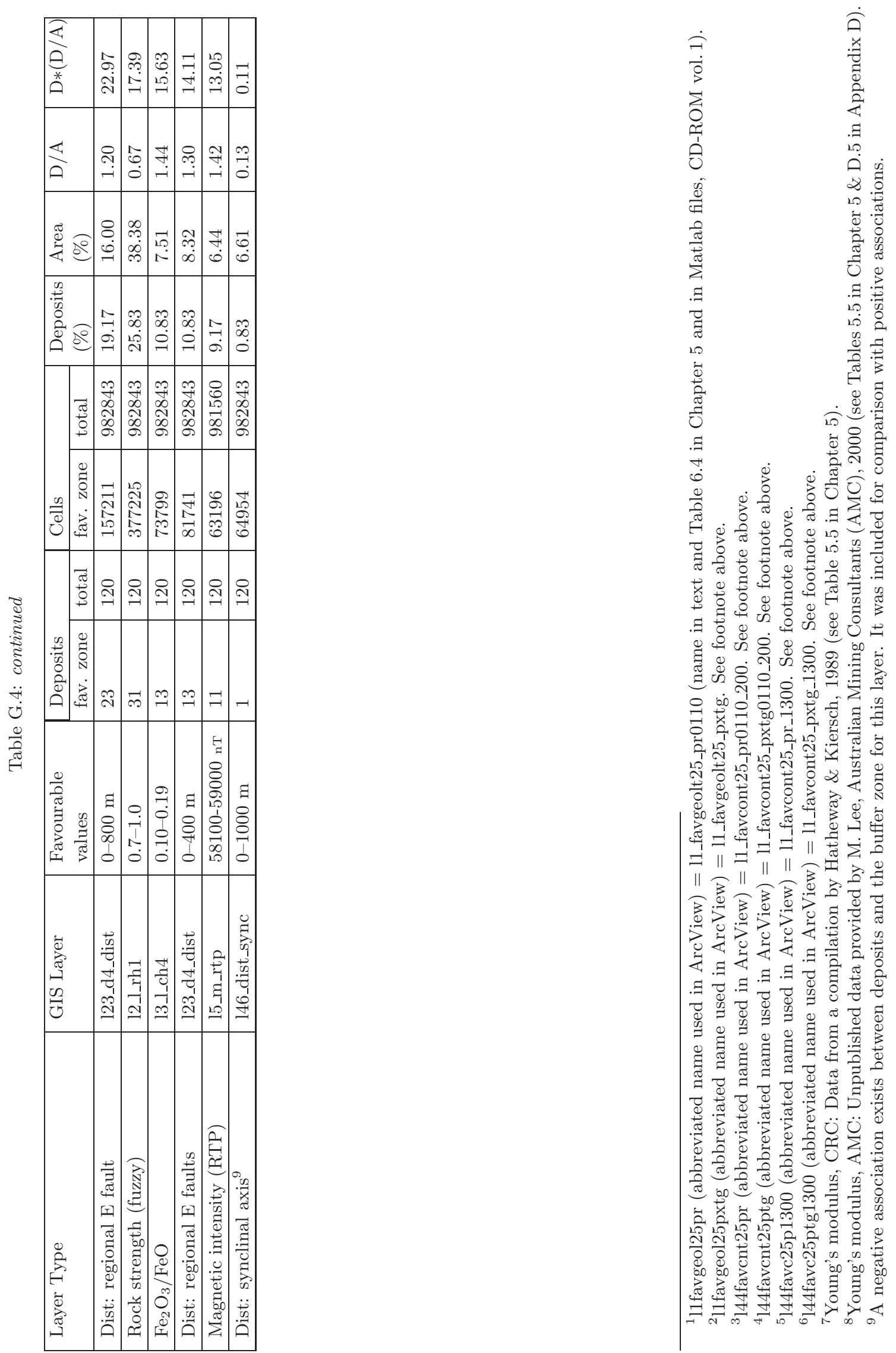
Appendix $\mathbf{H}$

Results of Neural Network

Experiments: Kalgoorlie Case Study 

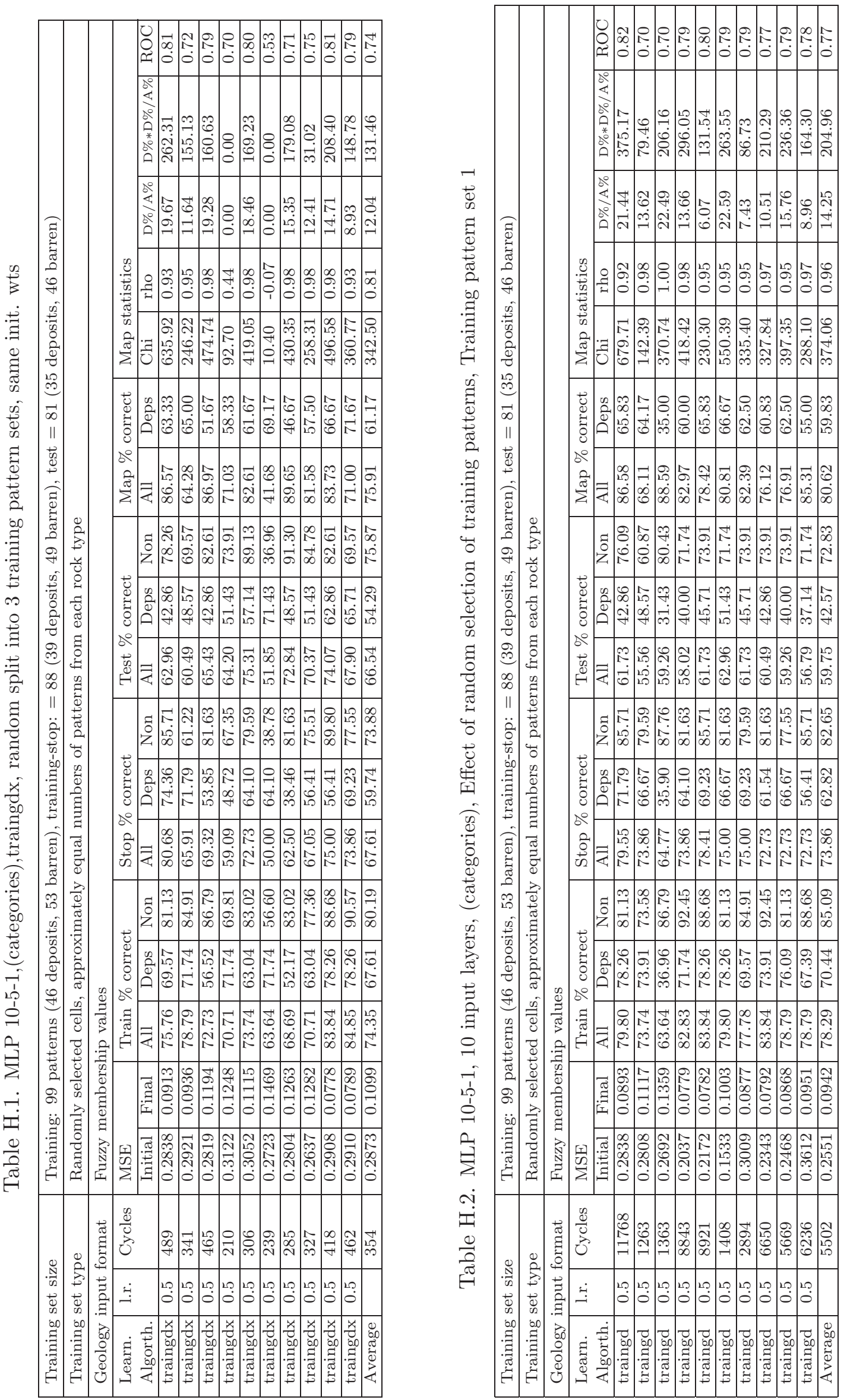

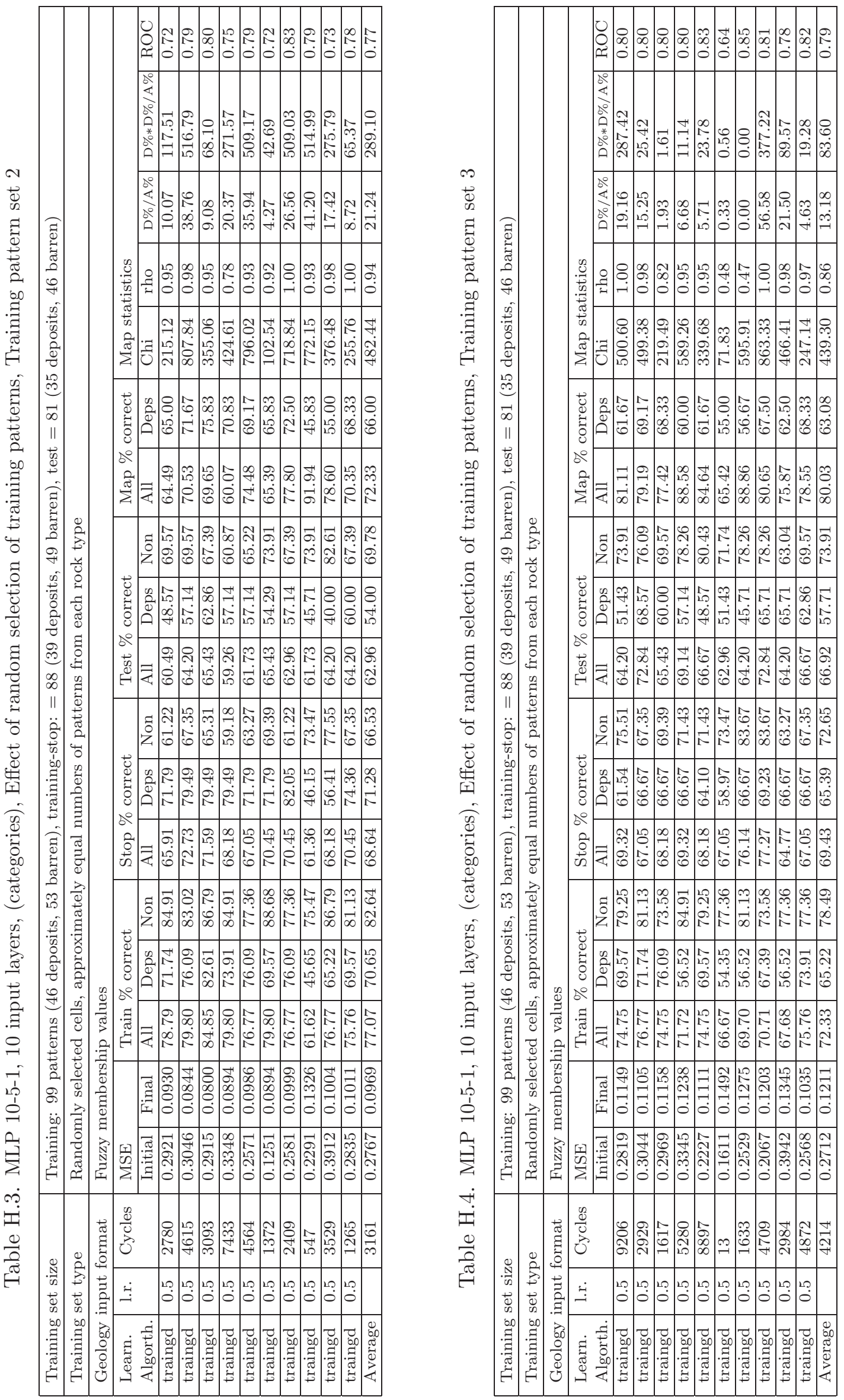

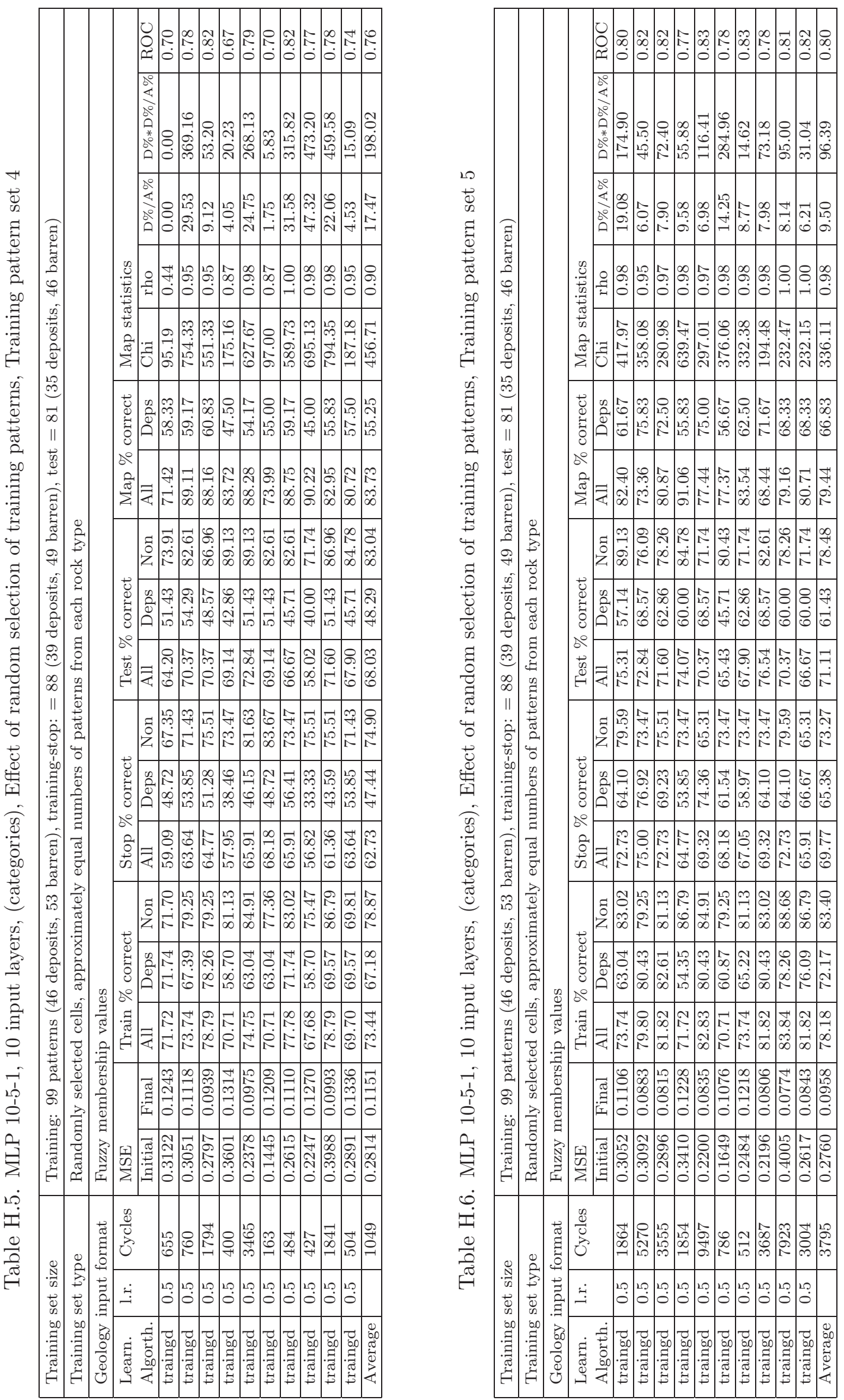

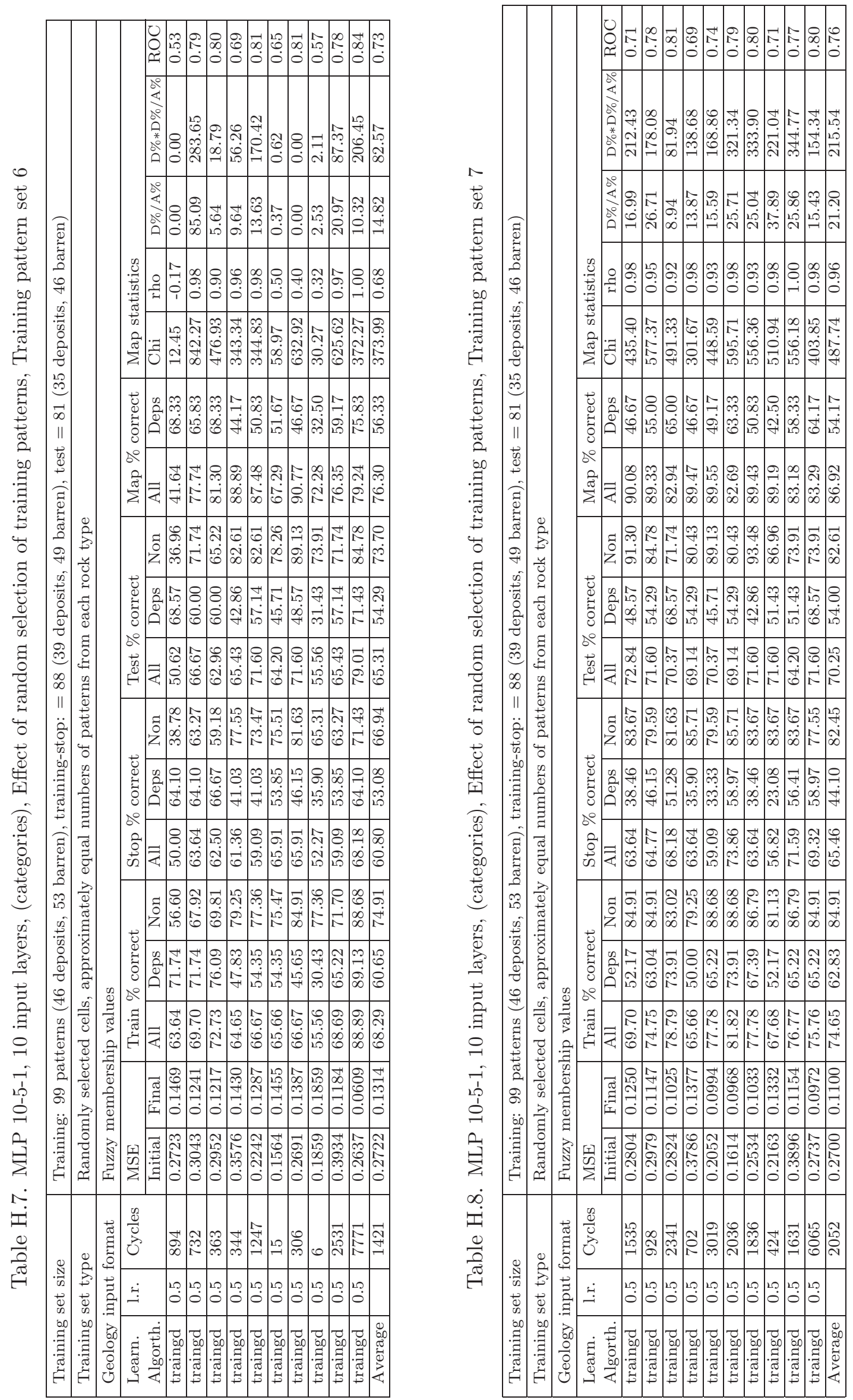

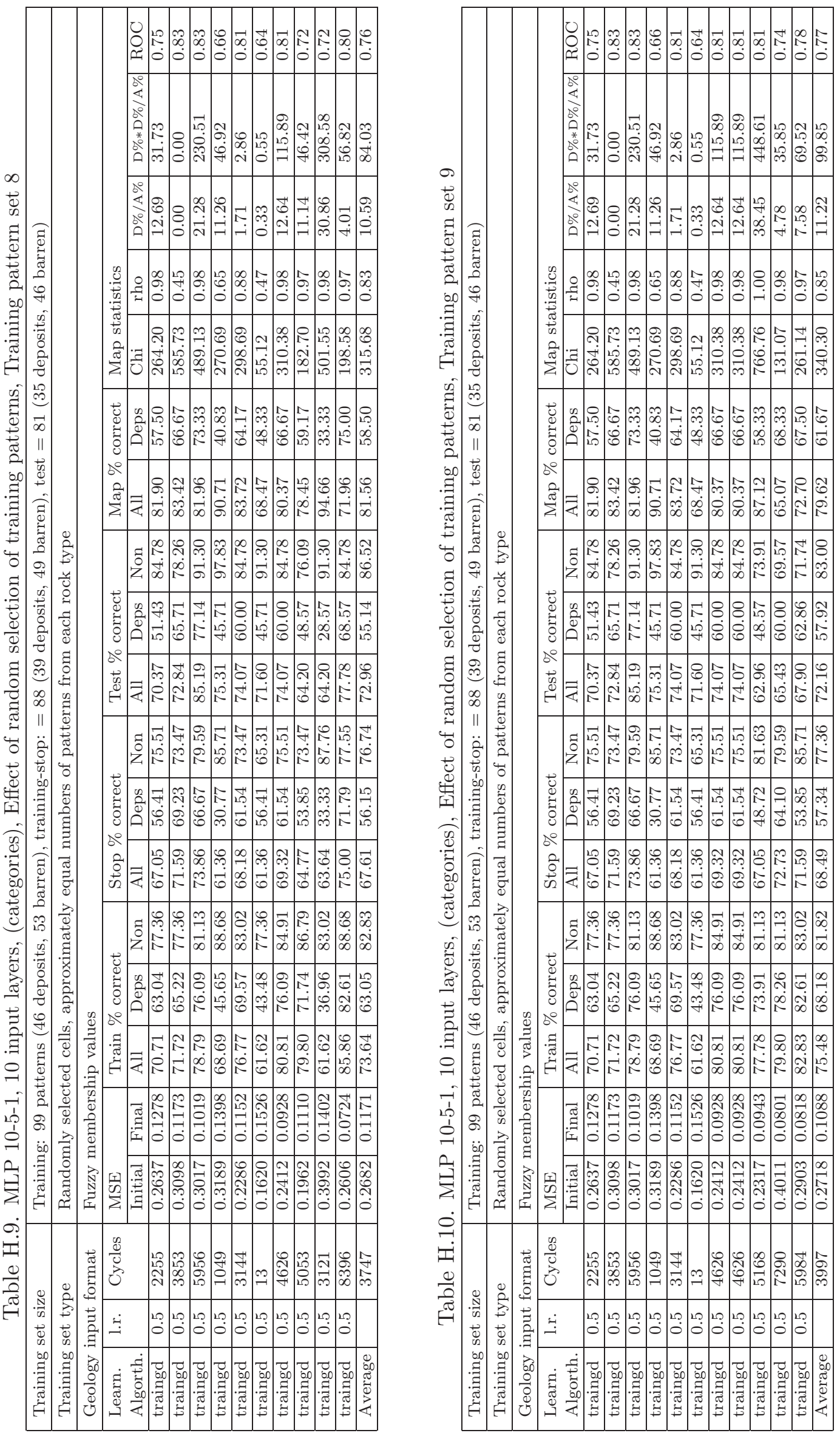

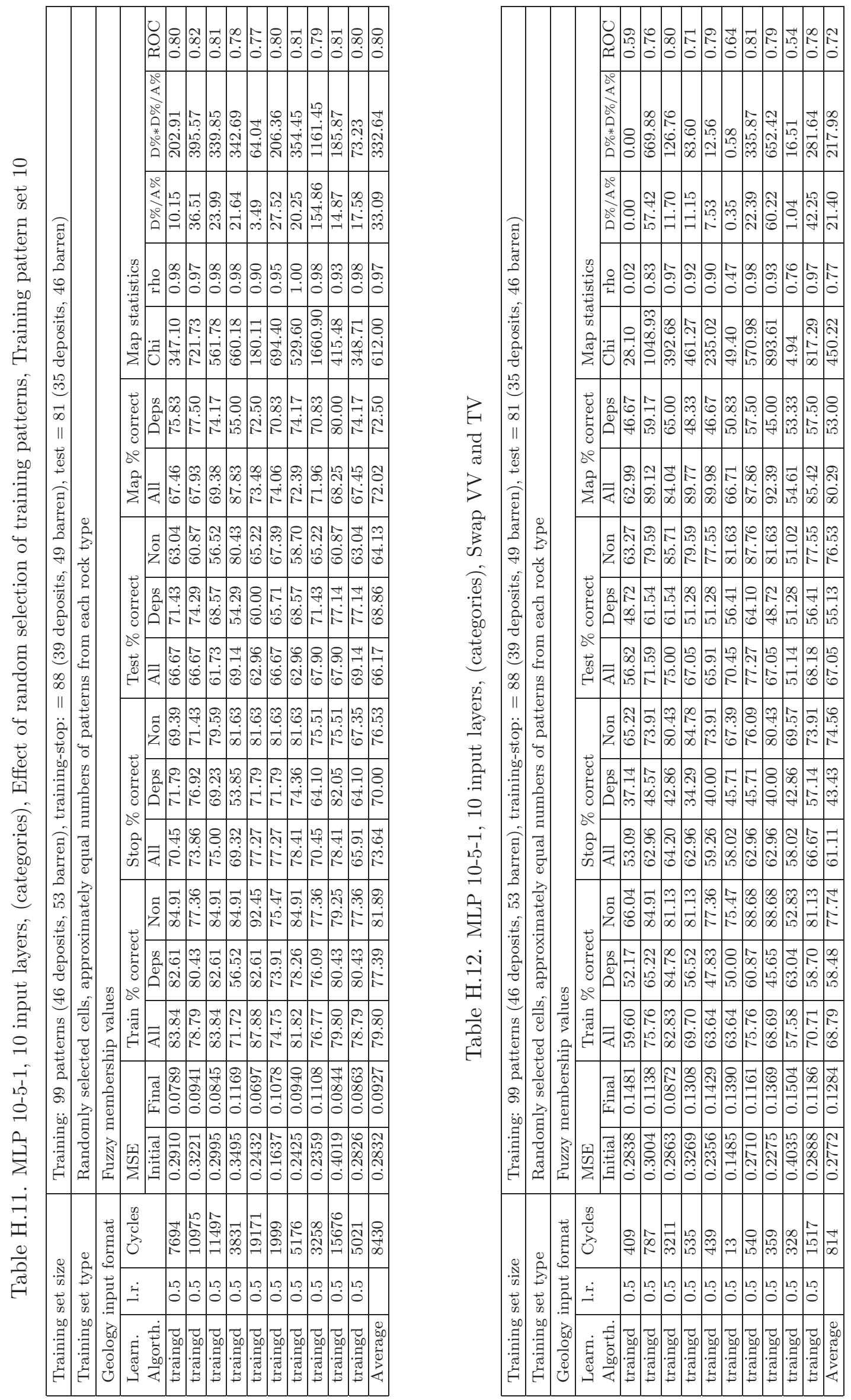
730

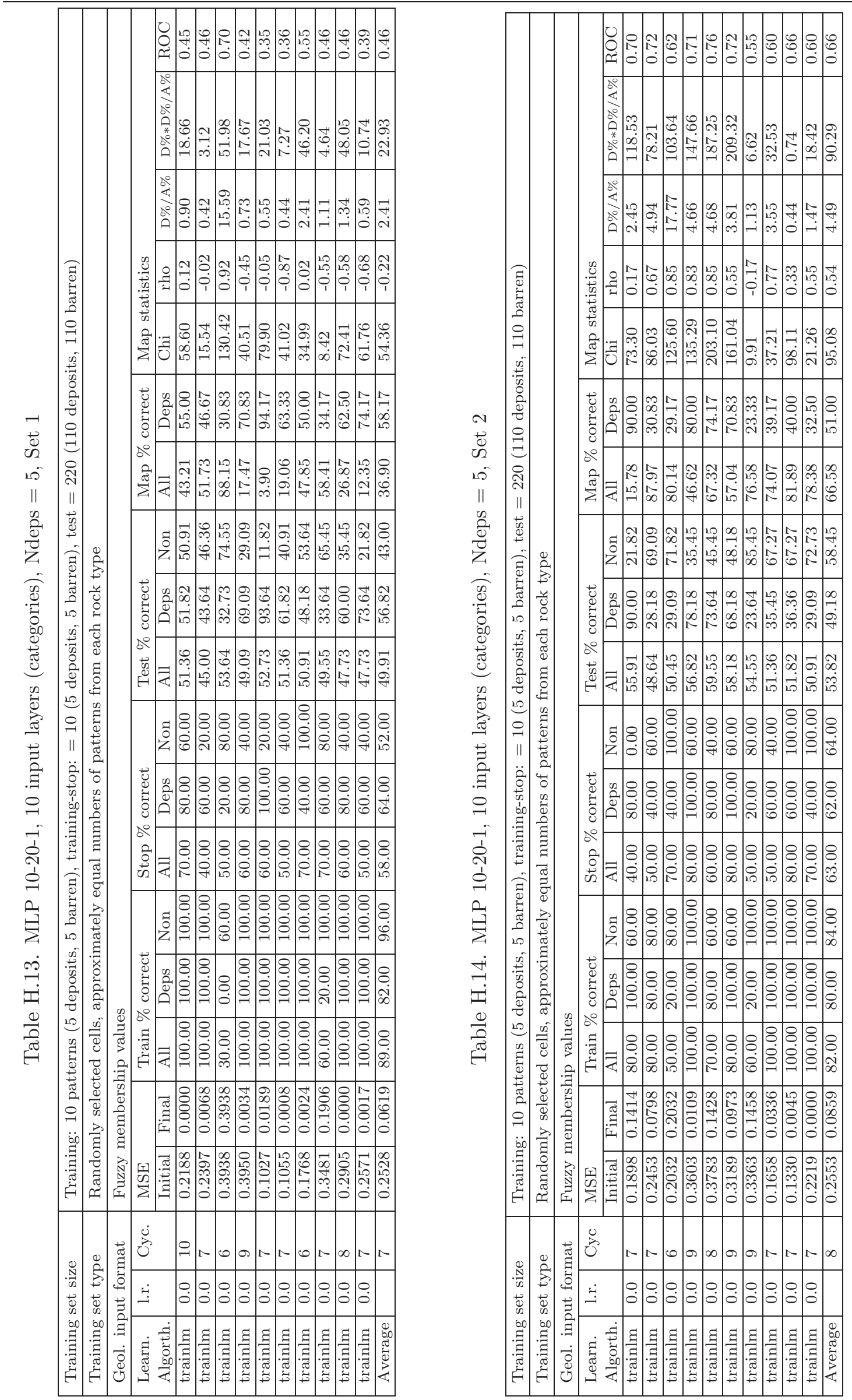



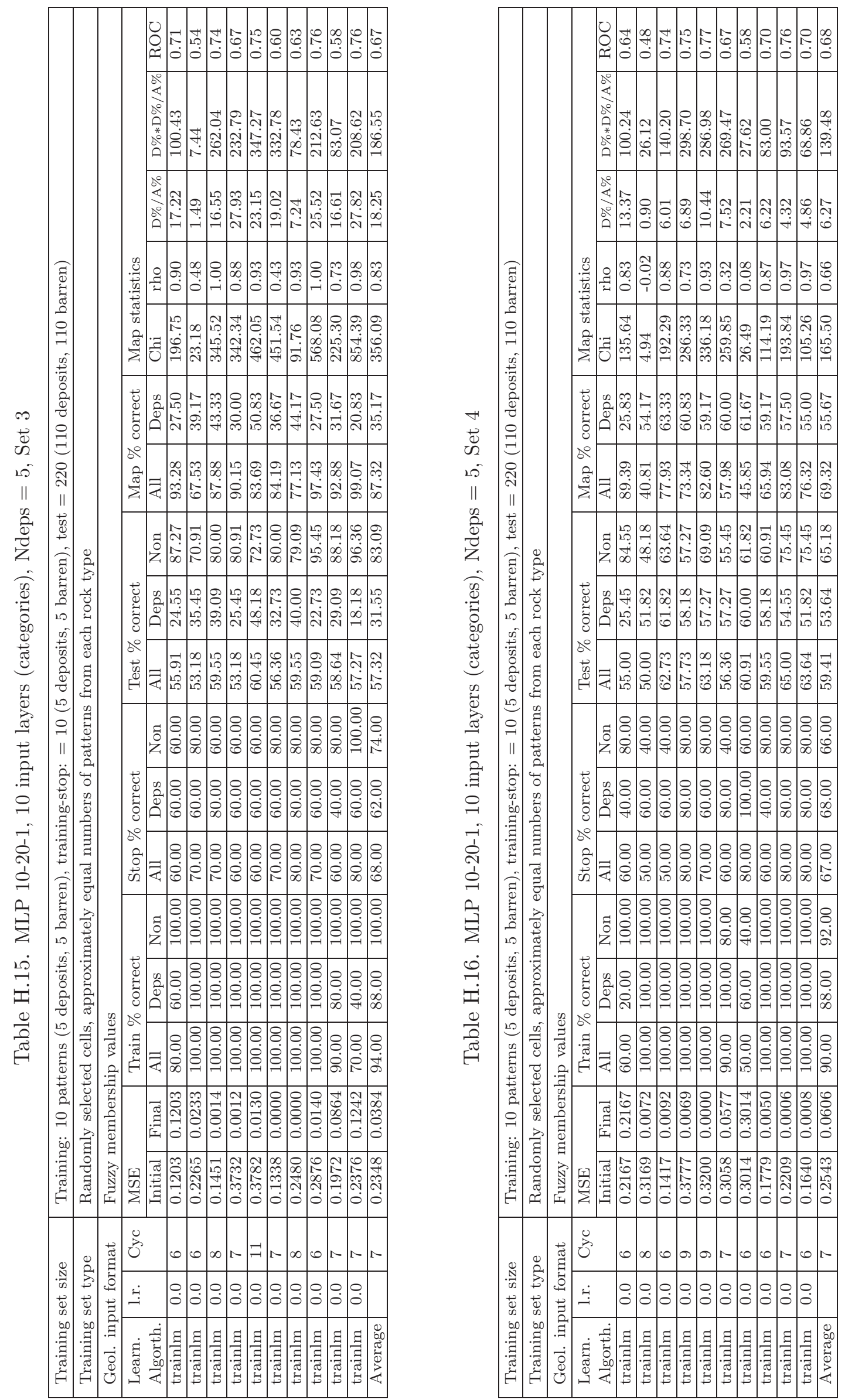

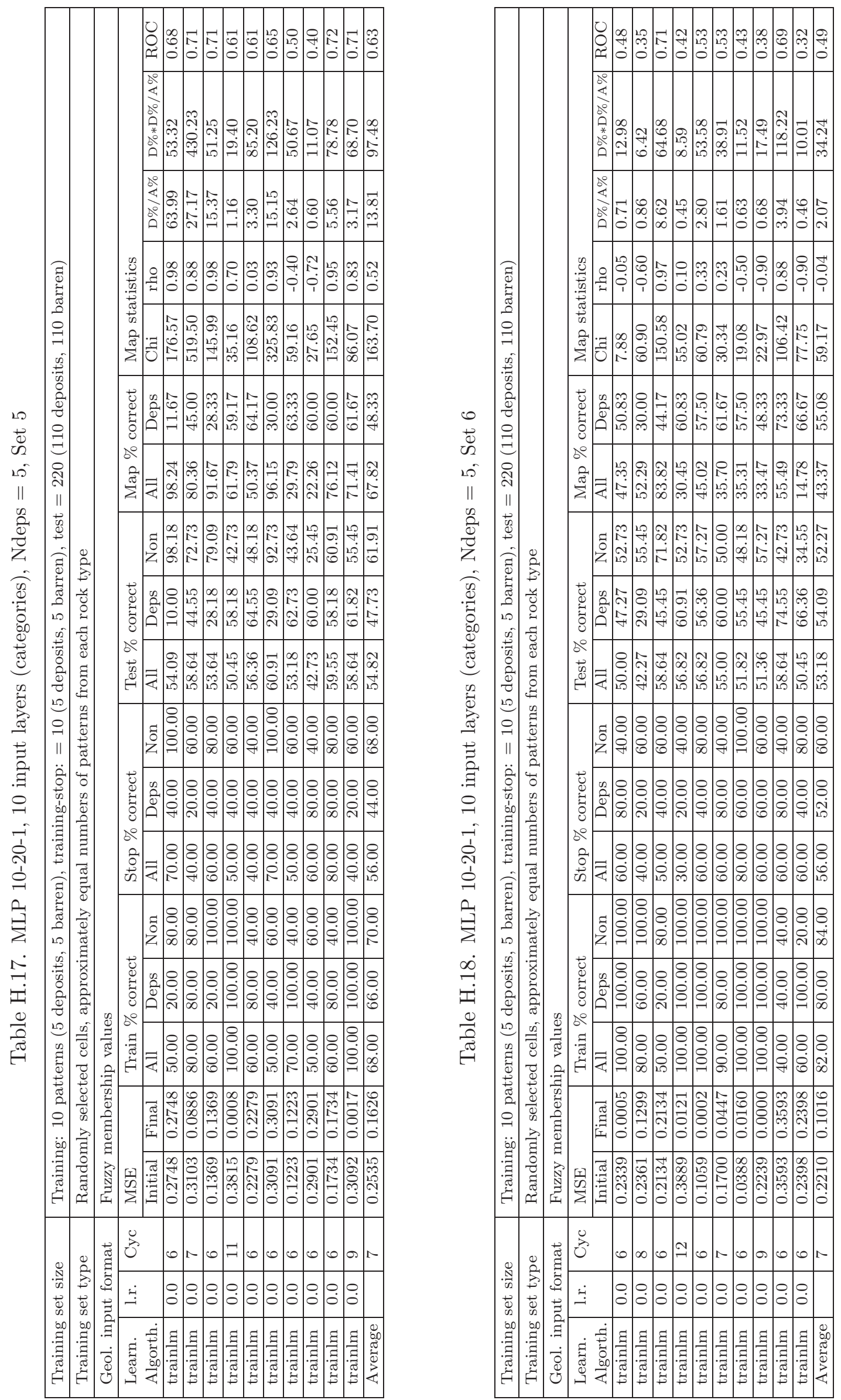


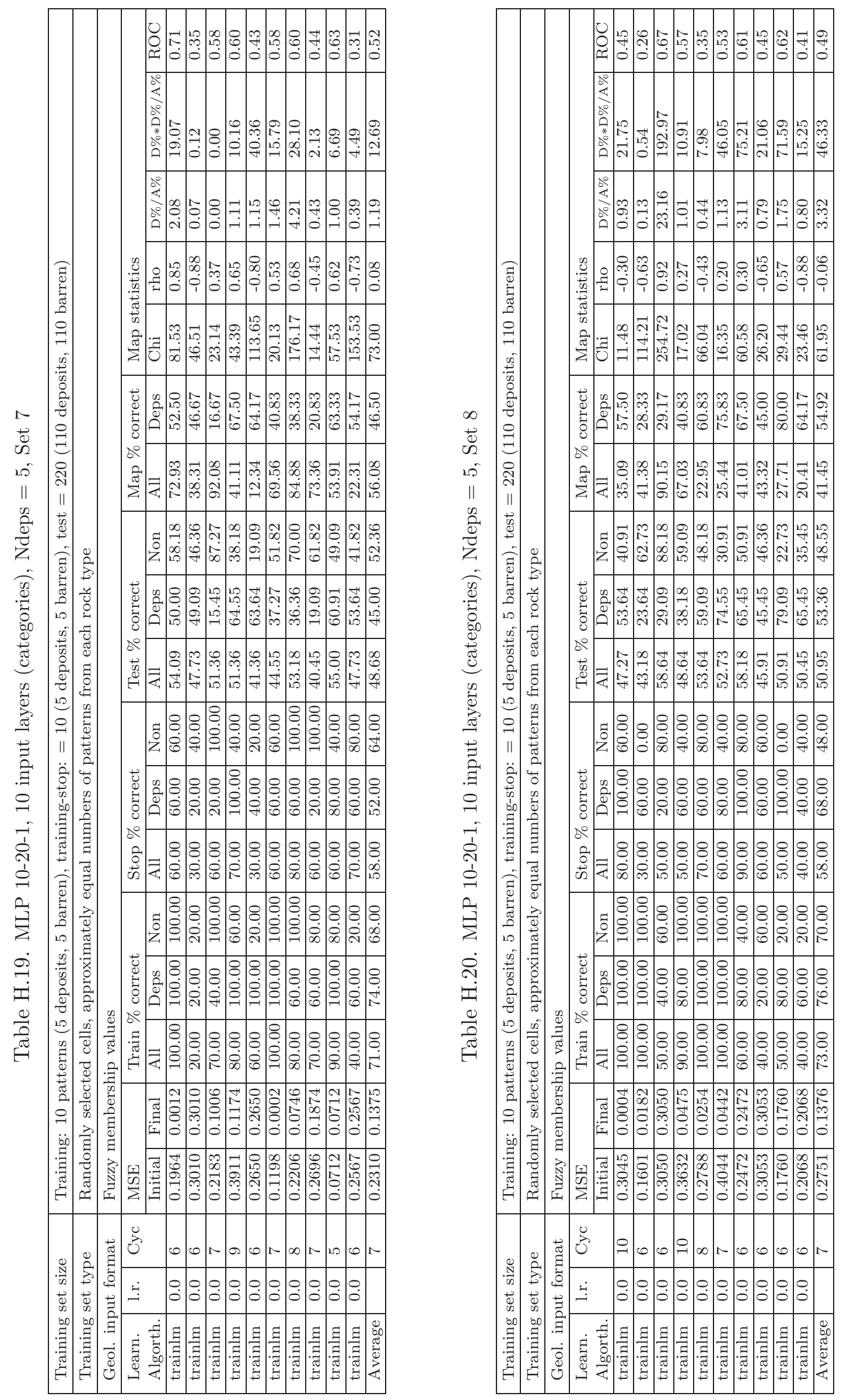




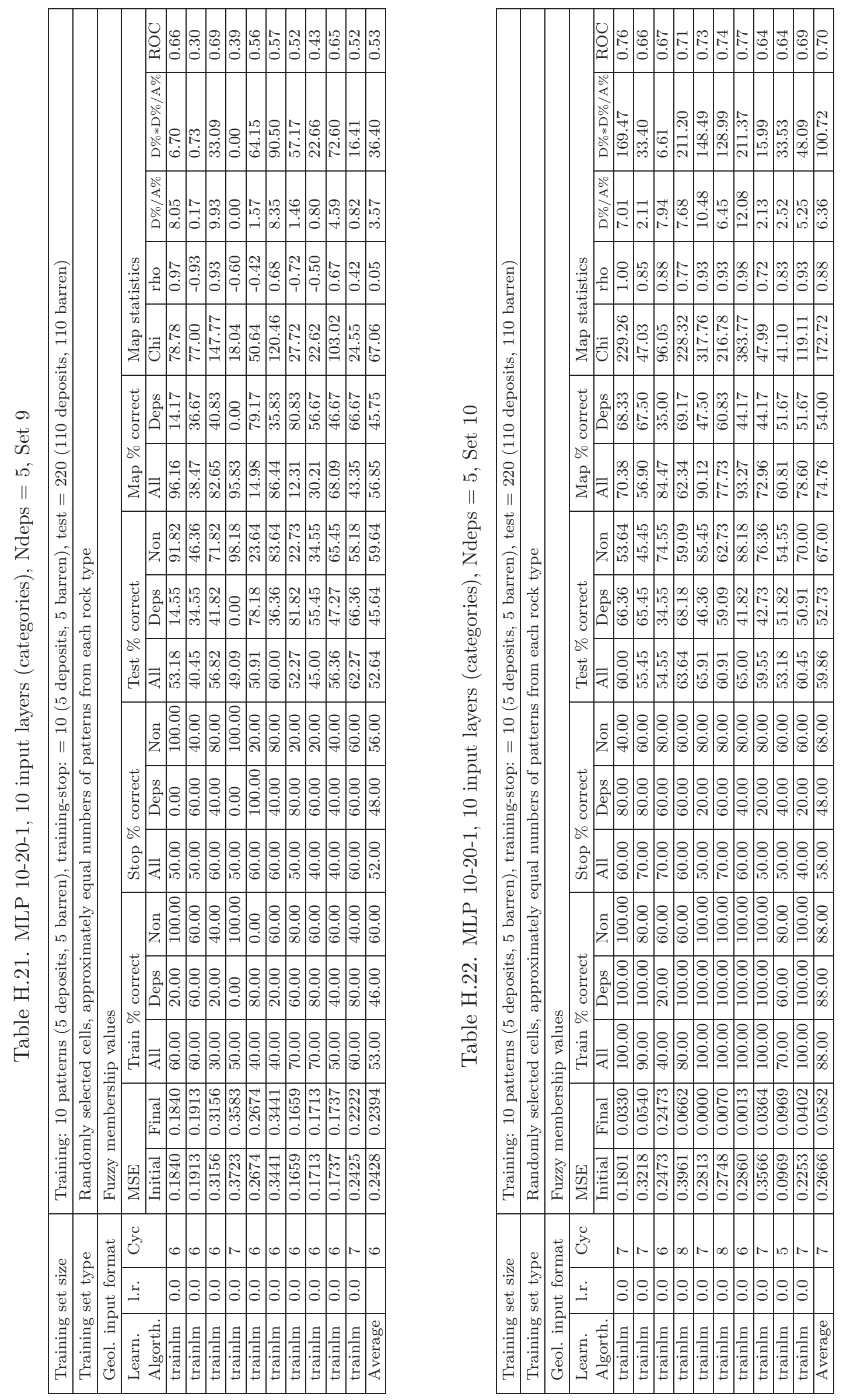



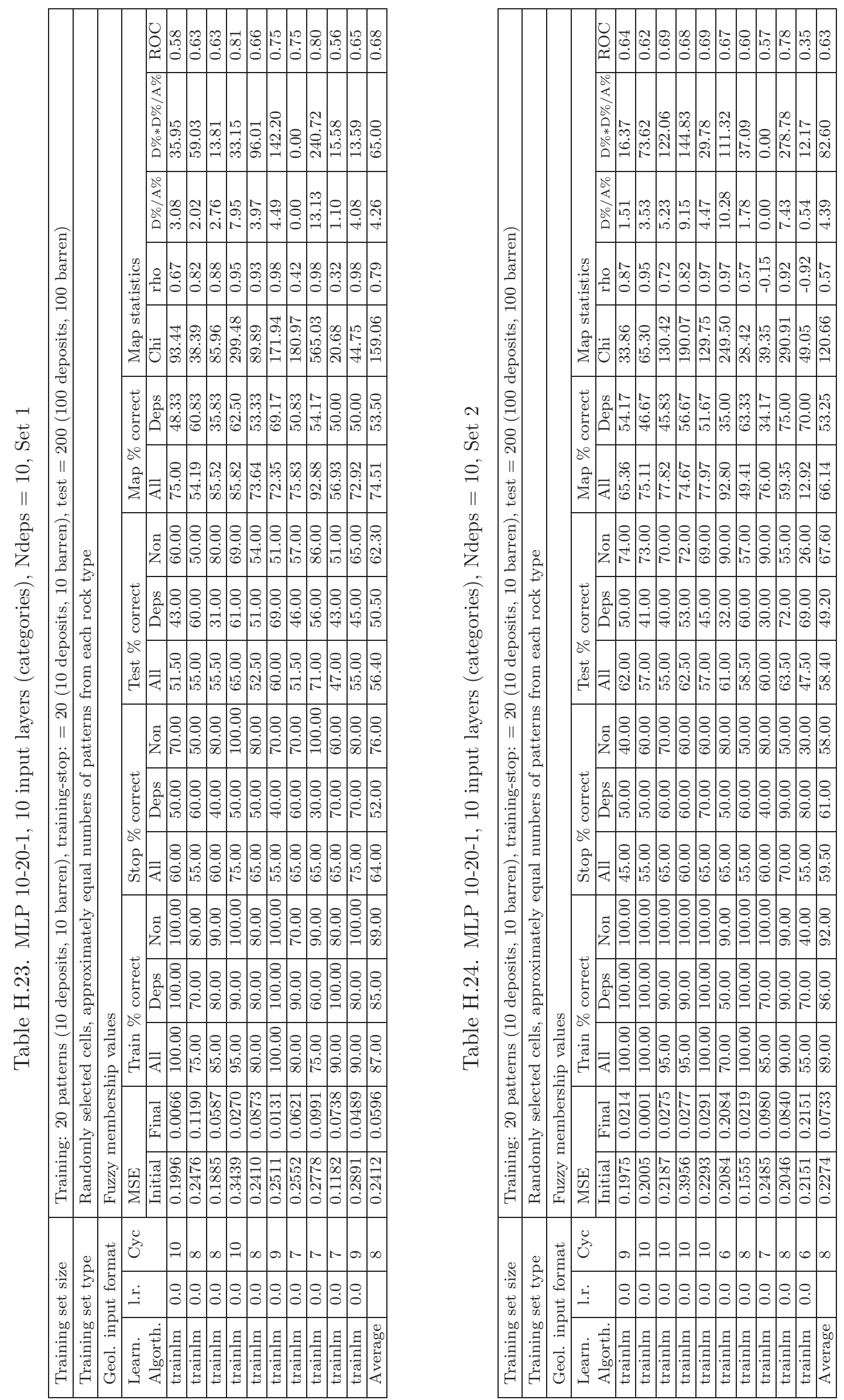

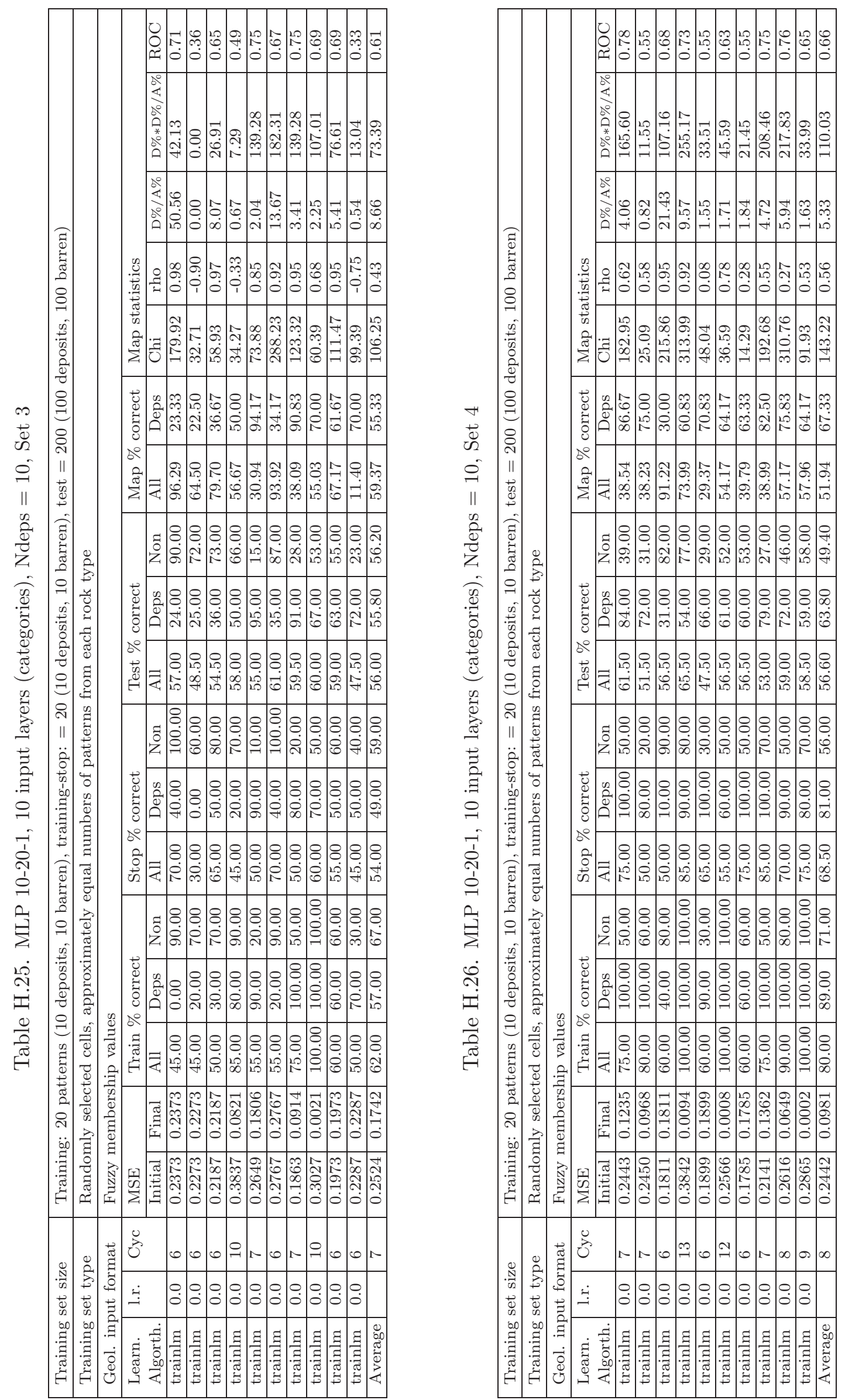

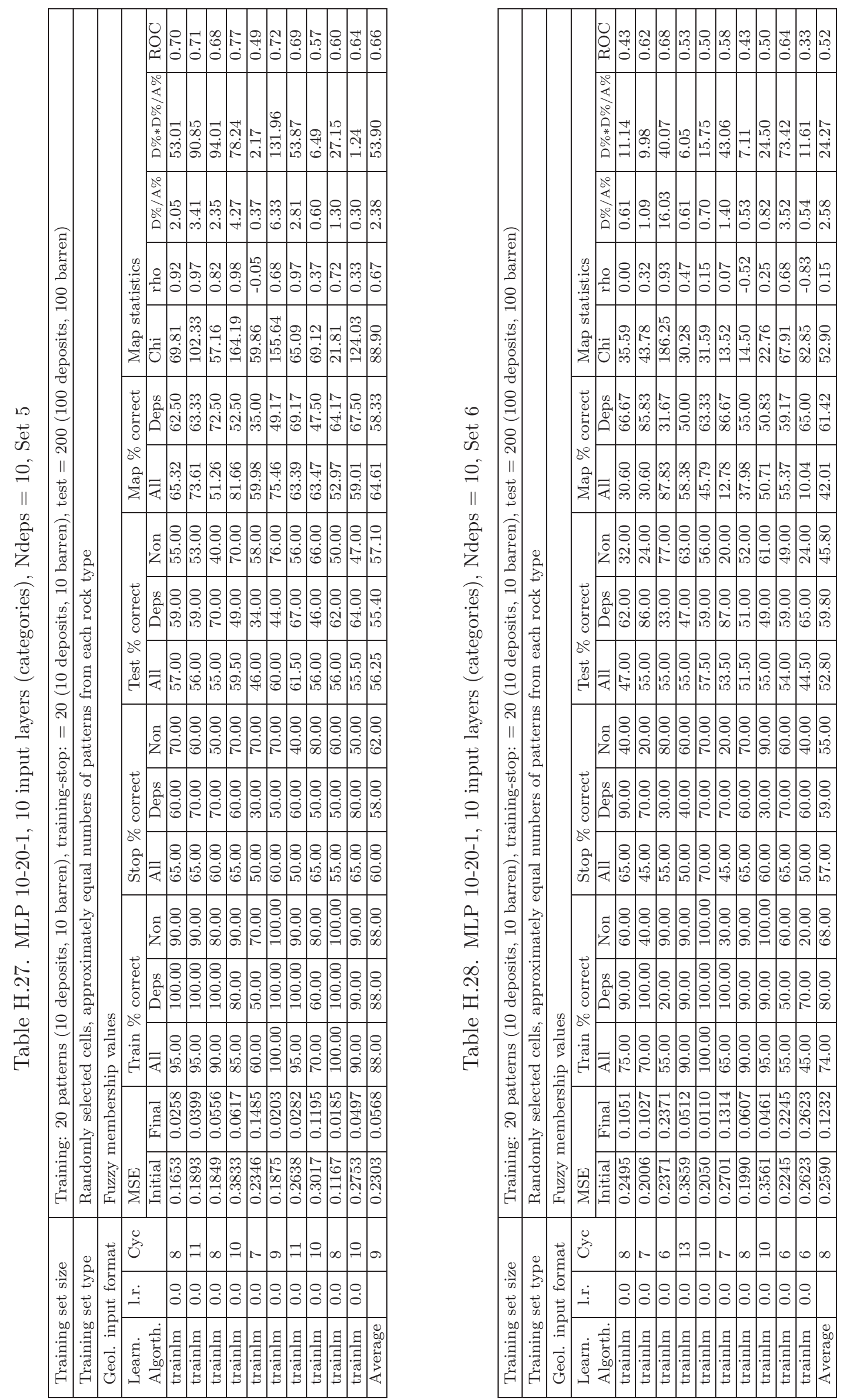


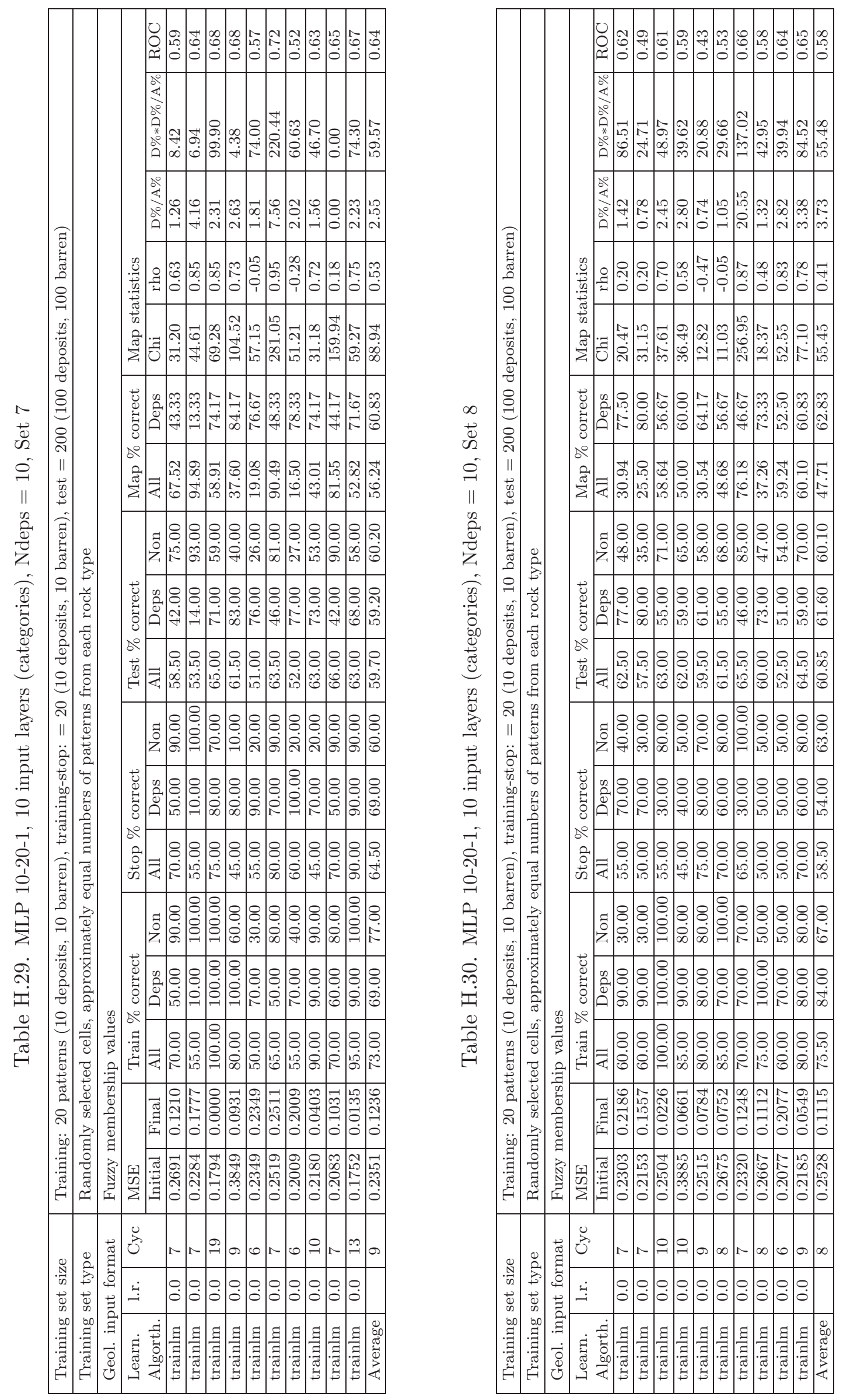



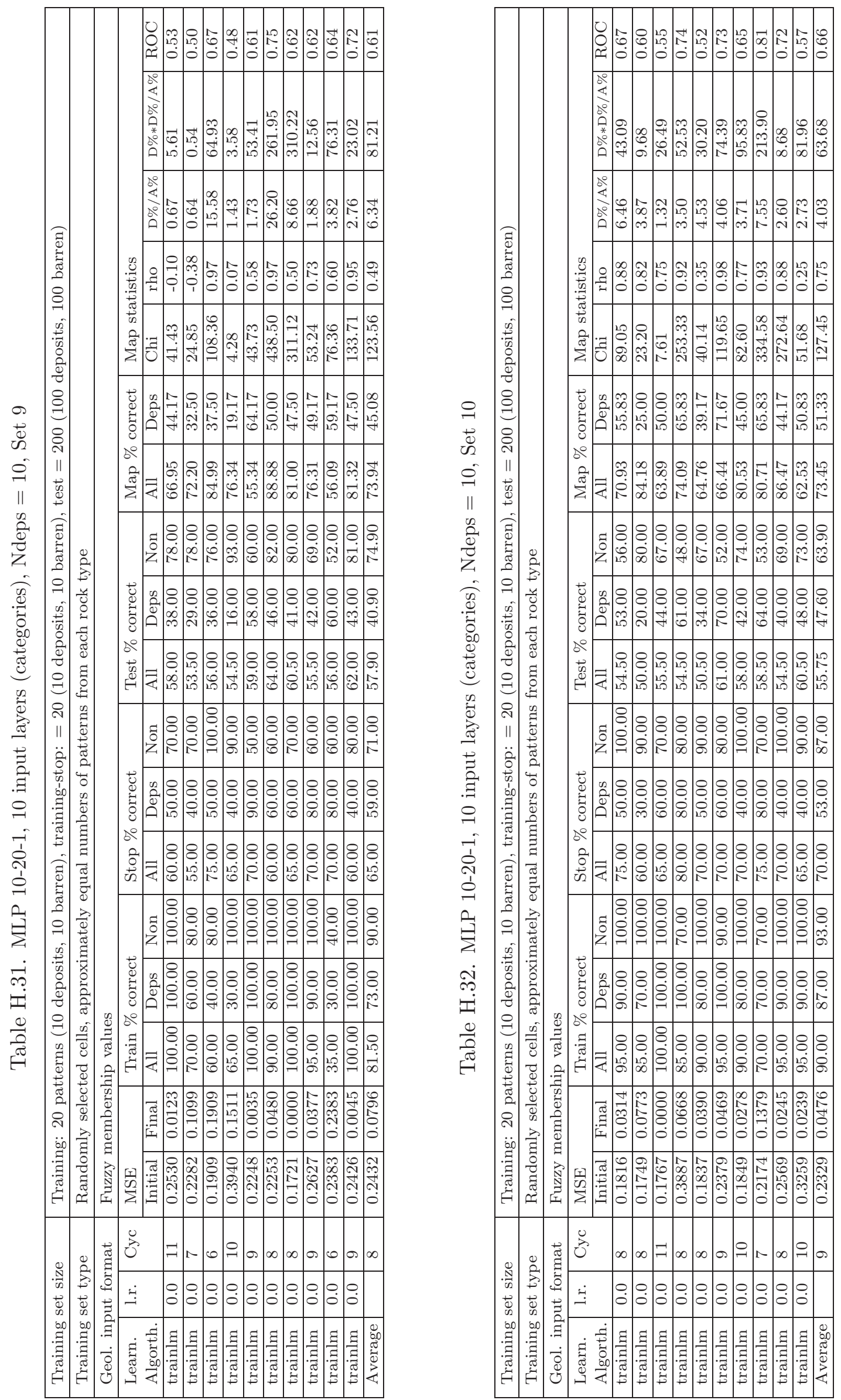

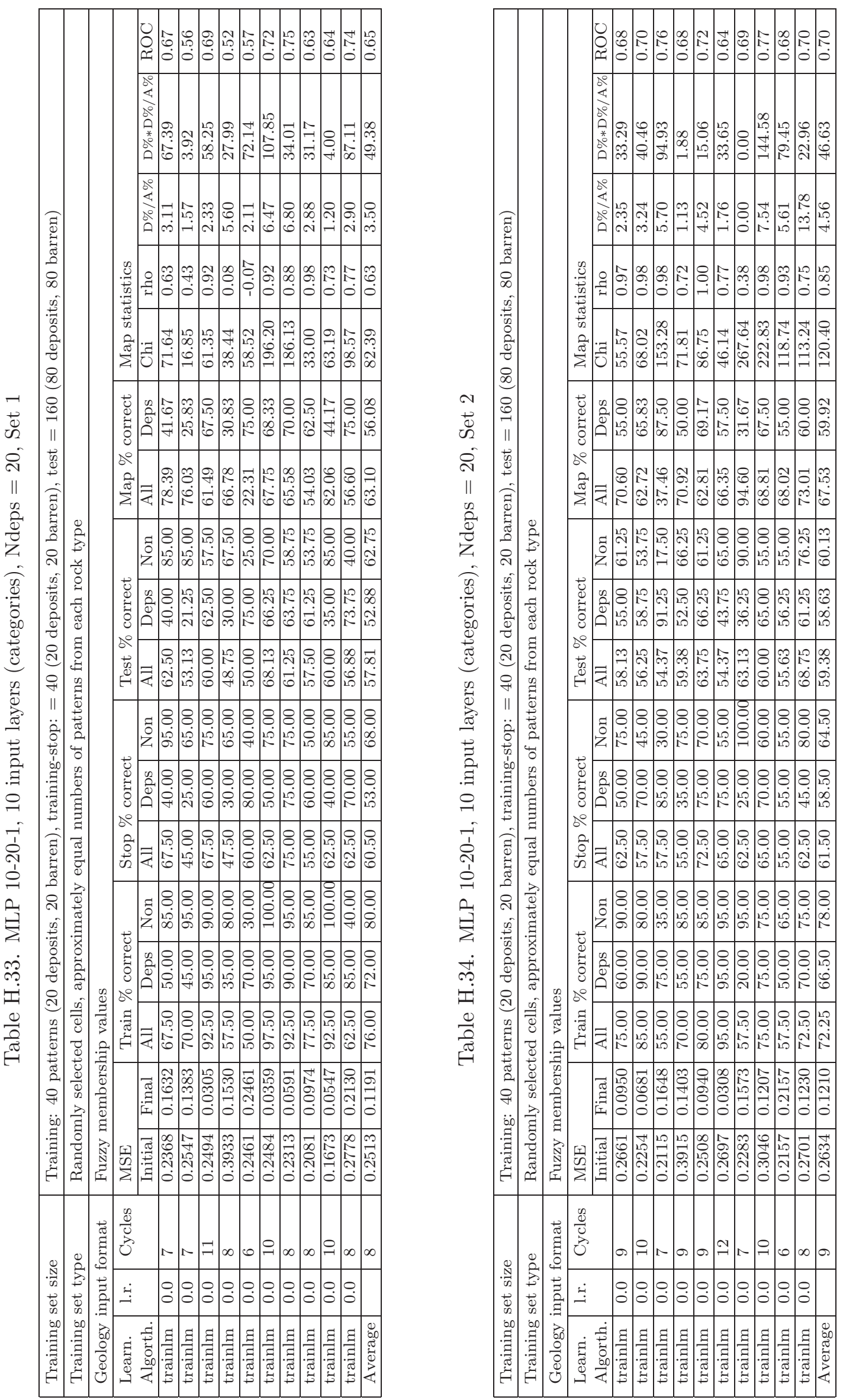

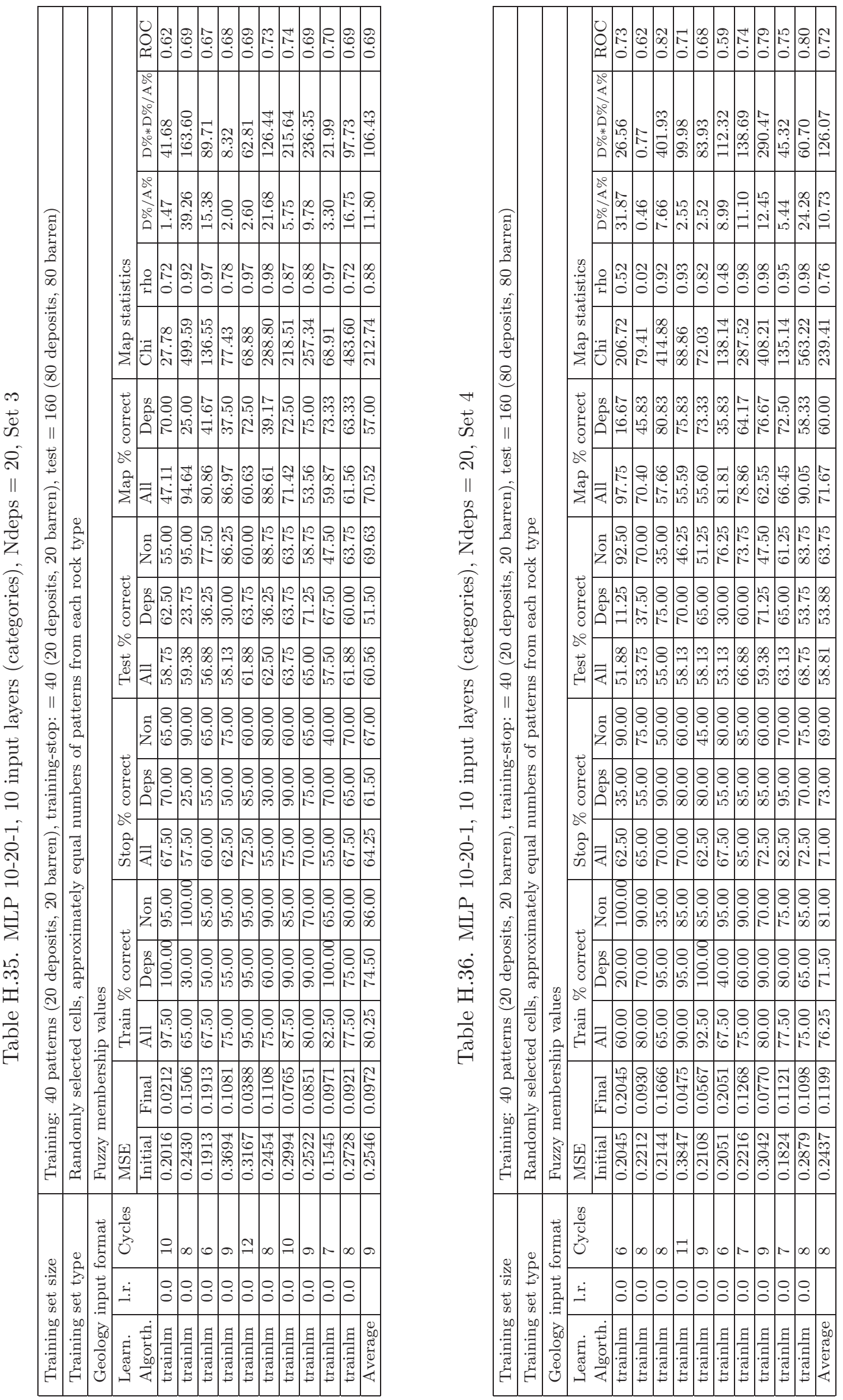

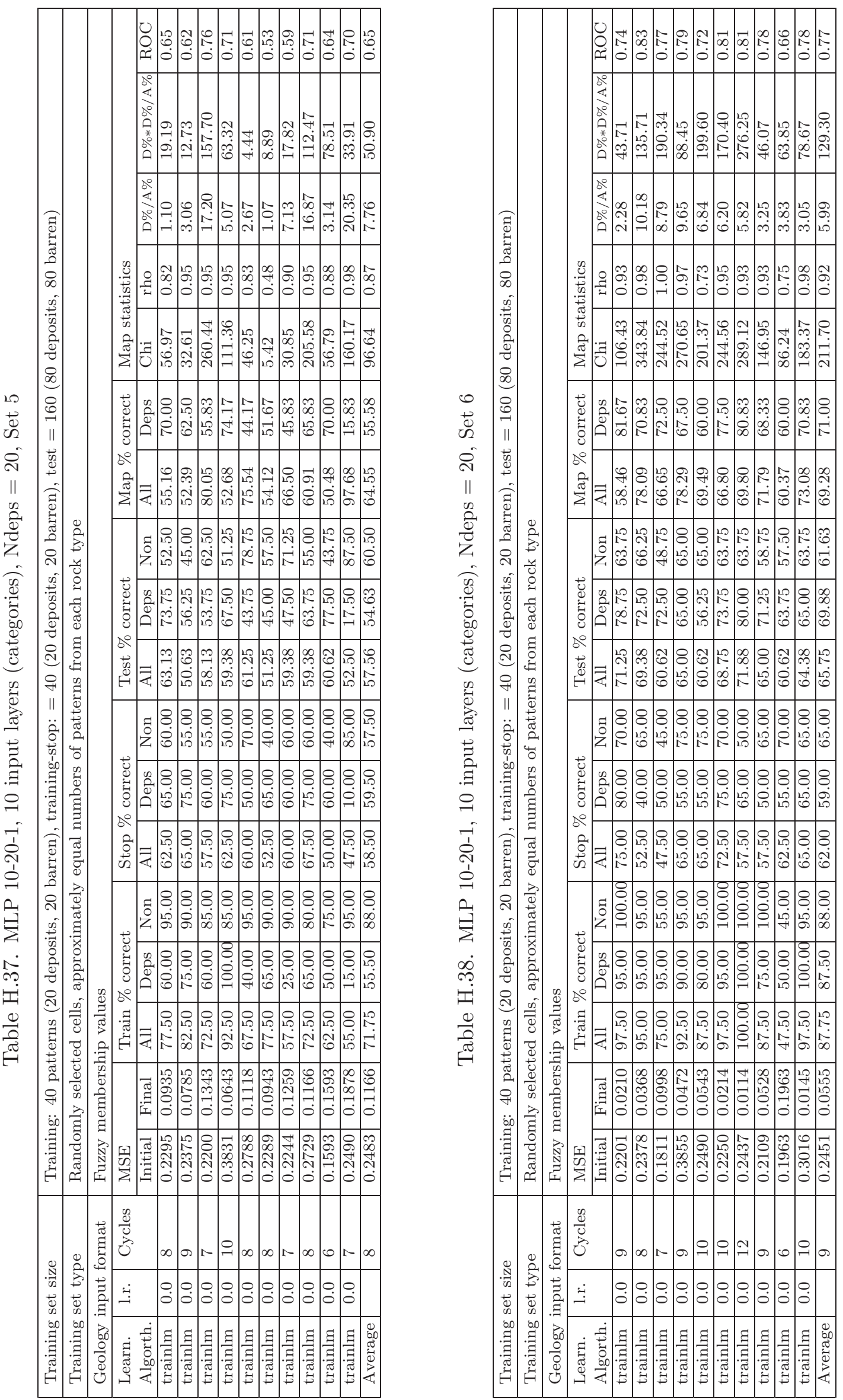

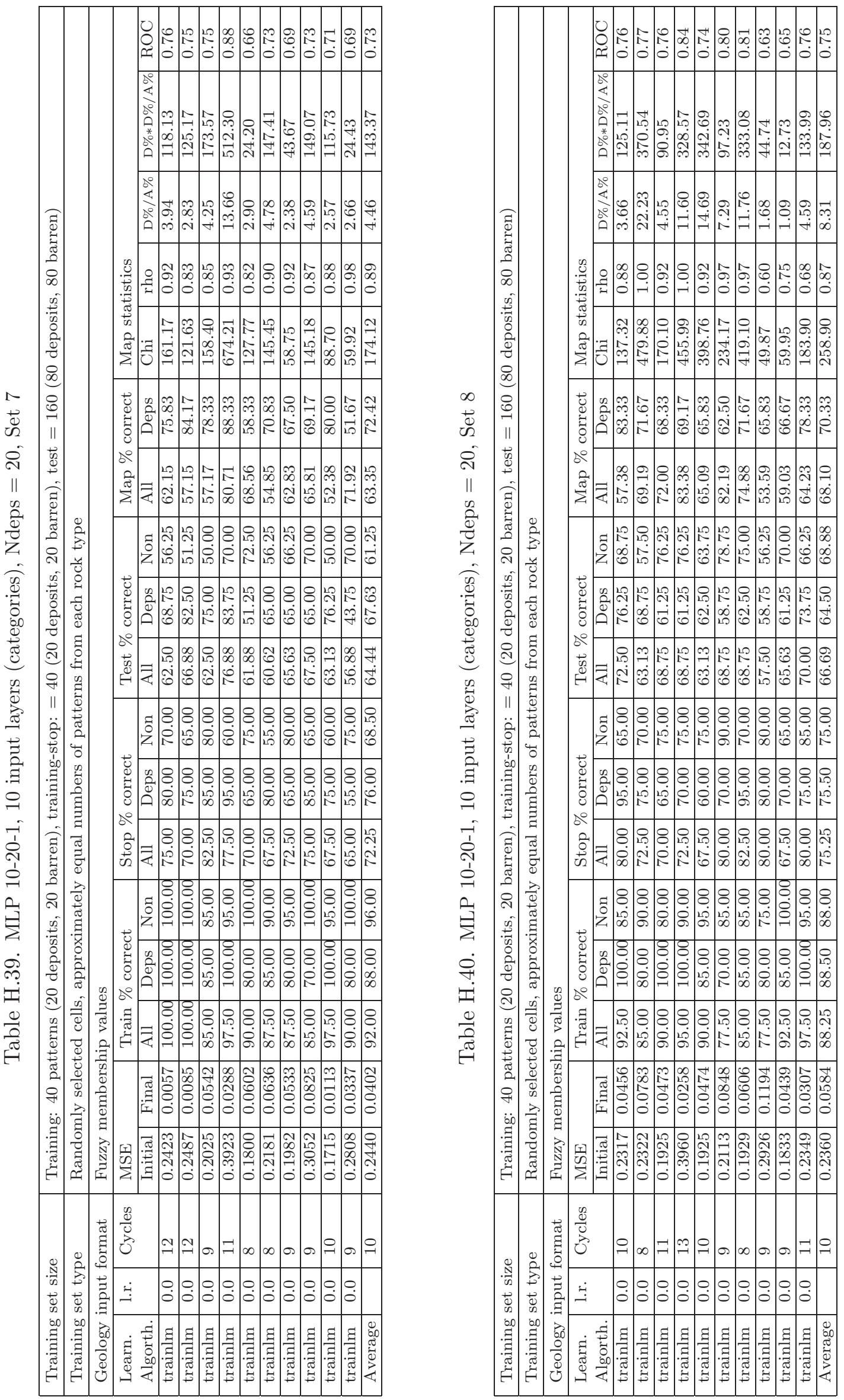

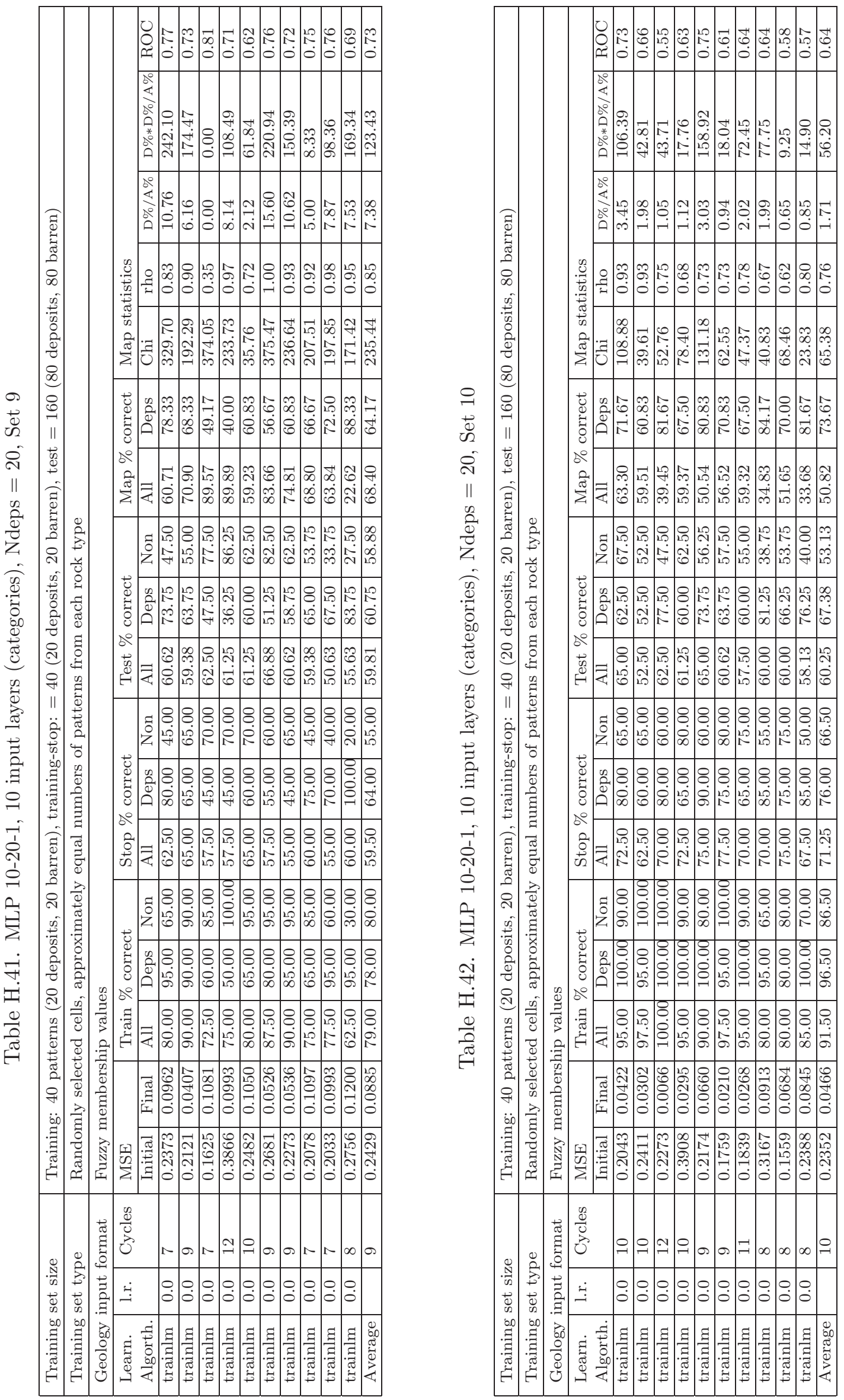

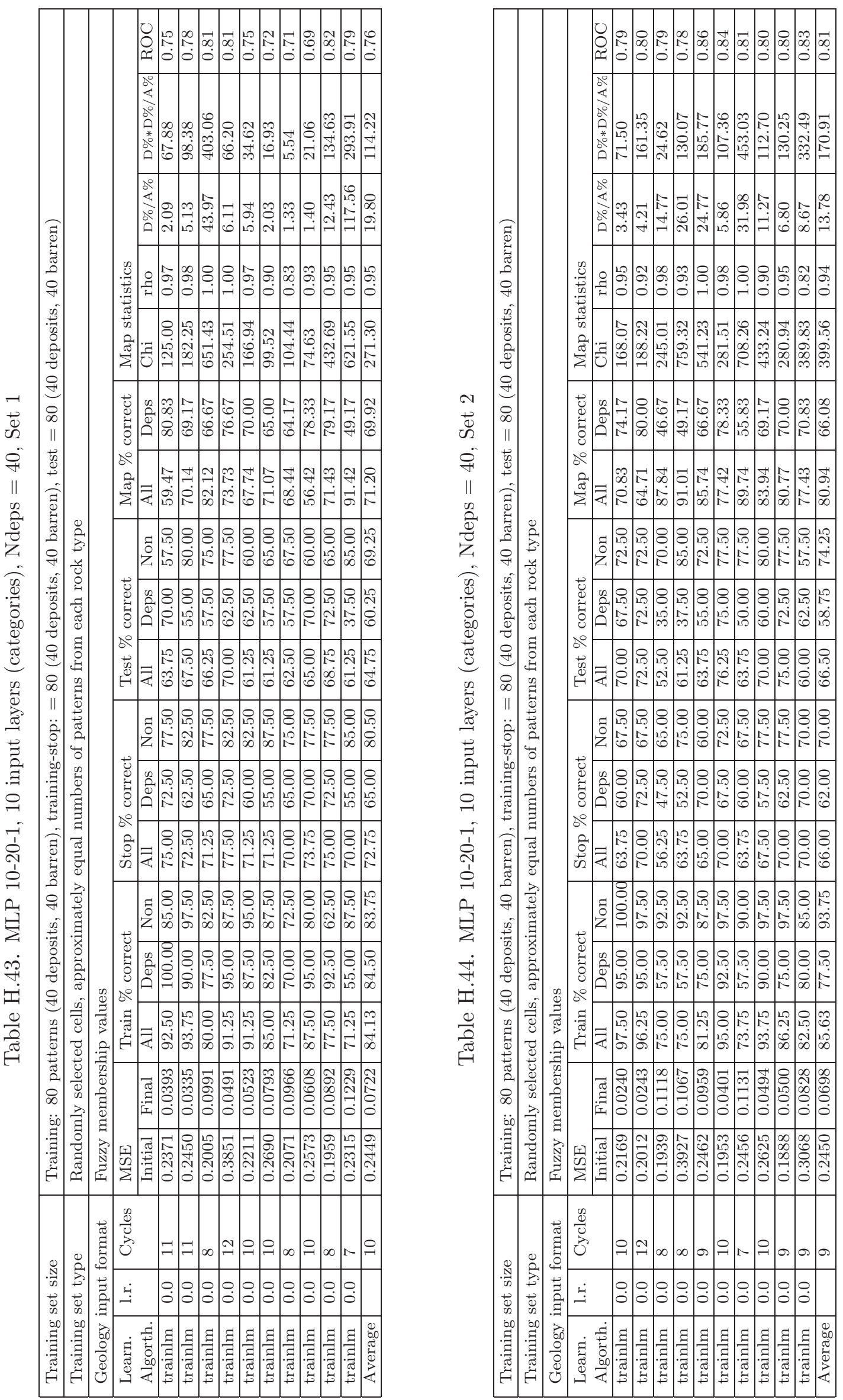

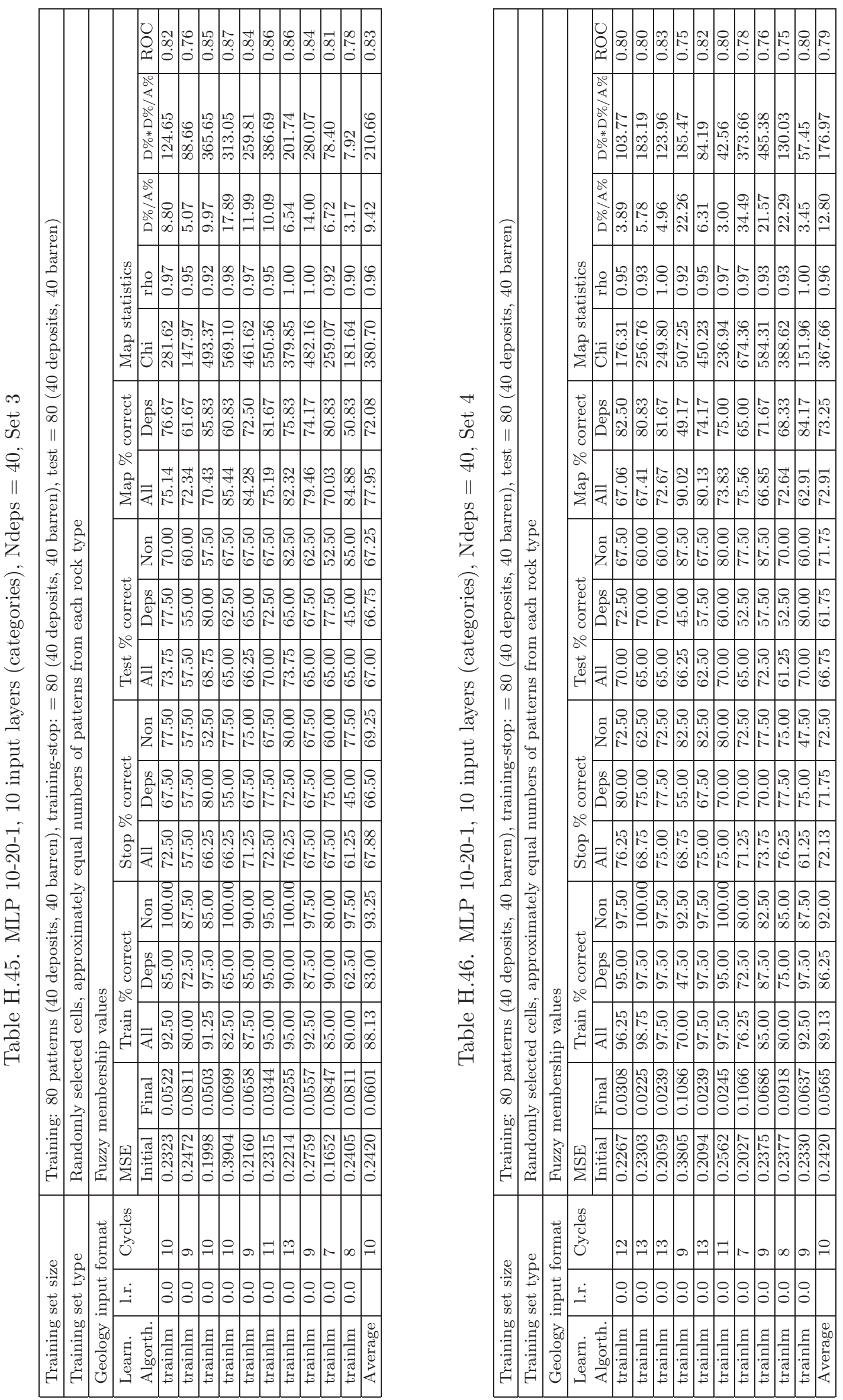

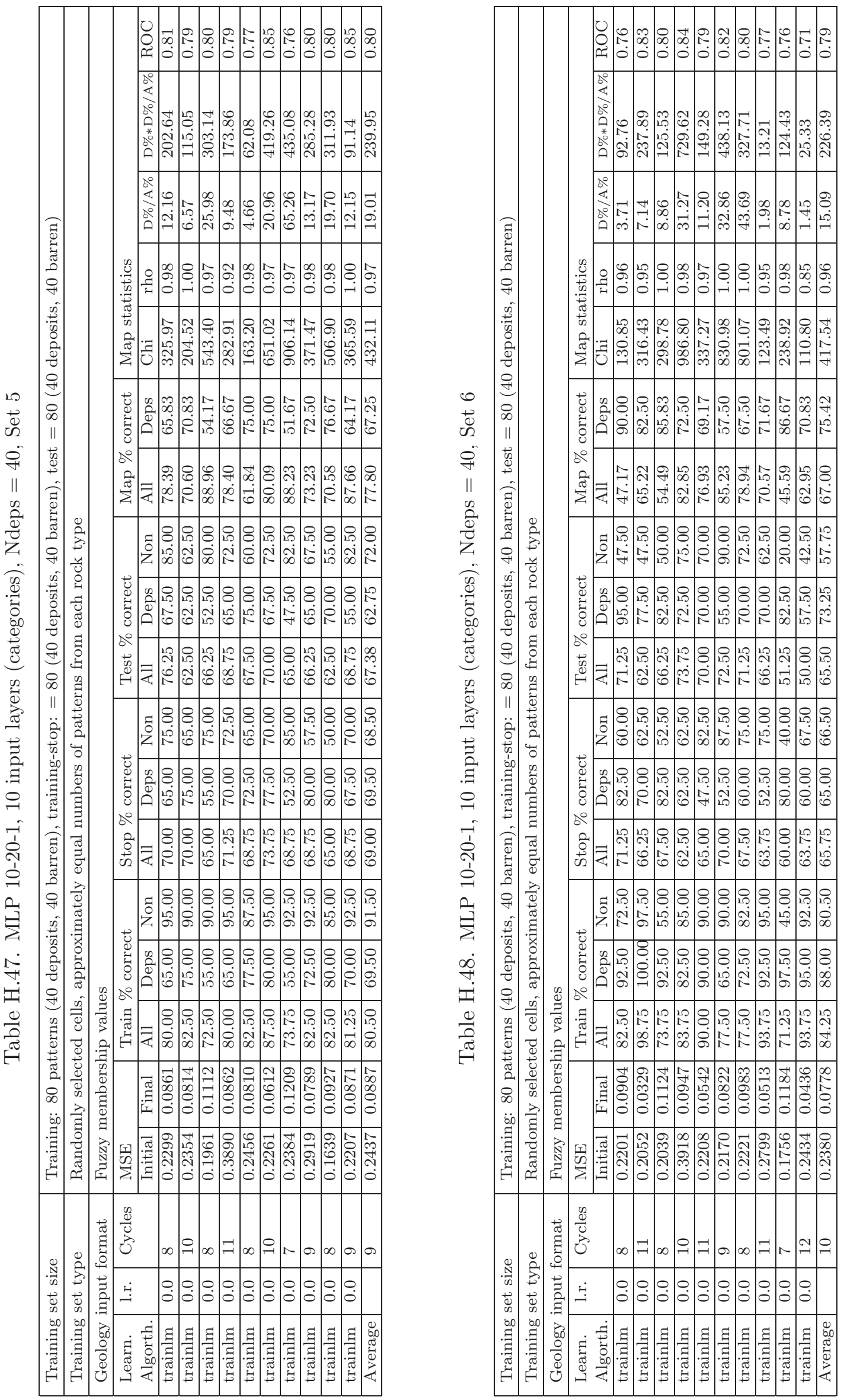

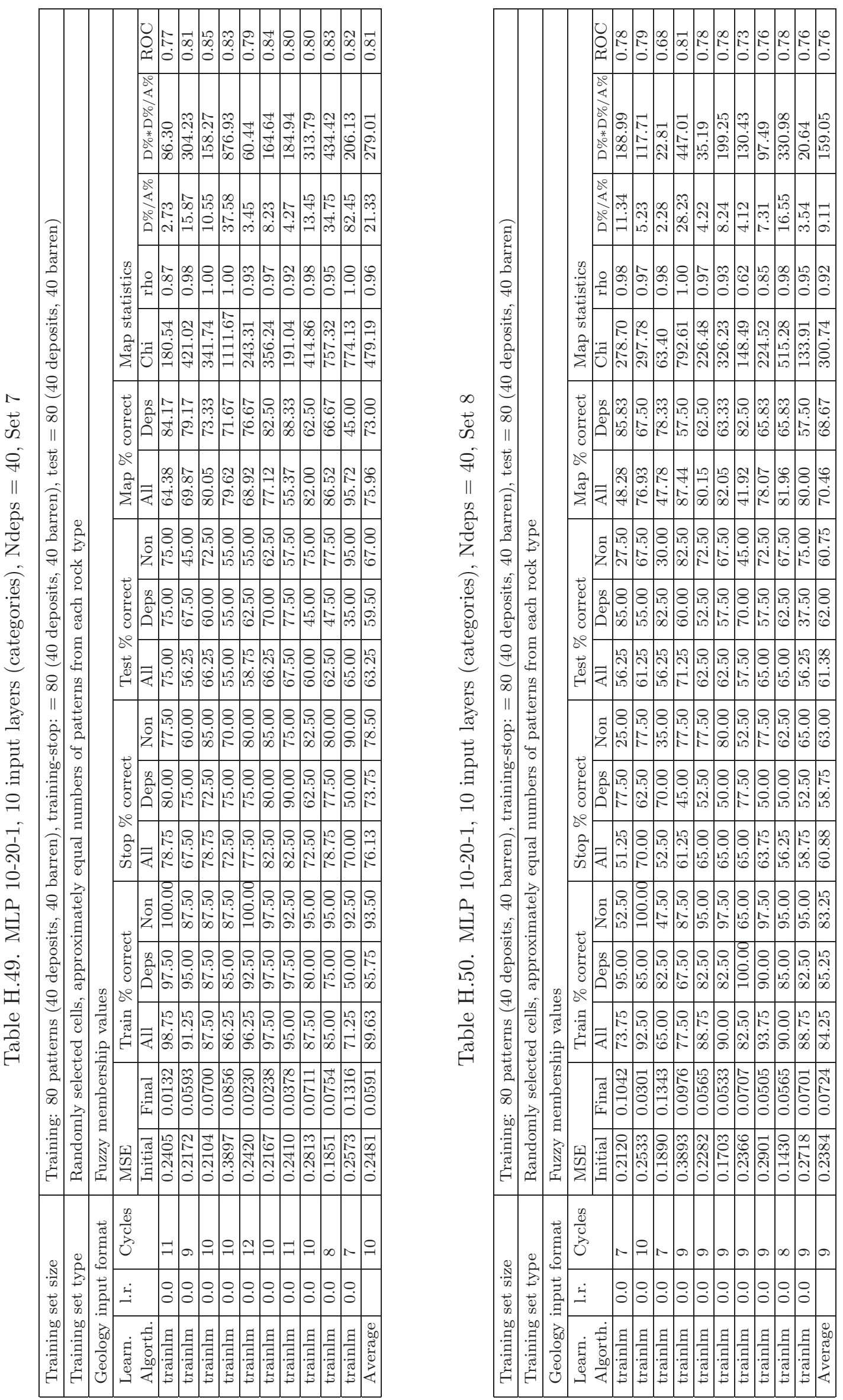

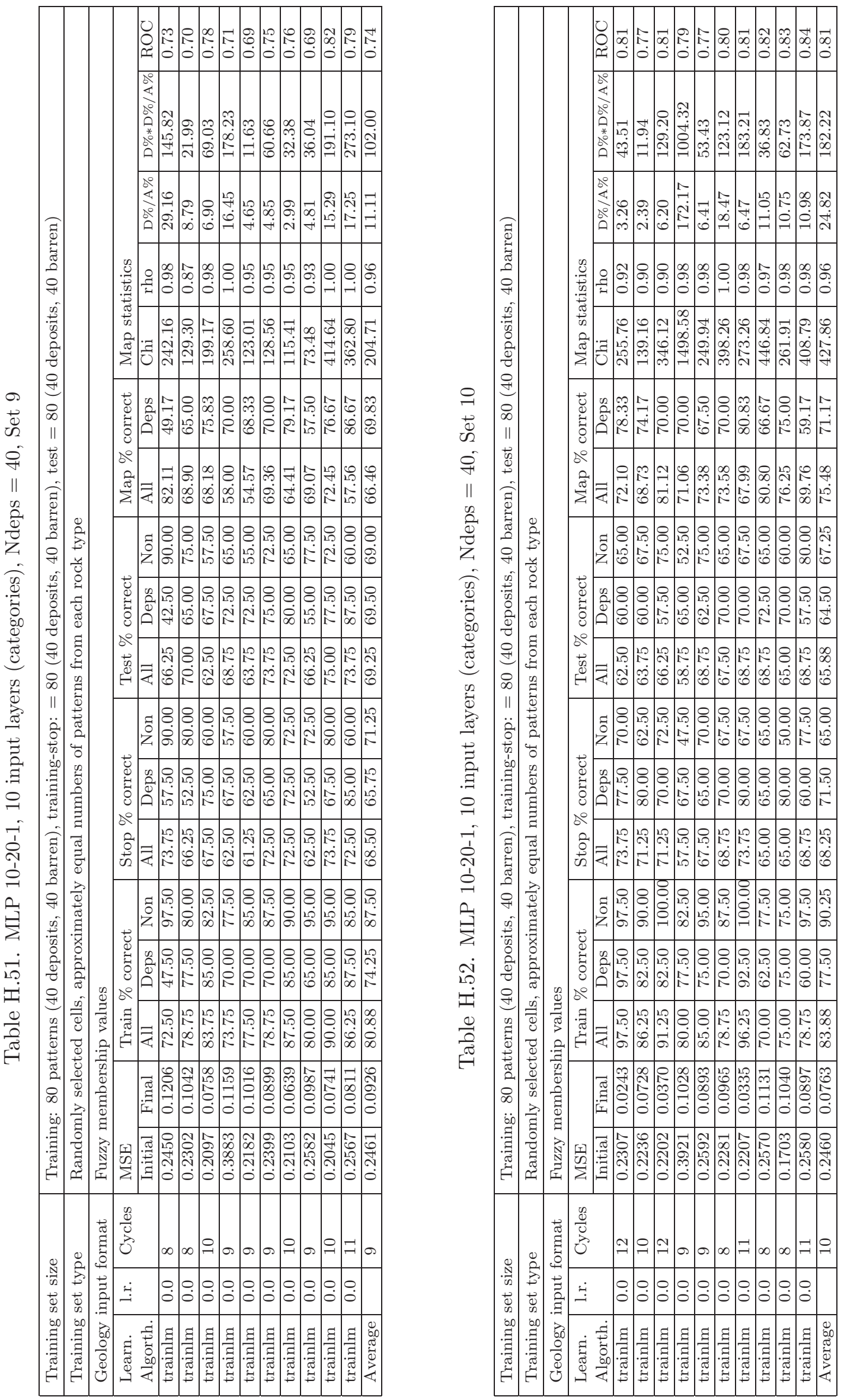

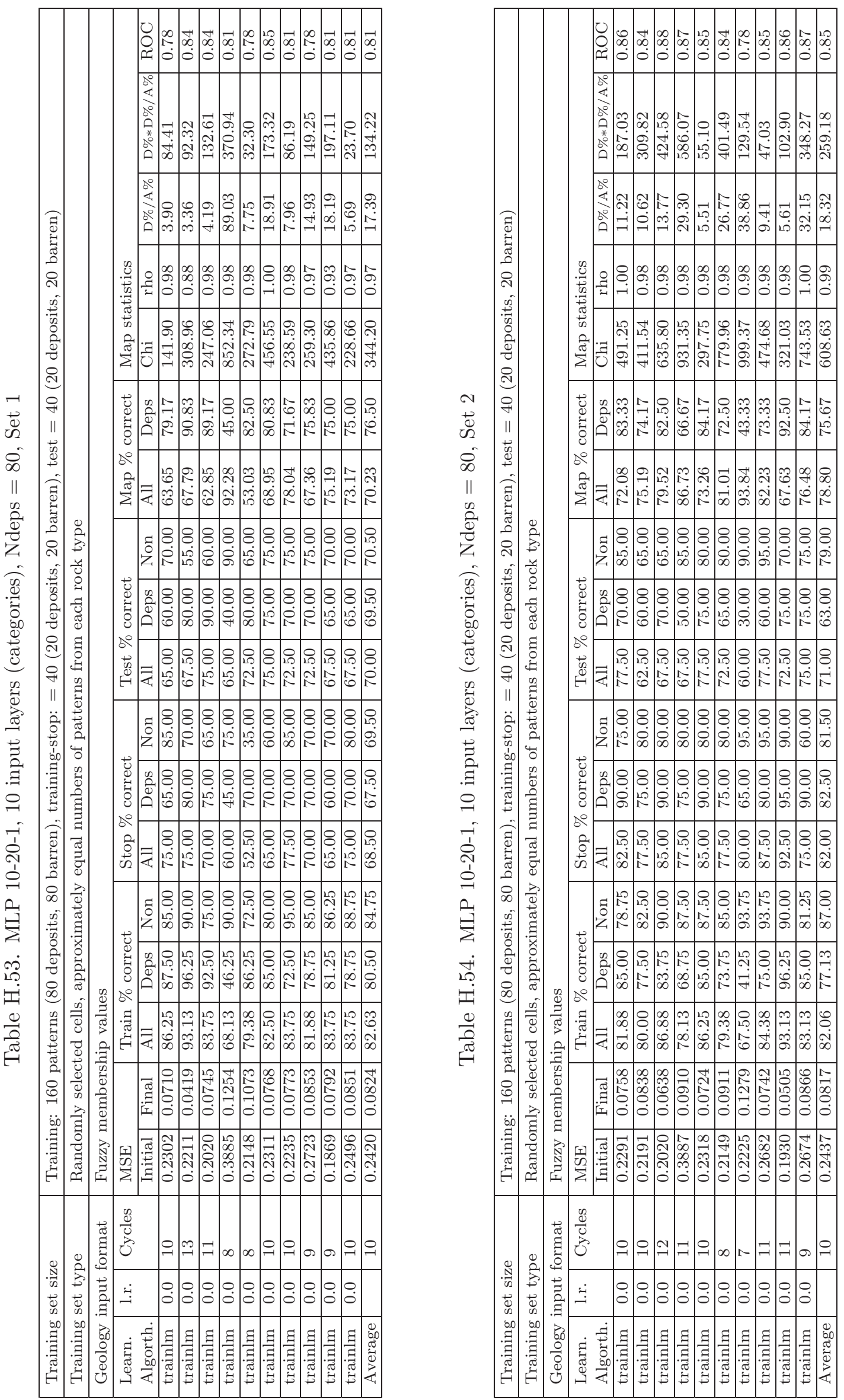

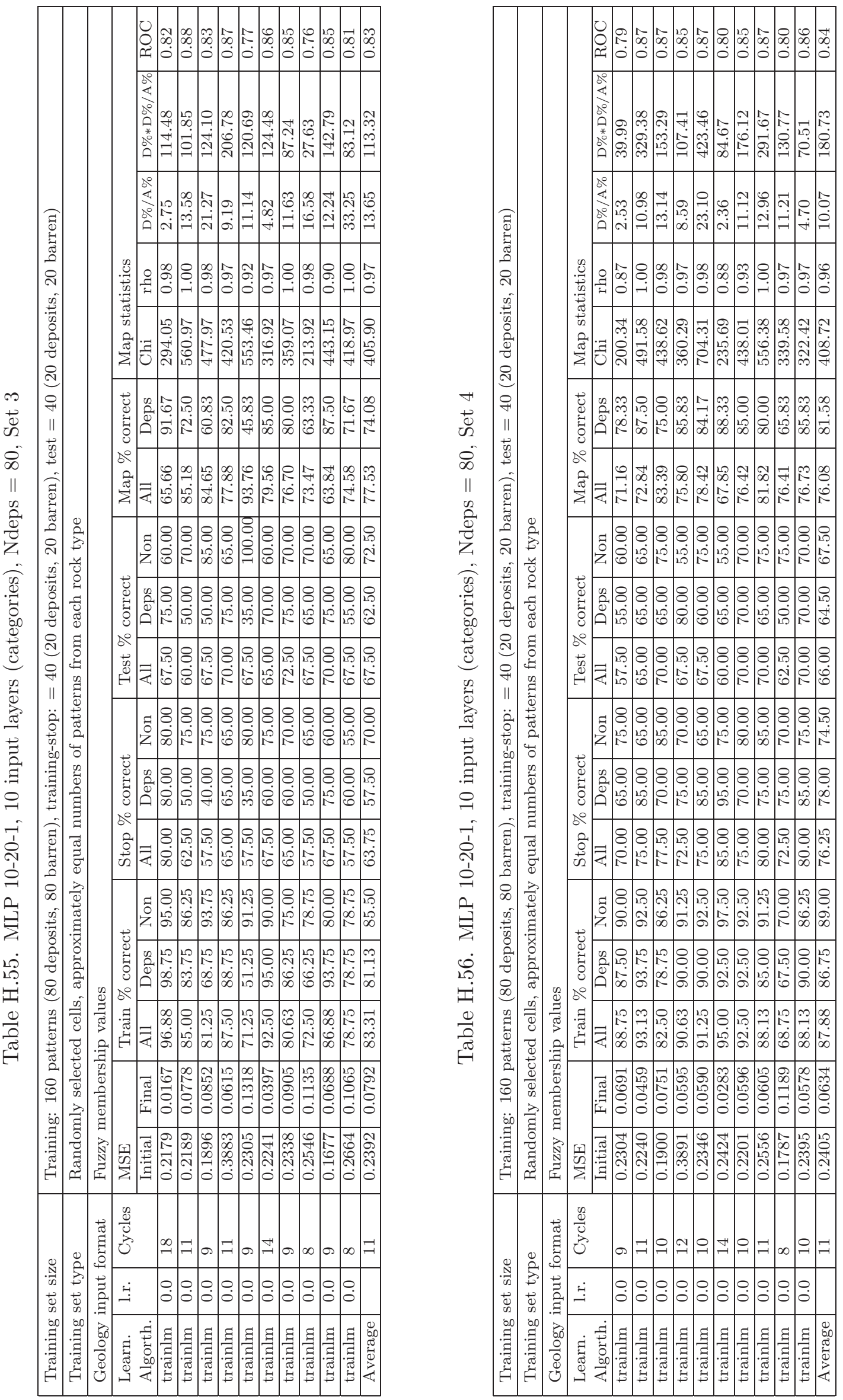

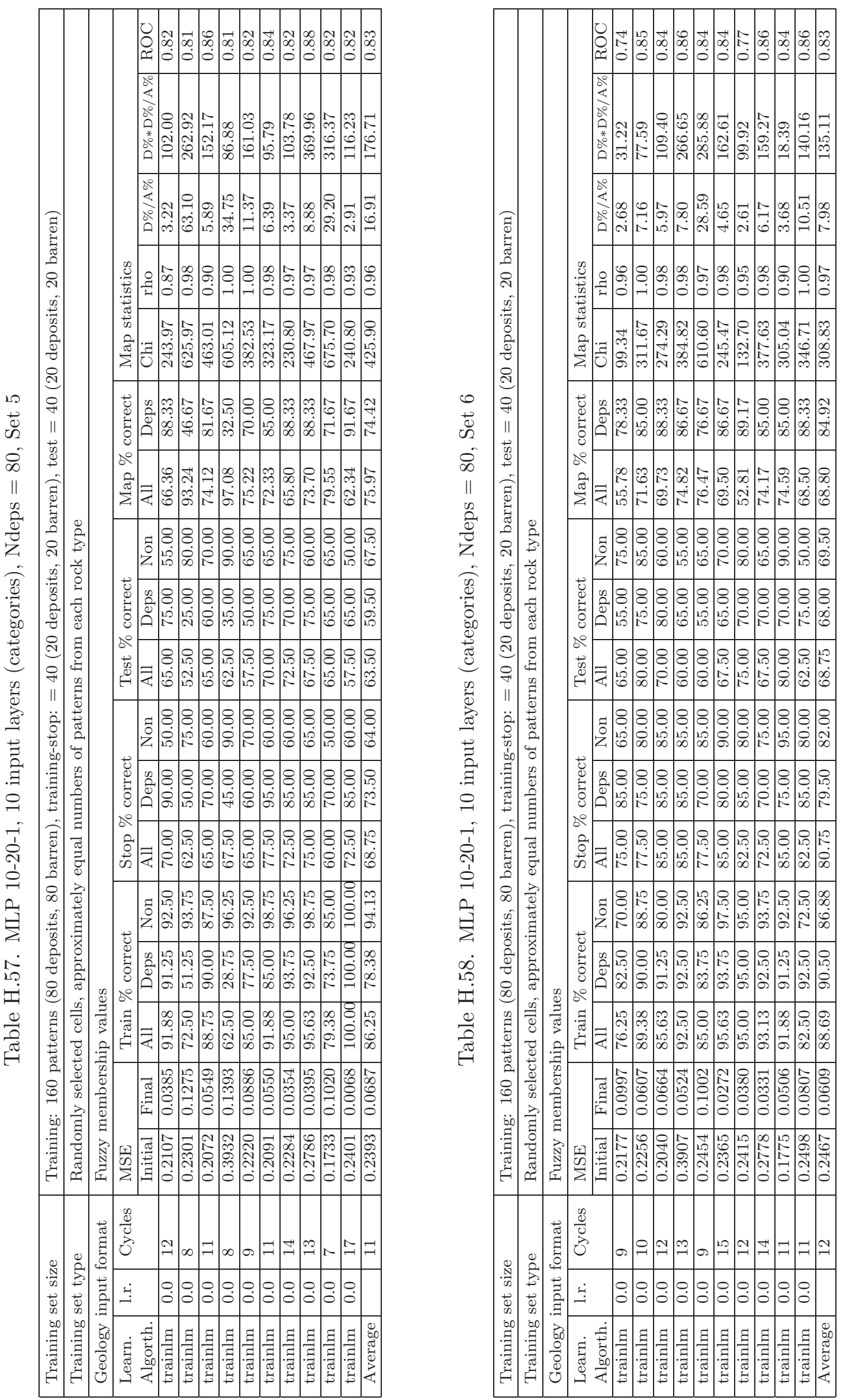

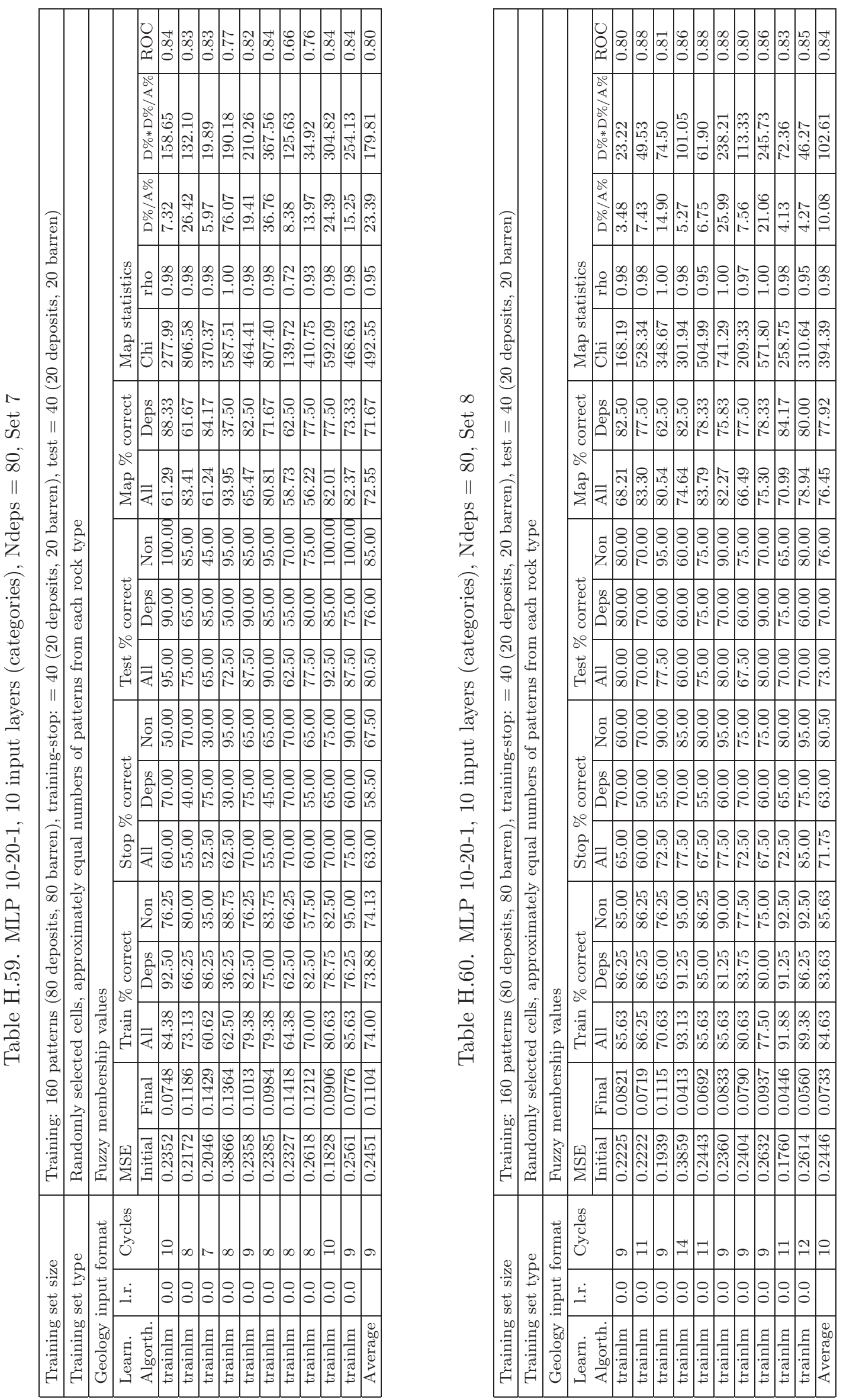

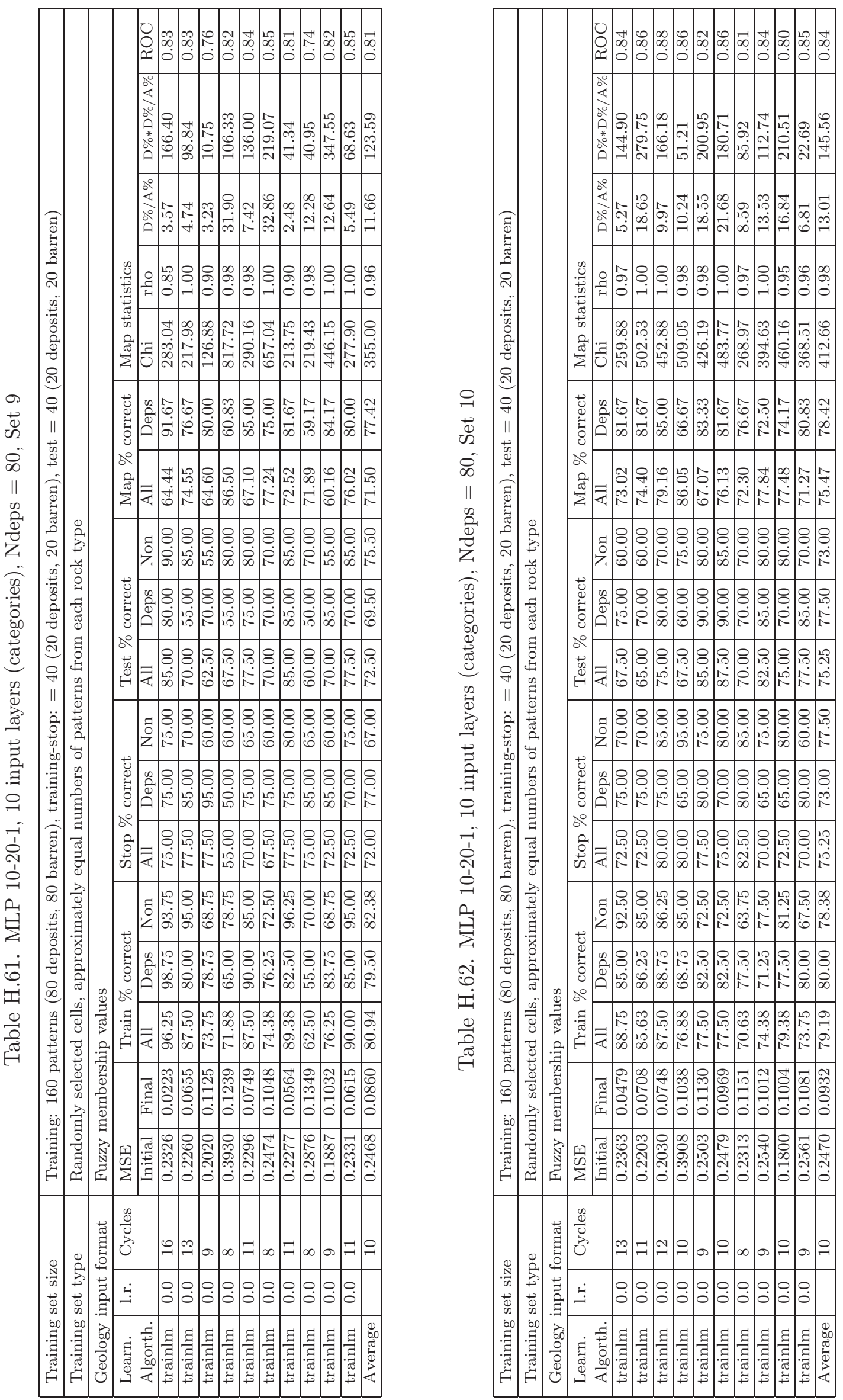

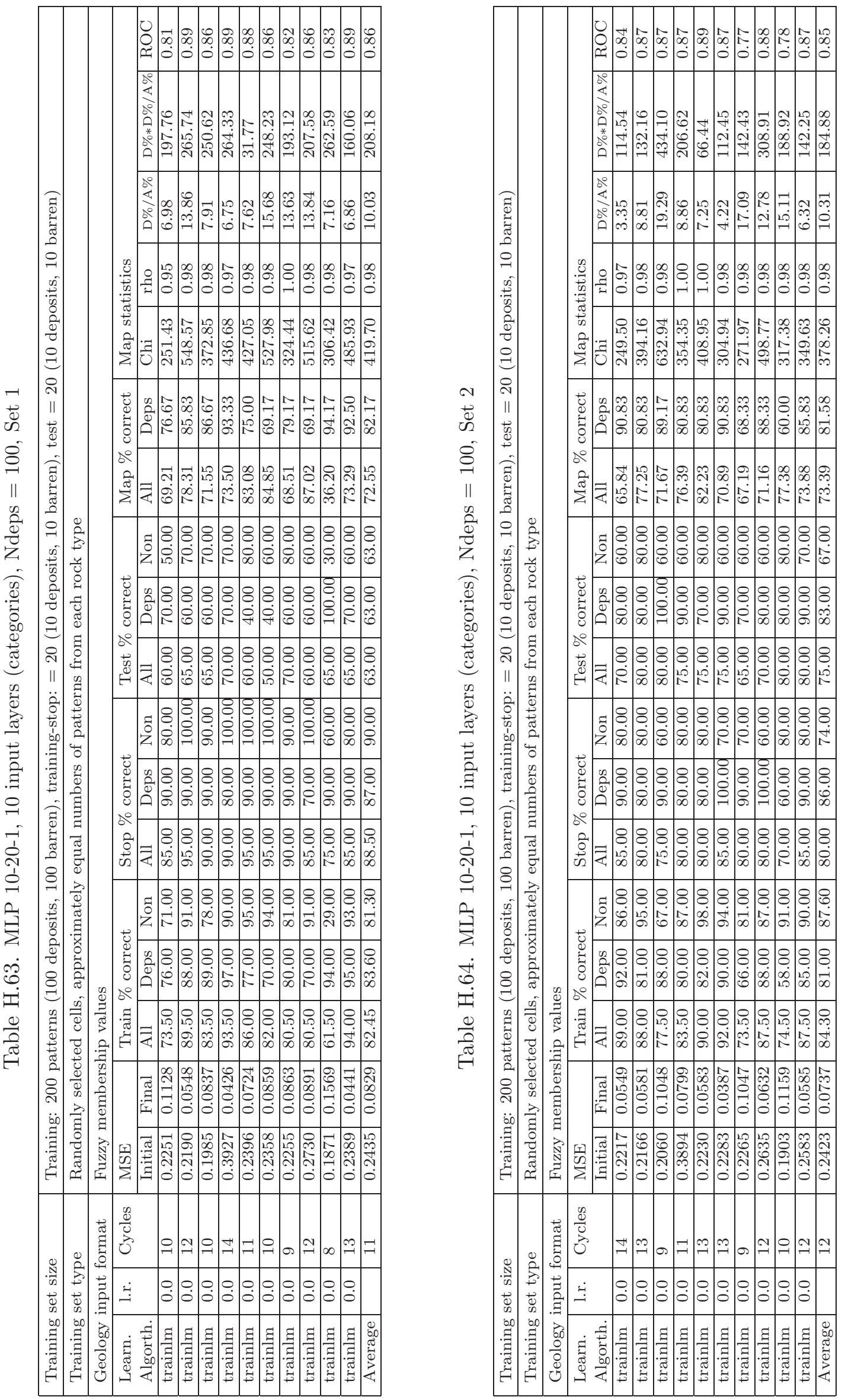

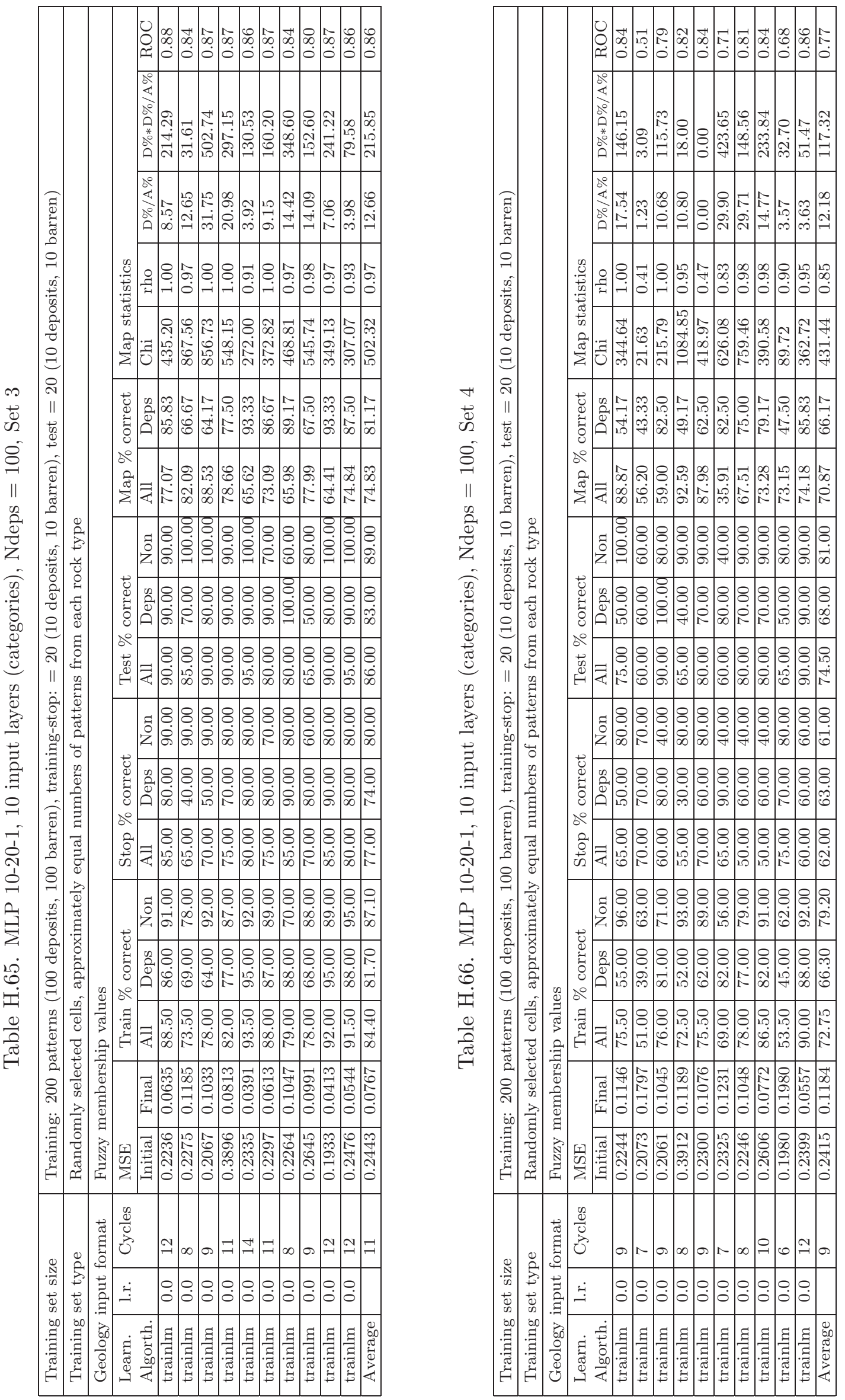

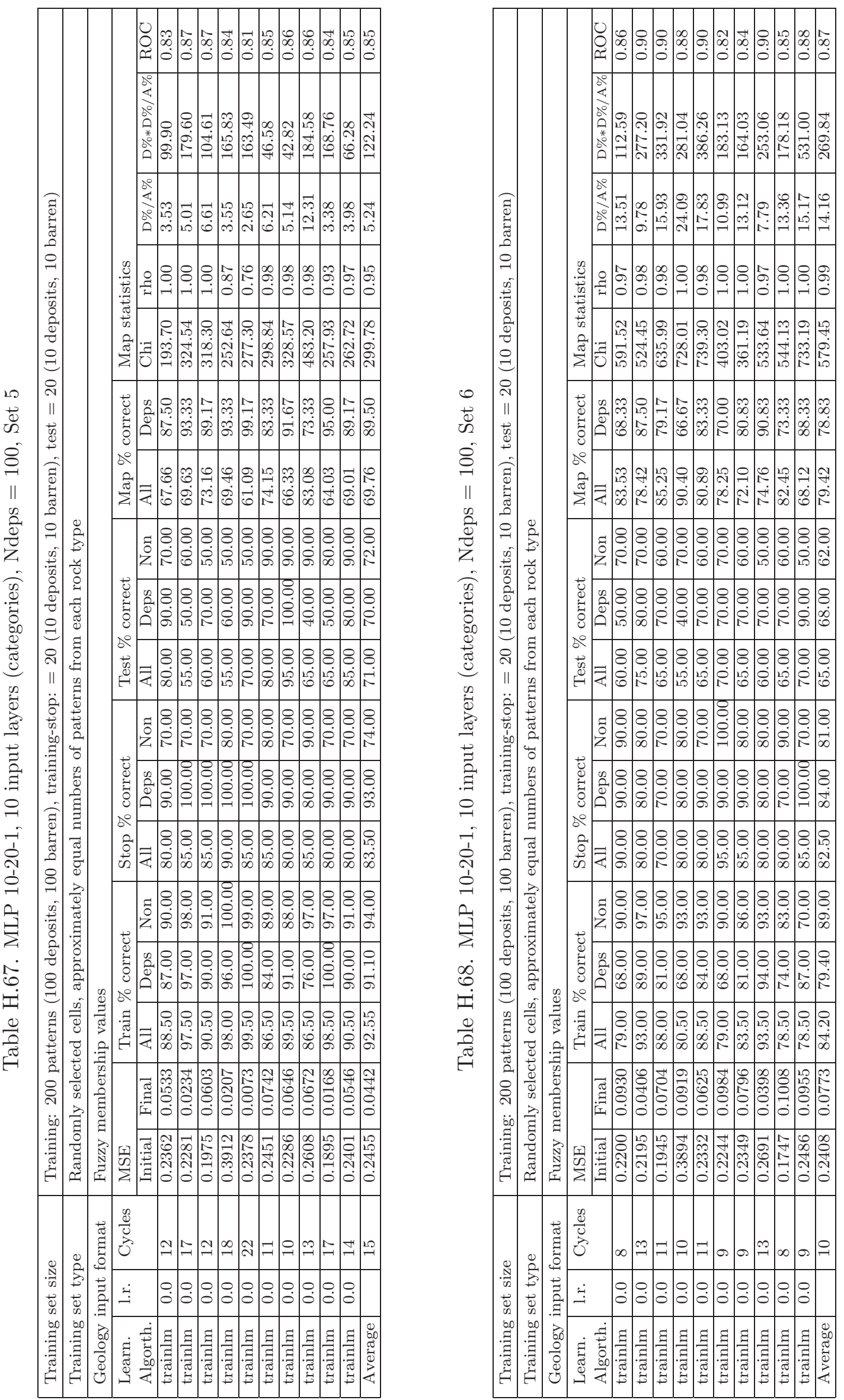

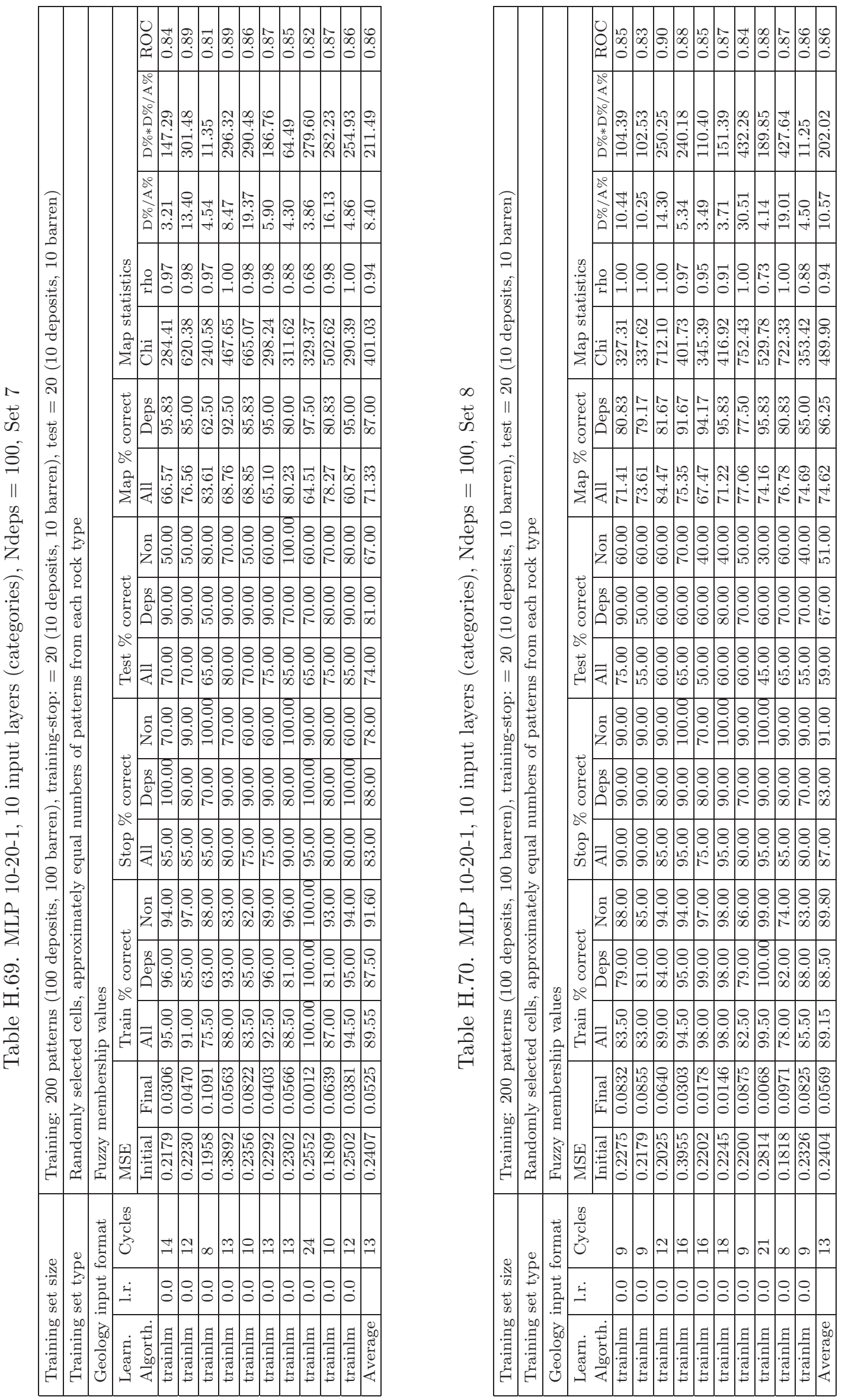

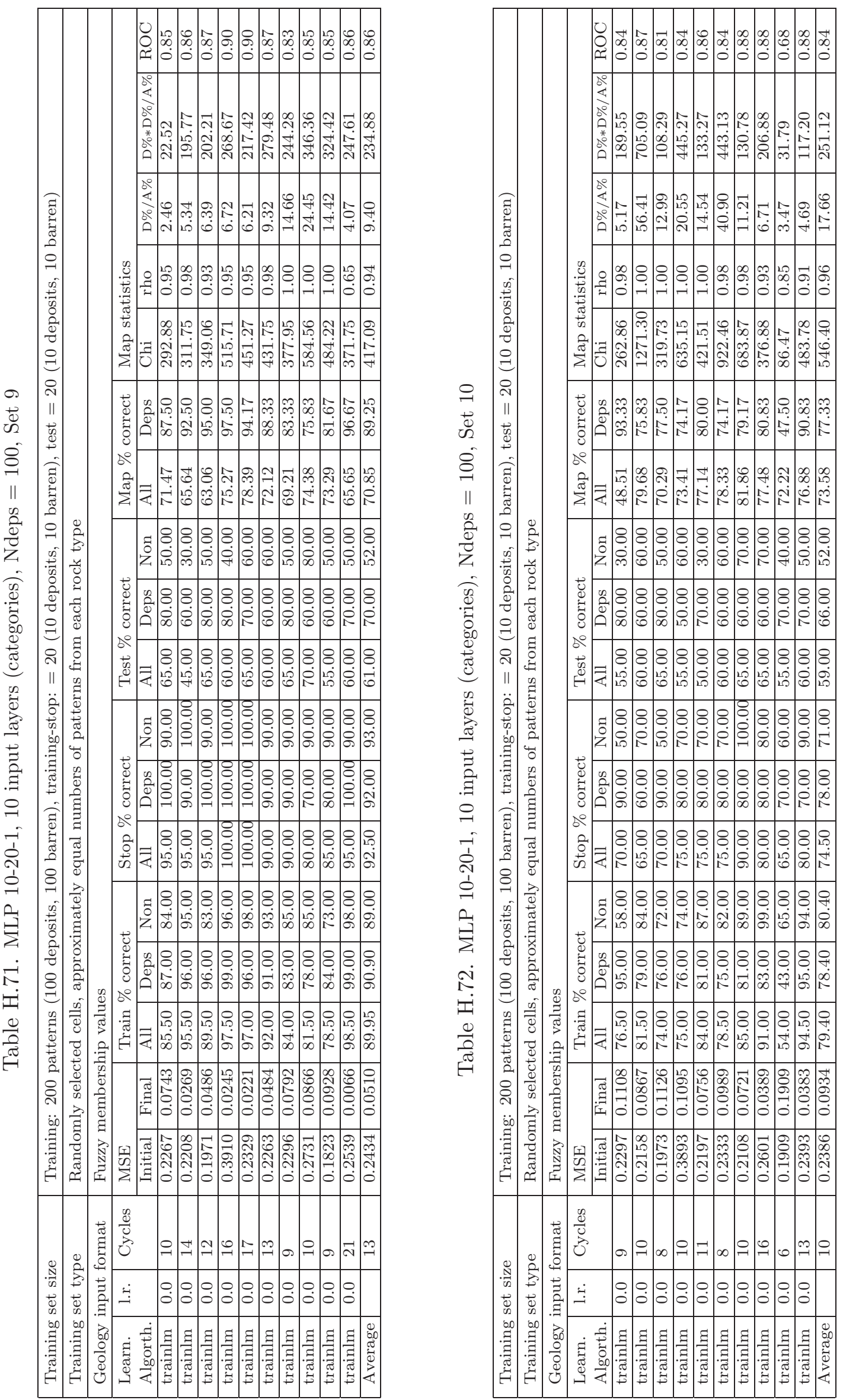


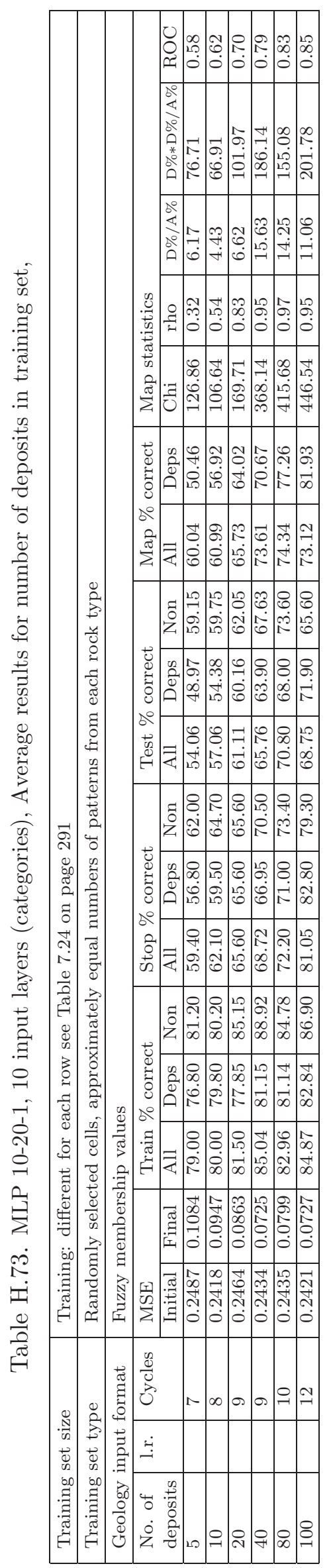



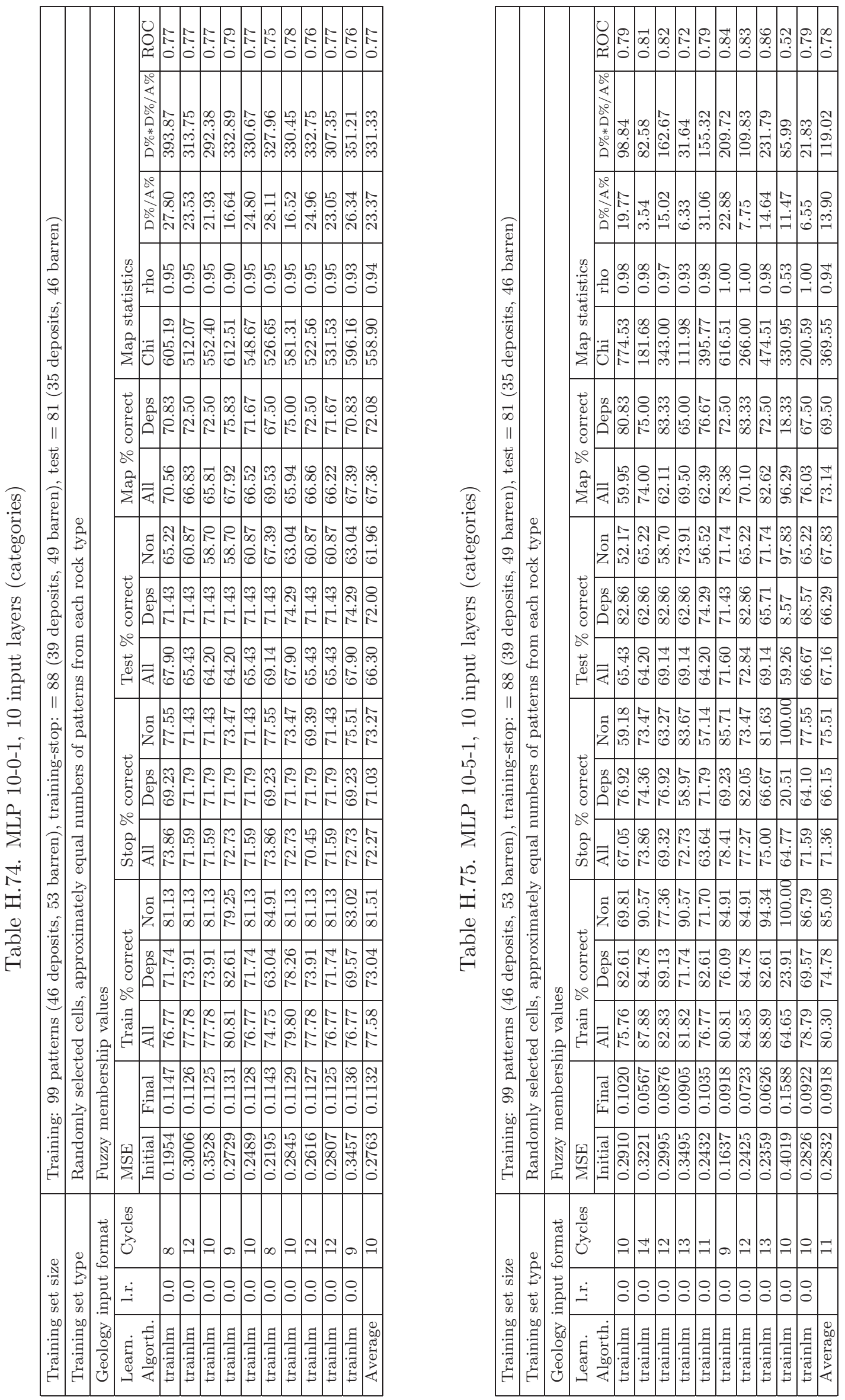

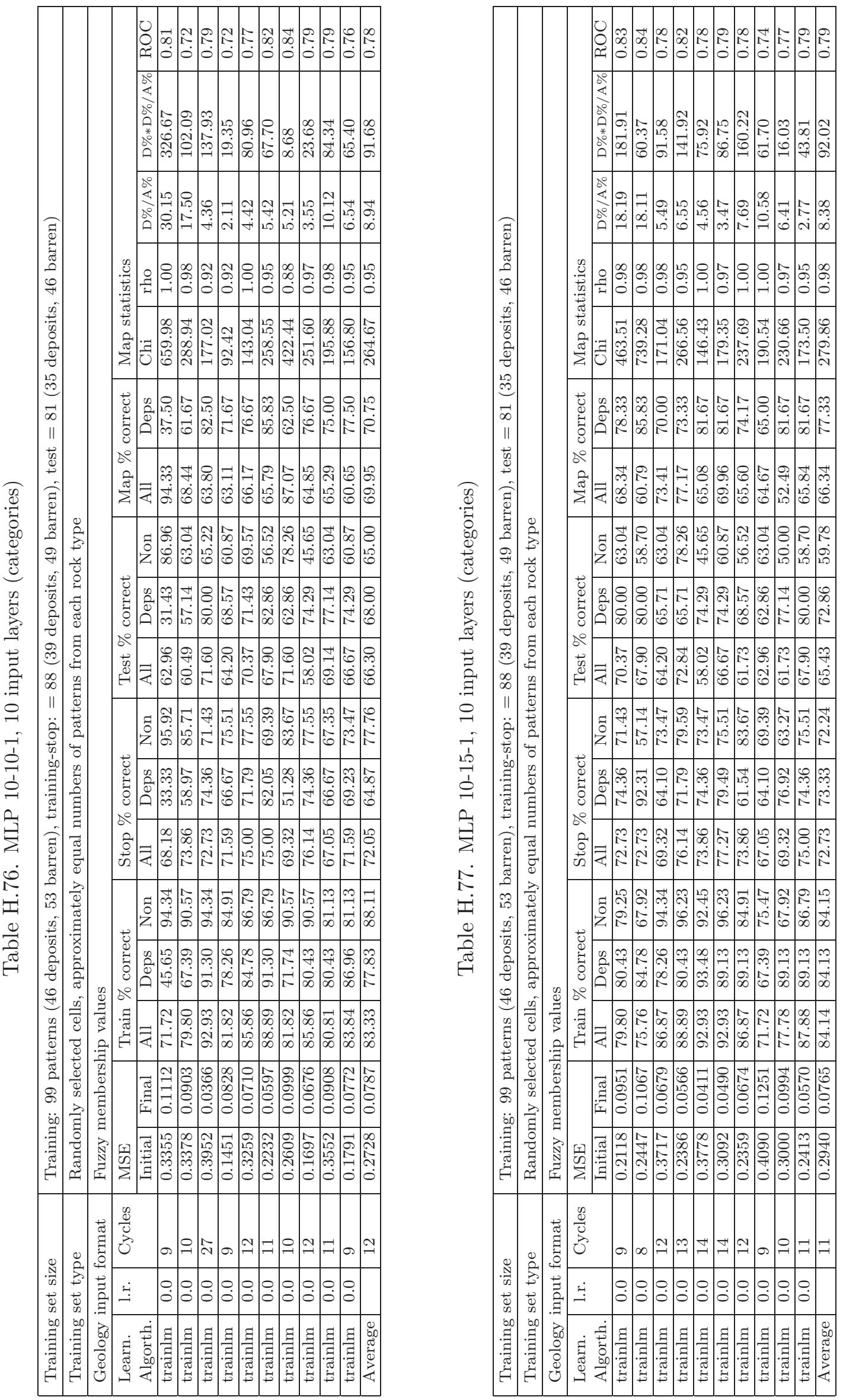

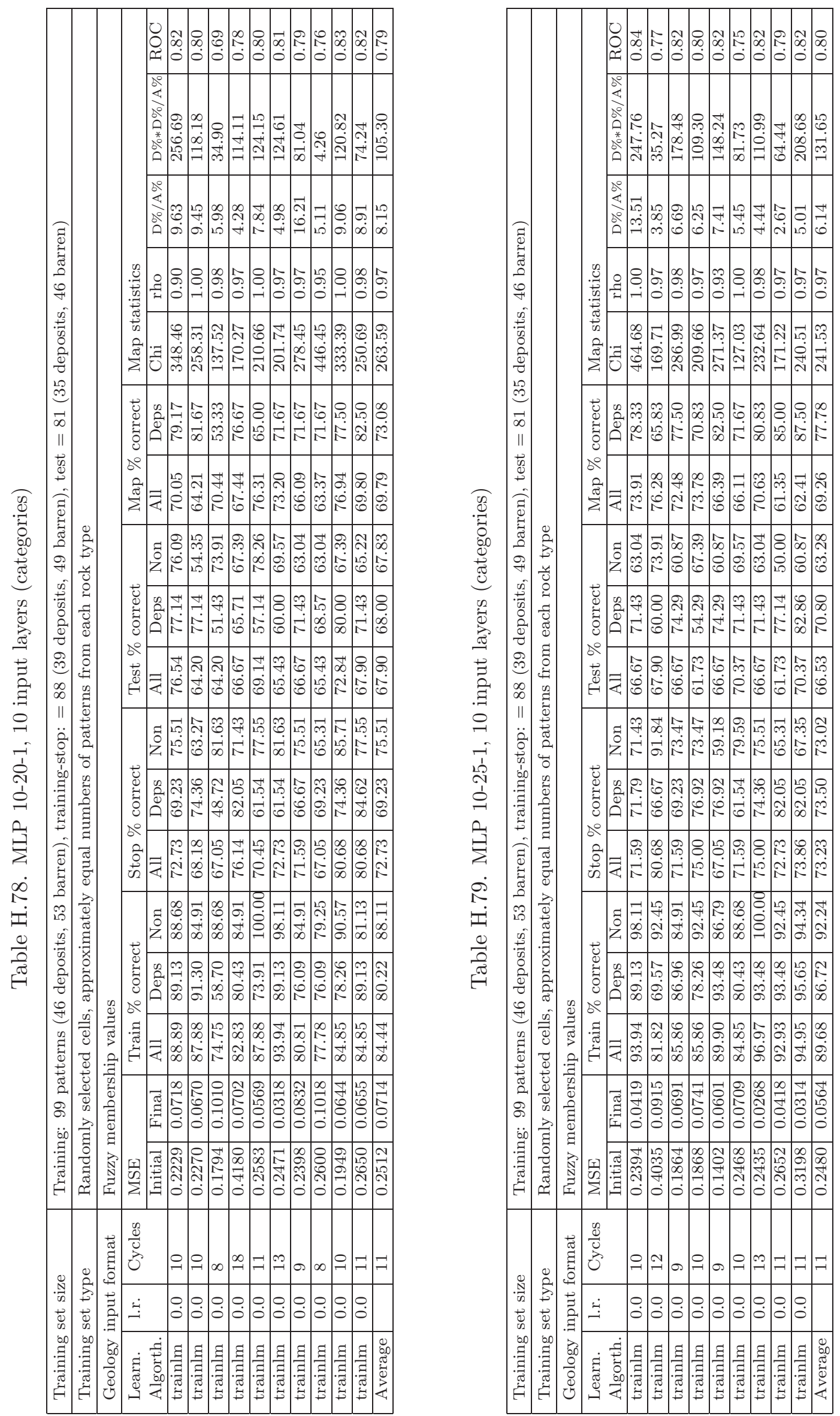

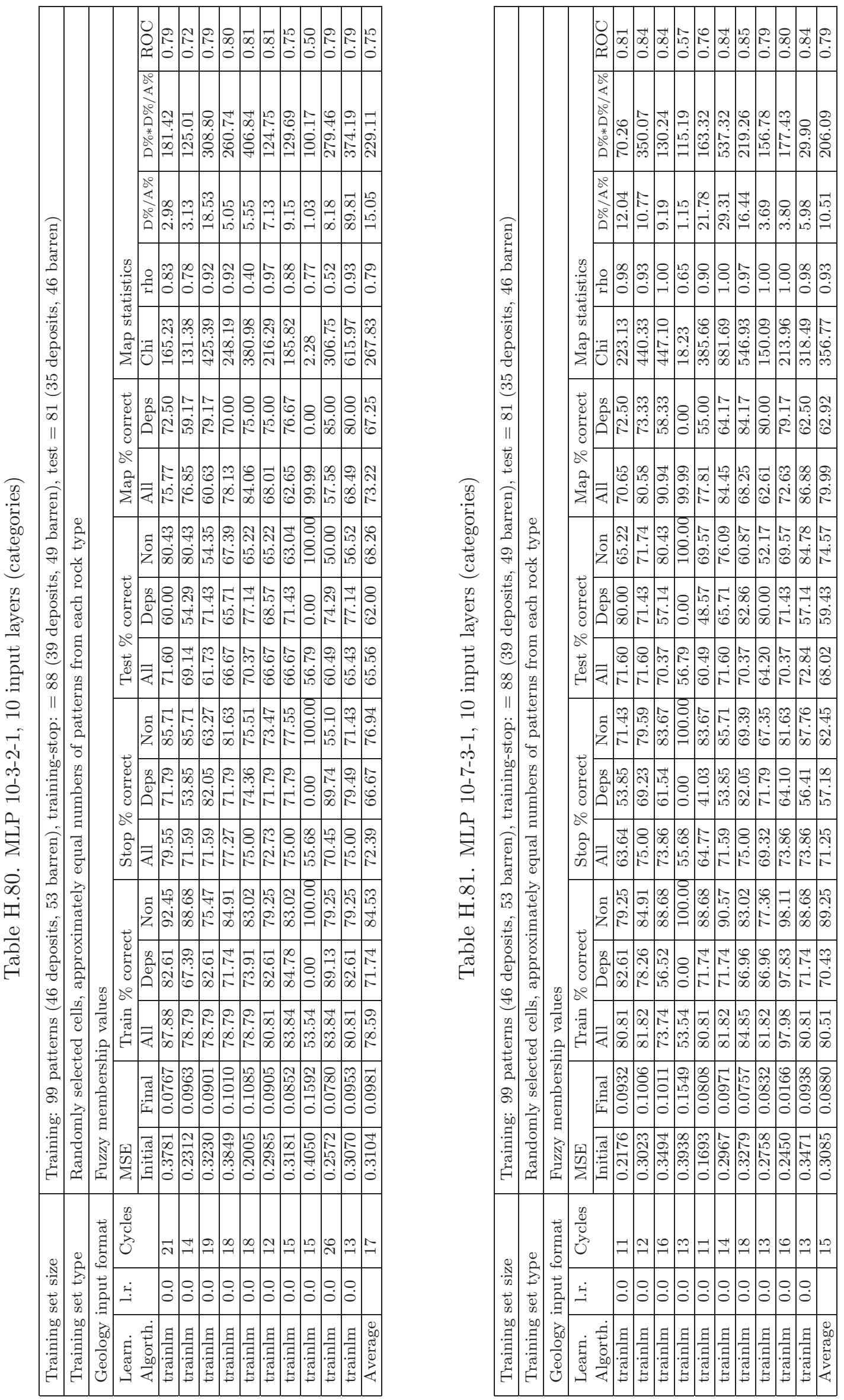

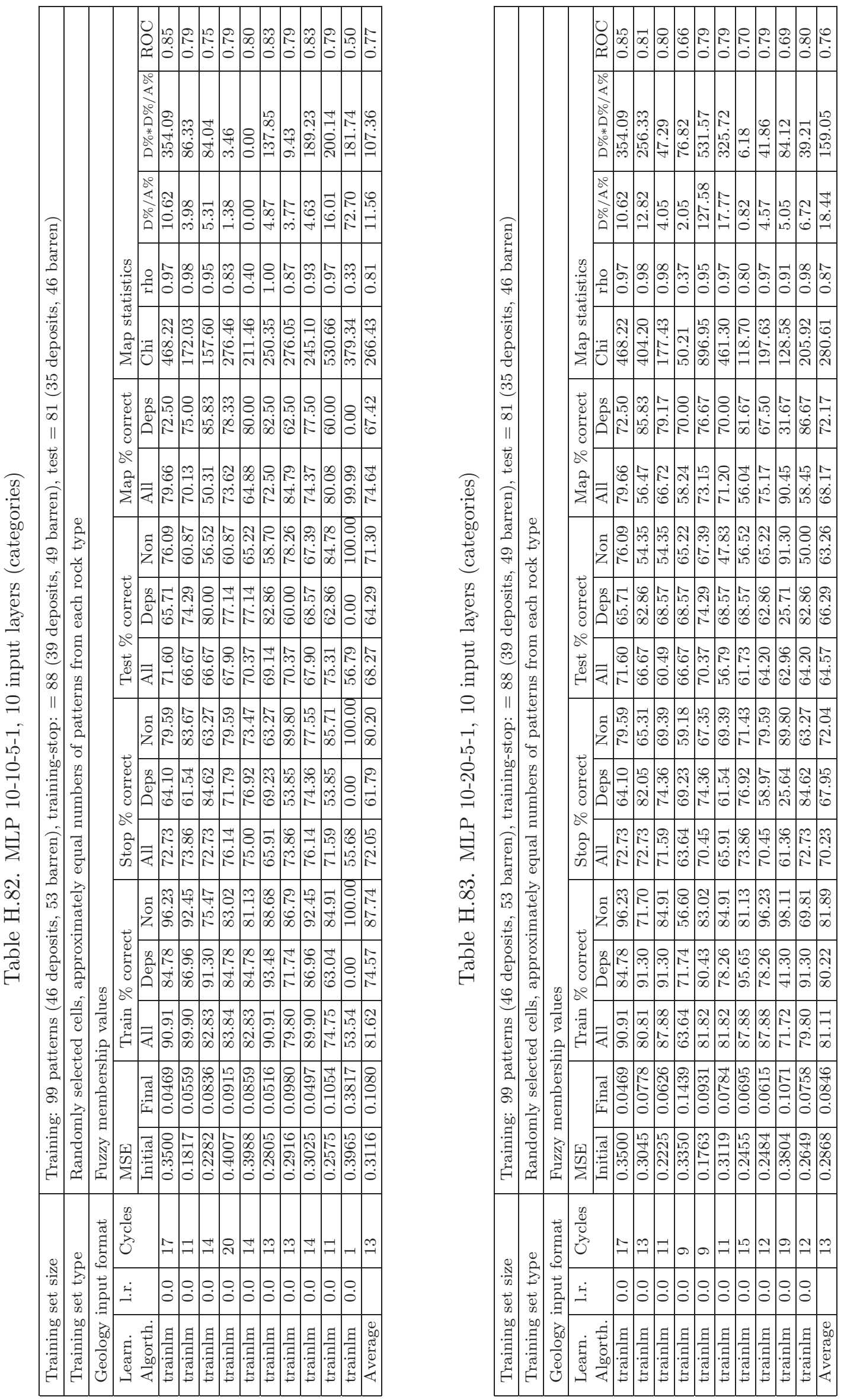

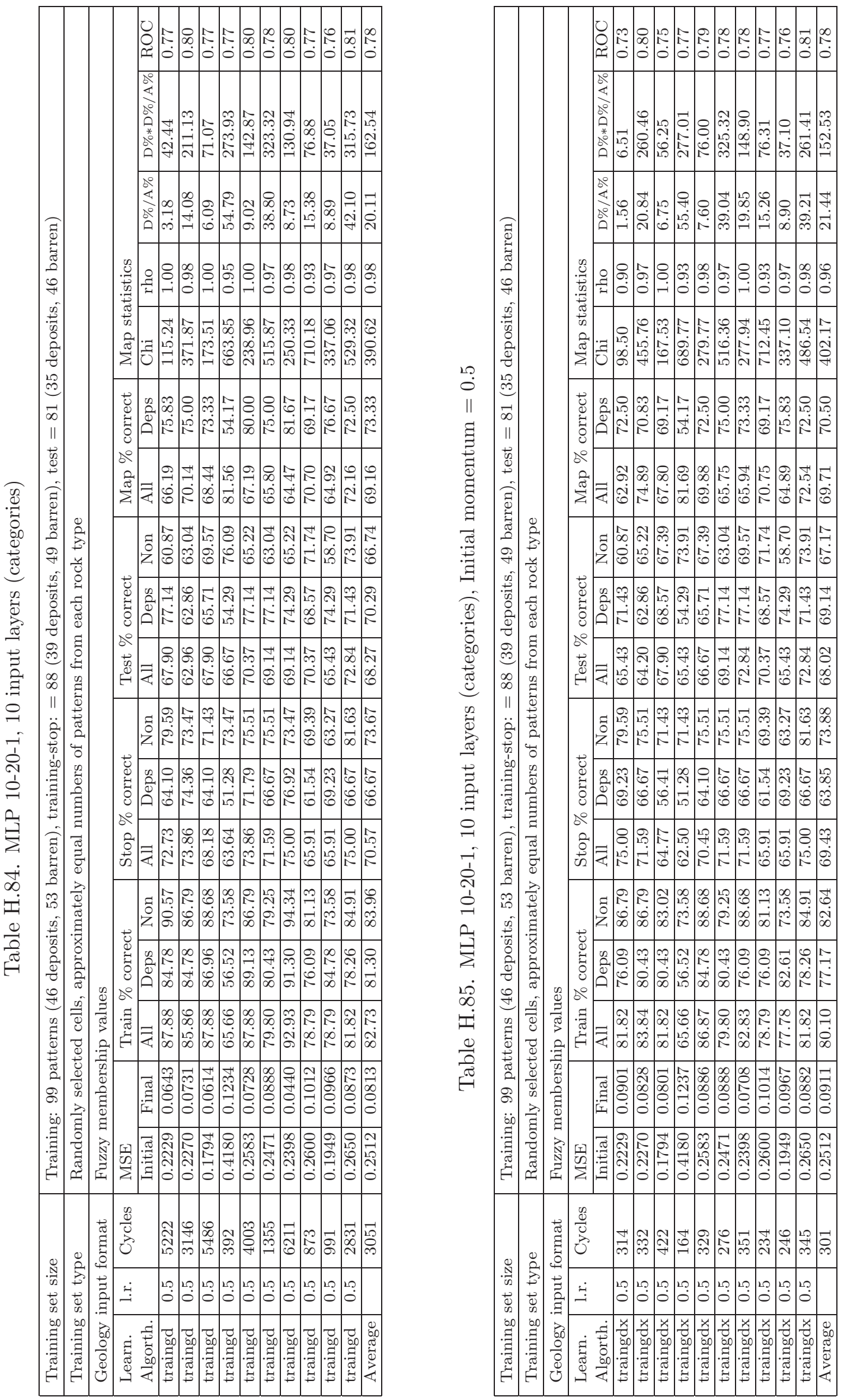

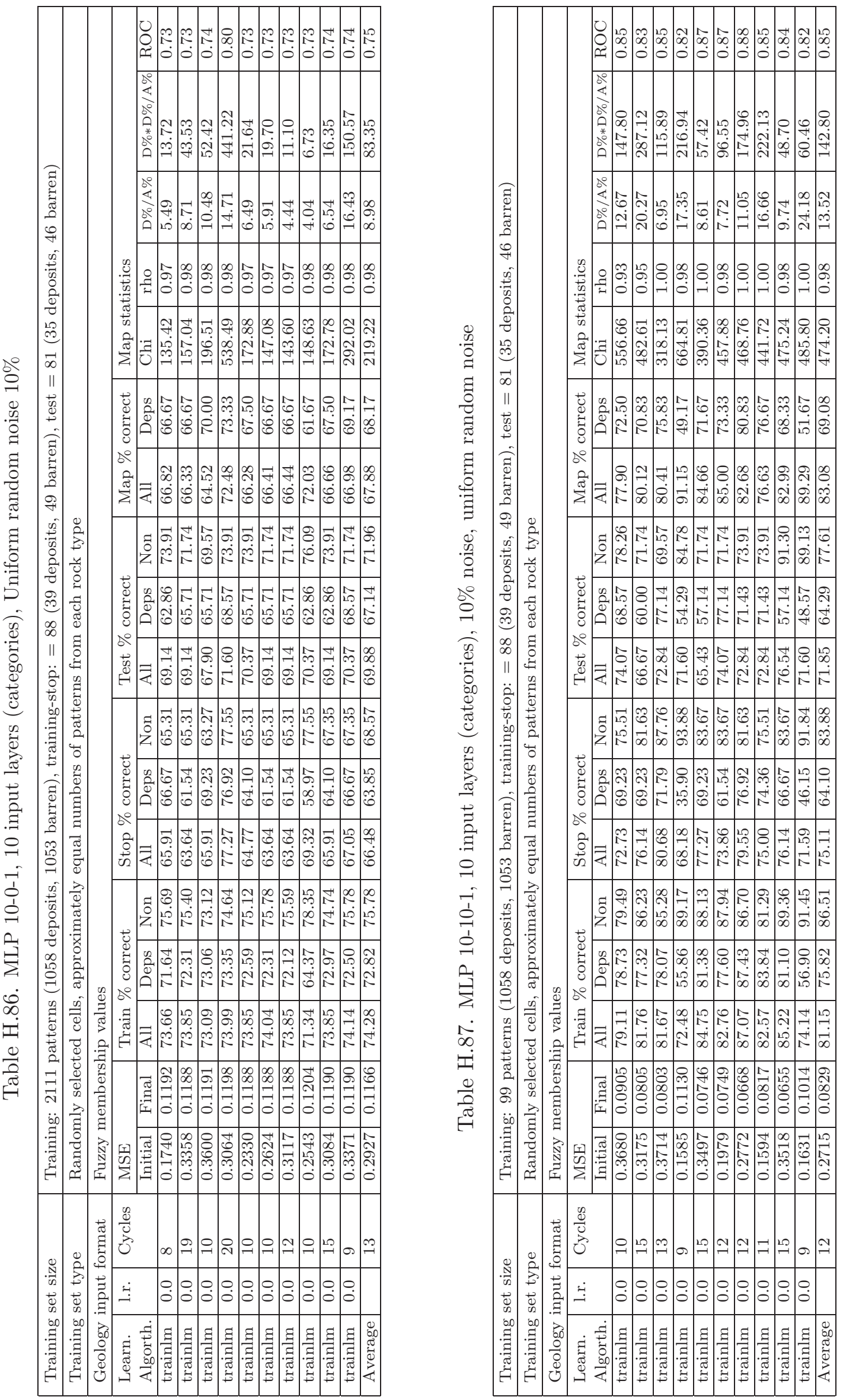

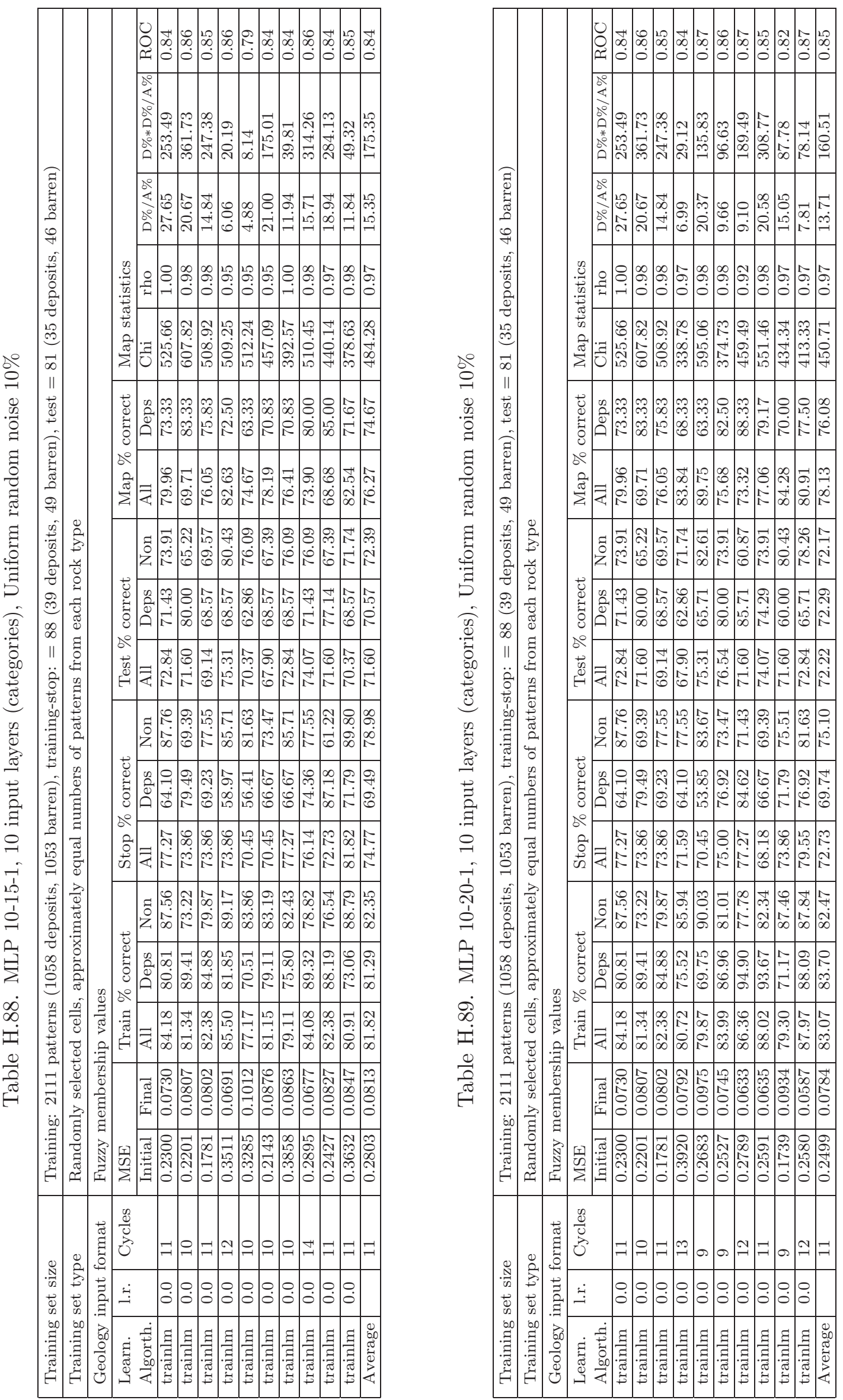

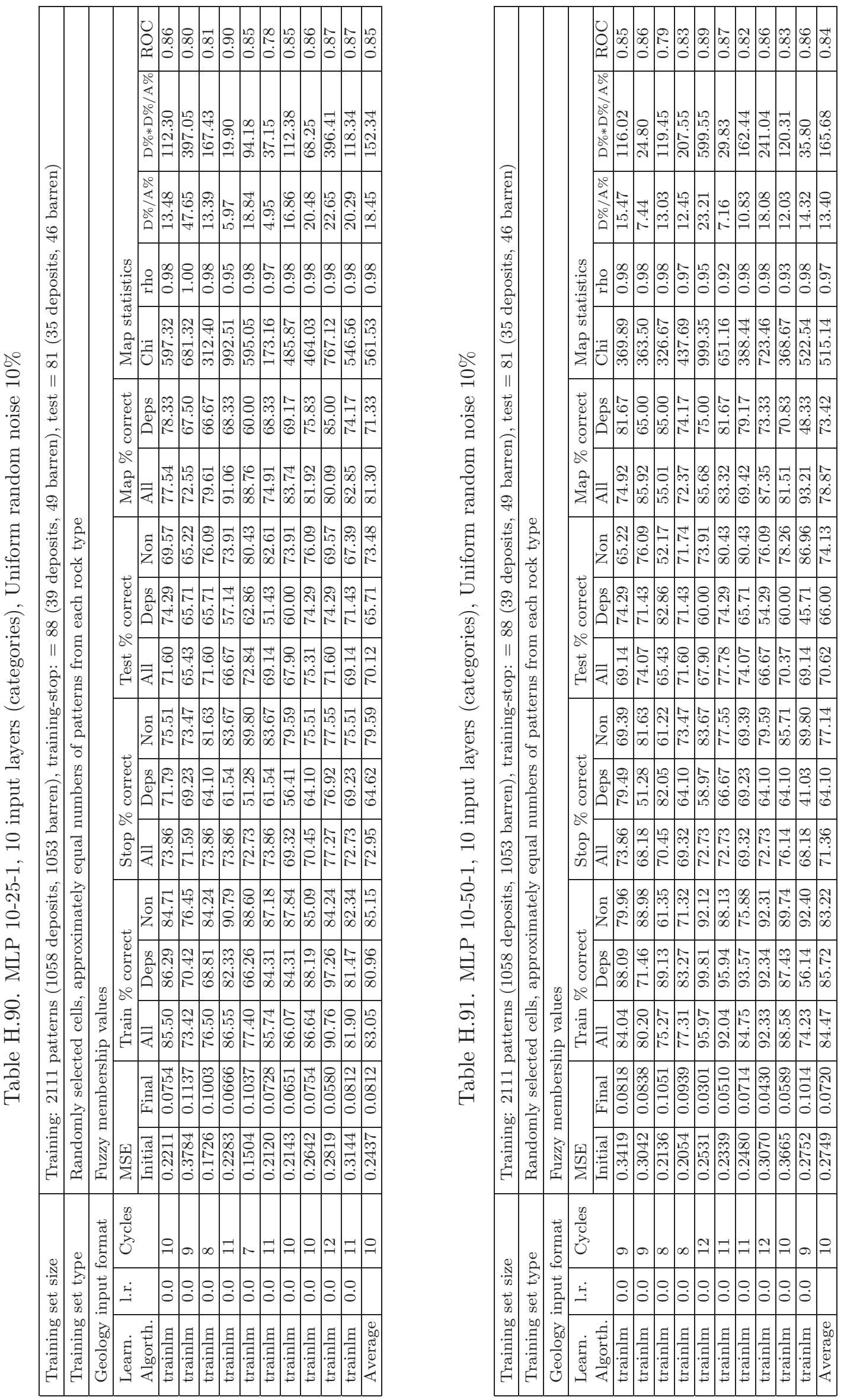

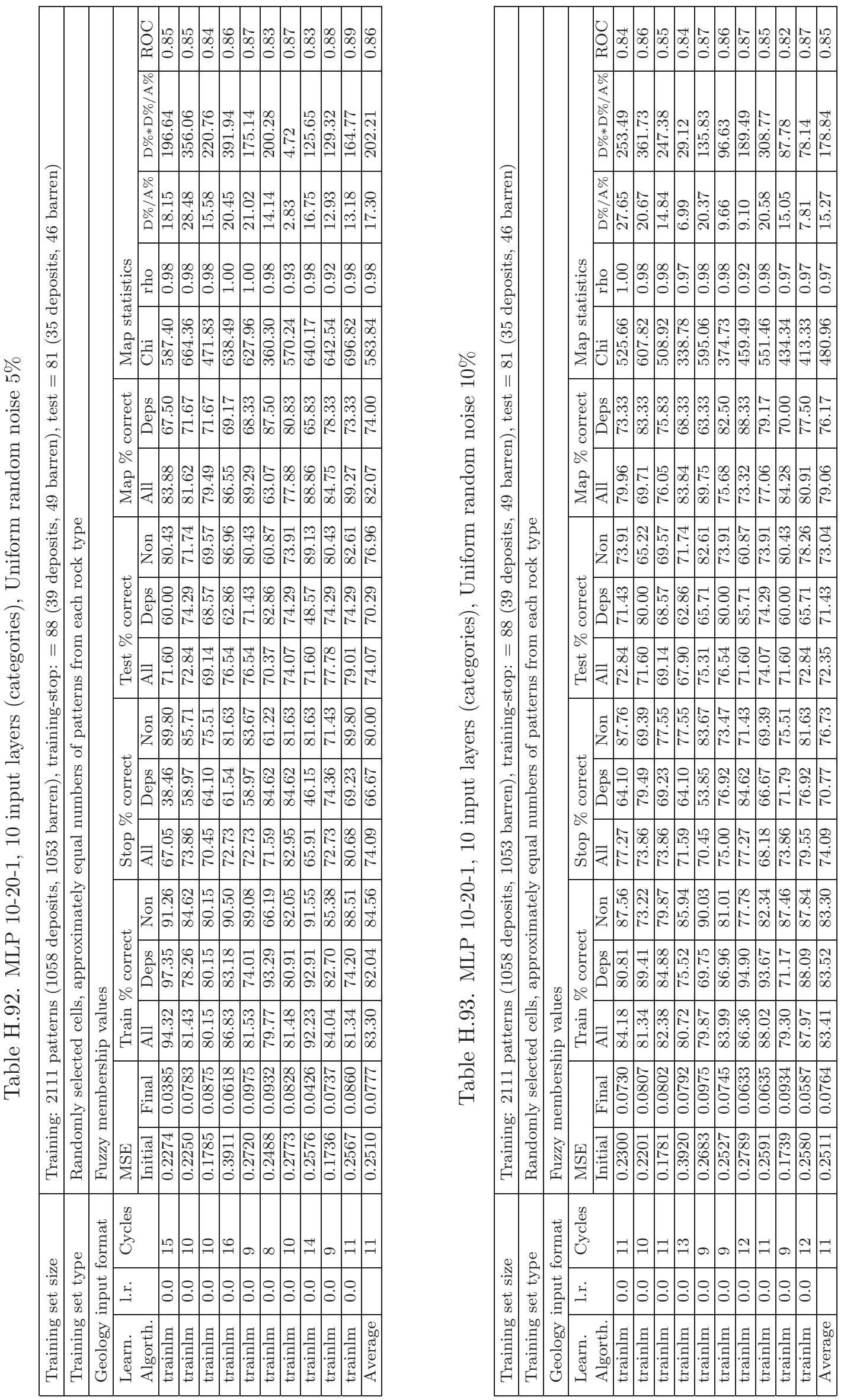

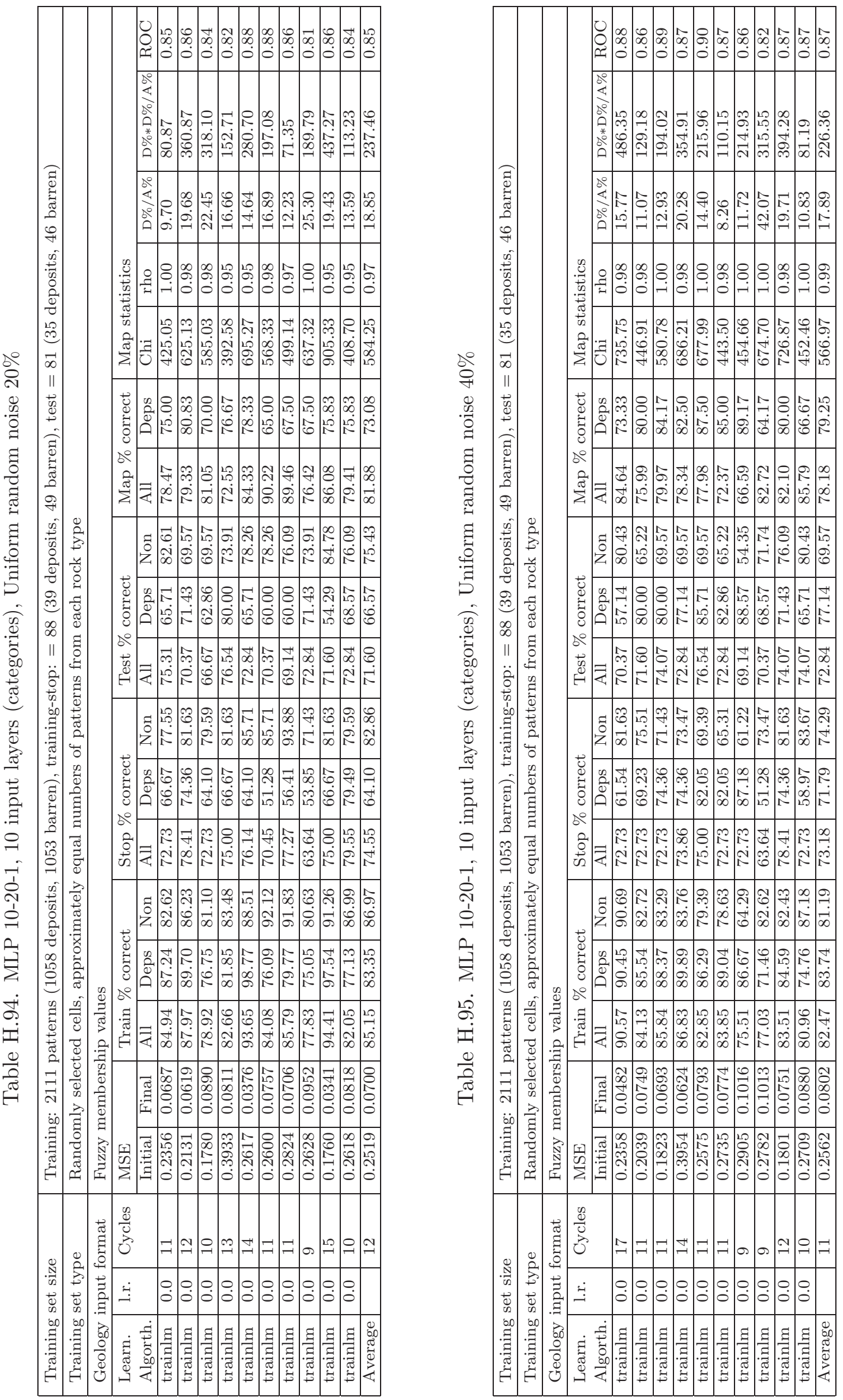

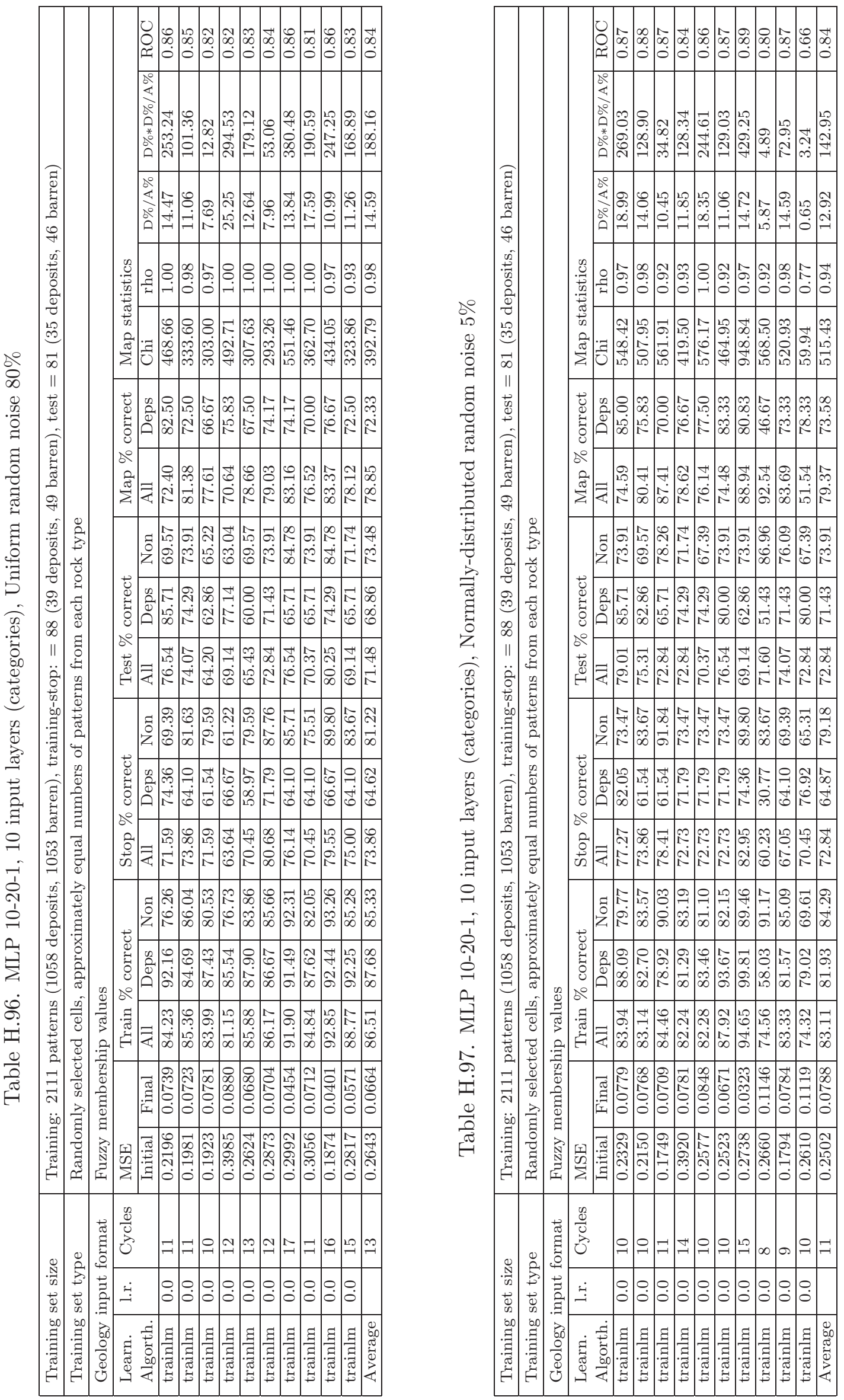

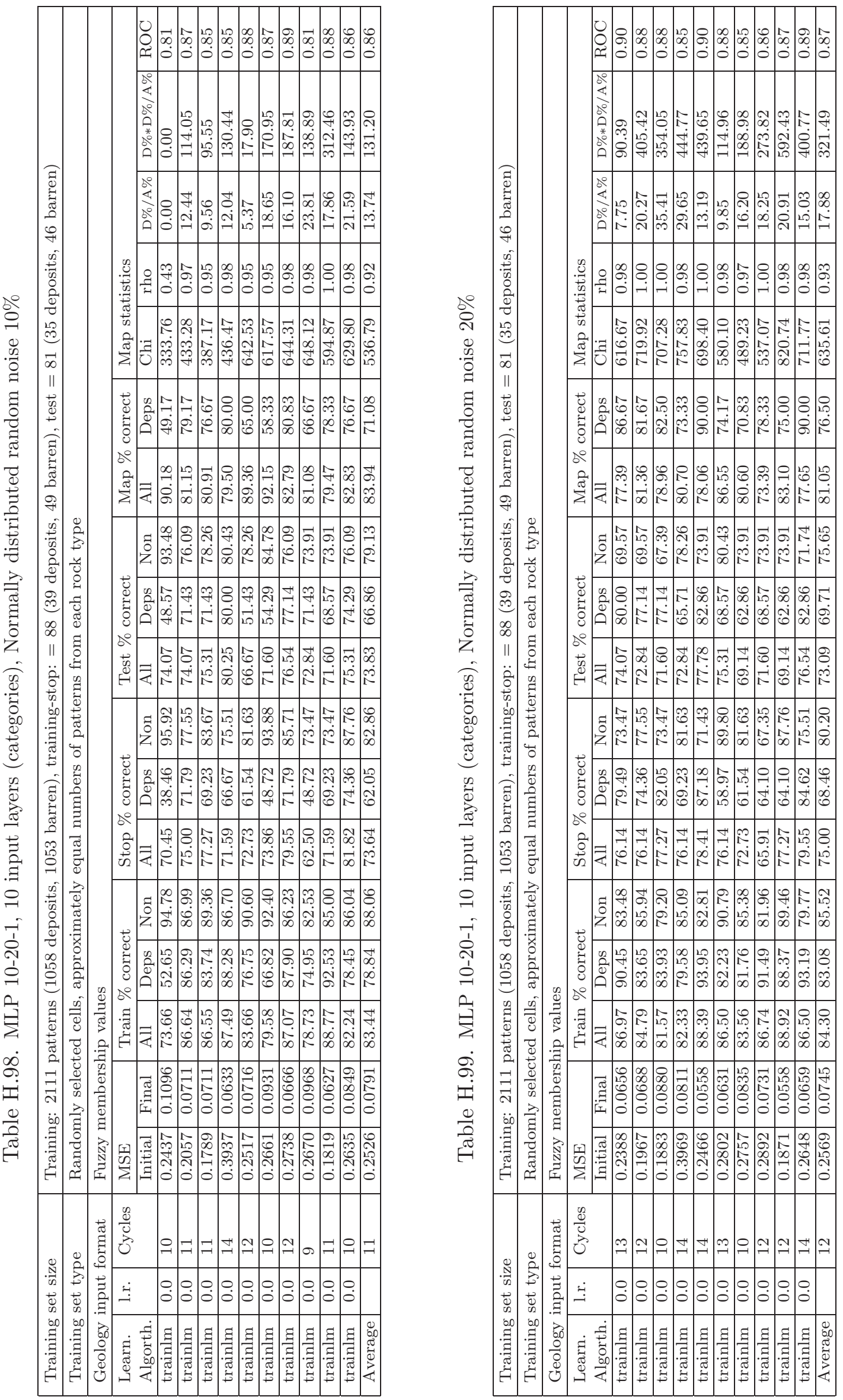

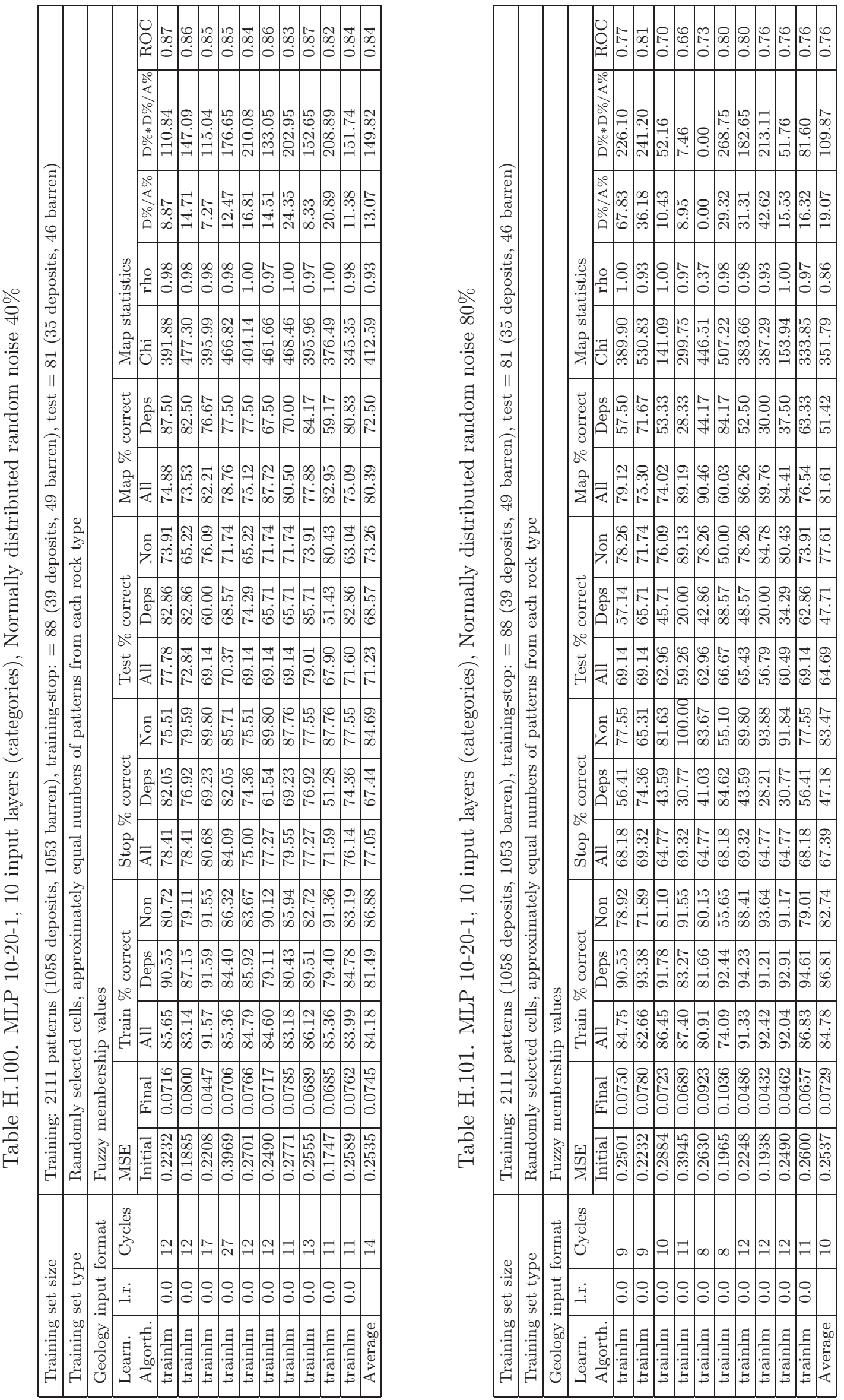

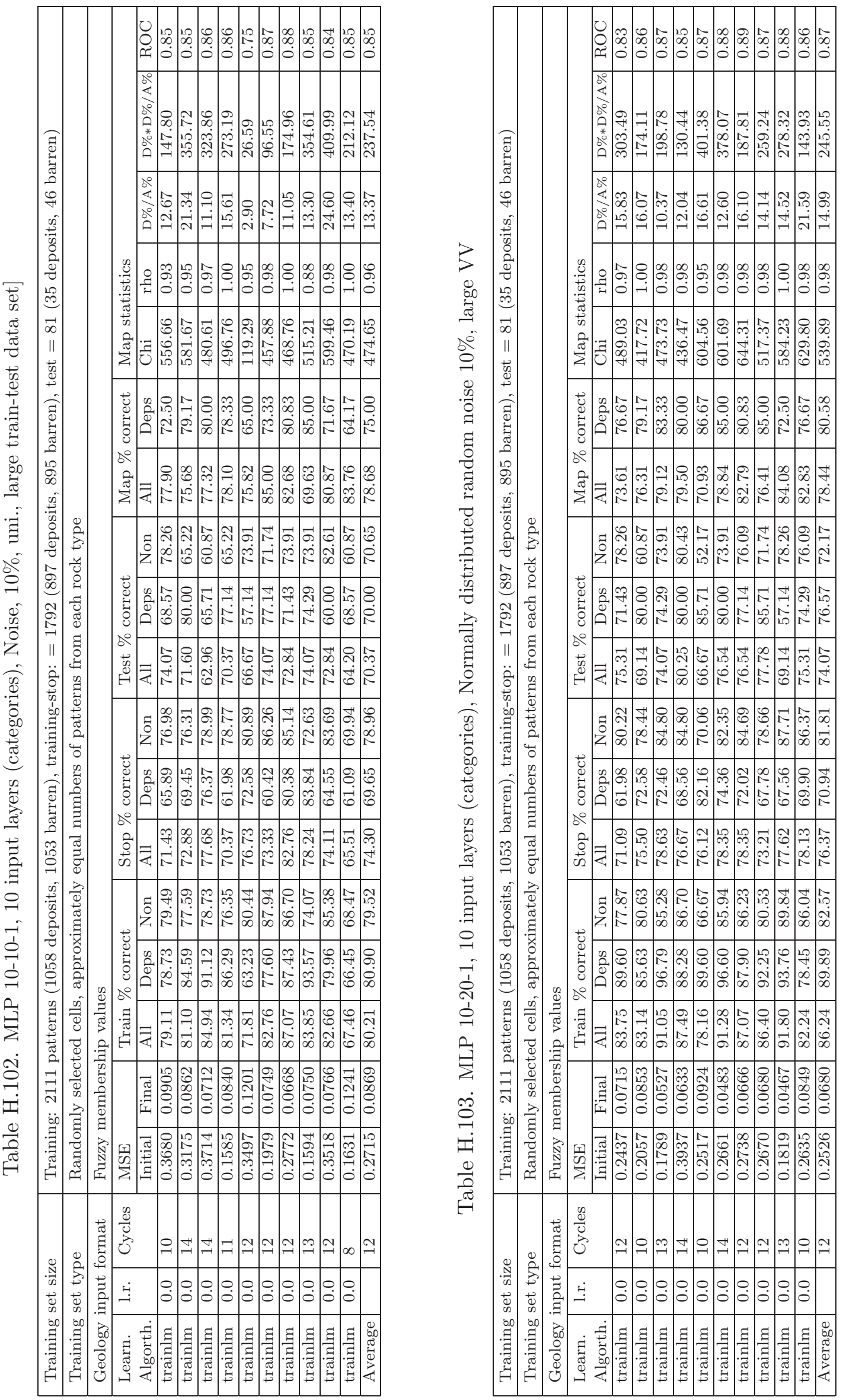

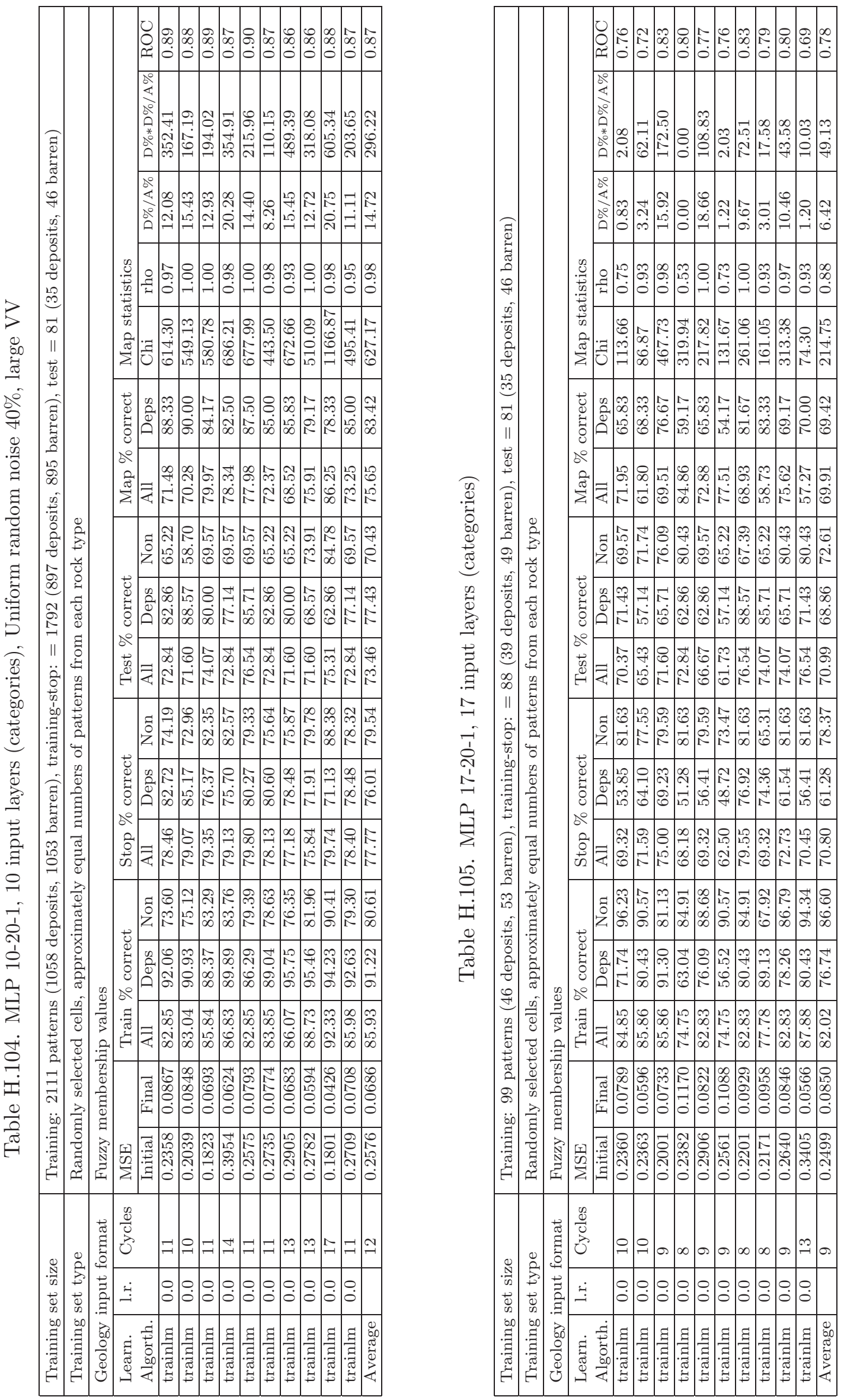

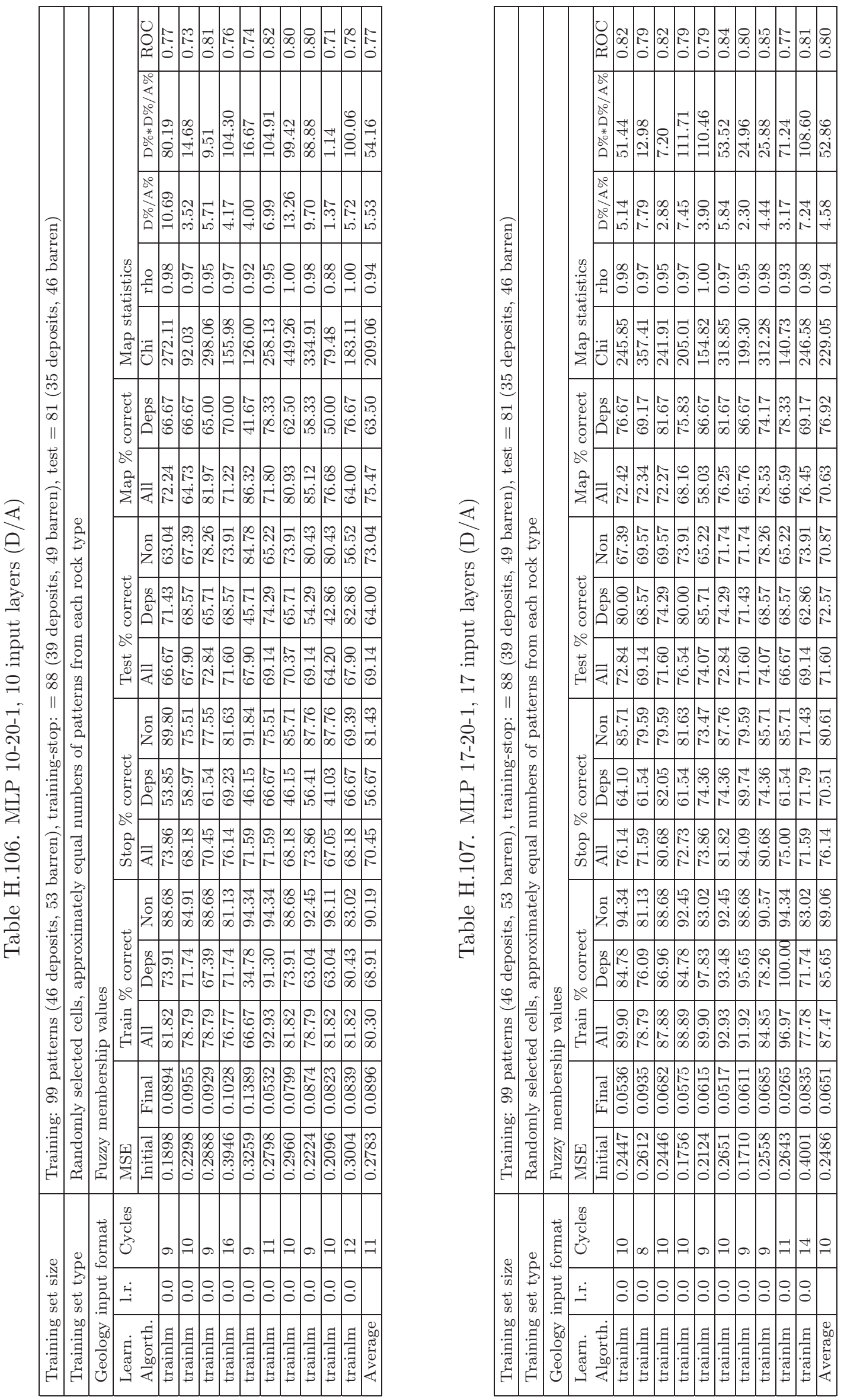

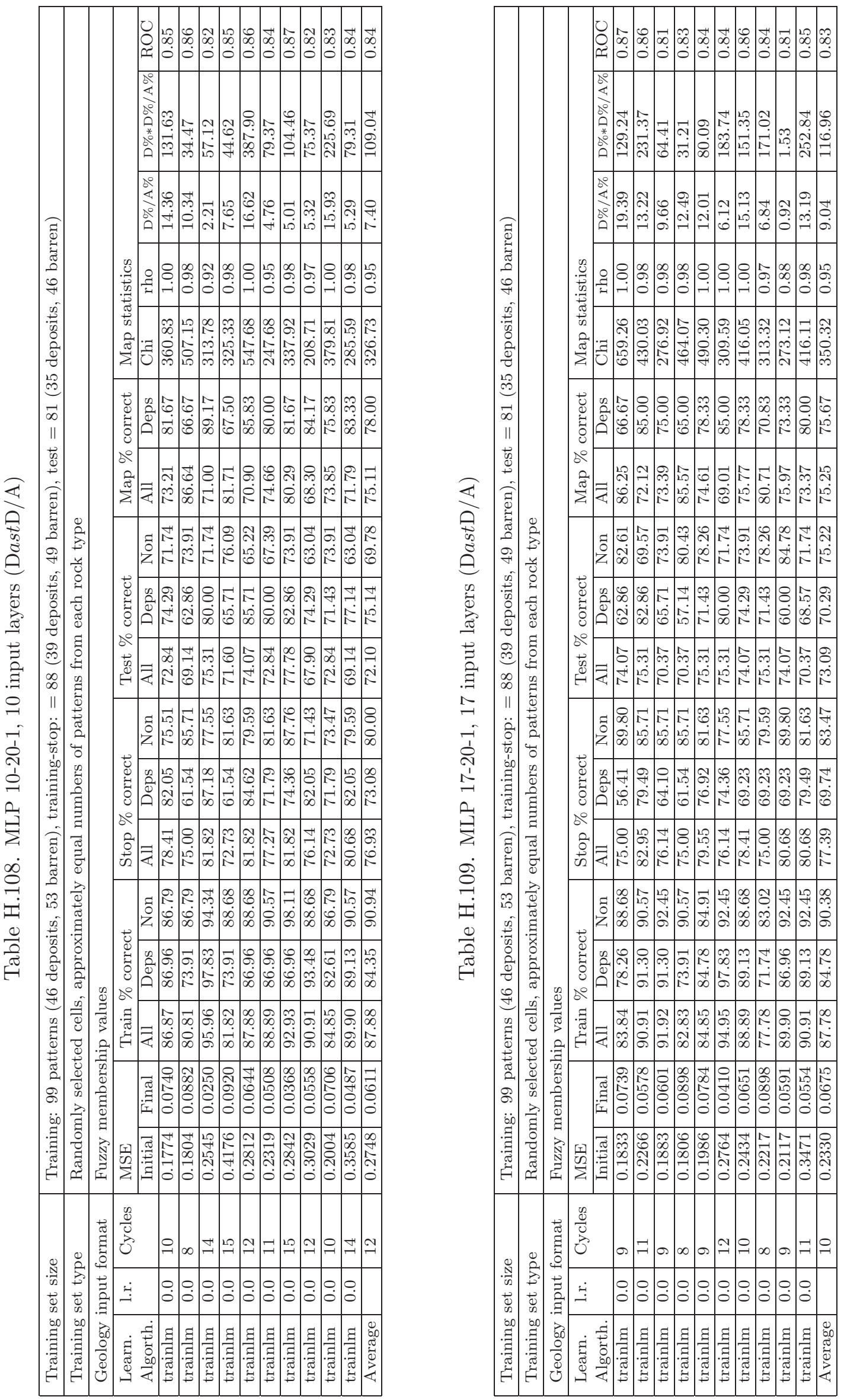

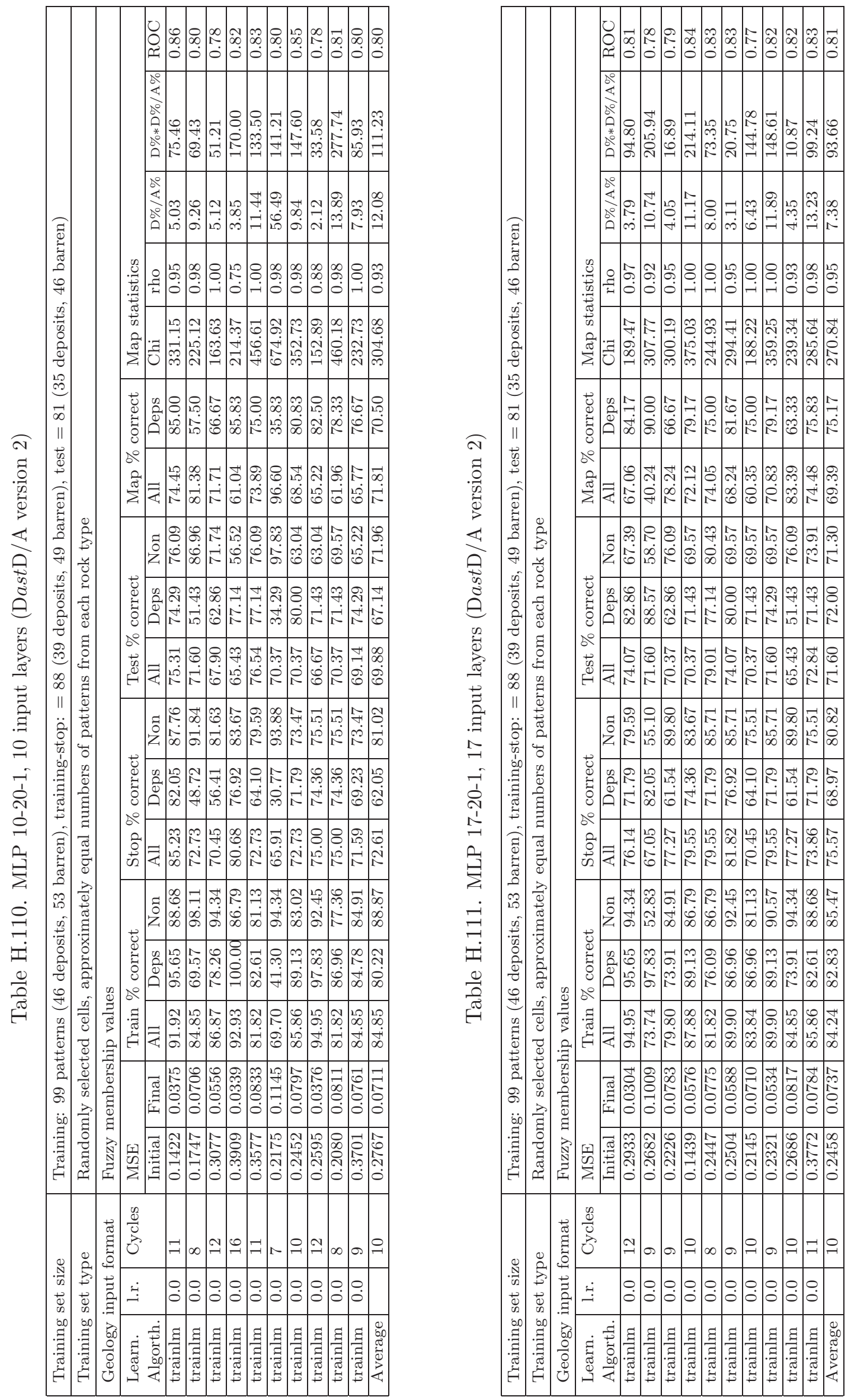

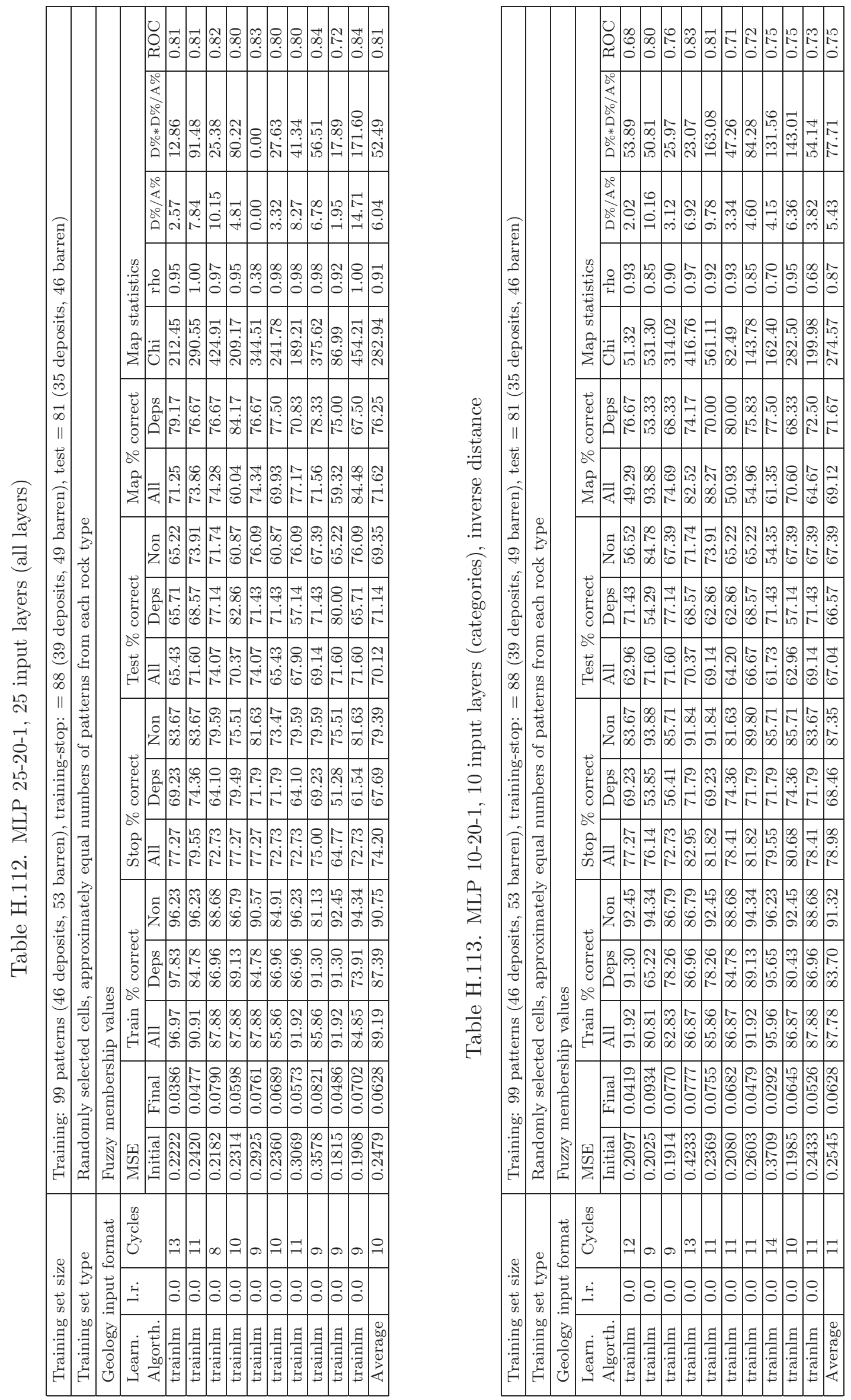

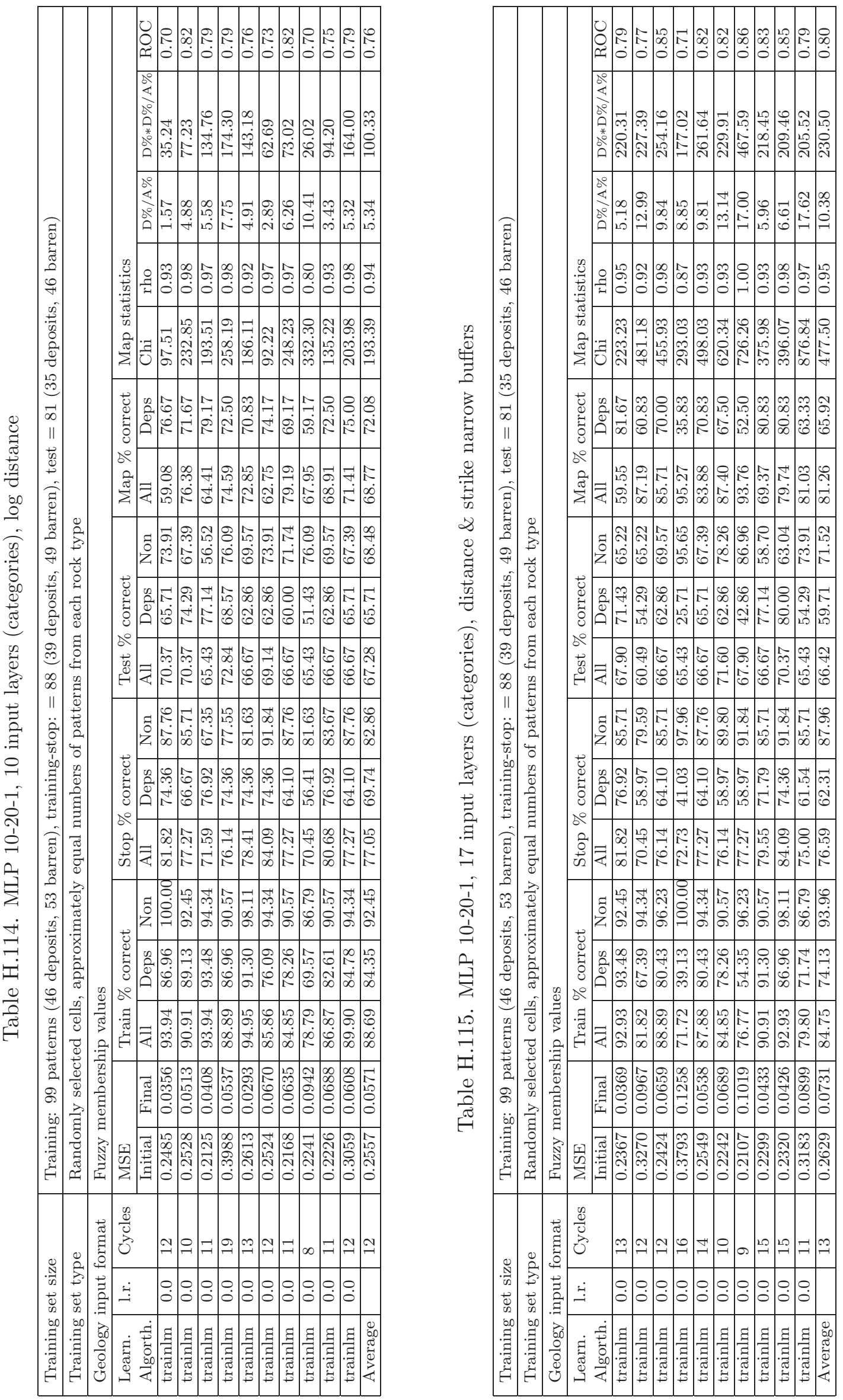

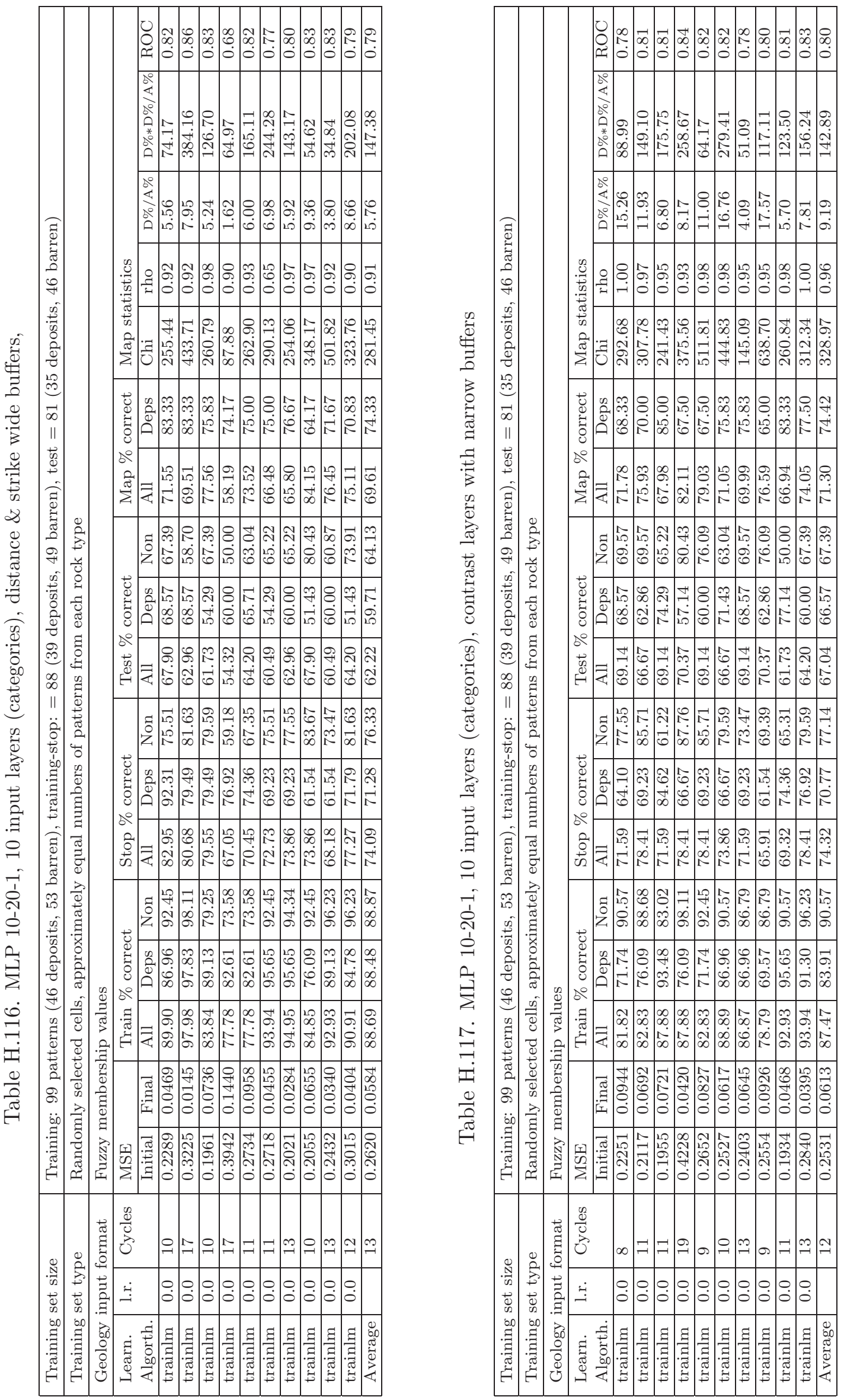

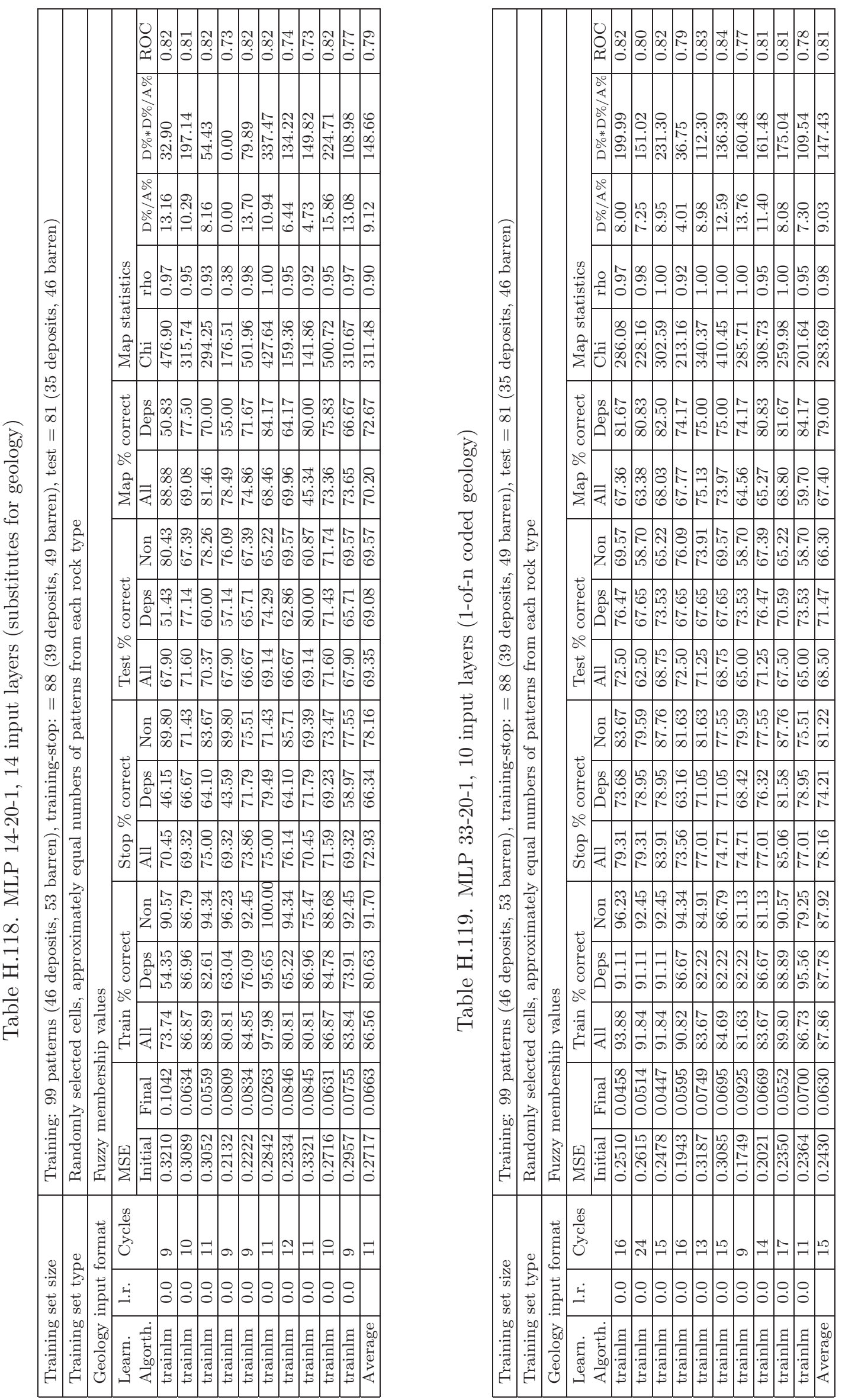

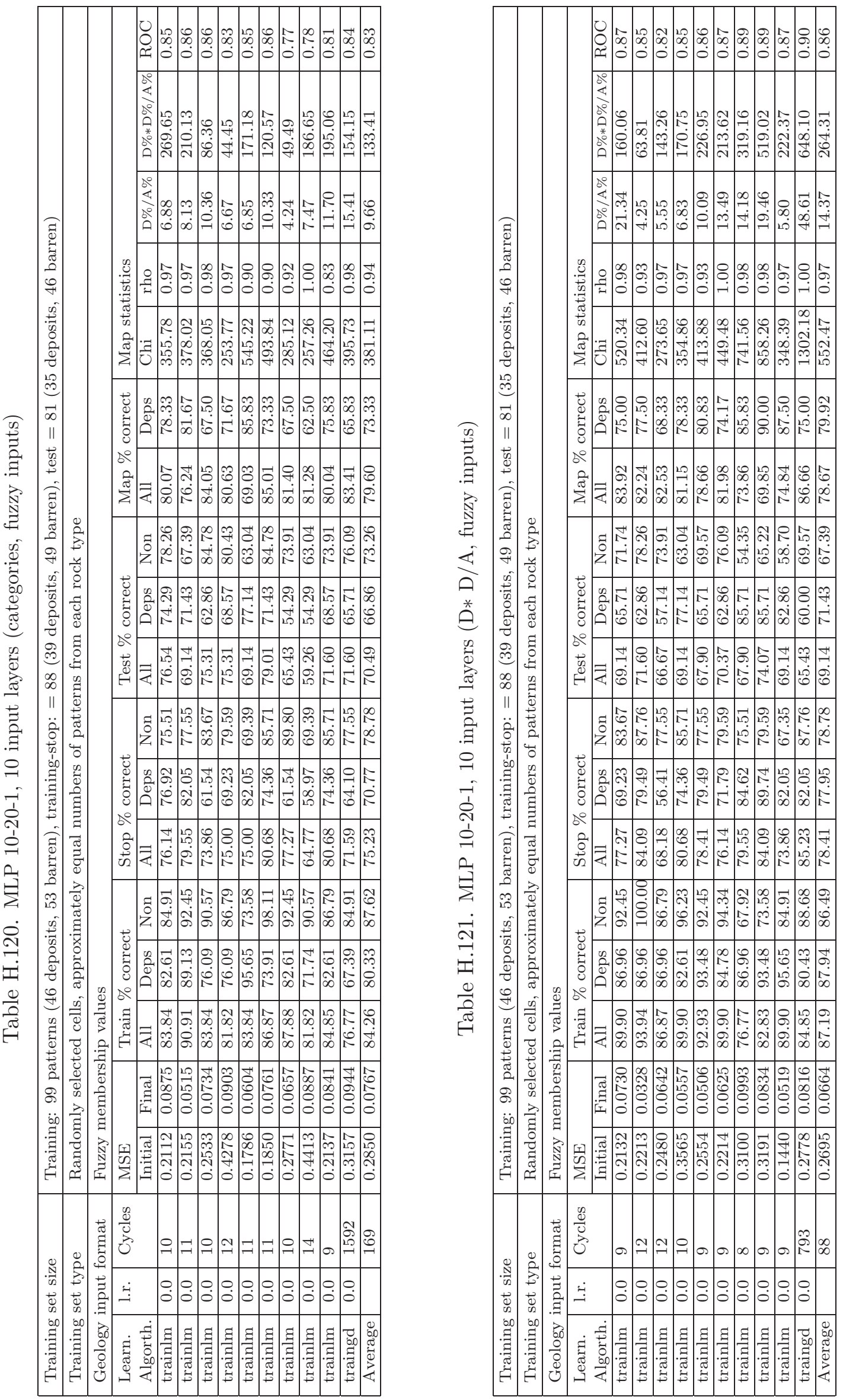

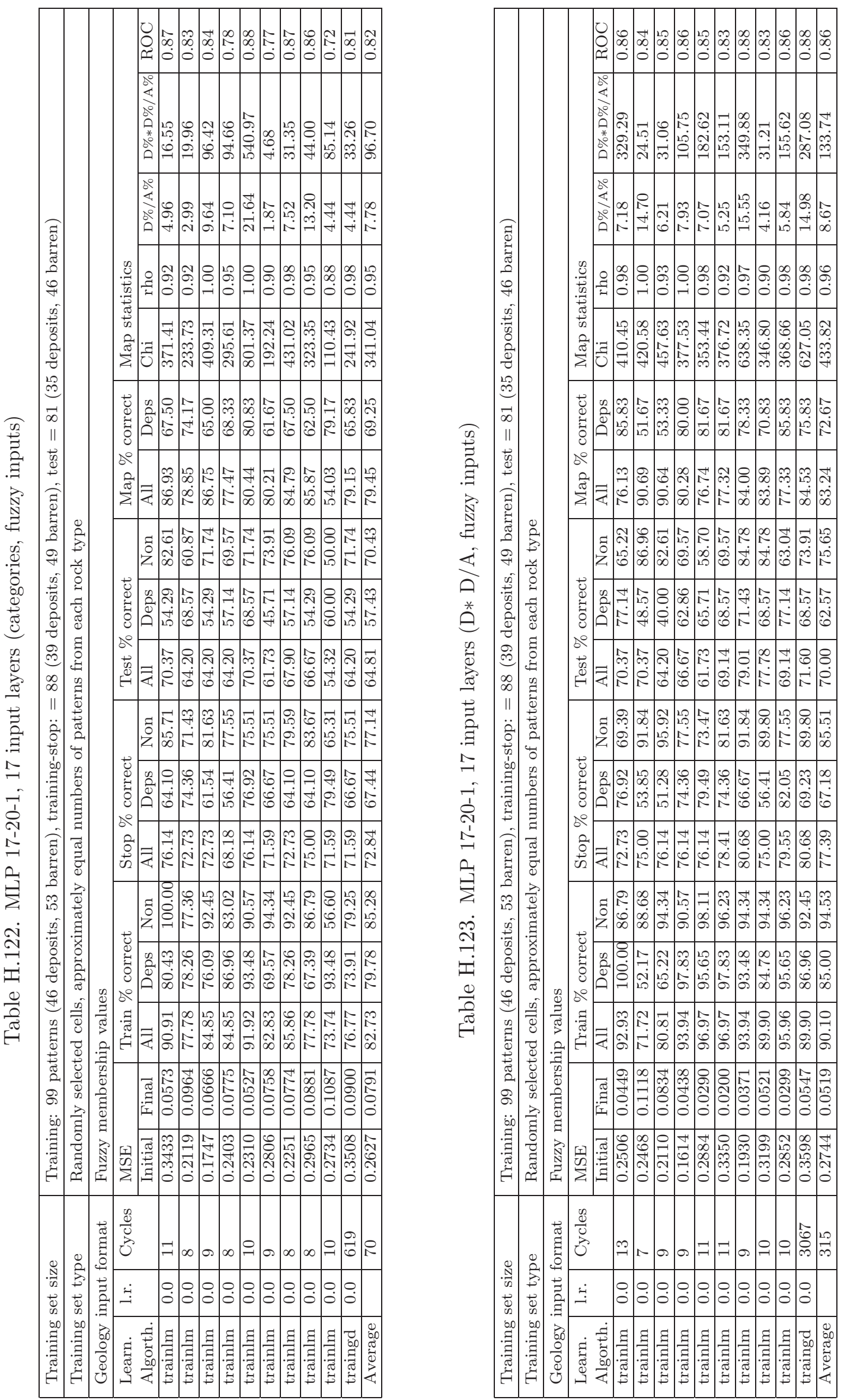
Appendix I

Results of Fuzzy Systems Experiments: Kalgoorlie Case Study

Results of Fuzzy Systems Experiments: Kalgoorlie Case Study 


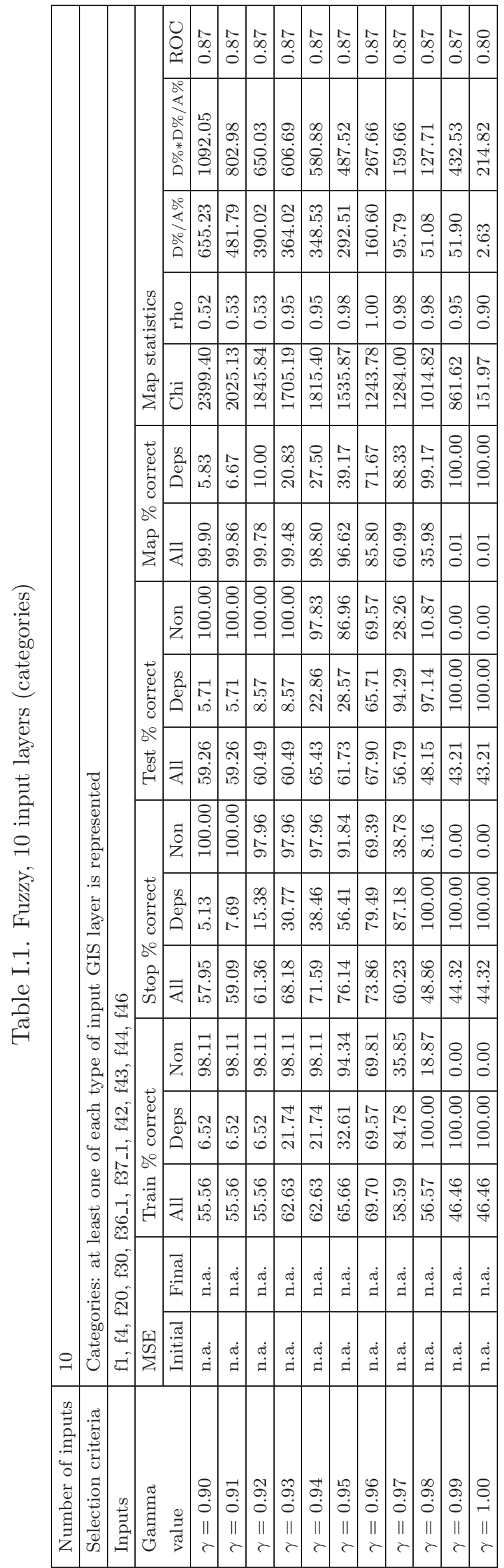




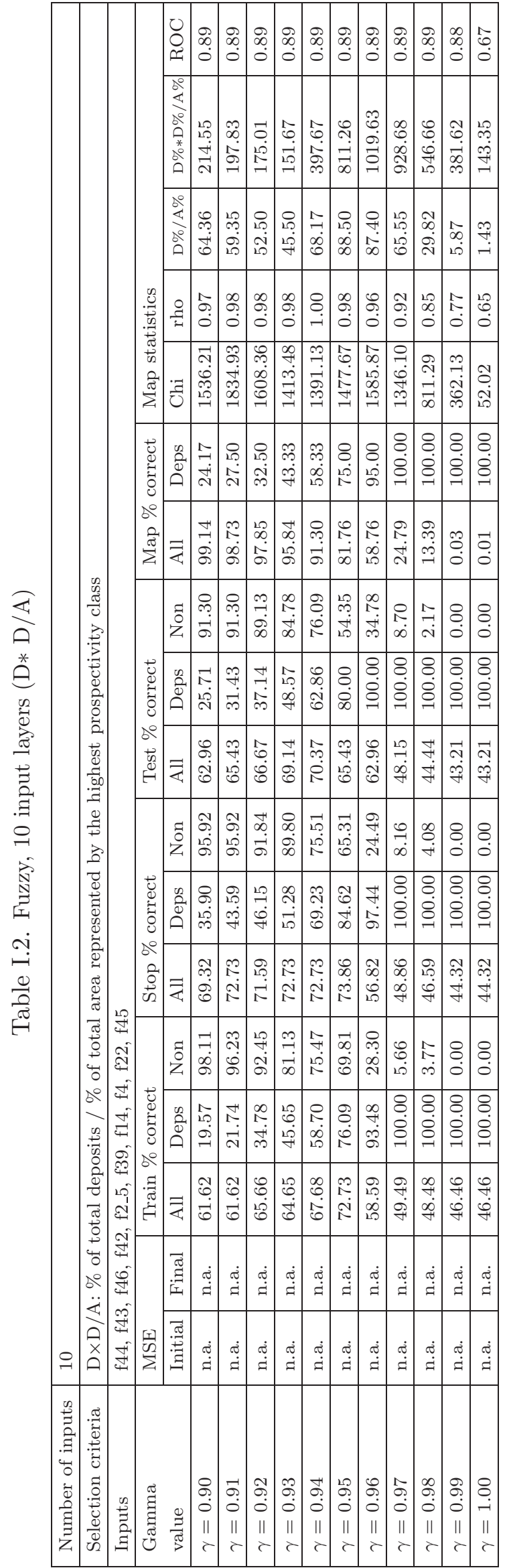




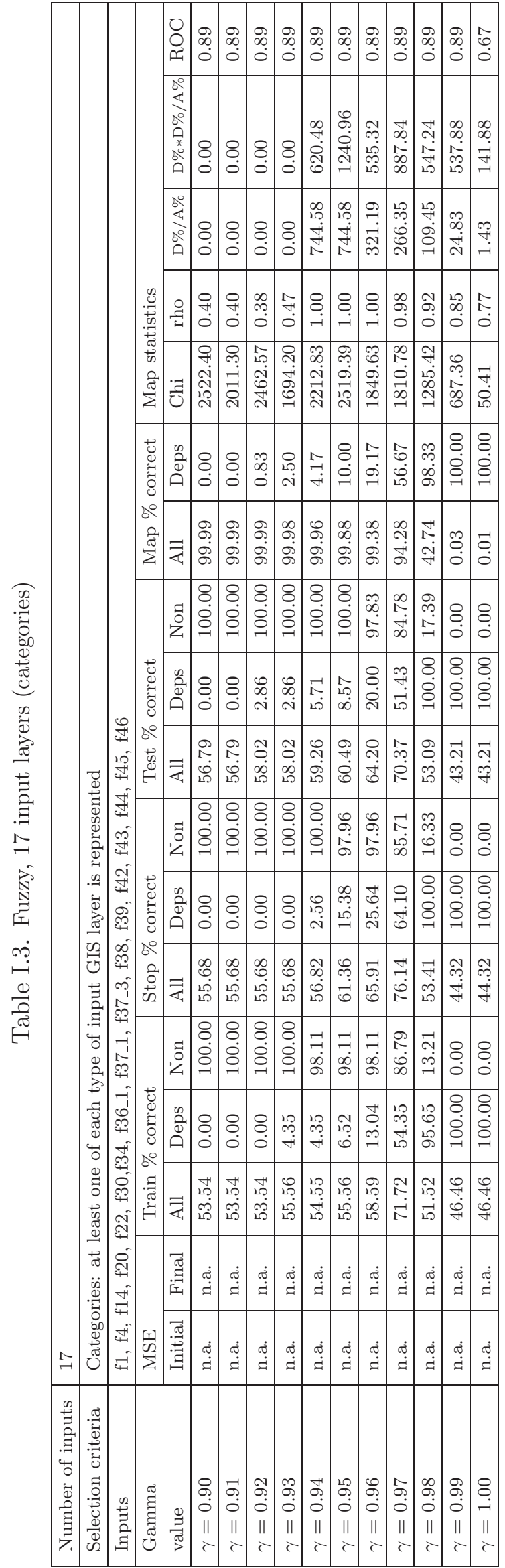




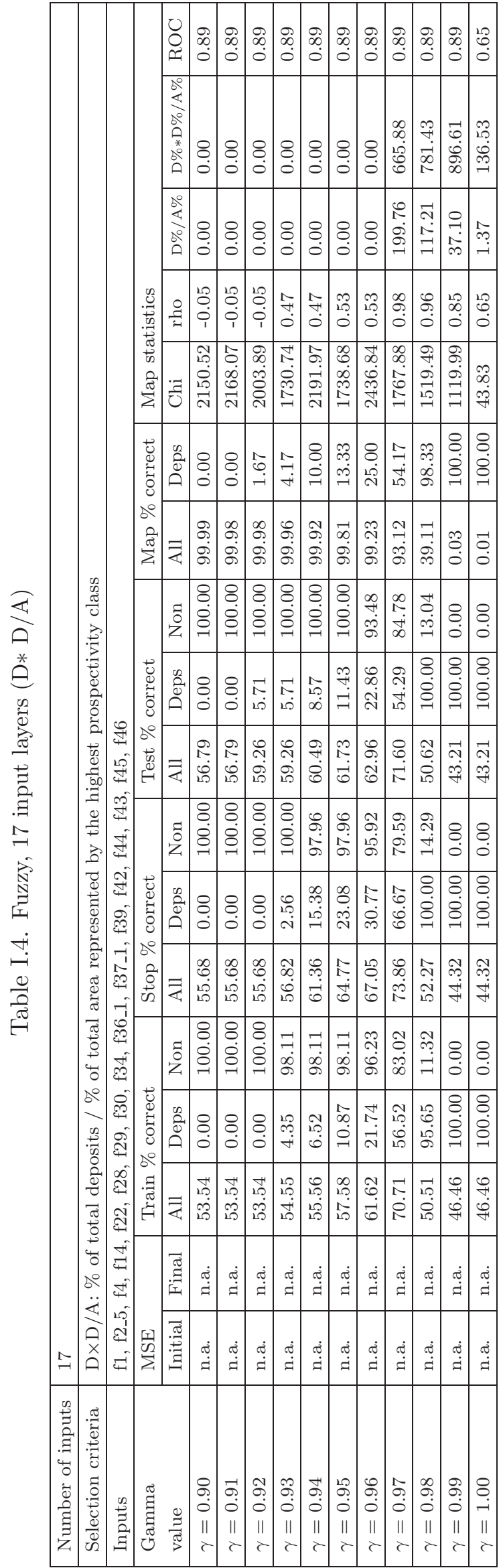




\section{Appendix J}

\section{Explanation of ArcView GIS Layer Names}

\section{J.1 Explanation of ArcView GIS Layer Names}

The directory "polygon_layers" contains vector data stored as grid files (suffix: .grd). The directory raster_layers contains raster data stored as shape files (suffix: .shp). Files with a ".avl "suffix are legend files and provide data used to colour the maps.

Table J.1: Explanation of ArcView GIS Layer Names

\begin{tabular}{|l|l|}
\hline Layer Name & Description \\
\hline Polygon Layers & (shape files) \\
\hline Augers & $\begin{array}{l}\text { Geochemistry; 13,503 hand-held auger drill holes, regolith, max. Au \& As assays } \\
(\mathrm{ppb}) \\
\text { Geochemistry; 112,366 percussion drill holes, max. Au \& As (ppb) at bottom of hole }\end{array}$ \\
AuAs & $\begin{array}{l}\text { Study area; geology clipped to area } \\
\text { Deposits: orogenic gold, 2603 mines, deposits and occurrences (1443 in study area) }\end{array}$ \\
Data_extent & $\begin{array}{l}\text { Deposits: as above but coverage is clipped to size of study area } \\
\text { Deps_all }\end{array}$ \\
$\begin{array}{l}\text { Deps_all_clip } \\
\text { Deps_1000tcg }\end{array}$ & $\begin{array}{l}\text { Faults: crustal-scale NNW } \\
\text { Faults: regional-scale NNW }\end{array}$ \\
D1 &
\end{tabular}


Table J.1: continued

\begin{tabular}{|l|l|}
\hline Layer Name & Description \\
\hline D3 & $\begin{array}{l}\text { Faults: regional-scale NE } \\
\text { Fuults: regional-scale E-W, oriented clipped to study area }\end{array}$ \\
D123 & $\begin{array}{l}\text { Faults: crustal-scale NNW, regional-scale NNW, and regional-scale NE } \\
\text { Faults }\end{array}$ \\
Ftruct_types & $\begin{array}{l}\text { Faults: all (not clipped to study area) } \\
\text { Folds }\end{array}$ \\
Folds_clipped & $\begin{array}{l}\text { Ground traces of anticlinal and synclinal axes (not clipped to study area) } \\
\text { Golid geology, all Goldfields Pty Ltd lease areas (completed 16.11.00). 108 rock types }\end{array}$ \\
Geology & $\begin{array}{l}\text { Mine outlines including open pits and mine dumps } \\
\text { Mineout-51 } \\
\text { Ozchem }\end{array}$
\end{tabular}

\section{Raster Layers (grid files)}

L1_l_ass_t25

L1_l_ass_t55

L1favgeol25pr

L2_l_rh1b

L2_l_rh2b

L2_l_rh3

L2_1_rh4

L2_l_rh5

L2_l_rh6

L2_l_rh7

L3_l_ch1

L3_l_ch2

L3_l_ch3

L3_l_ch4

L3_1_ch5

L4_l_pf

L4_l_pf_dist
Solid geology with a simplified 25 rock-type legend

Solid geology with a simplified 55 rock-type legend

Favourability of host rock-type based on $P$ (Deposit), fuzzy membership values

Rock strength - subjective estimate, fuzzy membership values

Rock strength - subjective estimate, 10 classes

Rock strength - Young's modulus, compilation by Hatheway (1989)

Rock strength - Youngs modulus, AMC Pty Ltd (Lee, 2000; unpublished data)

Rock strength - Uniaxial compressive strength

Rock strength - Uniaxial tensile strength

Rock strength - Fracture Toughness

Chemical ratio $-\mathrm{Fe}_{\text {total }}(\mathrm{wt} \%) \times \mathrm{Fe} /(\mathrm{Fe}+\mathrm{Mg}+\mathrm{Ca})$, second factor; elemental wt\%

Chemical ratio $-\mathrm{Fe} /(\mathrm{Fe}+\mathrm{Mg}+\mathrm{Ca})$, elemental wt\% ratio

Chemical ratio $-\mathrm{Fe}_{2} \mathrm{O}_{3} /\left(\mathrm{Fe}_{2} \mathrm{O}_{3}+\mathrm{FeO}\right)$

Chemical ratio $-\mathrm{Fe}_{2} \mathrm{O}_{3} / \mathrm{FeO}$

Chemical ratio $-\mathrm{Fe}^{3+} / \mathrm{Fe}^{2+}$, elemental wt\% ratio

Lithology; binary layer: porphyritic felsic intrusive rocks

Lithology; distance to nearest porphyritic felsic intrusive (rock types grouped; feldspar 
Table J.1: continued

\begin{tabular}{|c|c|}
\hline Layer Name & Description \\
\hline & $\begin{array}{l}\text { porphyry, feldspar quartz biotite porphyry, feldspar quartz hornblende porphyry, } \\
\text { feldspar } \\
\text { quartz porphyry, plagioclase }+/ \text { - hornblende porphyry }\end{array}$ \\
\hline L5_m_rtp & Airborne magnetic survey data; TMI, RTP, Kalgoorlie area \\
\hline L6_m_1vd & Airborne magnetic survey data; first vertical derivative \\
\hline L7_m_2vd & Airborne magnetic survey data; second vertical derivative \\
\hline L8_r_k & Airborne radiometric survey data; K-channel \\
\hline L9_r_th & Airborne radiometric survey data; Th-channel \\
\hline L10_r_tot & Airborne radiometric survey data; Th-channel \\
\hline L11_r_u & Airborne radiometric survey data; Th-channel \\
\hline L12_grav & Gravity; AGSO Bouguer gravity anomaly grid, Kalgoorlie area \\
\hline L13_lsat_tm & Landsat Thematic Mapper satellite image (composite) \\
\hline L14_m_z & Magnetic anomalies; z-scores for each rock type; $z=(x-$ mean $) / s$ \\
\hline L14_mz-1_dist & Magnetic anomalies; z-score $\leq-1$; distance to nearest magnetic anomaly \\
\hline L14_mz-1_bn01 & Magnetic anomalies; z-score $\leq-1$; binary layer; values; 0,1 \\
\hline L14_mz-1bn1nl & Magnetic anomalies; z-score $\leq-1$; binary layer; values; null, 1 \\
\hline L14_mz1_dist & Magnetic anomalies; z-score $\geq+1$; distance to nearest magnetic anomaly \\
\hline L14_mz1_lt1 & Magnetic anomalies; z-score $\geq+1$; distance to nearest anomaly $<1000 \mathrm{~m}$ \\
\hline L14_mz1_lt15 & Magnetic anomalies; z-score $\geq+1$; distance to nearest anomaly $<1500 \mathrm{~m}$ \\
\hline L14_mz1_lt2 & Magnetic anomalies; z-score $\geq+1$; distance to nearest anomaly $<2000 \mathrm{~m}$ \\
\hline L14_mz1_bn01 & Magnetic anomalies; z-score $\geq+1$; binary layer; values; 0,1 \\
\hline L14_mz1bn1nl & Magnetic anomalies; z-score $\leq+1$; binary layer; values; null, 1 \\
\hline L14_mz2_dist & Magnetic anomalies; z-score $\geq 2$; distance to nearest anomaly \\
\hline L14_mz2dist15 & Magnetic anomalies; z-score $\geq+2$, distance to nearest anomaly $<1500 \mathrm{~m}$ \\
\hline L14_mz2dist2 & Magnetic anomalies; z-score $\geq+2$, distance to nearest anomaly $<2000 \mathrm{~m}$ \\
\hline L14_mz2dist25 & Magnetic anomalies; z-score $\geq+2$, distance to nearest anomaly $<2500 \mathrm{~m}$ \\
\hline L14_mz2bn1nul & Magnetic anomalies; z-score $\geq+2$, binary layer; values; null, 1 \\
\hline L15_m_zint & Magnetic anomalies; rounded to integers \\
\hline L16_as_int & Auger drill hole arsenic (ppb), max. at bottom of hole; interpolation method 1 \\
\hline
\end{tabular}


Table J.1: continued

\begin{tabular}{|c|c|}
\hline Layer Name & Description \\
\hline L16_as_fint & $\begin{array}{l}\text { Auger drill hole arsenic (ppb); max. at bottom of hole; interpolation method } 2 \\
\text { (filtered) }\end{array}$ \\
\hline L17_au_int & Auger drill hole gold (ppb); max. at bottom of hole; interpolation method 1 \\
\hline L18_l_mdql & Lithology; binary layer: quartz dolerite and leucocratic dolerite \\
\hline L18_l_mdql-dist & Lithology; distance to nearest quartz dolerite and leucocratic dolerite \\
\hline L19_l_gsy & Lithology; binary layer: syenite and syenodiorite units \\
\hline L19_l_gsy-dist & Lithology; distance to nearest syenite and syenodiorite unit \\
\hline L20_f1_dist & Faults; distance to nearest crustal-scale NNW fault \\
\hline L20_s_crust & Faults; crustal-scale NNW faults (raster format) \\
\hline L21_s_shear & Faults; regional-scale NNW faults (raster format) \\
\hline L21_f2_dist & Faults; distance to nearest regional-scale NNW fault \\
\hline L22_d3_ns & $\begin{array}{l}\text { Faults; binary layer; regions north \& south of the nearest NE-trending regional-scale } \\
\text { fault }\end{array}$ \\
\hline L22_s_flt_ne & Faults: regional-scale NE faults (raster format) \\
\hline L22_d3_dist & Faults; distance to nearest regional-scale NE fault \\
\hline L23_s_flt_ne & Faults: regional-scale E-W faults (raster format) \\
\hline L23_d4_dist & Faults; distance to nearest regional-scale E-W fault \\
\hline L24_d_all_bin & Deposits; binary layer, all deposits \\
\hline L24_d_allbn_nd & Deposits; binary layer, all deposits, areas of Nodata excluded \\
\hline L24_d_bin1000 & Deposits; binary layer, deposits with total contained gold $\geq 1000 \mathrm{~kg}$ \\
\hline L24_d_bin3000 & Deposits; binary layer, deposits with total contained gold $\geq 3110 \mathrm{~kg}(100000 \mathrm{oz})$ \\
\hline L24_d_freq & Deposits; number of deposits in each $100 \times 100 \mathrm{~m}$ grid cell \\
\hline L25_d_tcg2 & Deposits; Total Contained Gold (TCG) per $100 \times 100$ m grid cell \\
\hline L25dtcg2_bak & Deposits; Total Contained Gold (TCG) per cell (Nodata areas shown in black) \\
\hline L25_d_oreton & Deposits, tonnes $(1000 \mathrm{~kg})$ of ore per $100 \times 100 \mathrm{~m}$ grid cell \\
\hline L25_f1c1_int & $\begin{array}{l}\text { Deposits: Gaussian filter, TCG/cell, central cell = original value, converted to inte- } \\
\text { gers }\end{array}$ \\
\hline L25_f1s1_int & $\begin{array}{l}\text { Deposits: Gaussian filter, TCG/cell, window sum }=\text { original value, converted to } \\
\text { integers }\end{array}$ \\
\hline
\end{tabular}

continued on next page 
Table J.1: continued

\begin{tabular}{|c|c|}
\hline Layer Name & Description \\
\hline L25_f2c1_int & $\begin{array}{l}\text { Deposits: directional Gauss. filter TCG/cell, central cell = original value, converted } \\
\text { to int. }\end{array}$ \\
\hline L25_f2s1_int & $\begin{array}{l}\text { Deposits: directional Gauss filter TCG/cell, window sum = original value, converted } \\
\text { to int. }\end{array}$ \\
\hline L25_tcg_f1c1 & $\begin{array}{l}\text { Deposits: Gaussian filter TCG per cell, central cell = original value, floating point } \\
\text { data }\end{array}$ \\
\hline L25_tcg_f1s1 & $\begin{array}{l}\text { Deposits: Gaussian filter TCG per cell, window sum }=\text { original value, floating point } \\
\text { data }\end{array}$ \\
\hline L25_tcg_f2c1 & Deposits: directional Gauss. filter TCG/cell, central cell value $=$ original value \\
\hline L25_tcg_f2s1 & $\begin{array}{l}\text { Deposits: directional Gauss filter TCG/cell, window sum = original value, floating } \\
\text { pt. data }\end{array}$ \\
\hline L26_anom_dst & Radiometric: distance to $\log 10(\mathrm{~K} / \mathrm{Th})$ anomalies \\
\hline L26_kdivth_lg & Radiometric: $\log 10(\mathrm{~K} / \mathrm{Th})$ \\
\hline L26_kth_anom & Radiometric: derived from L26_kdivth_lg anomalies in range $[1.13-2.21]=1$, rest $=0$ \\
\hline L26_kthanombn & Radiometric: binary anomalies $=1$, rest $=0$ \\
\hline L26_kthlg_dt5 & $\begin{array}{l}\text { Radiometric: distance to L26_kdivth_lg anomalies in range [1.13-2.21], buffer limit }= \\
5 \mathrm{~km}\end{array}$ \\
\hline L26_r_kdivth & Radiometric: $\mathrm{K} / \mathrm{Th}$ \\
\hline L27_anom_dst & Radiometric: distance to $\log 10(\mathrm{~K} /$ total $)$ anomalies \\
\hline L27_kdivto_lg & Radiometric: $\log 10(\mathrm{~K} /$ total $)$ \\
\hline L27_ktoanombn & Radiometric: binary anomalies $=1$, rest $=$ Null \\
\hline L27_ktolg_dt5 & $\begin{array}{l}\text { Radiometric: distance to L27_kdivtot_lg anomalies in range [4.68-5.06], buffer limit } \\
=5 \mathrm{~km}\end{array}$ \\
\hline L27_ktot_anom & $\begin{array}{l}\text { Radiometric: derived from L27_kdivtot_lg anomalies in range }[4.68-5.06]=1 \text {, rest }= \\
0\end{array}$ \\
\hline L27_r_kdivtot & Radiometric: $\mathrm{K} /$ total \\
\hline L28_s_f1str1k & Structure: strike of crustal-scale NW faults, buffer limit $=1 \mathrm{~km}$ \\
\hline L29_s_f2str & Structure: strike of regional-scale NW faults \\
\hline L29_s_f2str1k & Structure: strike of regional-scale NW faults, buffer limit $=1 \mathrm{~km}$ \\
\hline L30_s_f3str & Structure: strike of regional-scale NE faults \\
\hline L30_s_f3str1k & Structure: strike of regional-scale NE faults, buffer limit $=1 \mathrm{~km}$ \\
\hline
\end{tabular}


Table J.1: continued

\begin{tabular}{|c|c|}
\hline Layer Name & Description \\
\hline L31_s_f4str & Structure: strike of regional-scale E-W faults \\
\hline L31_s_f4str1k & Structure: strike of regional-scale E-W faults, buffer limit $=1 \mathrm{~km}$ \\
\hline L32_s_f2f3 & Structure: intersection of regional-scale NW \& NE faults \\
\hline L33_s_f23gdst & $\begin{array}{l}\text { Structure: distance to intersection of regional NW \& NE faults (grid-based calcula- } \\
\text { tion) }\end{array}$ \\
\hline L34_s_f23pdst & $\begin{array}{l}\text { Structure: distance to intersection of regional NW \& NE faults (vector-based calcu- } \\
\text { lation) }\end{array}$ \\
\hline L35_cnt25_200 & Lithology: contact type, 25 rock type legend, buffer limit $=200 \mathrm{~m}$ \\
\hline L35_ct25_1300 & Lithology: contact type, 25 rock type legend, buffer limit $=1300 \mathrm{~m}$ \\
\hline L35_l_rt25 & Lithology: contact type, 25 rock type legend \\
\hline L35_l_rt55 & Lithology: contact type, 55 rock type legend \\
\hline L36_l_rh1 & Rheology: contrast at lith. contact, percentile of subjective rankings \\
\hline L36_l_rh11k & Rheology: contrast at lith. contact, percentile of subjective rankings \\
\hline L36_l_rh2 & Rheology: contrast at lith. contact, subjective ranking \\
\hline L36_l_rh21k & Rheology: contrast at lith. contact, subjective rankings, buffer limit $=1 \mathrm{~km}$ \\
\hline L36_l_rh3 & Rheology: contrast at lith. contact, Young's modulus, Hatheway (1989) \\
\hline L36_l_rh31k & $\begin{array}{l}\text { Rheology: contrast at lith. contact, Young's modulus, Hatheway (1989), buffer limit } \\
=1\end{array}$ \\
\hline L36_l_rh4 & Rheology: contrast at lith. contact, Young's modulus, Lee (2000) \\
\hline L36_l_rh41k & $\begin{array}{l}\text { Rheology: contrast at lith. contact, Young's modulus, Lee (2000), buffer limit }=1 \\
\mathrm{~km}\end{array}$ \\
\hline L36_l_rh5 & Rheology: contrast at lith. contact, uniaxial compressive strength, Lee (2000) \\
\hline L36_l_rh51k & $\begin{array}{l}\text { Rheology: contrast at lith. contact, uniaxial compressive strength, buffer limit }=1 \\
\mathrm{~km}\end{array}$ \\
\hline L36_l_rh6 & Rheology: contrast at lith. contact, uniaxial tensile strength, Lee (2000) \\
\hline L36_l_rh61k & $\begin{array}{l}\text { Rheology: contrast at lith. contact, uniaxial tensile strength, Lee (2000), buffer }=1 \\
\mathrm{~km}\end{array}$ \\
\hline L36_l_rh7 & Rheology: contrast at lith. contact, fracture toughness, Lee (2000) \\
\hline L36_l_rh71k & $\begin{array}{l}\text { Rheology: contrast at lith. contact, fracture toughness, Lee }(2000) \text {, buffer limit }=1 \\
\mathrm{~km}\end{array}$ \\
\hline
\end{tabular}


Table J.1: continued

\begin{tabular}{|c|c|}
\hline Layer Name & Description \\
\hline L37_l_ch1 & $\begin{array}{l}\text { Chemistry: contrast at lith. contact, }\left(\mathrm{Fe}^{2+}+\mathrm{Fe}^{3+}\right) /\left(\mathrm{Fe}^{2+}+\mathrm{Fe}^{3+}+\mathrm{Mg}+\mathrm{Ca}\right) \text {, elemental } \\
\text { wt } \%\end{array}$ \\
\hline L37_l_ch11k & Chemistry: contrast at lith. contact, as above but $1 \mathrm{~km}$ buffer limit \\
\hline L37_l_ch2 & $\begin{array}{l}\text { Chemistry: contrast at lith. contact, } \quad\left(\mathrm{FeO}+\mathrm{Fe}_{2} \mathrm{O}_{3}\right) \text { wt\% oxide } \times \\
\left(\mathrm{Fe}^{2+}+\mathrm{Fe}^{3+}\right) /\left(\mathrm{Fe}^{2+}+\mathrm{Fe}^{3+}+\mathrm{Mg}+\mathrm{Ca}\right)\end{array}$ \\
\hline L37_l_ch21k & Chemistry: contrast at lith. contact, as above but $1 \mathrm{~km}$ buffer limit \\
\hline L37_l_ch3 & Chemistry: contrast at lith. contact, $\mathrm{Fe}_{2} \mathrm{O}_{3} /\left(\mathrm{Fe}_{2} \mathrm{O}_{3}+\mathrm{FeO}\right)$, wt\% oxides \\
\hline L37_l_ch31k & Chemistry: contrast at lith. contact, as above but $1 \mathrm{~km}$ buffer limit \\
\hline L37_l_ch4 & Chemistry: contrast at lith. contact, $\mathrm{Fe}_{2} \mathrm{O}_{3} / \mathrm{FeO}$, wt\% oxides \\
\hline L37_l_ch41k & Chemistry: contrast at lith. contact, as above but $1 \mathrm{~km}$ buffer limit \\
\hline L37_l_ch5 & Chemistry: contrast at lith. contact, $\mathrm{Fe}^{3+} / \mathrm{Fe}^{2+}$, elemental wt $\%$ \\
\hline L37_l_ch51k & Chemistry: contrast at lith. contact, as above but $1 \mathrm{~km}$ buffer limit \\
\hline L38_c_str & : strike of lithological contacts \\
\hline L38_c_str $1 \mathrm{k}$ & Lithology: strike of lithological contacts, buffer limit $=1 \mathrm{~km}$ \\
\hline L39_c_dist & Lithology: distance to lithological contacts \\
\hline L40_strk_d123 & Structure: strike of fault types; 1 (crustal NW), 2 (regional NW), \& 3 (regional NE) \\
\hline L42_f123_comb & Structure: fault types; 1 (crustal NW), 2 (regional NW), \& 3 (regional NE) \\
\hline L42_f1234_cmb & $\begin{array}{l}\text { Structure: fault types; } 1 \text { (crustal NW), } 2 \text { (regional NW), } 3 \text { (regional NE), \& } 4 \text { (re- } \\
\text { gional E-W) }\end{array}$ \\
\hline L42_f123den5k & Structure: total length of faults (crustal NE, regional NW, regional NE) \\
\hline L43_lith_div & Lithology: number of lithological contacts in window $5 \times 5 \mathrm{~km}$ around cell \\
\hline L44favcnt 2501 & $\begin{array}{l}\text { Lithology: favourability of nearest contact type based on } P(\text { Deposit }) \text {, fuzzy } \mathrm{m} / \mathrm{ship} \\
\text { values }\end{array}$ \\
\hline L45_l_meta & Lithology: metamorphic grade \\
\hline L46_dist_anti & Structure: distance to trace of axial plane of anticline \\
\hline L46_dist_sync & Structure: distance to trace of axial plane of syncline \\
\hline L46_folddist & Structure: distance to trace of axial plane of anticlines and synclines \\
\hline L46_foldnear & Structure: $1=$ nearest anticline, $2=$ nearest syncline \\
\hline
\end{tabular}


Table J.1: continued

\begin{tabular}{|c|c|}
\hline Layer Name & Description \\
\hline L47_grntd_dst & Lithology: distance to granitoid excluding distances $=0$ within granitoid itself \\
\hline L48_g_dst2200 & Lithology: distance to granitoid, buffer limit $=2200 \mathrm{~m}$ \\
\hline L49_l_umafic & Lithology: presence of ultramafic rocks (binary) \\
\hline L49_um_dist & Lithology: distance to ultramafic rocks \\
\hline L49_umdst2200 & Lithology: distance to ultramafic rocks, buffer limit $=2200 \mathrm{~m}$ \\
\hline L50_gtd_dist & Lithology: distance to granitoid including distances $=0$ within granitoid itself \\
\hline L50_granitoid & Lithology: presence of granitoids (binary) \\
\hline Mask100 & Study area \\
\hline Num_cell & Cell values $=$ cell number from top left to bottom right, $[1,1254,000]$ \\
\hline gdx17_10_r2 & $\begin{array}{l}\text { Neural network prospectivity map; } 17-10-1 \text { MLP, adaptive lrate \& momentum } \\
(\operatorname{traing} d x)\end{array}$ \\
\hline fuz_0510_98 & $\begin{array}{l}\text { Fuzzy system prospectivity map; } 10 \text {-inputs, input fuzzy } \mathrm{m} / \text { ship range }[0.5,1.0],(\gamma= \\
0.98)\end{array}$ \\
\hline
\end{tabular}

